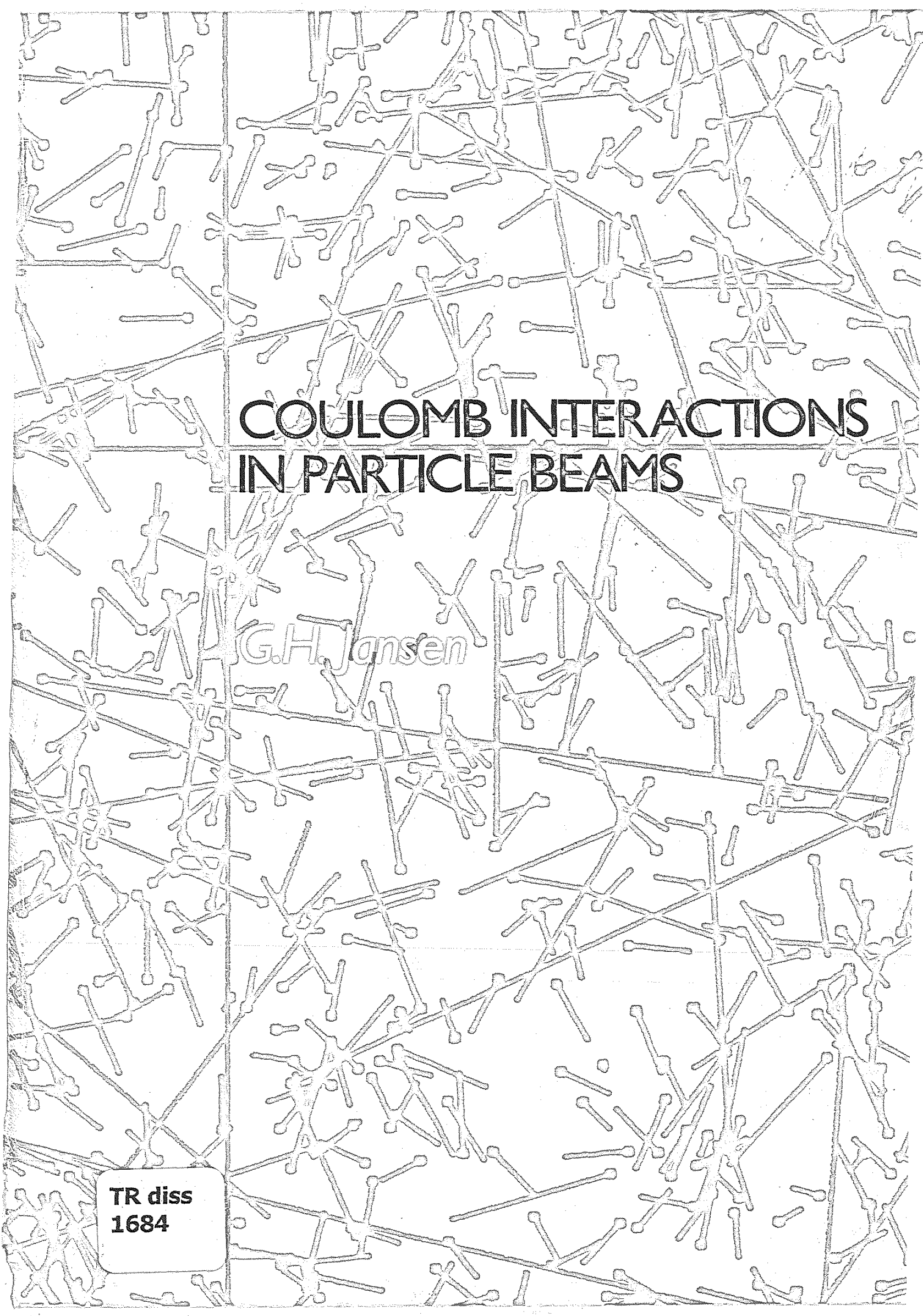




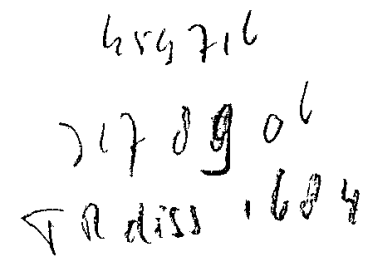

Coulomb Interactions in Particle Beams 


\title{
Coulomb Interactions in Particle Beams
}

\section{Proefschrift}

Ter verkrijging van de graad van doctor aan de

Technische Universiteit Delft, op gezag van de Rector Magnificus, Prof.drs. P.A. Schenck, in het openbaar te verdedigen ten overstaan van een commissie aangewezen door het College van Dekanen op donderdag 17 november 1988 te 16.00 uur

\author{
door \\ Gerrit Hermanus Jansen \\ Natuurkundig ingenieur \\ geboren te Hilversum
}

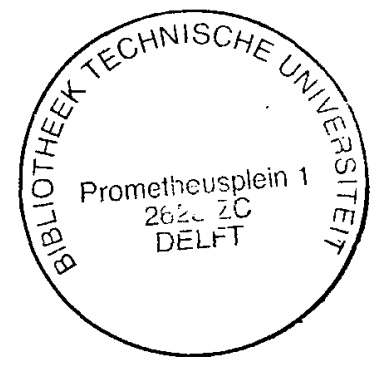


Dit proefschrift is goedgekeurd door de promotoren Prof.dr.ir. K.D. van der Mast

en

Prof.dr. J.M.J. van Leeuwen 
The cover shows on the background the by Monte Carlo simulation computed displacements of the electrons in the target plane of the IBMEL3 variable shaped spot electron beam lithography machine, operating at a $1 \mu \mathrm{A}$ beam current and a beam potential of $25 \mathrm{kV}$. The displacements are produced by the Coulomb interaction between the electrons during their flight through the column.

Cover design : (c) Geurt Naber, 1988 
To my parents 


\section{INTRODUCTION}

1.1 Introduction 7

1.2 Focussed particle beam systems 7

1.3 Classification of interaction phenomena 9

1.4 Organization of the chapters 11

2. HISTORICAL NOTES .

2.1 Introduction

2.2 Particle optics and particle interactions 13

$\begin{array}{lll}2.3 & \text { Boersch effect } & 15\end{array}$

2.4 Trajectory displacement effect 20

2.5 Space charge effect in low density beams 24

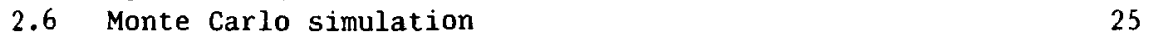

3. GENERAL BEAM PROPERTIES

$\begin{array}{llr}3.1 & \text { Introduction } & 27 \\ 3.2 & \text { Beam parameters } & 28 \\ 3.3 & \text { Classification of beams } & 30 \\ 3.4 & \text { Hamiltonian formalism and Liouville's theorem } & 31 \\ 3.5 & \text { Boltzmann equation } & 33 \\ 3.6 & \text { Conservation of beam emittance and brightness } & 34 \\ 3.7 & \text { Beam temperature } & 38 \\ 3.8 & \text { Thermodynamic limits for particle interaction effects } \\ 3.9 & \text { Debeye Screening } & 45 \\ 3.10 & \text { Potential energy relaxation } & 50\end{array}$

4. THE MANY BODY PROBLEM OP PARTICles INTERACTING THROUGH AN INVERSE SQUARE FORCE LAW

4.1 Introduction 53

4.2 Vlasov equation $\quad 54$

4.3 Fokker-Planck equation 56

4.4 Aspects of the diffusion approximation 59

4.5 Calculation of coefficients of dynamical friction and diffusion 61

$\begin{array}{ll}4.6 & \text { Coulomb logarithm } \\ 4.7 & 66\end{array}$

4.7 Discussion of the Fokker-Planck approach 70

4.8 Validity of the Fokker-Planck approach for particle beams 71

4.9 Holtsmark distribution $\quad 75$

$\begin{array}{ll}4.10 & \text { Conclusions }\end{array}$

5. CONCEPTS OP AN ANALYTICAL MODEL FOR STATISTICAL INTERACTIONS IN PARTICLE BRAMS

$\begin{array}{ll}5.1 & \text { Introduction }\end{array}$

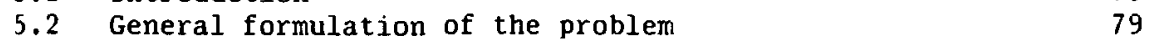

5.3 Reduction of the N-particle problem 82

5.4 Calculation of the displacement distribution 91

5.5 Moments and cumulants of the displacement distribution 94

5.6 On- and of $f$-axis reference trajectories 96

5.7 Models in 1,2 and 3 dimensions 98

5.8 Distribution of the interaction force in cylindrical beams 99

5.9 Representation in the k-domain 104

5.10 Addition of the effects generated in individual beam
segments

$\begin{array}{ll}5.11 \text { Slice method } & 114\end{array}$ 
6. TWO PARTICLE DYNAMICS

$\begin{array}{lll}6.1 & \text { Introduction } & 117\end{array}$

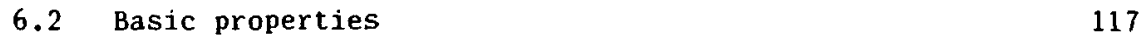

6.3 Coordinate representation in the orbital plane 121

6.4 Dynamics of a complete collision 124

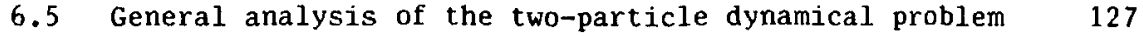

6.6 Numerical approach to the dynamical problem 130

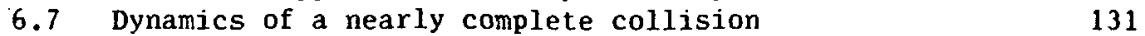

6.8 First order perturbation dynamics 134

6.9 Collisions with zero initial relative velocity 138

$\begin{array}{lll}6.10 & \text { Expressions for the longitudinal velocity shift } \Delta v_{z} & 141\end{array}$

$\begin{array}{lll}6.11 & \text { Expressions for the transverse velocity shift } \Delta v_{\perp} & 142\end{array}$

6.12 Expressions for the spatial shift $\Delta r \quad 143$

6.13 Coulomb-scaling

Appendix 6.A Mathematics of nearly complete collision dynamics 146

7. BOERSCH EFFECT

$\begin{array}{lll}7.1 & \text { Introduction } & 153\end{array}$

$\begin{array}{lll}7.2 & \text { General aspects } & 154\end{array}$

$\begin{array}{lll}7.3 & \text { Beam segment with a narrow crossover } & 155\end{array}$

7.4 Homocentric cylindrical beam segment 166

$\begin{array}{lll}7.5 & \text { Beam segment with a crossover of arbitrary dimensions } & 174\end{array}$

$\begin{array}{lll}7.6 & \text { Results for Gaussian angular and spatial distributions } 187\end{array}$

$\begin{array}{lll}7.7 & \text { Thermodynamic limits } & 191\end{array}$

8. STATISTICAL ANGULAR DEFLECTIONS

8.1 Introduction 193

$\begin{array}{ll}8.2 \text { General aspects } & 194\end{array}$

$\begin{array}{ll}\text { 8.3 Beam segment with a narrow crossover } & 195\end{array}$

$\begin{array}{ll}8.4 & \text { Homocentric cylindrical beam segment } 201\end{array}$

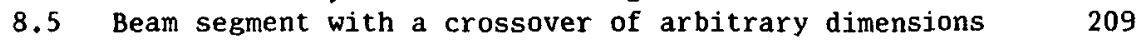

8.6 Application of the slice method 227

$\begin{array}{ll}8.7 & \text { Results for Gaussian angular and spatial distributions } 228\end{array}$

9. TRAJECTORY DISPIACEMENT EFPECT

9.1 Introduction $\quad 233$

$\begin{array}{lll}9.2 & \text { General aspects } & 234\end{array}$

9.3 Homocentric beam segment with a crossover $\quad 237$

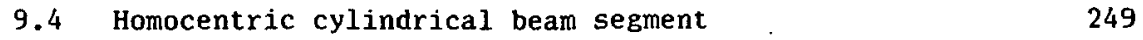

9.5 Beam segment with a crossover of arbitrary dimensions 251

$\begin{array}{ll}9.6 & \text { Trajectory displacement and angular deflection . } \\ \text { distribution }\end{array}$

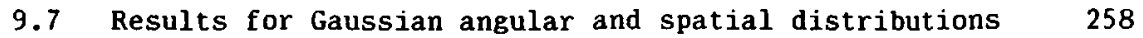

10. FURTHER INVESTIGATIONS ON STATISTICAL INTERACTIONS

$\begin{array}{lll}10.1 & \text { Introduction } & 259\end{array}$

10.2 Exact approach for off-axis reference trajectories 260

10.3 Approximating approach for of $f$-axis reference trajectories 265

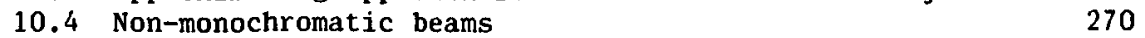

10.5 Beams in an external uniform axial electrostatic field 276

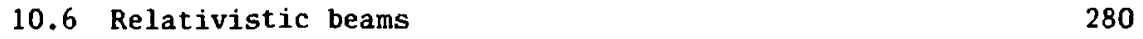

Appendix 10.A Distributions for the average reference trajectory 282 
11. SPACE CHARGR EFPECT IN LON DENSITY PARTICLE BEAMS

11.1 Introduction 285

$\begin{array}{ll}11.2 \text { General aspects } & 285\end{array}$

11.3 Beams with laminar flow 292

$\begin{array}{lll}11.4 & \text { First order perturbation theory } & 294\end{array}$

11.5 First order optical properties of the space charge lens 296

11.6 Third order geometrical aberrations of the space charge
lens

$\begin{array}{lll}11.7 & \text { Beam segment with a narrow crossover } & 303\end{array}$

11.8 Homocentric cylindrical beam segment 304

11.9 Addition of the effects generated in individual beam
segments

12. CALCULATION OF DIPFERENT SPOT- AND EDGE-WIDTH MRASURES

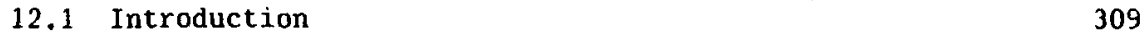

12.2 Spot-width obtained by knife-edge scans 309

12.3 Edge-width of a shaped spot 313

12.4 Trajectory displacement effect 316

$\begin{array}{ll}12.5 \text { Chromatic aberration } & 321\end{array}$

12.6 Spherical aberration $\quad 334$

$\begin{array}{lll}12.7 & \text { Space charge effect } & 336\end{array}$

12.8 Results for a truncated Gaussian angular distribution 338

13. monte carlo simulation OF PaRTicle beays

$\begin{array}{ll}13.1 \text { Introduction } & 343\end{array}$

$\begin{array}{lll}13.2 & \text { Source routine } & 344\end{array}$

13.3 Optical elements 346

13.4 Numerical ray-tracing $\quad 349$

13.5 Analytical ray-tracing 353

13.6 Simulation of large currents near the source 358

13.7 Correction of finite-size effects 359

$\begin{array}{ll}13.8 \text { Data analysis } & 362\end{array}$

13.9 Accuracy limitations of the MC-program 365

13.10 MC-simulation versus analytical modelling 367

$\begin{array}{ll}13.11 \text { Program organization and examples } & 368\end{array}$

Appendix 13.A Random number routine 373

Appendix 13.B Polynomial fit algorithm 376

14. COMPARISON OF ANALYTICAL RESULTS WITH MONTE CARLO SIMULATIONS

$\begin{array}{lll}14.1 & \text { Introduction } & 379\end{array}$

$\begin{array}{ll}14.2 \text { General aspects } & 379\end{array}$

14.3 Voltage and current dependencies for a fixed geometry 381

14.4 Geometry and current dependencies for a fixed beam voltage 388

14.5 Discussion of the results 389

15. COMPARISON OF RECENT THEORIES ON STATISTICAL INTERACTIONS

15.1 Introduction 393

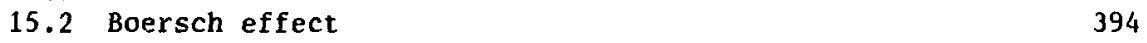

15.3 Statistical angular deflections 404

$\begin{array}{lll}15.4 & \text { Trajectory displacement effect } & 410\end{array}$

$\begin{array}{ll}15.5 \text { Conclusions } & 415\end{array}$ 
16. SUMAARY FOR THE ONE-MINUTE DESIGNER

16.1 Introduction

16.2 Physical aspects

419

16.3 Parameter dependencies

428

16.4 Equations for the Boersch effect

435

16.5 Equations for the trajectory displacement effect

440

16.6 Equations for the space charge effect

443

16.7 Addition of the effects generated in individual beam segments 


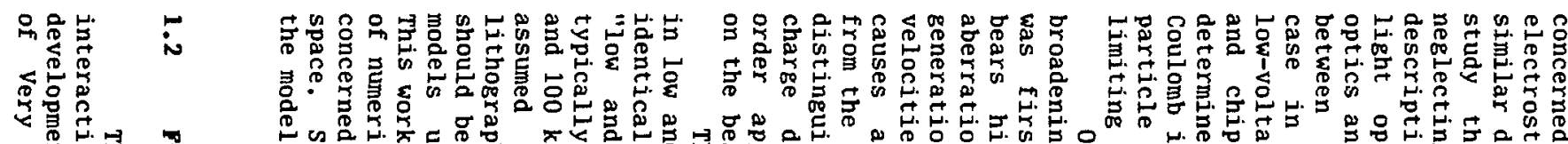

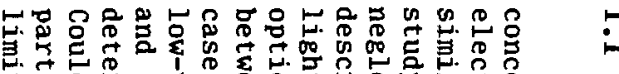

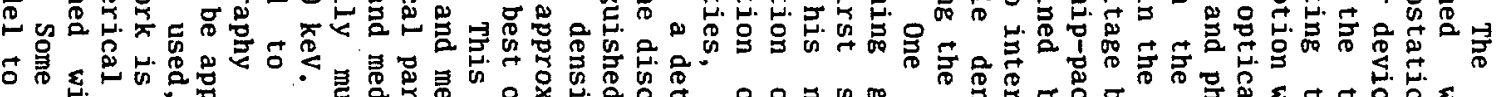

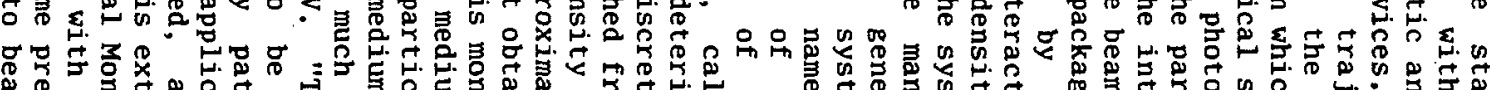

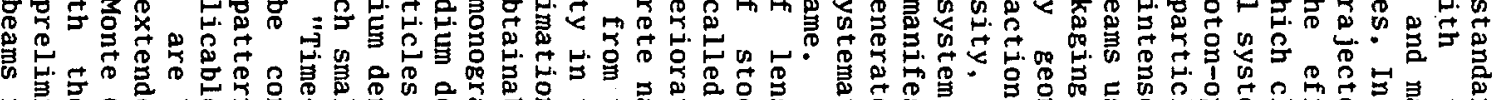

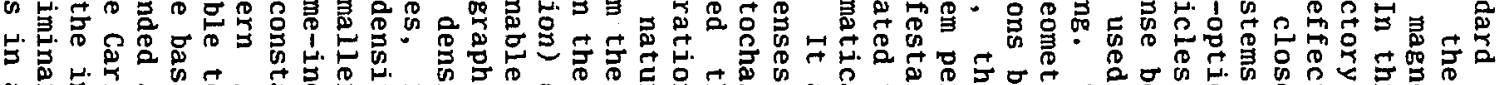

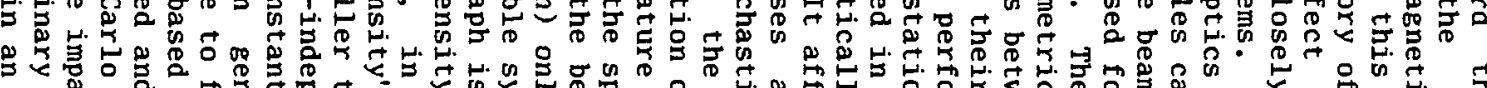

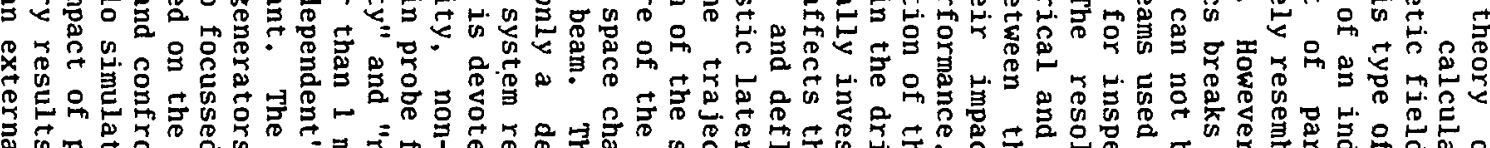

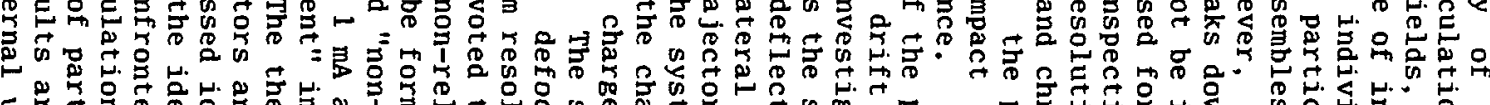

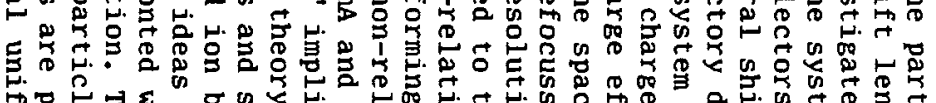

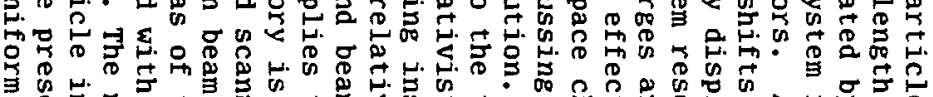

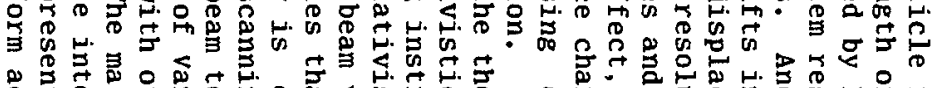

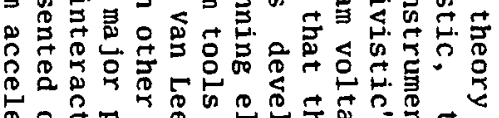
等

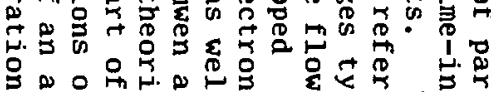

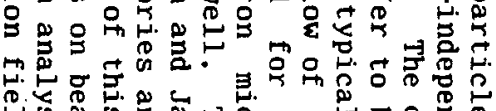

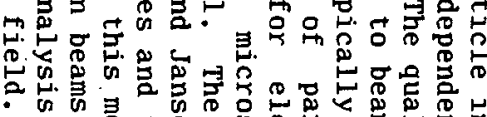
$\rightarrow \overrightarrow{0}$

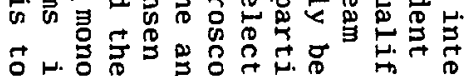

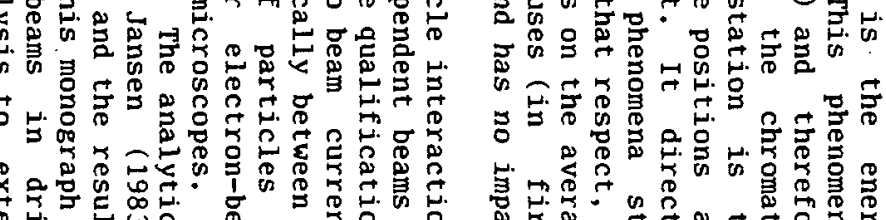

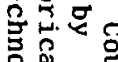

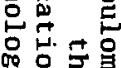

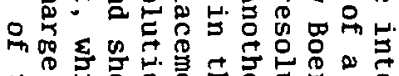

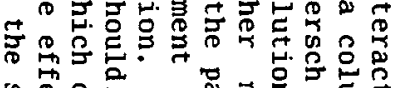

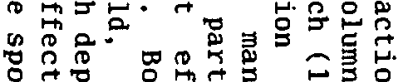

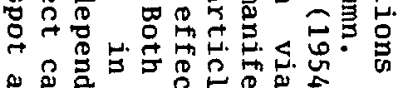

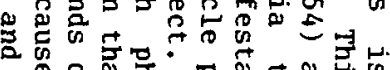

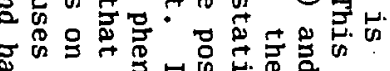

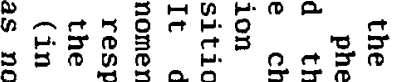

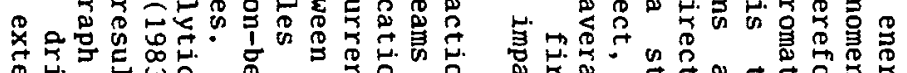

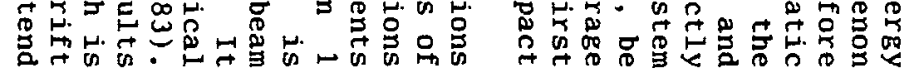

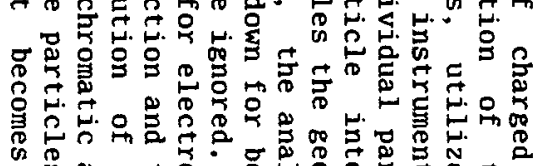
की

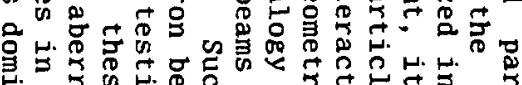

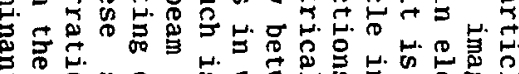

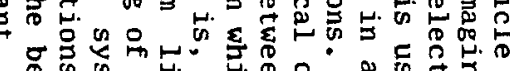

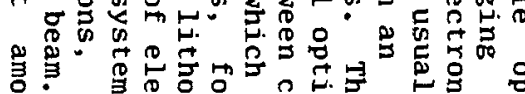

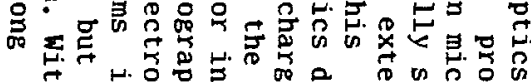

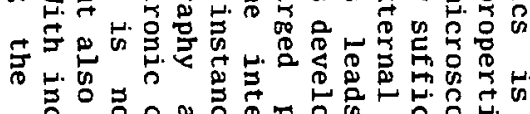

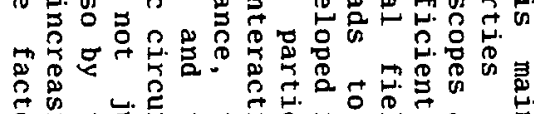

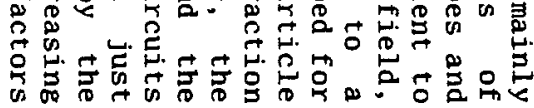


which is able to produce patterns with a minimum feature size below one micrometer. With the trend towards further reduction of the desired feature size, the usefulness of the conventional optical lithographic process becomes restricted by fundamental limits related to the wavelength of light. In order to overcome these limits, the technology of electron beam lithography was developed during the late sixties and seventies. It has been demonstrated that electron beam tools can produce patterns of features as small as tenths of nanometers. It became clear, however, that this technology suffers from some fundamental limits of its own. The main drawback of electron beam lithography is its limited machine throughput, due to the sequential nature of the exposure process. Therefore, at present, electron beams are primarily used for the production of masks, whereas direct slice writing is limited to special applications. The main objective of system designers is to decrease the feature size, while maintaining or increasing the throughput. As the exposure time is inversely proportional to the current in the probe, machine throughput can be improved by increasing the beam current. However, the deteriorating impact of both the Boersch effect and the trajectory displacement effect becomes more severe for larger currents, imposing an upper limit to the beam current at a certain resolution. The system resolution is not affected by the space charge effect, provided that its defocussing action can be compensated by proper lens adjustment. In this respect, it should be noted that difficulties arise in variable-shaped spot lithography systems, in which the beam current (and thus the space charge defocussing) changes on a spot-by-spot basis. An adequate theoretical description of the different phenomena involved, is indispensable in the optimization of the design of high throughput electron beam lithography machines.

Scanning electron microscopes generally operate at smaller beam currents than electron beam lithography machines. Consequently, the effects of particle interactions are less dominant. However, they can not be ignored in systems with high-brightness guns, operating at low beam voltages. In a scanning system, the brightness of the beam determines the obtainable signal to noise ratio (at a certain integration time per pixel). High brightness is thus desirable. Operation at a low beam voltage is necessary, in some applications, to prevent the specimen from electrical charging. Therefore, the systems employed for testing and inspection of electronic circuits and chips packaging, as well as the analytical systems for the research of isolators, typically operate around $1 \mathrm{kV}$. In the design of those systems, it should be anticipated that particle interactions may impact the performance of the beam considerably.

Focussed ion beam systems have gained interest during the last decade and are now widely studied in research laboratories. Their main application is mask-repair, while focussed ion beam systems for lithography and direct maskless implantation are under development. Presently, the highest brightness is produced by liquid metal ion sources. A severe disadvantage of the liquid metal ion source is its large energy spread. Focussed ion beam systems employing this type of gun are therefore usually limited by chromatic aberration. The effect of virtual source growth is observed as well, but does, in general, not linit the system performance. It is not clear, whether these phenomena should be entirely attributed to the Coulomb interactions between the particles in the beam. The observed current dependency indicates that they are at least partly responsible. 
In order to classify the different manifestations of the Coulomb interactions it is necessary to introduce some theoretical concepts first. Consider a set of test particles running, successively, along a specific trajectory in the beam, called the reference trajectory. Assume that they all have the same velocity and are well separated in space. Each of the test particles experiences the Coulomb repulsion of the particles by which it is surrounded, referred to as field particles. As a result, it will experience a displacement from its unperturbed path (which is the reference trajectory), both in position and velocity. This displacement is entirely determined by the initial coordinates of all field particles relative to the test particle (With "initial coordinates" we denote both the positions and the velocities at the start of the interaction). Clearly, different configurations of initial coordinates lead to different displacements. Accordingly, the displacements of the test particles, successively arriving at the target, will follow a certain distribution, which is related to the statistics of the beam.

The three-dimensional distributions of displacements in position and velocity, experienced by the test particles, represent the effect of Coulomb interactions. In general, a distribution can be characterized by its mean value and a measure for its width, e.g. the root mean square (rms) of the displacements from the mean value. The mean values of the distributions investigated here, correspond to the average shift in position and velocity of all test particles. These average shifts can be related to the smoothed-out distribution of charge in the beam and are therefore classified as manifestations of the space charge effect. The space charge effect is strictly deterministic. In other words, it does not depend on the statistics of the beam. On the other hand, the widths of the distribution of displacements of the test particles are directly related to the stochastic fluctuations in the charge density. These fluctuations occur as a consequence of the discrete (particle) nature of the beam. The distribution of displacements in position and velocity, relative to the average shift of the test particles, represent the so-called statistical effects. Summarizing: Space charge (or deterministic) effects are related to the smoothed-out charge density in the beam. Statistical effects are related to the stochastic fluctuations in the charge density, due to the discrete nature of the beam.

The magnitude of the different manifestations of coulomb interactions depends, to some extent, on the choice of the reference trajectory. The trajectory along the beam axis of a rotational symmetric beam represents a case which is of special interest. The strength of the electrostatic force, generated by the smoothed-out distribution of charge, is zero on this trajectory, due to the symmetry of the beam. The space charge effect is therefore absent and the resulting displacements are purely statistical. Particles moving along other trajectories in the beam, will be subject to the combined action of space charge and statistical effects.

Space charge effect and statistical effects are sometimes presented as the result of collective interaction and collisions between individual particles respectively. This interpretation is instructive for beams of very high density, in which every test particle is surrounded by a large number of field particles. The total force acting on an individual test particle is indeed produced by the simultaneous action of many field particles. Accordingly, it appears to be reasonable to distinguish between the influence of the system as a whole and the influence of the local neighbourhood. The former is 


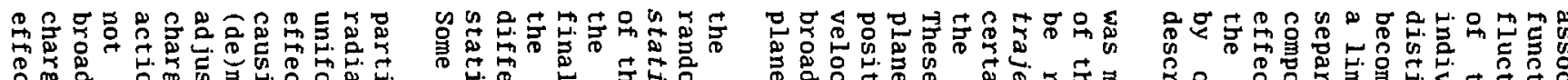

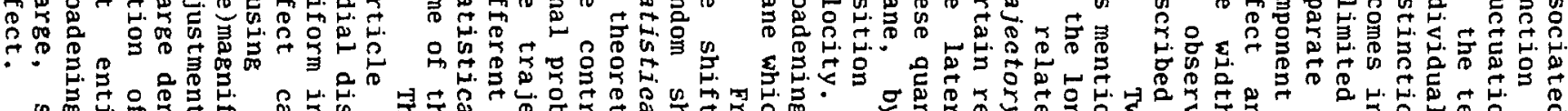

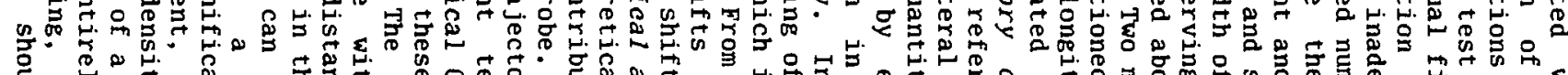

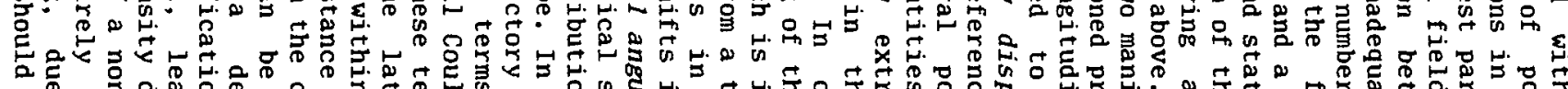

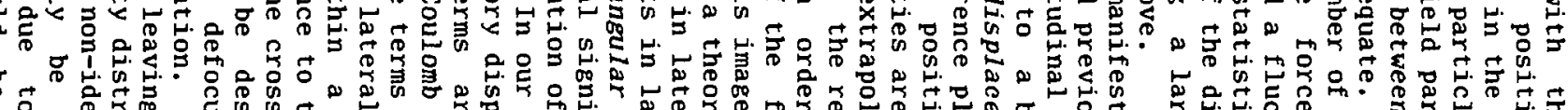

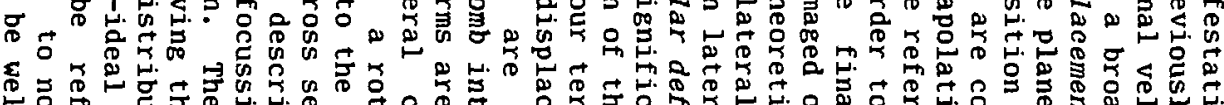

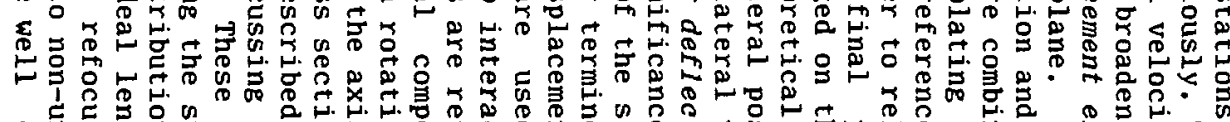

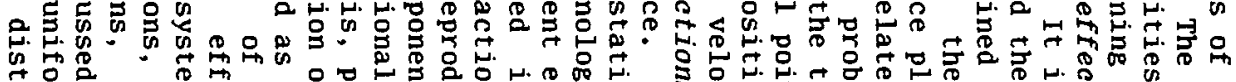

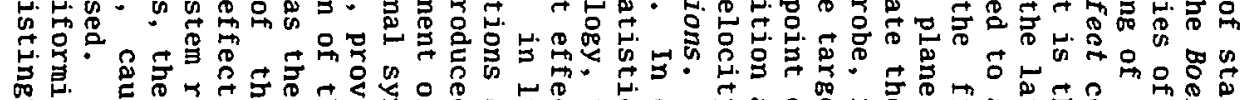

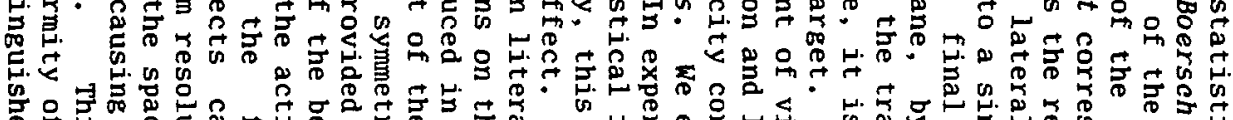

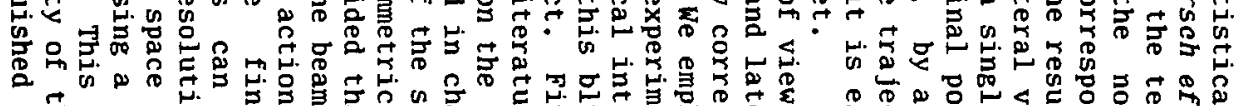

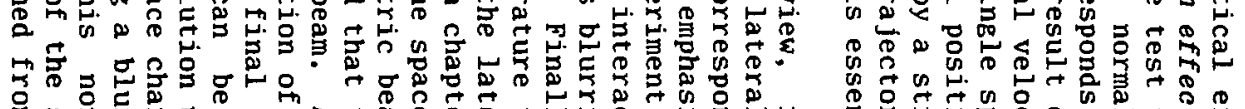

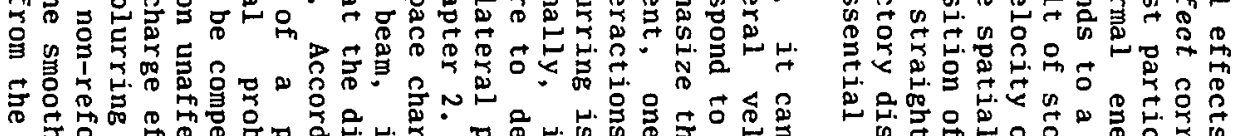

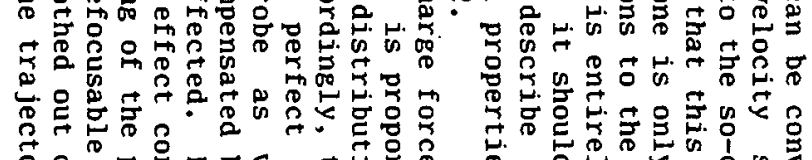

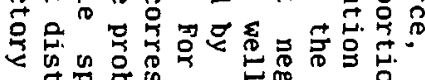

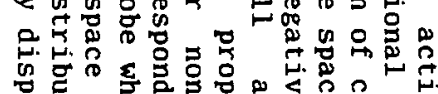

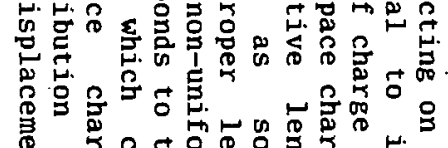

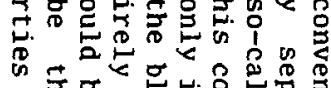

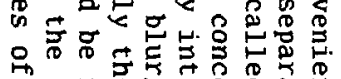

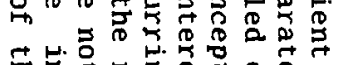

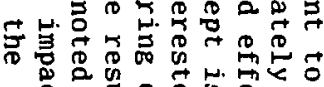

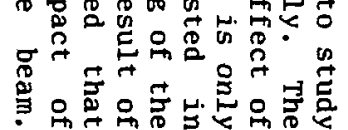

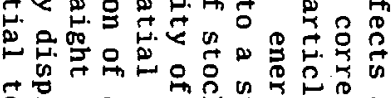

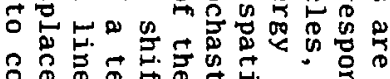

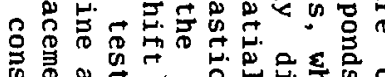

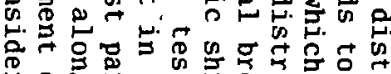

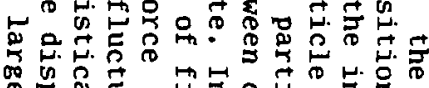

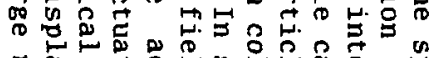

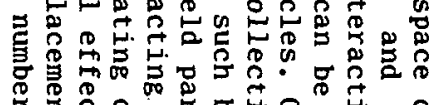
象若

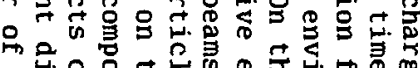

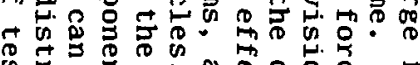

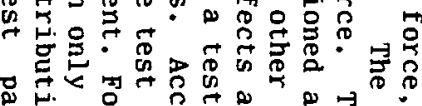

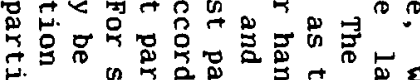

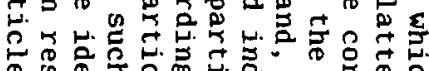

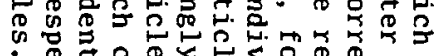

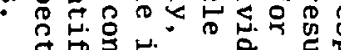

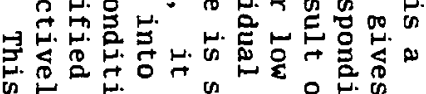

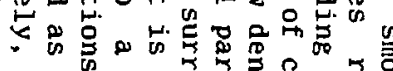

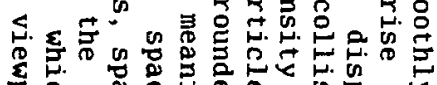
象 式官

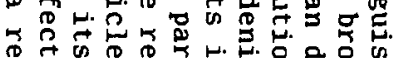

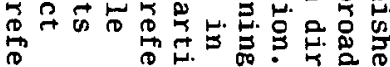

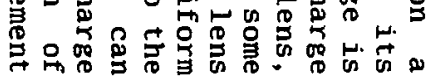

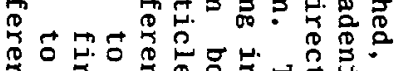

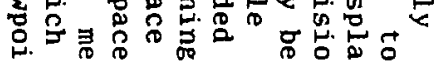

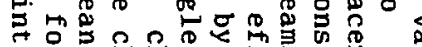

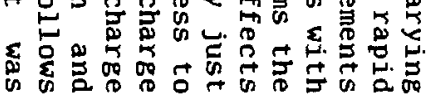


The chapters are organized such that one can, in principle, read each chapter separate from the others. Explicit references are therefore made when using the results presented elsewhere in this monograph. Some of the chapters start with a summary of the relevant results presented up to that point. Clearly, this causes some overlap in the text, but hopefully it improves the readability of this work, especially for those who do not wish to go through all the material.

Chapter 2 reviews the various contributions to the subject of Coulomb interactions in particle beams and gives reference to some related fields of physics as well. The history of the Boersch effect, the trajectory displacement effect, the space charge effect and the technique of numerical Monte carlo simulation are successively described, stressing the physical aspects involved. Chapter 3 introduces some general beam properties which are used throughout this monograph. Liouville's theorem, well known from statistical mechanics, is employed to derive the principle of emittance and brightness invariance. A thermodynamical description of the beam is considered, concentrating on the notion of beam temperature. The thermodynamical description of the beam is exploited to determine upper limits for the effect of statistical interactions, utilizing the concepts of relaxation of kinetic energy and relaxation of potential energy. Chapter 4 studies the $\mathrm{N}$-body problem of particles interacting through a Coulomb-type of interaction force, from a general perspective. It reviews the FokkerPlanck approach, which is used in plasma physics and stellar dynamics. The applicability of this approach to the problem of coulomb interactions in particle beams is examined. The Holtsmark distribution is introduced to describe the effect of statistical interactions in a cylindrical beam of moderate particle density. The chapters 2,3 and 4 , which approach the subject of Coulomb interactions from a general viewpoint, are intended to clarify the background of the analytical models presented in the remaining chapters and to elucidate the fundamental aspects involved.

Chapter 5 discusses the basic aspects of an analytical model for the statistical Coulomb interactions between the particles of a beam. Various methods of reducing this type of $\mathrm{N}$-body problem are examined. chosen is for a model based on two-particle interactions, which is accurate as long as the test particle is not involved in two or more strong interactions, simultaneously or successively, in the same beam segment. The statistical aspects of this so-called extended twoparticle model are described in detail. The Holtsmark distribution is derived within the model, as well as some related distributions, pertaining to a beam with small lateral dimensions relative to the average axial separation of the particles. Chapter 6 studies the dynamical problem of two particles, interacting through the coulomb force. The classic solution of the Kepler problem pertains to a collision which is complete, which means that the particles effectively come from infinity and recede to infinity. However, the extended twoparticle model requires knowledge of collisions which are possibly incomplete. It is shown that the problem can be solved analytically for nearly complete collisions as well as for weak collisions. An numerical method to treat strong incomplete collisions is also presented. Chapter 7 utilizes the material of the chapters 5 and 6 to calculate the Boersch effect in a beam segment in drift space. Chapter 8 is concerned with the ef fect of statistical angular deflections, while chapter 9 discusses the trajectory displacement effect. Chapter 10 is dedicated to some further investigations on statistical interactions to cover of $f$-axis reference trajectories, initially non-monochromatic beams, as well as uniform 
acceleration fields. The case of relativistic beams, in which the particles travel with a velocity comparable to the speed of light, is also investigated. An analytical model for the space charge effect in low density particle beams is described in chapter 11. The first and third order geometrical properties of the associated "space charge lens" are calculated. Chapter 12 translates the results from the previous chapters in terms of some experimental measures for the spot-width in source imaging (Gaussian beam) systems and the edge-width in aperture imaging (shaped beam) systems. The chapters 5 to 12 cover the main results of the analytical investigations.

Chapter 13 describes the principles of numerical Monte Carlo simulation of charged particle beams. An analytical ray tracing method is described, which provides an alternative to the usual third order numerical ray-tracing procedure. It is based on a decomposition of the full interaction into a sum of two-particle effects, quite similar to our analytical model of statistical interactions. While preserving the typical advantages of the Monte Carlo approach, it improves the speed of the program with one to two orders of magnitude, without loss of accuracy, for practical operating conditions. This approach is referred to as fast Monte Carlo simulation of charged particle beams. Several other features to improve the performance of the MC-program and to extend its applicability to high density beams are discussed.

Chapter 14 compares the analytically obtained expressions for the Boersch effect, the trajectory displacement effect and the space charge effect with the results of Monte Carlo simulations (using numerical ray-tracing). This material serves to verify the accuracy of the analytical models.

Chapter 15 confronts the extended two-particle approach with some other recent theories on statistical interactions. It focusses on a quantitative comparison of the different theories. It should be recalled that a qualitative discussion of the different approaches is incorporated in the chapters 2,4 and 5 .

Chapter 16 summarizes the results presented in this monograph which are relevant for the design of probe forming instruments. A review is given of the physical aspects of Coulomb interactions in particle beams and the basic theoretical parameters involved. The dependency of the various effects on the experimental parameters is explained from elementary physical considerations. Finally, an overview is given of the resulting equations for the calculation of the Boersch effect, the trajectory displacement effect and the space charge effect. 


\section{HISTORICAL NOTES}

\section{$2.1 \quad$ Introduction}

This chapter is concerned with the history of the subject of particle interactions in particle beams. Previous reviews, presented by Zimmermann (1970) and Rose and Spehr (1983), were rather short on the early theoretical work as well as the relation with activities in adjacent fields. Therefore, a somewhat more detailed reference is presented here. The subjects of the Boersch effect, the trajectory displacement effect, the space charge effect and the technique of numerical Monte Carlo simulation are treated separately. The various contributions, to each of these subjects, are arranged chronologically. The descriptions are only qualitative, emphasizing the physical aspects involved. A systematic discussion of the different approximations underlying the various analytical models, is included in chapter 5 (statistical interactions) and chapter 11 (space charge effect), while the reader is referred to chapter 15 for a quantitative comparison of the results of the different models for statistical interactions.

\subsection{Particle optics and particle interactions}

The branch of physics studying the properties of focussed electron or ion beams is called particle optics. It originates from the mid-twenties, when the focussing action of a magnetic field on an electron beam was first studied, Busch (1926). Particle optics, as it has developed ever since, is mainly concerned with the calculation of the trajectories of charged particles in an external electrostatic or magnetic field. The particles are essentially treated as independent, neglecting the effect of particle interactions. In this respect, it is completely analogous to the geometrical optics used for light optical systems. The theory is centred around the concept of a paraxial beam. In such a beam the particles flow near some axis on a trajectory which makes only a small angle with that axis. The ray equation, which follows from Maxwell's field equations using classical mechanics, is a second order differential equation, specifying the lateral ray coordinates as function of the axial coordinate. For a paraxial beam, it can be approximated by a power expansion in the angle and the distance to the axis. Truncation of the expansion after the first order terms (in this angle and distance) constitutes the so-called paraxial ray equation, which gives rise to Gaussian optics. It serves to relate the position of an object plane to the position of an image plane and to determine the magnification from the first to the second. The geometrical aberrations follow by taking the higher order terms of the ray equation into account, employing perturbation theory. In this approach it is sufficient to study the total deviation experienced by particles running along a few principal (first order) rays, which correspond to the independent solutions of the paraxial ray equation (One requires two principal rays for a rotational symmetric beam and four principal rays to treat the general case).

The performance of a probe forming system, as a whole, follows from the optical properties of its components, usually specified in terms of the first order imaging quantities and the aberration constants. The quality of the beam in the image plane can be derived from the total geometrical and chromatic aberrations, found by addition 
of the contribution of the individual components. This approach is extensively described in the various textbooks on electron optics, for instance see Glaser (1952), Klemperer (1953), Zvorykin (1961), Grivet (1965), Septier (1967), Szilagyi (1988) or for high energy beams Steffen (1965) and Lawson (1977).

The analogy with light optics breaks down for high intensity particle beams, in which the interaction between the particles can not be neglected. The significance of collective interaction, referred to as the space charge effect, was already recognized in the early stages of electron optics. It was understood that the space charge effect can dominate the emission properties in thermionic guns and may limit the minimum obtainable spot size in oscilloscope tubes. In the last device, defocussing and beam spreading, due to the space charge effect, were calculated by assuming laminar flow. This denotes that the beam is visualized as a fluid in which particle trajectories do not cross each other. In case of laminar flow, it is sufficient to consider the trajectory of a particle running along the edge of the beam. The space charge force acting on this particle can directly be expressed in terms of the macroscopic properties of the beam and the radial equation of motion can be solved by straightforward integration. The physics of space charge flow is discussed by Pierce (1954), Nagy and Szilagyi (1974) and Kirstein, Kino and Walters (1967). A comprehensive discussion of beam spreading and defocussing is given by Schwartz (1957) and van den Broek (1984). An introduction to the problem can as well be found in the standard textbooks on electron optics, for instance see Glaser (1952), Klemperer (1953), Hutter (1967), El-Kareh and El-Kareh (1970) or Szilagyi (1988). Despite the considerable attention given to space charge dominated beams during the past sixty years, the design of electron guns is still an important subject in the industrial research of picture tubes, van den Broek (1986b). However, the fundamental issues involved did not change since the first notice of these effects.

The importance of the interaction between the individual beam particles was not appreciated until the early fifties. Hines. (1951) and Mott-Smith (1952) analysed the effect of encounters between individual electrons on the velocity distribution in a traveling wave tube. The issue was initiated by Parzen and Goldstein (1951), who showed that the characteristics of such a device are related to the velocity distribution of the electrons.

At that time, the distinction between collective and individual Coulomb interactions was well established in the field of plasma physics, due to the work of Langmuir, Cowling, Landau, Chandrasekhar, Spitzer, Pines, Bohm and others. However, application of their results to particle beams is not straightforward, due to the distinct differences between the physics of neutralized plasma's near equilibrium and non-neutralized beams, having a short flight time, relative to the relaxation time of the interaction phenomena involved. The correspondence with the field of plasma physics is further discussed in chapter 4. An introduction into the problem of Coulomb interactions in plasmas is given by Bohm and Pines (1951), Pines and Bohm (1952), Ash and Gabor (1954), Trubnikov (1965), Sivukhin (1966) and Chapman and Cowling (1970). A general introduction to the principles of plasma physics is given by Spitzer (1962) and Ichimaru (1973). 
The subject of particle interactions in particle beams is strongly influenced by Boersch (1954). He carried out a systematic experimental investigation to the energy distribution of electrons in a high intensity focussed beam, produced by thermionic emission. Boersch measured the energy distribution as function of the beam current, for a fixed beam potential of $30 \mathrm{kV}$. The (half) Maxwellian energy distribution, expected on theoretical grounds, was only measured for very low current densities. With increasing current, a transition was observed to a broadened more symmetric distribution. This anomalous energy broadening is nowadays called the Boersch effect. Boersch concluded that the relevant beam parameter is the current density at the most dense point in the beam. He did not attribute the effect to the interaction between the individual electrons, but assumed that it was caused by collective longitudinal space charge oscillations, generated by the noise fed into the beam from the gun. Fack (1955) developed a model along these lines, but did not publish any quantitative results. The results obtained by Boersch were verified by Dietrich (1958), Miller and Dow (1961), Hartwig and Ulmer (1963a,b), Ulmer and Zimmermann (1964) and Simpson and Kuyatt (1966). These investigations are summarized by Simpson and Kuyatt (1966). All authors agreed that the effect exists and increases with the maximum current density in the beam. Ichinokawa $(1968,1969)$, however, suggested that the Boersch effect is not a genuine phenomenon, but an artifact of the retarding field analyser, related to its response to beam divergence. Ditchfield and Whelan (1975) repeated his experiment and concluded that his statement is incorrect, although the analyser response can lead to an apparent energy broadening in extreme cases. See also the discussion with the reviewers in the paper by Pfeiffer (1972). Many other investigations to the energy distribution in electron beams from thermionic guns followed, confirming the Boersch effect: Andersen and Mol (1968), Gaukler (1968), Andersen (1969), Hanszen and Lauer (1969), Pfeiffer (1971), Degenhardt and Koops (1982), Troyon (1987). The effect of energy broadening is also extensively observed in beams from field emission guns: Gadzuk and Plummer (1973), Swanson (1975), Gaukler, Speidel and Vorster (1975), Swanson (1975), Heinrich, Essig and Geiger (1977), Troyon (1976), Bell and Swanson (1979), Speidel, Kurz and Gaukler (1979), Groves (1981b), Speidel, Brauchle, Kramer and Schwab (1985), Takaoka and Ura (1986), Zinzindohoue (1986), Troyon and Zinzindohoue (1986), Troyon (1988). Similar experiments were carried out for different types of liquid metal ion sources: Krohn and Ringo (1975), Swanson, Schwind and Bell and Brady (1979), Swanson and Schwind and Bell (1980), Mayer (1985), Mair and Mulvey (1985), Umemura et al. (1986) and Ishitani et al. (1987b,c,d). It is not clear, whether the large energy spreads observed in these devices is entirely due to the Boersch effect.

In contrast to the experimental activity, the effort to explain the Boersch effect theoretically was rather limited during the decade following its discovery. It is interesting to note that no link was established to the work of Hines and Mott-Smith, mentioned previously, despite its close relation. Veith (1955) presented a qualitative analysis in case of space charge limited thermionic emission, based on the assumption that the Boersch effect is related to the dynamic behaviour of the space charge barier in front of the cathode, causing an amplification of shot noise. Lenz (1958) showed that a calculation, based on the transition of momentum through collisions between free electrons and weakly bounded electrons in a crossover region (thus assuming the presence of neutralizing ions), leads to the right order of magnitude in comparison to the observed energy broadening. Lenz 
considered the presence of weakly bounded electrons to be essential, based on the erroneous idea that collisions between free identical particles do not alter the energy distribution. He argued that colliding free identical particles exchange energy and momentum, leaving the macroscopic properties of the beam unchanged. However, this is only true for central collisions, which are very rare in practical beams. Schiske (1962) advocated an approach starting from the Holtsmark distribution. This is the probability distribution of the fluctuating component of the electrostatic force acting on a point charge surrounded by a random distributed cloud of identical point charges. It was first derived by Holtsmark (1919) in connection with his work on the Stark broadening of spectral lines. Schiske's discussions are based on a method described by Chandrasekhar (1943) to calculate the statistics of the gravitational field in stellar dynamics. Miller and Dow (1961) investigated the energy exchange between electrons in a (magnetically confined) crossed-field stream analyser and suggested that the Boersch effect, in such a device, is associated with multi-loop trajectories in the cathode region. It should be noted that the significance of the impact of the local interactions between the individual particles on the velocity distribution in the beam was, at that time, not generally recognized, for instance see Lindsay (1960).

Considerable progress to the understanding of the Boersch effect was made by Zimmermann $(1968,1969,1970)$. Proceeding on the ideas of Hartwig and Ulmer (1963a,b) and Ulmer and Zimmermann (1964), he developed the concept of relaxation of internal energy. The internal energy of a particle is defined as its kinetic energy in the frame of reference moving with the beam. The essential observation is that the axial internal energy spread of the particles is significantly reduced during acceleration. This is a consequence of the quadratic relation between kinetic energy and momentum. Notice that the energy spread in the laboratory system remains equal during acceleration, since every particle gains the same amount of energy. While the axial internal energy spread is reduced, the lateral internal energy spread is not affected and a non-equilibrium situation is generated. Due to the Coulomb interaction between the particles, relaxation will occur towards a more isotropic distribution of internal energy. Consequently, the longitudinal energy spread (in the laboratory system) increases at a rate which can be estimated from the average gain in axial momentum spread of a single particle, due to the interaction with the other particles in the beam. Zimmermann found that the root mean square ( $r m s$ ) energy spread increases with the square root of the current density.

The process of relaxation of internal energy in accelerated beams was already qualitatively described by Hines (1951), but his paper was apparently not known to Zimmermann, Hartwig or Ulmer. The result derived by Zimmermann for a cylindrical beam is identical to that of Mott-Smith (1952), which is, however, obtained by different reasoning. Zimmermann's approach is similar to the one used in plasma physics to describe coulomb interactions between the individual particles, see Trubnikov (1965) and Sivukhin (1966). In order to prevent the results from diverging, it is necessary to assume a maximum interaction radius, usually expressed in terms of an upper limit for the impact parameter. This is the closest distance of approach between two particles in the imaginary case that they do not interact. The choice of this upper limit seems somewhat arbitrary. However, the result is not sensitive to its exact value, since it depends only logarithmically on it.

The method employed by Zimmermann is less suited for beams which are not cylindrical, thus diverging beams, converging beams or beams containing a crossover. In such beams, the current density and the beam temperature associated with the transverse motion of the particles 
varies with the axial position in the beam (see section 3.7). This can be taken into account by averaging the result, obtained for a cylindrical slice, over the axial coordinate, but the physical ground for such a procedure seems questionable.

Loeffler (1969) calculated the Boersch effect in a beam segment in drift space with a narrow crossover, by considering the effect of weak binary encounters on a reference electron moving along the beam axis. He used first order perturbation theory to calculate the dynamics of two interacting particles, whereas Zimmermann (following the concepts of plasma physics) exploited the well-known solution of the full Keplerproblem. It should be noted that these approaches lead to identical expressions in the limit of weak complete collisions (see chapter 6). Loeffler considered the encounters between particles (in the geometry considered) to be a result of the crossover motion, which should be distinguished from the thermal motion. Consequently, he questioned the validity of the relaxation concept, on the ground that the Boersch effect exists in beams with a zero transverse temperature, such as beams with a stigmatic (point) crossover.

Loeffler's results diverge for zero beam angle and therefore do not apply to cylindrical beams. In this respect, his results are complementary to those obtained by Zimmermann, which are most suited for cylindrical beams. This was recognized by Zimmermann (1970), who embodied Loeffler's method for the crossover case. He argued that it basicly is in agreement with his own calculation, since both results demonstrate the same parameter dependency. He considered the distinction between thermal and crossover motion to be artificial. It should be added, that Zimmermann's and Loeffler's results only agree (qualitatively) in the high-current limit. In the low-current limit, Loeffler predicts a linear dependency on the beam current, whereas Zimmermann finds a square root dependency on this parameter, for all experimental conditions. In this context, it is important to notice that Loeffler expresses his result in terms of the median of the energy distribution, while Zimmermann, like most others, evaluate a root mean square (rms) value.

Ever since the contributions of Ulmer, Zimmermann and Loeffler the Boersch effect is generally believed to be generated by the Coulomb interactions between the individual beam particles. All subsequent theories, described in this section, are based on this assumption. Approaches, however deviating from the mainstream were taken by Fischer (1970) and Beck (1973). Fischer proceeded on the idea first formulated by Boersch (1954), that the phenomenon is caused by longitudinal space charge oscillations, generated by shot noise fed into the beam from the electron gun. In his approach, it is essential that the beam is neutralized. Thus for an electron beam, as considered by Fischer, the presence of a background of positive ions is assumed. This theory therefore does not explain the presence of the Boersch effect in beams at ultra high vacuum conditions, such as beams from field emission guns. Beck (1973) considered a non-neutralized electron beam and applied the theory for Brownian motion in an external field, described by Chandrasekhar (1943). In Beck's version of this model, the external field is produced by the (long-range) space charge force. The shortrange effects, caused by strong binary collisions, are expressed in terms of dynamical friction and diffusion (see section 4.3). Beck concluded that the initial velocity distribution can strongly be perturbed by the (long range) space charge effect, but that short range effects can be neglected in beams with practical current densities. Beck's results are confusing and inadequate, since he represents the transverse space charge velocity broadening as a contribution to the energy spread, ignoring the fact that this motion is not random, but 
systematic and can be largly compensated by refocussing.

Knauer $(1979 a, b)$ extended the work of Zimmermann for noncylindrical beams. He considered the transverse particle velocities to be a consequence of both thermal and crossover motion. This way, he obtained expressions which do not diverge in the limit of zero beam angle, as in Loeffler's approach. However, the physical basis for this procedure seems somewhat obscure. Knauer (1981) also discussed the interaction phenomena in a gun with a spherical cathode, introducing the concept of potential energy relaxation in collisionless threedimensional beams. "Three-dimensional" refers to beams in which the average axial separation of the particles is small compared to the lateral beam dimensions. "Collisionless" means that the beam trajectories do not cross, which is a consequence of the strong radial field near the cathode. Knauer treats the influence of near and distant neighbours separately, applying different statistical procedures. The physical basis for this approach appears to be questionable, but it can be demonstrated that the resulting dependency on the experimental parameters is independent of the statistical procedure followed. Knauer's process of potential energy relaxation leads to a two-third power dependency on the beam current.

Crewe (1978a) considered a beam in drift space, with a narrow crossover. He calculated the energy change of a central reference electron, due to a single (not necessarily weak) collision with another electron. For simplicity, he assumed that the total effect can be represented by a single collision, choosing adequate characteristic collision parameters. Crewe's expression for the energy change is inversely proportional to the axial separation of the particles and therefore increases linearly with the beam current.

A similar, but statistically more sophisticated model was developed by Rose and Spehr (1980). They stated that the main contribution stems from nearest neighbours, that is pairs of electrons having the smallest impact parameter relative to each other. They determined the effect on a (not necessarily central) reference particle, taking the probability distribution of impact parameters of nearest neighbour colliding particles into account. The improvement on Crewe's model is established by considering the entire nearest neighbour probability distribution, instead of picking just one characteristic value. The procedure of Rose and Spehr also solves some of the disadvantages of the model of Zimmermann and Knauer. This model includes an integration over all impact parameters, up to a somewhat arbitrary maximum value, as was mentioned previously. In the approach of Rose and Spehr, the contribution of large impact parameters is surpressed by a factor, which depends on the nearest neighbour probability distribution. In other words, colliding particles with a large impact parameter are unlikely to be the nearest neighbour of the reference particle and their contribution therefore vanishes in this approach. The accuracy of the model of Rose and Spehr depends on the validity of the assumption that the contribution of other particles than nearest neighbours can be neglected. It might be anticipated that their result underestimates the Boersch effect, in particular for high current densities. For practical current densities, the model of Rose and Spehr predicts a square root dependency on the beam current. In addition, it shows that the Boersch effect strongly depends on the size of crossover. Consequently, the effect can be reduced by forming beams with a astigmatic crossover.

De Chambost and Hennion (1979), Massey, Jones and Plumer (1981) and Sasaki $(1984,1986)$ presented models which are based on the calculation of the mean square of the fluctuations in the electrostatic force acting on a particle surrounded by randomly distributed point 
charges. This approach resembles the idea of Schiske (1962) and is, unlike the other models discussed so far, not based on two-particle collisions. A detailed description of a similar model in stellar dynamics is given by Chandrasekhar $(1941,1943)$. The basic assumption is that the time development of the distribution of field (or colliding) particles surrounding a test (or reference) particle can be described as a succession of independent states. The electrostatic force, acting on the test particle, is separated into a systematic (space charge) component, corresponding to the smoothed-out distribution of charge and a stochastic fluctuating component, depending on the instantaneous distribution of field particles. The former will be zero for a test particle which is surrounded by a spherical symmetric distribution of field particles. The latter varies randomly, and its instantaneous value during two successive independent states is assumed to show no correlation. The total effect of the fluctuations on the velocity distribution of the test particle, is found by adding statistically the deviations experienced during the subsequent independent states.

The models of this type encounter two inherent problems. Firstly, one is forced to introduce a lower limit for the distance between the test particle and its surrounding field particles, otherwise the mean square of the field fluctuations would diverge. The outcome depends critically on the value of this lower limit, but its choice seems arbitrary. Secondly, additional assumptions are required to estimate the speed of the fluctuations or, in other words; the size of the time intervals between the successive independent states. The approximations used to solve these problems vary for the three models mentioned, leading to significant differences in the results. The models of Massey et al. and Sasaki predict a square root dependency on the beam current, whereas the result of De Chambost and Hennion shows a $3 / 8$ power dependency. Massey et al. stated that the Boersch effect can be generated by potential energy relaxation, for which they used the model described above, as well as by velocity (internal kinetic energy) relaxation. For the latter they followed Knauer (1979a).

Van Leeuwen and Jansen (1983) presented a model for statistical interactions in a beam with a crossover, based on a reduction of the full N-particle problem to a sum of two-particle effects. Their model predicts the detailed energy distribution, whereas most earlier models evaluate only the (rms or median) width of the energy distribution, presupposing that its shape is Gaussian. According to van Leeuwen and Jansen, the energy distribution indeed is Gaussian for a significant range of operation, but takes on a quasi-Lorentzian form (Lorentzian core with exponential tails) in the limit of low current densities. The Lorentzian distribution falls of quadraticly, which implies that the tails of the distribution contain a substantial larger fraction of the particles than expected if a Gaussian shape is assumed. This is in agreement with the results obtained by Rose and Spehr (1980), who explicitly predicted such a non-Gaussian behaviour for low current densities. Groves, Hammond and Kuo (1979) noted non-Gaussian features in their numerically obtained results as well. According to van Leeuwen and Jansen the quasi-Lorentzian energy distribution is most adequately described by its Full Width at Half Maximum (FWHM). The rms and FWHM show different dependencies on the experimental parameters. The rmsvalue is proportional to the square root of the beam current, for all conditions. The FWHM-value shows an identical dependency in the Gaussian regime (FWHM=2.35 rms), but follows a linear dependency in the quasiLorentzian regime. For this regime, their outcome coincides with the result given by Loeffler (1969) for low current densities, which is however larger by a factor $\sqrt{2}$.

Gesley and Swanson (1984) analysed the energy shift and energy 
broadening generated in the vicinity of a spherical emitter with single file emission. In this model, all particles are uniformly accelerated along a line. The energy broadening is related to the distribution of initial distances between nearest neighbours, which is governed by shot noise. Gesley and Swanson exploited a semi-empirical equation to calculate the energy change of a particle involved in a binary interaction during acceleration. This equation still contains one unknown parameter. Some kind of unification of different theories is established by varying this parameter as well as another geometry dependent quantity.

Jansen, Groves and Stickel (1985) presented an equation which covers the results of a number of theories on the Boersch effect, all pertaining to the case of a beam segment with a crossover in drift space. It says that the relative rms energy spread $\left(=\left\langle\Delta \mathrm{E}^{2}>1 / 2 / E\right)\right.$ is directly proportional to the square root of the perveance (perveance $=I / V^{3} / 2$ ), as well as a function of three dimensionless parameters, which differs for each of the theories. The theories of van Leeuwen and Jansen (1983) and Rose and Spehr (1980) were found to be in close agreement with each other and with the results obtained by numerical Monte Carlo simulation.

Furukawa (1986) determined an upper limit for the Boersch effect, by assuming that it is caused by the conversion of potential energy into (internal) kinetic energy. Furukawa does not separate the generation of systematic.velocity components (space charge effect) and the generation of random velocity components (statistical effects). It is erroneous to assume that the former contributes to the energy broadening. His expression are therefore not very practical, although the actual energy spread will, indeed, always be much smaller than the value predicted by this upper limit.

Tang (1987) presented a model based on numerical integration of the so-called Fokker-Planck equation. His method resembles the ideas developed in plasma physics and stellar dynamics (see section 4.3). The time-evolution of the single-particle distribution function in 6dimensional phase space is studied under the influence of both the average space charge force as well as the fluctuating component of the interactions force. The impact of the latter is described as a diffusion process in velocity space, characterized by the coefficient of dynamical friction $\beta$ and the diffusion coefficient $D$. This approximation presupposes that the generated velocity distribution is Gaussian. The expressions used to calculate $\beta$ and $D$, as function of the macroscopic parameters of the beam, are based on weak binary collision. Accordingly, this approach does not improve on those of Zimmermann (1970) and Knauer (1979a), as far as the fundamental issues involved. However, Tang's discussion is useful in the sense that it clarifies the relation with some of the leading ideas in the field of plasma-physics and stellar dynamics.

\subsection{Trajectory displacement effect}

Loeffler (1964) was probably the first to mention that the encounters between the individual beam particles do not only affect the longitudinal velocity distribution, but also lead to stochastic displacements in the transverse direction. He observed that the demagnified image of a carbon microgrid became blurred when the beam current was increased. The major part of this blurring could be refocussed, but a significant part remained, even after refocussing. Loeffler identified these contributions respectively as the space charge 
effect and the transverse counterpart of the Boersch effect, here called the trajectory displacement effect. Loeffler's experimental results and early theoretical analysis are summarized by Hamish, Loeffler and Kaiser (1964).

Contrary to the Boersch effect, the published quantitative experimental data on the trajectory displacement effect is rather scarce. Most data pertains to high throughput electron beam lithography systems. Stickel and Pfeiffer (1978) measured the edge-width of the shaped spot in the IBM EL3 variable shaped beam column, as function of the target current and suggested a two-third power dependency on this parameter. Similar measurements of the edge-width of a shaped spot for different beam currents were presented by saitou et al. (1981), Veneklasen (1985) and Morita et al. (1985). Experimental data of the current dependency of the spot-size in a Gaussian beam system was given by Kelly, Groves and Kuo (1981). The current density profile of a focussed ion beam spot was measured by Cummings, Harriot, Chi and Ostermayer (1986) and Ward, Utlaut and Kubena (1987). They demonstrated the existence of long tails, which are probably due to the trajectory displacement effect. See Melngailis (1987) for a review on this matter. A number of theoretical discussions concern the impact of the statistical particle-particle interactions on the lateral properties of the beam. The distinction between the (deterministic) space charge effect and the (statistical) trajectory displacement effect is recognized in most contributions, but the terminology employed varies. In this section, the original terms are reproduced. Both the space charge effect and the trajectory displacement effect influence the lateral properties of the beam and are, in that respect, related. References to the literature, dealing with the space charge effect in low density particle beams, will be presented in section 2.5 . Most theoretical approaches to the trajectory displacement effect form an extension to models presented previously for the Boersch effect. Some of the physical principles were already described in the previous section and the discussion here is restricted to the new aspects.

Loeffler and Hudgin (1970) proceeded on an earlier approach to the Boersch effect in a beam segment with a narrow crossover, presented by Loeffler (1969). Three different phenomena of statistical interactions in a beam with a narrow crossover are distinguished, corresponding to each of the three components of the coulomb force, exerted by a colliding electron on a reference electron which runs along the beam axis. The Boersch effect, which corresponds to an energy change, is associated with the longitudinal component. The lateral force is separated in a component in the direction of the tranverse velocity of the colliding electron $\left(F_{\perp 1}\right)$ and a component perpendicular to this direction $\left(\mathbf{P}_{\perp 2}\right)$, see figure 2.1. The latter causes an "angular change" of the reference particle. The former does not produce an angular deflection, since its sign changes when the particles pass the point of closest approach, which will roughly coincide with the passage through the crossover. The angular deflection, experienced before reaching the crossover, is thus compensated in the traject following the crossover. This force component causes, therefore, only a lateral shift, called "trajectory displacement". The median width measures of the distributions of energy changes, angular changes and trajectory displacements are obtained by numerical integration over all possible trajectories of the colliding particles relative to the reference particle, employing a Monte Carlo method. Loeffler and Hudgin presented normalized curves, from which the magnitude of the different phenomena can be determined. All three curves clearly indicate the existence of two separate regimes, which show a different dependency on the experimental parameters. For high electron densities, all effects 


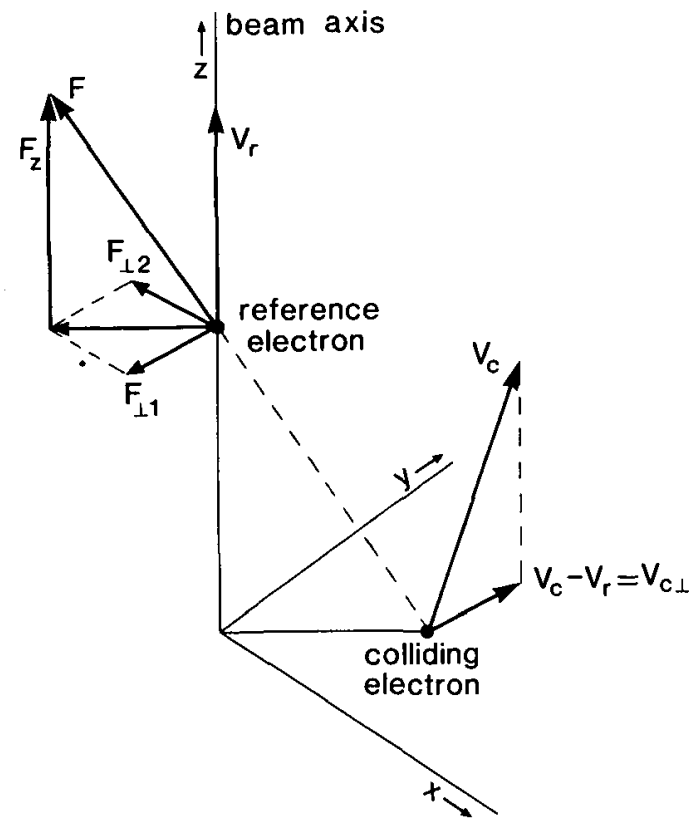

Figure 2.1 The three components of the force exerted by a colliding electron on a central reference electron, according to Loeffler (1969).

ultimately increase with approximately the square root of the beam current, but for low electron densities, different powers (varying between 1 and 3 ) are found for the separate effects.

The discussion presented by Massey, Jones and Plummer (1981), includes a calculation of the effect of statistical interactions on the lateral properties of the beam. Lateral displacement and angular deflection are expressed in terms of a single quantity called "trajectory aberration", which refers to the virtual broadening of the source. De Chambost (1982) presented a similar calculation, using the term "electron-electron effect". The central quantity in both models is the mean square of the fluctuations in the electrostatic interaction force acting on a particle. The approach is essentially the same as utilized for the Boersch effect. Accordingly, the same dependency on the beam current is obtained, which is, however, different for both models. The fundamental aspects were discussed in the previous section.

Knauer (1981), applied his model of potential energy relaxation in three dimensional collisionless beams, mentioned in the previous section, to estimate the "virtual source growth" in a gun with a spherical emitter. As for the Boersch effect, the result shows a twothird dependency on the beam current.

In the papers by Weidenhausen, Spehr and Rose (1985) and Spehr $(1985 a, b)$, two phenomena associated with the effect of statistical interactions on the lateral properties of a beam with a crossover are distinguished. Weidenhausen et al. presented a discussion of "stochastic ray deflections", which corresponds to the angular spread in the electron trajectories generated near a crossover. This effect is equivalent to the broadening of the distribution of transverse velocities and can be treated according to the closest encounter model, developed by Rose and Spehr (1980) for the Boersch effect. Stochastic 
ray deflections result in a spatial blurring in planes which are removed from the crossover. It can be neglected in source (crossover) imaging systems, but is possibly of significance in shaped beam columns, employing Koehler illumination. For practical current densities, the rms angular spread, caused by stochastic ray deflections, increases with the square root of the beam current.

Spehr (1985a) presented a calculation of the "stochastic probe broadening" effect, employing a thermodynamical description of the beam. Contrary to the effect of stochastic ray deflections, stochastic probe broadening is generated in the dilute parts of the beam. In these parts, the longitudinal beam temperature (which is possibly increased by the Boersch effect) exceeds the transverse beam temperature, which decreases with the inverse square of the beam radius (see section 3.7). Consequently, relaxation of internal energy, from the longitudinal to the lateral degrees of freedom, occurs as a result of the weak collisions between the beam particles. This process is treated in a similar way as in the model of Zimmermann (1970) and Knauer (1979a) for the Boersch effect, which corresponds to an energy transfer in the opposite direction. Spehr utilizes the concept of beam entropy to relate the energy (or heat) transfer, into the lateral degrees of freedom, to a broadening of the virtual crossover. The concept of "stochastic probe broadening" explains, according to Spehr, the blurring of the final probe observed in systems, in which the source (or source crossover) is imaged on the target. It should be emphasized that Spehr's model presupposes that the beam is non-monochromatic, since the probe broadening effect, predicted by this model, vanishes for a zero initial energy spread.

Van Leeuwen and Jansen (1983) presented a detailed calculation of the full "trajectory displacement distribution" for a beam with a crossover. The trajectory displacement, experienced by a reference particle, is expressed as a virtual displacement in the crossover plane, obtained by extrapolation of its final coordinates back to this plane. The general behaviour of the trajectory displacement distribution is similar to the behaviour of the energy distribution, produced by the Boersch effect. For high current densities, it is Gaussian and its width increases with the square root of the beam current. For low and moderate densities, it shows Lorentzian characteristics, leading to a two third power dependency on the beam current. The latter result is given by Jansen, van Leeuwen and van der Mast (1983) and forms a modification of the original work by van Leeuwen and Jansen (1983). Contrary to the Boersch effect, the Lorentzian type of regime prevails for practical operating conditions. Van Leeuwen and Jansen emphasized that the Boersch effect and the trajectory displacement effect stem from different type of collisions, leading to a different dependency on the experimental parameters. The Boersch effect results, predominantly, from collisions between particles having a large relative velocity. These collisions are complete, which means that the particles approach one another from a large separation and recede to a large separation. The Boersch effect is generated in the crossover area, where such collisions reach their point of closest approach. The effect, therefore, strongly depends on the size and shape of the crossover, but is insensitive to the length of the beam. The trajectory displacement effect, on the other hand, is usually dominated by weak, incomplete collisions between particles having a small relative velocity. It is not specifically generated in the vicinity of the crossover, but rather in the entire beam. Consequently, it depends on the beam length, but is insensitive to the size and shape of the crossover.

Venables and Cox (1987) reported on the development of a computer model to predict the performance of field emission gun 
scanning electron microscope columns, taking the trajectory displacement effect into account. For that they used the results of van Leeuwen and Jansen (1983) and Jansen, van Leeuwen and van der Mast (1983). The modelling indicates that the unexpected large spot sizes, observed by Venables and Janssen (1980) in the high resolution mode, can be attributed to the impact of the trajectory displacement effect.

Jansen (1988a) presented a set of equations to predict the

impact of the different manifestations of statistical interactions on the spot-size in Gaussian beam systems and the edge-width in shaped beam columns. The theoretical predictions for the trajectory displacement effect were found to be in good agreement with the edge-width measurements reported by Stickel and Pfeiffer (1978) and Veneklasen (1985). Jansen also presented a general form for the different types of distributions produced by the statistical interactions. According to this model, the distribution can be Gaussian, Holtsmarkian, Lorentzian or is of the so-called "pencil beam type". This type of distribution occurs in beams in which the axial separation of the particles is large compared to the lateral dimensions of the beam. Ward, Utlaut and Kubena (1988) independently obtained a similar general form, by generalizing the integral expression for the Holtsmark distribution.

\subsection{Space charge effect in low density particle beams}

The impact of the space charge effect on the properties of charged particles beams is extensively studied during the past 60 years. However, most publications deal with the beam spreading in high density beams, such as found in oscilloscope tubes and picture tubes, or describe the space charge effect occurring in the vicinity of the cathode of a thermionic gun. Relatively little attention is given to the space charge effect in low and medium density beams, such as found in electron microscopes and electron beam lithography systems. However, space charge effects may affect the performance of such systems. For instance, in a variable-shaped spot lithography column, the space charge effect causes a current-dependent defocussing which is difficult to compensate on a spot-by-spot basis. It, therefore, limits the edge resolution of these systems during normal operation.

The space-charge effect in low density electron beams is discussed by Meyer (1958), Crewe (1978b), Massey, Jones and Plummer (1981), De Chambost (1982), Sasaki (1982) and van den Broek (1986a). Crewe calculated the average radial force acting on a beam particle as function of its position and concluded that the radial equation of motion is identical to the one found for high density beams. This result is not surprising. The radial equation of motion of a particle, subject to the (average) space charge force, can straightforwardly be obtained from Gauss's theorem for the electrostatic field produced by a configuration of charges (see chapter 11). This law applies for arbitrary densities.

Although the same equation of motion applies to both high and low density beams, the analytical approach to solve this equation should be different for the distinguished cases. In general, the approximations used for high density beams do not apply to low density beams. In the former, it is valid to assume laminar flow and the problem can be solved by studying the trajectory of a particle running along the edge of the beam, as was mentioned previously. In the latter, the particle trajectories can cross each other as well as the beam axis and the laminar flow condition is not generally fulfilled. Therefore, a different approach is necessary. The problem can be solved analytically, 
by exploiting the fact that the deviations from the unperturbed trajectories (trajectories in absence of interaction) are small in such beams. This was recognized by the authors mentioned above. Using a first order perturbation approximation, they evaluated the trajectory of a test particle, by considering the space charge repulsion which would occur, when all particles would follow their unperturbed trajectories. The space charge perturbation in low density beams can be determined in a similar manner as the geometrical aberrations, starting from the paraxial ray equation, see for instance Meyer (1958) or Zvorykin (1961).

A straightforward solution can be obtained for a beam in field free drift space, with an uniform current density distribution in every cross section of the beam. In that case, the space charge effect corresponds to the action of an ideal negative lens and can thus be expressed in terms of a defocussing and a magnification effect. For nonuniform current density distributions, the space charge lens will be non-ideal. Consequently, the space charge effect can not entirely be refocussed. The non-refocusable blurring (blurring produced in the plane of best focus), can be described in terms of the geometrical aberrations of the space charge lens. Van den Broek (1984) studied the effect of non- uniform current density distribution on the spot growth numerically. He also obtained an analytical solution for the particular case of a parabolic space charge density distribution, employing first order perturbation theory, van den Broek (1986a). As most space charge phenomena, the non-refocusable space charge blurring is directly proportional to the beam current and is expected to dominate the trajectory displacement effect for high (non-uniform) current densities. The subject of the space charge effect in low density beams is investigated in chapter 11 .

\subsection{Monte Carlo simulation}

A novel approach to the calculation of interaction effects in particle beams was introduced by Groves, Hammond and Kuo (1979), Sasaki (1979,1982) and El-Kareh and Smither (1979). They independently developed a numerical technique, in which the trajectories of an ensemble (sample/bunch) of particles, with randomly chosen initial conditions, are traced through a system. The trajectories are determined by updating the position and velocity of every particle at regular time intervals, with each particle experiencing the coulomb repulsion of every other particle in the ensemble. Lenses and other optical elements can easily be incorporated in thin-lens approximation, which means that their action is represented by a deflection of the particles in a single plane. In order to obtain acceptable statistics, the tracing procedure is repeated for a number of bunches, each bunch starting with a different "seed" of initial conditions. The final coordinates, accumulated from all seeds, are processed in order to reduce the information to some characteristic quantities, as the width of the energy distribution, the defocussing distance and the spatial broadening in the plane of best focus.

This numerical approach is called Monte Carlo simulation, referring to the random initiation of the particle coordinates. The name is slightly misleading, since the ray-tracing itself is strictly deterministic, unlike the Monte Carlo models used to simulate the scattering of particles, bombarding a solid-state material. Such models are used in electron beam lithography to determine the proximity effect, see, for instance, Murata and Kyser (1987). The Monte Carlo approach described above, should also be distinguished from the Monte carlo 
calculation reported by Loeffler and Hudgin (1970). They employed a numerical Monte Carlo technique to approximate the integral over the coordinates of the colliding particles, within their binary encounter model. They did not attempt to solve the $\mathrm{N}$-body problem by straightforward numerical integration.

Monte Carlo simulations of electron beams in drift space were also reported by Dayan and Jones $(1981)$, Jones et al. $(1983,1985)$ and Tang (1983). Munro (1987) reported on a program written by Meisburger, which also covers uniform acceleration fields. Yau, Groves and Pease (1983) presented Monte Carlo simulations of different type of ion beams, emitted in a small cone from a spherical cathode, under influence of a spherical acceleration field. Ward (1984) and Ward, Utlaut and Kubena (1987,1988) employed Monte Carlo simulations to estimate the virtual source size growth of a liquid metal ion source. Narum and Pease (1986) utilized the Monte Carlo technique to simulate the interaction between the ions in a focussed ion beam, in presence of a postlens retarding field. Allee, Pehoushek and Pease (1988) used the Monte Carlo approach to model the acceleration zone in a laser irradiated thermionic and photoemissive electron source. Similar integration methods are extensively used in stellar dynamics, see for instance Aarseth (1972). Jansen (1987) introduced the concept of Fast Monte Carlo simulation, which is based on a reduction of the $\mathrm{N}$-body problem to binary interactions, for which analytical approximations are exploited. Some extensions to this model were reported by van der Mast and Jansen (1987). The principles of Monte Carlo simulation of particle beams will be discussed in chapter 13 .

The advantage of Monte Carlo simulation is its accuracy relative to the analytical models, which is a consequence of the minimum number of underlying physical assumptions. Obviously, this accuracy is only obtained when the numerical procedures are carried out with sufficient caution. This is not a trivial task and the quality of the simulation, of ten, is hard to judge from the presented results alone. A disadvantage of the Monte Carlo approach is that it does not give a direct appreciation of the physical mechanisms involved and does not lead to explicit equations, showing the dependencies on the experimental parameters. Consequently, the result of a single simulation only applies to the considered condition and every other condition requires a new simulation. Clearly, the analytical and the Monte Carlo approach are complementary in many respects and a more firm theoretical foundation can be obtained by their combination. This was recognized by Jansen, Groves and Stickel (1985) and Jansen and Stickel (1984), who compared the different analytical theories on particle interactions with the results of Monte Carlo simulations, for a simple beam geometry with a single crossover. 


\section{GENERAL BEAM PROPERTIES}

\subsection{Introduction}

This chapter discusses a number of well-known concepts used to model particle beams. It starts by surveying the experimental parameters, specifying the macroscopic properties of the beam. These parameters are then used to classify the different types of beams considered throughout this monograph.

After these preliminary sections we focus on some fundamental issues known from the field of statistical mechanics. Liouville's theorem is derived from Hamilton's equations of motion. This theorem covers the dynamics of an ensemble of systems in $6 \mathrm{~N}$-dimensional phasespace, $\mathbf{N}$ being the number of particles in each system. It states that a small volume $d V_{6 \mathrm{~N}}$, containing some $d N$ points (each representing a single system), may arbitrarily change its shape in the course of time, but maintains its volume. For a system of identical non-interacting particles a similar equation can be obtained covering the time development of a single system in 6-dimensional phase space. This equation, which is known as the collisionless Boltzmann equation, shows the same properties as Liouville's theorem in $6 \mathrm{~N}$-dimensional phase space. The phase space to which Liouville's theorem applies can be further reduced when the different degrees of freedom of the beam particles are uncoupled. This leads to the concept of conservation of emittance and conservation of brightness, which are of great importance to the theory of particle optics.

Next, the concept of beam temperature is introduced, which is an essential quantity in the thermodynamical description of the beam. In a rotational symmetric beam one has to distinguish two beam temperatures $T_{/ /}$and $T_{\perp}$, pertaining to the Iongitudinal and lateral degrees of freedom of the beam respectively. Non-equilibrium conditions may occur as a consequence of the compression and expansion of the beam volume. The expansion of the beam in axial direction during acceleration causes a decrease of the longitudinal beam temperature $T_{\|}$. The transverse beam temperature $T_{\perp}$ changes with the lateral dimensions of the beam, and is therefore affected by the focussing action of the lenses. The temperatures $T_{/ /}$and $T_{1}$ will be related to the geometrical properties of the beam and the conditions in the source.

Finally, the particular aspects of a beam of interacting particles are considered. In the thermodynamical description of the beam, Coulomb interactions cause a relaxation towards thermodynamic equilibrium. In this model one can represent the Boersch effect as a relaxation of kinetic energy, from the lateral degrees of freedom to the longitudinal degree of freedom. This physical picture seems adequate for beams with a narrow crossover, but is unable to explain the Boersch effect generated in a cylindrical beam with parallel rays. In order to complete the thermodynamical description of the beam, we will present a model for the relaxation of potential energy. The concept of Debeye screening will be utilized within this model and is therefore discussed in some detail.

Complete thermodynamic equilibrium is usually not reached in practical beams, due to their relatively low particle density and short time of flight. Thermodynamical considerations are therefore merely useful to provide insight in the physical mechanism involved and to determine upper limits for the impact of statistical interactions on the properties of the beam. 


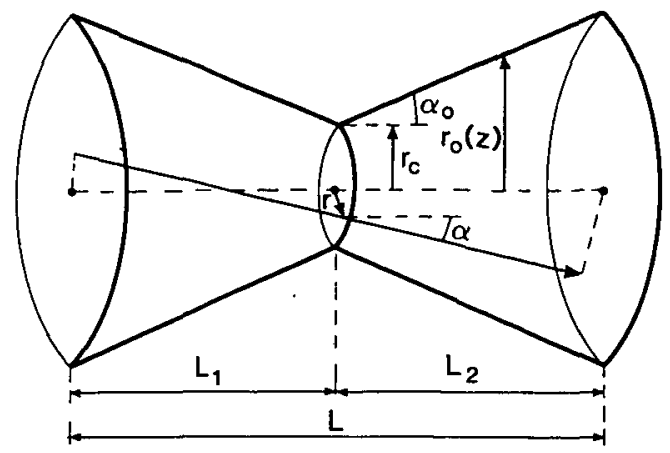

Figure 3.1 Definition of the experimental parameters for a beam segment with a crossover in drift space. The beam dimensions are specified by the crossover radius $r_{\mathrm{c}}$, the beam semi-angle $\alpha_{0}$ and the beam length $L$. The unperturbed trajectory of a particle is defined by the angle a and the radius $r$ in the crossover, as well as two azimuthal angles (which are not drawn in the figure).

\subsection{Beam parameters}

In most analytical models for particle interactions the beam is schematized as a succession of beam segments, separated by thin optical components. We will follow this approach and study the dynamics of a single beam segment. This section is concerned with the experimental parameters specifying the macroscopic condition of such a segment. It also introduces some additional physical parameters which will be used throughout this monograph.

Figure 3.1 shows a rotational symetric beam segment in drift space of length $L$ with a crossover. The particles, with mass $m$ and charge $\dot{e}$, enter the segment with an average energy $e V . V$ is called the beam potential. The energy distribution on entrance is $f_{E}(\Delta E)$. The width of this distribution is represented by $\Delta E_{0}$. The total beam current is $I$. The unperturbed trajectory of a particle is a straight line, which can be specified by four cylindrical coordinates: The angle $\alpha$ with the axial direction, the radial distance $r$ between the point of intersection with the crossover plane and the beam axis and two azimuthal angles. As the beam is rotational symmetric, the azimuthal angles will be uniformly distributed over $2 \pi$. The angular distribution is denoted as $f_{\alpha}(\alpha)$ and the spatial distribution as $f_{r}(r)$. The half-width of these distribution are represented by the crossover radius $r_{c}$ and beam semiangle $\alpha_{0}$ respectively. The beam radius at an arbitrary axial position 2 is denoted as $r_{0}(z)$. When $\alpha_{0}=0$, the beam is cylindrical and $r_{0}(z)=r_{c}$. The crossover position parameter $S_{c}$ and the image plane position parameter $S_{i}$ are defined as

$$
S_{c}=\frac{L_{1}}{L}, \quad S_{i}=\frac{L_{i}}{L}
$$

where $L_{1}$ is the distance from the entrance plane to the crossover plane and $L_{i}$ is the distance from the entrance plane to a plane which is optically conjugated to the target plarie. This plane, which is referred to as the image or the reference-plane, can be located inside or outside the beam segment. The (virtual) broadening observed in the image plane can directly be related to a broadening of the final probe, since it is optically conjugated to the target plane. In source (crossover) imaging 
systems the image plane coincides with the crossover plane, but this is not true for all segments of the beam in a shaped beam column, which employs Koehler illumination. Knowledge of $\mathrm{Si}$ is immaterial for the Boersch effect, but essential to determine the impact of the trajectory displacement effect on the final probe. The parameters $I, V, \Delta E_{0}, L, r_{c}$, $\alpha_{0}, s_{c}$ and $s_{i}$ are referred to as the experimental parameters. In most calculations we will assume that the initial energy spread does not significantly influence the effect of the interactions between the particles in the beam. Accordingly, we will usually consider the theoretical case of a monochromatic beam, which corresponds to $\Delta \mathrm{E}_{0}=0$.

We will now introduce a number of physical quantities which are frequently used and express them in terms of the experimental parameters. All presented relations are non-relativistic. The average axial velocity of the particles $\left\langle v_{\|}\right\rangle$is uniquely related to the beam potential as

$$
\left\langle\mathrm{v}_{\| /}\right\rangle=\sqrt{\frac{2 \mathrm{eV}}{\mathrm{m}}}
$$

Since we will usually consider monochromatic beams, we will of ten denote the average axial velocity of the particles simply as $v_{\| \prime}$ (or $v_{z}$ ) instead of $\left\langle v_{\| /}\right\rangle$. The average time of flight $T_{f}$ is equal to

$$
T_{\mathbf{f}}=\frac{\mathrm{L}}{\left\langle\mathrm{V}_{\| /}\right\rangle}
$$

It is here denoted as $T_{f}$, since the symbol $T$ will in this chapter be used to denote temperature. In the remaining chapters we will usually omit the subscript and denote the time of flight as $T$ instead of $T_{f}$. The linear particle density $\lambda$ is defined as

$$
\lambda=\frac{\mathrm{I}}{\mathrm{e}\left\langle\mathrm{v}_{\| /}\right\rangle}=\mathrm{I} \sqrt{\frac{\mathrm{m}}{2 \mathrm{e}^{3} \mathrm{~V}}}
$$

It is independent of the axial coordinate $z$, since the particle flow is assumed to be constant. The 3-dimensional particle density $n$, however, depends on $z$ through the beam radius $r_{0}(z)$

$$
n(z)=\frac{\lambda}{\pi r_{0}(z)^{2}}=\frac{I}{\pi r_{0}(z)^{2}} \sqrt{\frac{m}{2 e^{3} V}}
$$

If the current density distribution in the cross section of the beam at position $z$ is non-uniform, the local density $n$ will depend both on $z$ and on the radial coordinate $r$, thus $n=n(z, r)$. In that case equation (3.2.5) merely provides a measure for the average density at position 2 . The average axial separation of the particles $s$ and the average distance between the particles $d$ follow from $\lambda$ and $n$ respectively, using

$$
s=\lambda-1, d(z)=n(z)-1 / 3
$$

Finally, the total number of particles $N$, simultaneously present in the beam segment, is equal to

$$
N=\lambda L=I L \sqrt{\frac{m}{2 e^{3} V}}
$$

As a typical case, let us consider an electron beam with a crossover, 
with an uniform angular and spatial distribution and the following values for the experimental parameters: $I=1 \mu \mathrm{A}, \mathrm{V}=10 \mathrm{kV}, \mathrm{L}=0.1 \mathrm{~m}, \mathrm{~S}_{\mathbf{c}}=0.5$, $r_{c}=1 \mu \mathrm{m}$ and $\alpha_{0}=10 \mathrm{mR}$. By utilizing the equations $(3.2 .2)$ to $(3.2 .7)$ one obtains

$$
\begin{aligned}
& \left\langle\mathrm{v}_{/ /}\right\rangle=5.9 \times 10^{7} \mathrm{~m} / \mathrm{s}, \quad \mathrm{T}_{\mathrm{f}}=1.7 \times 10^{-9} \mathrm{~s}, \lambda=1.1 \times 10^{6} \mathrm{~m}^{-1}
\end{aligned}
$$

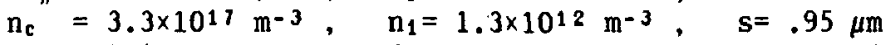

$$
\begin{aligned}
& \mathrm{d}_{\mathrm{c}}=1.4 \mu \mathrm{m} \quad, \quad \mathrm{d}_{1}=91 \mu \mathrm{m} \quad, \quad \mathrm{N}=1.1 \times 10^{5}
\end{aligned}
$$

The subscript $c$ refers to the condition in the crossover and the subscript 1 to the condition at the entrance plane. The values chosen for the experimental parameters are representative for the conditions encountered in the final beam sections of a state of the art, high throughput, electron beam lithography machine. Substantially higher beam currents can occur in the gun area.

\subsection{Classification of beans}

Having specified the experimental parameters, we are now able to classify different beams. Clearly, this can be done in number of ways, depending on what aspects of the bearn are considered. This section just explains the terminology used in this monograph and is not intended to review the classifications used in literature. We will distinguish beams by their geometry and by the particle density within the beam.

Two types of beam geometries are considered: Cylindrical beam segments and beam segments with a crossover. In the former the current density is constant along the beam axis. In the latter the current density varies with the axial position and reaches an maximum in the crossover. Divergent beams and convergent beams are in this representation included as limiting cases of a beam with a crossover. They correspond to the situation that the crossover coincides with entrance plane $\left(S_{c}=0\right)$ and the exit plane $\left(S_{c}=1\right)$ respectively. Both cylindrical beam segments and beam segments with a crossover are assumed to be rotational symmetric with respect to the beam axis, unless specified otherwise.

Homocentric beams are of special interest to the theory, since they represent a simple limiting case. A beam with a crossover is called homocentric, when all trajectories intersect each other in the crossover. The crossover is then stigmatic $\left(r_{c}=0\right)$. A cylindrical beam is called homocentric, when all trajectories are parallel $\left(\alpha_{0}=0\right)$. One could say that the trajectories intersect in infinity. Clearly, homocentric beams can not exist in reality, since they require infinite brightness (the concept of beam brightness will be discussed in section 3.6).

Another classification of beams is established on the basis of the particle density within the beam. In this classification one compares the average distance between the beam particles to the geometrical dimensions of the beam. A pencil beam is defined as a beam with small lateral dimensions relative to the axial separation of the particles s. Other beams are called extended beams. Related expressions, used in literature, are "single file regime" and "three dimensional regime", referring to the conditions encountered in pencil beams and extended beams respectively.

Throughout this monograph it is assumed that the particle density is low enough to neglect quantum mechanical effects. Accordingly we will treat the beam as a classic system and use Maxwell-Boltzmann statistics to describe its microscopic properties. This approach is justified as long as the de Broglie wavelength $\lambda_{B}$, associated with a 
particle, is short compared to the average separation of the particles d. In the frame of reference moving with the beam, the particle velocities are of the order $\alpha_{0}<v_{/ /}>$, where the average axial velocity $\left\langle\mathrm{v}_{/ /}\right\rangle$is given by equation (3.2.2). Accordingly, the appropriate de Broglie wavelength $\lambda_{B}$ is of the order

$$
\lambda_{B} \approx \frac{h}{m \alpha_{0}<v_{/ /}>}=\frac{h}{(2 m e)^{1 / 2}} \frac{1}{\alpha_{0} V^{1 / 2}}
$$

where $h$ is the Planck constant. For the example considered in the previous section (an electron beam with $\alpha_{0}=10 \mathrm{mR}$ and $V=10 \mathrm{kV}$ ) one finds $\lambda_{\mathrm{B}} \approx 1.2 \mathrm{~nm}$, which is indeed much smaller than the average separation of the particles $d_{c}$ at the most dense point in the beam.

Finally, relativistic effects are disregarded in most calculations, which is for an electron beam justified for voltages below approximately $100 \mathrm{kV}$. Accordingly, we ignore magnetic interactions and consider the effect of the mutual coulomb repulsion only. The possibility to include relativistic corrections is investigated in chapter 10.

\subsection{Hamiltonian formalism and Liouville's theorem}

Consider a non-relativistic system of $\mathbf{N}$ identical particles, interacting through the Coulomb force. Let us denote the position vector of particle $i$ as $r_{i}$ and its velocity vector as $v_{i}$. Both vectors have three components, corresponding to the three degrees of freedom of the particle. The micro-state $\Gamma(t)$ of the system at time $t$ is specified by the positions and velocities of all particles at time $t$ (constituting $6 \mathrm{~N}$ coordinates)

$$
\Gamma(t)=\left(r_{1}(t), r_{2}(t), \ldots, r_{N}(t) ; v_{1}(t), v_{2}(t), \ldots, v_{N}(t)\right)
$$

The micro-state of the system is represented by a point in $6 \mathrm{~N}-$ dimensional phase space $\left(\Gamma_{6 \mathrm{~N}}\right.$ space). Let $\Gamma\left(t_{0}\right)$ be the initial microstate on initial time to. The development of the micro-state in the course of time corresponds to a trajectory $\Gamma(t)$ in $\Gamma_{6 \mathrm{~N}}$ space, which starts at $\Gamma\left(t_{0}\right)$. This trajectory can, at least in principle, be obtained by integration of the equations of motion. This problem is the subject of classical mechanics.

We will start from the equations of motion in the Hamilton form, which can be derived from the Newtonian equations of motion, see for instance Goldstein (1980). Hamilton's equations are given by

$$
\frac{\partial H}{\partial p_{i}}=\dot{r}_{i} \quad, \quad \frac{\partial H}{\partial r_{i}}=-\dot{p}_{i} \quad(i=1,2, \ldots N)
$$

where $H$ is the Hamiltonian function, representing the total energy of the system, and $p_{i}$ is the conjugate momentum vector of particle $i$. Notice that the $2 \mathrm{~N}$ equations given by (3.4.2) are expressed in vector form, each.vector corresponding to three coordinates. Accordingly, the Hamilton equations constitute a set of $6 \mathrm{~N}$ first order differential equations in the phase space coordinates.

For the non-relativistic system of particles interacting through the coulomb force, considered here, the conjugate momentum of particle $i$ is simply given by $p_{i}=m v_{i}$ and the Hamilton function $H$ can be expressed as 


$$
H=\sum_{i=1}^{N}\left(\frac{p_{i}^{2}}{2 m}+V\left(r_{i}\right)\right)+\frac{1}{2} \sum_{\substack{i, j=1 \\ i \neq j}}^{N} \frac{C_{0}}{r_{i j}}
$$

where $V\left(r_{1}\right)$ is the potential energy of particle $i$ in an external field, $\mathrm{C}_{0}=\mathrm{e}^{2} / 4 \pi \mathrm{E}_{0}$ (the coulomb potential strength) and $\mathbf{r}_{\mathbf{i} \mathbf{j}}=\left|\mathbf{r}_{\mathbf{i}}-\mathbf{r}_{\mathbf{j}}\right|$, the distance between particle $i$ and particle $j$.

The dynamics of the system, with initial state $\Gamma\left(t_{0}\right)$, is fully specified by the equations $(3.4 .2)$ and $(3.4 .3)$. The representative trajectory in phase is uniquely determined by the initial micro-state of the system. Different initial states lead to different trajectories in $\Gamma_{6 \mathrm{~N}}$ space, which do not intersect. However, different micro-states may correspond to the same macroscopic condition of the system. This condition is defined by the value of the experimentally observable parameters. of special interest is the set of micro-states representing the same macroscopic condition. They can be considered as independent realizations of the same system. Such a set is called an ensemble of realizations or also an ensemble of systems (the reader should be alert to the different use of the word system in the various contexts). Instead of studying the properties of a single system over a period of time, it is mathematically more convenient to consider an ensemble of systems at one time and represent the macroscopic quantities of the system as an average over the members of the ensemble. The assumption that ensemble-averages are equivalent to time-averages constitutes the basis of statistical mechanics.

Let us examine the properties of an ensemble more closely. In $\Gamma_{6 \mathrm{~N}}$ space the members of the ensemble are, at a given instant of time, represented by a set of points. Let $\rho(\Gamma, t)$ describe the distribution of these points in phase space as function of time. This function is called the ensemble density function. The total number of systems in an ensemble does not change with time. Therefore, the ensemble density function must satisfy the equation of continuity

$$
\frac{\partial \rho}{\partial t}+\sum_{i=1}^{N}\left(\frac{\partial}{\partial \mathbf{r}_{i}}\left(\dot{\mathbf{r}}_{i} \rho\right)+\frac{\partial}{\partial \mathbf{v}_{i}}\left(\dot{\boldsymbol{v}}_{i} \rho\right)\right)=0
$$

which expresses that what flows into an element of volume either comes out again or builds up in the volume element. This equation can be simplified using the fact that the velocity of a representative point in phase space is divergence free

$$
\sum_{i=1}^{N}\left(\frac{\partial \dot{\mathbf{r}}_{\mathbf{i}}}{\partial \mathbf{r}_{\mathbf{i}}}+\frac{\partial \dot{\mathbf{v}}}{\partial \mathbf{v}_{\mathbf{i}}}\right)=0
$$

which is a direct consequence of the Hamilton equations (3.4.2). Substitution of equation (3.4.5) into the equation of continuity (3.4.4) gives the celebrated theorem of Liouville

$$
\frac{\partial \rho}{\partial t}+\sum_{i=1}^{N}\left(\dot{\mathbf{r}}_{\mathbf{i}} \cdot \frac{\partial \rho}{\partial \mathbf{r}_{\mathbf{i}}}+\dot{\mathbf{v}}_{\mathbf{i}} \cdot \frac{\partial \rho}{\partial \mathbf{v}_{\mathbf{i}}}\right)=\frac{\mathrm{d} \rho}{\mathrm{dt}}=\mathbf{0}
$$

where $\mathrm{d} \rho / \mathrm{dt}$ is the total (or hydrodynamic) derivative. The Liouville theorem states that the density of representative points $\rho$ is constant along a flow line. A small volume $\mathrm{d} V_{6 \mathrm{~N}}$ in phase space, containing $\mathrm{d} N$ 
points, may arbitrarily change its shape in the course of time, but maintains its volume, since the density of points $\rho=d N / d V_{6 N}$ is constant. Thus $\mathrm{d} V_{6 \mathrm{~N}} / \mathrm{dt}=0$. Notice that points within the volume can not cross the volume boundary, because phase space trajectories do not intersect.

For the particular case of a system of $\mathrm{N}$ particles interacting through the coulomb force, Liouville's theorem can be written as

$$
\frac{\partial \rho}{\partial t}+\sum_{i=1}^{N} v_{i} \cdot \frac{\partial \rho}{\partial r_{i}}+\frac{1}{m} \sum_{i=1}^{N}\left(\sum_{j \neq i}^{N} \frac{C_{0}\left(r_{i}-r_{j}\right)}{\left|r_{i}-r_{j}\right|^{3}}+\mathbf{F}_{i}\right) \cdot \frac{\partial \rho}{\partial v_{i}}=0
$$

where $F_{i}$ is the (conservative) external force acting on particle $i$.

Equation (3.4.7) applies to the general case of a system of $N$ identical particles interacting through the Coulomb force. Now, consider the particular case of a particle beam in a probe forming optical instrument. Particles are continuously emitted from the source, producing a constant flow, and are "absorbed" at the target. The composition of the beam changes continuously, while the total number of particles, present at one time, will approximately be constant. However, equation (3.4.7), as it was derived here, refers to a fixed system of $N$ particles. In order to solve this conceptual problem it is useful to visualize the beam as a succession of bunches and follow the motion of each bunch separately in the frame of reference moving with the beam. The bunches are assumed to be short compared to the total beam length, but long enough to represent the macroscopic condition of the beam. Thus, the bunches reaching the target in the course of time can be considered as different realizations of the same beam. In this view the properties of the beam, observed at the target, represent an average over the bunches reaching the target during the period of observation. clearly, this physical interpretation directly reflects the mathematical procedure of calculating a macroscopic property as an average over the members of an ensemble (assuming that beam-bunches and ensemble-members can be considered as equivalent).

Equation (3.4.7) is here presented as the fundamental equation covering the dynamic behaviour of a non-relativistic system of particles interacting through the Coulomb force. It should be noted that the same equation arises in the theory of stellar dynamics, for instance see Chandrasekhar (1942a), Gilbert (1972). The only mutation required is the replacement of the coulomb constant $\mathrm{C}_{0}$ by its gravitational counterpart. This constant depends on the mass, which may vary per "particle".

\subsection{Boltzmann equation}

Liouville's theorem in $\Gamma_{6 \mathrm{~N}}$, as given by $(3.4 .7)$, is not practical for the actual evaluation of the beam properties, due to the fact that $\mathrm{N}$ is, in general, a large number. It would be more convenient to have an equation for the single-particle distribution function $f(r, v, t)$, corresponding to the coordinates of the beam particles in the 6 dimensional phase space $\left(\Gamma_{6}\right.$ space or $\mu$-space). Formally, this function can be obtained from the ensemble density $\rho(\Gamma, t)$, by integration over the coordinates of all particles, but one

$$
f\left(r_{1}, v_{1}, t\right)=\int \rho(\Gamma, t) d r_{2} d r_{3} \cdots d r_{N} d v_{2} d v_{3} \cdots d v_{N}
$$

which can be envisioned as a projection of the coordinates in $\Gamma_{6} \mathrm{~N}$ space 
on the subspace $\Gamma_{6}$. Accordingly, the fundamental equation covering the dynamical behaviour of $f(r, v, t)$ can be found from Liouville's theorem (3.4.7), by a similar integration.

Let us examine the result of such an integration for the different terms in equation (3.4.7) separately. The first term, $\partial \rho / \partial t$, transforms directly to af/at. Next, consider the terms included within a single summation (over index i)

$$
\sum_{i=1}^{N} v_{i} \cdot \frac{\partial \rho}{\partial r_{i}} \quad \text { and } \quad \frac{1}{m} \sum_{i=1}^{N} F_{i} \cdot \frac{\partial \rho}{\partial v_{i}}
$$

All terms $i \neq 1$ vanish, since it is allowed to assume, without loss of generality, that $\rho$ is zero at the integration boundaries. The other terms (corresponding to $i=1$ ) can directly be expressed into the derivatives of the single particle function $f$. Finally, consider the terms included within a double summation (over indices $i$ and $j$ ), which correspond to the coulomb interaction between the particles. These terms depend on the position of a particle $i$, relative to the other particles $j$ in the system. Consequently, the results obtained after integration, can not be expressed in the single particle function alone. Evaluation of these terms requires the knowledge of the two-particle correlation function, which specifies the conditional probability that a particle $j \quad(\neq 1)$ is located at $r_{j}$, if particle 1 is located at $r_{1}$. Formally, this function can be found by integrating Liouville's theorem (3.4.7) over the coordinates of all particles, but two. However, as it turns out, this requires the knowledge of the three-particle correlation function and a straightforward explicit solution is still not obtained. In general, the equation for the n-particle distribution involves the $(n+1)$-particle distribution. The resulting set of coupled equations is referred to as the BBGKY hierarchy (for Bogolioubov, Born, Green, Kirkwood and Yvon). Formally speaking, the problem of determining the impact of particle interactions on the time-evolution of the singleparticle function $f(r, v, t)$ is to find a method of truncating the BBGKY hierarchy. A discussion of the approach to this problem taken in plasma physics and stellar dynamics is postponed to the next chapter and the interaction term is here left unspecified as $[\partial f / \partial t]_{1}$.

Hence, the resulting equation for the single-particle distribution function $f(r, v, t)$ can be expressed as

$$
\frac{d f}{d t}=\frac{\partial f}{\partial t}+v \cdot \frac{\partial f}{\partial r}+\frac{F}{m} \cdot \frac{\partial f}{\partial v}=\left[\frac{\partial f}{\partial t}\right]_{i}
$$

This is the Boltzmann transport equation, sometimes also referred to as Liouville's theorem in $\Gamma_{6}$ space (especially when $[\partial f / \partial t]_{i}=0$ ) or as the kinetic equation. The quantity $\mathrm{df} / \mathrm{dt}$ is the total (or hydrodynamic) derivative. Boltzmann's equation states that the rate of change of $f$ $(=\mathrm{df} / \mathrm{dt})$ along a free (unperturbed) particle trajectory is entirely the result of the interaction between the particles.

\subsection{Conservation of beam emittance and brightness}

Now we will focus on the properties of a system of $N$ identical particles, which do not interact. Thus we take $[a f / \partial t]_{i}=0$. The resulting (collisionless) Boltzmann equation (3.5.2) shows the same features in $\Gamma_{6}$ space as the Liouville equation $(3.4 .6)$ in $\Gamma_{6 N}$ space (which is, however, also valid for interacting particles). Thus, the time-invariance of the 
phase space volume enclosing a group of representative points applies to $\Gamma_{6}$ space as well: $d V_{6} / d t=0$. A further reduction of the phase space to which Liouville's theorem applies is achieved when the three degrees of freedom of a particle are uncoupled. Liouville's theorem then pertains to the three subspaces $\Gamma_{2}$ in $x, y$ and $z$-direction separately.

In a beam of non-interacting particles, the two lateral degrees of freedom will in general be independent of the longitudinal degree of freedom. Consequently, Liouville's theorem applies to the subspaces $\Gamma_{4}$ and $\Gamma_{2}$ of lateral and longitudinal coordinates respectively: $\mathrm{d} V_{/ /} / \mathrm{dt}=0$ and $\mathrm{d} V_{\perp} / \mathrm{dt}=0$. The two lateral-degrees of freedom may be independent too, but this is not required in the further analysis.

First, let us investigate the consequences of Liouville's theorem in the subspace of axial coordinates $\Gamma_{2}$. Consider a small volume $\Delta V_{2}$, occupied by $N$ particles. Let $\Delta r_{/ \prime}$ be the axial spatial length occupied by the particles and $\Delta v_{\|}$the corresponding length in axial velocity space. Liouville's theorem implies that $\Delta V_{2}=\Delta r_{\|} \Delta v_{\|}$is conserved along the particle trajectories. This statement can be expressed in terms of the experimental parameters of the beam. For $\mathrm{N}$ large enough one may write

$$
\Delta \mathbf{r}_{\|} \approx N s_{\mathbf{v}}\left(\Delta \mathbf{v}_{\| /}\right) \sim s \sim\left\langle\mathbf{v}_{/ /}\right\rangle / \mathrm{I}
$$

in which $s_{v}\left(\Delta v_{/ l}\right)$ denotes the average axial separation of the particles within the velocity range $\Delta v_{/ /}$, s the average axial separation of all particles and I the beam current. The last step follows with equations (3.2.6) and (3.2.4). The velocity range $\Delta v_{/ /}$can be expressed in the corresponding range in normal kinetic energy : $\Delta \mathrm{v}_{/ / /}=\Delta \mathrm{E}_{/ /} / \mathrm{mv}_{/ /}$. Hence, $\Delta v_{\|} \Delta r_{/ /} \sim \Delta E_{/ /} / I$. Accordingly, Liouville's theorem, applied to the subspace of axial coordinates, gives

$$
\Delta V_{2}=\Delta v_{/ /} \Delta r_{\|}=\text {constant } \quad \Rightarrow \quad \Delta E_{/ /} / I=\text { constant }
$$

The second equation expresses that the normal energy spread of the group of $N$ particles is conserved during the flight as long as the beam current associated with these particles is constant (and visa versa).

Next, consider a small volume $\Delta V_{4}$ in the the subspace of lateral coordinates $\Gamma_{4}$, occupied by $N$ particles. The trajectories of these particles constitute a bundle of rays within the beam. Let $\Delta r_{\perp}{ }^{2}$ be the surface area occupied by the cross section of the bundle in an arbitrary reference plane, perpendicular to the beam axis. Let $\Delta v_{\perp}^{2}$ be the corresponding surface area in lateral velocity space. Liouville's theorem implies that the volume $\Delta V_{4}=\Delta r_{\perp}{ }^{2} \Delta v_{\perp}{ }^{2}$ is independent of the choice of the reference plane. In particle optics this principle is known as invariance of emittance. As an illustration of this principle, consider the single lens system of figure 3.2. A rotational symmetric paraxial beam, emitted from the object at $z=z_{0}$, is imaged by a thin ideal (that is aberration free) lens located at $z=z_{1}$ to the image plane at $2=z_{1}$. Assume that all particles drift with the same axial velocity $v_{z}$. Due to the rotational symmetry it is sufficient to study the rays located in a meridian plane, which is a plane through the beam axis. A meridian ray is fully specified by a radial distance $r$, at some axial position $z$ and its derivative with respect to $z$, denoted as $r^{\prime}$. Thus the appropriate phase space coordinates are $r$ and $r^{\prime}$. Notice that for a paraxial beam in drift space $v_{\perp}=r^{\prime} v_{/ /}=\tan (\alpha) v_{/ /} \sim v_{/ /}$. Now consider the four rays emitted from the object plane with values for $r$ and $r^{\prime}$ respectively given by $\left(r=r_{0}, r^{\prime}=r_{\delta}\right), \quad\left(r=r_{0}, r^{\prime}=-r \delta\right), \quad\left(r=-r_{0}, r^{\prime}=r_{\delta}\right)$ and $\left(r=-r_{0}, r^{\prime}=-r \delta\right)$, see figure 3.2. The phase space volume enclosed by these four rays is depicted by the hatched areas in the diagrams of figure 3.3. Such plots are known as phase-space diagrams or emittance plots. 


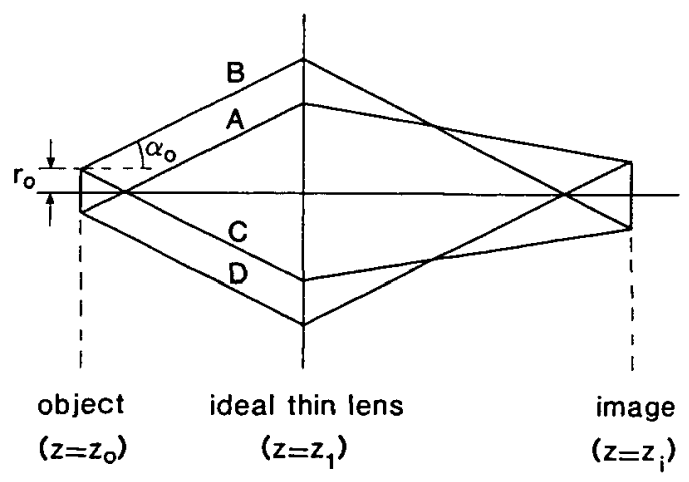

Figure 3.2 Schematic view of a section of a rotational symmetric paraxial beam with a thin ideal lens. The lateral coordinates of the rays $\mathrm{A}, \mathrm{B}, \mathrm{C}$ and $\mathrm{D}$ enclose a volume of phase space, which is independent of $z$ (see also figure 3.3).

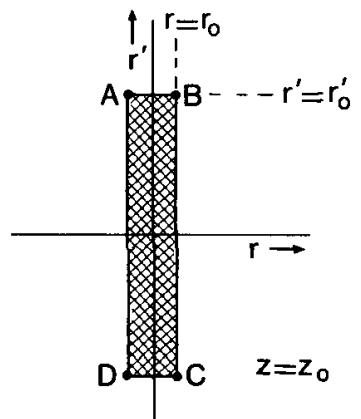

fig. 3.3a

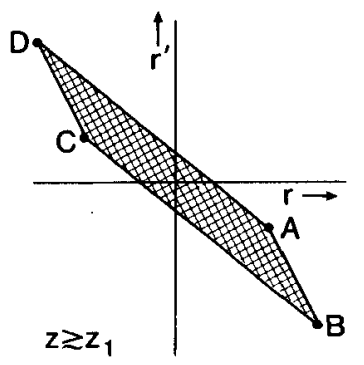

fig. $3.3 \mathrm{c}$

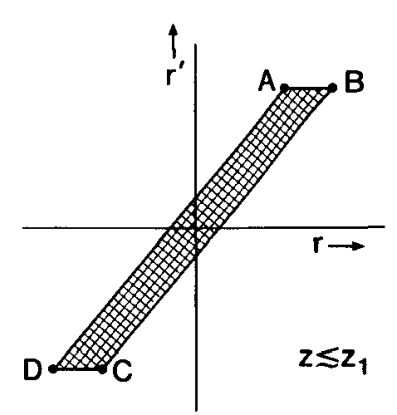

fig. 3.3b

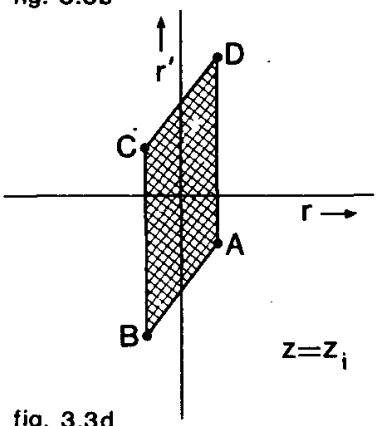

Pigure 3.3 Emittance plots representing the volume of phase space of. lateral coordinates enclosed by the rays $\mathrm{A}, \mathrm{B}, \mathrm{C}$ and $\mathrm{D}$ (shown in figure 3.2), at four different locations in the beam. The surface of the hatched area is the same for all plots. 
Diagram 3.3a refers to the object plane $\left(z=z_{0}\right)$; diagrams $3.3 b$ and $3.3 c$ refer to the object and image principal planes of the lens respectively, which are both located at $z=z_{1}$, since the lens is assumed to be thin; diagram $3.3 \mathrm{~d}$ refers to the image plane $\left(z=z_{i}\right)$. Invariance of emittance implies that the surface of the hatched areas is identical in all diagrams.

Although the principle of emittance invariance can be applied to arbitrary planes, it is particularly useful to obtain relations between the properties of the beam in planes which are optically conjugated. Such planes are related as image and object planes by the optical system. Let us investigate this more closely. Consider the object and image plane of a system containing an arbitrary number of optical, not necessarily thin, components. Assume, as previously, that the beam is paraxial. However, the beam can be non-rotational symmetric and is not necessarily entirely located in drift space (Thus the beam potential may differ in image and object plane). Consider a small bundle of rays. Let us denote $d S=d r_{\perp}{ }^{2}$, which is the surface occupied by the bundle in a plane perpendicular to the beam axis. The corresponding volume in lateral velocity space can be expressed in terms of the solid angle $d \Omega$ formed by the bundle in that plane: $d \Omega=\Delta v_{\perp}{ }^{2} / \pi v_{/}{ }^{2}$. As the analysis here is restricted to the object and image plane, the width of the angular distribution can be considered as independent of the radial position $r$, as can, for instance, be seen from figures $3.3 \mathrm{a}$ and $3.3 \mathrm{~d}$. Consequently, the emittance in these planes is simply equal to the product of $d \Omega$ and dS. Thus, for the object and image plane, one finds

$$
\Delta V_{4}=\Delta \mathrm{v}_{\perp}{ }^{2} \Delta \mathrm{r}_{\perp}{ }^{2}=\pi \mathrm{v}_{/ /}{ }^{2} \mathrm{~d} \Omega \mathrm{dS}=(2 \pi \mathrm{e} / \mathrm{m}) \mathrm{Vd} \Omega \mathrm{dS}=\text { constant }
$$

utilizing the non-relativistic relation $\mathrm{v}_{/ /}{ }^{2}=2 \mathrm{eV} / \mathrm{m}$. The values of $\mathrm{V}, \mathrm{d} \Omega$ and dS may vary for different conjugated planes, but invariance of emittance implies that their product is the same. From equation (3.6.2) it follows that the spatial magnification from object to image plane $\mathrm{Mr}_{\mathrm{r}}$ $\left(=\sqrt{d S_{i}} / \sqrt{d S_{0}}\right)$ is related to the corresponding angular magnification $M_{\alpha}$ $\left(=\sqrt{a \Omega_{i}} / \sqrt{d \Omega_{0}}\right)$ as

$$
M_{r} M_{\alpha}=\sqrt{\frac{V_{0}}{V_{i}}}
$$

which is known as the law of Helmholtz-Lagrange.

An alternative way to express the invariance of emittance is obtained by introducing the concept of beam brightness. The normalized differential brightness of a bundle of rays, carrying a current $d I$, is defined as

$$
\beta_{\mathrm{a}}=\frac{\mathrm{dI}}{\mathrm{V} \mathrm{d} \Omega \mathrm{dS}}
$$

It is called differential brightness, because it is a function of the infinitesimal quantities $d I, d \Omega$ and $d S$, whereas the average (or total) brightness is a function of $I, \Omega$ and $S$. It is referred to as normalized, in order to distinguish: it from the usual differential brightness $\beta=(d I / d \Omega d S)$, which differs by a factor $V$. As the current $d I$ remains constant during the flight, conservation of emittance, expressed by equation (3.6.2), implies conservation of normalized differential brightness $\beta_{p}$. The reader is referred to Lejeune and Aubert (1980) for a more detailed discussion on the subject of emittance and brightness invariance.

Brightness is an important concept in the description of 
particle beams in probe forming instruments. It is used to characterize the gun performance. Given the brightness of the beam near the gun, a lower limit for the probe size in the target plane can be obtained as function of the beam current and the beam angle at this location. In order to explain this statement, consider a beam in the vicinity of the target plane, with a beam semi-angle $\alpha_{p}$, beam current $I$ and beam potential $V$, Let $\beta_{\mathrm{n}}$ be the normalized brightness of the gun. Using equation (3.6.4), with $d \Omega=\Omega=\pi \alpha_{p}{ }^{2}$ and $d S=S=\pi r_{p}{ }^{2}$, one finds that the probe radius $r_{p}$ should satisfy the following relation

$$
r_{p} \geq \frac{1}{\pi \alpha_{p}} \sqrt{\frac{I}{\beta_{n} V}}
$$

The actual probe radius $r_{p}$ will be larger than the value given by the right hand side of equation (3.6.5), due to the aberrations of the optical components of the system and the effect of particle interactions. It should be noted that the aberrations of the optical components do not affect the differential brightness, which is invariant even in the presence of external (conservative) fields. But they do cause a reduction of the average brightness, which is the relevant parameter in equation (3.6.5). On the other hand, the presence of particle interactions renders Liouville's theorem inapplicable in $\Gamma_{6}$ space. Accordingly the differential brightness is not invariant, but deteriorates during the flight of the particles through the system.

\subsection{Beam temperature}

The velocity distribution of the particles within the beam depends in general on the position $r$ within the beam, as is formally expressed by the single particle distribution function $f(r, v, t)$, given by equation (3.5.1). The properties of the local velocity distribution are of ten specified in terms of a beam temperature. In this section we will follow this approach and utilize some principles originating from the kinetic theory of gases.

For an isolated gas in thermodynamic equilibrium, it is well known that the particle velocities are distributed according to the Maxwell-Boltzmann distribution

$$
f(v)=\left(\frac{m}{2 \pi k_{B} T}\right)^{3 / 2} e^{-m v^{2} / 2 k_{B} T}
$$

where $k_{B}$ is the Boltzmann constant. This is a three dimensional timeindependent Gaussian distribution with zero average and a mean square value $\left\langle v^{2}\right\rangle=3 k_{B} T / m$. The zero average value indicates that the gas is at rest, from a macroscopic point of view. According to equation (3.7.1) the average kinetic energy per particle is related to the temperature $T$ as

$$
\frac{1}{2} m\left\langle v^{2}\right\rangle=\frac{1}{2} m\left(\left\langle v_{x}^{2}\right\rangle+\left\langle v_{y}^{2}\right\rangle+\left\langle v_{z}^{2}\right\rangle\right)=\frac{3}{2} k_{B} T
$$

This equation expresses that a so-called thermal energy of $k_{B} T / 2$ is associated with every degree of freedom. In absence of external forces the velocity distribution will show no correlation with the spatial coordinates.

Now, let us return to the case of a rotational symmetric particle beam. Clearly, relation (3.7.2) can not straightforwardly be 


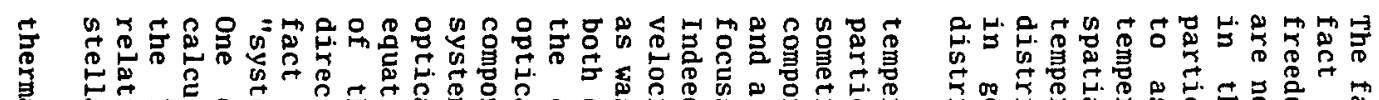

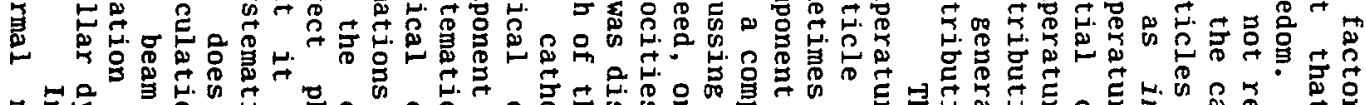

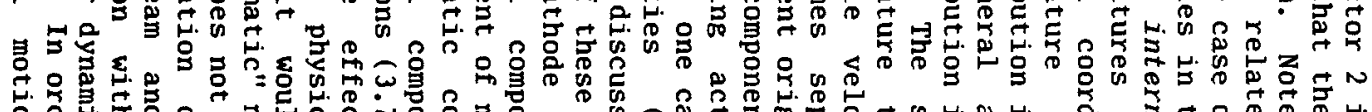

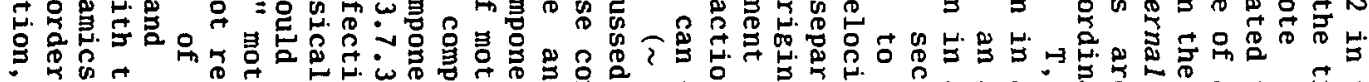

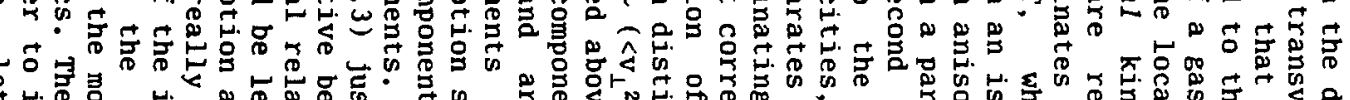

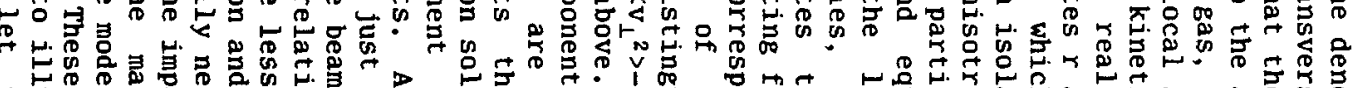

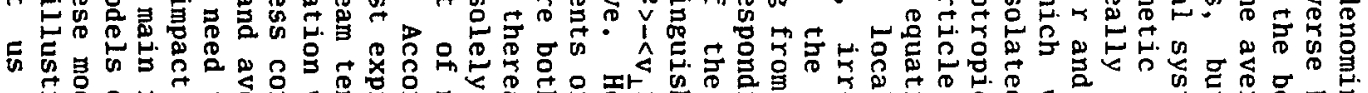

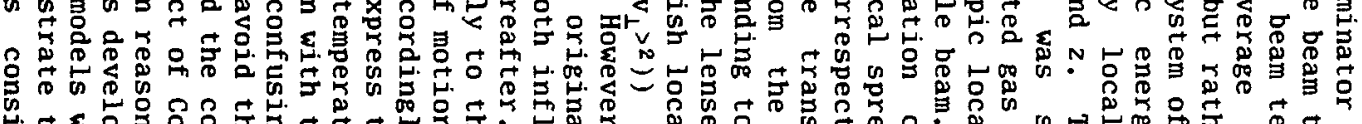

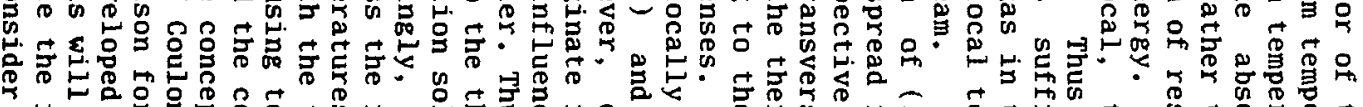

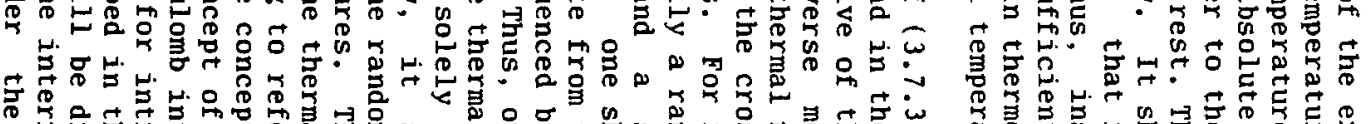

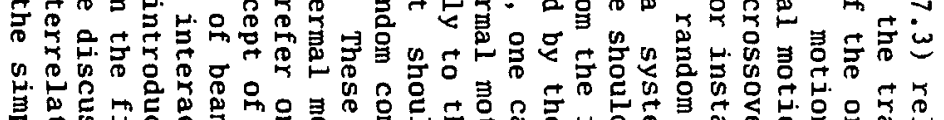

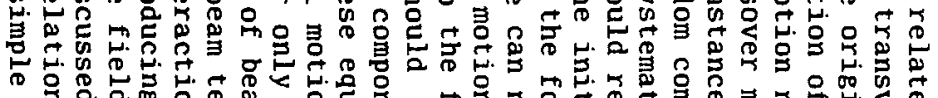

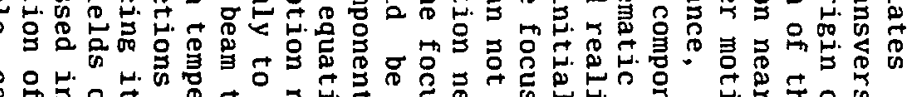

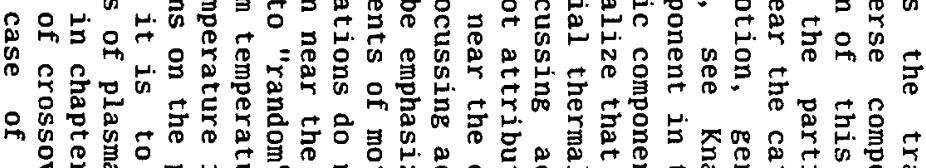

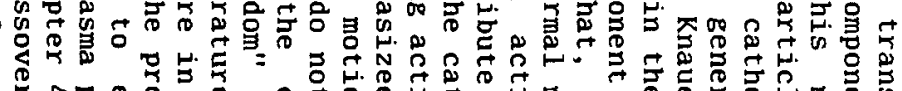

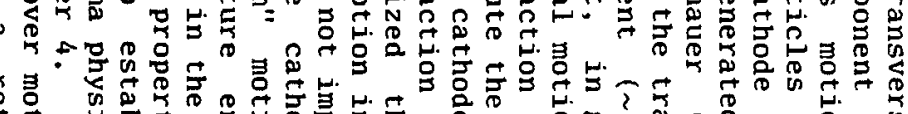
等

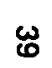

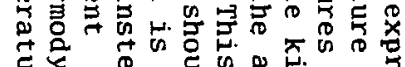

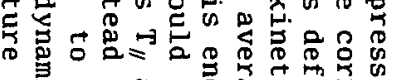

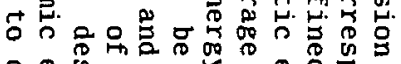

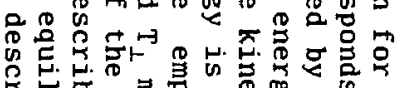

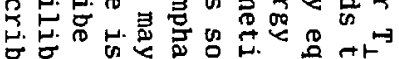

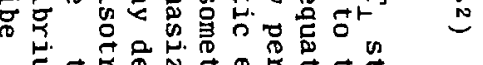

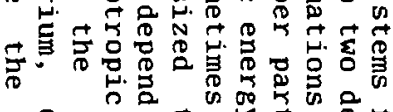

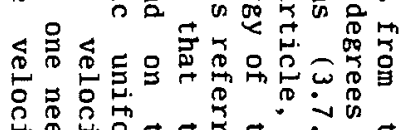

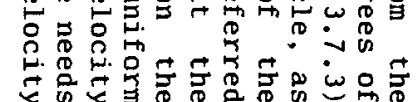

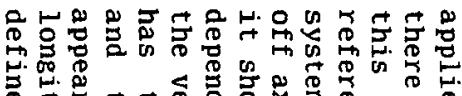

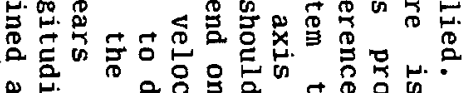

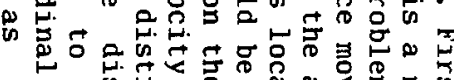

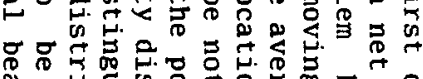

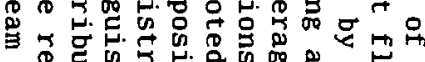

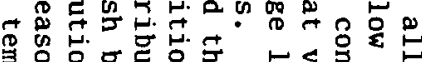

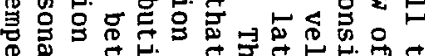

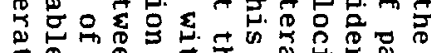

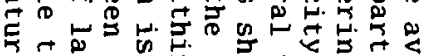
万人

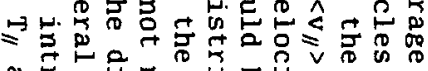

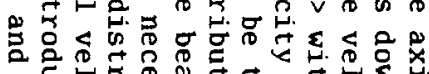

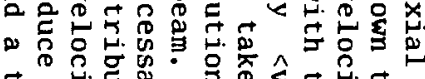

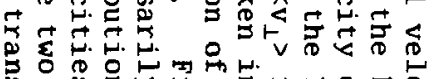

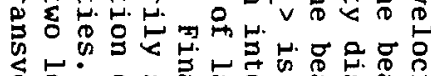

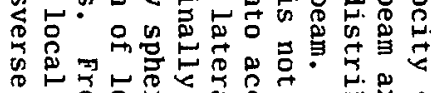

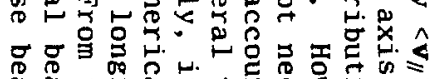

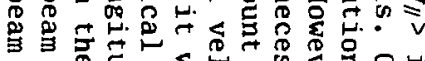

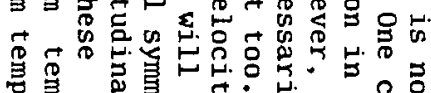

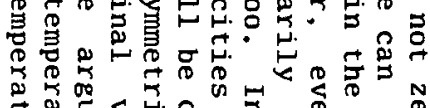

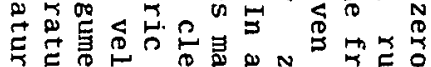
万ิ

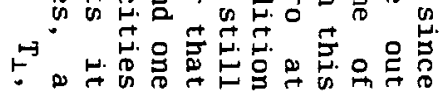


symmetric beam segment with a narrow crossover. Assume that the crossover is an image of a thermionic source (or source crossover). Let the angular distribution be uniform, with a beam semi-angle $\alpha_{0}$. According to the second equation of $(3.7 .3)$, the transverse beam temperature in the crossover can now be expressed as

$$
\mathrm{T}_{\perp \perp}=\frac{\mathrm{mv}_{l /}^{2}}{2 \mathrm{k}_{B}}\left\langle\alpha^{2}\right\rangle=\frac{\mathrm{eV}}{2 \mathrm{k}_{\mathrm{B}}} \alpha_{0}^{2}
$$

using $\left\langle\alpha^{2}\right\rangle=\alpha_{0}{ }^{2} / 2$ and equation (3.2.2). For a Gaussian angular distribution one obtains the same result when defining $\alpha_{0}=2 \sigma_{\alpha}$ (with $\left.\left\langle\alpha^{2}\right\rangle=2 \sigma_{\alpha}^{2}=\alpha_{0}^{2} / 2\right)$. The crossover temperature $\mathrm{T}_{\perp}$ can be regarded as the result of the initial thermal motion near the source, but might as well be expressed in terms of the crossover motion related to the focussing action of the preceding lenses.

The definition of beam temperature allows us to describe the properties of the beam in terms of thermodynamical quantities, which obey thermodynamical relations (By thermodynamical quantities we mean temperature, volume, particle density, entropy, etc). We will not pursue this approach in detail and just note that such a description is equivalent to the description in terms of geometrical properties, as well as to the description in terms of statistical mechanical properties, such as the single-particle distribution function $f(r, v, t)$. As an illustration of the equivalence between the thermodynamical description of the beam and the statistical mechanical approach, we will show that the implications of Liouville's theorem, discussed in section 3.6, can also be understood from thermodynamical considerations. The application of Liouville's theorem to a rotational symmetric beam of identical non-interacting particles, lead to the conclusion that the phase space volumes $\Delta V_{l l}$ and $\Delta V_{1}$ are invariant. In order to obtain this result within the thermodynamical description, one should visualize the beam as an isolated ideal atomair gas of volume $V$ and temperature $T$. As it flows through the system it changes its volume $V$. The corresponding change in the temperature $T$ can be found from

$$
\mathrm{TV}^{2} \text { If }=\text { constant }
$$

which is the adiabatic equation for an ideal gas with $f$ degrees of freedom. Since the longitudinal and the lateral motion of the particles are independent, equation (3.7.5) should be applied separately to the longitudinal degree of freedom $\left(f_{\| /}=1\right)$ and the lateral degrees of freedom $\left(f_{\perp}=2\right)$. By substitution of equations $(3.7 .3) ; \quad v_{/ /} \sim \Delta r_{/ /}$and $v_{\perp} \sim \Delta r_{\perp}{ }^{2}$ into equation $(3.7 .5)$ one finds

$$
\left\langle\Delta v_{/ /}^{2}\right\rangle \Delta r_{/ /}^{2}=\text { constant, }\left\langle\Delta v_{\perp}^{2}\right\rangle \Delta r_{\perp}^{2}=\text { constant }
$$

which is equivalent to the equations $(3.6 .1)$ and $(3.6 .2)$ respectively, obtained from Liouville's theorem, q.e.d.

We like to investigate the dependency of the beam temperatures $T_{/ \prime}$ and $T_{\perp}$ on the experimental parameters. We will first focus on the behaviour of the longitudinal beam temperature $T_{\| /}$and next consider the transverse beam temperature $T_{\perp}$. In general, these temperatures are determined by the emission process, the acceleration of the beam, and the focal action of the optical components of the system. Let us examine each of these elements for the specific case of an electron beam produced by a thermionic source. The velocity distribution of the electrons emitted from the cathode, with temperature $T_{c a}$, is given by the so-called half Maxwell-Boltzmann distribution 


$$
f_{i}\left(v_{i}\right)=\frac{1}{2 \pi}\left(\frac{m}{k_{B} T_{c a}}\right)^{2} e^{-m v_{i} 2 / 2 k_{B} T_{c a}} v_{i / /} \theta\left(v_{i} / /\right)
$$

in which the subscript $i$ refers to the initial situation, before acceleration. The step-function $\theta(x)$ is defined as

$$
\theta(x)= \begin{cases}1 & \text { for } x \geqslant 0 \\ 0 & \text { for } x<0\end{cases}
$$

Equation (3.7.7) is also used to describe the velocity distribution of the particles of a gas escaping from a container into vacuum through a small hole, when the velocities of the particles inside the container obey the Maxwell-Boltzmann distribution given by equation (3.7.1). The step function accounts for the fact that only those electrons, which have a positive axial velocity, will emerge from the cathode. The factor $v_{i / l}$ stems from the fact that the electrons which leave the cathode surface area $d S$, between times $t$ and $t+d t$, with an axial velocity $v_{i} /$. come from a depth $d z=v_{i / /} d t$. This group of electrons fills an element of volume of coordinate space dr, which is equal to

$$
\mathrm{dr}=\mathbf{v}_{\mathbf{i} / /} \mathrm{dSdt}
$$

The number of electrons passing through $d S$ in time $d t$, with an axial velocity between $v_{i} /$ and $v_{i} / d v_{i} /$, follows by multiplication with the density inside the metal of particles within this velocity range. Consequently, the velocity distributions inside and outside the metal differ by a factor $v_{i / /}$. Clearly, the normalization constant appearing in the velocity distributions differs too. As a result of the additional factor $v_{i / /}$, equation (3.7.7) fulfils Lambert's law (or cosine law): The angular current density emitted in any direction is proportional to the cosine of the angle between this direction and the normal. Finally, we note that equation (3.7.7) presupposes that the workfunction is constant along the emitting part of cathode surface and disregards the effect of energy dependent reflections of the electrons at the inner surface of the cathode. For a detailed description of the theory of thermionic emission, the reader is referred to Nottingham (1956), Lindsay (1960), Franzen and Porter (1975) and Lauer (1982).

By applying the definitions of beam temperature, given by equations $(3.7 .3)$, to the velocity distribution of equation $(3.7 .7)$, one finds

$$
\mathrm{T}_{\mathrm{i} / /}=2(1-\pi / 4) \mathrm{T}_{\mathrm{ca}} \approx 0.429 \mathrm{~T}_{\mathrm{ca}}, \quad \mathrm{T}_{\mathrm{i}_{\perp}}=\mathrm{T}_{\mathrm{ca}}
$$

Thus, the transverse beam temperature of the electrons leaving the cathode is equal to the cathode temperature, but a smaller value is found for the longitudinal beam temperature.

After emission, the electrons are accelerated in the axial direction by a potential $v$. The final axial velocity of an electron $v_{f} /$ is related to its initial axial velocity $\nabla_{i} /$ as

$$
v_{f} \|^{2}=\nabla_{i} / /^{2}+2 \mathrm{eV} / \mathrm{m}
$$

The transverse velocity of the electrons remains unchanged, disregarding the focussing properties of the gun. Conservation of the number of particles implies

$$
f_{f}\left(v_{f}\right) d v_{f}=f_{i}\left(v_{i}\right) d v_{i}
$$


By substituting equations (3.7.7) and (3.7.10) in the right hand of equation (3.7.11), one finds for the velocity distribution after acceleration

$$
f_{f}\left(v_{f}\right)=\frac{1}{2 \pi}\left(\frac{m}{k_{B} T_{c a}}\right)^{2} e^{-\left(m v_{f}{ }^{2}-2 e V\right) / 2 k_{B} T_{c a}} v_{f / l} \theta\left(v_{f} / /^{2}-2 e V / m\right)
$$

As far as the lateral component of velocity is concerned, this distribution is identical to the initial distribution, described by equation (3.7.7). However, it is significantly contracted in the axial direction. Utilizing equations $(3.7 .10)$ and $(3.7 .11)$ one may write

$$
\begin{aligned}
\left\langle\mathrm{v}_{\mathrm{f}} \|^{2}\right\rangle & =\left\langle\mathrm{v}_{\mathrm{i}} \|^{2}+2 \mathrm{eV} / \mathrm{m}\right\rangle=\left\langle\mathrm{v}_{1 / \|^{2}}\right\rangle+2 \mathrm{eV} / \mathrm{m} \\
\left\langle\mathrm{v}_{\mathrm{f}}\right\rangle^{2} & =\left\langle\left(\mathrm{v}_{\mathrm{i} / \|^{2}}+2 \mathrm{eV} / \mathrm{m}\right)^{1 / 2}\right\rangle \mathbf{2} \\
& =(2 \mathrm{eV} / \mathrm{m})\left\{1+(\mathrm{m} / 4 \mathrm{eV})\left\langle\mathrm{v}_{1} \|^{2}\right\rangle-(\mathrm{m} / 4 \mathrm{eV})^{2}\left\langle\mathrm{v}_{\mathrm{i}} \|^{4}\right\rangle+\cdots\right\}^{2} \\
& =\left\langle\mathrm{v}_{\mathrm{i}} \|^{2}\right\rangle+2 \mathrm{eV} / \mathrm{m}+(\mathrm{m} / 8 \mathrm{eV})\left(\left\langle\mathrm{v}_{1} \|^{4}\right\rangle-\left\langle\mathrm{v}_{1} \|^{2}\right\rangle^{2}\right)+\cdots
\end{aligned}
$$

Hence, it follows from equations $(3.7 .3)$ and $(3.7 .7)$ that

$$
T_{f} \approx \frac{m^{2}}{8 k_{B} e V}\left(\left\langle v_{i} \|^{4}\right\rangle-\left\langle v_{i} \|^{2}\right\rangle 2\right)=\frac{k_{B} T_{c a}}{2 e V} T_{c a}
$$

assuming that $\mathrm{eV} \gg \mathrm{m}\left\langle\mathrm{v}_{\mathrm{i}} \|^{2}\right\rangle$. Comparison with equation (3.7.9) learns that the acceleration leads to a decrease of the longitudinal beam temperature by factor $\mathrm{k}_{\mathrm{B}} \mathrm{T}_{\mathrm{c} a} /(4-\pi) \mathrm{eV} \approx 1.16 \mathrm{k}_{\mathrm{B}} \mathrm{T}_{\mathrm{c}} \mathrm{a} / \mathrm{eV}$. In practical beams this factor is of the order 10-5. Thus, in absence of interactions, the electrons leaving the thermionic gun, show a negligible axial velocity spread compared to the lateral velocity spread. The latter corresponds to the temperature $T_{c a}$ of the cathode surface.

The reduction of axial velocity spread, due to the acceleration, is a phenomenon which is of crucial importance to the theory of statistical Coulomb interactions between the beam particles.- It can be derived in different ways. It is sometimes presented as a consequence of the quadratic relation between kinetic energy and velocity. This interpretation might be clear from the derivation given above, but can also directly be understood from the following argument. Consider two particles moving in the axial direction with velocities $v$ and $v+\Delta v$ respectively. The difference in the kinetic energy of the particles $\Delta E$ is given by

$$
\Delta E=\frac{1}{2} m\left[(v+\Delta v)^{2}-v^{2}\right] \approx m v \Delta v
$$

assuming that $v>>\Delta v$. The acceleration of the particles does not affect the value of $\Delta E$, since both particles gain the same amount of energy. However, the velocity $v$ is (by definition) increased during acceleration. Consequently, $\Delta v$ must decrease proportionally in order to leave the value of the right hand side of equation (3.7.14) unaffected.

Another way to understand the same phenomenon, is to compare the expressions for the increment in energy $E_{f}-E_{i}$ and the increment in momentum $m\left(v_{f}-v_{i}\right)$ experienced by a particle during acceleration with a force $F_{a}$ along a distance $z_{1}-z_{0}$ over a time interval $t_{f}-t_{i}$

$$
E_{f}-E_{i}=\int_{z_{0}}^{z_{1}} d z F_{a}, \quad m\left(v_{f}-v_{i}\right)=\int_{t_{i}}^{t_{f}} d t F_{a}
$$

All particles in the beam travel the same distance $z_{1}-z_{0}$ and 
consequently gain equal amounts of energy. However, the flight time $t_{f}-t_{i}$ depends on the initial velocity $v_{i}$ of a particle. Particles with a relatively large initial velocity will have a relatively short flight time, while particles with a relatively small initial velocity will have a relatively long flight time. Accordingly, fast particles gain less velocity than slow particles, causing a reduction of the velocity spread.

The decrease in velocity spread due to the acceleration, can also be presented as a consequence of Liouville's theorem. It was found that the application of this theorem to the subspace $\Gamma_{2}$ of axial coordinates, implies that the volume $\Delta V_{2}=\Delta r_{\|} \Delta v_{\| /}$is invariant, see equation (3.6.1). It was also found that the length $\Delta r_{\| \prime}$ is directly proportional to the axial velocity $v_{/ /}$. Thus $\Delta r_{/ \prime}$ increases during acceleration. As $\Delta r_{\|} \Delta v_{\|}$is invariant, the axial velocity spread $\Delta v_{\|}$ decreases accordingly, q.e.d. Finally, we recall that equation (3.6.1) is equivalent to the adiabatic equation for an 1-dimensional ideal gas, which is given by equation (3.7.5) (taking $f=1$ ). Thus, in the thermodynamical description of the beam, one may envision the reduction of the axial velocity spread as a "cooling-effect" caused by the adiabatic expansion of the "gas" of charged particles during acceleration.

In general Liouville's theorem, applied to the appropriate phase space, expresses that an expansion of the spatial volume must coincide with a compression of the velocity space. Clearly, this principle does not only affect the longitudinal beam temperature, but the transverse beam temperature as well. Initially, the transverse beam temperature $T_{\perp}$ is identical to the cathode temperature $T_{c a}$, as is expressed by the second equation of (3.7.9). As the particles flow through the system, their lateral separation changes with the beam radius. Conservation of emittance, which is a consequence of Liouville's theorem, implies that the transverse beam temperature $T_{\perp}$, being directly proportional to the transverse velocity spread, increases with decreasing beam radius and visa versa. To illustrate this point, consider the beam geometry of figure 3.4 and the corresponding emittance plots of figure 3.5. The crossover plane is an image of the source, while the plane $P$ is an image of the entrance pupil. For this particular combination of planes, emittance invariance can be expressed as $\Delta r_{p}^{\prime} r_{p}=\Delta r_{c}^{\prime} r_{c}$. Using equation (3.7.3) and $\Delta v_{1}=v_{\| /} \Delta r^{\prime}$, one finds for the relation between the transverse beam temperatures in these planes

$$
T_{\perp p}=\frac{\Delta r_{p}^{\prime}}{\Delta r_{c}^{\prime 2}} T_{\perp c}=\frac{r_{c}^{2}}{r_{p}^{2}} T_{\perp c}
$$

For other planes than the set of planes considered here, this relation holds only approximately, due to edge effects, as can be seen from figure 3.5. Thus, in general

$$
T_{\perp} \approx \frac{r_{c}^{2}}{r_{0}(z)^{2}} T_{\perp c}
$$

Notice that the transverse beam temperature is identical in all points of a plane perpendicular to the beam axis. Thus, the transverse beam temperature $T_{\perp}$ depends on the axial position $z$, but not on the radial coordinate $r$, By combining the equations (3.7.4) and (3.7.17) one obtains

$$
T_{\perp} \approx \frac{e V}{2 k_{B}} \frac{\left(\alpha_{0} r_{c}\right)^{2}}{r_{0}(z)^{2}}
$$




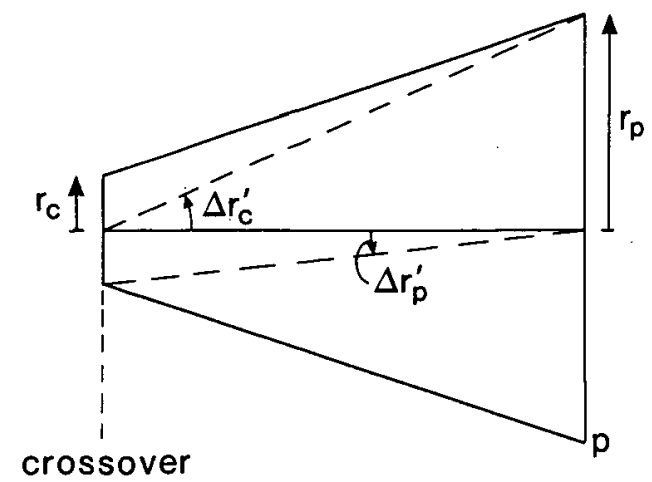

Figure 3.4 Schematic view of a beam section between a crossover and a plane $P$, which is an image of the entrance pupil of the system.

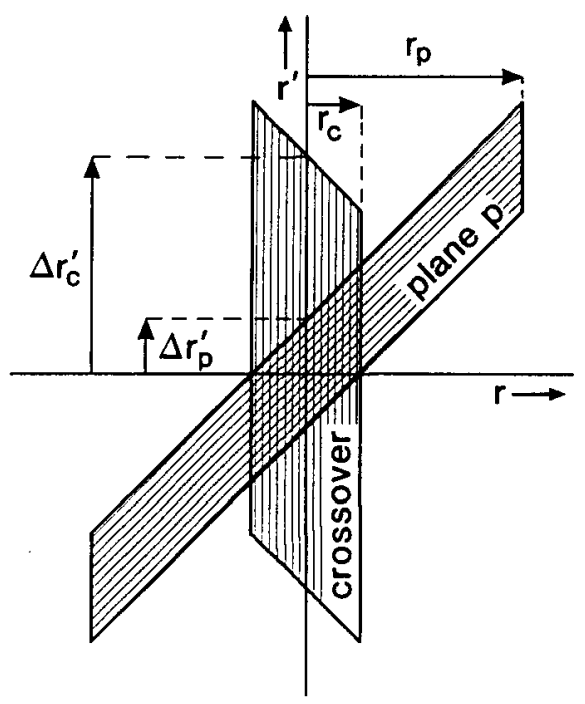

Figure 3.5 Emittance plots corresponding to the crossover plane and the plane $P$, shown in figure 3.4 
This equation relates the transverse beam temperature $T_{1}$, at an arbitrary location within the beam segment, to the value of the geometrical parameters. Alternatively, this relation can be expressed in terms of the normalized brightness $\beta_{n}$, given by equation $(3.6 .4)$, and the current density $J=I / \pi r_{0}(z)^{2}$

$$
\beta_{\mathrm{n}}=\frac{\mathrm{I}}{\mathrm{V} \pi \alpha_{0}^{2} \pi \mathrm{r}_{\mathrm{c}}^{2}} \approx \frac{\mathrm{eJ}}{2 \pi \mathrm{k}_{\mathrm{B}} \mathrm{T}_{\perp}}
$$

in which it was assumed that the current density is constant in every cross section of the beam (Accordingly, differential brightness and total brightness are not distinguished). As $\beta_{n}$ is conserved, the ratio of $\mathrm{J}$ and $\mathrm{T}_{\perp}$ is conserved too. In other words, conservation of normalized brightness (or emittance) implies that $T_{\perp}$ is directly proportional to $\mathrm{J}$. Equation (3.7.19) is equivalent to Langmuir's equation for the maximum current density obtainable in the spot of a cathode-ray tube, Langmuir (1937). Langmuir's result pertains to the case of a half MaxwellBoltzmann distribution and therefore differs by a factor 2 . In addition, we note that equation $(3.7 .19)$, unlike Langmuir's equation, requires that $\mathrm{eV} / \mathrm{k}_{\mathrm{B}} \mathrm{T} \gg>1$.

When the total emittance is conserved through the entire system, equation (3.7.17) can be generalized to include all segments of the beam. As a result, one can relate the transverse beam temperature $T_{1}$, at an arbitrary location in the beam, to the temperature of the cathode $T_{c}$ a , using

$$
T_{\perp} \approx \frac{r_{c a}}{r_{0}(z)^{2}} T_{c a}
$$

where $r_{c a}$ is the radius of the beam at the cathode. It should be emphasized that this equation presupposes conservation of the total emittance. Accordingly, lens aberrations, beam limiting apertures and particle interactions are assumed to be absent.

Equations $(3.7 .13),(3.7 .18)$ and $(3.7 .20)$ yield the main results of this section. From equation (3.7.20) it follows that the transverse beam temperature is large in crossovers and small in the dilute parts of the beam. In practical beams, the maximum value of $r_{0} / r_{c a}$ is of the order $10^{2}$. Thus, the corresponding reduction of the transverse beam temperature, relative to the cathode temperature $T_{c}$ a, is of the order $10^{4}$. According to equation (3.7.13), the longitudinal beam. temperature $T_{l}$ is of the order of $10^{-5}$ times $T_{c a}$. Thus, disregarding the effect of particle interactions, one may in general assume that $T_{/ /}<T_{\perp}$, even in the dilute parts of the beam. The difference between both temperatures will be the largest in the crossover area, where the beam radius is the smallest.

\subsection{Thermodynamic limits for particle interaction effects}

In the previous section it was demonstrated that non-equilibrium situations in particle beams may occur, as a consequence of the expansion and compression of the beam volume, under influence of the acceleration in the gun-area and the focussing action of lenses. Coulomb interactions between the beam particles were disregarded so far. However, they do affect the velocity distribution of the particles, as well as their spatial distribution and thus change the macroscopic properties of the beam. In the thermodynamical description of the beam, the particle interactions cause a relaxation towards equilibrium. Within 
this model one can estimate the effect of particle interactions, by assuming that the relaxation is complete, which means that thermodynamic equilibrium is established during the flight. As we are primarily interested in the principle aspects involved, the analysis will be restricted to the generation of energy spread (the Boersch effect).

The speed of the relaxation process depends on the frequency of the collisions and the average impact per collision. Consequently, the relaxation times for the different interaction effects, depend on the particle density in the beam and the velocity distribution of the particles. The question, whether equilibrium will be established or not, can be answered by comparing the appropriate relaxation times with the time of flight $T_{f}$, given by equation (3.2.3). Anticipating on the results of sections 4.8 and 7.7 , we mention that the equilibrium condition is in general not reached in practical beams. Consequently, equilibrium thermodynamics, as will be employed here, is not suited for an accurate quantitative calculation of the particle interaction effects and the results merely serve to determine upper limits.

In section 3.7, it was demonstrated that the longitudinal beam temperature $T_{\|}$is in general expected to be negligible compared to the maximum transverse beam temperature $T_{\perp}$, which occurs in the crossover area's of the beam. Now, assume that thermodynamic equilibrium is reached in the crossover. The corresponding equilibrium temperature $T_{e}$ will be given by

$$
\mathrm{T}_{\mathrm{B}}=\frac{2}{3} \mathrm{~T}_{\mathrm{C}_{\perp}}=\frac{2 \mathrm{eV}}{3 \mathrm{k}_{\mathrm{B}}}\left\langle\alpha^{2}\right\rangle
$$

utilizing equation (3.7.4). The factor $2 / 3$ stems from the fact that the transverse beam temperature corresponds to two degrees of freedom and the longitudinal to one. The corresponding normal root mean square (rms) energy spread $\left\langle\Delta \mathrm{E}_{/ /}{ }^{2}\right\rangle 1 / 2$ can be found by employing the nonrelativistic relation

$$
\frac{<\Delta \mathrm{E}_{/ / 2}>_{1 / 2}}{\mathrm{eV}} \approx \frac{2<\Delta \mathrm{V}_{/ /}^{2}>1 / 2}{\mathrm{v}_{/ /}}=\left(\frac{2 \mathrm{k}_{\mathrm{B}} \mathrm{T} / /}{\mathrm{eV}}\right)^{1 / 2}
$$

utilizing equations (3.7.14) and (3.7.3). The first step presupposes that $\left\langle\Delta \mathrm{E}_{/ /}{ }^{2}>1 / 2<<\mathrm{eV}\right.$. From equations $(3.8 .1)$ and $(3.8 .2)$ one finds that the equilibrium condition leads to an energy spread

$$
\frac{<\Delta E_{\| / 2}^{2}>1 / 2}{e V} \approx \frac{2}{\sqrt{3}}<\alpha^{2}>1 / 2=(2 / 3) 1 / 2 \alpha_{0}
$$

In the last step we used $\left\langle\alpha^{2}\right\rangle=\alpha_{0} 2 / 2$, which pertains to the case of an uniform angular distribution. For a Gaussian angular distribution one should use $\left\langle\alpha^{2}\right\rangle=2 \sigma_{\alpha}{ }^{2}$. Equation (3.8.3) imposes an upper limit to the energy spread generated by conversion of kinetic energy from the lateral degrees of freedom to the longitudinal degree of freedom. For the numerical example of section $3.2\left(\mathrm{~V}=10 \mathrm{kV}, \alpha_{0}=10 \mathrm{mR}\right)$, one obtains $<\Delta E_{/ /}{ }^{2}>1 / 2=82 \mathrm{eV}$.

Equation (3.8.3) does not impose a real upper limit to total generated energy spread under all circumstances, as is suggested by some authors, for instance see Crewe (1978a), Knauer (1979a) and Rose and Spehr (1983). It just refers to the transition of kinetic energy from one degree of freedom to another (relaxation of kinetic energy) and does not account for the conversion of potential energy into random velocity components (relaxation of potential energy).

In order to clarify this statement, consider the extreme case of a monochromatic homocentric cylindrical beam. In such a beam all 
particles move initially with identical velocity along parallel trajectories. Observed in the frame of reference moving with the beam, the particles are at rest. Consequently, there is no internal kinetic energy to be converted. Indeed, equation (3.8.3) predicts a zero upper limit for the generated energy spread, since $\alpha_{0}=0$. In other words, according to this analysis the Boersch effect does not occur in such a beam.

However, some energy spread can be generated in such a beam, due to the relaxation of potential energy. The particles entering the beam all have the same axial velocity, but are otherwise randomly distributed over the beam volume. The mutual potential energy of a pair of particles depends on their relative position. As these positions are assumed to be randomly distributed, the potential energy will vary for each pair of particles. Near neighbours can have a substantial potential energy. Due to the Coulomb interaction during the flight, part of this potential energy will be redistributed into kinetic energy. As the associated velocity components are fully stochastic, the potential energy relaxation causes an increase of the velocity spread. The increase of the axial velocity spread corresponds to the Boersch effect.

It would be convenient to have an expression for the upper limit of the Boersch effect caused by potential energy relaxation, similar to equation (3.8.3), specifying the upper limit for the relaxation of kinetic energy. In order to derive such an expression, we have to introduce the concept of Debeye screening first. This will be done in the next section. As the subject of screening is of general importance to the theory of particle interactions, we will investigate its applicability to a system of identical charged particles in some detail. In section 3.10 we will return to the main line of this chapter and consider the thermodynamic limit for the process of potential energy relaxation.

\subsection{Debeye screening}

We wish to calculate the effective potential field of a fixed charged particle, surrounded by other charged particles, which are statistically distributed. This problem was first considered by Debeye and Huickel (1923). They demonstrated that the potential field of an ion in a strong electrolyte is effectively screened by the cloud of particles surrounding it. The effective force range of the ion is therefore confined within a certain characteristic length, which is determined by the charge density and temperature of the medium. This length is called the Debeye screening length $\lambda_{D}$. Here we will study the applicability of this concept to a non-neutralized system of identical charged particles.

Let $N$ be the total number of particles in the system, $n$ the average particle density, e the particle charge and $m$ the particle mass. Consider a test particle located in the origin $(\mathbf{r}=0)$ of a coordinate system. The coulomb potential field of the test particle is given by

$$
\Phi(\mathbf{r})=C_{c} / r
$$

where $C_{c}=e / 4 \pi \varepsilon_{0}$ (note: $C_{0}=e C_{c}$ ). The other particles are referred to as field particles. The spatial distribution of the field particles around the origin will be affected by the presence of the electrostatic field of the test particle and consequently deviates from an uniform distribution. This redistribution of the particles around the origin creates an electrostatic field, which partly compensates the original 
field of the test particle. The effective screened potential $\Phi_{s}(\mathbf{r})$ of the test particle, is now defined as the sum of the original and the induced potential. The Poisson equation for this potential can be written as

$$
\nabla^{2} \Phi_{s}(r)=\frac{e}{\epsilon_{0}} \delta(r)+\frac{e}{E_{0}} n_{d}(r)
$$

where $\delta(x)=\delta(x) \delta(y) \delta(z)$ is the three-dimensional delta function. The quantity $n_{d}(r)$ is the local deviation from the average particle density $n$, which is induced by the test particle

$$
n_{d}(r)=n_{m}(r)-n \text { with } n_{m}(r)=\sum_{i=1}^{N} \text { e } \delta\left(r-r_{i}\right)
$$

Presupposing thermodynamic equilibrium, the microscopic distribution $\mathrm{n}_{\mathrm{m}}(\mathbf{r})$ will obey Maxwell-Boltzmann statistics (as usual we ignore quantum-mechanical effects, assuming that the particle density is low enough)

$$
n_{m}(r)=n e^{-e \Phi_{s}(r) / k_{B} T}
$$

Substitution into equation $(3.9 .3)$ yields

$$
n_{d}(r)=n\left(e^{-e \Phi_{s}(r) / k_{B} T}-1\right) \approx-\frac{n e \Phi_{s}(r)}{k_{B} T}
$$

The expansion of the exponential function requires $e_{s}(r) / k_{B} T \ll<$, which means that the potential energy of the particles is assumed to be much smaller than their kinetic energy. Substitution of equation (3.9.5) into the Poisson equation $(3.9 .2)$ yields

$$
\nabla^{2} \Phi_{s}(r)+\Phi_{s}(r) / \lambda_{D}^{2}=\frac{e}{\epsilon_{0}} \delta(r)
$$

where $\lambda_{D}$ is the Debeye screening length, defined as

$$
\lambda_{D}=\left(\frac{E_{0} k_{B} T}{e^{2} n}\right)^{1 / 2}
$$

The solution of equation $(3.9 .6)$, with the boundary condition that $\Phi_{s}(r)$ vanishes at infinity, is

$$
\Phi_{s}(r)=\frac{C_{c}}{r} e^{-r / \lambda_{D}}
$$

Thus, for distances $r<<\lambda_{D}$ the effective potential is essentially equal to the bare Coulomb potential, given by equation (3.9.1), while it is strongly reduced for distances $r \geqslant \lambda_{D}$. Thus, roughly speaking, one may say that, according to equation (3.9.8), the effective interaction range of the test particle is limited to the Debeye distance $\lambda_{D}$.

We will continue this section by considering the validity of the screening concept for non-neutralized systems. We have assumed that the main effect of the Coulomb interaction is a microscopic redistribution of the particles, while the macroscopic dimensions of the system remain unchanged. Accordingly, we considered the average density $n$ to be constant. Clearly, for a non-neutralized system of charged particles in a field free space, this can not be entirely true. The collective Coulomb repulsion will cause the system to expand under influence of its 
own space charge, leading to a lower average density. A way out of this problem is to assume that the time scale of the collective process is much longer then the relaxation time associated with the discrete particle effects.

Let us investigate whether such an assumption is justified or not. In order to estimate the time scale of the collective process, we consider a sphere of radius $R$ within the particle gas. The force $F$ acting on a particle located on the surface of the sphere is given by

$$
F=\frac{e^{2} n R}{3 \varepsilon_{0}}
$$

as follows with Gauss's theorem. We now demand that the displacement of the particle, due to the force $F$, during some time interval $T_{f}$, which is representative for the time scale of the system, is small compared to $R$

$$
\frac{\mathrm{FT}_{\mathrm{f}}^{2}}{2 \mathrm{~m}} \ll \mathrm{R}
$$

For the case of a particle beam $T_{f}$, should be chosen equal to the time of flight, which is given by equation (3.2.3). Substitution of equation $(3.9 .9)$ into equation $(3.9 .10)$ yields

$$
T_{f} \ll \frac{\sqrt{6}}{\omega_{p}}
$$

where $\omega_{p}$ is given by

$$
\omega_{p}=\left(\frac{\mathrm{e}^{2} \mathrm{n}}{\mathrm{m} \varepsilon_{0}}\right)^{1 / 2}
$$

This quantity is known as the plasma frequency.

Now, let us estimate the time scale associated with the discrete particle effects. The derivation of the screened potential relies upon the assumption that the density distribution of the field particles, under influence of the electrostatic field of the test particle, obeys Maxwell-Boltzmann statistics, see equation (3.9.4). The relaxation time required for the system to establish this equilibrium condition, can roughly be estimated as the time in which a particle, with a typical thermal velocity $V_{\otimes}\left(k_{B} T / m\right) 1 / 2$, travels over the Debeye distance $\lambda_{D}$. Thus, thermodynamic equilibrium requires

$$
T_{f} \gg \frac{\lambda_{D}}{\left(k_{B} T / m\right)^{1 / 2}}
$$

Substitution of the expression for $\lambda_{\mathrm{D}}$, given by equation (3.9.7), yields

$$
T_{f} \gg \frac{1}{\omega_{p}}
$$

where $\omega_{p}$ is again given by equation (3.9.12). Debeye screening requires the relaxation time $1 / \omega_{p}$ to be short compared to the timescale of the system $\mathbf{T}_{\mathbf{f}}$.

Clearly, the constraints of equations (3.9.11) and $(3.9 .14)$ can not be fulfilled simultaneously. This analysis shows that, in a system of identical charged particles, collective effects and discrete particle effects take place on the same time scale, characterized by the quantity $1 / \omega_{\mathrm{p}}$. Thus, one may not assume that the redistribution of nearest neighbours occurs much faster than the spreading under influence 


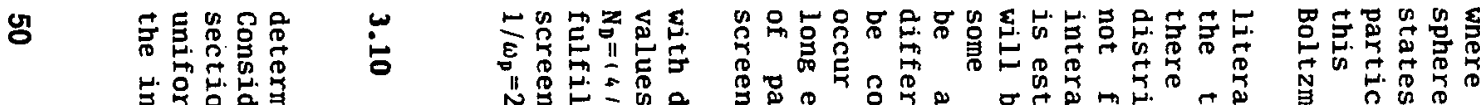

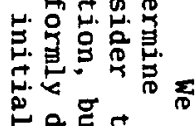

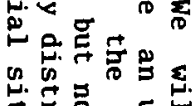

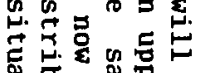

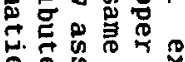

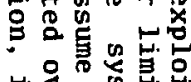

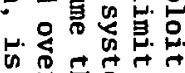

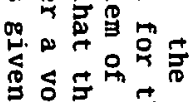

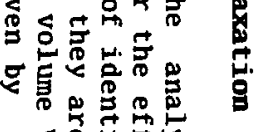

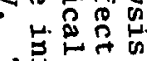

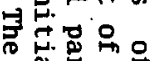

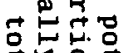

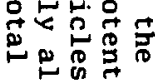

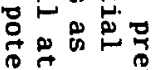

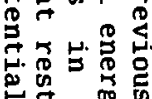

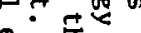
$\overbrace{0}^{\circ}$

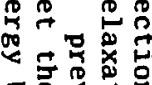

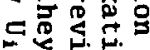

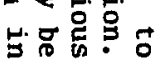

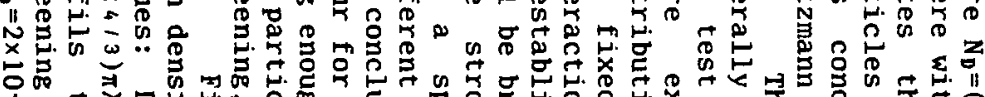

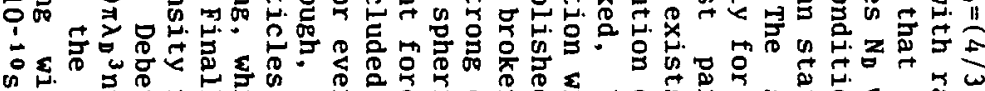

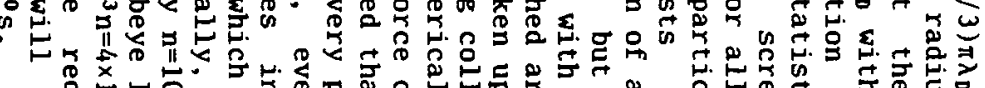

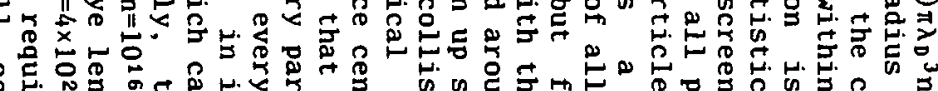

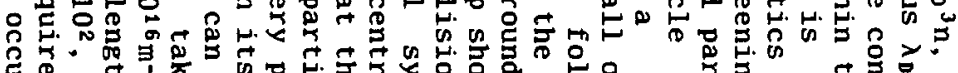

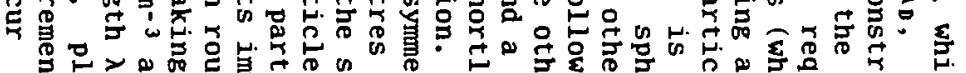

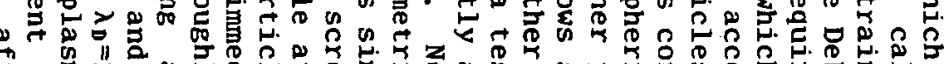

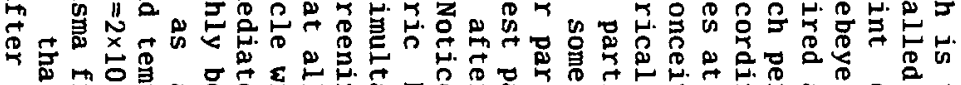

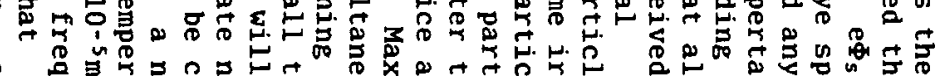

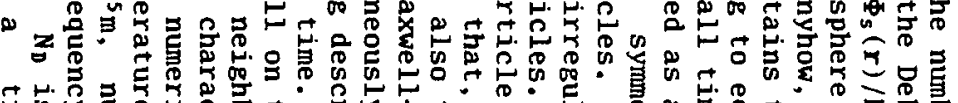

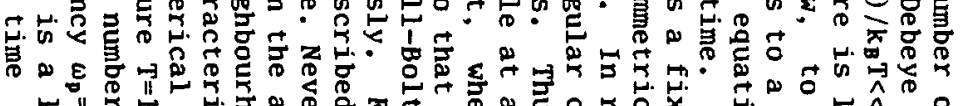

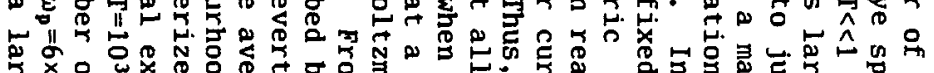

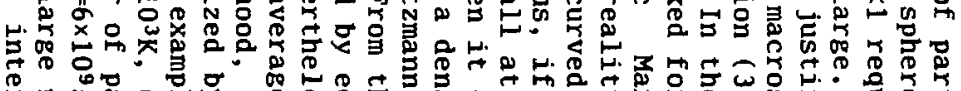

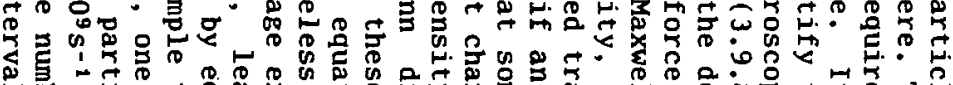
等

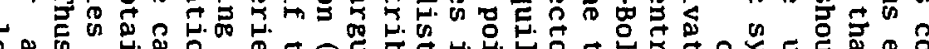

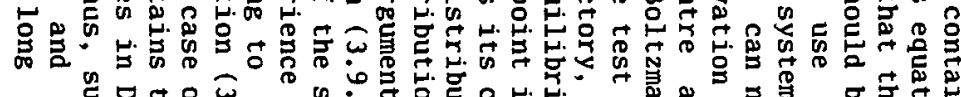

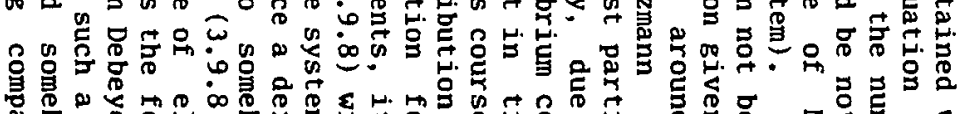

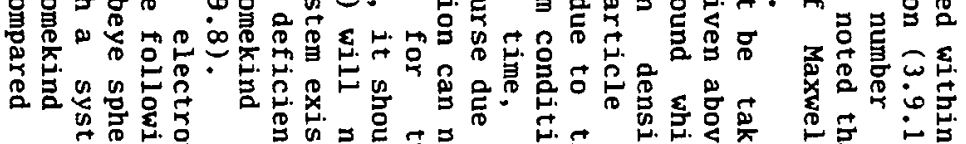

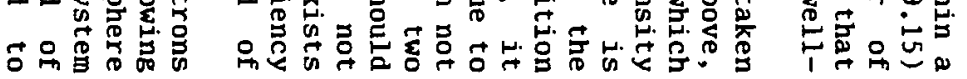

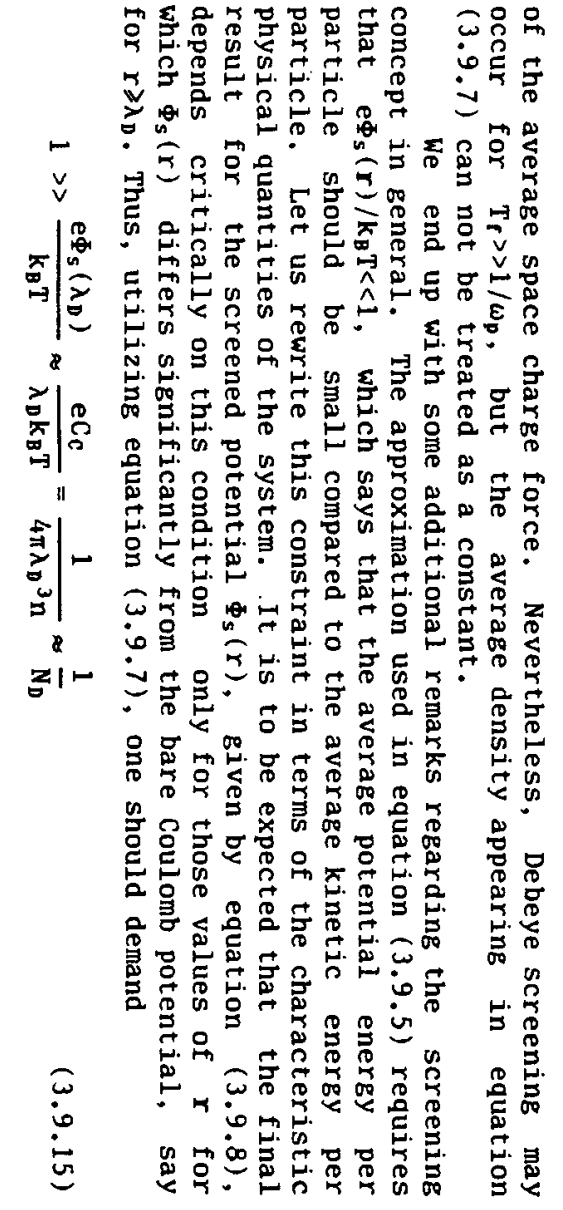




$$
U_{i}=\frac{1}{2} \sum_{\substack{i, j=1 \\ i \neq j}}^{N} \frac{C_{0}}{r_{i j}} \approx \frac{1}{2} n^{2} \int_{V} d r_{i} \int_{V} d r_{j} \frac{C_{0}}{r_{i j}}
$$

where $r_{i j}=\left|r_{i}-r_{j}\right|$, the distance between particle $i$ and $j$. For a large volume $v$, one may ignore edge-effects and approximate

$$
U_{i} \approx \frac{1}{2} n^{2} V \int_{V} d r \frac{C_{0}}{r}
$$

Notice that the integral in equation (3.10.2) diverges when one takes $V$ infinitely large. In other words, the total potential energy per unit of volume $U_{i} / V$ increases with the size of the system, This is a consequence of the long range of the Coulomb force.

Now, assume that the particles start interacting. As a result, part of the initial potential energy $U_{i}$ will be converted into thermal energy, which is related to the random components of motion. At the same time, the system will expand under influence of its average space charge. This expansion corresponds to a systematic motion and has no direct impact on the thermal energy of the particles. As we are primarily interested in obtaining an upper limit for the resulting temperature of the system, we will ignore the average space charge effect entirely and assume that system will reach thermodynamic equilibrium while maintaining its average density $n$.

In thermodynamic equilibrium, the microscopic density distribution $n_{m}(r)$ obeys Maxwell-Boltzmann statistics and is given by equation (3.9.4). Accordingly, the total potential energy $U_{e}$, in the equilibrium situation, can be expressed as

$$
U_{e} \approx \frac{1}{2} n^{2} V \cdot \int_{V} d r \frac{C_{0}}{r} e^{-e \Phi_{s}(r) / k_{B} T}
$$

Conservation of energy implies that the difference in potential energy between the initial and the equilibrium situation should be identical to the resulting total thermal energy of the system

$$
\frac{3}{2} n V k_{B} T=U_{i}-U_{B}
$$

using that $N=n V$. Substitution of equations (3.10.2) and (3.10.3) into equation $(3.10 .4)$ yields

$$
\frac{3}{2} \mathrm{nVk}_{B} \mathrm{~T}=\frac{1}{2} \mathrm{n}^{2} \mathrm{~V} \int_{V} \mathrm{dr} \frac{\mathrm{C}_{0}}{\mathrm{r}}\left(1-\mathrm{e}^{-\mathrm{e} \Phi_{S}(\mathrm{r}) / \mathrm{k}_{B} \mathrm{~T}}\right)
$$

As the volume $\mathrm{V}$ is large, one may approximate this equation as

$$
\frac{3}{2} \mathrm{nVk}_{B} \mathrm{~T}=\frac{1}{2} \mathrm{n}^{2} \mathrm{~V} \int_{0}^{\infty} 4 \pi r^{2} \mathrm{dr} \frac{\mathrm{C}_{0}}{\mathrm{r}}\left(1-\mathrm{e}^{-\mathrm{e} \Phi_{s}(r) / k_{B} \mathrm{~T}}\right)
$$

employing spherical coordinates. The integral in equation (3.10.6) is finite, despite the fact that we took $V$ infinitely large. This is a result of the fact that the screened potential $\Phi_{s}(r)$ decreases exponentially for $r \gg>\lambda_{0}$, as can be seen from equation (3.9.8).

By combining equations $(3.9 .7),(3.9 .8)$ and $(3.10 .6)$ and 
substituting $x=r / \lambda_{D}$, one obtains

$$
\int_{0}^{\infty}\left[1-\exp \left(\frac{-1}{4 \pi n \lambda_{D}{ }^{3}} \frac{\exp (-x)}{x}\right)\right] x d x=3
$$

This equation was solved numerically. It yields

$$
4 \pi n \lambda_{D}{ }^{3}=a \quad \text { with } \quad a=.2851
$$

With equation (3.9.7), one finds for the final thermal energy per particle

$$
\frac{3}{2} k_{B} T=\frac{3}{2}\left(4 \pi a^{2}\right)^{1 / 3} C_{0} n^{1 / 3}
$$

which depends on the average particle density $n$ only.

Let us now return to the case of a homocentric cylindrical particle beam. From equations (3.10.9) and (3.8.2), one obtains as an upper limit for the energy spread generated by potential energy relaxation

$$
\frac{<\Delta E_{/ / 2}^{2}>1 / 2}{\mathrm{eV}}=\left(32 \mathrm{a}^{2}\right)^{1 / 6} \frac{\mathrm{C}_{0}^{1 / 2} \mathrm{n}^{1 / 6}}{(\mathrm{eV})^{1 / 2}}
$$

Substitution of the expression for the density $n$, given by equation $(3.2 .5)$, yields

$$
\frac{<\Delta E_{/^{2}>1 / 2}}{e^{V}}=C_{P E} \frac{J^{1 / 6}}{V^{1 / 12}} \quad, \quad C_{P E}=\frac{a^{1 / 3}}{2^{1 / 4} \pi^{1 / 3}} \frac{m^{1 / 12} e^{1 / 4}}{E_{0}^{1 / 2}}
$$

where $J\left(=I / \pi r_{0}{ }^{2}\right)$ is the current density in the beam. For the case of electrons one finds $C_{P E}=7.972 \times 10^{-3}$. Taking as a numerical example $J=100 \mathrm{~A} / \mathrm{m}^{2}$ and $V=10 \mathrm{kV}$, equation $(3.10 .11)$ yields $\left\langle\Delta E_{/ /}{ }^{2}>1 / 2=0.80 \mathrm{eV}\right.$. 


\section{THE MAKY BODY PROBLem OF PARTICles INTBRACTING THROUGH an IMVERSE SQUARE FORCE LAN}

\subsection{Introduction}

In the previous chapter the Boltzmann equation was introduced as the fundamental equation for the time development of the single-particle distribution function $f(r, v, t)$ in $\Gamma_{6} \mathrm{~N}$ space. Its implications were studied for the specific case of a system of identical particles, which do not interact. In this chapter, the more complex problem is considered of a system of identical particles, interacting through a force which is proportional to the inverse square of the distance between the particles. This type of $\mathrm{N}$-body problem is extensively studied in plasma physics as well as in stellar dynamics. The fundamental aspects of the approaches taken in these fields are outlined, starting from the Boltzmann equation. Their applicability to the particular problem of Coulomb interactions in particle beams is investigated.

The main part of this chapter is concerned with the so-called Fokker-Planck approach. This approach relies on the assumption that the action of the fluctuating component of the interaction force can be described in terms of a diffusion process in velocity space. This assumption is justified for systems of high particle density, operating at a high temperature, near thermodynamic equilibrium. Accordingly, the Fokker-Planck approach is suited for plasmas, in which these conditions are usually fulfilled. It is shown that Fokker-Planck approach is, in general, not suited to describe the impact of the statistical interactions in particle beams. The main reason. for this failure is that a particle beam is relatively "cold" compared to a plasma and usually operates at a low density, while the particles have a short flight time relative to the characteristic relaxation times involved. This also indicates that thermodynamic equilibrium is not reached in practical beams. Accordingly, Debeye screening usually does not occur.

The Fokker-Planck approach may be adequate to evaluate the energy spread generated in the vicinity of a narrow crossover in a beam of relatively high density, where the conditions of a plasma are, to some extent, reproduced. The root mean square (rms) energy spread can be determined from the velocity-diffusion coefficient used in the FokkerPlanck equation. The resulting expression is, apart from a numerical factor, indentical to the one presented by Zimmermann (1970) and Knauer (1979a) for a cylindrical beam segment.

The last part of this chapter is concerned with the Holtsmark distribution, which is the probability distribution of the electric field strength acting on some point in a gas composed of randomly distributed point charges. It is shown that the distribution of velocities generated in an extended homocentric cylindrical beam segment is Holtsmarkian, provided that the perturbations are small. This knowledge can be exploited to calculate the energy spread and the angular deflections generated in a nearly homocentric cylindrical beam of relatively low density. The dependency of the energy spread on the experimental parameters is made explicit.

The method centred around the Holtsmark distribution is suited to describe the effect of potential energy relaxation in a homocentric cylindrical beam of low particle density, whereas the Fokker-Planck approach can be used to describe the effect of kinetic energy relaxation in a beam segment with a narrow crossover of high particle density. A more refined model will be presented in chapter 5 , which is suited to calculate the impact of the statistical interactions, in any type of beam geometry, for any practical particle density. It combines some of the features of the two approaches which are discussed here. 
Consider a system of $N$ identical particles in which the force $F_{i j}$ exerted by particle $j$ on particle $i$ can be expressed as

$$
F_{i j}=C_{0} \frac{r_{i j}}{r_{i j}{ }^{3}}
$$

where the relative position $\mathbf{r}_{\mathbf{i}}=\mathbf{r}_{\mathbf{i}}-\mathbf{r}_{\mathbf{j}}$ and $\mathrm{C}_{0}$ is a physical constant, which depends on the type of interaction. For a system of identical charged particles $\mathrm{C}_{0}=\mathrm{e}^{2} / 4 \pi \varepsilon_{0}$, where $e$ denotes the particle charge. For a stellar system $\mathrm{C}_{0}=-\mathrm{Gm}^{2}$, where $\mathrm{G}$ is the gravitational constant and $\mathrm{m}$ the "particle" mass. Clearly, in practical stellar systems the mass varies per star and $C_{0}$ depends on the pair of stars considered, thus $C_{0}=C_{0_{i} j}$. A similar complication arises in multi-component plasmas. Although the calculations for such systems are more elaborate than those for a system consisting of identical particles, the fundamental issues involved are the same. For simplicity we concentrate on the case of identical charged particles. The calculations presented here are non-relativistic. Accordingly, magnetic interactions are ignored.

The total force on particle $i$ is equal to the sum of the forces exerted by all other particles

$$
\mathbf{F}_{\mathbf{i}}=\mathrm{C}_{\mathbf{0}} \sum_{j \neq i}^{N} \frac{\mathbf{r}_{\mathbf{i} \mathbf{j}}}{\mathbf{r}_{\mathbf{i} \mathbf{j}}{ }^{3}}
$$

assuming that external forces are absent. Due to the long range of the interaction force, the number of particles which significantly contribute to the total force $F_{i}$ on particle $i$, will in general be large. In other words, every particle interacts with many other particles simultaneously. Most of these interactions will be weak, which means that they cause only small changes in the velocity of the colliding particles. Strong collisions, which correspond to large changes in velocity, will be relatively rare. Consequently, the trajectories in coordinate space follow smoothly curved lines with continuously varying curvature. It should be noted that this situation is quite different from the one encountered in non-ionized gases. At normal densities, the interaction between neutrals is limited to infrequent, strong collisions between pairs. The collisions are well separated in time, which means that collisions involving more than two particles simultaneously are rare. Accordingly, the particles follow a broken line in space. This motion can adequately be characterized by quantities as the "mean free path" (average distance between two collisions) and the "collision frequency" (number of collisions per time unit), which are of central importance to the kinetic theory of nonionized gases. For instance, see Chapman and Cowling (1970). It might be clear that such concepts are less suited for the description of ionized gases. Thus, the theory for plasmas and stellar dynamics requires a different approach than taken in the classical kinetic theory of gases.

As every particle interacts simultaneously with many other particles, both near and remote, it appears to be justifiable to distinguish between the influence of the system as a whole and the influence of the immediate local neighbourhood. The former will be a smoothly varying function of position and time, which can be expressed in terms of a potential $\Phi(r, t)$, while the latter will be subject to 
relatively rapid fluctuations. Accordingly, the total interaction force on particle $i$ is divided into two components

$$
\mathbf{P}_{i}=\mathbf{F}_{a v, i}+\mathbf{F}_{f, i}=-\frac{\partial}{\partial r_{i}} \Phi\left(r_{i}, t\right)+c_{0} \sum \frac{r_{i j}}{r_{i j}{ }^{3}}
$$

in which the summation in the second term on the right hand sight is now limited to neighbours. The potential $\Phi(r, t)$ corresponds to the smoothed-out distribution of charge. It can be related to the singleparticle distribution function $f(r, v, t)$ as

$$
\Phi(r, t)=c_{0} \int d v_{1} \int d r_{1} \frac{f\left(r_{1}, v_{1}, t\right)}{\left|r-r_{1}\right|}
$$

This potential is a function of the absolute position within the system $r$ and time $t$ only and is, in that respect, equivalent to an external, time dependent, electrostatic potential. Thus, the first component in equation (4.2.3) may be treated as an external force. The second component can not directly be expressed in terms of the macroscopic properties of the system. Formally, the summation over $j$ refers to near neighbours. However, it is difficult to define an adequate criterion to distinguish near neighbours from the other particles in the system. In fact, the second term just represents the remaining force after substraction of the average component $F_{\mathrm{a}}, \mathrm{i}$. One usually identifies this component with the force exerted during collisions. We prefer the more general term "fluctuating force component" and denote it as $\mathbf{F}_{\mathrm{f}}$.

Using equation (4.2.3), Boltzmann's equation (3.5.2) can now be expressed as

$$
\frac{d f}{d t}=\frac{\partial f}{\partial t}+v \cdot \frac{\partial f}{\partial r}-\frac{1}{m} \frac{\partial \Phi}{\partial r} \cdot \frac{\partial f}{\partial v}=\left[\frac{\partial f}{\partial t}\right]_{f}
$$

in which $[\partial f / \partial t]_{f}$ is the rate of change of $f(r, v, t)$ due to the fluctuating component of the interaction force. Disregarding this term $\left([\partial \mathrm{f} / \partial \mathrm{t}]_{\mathrm{f}}=0\right)$, equation $(4.2 .5)$ becomes identical to the so-called nonrelativistic vlasov equation for a single component plasma

$$
\frac{d f}{d t}=\frac{\partial f}{\partial t}+v \cdot \frac{\partial f}{\partial r}-\frac{1}{m} \frac{\partial \Phi}{\partial r} \cdot \frac{\partial t}{\partial v}=0
$$

In combination with equation (4.2.4), the Vlasov equation offers a selfconsistent description of the system in collisionless continuum approximation, determining the time-development of the single-particle distribution function $f(r, v, t)$, under influence of the average interaction force. Clearly, the Vlasov' equation does not account for the fluctuations due to the discreteness of the particles, referred to as collisional effects. Thus it is unable to describe an approach of the system towards equilibrium. In stellar dynamics, the vlasov equation (usually referred to as collisionless Boltzmann equation) is used to describe the evolution of stellar systems which can be regarded as collisionless, which means that their characteristic relaxation times are large compared to the total timescale of the universe ( $\$ 10^{10}$ years). In particular, it is used to describe the dynamics of Galaxies, see for instance Chandrasekhar (1942a) and Contoupolos (1972). In plasma physics, one usually deals with neutralized, multi-component systems. In such systems, the average interaction force $F_{a v}$ is responsible for the occurrence of collective plasma oscillations. 
Let us now consider the fluctuations in the interaction force. Equation $(4.2 .3)$ expresses that the fluctuating component of the total force, acting on a test particle, is related to the distribution of its neighbour field particles, which is a rapidly changing function of time. A rough estimation of the correlation time of the fluctuations $\tau_{f}$ is given by the ratio between the average separation of the particles and their average relative velocity

$$
T_{f}=n^{-1 / 3 /<v^{2}>1 / 2 \approx n-1 / 3}\left(m / k_{B} T\right)^{1 / 2}
$$

where $\mathrm{n}$ is the average particle density and $\mathrm{m}$ is the particle mass. The relation $\left\langle v^{2}\right\rangle_{a} k_{B} T / m$ was utilized, assuming thermal equilibrium. In terms of collisions, if can be regarded as a measure for the average collision duration. As a numerical example, consider the case of electrons in a plasma with density $\mathrm{n}=10^{16} \mathrm{~m}^{-3}$ and temperature $\mathrm{T}=10^{5} \mathrm{~K}$. From equation (4.3.1) one finds $T_{f} 4 \cdot 10^{-12} \mathrm{~s}$. This time will be short compared to the time in which any of the macroscopic parameters of the system change appreciable. Thus, in general, the fluctuations in the interaction force will occur with extreme rapidity compared to the rate of change of the average force $\mathbf{P a v}_{\mathrm{av}}$, which is related to the time-development of the (macroscopic) single-particle distribution function $f(r, v, t)$, as expressed by equations $(4.2 .3)$ and $(4.2 .4)$.

From these considerations, it appears to be reasonable to assume that there exists a time interval $\Delta t$, which is long compared to the correlation time of the fluctuations $\tau_{\varepsilon}$, but short enough to neglect the variation in $F_{a v}$. The velocity change of the test particle, during this time interval, can then be expressed as

$$
\Delta v=P_{a v}(t) \Delta t+\delta v(t ; \Delta t), \delta v(t ; \Delta t)=\int_{t}^{t+\Delta t} P_{f}\left(t^{\prime}\right) d t^{\prime}
$$

For the conditions stated $\left(\Delta t>>\tau_{f}\right)$, the displacements $\delta \nabla(t ; \Delta t)$ and $\delta v(t+\Delta t ; \Delta t)$, experienced by the test particle in successive time intervals, are expected to show no correlation. To put it more generally, the displacements experienced in disjoint time intervals can be considered as statistically independent. Thus, on this time scale the motion in velocity space can be visualized as a succession of random microscopic jumps superimposed on a smooth systematic motion, determined

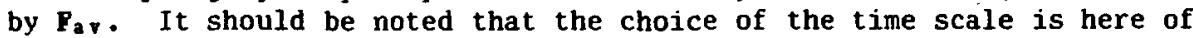
crucial importance. On a finer time scale, $\Delta t$ becomes of the same order as the correlation time of the fluctuations (or collision duration) $\tau_{f}$. Consequently, the displacements, experienced during successive time intervals, will be correlated, since they are caused by the interaction within the same complexion of neighbours (or in other words the same collision). Using a more course grained time scale, implies that the

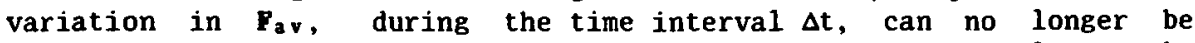
neglected, while the size of jumps $\delta v$, due to $P_{f}$, can no longer be regarded as microscopic.small. Consequently, the separation between $P_{a} v$ and $F_{f}$ becomes useless. The constraints on the time interval $\Delta t$ can be summarized as

$$
\Delta t \gg \tau_{f}, \quad f(r, v, t+\Delta t) \approx f(r, v, t), \quad \delta v(t ; \Delta t) \ll<\left(k_{B} T / m\right) 1 / 2
$$

It is essential to the theory, presented in the remainder of this section, that a time interval $\Delta t$ exists, which fulfils these requirements. 
Although the velocity displacements $\delta \mathrm{v}$, experienced in disjoint time intervals, are uncorrelated, there may be a systematic tendency for velocities to jump in a specific direction. Such should be expected when a test particle has an excess velocity relative to the field particles surrounding it, or in other words, a velocity which is large compared to the average thermal velocity $\left(\mathrm{k}_{\mathrm{B}} \mathrm{T} / \mathrm{m}\right)^{1 / 2}$. In that case, the field particles will on the average decelerate the test particle. This means that the average of the velocity displacements $\delta \mathbf{v}$, experienced by the test particle during a certain period of time, will be non-zero, while the resulting velocity change is directed opposite to the velocity of the test particle. This phenomenon is referred to as dynamical friction.

Under the conditions stated by the equations (4.3.3), the timedevelopment of the single-particle distribution function $f(r, v, t)$, under influence of the fluctuating component of the interaction force, can be described by the integral equation

$$
f_{f}(r, v, t+\Delta t)=\int d \delta v w_{\Delta t}(r, v-\delta v ; \delta v) f_{f}(r, v-\delta v, t)
$$

where $W_{\Delta t}(r, v ; \delta v)$ represents the transition probability that $v$ changes by an increment $\delta \mathbf{v}$, during the time interval $\Delta t$. This function is normalized such that

$$
\int d \delta v w_{\Delta t}(r, v ; \delta v)=1
$$

The assumption underlying equation $(4.3 .4)$, is that the motion of a particle in phase space, from its location $(\mathbf{r}, \mathbf{v})$ at time to $(\mathbf{r}, \mathbf{v}+\delta \mathbf{v})$ at time $t+\Delta t$, depends only on its instantaneous position in phase space $(\mathbf{r}, \mathbf{v})$ and is entirely independent of its previous coordinates. In general, a stochastic process with this characteristic, namely that what happens at a given instant of time depends only on the state of the system at that time, is called a Markoff process. The validity of the Markof $f$ assumption depends on the choice of the time interval $\Delta t$, as was discussed previously.

The transition probability function $\mathrm{w}_{\Delta t}(r, v ; \delta v)$ represents the essential stochastic nature of the interaction process. Notice that it accounts for jumps in velocity only. Clearly, the fluctuations in the interaction force cause random spatial displacements $\delta \mathbf{r}$ within the time interval $\Delta t$ as well. However, these displacements are of the order $\Delta t^{2}$ and can thus be neglected if $\Delta t$ is small enough. Spatial displacements, without the time interval $\Delta t$, are represented through the change in velocity $\delta v$ and need not be considered separately. Basicly, this approach expresses that the trajectories of the particles in coordinate space can adequately be described as a concatenation of straight lines, provided that $\Delta t$ is chosen short enough. In this context, it should be added that one of ten studies extended homogeneous systems, in which the microscopic spatial displacements can be disregarded anyhow (from a macroscopic point of view).

Equation $(4.3 .4)$ is true irrespective of the size of the jump $\delta \mathrm{v}$. We will now exploit the fact that the jumps in velocity are expected to be small. Expanding equation $(4.3 .4)$ in Taylor series with respect to $t$ and $v$, one finds (notation: $f=f_{f}(r, v, t) ; w_{\Delta t}=w_{\Delta t}(r, v ; \delta v)$ ) 


$$
\begin{aligned}
& f+\Delta t \frac{\partial f}{\partial t}+\cdots=\int d \delta v\left[1-\sum_{k} \delta v_{k} \frac{\partial}{\partial v_{k}}+\frac{1}{2} \sum_{k, 1} \delta v_{k} \delta v_{1} \frac{\partial^{2}}{\partial v_{k} \partial v_{1}}+\cdots\right] w_{\Delta t} f \\
& =f-\sum_{k} \frac{\partial}{\partial v_{k}}\left[\int d \delta v \delta v_{k} w_{\Delta t}\right] f+\frac{1}{2} \sum_{k, 1} \frac{\partial^{2}}{\partial v_{k} \partial v_{1}}\left[\int d \delta v \delta v_{k} \delta v_{1} w_{\Delta t}\right] f+\cdots
\end{aligned}
$$

in which the summation indices $k, 1=1,2,3$ refer to the components of $\delta v$. Defining the so-called first and second jump-moments as

$$
\left\langle\delta v_{k}\right\rangle=\int d \delta v \delta v_{k} w_{\Delta t}, \quad\left\langle\delta v_{k} \delta v_{1}\right\rangle=\int d \delta v \delta v_{k} \delta v_{1} w_{\Delta t}
$$

one may write

$$
\left[\frac{\partial f}{\partial t}\right]_{f} \approx \sum_{k} \frac{\partial}{\partial v_{k}}\left[\frac{\left\langle\delta v_{k}\right\rangle}{\Delta t} f\right]+\frac{1}{2} \sum_{k, 1} \frac{\partial^{2}}{\partial v_{k} \partial v_{1}}\left[\frac{\left\langle\delta v_{k} \delta v_{1}\right\rangle}{\Delta t} f\right]
$$

Equation (4.3.7) relates the short-time stochastic behaviour of the test particle, expressed in the jump moments of its velocity, to the long time behaviour of the single-particle distribution function $f(r, v, t)$. The principal advantage of this approximation above the original problem, expressed by equation (4.3.4), is that it directly relates the rate of change of the single-particle distribution function $f(r, v, t)$ to the values of the jump moments $\left\langle\delta v_{k}\right\rangle$ and $\left\langle\delta v_{k} \delta v_{l}\right\rangle$ $(k, l=1,2,3)$, which can be obtained without detailed knowledge of the entire transition probability function $w_{\Delta t}(r, v ; \delta v)$. The jump-moments can be estimated directly, by studying the collisional processes between a test particle and its surrounding field particles over the time interval $\Delta t$. This is a problem of particle dynamics, which will be considered in section 4.5 .

From the preceding analysis, it follows that the right hand side of equation (4.2.5) can be expressed as (using the conventions of vector and tensor analysis)

$$
\left[\frac{\partial f}{\partial t}\right]_{f}=-\frac{\partial J}{\partial v} \quad, \quad J=-\beta v f-D \frac{\partial f}{\partial v}
$$

where $\beta$ is the so-called coefficient of dynamical friction and $D$ is the diffusion tensor (which is a tensor of the second rank with elements $\left.D_{x_{1}}\right)$. The vector $J$ is called the collision flux. It represents the flux in velocity space due to the fluctuating component of the interaction force. By comparison with equation (4.3.7), it follows that $\beta$ and $D$ are related to the jump-moments, defined by the equations $(4.3 .6)$, as

$$
-\beta v_{k}=\frac{\left\langle\delta v_{k}\right\rangle}{\Delta t}, \quad D_{k l}=\frac{\left\langle\delta v_{k} \delta v_{1}\right\rangle}{2 \Delta t}
$$

The dynamical friction force is related to the systematic part of the velocity jumps of the test particle. The notation $(-\beta v)$ expresses that this force is expected to be proportional to minus the velocity of the particle, similar to the friction force given by Stokes law. The elements of the diffusion tensor $D_{\mathrm{x}_{1}}$ are related to the purely stochastic part of the velocity jumps of the test particle. In equation (4.3.8), it was assumed that the tensor $D$ is independent of the velocity v. In general, it should be emphasized that the concept of dynamical friction and diffusion relies on the assumption that $\beta$ and $D$ are $a$ function of the macroscopic properties of the system only. This is equivalent to the assumption that both $f(r, v, t)$ and $w_{\Delta t}=w_{\Delta t}(r, v ; \delta v)$ are slowly varying functions of $r$ and $v$. The properties of $\beta$ and $D$ and their 
dependency on the macroscopic parameters will be discussed in section 4.5 .

Equations (4.3.8) and (4.3.9) constitute the main results of this section. They describe the impact of the fluctuations in the interaction force (usually referred to as collisional effects) in socalled diffusion approximation. Combining equations (4.2.5) and $(4.3 .8)$, one finds

$$
\frac{d f}{d t}=\frac{\partial f}{\partial t}+v \cdot \frac{\partial f}{\partial r}+\frac{1}{m} \frac{\partial \Phi}{\partial r} \cdot \frac{\partial f}{\partial v}=\beta \frac{\partial v f}{\partial v}+D \frac{\partial^{2} f}{\partial v^{2}}
$$

which is an equation of the Fokker-Planck form. In combination with the equations (4.2.4) and (4.3.9), the Fokker-Planck equation (4.3.10) offers a self-consistent description of the system in diffusion approximation, determining the time-development of the single-particle distribution function $f(r, v, t)$, under influence of the combined action of the average interaction force and the fluctuating force component, related to the discreteness of the particles. The remaining task, is to calculate the coefficient of dynamical friction $\beta$ and diffusion coefficient $D$, as function of the macroscopic properties of the plasma.

For a general discussion of the Fokker-Planck equation, the reader is referred to van Kampen (1981). A comprehensive review on related topics as random walks, diffusion, Brownian motion and stellar dynamics is given by Chandrasekhar (1943). The Fokker-Planck equation and its application in plasma physics is discussed by Trubnikov (1965), Sivukhin (1966) and Ichimaru (1973).

\subsection{Aspects of the diffusion approximation}

The Fokker-Planck equation (4.3.10) presupposes that the action of the fluctuating component of the interaction force can adequately be described as a diffusion process in velocity space. In this section we will elucidate some aspects of this so-called diffusion approximation.

The rate of change of the single-particle distribution function $f(r, v, t)$, due to the fluctuations in the interaction force, is given by equation (4.3.8). In order to clarify the structure of this equation, it is instructive to consider an ordinary spatial diffusion process in an atomair gas. Let $\rho(\mathbf{r})$ be the mass density of the gas at position $r, J_{m}$ the total mass flow at this location and $D_{r}$ the diffusion coefficient (As the gas is isotropic, one finds for the tensor elements $D_{i j}=D \delta_{i j}$ and $D$ reduces to a scalar). The mass flow $J_{m}$ is given by

$$
J_{m}=v \rho-D_{r} \frac{\partial \rho}{\partial r}
$$

The first term represents the mass flow due to convection. The second term specifies the contribution of diffusion. It expresses that the mass flow, due to diffusion, is proportional to minus the gradient of the mass density. This is known as Fick's law. Continuity requires

$$
\frac{\partial \rho}{\partial t}=-\frac{\partial J_{m}}{\partial r}
$$

The so-called general diffusion equation follows by substituting equation (4.4.1) into equation $(4.4 .2)$. Here, we merely want to show that equations $(4.4 .1)$ and $(4.4 .2)$ have the same structure as equations $(4.3 .8)$. The coefficients $v$ and $D_{r}$, appearing in equation $(4.4 .1)$, can 
be expressed in terms of the microscopic spatial jump $\Delta r$ of a test particle, experienced during the period $\Delta t$

$$
v_{k}=\frac{\left\langle\delta r_{k}\right\rangle}{\Delta t}, \quad D_{k 1}=\frac{\left\langle\delta r_{k} \delta r_{1}\right\rangle}{2 \Delta t}
$$

similar to equations (4.3.9). One easily verifies that equations $(4.4 .1),(4.4 .2)$ and $(4.4 .3)$ are completely analogous to equations (4.3.8) and (4.3.9). Formally, the last set of equations can be obtained from the first set, by replacing the spatial coordinates by velocities : $r \rightarrow v$ (thus $r_{k} \rightarrow v_{k}$ and $r_{1} \rightarrow v_{1}$ ).

In the derivation of equation (4.3.8), the expansion in $\delta \mathbf{v}$ was truncated after the second order term. This implies that the jumps in velocity $\delta v$ should be small. This condition is expressed by the last constraint in equations (4.3.3). Clearly, the smaller one takes the time period $\Delta t$, the smaller the corresporiding velocity jump $\delta \mathrm{v}$. However, the Markoff assumption requires that $\Delta t$ is chosen large enough to guarantee that the velocity displacements, experienced in successive time intervals, are not correlated. This condition is expressed by the first constraint in equations (4.3.3). In fact, it says that the velocity displacements, experienced in disjoint time-intervals, should not be generated by the same collision. The combination of the first and the third constraint in equations (4.3.3) implies that the effect of strong collisions can not be taken into account within the diffusion approximation, since a strong collision results in a large velocity displacement $\delta \mathbf{v}$ within the correlation time $\tau_{\mathrm{f}}$.

Another way to visualize the same problem, is the following. Equation (4.3.8) expresses that the increment of the number of particles, contained within a volume element in velocity space, is equal to the net flux through the volume boundaries. This is accurate when the path of the test particle in velocity space can be regarded as continuous, despite the fact that one is effectively sampling with a period $\Delta t$. The continuity condition is violated during strong collision, in which the velocity of the particle can change appreciable within the period $\Delta t$. Let the velocity of the test particle at time $t$ be $v$. If a strong collisions occurs during the next time interval $\Delta t$, the particle is in fact "annihilated" at point $v$ and "created" at some remote point $\mathbf{v}+\delta \mathbf{v}$, without passing through the intermediate points in velocity space. Accordingly, we conclude, once again, that the effect of strong collisions can not be taken into account by means of equation (4.3.8).

The stationary solution of the diffusion equation is a Gaussian distribution. This follows straightforwardly from equation (4.3.8). For simplicity, let us consider a homogeneous isotropic system, for which $\mathrm{D}_{k 1}=\mathrm{D} \delta_{k 1}, \quad \mathrm{f}(\mathbf{r}, \mathbf{v}, t)=\mathrm{nf}(\mathbf{v}, \mathrm{t})$ and $\mathrm{D}$ and $\beta$ are constant. A stationary solution $f_{s}$ implies $\left[\partial f_{s} / \partial t\right]_{f}=0$. By integration of the individual equations for each of the three components of velocity, one finds for the total velocity distribution

$$
f_{s}(v)=C e^{-\beta v_{x}^{2} / 2 D} e^{-\beta v_{y}^{2} / 2 D} e^{-\beta v_{z}^{2} / 2 D}=C e^{-\beta v^{2} / 2 D}
$$

where $C=(\beta / 2 \pi D)^{3 / 2}$, as is required by normalization. Thus, the stationary velocity distribution is a three-dimensional Gaussian distribution. This could be expected on the basis of the so-called central limit theorem, which says that the probability distribution of the sum of a great number of independent identical stochastic variables (in this case the sum of all microscopic velocity jumps experienced by 
the test particle during a certain period of time) will be Gaussian. Notice that no stationary solution exists when the dynamical friction coefficient $\beta$ is zero. Without dynamical friction, there is no tendency to equilibrium.

From statistical mechanics, one knows that the equilibrium solution must be Maxwellian

$$
f_{B}(v)=\left[\frac{m}{2 \pi k_{B} T}\right]^{3 / 2} e^{-m v^{2} / 2 k_{B} T}
$$

which is identical to the stationary solution given by equation $(4.4 .4)$, provided that

$$
D / \beta=k_{B} T / m
$$

Thus, the assumption that the stationary solution of the diffusion equation (4.2.7) is Maxwellian, implies that the coefficient of dynamical friction $\beta$ and the diffusion coefficient $D$ are related according to equation $(4,4.6)$. It expresses that the equilibrium condition implies a balance between fluctuations and damping, represented by $D$ and $\beta$ respectively. Equation $(4.4 .6)$ is an example of the so-called fluctuation-dissipation theorem.

It should be noted that it is not trivial to identify the stationary solution of the diffusion equation $(4.4 .4)$ with the equilibrium solution (4.4.5). In fact, it is a principle task of the theory to demonstrate that an arbitrary initial state of the system, will eventually $(t+\infty)$ lead to the stationary equilibrium solution given by equation (4.4.5). We will not pursue this problem and assume that this is indeed the case.

\subsection{Calculation of coefficients of dynamical friction and diffusion}

In the previous sections, the calculation of the evolution of the single-particle distribution function $f(r, v, t)$, under influence of the fluctuating component of the interaction force, was reduced to the evaluation of the coefficient of dynamical friction $\beta$ and the diffusion tensor $D$, which are related to the first and second jump-moments of the particle velocities respectively, as expressed by equation (4.3.9). In this section we will consider the dynamical problem of calculating these jump moments, defined by equation (4.3.6).

In plasma-physics, one usually identifies the effect of the fluctuating component of the interaction force, acting on a particle during the time interval $\Delta t$, with the impact of all two-particle collisions experienced by the particle in that period. We will follow this approach. Consider a homogeneous system of identical charged particles with density $n$. Assume that the velocity distribution $f(v)$ is isotropic and that the velocities show no correlation with the position within the system (molecular chaos). Let us focus on a single test particle and calculate its interaction with the other particles in the system, called field particles. First, we will determine the impact of a collision with a single field particle. This leads to an expression for the velocity shift of the test particle as function of the relative initial position and velocity of the two particles. Next, we will determine the mean velocity shift and mean square velocity shift, experienced by the test particle over a period of time $\Delta t$, by averaging over all possible relative coordinates of test and field particle, taking their probability distribution into account. It is assumed that 


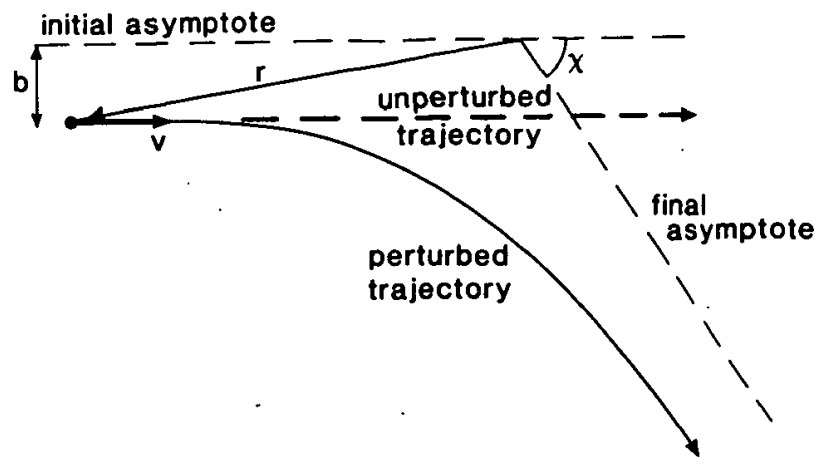

Figure 4.I Relative coordinates of two particles with interaction (perturbed trajectory) and without interaction (unperturbed trajectory). The parameter $b$ is the impact parameter and $x$ is the deflection angle for a complete collision.

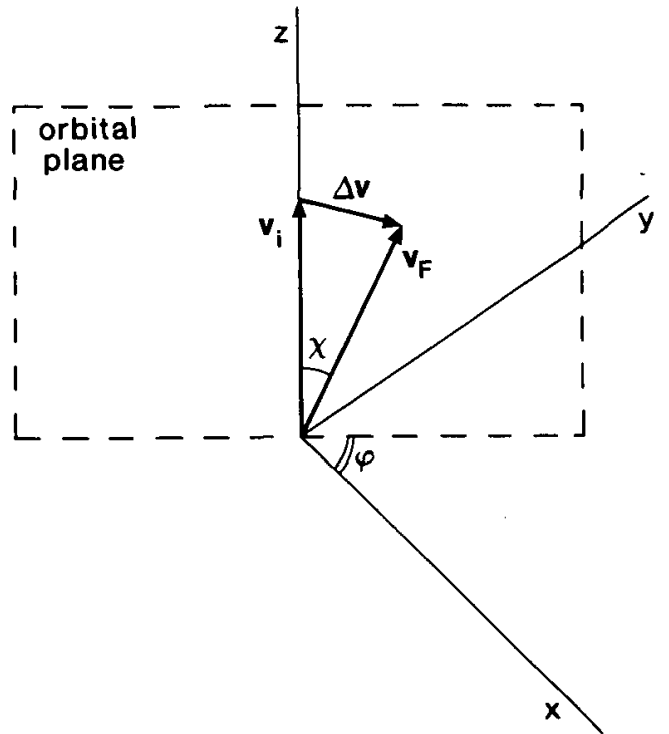

Figure 4.2 Orientation of the orbital plane with respect to an orthogonal coordinate system, with a 2 -axis which is directed along the initial relative velocity $v_{1}$. 
the system operates near thermodynamic equilibrium, which implies that the velocity distribution of the particles is (nearly) Maxwellian.

Consider a collision between the test particle and a single field particle. Let us denote the initial position and velocity of the test particle as $r_{t}$ and $v_{t}$ respectively and the corresponding coordinates of the field particle as $r_{f}$ and $v_{f}$. As the interaction force is central, the motion of the particles takes place in a plane, referred to as the orbital plane. The collision can best be described in terms of the relative position $r=r_{f}-r_{t}$ and relative velocity $v=v_{f}-v_{t}$. The relative position vector follows a hyperbola in the orbital plane, see figure 4.1. The deflection angle $\chi$ specifies the angle between the asymptotes of the hyperbola. It is given by

$$
\tan (x / 2)=\frac{b_{\perp}}{b} \quad \text { and } \quad b_{\perp}=\frac{2 C_{0}}{\mathrm{mv}^{2}}
$$

where $v=\left|v_{f}-v_{t}\right|$ is the magnitude of the relative velocity and $b$ is the impact parameter, which is defined as the closest distance of approach for the imaginary case that the particles do not interact. The quantity $b_{\perp}$ is the value of the impact parameter which leads to a 90 degrees deflection $(x=\pi / 2)$. For a derivation of equation $(4.5 .1)$, the reader is referred to section 6.4 . It should be emphasized that equation $(4.5 .1)$ pertains to a complete collision, that is a collision for which the initial and final state approach the asymptotic conditions. In such a collision, both the initial and final energy will be entirely kinetic. Thus, conservation of energy implies that only the direction of the relative velocity is changed and not its magnitude. Accordingly, a complete collision is sufficiently specified by the deflection angle $\chi$ only (as far as the motion in the orbital plane is concerned).

Let us define the direction of the initial relative velocity $v=v_{f}-v_{t}$ as the $z$-direction. Let $\varphi$ be the angle between the orbital plane and the $x$-axis, see figure 4.2. The three components of the velocity displacement of the test particle, can now be expressed as

$$
\begin{aligned}
\Delta v_{x}=\frac{1}{2} v \sin \chi \cos \varphi=v \frac{\tan (\chi / 2)}{1+\tan ^{2}(\chi / 2)} \cos \varphi & =\frac{v \sqrt{ }}{1+q} \cos \varphi \\
\Delta v_{y}=\frac{1}{2} v \sin \chi \sin \varphi & =\frac{v \sqrt{ }}{1+q} \sin \varphi \quad(4.5 .2) \\
\Delta v_{z}=\frac{1}{2} v(\cos \chi-1)=v \frac{-\tan ^{2}(\chi / 2)}{1+\tan ^{2}(\chi / 2)}, & =\frac{v}{1+q}
\end{aligned}
$$

where $q=\left(b / b_{\perp}\right)^{2}$. The factor $1 / 2$ is included to account for the fact that the change in the velocity of the test particle is half the change in the relative velocity of the particles, as follows from conservation of momentum (for particles with identical mass).

The number of collisions $\mathrm{dN}_{c}$, experienced by the test particle during the time interval $\Delta t$, with an impact parameter between $b$ and $b+d b$, azimuth between $\varphi$ and $\varphi+d \varphi$ and relative velocity between $v$ and $\mathbf{v}+\mathrm{d} \mathbf{v}$, is equal to

$$
\mathrm{dN}_{c}=n f\left(v^{+} \mathbf{v}_{t}\right) d v \quad v \Delta t d \varphi \text { bd b }
$$

using that $v_{f}=v^{+} v_{t}$. Accordingly, the average of an arbitrary collision quantity $A=A(b, v, \varphi)$ (such as $\Delta v_{x}, \Delta v_{y}, \Delta v_{z}, \Delta v_{x}{ }^{2}, \ldots$ ) over all collisions, experienced during time interval $\Delta t$, can be expressed as 


$$
\langle\langle A\rangle\rangle=\int \mathrm{d} \mathbf{v}\left(\mathbf{v}+\nabla_{t}\right)\langle A(v)\rangle, \quad\langle A(v)\rangle=n v \Delta t \int_{0}^{2 \pi} d \varphi \int_{0}^{b_{m}} b d b A(b, v, \varphi) \quad(4.5 .4)
$$

For convenience of notation we separated the average over the spatial coordinates $b$ and $\varphi$, represented as $\langle A(v)\rangle$, from the total average $\langle<A\rangle$, which also includes an average over the relative velocity $v$ (the reader should be alert that the notation $<\ldots . .>$ will also be used to indicate cumulants, e.g. in section 5.5. Here it just refers to a double average). The upper integration boundary of the impact parameter $b$ is denoted as $b_{m}$. In order to include all field particles, one should take $b_{m}=\infty$. However, this would lead to a divergence of the $b$-integral and one is forced to introduce some finite value for $b_{m}$. We will come back to this point later on. From equations $(4.5 .2)$ and $(4.5 .4)$, one finds by integration

$$
\begin{aligned}
& \left\langle\Delta v_{x}\right\rangle=\left\langle\Delta v_{y}\right\rangle=0 \\
& \frac{\left\langle\Delta v_{z}\right\rangle}{\Delta t}=-\pi n b_{\perp}{ }^{2} v^{2} \ln \left(1+q_{m}\right)=-\frac{8 \pi n C_{0}^{2}}{m^{2} v^{2} \Lambda_{c}} \\
& \left\langle\Delta v_{k} \Delta v_{1}\right\rangle=0 \quad \text { for } \quad k \neq 1 \\
& \frac{\left\langle\Delta v_{x}{ }^{2}\right\rangle}{\Delta t}=\frac{\left\langle\Delta v_{y}^{2}\right\rangle}{\Delta t}=\frac{1}{2} \pi n b_{\perp}{ }^{2} v^{3}\left(\ln \left(1+q_{m}\right)-\frac{q_{m}}{1+q_{m}}\right) \approx \frac{4 \pi n C_{0} 2}{m^{2} v} \Lambda_{C} \\
& \frac{\left\langle\Delta v_{z}{ }^{2}\right\rangle}{\Delta t}=\pi n b_{\perp}{ }^{2} v^{3} \frac{q_{m}}{1+q_{m}} \approx \frac{8 \pi n C_{0}^{2}}{m^{2} v}
\end{aligned}
$$

where $q_{m}=\left(b_{m} / b_{\perp}\right)^{2}$ and $\Lambda_{c}$ is the so-called Coulomb logarithm, defined as

$$
\Lambda_{c}=\frac{1}{2} \int_{0}^{q_{m}} d q \frac{1}{1+q}=\ln \left[\left(1+q_{m}\right)^{1 / 2}\right] \approx \ln \left(q_{m} 1 / 2\right)=\ln \left(b_{m} / b_{\perp}\right)
$$

All approximations expressed in equations of (4.5.5) and (4.5.6), require $\mathfrak{f}_{m}>>1$ (thus $b_{m} \gg b_{\perp}$ ). Notice that the equations $(4.5 .5)$, as well as equations $(4.5 .2)$, fulfil the relation

$$
\left\langle\Delta v_{z}\right\rangle=-\left\langle\Delta v_{x}{ }^{2}+\Delta v_{y}{ }^{2}+\Delta v_{z}{ }^{2}\right\rangle / v \approx-\left\langle\Delta v_{x}{ }^{2}+\Delta v_{y}{ }^{2}\right\rangle / v
$$

which follows from the fact that the magnitude of the relative velocity is conserved in every complete collision: $|\mathbf{v}+\Delta \mathbf{v}|=\mathbf{v}$.

The Coulomb logarithm $\Lambda_{c}$ diverges logarithmically with the maximum value of the impact parameter $b_{m}$. The quantities in equation (4.5.5) which are proportional to $\wedge_{c}$, diverge accordingly. In plasmaphysics one usually takes $b_{m}$ equal to the Debeye screening length, which was introduced in section 3.9. This choice is somewhat arbitrary, in particular as far as the exact numerical value is concerned. The physical basis of the divergence of the Coulomb logarithm $\Lambda_{c}$ is investigated in the next section, leading to the conclusion that different expressions for $b_{m}$ should be used to treat the cases of neutralized and non-neutralized systems. At this point, it is sufficient to realize that the final results are not very sensitive to the exact value of $b_{m}$, since it appears under the logarithm in the expression for $\Lambda_{c}$. Furthermore, it is important to observe that the higher jump-moments than the second are finite, even for $b_{m}+\infty$. This can directly be verified from the equations $(4.5 .2)$ and equation (4.5.4). 


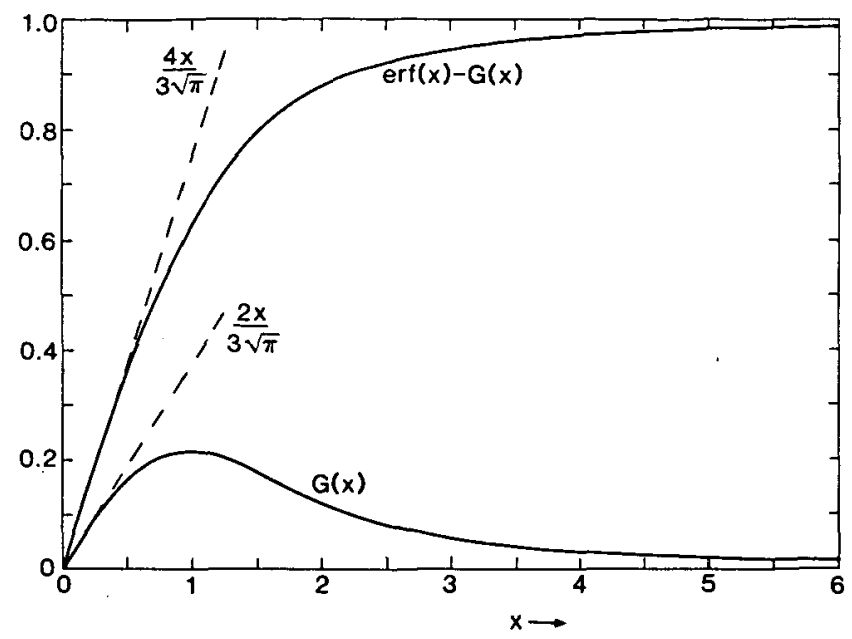

Figure 4.3 The functions $\mathrm{G}(\mathrm{x})$ and $\operatorname{erf}(\mathrm{x})-\mathrm{G}(\mathrm{x})$, defined by equation (4.5.9).

The final step in the calculation of the jump-moments, is to evaluate the average of the quantities given by equations (4.5.5) over the velocity distribution of the field particles, as expressed by the first equation of $(4.5 .4)$. Notice that the Coulomb logarithm $\Lambda_{c}$ depends on the relative velocity $v$ through $b_{1}$, see equations $(4.5 .6)$ and (4.5.1). However, since the exact value of $b_{m}$ is somewhat arbitrary anyhow, one usually treats $\Lambda_{c}$ as a constant by substituting some characteristic value for the relative velocity. We will follow this approach. The orientation of the coordinate system, used so far, depends on the direction of the relative velocity $v=v_{f}-v_{t}$. Consequently, it will vary with $v_{f}$. Therefore, we now choose a new coordinate system, in which the $z$-axis is in the direction of the test particle $v_{t}$. Assuming that the velocity distribution of the field particles is Maxwellian, one finds after a lengthy, but straightforward calculation (see, for instance, Chandrasekhar (1942a) or Trubnikov (1965))

$$
\begin{aligned}
& \frac{\left\langle\left\langle\Delta v_{z}\right\rangle\right\rangle}{\Delta t}=-\frac{8 \pi n C_{0}^{2} \Lambda_{C}}{m^{2} v_{T}^{2}} G\left(v_{t} / v_{T}\right) \\
& \frac{\left\langle\left\langle\Delta v_{x}^{2}\right\rangle\right\rangle}{\Delta t}=\frac{\left\langle\left\langle\Delta v_{y}^{2}\right\rangle\right\rangle}{\Delta t}=\frac{4 \pi n C_{0}^{2} \Lambda_{C}}{m^{2} v_{t}}\left[\operatorname{erf}\left(v_{t} / v_{T}\right)-G\left(v_{t} / v_{T}\right)\right] \\
& \frac{\left\langle\left\langle\Delta v_{z}^{2}\right\rangle\right\rangle}{\Delta t}=\frac{8 \pi n C_{0}^{2} \Lambda_{C}}{m^{2} v_{t}} G\left(v_{t} / v_{T}\right)
\end{aligned}
$$

in which $v_{T}=\left(2 \mathrm{kBT}_{\mathrm{B}} / \mathrm{m}\right)^{1 / 2}$ is the thermal velocity and

$$
\operatorname{erf}(x)=\frac{2}{\sqrt{n}} \int_{0}^{x} d t e^{-t^{2}}, \quad G(x)=\frac{\operatorname{erf}(x)-x\{d[\operatorname{erf}(x)] / d x\}}{2 x^{2}}
$$

The functions $G(x)$ and $\operatorname{erf}(x)-G(x)$ are plotted in figure 4.3. For small arguments, one $f$ inds by means of power expansion

$$
\lim _{x \rightarrow 0} G(x)=\frac{2 x}{3 \sqrt{\pi}}, \quad \lim _{x \neq 0}[\operatorname{erf}(x)-G(x)]=\frac{4 x}{3 \sqrt{\pi}}
$$


Thus, for $v_{t} / v_{T}<<1$ equations $(4.5 .8)$ can be approximated by

$$
\begin{aligned}
& \frac{\left\langle\left\langle\Delta v_{z}\right\rangle\right\rangle}{\Delta t}=-\frac{16 \pi^{1 / 2} n C_{0}{ }^{2} \Lambda_{C}}{3 m^{2} v_{T}{ }^{3}} v_{t}\left(=-\beta v_{t}\right) \\
& \frac{\left\langle\left\langle\Delta v_{x}{ }^{2}\right\rangle\right\rangle}{\Delta t}=\frac{\left\langle\left\langle\Delta v_{y}{ }^{2}\right\rangle\right\rangle}{\Delta t}=\frac{\left\langle\left\langle\Delta v_{z}^{2}\right\rangle\right\rangle}{\Delta t}=\frac{16 \pi^{3} / 2 n C_{0}^{2} \Lambda_{c}}{3 m^{2} v_{T}} \quad(=2 D)
\end{aligned}
$$

The diffusion tensor elements can be expressed as $D_{k 1}=\delta_{k 1} D$, which corresponds to an isotropic diffusion process. This is not surprising, since it was assumed that the velocity distribution of the field particles is spherical symmetric. The diffusion constant $D$ is independent of $v_{t}$, while the dynamical friction $\left(=\left\langle\left\langle\Delta v_{z}\right\rangle\right\rangle / \Delta t\right)$ is proportional to $-v_{t}$, as assumed in equation (4.3.8). Notice that the equations (4.5.11) indeed fulfil relation (4.4.6), justifying, a posteriori, the assumption that the equilibrium velocity distribution of the field particles is Maxwellian.

\subsection{Coulomb logarithm}

The coulomb logarithm, defined by equation $(4.5 .6)$, depends on the ratio $b_{m} / b_{1}$, where $b_{m}$ denotes the maximum value of the impact parameter considered in the calculation of the velocity jump-moments and $b_{\perp}$ is the value of the impact parameter, corresponding to a 90 degrees deflection, as defined by equation (4.5.1). If one takes $b_{m}$ equal to the the Debeye screening length $\lambda_{D}$, given by equation $(3.9 .7)$, equation $(4.5 .6)$ transforms to

$$
\Lambda_{C} \approx \ln \left(\lambda_{D} / b_{\perp}\right) \approx \ln \left(\frac{2 \pi m<v^{2}>\left(k_{B} T / 1 / 2 e_{0}{ }^{3 / 2}\right.}{e^{3} n^{1 / 2}}\right) \approx \ln \left(\frac{6 \pi\left(k_{B} T \varepsilon_{0}\right)^{3 / 2}}{e^{3} n^{1 / 2}}\right)
$$

using that $\left\langle\mathrm{v}^{2}\right\rangle=3 \mathrm{k}_{\mathrm{B}} \mathrm{T} / \mathrm{m}$. Taking as a numerical example the case of electrons in a plasma, with $n=10^{16} \mathrm{~m}^{-3}, T=10^{5} \mathrm{~K}$, one finds $\lambda_{\mathrm{D}} / \mathrm{b}_{\perp}=2 \times 10^{6}$ and $L_{c} \approx 14$.

The procedure of cutting of the interaction range at the Debeye length is introduced to obtain non-divergent results. This method appears to be rather artificial and we wish to investigate its physical basis in more detail. The origin of the divergence is not the long range of the coulomb force, as is of ten stated, but rather the utilization of equation (4.5.1) beyond its range of applicability. This equation specifies the angle of deflection $x$ in a binary collision, defined as the angle between the asymptotes of the hyperbola, describing the relative motion of the particles in the orbital plane. Accordingly, its applicability requires that the collision is complete within the time interval $\Delta t$. This implies that the distance vat, over which the collision takes place, should be large compared to the distance of closest approach, which is (for most collisions) of the same magnitude as the impact parameter $b$. Thus, the applicability of equation (4.5.1) requires v $\Delta t \gg b$. However, this condition is certainly not fulfilled for very remote collisions, that is collisions with a large value of the impact parameter $b$. By using equation (4.5.1) to compute the effect of such collisions, one overestimates their contribution to the total deviation of the test particle. This causes the divergence of the results given by the equations $(4.5 .5)$.

From this analysis the remedy appears to be simple. The usage of equation (4.5.1) should be restricted to those impact parameters for 


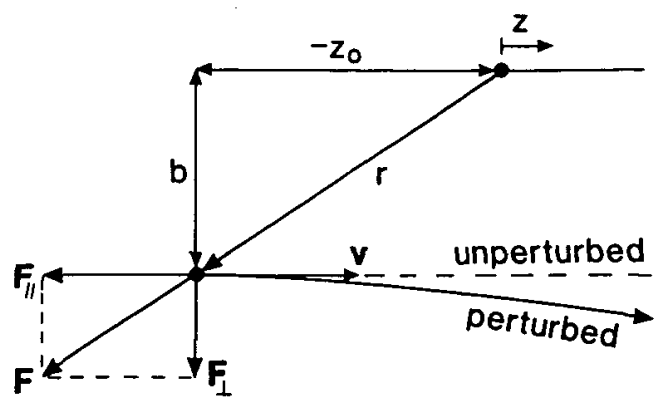

Figure 4.4 Initial relative coordinates of two particles involved in a weak collision. The force $P$ acting on the test particle is separated in a component $F_{\| /}$, parallel to the initial relative velocity $\nabla$ and a component $P_{\perp}$, perpendicular to this direction.

which the corresponding collision is nearly complete, say $b<c_{1} v \Delta t$, where $c_{1} \ll<1$. For larger values one should employ a more refined particle dynamics, which takes the length of the interaction into account. For large values of $b$, say $b>c_{2} v \Delta t$, where $c_{2} \gg 1$, the deviations from the unperturbed trajectories will be small and first order perturbation theory is appropriate to solve the dynamical problem. In this approach one calculates the interaction force, as if the particles were running along their unperturbed trajectories. Non-divergent results for the jump-moments can be obtained, by using complete collision dynamics for small b-values and first order perturbation dynamics for large b-values. In this approach, one avoids the introduction of an artificial upper limit for b.

Let us consider the first order perturbation approximation for the dynamical problem more closely. As before, we define the direction of the relative velocity $v=v_{f}-v_{t}$ as the $z$-direction. Assume that the test-particle is located in the origin of the coordinate system. The unperturbed distance between the particles is now given by $\tilde{r}(t)=\left[\tilde{z}(t)^{2}+b^{2}\right]^{1 / 2}=\left[(v t)^{2}+b^{2}\right]^{1 / 2}$, defining $t=0$ as the moment of closest approach $(\tilde{r}=b)$, see figure 4.4 . The force acting on the test particle can be expressed in terms of a component parallel to $v$ ( $z$-direction) and a component perpendicular to $v$. In first order perturbation approximation, these components are equal to

$$
\tilde{F}_{\perp}(t)=\frac{C_{0} b}{\tilde{r}(t)^{3}}, \quad \tilde{F}_{/ /}(t)=\frac{C_{0} v t}{\tilde{r}(t)^{3}}
$$

For the corresponding components of the velocity shift of the test particle, one finds

$$
\begin{aligned}
& \Delta \tilde{v}_{\perp}=\int_{t_{0}}^{t_{0}+\Delta t} d t \frac{\tilde{F}_{\perp}(t)}{m}=\frac{c_{0}}{m v b}\left(\frac{z_{0}+v \Delta t}{\left[b^{2}+\left(z_{0}+v \Delta t\right)^{2}\right]^{1 / 2}}-\frac{z_{0}}{\left[b^{2}+z_{0}^{2}\right]^{1 / 2}}\right) \\
& \Delta \tilde{v}_{/ /}=\int_{t_{0}}^{t_{0}+\Delta t} d t \frac{1}{m} \frac{\tilde{F}_{/ /}(t)}{m v}=-\frac{c_{0}}{m v}\left(\frac{1}{\left[b^{2}+\left(z_{0}+v \Delta t\right)^{2}\right]^{1 / 2}}-\frac{1}{\left[b^{2}+z_{0}^{2}\right]^{1 / 2}}\right)
\end{aligned}
$$

where $z_{0}=v_{0}$. The required $x, y$ and $z$ components of the velocity shift, now follow as $\Delta \mathrm{v}_{x}=\Delta \tilde{v}_{\perp} \cos \varphi, \Delta \mathrm{v}_{\mathrm{y}}=\Delta \tilde{v}_{\perp} \sin \varphi$ and $\Delta \mathrm{v}_{\mathrm{z}}=\Delta \tilde{\mathrm{v}}_{/ 1}$. The resulting expressions constitute an alternative to those of equation (4.5.2). They 
apply to remote collisions, that is to $b>c_{2} v \Delta t$. Notice that both sets of expressions become identical in the limit of weak complete interactions, which corresponds to $: z_{0} \rightarrow \infty,\left(z_{0}+v \Delta t\right) \rightarrow \infty, q \rightarrow \infty$.

One can now perform the first step of the calculation of the first and second order velocity jump moments, which is described by the second equation of $(4.5 .4)$, in alternative way by splitting the bintegration into two parts

$$
\langle A(v)\rangle=n \int_{0}^{2 \pi} d \varphi\left(v \Delta t \int_{0}^{b_{1}} b d b A_{n}(b, v, \varphi)+\int_{b_{1}}^{\infty} b d b \int_{-\infty}^{\infty} d z_{0} A_{\Gamma}\left(z_{0}, b, v, \varphi\right)\right)
$$

where $b_{1}=c v \Delta t$, with $c_{1}<c<c_{2}, A_{n}$ is the expression of the quantity $A$ (which stands for $\Delta v_{x}, \Delta v_{y}, \Delta v_{z}, \Delta v_{x}{ }^{2}, \ldots$ ) obtained for a near (complete) collision and $A_{r}$ the alternative expression for a remote collision, obtained by first order perturbation theory. A calculation along these lines was carried out by Sivukhin (1966). We will summarize his reasoning.

It is sufficient to consider the calculation of $\left\langle\Delta v_{x}{ }^{2}\right\rangle=\left\langle\Delta v_{y}{ }^{2}\right\rangle=$ $\left\langle\Delta v_{\perp}{ }^{2}\right\rangle / 2$, since $\left\langle\Delta v_{z}{ }^{2}\right\rangle$ can be neglected for $\left.\left.\Lambda_{C}\right\rangle\right\rangle 1$, while the correct value of $\left\langle\Delta v_{z}\right\rangle$ follows from $\left\langle\Delta v_{\perp}{ }^{2}\right\rangle$, using equation (4.5.7). Clearly, the first term of equation $(4.6 .4)$ leads to the expressions for $\left\langle\Delta v_{x}{ }^{2}\right\rangle$ and $\left\langle\Delta v_{y}{ }^{2}\right\rangle$ given by $(4.5 .5)$, where $b_{m}$ is now replaced by $b_{1}$. The second term in equation (4.6.4) can be scaled in such a way, that one obtains an expression, which is identical to that of the first term, but in which $\Lambda_{c}$ is replaced by a two dimensional integral, which contains the ratio $b_{1} / v \Delta t$ as the only free parameter. Accordingly, one may conclude that equation (4.5.5) remains true, provided that the Coulomb logarithm is replaced by

$$
\Lambda_{c}=\ln \left(b_{1} / b_{1}\right)+f\left(v \Delta t / b_{1}\right)
$$

where

$$
f(u)=\int_{0}^{u} \frac{d x}{x^{2}} \int_{-\infty}^{\infty} d y\left(\frac{x+y}{\left(1+(x+y)^{2}\right]^{1 / 2}}-\frac{y}{\left[1+y^{2}\right]^{1 / 2}}\right)^{2}
$$

By means of an asymptotic analysis, it can be demonstrated that $f(u) \approx \ln (u)$ for $u>2$. Thus, by using a proper value for the free parameter $b_{1}$ (that is $b_{1}<<v \Delta t$ ), one may approximate $(4.6 .5)$ as

$$
\Lambda_{c}=\ln \left(v \Delta t / b_{\perp}\right)
$$

According to this analysis $b_{m}=v \Delta t$.

We have now removed the divergence in a natural way, but obtained a new problem instead, namely that the Coulomb logarithm $\Lambda_{c}$ depends on the time interval $\Delta t$. This quantity has been considered as arbitrary so far, the only constraints on it being expressed by the equations (4.3.3). At this point, one should realize that equations (4.6.3) are valid as long as the particles follow approximately their unperturbed trajectories, which are straight lines. However, the two particles considered, are not the only particles in the system. The interaction with the other particles will cause (random) deviations from the unperturbed trajectories. When such a deviation occurs, the interaction between the two particles is effectively broken up and starts again along a new trajectory. Let us assume that the average time period between two successive interruptions is $\tau_{0}$. In general, this will be a small time interval compared to $\Delta t$. Thus, the interaction is on 
the average broken up $\Delta t / \tau_{0}$ times during the time interval $\Delta t$. The corresponding $\Delta t / \tau_{0}$ deviations, built up during the periods between two successive interruptions, should be regarded as statistically independent. Accordingly, one has to replace the expression for $\left\langle\Delta v_{\perp}{ }^{2}\right\rangle \Delta t$, calculated over the time interval $\Delta t$, by $\left(\Delta t / \tau_{0}\right) \times\left\langle\Delta v_{\perp}{ }^{2}\right\rangle_{\tau 0}$, where $\left\langle\Delta v_{\perp}{ }^{2}\right\rangle_{T 0}$ is calculated over the time interval $\tau_{0}$. In this approximation, the coulomb logarithm $\wedge_{c}$ becomes equal to

$$
\Lambda_{C} \approx \ln \left[\left\langle\mathrm{V}^{2}>1 / 2 \tau_{0} / b_{\perp}\right] \approx \ln \left[\left(k_{B} T / m\right)^{1 / 2} T_{0} / b_{\perp}\right]\right.
$$

as follows with equations (4.5.5) and (4.6.6). As usual, we replaced the velocity $v$ in the argument of the logarithm by the average value $\left\langle v^{2}>1 / 2\right.$. Equations $(4.6 .7)$ corresponds to $b_{m}=\left(k_{B} T / m\right) 1 / 2 \tau_{0}$. The remaining problem is now the estimation of $\tau_{0}$.

In neutralized plasmas, the collective response to the motion of the individual charged particles, leads to collective harmonic oscillations of the electrons, see for instance Pines and Bohm (1952) or Ichimaru (1973). The eigen-frequency of the system of electrons embedded in the neutralizing background of positive ions is equal to the plasma frequency $\omega_{p}$, given by equation (3.9.12). The organized (collective) motion will interfere with the random (thermal) motion of the individual particles, with a period of approximately $1 / \omega_{p}$. Thus, for neutralized systems, it appears to be reasonable to take $\tau_{0}=1 / \omega_{\mathrm{p}}$. Accordingly, one finds $b_{m}=\lambda_{D}$, the Debeye screening distance. Thus, the procedure of cutting of the interaction range at the Debeye distance, seems justified for neutralized systems and the Coulomb logarithm $\Lambda_{c}$ should be calculated from equation (4.6.1).

on the other hand, for non-neutralized systems, such as the system of identical charged particles considered in this chapter, it appears to be more appropriate to identify $\tau_{0}$ with the correlation time of the fluctuations in the interaction force $\tau_{f}$, given by equation (4.3.1). Accordingly, one finds $b_{m}=n^{-1 / 3}$, which is the average distance between the particles in the system. Thus, for non-neutralized systems the Coulomb logarithm $\Lambda_{c}$ should be calculated as

$$
\Lambda_{c} \approx \ln \left(\frac{n^{-1 / 3}}{b_{\perp}}\right) \approx \ln \left(\frac{m<v^{2}>}{2 C_{0} n^{1 / 3}}\right) \approx \ln \left(\frac{3 k_{B} T}{2 C_{0} n^{1 / 3}}\right)
$$

using the same approximations as in equation (4.6.1). It should be emphasized, that the phenomenon of Debeye screening can occur in nonneutralized systems, as was discussed in section 3.6. The reason for choosing $b_{m}=n^{-1 / 3}$ is not the absence of screening, but is rather related to the absence of collective oscillations. Notice that for systems in which the phenomenon of Debeye screening does occur, the distance $\lambda_{D}$ will be large compared to the average interparticle distance $n^{-1 / 3}$ (otherwise the Debeye sphere does not contain many particles, contradicting the premises of the screening-concept, see equation $(3.9 .15))$. Finally, we note that equation $(4.6 .8)$ also applies to stellar systems (replacing $\mathrm{C}_{0}$ by $\mathrm{Gm}^{2}$ ), which should be regarded as nonneutralized, see Chandrasekhar $(1941,1942 \mathrm{a})$. Similar results (but differing by a numerical factor) are given by Chandrasekhar and Von Neumann (1942b,1943). These results are, however, obtained by entirely different reasoning. 
In the previous sections the main issues related to the FokkerPlanck type of equation (4.3.10) were discussed, stressing the aspects concerning the fluctuating component of the interaction force. In this section, we will concentrate on the relationship between the various concepts introduced so far, with the objective to clarify the global philosophy of the Fokker-Planck approach.

The fundamental presupposition of the Fokker-Planck approach is that the action of the fluctuating component of the interaction force can be described in terms of a diffusion process in velocity space, characterized by the coefficient of dynamical friction $\beta$ and the diffusion tensor $D$. It is assumed that these quantities are determined by the macroscopic properties of the system only. This approach is justified when there exists a time interval $\Delta t$, which is long compared to the correlation time of the fluctuations $\tau_{f}$, but small enough to neglect the variation in the macroscopic properties of the system, represented by $f(r, v, t)$ and small enough to consider the jumps in the velocity $\delta v(t ; \Delta t)$ as microscopic. These constraints on $\Delta t$ are expressed by equations (4.3.3). Spatial displacements, within the interval $\Delta t$, are ignored on the argument that they are of the second order in $\Delta t$ and thus negligible compared to the first order shift $\delta v \Delta t$. The stationary solution of the diffusion equation is a Gaussian (velocity) distribution, which is consistent with the assumption that the velocity of the test particle is built up by a large number of uncorrelated microscopic shifts $\delta \mathbf{v}$ (central limit theorem). The stationary solution is Maxwellian, provided that $\beta$ and $D$ are related by equation $(4.4 .6)$. Therefore, the model is suited to describe the impact of particle interactions in a system which operates near thermodynamic equilibrium.

The diffusion approximation reduces the problem of determining the effect of the fluctuations in the interaction force on the single particle probability function $f(r, v, t)$, to the calculation of the coefficient of dynamical friction $\beta$ and the diffusion tensor $D$, which are related to the first and second velocity jump-moments respectively. These jump-moments, which are specified by the equations (4.5.11), are proportional to the Coulomb logarithm $\Lambda_{c}$. For neutralized systems (such as plasmas), this quantity is given by equation (4.6.1) and for nonneutralized systems (such as systems of identical charged particles and stellar systems) it is given by equation (4.6.8). The argument of the logarithm, appearing in these expressions, may be of $f$ by a factor, say 2 to 5 . If one denotes this factor as $f$, the relative error in $\Lambda_{c}$ can be expressed as $1+\ln (f) / \Lambda_{c}$. Taking as an example $\Lambda_{c}=10$, one finds a relative error in $\Lambda_{c}$ of $7 \%$ for $f=2$ and $16 \%$ for $f=5$. Clearly, the sensitivety of $\wedge_{c}$, for the error factor $f$, decreases with increasing values of $\Lambda_{c}$.

The Coulomb logarithm $\Lambda_{c}$ is an important quantity within the framework of the Fokker-Planck approach. The validity of the diffusion approximation requires that $\Lambda_{c} \gg 1$. The argument is twofold:

Firstly, one should recall that the first and second jumpmoments, given by equation $(4.5 .11)$, diverge proportionally to $\Lambda_{c}$, while higher moments are finite, irrespective of the value of $\Lambda_{c}$ (since they are independent of $b_{m}$ ). Accordingly, the higher jump moments can be neglected relative to the first and second one, provided that $\Lambda_{c}$ is large enough. This condition guarantees that the time-evolution of the single particle distribution function $f(r, v, t)$ is indeed dominated by the first and second jump-moments, as assumed in the Fokker-Planck approach.

Secondly, the Coulomb-logarithm is a measure for the relative contribution of (weak) remote interactions compared to (strong) near 
interactions. Defining all interactions with $b>2 b_{\perp}$ as remote and all interactions with $b<2 b_{\perp}$ as near, one finds for the ratio of their contributions to the Coulomb logarithm (see equation $(4.5 .6)$ )

$$
\frac{\Lambda_{c}, \text { remote }}{\Lambda_{c}, \text { near }}=\int_{4}^{q_{m}} \frac{d q}{1+q} / \int_{0}^{4} \frac{d q}{1+q}=\frac{\ln \left(1+q_{m}\right)-\ln 5}{\ln 5} \approx 1.2 \Lambda_{c}-1 \approx \Lambda_{c}
$$

assuming that $\Lambda_{C} \gg 1$. This equation shows that the total contribution of remote interactions is approximately $\Lambda_{C}$ times larger than the contribution of near interactions. Thus, when $\Lambda_{c} \gg>1$, the jumps in the velocity of a test particle will, in general, be small and its motion in velocity space can be considered as quasi-continuous. We recall that this condition is essential for the validity of the diffusion approximation.

The calculation of the coefficient of dynamical friction $\beta$ and the diffusion tensor $D$, is based on a reduction of the $\mathrm{N}$-body problem to two-particle interactions. In this procedure the interaction between the test particle and a particular field particle is calculated, as if they are the only particles in the system. The total deviation of the test particle, due to the interactions with all field particles during the time interval $\Delta t$, is computed as the sum of all separate two-particle effects occurring in that period. This procedure, which is sometimes referred to as the binary interaction approximation, would be justified when all collisions would be well separated in time. However, in reality many of the two-particle interactions occur simultaneously and not successively, due to the long range of the interaction force. This rises the question whether the binary interaction approximation is valid or not.

In defense of the binary interaction approximation, it should be noted that most interactions are weak, provided that $\Lambda_{c} \gg 1$. The interactions which occur simultaneously are therefore predominantly remote interactions, causing small deviations in the velocities of the particles involved. These deviations can be estimated by means of first order perturbation dynamics, in which one evaluates the effect of the interaction force experienced along the unperturbed trajectories. This is expressed by equations (4.6.3). As forces are additive, the reduction to binary interactions is justified for this type of interactions. In general, one may say that the binary interaction approximation is justified, as long as strong interactions are rare $\left(\Lambda_{C} \gg 1\right)$. Notice that this condition is required anyhow, to justify the diffusion approximation.

\subsection{Validity of the Fokker-Planck approach for particle beams}

In this section we will study whether the Fokker-Planck approach is suited to describe the effect of statistical interactions in particle beams or not. To that intent, let us consider a rotational symmetric beam segment in drift space with an uniform density distribution in every cross section of the beam. The macroscopic condition of the beam segment is specified by the values of the experimental parameters $I, V$, $\Delta E_{0}$ (or alternatively cathode temperature $\mathrm{T}_{\mathrm{c}}$ ), $\mathrm{L}, \mathrm{r}_{\mathrm{c}}, \alpha_{0}, S_{\mathrm{c}}$ and $S_{\mathrm{i}}$, defined in section 3.2. First, we will express the characteristic theoretical quantities, introduced in this chapter, in terms of the experimental parameters. Next, we will investigate whether the various assumptions underlying the Fokker-Planck approach are justified for normal operating conditions. 
The physical quantities to be examined, are the correlation time of the fluctuations in the interaction force $\tau_{\mathrm{f}}$, the Debeye length $\lambda_{p}$, the plasma frequency $\omega_{p}$ and the Coulomb logarithm $\Lambda_{c}$, defined by the equations $(4.3 .1),(3.9 .7),(3.9 .12)$ and $(4.6 .8)$ respectively. Given the type of particles, these quantities depend on two parameters only : The particle density $n$ and the beam temperature $T$. The particle density $n$ is a function of the axial position $z$, through the beam radius $r_{0}(z)$, as expressed by equation (3.2.5). The temperature in a rotational symmetric beam of non-interacting particles was studied in section 3.7 . It was concluded that one should, in general, distinguish two temperatures, $\mathrm{T}_{/ /}$and $\mathrm{T}_{\perp}$, corresponding to the longitudinal and lateral degrees of freedom respectively. These temperatures are specified in terms of the experimental parameters by equations (3.7.13) and (3.7.18). Assuming, for the moment, that it is useful to distinguish different values for $\tau_{f}, \lambda_{D}$ and $\Lambda_{C}$ in axial and lateral direction, one finds from equations $(4.3 .1),(3.9 .7),(3.9 .12)$ and $(4.6 .8)$

$$
\begin{aligned}
& \tau_{\mathrm{f} / /}=\frac{2 \pi^{1 / 3}(\mathrm{meV})^{1 / 2}}{\lambda^{1 / 3} \mathrm{k}_{B} T_{\mathrm{ca}}} r_{0}(z)^{2 / 3}, \quad \tau_{f_{\perp}}=\frac{21 / 2 \pi^{1 / 3} \mathrm{~m}^{1 / 2}}{\lambda^{1 / 3}(\mathrm{eV})^{1 / 2} \alpha_{0} \mathrm{r}_{\mathrm{c}}} r_{0}(z)^{5 / 3} \\
& \lambda_{D \|}=\left(\frac{\pi \epsilon_{0}\left(k_{B} T_{C a}\right)^{2}}{4 \mathrm{e}^{3} \mathrm{~V} \lambda^{1 / 3}}\right)^{1 / 2} \frac{1}{r_{0}(z)} \quad, \quad \lambda_{D_{\perp}}=\left(\frac{\pi \epsilon_{0} V \alpha_{0}{ }^{2} r_{c}{ }^{2}}{2 \mathrm{e} \lambda}\right)^{1 / 2} \\
& \omega_{p}=\left(\frac{e^{2} \lambda}{\pi \epsilon_{0} m}\right)^{1 / 2} \frac{1}{r_{0}(z)} \text {. } \\
& \Lambda_{c / /}=\ln \left(\frac{\pi^{4 / 3} \varepsilon_{0}\left(k_{B} T_{c a}\right)^{2} r_{0}(z)^{2 / 3}}{e^{3} V \lambda^{1 / 3}}\right), \quad \Lambda_{c_{\perp}}=\ln \left(\frac{2 \pi^{4 / 3} \varepsilon_{0} V \alpha_{0}^{2} r_{c}{ }^{2}}{e \lambda^{1 / 3} r_{0}(z)^{4 / 3}}\right)
\end{aligned}
$$

where $\lambda$ is the linear particle density, given by equation (3.2.4). Notice that all quantities depend on the axial coordinate $z$, except $\lambda_{D_{\perp}}$. Defining $N_{D}$ as the total number of particles in the Debeye box with sides $\lambda_{D_{\perp}}, \lambda_{D_{\perp}}$ and $\lambda_{D_{/ /}}$, one obtains

$$
N_{D}=\frac{\pi^{1 / 2} \varepsilon_{0}^{3 / 2} V^{1 / 2} \alpha_{0}^{2} r_{c}^{2}}{4 e^{5 / 2} \lambda^{1 / 2}} \frac{1}{r_{0}(z)}
$$

Equations (4.8.1) and (4.8.2) express the physical quantities which are relevant to the Fokker-Planck approach, in terms of the experimental parameters of the beam.

Let us now consider the same numerical example as in section 3.2 , that is an electron beam with $I=1 \mu \mathrm{A}, V=10 \mathrm{kV}, L=0.1 \mathrm{~m}, S_{\mathrm{c}}=0.5, r_{\mathrm{c}}=1 \mu \mathrm{m}$ and $\alpha_{0}=10 \mathrm{mR}$, and take $\mathrm{k}_{\mathrm{B}} \mathrm{T}_{\mathrm{c}}=0.5 \mathrm{eV}$ (equal to the transiverse temperature in the crossover). From equations $(4.8 .1)$ and $(4.8 .2)$ one obtains

$$
\begin{aligned}
& \tau_{\mathrm{f} / / \mathrm{c}}=1.5 \times 10^{-9} \mathrm{~s} \quad, \quad \tau_{\mathrm{f} / 1}=9.2 \times 10^{-8} \mathrm{~s} \\
& T_{f \perp C}=4.9 \times 10^{-12} \mathrm{~S}, \quad \tau_{f_{\perp 1}}=1.5 \times 10^{-1} \mathrm{~s} \\
& l_{\mathrm{D} / \mathrm{C}}=.032 \mu \mathrm{m} \quad, \quad l_{\mathrm{D} / / 1}=16 \mu \mathrm{m} \\
& \lambda_{D_{\perp}}=9.1 \mu \mathrm{m} \\
& 1 / \omega_{p c}=3.1 \times 10^{-11} \mathrm{~s} \quad, \quad 1 / \omega_{p 1}=1.5 \times 10^{-8} \mathrm{~s} \\
& \Lambda_{c / / c}=2.0 \times 10^{-5}, \quad \Lambda_{c / / 1}=7.2 \times 10^{-2} \\
& \Lambda_{C_{\perp C}}=6.2 \quad, \quad \Lambda_{C_{\perp} 1}=7.8 \times 10^{-3} \\
& N_{D C}=.89 \quad, \quad N_{D 1}=1.8 \times 10^{-3}
\end{aligned}
$$

where the subscript $c$ refers to the conditions in the crossover and the subscript 1 to the conditions at the entrance plane of the beam segment. In the calculation of $\Lambda_{c / / c}, \Lambda_{c / 11}$ and $\Lambda_{c_{\perp 1}}$, the arguments of the logarithm turned out to be smaller than 1 and we used the full 
expression $\Lambda_{D}=\ln \left[\left(1+q_{m}\right)^{1 / 2}\right]$, instead of the usual approximation $\Lambda_{D}=\ln \left[q_{m}{ }^{1 / 2}\right]$, see equation $(4.5 .6)$.

Let us investigate what is to be learned from these numbers. The values found for the correlation time of the fluctuations in the interaction force $\tau_{f}$, indicate that most collisions take place in the crossover, mainly due to the transverse motion of the particles. In order to get an impression of the average number of collisions experienced by a particle during its flight through the beam segment, these values should be compared to the flight time $T_{f}$, given by equation (3.2.3). For the quantities referring to the crossover, it is more appropriate to compare them to the flight time through the crossover area $T_{f c}$. As a measure for this quantity, we use

$$
\mathrm{T}_{\mathrm{fc}} \approx 2 \mathrm{r}_{\mathrm{c}} / \alpha_{\mathrm{O}} \mathrm{v}_{\|}
$$

which is the time that a particle travels over the distance $2 r_{c} / \alpha_{0}$. For the numerical example, one finds from equations (3.2.3) and (4.8.3)

$$
T_{f}=1.710^{-9} \mathrm{~s}, \quad T_{f c}=3.410^{-12} \mathrm{~s}
$$

Thus, the correlation time of the fluctuations if can not be considered as short compared to the appropriate flight time (e.g. $\left.T_{f} / \tau_{f / / 1}=1.8 \times 10^{-2}, T_{f c} / \tau_{f} c=0.69\right)$. Accordingly, the number of collisions is in general not a large number.

The inverse of the plasma frequency $\omega_{p}$ is the relevant measure for the relaxation time of the system to reach thermodynamic equilibrium, as was shown in section 3.9. From the numbers of the numerical example, one finds that thermodynamic equilibrium will, in general, not be reached $\left(T_{f} \omega_{p 1}=T_{f c} \omega_{p c}=.11\right)$. This implies that Debeye screening, which presupposes thermodynamic equilibrium, will in general not occur. In this context, it should be noted that the condition that the number of particles in the Debeye box $N_{p}$ is large, which is expressed by equation (3.9.15), is not fulfilled either.

The validity of the Fokker-Planck approach requires that the Coulomb logarithm is large, as was pointed out in the previous section. clearly, the only parameter fulfilling this condition is $\Lambda_{c_{\perp} c}$.

- In general, one sees that the basic requirements of the FokkerPlanck approach, expressed by the equations (4.3.3), are not fulfilled. The correlation time of the fluctuations $r_{f}$ is not small compared to the total timescale of the system $T_{f}$ or $T_{f}$. Accordingly, the macroscopic condition of a bunch of particles in the beam, determining the coefficient of dynamical friction $B$ and diffusion tensor $D$, changes appreciable during the time interval $T_{f}$. In other words, $\beta$ and $D$ can not be considered as constant during the typical period of a collision. In addition, the contribution of strong interactions, corresponding to large jumps in velocity, can, in general, not be ignored. These results can be summarized by the statement that the motion in velocity space can not be considered as a succession of a large number of microscopic random displacements, superimposed on a smooth systematic motion.

As the number of collisions is, in general, not large, while the effect of strong collisions can not be ignored, the resulting velocity distribution is not necessarily Gaussian. This is equivalent to the assertion that the higher jump moments than the second can not be disregarded, which implies that the diffusion approximation is inaccurate.

Finally, we note that for a particle beam, one is not only interested in the velocity distribution of the particles, but also in the distribution of stochastic spatial displacements (trajectory displacement effect). As the Fokker-Planck approach neglects the spatial 
displacements which are generated during the collision (by just taking the resulting velocity change into account), this method is not suited to describe the trajectory displacement effect.

One may object, that this is just a single numerical example and dispute the generality of the conclusions. However, examining the dependency of equations $(4,8,1)$ on the experimental parameters, one should conclude, that the conditions described above, do not change drastically within the practical range of operating conditions, encountered in the systems of interest (see section 1.1). The main insufficiency arises from the fact that beams are relatively cold compared to a plasma, while the flight times of the beam particles are short compared to the relevant relaxation times. Accordingly, the number of collisions is limited, contradicting the basic philosophy of the diffusion approximation.

The requirements of the Fokker-Planck approach are, to a certain extent, fulfilled in the crossover area, which is the "hottest" section of the beam. Thus, the Fokker-Planck approach may give a reasonable approximation for the Boersch effect, generated in this part of the beam. From the second equation of $(4.5 .11)$ one finds, using $\mathrm{n} \Delta \mathrm{t}=\mathrm{J}_{\mathrm{c}} \Delta z / \mathrm{ev}_{/ /}{ }^{2}$ and $\mathrm{v}_{\mathrm{T}}=\left(2 \mathrm{k}_{\mathrm{g}} \mathrm{T}_{\perp \mathrm{c}} / \mathrm{m}\right)^{1 / 2}$

$$
<\Delta \mathrm{E}^{2}>1 / 2=\mathrm{mv}_{/ /}<\Delta v_{/ /}^{2}>1 / 2=\frac{2^{7 / 4} \pi^{1 / 4}}{3^{1 / 2}} \frac{\mathrm{C}_{0} \mathrm{~m}^{1 / 4}}{\mathrm{e}^{1 / 2}} \frac{\left(\mathrm{J}_{\mathrm{c}} \Delta \mathrm{z}\right)^{1 / 2}}{\left(\mathrm{k}_{B} \mathrm{~T}_{\perp \mathrm{c}}\right)^{1 / 4}}{\Lambda_{\mathrm{C}_{\perp} \mathrm{c}^{1 / 2}}}
$$

assuming that the major effect comes from the motion in the lateral directions. The Coulomb logarithm $\Lambda_{c_{\perp}}$ is given by equations (4.8.1). Substituting $r_{0}=r_{c}$ and $\lambda=\pi r_{c}^{2} J_{c} /\left(2 e^{3} V / m\right)^{1 / 2}$ (see equation $(3.2 .4)$ ), it can be expressed as

$$
\Lambda_{\perp c}=\ln \left(\frac{2 \pi^{4 / 3} \varepsilon_{0} V^{7 / 6} \alpha_{0}^{2}}{\mathrm{e}^{1 / 2} \mathrm{~m}^{1 / 6} \mathrm{~J}_{\mathrm{c}}^{1 / 3}}\right)=\ln \left[\frac{\mathrm{k}_{\mathrm{BT}} \mathrm{T}_{\perp c}}{\mathrm{C}_{0}}\left(\frac{\mathrm{eV}}{\mathrm{m}}\right)^{1 / 6}\left(\frac{\mathrm{e}}{\mathrm{J}_{\mathrm{c}}}\right)^{1 / 3}\right]
$$

in which equation $(3.7 .4)$ was utilized to express the beam semi-angle $\alpha_{0}$ in terms of the temperature $T_{\perp c}$. Apart from a numerical factor, equations (4.8.4) and (4.8.5) are identical to the results given by Zimmermann (1970) and Knauer (1979a), for a cylindrical beam segment of length $\Delta z$ (Their results are larger by a factor $(3 \pi)^{1 / 2 / 25 / 4} \approx 1.29$. In addition, Zimmermann's argument of the Coulomb logarithm is smaller by a factor 2.22). The related models of Zimmermann and Knauer will be further discussed in chapter 15.

Dynamical friction, specified by the first equation of (4.5.1.), can be disregarded since the (test) particles do not have a large excess velocity relative to the surrounding (field) particles. Therefore, it is sufficient to consider the second moment of the distribution of velocity shifts only, provided that the distribution is indeed Gaussian, as implicitly assumed within the diffusion approximation.

The next step would be the calculation of the single particle distribution function $f(r, v, t)$, as it develops during the time of flight. This function can be obtained by solving the Fokker-Planck equation (4.3.10). However, since one is mainly interested in the generated energy spread, one can omit this step. All relevant information is contained in the second jump moment, given by equation $(4.8 .4)$. In general, one may say that the primary objective of a theory for statistical Coulomb interactions in particle beams, is to calculate the transition probability function $w_{\Delta t}$, specified by the equations (4.3.4) and (4.3.5), rather than the evolution of the single-particle distribution function $f(r, v, t)$. In the specific case that the diffusion approximation is justified, the transition probability function $w_{\Delta t}$ is Gaussian and one can suffice to calculate its second moment. 
We now like to report on a method for the calculation of collisional effects which can, in some cases, be used where the FokkerPlanck approach fails. Consider the extreme case of a monochromatic homocentric cylindrical beam, in which the particles move with identical velocities along parallel rays. observed in the frame of reference moving with the beam, the particles are initially at rest. Random velocities will be generated, due to the relaxation of potential energy, as was discussed in the sections 3.8. and 3.10 . Since the effective beam temperatures are initially zero, the Fokker-Planck approach is clearly not suited to describe the effect of statistical interactions in such a beam.

Let us assume that the particle density is not so high, that the beam will significantly be deformed by the space charge effect. We thus postulate, that the displacements, caused by the coulomb interaction between the particles in the beam, are small compared to the average separation of the particles. For this condition, the generated displacements in velocity and positions, can be calculated in first order perturbation approximation, as was outlined in section 4.6, see equation (4.6.3). This means that the real interaction force, acting on a particle, is approximated by the force, which would act on that particle, when all particles would follow their unperturbed trajectories. Conform this approximation, one can directly relate the distribution of the deviations, caused by the statistical interactions, to the distribution of the fluctuation component of the interaction force which would occur in the unperturbed beam.

For the specific case of particles which are at rest relative to each other (as in our example), this distribution was first calculated by Holtsmark (1919). He considered the problem of determining the probability $\rho(\boldsymbol{F})$, that a given electric field strength $F$, acts at a

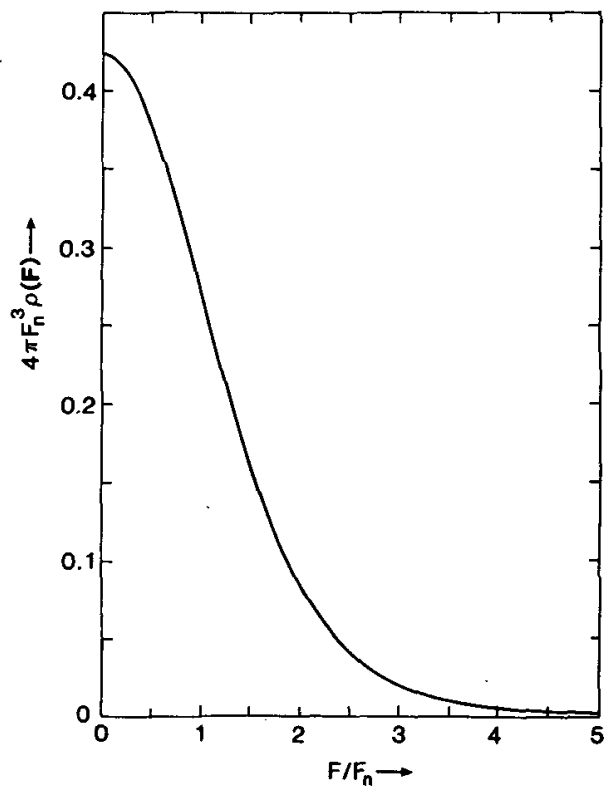

Figure 4.5 The Holtsmark distribution, defined by equation (4.9.1). 
point in a gas of density $n$, composed of randomly distributed ions. His result can be expressed as

$$
\rho(F)=\frac{1}{2 \pi^{2} F} \int_{0}^{\infty} k d k \sin (F k) e^{-\left(F_{n} k\right)^{3 / 2}}
$$

where $F=|F|$ and $F_{n}$ is the normalized field strength, given by

$$
F_{n}=(4 / 15)^{2 / 3} 2 \pi C_{0} \quad n^{2 / 3}
$$

The Holtsmark distribution is plotted in figure 4.5. A comprehensive discussion of the Holtsmark distribution is given by Chandrasekhar (1941,1943). A derivation of the Holtsmark distribution, in the form of equation $(4.9 .1)$, is included in section 5.8 .

The essential observation of this section is the following. For a monochromatic homocentric cylindrical beam of low particle density one may assume that velocity displacement $\Delta v$, experienced by a particle during the time of flight $T_{f}$, is simply given by

$$
\Delta \mathbf{v} \approx \mathbf{F T} \mathbf{f} / \mathrm{m} \text {. }
$$

since $\mathbf{F}$ is constant, due to the fact that the particles are initially at rest relative to each other and do not experience large displacements during the flight. The distribution of $F$ is given by equation (4.9.1). The distribution of velocity displacements $\Delta v$, denoted as $\rho_{v}(\Delta \mathbf{v})$, now follows as

$$
\rho_{\mathbf{v}}(\Delta \mathbf{v})=\left(\frac{\mathrm{m}}{\mathrm{T}_{\mathrm{f}}}\right)^{3} \rho\left(P=\mathrm{m} \Delta \mathbf{v} / \mathrm{T}_{\mathrm{f}}\right)
$$

The factor $\left(\mathrm{m} / \mathrm{T}_{\mathrm{f}}\right)^{3}$ guarantees normalization of the 3-dimensional distribution $\rho_{v}(\Delta v)$. Equation $(4.9 .4)$ expresses that the distribution of velocity displacements $\rho_{v}(\Delta v)$ and the distribution of the fluctuating component of the interaction force $\rho(P)$ are entirely correlated. Since $\rho(F)$ is a Holtsmark distribution, $\rho_{\mathbf{v}}(\Delta \mathbf{v})$ is a Holtsmark distribution too.

The properties of the Holtsmark distribution are quite different from those of a Gaussian distribution. Its asymptotic behaviour is specified by

$$
\lim _{\mathrm{FiO}} \rho(F)=\frac{1}{3 \pi^{2} F_{n}^{3}}, \quad \lim _{\mathrm{F} \rightarrow \infty} \rho(F)=\frac{15}{29 / 2 \pi^{3 / 2}} \frac{F_{n} 3 / 2}{F^{9 / 2}}
$$

The behaviour for $F+\infty$ shows that the Holtsmark distribution has distinctive non-Gaussian features. Its long tails lead to a divergence of the second and higher moments

$$
\left\langle F^{m}\right\rangle=\int_{0}^{\infty} 4 \pi F^{2} d F F^{m} \rho(F) \rightarrow \infty \text { for } m \geqslant 2
$$

This divergence is a consequence of the assumption of complete randomness of the particle distribution, in all elements of volume. This assumption is not valid in the very immediate neighbourhood of a (test) particle. Accordingly, the Holtsmark distribution predicts to high probabilities for large values of F. Holtsmark (1919) avoided this problem by considering the distribution of the field strength $F$ in a gas, consisting of dipoles or quadrupoles, instead of simple (monopole) 
ions. Chandrasekhar (1941) took a different approach by postulating that no particles with velocity $v$ occur within a sphere with radius $\mathrm{r}=2 \mathrm{C}_{\mathrm{om}} / \mathrm{v}^{2}$, while the distribution is random for all distances larger than this critical distance.

From equations $(4.9 .1)$ and $(4.9 .2)$, it follows that the distribution of $F$ scales with $n^{2 / 3}$. This can directly be understood from the fact that the Coulomb force scales with the inverse square of the average interparticle distance, which is equal to $n^{-1 / 3}$. For the particular case of a particle beam, one should expect that the interaction effects increase with the $2 / 3$ power of the current density J. More specifically, the (arbitrary) width of the energy distribution $\Delta E$, generated in a homocentric cylindrical beam, scales as

$$
\Delta E \sim m v_{/ /} \frac{F_{n} \Delta t}{m}=F_{n} \Delta z=\pi(8 / 15) 2 / 3 \frac{C_{0} m^{1 / 3}}{e} \frac{\mathrm{J}^{2} / 3 \Delta z}{V^{1 / 3}}
$$

using that $n=J / e v_{\mu}$. We emphasize that this result relies on the assumption that the displacements are small compared to the average interparticle distance $n^{-1 / 3}$. In addition, it is assumed that the beam is extended (three-dimensional), which guarantees that every test particle is surrounded by a spherical symmetric cloud of field particles.

Clearly, equation (4.9.7) shows a different dependency on the experimental parameters as equation (4.8.4), obtained for the beam area in the vicinity of a crossover, employing the diffusion approximation (note that $2 \mathrm{k}_{\mathrm{B}} \mathrm{T}_{\perp \mathrm{C}}=\mathrm{eV \alpha _{ \textrm {a } }}{ }^{2}$ ). The former predicts a $2 / 3$ power dependency on the current density and a linear dependency on the length $\Delta z$, while the latter is proportional to the square root of both parameters. The difference in the dependency on the length $\Delta z$, stems from the fact that the diffusion approach presupposes that the velocity change of a test particle is built up by a large number of independent collisions, while the present analysis assumes that it results from the interaction within a certain complexion of neighbour field particles, which does not change during the time of flight.

\subsection{Conclusions}

Summarizing the results of this chapter, it appears that the methods employed in plasma physics and stellar dynamics are, in general, not. suited to describe all aspects of statistical coulomb interactions in particle beams, for all practical values of the experimental parameters. The diffusion approximation (or Fokker-Planck approach) is suited to calculate the velocity broadening in sections of the beam, characterized by a strong transverse particle motion ("hot" sections) and a high particle density. Such conditions are of ten encountered in the crossover area's. The analysis of the previous section, which utilizes the Holtsmark distribution, is more appropriate for beam sections with little particle motion and a low particle density. These conditions are encountered in (nearly) homocentric cylindrical beams. Both approaches lead to different types of distributions, while the widths of these distributions follow different dependencies on the experimental parameters. Concerning the fundamental issues involved, it should be realized that the two approaches are based on different physical mechanism's. In the diffusion approximation one determines the transition of kinetic energy by complete collisions from one degree of freedom to another. In this view, the statistical effects are the result of a relaxation of kinetic energy. The approach centred around 


\section{CONCEPTS OF AN ARALYTICAL MODEL FOR STATISTICAL INTERACTIONS IK PARTICLE BRAMS}

\subsection{Introduction}

This chapter discusses the fundamental aspects of an analytical model describing the impact of statistical Coulomb interactions on the properties of a beam of identical charged particles. It starts with a general analysis of this type of $\mathrm{N}$-particle problem. Various methods of reducing the $\mathrm{N}$-particle problem are investigated, leading to the conclusion that the so-called extended two-particle approximation, introduced by van Leeuwen and Jansen (1983), is the least restrictive. The mathematical framework of this model is worked out in detail, demonstrating the feasibility of a direct calculation of the entire distribution of displacements in velocity and position. The model is exploited to determine the distribution of the fluctuating component of the interaction force in an extended beam as well as in a pencil beam. For the former one finds the Holtsmark distribution. The analysis of this chapter indicates, in general terms, what types of distributions are produced by the statistical interactions. It also predicts the dependency of the width of these distributions on the particle density within the beam. The material of this chapter provides the basic concepts required for the calculation of the Boersch effect, the effect of statistical angular deflections and the trajectory displacement effect, carried out in the next chapters.

\subsection{General formulation of the problem}

Consider the beam of a probe forming instrument, e.g. a scanning electron microscope or an electron beam pattern generator. Particles are continuously emitted from the cathode and accelerated towards the anode. From there they drift through the remaining part of the column. The direction of the particles is altered by the lenses and the deflectors in the column, depending on their lateral position within the beam and their velocity. As a consequence each particle experiences a varying density of its surrounding neighbours, the highest density being reached at the location of the crossovers. At the end of the system the particles hit the target, where they are removed from the beam.

We schematize the beam as a succession of beam segments, separated by optical components. We will study the impact of the interaction between the particles for a single beam segment. The object of the theory is to evaluate the statistical effects as function of the experimental parameters $I, V, \Delta E_{0}, L, r_{c}, \alpha_{c}, S_{c}$ and $S_{i}$, defined in section 3.2. It is assumed that the total effect generated in the entire beam can be represented as a sum of the effects generated in the individual beam segments. This is not a trivial assumption, as will be discussed in some detail at the end of this chapter. The beam segments are supposed to be rotational symmetric.

We postulate that no external forces act on the particles during the flight through the individual beam segments, which constitute the beam. Thus we regard the acceleration region in the gun as well as the lens and deflector areas as infinitely thin. Accordingly, the unperturbed trajectories of the particles can be visualized as straight lines which are broken at the location of the lenses and deflectors. The problem of determining the effect of statistical interactions in presence of an uniform axial electrostatic field will be discussed in 
chapter 10.

As a result of the acceleration in the source the axial velocity spread is in general several orders of magnitude smaller than the lateral velocity spread, as was shown in section 3.7 . For simplicity we now assume that the initial axial velocity spread can be neglected entirely and postulate that the beam is initially monochromatic with respect to the normal energy. This implies that the initial axial velocity is the same for all particles. The validity of this simplifying assumption will be verified in chapter 10 .

The basic approach to the problem is to consider the coulomb interaction of a single test (or reference) particle with all other particles in the beam, referred to as field (or colliding) particles. Due to this interaction the test particle will experience a deviation from its unperturbed trajectory. From now on we will use the term "displacement" to denote any deviation in position or velocity from the unperturbed values. The displacement of the test particle is fully determined by the initial coordinates of the field particles relative to the test particle. Due to the stochastic nature of the beam another test particle, running along the same trajectory some time later, will be surrounded by a different configuration of field particles and consequently experiences a different displacement.

Consider a large set of test particles running successively with identical velocity along a specific trajectory in the beam, called the reference trajectory. Assume that the test particles are well separated and can be considered as independent. Our problem now consists of two parts. First we wish to determine the total influence of a given set of field particles on the path of a specific test particle. This is called the dynamical part of the problem. Next we want to determine the distribution of displacements in velocity and position of the entire set of test particles successively arriving at the end of the beam segment. This evaluation represents the statistical part of the problem. It effectively corresponds to a time-average. It is now assumed that this time-average can be replaced by an average over all possible configurations of field particles relative to the single test particle considered in the dynamical part of the problem. This effectively corresponds to an ensemble average. The concepts of time and ensemble average were discussed in section 3.4 .

In general, the outcome of the dynamical and the statistical part of the calculation will, to a certain extent, depend on the choice of the reference trajectory. As we are primarily interested in the effect of statistical interactions it is advantageous to take the beam axis as reference trajectory. Due to the rotational symmetry of the beam no systematic forces act on the test particles moving along this central trajectory. Accordingly, the displacements experienced by the particles are purely stochastic, which means that their average displacement is zero. Particles moving along other trajectories in the beam will be subject to the combined action of space charge and statistical effects. The evaluation of the statistical effects experienced along such of $f-$ axis trajectories is more involved. Therefore, most calculations are restricted to the on-axis reference trajectory, assuming that the results obtained for this trajectory are representative for other trajectories as well. The validity of this assumption will be verified in chapter 10 , by evaluating the average effect generated along all possible reference trajectories in the beam.

Figure 5.1 shows the unperturbed trajectories of a test particle and a field particle at the moment that the field particle passes the $x, y-p l a n e$ of a fixed coordinate system within the beam segment, referred to as the laboratory system. The z-axis of this system coincides with the beam axis. The particles run in the positive z-direction. The $x, y-$ 


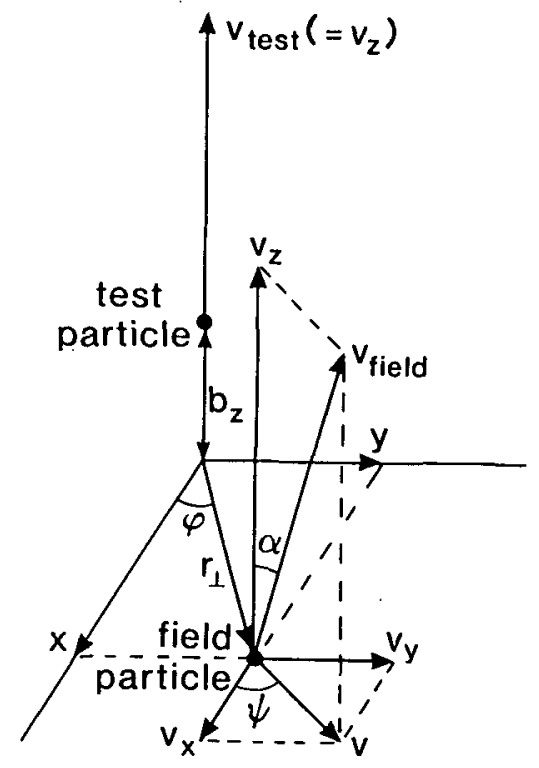

Figure 5.1 Unperturbed coordinates of an on-axis test particle and a field particle at the moment that the field particle passes the $x, y-p l a n e$ of the laboratory system.

plane is assumed to coincide with the crossover plane, unless specified otherwise. For convenience of notation the set of relative coordinates of a single field particle with respect to the test particle is abbreviated as

$$
\xi=\left\{x, y, b_{z}, v_{x}, v_{y}\right\} \text { or } \xi=\left\{r_{\perp}, \varphi, b_{z}, v, \psi\right\}
$$

using rectangular or cylindrical coordinates respectively. The different sets of coordinates are related by $x=r_{\perp} \cos \varphi, \quad y=r_{\perp} \sin \varphi, \quad v_{x}=v \cos \psi$ and $v_{y}=v \sin \psi$. The quantity $r_{\perp}$ is the modulus of the projection of the relative position vector $r=r_{f i e l d}-r_{t e s t}$ on the $x, y$-plane. The relative velocity $v=v_{f}$ ield $-v_{t e s t}$ is directed perpendicular to the beam axis, since it is assumed that all particles run with identical axial velocity $v_{z}$. Accordingly, its modulus $v$ can directly be related to the angle $a$ between the unperturbed velocity of the field particle and the $z-$ direction $\left(v=v_{z} \tan \alpha \approx v_{z} \alpha\right)$. The vector $\xi$ gives a complete specification of the unperturbed trajectory of the field particle relative to that of the test particle. By specifying $\xi_{1}, \xi_{2}, \cdots, \xi_{N-1}$ of all $N-1$ field particles the unperturbed coordinates of all particles can be determined at any moment.

Given the configuration of field particles, specified by the set $\xi_{1}, \xi_{2}, \cdots, \xi_{N-1}$, the test particle will experience a certain displacement from the reference trajectory (which is its unperturbed path). The displacement in velocity is expressed in terms of its components $\Delta v_{x}, \Delta v_{y}$ and $\Delta v_{z}$. The spatial displacement can best be expressed in terms of a virtual displacement in the plane which is optically conjugated to the target plane. The location of this (image) plane is specified by the parameter $S_{i}$, see equation (3.2.1). The virtual displacement is determined by extrapolating the final position 
of the test particle towards the image plane by a straight line along its final velocity. The quantities $\Delta x$ and $\Delta y$ are the $x$ - and $y$-component of the virtual radial displacement $\Delta r$ in the image plane. The trajectory displacement effect is related to the generation of these displacements. The Boersch effect corresponds to the generation of a spread in axial velocities and the relevant displacement therefore is $\Delta v_{2}$. In general, we will denote the set of relevant displacements by the vector $\Delta \eta$. The reader should be alert that the quantity $\Delta \eta$ may represent a simple scalar (as in the case of the Boersch effect), but is in general a vector.

The dynamical part of the problem now consists of the calculation of $\Delta \eta$ as a function of $\xi_{1}, \xi_{2}, \cdots, \xi_{N-1}$

$$
\Delta \boldsymbol{\eta}=\Delta \boldsymbol{\eta}\left(\boldsymbol{\xi}_{1}, \boldsymbol{\xi}_{2}, \cdots, \boldsymbol{\xi}_{\mathrm{N}}-1\right)
$$

The statistical part of the problem is the determination of the probability $\mathrm{P}_{N}\left(\xi_{1}, \xi_{2}, \cdots, \xi_{N-1}\right)$ of the configuration $\xi_{1}, \xi_{2}, \cdots, \xi_{N-1}$ and the evaluation of the distribution of displacements $\rho(\Delta \eta)$ from its definition

$$
\rho(\Delta \eta)=\int d \xi_{1} d \xi_{2} \cdots d \xi_{N-1} P_{N}\left(\xi_{1}, \xi_{2}, \cdots, \xi_{N-1}\right) \delta\left[\Delta \eta-\Delta \eta\left(\xi_{1}, \xi_{2}, \cdots, \xi_{N-1}\right)\right]
$$

in which $\delta[x]$ is the (multi-dimensional) delta-Dirac function. The distribution $\rho(\Delta \eta)$ is normalized when $\operatorname{PN}_{N}\left(\xi_{1}, \xi_{2}, \cdots, \xi_{N-1}\right)$ is normalized

$$
\int d \Delta \eta \rho(\Delta \eta)=\int d \xi_{1} d \xi_{2} \cdots d \xi_{N-1} P_{N}\left(\xi_{1}, \xi_{2}, \cdots, \xi_{N-1}\right)
$$

As we took the beam axis as reference trajectory the mean value $\langle\Delta \eta\rangle$ of the distribution $\rho(\Delta \eta)$ will be zero. However, the second moment or mean square width $\left\langle\Delta \eta^{2}\right\rangle$ of the distribution $\rho(\Delta \eta)$ does not vanish

$$
\begin{aligned}
\left\langle\Delta \eta^{2}\right\rangle & =\int \mathrm{d} \Delta \eta \rho(\Delta \eta) \Delta \eta^{2} \\
& =\int \mathrm{d} \xi_{1} \mathrm{~d} \xi_{2} \cdots \mathrm{d} \xi_{N-1} P_{N}\left(\xi_{1}, \xi_{2}, \cdots, \xi_{N-1}\right)\left[\Delta \eta\left(\xi_{1}, \xi_{2}, \cdots, \xi_{N-1}\right)\right]^{2}
\end{aligned}
$$

The problem of calculating the effect of statistical interactions now consists of the evaluation of the equations (5.2.2) and (5.2.3). The resulting distribution $\rho(\Delta \eta)$ contains all the desired information.

\subsection{Reduction of the N-particle problem}

A straightforward analytical evaluation of the equations (5.2.2) and (5.2.3) does not appear to be feasible and one is forced to introduce some simplifying assumptions. In this section we wish to study the different alternatives to solve this problem. The discussion is restricted to the fundamental aspects involved. The reader is referred to chapter 2 for a chronological review of the various approaches discussed in literature and to chapter 15 for a quantitative comparison of the results of some recent theories.

All approaches discussed here have in common that the initial coordinates of the field particles are assumed to be identical statistically independent quantities 


$$
P_{N}\left(\xi_{1}, \xi_{2}, \cdots, \xi_{N-1}\right)=\prod_{i=1}^{N-1} P_{2}\left(\xi_{i}\right)
$$

where $P_{2}(\xi)$ is the probability that a field particle has coordinates $\xi$ relative to the test particle. For the on-axis reference trajectory in a rotational symmetric beam segment with a crossover in the $x, y$-plane $\mathrm{P}_{2}(\xi)$ can be expressed as

$$
\int d \xi P_{2}(\xi)=\int_{0}^{\infty} d v f_{v}(v) \int_{0}^{2 \pi} \frac{d \Phi}{2 \pi} \int_{0}^{\infty} d r_{\perp} f_{r}\left(r_{\perp}\right) \int_{-S_{c} L}^{\left(1-S_{c}\right) L} \frac{d b_{z}}{L}
$$

anticipating that the magnitude of the displacement caused by a single field particle $|\Delta \eta|$ depends on the angles $\psi$ and $\varphi$ only through the relative angle $\Phi=\psi-\varphi$. The crossover-location parameter $S_{c}$ is defined by equation (3.2.1). The distributions $f_{r}\left(r_{\perp}\right)$ and $f_{v}(v)$ are related to the current density and angular distribution in the crossover plane respectively. When these distributions are uniform one should use

$$
f_{r}\left(r_{\perp}\right)=\frac{2 r_{\perp}}{r_{c}^{2}} \theta\left(r_{c}-r_{\perp}\right), \quad f_{v}(v)=\frac{2 v}{v_{0}^{2}} \theta\left(v_{0}-v\right)
$$

where $v_{0} \approx v_{z} \alpha_{0}$ and $\theta(x)$ is the step-function defined by equation $(3.7,8)$. In case of Gaussian distributions one should use

$$
f_{r}\left(r_{\perp}\right)=\frac{2 r_{\perp}}{r_{c}^{2}} e^{-\left(r_{\perp} / r_{c}\right)^{2}}, \quad f_{v}(v)=\frac{2 v}{v_{0}^{2}} e^{-\left(v / v_{0}\right)^{2}}
$$

Notice that the $\sigma^{2}$-value of a two-dimensional Gaussian distribution $f(r)$ is equal to half its mean square value: $\sigma^{2}=\left\langle r^{2}\right\rangle / 2$.

Equation (5.3.2) treats the current density and angular distribution as independent. This assumption is justifiable at the location of a crossover (which is an image of the source or the entrance pupil of the system) provided that the effect of lens aberrations can be neglected. It should be noted, however, that even in absence of aberrations this approach still ignores the fact that the illumination of the edge of the crossover may differ from the illumination of the centre of the crossover. This leads to a correlation in the boundaries of the angular and spatial distribution, which may become significant for small beam angles. Furthermore, we emphasize that equation (5.3.2) pertains to the on-axis reference trajectory. For an of $f$-axis reference trajectory one may not combine $\psi$ and $\varphi$ to $\Phi$ and one has to account for the fact that the distributions of the relative coordinates $r$ and $v$ are non-rotational symmetric. The symmetry can be restored by considering the average over all off-axis reference trajectories, but the effective distributions of $f_{r}\left(r_{\perp}\right)$ and $f_{v}(v)$ will differ from those of equations (5.3.3) and (5.3.4).

Equation (5.3.1) implies that all correlations in the coordinates of the field particles are ignored. In practical systems statistical correlations between the particle coordinates are determined both by the initial probability distribution of the particles leaving the cathode and the dynamics under influence of the Coulomb force during the time of flight. The last "source" of correlation is closely related to the concept of Debeye screening, which was discussed in section 3.9. Screening of the test particle prerequires that the interaction takes place over a sufficiently long time period to approach thermodynamic equilibrium. It corresponds to a redistribution of the field particles 
surrounding the test particle under influence of the electrostatic field of the test particle. In terms of the analysis of this chapter the screening, built up during the flight through the beam sections preceding the one considered, corresponds to correlations within the initial configuration $\xi_{1}, \xi_{2}, \cdots, \xi_{N-1}$. Equation (5.3.1) ignores these correlations as well as those related to the emission process. This simplification may lead to an overestimation of the probability of large displacements $\Delta \eta$ and the tails of the resulting displacement distribution $\rho(\Delta \boldsymbol{\eta})$ should be viewed with some caution.

Within the approximation of equation (5.3.1) one can in principle account for the screening of the test-particle in an approximate way by limiting the effective interaction range of the test particle to the Debeye distance. While the coordinates of the field particles are chosen randomly (that is independent of the coordinates of the test particle) it is then assumed that only those field particles interact with the test particle which have an unperturbed trajectory which intersects the Debeye sphere of the test particle. For simplicity these interactions are calculated from the unscreened (bare) Coulomb potential, while the influence of more distant field particles is neglected entirely. This procedure corresponds to a truncation of the integration boundaries of equation (5.3.2). We recall that the analysis of section 4.9 lead to the conclusion that the conditions required for Debeye screening are in general not fulfilled in practical beams. Accordingly, we will assume that that Debeye screening does not occur and identify the integration boundaries in equation (5.3.2) with the boundaries of the beam.

The dynamical problem of evaluating the displacement of the test particle $\Delta \boldsymbol{\eta}\left(\xi_{1}, \xi_{2}, \cdots, \xi_{N}-1\right)$ for the configuration of field particles $\xi_{1}, \xi_{2}, \cdots, \xi_{\mathrm{N}-1}$ may in general be expressed as an integral over time

$$
\Delta \boldsymbol{\eta}\left(\xi_{1}, \xi_{2}, \cdots, \xi_{N-1}\right)=\int_{t_{i}}^{t_{f}} d t G_{N}\left(\xi_{1}, \xi_{2}, \cdots, \xi_{N-1}, t\right)
$$

in which $t_{i}$ and $t_{f}$ the initial and final time of the interaction, corresponding to the moment that the test particle enters and leaves the beam segment respectively. In the case of the Boersch effect $\Delta \eta=\Delta v_{z}$ and $\mathbf{G}_{\mathbf{N}}$ is directly related to the axial component of the interaction force $F_{z}$ acting on the test particle

$$
\mathbf{G}_{N}\left(\xi_{1}, \xi_{2}, \cdots, \xi_{N-1}, t\right)=F_{2}\left(\xi_{1}, \xi_{2}, \cdots, \xi_{N-1}, t\right) / m
$$

with $m$ the particle mass. In the case of the trajectory displacement effect in a rotational symmetric beam one should consider $\Delta \eta=|\Delta r|=\left(\Delta x^{2}+\Delta y^{2}\right)^{1 / 2}$. The lateral displacement $\Delta r$ can be related to the lateral component of the interaction force $F_{\perp}$ as

$$
\begin{aligned}
\Delta r\left(\xi_{1}, \xi_{2}, \cdots, \xi_{N-1}\right) & =\int_{t_{i}}^{t_{f}} d t^{\prime} \int_{t_{i}}^{t^{\prime}} d t F_{\perp}\left(\xi_{1}, \xi_{2}, \cdots, \xi_{N-1}, t\right) / m \\
& =\int_{t_{i}}^{t_{f}} d t\left(t_{f}-t\right) F_{L}\left(\xi_{1}, \xi_{2}, \cdots, \xi_{N-1}, t\right) / m
\end{aligned}
$$

as follows by changing the order of the integration. Accordingly, the function $G_{N}$ is for this case equal to

$$
\mathbf{G}_{N}\left(\xi_{1}, \xi_{2}, \cdots, \xi_{N-1}, t\right)=\left(t_{f}-t\right) F_{\perp}\left(\xi_{1}, \xi_{2}, \cdots, \xi_{N-1}, t\right) / m
$$


The computation of the interaction force $F\left(\xi_{1}, \xi_{2}, \cdots, \xi_{N-1}, t\right)$ at time $t$ requires the knowledge of the actual position $r_{i}$ of field particle $i$ relative to the test particle $\left(\mathbf{r}_{i}=\mathbf{r}_{\mathrm{f} \text { ield, }}-\mathbf{r}_{\mathrm{tes}}\right)$ at that time

$$
F\left(\xi_{1}, \xi_{2}, \cdots, \xi_{N-1}, t\right)=-c_{0} \sum_{i=1}^{N-1} \frac{r_{i}\left(\xi_{1}, \xi_{2}, \cdots, \xi_{N-1}, t\right)}{r_{i}\left(\xi_{1}, \xi_{2}, \cdots, \xi_{N-1}, t\right)^{3}}
$$

where $C_{0}=e^{2} / 4 \pi \varepsilon_{0}$, with $e$ the particle charge. However, the computation of the positions at time $t$ requires the knowledge of the particle trajectories under influence of the interaction force $F\left(\xi_{1}, \xi_{2}, \cdots, \xi_{N-1}, t\right)$ up to that time. This coupling is the essence of the many particle problem.

So far we only translated the dynamical problem into the evaluation of $F\left(\xi_{1}, \xi_{2}, \cdots, \xi_{N-1}, t\right)$. At this point one has to introduce some principle assumptions in order to proceed towards an analytical solution of the $\mathrm{N}$-particle problem. The different alternatives appear to be the following:

\section{First order perturbation approximation.}

In this approximation it is assumed that the deviations from the unperturbed trajectories are small. The actual interaction force $\mathbf{F}\left(\xi_{1}, \xi_{2}, \cdots, \xi_{N-1}, t\right)$ acting on the test particle, which is a function of the actual positions $r_{i}\left(\xi_{1}, \xi_{2}, \cdots, \xi_{N-1}, t\right)$, is now approximated by the interaction force which would act on the test particle when all particles would follow their unperturbed trajectories. As these trajectories are known beforehand (from the specification of $\left.\xi_{1}, \xi_{2}, \cdots, \xi_{\mathrm{N}-1}\right)$ the problem is now decoupled.

The unperturbed position $\tilde{r}_{i}$ of a particle $i$ at time $t$ depends only on $\xi_{i}$ and $t$. Accordingly, the first order perturbation approximation can be expressed as

$$
\mathbf{r}_{i}\left(\xi_{1}, \xi_{2}, \cdots, \xi_{N-1}, t\right)=\tilde{r}_{i}\left(\xi_{i}, t\right)
$$

and equation $(5.3 .8)$ yields

$$
\mathbf{P}\left(\boldsymbol{\xi}_{1}, \boldsymbol{\xi}_{2}, \cdots, \boldsymbol{\xi}_{N-1}, t\right)=-\mathrm{C}_{0} \sum_{i=1}^{N-1} \frac{\tilde{\mathbf{r}}_{i}\left(\boldsymbol{\xi}_{i}, t\right)}{\tilde{\mathbf{r}}_{1}\left(\boldsymbol{\xi}_{i}, t\right)^{3}}
$$

Consequently, the function $G_{N}\left(\xi_{1}, \xi_{2}, \cdots, \xi_{N-1}, t\right)$, being proportional to $\mathbf{F}\left(\xi_{1}, \xi_{2}, \cdots, \xi_{N-1}, t\right)$, can be written as

$$
G_{N}\left(\xi_{1}, \xi_{2}, \cdots, \xi_{N}-1, t\right)=\sum_{i=1}^{N-1} G_{2}\left(\xi_{i}, t\right)
$$

in which $G_{2}$ is the equivalence of $G_{N}$ for a beam consisting of only the test particle and a single field particle $(N=2)$. It follows from equation (5.3.5) that the displacement $\Delta \eta$ of the test particle can be expressed as

$$
\Delta \eta\left(\xi_{1}, \xi_{2}, \cdots, \xi_{N-1}\right)=\sum_{i=1}^{N-1} \Delta \eta_{2}\left(\xi_{i}\right)
$$


in which and $\Delta \eta_{2}$ denotes to the displacement caused by the interaction with a single field particle. The calculation of $\Delta \eta_{2}$ is a two-particle problem. In first order perturbation approximation $\Delta \eta_{2}$ follows from the equations (5.3.5) and either equation (5.3.6) or (5.3.7), taking $N=2$. This calculation can be carried out analytically as will be shown in chapter 6 .

It should be emphasized that the reduction of the $\mathrm{N}$-particle dynamical problem to $\mathrm{N}-1$ two-particle problems, expressed by equation (5.3.12), is entirely justified as long as all particles follow approximately their unperturbed trajectories, which requires that the interaction between the particles is weak. The validity of this approach is a direct consequence of the fact that forces are additive. However, the first order perturbation approximation clearly leads to erroneous results in case some of the interactions cause significant deviations from the unperturbed trajectories of either the test particle or the field particles. Equation (5.3.9) is no longer true for all particles and the actual interaction force consequently differs from the interaction force computed from the unperturbed trajectories. Thus the first order perturbation approximation provides an accurate analysis in case all interactions are weak, but breaks down otherwise.

In order to solve the statistical part of the problem it is assumed that the field particles are statistically independent, as expressed by equation (5.3.1). Notice that this assumption is consistent with the approximation that the particles run along their unperturbed trajectories (presupposing that no correlations occur as a consequence of the emission process). By substitution of the equations (5.3.1) and (5.3.12) into equation (5.2.5) one finds for the mean square displacement

$$
\left\langle\Delta \eta^{2}\right\rangle=(N-1) \int \mathrm{d} \xi \mathrm{P}_{2}(\xi) \Delta \eta_{2}(\xi)^{2}
$$

utilizing that $P_{2}(\xi)$ is normalized and that the mean displacement $\langle\Delta \eta\rangle=0$. It should be anticipated that a straightforward evaluation of equation (5.3.13) in general leads to divergent results, since the expression for $\Delta \eta_{2}(\xi)$, based on first order perturbation dynamics, overestimates the effect of strong collisions. To prevent this divergence one should restrict the integration domain to those values of $\xi$ which correspond to weak collisions, assuming that the effect of strong collisions can be neglected.

Loeffler (1969) used an alternative statistical procedure to avoid divergence of $\left\langle\Delta \eta^{2}\right\rangle$, while employing the first order perturbation approximation. He argued that this divergence is a consequence of the use of the continuous probability distribution $\mathrm{P}_{2}(\xi)$. which disregards the discrete nature of the beam. In Loeffler's approach one accounts for the finite number of particles $N$ within the beam by computing the $n^{\text {th }}$ largest displacement $\Delta \eta_{n}$ out of the set of $N-1$ displacements experienced by the test particle due to the two-particle interactions with the other $\mathrm{N}-1$ particles. This displacement is defined as

$$
N\left(\left|\Delta \eta_{2}\right|>\left|\Delta \eta_{n}\right|\right)=n-1 / 2
$$

with

$$
N\left(\left|\Delta \eta_{2}\right|>\left|\Delta \eta_{n}\right|\right)=(N-1) \int_{\left|\Delta \eta_{2}(\xi)\right|>\left|\Delta \eta_{n}\right|} \operatorname{d\xi } P_{2}(\xi)
$$

in which the integration domain of $\xi$ is limited to that part in which 
the condition $\left|\Delta \eta_{2}(\xi)\right|>\left|\Delta \eta_{n}\right|$ is fulfilled. The total displacement of the test particle follows by adding up all displacements up to the $(\mathrm{N}-1)^{\text {t. }}$ largest one

$$
\left\langle\Delta \eta^{2}\right\rangle=\sum_{n=1}^{N-1} \Delta \eta_{n}^{2}
$$

Loeffler states that this procedure leads to a median value of the total interaction distribution, rather than a mean square displacement. The validity of this statement seems questionable.

\section{Closest encounter approximation}

This approach assumes that the displacement of the test particle $\Delta \eta$ is dominated by the single strong interaction with its nearest neighbour field particle, which is defined as the particle with the smallest impact parameter with respect to the test particle. It was proposed by Rose and Spehr (1980) and is also used by Weidenhausen, Spehr and Rose (1984). We recall that the impact parameter b of a twoparticle collision is the closest distance of approach in absence of interaction. In terms of the components of $\xi_{i}$, defined in equation (5.2.1), it can be expressed as

$$
b(\xi)=\left[b_{z}^{2}+b_{r^{2}}^{2}\right]^{1 / 2}=\left[b_{z}^{2}+r^{2} \sin ^{2}(\Phi)\right]^{1 / 2}
$$

$(\Phi=\psi-\varphi)$ as can be seen from figure 5.2 . Let $b_{n}$ be the impact parameter

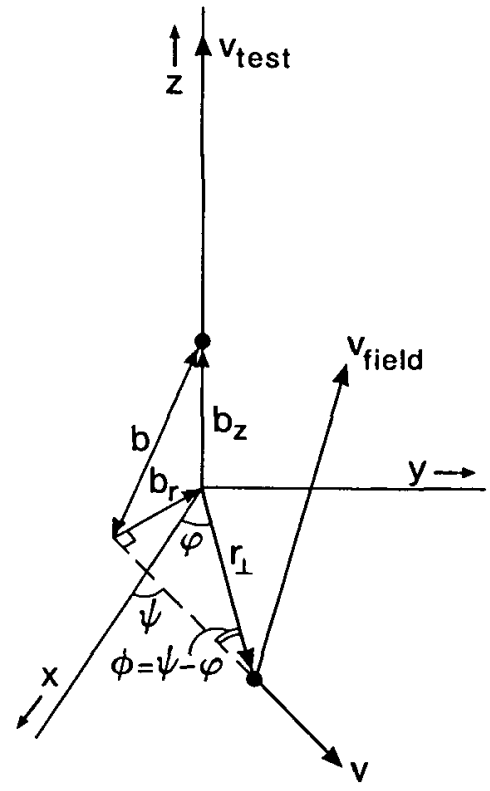

Figure 5.2 Unperturbed trajectories of a test particle and a field particle in the laboratory system. The impact parameter b is the closest distance of approach in absence of interaction. 
of the nearest neighbour and $\xi_{\mathrm{n}}$ the coordinates of this particle relative to the test particle. The closest encounter approximation reduces the $\mathrm{N}$-particle dynamical problem to a two-particle problem, using

$$
\Delta \boldsymbol{\eta}\left(\xi_{1}, \xi_{2}, \cdots, \xi_{N-1}\right)=\Delta \eta_{2}\left(\xi_{n}\right)
$$

In this model one may not use first order perturbation theory to calculate the two-particle displacement $\Delta \boldsymbol{\eta}_{2}\left(\xi_{\mathrm{n}}\right)$, since this displacement is not necessarily small. Instead, one should use the analytical solution of the classical Kepler problem which is also valid for strong interactions. It should be noted that the usual solution of this problem prerequires the collision to be complete (see chapter 6), which implies that the initial and final separation of the particles is large compared to the impact parameter $b$. This assumption is consistent with the closest encounter approach which focusses on the collision with the smallest value of $b$. Such a collision will in general be complete provided that the diameter of the crossover is small compared to diameter of the beam at the start and the end of the beam segment. Thus the closest encounter approach based on complete collisions seems an adequate approach for beams with a narrow crossover.

In order to solve the statistical part of the problem one has to determine the probability that an arbitrary field particle with impact parameter $b_{n}$ and coordinates $\xi_{n}$ is the nearest neighbour of the test particle. Assuming again that the particles are statistically independent, this probability can be expressed as

$$
W_{2}\left(\xi_{n}, b_{n}\right)=\left[(N-1) \int_{b\left(\xi_{n}\right)=b_{n}}^{d} d \xi_{n} P_{2}\left(\xi_{n}\right)\right] \times\left[1-\int_{b(\xi)<b_{n}}^{d \xi} P_{2}(\xi)\right]^{N-2}
$$

The first term is identical to the probability that an arbitrary field particle has an impact parameter $b_{n}$ and coordinates $\xi_{n}$, while the second term is identical to the probability that all other fields particles have a larger impact parameter. The mean square displacement of the test particle can now be computed as

$$
\left\langle\Delta \eta^{2}\right\rangle=\int \mathrm{d} b_{n} \int \mathrm{d} \xi_{\mathrm{a}} \mathrm{W}_{2}\left(\xi_{\mathrm{n}}, \mathrm{b}_{\mathrm{n}}\right) \Delta \boldsymbol{\eta}_{2}\left(\boldsymbol{\xi}_{\mathrm{n}}\right)^{2}
$$

which shows the same structure as equation $(5.3 .13)$ but is mathematically more complex.

The closest encounter approach presupposes that the main contribution to the total displacement of the test particle stems from a single collision with its nearest neighbour fieid particle. This assumption is justified when this single collision is strong relative to the interactions with all other field particles. However, it breaks down in case the effect is built up by a large number of weak interactions. Such might for instance be expected for a cylindrical beam at low current density. Clearly, it does not handle the case of multiple strong interactions either.

\section{Extended two-particle approximation.}

In this approach one follows the same statistical procedure as in the first order perturbation approximation, which is expressed by the equations (5.3.1) and (5.3.12). However, the dynamical problem of calculating the two-particle displacement $\Delta \eta_{2}(\xi)$ is not solved in first 
order perturbation approximation, but rather by an exact analysis of the Kepler problem, which takes the collision duration into account. Unfortunately, it is not possible to obtain the exact solution of this problem in an explicit form, as will be demonstrated in chapter 6, and one is forced to evaluate the integral of equation (5.3.13) numerically. Since strong interactions are now taken into account properly this integral will not diverge, as in the first order perturbation approximation. Explicit analytical solutions can be derived for the limiting cases in which it is allowed to use either first order perturbation dynamics or (nearly) complete collision dynamics. This method, here referred to as the extended two-particle approximation, was first presented by van Leeuwen and Jansen (1983).

In this section we will only consider the general philosophy of the extended two-particle model, while the mathematical basis is the subject of the remaining sections of this chapter. The model can in a way be regarded as a synthesis of the two methods outlined so far. In case all interactions are weak it clearly shows the same features as the first order perturbation approximation. It uses the same statistical procedure, while the approach to the dynamical part of the problem leads to identical results for weak interactions. In case the displacement of the test particle is dominated by a single complete collision it should lead to identical results as obtained with the closest encounter approximation. The dynamics of that single collision is handled alike. The statistical method is somewhat different since the extended twoparticle model includes the (weak) interaction with the other particles as well. It should be noted that the calculation of these interactions will not be accurate since the strong interaction causes the test particle to deviate significantly from its unperturbed trajectory. However, since the total displacement of the test particle is dominated by that single strong interaction anyhow (as assumed in the closest encounter approximation) the exact contribution of the weak collision taking place simultaneously is immaterial. Accordingly, one should expect that, for those conditions for which the closest encounter approximation is justified, both methods yield the same results.

From the preceding analysis it follows that the extended twoparticle approximation covers all those cases in which either the first order perturbation approximation is justified (multiple weak interactions) or the closest encounter approximation is justified (single strong complete collision). Furthermore, we note that the exact two-particle analysis provides accurate results even for strong but incomplete collisions, which are not covered by the other methods. The extended two-particle approximation breaks down when the test particle is involved in two or more strong collisions simultaneously or successively in the same beam segment.

\section{Mean square field fluctuation approximation}

In the previous approaches the displacement of the test particle was first calculated for a specific configuration of field particles and averaged over all possible configurations next. We will now consider an approach in which this order is interchanged. The basic assumption of this approach is that the time development of the distribution of field particles surrounding the test particle can be described as a succession of independent states. Accordingly, equation (5.3.5) is rewritten as 


$$
\Delta \eta\left(\xi_{1}, \xi_{2}, \cdots, \xi_{N-1}\right)=\sum_{s=1}^{N_{s}} \Delta t_{s} G_{N}\left(\xi_{1}, s \ldots \xi_{N-1, s}\right)
$$

in which the configuration of field particles in state number $s$ is specified by $\xi_{1}, s, \xi_{2}, s, \cdots, \xi_{N-1}, s . N_{s}$ denotes the total number of independent states encountered by the test particle on its way through the beam segment and $\Delta t_{s}$ the duration of state number $s$ (note: $t_{i}=t_{1}$ and $\left.t_{f}=t_{N_{s}}+\Delta t_{N_{s}}\right)$. The ensemble-average is now carried out over the conf iguration $\xi_{1}, s, \xi_{2}, s, \cdots, \xi_{N-1}, s$. The mean square displacement follows as

$$
\left\langle\Delta \eta^{2}\right\rangle=\sum_{s=1}^{N_{s}} \Delta t_{s}{ }^{2}\left\langle G_{N}^{2}\right\rangle_{s}
$$

in which

$$
\left\langle\mathbf{G}_{N}{ }^{2}\right\rangle_{s}=\int \mathrm{d} \xi_{1}, \mathrm{~s} \cdots \mathrm{d} \xi_{N-1, s} P_{N, s}\left(\xi_{1}, s, \cdots, \xi_{N-1, s}\right)\left[G_{N}\left(\xi_{1}, s, \cdots, \xi_{N-1, s}\right)\right]^{2}
$$

$P_{N, s}\left(\xi_{1, s}, \xi_{2}, s, \cdots, \xi_{N-1, s}\right)$ represents the probability of the configuration $\xi_{1}, s, \xi_{2}, s, \cdots, \xi_{N-1}$, s occurring in state $s$.

The evolution of the system in the course of time is represented by the set of quantities $\Delta t_{s}$ and $P_{N, s}\left(\xi_{1}, s, \xi_{2}, s, \cdots, \xi_{N}-1, s\right)$ where $s=1,2, \cdots, N_{s}$. The configuration $\xi_{1}, s, \xi_{2}, s, \cdots, \xi_{N-1, s}$ is considered to be fixed during the period $\Delta t_{s}$. Thus the motion of the field particles relative to the test particle is in fact represented as a series of instantaneous rearrangements, every rearrangement corresponding with the transition to a new independent state. As the configuration of field particles in state $s$ is considered to be fixed during the time interval $\Delta t_{s}$ one may write

$$
G_{N}\left(\xi_{1}, s, \xi_{2}, s, \cdots, \xi_{N-1, s}\right)=\sum_{i=1}^{N-1} G_{2}\left(\xi_{i}, s\right)
$$

since forces are additive. It should be emphasized that equation (5.3.24) does not rely on the assumption that the interactions are weak, unlike the similar equation (5.3.11).

While the reduction to two-particle interactions, given by equation (5.3.24), now comes out in a rather natural way, it should be anticipated that difficulties might arise with the determination of the set of quantities $\Delta t_{s}$ and $P_{N, s}\left(\xi_{1}, s, \xi_{2}, s, \cdots, \xi_{N-1, s}\right)$ where $s=1,2, \cdots, N_{s}$. Let us assume that the particles within the state $s$ are statistically independent. Thus similar to equation $(5.3 .1)$ it is assumed that

$$
P_{N, s}\left(\xi_{1, s}, \xi_{2}, s, \cdots, \xi_{N-1, s}\right)=\prod_{i=1}^{N-1} P_{2}, s\left(\xi_{i, s}\right)
$$

where $\mathrm{P}_{2,5}(\xi)$ is the probability that a field particle has coordinates $\xi$ relative to the test particle in state $s$. Combining equations (5.3.23), $(5.3 .24)$ and $(5.3 .25)$ one obtains 


$$
\left\langle\mathbf{G}_{N}{ }^{2}\right\rangle_{s}=(N-1) \int d \xi_{s} P_{2}, s\left(\xi_{s}\right) G_{2}\left(\xi_{s}\right)^{2}
$$

using that $\left\langle\mathbf{G}_{N}\right\rangle_{s}=0$. Equations $(5.3 .22)$ and $(5.3 .26)$ now represent the essence of the problem. Equation $(5.3 .26)$ is directly related to the mean square field force occurring in state $s$, as can be seen from the equations (5.3.5), (5.3.6) and (5.3.7) (taking $N=2)$. Equation $(5.3 .22)$ expresses that the displacements of the test particle experienced in the different states are assumed to be independent. Accordingly, they are added quadraticly. Notice that the individual terms are proportional to $\Delta t_{s}{ }^{2}$. This quadratic dependency on $\Delta t_{s}$ is a consequence of the fact that displacement experienced in state $s$ does not occur instantaneously, but builds up during the entire time interval $\Delta t_{s}$. As a result of this quadratic dependency on $\Delta t_{s}$, the outcome of equation (5.3.22) depends critically on the choice of the set of intervals $\Delta t_{s}$ where $s=1,2 \cdots, N_{s}$. In other words, the correlation time of the fluctuations is a critical parameter within this model.

Another problem is related to the evaluation of equation $(5.3 .26)$. As $G_{2}\left(\xi_{s}\right)$ is proportional to the coulomb force exerted by the field particle on the test particle, the integral diverges for small interparticle distances if one assumes that distribution of field particles $P_{2}, s\left(\xi_{s}\right)$ is fully random. One is forced to introduce a lower limit for the interparticle distance to prevent this divergence. This complication is a direct consequence of the assumption that the different states are entirely independent. In reality, two particles which are very near to each other in state $s$ will have increased their separation in state $s+1$. Their positions in state $s+1$ are thus correlated. Contrary to the two-particle models this effect is ignored in this model and the resulting divergence of equation $(5.3 .26)$ has to be removed artificially.

Models employing the mean square field fluctuation approximation are presented by De Chambost and Hennion (1979), Sasaki $(1984,1986)$ and Massey, Jones and Plumer (1981). The last model pertains specifically to a cylindrical beam geometry. It deviates from the model described here in the sense that the different states $s=1,2, \cdots, N_{s}$ are not treated as independent, but, oppositely, as entirely correlated. Accordingly, the summation of the contributions of the different states is performed by using

$$
\left\langle\Delta \eta^{2}\right\rangle 1 / 2=\sum_{S=1}^{N_{s}} \Delta t_{s}\left\langle G_{N} 2\right\rangle_{s} 1 / 2
$$

instead of equation (5.3.22). In this model the choice of the time intervals $\Delta t_{s}$ (with $s=1,2, \cdots, N_{s}$ ) is not critical, due to the linear dependency on this parameter. For low particle densities this model yields the same results as the first order perturbation approximation, provided that the divergence related to strong interactions is solved in the same way.

\subsection{Calculation of the displacement distribution}

We will now concentrate on the so-called extended two particle approximation, which appears to be the least restrictive one of the four alternative approaches outlined in the previous section. In this section we will demonstrate that this model permits a direct calculation 
of the entire displacement distribution $\rho(\Delta \eta)$. We will follow the analysis given by van Leeuwen and Jansen (1983).

The two-particle approximation relies on the assumption that the total displacement of the test particle can be computed as the sum of the displacements due to pair collisions, as expressed by equation (5.3.12). In addition it is assumed that the field particles are statistically independent, as expressed by equation (5.3.1). By substitution of these relations into equation $(5.2 .3)$ one obtains for the displacement distribution

$$
\rho(\Delta \eta)=\int d \xi_{1} d \xi_{2} \cdots d \xi_{N-1} \prod_{i=1}^{N-1} P_{2}\left(\xi_{i}\right) \delta\left(\Delta \eta-\sum_{i=1}^{N-1} \Delta \eta_{2}\left(\xi_{i}\right)\right)
$$

Let $n$ be the number of components of the displacement $\Delta \eta$. The $\delta$-function is then $n$-dimensional and can be represented as

$$
\delta(x)=\frac{1}{(2 \pi)^{n}} \int \mathrm{dk} \mathrm{e}^{\mathbf{i k \cdot x}}
$$

in which both $x$ and $k$ are $n$-component vectors. Utilizing this representation, equation $(5.4 .1)$ transforms to

$$
\rho(\Delta \eta)=\frac{1}{(2 \pi)^{n}} \int d k e^{i k \cdot \Delta \eta} A_{N}(k)
$$

in which

$$
A_{N}(k)=\left[\int d \xi P_{2}(\xi) e^{-i k \cdot \Delta \eta_{2}(\xi)}\right]^{N-1}
$$

A further reduction is possible by making use of the fact that $\mathrm{N}$ is in general a large number. Utilizing the normalization of $P_{2}(\xi)$ one may rewrite equation $(5.4 .4)$ as

$$
A_{N}(k)=\left[1-\int \mathrm{d} \xi P_{2}(\xi)\left(1-\mathrm{e}^{-i k \cdot \Delta \eta_{2}(\xi)}\right)\right]^{N-1}
$$

The integral in equation (5.4.5) will be small for most $\xi$, in particular for those values of $\xi$ which correspond to a large separation of the test and the field particle $\left(\Delta \eta_{2}(\xi) \rightarrow 0\right)$. Recalling that $P_{2}(\xi)$ is normalized, it becomes clear that the integral will fall off proportional to $N$, since the total displacement of the test particle is mainly caused by a few neighbour field particles. This can also be observed from equation (5.3.2), which is inversely proportional to the beam length $L$ (which is proportional to $N$ ). We make the dependency on $N$ explicit by defining

$$
P_{2}(\xi)=\frac{1}{L} p(\xi)=\frac{\lambda}{N} p(\xi)
$$

in which $\lambda$ is the linear particle density defined by equation $(3.2 .4)$. Accordingly, equation $(5.4 .5)$ transforms to

$$
A_{N}(k)=\left[1-\frac{\lambda}{N} \int d \xi p(\xi)\left(1-e^{-i k \cdot \Delta \eta_{2}(\xi)}\right)\right]^{N-1} \approx e^{-\lambda p(k)}
$$

using that $\mathrm{N}$ is a large number. The function $\mathbf{p}(\mathbf{k})$ is here defined as 


$$
p(k)=\int d \xi p(\xi)\left(1-e^{-i k \cdot \Delta \eta_{2}(\xi)}\right)
$$

Combining equations $(5.4 .3)$ and (5.4.7) the displacement distribution $\rho(\Delta \eta)$ becomes

$$
\rho(\Delta \eta)=\frac{1}{(2 \pi)^{n}} \int d k e^{i k \cdot \Delta \eta-\lambda p(k)}
$$

The equations (5.4.8) and (5.4.9) constitute the major results of this section. One sees that the function $p(k)$ is fully equivalent to $\rho(\Delta \eta)$. The evaluation of $p(k)$ involves only two-particle dynamics, represented by $\Delta \eta_{2}$, and two-particle statistics, represented by $p(\xi)$.

It is often useful to separate the two-particle analysis from the $p(k)$ transform. For that purpose, the two-particle distribution function $\rho_{2}(\Delta \eta)$ is defined as

$$
\rho_{2}(\Delta \eta)=\int \mathrm{d} \xi p(\xi) \delta\left[\Delta \eta-\Delta \eta_{2}(\xi)\right]
$$

which is basicly the displacement distribution for a system containing only two particles, the test particle and a single field particle. Equation $(5.4 .8)$ can now be expressed as

$$
p(k)=\int d \Delta \eta \rho_{2}(\Delta \eta)\left(1-e^{-i k \cdot \Delta \eta}\right)
$$

The evaluation of the displacement distribution $\rho(\Delta \eta)$ now consists of three steps. The first step, expressed by equation $(5.4 .10)$, is the calculation of the two-particle distribution function $\rho_{2}(\Delta \eta)$, employing two-particle dynamics (represented by $\Delta \eta_{2}(\xi)$ ) and two-particle statistics (represented by $p(\xi))$. The second step, expressed by equation (5.4.11), is the transformation of $\rho_{2}(\Delta \eta)$ to the k-domain, which results in the function $p(k)$. The third step, expressed by equation (5.4.9), can be considered as a back-transformation of $p(k)$ to the $\Delta \eta$-domain, which leads to the wanted $N$-particle displacement distribution $\rho(\Delta \boldsymbol{\eta})$. As far as the dependency on the experimental parameters is concerned it should be noted that the two-particle distribution function $\rho_{2}(\Delta \eta)$ and its transform $p(k)$ are entirely determined by geometrical parameters only, which are represented by $p(\xi)$. The particle density, expressed in terms of $\lambda$, enters the model in the evaluation of $\rho(\Delta \eta)$, using equation (5.4.9). Accordingly, a particular beam geometry requires only a single computation of the function $p(k)$, which is sufficient to evaluate the distribution $\rho(\Delta \boldsymbol{\eta})$ for a range of $\lambda$-values.

We emphasize that, given a certain value of $\lambda$, each of the three functions $\rho_{2}(\Delta \eta), p(k)$ and $\rho(\Delta \eta)$ contains all relevant information. Accordingly, these functions should be regarded as equivalent representations of the problem, related by simple Fourier-type of integral transformations. The three-step approach of the model is not only convenient from a mathematical point of view, but clarifies the physical aspects of the problem as well. In fact we will see that some of the characteristic features of $\rho(\Delta \eta)$ can best be described in terms of the corresponding properties of $p(k)$ and $\rho_{2}(\Delta \eta)$.

With respect to the relation between the functions $\rho_{2}(\Delta \eta), p(k)$ and $\rho(\Delta \eta)$, one may add, that given the displacement distribution $\rho(\Delta \eta)$, the function $p(k)$ can be retrieved using the inverse Fourier transformation of equation (5.4.9) 


$$
e^{-\lambda p(k)}=\int d \Delta \eta \rho(\Delta \eta) e^{-i k \cdot \Delta \eta}
$$

Similarly, one can retrieve $\rho_{2}(\Delta \eta)$ from $p(k)$ using

$$
\rho_{2}(\Delta \eta)=\frac{1}{(2 \pi)^{n}} \int d k[L-p(k)] e^{-i k \cdot \Delta \eta}
$$

The length $\mathrm{L}$ appears in this expression due to the fact that $\rho_{2}(\Delta \eta)$ is not normalized to 1 but to $\mathrm{L}$. This can be seen from the equations $(5.4 .6)$ and $(5.4 .10)$

$$
\int d \Delta \eta \rho_{2}(\Delta \eta)=\int d \xi p(\xi)=L \int d \xi P_{2}(\xi)=L
$$

The practical value of the equations $(5.4 .12)$ and $(5.4 .13)$ is limited, since one is primarily interested in determining $\rho(\Delta \eta)$ through $p(k)$ and $\rho_{2}(\Delta \eta)$, instead of the other way around.

In the remainder of this chapter the features of the model will be further investigated, emphasizing the fundamental aspects. The relation between the characteristic properties of the functions $\rho_{2}(\Delta \eta)$, $p(k)$ and $\rho(\Delta \eta)$ will be discussed in the next section. The aspects of the model related to the choice of the reference trajectory (on- or of $f$ axis) are considered in section 5.6. In the presentation given so far the dimension $n$ of the displacement $\Delta \eta$ was left unspecified. Section 5.7 presents the basic equations for $n=1,2$ and 3 . These results are employed in section 5.8 to determine the distribution of the fluctuating component of the interaction force in a cylindrical beam. The significance of the representation in the $k$-domain is the subject of section 5.9. Finally, it will be shown in section 5.10 that the model gives a clear indication how to add the results obtained for the individual beam segments.

\subsection{Moments and cumulants of the displacement distribution}

In this section we will define the moments and cumulants of the displacement distribution $\rho(\Delta \eta)$. We will also investigate their relation with the moments of the two-particle distribution $\rho_{2}(\Delta \eta)$ as well as the relation with the properties of the function $p(k)$. The material of this section may appear to be rather "technical". However, the resulting relations are of great significance to the model and will be used extensively.

For convenience of notation the analysis of this section is restricted to the case of an one-dimensional displacement $\Delta \eta \quad(n=1)$. However, all results can straightforwardly be extended to cover the general case of a multi-dimensional displacement $\Delta \boldsymbol{\eta}(\mathbf{n}>1)$.

The $m^{\text {th }}$ moment of the displacement distribution $\rho(\Delta \eta)$ is defined as

$$
\left\langle\Delta \eta^{m}\right\rangle=\int \mathrm{d} \Delta \eta \rho(\Delta \eta) \Delta \eta^{\mathrm{m}}
$$

clearly, the zero order moment $(m=0)$ is related to the normalization of $\rho(\Delta \eta)$ and should be equal to 1 . Substitution of equation (5.4.9) into equation $(5.5 .1)$, taking $m=0$, yields 


$$
\begin{aligned}
1 & =\int d \Delta \eta \rho(\Delta \eta)=\int d \Delta \eta \int \frac{d k}{2 \pi} e^{i k \cdot \Delta \eta-\lambda p(k)} \\
& =\int d k \delta(k) e^{-\lambda p(k)}=\left[e^{-\lambda p(k)}\right]_{k=0}
\end{aligned}
$$

utilizing the representation of the $\delta$-function given by equation (5.4.2). Equation (5.5.2) states that the normalization of $\rho(\Delta \eta)$ requires that $p(k=0)=0$. Notice that the property $p(k=0)=0$ is guaranteed by the definition of $p(k)$, see equation $(5.4 .8)$.

In the same way one finds for the first moment $(m=1)$ of $\rho(\Delta \eta)$, using equations and $(5.5 .1)$ and $(5.4 .9)$

$$
\begin{aligned}
\langle\Delta \eta\rangle & =\int \mathrm{d} \Delta \eta \Delta \eta \rho(\Delta \eta)=\int \mathrm{d} \Delta \eta \Delta \eta \int \frac{\mathrm{dk}}{2 \pi} \mathrm{e}^{i k \cdot \Delta \eta-\lambda \mathrm{p}(\mathrm{k})} \\
& =-i \int \mathrm{d} \Delta \eta \int \frac{\mathrm{dk}}{2 \pi} \frac{\partial}{\partial k}\left[\mathrm{e}^{i k \cdot \Delta \eta}\right] \mathrm{e}^{-\lambda p(k)}
\end{aligned}
$$

Integration by parts, using that $p(k)+\infty$ at the $k$-integration boundaries, results in

$$
\begin{aligned}
\langle\Delta \eta\rangle & =i \int d \Delta \eta \int \frac{d k}{2 \pi} \frac{\partial}{\partial k}\left[e^{-\lambda p(k)}\right] e^{i k \cdot \Delta \eta} \\
& =i \int d k \delta(k) \frac{\partial}{\partial k}\left[e^{-\lambda p(k)}\right]=i\left[\frac{\partial}{\partial k} e^{-\lambda p(k)}\right]_{k=0}
\end{aligned}
$$

again using equation (5.4.2). By repetition of the same mathematical procedure it can in general be proven that the $\mathrm{m}^{\mathrm{th}}$ moment of $\rho(\Delta \eta)$ is given by

$$
\left\langle\Delta \eta^{m}\right\rangle=i^{m}\left[\frac{\partial^{m}}{\partial k^{m}} e^{-\lambda p(k)}\right]_{k=0}
$$

Thus all moments of $\rho(\Delta \eta)$ are related to the properties of $p(k)$ at $k=0$. Accordingly, one may write

$$
\mathrm{e}^{-\lambda p(k)}=\sum_{m=0}^{\infty} \frac{k^{m}}{m !}\left[\frac{\partial^{m}}{\partial k^{m}} e^{-\lambda p(k)}\right]_{k=0}=\sum_{m=0}^{\infty} \frac{(-i k)^{m}}{m !}\left\langle\Delta \eta^{m}\right\rangle
$$

Thus the function $\exp [-\lambda p(k)]$ can be expressed as a power expansion in $k$ in which the coefficients are the moments of the distribution $\rho(\Delta \eta)$. A function with this characteristic is called "moment generating function", for instance see van Kampen (1981). Notice that equation (5.5.5) can also be derived directly from equation $(5.4 .12)$, by power expansion of the exponential function.

Taking the logarithm on both sides of equation (5.5.5) one finds by means of power expansion (using $\ln (1+x)=x-x^{2} / 2+\cdots+(-1)^{j+1} x^{j} / j$ )

$$
-\lambda p(k)=\sum_{j=1}^{\infty} \frac{(-1)^{j+1}}{j}\left[\sum_{m=1}^{\infty} \frac{(-i k)^{m}}{m !}\left\langle\Delta \eta^{m}\right\rangle\right]^{j}=\sum_{m=1}^{\infty} \frac{(-i k)^{m}}{m !}\left\langle\Delta \eta^{m}\right\rangle>(5.5 .6)
$$

in which $\left\langle\left\langle\Delta \eta^{m} \gg>\right.\right.$ is called the $m^{t h}$ cumulant of $\rho(\Delta \eta)$. All cumulants are implicitly defined by equation (5.5.6). The first four cumulants are related to the moments of $\rho(\Delta \eta)$ as 


$$
\begin{aligned}
& <\langle\Delta \eta\rangle\rangle=\langle\Delta \eta\rangle \\
& \left\langle\left\langle\Delta \eta^{2}\right\rangle\right\rangle=\left\langle\Delta \eta^{2}\right\rangle-\langle\Delta \eta\rangle^{2} \\
& \left\langle\left\langle\Delta \eta^{3}\right\rangle=\left\langle\Delta \eta^{3}\right\rangle-3\left\langle\Delta \eta^{2}\right\rangle\langle\Delta \eta\rangle+2\langle\Delta \eta\rangle^{3}\right. \\
& \left\langle\left\langle\Delta \eta^{4}\right\rangle=\left\langle\Delta \eta^{4}\right\rangle-4\left\langle\Delta \eta^{3}\right\rangle\langle\Delta \eta\rangle-3\left\langle\Delta \eta^{2}\right\rangle^{2}+12\left\langle\Delta \eta^{2}\right\rangle\langle\Delta \eta\rangle^{2}-6\langle\Delta \eta\rangle^{4}\right.
\end{aligned}
$$

Notice that the first cumulant and first moment are identical, while the second cumulant is identical to the mean square spread (or variance) of the distribution. According to equation (5.5.6) the function $-\lambda p(k)$ can be regarded as the "cumulant generating function" of the distribution $\rho(\Delta \eta)$.

So far we concentrated on the displacement distribution $\rho(\Delta \eta)$ and its relation with the function $p(k)$. We now like to investigate the relation with the properties of the two-particle distribution $\rho_{2}(\Delta \eta)$. The $\mathrm{m}^{\mathrm{t}} \mathrm{m}$ moment of the this distribution is defined as

$$
\left\langle\Delta \eta_{2} m\right\rangle=\int d \Delta \eta \rho_{2}(\Delta \eta) \Delta \eta_{2} m=\int d \xi p(\xi) \Delta \eta_{2} m(\xi)
$$

in which we utilized the definition $\rho(\Delta \eta)$, given by equation (5.4.10). The moment generating function of $\rho_{2}(\Delta \eta)$ is $p(k)$, as follows by power expansion of the exponential function in equation (5.4.11)

$$
-p(k)=\sum_{m=1}^{\infty} \frac{(-i k)^{m}}{m !}\left\langle\Delta \eta_{2}{ }^{m}\right\rangle
$$

By comparison of the equations $(5.5 .6)$ and $(5.5 .9)$ one sees that the cumulants of $\rho(\Delta \eta)$ are directly related to the moments of $\rho_{2}(\Delta \eta)$ as

$$
\left\langle\left\langle\Delta \eta^{m}\right\rangle>=\lambda\left\langle\Delta \eta 2^{m}\right\rangle\right.
$$

which is an important result. For $m=1$ and $m=2$ it yields,- employing equations $(5.5 .7)$ and $(5.5 .8)$

$$
\begin{aligned}
& \langle\langle\Delta \eta\rangle\rangle=\langle\Delta \eta\rangle=\lambda \int \mathrm{d} \xi \mathrm{p}(\xi) \Delta \eta_{2}(\xi) \\
& \left\langle\left\langle\Delta \eta^{2}\right\rangle\right\rangle=\left\langle\Delta \eta^{2}\right\rangle-\langle\Delta \eta\rangle^{2}=\lambda \int \mathrm{d} \xi \mathrm{p}(\xi) \Delta \eta_{2}(\xi)^{2}
\end{aligned}
$$

For the on-axis reference trajectory one finds $\langle\Delta \eta\rangle=0$ and the second equation becomes identical to equation (5.3.13), obtained by direct calculation (note $\lambda p(\xi)=N P_{2}(\xi) \approx(N-1) P_{2}(\xi)$, see equation $(5.4 .6)$ ).

\subsection{On- and off-axis reference trajectories}

Equation (5.4.8), defining the function $p(k)$, can be split up into a real and an imaginary part (using $1-\exp (i x)=2 \sin ^{2}(x / 2)+i \sin (x)$ )

$$
p(k)=\operatorname{pr}(k)+i p_{i}(k)
$$

in which 


$$
\begin{aligned}
& \operatorname{Pr}(k)=2 \int d \Delta \eta \rho_{2}(\Delta \eta) \sin ^{2}\left(k \cdot \Delta \eta_{2} / 2\right) \\
& \operatorname{pi}_{i}(k)=\int d \Delta \boldsymbol{j} \rho_{2}(\Delta \eta) \sin \left(k \cdot \Delta \eta_{2}\right)
\end{aligned}
$$

By comparison with equation $(5.5 .6)$ these functions can be identified as (restricting ourselves again to the one-dimensional case)

$$
\begin{aligned}
-\lambda p_{r}(k)= & \sum_{j=1}^{\infty} \frac{(-1) j k^{2} j}{(2 j) !}\left\langle\left\langle\Delta \eta^{2} j\right\rangle\right\rangle=\frac{k^{2}}{2}\left\langle\left\langle\Delta \eta^{2}\right\rangle\right\rangle-\frac{k^{4}}{24}\left\langle\left\langle\Delta \eta^{4}\right\rangle\right\rangle+\ldots \\
-\lambda p_{i}(k)= & \sum_{j=1}^{\infty} \frac{(-1) j k^{2 j-1}}{(2 j-1) !}\left\langle\left\langle\Delta \eta^{2} j-1\right\rangle\right\rangle=-k\langle\Delta \eta\rangle+\frac{k^{3}}{6}\left\langle\left\langle\Delta \eta^{3}\right\rangle\right\rangle+\cdots
\end{aligned}
$$

using that $\langle\langle\Delta \eta\rangle\rangle=\langle\Delta \eta\rangle$. In general $p_{r}(-k)=p_{r}(k)$, while $p_{i}(-k)=-p_{i}(k)$, as can be seen from either equation (5.6.2) or equation (5.6.3). Thus $p_{r}(k)$ is a symmetric function and $p_{i}(k)$ is an anti-symmetric function in $k$.

For the on-axis reference trajectory in a rotational symmetric beam segment the imaginary part $p_{i}(k)$ will vanish and $p(k)=p_{r}(k)$. In that case all statistical interaction effects are represented by the real part of $p(k)$. For an of $f$-axis reference trajectory $p_{i}(k)$ does not vanish and affects the displacement distribution $\rho(\Delta \eta)$, which can now be expressed as

$$
\rho(\Delta \eta)=\frac{1}{(2 \pi)^{a}} \int \mathrm{d} k \mathrm{e}^{i k \cdot\left(\Delta \eta-\lambda p_{i}(k) k / k^{2}\right)-\lambda p_{r}(k)}
$$

as follows from equations $(5.4 .9)$ and $(5.6 .1)$. Notice that $\rho(\Delta \eta)$ is always real as a consequence of the symmetry properties $p_{r}(-k)=p_{r}(k)$ and $p_{i}(-k)=-p_{i}(k)$. The first term of the expansion of $\lambda p_{i}(k)$ is equal to $k \cdot\langle\Delta \eta\rangle$, as can be seen from equation (5.6.3). Ignoring higher order terms equation (5.6.4) transforms to

$$
\rho(\Delta \eta) \approx \frac{1}{(2 \pi)^{n}} \int d k e^{i k \cdot\left(\Delta \eta-\langle\Delta \eta)-\lambda p_{r}(k)\right.}
$$

This equation shows that the first order effect of an of $f$-axis reference trajectory is the occurrence of an uniform shift in $\rho(\Delta \boldsymbol{\eta})$ by a value $\langle\Delta \eta\rangle$. This shift corresponds (by definition) to the space charge effect. In the approximation of equation $(5.6 .5)$ the effect of statistical interactions is entirely represented by the real part of $p(k)$ only. Accordingly, the statistical interactions experienced along some offaxis reference trajectory can be described by the same set of equations as used for the on-axis reference trajectory. However, even in this approximation, the resulting shifted distribution $\rho(\Delta \eta-\langle\Delta \eta\rangle)$ may differ from the one obtained for the on-axis reference trajectory, due to the differences in $p(\xi)$, affecting $\rho(\Delta \eta-\langle\Delta \eta\rangle)$ through $\operatorname{pr}_{r}(k)$. By including non-vanishing higher order terms in the expansion of $p_{i}(k)$ the statistical displacement distribution $\rho(\Delta \eta-\langle\Delta \eta\rangle)$ becomes asymmetric (which means $\rho(\Delta \boldsymbol{\eta}-\langle\Delta \boldsymbol{\eta}\rangle) \neq \rho(-\Delta \boldsymbol{\eta}+\langle\Delta \boldsymbol{\eta}\rangle)$ ), as can be seen from equation $(5.6 .4)$.

In the remaining of this chapter we will ignore the imaginary part of $p(k)$ by stating $p(k)=p_{r}(k)$. As we limit ourselves here to the effect of statistical interactions this approach is exact for the on- 
axis reference trajectory and correct up to (but not including) third order terms in $\mathbf{k}$ for other reference trajectories.

\subsection{Models in 1, 2 and 3 dimensions}

The main relations of the model are represented by the equations $(5.4 .9),(5.4 .10)$ and $(5.4 .11)$, defining the functions $\rho(\Delta \eta), \rho_{2}(\Delta \eta)$ and $p(k)$ respectively. The dimension $n$ of the displacement $\Delta \boldsymbol{\eta}$ was left unspecified so far. We will now consider these equations for the cases $\mathrm{n}=1,2$ and 3 specifically.

Using scalar notation the equations $(5.4 .10),(5.4 .11)$ and (5.4.9) can be expressed as

$\rho_{2}\left(\Delta \eta_{1}, \cdots, \Delta \eta_{\mathrm{n}}\right)=\int \mathrm{d} \xi \mathrm{p}(\xi) \delta\left[\Delta \eta_{1}-\Delta \eta_{2}, 1(\xi)\right] \cdots \delta\left[\Delta \eta_{\mathrm{n}}-\Delta \eta_{2}, \mathrm{n}(\xi)\right]$

$p\left(k_{1}, \cdots, k_{n}\right)=\int d \Delta \eta_{1} \cdots d \Delta \eta_{n} \rho_{2}\left(\Delta \eta_{1}, \cdots, \Delta \eta_{n}\right)\left(1-e^{-i\left(k_{1} \cdot \Delta \eta_{1}+\cdots+k_{n} \cdot \Delta \eta_{n}\right)}\right)$

$\rho\left(\Delta \eta_{1}, \cdots, \Delta \eta_{n}\right)=\frac{1}{(2 \pi)^{n}} \int d k_{1} \cdots d k_{n} e^{i\left(k_{1} \cdot \Delta \eta_{1}+\cdots+k_{n} \cdot \Delta \eta_{n}\right)-\lambda p\left(k_{1}, \cdots, k_{n}\right)}$

where $\Delta \eta_{1}, \Delta \eta_{2}, \cdots, \Delta \eta_{n}$ are the components of $\Delta \eta_{;} \quad \Delta \eta_{2}, 1, \Delta \eta_{2}, 2, \cdots, \Delta \eta_{2}, \mathrm{n}$ the components of $\Delta \eta_{2}$ and $k_{1}, k_{2}, \cdots, k_{n}$ the components of $k$.

For $n=1$ these equations transform to

$$
\begin{aligned}
& \rho_{2}(\Delta \eta)=\int \mathrm{d} \xi \mathrm{p}(\xi) \delta\left[\Delta \eta-\Delta \eta_{2}(\xi)\right] \\
& p(k)=2 \int_{-\infty}^{\infty} \mathrm{d} \Delta \eta \rho_{2}(\Delta \eta) \sin ^{2}(\mathrm{k} \Delta \eta / 2) \\
& \rho(\Delta \eta)=\frac{1}{\pi} \int_{0}^{\infty} \mathrm{dk} \cos (\mathrm{k} \Delta \eta) \mathrm{e}^{-\lambda \mathrm{p}(\mathrm{k})}
\end{aligned}
$$

In equation (5.7.5) we ignored the imaginary part of $p(k)$, conform the discussion of the previous section. Accordingly, $p(k)=p(-k)$, which was used to obtain equation (5.7.6). This set of equations will be used to describe the Boersch effect, taking $\Delta \eta=\Delta v_{z}$.

For $n=2$ the equations $(5.7 .1),(5.7 .2)$ and $(5.7 .3)$ can be reduced to one-dimensional integrals, provided that the distributions $\rho\left(\Delta \eta_{1}, \Delta \eta_{2}\right)$ and $\rho_{2}\left(\Delta \eta_{1}, \Delta \eta_{2}\right)$ are functions of $\Delta \eta=\left(\Delta \eta_{1}^{2}+\Delta \eta_{2}^{2}\right)^{1 / 2}$ only. This is to be expected in case of rotational symetry. The two-particle distribution of $\Delta \eta$ is given by

$$
\rho_{2}(\Delta \eta)=2 \pi \Delta \eta \rho_{2}(\Delta \eta)=\int \mathrm{d} \xi \mathrm{p}(\xi) \delta\left[\Delta \eta-\Delta \eta_{2}(\xi)\right]
$$

Employing cylindrical coordinates the function $p(k)$ can be expressed as

$$
p(k)=p(k)=\int_{0}^{\infty} \Delta \eta d \Delta \eta \int_{0}^{2 \pi} d \varphi \frac{\rho_{2}(\Delta \eta)}{2 \pi \Delta \eta}\left(1-e^{-i k \Delta \eta \cos \varphi}\right)
$$


which is identical to

$$
p(k)=\int_{0}^{\infty} d \Delta \eta \rho_{2}(\Delta \eta)\left[1-J_{0}(k \Delta \eta)\right]
$$

utilizing the integral representation of the Bessel function of the zero order $\mathrm{J}_{0}$,

$$
J_{0}(x)=\int_{0}^{2 \pi} \frac{d \varphi}{2 \pi} e^{i x \cos \varphi}
$$

By similar reasoning one finds for the displacement distribution

$$
\rho(\Delta \eta)=2 \pi \Delta \eta \rho(\Delta \eta)=\Delta \eta \int_{0}^{\infty} k d k J_{0}(k \Delta \eta) e^{-\lambda p(k)}
$$

The equations $(5.7 .7),(5.7 .8)$ and $(5.7 .10)$ will be used to describe the trajectory displacement effect in a rotational symmetric beam, taking $\Delta \eta=\Delta r$.

Finally for $n=3$ we consider the situation that the distributions $\rho\left(\Delta \eta_{1}, \Delta \eta_{2}, \Delta \eta_{3}\right)$ and $\rho_{2}\left(\Delta \eta_{1}, \Delta \eta_{2}, \Delta \eta_{3}\right)$ are functions of $\Delta \eta=\left(\Delta \eta_{1}{ }^{2}+\Delta \eta_{2}^{2}+\Delta \eta_{3}^{2}\right)^{1 / 2}$ only, which is to be expected in case of spherical symmetry. Employing spherical coordinates, equations ( $5.7 \cdot 1)$, $(5.7 .2)$ and $(5.7 .3)$ transform to

$$
\begin{aligned}
& \rho_{2}(\Delta \eta)=4 \pi \cdot \Delta \eta^{2} \rho_{2}(\Delta \eta)=\int \mathrm{d} \xi \mathrm{p}(\xi) \delta\left[\Delta \eta-\Delta \eta_{2}(\xi)\right] \\
& p(k)=p(k)=\int_{0}^{\infty} \mathrm{d} \Delta \eta \rho_{2}(\Delta \eta)\left(1-\frac{\sin (k \Delta \eta)}{k \Delta \eta}\right) \\
& \rho(\Delta \eta)=4 \pi \Delta \eta^{2} \rho(\Delta \eta)=\frac{2 \Delta \eta}{\pi} \int_{0}^{\infty} k d k \sin (k \Delta \eta) e^{-\lambda p(k)}
\end{aligned}
$$

This set of equations will be used to derive the Holtsmark distribution, previously introduced in equation (4.9.1).

\subsection{Distribution of the interaction force in cylindrical beams}

We will now employ the basic equations of the extended twoparticle model to compute the probability distribution of the fluctuating electrostatic interaction force acting on a test particle located on the axis of a cylindrical beam of charged particles. Doing so we make a little detour from the main line of this chapter, which is primarily concerned ith the fundamental concepts of the model. This intermezzo serves as a demonstration of the practical merits of this approach, while it also provides some tools to explain the results obtained for the Boersch effect (discussed in chapter 7 ) and the trajectory displacement effect (discussed in chapters 8 and 9).

We will assume that the beam can be regarded as infinitely long compared to the average separation of the particles. If one assumes, in addition, that the diameter of the beam is infinitely large, the problem simplifies substantially, since the distribution of field particles 
surrounding the test particles may then be considered as spherical symmetric. Accordingly, the wanted probability distribution of the interaction force is expected to follow the Holtsmark distribution. First, we will investigate whether this result can be reproduced within the model and next we will study the more complex problem of a beam with a finite, possibly small, diameter.

Consider a test particle surrounded by an infinitely large spherical symmetric cloud of field particles with density $n_{d}$. The appropriate model to treat this three dimensional case consists of the equations $(5.7 .11),(5.7 .12)$ and $(5.7 .13)$. Instead of the probability distribution of displacements $\Delta \eta$, we will determine the distribution of the interaction force $\mathbf{F}$, thus we take $\Delta \boldsymbol{\eta}=\mathbf{F}$. In addition we have to replace the linear electron density $\lambda$ by the three dimensional particle density $n_{d}$, which is the relevant measure for the particle density in the present case. Both quantities can be related through the total number of particles $\mathbf{N}$

$$
\lambda L=N=(4 / 3) \pi L^{3} n_{d}
$$

in which $L$ now denotes the radius of the sphere of field particles $(L+\infty)$. The magnitude of the force exerted by a field particle at distance $r$ from the test particle is given by

$$
F=\Delta \eta_{2}=C_{0} / r^{2}, \quad C_{0}=e^{2} / 4 \pi \varepsilon_{0}
$$

From equation (5.7.11) one finds for the two-particle distribution of the interaction force (using that $p(\xi)$ is normalized to $L$ )

$$
\rho_{2}(F)=L \int_{0}^{\infty} \frac{4 \pi r^{2} d r}{(4 / 3) \pi L^{3}} \delta\left(F-C_{0} / r^{2}\right)=\frac{3 C_{0}{ }^{3 / 2}}{2 L^{2} F^{5 / 2}}
$$

Substitution of this expression into equation (5.7.12) yields

$$
p(k)=\frac{3 C_{0} 3 / 2 k^{3 / 2}}{2 L^{2}} \int_{0}^{\infty} d x \frac{x-\sin (x)}{x^{7 / 2}}=\frac{\pi^{1 / 2}\left(2 C_{0}\right)^{3 / 2} \lambda}{5 L^{2}} k^{3 / 2}
$$

in which we used the identity

$$
\int_{0}^{\infty} d x \frac{x-\sin (x)}{x^{7 / 2}}=\frac{8}{15} \int_{0}^{\infty} d x \frac{\cos (x)}{x^{1 / 2}}=\frac{8}{15} \sqrt{\frac{\pi}{2}}
$$

which follows after several integration by parts. By means of equation (5.8.1) one can rewrite equation $(5.8 .4)$ as

$$
\lambda p(k)=\left(F_{n} k\right)^{3 / 2}, \quad F_{n}=(4 / 15)^{2 / 3}\left(2 \pi C_{0}\right) n_{d} 2 / 3
$$

Finally, the desired distribution follows from equation (5.7.13)

$$
\rho(F)=\frac{1}{2 \pi^{2} F} \int_{0}^{\infty} k d k \sin (k F) e^{-\left(F_{n} k\right)^{3 / 2}}
$$

which is indeed identical to the Holtsmark distribution introduced in section 4.9 , see equations $(4.9 .1)$ and $(4.9 .2)$. The $3 / 2$ power dependency on $\mathrm{k}$ is characteristic for the Holtsmark distribution. 
Now consider a beam with finite radius $r_{0}$. As the distribution $\rho(F)$ is not expected to be spherical symmetric one has to determine the distribution of the axial force component $F_{/ /}$and the lateral force component $F_{\perp}$ separately. Let us consider $\rho\left(F_{\perp}\right)$ first. The twodimensional variant of the model is given by the equations (5.7.7), $(5.7 .8)$ and $(5.7 .10)$. In terms of the coordinates represented by $\xi$, see equation (5.2.1) and figure 5.1, one finds

$$
\Delta \eta_{2}=F_{\perp}=\frac{C_{0} r}{\left(r^{2}+z^{2}\right)^{3 / 2}}=\frac{C_{0}}{r^{2}} \frac{1}{\left(1+(z / r)^{2}\right)^{3 / 2}}
$$

analogous to equation (5.8.2). The corresponding two-particle force distribution follows from equation (5.7.7), assuming an uniform current density distribution within the beam

$$
\rho_{2}\left(F_{\perp}\right)=\int_{0}^{\infty} 2 \mathrm{~d} z \int_{0}^{r_{0}} \frac{2 r d r}{r_{0}^{2}} \delta\left(F_{\perp}-\frac{C_{0}}{r^{2}} \frac{1}{\left(1+(z / r)^{2}\right)^{3 / 2}}\right)
$$

Substituting

$$
s=\frac{z}{r}, \quad t=\frac{C_{0}}{r^{2}} \frac{1}{\left(1+s^{2}\right)^{3 / 2}}
$$

this transforms to

$$
\rho_{2}\left(F_{\perp}\right)=\frac{2 C_{0}^{3 / 2}}{r_{0}^{2}} \int_{0}^{\infty} \frac{d s}{\left(1+s^{2}\right)^{9 / 4}} \int_{t_{0}}^{\infty} \frac{d t}{t^{5 / 2}} \delta\left[F_{\perp}-t\right]
$$

where $t_{0}=C_{0} / r_{0}^{2}\left(1+s^{2}\right)^{3 / 2}$. Using the definition of the $\delta$-function this becomes

$$
\rho_{2}\left(F_{\perp}\right)=\frac{2 C_{0}^{3 / 2}}{r_{0}^{2} F_{\perp} 5 / 2} \int_{0}^{\infty} \frac{d s}{\left(1+s^{2}\right)^{9 / 4}} \theta\left(F_{\perp}-\frac{C_{0}}{r_{0}^{2}\left(1+s^{2}\right)^{3 / 2}}\right)
$$

where $\theta(x)$ is the step-function. A further reduction in general terms does not appear to be feasible and we will suffice to investigate two extreme cases: $r_{0}+\infty$ and $r_{0} \rightarrow 0$. In the classification of section 3.3, the former represents an (extreme) extended cylindrical beam, while the latter corresponds to an (extreme) pencil-beam. For $r_{0}=0$ all particles are in a row on the beam axis. For $r_{0} \rightarrow \infty$ the conditions underlying the Holtsmark distribution are reproduced and one should expect to obtain its two-dimensional variant.

For $r_{0}+\infty$ the argument of the step-function in equation (5.8.12) is positive for all positive $F_{\perp}$ and one directly obtains

$$
\rho_{2}\left(F_{\perp}\right)=\frac{C_{0}{ }^{3 / 2}}{r_{0}^{2} F_{\perp}^{5 / 2}} I_{1}
$$

in which $I_{1}$ represents

$$
I_{1}=\int_{0}^{\infty} \frac{2 \mathrm{ds}}{\left(1+s^{2}\right)^{9 / 4}}=\frac{12 \sqrt{1} \Gamma(3 / 4)}{5 \Gamma(1 / 4)}=1.4378
$$

with $\Gamma(x)$ the Gamma-function. The function $p(k)$ now follows from equation $(5.7 .8)$ 


$$
p(k)=\frac{C_{0}{ }^{3 / 2}}{r_{0}^{2}} I_{1} I_{2} k^{3 / 2}
$$

in which $I_{2}$ represents

$$
I_{2}=\int_{0}^{\infty} \mathrm{dx} \frac{1-J_{0}(x)}{x^{5 / 2}}=\frac{4}{9} \int_{0}^{\infty} \mathrm{dx} \frac{J_{0}(x)}{x^{1 / 2}}=\frac{2 \sqrt{2} \Gamma(1 / 4)}{9 \Gamma(3 / 4)}=0.92982
$$

employing integration by parts (using $d J_{0}(x) / d x=-J_{1}(x)$ and $\left.d\left[\mathrm{xJ}_{1}(x)\right] / d x=x_{0}(x)\right)$. By substituting $n_{d}=\lambda / \pi r_{0}^{2}$ one obtains

$$
\lambda p(k)=2 \pi C_{0}{ }^{3 / 2} n_{d} \quad I_{1} I_{2} k^{3 / 2}=F_{n} 3 / 2 k^{3 / 2}
$$

which is identical to equation (5.8.6). From equation (5.7.10) one finds for the distribution of the lateral interaction force in an extended cylindrical beam $\left(r_{0}+\infty\right)$

$$
\rho\left(F_{\perp}\right)=\frac{1}{2 \pi} \int_{0}^{\infty} k d k J_{0}\left(k F_{\perp}\right) e^{-\left(F_{n} k\right)^{3 / 2}}
$$

which is the two-dimensional variant of the Holtsmark distribution given by equation $(5 \cdot 8.7)$. It specifies the distribution of the interaction force component in a plane perpendicular to the beam axis. (In fact the same result follows for a plane of arbitrary orientation, since the distribution of field particles is for $r_{0 \rightarrow \infty}$ effectively spherical symmetric)

Now consider equation (5.8.12) for $r_{0 \rightarrow 0}$, that is for a cylindrical pencil beam. The argument of the step-function is only positive for large values of $s$, that is $s>s_{0}$, where

$$
s_{0}=\left[\left(C_{0} / r_{0}^{2} F_{1}\right)^{2 / 3}-1\right]^{1 / 2}
$$

The integral in equation $(5.8 .12)$ can now be approximated as

$$
\int_{s_{0}}^{\infty} \frac{d s}{\left(1+s^{2}\right)^{9 / 4}} \approx \int_{s_{0}}^{\infty} \frac{d s}{s^{9 / 2}}=\frac{2}{7 s_{0}^{7 / 2}}
$$

Accordingly, one obtains for the two-particle distribution of $F_{\perp}$ in a pencil beam

$$
\rho_{2}\left(F_{\perp}\right)=\frac{4 C_{0}^{1 / 3} r_{0}^{1 / 3}}{7 F_{\perp}^{4 / 3}}
$$

The function $p(k)$ follows from equation (5.7.8)

$$
\mathrm{p}(\mathrm{k})=(4 / 7) \mathrm{C}_{0}{ }^{1 / 3} \mathrm{ro}^{1 / 3} \mathrm{I}_{3} \mathrm{k}^{1 / 3}
$$

in which $I_{3}$ represents

$$
I_{3}=\int_{0}^{\infty} d x \frac{1-J_{0}(x)}{x^{4 / 3}}=3 \int_{0}^{\infty} d x \frac{J_{1}(x)}{x^{1 / 3}}=\frac{18 \Gamma(5 / 6)}{2^{1 / 3} \Gamma(1 / 6)}=2.8972
$$

as follows by integration by parts. The $1 / 3$ power dependency on $k$, expressed by equation $(5.8 .19)$, is characteristic for the distribution 
of the transverse component of the interaction force in a pencil beam. From equation $(5.7 .10)$ one finds for this distribution

$$
\rho\left(\mathbf{P}_{\perp}\right)=\frac{1}{2 \pi} \int_{0}^{\infty} k d k J_{0}\left(k F_{\perp}\right) e^{-(4 / 7) C_{0} 1 / 3 r_{0}{ }^{1 / 3} I_{3} \lambda k^{1 / 3}}
$$

Notice that the width of this distribution tends to zero for $r_{0} \rightarrow 0$. The physical reason is obvious: For $r_{0}=0$ all particles are on a row and the interaction force does not have a transverse component $\mathbf{F}_{\perp}$.

Let us now consider the distribution of the axial force component $\rho\left(F_{\| /}\right)$. As the calculation follows the same lines of thought as the calculation of $\rho\left(F_{\perp}\right)$ we will not present it in detail, but only give the main results. From equation $(5.7 .4)$ one finds for the two-particle force distribution, analogous to equation (5.8.9)

$$
\rho_{2}\left(F_{/ /}\right)=\int_{-\infty}^{\infty} d z \int_{0}^{r_{0}} \frac{2 r d r}{r_{0}^{2}} \delta\left(F_{/ /}-\frac{C_{0}}{r^{2}} \frac{(z / r)}{\left(1+(z / r)^{2}\right)^{3 / 2}}\right)
$$

in which $F_{/ /}$carries form $-\infty$ to $+\infty$ (note $\rho_{2}\left(\left|F_{/ /}\right|\right)=2 \rho_{2}\left(F_{/ /}\right)$). Similar to the derivation of equation $(5.8 .11)$ this can be transformed to

$$
\rho_{2}\left(F_{/ /}\right)=\frac{C_{0} 3 / 2}{r_{0}^{2} F_{/ /}^{5 / 2}} \int_{0}^{\infty} \frac{s^{3 / 2} d s}{\left(1+s^{2}\right)^{9 / 4}} \theta\left(\left|F_{/ /}\right|-\frac{C_{0}}{r_{0}^{2}\left(1+s^{2}\right)^{3 / 2}}\right)
$$

We will again investigate the extreme cases $r_{0}+\infty$ and $r_{0} \rightarrow 0$. For $r_{0}+\infty$ the argument of the step-function is positive for all positive $F_{/ /}$and equation $(5.8 .22)$ yields

$$
\rho_{2}\left(F_{/ /}\right)=\frac{C_{0}{ }^{3 / 2}}{r_{0}^{2} F_{/ /}^{5 / 2}} \int_{0}^{\infty} \frac{s^{3 / 2} d s}{\left(1+s^{2}\right)^{9 / 4}}=\frac{2 C_{0}{ }^{3 / 2}}{5 r_{0}^{2} F_{/ /}^{5 / 2}}
$$

The function $p(k)$ follows from equation (5.7.5)

$$
p(k)=\frac{2^{5 / 2} C_{0}^{3 / 2}}{5 r_{0}^{2}} k^{3 / 2} \int_{0}^{\infty} d x \frac{\sin ^{2}(x)}{x^{5 / 2}}=\frac{21 / 2 \pi^{1 / 2} C_{0} 3 / 2}{15 r_{0}^{2}} k^{3 / 2}
$$

showing the $3 / 2$ power dependency on $k$ characteristic for the Holtsmark distribution. Substituting $n_{d}=\lambda / \pi r_{0}{ }^{2}$ one obtains again equation (5.8.15). By means of equation $(5.7 .6)$ one finds for the distribution of the axial force component in an extended cylindrical beam

$$
\rho\left(F_{/ /}\right)=\frac{1}{\pi} \int_{0}^{\infty} \mathrm{dk} \cos \left(k F_{/ /}\right) \mathrm{e}^{-\left(F_{\mathrm{D}} k\right) 3 / 2}
$$

which is the one-dimensional variant of the Holtsmark distribution given by equation $(5,8,7)$.

Finally, consider equation (5.8.22) for $r_{0} \rightarrow 0$, that is for a cylindrical pencil beam. Analogous to equation $(5.8 .18)$ one finds in this limit 


$$
\rho_{2}\left(F_{1 /}\right)=\frac{C_{0} 1 / 2}{2 F_{/ / 3 / 2}}
$$

The function $p(k)$ follows from equation (5.7.5)

$$
p(k)=\left(2 \pi c_{0}\right)^{1 / 2} k^{1 / 2}
$$

This k-dependency is characteristic for the distribution of the axial component of the interaction force in a pencil beam. From equation $(5.7 .6)$ one finds for this distribution

$$
\rho\left(F_{\| /}\right)=\frac{1}{\pi} \int_{0}^{\infty} d k \cos \left(k F_{/ l}\right) e^{-\left(2 \pi C_{0}\right) 1 / 2 \lambda k^{1 / 2}}
$$

The width of the distribution tends to a non-zero finite value for $r_{0} \rightarrow 0$, in agreement with the fact that the longitudinal component of the interaction force does not vanish in case all particles are on a row.

\subsection{Representation in the $k$-domain}

We will now proceed with the discussion of the fundamental aspects of the model. From the equations constituting the model (see section 5.7) it is clear that all information regarding the interaction distribution $\rho(\Delta \eta)$ is also present in the function $p(k)$ as well as in the two-particle distribution $\rho_{2}(\Delta \eta)$. In this section we wish to demonstrate that the representation by the function $p(k)$ in the k-domain provides a simple way of describing most of the characteristic properties of $\rho(\Delta \eta)$.

Suppose the quantity $\lambda p(\mathbf{k})$ is for all $\mathbf{k}$ given by

$$
\lambda p(k)=A_{\gamma}|k|^{\gamma}
$$

where $\gamma$ is a numerical constant and $A_{\gamma}$ a quantity which depends on the experimental parameters. As $\mathbf{p}(\mathbf{k}=\mathbf{0})=0$, the corresponding distribution $\rho(\Delta \eta)$ is normalized, see equation $(5.5 .2)$. In the representation of equation (5.9.1) the small k-behaviour of $p(k)$ can be expressed as

$$
\gamma=2, \quad A_{2}=\left\langle\left\langle\Delta \eta^{2}\right\rangle\right\rangle / 2 n=\lambda\left\langle\Delta \eta_{2}^{2}\right\rangle / 2 n \quad(n=1,2,3)
$$

as follows from equations $(5.6 .2)$ or $(5.6 .3)$, assuming that $p(k)=p_{r}(k)$ and that $\rho(\Delta \eta)$ is symmetric. In the second step we used that the second cumulant $\left\langle\left\langle\Delta \eta^{2}\right\rangle\right\rangle$ of $\rho(\Delta \eta)$ is identical to the second moment $\left\langle\Delta \eta_{2}{ }^{2}\right\rangle$ of $\rho_{2}(\Delta \eta)$, see equation $(5.5 .10)$. Notice that $\left\langle\left\langle\Delta \eta^{2}\right\rangle\right\rangle=\left\langle\Delta \eta^{2}\right\rangle$ since $\langle\Delta \eta\rangle=0$, see equation $(5 \cdot 5.7)$. For the general case of a n-component displacement $\Delta \eta$ one finds from equations $(5.7 .3),(5.9 .1)$ and $(5.9 .2)$

$$
\rho(\Delta \eta)=\rho\left(\Delta \eta_{1}, \Delta \eta_{2}, \cdots, \Delta \eta_{0}\right)=\frac{1}{(2 \pi)} \prod_{j=1}^{n} \int d k_{j} e^{i k_{j} \cdot \Delta \eta_{j}-A_{2} k_{j}{ }^{2}}
$$

using that $k^{2}=k_{1}^{2}+\cdots+k_{n}^{2}$. Integration yields 


$$
\rho(\Delta \eta)=\prod_{j=1}^{n} \frac{1}{\left(4 \pi A_{2}\right)^{1 / 2}} e^{-\Delta j^{2} / 4 A_{2}}=\frac{1}{\left(4 \pi A_{2}\right)^{n / 2}} e^{-\Delta \eta^{2} / 4 A_{2}}
$$

which is a n-dimensional Gaussian distribution. The mean square of this distribution $\left\langle\Delta \eta^{2}\right\rangle$ is equal to $2 \mathrm{nA}_{2}$, which is consistent with equation $(5.9 .2)$. We conclude that Gaussian behaviour prevails if the integral for $\rho(\Delta \eta)$ is dominated by the small k-behaviour of $p(k)$. This will be true for large values of the linear particle density $\lambda$.

As a practical measure for the width of the displacement distribution we will of ten use the Full Width at Half Maximum (FWHM). In some applications one is also interested in the value of the median Full Width ( $\mathrm{FW}_{50}$ ), which is the smallest width containing $50 \%$ of the particles. For the distribution of equation $(5.9 .3)$ one finds

$$
\begin{aligned}
& \text { FWHM }_{\Delta \eta} / A_{2} 1 / 2=4(\ln 2)^{1 / 2}=3.3302 \quad(n=1,2,3)
\end{aligned}
$$

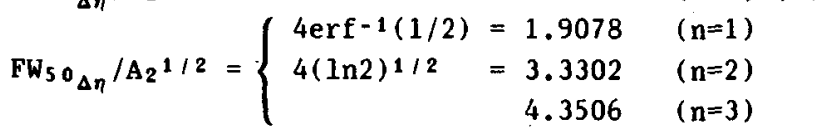

in which $\operatorname{erf}^{-1}(x)$ denotes the inverse of the Fresnel error-function.

Anticipating on the results of chapter 7 we note that a linear power-dependency in $k(\gamma=1)$ is relevant for the theory of the Boersch effect. We will therefore examine this case. By substituting equation (5.9.1) with $\gamma=1$ into equations $(5.7 .6),(5.7 .10)$ and $(5.7 .13)$, which specify the distribution $\rho(\Delta \boldsymbol{\eta})$ in 1,2 and 3 dimensions respectively, one finds after integration

$$
\begin{array}{ll}
\rho(\Delta \eta)=\frac{1}{\pi A_{1}\left[1+\left(\Delta \eta / A_{1}\right)^{2}\right]} & (n=1) \\
\rho(\Delta \eta)=\frac{1}{2 \pi A_{1}{ }^{2}\left[1+\left(\Delta \eta / A_{1}\right)^{2}\right]^{3 / 2}} & (n=2) \\
\rho(\Delta \eta)=\frac{1}{\pi^{2} A_{1}{ }^{3}\left[1+\left(\Delta \eta / A_{1}\right)^{2}\right]^{2}} & (n=3)
\end{array}
$$

which are known as Lorentz (or Cauchy) distributions. The FWHM and FW50 values of these distributions are given by

$$
\begin{aligned}
& \text { FWHM }_{\Delta \eta} / A_{1}=2(2 /(n+1)-1) 1 / 2 \\
& \text { FW }_{50_{\Delta \eta}} / A_{1}= \begin{cases}2 & (n=1,2,3) \\
2 \sqrt{3}=3.4641 & (n=1) \\
4.5389 & (n=2) \\
& (n=3)\end{cases}
\end{aligned}
$$

The Lorentzian distributions do not have finite second or higher moments, as can be verified from equation $(5.5 .1)$. This is in general true for all distributions corresponding to $\gamma$-values $<2$. We will come back to this point at the end of this section. In terms of equation (5.9.1), one can represent the Lorentzian distributions (5.9.5), $(5.9 .6)$ and $(5.9 .7)$ in the $k$-domain as

$$
\gamma=1, \quad A_{1}=\text { FWHM }_{\Delta \eta} / 2(22 /(n+1)-1)^{1 / 2} \quad(n=1,2,3)
$$

The cases $\gamma=2$ (Gaussian distribution) and $\gamma=1$ (Lorentzian distribution) appear to be the only cases for which equation $(5.7 .3)$ can be evaluated analytically. 
The Holtsmark distribution corresponds to the intermediate case $\gamma=3 / 2$, as can be seen from equation (5.8.6). The Holtsmark distribution pertains to the fluctuating component of the total interaction force $P$ in an extended homocentric cylindrical beam segment. In such a beam the displacement of the test particle $\Delta \eta$ experienced during the flight is directly proportional to $\mathbf{F}$, or a component of $\mathbf{F}$, provided that all particles follow approximately their unperturbed trajectories, see equation (4.9.3). The displacement distribution $\rho(\Delta \boldsymbol{\eta})$ generated in a homocentric cylindrical beam is therefore expected to be Holtsmarkian for small current densities, which are, however, large enough to consider the beam as extended. In the chapters 7,8 and 9 it will be demonstrated that the Holtsmark distribution also appears for other beam geometries, provided that the beam is extended and that the perturbations experienced by the particles are small.

According to equation $(5.8 .4)$ the Holtsmark distribution can in the $k$-domain be represented as

$$
\gamma=3 / 2, A_{3 / 2}=(4 / 15)\left(2 \pi C_{0}\right)^{3 / 2} n_{d} \quad(n=1,2,3)
$$

The corresponding displacement distributions in 1,2 and 3 dimensions are given by the equations $(5.8 .25),(5.8 .16)$ and $(5.8 .7)$ respectively. The FWHM and FWso values of these distributions can be retrieved from the data of table 5.1.

other $k$-dependencies are found in beams which have a small transverse dimension compared to the axial separation of the particles, referred to as pencil beams. In the limit of low particle density the distribution of displacements generated in a pencil beam shows again the same form as the distribution(s) of the interaction force, or one of its components. According to the equations (5.8.19) and (5.8.27) these distributions can in the $k$-domain be represented as

$$
\begin{array}{ll}
\gamma=1 / 3, A_{1 / 3}=\left(2 \pi C_{0}\right)^{1 / 2 \lambda} & (n=1) \\
\gamma=1 / 2, A_{1 / 2}=(4 / 7) I_{3} C_{0} 1 / 3 r_{0} 1 / 3 \lambda & (n=2)
\end{array}
$$

in which $I_{3}=2.8972$. The corresponding distribution for $n=1$ (axial force component) is given by equation (5.8.28) and for $n=2$ (lateral force component) by equation (5.8.20). These distributions are relevant for the Boersch effect and the trajectory displacement effect generated in low-density pencil beams. The FWHM and FW50 values can be determined from table 5.1.

Table 5.1. The Full Width at Half Maximum (FWHM) and the Full Width median ( $\mathrm{FW}_{5 \mathrm{O}}$ ) of the different types of 1-, 2- and 3-dimensional distributions generated by statistical interactions. The general form of the distribution is given by equation (5.9.12).

\begin{tabular}{|c|c|c|c|c|c|c|}
\hline & \multicolumn{3}{|c|}{${\text { FWHM } / \mathrm{A}_{\gamma}{ }^{1 / \gamma}}^{1 \gamma}$} & \multicolumn{3}{c|}{ FW $_{50} / \mathrm{A}_{\gamma}{ }^{1 / \gamma}$} \\
\hline$\gamma$ & $\mathrm{n}=1$ & $\mathrm{n}=2$ & $\mathrm{n}=3$ & $\mathrm{n}=1$ & $\mathrm{n}=2$ & $\mathrm{n}=3$ \\
\hline $1 / 3$ & .063581 & .013013 & .0049493 & 3.5751 & 6.8096 & 9.1538 \\
\hline $1 / 2$ & .44711 & .17997 & .10229 & 2.5677 & 4.7478 & 6.3418 \\
\hline 1 & 2 & 1.5328 & 1.2872 & 2 & 3.4641 & 4.5289 \\
\hline $3 / 2$ & 2.8775 & 2.6554 & 2.5128 & 1.9379 & 3.3206 & 4.2956 \\
\hline 2 & 3.3302 & 3.3302 & 3.3302 & 1.9078 & 3.3302 & 4.3506 \\
\hline
\end{tabular}


The complete list of k-dependencies encountered in the calculation of statistical effects exists of the following $\gamma$-values:

$\begin{array}{lll}\gamma=2 & - & \text { Gaussian distribution } \\ \gamma=3 / 2 & - & \text { Holtsmark distribution } \\ \gamma=1 & - & \text { Lorentzian distribution } \\ \gamma=1 / 2 & - & \text { distribution of } F_{/ \prime} \text { in a pencil beam } \\ \gamma=1 / 3 & - & \text { distribution of } F_{\perp} \text { in a pencil beam }\end{array}$

The corresponding distributions in one and two dimensions are depicted in the figures 5.3 and 5.4 respectively. These figures clearly show that a small $\gamma$-value leads to a distribution with a narrow core and long tails. In figure 5.4 we included a plot corresponding to the value $\gamma=1 / 2$, which is relevant for the angular deflection distribution in a beam segment with a narrow crossover and low current density, as will be shown in section 8.3 .

In the remaining part of this section we will investigate how the width and the tails of the displacement distribution $\rho(\Delta \eta)$ depend on the parameter $\gamma$ and the experimental parameters represented by $\mathrm{A} \gamma$. Substitution of equation (5.9.1) into equation $(5.4 .9)$ yields

$$
\rho(\Delta \eta)=\frac{1}{(2 \pi)^{n}} \int \mathrm{d} k \mathrm{e}^{\mathrm{ik} \cdot \Delta \boldsymbol{\eta}-\mathrm{A}_{\boldsymbol{\gamma}}|\mathbf{k}|^{\gamma}}
$$

By introducing the scaling

$$
\overline{\mathbf{k}}=\mathbf{A}_{\gamma}{ }^{1 / \gamma} \mathbf{k}, \Delta \bar{\eta}=A_{\gamma}{ }^{-1 / \gamma} \Delta \eta, \bar{\rho}(\Delta \bar{\eta})=A_{\gamma}{ }^{n / \gamma} \rho(\Delta \eta)
$$

equation (5.9.12) transforms to

$$
\bar{\rho}(\Delta \bar{\eta})=\frac{1}{(2 \pi)^{n}} \int \mathrm{d} \overline{\mathbf{k}} \mathrm{e}^{\mathrm{ik} \cdot \Delta \overline{\boldsymbol{\eta}}-|\overline{\mathbf{k}}|^{\gamma}}
$$

Since we have absorbed the parameter $A_{\gamma}$ in the scaling, equation (5.9.14) does not depend on the experimental parameters. Accordingly, the Full Width at Half Maximum of $\bar{\rho}(\Delta \bar{\eta})$, indicated as $F W H M_{\gamma}$, is a function of the numerical factor $\gamma$ only. This function is depicted in figure $5.5 \mathrm{a}$, for $n=1,2$ and 3 . Table 5.1 gives the corresponding numerical values for some specific cases, which are relevant to the theory of statistical interactions. From equation (5.9.13) it follows that the unscaled FWHM is given by

$$
\text { FWHM }_{\Delta \eta}=A_{\gamma}{ }^{1 \gamma} \text { FWHMr }
$$

This equation expresses the FWHM of $\rho(\Delta \eta)$ in terms of the experimental parameters (represented by $A_{\gamma}$ ) and the parameter $\gamma$, which is a constant provided that the shape of $\rho(\Delta \eta)$ remains unchanged. Equation (5.9.15) is the generalization of equations (5.9.4) and (5.9.8) which apply to the cases $\gamma=2$ and $\gamma=1$ respectively. Equation $(5.9 .15)$ can be modified to cover other width-measures as well, such as the median width $\mathrm{FW}_{50}$. The corresponding numerical values of $F W_{50 \gamma}$ are plotted in figure $5.5 \mathrm{~b}$. It is important to observe that the ratio $F W H W_{\gamma} / F W_{50 r}$ is not constant, but varies with $\gamma$, as is shown in figure $5.5 \mathrm{c}$.

The parameter Ar is for all conditions directly proportional to the beam current $I$, as can be seen from equations $(5.9 .2),(5.9 .10)$ and (5.9.11), using that $\lambda$ is directly proportional to $I$, as expressed by equation (3.2.4). Accordingly, the FWHM and $\mathrm{FW}_{50}$ of the energy distribution generated by the Boersch effect and the trajectory displacement distribution are proportional to $I^{1 / 2}$ in the Gaussian 


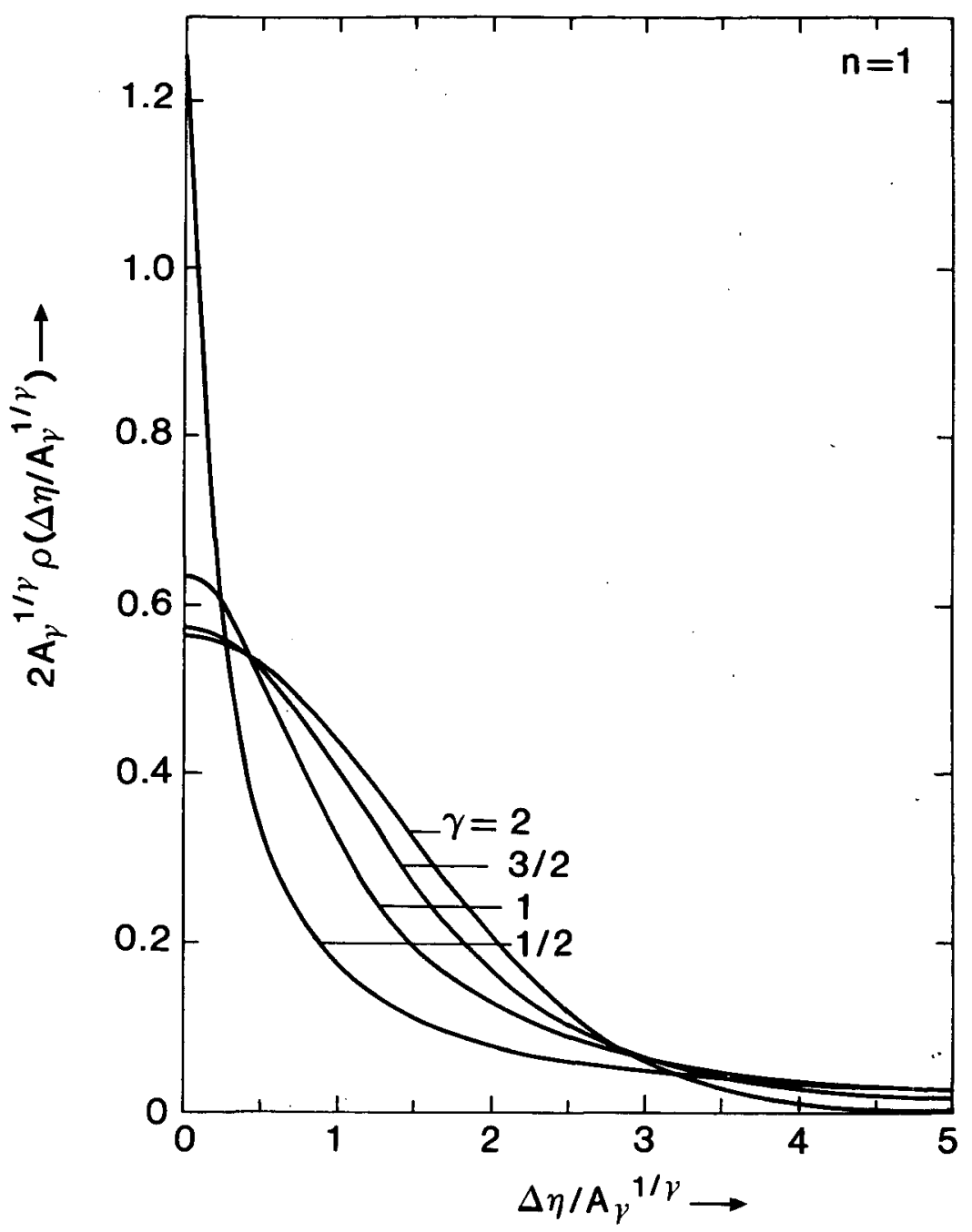

Figure 5.3 The different types of 1-dimensional displacement distributions generated by statistical interactions. 


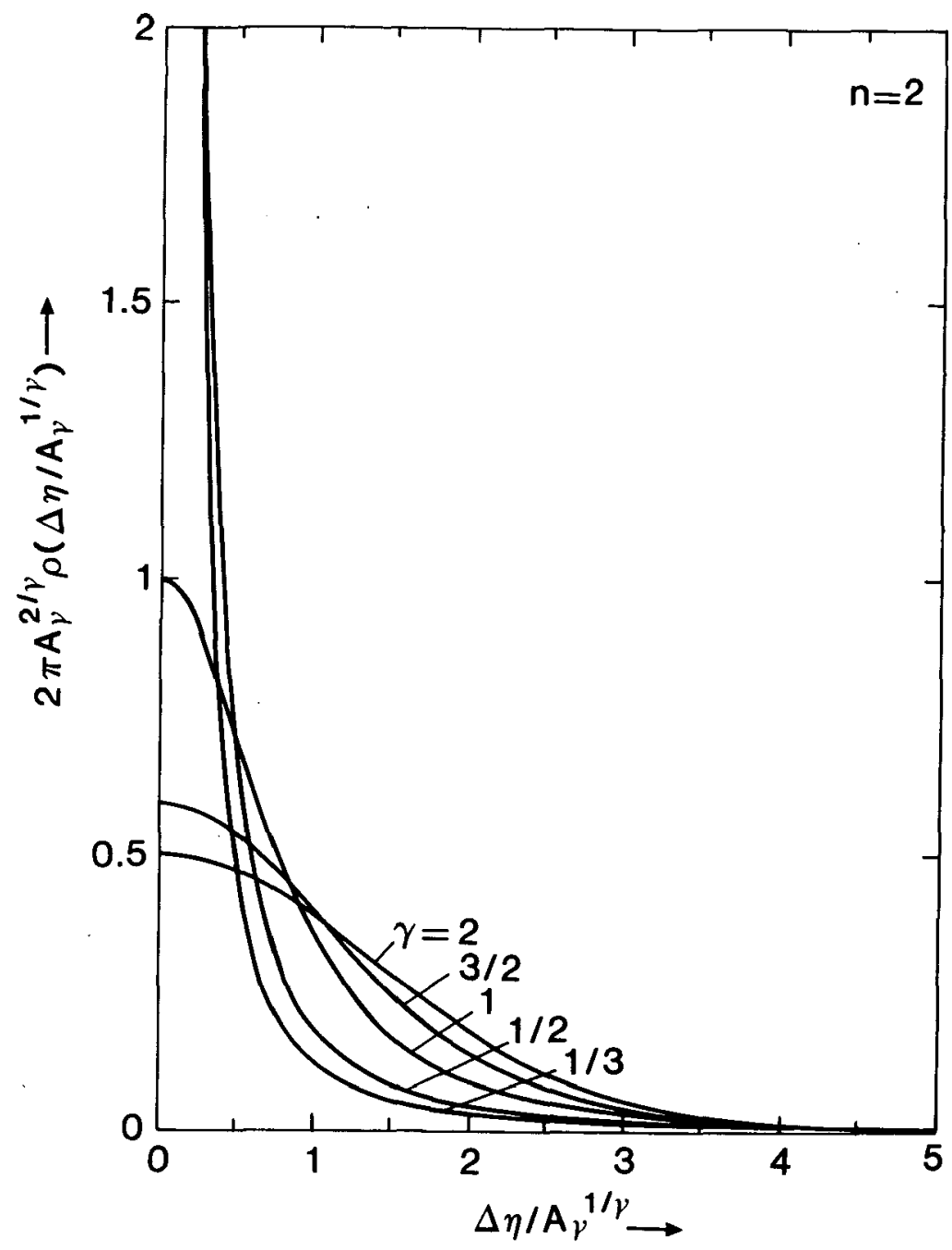

Figure 5.4 The different types of 2-dimensional displacement distributions generated by statistical interactions. 


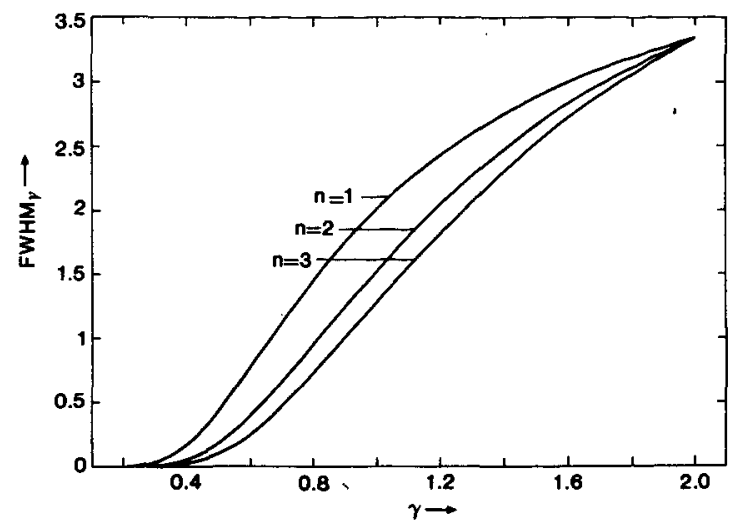

fig.5.5a

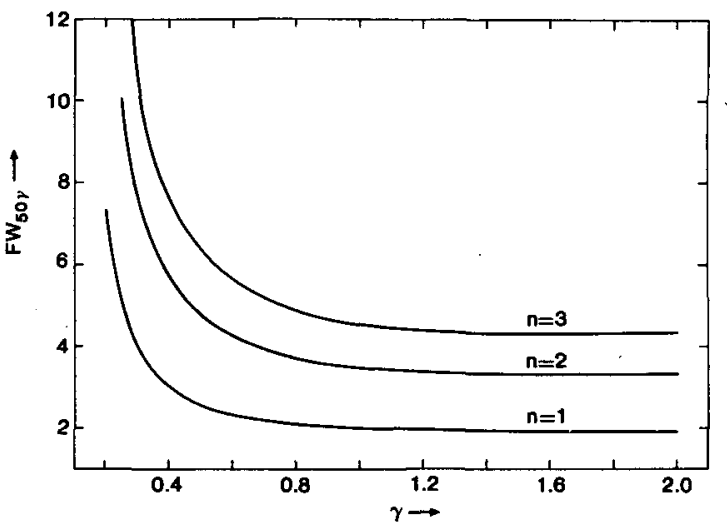

fig.5.5b

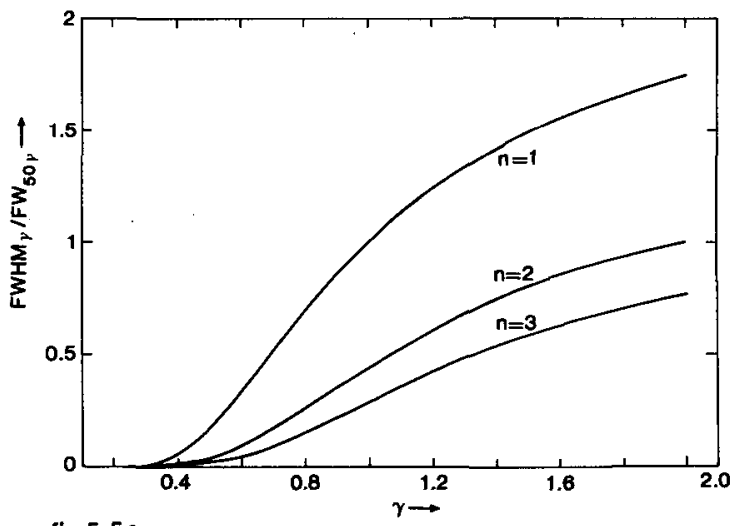

fig.5.5c

Pigure 5.5 Different width-measures of the 1-, 2- and 3-dimensional scaled distribution of displacements as function of the parameter $\gamma$, which defines the shape of the distribution. The figures $5.5 \mathrm{a}$ and $5.5 \mathrm{~b}$ give the Full Width at Half Maximum (FWHM) and the Full Width median (FWso) respectively, while the ratio of these quantities is plotted in figure $5.5 \mathrm{c}$. 
regime $(\gamma=2)$, proportional to $\mathrm{I}^{2 / 3}$ in the Holtsmark regime $(\gamma=3 / 2)$ and proportional to $I$ in the Lorentzian regime $(\gamma=1)$. For pencil beams the Boersch effect increases with $I^{2}(\gamma=1 / 2)$ and the trajectory displacement effect increases with $I^{3}(\gamma=1 / 3)$.

The fall-off in the tails of the distribution $\rho(\Delta \boldsymbol{\eta})$ is determined by the small k-behaviour, as can be seen from equation (5.9.12). For large values of $\Delta \eta=|\Delta \eta|$ one may expand the second part of the exponential function appearing in this equation. Substituting $x=\Delta \eta k$ one obtains

$$
\lim _{\Delta \eta \rightarrow \infty} \rho(\Delta \eta) \cong \frac{1}{(2 \pi \Delta \eta)^{n}} \int d x e^{i x \Delta \eta / \Delta \eta}\left[1-A_{\gamma}(x / \Delta \eta)^{\gamma}\right]
$$

From this equation it can be understood that the fall-of $f$ in the tails is given by

$$
\lim _{\Delta \eta+\infty} \rho(\Delta \eta) \sim \frac{A \gamma}{\Delta \eta^{n+\gamma}} \quad(0<\gamma<2 ; n=1,2,3)
$$

It should be emphasized that equation (5.9.16) does not cover the case $\gamma=2$, which corresponds to a Gaussian distribution. Notice that equation (5.9.16) is in agreement with equations (5.9.5), (5.9.6) and (5.9.7), which apply to the case $\gamma=1$ (Lorentzian distribution) with $n=1,2,3$ respectively. The contribution of the tails to the $\mathrm{m}^{\mathrm{t}} \mathrm{h}$ moment of the $\mathrm{n}$ dimensional distribution $\rho(\Delta \eta)$ can now be expressed as

$$
\left\langle\Delta \eta^{\mathrm{m}}\right\rangle \sim \int_{\Delta \eta_{0^{-}}}^{\infty} \Delta \eta^{\mathrm{n}-1} \mathrm{~d} \Delta \eta \rho(\Delta \eta) \Delta \eta^{\mathrm{m}} \sim \int_{\Delta \eta_{0}}^{\infty} \mathrm{d} \Delta \eta \frac{\mathrm{Ar}}{\Delta \eta^{1+\gamma-\mathrm{m}}}
$$

in which $\Delta \eta_{0}$ should be chosen large enough to guarantee the validity of equation (5.9.16). Equation (5.9.17) makes clear that no finite second or higher moments exist for $\gamma<2$. Only the Gaussian distribution $(\gamma=2)$ has a finite value for the second moment (while the third and higher cumulants of a Gaussian distribution are zero).

Anticipating on the results presented in chapters 7,8 and 9 we note that the approximation of the function $p(k)$ by equation (5.9.1) will only be accurate within a certain range of $k$-values. The functions $p(k)$ obtained in the actual calculations for the Boersch effect and the trajectory displacement effect are all proportional to $k^{2}$ for small $k$, proportional to $k^{3 / 2}$ or $k$ for intermediate $k$-values and proportional to $k^{1 / 2}$ (Boersch effect) or $k^{1 / 3}$ (trajectory displacement effect) for very large $k$-values. The particle density, expressed in the linear particle density $\lambda$, determines what part of the function $p(k)$ dominates the displacement distribution $\rho(\Delta \eta)$. In general the small k-behaviour is dominant for high values of $\lambda$ and the large $k$-behaviour is dominant for small values of $\lambda$, as can be seen from equation $(5.4 .9)$.

\subsection{Addition of the effects generated in individual beam segments}

The model outlined in the previous section enables us to calculate the effect of statistical interactions in an individual beam segment, which contains a single crossover or is cylindrical. A practical beam usually consists of several of such beam beam segments. The question now arises how to determine the effect generated in the total system from the results obtained for the individual segments. Consider a beam which is partitioned in $N_{b}$ beam segments. Let 
the statistical displacement distribution of segment $j \quad\left(j=1 \cdots N_{b}\right)$ be given by

$$
\rho_{j}(\Delta \eta)=\frac{1}{(2 \pi)^{2}} \int \mathrm{d} k \mathrm{e}^{i k \cdot \Delta \eta-\lambda_{j} p_{j}(k)}
$$

using the representation of equation (5.4.9). All information regarding segment $j$ is contained in the quantity $\lambda_{j} p_{j}(k)$. We now make the following two assumptions:

(1) The displacements $\Delta \boldsymbol{\eta}_{j}$ (with $\mathrm{j}=1 \cdots \mathrm{N}_{\mathrm{b}}$ ) experienced by the test particle in the successive beam segments are not correlated.

(2) All distributions $\rho_{j}(\Delta \eta)$ (with $j=1 \cdots N_{b}$ ) can be represented by equation (5.9.1) with identical values for the $k$-dependency parameter $\gamma$. This implies that the distributions are assumed to be congruent.

To start with let us consider a system consisting of two beam segments. Assumption (1) implies that the distribution of statistical effects generated in the total beam can be expressed as a convolution of the distributions corresponding to the individual beam segments

$$
\rho(\Delta \eta)=\int d \Delta \eta^{\prime} \rho_{1}\left(\Delta \eta^{\prime}\right) \rho_{2}\left(\Delta \eta-\Delta \eta^{\prime}\right)
$$

Substitution of the expressions for $\rho_{1}(\Delta \eta)$ and $\rho_{2}(\Delta \eta)$ given by equation $(5.10 .1)$ yields

$$
\rho(\Delta \eta)=\int d \Delta \eta^{\prime} \int \frac{d k}{(2 \pi)} \int \frac{d k^{\prime}}{(2 \pi)^{0}} e^{i k \cdot \Delta \eta^{\prime}+i k^{\prime} \cdot\left(\Delta \eta-\Delta \eta^{\prime}\right)-\lambda_{1} p_{1}(k)-\lambda_{2} p_{2}\left(k^{\prime}\right)}(5.10 .3)
$$

Conform the representation of the $\delta$-function given by equation (5.4.2) one may write

$$
\delta\left(\mathbf{k - k ^ { \prime } )}=\int \frac{\mathrm{d} \Delta \boldsymbol{\eta}^{\prime}}{(2 \pi)^{\mathbf{n}}} \mathrm{e}^{i \Delta \boldsymbol{\eta}^{\prime} \cdot\left(\mathbf{k}-\mathbf{k}^{\prime}\right)}\right.
$$

Accordingly, equation (5.10.3) transforms to

$$
\rho(\Delta \eta)=\frac{1}{(2 \pi) \mathbf{n}} \int d k \mathrm{e}^{i k \cdot \Delta \eta-\left[\lambda_{1} p_{1}(k)+\lambda_{2} p_{2}(k)\right]}
$$

By comparison with equation $(5.10 .1)$ one finds that the total distribution can in the $k$-domain be represented as

$$
[\lambda p(k)]_{T}=\lambda_{1} p_{1}(k)+\lambda_{2} p_{2}(k)
$$

For a system consisting of $\mathrm{N}_{b}$ beam segments this expression can be generalized to

$$
[\lambda p(k)]_{T}=\sum_{j=1}^{N_{b}} \lambda_{j} p_{j}(k)
$$

Thus assumption (1) implies that the representation of the total displacement distribution in the $k$-domain can be obtained as the sum of all functions $\lambda p(k)$ corresponding to the individual beam segments.

We like to translate this result in terms of the distributions $\rho_{j}(\Delta \boldsymbol{l})$ where $j=1 \cdots \mathrm{N}_{\mathrm{b}}$. For that we need assumption (2). Following equation $(5.9 .15)$ one may express the FWHM of the distribution $\rho_{j}(\Delta \eta)$ as 


$$
\mathrm{FWHM}_{\mathbf{j}}=\mathrm{A}_{\mathbf{j}}{ }^{1 / \gamma} \mathrm{FWHM}
$$

in which $A_{j}$ corresponds to the representation in $k$-space of the distribution of segment $j$ by means of equation (5.9.1). Equation (5.10.6) implies that the parameter AT, corresponding to the total effect, is a linear sum of the parameters $A_{j}$ corresponding to the individual beam segments. Assumption (2) implies that the parameter FWHMr has the same numerical value in all segments. Consequently, the FWHM of the total displacement distribution can be expressed as

$$
\mathrm{FWHM}_{T}=\left(\sum_{j=1}^{N_{\mathrm{b}}} \mathrm{A}_{j}\right)^{1 / \gamma} \mathrm{FWHM}_{\gamma}=\left(\sum_{j=1}^{N_{\mathrm{D}}}\left(\mathrm{A}_{j}{ }^{1 / \gamma} \text { FWHM }\right)^{\gamma}\right)^{1 / \gamma}
$$

By substitution of equation $(5.10 .7)$ one obtains

$$
\operatorname{FWHM}_{T}=\left(\sum_{j=1}^{N_{b}} \text { FWHM }_{j} \gamma\right)^{1 / \gamma}
$$

Thus Gaussian distributions $(\gamma=2)$ should be added quadraticly, Lorentzian distributions $(\gamma=1)$ should be added linearly, Holtsmark distributions $(\gamma=3 / 2)$ with a $3 / 2$ power, etc. Since the power-dependency on $k$ does not change by the summation procedure of equation $(5.10 .6)$, the total distribution will be of the same kind as the distributions generated in the individual beam segments. In other words, the distributions are "stable".

In case assumption (2) is not valid (e.g. not all distributions are congruent) equation (5.10.8) can not be applied. However, as long as assumption (1) is valid, equation (5.10.6) still holds and the total function $p(k)$ can straightforwardly be determined from the individual contributions. The total displacement distribution follows as usual by means of equation (5.4.9). Clearly, as the power-dependency on $k$ varies one can not beforehand predict the resulting $k$-dependency. Accordingly the shape of the resulting distribution depends on the ratio of the individual contributions and one can not simply translate equation (5.10.6). into a summation rule for the FWHM values. This is only a practical problem and not a principle one, since the resulting FWHM (or any other width) can still be determined from the total $p(k)$ function by means of equation $(5.4 .9)$.

In general, principle problems do occur when assumption (1) is not valid (which means that the displacements experienced in successive beam segments are correlated). However, there is one exception, namely the extreme case of full correlation. Let us investigate this case in more detail. Full correlation means that the displacement $\Delta \boldsymbol{\eta}_{j}$ experienced by the test particle in segment $j$ is fully determined by the displacement experienced in the previous segment. Thus one may write

$$
\Delta \boldsymbol{\eta}_{\mathbf{j}}=\mathbf{C}_{\mathbf{j}}\left(\Delta \boldsymbol{\eta}_{\mathbf{1}}\right)
$$

in which $\mathbf{C}_{j}$ some (deterministic) function. Accordingly, the total displacement experienced in the entire system is given by

$$
\Delta \eta=\sum_{j=1}^{N_{b}} \Delta \eta_{j}=\sum_{j=1}^{N_{b}} c_{j}\left(\Delta \eta_{1}\right)
$$

and the total displacement distribution can be expressed as 


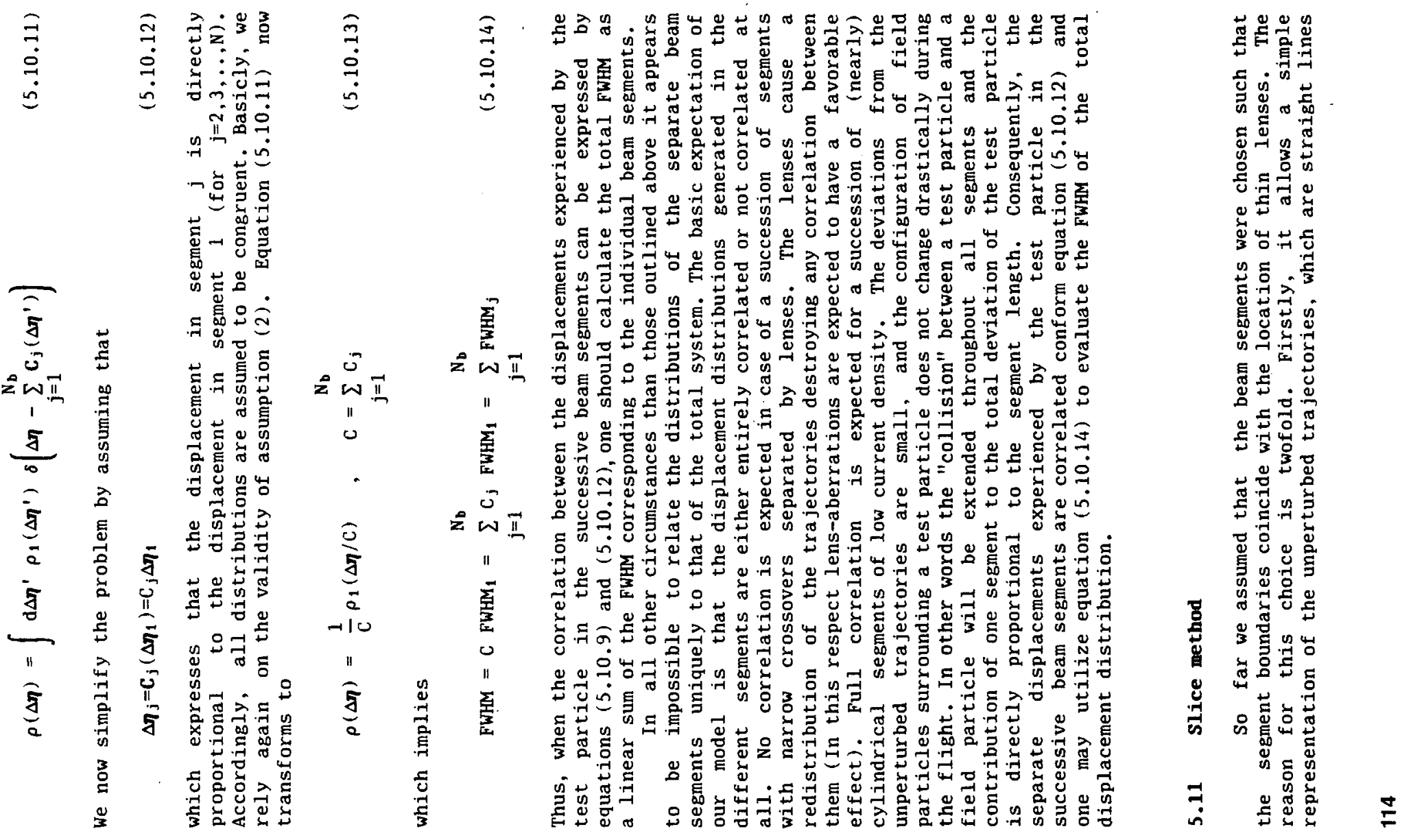




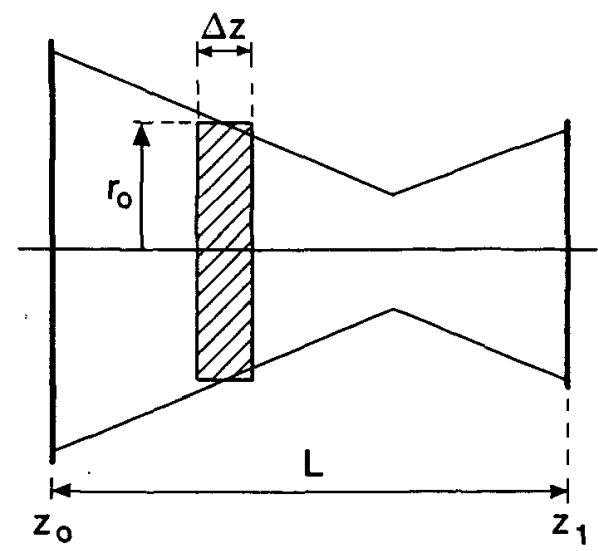

Figure 5.6 Schematic representation of the subdivision of a beam segment into thin cylindrical slices.

due to the absence of optical components within the segment. Secondly, we expect that the displacements generated in the individual segments, separated by lenses, show no correlation. This allows us to determine the total effect from equation $(5.10 .6)$ or equation (5.10.8). Considering the alternatives methods of partitioning the beam it becomes clear that it is not practical to extend the segments over a larger part of the beam and include lenses, primarily due to complications with. the representation of the unperturbed trajectories. However, one can easily choose shorter segments than those defined by the distances between the optical components. In this section we will study this alternative in some detail.

Let us subdivide a beam segment between two thin optical components into slices of length $\Delta z$, see figure 5.5 . The displacement distribution $\rho(\Delta \eta)$ is now evaluated per slice. If one assumes that the displacements of the test particle experienced in the individual slices are entirely correlated one may use equation (5.10.14) to determine the FWHM of the total displacement distribution generated in the whole segment. This approach, referred to as the slice method, is justified if the displacements from the unperturbed trajectories are small, which will be true for low current densities.

The slice-method has some distinct advantages. By choosing the slices thin enough one may treat them as cylindrical. Accordingly, one can utilize the expression obtained for a cylindrical beam segment to calculate the effect in any beam geometry, using

$$
F W H M=\sum_{s=1}^{N_{s}} F_{W H M_{P}}\left(I, V, r_{0}, \Delta z_{s}\right) \approx \int_{z_{0}}^{z_{1}} d z\left(\frac{\operatorname{FWHM}_{P}\left(I, V, r_{0}, \Delta z\right)}{\Delta z}\right)
$$

where FWHMP $_{P}\left(I, V, r_{0}, \Delta z\right)$ is the FWHM of the distribution generated in a cylindrical slice (with parallel rays) of length $\Delta z$, radius $r_{0}$, carrying a current $I$ at potential $V$ (supposing an uniform current density distribution). This expression is expected to be linear in $\Delta z$. Accordingly, the integrant $\operatorname{FWHM}_{\mathrm{P}}\left(\mathrm{I}, \mathrm{V}, \mathrm{r}_{0}, \Delta z\right) / \Delta z$ will only depend on $z$ through the beam radius $r_{0}$. 
In the chapters 7,8 and 9 we will use the slice-method to relate the results obtained for a beam segment with a crossover to those obtained for a cylindrical beam segment. It should be emphasized that the validity of equation (5.11.1) requires the current density to be low enough to guarantee that the displacements from the unperturbed trajectories are small. Returning to the analysis of section 5.3 one may note that the slice-method resembles the procedure of Massey, Jones and Plummer (1981), which is a variant of the "mean square field fluctuation approximation", see equation (5.3.27). However, by considering the FWHM-value instead of a rms-value, we avoid the divergence related to close encounters in a natural way. 


\section{TWO-PARTICLE DYNAMICS}

\subsection{Introduction}

This chapter studies the dynamical problem of two particles interacting through the Coulomb force in abserce of external fields. The unperturbed motion of the particles in the laboratory system is represented by the so-called geometrical parameters. These parameters are transformed to coordinates in the orbital plane. The calculation of the particle motion in this plane constitutes the well known Kepler problem, which was solved by Newton (1687). For the repulsive Coulomb force considered here, the solution of this problem predicts that the particles will follow a hyperbolic orbit, which is in polar coordinates represented by a function $r(\theta)$. This function gives sufficient information to determine the effect of a collision which is complete in the sense that the particles effectively come from infinity and recede to infinity. However, additional information is required to describe incomplete collisions. Given an arbitrary initial condition, one has to determine each of the polar coordinates $r$ and $\theta$, as well as there derivatives as function of time $t$. The main part of the chapter is dedicated to the solution of this problem. Integration of the equations of motion results in a function $t(r)$, which implicitly specifies the function $r(t)$, required in the calculation. Unfortunately, the function $t(r)$ can not be inverted, which prohibits an exact analytical calculation. Three alternative methods are studied to solve this problem. The first method exploits a numerical routine to perform the inversion. The second method is based on a power expansion in $r$ of the exact solution $t(r)$ and applies to nearly complete collisions. The third method is based on first order perturbation theory, which is a valid approximation for weak interactions. The last two methods provide explicit equations which express the velocity and spatial displacements of the particles in terms of the geometrical parameters.

\subsection{Basic properties}

Consider a test particle moving along the axis of a beam segment of arbitrary geometry. The particle interacts with a single field particle which follows some of $f$-axis trajectory. External forces are assumed to be absent. Figure 6.1 depicts the unperturbed trajectories of the particles at the moment that the field particle passes the $x, y$-plane of the laboratory system. The $z$-axis of the laboratory system coincides with the unperturbed path of the test particle (which is the beam axis). For a beam segment with a crossover the laboratory system is usually chosen such that the $x, y$-plane coincides with the crossover plane. The axial location of this plane is specified by the parameter $S_{c}$, see equation (3.2.1). In this chapter we will use the parameter $S_{c}$, in a more general sense, to define the axial location $z=0$ of the $x, y$-plane, irrespective of the beam geometry.

It is assumed that both particles have the same axial velocity. Accordingly, the relative coordinates are completely specified by

$$
\xi=\left(r_{1}, \varphi, b_{2}, v, \psi\right)
$$

as was stated previously by equation (5.2.1). This set of parameters defines the unperturbed motion of the particles, which shows only geometrical properties. Therefore, we will here refer to these 


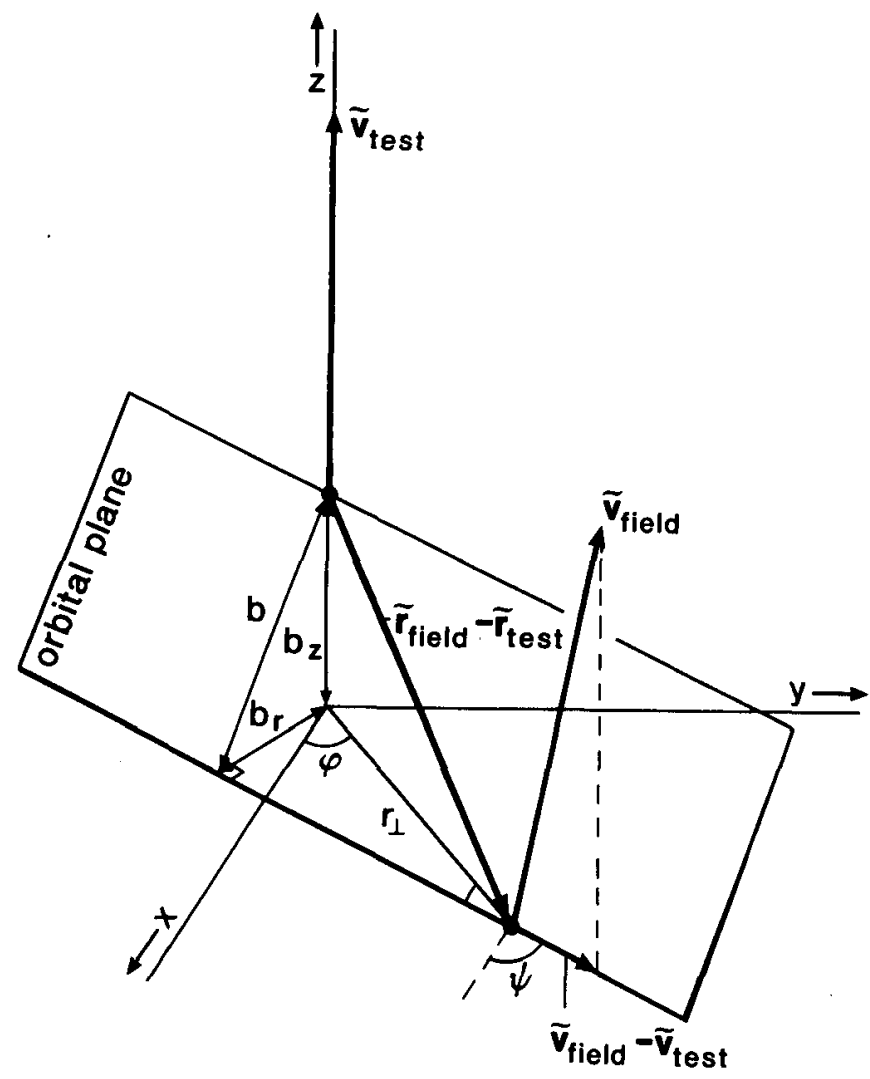

Figure 6.1 Unperturbed trajectories of a test particle and a field particle in the laboratory system. The orbital plane is spanned by the relative unperturbed velocity vector $\hat{\nabla}_{f} \mathrm{ll}_{\mathrm{d}} \hat{\mathrm{v}}_{\mathrm{tes}} \mathrm{t}$ and the relative unperturbed position vector Frield-Fist.

quantities as the geometrical variables. From the geometrical variables $\xi$ one can obtain the unperturbed coordinates of the particles at any moment. The moment that the field particle passes through the $x, y-p$ lane (shown in figure 6.1 ) is defined as $t=0$.

Due to the mutual Coulomb interaction during the flight the particles will deviate from their unperturbed trajectories. The experienced deviations are determined by the initial relative coordinates of the particles (specified by the geometrical variables and the initial time $t_{i}$ ) and the time of flight $T$, which is equal to

$$
T=t_{i}-t_{i}=L / v_{2}
$$

with $L$ the length of the beam segment and $v_{z}$ the axial velocity of the particles. The initial and final time of the interaction, $t_{i}$ and $t_{f}$, can be expressed as 


$$
t_{i}=-s_{c} T, \quad t_{r}=\left(1-S_{c}\right) T
$$

in which the start and the end of the interaction is identified with the moments that the field particle passes the entrance and the exit plane of the beam segment respectively.

The variables specifying the deviations from the unperturbed trajectories, here referred to as the dynamical variables, are abbreviated by

$$
\Delta \boldsymbol{\eta}=\left(\Delta \mathrm{x}, \Delta \mathrm{y} ; \Delta \mathbf{v}_{\mathbf{z}}\right)
$$

using the same notation as in chapter 5. The quantities $\Delta x$ and $\Delta y$ are the virtual spatial displacements in the plane which is optically conjugated to the target plane. This plane is called the image or also the reference plane. The axial location of the image plane is specified by the quantity $S_{i}$, defined by equation (3.2.1). The displacements $\Delta x$ and $\Delta y$ are the relevant dynamical variables for the calculation of the trajectory displacement effect. For a test particle running along the axis of a rotational symmetric beam it is sufficient to determine the radial displacement $\Delta r=\left(\Delta x^{2}+\Delta y^{2}\right)^{1 / 2}$. The axial velocity displacement $\Delta v_{z}$ is the relevant dynamical variable for the calculation of the Boersch effect. In general we will denote all "deviations from the unperturbed trajectory", both in position and velocity, as "displacements" or "shifts".

The basic problem considered in this chapter is to determine the dynamical variables $\Delta \eta$ as function of the geometrical variables $\xi$, the initial time $t_{i}$ and the interaction time $T$. The set of geometrical parameters given by equation $(6.2 .1)$ is clearly not the most general possible, since it assumes that the unperturbed trajectory of the test particle coincides with the beam axis, while it ignores any difference in axial velocity. However, the analysis of this chapter can be generalized to include those cases. The only required modification concerns the transformation from the laboratory system to coordinates in the orbital plane, as will be indicated in the next section.

This section covers the general formulation of the problem. We will first discuss the relation between the coordinate representations in the laboratory system and the centre of mass system and we will next review the physical constants of motion.

The relative position vector $\mathbf{r}$ and the relative velocity vector $v$ are defined as

$$
\mathbf{r}=\mathbf{r}_{\mathrm{c}}-\mathbf{r}_{\mathrm{t}}, \mathbf{v}=\mathbf{v}_{\mathrm{c}}-\mathbf{v}_{\mathrm{t}}
$$

in which the subscript $c$ refers to the field (or colliding) particle and the subscript $t$ to the test particle. The unperturbed coordinates of the particles will be indicated with a tilde $(\sim)$. Thus the unperturbed relative position is $\tilde{\mathbf{r}}$ and the unperturbed relative velocity is $\tilde{\mathbf{v}}$. The coordinates referring to the time $t=0$ will be indicated with the subscript 0 and the coordinates referring to the initial time $t_{i}$ and final time $t_{f}$ with the subscripts $i$ and $f$ respectively. The unperturbed relative position and velocity at time $t=0$ are related to the geometrical variables as

$$
\tilde{\mathbf{r}}_{0}=\left(\begin{array}{c}
r_{1} \cos \varphi \\
r_{1} \sin \varphi \\
-b_{2}
\end{array}\right), \quad \tilde{\mathbf{v}}_{0}=\left(\begin{array}{c}
v \cos \psi \\
v \sin \psi \\
0
\end{array}\right)
$$

The initial relative coordinates follow from 


$$
\mathbf{r}_{\mathbf{i}}=\tilde{\mathbf{r}}_{0}+\mathrm{t}_{\mathbf{i}} \tilde{\mathbf{v}}_{0}, \quad \mathbf{v}_{\mathbf{i}}=\tilde{\mathbf{v}}_{0}
$$

The unperturbed velocity remains constant by definition, as expressed by the second equation. The magnitude $|\tilde{v}|$ of the unperturbed velocity is identical to the geometrical parameter $v$ (thus $v_{i}=\hat{v}_{0}=v$ ).

The centre of mass coordinates are defined as

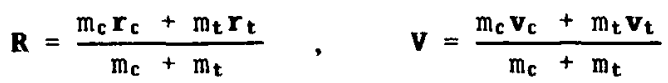

in which $m_{c}$ and $m_{t}$ the mass of the field and test particle respectively. We will consider the case of identical particles, thus $m_{c}=m_{t}=m_{\text {. }}$ Accordingly, equations $(6.2 .8)$ reduce to

$$
\mathbf{R}=\frac{1}{2}\left(\mathbf{r}_{\mathbf{c}}+\mathbf{r}_{\mathrm{t}}\right), \quad \mathbf{V}=\frac{1}{2}\left(\mathbf{v}_{\mathbf{c}}+\mathbf{v}_{\mathrm{t}}\right)
$$

At $t=0$ the centre of mass coordinates are given by

$$
\mathbf{R}_{0}=\frac{1}{2}\left(\begin{array}{c}
r_{\perp} \cos \varphi \\
r_{\perp} \sin \varphi \\
b_{2}
\end{array}\right) \quad, \quad \mathbf{v}=\frac{1}{2}\left(\begin{array}{c}
v_{\perp} \cos \psi \\
v_{\perp} \sin \psi \\
2 v_{z}
\end{array}\right)
$$

As external forces are assumed to be absent the total momentum of the two-particle system is conserved. Consequently, the velocity of the centre of mass remains constant irrespective of the interaction between the particles and equation (6.2.10) applies to both the unperturbed and the perturbed trajectories. Accordingly, the position $\mathbf{R}$ of the centre of mass at time $t$ is (independent of the interaction) given by

$$
\mathbf{R}(\mathbf{t})=\mathbf{R}_{0}+\mathbf{t} \mathbf{V}
$$

and the coordinates of field and test particle at time $t$ can be retrieved from

$$
\begin{aligned}
& r_{c}(t)=R(t)+\frac{1}{2} r(t), \quad v_{c}(t)=v(t)+\frac{1}{2} \mathbf{v}(t) \\
& r_{t}(t)=R(t)-\frac{1}{2} r(t), \quad v_{t}(t)=v(t)-\frac{1}{2} v(t)
\end{aligned}
$$

If one knows the relative coordinates $r(t)$ and $v(t)$ as function of time one can determine the corresponding coordinates of the individual particles by means of the equations $(6.2 .10),(6.2 .11)$ and $(6.2 .12)$.

The absence of external forces also implies that the total energy and the angular momentum of the two-particle system are conserved. The total kinetic energy (in the laboratory system) is defined as

$$
E_{k i n, L}=\frac{1}{2} m_{c} v_{c}{ }^{2}+\frac{1}{2} m_{t} v_{t}{ }^{2}=\frac{1}{2}\left(m_{c}+m_{t}\right) v^{2}+\frac{1}{2} \frac{m_{c} m_{t}}{m_{c}+m_{t}} v^{2}
$$

The first term on the right hand side of equation (6.2.13) corresponds to the kinetic energy of the centre of mass, while the second term corresponds to the kinetic energy observed in a reference system moving with the centre of mass (called centre of mass system). The first term is constant and can be disregarded in the further analysis. The total energy in the centre of mass system is equal to (using $m_{c}=m_{t}=m$ ) 


$$
\mathrm{E}=\frac{1}{4} \mathrm{mv^{2 }}+\frac{\mathrm{C}_{0}}{\mathrm{r}}
$$

in which $C_{0}=e^{2} / 4 \pi \varepsilon_{0}$. The value of $E$ is conserved during the encounter and can be determined from the initial coordinates given by equation $(6.2 .7)$

$$
E=\frac{1}{4} m v_{i}^{2}+\frac{C_{0}}{r_{i}}
$$

The angular momentum $J_{\mathrm{L}}$ relative to the origin of the laboratory system is defined as

$$
J_{L}=m_{c} r_{c} \times v_{c}+m_{t} r_{t} \times v_{t}=\left(m_{c}+\dot{m}_{t}\right) R \times V+\frac{m_{c} m_{t}}{m_{c}+m_{t}} \mathbf{r} \times v
$$

The first term, specifying the angular momentum of the centre of mass, is constant as follows by substitution of equation $(6.2 .11)$. Since $J_{L}$ is conserved as a whole, the second term, specifying the angular momentum relative to the centre of mass, is constant too. Using $m_{c}=m_{t}=m$ one finds for this term

$$
\mathrm{J}=\frac{1}{2} \operatorname{mrxv}
$$

The vector $\mathbf{J}$ can be determined from the initial coordinates. Using equations $(6.2 .7)$ and $(6.2 .6)$ one obtains

$$
J=\frac{1}{2} m r_{i} \times v_{i}=\frac{1}{2} m \tilde{\mathbf{r}}_{0} \times \tilde{v}_{0}=\frac{1}{2} m v\left(\begin{array}{l}
b_{z} \sin \varphi \\
-b_{z} \cos \varphi \\
r_{1} \sin \Phi
\end{array}\right)
$$

with $\Phi=\psi-\varphi$. The modulus of this vector is

$$
\mathrm{J}=\frac{1}{2} \mathrm{mbv}_{\mathrm{i}}=\frac{1}{2} \mathrm{mb} \tilde{v}_{0}
$$

where $b$ is the impact parameter, which is given by

$$
b=\left(b_{z}^{2}+b_{r}^{2}\right)^{1 / 2}=\left(b_{z}^{2}+r_{\perp}^{2} \sin ^{2} \Phi\right)^{1 / 2}
$$

as can be seen from figure 6.1. Conservation of angular momentum in the centre of mass system implies that the relative motion of the particles takes place in a plane perpendicular to the vector $\mathbf{r x v}$, which is the plane through $\mathbf{r}$ and $\mathbf{v}$. This plane is called the orbital plane and is indicated in figure 6.1. From equation (6.2.14) and equation (6.2.17) one sees that the relative coordinates $\mathbf{r}$ and $\mathbf{v}$ obey the same relations as the coordinates of a single particle with reduced mass $\mathrm{m} / 2$ moving under influence of a fixed force centre. The two-particle problem is thus reduced to this single particle problem.

\subsection{Coordinate representation in the orbital plane}

In order to describe the motion in the orbital plane it is convenient to construct an orthonormal reference system in this plane 


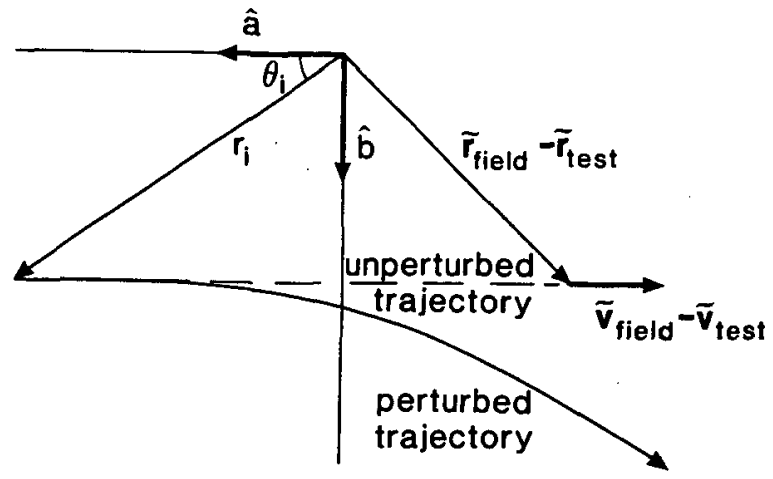

Figure 6.2 The relative perturbed and unperturbed trafectory of the field and the test particle and the orthonormal coordinate system $(\mathbf{a}, \bar{b})$ in the orbital plane.

$$
\hat{\mathbf{a}}=-\frac{\tilde{\mathbf{v}}_{0}}{\left|\tilde{\mathbf{v}}_{0}\right|} \quad, \quad \hat{\mathrm{D}}=\frac{\tilde{\mathbf{r}}_{0}-\left(\tilde{\mathbf{r}}_{0} \cdot \hat{\mathbf{a}}\right) \cdot \hat{\mathbf{a}}}{\left|\tilde{\mathbf{r}}_{0}-\left(\tilde{\mathbf{r}}_{0} \cdot \hat{\mathbf{a}}\right) \cdot \hat{\mathbf{a}}\right|}
$$

The vector $\hat{a}$ is directed opposite to the unperturbed relative velocity and the vector $\hat{b}$ points towards the perihelion (point of closest approach) of the unperturbed trajectory, see figure 6.2. By substitution of equations $(6.2 .6)$ one obtains for the vectors $\hat{a}$ and $\bar{b}$ in terms of the coordinates of the laboratory system

$$
\hat{a}=-\left(\begin{array}{c}
\sin \psi \\
\cos \psi \\
0
\end{array}\right), \quad \delta=\frac{1}{b}\left(\begin{array}{c}
r_{\perp}(\cos \psi-\cos \psi \cos \Phi) \\
r_{\perp}(\sin \psi-\sin \psi \cos \Phi) \\
-b_{z}
\end{array}\right)
$$

with $\Phi=\psi-\varphi$. These equations constitute the coordinate transformation from the $(\hat{a}, \hat{b})$ coordinate system to the laboratory system. We recall that the analysis is restricted to the specific case of an on-axis test particle, which has the same axial velocity as the field particle. For the general case one can still use equations $(6.3 .1)$ to perform the transformation from laboratory system to coordinates in the orbital plane, but the representation of $\tilde{\mathbf{r}}_{0}, \tilde{\mathbf{v}}_{0}, R$ and $\mathbf{V}$ differs from equations $(6.2 .6)$ and $(6.2 .10)$. Accordingly, equations $(6.3 .2)$ become different too. We emphasize that the only modification required to treat the general case concerns the coordinate transformation from the laboratory system to the $(\hat{a}, \hat{b})$ coordinate system in the orbital plane, which is in all cases fully specified by equations $(6.3 .1)$ and $(6.2 .5)$.

The unperturbed relative trajectory can in the $(\hat{a}, \hat{b})$ coordinate system be represented as

$$
\tilde{\mathbf{r}}(t)=\left(a_{0}-\tilde{v}_{0} t\right) \hat{a}+b \hat{b}
$$

where 


$$
\begin{aligned}
& a_{0}=\left(\tilde{r}_{0} \cdot \hat{a}\right)=-r_{\perp} \cos \Phi \\
& b=\left(\tilde{r}_{0} \cdot \hat{b}\right)=\left(b_{2}{ }^{2}+r_{\perp}{ }^{2} \sin ^{2} \Phi\right)^{1 / 2}
\end{aligned}
$$

The impact parameter $b$ was already introduced by equation (6.2.20) and is repeated for convenience. Notice that the unperturbed relative trajectory is (by definition) directed parallel to the a-vector, as is depicted in figure 6.2.

We will use the polar coordinates $r$ and $\theta$ to describe the perturbed relative trajectory in the orbital plane. The initial polar coordinates are given by

$$
\begin{aligned}
& r_{i}=\left[\left(a_{0}-\tilde{v}_{0} t_{i}\right)^{2}+b^{2}\right]^{1 / 2} \\
& \theta_{i} \doteq \frac{1}{2} \pi\left(1+\sigma_{i}\right)-\sigma_{i} \arcsin \left(b / r_{i}\right) \\
& \sigma_{i}=\operatorname{sign}\left(\tilde{v}_{0} t_{i}-a_{0}\right)
\end{aligned}
$$

with $\operatorname{sign}(x)=+1$ for $x \geqslant 0$ and $\operatorname{sign}(x)=-1$ for $x<0$. The sign-parameter $\sigma_{i}$ is negative in case the collision starts before the perihelion and is positive otherwise. The case $\sigma_{i}=-1$ is depicted in figure 6.2.

The dynamical problem now consists of the calculation of the polar coordinates $r$ and $\theta$ as function of time under influence of the mutual Coulomb repulsion. This problem will be considered in the next sections. The remaining part of this section is concerned with the back-transformation to the laboratory system. From the polar coordinates $r(t)$ and $\theta(t)$ and their derivatives $\dot{r}(t)$ and $\dot{\theta}(t)$ at time $t$ one can retrieve the relative position vector $r(t)$ and relative velocity vector $v(t)$ in the laboratory-system by using

$$
\begin{aligned}
& r(t)=a(t) \hat{a}+b(t) \hat{b} \\
& \mathbf{v}(t)=\dot{a}(t) \hat{a}+\dot{b}(t) b
\end{aligned}
$$

where the vectors $\hat{a}$ and $\bar{b}$ are given by equation (6.3.2) and

$$
\begin{aligned}
& \mathbf{a}(t)=r(t) \cos \theta(t) \\
& \mathbf{b}(t)=r(t) \sin \theta(t) \\
& \dot{a}(t)=\dot{r}(t) \cos \theta(t)-r(t) \dot{\theta}(t) \sin \theta(t) \\
& \dot{b}(t)=\dot{r}(t) \sin \theta(t)+r(t) \dot{\theta}(t) \cos \theta(t)
\end{aligned}
$$

The coordinates of the individual particles follow through the centre of mass motion by means of equations (6.2.12).

The displacement from the unperturbed trajectory at time $t$ follows from the equations $(6.3 .6)$ by substracting the unperturbed coordinates given by equation $(6.3 .3)$

$$
\begin{aligned}
& \Delta r(t)=\Delta a(t) \hat{a}+\Delta b(t) \hat{b} \\
& \Delta v(t)=\Delta \dot{a}(t) \hat{a}+\Delta \dot{b}(t) \hat{b}
\end{aligned}
$$

in which 


$$
\begin{aligned}
\Delta a(t) & =a(t)-\left(a_{0}-\tilde{v}_{0} t\right), & \Delta b(t) & =b(t)-b \\
\Delta \dot{a}(t) & =\dot{a}(t)+\tilde{v}_{0}, & \Delta \dot{b}(t) & =\dot{b}(t)
\end{aligned}
$$

The virtual spatial displacement $\Delta \mathbf{r}_{\mathbf{r}}$ in the reference plane follows using

$$
\Delta r_{r}(t)=\Delta r(t)-\left(t-t_{r}\right) \Delta v(t), \quad t_{r}=\left(S_{i}-S_{c}\right) T
$$

see equations $(6.2 .3)$ and $(3.2 .1)$. Finally, one can determine the displacements of the individual particles by means of

$$
\begin{aligned}
\Delta r_{c}(t) & =\frac{1}{2} \Delta r(t), & \Delta v_{c}(t) & =\frac{1}{2} \Delta v(t) \\
\Delta r_{t}(t) & =-\frac{1}{2} \Delta r(t), & \Delta v_{t}(t) & =-\frac{1}{2} \Delta v(t)
\end{aligned}
$$

as follows from equations (6.2.12), using that the centre of mass motion is identical for the perturbed and the unperturbed trajectories.

\subsection{Dynamics of a complete collision}

In terms of the polar coordinates $r$ and $\theta$ equations (6.2.14) and $(6.2 .17)$ become

$$
\begin{aligned}
& E=\frac{1}{4} m\left(\dot{r}^{2}+r^{2} \dot{\theta}^{2}\right)+\frac{C_{0}}{r} \\
& J=\frac{1}{2} m r^{2} \dot{\theta}
\end{aligned}
$$

The quantities $\mathrm{E}$ and $\mathrm{J}$ are constants of motion. Their values are specified by the equations $(6.2 .15)$ and $(6.2 .19)$. The relative motion in the orbital plane has four degrees of freedom. Accordingly, it is fully determined by the values of $E, J$ and the initial polar coordinates $r_{i}$ and $\theta_{i}$. The initial polar coordinates are specified by the equations $(6.3 .5)$.

The equations $(6.4 .1)$ and $(6.4 .2)$ can be simplified by introducing the micro-scaled variables

$$
\rho=r / d_{s}, \quad \tau=t / t_{s}
$$

where the micro-scale distance $d_{s}$ and the micro-scale time $t_{s}$ are defined as

$$
\mathrm{d}_{\mathrm{s}}=\mathrm{J} / \sqrt{\mathrm{mE}}, \quad \mathrm{t}_{\mathrm{s}}=\mathrm{J} / 2 \mathrm{E}
$$

Employing this scaling equations $(6.4 .1)$ and (6.4.2) transform to

$$
\begin{aligned}
& 1=\left(\frac{d \rho}{d \tau}\right)^{2}+\rho^{2}\left(\frac{d \theta}{d \tau}\right)^{2}+\frac{2}{\rho-\sqrt{q}} \\
& 1=\rho^{2}\left(\frac{d \theta}{d \tau}\right)
\end{aligned}
$$

where $q$ is defined as 


$$
q=\frac{4 J^{2} E}{C_{0}^{2} m}
$$

Elimination of the differential $d \tau$ from equations $(6.4 .5)$ and $(6.4 .6)$ yields

$$
d \theta=\frac{d \rho}{\rho\left(\rho^{2}-2 \rho / \sqrt{9}-1\right)^{1 / 2}}
$$

which is a non-linear first order differential equation in $\rho$ and $\theta$. Integration yields

$$
\rho=\frac{\sqrt{q}}{\epsilon \cos \left(\theta-\theta_{p}\right)-1} \quad, \quad \varepsilon=(1+q)^{1 / 2}
$$

The parameter $E$ is known as the eccentricity. The integration constant $\theta_{p}$ corresponds to the perihelion angle. By removing the scaling in equations $(6.4 .9)$ by means of equations $(6.4 .3)$ and $(6.4 .4)$ one finds

$$
r=\frac{r_{p}(\varepsilon-1)}{\varepsilon \cos \left(\theta-\theta_{p}\right)-1}
$$

where $r_{p}$ is the perihelion distance

$$
r_{p}=r\left(\theta_{p}\right)=\frac{\mathrm{J}}{\sqrt{\mathrm{mE}}} \sqrt{\varepsilon+1}
$$

Equation (6.4.10) describes the relative trajectory of two identical charged particles interacting through the coulomb force. It is the same as the trajectory-equation of a single particle with reduced mass $\mathrm{m} / 2$ moving under the influence of a fixed force centre. The trajectoryequation (6.4.10) represents a hyperbolic orbit, as is depicted in figure 6.3. The orbit is symmetrical with respect to the line through the origin and the perihelion, called apse-line.

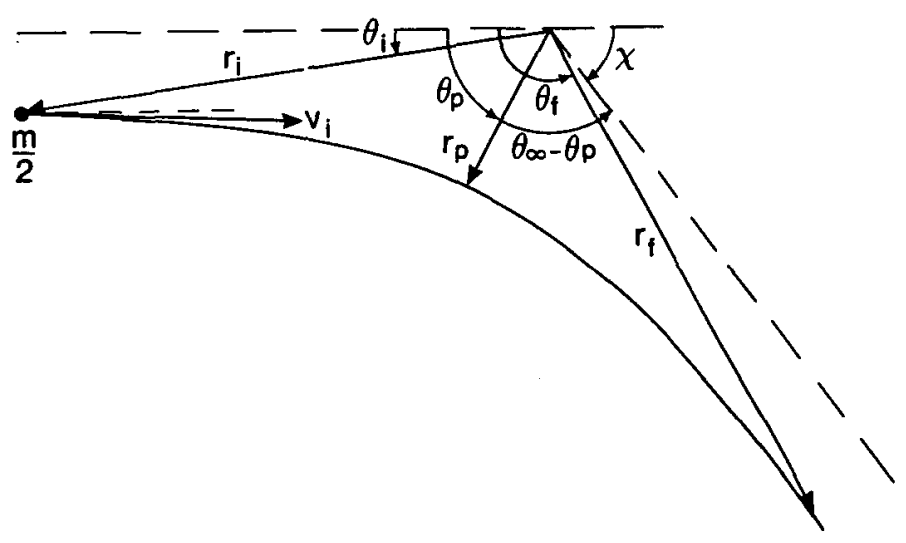

Figure 6.3 The trajectory of a particle with reduced mass $\mathrm{m} / 2$, moving under influence of a fixed force centre. The polar coordinates with the subscripts $i, p$ and $f$ refer to the initial, the perihelion and the final situation respectively. The initial and final directional asymptotes correspond to the polar angles $\theta_{-\infty}(=0)$ and $\theta_{\infty}$ respectively. 
The asymptotes of the orbit can be found from equation $(6.4 .10)$ by taking $r+\infty$. The polar angles of the initial and $f$ inal asymptote are denoted as $\theta_{-\infty}$ and $\theta_{\infty}$ respectively. By definition $\theta_{-\infty}=0$, while $\theta_{\infty}$ follows from

$$
1 / \varepsilon=\cos \left(\theta_{\infty}-\theta_{p}\right)=\cos \left(\theta_{\infty} / 2\right)
$$

where the symmetry of the trajectory with respect to the apse-line was used. Accordingly, one finds

$$
\begin{aligned}
& \cos \left(\theta_{\infty}\right)=2 \cos ^{2}\left(\theta_{\infty} / 2\right)-1=\frac{1-q}{1+q} \\
& \sin \left(\theta_{\infty}\right)=\left[1-\cos ^{2}\left(\theta_{\infty}\right)\right]^{1 / 2}=\frac{2 \sqrt{q}}{1+q}
\end{aligned}
$$

while the deflection angle $\chi$ can be expressed as $\left(\chi=\pi-\theta_{\infty}\right)$

$$
\tan \left(\frac{x}{2}\right)=\tan \left(\frac{\pi-\theta_{\infty}}{2}\right)=\left(\frac{\cos ^{2}\left(\theta_{\infty} / 2\right)}{1-\cos ^{2}\left(\theta_{\infty} / 2\right)}\right)^{1 / 2}=\frac{1}{\sqrt{4}}
$$

By substitution of equations $(6.2 .15)$ and $(6.2 .19)$ into equation (6.4.7) one obtains for the parameter $q$

$$
q=\left(\frac{m b v_{i}^{2}}{2 C_{0}}\right)^{2}\left(1+\frac{4 C_{0}}{m v_{i}^{2} r_{i}}\right)
$$

For the asymptotic condition $r_{i}+\infty$ this results in

$$
q=q_{c}=\left(\frac{m b v_{i}^{2}}{2 c_{0}}\right)^{2}
$$

Substitution into equation (6.4.14) yields the well known expression for the deflection angle of a complete collision

$$
\tan \left(\frac{x}{2}\right)=\frac{b_{\perp}}{b}, \quad b_{\perp}=\frac{2 c_{0}}{m v_{i}^{2}}
$$

The initial and final state of a complete collision approach the asymptotic conditions. Accordingly, the initial and final energy are both entirely kinetic and conservation of energy implies that the magnitude of the relative velocity before and after the collision is the same. Thus the dynamical effect of the encounter is a directional change of the relative velocity which is fully specified by the deflection angle $x$.

Equation (6.4.17) expresses the deflection angle $\chi$ as function of the impact parameter $b$ and the initial relative velocity $v_{i}$. As an alternative one often describes the effect of two-particle collisions in terms of a differential cross section $\sigma\left(x, v_{i}\right)$, which is the ratio of the flux of (test) particles scattered into a solid angle $2 \pi \sin (\chi) \mathrm{d} \chi$ and the incoming flux per unit area, see for instance Gryzinski $(1964 a, b, c)$. From equation (6.4.17) one obtains for the corresponding differential cross section

$$
\sigma\left(\chi, v_{i}\right)=\frac{2 \pi b d b}{2 \pi \sin (x) d x}=\left(\frac{C_{0}}{m v_{i}^{2}}\right)^{2} \frac{1}{\sin ^{4}(x / 2)}
$$

which is known as Rutherfords scattering law. We will not use the concept of the differential cross section in the further analysis. Instead we will express the displacements of the test particle directly in terms of the geometrical parameters. 
Let us now consider the general problem of a collision starting with an arbitrary (not necessary large) separation $r_{i}$ and relative velocity $v_{i}$, which takes place over some finite time $T$. Neither the initial nor the final situation necessarily approach the asymptotic state and the collision is in general incomplete. Accordingly, it is not sufficient to determine the asymptotic deflection angle $x$ only. In fact, one needs to know each of the polar coordinates $r$ and $\theta$, as well as their derivatives $\dot{r}$ and $\dot{\theta}$, explicitly as function of time $t$.

obtains

By eliminating $d \theta / d T$ from equations $(6.4 .5)$ and $(6.4 .6)$ one

$$
\frac{d \rho}{d \tau}=\frac{H(\rho)}{\rho} \operatorname{sign}\left(\tau-\tau_{p}\right)
$$

with $\tau_{p}$ the (scaled) time of perihelion passage. The function $H(\rho)$ is defined as

$$
H(\rho)=\left(\rho^{2}-\frac{2 \rho}{\sqrt{9}}-1\right)^{1 / 2}
$$

Integration of equation $(6.5 .1)$ yields

$$
\left|\tau-\tau_{p}\right|=T(\rho)=H(\rho)+\frac{1}{\sqrt{7}} \ln \left[\sqrt{\frac{q}{q+1}}\left(H(\rho)+\rho-\frac{1}{\sqrt{7}}\right)\right]
$$

This equation specifies the time $\tau$ relative to the time of perihelion passage $T_{p}$ as function of the scaled separation of the particles $\rho$. However, actually one requires $\rho$ as function of time. Therefore, one has to evaluate the inverse function of $T(\rho)$, denoted as $\rho(|\tau-\tau p|)$. Unfortunately, this inversion can not be performed analytically without introducing approximations. This problem will be discussed in detail in the next sections. For the moment it is assumed that one can indeed evaluate the function $\rho\left(\left|\tau-\tau_{p}\right|\right)$, either by numerical inversion or using some analytical approximation.

The calculation now proceeds as follows. The initial value $\rho_{i}$, obtained from equations $(6.3 .5)$ and $(6.4 .3)$, specifies the initial (scaled) time $\tau_{i}$ relative to the time of perihelion passage $\tau_{p}$

$$
\tau_{i}-\tau_{p}=\sigma_{i} T\left(\rho_{i}\right)
$$

in which the sign-parameter $\sigma_{i}$ is defined by the last equation of (6.3.5). The final time $\tau_{f}$ relative to $\tau_{p}$ follows by adding the total scaled time of flight $\tau_{T}$

$$
\tau_{t}-\tau_{p}=\tau_{i}-\tau_{p}+\tau_{T}=\sigma_{i} T\left(\rho_{i}\right)+T_{T}, \quad \tau_{T}=T / t_{S}
$$

The final value $\rho_{f}$ is now determined by evaluating $\rho\left(\left|\tau_{f}-\tau_{p}\right|\right)$, for which one requires the analysis of the next sections. In order to take care of the signs we use the auxiliary sign-parameters $\sigma_{i}$ and $\sigma_{\mathrm{f}}$, defined as

$$
\begin{aligned}
& \sigma_{i}=\operatorname{sign}\left(-r_{i} \cdot \hat{a}\right)=\operatorname{sign}\left(\tilde{v}_{0} t_{i}-a_{0}\right) \\
& \sigma_{f}=\operatorname{sign}\left(\sigma_{i} T\left(\rho_{i}\right)+\tau_{T}\right)
\end{aligned}
$$




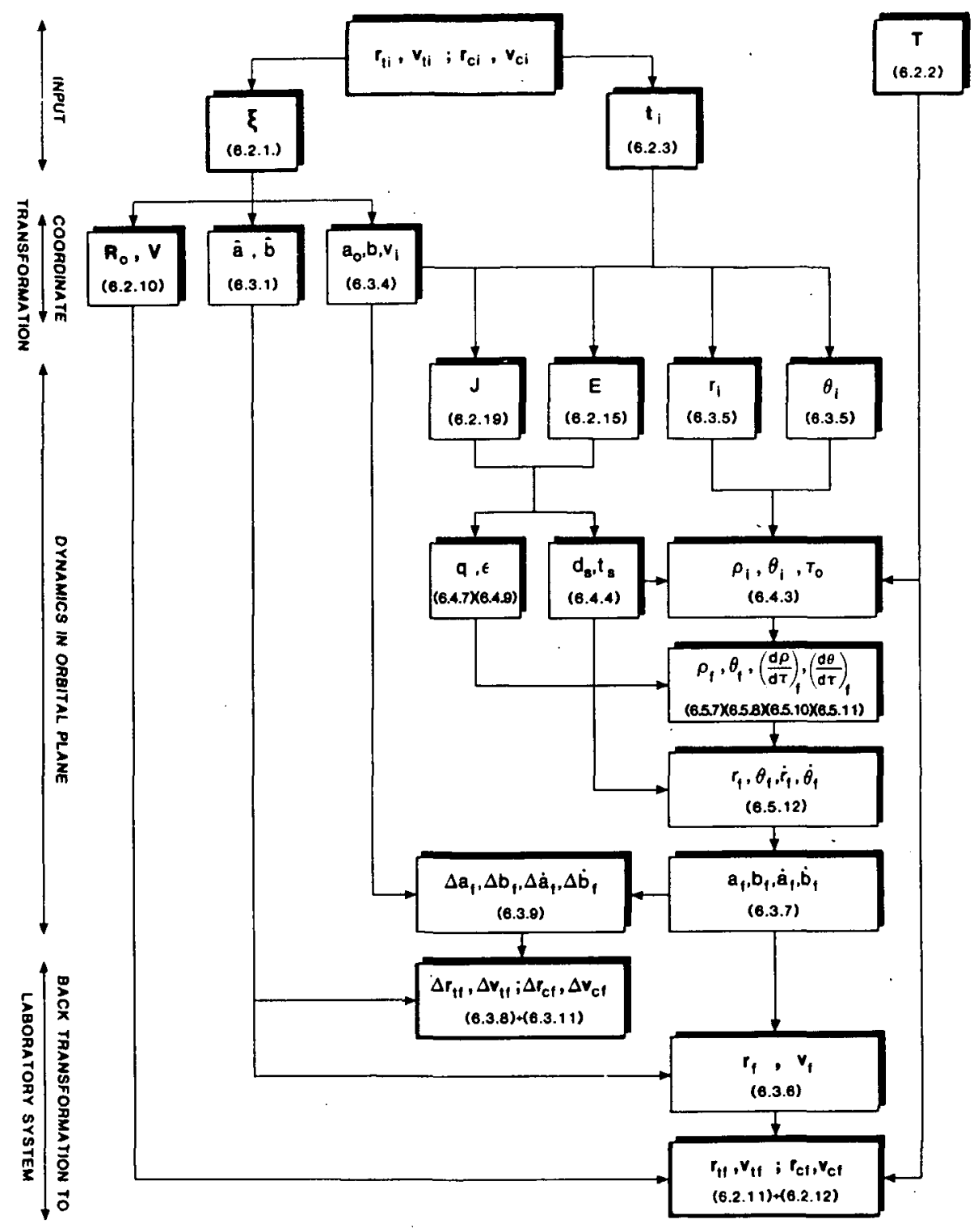

Pigure 6.4 Flow-diagram for the calculation of the final coordinates of the test and the field particle from their initial coordinates and the time of flight $T$. The equation numbers in the boxes refer to the steps in the calculation which yield the quantity (or quantities) indicated in the same box. 
(for convenience we repeated the definition of $\sigma_{i}$, presented in equation $(6.3 .5))$. The following cases are now distinguished:

$\sigma_{1}=-1, \sigma_{f}=+1$ : Collision starts before the perihelion and ends after it $\left(\tau_{i}-\tau_{p}<0\right.$ and $\left.\tau_{f}-\tau_{p}>0\right)$

$\sigma_{i}=-1, \sigma_{f}=-1$ : Collision starts before the perihelion and ends before it $\left(\tau_{i}-\tau_{p}<0\right.$ and $\left.\tau_{f}-\tau_{p}<0\right)$

$\sigma_{i}=+1, \sigma_{f}=+1$ : Collision starts after the perihelion and ends after it $\left(\tau_{i}-\tau_{p}>0\right.$ and $\left.\tau_{f}-\tau_{p}>0\right)$

The final value $\rho_{f}$ is in all cases given by

$$
\rho_{\mathrm{f}}=\rho\left[\sigma_{\mathrm{f}}\left(\sigma_{\mathrm{i}} T\left(\rho_{\mathrm{i}}\right)+\tau_{\mathrm{T}}\right)\right]=\rho\left[\left|\sigma_{\mathrm{i}} T\left(\rho_{\mathrm{i}}\right)+\tau_{\mathrm{T}}\right|\right]
$$

The corresponding polar angle $\theta_{f}$ follows by means of the ray equation $(6.4 .9)$ and can be expressed as

$$
\theta_{f}=\theta_{i}-\sigma_{i} A\left(\rho_{i}\right)+\sigma_{f} A\left(\rho_{f}\right)
$$

where the function $A(x)$ is defined as

$$
A(x)=\arccos [(1+\sqrt{\mathbb{q}} / x) / \varepsilon]
$$

The remaining quantities to be determined are the final values of the derivatives of the polar coordinates $\rho$ and $\theta$ with respect to time $T$. By means of equation (6.5.1) one obtains

$$
\left(\frac{d \rho}{d \tau}\right)_{f}=\frac{\sigma_{f} H\left(\rho_{f}\right)}{\rho_{f}}
$$

in which the function $H(\rho)$ is given by equation (6.5.2). The derivative of the polar angle, follows from the conservation of angular momentum, expressed by equation $(6.4 .6)$

$$
\left(\frac{d \theta}{d \tau}\right)_{f}=\frac{1}{\rho_{\mathrm{f}}^{2}}
$$

Finally, the unscaled polar coordinates at $t_{f}$ are retrieved as

$$
r_{f}=d_{s} \rho_{f}, \quad \dot{r}_{f}=\frac{d_{s}}{t_{s}}\left(\frac{d \rho}{d \tau}\right)_{f}, \quad \dot{\theta}_{f}=\frac{1}{t_{s}}\left(\frac{d \theta}{d \tau}\right)_{f}
$$

in which the scale-parameters $d_{s}$ and $t_{s}$ are defined by equation $(6.4 .4)$. Notice that the (unscaled) angle $\theta_{f}$ is directly given by equation (6.5.8). This completes the calculation of the dynamics in the orbital plane.

Next, the final polar coordinates $r_{f}, \dot{r}_{f}, \theta_{f}$ and $\dot{\theta}_{f}$. in the orbital plane have to be transformed to coordinates in the laboratory system. This is done by performing the following steps. The polar coordinates are transformed to the rectangular coordinates of the $(\hat{a}, \hat{b})$ system in the orbital plane by means of equation (6.3.7). The final relative position $r_{f}$ and velocity $\nabla_{f}$ in terms of the coordinates of the laboratory system now follow with equation (6.3.6). The final coordinates of the individual particles can then be determined through the centre of mass motion, using equations $(6.2 .11)$ and $(6.2 .12)$. The complete calculation scheme is summarized by the flow-diagram of figure 6.4 .

The calculation outlined above can be carried out analytically apart from one step, namely the evaluation of the polar coordinate $r$ as 
tunction of time, which is implicitly given by equation (6.5.7). We will consider three alternative methods to solve this problem. In the first method the inversion is performed numerically. Section 6.6 is concerned with an algorithm which serves this purpose. In the second method, the inverted function is approximated by expanding equation (6.5.3) in powers of $\rho$. This approach is valid when both $\rho_{i}$ and $\rho_{f}$ are large compared to unity, which is the case for complete or nearly complete collisions. This approximation is studied in section 6.7. The third method is based on first order perturbation theory, which constitutes a valid approximation for weak interactions. It is described in section 6.8. Special care has to be taken to deal with the case $v_{1}=0$, which means that the particles are initially at rest in the centre of mass system. This problem will be considered separately in section 6.9.

\subsection{Numerical approach to the dynamical problem}

Given the value of time $T=\left|T-T_{p}\right|$ one has to determine the value of $\rho$ which fulfils (reproducing equation (6.5.3))

$$
T=T(\rho)=\mathrm{H}(\rho)+\frac{1}{\sqrt{\mathrm{q}}} \ln \left[\sqrt{\mathrm{q}+1}\left(\mathrm{H}(\rho)+\rho-\frac{1}{\sqrt{\mathrm{q}}}\right)\right]
$$

in which $H(\rho)$ is given by equation $(6.5 .2)$. For $T=0$ the solution is $\rho=\rho_{p}$, where

$$
\rho_{p}=\frac{1}{\sqrt{q}}\left[1+(1+q)^{1 / 2}\right]
$$

the scaled perihelion distance. One easily verifies that $T\left(\rho_{p}\right)=0$ from the fact that $H\left(\rho_{p}\right)=0$, while the argument of the logarithm in equation (6.6.1) becomes equal to 1 . For $\rho>\rho_{p}$ the value of $T(\rho)$ increases monotonically with $\rho$.

As a first approximation we ignore the logarithm in equation (6.6.1) and solve the equation $T=\mathrm{H}\left(\rho_{1}\right)$. This gives

$$
\rho_{1}=\frac{1}{\sqrt{9}}\left(1+\left[1+q\left(1+T^{2}\right)\right]^{1 / 2}\right)
$$

It provides the exact solution for $T=0$ but overestimates $\rho$ for $T>0$. Starting with $\rho=\rho_{1}$ one can determine the exact solution of equation (6.6.1) numerically, using the iteration process

$$
\rho_{k+1}=\rho_{k}+\frac{T-T\left(\rho_{k}\right)}{[d T / d \rho]_{\rho_{k}}}
$$

which is known as the Newton-Raphson method, for instance see Pipes and Harvill (1983). Using $d T / d \rho=\rho / H(\rho)$ one obtains

$$
\rho_{k+1}=\rho_{k}+\frac{H\left(\rho_{k}\right)}{\rho_{k}}\left[T-T\left(\rho_{k}\right)\right]
$$

This process is convergent for any start value $\rho>\rho_{p}$. A good start value is provided by equation $(6.6 .3)$. 


\subsection{Dynamics of a nearly complete collision}

In this section we will determine the inverse function of $T(\rho)$, denoted as $\rho(T)$, by expanding equation $(6.5 .3)$ into powers of $\rho$. Using the series representations

$$
\begin{aligned}
& (1+\delta) 1 / 2=1+\frac{1}{2} \delta-\frac{1}{8} \delta^{2}+\frac{1}{16} \delta^{3}+0\left(\delta^{4}\right) \\
& \ln (1+\delta)=\delta-\frac{1}{2} \delta^{2}+0\left(\delta^{3}\right)
\end{aligned}
$$

(for $\delta<1$ ) one obtains with equation (6.5.2)

$$
\begin{aligned}
& H(\rho)=\rho-\frac{1}{\sqrt{q}}-\frac{q+1}{2 q} \rho-1-\frac{q+1}{2 q^{3 / 2}} \rho^{-2}+0(\rho-3) \\
& \ln \left[\sqrt{\frac{q}{q+1}}\left(H(\rho)+\rho-\frac{1}{\sqrt{q}}\right)\right]=\ln \left[\sqrt{\frac{q}{q+1}} 2 \rho\right]-\frac{1}{\sqrt{q}} \rho-1-\frac{q+3}{4 q} \rho-2+0(\rho-3)
\end{aligned}
$$

Accordingly, equation $(6.5 .3)$ can be written as

$$
T(\rho)=\rho+\frac{1}{\sqrt{q}}\left[\ln \left(\sqrt{\frac{q}{q+1}} 2 \rho\right)-1\right]-\frac{q+3}{2 q} \rho^{-1}-\frac{3 q+5}{4 q^{3 / 2}} \rho^{-2}+0(\rho-3)
$$

The successive terms will decrease in magnitude provided that $\rho \gg 1$ and $q$

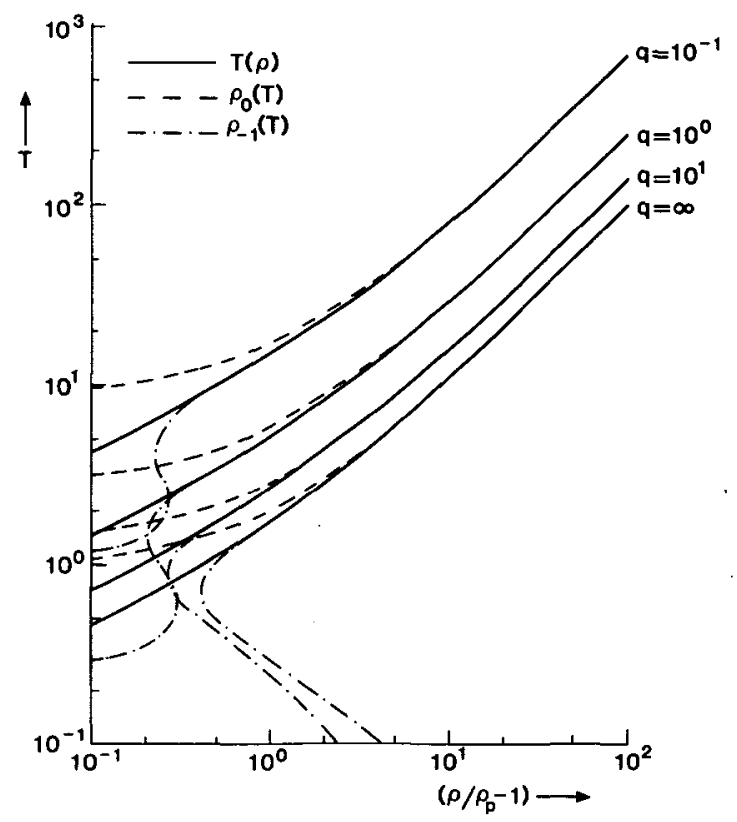

Pigure 6.5 The function $T(\rho)$, defined by equation (6.6.1), and the approximations for its inverse function $\rho(T)$ which are used for nearly complete collisions ( $T \gg>1)$. The approximating functions $\rho_{0}(T)$ and $\rho_{-1}(T)$ are accurate up to and including terms of the order $T^{0}$ and $T^{-1}$ respectively and are obtained from equation (6.7.4). 
are finite and non-zero. The inverse function of $T(\rho)$ can now be approximated as

$$
\rho(T)=T-\frac{1}{\sqrt{q}}\left[1-\frac{1}{\sqrt{q}} T^{-1}\right]\left[\ln \left(\sqrt{\frac{q}{q+1}} 2 T\right)-1\right]+\frac{q+3}{2 q} T^{-1}+0\left(\frac{\ln T}{T^{2}}\right)(6.7 .4)
$$

Figure 6.5 compares the exact relation between $\rho$ and $T$, given by equation (6.5.3) with the approximation of equation (6.7.4). It also shows the zero-order approximation obtained by ignoring the terms in equation (6.7.4) proportional to $T^{-1}$. The figure shows that equation $(6.7 .4)$ yields accurate results for $\rho / \rho_{p}-1 \gtrless 0.5$, thus $\rho \gtrsim 1.5 \rho_{p}$.

The calculation which was outlined in section 6.5 can now be performed analytically. We have done so for the case $\sigma_{i}=-1$ and $\sigma_{f}=+1$ (interaction starts before the perihelion and ends after it), as is described in some detail in appendix 6.A. In general terms the calculation proceeds as follows. The final scaled polar coordinates $\rho_{f}$, $\theta_{f}$ and their derivatives $[\mathrm{d} \rho / \mathrm{d} \tau]_{f}$ and $[\mathrm{d} \theta / \tau]_{\mathrm{f}}$ are determined as function of the initial scaled polar coordinates $\rho_{i}$ and $\theta_{i}$ and the scaled time of flight $\tau_{T}$. By substitution of these expressions into equations $(6.3 .7)$, removing the scaling defined by equations $(6.4 .3)$ and $(6.4 .4)$, one obtains the final values for the coordinates $a, b$ and their derivatives $\dot{a}$ and $b$. The deviations from the unperturbed trajectories, represented by $\Delta \mathrm{a}, \Delta \mathrm{b}, \Delta \dot{\mathrm{a}}$ and $\Delta \dot{\mathrm{b}}$, then follow with equations $(6.3 .9)$. This way one eventually finds (see appendix 6.A)

$$
\begin{aligned}
& \Delta a_{f} \cong \frac{2\left(T v_{i}-r_{i}\right)}{1+q_{c}}-\frac{1-q_{c}}{1+q_{c}}\left[2 h \Lambda_{c}-h \frac{T v_{i}}{r_{i}}\right]+\frac{2 \sqrt{q_{c}}}{1+q_{c}}\left[b-\frac{2 b}{1+q_{c}}\left(\frac{T v_{i}}{r_{i}}-1\right)\right] \\
& \Delta b_{f} \cong \frac{2 \sqrt{q_{c}}}{1+q_{c}}\left[T v_{i}-r_{i}-2 h \Lambda_{c}+\frac{2 h}{1+q_{c}}\left(\frac{T v_{i}}{r_{i}}-1\right)\right] \\
& \Delta \dot{a}_{f} \cong \frac{2 v_{i}}{1+q_{c}}+\frac{1-q_{c}}{1+q_{c}}\left[\frac{h v_{i}}{r_{i}}-\frac{h v_{i}}{T v_{i}-r_{i}}\right]-\frac{4 q_{c}}{\left(1+q_{c}\right)^{2}} \frac{h v_{i}}{r_{i}} \\
& \Delta \dot{b}_{f} \cong \frac{2 \sqrt{q_{c}}}{1+q_{c}}\left[v_{i}+\frac{h v_{i}}{r_{i}} \frac{2}{1+q_{c}}-\frac{h v_{i}}{T v_{i}-r_{i}}\right]
\end{aligned}
$$

in which $\Lambda_{c}$ and $h$ are defined as

$$
\Lambda_{c}=\ln \left(\sqrt{\frac{q_{c}}{q_{c}+1}} \frac{2}{b} \sqrt{r_{i}\left(T v_{i}-r_{i}\right)}\right)-1, \quad h=\frac{2 C_{0}}{m_{i}^{2}}
$$

and $q_{c}$ is given by equation $(6.4 .16)$ (note $\sqrt{q_{c}}=b / h$ ). The predictions of equations $(6.7 .5)$ were compared with the exact results obtained by numerical calculation, using the method outlined in section 6.6. It was found that equations $(6.7 .5)$ indeed yield correct results provided that

$$
r_{i} \gg b \text { and } T v_{i}-r_{i} \gg b \text { and } h / r_{i} \ll 1
$$

The collisions for which these conditions are fulfilled are called nearly complete collisions.

The equations (6.7.5) are valid for arbitrary values of $q_{c}$ $\left(q_{c} \geqslant 0\right)$, and thus apply to both strong and weak collisions (as long as they are nearly complete). The case $q_{c}=0$ corresponds to a central collisions $(b=0)$, while weak interactions are found for $\sqrt{q_{c}}>1$, as can be seen from equation $(6.4 .14)$. Let us investigate both cases. For $\sqrt{q_{c}} \gg 1$ equations $(6.7 .5)$ transform to 


$$
\begin{aligned}
& \Delta a_{f} \cong h\left[\ln \left(\frac{4 r_{i}\left(T v_{i}-r_{i}\right)}{b^{2}}\right)-\frac{T v_{i}}{r_{i}}\right] \\
& \Delta b_{f} \cong \frac{2 h\left(T v_{i}-r_{i}\right)}{b}\left\{1-\frac{2 h}{T v_{i}-r_{i}}\left[\ln \left(\frac{4 r_{i}\left(T v_{i}-r_{i}\right)}{b^{2}}\right)-1\right]\right\} \cong \frac{2 h\left(T v_{i}-r_{i}\right)}{b} \\
& \Delta \dot{a}_{f} \cong h v_{i}\left(\frac{1}{T v_{i}-r_{i}}-\frac{1}{r_{i}}\right) \\
& \Delta \dot{b}_{f} \cong \frac{2 h v_{i}}{b}\left(1-\frac{h}{T v_{i}-r_{i}}\right) \cong \frac{2 h v_{i}}{b}
\end{aligned}
$$

These equations apply to weak nearly complete collisions. $(6.7 .5)$

For a central collision $\left(q_{c}=0\right)$ one obtains from equations

$$
\begin{aligned}
& \Delta a_{f} \cong 2\left(T v_{i}-r_{i}\right)-h\left[\ln \left(\frac{4 r_{i}\left(T v_{i}-r_{i}\right)}{h^{2}}\right)-2-\frac{T v_{i}}{r_{i}}\right] \\
& \Delta \dot{a}_{f} \cong 2 v_{i}+h v_{i}\left(\frac{1}{r_{i}}-\frac{1}{T v_{i}-r_{i}}\right) \\
& \Delta b_{f}=\Delta \dot{b}_{f}=0
\end{aligned}
$$

The motion is one-dimensional and takes place in the â-direction. Notice that the expression for $\Delta \dot{a}_{f}$ also follows directly from conservation of energy.

The coordinates $\Delta a_{f}, \Delta b_{f}, \Delta \dot{a}_{f}$ and $\Delta \dot{b}_{f}$ refer to the condition at time $t_{\mathrm{f}}$, that is the time that the field particle leaves the beam segment. In order to determine the virtual spatial displacement in the reference plane ..one has to extrapolate the final coordinates over the time interval $T_{1}=t_{f}-t_{r}$, as is expressed by equations $(6.3 .10)$. One can perform this extrapolation for the coordinates in the orbital plane, using

$$
\Delta a_{r}=\Delta a_{f}-T_{1} \Delta \dot{a}_{f}, \quad \Delta b_{r}=\Delta b_{f}-T_{1} \Delta \dot{b}_{f}
$$

Substitution of equations $(6.7 .5)$ yields

$$
\begin{aligned}
\Delta a_{r} \cong & 2 \frac{\left(T-T_{1}\right) v_{i}-r_{i}}{1+q_{c}}-h \frac{1-q_{c}}{1+q_{c}}\left[2 \Lambda_{c}-\frac{\left(T-T_{1}\right) v_{i}}{r_{i}}-\frac{v_{i} T_{1}}{T v_{i}-r_{i}}\right] \\
& +\frac{2 q_{c} h}{1+q_{c}}\left[1-\frac{2}{1+q_{c}}\left[\frac{\left(T-T_{1}\right) v_{i}}{r_{i}}-1\right)\right] \\
\Delta b_{r} \cong & \frac{2 \sqrt{q_{c}}}{1+q_{c}}\left[\left(T-T_{1}\right) v_{i}-r_{i}-2 h \Lambda_{c}+\frac{2 h}{1+q_{c}}\left(\frac{\left(T-T_{1}\right) v_{i}}{r_{i}}-1\right)+h \frac{T_{1} v_{i}}{T v_{i}-r_{i}}\right]
\end{aligned}
$$

These equations specify the virtual spatial displacement in an arbitrary reference plane (defined by $T_{1}$ ), which is required for the calculation of the trajectory displacement effect.

The equations $(6.7 .5)$ and $(6.7 .11)$ constitute the main results of this section. It should be emphasized that they pertain to a nearly complete collision, which implies that the conditions expressed by equations $(6.7 .7)$ should be fulfilled. We will conclude this section by bringing the results in an alternative form, which is sometimes more convenient. Equations $(6.7 .5)$ and $(6.7 .11)$ contain the initial distance 
$\mathbf{r}_{\mathbf{i}}$, which is related to the geometrical parameters through the first equation of $(6.3 .5)$. In order to make the dependency on the geometrical parameters explicit we now approximate $r_{i}$ and its reciprocal value as

$$
\begin{aligned}
& r_{i}=a_{0}-v_{i} t_{i}+\frac{b^{2}}{2\left(a_{0}-v_{i} t_{i}\right)}+\cdots \approx a_{0}-v_{i} t_{i} \\
& \frac{1}{r_{i}}=\frac{-1}{v_{i} t_{i}}+\frac{a_{0}}{\left(v_{i} t_{i}\right)^{2}}+\cdots \approx \frac{-1}{v_{i} t_{i}}
\end{aligned}
$$

These approximations rely on the conditions expressed by equations (6.7.7). The approximation used in the second equation of (6.7.12) requires in addition that $a_{0}<<v_{i} t_{i}$, which implies that the $x, y$-plane of the laboratory system should be located somewhere near the middle of the beam segment (thus $S_{c} \approx 1 / 2$ ). The quantities $t_{i}$ and $T_{1}=t_{f}-t_{r}$ can be expressed in terms of the parameters $S_{c}$ and $S_{i}$ and the time of flight $T$, see equations $(6.2 .3)$ and $(6.3 .10)$. Furthermore, $v_{i}$ is equal to the geometrical parameter $v$. Accordingly, $\Delta \mathrm{a}_{r}$ and $\Delta \mathrm{b}_{r}$, given by equation $(6.7 .11)$ and the derivatives $\Delta \dot{a}_{f}$ and $\Delta \dot{b}_{f}$, given by equations $(6.7 .5)$, can be expressed as

$$
\begin{aligned}
& \Delta a_{r} \cong 2 \frac{\left(S_{i}-S_{c}\right) T v-a_{0}}{1+q_{c}}-h \frac{1-q_{c}}{1+q_{c}}\left[2 \Lambda_{c}-\frac{S_{i}}{S_{c}}-\frac{1-S i}{1-S_{c}}\right]+h \frac{2 q_{c}}{1+q_{c}}\left[1-\frac{2}{1+q_{c}}\left(\frac{S_{i}}{S_{c}}-1\right)\right] \\
& \Delta b_{r} \cong \frac{2 \sqrt{q_{c}}}{1+q_{c}}\left[\left(S_{i}-S_{c}\right) T v-a_{0}-2 h \Lambda_{c}+\frac{2 h}{1+q_{c}}\left(\frac{S_{i}}{S_{c}}-1\right)+h \frac{1-S_{i}}{1-S_{c}}\right] \\
& \Delta \dot{a}_{f} \cong \frac{2 v}{1+q_{c}}+\frac{1-q_{c}}{1+q_{c}} \frac{h}{T}\left(\frac{1}{S_{c}}-\frac{1}{1-S_{c}}\right)-\frac{4 q_{c}}{\left(1+q_{c}\right)^{2}} \frac{h}{S_{c} T} \\
& \Delta \dot{b}_{f} \cong \frac{2 \sqrt{q_{c}}}{1+q_{c}}\left[v+\frac{h}{T}\left(\frac{2}{S_{c}\left(1+q_{c}\right)}-\frac{1}{1-S_{c}}\right)\right]
\end{aligned}
$$

where $\Lambda_{c}$, which is defined by equation $(6.7 .6)$, is now expressed as

$$
\Lambda_{c}=\ln \left(\sqrt{\frac{q_{c}}{q_{c}+1}} \frac{2 v T}{b} \sqrt{S_{c}\left(1-S_{c}\right)}\right)-1
$$

Equations (6.7.13) specify the deviation from the unperturbed trajectories as function of the geometrical parameters $\xi=\left(r_{1}, \varphi, b_{2}, v, \psi\right)$, the time of flight $T$, and the parameters $S_{c}$ and $S_{i}$. The quantities ao, $q_{c}$ and $h$ are determined by $\xi$ through equations $(6.3 .4),(6.4 .16)$ and $(6.7 .6)$ respectively. Note that the expressions for $\Delta a_{r}$ and $\Delta b_{r}$ diverge logarithmically (through $\Lambda_{c}$ ) for $T+\infty$, while the derivatives $\Delta \dot{a}_{f}$ and $\Delta \dot{b}_{f}$, are finite for $T \rightarrow \infty$. The values of $\Delta \dot{a}_{f}$ and $\Delta \dot{b}_{f}$ for $T \rightarrow \infty$ are directly related to the value of the asymptotic polar angle $\theta_{\infty}$, as can be seen from equations $(6.4 .13)$.

\subsection{First order perturbation dymamics}

Another method to solve the two-particle dynamical problem by analytical means is given by the so-called first order perturbation approximation, which is valid for weak collisions. This approach was discussed previously in section 5.3, were we considered the $\mathrm{N}$-particle dynamical problem from a general perspective. A two-particle collision is called weak if either the eccentricity $\epsilon$ is large or the perihelion 
is already passed at the start of the collision or the perihelion is not reached during the flight. In terms of the parameters defined in the previous sections these conditions can be expressed as

$$
\epsilon=\sqrt{q+1} \gg 1
$$

or

or

$$
\sigma_{i}=+1 \text { and } r_{i} \gg b
$$

$$
\sigma_{f}=-1 \text { and } \cdot r_{i}-T v_{i} \gg b
$$

The deflection from the unperturbed trajectory will be small in each of these cases. Accordingly, one may approximate the real force acting on the particles by the force which would act when the particles would follow their unperturbed trajectories. This force can be determined using

$$
\tilde{P}(t)=\frac{C_{0}\left(v_{i} t-a_{0}\right)}{\tilde{r}(t)^{3}} \hat{a}-\frac{C_{0} b}{\tilde{r}(t)^{3}} \delta
$$

in which the unperturbed distance between the particles $\tilde{r}(t)$ is given by equation (6.3.3). In first order perturbation approximation one computes the displacements in position and velocity experienced by the test particle as

$$
\begin{aligned}
& \Delta \nabla_{t f}=\int_{t_{i}}^{t_{f}} d t \frac{\tilde{F}(t)}{m} \\
& \Delta r_{t f}=\int_{t_{i}}^{t_{f}} d s \cdot \int_{t_{i}}^{s} d t \frac{\tilde{F}(t)}{m}=\int_{t_{i}}^{t_{f}} d t\left(t_{f}-t\right) \frac{\tilde{F}(t)}{m}
\end{aligned}
$$

The right hand side of the equation for $\Delta r$ was obtained by changing the order of the integration. Integration of equations $(6.8 .3)$ yields

$$
\begin{aligned}
\Delta v_{t f}=\frac{c_{0}}{m v_{i}} & \left\{\left[\frac{1}{r_{i}}-\frac{1}{r_{f}}\right] \hat{a}-\left[\frac{a_{i}}{b r_{i}}+\frac{T v_{i}-a_{i}}{b r_{f}}\right] \hat{b}\right\} \\
\Delta r_{t f}=\frac{c_{0}}{m v_{i}^{2}} & \left\{\left[\sigma_{i} \ln \left(\frac{|a|+r_{i}}{b}\right)-\sigma_{f} \ln \left(\frac{\left|T v_{i}-a_{i}\right|+r_{f}}{b}\right)+\frac{T v_{i}}{r_{i}}\right] \hat{a}\right\} \\
& \left.+\left[\frac{b^{2}-a_{i}\left(T v_{i}-a_{i}\right)}{b r_{i}}-\frac{r_{f}}{b}\right] \hat{b}\right\}
\end{aligned}
$$

in which

$$
\begin{array}{lll}
a_{1}=a_{0}-v_{i} t_{i} & & \\
\sigma_{i}=\operatorname{sign}\left(-a_{i}\right) & , & \\
r_{i}=\left[a_{i}^{2}+b^{2}\right]^{1 / 2} & , &
\end{array}
$$

similar to equations $(6.3 .4),(6.3 .5)$ and $(6.5 .6)$.

Utilizing the equations $(6.3 .8)$ and $(6.3 .11)$ one can express the results of equations $(6.8 .4)$ in terms of the coordinates of the $(\hat{a}, \hat{b})$ reference system 


$$
\begin{aligned}
& \Delta a_{f} \cong-h\left[\sigma_{i} \ln \left(\frac{\left|a_{i}\right|+r_{i}}{b}\right)-\sigma_{f} \ln \left(\frac{\left|T v_{i}-a_{i}\right|+r_{f}}{b}\right)+\frac{T v_{i}}{r_{i}}\right] \\
& \Delta b_{f} \cong-\frac{h}{b}\left(\frac{b^{2}-a_{i}\left(T v_{i}-a_{i}\right)}{r_{i}}-r_{f}\right) \\
& \Delta \dot{a}_{f} \cong-h v_{i}\left(\frac{1}{r_{i}}-\frac{1}{r_{f}}\right) \\
& \Delta \dot{b}_{f} \cong \frac{h v_{i}}{b}\left(\frac{a_{i}}{r_{i}}+\frac{T v_{i}-a_{i}}{r_{f}}\right)
\end{aligned}
$$

in which the length $h$ is given by equation (6.7.6). Equations (6.8.6), which apply to weak interactions, should be compared to equations $(6.7 .5)$, which are valid for nearly complete collisions. Both sets of equations should yield the same results for collisions which are both weak and nearly complete. According to equations (6.7.7) and (6.8.5) a nearly complete collision implies that

$$
\sigma_{i}=-1, \quad \sigma_{f}=+1, \quad a_{i} \approx r_{i}, \quad T v_{i}-a_{i} \approx r_{f}
$$

Substitution in equations $(6.8 .6)$ yields

$$
\begin{aligned}
& \Delta a_{f} \cong h\left[\ln \left(\frac{4 r_{i} r_{f}}{b^{2}}\right)-\frac{T v_{i}}{r_{i}}\right] \\
& \Delta b_{f} \cong \frac{2 h r_{f}}{b}\left(1-\frac{b^{2}}{2 r_{i} r_{f}}\right) \cong \frac{2 h r_{f}}{b} \\
& \Delta \dot{a}_{f} \cong h v_{i}\left(\frac{1}{r_{f}}-\frac{1}{r_{i}}\right) \\
& \Delta \dot{b}_{f} \cong \frac{2 h v_{i}}{b}
\end{aligned}
$$

which is indeed identical to equations (6.7.8), provided that one may identify $r_{f}$ with $T_{i}-r_{i}$. For a weak nearly complete collision both quantities are, in fact, related by

$$
r_{f}=T v_{i}-r_{i}+\frac{1}{2} b^{2}\left(\frac{1}{r_{i}}-\frac{1}{r_{i}}\right)+\cdots \approx T v_{i}-r_{i}
$$

as follows by eliminating $a_{i}$ from the expanded expressions for $r_{i}$ and $r_{f}$ given by equations $(6.8 .5)$. We have just demonstrated that there are two routes to obtain equations $(6.8 .8)$. In sections 6.7 we started by considering a nearly complete collision, which fulfils equations $(6.7 .7)$, and took the limit $\sqrt{q_{c}}+\infty$ next, which refers to weak interactions. In this section we interchanged this order, thus starting by assuming that the interaction is weak and considering the limit of complete collisions next.

A weak central collision corresponds to $b=0$ and $\sigma_{i}=\sigma_{f}$ (the perihelion is not passed during the collision). Accordingly, one finds from equations $(6.8 .6)$ and $(6.8 .5)$ for this case 


$$
\begin{aligned}
& \Delta a_{f} \cong-h\left[\sigma_{i} \ln \left(\frac{r_{i}}{r_{f}}\right)+\frac{T v_{i}}{r_{i}}\right], \quad \Delta \dot{a}_{f} \cong-h v_{i}\left(\frac{1}{r_{i}}-\frac{1}{r_{f}}\right) \\
& \Delta b_{f}=\Delta \dot{b}_{f}=0, \quad r_{i}=\left|a_{i}\right|, \quad r_{f}=\left|T v_{i}-a_{i}\right|
\end{aligned}
$$

The reader should notice that equations $(6.7 .9)$ and equations $(6.8 .10)$ both apply to a central collision. However, the former refers to a nearly complete collision in which the perihelion is passed $\left(r_{i}\right)>h$, $\left.r_{f} \gg h, \quad \sigma_{i}=-1, \sigma_{f}=+1\right)$, while the latter refers to a weak incomplete collision in which the perihelion is not reached $\left(r_{i} \neq r_{f}, \sigma_{i}=\sigma_{f}\right)$.

The virtual displacements in the reference plane follow again by means of equations $(6.7 .10)$. Substitution of equations $(6.8 .6)$ gives

$$
\begin{aligned}
& \Delta a_{r} \cong-h\left[\sigma_{i} \ln \left(\frac{\left|a_{i}\right|+r_{i}}{b}\right)-\sigma_{f} \ln \left(\frac{\left|T v_{i}-a_{i}\right|+r_{f}}{b}\right)+\frac{\left(T-T_{i}\right) v_{i}}{r_{i}}+\frac{T_{1} v_{i}}{r_{f}}\right] \\
& \Delta b_{r} \cong-\frac{h}{b}\left[r_{i}-r_{f}-\frac{\left(T-T_{i}\right) v_{i} a_{i}}{r_{i}}+\frac{\left(v_{i} T-a i\right) T_{i} v_{i}}{r_{f}}\right]
\end{aligned}
$$

which should be compared with equations (6.7.11).

The equations $(6.8 .6)$ and $(6.8 .11)$ constitute the main results of this section. They are analogous to equations (6.7.5) and (6.7.11), derived for a nearly complete collision. One would like to bring equations $(6.8 .6)$ and $(6.8 .11)$ in the same form as equations $(6.7 .13)$. However, for a weak collision one may not approximate $r_{i}$ by means of equation (6.7.12), since $b$ is not necessarily small compared to $\mathrm{Tv}_{\mathrm{i}}-\mathrm{a}_{0}$. Thus, one has to use the full expression for both $r_{i}$ and $r_{f}$ given by equations $(6.8 .5)$. One may replace the quantities $t_{i}$ and $T_{1}=t_{f}-t_{r}$ in equations $(6.8 .5),(6.8 .6)$ and $(6.8 .11)$ by the parameters $S_{i}$ and $S_{c}$ and the time of flight $T$, using equations $(6.2 .3)$ and $(6.3 .10)$. The equations (6.8.5) transform to (denoting $v_{i}$ as $v$ )

$$
\begin{aligned}
& a_{i}=S_{c} v T+a_{0} \\
& \sigma_{i}=\operatorname{sign}\left[-S_{c} T v-a_{0}\right], \quad \sigma_{f}=\operatorname{sign}\left[\left(1-S_{c}\right) T v-a_{0}\right] \\
& r_{i}=\left[\left(S_{c} T v+a_{0}\right)^{2}+b^{2}\right]^{1 / 2}, \quad r_{f}=\left\{\left(\left(1-S_{c}\right) T v-a_{0}\right)^{2}+b^{2}\right\}^{1 / 2}
\end{aligned}
$$

where $a_{0}$ and the impact parameter $b$ are given by equations (6.3.4). Equations $(6.8 .6)$ and $(6.8 .11)$ transform accordingly. As the modifications are minor, we leave this to the reader.

We finish this section with a note regarding the fundamental aspects of the first order perturbation approximation which was utilized here. Formally speaking, the first order perturbation method corresponds to an expansion of equation $(6.5 .3)$ in the parameter $1 / \sqrt{\mathrm{q}}$. Utilizing equations $(6.7 .1)$ one can express equation $(6.5 .3)$ for $(1 / \sqrt{\mathfrak{q}}) \rightarrow 0$ as

$$
\begin{aligned}
T(\rho)= & \left(\rho^{2}-1\right)^{1 / 2}+\frac{1}{\sqrt{7}}\left[\ln \left(\rho^{2}-1\right)^{1 / 2}-\frac{1}{2}-\rho+\frac{\rho}{\left(\rho^{2}-1\right)^{1 / 2}}-\frac{\rho^{2}}{2\left(\rho^{2}-1\right)}\right] \\
& +\frac{1}{q}\left[\frac{2 \rho}{\rho-1}\left(1-\frac{\left(\rho^{2}-1\right)^{1 / 2}}{\rho}\right)-\frac{\rho^{2}}{2\left(\rho^{2}-1\right)^{1 / 2}}\right]+0\left(q^{-3 / 2}\right)
\end{aligned}
$$

The first term corresponds to the unperturbed trajectory, as can be verified by removing the scaling (notice that for $1 / \sqrt{q}=0$ one may approximate $d_{s}=b$ and $\left.v_{s}=v_{i}\right)$. In figure 6.5 the solid line for $q=\infty$ depicts the relation between $\rho$ and $T$ given by. this term. In first order 
perturbation approximation one includes the term in equation

$(6,8,13)$ which is linear in $1 / \sqrt{9}$ and ignores higher order terms. This approach constitutes an alternative for the derivation of equations (6.8.6) outlined above, which was, however, preferred for its simplicity.

\subsection{Collisions with zero initial relative velocity}

In this section we will consider the case $v_{i}=0$. This means that the particles are initially at rest in the centre of mass system. During the time of flight $T$, part of their potential energy will be converted into kinetic energy. The generated relative velocity will be directed along the line joining the two particles. The particle motion is thus limited to a line rather than to a plane. Consequently, the representation in the $(\hat{a}, \hat{b})$ reference system, defined by equations (6.3.1), is inapplicable and the analysis of the previous sections should be modified.

From equation (6.2.1) it follows that the set of relevant geometrical variables reduces to (taking $v=0$ )

$$
\xi=\left(r_{\perp}, \varphi, b_{2}\right)
$$

see also figure 6.1. As the relative motion of the particles takes place along the line joining their centres, one can suffice by calculating the relative position $r$ and its derivative $\dot{r}$. Taking $\dot{\theta}=0$ for all time $t$ equation $(6.4 .1)$ becomes

$$
\mathrm{E}=\frac{1}{4} \dot{\mathrm{m}} \dot{\mathrm{r}}^{2}+\frac{\mathrm{C}_{0}}{\mathrm{r}}
$$

The energy $\mathrm{E}$ in the centre of mass is conserved during the interaction and thus identical to its initial value

$$
E=C_{0} / r_{i} \quad, \quad r_{i}=\left(b_{z}^{2}+r_{\perp}^{2}\right) i / 2
$$

analogous to equations $(6.2 .15)$ and $(6.3 .5)$. To simplify the equations we introduce the micro-scaling quantities

$$
d_{s}=\frac{C_{0}}{E}\left(=r_{i}\right), \quad v_{s}=2 \sqrt{\frac{E}{m}}, \quad t_{s}=\frac{m^{1 / 2} C_{0}}{2 E^{3 / 2}}
$$

which will be used instead of the quantities defined by equations (6.4.4). One sees that $d_{s}$ is equal to the initial distance $r_{i}$, while $v_{s}$ is the relative velocity for $r \rightarrow \infty$. Employing this scaling one can rewrite equation $(6.9 .2)$ as

$$
1=\left(\frac{\mathrm{d} \rho}{\mathrm{d} \tau}\right)^{2}+\frac{1}{\rho}
$$

integration yields

$$
\left|\tau-\tau_{i}\right|=T(\rho)=\sqrt{\rho^{2}-\rho}+\frac{1}{2} \ln \left[2 \sqrt{\rho^{2}-\rho}+2 \rho-1\right]
$$

which should be compared with equation $(6.5 .3)$.

Clearly, we face the same problem as before: The function $T(\rho)$, defined by equation (6.9.6), can not be inverted analytically without introducing approximations. We will investigate the same approaches to 
this problem as described in the sections $6.6,6.7$ and 6.8 , namely numerical inversion, power expansion for large $p$ (which is justified for nearly complete collisions) and first order perturbation theory (which is justified for weak collisions).

The numerical approach described in section 6.6 is also applicable to the present problem. The iteration process of equation $(6.6 .5)$ can again be used, where the function $H(\rho)$ now represents

$$
H(\rho)=\sqrt{\rho^{2}-\rho}
$$

A suitable start value of the iteration process is given by $\rho_{1}=1$.

A collision is called half-complete if the final scaled distance $\rho_{f} \gg 1$, which means $r_{f} \gg r_{i}$. For this condition one may expand equation $(6.9 .6)$ in a power series in $\rho$. Using equations $(6.7 .1)$ one finds

$$
T(\rho)=\rho+\frac{1}{2}[\ln (4 \rho)-1]-\frac{3}{8} \cdot \frac{1}{\rho}-\frac{5}{32} \frac{1}{\rho^{2}}+0(\rho-3)
$$

Accordingly, the inverted function can be approximated as

$$
\rho(T)=T-\frac{1}{2}\left(1-\frac{1}{T}\right)[\ln (4 T)-1]+\frac{3}{8} \frac{1}{T}+0\left(\frac{\ln T}{T^{2}}\right)
$$

Equations (6.9.8) and $(6.9 .9)$ are analogous to equations $(6.7 .3)$ and $(6.7 .4)$ respectively. Equation $(6,9.9)$ gives the final scaled distance $\rho$ as function of the scaled interaction time $T$. In addition, one requires the derivative of $\rho$ with respect to time. Substitution of equation (6.9.9) into equation $(6.9 .5)$ yields

$$
\left(\frac{\mathrm{d} \rho}{\mathrm{d} \tau}\right)=1-\frac{1}{2 T}\left[1+\frac{1}{2 T}\left(\ln (4 T)-\frac{1}{2}\right)\right]+0\left(T^{-3}\right)
$$

Equations (6.9.9) and (6.9.10) provide a complete specification of the final situation as function of the scaled time $T$. The next step is to remove the scaling using equations $(6.4 .3)$ and $(6.9 .4)$

$$
\begin{aligned}
& r_{f} \cong \frac{2 A T}{r_{i}^{1 / 2}}-\frac{r_{i}}{2}\left(1-\frac{r_{i}^{3 / 2}}{4 A T}\right)\left[\ln \left(\frac{8 A T}{r_{i}^{3 / 2}}\right)-1\right]+\frac{3}{16} \frac{r_{i}^{5 / 2}}{A T} \\
& v_{f} \cong \frac{2 A}{r_{i}^{1 / 2}}-\frac{r_{i}}{2 T}\left\{1+\frac{r_{i}^{3 / 2}}{4 A T}\left[\ln \left(\frac{8 A T}{r_{i}^{3 / 2}}\right)-\frac{1}{2}\right]\right\}
\end{aligned}
$$

in which $A=\left(C_{0} / m\right)^{1 / 2}$ and $T$ the time of flight given by equation (6.2.2). As the initial relative velocity $v$ is zero the change in relative velocity is simply given by

$$
\Delta v_{f}=v_{f}
$$

The virtual displacement in the reference plane follows from

$$
\Delta r_{r}=\left(r_{f}-r_{i}\right)-T_{i} v_{f}
$$

with $T_{1}=t_{f}-t_{r}=\left(1-S_{i}\right) T$, see equations $(6,3.10)$ and $(6,2.3)$. Substitution of equation (6.9.11) into equations $(6.9 .13)$ yields

$$
\Delta r_{r}=S_{i} \frac{2 A T}{r_{i} 1 / 2}-\frac{r_{i}}{2}\left[S_{i}+\ln \left(\frac{8 A T}{r_{i}^{3 / 2}}\right)\right]+\frac{r_{i} 5 / 2}{8 A T}\left[\left(2-S_{i}\right) \ln \left(\frac{8 A T}{r_{i}^{3 / 2}}\right)+\frac{S_{i}}{2}\right]
$$

The velocity and spatial displacement of the test particle follow from 


$$
\Delta v_{\perp t} \cong v_{f} \frac{r_{\perp}}{2 r_{i}}, \quad \Delta v_{z t} \cong v_{f} \frac{b_{z}}{2 r_{i}}, \quad \Delta r_{\perp t} \cong \Delta r_{r} \frac{r_{1}}{2 r_{i}}
$$

We emphasize that equations $(6.9 .11)$ and $(6.9 .14)$ apply to nearly halfcomplete collisions, which implies $T \gg 1$. Using unscaled quantities this condition can be expressed as

$$
T \gg r_{i}{ }^{3 / 2 / 2 A}
$$

as follows with the last equation of $(6.9 .4)$.

Finally, we will employ perturbation theory to determine the velocity shift of the test particle in the case $v=0$. In this approach one assumes that the relative distance $r$ changes very little during the interaction, thus $r_{f} \approx r_{i}$. In terms of the scaled coordinates used above this means $\rho \approx 1$. Power expansion of equation $(6.9 .6)$ around $\rho=1$ gives, using equations $(6.7 .1)$

$$
T(\rho)=(p-1)^{1 / 2}+\frac{1}{3}(\rho-1)^{3 / 2}+0\left[(p-1)^{2}\right]
$$

analogous to equation (6.8.13), derived for the general case $v \neq 0$ (note, however, that in the general case $\rho$ can be large compared to 1). By inversion one obtains

$$
\rho(T)=1+\frac{1}{4} T^{2}-\frac{1}{48} T^{4}+0\left(T^{5}\right)
$$

The derivative of $\rho$ with respect to $T$ follows with equation $(6.9 .5)$

$$
\left(\frac{\mathrm{d} \rho}{\mathrm{d} T}\right)=\frac{1}{2} T-\frac{1}{12} T^{3}+0\left(T^{4}\right)
$$

By removing the scaling in equations (6.9.18) and (6.9.19), using equations $(6.4 .3)$ and $(6.9 .4)$, one finds

$$
\begin{aligned}
& r_{f} \cong r_{i}+\frac{A^{2} T^{2}}{r_{i}^{2}}-\frac{1}{3} \frac{A^{4} T^{4}}{r_{i}^{5}} \\
& v_{f} \cong 2 \frac{A^{2} T}{r_{i}^{2}}-\frac{4}{3} \frac{A^{4} T^{3}}{r_{i}^{5}}
\end{aligned}
$$

where $A=\left(C_{0} / m\right)^{1 / 2}$. The virtual displacement in the reference plane follows with equations $(6.9 .13)$

$$
\Delta r_{r}=\left(2 S_{i}-1\right) \frac{A^{2} T^{2}}{r_{i}^{2}}-\left(\frac{4 S_{i}}{3}-1\right) \frac{A^{4} T^{4}}{r_{i}^{5}}
$$

First order perturbation dynamics is achieved by truncating the expansions in equations (6.9.20) and (6.9.21) after the terms proportional to $\left(A^{2} / r_{i}^{2}\right)$. This quantity corresponds to the acceleration of the individual particles at $t=t_{i}$. The spatial displacement $\Delta r_{\perp t}$ and the velocity displacements $\Delta v_{\perp t}$ and $\Delta v_{2 t}$ experienced by the test particle can be determined by means of equations $(6.9 .15)$. 
In this section we will present some explicit expressions for the axial velocity shift of the test particle $\Delta v_{z}$ in terms of the geometrical parameters. We will employ the results obtained in sections $6.7,6.8$ and 6.9. Expressions for the transverse velocity shift $\Delta v_{\perp}$ and the spatial shift $\Delta r$ will be presented in sections 6.11 . and 6.12 respectively.

By utilizing equations $(6.3 .8)$ and $(6.3 .11)$ one can express the transformation of the displacements of the test particle from coordinates in the $(\hat{a}, \hat{b})$ reference system to coordinates in the laboratory system as

$$
\begin{aligned}
& \Delta \mathbf{r}_{\mathrm{tr}}=-\left(\Delta \mathrm{a}_{\mathrm{r}} \hat{\mathrm{a}}+\Delta \mathrm{b}_{\mathrm{r}} \hat{\mathrm{b}}\right) / 2 \\
& \Delta \mathbf{v}_{\mathrm{tf}}=-\left(\Delta \dot{\mathrm{a}}_{\mathrm{f}} \hat{\mathrm{a}}+\Delta \dot{\mathrm{b}}_{\mathrm{f}} \hat{b}\right) / 2
\end{aligned}
$$

in which $\hat{a}$ and $\hat{b}$ are given by equations (6.3.2). $\Delta r_{t r}$ is the virtual lateral displacement in the reference plane and $\Delta v_{t f}$ is the final velocity displacement. The latter is the relevant dynamical parameter for the Boersch effect, while the former is relevant for the trajectory displacement effect. For covenience of notation we will from now on omit the indices $t$ (for test-particle), $r$ (for reference plane) and $f$ (for final). The reader should be alert that all results apply to the coordinates of the test particle at $t=t_{f}$, while the spatial displacements are extrapolated to the reference plane.

Equations (6.3.2) express the vectors $\hat{a}$ and $\bar{b}$ in terms of the coordinates of the laboratory system. The vector â has no $z$-component due to the fact that the particles have the same axial velocity. Consequently, the velocity shift $\Delta v_{z}$ is entirely determined by the quantity $\Delta \mathrm{b}_{\mathrm{f}}$ only

$$
\Delta \mathbf{v}_{\mathbf{z}}=-\Delta \dot{b}_{\mathrm{f}} \mathrm{b}_{\mathbf{z}} / 2 \mathrm{~b}
$$

as follows from equations (6.3.2) and (6.10.1). For a nearly complete collision one finds with equations $(6.7 .13),(6.4 .16)$ and $(6.7 .6)$

$$
\Delta v_{z} \cong \frac{m v^{3} b_{z}}{2 C_{0}\left(1+q_{c}\right)}\left[1+\frac{2 C_{0}}{m v^{3} T}\left(\frac{2}{S_{c}\left(1+q_{c}\right)}-\frac{1}{1-S_{c}}\right)\right]
$$

where $q_{c}$ is given by equation $(6.4 .16)$. A complete collision corresponds to $T+\infty$. In this limit equation $(6.10 .3)$ becomes

$$
\Delta \mathrm{v}_{\mathrm{z}} \cong \frac{\mathrm{mv}^{3} \mathrm{~b}_{\mathrm{z}}}{2 \mathrm{C}_{0}\left(1+\mathrm{q}_{\mathrm{c}}\right)}
$$

independent of $T$ and $S_{c}$.

The effect of a weak collision can be determined from equations $(6.10 .2),(6.8 .6),(6.8 .12)$ and $(6.7 .6)$

$$
\Delta v_{z} \cong \frac{C_{0} b_{z}}{m v^{2}}\left[\frac{S_{c} T v+a_{0}}{\left(\left(S_{c} T v+a_{0}\right)^{2}+b^{2}\right]^{1 / 2}}+\frac{\left(1-S_{c}\right) T v-a_{0}}{\left\{\left(\left(1-S_{c}\right) T v-a_{0}\right)^{2}+b^{2}\right\}^{1 / 2}}\right]
$$

where $a_{0}$ and $b$ are given by equations $(6,3,4)$. For a collision which is both weak and complete one obtains

$$
\Delta v_{z} \cong \frac{2 C_{0} b_{z}}{m^{2} b^{2}}
$$

as follows from equation $(6.10 .4)$, taking $q_{c} \gg 1$, as well as from 
equation $(6.10 .5)$, using $\mathrm{T} \rightarrow \infty$.

The case $v=0$ requires a separate treatment, as was discussed in section 6.9. For a half-complete collision one obtains from equations $(6.9 .11),(6.9 .15)$ and $(6.9 .3)$

$$
\Delta v_{z} \cong\left(\frac{C_{0}}{m}\right)^{1 / 2} \frac{b_{z}}{\left(b_{z}^{2}+r_{\perp}^{2}\right)^{3 / 6}}-\frac{b_{z}}{4 T} \quad(v=0) \quad(6.10 .7)
$$

in which all terms of equation $(6.9 .11)$ were included up to and including those of the order $\mathrm{T}^{-1}$. In the limit $\mathrm{T}+\infty$ one may ignore the second term in equation $(6.10 .7)$. The remaining term expresses that the initial potential energy is completely converted into kinetic energy.

For a weak collision only a small fraction of the initial potential energy will be converted into kinetic energy. From equations $(6.9 .20),(6.9 .15)$ and $(6.9 .3)$ one obtains for this case

$$
\Delta v_{z} \cong \frac{c_{0}}{m} \frac{b_{z} T}{\left(b_{z}^{2}+r_{1}^{2}\right)^{3 / 2}}
$$

in which only the most significant term of equation $(6.9 .20)$ was included. Equation (6.10.8) presupposes that the force acting on the test particle is approximately constant during the time of flight $T$ (first order perturbation theory). Equations $(6.10 .7)$ and $(6.10 .8)$ are relevant for monochromatic homocentric cylindrical beam sections, in which the particles are initially at rest in the frame of reference moving with the beam.

Most theories on the Boersch effect which are based on twoparticle dynamics utilize equation $(6.10 .4)$ or equation $(6.10 .6)$. Loeffler (1969) started from equation (6.10.5), but used equation (6.10.6) in the actual calculation (we note that Loeffler's equation (4) follows with $\Delta E=m v_{z} \Delta v_{z}, \quad \varphi=\Phi-\pi, \quad \alpha L_{1}=S_{c} T v$ and $\left.\alpha L_{2}=\left(1-S_{c}\right) T v\right)$. All expressions yield $\Delta v_{z}=0$ for $b_{z}=0$. This can be understood from the fact that the orbital plane is then oriented perpendicular to the beam axis, see figure 6.1. Consequently, the displacements have no component in the axial direction.

\subsection{Expressions for the transverse velocity shift $\Delta v_{\perp}$}

Using equations $(6.10 .1)$ and $(6.3 .2)$ one can express the transverse velocity shift $\Delta v_{\perp}$ as

$$
\Delta v_{\perp}=\left(A_{v} 2+B_{v}^{2} \frac{\left[r_{\perp} \sin (\Phi)\right]^{2}}{b^{2}}\right)^{1 / 2}, A_{v}=\left|\Delta \dot{a}_{f} / 2\right|, B_{v}=\left|\Delta b_{f} / 2\right| \quad(6.11 .1)
$$

In case $r_{\perp}=0$ one simply finds $\Delta v_{\perp}=A_{v}$ and $\Delta v_{\perp}$ is entirely determined by $\Delta \dot{a}_{f}$ only. This can be understood from the fact that the orbital plane is for $r_{\perp}=0$ parallel to the beam axis, see figure 6.1 .

For a nearly complete collision one obtains by substitution of equations $(6.7 .13)$ into equation $(6.11 .1)$

$$
\begin{aligned}
\Delta v_{\perp} \cong \frac{v}{1+q_{c}}\left\{\left[1+\frac{2 c_{0}}{m v^{3} T}\left[\frac{1-q_{c}}{2}\left(\frac{1}{S_{c}}-\frac{1}{1-S_{c}}\right)-\frac{2 q_{c}}{1+q_{c}} \frac{1}{S_{c}}\right]\right]^{2}\right. \\
\left.+q_{c}\left(\frac{r_{\perp} \sin (\Phi)}{b}\right)^{2}\left[1+\frac{2 C_{0}}{m v^{3} T}\left(\frac{2}{S_{c}\left(1+q_{c}\right)}-\frac{1}{1-S_{c}}\right)\right]^{2}\right\}^{1 / 2}(6.11 .2)
\end{aligned}
$$

with $q_{c}$ given by equation $(6.4 \cdot 16)$. A complete collision corresponds to 
$T+\infty$. In this limit equation $(6.11 .2)$ becomes

$$
\Delta v_{\perp} \cong \frac{v}{1+q_{c}}\left[1+q_{c}\left(\frac{r_{\perp} \sin (\Phi)}{b}\right)^{2}\right]^{1 / 2}
$$

independent of $\mathrm{T}$ and $\mathrm{S}_{\boldsymbol{c}}$.

For a weak collision one finds from equations $(6.8 .6),(6.8 .12)$ and $(6.11 .1)$

$$
\Delta v_{\perp} \equiv \frac{c_{0}}{m v b}\left[\left(\frac{b}{r_{i}}-\frac{b}{r_{f}}\right)^{2}+\left(\frac{r_{\perp} \sin (\Phi)}{b}\right)^{2}\left(\frac{S_{c} T v+a_{0}}{r_{i}}+\frac{\left(1-S_{c}\right) T v-a_{0}}{r_{f}}\right)^{2}\right]^{1 / 2}(6.11 .4)
$$

with $r_{i}=\left[\left(S_{c} T v+a_{0}\right)^{2}+b^{2}\right]^{1 / 2}, \quad r_{f}=\left\{\left[\left(1-S_{c}\right) T v-a_{0}\right]^{2}+b^{2}\right\}^{1 / 2}$ and $a_{0}$ and $b$ given by equations $(6.3 .4)$. Note that $\Delta v_{1}=0$ in case $S_{c}=1 / 2$ and $r_{\perp}=0$. Thus weak collisions in a point crossover $\left(r_{c}=0\right)$ which is located in the middle of a beam segment $\left(S_{c}=1 / 2\right)$ do not result in angular deflections of the test particle. This effect is due to the symmetry of the trajectories with respect to the crossover. The angular displacement experienced by the test particle in the first half of the beam segment is exactly cancelled during its flight through the second half of the beam segment. For a collision which is both weak and complete one obtains

$$
\Delta v_{\perp} \cong \frac{2 C_{0} r_{\perp} \sin (\Phi)}{m^{2}}
$$

as follows from equation (6.11.4) taking $q_{c} \gg>1$, as well as from equation $(6,11.3)$, taking $\mathrm{T} \rightarrow \infty$.

The case $\mathrm{v}=0$ requires a separate treatment. For a half-complete collision it follows from equations $(6.9 .11),(6.9 .15)$ and $(6.9 .3)$

$$
\Delta v_{\perp} \cong\left(\frac{C_{0}}{m}\right)^{1 / 2} \frac{r_{\perp}}{\left(b_{z}^{2}+r_{\perp}^{2}\right)^{3 / 4}}-\frac{r_{\perp}}{4 T} \quad(v=0)
$$

in which all terms of equation (6.9.11) were included up to and including those of the order $\mathrm{T}^{-1}$. For a weak collision one obtains from equations $(6.9 .20),(6.9 .15)$ and $(6.9 .3)$

$$
\Delta v_{\perp} \cong \frac{C_{0}}{m} \frac{r_{\perp} T}{\left(b_{z}^{2}+r_{\perp}^{2}\right)^{3 / 2}}
$$

in which the most significant term of equation $(6.9 .20)$ was included only.

\subsection{Expressions for the spatial shift $\Delta r$}

Using equations $(6.10 .1)$ and $(6.3 .2)$ one can express the virtual radial displacement of the test particle in the reference plane as

$$
\Delta r=\left(A_{r}{ }^{2}+B_{r}{ }^{2} \frac{\left[r_{\perp} \sin (\Phi)\right]^{2}}{b^{2}}\right)^{1 / 2}, \quad A_{r}=\left|\Delta a_{r} / 2\right|, B_{r}=\left|\Delta b_{r} / 2\right| \quad(6.12 .1)
$$

similar to equation (6.11.1) referring to the transverse velocity shift $\Delta v_{\perp}$. In case $r_{\perp}=0$ one finds $\Delta r=A_{r}$ and $\Delta r$ is entirely determined by $\Delta a_{r}$ only.

For a nearly complete collision the quantities $A_{r}$ and $B_{r}$ follow directly from equations $(6.7 .13),(6.4 .16)$ and $(6.7 .6)$ 


$$
\begin{aligned}
& A_{r} \cong\left|\frac{\left(S_{i}-S_{c}\right) T v-a_{0}}{1+q_{c}}-\frac{b}{2 \sqrt{q_{c}}} \frac{1-q_{c}}{1+q_{c}}\left[2 \Lambda_{c}-\frac{S_{i}}{S_{c}}-\frac{1-S_{i}}{1-S_{c}}\right]+\frac{b-\sqrt{q_{c}}}{1+q_{c}}\left[1-\frac{2}{1+q_{c}}\left(\frac{S_{i}}{S_{c}}-1\right)\right]\right| \\
& B_{r} \cong\left|\frac{\sqrt{q_{c}}}{1+q_{c}}\left[\left(S_{i}-S_{c}\right) T v-a_{0}\right]-\frac{2 b}{1+q_{c}}\left[\Lambda_{c}-\frac{1}{1+q_{c}}\left(\frac{S_{i}}{S_{c}}-1\right)-\frac{1}{2} \frac{1-S_{i}}{1-S_{c}}\right]\right|
\end{aligned}
$$

where $q_{c}$ is given by equation $(6.4 .16), \Lambda_{c}$ by equation $(6.7 .14)$ and $a_{0}$ and $b$ by equation $(6.3 .4)$. It should be noted that the assumption that the collision is nearly complete implies that $S_{c} \approx 1 / 2$, see equations (6.7.7). Equations $(6.12 .2)$ simplify considerably for the case $S_{c}=S_{i}$, which implies that the reference plane is identical to the $x, y-p l a n e$ of the laboratory system (which coincides with the crossover plane)

$$
\begin{aligned}
& \left.A_{r} \cong \mid \frac{a_{0}}{1+q_{c}}+\frac{b}{\sqrt{q_{c}}} \frac{1-q_{c}}{1+q_{c}}\left[\Lambda_{c}-1\right]-\frac{b \sqrt{q_{c}}}{1+q_{c}}\right] \mid \quad\left(S_{c}=S_{i}\right) \\
& B_{r} \cong\left|\frac{a_{0}-\sqrt{q_{c}}}{1+q_{c}}+\frac{2 b}{1+q_{c}}\left[\Lambda_{c}-\frac{1}{2}\right]\right|
\end{aligned}
$$

A further reduction is achieved for the case of a point crossover $\left(r_{c}=0\right.$ thus $r=0)$. From equations $(6.12 .1),(6.12 .3)$ and $(6.7 .14)$ one obtains for $r_{\perp} \stackrel{1}{=} 0$

$$
\Delta r \cong\left|\frac{b_{z}}{\sqrt{q_{c}}}\left\{\frac{1-q_{c}}{1+q_{c}}\left\{\ln \left(\sqrt{\frac{q_{c}}{q_{c}+1}} \frac{2 v T}{b_{z}} \sqrt{S_{c}\left(1-S_{c}\right)}\right\}-2\right]-\frac{q_{c}}{1+q_{c}}\right\}\right|\left(r_{\perp}=0\right)
$$

previously published by van Leeuwen and Jansen (1983) (their equation (4.9) follows by substituting $S_{c}=1 / 2$ ).

For a weak collision the quantities $A_{r}$ and $B_{r}$ follow from equations $(6.8 .11),(6.8 .12)$ and $(6.7 .6)$

$$
\begin{aligned}
& A_{r} \cong \frac{C_{0}}{m v^{2}}\left|\sigma_{i} \ln \left(\frac{\left|S_{c} v T+a_{0}\right|+r_{i}}{b}\right)-\sigma_{i} \ln \left(\frac{\left|\left(1-S_{c}\right) v T-a_{0}\right|+r_{f}}{b}\right)+v T\left(\frac{S_{i}}{r_{i}}+\frac{1-S_{i}}{r_{f}}\right)\right| \\
& B_{r} \cong \frac{C_{0}}{m v^{2} b}\left|r_{i}-r_{f}-v T\left[\frac{S_{i}\left(S_{c} v T+a_{0}\right)}{r_{i}}-\frac{\left(1-S_{i}\right)\left[\left(1-S_{c}\right) v T-a_{0}\right]}{r_{i}}\right]\right|
\end{aligned}
$$

where $r_{i}, r_{f}, \sigma_{i}$ and $\sigma_{f}$ are given by equations $(6.8 .12)$ and $a_{0}$ and $b$ by equations $(6.3 .4)$. For the case of a point crossover $\left(r_{c}=0\right.$ thus $\left.r_{\perp}=0\right)$ one finds from equations $(6.12 .1),(6.12 .5)$ and $(6.8 .12)$

$$
\Delta r \cong \frac{C_{0}}{m v^{2}}\left|\ln \left(\frac{\left[S_{c} v T+r_{i}\right]\left[\left(1-S_{c}\right) v T+r_{f}\right]}{b_{z}^{2}}\right)-v T\left(\frac{S_{i}}{r_{i}}+\frac{1-S_{i}}{r_{i}}\right)\right| \quad\left(r_{\perp}=0\right)(6.12 .6)
$$

with $r_{i}=\left[\left(S_{c} v T\right)^{2}+b_{z}^{2}\right]^{1 / 2}$ and $r_{f}=\left\{\left(\left(1-S_{c}\right) v T\right]^{2}+b_{z}\right\}^{1 / 2}$. Equation (6.12.6) becomes independent of $S_{i}$ for $S_{c}=1 / 2$, which implies $r_{i}=r_{f}$. This is a consequence of the fact that the transverse velocity of the test particle is not changed $\left(\Delta v_{1}=0\right)$, as can be verified from equation $(6.11 .4)$. Its final transverse velocity is identical to its initial transverse velocity and the resulting effect is a radial shift only. Accordingly, the virtual displacement of the test particle $\Delta r$ is independent of the choice of the reference plane. It should be emphasized that this phenomenon occurs only for a point crossover located in the middle of the beam segment, thus $S_{c}=1 / 2$ and $r_{c}=0$ (implying $r:=0$ ). For ${ }^{\perp}$ collision which is both weak and nearly complete it can be 
proven that

$$
\begin{aligned}
\Delta r \cong \frac{2 C_{0}}{m v^{2}}\left\{\left[\ln \left(\frac{2 v T \sqrt{S_{c}\left(1-S_{c}\right)}}{b}\right)-\frac{1}{2}\left(\frac{S_{i}}{S_{c}}+\frac{1-S_{i}}{1-S_{c}}\right)\right]^{2}\right. \\
\left.+\left[\frac{\left[v T\left(S_{i}-S_{c}\right)+r_{\perp} \cos (\Phi)\right] r_{\perp} \sin (\Phi)}{b^{2}}\right]^{2}\right\}^{1 / 2}
\end{aligned}
$$

This result follows from equations $(6.12 .1)$ and $(6.12 .2)$, taking $\sqrt{\varphi_{c}}+\infty$, as well as from equations $(6.12 .1)$ and $(6.12 .5)$, taking $r_{i}=S_{c} v T+a_{0}$ and $r_{f}=\left(1-S_{c}\right)$ vT-a $a_{0}$ with $a_{0}=-r_{\perp} \cos (\Phi)$.

The case $v=0$ requires a separate treatment as was discussed in section 6.9. For a half-complete collision one obtains from equations $(6.9 .14),(6.9 .15)$ and $(6.9 .3)$

$$
\Delta r \equiv\left|\frac{\left(C_{0} / m\right)^{1 / 2} r_{\perp} T S_{i}}{\left(b_{z}^{2}+r_{\perp}^{2}\right)^{3 / 4}}-\frac{r_{\perp}}{4}\left[S_{i}+\ln \left(\frac{8\left(C_{0} / m\right)^{1 / 2} T}{\left(b_{z}^{2}+r_{\perp}^{2}\right)^{3 / 4}}\right)\right]\right| \quad(v=0) \quad(6.12 .8)
$$

in which the two most significant terms of equation (6.9.14) were included only. For a weak collision one finds from equations $(6.9 .21)$, $(6.9 .15)$ and $(6,9.3)$

$$
\Delta r \cong\left|\frac{C_{0}}{m} \frac{r_{\perp} T^{2}\left(S_{i}-1 / 2\right)}{\left(b_{z}^{2}+r_{\perp}^{2}\right)^{3 / 2}}\right| \quad(v=0)
$$

in which the most significant term of equation $(6.9 .20)$ was included only. Equation (6.12.9) shows that a weak collision has (in first order perturbation approximation) no resulting effect in case $s_{i}=1 / 2$. Thus the virtual displacement in a reference plane located in the middle of the beam segment is zero.

\subsection{Coulomb-scaling}

In the analytical as well as in the numerical calculations on Coulomb interactions it is often convenient to scale the equations. Scaling usually simplifies the notation and may lead to a reduction of the number of independent parameters. In the succeeding chapters we will frequently use the so-called coulomb or $\delta, v$-scaling, in which length and velocity are scaled with $\delta$ and $v$ respectively, where

$$
\delta=\left(\frac{4 \mathrm{C}_{0} \mathrm{~T}^{2}}{\mathrm{~m}}\right)^{1 / 3} \quad, \quad \nu=\left(\frac{4 \mathrm{C}_{0}}{\mathrm{mT}}\right)^{1 / 3}
$$

The aim of this scaling is to establish equations which do not explicitly depend on $\mathrm{T}, \quad \mathrm{C}_{0}$ or $\mathrm{m}$. For an electron beam with $\mathrm{V}=10 \mathrm{kV}$ and $\mathrm{L}=0.1 \mathrm{~m}$ one finds $\delta=14.2 \mu \mathrm{m}$ and $\nu=8.4410^{3} \mathrm{~m} / \mathrm{s}$, which can be considered as typical values. Quantities scaled with $\delta$ and/or $v$ are indicated with an asterisk.

From equations $(6.13 .1)$ it can directly be seen that the quantities $\delta$ and $v$ fulfil the relations

$$
\delta / \nu=\mathrm{T} \quad \delta \nu^{2}=4 \mathrm{C}_{0} / \mathrm{m}
$$

Thus the scale-measure for time $\delta / \nu$ is identical to the time of flight $T$. The total energy $E$ and angular momentum $J$ in the centre of mass system, given by equations $(6.2 .15)$ and $(6.2 .19)$ respectively, can now be expressed as 


$$
\begin{aligned}
& \mathrm{E}^{*}=\frac{4 \mathrm{E}}{\mathrm{m} \nu^{2}}=\mathrm{v}^{* 2}+1 / \mathrm{r}_{\mathrm{i}}^{*} \\
& \mathrm{~J}^{*}=\frac{2 \mathrm{~J}}{\mathrm{~m} \delta v}=\mathrm{b}^{*} \mathrm{~V}^{*}
\end{aligned}
$$

where $\mathrm{v}^{*}=\mathrm{v} / \nu, \mathrm{b}^{*}=\mathrm{b} / \delta$ and the scaled initial separation $\mathrm{r}_{\mathrm{i}}^{*}$ is given by

$$
r_{i}^{*}=\frac{r_{i}}{\delta}=\left[\left(S_{c} V^{*}+a_{0}^{*}\right)^{2}+b^{* 2}\right]^{1 / 2}
$$

as follows from equations $(6.8 .12)$. The parameter $q$, defined by equation $(6.4 .7)$, and its equivalent $q_{c}$ for a complete collision $\left(r_{i}+\infty\right)$ can be expressed as

$$
q=4 J^{* 2} E^{*}=q_{c}\left(1+1 / v^{* 2} r_{i}^{*}\right), \quad q_{c}=\left(2 b^{*} v^{* 2}\right)^{2}
$$

which are the scaled versions of equations (6.4.15) and (6.4.16).

It can straightforwardly be demonstrated that by employing the $\delta, v$-scaling the basic equations presented in the sections $6.2,6.3$ and 6.4 reduce to a form which does not contain $T, C_{0}$ and $m$ explicitly. As a consequence one may formally express the displacements experienced by the test particle as

$$
\begin{aligned}
& \Delta r=\delta \Delta r^{*}\left(r_{\perp}{ }^{*}, \varphi, b_{z}{ }^{*}, v^{*}, \psi, S_{c}, S_{i}\right) \\
& \Delta \mathbf{v}=v \Delta v^{*}\left(r_{\perp}{ }^{*}, \varphi, b_{z}{ }^{*}, v^{*}, \psi, S_{c}, S_{i}\right)
\end{aligned}
$$

with $\mathrm{r}^{*}=\mathrm{r} / \delta, \quad \mathrm{b}_{\mathrm{z}}^{*}=\mathrm{b}_{\mathrm{z}} / \delta, \mathrm{v}^{*}=\mathrm{v} / v$. The reader may verify that the functions $t r^{*}$ and $\Delta v^{*}$ are indeed independent of $T, C_{0}$ and $m$ by introducing the $\delta, \nu$-scaling in the expressions for $\Delta v_{z}, \Delta r$ and $\Delta v_{1}$ presented in sections $6.10,6,11$ and 6.12 .

\section{Appendix 6.A Mathematics of nearly complete collision dynamics}

This appendix is concerned with the analytical calculation of the dynamics of a nearly complete collision. Given the initial polar coordinates $\rho_{i}$ and $\theta_{i}$ and the scaled time of flight $\tau_{T}$ we shall compute the final scaled polar coordinates $\rho_{f}, \theta_{f}$ and their derivatives $[d \rho / d \tau]_{f}$ and $[d \theta / \tau]_{f}$. These quantities will then be used to determine the final values of the coordinates $a, b$ and their derivatives $\dot{a}$ and $\dot{b}$. Finally, the displacements of the particles follow in terms of the quantities $\Delta a$, $\Delta \mathrm{b}, \Delta \dot{\mathrm{a}}$ and $\Delta \dot{\mathrm{b}}$. Since the calculation pertains to a nearly complete collision it is assumed that the conditions stated by equations $(6.7 .7)$ are fulfilled.

Substitution of equations $(6.7 .3)$ and $(6.7 .4)$ into equation $(6.5 .7)$ yields

$$
\rho_{r}=\tau_{T}-\rho_{i}-\frac{2 \Lambda}{\sqrt{q}}\left(1-\frac{1}{\sqrt{q}} \frac{1}{\tau_{T}-\rho_{i}}\right)+\frac{q+3}{2 q}\left(\frac{1}{\rho_{i}}+\frac{1}{\tau_{T}-\rho_{i}}\right)+0\left(\frac{\ln \rho_{i}}{\rho_{i}^{2}}\right)+0\left(\frac{\ln \left(\tau_{T}-\rho_{i}\right)}{\left(\tau_{T}-\rho_{i}\right)^{2}}\right)
$$

where $\Lambda$ is defined as 


$$
\Lambda=\ln \left(\sqrt{\frac{q}{q+1}}_{2} \sqrt{\rho_{\mathrm{i}}\left(\tau_{\mathrm{T}}-\rho_{\mathrm{i}}\right)}\right)-1
$$

Equation (6.A.1) gives the final polar coordinate $\rho_{f}$ as function of its initial value $\rho_{i}$ and the scaled time of flight $T_{T}$.

The objective of the calculation is to evaluate equations (6.3.7) at $t=t_{f}$, which leads to the final coordinates $a_{f}, b_{f}$ and their derivatives $\dot{a}_{f}$ and $\dot{b}_{f}$. In addition to the final polar distance $\rho_{f}$, one requires the final polar angle $\theta_{f}$ and the final values of the derivatives $[d p / d \tau]_{\text {f }}$ and $[d \theta / d \tau]_{f}$. Following the calculation scheme outlined in section 6.5 (see also figure 6.4) we shall express these quantities in terms of power series in $\rho_{i}, \theta_{i}$ and $\tau_{T}-\rho_{i}$, similar to equation (6.A.1).

In order to obtain a consistent calculation one should beforehand specify to which order in $\rho_{i}, \theta_{i}$ and $\tau_{T}-\rho_{i}$ the various equations are to be expanded. We introduce the following notation to classify the oxder of a term containing some combination of the quantities $\rho_{i}, \theta_{i}$ and $\tau_{T}-\rho_{i}$

$$
0_{-n}=0\left(\frac{\theta_{i}{ }^{1}}{\rho_{i}{ }^{k}\left(\tau_{T}-\rho_{i}\right)^{m}}\right), \quad n=k+1+m
$$

(notice that $\left.\theta_{i} \sim 1 / \rho_{i}\right)$. This classification does not account for logarithmic dependencies and a term $0_{-n}$ may contain a factor $\left[\ln \left(\rho_{i}\right)\right]^{r}\left[\ln \left(\tau_{T}-\rho_{i}\right)\right]^{s}$, with $r$ and some finite positive number.

our aim is to determine the coordinates $a_{f}$ and $b_{f}$ up to and including terms of $0_{-1}$. Accordingly, the expression for the polar distance $\rho_{\mathrm{f}}$ in equation (6.A.1) was developed to this order. Since the highest order term occurring in the expansion of $\rho_{f}$ is of $o_{1}$, one should compute $\sin \left(\theta_{f}\right)$ and $\cos \left(\theta_{f}\right)$ up to and including terms of $0_{-2}$. This guarantees that all terms of $0_{-1}$ will be present in $a_{f}$ and $b_{f}$, as can be seen from equations $(6,3.7)$. The derivatives $\dot{a}_{f}$ and $\dot{b}_{f}$ have to be expanded up to and including terms of $0_{-2}$, because they are multiplied with terms of $0_{1}$.in the extrapolation of the final coordinates to virtual coordinates in the reference plane. As a consequence one should determine $[d \rho / d \tau]_{f}$ up to and including terms of $0_{-2}$ and $[d \theta / d \tau]_{f}$ up to and including terms $0_{-3}$, see again equations $(6,3.7)$.

The final polar angle $\theta_{f}$ is specified by equations $(6.5 .8)$ and $(6.5 .9)$. By using the series expansion

$$
\arccos (x+\delta)=\arccos (x)-\frac{\delta}{\left(1-x^{2}\right)^{1 / 2}}-\frac{\delta^{2} x}{2\left(1-x^{2}\right)^{3 / 2}}+O\left(\delta^{3}\right)
$$

(for $\delta<1$ ) one obtains

$$
\theta_{f}=\theta_{1}+\arccos \left(\frac{1-q}{1+q}\right)-\left(\frac{1}{\rho_{i}}+\frac{1}{\rho_{f}}\right)-\frac{1}{2 \sqrt{q}}\left(\frac{1}{\rho_{i}}+\frac{1}{\rho_{f}^{2}}\right)+0_{-3}
$$

using that $2 \arccos (1 / \varepsilon)=\arccos [(1+q) /(1-q)]$, as follows from the definition of the eccentricity $\epsilon$, given by the second equation of $(6.4 .9)$, and the dynamical result of equations $(6.4 .13)$. Instead of the polar angle $\theta_{f}$ itself, one rather needs the related functions $\sin \left(\theta_{f}\right)$ and $\cos \left(\theta_{f}\right)$. By using the relations 


$$
\begin{aligned}
& \cos \left[\arccos \left(\frac{1-q}{1+q}\right)+\delta\right]=\frac{1-q}{1+q} \cos \delta-\frac{2 \sqrt{q}}{1+q} \sin \delta \\
& \sin \left[\arccos \left(\frac{1-q}{1+q}\right)+\delta\right]=\frac{2 \sqrt{q}}{1+q} \cos \delta+\frac{1-q}{1+q} \sin \delta
\end{aligned}
$$

(as follows with $\sin \{\arccos [(1-q) /(1+q)]\}=2 \sqrt{q} /(1+q)$ ) and the expansions

$$
\cos (\delta)=1-\delta^{2} / 2+O\left(\delta^{4}\right), \quad \sin (\delta)=\delta+O\left(\delta^{3}\right)
$$

one finds from equation (6.A.4)

$$
\cos \left(\theta_{f}\right)=\frac{1-q}{1+q} P-\frac{2 \sqrt{q}}{1+q} Q, \quad \sin \left(\theta_{f}\right)=\frac{2 \sqrt{q}}{1+q} P+\frac{1-q}{1+q} Q
$$

where $\mathrm{P}$ and $\mathrm{Q}$ stand for

$$
\begin{aligned}
& \mathrm{P}=1-\frac{1}{2} \theta_{i} 2+\frac{1}{2}\left(\frac{1}{\rho_{i}}-\frac{1}{\rho_{f}}\right)^{2}+\theta_{i}\left(\frac{1}{\rho_{i}}+\frac{1}{\rho_{f}}\right)+0_{-3} \\
& Q=\theta_{i}-\left(\frac{1}{\rho_{i}}+\frac{1}{\rho_{f}}\right)-\frac{1}{2 \sqrt{q}}\left(\frac{1}{\rho_{i}^{2}}+\frac{1}{\rho_{f}^{2}}\right)+0_{-3}
\end{aligned}
$$

Notice that equations (6.A.5) transform to equations $(6.4 .13)$ for $P=1$ and $Q=0$. These values for $P$ and $Q$ are indeed found from equations (6.A.6) in the limit $\rho_{i}+\infty, \rho_{f}+\infty$ and $\theta_{i} \rightarrow 0$. In order to evaluate the equations (6.A.6) one needs an expression for $1 / \rho_{f}$ in terms of $\rho_{i}$ and $T$. From equation (6.A.1) it follows (using $(1+\delta)-1=1-\delta+0\left(\delta^{2}\right)$ )

$$
\frac{1}{\rho_{f}}=\frac{1}{\tau_{T}-\rho_{i}}+\frac{1}{\left(\tau_{T}-\rho_{i}\right)^{2}} \frac{2 \Lambda}{\sqrt{q}}+0_{-3}
$$

Substitution into the equations (6.A.6) yields

$$
\begin{aligned}
& \mathrm{P}=1-\frac{1}{2}\left(\frac{1}{\rho_{i}}+\frac{1}{\tau_{T}-\rho_{i}}\right)^{2}-\frac{1}{2} \theta_{i}^{2}+\theta_{i}\left(\frac{1}{\rho_{i}}+\frac{1}{\tau_{T}-\rho_{i}}\right)+0_{-3} \\
& Q=\theta_{i}-\left(\frac{1}{\rho_{i}}+\frac{1}{\tau_{T}-\rho_{i}}\right)-\frac{1}{2 \sqrt{q}}\left(\frac{1}{\rho_{i}{ }^{2}}+\frac{1+4 \Lambda}{\left(\tau T-\rho_{i}\right)^{2}}\right)+0_{-3}
\end{aligned}
$$

According to the second equation of $(6.3 .5)$ the initial polar angle $\theta_{i}$ is related to $\rho_{i}$ and the scaled impact parameter $\beta$ as

$$
\theta_{i}=\arcsin \left(\beta / \rho_{i}\right)=\beta / \rho_{i}+0.3, \quad \beta=b / d_{s}
$$

Substitution into equations $(6 . A .8)$ yields

$$
\begin{aligned}
& P=1-\frac{1}{2}\left(\frac{\beta-1}{\rho_{1}}-\frac{1}{\tau_{T}-\rho_{i}}\right)^{2}+0_{-3} \\
& Q=\frac{\beta-1}{\rho_{1}}-\frac{1}{\tau_{T}-\rho_{i}}-\frac{1}{2 \sqrt{q}}\left(\frac{1}{\rho_{1}}+\frac{1+4 \Lambda}{\left(\tau_{T}-\rho_{1}\right)^{2}}\right)+0-3
\end{aligned}
$$

which gives the $f_{i n a l}$ values $\sin \theta_{f}$ and $\cos \theta_{f}$ in terms of $\rho_{i}, T_{T}$ and $\beta$, using equations (6.A.5).

The next step is to determine the derivatives of $[\mathrm{d} \rho / \mathrm{d} \tau]_{\mathrm{f}}$ and $[d \theta / d \tau]_{f}$. Combining equation $(6.5 .10)$ and the first equation of $(6.7 .2)$ 
one obtains

$$
\left(\frac{d \rho}{d \tau}\right)_{f}=\frac{H\left(\rho_{f}\right)}{\rho_{f}}=1-\frac{1}{\sqrt{q}} \rho_{f}^{-1}-\frac{q+1}{2 q} \rho_{f}-2+0-3
$$

With equation (6.A.7) it now follows that

$$
\left(\frac{d \rho}{d \tau}\right)_{f}=1-\frac{1}{\left(\tau_{T}-\rho_{i}\right)} \frac{1}{\sqrt{q}}-\frac{1}{\left(\tau_{T}-\rho_{i}\right)^{2}} \frac{4 \Lambda+q+1}{2 q}+0_{-3}
$$

Substitution of equation (6.A.7) into equation (6.5.11) yields

$$
\left(\frac{d \theta}{d \tau}\right)_{f}=\frac{1}{\left(\tau_{T}-\rho_{i}\right)^{2}}+\frac{1}{\left(\tau_{T}-\rho_{i}\right)^{3}} \frac{4 \Lambda}{\sqrt{q}}+0_{-4}
$$

The equations (6.A.1), (6.A.5), (6.A.10), (6.A.12) and (6.A.13) constitute a complete set of expressions from which the final values of the scaled polar coordinates and their derivatives with respect to the scaled time $\tau$ can be determined from the initial value $\rho_{i}$, the scaled impact parameter $\beta$ (or alternatively the polar angle $\theta_{i}$ ) and the time of flight $T_{T}$. The only additional physical parameter appearing in the expression is the eccentricity related constant $q$, which specifies the strength of the interaction, as can be understood from equation $(6.4 .14)$. This equation indicates that large values of $q$ correspond to weak interactions.

The second part of the calculation consists of the evaluation of the coordinates $a, b$, and their derivatives $\dot{a}$ and $b$. We will denote their micro-scaled equivalents as $\alpha, \beta, \dot{\alpha}$ and $\dot{\beta}$. By scaling equations (6.3.7) transform to ( for $t=t_{f}$ )

$$
\begin{aligned}
& \alpha_{\mathrm{f}}=\mathrm{a}_{\mathrm{f}} / \mathrm{d}_{\mathrm{s}}=\rho_{\mathrm{f}} \cos \left(\theta_{\mathrm{f}}\right) \\
& \beta_{\mathrm{f}}=\mathrm{b}_{\mathrm{f}} / \mathrm{d}_{\mathrm{s}}=\rho_{\mathrm{f}} \sin \left(\theta_{\mathrm{f}}\right) \\
& \dot{\alpha}_{\mathrm{f}}=\dot{\mathrm{a}}_{\mathrm{f}} / \mathrm{v}_{\mathrm{s}}=\left(\frac{\mathrm{d} \rho}{\mathrm{d} \tau}\right)_{\mathrm{f}} \cos \left(\theta_{\mathrm{f}}\right)-\rho_{\mathrm{f}}\left(\frac{\mathrm{d} \theta}{\mathrm{d} \tau}\right)_{\mathrm{f}} \sin \left(\theta_{\mathrm{f}}\right) \\
& \beta_{\mathrm{f}}=\beta_{\mathrm{f}} / \mathrm{v}_{\mathrm{s}}=\left(\frac{\mathrm{d} \rho}{\mathrm{d}_{\mathrm{f}}}\right)_{\mathrm{f}} \sin \left(\theta_{\mathrm{f}}\right)+\rho_{\mathrm{f}}\left(\frac{\mathrm{d} \theta}{\mathrm{d} \tau}\right)_{\mathrm{f}} \cos \left(\theta_{\mathrm{f}}\right)
\end{aligned}
$$

with $v_{s}=d_{s} / t_{s}$, see equation $(6.4 .4)$. By substitution of equations (6.A.5) into equations (6.A.14) one obtains

$$
\begin{array}{lll}
\alpha_{f}=\frac{1-q}{1+q} A-\frac{2 \sqrt{q}}{1+q} B & \beta_{f}=\frac{2 \sqrt{q}}{1+q} A+\frac{1-q}{1+q} B \\
\dot{\alpha}_{f}=\frac{1-q}{1+q} C-\frac{2 \sqrt{q}}{1+q} D & \dot{\beta}_{f}=\frac{2 \sqrt{q}}{1+q} C+\frac{1-q}{1+q} D
\end{array}
$$

in which $A, B, C$ and $D$ are defined as

$$
\begin{array}{ll}
A=\rho_{f} P & B=\rho_{f} Q \\
C=\left(\frac{d \rho}{d \tau}\right)_{f} P-\rho_{f}\left(\frac{d \theta}{d \tau}\right)_{f} Q, & D=\left(\frac{d \rho}{d \tau}\right)_{f} Q+\rho_{f}\left(\frac{d \theta}{d \tau}\right)_{f} P
\end{array}
$$

Substitution of equations (6.A.1), (6.A.5), (6.A.10), (6.A.12) and (6.A.13) yields 


$$
\begin{aligned}
& A=\tau_{T}-\rho_{i}-\frac{2 \Lambda}{\sqrt{q}}+\frac{1}{\rho_{i}}\left(\frac{3(q+1)}{2 q}-\beta\right)+\frac{1}{\tau_{T}-\rho_{i}} \frac{4 \Lambda+3}{2 q}-\frac{\tau_{T}-\rho_{i}}{2 \rho_{i}^{2}}(\beta-1)^{2}+0_{-2} \\
& B=-1+\frac{\beta-1}{\rho_{i}}\left(\tau_{T}-\rho_{i}-\frac{2 \Lambda}{\sqrt{q}}\right)-\frac{1}{2 \sqrt{q}}\left(\frac{1}{\tau_{T}-\rho_{i}}+\frac{\tau_{T}-\rho_{i}}{\rho_{i}^{2}}\right)+0.2 \\
& C=1-\frac{1}{\tau_{T}-\rho_{i}} \frac{1}{\sqrt{q}}-\frac{(\beta-1)^{2}}{2 \rho_{i}^{2}}-\frac{1}{\left(\tau_{T}-\rho_{i}\right)^{2}} \frac{4 \Lambda+1}{q}-\frac{2(\beta-1)}{\rho_{i}\left(\tau_{T}-\rho_{i}\right)}+0_{-3} \\
& D=\frac{\beta-1}{\rho_{i}}-\frac{1}{2 \sqrt{q}}\left(\frac{1}{\rho_{i}^{2}}+\frac{2(\beta-1)}{\rho_{i}\left(\tau_{T}-\rho_{i}\right)}-\frac{1}{\left(\tau_{T}-\rho_{i}\right)^{2}}\right)+0-3
\end{aligned}
$$

The equations (6.A.15) and (6.A.17) specify the final coordinates in the $(\hat{a}, \hat{b})$ reference system as function of the initial coordinates $\rho_{i}$ and $\beta$, the time of flight $\tau_{T}$ and the parameter $q$. All coordinates are still scaled and the next step is to remove this scaling. The scale parameters $d_{s}$ and $t_{s}$ are given by equation $(6.4 .4)$. At this point it should be noted that $d_{s}$ and $t_{s}$ depend on the energy $E$ and therefore on the initial distance $r_{i}$, see equation (6.2.15). This is also the case for the parameter $q$, as can be seen from equation $(6.4 .15)$. These dependencies can be made explicit by using

$$
\begin{aligned}
& \mathrm{d}_{s}=\frac{\mathrm{J}}{\sqrt{\mathrm{mE}}}=\mathrm{b}\left(1+\frac{2 \mathrm{~h}}{\mathrm{r}_{\mathrm{i}}}\right)^{-1 / 2}=\mathrm{b}\left(1-\frac{\mathrm{h}}{\mathrm{r}_{\mathrm{i}}}+\frac{3}{2} \frac{\mathrm{h}^{2}}{\mathrm{r}_{\mathrm{i}}{ }^{2}}+0_{-3}\right) \\
& \mathrm{v}_{\mathrm{s}}=\frac{\mathrm{d}_{\mathrm{s}}}{\mathrm{t}_{\mathrm{s}}}=\frac{2 \sqrt{\mathrm{E}}}{\sqrt{\mathrm{m}}}=\mathrm{v}_{\mathrm{i}}\left(1+\frac{2 \mathrm{~h}}{\mathrm{r}_{\mathrm{i}}}\right)^{1 / 2}=\mathrm{vi}\left(1+\frac{\mathrm{h}}{\mathrm{r}_{\mathrm{i}}}-\frac{1}{2} \frac{\mathrm{h}^{2}}{\mathrm{r}_{\mathrm{i}}{ }^{2}}+0_{-3}\right) \\
& \mathrm{q}=\mathrm{q}_{\mathrm{c}}\left(1+\frac{2 \mathrm{~h}}{\mathrm{r}_{\mathrm{i}}}\right)
\end{aligned}
$$

in which $h$ and $q_{c}$ are defined as

$$
\mathrm{h}=\frac{2 \mathrm{C}_{0}}{\mathrm{mv}_{\mathrm{i}}^{2}} \quad, \quad \mathbf{q}_{\mathrm{c}}=\left(\frac{\mathrm{b}}{\mathrm{h}}\right)^{2}=\left(\frac{\mathrm{mbv}_{\mathrm{i}}{ }^{2}}{2 \mathrm{C}_{0}}\right)^{2}
$$

Substitution of equations (6.A.18) into equations (6.A.15) and (6.A.16) finally gives (after some reorganization of the resulting terms)

$$
\begin{aligned}
& a_{f}=\frac{1-q_{c}}{1+q_{c}}\left[T v_{i}-r_{i}-2 h \Lambda_{c}+h \frac{T v_{i}}{r_{i}}\right]+\frac{2 \sqrt{q_{c}}}{1+q_{c}}\left[b-\frac{2 b}{1+q_{c}}\left(\frac{T v_{i}}{r_{i}}-1\right)\right]+0_{-1} \\
& b_{f}=\frac{2 \sqrt{q_{c}}}{1+q_{c}}\left[T v_{i}-r_{i}-2 h \Lambda_{c}+\frac{2 h}{1+q_{c}} \frac{T v_{i}}{r_{i}}\right]-\frac{1-q_{c}}{1+q_{c}}\left[b+\frac{2 b}{1+q_{c}}\right]+0-1 \\
& \dot{a}_{f}=\frac{1-q_{c}}{1+q_{c}}\left[v_{i}+\frac{h v_{i}}{r_{i}}-\frac{h v_{i}}{T v_{i}-r_{i}}\right]-\frac{4 q_{c}}{\left(1+q_{c}\right)^{2}} \frac{h v_{i}}{r_{i}}+0_{-2} \\
& \dot{b}_{f}=\frac{2-\sqrt{q_{c}}}{1+q_{c}}\left[v_{i}+\frac{h v_{i}}{r_{i}} \frac{2}{1+q_{c}}-\frac{h v_{i}}{T v_{i}-r_{i}}\right]+0-2
\end{aligned}
$$

in which $\Lambda_{c}$ is defined as

$$
\Lambda_{c}=\ln \left(\sqrt{\frac{q_{c}}{q_{c}+1}} \frac{2}{b} \sqrt{r_{i}\left(T v_{i}-r_{i}\right)}\right)-1
$$


similar to equation (6.A.2). The expansions of equations (6.A.20) include one order less than those of equations (6.A.17). We did carry out the expansion one order further, but the resulting expressions became impractically large and are therefore omitted.

The deviations from the unperturbed coordinates follow by equations (6.3.9). The equation for $\Delta a$ contains the quantity $a_{0}-v_{i} t$ (note $v_{i}=\tilde{v}_{0}$ ). For $t=t_{i}$ one may write (using that $\sigma_{i}=-1$ and $\sigma_{i}=+1$ )

$$
a_{0}-v_{i} t_{f}=a_{0}-v_{i} t_{i}-v_{i} T=r_{i}-v_{i} T-\frac{b^{2}}{2 r_{i}}+0-2
$$

as follows by expanding and inverting the expression for $r_{i}$ given by equation (6.3.5) and using that $T=t_{f}-t_{i}$. By substitution of equations (6.A.20) into equations $(6.3 .9)$ one obtains

$$
\begin{aligned}
& \Delta a_{f}=\frac{2\left(T v_{i}-r_{i}\right)}{1+q_{c}}-\frac{1-q_{c}}{1+q_{c}}\left[2 h \Lambda_{c}-h \frac{T v_{i}}{r_{i}}\right]+\frac{2 \sqrt{q_{c}}}{1+q_{c}}\left[b-\frac{2 b}{1+q_{c}}\left(\frac{T v_{i}}{r_{i}}-1\right)\right]+0-1 \\
& \Delta b_{f}=\frac{2 \sqrt{q_{c}}}{1+q_{c}}\left[T v_{i}-r_{i}-2 h \Lambda_{c}+\frac{2 h}{1+q_{c}}\left(\frac{T v_{i}}{r_{i}}-1\right)\right]+0_{-1} \\
& \Delta \dot{a}_{f}=\frac{2 v_{i}}{1+q_{c}}+\frac{1-q_{c}}{1+q_{c}}\left[\frac{h v_{i}}{r_{i}}-\frac{h v_{i}}{T v_{i}-r_{i}}\right]-\frac{4 q_{c}}{\left(1+q_{c}\right)^{2}} \frac{h v_{i}}{r_{i}}+0-2 \\
& \Delta \dot{b}_{f}=\frac{2 \sqrt{q_{c}}}{1+q_{c}}\left[v_{i}+\frac{h v_{i}}{r_{i}} \frac{2}{1+q_{c}}-\frac{h v_{i}}{T v_{i}-r_{i}}\right]+0.2
\end{aligned}
$$

which completes the calculation. 


\section{BOERSCH EFPECT}

\subsection{Introduction}

In this chapter the Boersch effect will be calculated by means of the extended two-particle approach. This approach was outlined in chapter 5. It utilizes the two-particle dynamics of chapter 6 . The analysis presented here pertains to a rotational symmetric beam segment in drift space.

The chapter starts with a summary of the relevant results obtained so far. This material is then used to calculate the Boersch effect in two specific beam geometries which are most suited for an analytical treatment of the problem. One is a beam segment with a narrow crossover and the other is a homocentric cylindrical beam segment. In the former the Boersch effect is generated by relaxation of kinetic energy, in the latter by relaxation of potential energy. The general case of a beam segment with a crossover of arbitrary dimensions is considered next. The angular and spatial distribution in the crossover are taken uniform in most calculations. However, Gaussian distribution(s) are considered too, leading to the definition of some effective width measures for which the results found for uniform distributions do apply. For all geometries the full energy distribution, produced by the Boersch effect, is computed. Explicit expressions are presented for the Full width at Half Maximum (FWHM) of this distribution, as well as the Full width median value $\left(\mathrm{FW}_{50}\right)$ and the root mean square value (rms). The number of independent parameters in the model is reduced by utilizing scaled (dimensionless) quantities. This also simplifies the notation of the intermediate results. In the final results the scaling is removed in order to make the dependency on the experimental parameters explicit.

A computer program has been developed which can perform every step of the calculation numerically, following the scheme of the extended two-particle approach. First, it calculates the two-particle distribution, utilizing the analytical results for the two-particle dynamical problem. Next, it performs two steps, each corresponding to a Fourier-type of integral transform. This finally yields the manyparticle velocity displacement distribution, which is equivalent to the energy distribution generated by the Boersch effect. The most surprising result of these investigations is probably that one has to distinguish a variety of regimes depending on the particle density in the beam and the beam geometry. Each regime corresponds to a different type of energy distribution. In the general case of a beam segment of arbitrary dimensions one ends up with four regimes: The Gaussian regime, the Lorentzian regime, the Holtsmark regime and the pencil beam regime. The widths of the distinguished energy distributions show different dependencies on the experimental parameters.

Where possible the numerical calculations are verified by analytical means. Analytical results for the width of the energy distribution can be obtained for all limiting cases were a single regime is dominant. A direct analytical calculation of the behaviour of the energy distribution in the transition areas between the regimes seems not possible. The single exception is the transition between Gaussian and Lorentzian regime in a beam segment with a narrow crossover. In order to increase the applicability of the model, the numerical data for the FWHM and the FW5o of the energy distribution is approximated with analytical expressions, covering the entire range of operating conditions. 
The Boersch effect corresponds to a broadening of the distribution of axial velocities $\rho\left(\Delta v_{2}\right)$ due to statistical Coulomb interactions between the beam particles. We will utilize the extended two-particle approach to compute this phenomenon. This model was outlined in chapter 5 . The dynamical part of the problem consists of the calculation of the axial velocity shift $\Delta \mathrm{v}_{z}$ experienced by the test particle due to the interaction with a single field particle. This problem was studied in chapter 6 . The shift $\Delta v_{2}$ can be expressed as a function of the geometrical variables $=\left(r_{1}, b_{2}, v, \Phi\right)$, the time of flight $T$ and the initial time $t_{i}=-S_{c} T$, see equations $(6.2 .1),(6.2 .2)$ and (6.2.3) respectively and figure 5.1 (note $\Phi=\psi-\varphi)$. Explicit analytical equations can be determined for complete collisions and for weak collisions. For a complete collision we found

$$
\Delta v_{z} \equiv \frac{m v^{3} b_{z}}{2 C_{0}\left[1+\left(b_{z}^{2}+r_{\perp}^{2} \sin ^{2} \Phi\right)\left(m v^{2} / 2 C_{0}\right)^{2}\right]}
$$

see equations $(6.10 .4),(6.4 .16)$ and $(6.3 .4)$. The effect of a weak collision can be described by (reproducing equation $(6.10 .5)$ )

$$
\Delta v_{z} \cong \frac{C_{0} b_{z}}{m b^{2}}\left[\frac{s_{c} T v+a_{0}}{\left[\left(S_{c} T v+a_{0}\right)^{2}+b^{2}\right]^{1 / 2}}+\frac{\left(1-S_{c}\right) T v-a_{0}}{\left\{\left(\left(1-S_{c}\right) T v-a_{0}\right)^{2}+b^{2}\right\}^{1 / 2}}\right]
$$

in which

$$
a_{0}=-r_{\perp} \cos \Phi, \quad b=\left(b_{z}^{2}+r_{\perp}{ }^{2} \sin ^{2} \Phi\right) 1 / 2
$$

Equations $(7.2 .1)$ and $(7.2 .2)$ apply to the case that the initial relative velocity of the particles is non-zero $(v \neq 0)$. In case $v=0$ we found for a half-complete collision

$$
\Delta v_{z} \cong\left(\frac{C_{0}}{m}\right)^{1 / 2} \frac{b_{2}}{\left(b_{2}^{2}+r_{\perp}^{2}\right)^{3 / 4}} \quad(v=0)
$$

as follows from equation (6.10.7), taking $\mathrm{T} \rightarrow \infty$. For a weak collision we found (reproducing equation $(6.10 .8)$ )

$$
\Delta v_{z} \equiv \frac{C_{0}}{m} \frac{b_{z} T}{\left(b_{z}^{2}+r_{\perp}^{2}\right)^{3 / 2}} \quad(v=0)
$$

Equations (7.2.1) and (7.2.2) will be used in the calculation of the Boersch effect generated in a beam segment with a crossover, while equations $(7.2 .4)$ and $(7.2 .5)$ will be used for a homocentric cylindrical beam segment. Collisions which are neither complete nor weak will be evaluated numerically, following the procedure outlined in section 6.6 .

The statistical part of the problem consists of the evaluation of equations $(5.7 .4),(5.7 .5)$ and $(5.7 .6)$. Taking $\Delta \eta=\Delta v_{z}$ these equations become

$$
\begin{aligned}
& \rho_{2}\left(\Delta v_{z}\right)=\int_{0}^{v_{0}} \frac{2 v d v}{v_{0}{ }^{2}} \int_{0}^{2 \pi} \frac{d \Phi}{2 \pi} \int_{0}^{r_{c}} \frac{2 r_{\perp} d r_{\perp}}{r_{c}{ }^{2}} \int_{-S_{c} L}^{\left(1-S_{c}\right) L} d b_{z} \delta\left[\Delta v_{z}-\Delta v_{z}\left(v, \Phi, r_{\perp}, b_{z}\right)\right] \\
& (7.2 .6) \\
& p(k)=2 \int_{-\infty}^{\infty} d \Delta v_{z} \rho_{2}\left(\Delta v_{z}\right) \sin ^{2}\left(k \Delta v_{z} / 2\right)
\end{aligned}
$$




$$
\rho\left(\Delta v_{z}\right)=\frac{1}{\pi} \int_{0}^{\infty} d k \cos \left(k \Delta v_{z}\right) e^{-\lambda p(k)}
$$

in which we expressed $p(\xi) d \xi$ directly in terms $v, \Phi, r_{\perp}$ and $b_{z}$, using equations (5.4.6) and (5.3.2). The distribution in $v$ and $r_{\perp}$ are here taken uniform with a cut-of $f$ at $v_{0}=\alpha_{0} v_{z}$ and $r_{c}$ respectively, as prescribed by equation (5.3.3). In section 7.6 we will consider the case that one of these distribution or both of them are Gaussian. Equations $(7.2 .6),(7.2 .7),(7.2 .8)$ constitute the basis of our three-step approach, which was discussed in section 5.4. The function $\rho_{2}\left(\Delta v_{z}\right)$ is called the two-particle distribution. The function $p(k)$ represents its transform to the k-domain. Both functions are entirely determined by the geometrical properties of the beam only, represented by $v_{0}, r_{c}$ and $L$. The linear particle density $\lambda$ enters the model in the last step, which yields the (N-particle) displacement distribution $\rho\left(\Delta v_{z}\right)$.

We recall that our model for statistical interactions relies on the following assumptions :

- The field particles can be considered as statistically independent, as expressed by equation (5.3.1).

- The total displacement of the test particle is equal to the sum of all displacements experienced in the two-particle interactions with the individual field particles, as expressed by equation (5.3.12).

- Magnetic interactions and relativistic effects can be ignored. Accordingly, the effect is assumed to be entirely the result of Coulomb interactions.

In this chapter we make some further simplifications which facilitate the calculations considerably :

- The reference trajectory, which is the unperturbed trajectory of the test-particles, coincides with the beam axis. It is assumed that the effect experienced by the test-particles running along this (on-axis) trajectory is representative for other (off-axis) trajectories as well.

- The beam is initially monochromatic with respect to the normal energy of the particles, which implies that all particles run initially with identical axial velocity. It is assumed that the impact of statistical interactions on the properties of the beam does not depend on the initial energy spread, which will be present in practical beams.

In chapter 10 we will present the results of some further calculations, which do not rely on these simplifications. The results confirm the validity of the approach taken here.

Finally, we emphasize that all calculations are restricted to beams which fulfil the following conditions :

- The beam is rotational symmetric.

- The beam is paraxial, which means that $\operatorname{tg} \alpha_{0} a_{0}$ and $r_{c}$ are small.

- External forces are absent, thus the particles fly through drift space.

\subsection{Beam segment with a narrow crossover}

To indicate whether a crossover should be considered as narrow or not we introduce the characteristic beam geometry quantities $K, K_{1}$ and $\mathrm{K}_{2}$ 


$$
\mathrm{K}=\frac{\alpha_{0} \mathrm{~L}}{2 \mathrm{r}_{\mathrm{c}}} \quad, \quad \mathrm{K}_{1}=2 \mathrm{KS}_{\mathrm{c}} \quad, \quad \mathrm{K}_{2}=2 \mathrm{~K}\left(1-\mathrm{S}_{\mathrm{c}}\right)
$$

employing the experimental parameters defined in section $3.2 . K_{1}$ is the ratio between the beam radius at the start of the beam segment and the crossover radius. $K_{2}$ is the corresponding ratio referring to the end of the beam segment. A crossover is called narrow if it has the characteristic $K \gg>1$. This condition implies $K_{1} \gg 1$ and $K_{2} \gg 1$, provided that the crossover is located somewhere near the middle of the beam segment $\left(S_{c} \approx 0.5\right)$.

In this section we will calculate the Boersch effect in a beam segment with the characteristics $K_{1} \gg>1$ and $K_{2} \gg 1$. Physically this implies that the main effect comes from collisions which are complete or nearly complete. Accordingly, one may use equation (7.2.1) to determine the shift $\Delta \mathrm{v}_{z}$ of the test particle caused by the interaction with a single field particle. It should be noted that the initial and final potential energy of two particles involved in a complete collision is effectively zero. A complete collision does not affect the total kinetic energy of the particles, but merely leads to directional changes and a redistribution of the total kinetic energy. Accordingly, a calculation based on this type of collisions implicitly assumes that the Boersch effect stems from a conversion of kinetic energy from one degree of freedom into another (more specifically from the lateral degrees of freedom into the longitudinal degree of freedom). This approach is justified for the present case of a narrow crossover as will be shown in section 7.5, were we treat the general case, using full collision dynamics.

The program of this section is to evaluate the two-particle distribution $\rho_{2}\left(\Delta v_{z}\right)$ from equations (7.2.1) and (7.2.6). This distribution will be used to determine the $\mathrm{N}$-particle displacement distribution $\rho\left(\Delta \mathrm{v}_{z}\right)$ by means of equations $(7.2 .7)$ and $(7.2 .8)$. It is convenient to introduce the following scaling-quantities for length and velocity respectively

$$
\mathrm{d}_{0}=\frac{\mathrm{C}_{0}}{\mathrm{mv}_{0}^{2}} \quad, \quad \mathrm{v}_{0}=\alpha_{0} \mathrm{v}_{2}
$$

$v_{0}$ is the maximum transverse velocity of a particle and $d_{0}$ is the closest distance of approach for two particles starting out with a relative velocity $2 \mathrm{v}_{0}$. The objective of this scaling is to simplify the equations by isolating key-combinations of experimental parameters. For an electron beam with $\mathrm{V}=10 \mathrm{kV}$ and $\alpha_{0}=10 \mathrm{mR}$ one finds $\mathrm{d}_{0}=0.72 \mathrm{~nm}$ and $v_{0}=5.9 \times 10^{5} \mathrm{~m} / \mathrm{s}$, which can be considered as typical values. All quantities scaled with $d_{0}$ and/or $v_{0}$ are headed with a bar.

Employing the do, $v_{0}-s c a l i n g$ equation $(7.2 .1)$ transforms to

$$
\Delta \bar{v}_{z}=\frac{\Delta v_{z}}{v_{0}}=\frac{\bar{v}^{3} \bar{b}_{z}}{2\left[1+\left(\bar{b}_{z}^{2}+\bar{r}_{\perp}^{2} \sin ^{2} \Phi\right) \bar{v}^{4} / 4\right]}
$$

The scaled two-particle distribution $\bar{\rho}_{2}\left(\Delta \vec{v}_{z}\right)$ follows with equation $(7.2 .6)$

$$
\bar{\rho}_{2}\left(\Delta \bar{v}_{z}\right)=\frac{v_{0}}{d_{0}} \rho_{2}\left(\Delta v_{z}\right)=\int_{0}^{1} 2 \bar{v} d \bar{v} \int_{0}^{2 \pi} \frac{d \Phi}{2 \pi} \int_{0}^{\frac{\bar{r}_{c}}{2 \bar{r}_{\perp}} d \bar{r}_{\perp}} \frac{\bar{r}_{c}{ }^{2}}{d} \int_{-\infty}^{\infty} \delta\left[\Delta \bar{v}_{z}-\Delta \bar{v}_{z}\left(\bar{v}, \Phi, \bar{r}_{\perp}, \bar{b}_{z}\right)\right]
$$

in which $\Delta \bar{v}_{z}\left(\bar{v}, \Phi, \bar{r}, \bar{b}_{z}\right)$ is given by equation $(7.3 .3)$ and $\bar{r}_{c}$ is the scaled 
crossover radius. In terms of the experimental parameters this parameter can be expressed as

$$
\bar{r}_{c}=\frac{r_{c}}{d_{0}}=\frac{8 \pi \epsilon_{0}}{e} \alpha_{0}{ }^{2} V_{c}
$$

as follows with equations (7.3.2). We evaluated the integrals of equation (7.3.4) numerically for different values of the scaled crossover radius $\bar{r}_{c}$. The results are shown in figure 7.1 .

$$
\text { It would be convenient to evaluate equation (7.3.4) }
$$
analytically. However, this is only possible in some special cases. For a homocentric beam $\left(\bar{r}_{c}=0\right)$, equation $(7.3 .4)$ reduces to a twodimensional integral over $\overrightarrow{\mathbf{v}}$ and $\bar{b}_{z}$, which can be carried out analytically. For $\bar{r}_{c} \neq 0$ one can obtain a good analytical approximation when $\bar{r}_{c} \Delta \bar{v}_{z} \gg 1$ (large crossover and/or large velocity shift), or oppositely when $\bar{r}_{\mathrm{c}} \Delta \overline{\mathrm{v}}_{\mathrm{z}}<<1$. In order to investigate these individual cases we will first bring equation (7.3.4) in a different form.

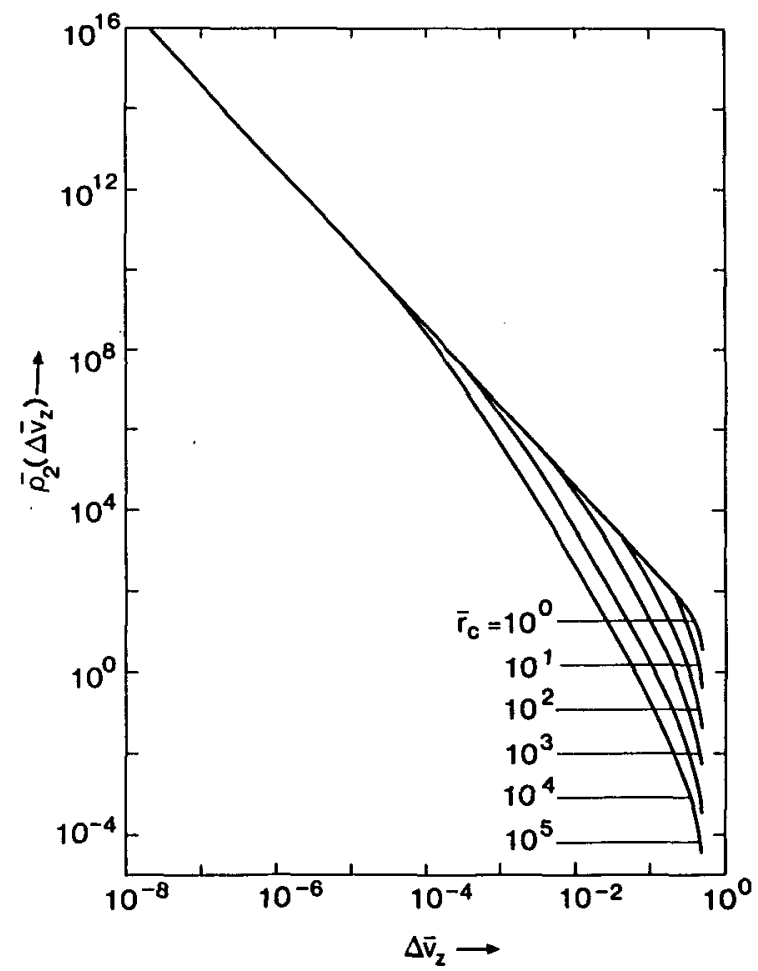

Figure 7.I The scaled two-particle distribution $\bar{\rho}_{2}\left(\Delta \bar{v}_{2}\right)$ based on complete collisions, for different values of the scaled crossover radius $\overline{\mathrm{Y}}_{\mathrm{t}}$. 
One can rewrite equation (7.3.4) using the following identity

$$
\int_{0}^{2 \pi} \frac{d \Phi}{2 \pi} \int_{0}^{\frac{\bar{r}_{c}}{2 \bar{r}_{\perp}} d \bar{r}_{\perp}} \frac{4}{\bar{r}_{c}{ }^{2}} \int_{0}^{1} d y \sqrt{1-y^{2}}
$$

with $y=\left(\bar{r}_{f} / \bar{r}_{c}\right) \sin \Phi$. This is allowed since the expression for $\Delta \bar{v}_{z}$, given by equation (7.3.3), depends on $\bar{r}_{1}$ and $\Phi$ only through the combination $\overline{\mathbf{r}}_{1} \sin \Phi$. In addition we substitute

$$
z=\bar{b}_{z} \bar{v}^{2} / 2
$$

Equation (7.3.4) now transforms to

$$
\bar{\rho}_{2}\left(\Delta \bar{v}_{z}\right)=\frac{16}{\pi} \int_{0}^{\frac{d}{d} \bar{v}} \int_{0}^{1} d y \sqrt{1-y^{2}} \int_{0}^{\infty} d z \delta\left(\Delta \bar{v}_{z}-\frac{z \bar{v}}{z^{2}+R^{2}}\right), \quad \Delta \bar{v}_{z} \geqslant 0
$$

where $R=R(\bar{v}, y)$ is defined as

$$
R(\bar{v}, y)=\left(1+\bar{v}^{4} y^{2} \bar{r}_{c}^{2 / 4}\right)^{1 / 2}
$$

As the distribution $\bar{\rho}_{2}\left(\Delta \bar{v}_{z}\right)$ is symmetric in $\Delta \bar{v}_{z}$ it is sufficient to evaluate it for positive values of $\Delta \bar{v}_{z}$. Accordingly, we limited the $z-$ integration in equation $(7.3 .6)$ to positive values only. One sees that the second term in the argument of the $\delta$-function has a maximum for $z=R$. We now split the $z$-integration into the intervals $[0, R]$ and $[R, \infty]$ and substitute $z=R^{2} / z$ in the second interval. It is here essential to note that the integrant is not affected by this substitution. The $z-$ integral of equation $(7.3 .6)$ now transforms to

$$
\int_{0}^{R} d z\left(1+\frac{R^{2}}{z^{2}}\right) \delta\left(\Delta \bar{v}_{z}-\frac{z \bar{v}}{z^{2}+R^{2}}\right)=\int_{u=0}^{u=1}-2 R d\left(\frac{\sqrt{1-u^{2}}}{u}\right) \delta\left(\Delta \bar{v}_{z}-\frac{u \bar{v}}{2 R}\right)
$$

In the last step we substituted $u=2 R z /\left(z^{2}+R^{2}\right)$. Equation $(7.3 .6)$ becomes

$$
\bar{\rho}_{2}\left(\Delta \bar{v}_{z}\right)=\frac{32}{\pi} \int_{0}^{1} \frac{d \bar{v}}{\bar{v}} \int_{0}^{1} d y \sqrt{1-y^{2}} \int_{0}^{1} \frac{R(\bar{v}, y) d u}{u^{2}\left(1-u^{2}\right)^{1 / 2}} \delta\left(\Delta \bar{v}_{z}-\frac{u \bar{v}}{2 R(\bar{v}, y)}\right)
$$

which can be rewritten as (for $0<\Delta \bar{v}_{z}<1 / 2$ )

$$
\bar{\rho}_{2}\left(\Delta \bar{v}_{z}\right)=\frac{16}{\pi \Delta \bar{v}_{z}^{2}} \int_{2 \Delta \bar{v}_{z}}^{1} d \bar{v} \int_{0}^{\left.\min \left(1,\left(1-4 \Delta \bar{v}_{z}^{2} / \bar{v}^{2}\right)^{1 / 2} / \Delta \bar{v}_{z} \bar{v}_{c}\right)\right]} \frac{\left(1-y^{2}\right)^{1 / 2}}{\left[1-\Delta \bar{v}_{z}^{2}\left(4 / \bar{v}^{2}-\bar{v}^{2} y^{2} \bar{r}_{c}^{2}\right)\right]^{1 / 2}}
$$

Equation (7.3.9) will be used later on to evaluate the function $p(k)$, while equation $(7.3 .10)$ is most suited to study the special cases mentioned previously. For a homocentric beam $\left(\bar{r}_{c}=0\right)$ equation $(7.3 .10)$ becomes

$$
\bar{\rho}_{2}\left(\Delta \bar{v}_{z}\right)=\frac{4\left(1-4 \Delta \bar{v}_{z}^{2}\right) 1 / 2}{\Delta \bar{v}_{z}^{2}} \quad\left(\bar{r}_{c}=0, \quad 0<\Delta \bar{v}_{z}<1 / 2\right)
$$


For small values of $\bar{r}_{c}$ and small values of $\Delta \bar{v}_{z}\left(\bar{r}_{c} \Delta \bar{v}_{z} \ll<\right)$ one finds from equation $(7.3 .10)$

$$
\bar{\rho}_{2}\left(\Delta \bar{v}_{z}\right) \cong \frac{4}{\Delta \bar{v}_{z}^{2}} \quad\left(\bar{r}_{\mathrm{c}} \Delta \overline{\mathrm{v}}_{z}<<1,0<\Delta \overline{\mathrm{v}}_{\mathrm{z}}<1 / 2\right)
$$

For the opposite case $\left(\overline{\mathrm{r}}_{\mathrm{c}} \Delta \overline{\mathrm{v}}_{z} \gg>1\right)$ equation $(7.3 .10)$ can be approximated as

$$
\bar{\rho}_{2}\left(\Delta \overline{\mathrm{V}}_{\mathbf{z}}\right) \cong \frac{8}{\Delta \overline{\mathrm{V}}_{\mathrm{z}}{ }^{3} \overline{\mathrm{r}}_{\mathrm{c}}} \ln \left(\frac{1}{2 \Delta \overline{\mathrm{v}}_{\mathrm{z}}}\right) \quad\left(\overline{\mathrm{r}}_{\mathrm{c}} \Delta \overline{\mathrm{v}}_{\mathbf{z}}>>1,0<\Delta \overline{\mathrm{v}}_{\mathbf{z}}<1 / 2\right)
$$

The behaviour of the two-particle function $\bar{\rho}_{2}\left(\Delta \bar{v}_{2}\right)$ described by equations $(7.3 .11),(7.3 .12)$ and $(7.3 .13)$ is confirmed by the numerical data plotted in figure 7.1 .

We now proceed with the calculation of the function $p(k)$ and the displacement distribution $\rho\left(\Delta v_{z}\right)$. According to equation (7.2.7) one can express the scaled function $\overline{\mathrm{p}}(\bar{k})$ as

$$
\bar{p}(\bar{k})=\frac{p(k)}{d_{0}}=2 \int_{-\infty}^{\infty} d \Delta \bar{v}_{2} \bar{\rho}_{2}\left(\Delta \bar{v}_{z}\right) \sin ^{2}\left(\bar{k} \Delta \bar{v}_{z} / 2\right)
$$

with $\overline{\mathbf{k}}=\mathrm{kv}_{0}$. Substitution of equation (7.3.9) and partial integration with respect to $u$, yields

$$
\bar{p}(\bar{k})=\bar{k} \frac{32}{\pi} \int_{0}^{l} d \bar{v} \int_{0}^{1} d y \sqrt{1-y^{2}} \int_{0}^{1} d u \frac{\left(1-u^{2}\right)^{1 / 2}}{u} \sin \left(\frac{\bar{k} \bar{v} u}{\left(4+\bar{v}^{4} y^{2} \bar{r}_{c}^{2}\right)^{1 / 2}}\right)
$$

This expression was evaluated numerically for different values of the scaled crossover radius $\bar{r}_{c}$. The results are shown in figure 7.2 . One sees that the dependency on $\bar{k}$ is quadratic for small values of $\bar{k}$, particularly in combination with large $\bar{r}_{c}$. The dependency becomes linear for large $\bar{k}$-values.

The integrals in equation (7.3.15) can be simplified for the extreme cases $\vec{k} \rightarrow 0$ and $\vec{k} \rightarrow \infty$. For $\bar{k} \rightarrow 0$ one may replace the sin-function by its argument and carry out the u-integration, which yields a factor $\pi / 4$. The integration over $\bar{v}$ can be performed next and one obtains

$$
\overline{\mathrm{p}}(\overline{\mathrm{k}})=\frac{1}{2} \overline{\mathrm{p}}_{2}\left(\overline{\mathrm{r}}_{\mathrm{c}}\right) \overline{\mathrm{k}}^{2}, \quad \overline{\mathrm{p}}_{2}\left(\overline{\mathrm{r}}_{\mathrm{c}}\right)=\frac{8}{\overline{\bar{r}}_{\mathrm{c}}} \int_{0}^{1} \mathrm{dy} \frac{\sqrt{1-\mathrm{y}^{2}}}{\mathrm{y}} \sinh ^{-1}\left(\frac{\overline{\mathrm{r}}_{\mathrm{c}} \mathrm{y}}{2}\right)(\overline{\mathrm{k}} \rightarrow 0)
$$

(note that $\sinh ^{-1}(x)=\ln \left[x+\left(1+x^{2}\right)^{1 / 2}\right]$ ). For a homocentric beam $\bar{r}_{c}=0$ and one finds $\bar{p}_{2}=\pi$. The function $\bar{p}_{2}\left(\bar{r}_{c}\right) / \pi$ is drawn in figure 7.3 . For later use we fitted this function with

$$
\overline{\mathbf{p}}_{2 \mathrm{a}}\left(\overline{\mathrm{r}}_{\mathrm{c}}\right)=\frac{\pi}{1+\pi \bar{r}_{\mathrm{c}} /\left\{2 \ln \left[.8673\left(114.6+\overline{\mathrm{r}}_{\mathrm{c}}\right)\right]\right\}^{2}}
$$

which has the same asymptotic behaviour for $\overline{\mathbf{r}}_{\mathrm{c}} \rightarrow 0$ and $\overline{\mathbf{r}}_{\mathrm{c}}+\infty$ as $\overline{\mathbf{p}}_{2}\left(\overline{\mathbf{r}}_{\mathrm{c}}\right)$. For comparison we included the fit $\bar{p}_{2 a}\left(\bar{r}_{c}\right) / \pi$ in figure 7.3 . The accuracy of the fit is better than $2 \%$ for all $\bar{r}_{c}$ values.

$$
\text { In the limit } \bar{k} \rightarrow \infty \text { one can simplify equation }(7.3 .15) \text { by }
$$

exploiting the following representation of the $\delta$-function 


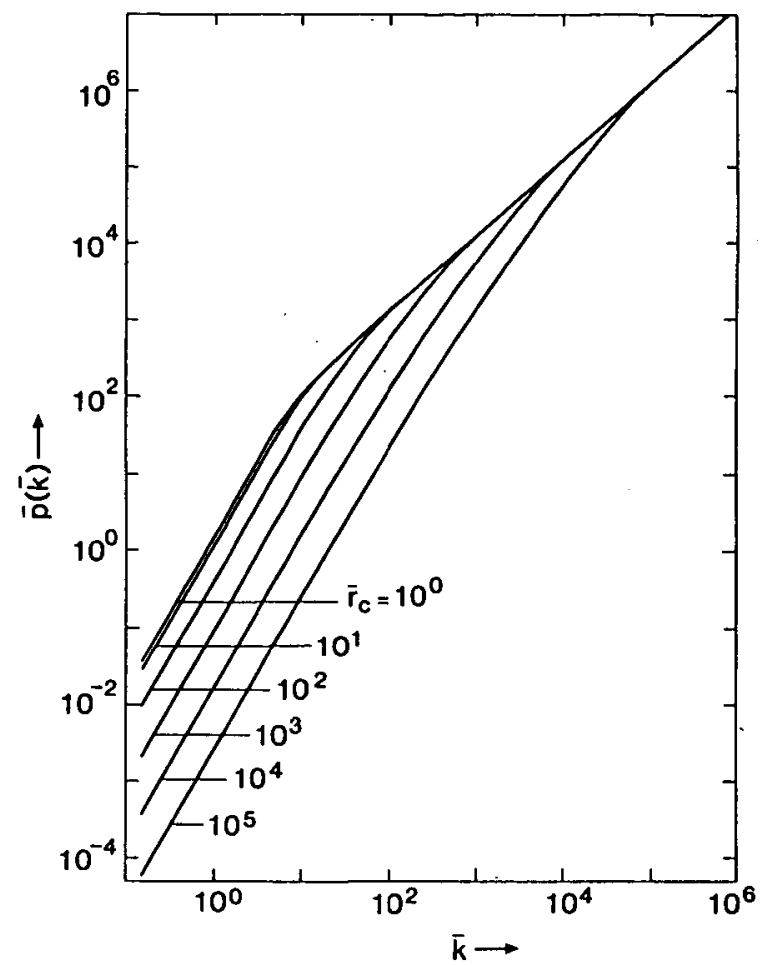

Figure 7.2 The scaled function $\overline{\mathrm{p}}(\bar{K})$ corresponding to the distribution of axial velocity displacements generated by complete collisions, for different values of the scaled crossover radius $\overline{\mathbf{r}}_{\mathrm{c}}$.

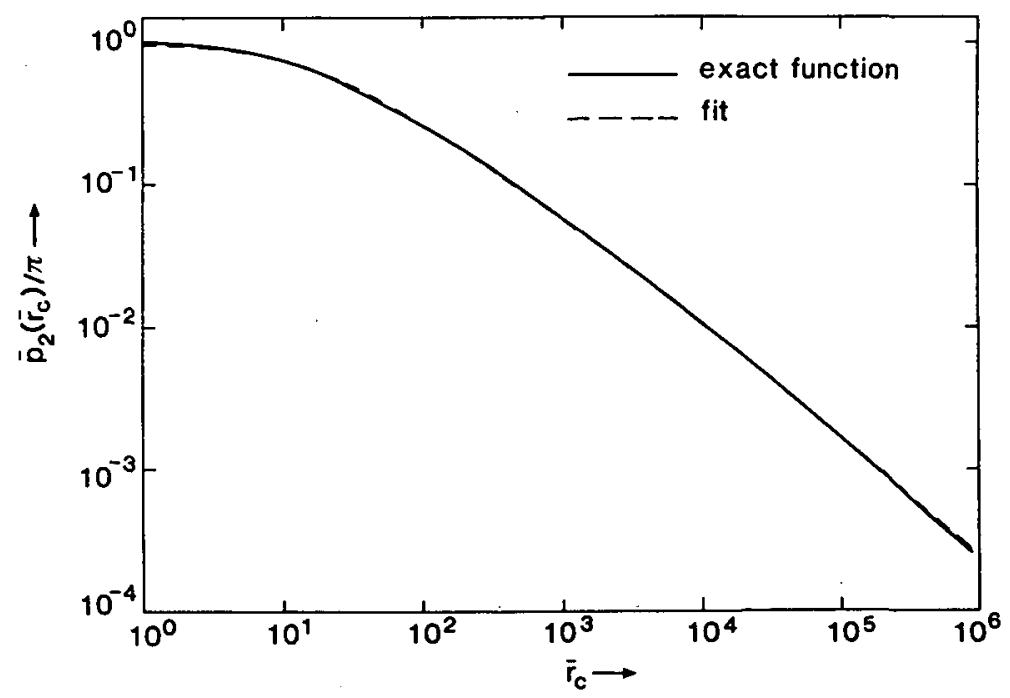

Pigure 7.3 The function $\overline{\mathrm{p}}_{2}\left(\overline{\mathrm{r}}_{\mathrm{c}}\right)$ defined by equation (7.3.16) and its approximation given by equation (7.3.17). This function gives the $\bar{Y}_{c}$-dependency of the function $\bar{p}(\bar{k})$, plotted in figure 7.2 , for $\mathrm{k} \rightarrow 0$. 


$$
\delta(x)=\lim _{k \rightarrow \infty} \frac{\sin (k x)}{\pi x}
$$

see for comparison equation (5.4.2). The $u$ and $\bar{v}$ integration can now successively be performed (note that the u-integration carries only over half the $\delta$-function), while the y-integration yields again a factor $\pi / 4$. Accordingly, one finds

$$
\overline{\mathrm{p}}(\overline{\mathrm{k}})=\overline{\mathrm{p}}_{\infty} \overline{\mathrm{k}}, \quad \overline{\mathrm{p}}_{\infty}=4 \pi \quad(\overline{\mathrm{k}}+\infty)
$$

The derivation of equation (7.3.19) makes clear that the large $\bar{k}$-behaviour of $\bar{p}(\bar{k})$ comes from the small $\Delta \bar{v}_{z}$ behaviour of the twoparticle distribution $\bar{\rho}_{2}\left(\Delta \bar{v}_{z}\right)$, described by equation $(7.3 .12)$.

In order to achieve a simple expression for the function $\bar{p}(\bar{k})$, we approximate equation $(7.3 .15)$ by

$$
\bar{p}_{a}(\bar{k})=\bar{p}_{\infty} \sqrt{\bar{k}^{2}+\left[\bar{p}_{\infty} / \bar{p}_{2}\left(\bar{r}_{c}\right)\right]^{2}}-\bar{p}_{\infty}{ }^{2} / \bar{p}_{2}\left(\bar{r}_{c}\right)
$$

in which $\bar{p}_{2}\left(\bar{r}_{c}\right)$ and $\bar{p}_{\infty}$ given by equations $(7.3 .16)$ and (7.3.19) respectively. The function $\bar{p}_{\mathbf{a}}(\bar{k})$ has the same asymptotic behaviour as the original function $\bar{p}(\bar{k})$. Figure 7.4 shows both functions for different values of $\bar{r}_{c}$. One sees that the fit is accurate for small and for large $\bar{k}$-values, but shows a significant deviation from the real function for intermediate values of $\vec{k}$, especially in combination with large values of $\bar{r}_{\mathrm{c}}$.

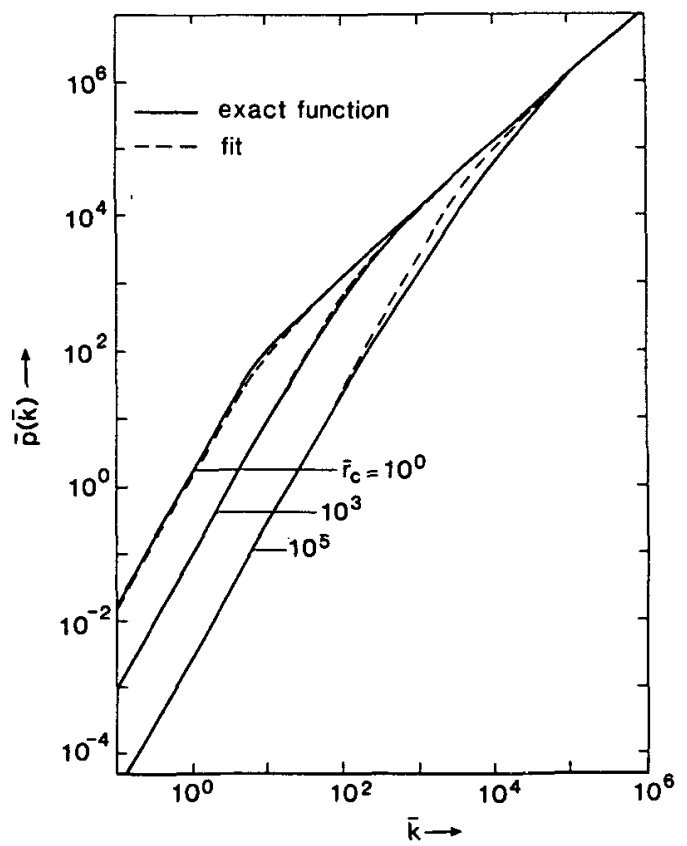

Figure 7.4 The exact scaled function $\overline{\mathrm{p}}(\mathrm{k})$ for complete collisions and its approximation given by equation (7.3.20). 
The final step consists of the calculation of the displacement distribution $\rho\left(\Delta v_{z}\right)$. By scaling equation $(7.2 .8)$ one obtains

$$
\vec{\rho}\left(\Delta \bar{v}_{z}\right)=\operatorname{vo\rho }\left(\Delta \mathrm{v}_{z}\right)=\frac{1}{\pi} \int_{0}^{\infty} \mathrm{d} \bar{k} \cos \left(\bar{k} \Delta \bar{v}_{z}\right) e^{-\bar{\lambda} \bar{p}(\bar{k})}
$$

where $\bar{\lambda}$ is the scaled linear particle density, which is given by

$$
\bar{\lambda}=\lambda d_{0}=\frac{c_{0}}{e m} \frac{I}{\alpha_{0}^{2} V_{z}^{3}}=\frac{m^{1 / 2}}{2^{7 / 2} \pi \varepsilon_{0} \mathrm{e}^{1 / 2}} \frac{I}{\alpha_{0}^{2} V^{3 / 2}}
$$

as follows with equations (3.2.4) and (7.3.2).

The advantage of the approximation given by equation (7.3.20) is that it permits to do the $\bar{k}$-integration analytically. Substitution into equation (7.3.21) and integration yields

$$
\bar{\rho}_{\mathrm{a}}\left(\Delta \overline{\mathrm{v}}_{\mathrm{Z}}\right)=\frac{\alpha \beta \exp (\alpha \beta)}{\pi\left(\Delta \bar{v}_{2}^{2}+\alpha^{2}\right)^{1 / 2}} K_{1}\left[\beta\left(\Delta \bar{v}_{\mathrm{Z}}^{2}+\alpha^{2}\right)^{1 / 2}\right]
$$

with $K_{1}$ the modified Bessel function and $\alpha$ and $\beta$ defined as

$$
\alpha=\lambda \overline{\mathbf{p}}_{\infty}, \quad \beta=\overline{\mathbf{p}}_{\infty} / \overline{\mathbf{p}}_{2}\left(\overline{\mathbf{r}}_{\mathbf{c}}\right)
$$

The shape of the distribution $\bar{\rho}_{\mathrm{a}}\left(\Delta \overline{\mathrm{v}}_{z}\right)$, given by equation (7.3.23), depends on the values of $\bar{\lambda}$ and $\bar{r}_{c}$ through $\alpha$ and $\beta$. One should distinguish two extreme cases:

I) Gaussian regime. For $\lambda \gg>\bar{p}\left(\bar{r}_{c}\right) / \bar{p}_{\infty}{ }^{2}$ (or $\alpha \beta \gg>1$ ) and $\Delta \bar{v}_{z}<<\lambda \bar{p}_{\infty}$ (or $\left.\Delta \overline{\mathbf{v}}_{\mathbf{z}}<<\alpha\right)$ one may utilize that

$$
\lim _{x \rightarrow \infty} K_{1}(x)=(\pi / 2 x)^{1 / 2} \exp (-x)[1+0(1 / x)]
$$

in which $0(1 / x)$ stands for terms of the order $1 / x$. Equation (7.3.23) now transforms to (approximating $\Delta \bar{v}_{z}{ }^{2}+\alpha^{2} \cong \Delta \bar{v}_{z}{ }^{2}$ )

$$
\bar{\rho}_{\mathrm{a}}\left(\Delta \overline{\mathrm{v}}_{\mathrm{z}}\right)=\frac{1}{(2 \pi)^{1 / 2} \sigma} \exp \left[-\Delta \overline{\mathrm{v}}_{\mathrm{z}}{ }^{2} / 2 \sigma^{2}\right), \sigma^{2}=\frac{\alpha}{\beta}=\lambda \overline{\mathrm{p}}_{2}\left(\overline{\mathrm{r}}_{\mathrm{c}}\right)
$$

which is an one-dimensional Gaussian distribution.

II) Lorentzian regime. For $\bar{\lambda}<<\overline{\mathrm{p}}\left(\overline{\mathbf{r}}_{\mathrm{c}}\right) / \overline{\mathrm{p}}_{\infty}^{2}$ (or $\alpha \beta<<1$ ) and $\Delta \overline{\mathrm{v}}_{z}<<\overline{\mathbf{p}}^{2}\left(\overline{\mathrm{r}}_{\mathrm{c}}\right) / \overline{\mathrm{p}}_{\infty}$ (or $\left.\Delta \bar{v}_{z}<<1 / \beta\right)$ one may use that

$$
\lim _{x \rightarrow 0} K_{1}(x)=1 / x+0(x), \quad \lim _{x \rightarrow 0} \exp (x)=1+0(x)
$$

Accordingly, equation $(7.3 .23)$ transforms to

$$
\bar{\rho}_{\mathrm{a}}\left(\Delta \overline{\mathrm{v}}_{\mathrm{z}}\right)=\frac{1}{\pi \alpha\left[1+\left(\Delta \overline{\mathrm{v}}_{\mathrm{z}} / \alpha\right)^{2}\right]}
$$

which is an one-dimensional Lorentzian distribution.

Summarizing the results so far, we found that the distribution of the scaled axial velocity displacements $\bar{\rho}\left(\Delta \bar{v}_{z}\right)$ generated in a beam segment with a narrow crossover, is Gaussian for large values of the scaled linear particle density $\pi$ and Lorentzian for small $\lambda$-values. This behaviour is related to the quadratic and linear behaviour of the function $\bar{p}(\bar{k})$ for small $\bar{k}$ and large $k$ respectively. 
The mean square value $\left\langle\Delta \overline{\mathbf{v}}_{z}{ }^{2}\right\rangle$ of the distribution $\bar{\rho}\left(\Delta \bar{v}_{z}\right)$ can be determined in several ways. One way is to use its definition

$$
\left\langle\Delta \bar{v}_{Z}^{2}\right\rangle=\int d \Delta \bar{v}_{z} \bar{\rho}\left(\Delta \bar{v}_{Z}\right) \Delta \bar{v}_{z}^{2}
$$

and substitute equation (7.3.23) for $\bar{\rho}\left(\Delta \bar{v}_{z}\right)$. Another way is to determine $\left\langle\Delta \bar{v}_{z}{ }^{2}\right\rangle$ from the two-particle distribution $\bar{\rho}_{2}\left(\Delta \bar{v}_{2}\right)$, given by equation (7.3.9), using

$$
\left\langle\Delta \bar{v}_{z} 2\right\rangle=\pi \cdot d \Delta \bar{v}_{2} \bar{\rho}_{2}\left(\Delta \bar{v}_{z}\right) \Delta \bar{v}_{z}^{2} \text {. }
$$

which is based on equation (5.5.10) (with $m=2$ ). The usual way to calculate $\left\langle\Delta \overline{\mathrm{v}}_{\mathrm{z}}{ }^{2}\right\rangle$ is to evaluate

$$
\left\langle\Delta \bar{v}_{z}{ }^{2}\right\rangle=\frac{\pi}{d_{0}} \int \mathrm{d} \xi p(\xi)\left[\Delta \bar{v}_{z}(\xi)\right]^{2}
$$

as follows from the second equation of (5.5.11) with $\Delta \eta=\Delta v_{z}$. The probability distribution $p(\xi)$ is defined by equations (5.4.6) and (5.3.2), while equation $\Delta \bar{v}_{z}(\xi)$ is (for complete collisions) given by equation (7.3.3). Finally, one may determine $\left\langle\Delta \bar{v}_{z}{ }^{2}\right\rangle$ from the small $\bar{k}$-behaviour of the function $\overline{\mathbf{p}}(\bar{k})$. We will utilize this method. Substitution of equation (7.3.16) into equation (5.5.4) (with $m=2$ ) directly yields

$$
\left\langle\Delta \overline{\mathrm{v}}_{\mathrm{z}} 2\right\rangle=\overline{\mathrm{p}} \overline{\mathrm{p}}_{2}\left(\overline{\mathrm{r}}_{\mathrm{c}}\right)
$$

It should emphasized that this relation is generally true irrespective of the shape of the distribution $\bar{\rho}\left(\Delta \bar{v}_{z}\right)$.

Other measures for the width of the distribution in general do depend on the shape of the distribution. As a practical width measure we consider the Full Width at Half Maximum (FWHM). The FWHM of the Gaussian distribution $(7.3 .25)$ is equal to

$$
\overline{\text { FWHMG }}_{G}=2(2 \ln 2)^{1 / 2} \sigma=2(2 \pi \ln 2)^{1 / 2} \operatorname{PCE}\left(\bar{Y}_{\mathrm{C}}\right) \lambda^{1} / 2
$$

where the function $P_{C E}\left(\bar{r}_{c}\right)$ is defined as

$$
\operatorname{PCE}\left(\bar{r}_{\mathrm{C}}\right)=\left[\overline{\mathrm{p}}_{2}\left(\overline{\mathrm{r}}_{\mathrm{C}}\right) / \pi\right]^{1 / 2}
$$

The subscript $C E$ indicates that the function refers to the Energy distribution generated in a beam segment with a Crossover. The FWHM of the Lorentzian distribution given by equation $(7.3 .26)$ is equal to

$$
\overline{\mathrm{FWHM}}_{\mathbf{L}}=2 \alpha=2 \pi \overline{\mathrm{p}}_{\infty}=8 \pi \bar{\lambda}
$$

Clearly, equations $(7.3 .31)$ and $(7.3 .33)$ show different dependencies on the parameters $\bar{\lambda}$ and $\vec{r}_{c}$, and therefore lead to different dependencies on the experimental parameters. The dependency of the FWHM of $\bar{\rho}\left(\Delta \bar{v}_{z}\right)$ on $\lambda$ and $\bar{r}_{c}$ is depicted in figure 7.5 . The input for this figure was determined numerically using the exact function $\bar{p}(\bar{k})$. Thus the results do not rely on the approximation contained in equation (7.3.20). The thermodynamic limit indicated in figure 7.5 will be discussed in section 7.7 .

Anticipating on the analysis of section 7.5 we mention that the equations ( 7.3 .31$)$ and $(7.3 .33)$ can be interpolated using 


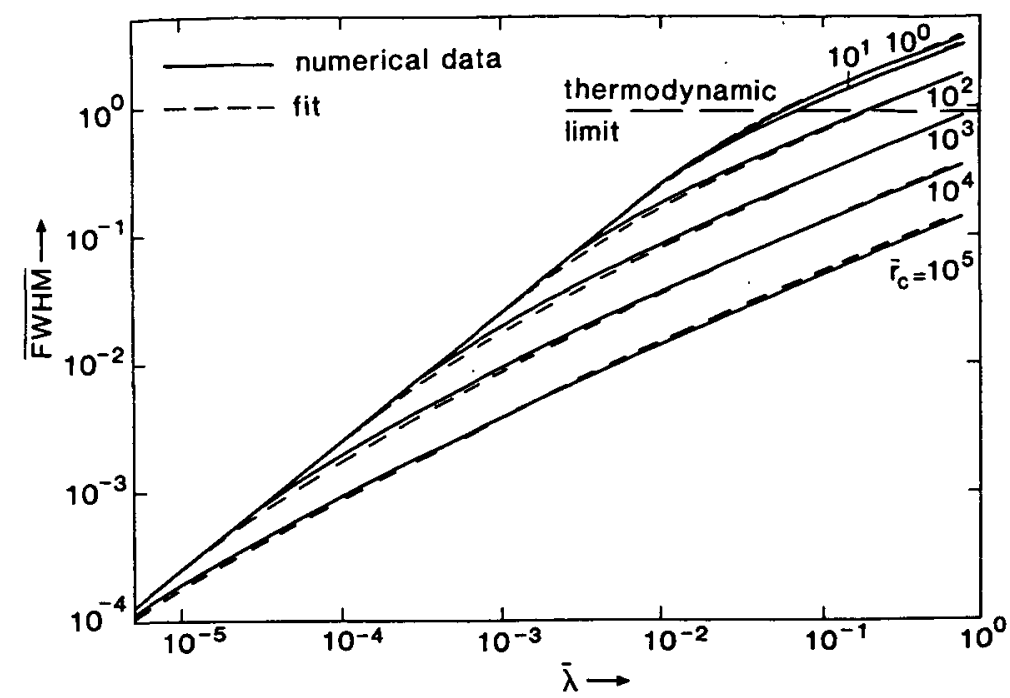

Figure 7.5 The FWHM of the scaled distribution of axial velocity displacements $\bar{\rho}\left(\Delta \bar{v}_{z}\right)$ based on complete collisions, for different values of the scaled crossover radius $\bar{r}_{\mathrm{r}}$. The depicted fit is defined by equations (7.3.34) and (7.3.35). The thermodynamic 1imit is given by equation $(7.7 .2)$ and refers to the relaxation of kinetic energy.

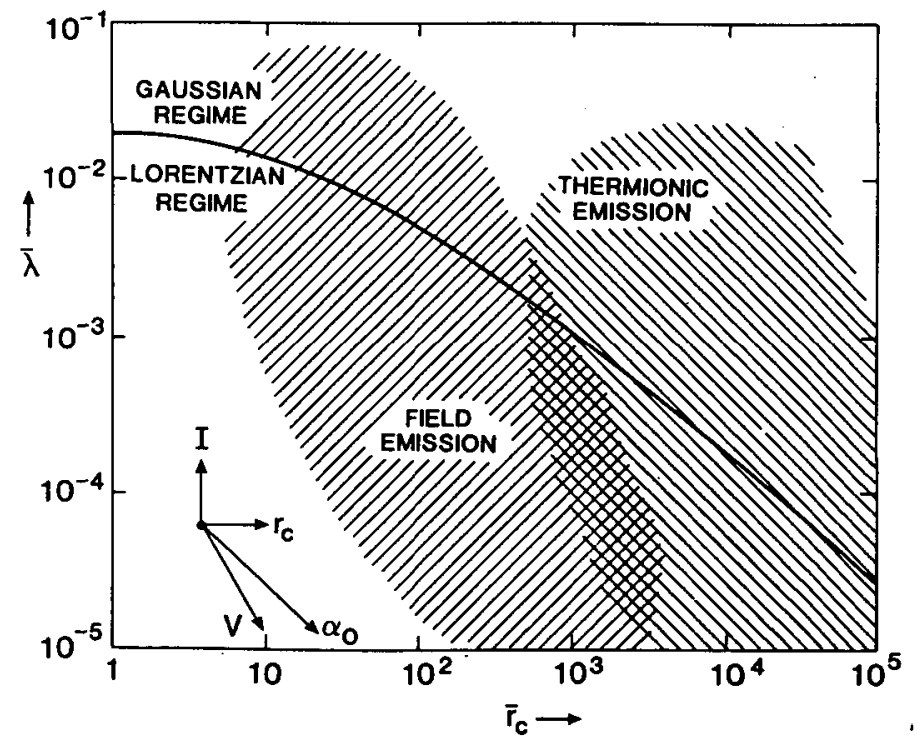

Pigure 7.6 Practical operating conditions, in terms of the quantities $\bar{r}_{c}$ and $\lambda$, for a beam segment with a narrow crossover $(\mathrm{K}>1)$. The solid line separates the regimes leading to a Gaussian and a Lorentzian type of energy distribution. The effect of varying each of the experimental parameters is indicated by the arrows. 
where the function $G_{C E}\left(\pi, \bar{r}_{c}\right)$ is defined as

$$
\mathrm{G}_{\mathrm{CE}}\left(\bar{\lambda}, \overline{\mathrm{r}}_{\mathrm{c}}\right)=\left[\frac{1}{\operatorname{PCE}_{\mathrm{E}}\left(\overline{\mathrm{r}}_{\mathrm{c}}\right)^{4}}+1.57510-3 \frac{\overline{\mathrm{r}}_{\mathrm{c}}^{4 / 3}}{\bar{\lambda}^{2 / 3}}+\frac{7.60610^{-4}}{\lambda^{2}}\right]^{-1 / 4}
$$

The function $P_{C E}\left(\bar{r}_{C}\right)$ is defined by equation (7.3.32). The fit represented by equations $(7.3 .34)$ and $(7.3 .35)$ is also plotted in figure 7.5. The accuracy of the fit is better than $5 \%$ over the entire range of operating conditions. In the Gaussian regime $\lambda \gg P_{C E}\left(\bar{r}_{C}\right)^{2} / 16 \pi$ and one may approximate $G_{C E}\left(\bar{X}, \bar{r}_{C}\right) \approx P_{C E}\left(\bar{r}_{C}\right)$. This implies that FWHM $\approx F_{W H M}$, which is given by equation (7.3.31). In the Lorentzian regime $\lambda<<P_{C E}\left(\bar{r}_{C}\right)^{2} / 16 \pi$ and one may approximate $G_{C E}\left(\bar{X}, \bar{r}_{C}\right) 6.022{ }^{1} 1 / 2$. This implies that FWHM $\approx$ FWHM $_{L}$, which is given by equation $(7.3 .33)$. The second term between brackets in the right hand side of equation (7.3.35) is included to improve the quality of the fit. Its origin will be clarified in section 7.5 .

Equations (7.3.31) through (7.3.35) provide a complete specification of the FWHM of the generated axial velocity distribution $\rho\left(\Delta v_{z}\right)$ in terms of the scaled coordinates defined by equations (7.3.2). In order to make the dependency on the experimental parameters explicit we will now remove this scaling. The properties of the scaled velocity distribution $\bar{\rho}\left(\Delta \bar{v}_{2}\right)$ can be translated in terms of the corresponding energy distribution, using

$$
\Delta \mathrm{E} \cong \mathrm{mv}_{\mathrm{z}} \Delta \mathrm{v}_{\mathrm{z}}=\mathrm{mv}_{\mathrm{z}}^{2} \alpha_{0} \Delta \overline{\mathrm{v}}_{\mathrm{z}}=\mathrm{E} 2 \alpha_{0} \Delta \overline{\mathrm{v}}_{\mathrm{z}}
$$

assuming that $v_{z} \gg \Delta V_{z}$. Accordingly, equation (7.3.34) can be expressed as

$$
\frac{F W H M_{E}}{E}=C_{C G E} G_{C E}\left(\pi, \bar{r}_{C}\right) \sqrt{\frac{I}{V^{3 / 2}}}, \quad C_{C G E}=\left(\frac{8(\ln 2)^{2} m}{\varepsilon_{0^{2}} \mathrm{e}}\right)^{1 / 4}(7.3 .37)
$$

The ratio $I / V^{3 / 2}$ is known as the perveance. In the case of electrons one finds $C_{C G E}=726.62$ in SI-units (note: the subscript CGE indicates that the constant applies to a beam geometry with a Crossover and refers to the Gaussian Energy distribution). Equation ( $\overline{7} .3 .37$ ) yields the main result of this section. It gives the FWHM of the energy distribution generated in a beam segment with a narrow crossover for the entire range of operating conditions. The operating conditions are defined by the parameters $\lambda$ and $\bar{r}_{c}$. Figure 7.6 indicates which values of $\lambda$ and $\bar{r}_{c}$ apply to practical systems. From the figure one sees that systems employing thermionic emission predominantly operate in the Gaussian regime and in the transition area between the Gaussian and the Lorentzian regime, whereas systems with a field emission gun predominantly operate in the Lorentzian regime. We emphasize that the analysis of this section pertains to a beam segment with a narrow crossover $(K>>1)$, for which one may assume that the Boersch effect is generated by complete collisions. In section 7.5, where we treat the general case of a beam with a crossover in which $K$ is not necessarily large, it will be shown that one should in general distinguish four regimes. In addition to the Gaussian regime and the Lorentzian regime one finds, for small K-values, a Holtsmark regime and a pencil beam regime, corresponding to medium and small values of $\pi$ respectively.

We conclude this section by investigating the dependency on the experimental parameters in the different regimes covered by equation 


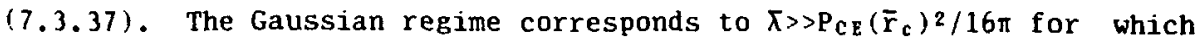
one may approximate that $\mathrm{G}_{C E}\left(\bar{\lambda}, \bar{r}_{c}\right) \approx \mathrm{P}_{C E}\left(\bar{r}_{c}\right)$, as was mentioned before. The function $P_{C E}\left(\bar{r}_{c}\right)$, defined by equation (7.3.32), is for all practical purposes best described by the fit given by equation (7.3.17). For small values of $\bar{r}_{c}$ one obtains $P_{C E}\left(\bar{r}_{c}\right) \approx 1$. For these conditions equation (7.3.37) transforms to

$$
\frac{F W H M_{E}}{E}=C_{C G E} \sqrt{\frac{I}{V^{3 / 2}}} \quad\left(\pi>P_{C E}\left(\bar{r}_{C}\right)^{2} / 16 \pi, \bar{r}_{C}<10\right)
$$

For large values of $\bar{r}_{c}$ one finds from equation $(7,3.17)$

$$
\operatorname{PCE}\left(\bar{r}_{\mathrm{C}}\right) \approx \frac{9.20}{\left(\pi \bar{r}_{\mathrm{c}}\right)^{1 / 2}}\left[1+.217 \ln \left(1+\bar{r}_{\mathrm{c}} / 114.6\right)\right]
$$

Accordingly, equation $(7.3 .37)$ yields

$$
\begin{aligned}
& \frac{\text { FWHME }}{E}=C^{*} \operatorname{cgE} \frac{I^{1 / 2}}{V^{5 / 4 r_{c}}{ }^{1 / 2} \alpha_{0}}[1+\left..217 \ln \left(1+\bar{r}_{c} / 114.6\right)\right] \\
&\left(\pi>>P_{C E}\left(\bar{r}_{c}\right)^{2} / 16 \pi, \bar{r}_{c} \geqslant 100\right)
\end{aligned}
$$

where the constant $C^{\star} \mathrm{CGE}$ is given by

$$
\mathrm{C}^{*} \mathrm{CGE}=9.20 \frac{(\ln 2)^{1 / 2}}{2^{3 / 4} \pi} \frac{(\mathrm{me})^{1 / 4}}{\varepsilon_{0}}
$$

In the case of electrons one finds $C^{*} C G=0.1012$ in SI-units.

The Lorentzian regime corresponds to $\bar{\lambda}<<\mathrm{P}_{\mathrm{cE}}\left(\bar{r}_{\mathrm{c}}\right)^{2 / 16 \pi}$. Equation (7.3.37) now transforms to

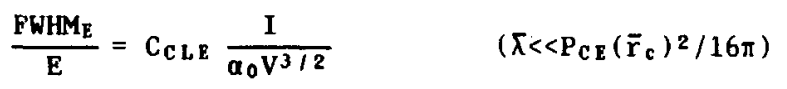

where the constant $C_{C L E}$ is given by

$$
C_{C L E}=\left(\frac{2 m}{\varepsilon_{0}^{2} e}\right)^{1 / 2}
$$

In the case of electrons one finds $C_{C L E}=3.8085 \times 10^{5}$ in SI-units.

\subsection{Homocentric cylindrical beam segment}

In this section we will calculate the Boersch effect in a homocentric cylindrical beam. As before we assume that the beam is monochromatic. Consequently, all particles run initially with identical velocities along parallel trajectories. Thus in the frame of reference moving with the beam the particles are initially at rest. Due to the Coulomb interaction part of their mutual potential energy will be converted into kinetic energy. This process causes a generation of random velocity components. The generated spread of axial velocities corresponds to the Boersch effect.

From this analysis it becomes clear that "relaxation of potential energy" is the mechanism which generates the Boersch effect in this type of beam geometry. This mechanism should be distinguished from "relaxation of kinetic energy", which is dominant in a beam segment with a narrow crossover, as was discussed in the previous section. In this respect both geometries should considered as opposite. Accordingly, different types of collisions are involved. Complete collisions generate 
most of the effect in a beam segment with a narrow crossover, while weak incomplete collisions are dominant in a homocentric cylindrical beam.

For the case of a cylindrical beam one can not apply the scaling defined by equations $(7.3 .2)$, because $v_{0}=0$. Instead we will use the $\delta, v-$ scaling given by equations (6.13.1). The scaled two-particle distribution $\rho_{2}{ }^{*}\left(\Delta v_{2}{ }^{*}\right)$ follows from equation $(7.2 .6)$

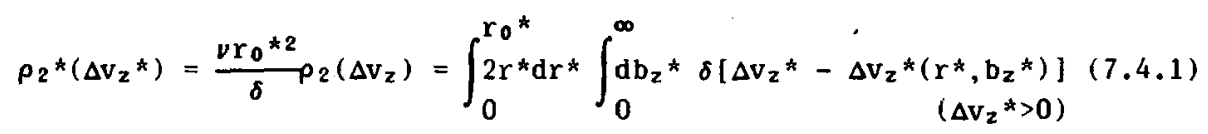

It is sufficient to consider the case $\Delta v_{z}{ }^{*}=\left(\Delta v_{z} / v\right)>0$, thus limiting the $b_{z}{ }^{*}$-integration to positive $b_{z}^{*}$-values. The function $\Delta v_{z}^{*}\left(r^{*}, b_{z}{ }^{*}\right)$ can be determined analytically for weak collisions and half-complete collisions, see equations $(7.2 .5)$ and $(7.2 .4)$, respectively. In all other cases one has to compute $\Delta v_{z}{ }^{*}\left(r^{*}, b_{z}{ }^{*}\right)$ numerically, following the method which was outlined in section 6.6. We evaluated equation (7.4.1) numerically on the basis of the exact analysis of the function $\Delta v_{z}{ }^{*}\left(r^{*}, b_{z}{ }^{*}\right)$. The outcome is presented in figure 7.7 .

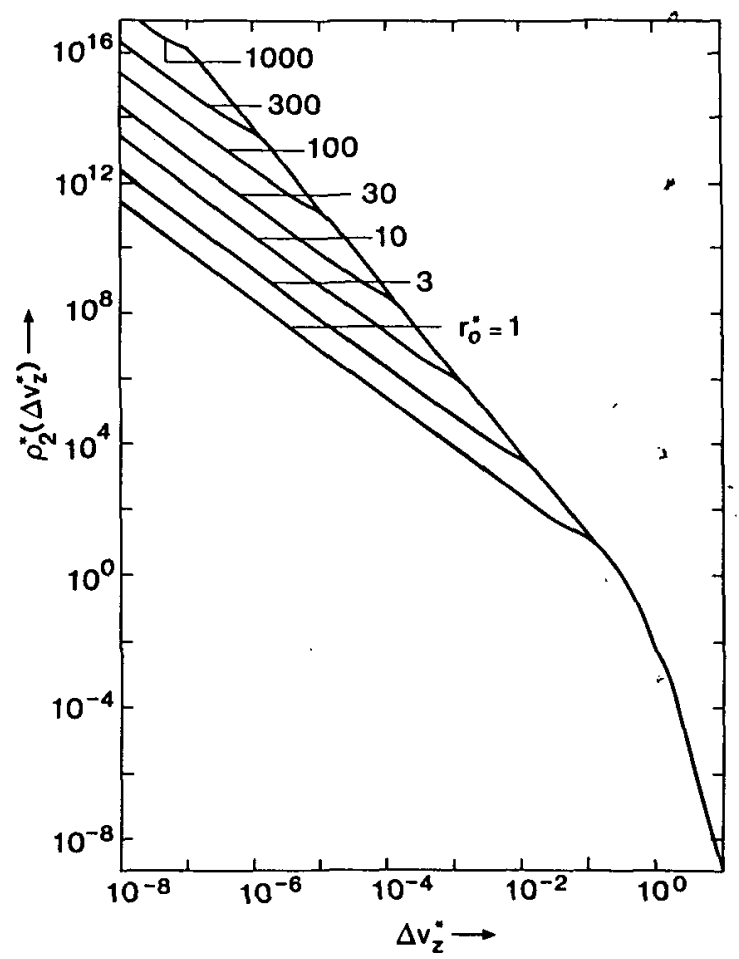

Figare 7.7 The scaled two-particle distribution $\rho_{2} *\left(\Delta v_{z} *\right)$ for a howocentric cylindrical beam segment, plotted for different values of the scaled beam radius $r_{0} *$. 
It should be anticipated that the function $\rho_{2} *\left(\Delta v_{z}^{*}\right)$ is determined by weak interactions for most of the $\Delta v_{2}^{*}$-range plotted in figure 7.7 , while its behaviour for very large $\Delta \mathbf{v}_{\mathbf{z}}{ }^{*}$-values stems from half-complete collisions. We will verify this statement by evaluating equation (7.4.1) analytically, employing the approximations given by equations $(7.2 .5)$ and $(7.2 .4)$ respectively. Let us first consider the limit of weak interactions. We recall that equation (7.2.5) is based on the first order perturbation approximation

$$
\Delta \mathbf{v}_{\mathbf{z}} \cong \mathrm{F}_{/ /} \mathrm{T} / \mathrm{m}
$$

Thus instead of evaluating $\rho_{2}{ }^{*}\left(\Delta v_{z}{ }^{*}\right)$ directly from equations $(7.4 .1)$ and (7.2.5) one may as well determine $\rho_{2} *\left(\Delta v_{z} *\right)$ from the distribution of axial forces $\rho_{2}\left(F_{/ 1}\right)$ occurring in a cylindrical beam, by using

$$
\rho_{2}{ }^{*}\left(\Delta \mathrm{v}_{2}{ }^{*}\right)=\frac{\nu \mathrm{r}_{0}^{* 2}}{\delta} \rho_{2}\left(\Delta \mathrm{v}_{2}\right)=\frac{\nu \mathrm{r}_{0}^{* 2}}{\delta} \frac{\mathrm{m}}{\mathrm{T}} \rho_{2}\left(\mathrm{~F}_{\|}=\Delta \mathrm{v}_{2} \mathrm{~m} / \mathrm{T}\right)
$$

The calculation of $\rho_{2}\left(F_{\mu}\right)$ was carried out in section 5.8. Equation $(5.8 .23)$ specifies $\rho_{2}\left(F_{/ \prime}\right)$ for an extended beam $\left(r_{0}+\infty\right)$. Substitution into equation $(7.4 .3)$ yields

$$
\rho_{2}{ }^{*}\left(\Delta v_{z}{ }^{*}\right)=\frac{1}{20 \Delta v_{z}{ }^{5 / 2}} \quad\left(\Delta v_{z}^{*}<<1, r_{0}{ }^{* 2} \Delta v_{z} *>>1\right)
$$

in which we utilized the definitions of $\delta$ and $\nu$ given by equations $(6.13 .1)$.

For a pencil-beam $\left(r_{0} \rightarrow 0\right)$ one should use equation (5.8.26). Substitution into equation $(7.4 .3)$ yields

$$
\rho_{2} *\left(\Delta v_{z}^{*}\right)=\frac{r_{0} * 2}{4 \Delta v_{z}^{* 3 / 2}} \quad\left(\Delta v_{z}^{*}<<1, r_{0} * 2 \Delta v_{z}^{*}<<1\right)
$$

One may verify from figure 7.7 that the small and the intermediate $\Delta \mathrm{v}_{\mathrm{z}}{ }^{*}$-behaviour of $\rho_{2}{ }^{*}\left(\Delta \mathrm{v}_{\mathrm{z}}{ }^{*}\right)$ indeed follows equations (7.4.4) and $(7.4 .5)$.

In order to investigate the large $\Delta v_{2}{ }^{*}$-behaviour of $\rho_{2} *\left(\Delta v_{2}{ }^{*}\right)$ we scale the expression for $\Delta v_{z}$ given by equation $(7.2 .4)$ and substitute the result into equation $(7,4.1)$. This way one obtains

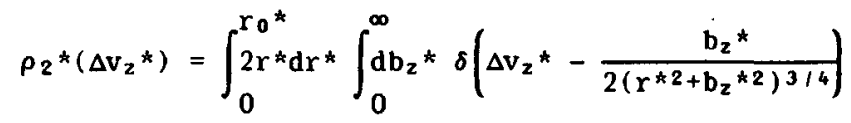

By substituting

$$
s=\frac{b_{z}^{*}}{r^{*}} \quad, \quad t=\frac{s}{2 r^{* 1 / 2}\left(1+s^{2}\right)^{3 / 4}}
$$

and carrying out the t-integration, equation $(7.4 .6)$ transforms to

$$
\rho_{2}{ }^{*}\left(\Delta v_{z}{ }^{*}\right)=\frac{1}{16 \Delta v_{z}{ }^{* 1}} \int_{0}^{\infty} d s \frac{s^{6}}{\left(1+s^{2}\right)^{9 / 2}} \theta\left(\Delta v_{z} *-\frac{s}{2 r_{0}{ }^{* 1 / 2}\left[1+s^{2}\right]^{3 / 4}}\right)
$$

in which $\theta(x)$ the step-function defined by equation (3.7.8).

For an extended beam $\left(r_{0}{ }^{*}+\infty\right)$ the argument of the step-function is positive for all positive values of $\Delta v_{z}{ }^{*}$ and one obtains 


$$
\rho_{2}{ }^{*}\left(\Delta V_{z}{ }^{*}\right)=\frac{1}{16 \Delta v_{z}{ }^{* 7}} \int_{0}^{\infty} d s \frac{s^{6}}{\left(1+s^{2}\right)^{9 / 2}}=\frac{1}{112} \frac{1}{\Delta v_{z}^{* 7}}
$$

This asymptote gives a good approximation of $\rho_{2} *\left(\Delta \mathrm{v}_{\mathrm{z}}{ }^{*}\right)$ for $\Delta \mathrm{v}_{\mathrm{z}}{ }^{*}$, as can be verified from figure 7.7 .

For a pencil beam $\left(r_{0}^{*} \rightarrow 0\right)$ the argument of the step-function is positive for $s>s_{0} \approx 1 /\left(4 \Delta v_{z}{ }^{2} r_{0}{ }^{*}\right)$. Thus the lower integration boundary of the integral in equation $(7.4 .8)$ should be replaced by $s_{0}$. As $s_{0} \gg 1$ one may approximate

$$
\rho_{2}{ }^{*}\left(\Delta v_{2}{ }^{*}\right)=\frac{1}{16 \Delta v_{2} * 1} \int_{s_{0}=1 /\left(4 \Delta v_{z}{ }^{* 2} r_{0} *\right)}^{\infty}=\frac{r_{0} * 2}{2 \Delta v_{z}^{* 3}}
$$

However, this asymptote is not reached for normal operating conditions $\left(r_{0} * 1\right)$ and will be disregarded in the remaining analysis. Equations $(7.4 .4),(7.4 .5)$ and $(7.4 .9)$ provide a complete analysis of the behaviour of the two-particle distribution $\rho_{2}{ }^{*}\left(\Delta v_{z}{ }^{*}\right)$.

The next step is to determine the scaled function $p^{*}\left(k^{*}\right)$ which follows from equation $(7,2.7)$

$$
\mathrm{p}^{*}\left(\mathrm{k}^{*}\right)=\frac{r_{0^{* 2}}}{\delta} \mathrm{p}(\mathrm{k})=4 \int_{0}^{\infty} \mathrm{d} \Delta \mathrm{v}_{\mathrm{z}}{ }^{*} \rho_{2} *\left(\Delta \mathrm{v}_{\mathrm{z}}^{*}\right) \sin ^{2}\left(\mathrm{k}^{*} \Delta \mathrm{v}_{\mathrm{z}}{ }^{*} / 2\right)
$$

with $k^{*}=k \nu$. We evaluated the integral of equation $(7.4 .11)$ numerically from the numerical data of $\rho_{2}{ }^{*}\left(\Delta v_{z}{ }^{*}\right)$. The results are shown in figure 7.8 .

The behaviour of the function $p^{*}\left(k^{*}\right)$ can be understood from the analysis of the two-particle distribution $\rho_{2}^{*}\left(\Delta v_{z}^{*}\right)$. Substitution of equation $(7.4 .4)$ into equation $(7.4 .11)$ yields

$$
p^{*}\left(k^{*}\right)=\frac{1}{10 \sqrt{2}} k^{* 3 / 2} \int_{0}^{\infty} d x \frac{\sin ^{2}(x)}{x^{5 / 2}}=\frac{(2 \pi)^{1 / 2}}{15} k^{* 3 / 2}
$$

which provides an accurate approximation for $k * \gtrsim 10$ and $k * \lll 10 r_{0} * 2$. This result can also be obtained from equation (5.8.24), which refers to the distribution of axial forces in extended beam.

Substitution of equation (7.4.5) into equation $(7.4 .11)$ yields

$$
p^{*}\left(k^{*}\right)=\frac{r_{0} * 2}{\sqrt{2}} k^{* 1 / 2} \int_{0}^{\infty} d x \frac{\sin ^{2}(x)}{x^{3 / 2}}=\sqrt{\frac{\pi}{2}} r_{0} * 2 k^{* 1 / 2}
$$

which provides an accurate approximation for $k * 10 \mathrm{r}_{0} * 2$. This result is equivalent to equation (5.8.27), referring to the distribution of axial forces in a pencil beam.

For small $k^{*}$-values the function $p^{*}\left(k^{*}\right)$ shows a quadratic $k^{*}$-dependency, which can be described as

$$
\lim _{k \rightarrow 0} p^{*}\left(k^{*}\right)=\frac{1}{2} p_{2} *\left(r_{0}\right)^{*} k^{\star 2} \quad, \quad p_{2} *(\infty)=0.1513
$$

The small $k^{*}$-behaviour of the function $p^{*}\left(k^{*}\right)$ is directly related to the 


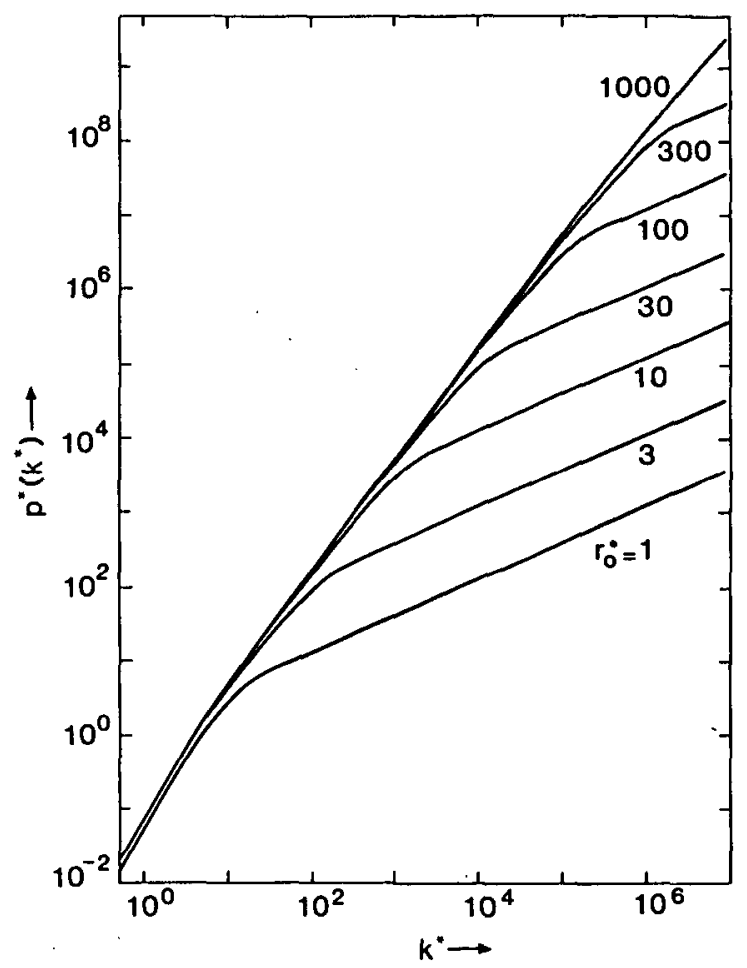

Figure 7.8 The scaled function $\mathrm{p}^{*}\left(\mathrm{k}^{*}\right)$ corresponding to the distribution of axial velocity displacements generated in a homocentric cylindrical beam segment, plot ted for different values of the scaled beam radius $r_{0}{ }^{*}$.

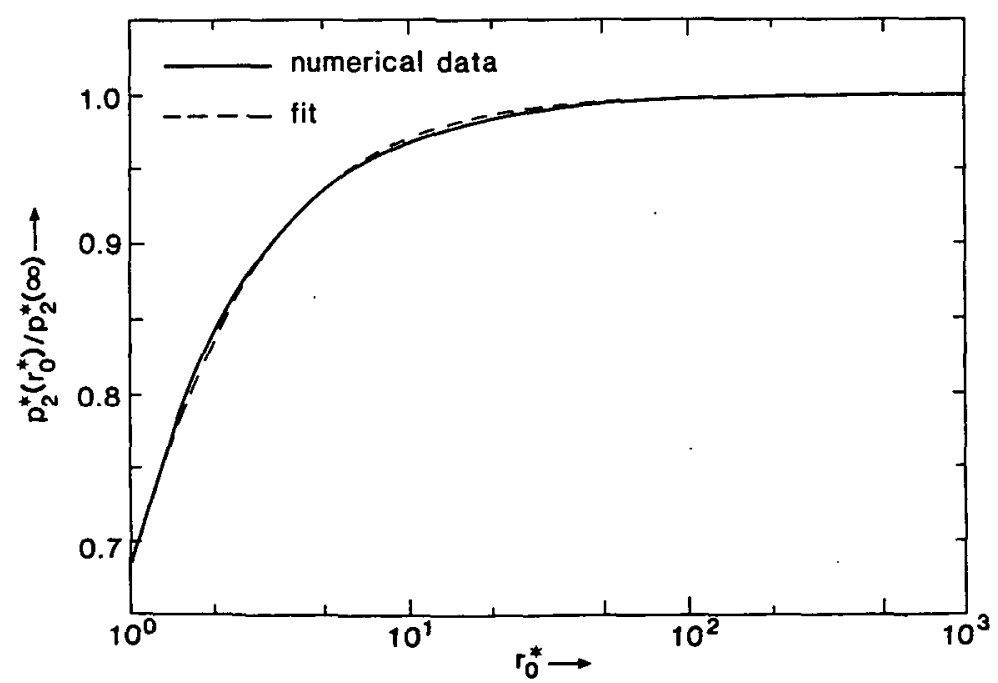

Pigure 7.9 The function $\mathrm{p}_{2}\left(\mathrm{r}^{*}\right)$, defined by equation (7.4.14) and its approximation given by equation (7.4.16). This function gives the $\mathrm{r}_{0}^{\star}$-dependency of the function $\mathrm{p}^{*}\left(\mathrm{k}^{\star}\right)$, plotted in figure 7.8 , for $k^{k}+0$. 
second moment $\left\langle\Delta \mathrm{v}_{\mathrm{z}} 2^{* 2}\right\rangle$ of the two-particle distribution $\rho_{2} *\left(\Delta \mathrm{v}_{\mathrm{z}}{ }^{*}\right)$, as follows by expanding the sin-function in equation $(7.4 .11)$ for small $k^{*}$. By comparing this expansion with equation $(7.4 .14)$ one obtains

$$
\left\langle\Delta v_{z_{2}} * 2\right\rangle=p_{2}^{*}\left(r_{0}^{*}\right)
$$

The quantity $\left\langle\Delta v_{z 2} * 2\right\rangle$ does not stem from either weak or half-complete collisions, but is built up over the entire $\Delta \mathrm{v}_{2}{ }^{*}$-range. This prohibits an analytical estimation of its value. We evaluated the function $p_{2}{ }^{*}\left(r_{0}^{*}\right) / p_{2}{ }^{*}(\infty)$ numerically. The result is depicted in figure 7.9 . For later use we fitted the numerical data with the function

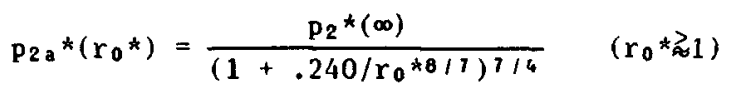

which is also plotted in figure 7.9. The set of equations (7.4.12), $(7.4 .13)$ and $(7.4 .14)$ provides a complete analysis of the behaviour of the function $p^{*}\left(k^{*}\right)$.

The final step consist of the calculation of the displacement distribution $\rho^{*}\left(\Delta v_{z}^{*}\right)$. By scaling equation $(7.2 .8)$ one obtains

$$
\rho^{\star}\left(\Delta \mathrm{v}_{\mathrm{z}}^{*}\right)=\nu \rho\left(\Delta \mathrm{v}_{\mathrm{z}}\right)=\frac{1}{\pi} \int_{0}^{\infty} \mathrm{d} \mathrm{k}^{*} \cos \left(\mathrm{k}^{\star} \Delta \mathrm{v}_{\mathrm{z}}{ }^{*}\right) \mathrm{e}^{-4 \lambda^{\star} \mathrm{p}^{\star}\left(\mathrm{k}^{\star}\right)}
$$

where $\lambda^{*}$ is the scaled linear particle density for a cylindrical beam, which is given by

$$
\lambda^{*}=\lambda \frac{\delta}{4 \mathrm{r}_{0}^{* 2}}=\frac{\mathrm{C}_{0}}{\mathrm{em}} \frac{\mathrm{IL}^{2}}{\mathrm{r}_{0}^{2} \mathrm{~V}_{\mathrm{z}}^{3}}=\frac{m^{1 / 2}}{2^{7 / 2} \pi \varepsilon_{0} \mathrm{e}^{1 / 2}} \frac{\mathrm{IL}^{2}}{\mathrm{r}_{0}^{2} \mathrm{~V}^{3 / 2}}
$$

analogous to $\lambda$, used for a beam segment with a crossover, see equation (7.3.22). The second moment of this distribution follows from the function $p^{*}\left(k^{*}\right)$ using equation $(5.5 .4)$ (with $m=2$ )

$$
\left\langle\Delta \mathrm{v}_{\mathrm{z}} * 2\right\rangle=4 \mathrm{p}_{2} *\left(\mathrm{r}_{0} *\right) \lambda^{*}
$$

This relation can also be obtained from equation $(7.4 .15)$, using equation $(5,5,10)$.

We evaluated the FWHM of $\rho^{*}\left(\Delta v_{z}{ }^{*}\right)$ numerically from the numerical data of $p^{*}\left(k^{*}\right)$. The results are plotted in figure 7.10. A key-parameter for the interpretation of this data is $\chi_{p}=4 \lambda^{*} r_{0}{ }^{* 3}$, which is called the pencil beam factor for a cylindrical beam. It determines whether a beam should be considered as a pencil beam $\left(\chi_{p}<<1\right)$ or an extended beam $\left(\chi_{p} \gg>1\right)$. The physical significance of $\chi_{p}$ becomes evident by removing the scaling. Using equation $(7.4 .18)$ and equations $(6.13 .1)$ one obtains

$$
x_{p}=4 \lambda{ }^{*} r_{0}{ }^{* 3}=\lambda r_{0}
$$

Thus $\chi_{p}$ is the ratio between the beam radius $r_{0}$ and the average axial separation of the particles $1 / \lambda$. The following regimes should be distinguished in the data of figure 7.10 :

I) Gaussian regime. For $\lambda^{\star} \gtrsim 1$ and $\chi_{p} \gg>1$ the distribution $\rho^{*}\left(\Delta v_{z}{ }^{*}\right)$ is Gaussian with a FWHM given by 


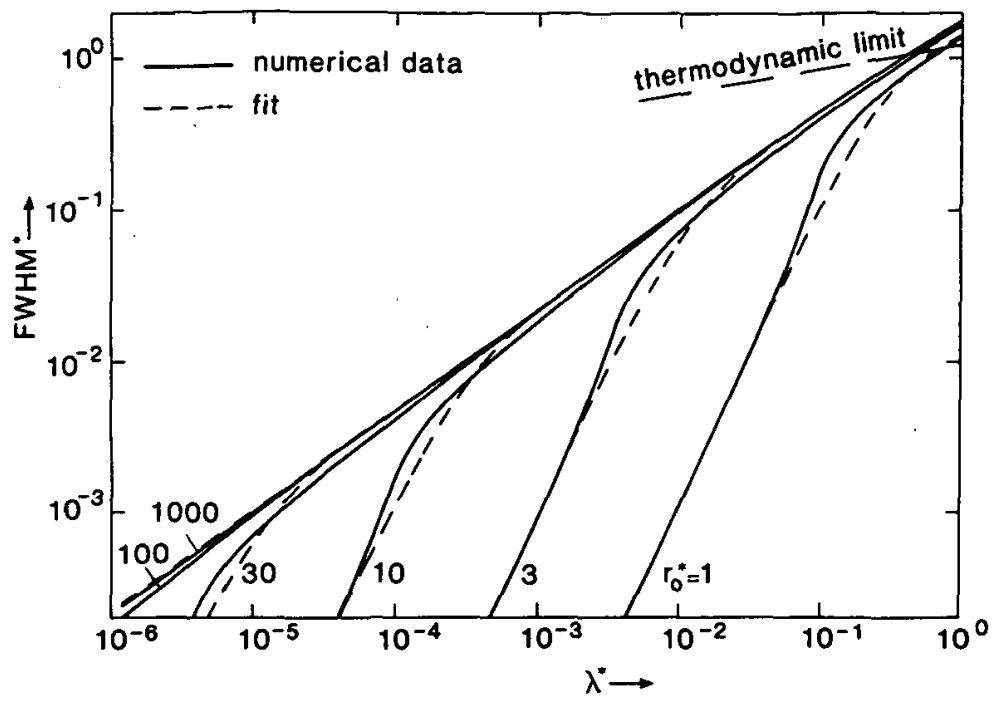

Figure 7.10 The FWHM of the scaled distribution of axial velocity displacements $\rho^{*}\left(\Delta_{\mathrm{Z}}{ }^{*}\right)$ generated in a homocentric cylindrical beam segment, for different values of the scaled beam radius $r_{0}{ }^{*}$. The depicted fit is defined by equations (7.4.27) and (7.4.28). The thermodynamic limit is given by equation (7.7.4) and refers to the relaxation of potential energy.

$$
\begin{aligned}
\text { FWHMG }^{*} & =4\left[2 p_{2} *(\infty) \ln 2\right]^{1 / 2} \operatorname{PPE}\left(r_{0}^{*}\right) \lambda^{* 1 / 2} \\
& =1.832 \operatorname{PPE}\left(r_{0}^{*}\right) \lambda^{* 1 / 2}
\end{aligned}
$$

where the function $\operatorname{Pp}_{P}\left(r_{0}^{*}\right)$ is defined as

$$
\operatorname{PPE}\left(r_{0}^{*}\right)=\left[p_{2} *\left(r_{0}^{*}\right) / p_{2} *(\infty)\right]^{1 / 2}
$$

with $\mathrm{p}_{2}{ }^{*}\left(\mathrm{r}_{0}{ }^{*}\right)$ and $\mathrm{p}_{2}{ }^{*}(\infty)$ defined by equation $(7.4 .14)$. The subscript $P E$ indicates that the result refers to the Energy distribution generated in a homocentric cylindrical beam segment in which the particles follow Parallel trajectories. The Gaussian behaviour of $\rho^{\star}\left(\Delta v_{z}{ }^{*}\right)$ stems from the quadratic behaviour of $p^{\star}\left(k^{*}\right)$ for small $k^{*}$ values.

II) Holtsmark regime. For $\lambda^{*}<<1$ and $\chi_{p} \gg>1$ the distribution $\rho^{*}\left(\Delta v_{z}\right)^{*}$ becomes identical to the 1-dimensional variant of the Holtsmark distribution. Substitution of equation (7.4.12) into equation (7.4.17) yields

$$
\rho^{*}\left(\Delta \mathrm{v}_{\mathrm{Z}}^{*}\right)=\frac{1}{\pi} \int_{0}^{\infty} \mathrm{d} \mathrm{k}^{*} \cos \left(\mathrm{k}^{*} \Delta \mathrm{V}_{\mathrm{Z}}{ }^{*}\right) \mathrm{e}^{-(4 \sqrt{2 \pi} / 15) \lambda^{*} \mathrm{k}^{* 3 / 2}}
$$

analogous to equation $(5.8 .25)^{\circ}$, specifying the distribution of the axial force component $F_{\|}$in an extended cylindrical beam. The shape of this type of distribution is depicted in figure 5.3 (curve corresponding to $\gamma=3 / 2)$. The FWHM of the distribution of equation $(7.4 .23)$. is equal to 
as follows with equation (5.9.15) and table 5.1 .

III) Pencil beam regime. For $\chi_{p} \ll 1$ the distribution $\left.\rho^{*}\left(\Delta v_{z}\right)\right)$ is determined by the large $k^{*}$-behaviour specified by equation (7.4.13). Substitution into equation $(7.4 .17)$ yields

$$
\rho^{*}\left(\Delta \mathrm{v}_{\mathrm{z}}^{*}\right)=\frac{1}{\pi} \int_{0}^{\infty} \mathrm{d} \mathrm{k}^{*} \cos \left(\mathrm{k}^{*} \Delta \mathrm{v}_{\mathrm{z}} *\right) \mathrm{e}^{-2 \sqrt{2 \pi} \mathrm{r}_{0} * 2 \lambda^{*} \mathrm{k}^{* 1 / 2}}
$$

analogous to equation (5.8.28), specifying the distribution of the axial force component $F_{/ /}$in a pencil beam. $A$ plot of this type of distribution is given by figure 5.3 (curve for $\gamma=1 / 2$ ). The FWHM of the distribution of equation $(7.4 .25)$ follows again with equation (5.9.15) and table 5.1

$$
\text { FWHMP }^{*}=.447118 \pi r_{0}^{* 4} \lambda * 2=11.237 r_{0}^{* 4} \lambda^{* 2}
$$

It can directly be verified that equations (7.4.21), (7.4.24) and (7.4.26) provide a complete description of the different regimes shown in figure 7.10 . In order
$(7.4 .26)$ we use

In order to interpolate equations $(7.4 .21),(7.4 .24)$ and

$$
\mathrm{FWHM}^{*}=\mathrm{FWHM}_{\mathrm{H}}^{*} \mathrm{HPE}_{\mathrm{PE}}\left(\lambda^{*}, r_{0}{ }^{*}\right)
$$

where $\mathrm{FWHM}_{\mathrm{H}}$ * is given by equation (7.4.24) and the function $\mathrm{H}_{\mathrm{PE}}\left(\lambda^{*}, \mathrm{r}_{0} *\right)$ is defined as

$$
\mathrm{H}_{\mathrm{PE}}\left(\lambda^{*}, \mathrm{r}_{0^{*}}\right)=\left\{\left[1+\left(\frac{\mathrm{FWHM}_{\mathrm{H}^{*}}}{\mathrm{FWHM}_{G^{*}}}\right)^{6}\right]^{1 / 4}+\left(\frac{\mathrm{FWHM}_{\mathrm{H}}^{*}}{\mathrm{FWHM}_{\mathrm{P}^{*}}}\right)^{3 / 2}\right\}^{-2 / 3}
$$

which is identical to

$$
H_{P E}\left(\lambda^{*}, r_{0}^{*}\right)=\left[\left(1+2.997 \frac{\lambda^{*}}{\operatorname{PPE}_{P}\left(r_{0}^{*}\right)^{6}}\right)^{1 / 4}+1.386 \frac{1}{\chi_{p}^{2}}\right]^{-2 / 3}
$$

with $\chi_{p}=4 \lambda^{*} r_{0} * 3$, as specified by equation $(7.4 .20)$. The function $P_{P E}\left(r_{0}^{*}\right)$ is specified by equation $(7.4 .22)$ and the fit-function given by equation (7.4.16). The function $\mathrm{HPE}_{\mathrm{PE}}\left(\lambda^{*}, \mathrm{r}_{0^{*}}\right)$ is similar to the function $G_{C E}\left(\bar{\lambda}, \bar{r}_{C}\right)$ given by equation $(7.3 .35)$. However, in the present case we took $F_{W H M}{ }^{*}$ as reference, instead of FWHM $_{\mathrm{G}}{ }^{*}$, because the Holtsmark regime is dominant for practical operating conditions. For comparison the fit of equation $(7.4 .27)$ is included in figure 7.10 .

Equations $(7.4 .21)$ through $(7.4 .28)$ give a complete specification of the FWHM of the generated axial velocity distribution $\rho^{*}\left(\Delta v_{z}{ }^{*}\right)$ in terms of the scaled coordinates defined by equations $(6.13 .1)$. The remaining task is to remove the scaling in order to make the dependency on the experimental parameters explicit. The properties of the scaled velocity distribution $\rho^{*}\left(\Delta v_{z}{ }^{*}\right)$ can be related to the corresponding energy distribution using

$$
\Delta E \cong \mathrm{mv}_{z} \Delta \mathrm{v}_{z}=\mathrm{E} \frac{2 v}{v_{z}} \Delta \mathrm{v}_{z}^{*}=\mathrm{E}\left(\frac{4 \mathrm{e}}{\pi \epsilon_{0}} \frac{1}{\mathrm{LV}}\right)^{1 / 3} \Delta \mathrm{v}_{\mathrm{z}}{ }^{*}
$$


The general expressions of equation (7.4.27) transforms to

$\frac{F_{W H M_{E}}}{E}=C_{P H E} H_{P E}\left(\lambda^{*}, r_{0}^{*}\right) \frac{I^{2 / 3} L}{V^{4 / 3} r_{0}^{4 / 3}}, \quad C_{P H E}=2.8775 \frac{m^{1 / 3}}{(15 \pi)^{2 / 3} E_{0}} \quad(7.4 .30)$

In the case of electrons one finds $\mathrm{C}_{\mathrm{PHE}}=2.4147$ in SI-units (note: the subscript PHE indicates that the constant applies to a Parallel beam and refers to the Holtsmark type of Energy distribution).

For the Holtsmark-regime $\left(\lambda^{*}<<1\right.$ and $\left.\chi_{p}>>1\right)$ one may approximate $\mathrm{H}_{\mathrm{PE}}\left(\lambda^{*}, \chi_{\mathrm{p}}\right)=1$. Accordingly, equation $(7.4 .30)$ yields directly

$$
\frac{F W H M}{E}=C_{P H E} \frac{I^{2 / 3} L}{V^{4 / 3} r_{0}^{4 / 3}} \quad\left(\lambda^{*}<<1, \chi_{p}>>1\right)
$$

For the Gaussian regime $\left(\lambda * \gtrsim 1\right.$ and $\left.\chi_{p} \gg>1\right)$ equation $(7.4 .30)$ transforms to

$$
\frac{F W H M_{E}}{E}=C_{P G E} P_{P E}\left(r_{0}^{*}\right) \frac{I^{1 / 2} L^{2 / 3}}{V^{13 / 12 r_{0}}} \quad\left(\lambda \star \gtrsim 1, \chi_{P} \gg>1\right)
$$

where the constant $C_{P G E}$ is given by

$$
C_{P G E}=\frac{217 / 12\left[p_{2}^{*}(\infty) \ln 2\right]^{1 / 2}}{\pi^{5 / 6}} \frac{e^{1 / 12 m^{1 / 4}}}{E_{0} 5 / 6}
$$

in which $\mathrm{p}_{2} *(\infty)$ is defined by equation $(7.4 .14)$. Equation (7.4.32) can also be derived directly from equations (7.4.21) and (7.4.29). In the case of electrons one finds $C_{P G E}=0.4537$ in SI-units.

For the Pencil beam regime $\left(\chi_{p}<<1\right)$ equation $(7.4 .30)$ yields

$$
\frac{F_{W H M_{E}}}{E}=C_{P P E} \frac{I^{2} L}{V^{2}} \quad\left(x_{p}<<1\right)
$$

where the constant $C_{P P E}$ is given by

$$
C_{P P E}=0.44711 \frac{\mathrm{m}}{4 \varepsilon_{0} \mathrm{e}^{2}}
$$

which can also be derived directly from equations $(7.4 .26)$ and $(7.4 .29)$. In the case of electrons one finds $C_{P P E}=4.4800 \times 10^{17}$ in SI-units.

\subsection{Beam segment with a crossover of arbitrary dimensions}

In the previous two sections the Boersch effect was calculated for the extreme cases of a beam segment with a narrow crossover and a homocentric cylindrical beam segment respectively. In this section we like to investigate the general case of a beam segment with a crossover in which the parameter $K$, defined by equation (7.3.1), has some arbitrary value between, say $10^{\circ}$ and $10^{4}$. Unfortunately, this case is less suited for an analytical treatment and one has to rely strongly on numerical calculations. In order to establish results which are of practical merit the final numerical data will be fitted with analytical expressions. Our aim is to obtain a set of expressions which for $\mathrm{K} \rightarrow \infty$ (narrow crossover) yield the results of section 7.3 and for $K \rightarrow 0$ (homocentric cylindrical beam) yield the results of section 7.4 .

We will again use the scaling of equation (7.3.2). The scaled two-particle distribution $\bar{\rho}_{2}\left(\Delta \bar{v}_{2}\right)$ is defined by equation $(7.3 .4)$. As $\mathrm{K}$ is not necessarily large, only a fraction of the collisions will be 
complete. Accordingly, one may not use equation (7.3.3) for the entire integration domain of equation (7.3.4). The conditions for which equation (7.3.3) is valid can be expressed as

$$
\overline{\mathrm{r}}_{\mathrm{c}} \mathrm{K}_{1} \overline{\mathrm{v}}+\overline{\mathrm{a}}_{0} \gg \overline{\mathrm{b}} \text { and } \overline{\mathrm{r}}_{\mathrm{c}} \mathrm{K}_{2} \overline{\mathrm{v}}-\overline{\mathrm{a}}_{0} \gg \bar{b} \text { and } \overline{\mathrm{r}}_{\mathrm{c}} \mathrm{K}_{\mathrm{v}}{ }^{3} \gg 2
$$

as follows from equation (6.7.7) using equations $(6.3 .5),(6.2 .3)$, $(6.7 .6),(7.3 .1)$ and $(7.3 .2)$. We defined $\overline{\mathbf{a}}_{0}=\mathrm{a}_{0} / \mathrm{d}_{0}$ and $\bar{b}=\mathrm{b} / \mathrm{d}_{0}$, where $\mathrm{a}_{0}$ and $b$ are given by equation (7.2.3). Equation (7.3.3) predicts too large values of $\Delta \bar{v}_{z}$ in case the constraints of equation (7.5.1) are violated.

For collisions which are weak one may use equation (7.2.2). By scaling it transforms to

$$
\Delta \bar{v}_{z} \cong \frac{\bar{b}_{z}}{\bar{v} \bar{b}^{2}}\left[\frac{\bar{r}_{c} K_{1} \bar{v}+\bar{a}_{0}}{\left[\left(\bar{r}_{c} K_{1} \bar{v}+\bar{a}_{0}\right)^{2}+\bar{b}^{2}\right]^{1 / 2}}+\frac{\bar{r}_{c} K_{2} \bar{v}-\bar{a}_{0}}{\left\{\left(\bar{r}_{c} K_{2} \bar{v}-\bar{a}_{0}\right)^{2}+\bar{b}^{2}\right)^{1 / 2}}\right]
$$

Notice that this expression depends on $K_{1}$ and $K_{2}$, unlike equation (7.3.3). We recall that a collision is weak when the eccentricity $E$ is large or the perihelion is not reached during the flight or the perihelion is already passed at the start of the collision, as expressed by equation $(6.8 .1)$. In terms of the scaled parameters used here these conditions transform to

or

$$
\overline{\bar{v}} 2 \gg 2
$$

or

$$
\left[\left(\bar{r}_{c} K_{1} \bar{v}+\bar{a}_{0}\right)^{2}+\bar{b}^{2}\right]^{1 / 2} \gg \bar{r}_{c} K \bar{v}
$$

$$
\left[\left(\overline{\mathrm{r}}_{\mathrm{c}} \mathrm{K}_{2} \overline{\mathrm{v}}-\overline{\mathrm{a}}_{0}\right)^{2}+\bar{b}^{2}\right]^{1 / 2} \gg \overline{\mathrm{r}}_{\mathrm{c}} \mathrm{K} \overline{\mathrm{v}}
$$

In the calculation of the two-particle distribution $\bar{\rho}_{2}\left(\Delta \bar{v}_{z}\right)$ from equation (7.3.4), our computer program determines for every two-particle collision whether all constraints of equation (7.5.1) are satisfied or not. If so the collision is complete and it determines the displacement $\Delta \overline{\mathbf{v}}_{\mathbf{z}}$ from equation $(7,3.3)$. If not it tests next whether one or more of the constraints of equation (7.5.3) are satisfied or not. If so the collision is weak and it evaluates the corresponding displacement $\Delta \overline{\mathbf{v}}_{z}$ from equation (7.5.2). For collisions which are neither complete nor weak the displacement $\Delta \overline{\mathbf{v}}_{\mathbf{z}}$ is computed by means of the numerical approach which was outlined in section 6.6 and is referred to as full collision dynamics. Clearly, one may use the full collision dynamics for all types of collisions, but, if applicable, the analytical expressions are preferred since their evaluation requires typically 5 times less computation time.

To start with, we will consider the case that the crossover is located in the middle of the beam segment, thus $S_{c}=0.5$ and $K=K_{1}=K_{2}$. At the end of this section we will indicate how the results can be generalized to include other beam geometries. The two-particle distribution $\bar{\rho}_{2}\left(\Delta \bar{v}_{z}\right)$ was evaluated numerically from equation $(7.3 .4)$ for $K=0.1,0.2,0.5,1,2,5,10,20,50,100,200,500,1000,2000$, 5000 and 10,000 . For every $K$ we considered the cases $\bar{r}_{c}=10^{\circ}, 10^{1}, 10^{2}$, $10^{3}, 10^{4}$ and $10^{5}$. Figures $7.11 \mathrm{a}, 7.11 \mathrm{~b}$ and $7.11 \mathrm{c}$ depict the results obtained for $K=1, \quad K=100$ and $K=10,000$ respectively. These figures should be compared to figure 7.1 , which is computed on the bases of complete collisions. The plots are identical for large values of $\Delta \bar{v}_{z}$. However, significant differences occur for small and intermediate $\Delta \bar{v}_{z}$-values, especially in combination with small $\bar{r}_{c}$-values. The figures 7.11 show that the values obtained for $\bar{\rho}_{2}\left(\Delta \bar{v}_{z}\right)$ increase with $K$ and ultimately approach the values of figure 7.1 for $K+\infty$. 


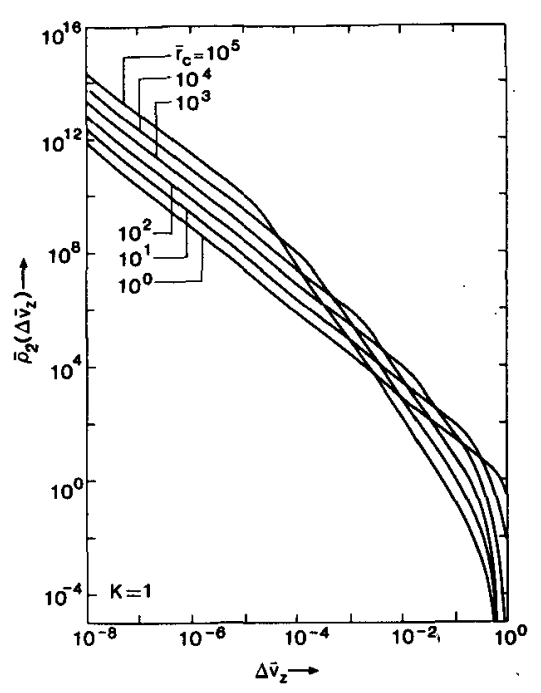

fig.7.11a

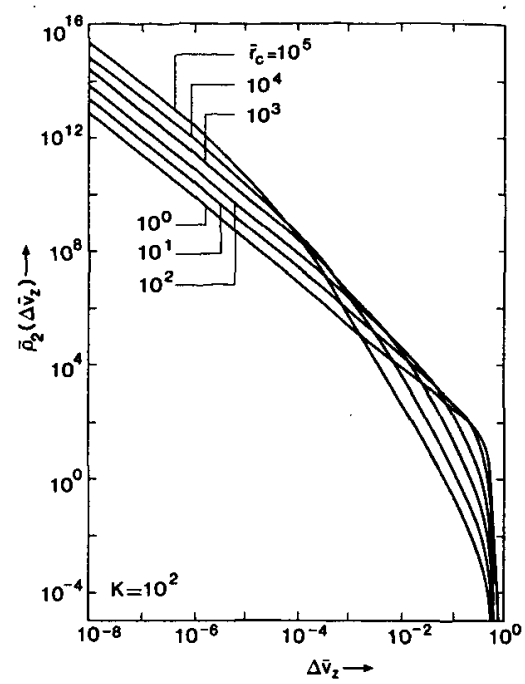

fig.7.11b

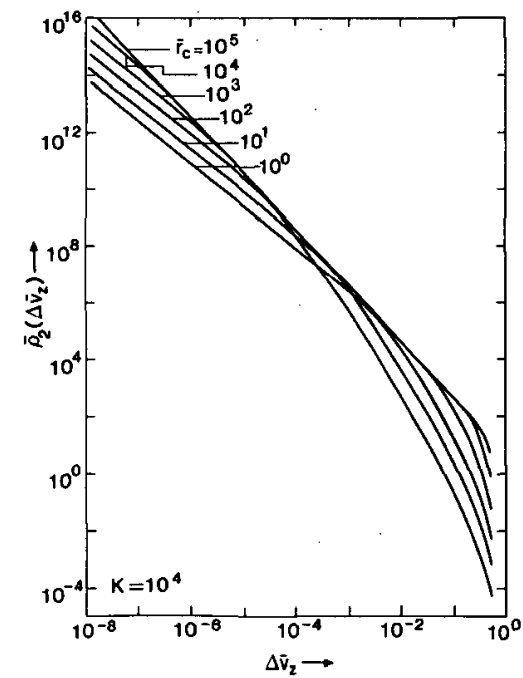

tig.7.11c

Figure 7.11 The scaled two-particle distribution $\bar{\rho}_{2}\left(\Delta \bar{v}_{2}\right)$ for a bean segment with a crossover in the middle, plotted for different values of the scaled crossover radius $\bar{r}_{c}$. The figures $7.11 \mathrm{a}, 7.11 \mathrm{~b}$ and $7.11 \mathrm{c}$ pertain to different values of the beam geometry parameter $\mathrm{K}=\alpha_{0} \mathrm{~L} / 2 \mathrm{r}_{\mathrm{c}}$, as is indicated. The plots should be compared to those of figure 7.1 , derived on the basis of complete collisions. 
Let us investigate whether the results depicted in the figures $7.11 \mathrm{a}, 7.11 \mathrm{~b}$ and $7.11 \mathrm{c}$ can be understood by analytical means. The reader might verify that equation (7.3.13), derived on the basis of complete collisions, provides an accurate approximation of the numerical data of $\bar{\rho}_{2}\left(\Delta \bar{v}_{z}\right)$ for $\Delta \bar{v}_{z}\left(K \bar{r}_{c} / 2\right)^{1 / 3}>>1$. This makes clear that the large $\Delta \bar{v}_{z}$-behaviour of $\bar{\rho}_{2}\left(\Delta \bar{v}_{z}\right)$ [thus $\Delta \bar{v}_{z}>>\left(2 / K \bar{r}_{c}\right)^{1 / 3}$ ] is determined by strong complete collisions. Small displacements $\Delta \bar{v}_{2}$ correspond to weak, predominantly. incomplete collisions with more distant field particles. The plots of the figures 7.11 show that the contribution of these collisions to $\bar{\rho}_{2}\left(\Delta \bar{v}_{2}\right)$ is very sensitive to the value of the beam geometry parameter $\mathrm{K}$.

In order to analyse the small $\Delta \vec{v}_{z}$-behaviour in a quantitative way we will evaluate equation $(7.3 .4)$ on the basis of weak interactions, for which one may use equation (7.5.2). An exact solution of this problem does not appear to be feasible and we will restrict the discussion to some special cases. We will first investigate the limit $K \rightarrow 0$ and next the limit $K \gg>1$. For $K \rightarrow 0$ one may approximate equation $(7.5 .2)$ as (taking $K_{1}=K_{2}=K$ )

$$
\Delta \bar{v}_{z} \cong \frac{\bar{b}_{z} \bar{r}_{c} K}{\left(\bar{b}_{z}{ }^{2}+\bar{r}_{\perp}{ }^{2}\right)^{3 / 2}} \quad(K \rightarrow 0)
$$

using equations $(7,2,3)$. This results is identical to the scaled version of equation (7.2.5), derived for $v=0$. This expression was used in the previous section to evaluate the contribution of weak interactions in a homocentric cylindrical beam segment. The corresponding results for the two-particle distribution $\rho_{2}\left(\Delta v_{z}\right)$ are expressed by equations $(7.4 .4)$ and $(7.4 .5)$. Apparently, one may use these results to describe the limit $\mathrm{K} \rightarrow 0$. However, one has to transform the results from the $\delta, \nu$-scaling used in section 7.4 to the $d_{0}, v_{0}-s c a l i n g$ used here. From equations $(6.13 .1)$ and $(7.3 .2)$ it follows that the different sets of scaling-quantities are related as

$$
\begin{array}{lll}
\mathrm{d}_{0}=\frac{1}{4 \mathrm{v}_{0}{ }^{2}} \delta & , & \mathrm{v}_{0}=\mathrm{v}_{0}^{*} v \\
\delta=\left(4 \overline{\mathrm{r}}_{\mathrm{c}} \mathrm{K}\right)^{2 / 3} \mathrm{~d}_{0} & , & v=\left(\frac{2}{\overline{\mathrm{r}}_{\mathrm{c}} \mathrm{K}}\right)^{1 / 3} \mathrm{v}_{0}
\end{array}
$$

Accordingly, the different sets of parameters can be related using

$$
\begin{aligned}
& \bar{r}_{c}=4 v_{0} * 2 r_{c}{ }^{*}, \quad K=\frac{v_{0}^{*}}{2 r_{c}}, \quad \pi=\frac{r_{0}^{* 2}}{v_{0}^{* 2}} \lambda^{*} \\
& r_{c}^{*}=\left(\frac{\bar{r}_{c}}{16 K^{2}}\right)^{1 / 3}, \quad v_{0}^{*}=\left(\bar{r}_{c} K / 2\right)^{1 / 3}, \lambda^{*}=4 \pi K^{2}\left(\bar{r}_{c} / \bar{r}_{0}\right)^{2}
\end{aligned}
$$

in which $r_{c}$ denotes the crossover radius and $r_{0}$ the radius of the cylindrical beam. Clearly, both quantities become identical for $k \rightarrow 0$.

With equations $(7.5 .5)$ and $(7.5 .6)$ one can rewrite equations $(7.4 .4)$ and $(7.4 .5)$ as

$$
\begin{aligned}
& \bar{\rho}_{2}\left(\Delta \bar{v}_{z}\right)=\frac{2^{5 / 2} \mathrm{~K}^{3 / 2}}{5 \bar{r}_{\mathrm{c}}{ }^{1 / 2} \Delta \overline{\mathrm{V}}_{\mathrm{z}} 5 / 2} \quad\left[\Delta \overline{\mathrm{V}}_{\mathrm{Z}}\left(\overline{\mathrm{r}}_{\mathrm{c}} \mathrm{K} / 2\right) 1 / 3<<1, \Delta \overline{\mathrm{V}}_{\mathrm{z}} \overline{\mathrm{r}}_{\mathrm{c}} 2 / 3 /(32 \mathrm{~K}) 1 / 3>>1\right] \\
& \bar{\rho}_{2}\left(\Delta \bar{v}_{z}\right)=\frac{\left(\overline{\mathrm{r}}_{\mathrm{c}} \mathrm{K} / 2\right)^{1 / 2}}{\Delta \overline{\mathrm{v}}_{\mathrm{z}}{ }^{3 / 2}} \quad\left[\Delta \overline{\mathrm{V}}_{\mathrm{z}}\left(\overline{\mathrm{r}}_{\mathrm{c}} \mathrm{K} / 2\right)^{1 / 3}<<1, \Delta \overline{\mathrm{V}}_{\mathrm{z}} \overline{\mathrm{r}}_{\mathrm{c}} 2 / 3 /(32 \mathrm{~K}) 1 / 3<<1\right]
\end{aligned}
$$

using the definition of the scaled two-particle distribution $\bar{\rho}_{2}\left(\Delta \bar{v}_{2}\right)$ 
given by equation (7.3.4). We note that these equations can also be obtained directly from equations $(7.3 .4)$ and $(7.5 .4)$. The asymptotic behaviour described by equation (7.5.8) agrees with the data of the figures $7.11 \mathrm{a}, 7.11 \mathrm{~b}$ and $7.11 \mathrm{c}$. The behaviour predicted by equation (7.5.7) becomes only manifest for extreme small $\mathrm{K}$-values in combination with large $\overline{\mathbf{r}}_{c}$-values, see figure $7.11 \mathrm{a}$.

$\mathrm{K}_{1}=\mathrm{K}_{2}=\mathrm{K}$ )

For $\mathrm{K} \gg>1$ one may approximate equation (7.5.2) as (taking again

$$
\Delta \bar{v}_{z}=\frac{2 \bar{r}_{c} K}{\bar{b}_{z}\left(\left(\bar{r}_{c} K \bar{v}\right)^{2}+\bar{b}_{z}^{2}\right]^{1 / 2}} \quad\left(K>>1, \bar{\sigma}_{z}>>\bar{r}_{\perp}, \bar{v} \neq 0\right)
$$

in which it was assumed that $\bar{v} \neq 0$ and $\bar{b}_{z} \gg \bar{r}_{\perp}$, thus $b \approx b_{z}$. This is justified since we are considering weak and therefore remote interactions. From equations (7.3.4) and (7.5.9) one obtains (for $\Delta \bar{v}_{z}>0$ )

$$
\bar{\rho}_{2}\left(\Delta \bar{v}_{z}\right)=\int_{0}^{1} 2 \bar{v} d \bar{v} \int_{0}^{\infty} d \bar{h}_{z} \delta\left(\Delta \bar{v}_{z}-\frac{2 \bar{r}_{c} K}{\bar{b}_{z}\left[\left(\bar{r}_{c} K \bar{v}\right)^{2}+\bar{b}_{z}\right]^{1 / 2}}\right)
$$

By substituting

$$
s=\frac{b_{z}}{\bar{r}_{c} K \bar{v}} \quad, \quad t=\frac{2}{\bar{r}_{c} K s\left(1+s^{2}\right)^{1 / 2}} \frac{1}{\bar{v}^{2}}
$$

and carrying out the t-integration. equation $(7.5 .10)$ transforms to

$$
\bar{\rho}_{2}\left(\Delta \bar{v}_{z}\right)=\frac{2^{3 / 2}}{\left(\bar{r}_{c} K\right)^{1 / 2} \Delta \bar{v}_{z}^{5 / 2}} \int_{0}^{\infty} \frac{d s}{s^{3 / 2}\left(1+s^{2}\right)^{3 / 4}} \theta\left(\Delta \bar{v}_{z}-\frac{2}{\bar{r}_{c} K s\left(1+s^{2}\right)^{1 / 2}}\right)
$$

in which $\theta(x)$ is the step-function. Let $s_{0}$ be a value of the integration variable $s$ for which the argument of the step function becomes zero

$$
\mathrm{s}_{0}{ }^{4}-\mathrm{s}_{0}^{2}-\left(2 / \overline{\mathrm{r}}_{\mathrm{c}} \mathrm{K} \Delta \overline{\mathrm{v}}_{\mathrm{Z}}\right)^{2}=0
$$

This equation has only a single positive root, which defines the lower integration boundary of equation (7.5.12). For $\bar{r}_{c} K \Delta \bar{v}_{z} \ll<$ one finds $s_{0} \approx\left(2 / \bar{r}_{c} K \Delta \bar{v}_{z}\right) 1 / 2$. Thus $s_{0} \gg>1$. Accordingly, the integral in equation (7.5.12) yields approximately $1 / 2 \mathrm{~s}_{0}{ }^{2}=\overline{\mathrm{r}}_{\mathrm{c}} \mathrm{K} \Delta \overline{\mathrm{v}}_{\mathrm{z}} / 4$ and one retrieves equation $(7.5 .8)$. For $\overline{\mathrm{r}}_{\mathrm{c}} \mathrm{K} \Delta \overline{\mathrm{v}}_{\mathrm{z}} \gg>1$ one finds $\mathrm{sos}_{0}\left(2 / \overline{\mathrm{r}}_{\mathrm{c}} \mathrm{K} \Delta \overline{\mathrm{v}}_{\mathrm{z}}\right)^{1 / 4}$. Thus $\mathrm{s}_{0}<<1$. Accordingly, the integral in equation (7.5.12) is approximately equal to $2 / J_{S_{0}}=\left(2 \bar{r}_{c} K \Delta \bar{v}_{Z}\right)^{1 / 2}$ and one obtains

$$
\bar{\rho}_{2}\left(\Delta \bar{v}_{z}\right)=\frac{4}{\Delta \bar{v}_{z}^{2}} \quad\left(K>>1, \quad \bar{r}_{c} K \Delta \bar{v}_{z}>>1, \Delta \bar{v}_{z}<1 / 2\right)
$$

which is identical to equation (7.3.12). Apparently, this dependency stems from collisions which are both weak and complete. Accordingly, the result does not depend on $K$. The different regimes of the two-particle distribution $\bar{\rho}_{2}\left(\Delta \bar{v}_{z}\right)$ are fully specified by equations $(7.3 .13),(7.5 .7)$, $(7.5 .8)$ and $(7.5 .13)$.

We now proceed with the calculation of the function $\bar{p}(\bar{k})$ defined by equation $(7,3.14)$. This equation was evaluated numerically from the data of $\bar{\rho}_{2}\left(\Delta \bar{v}_{z}\right)$. The results for $K=1,100$ and 10,000 are shown in figures $7.12 \mathrm{a}, 7.12 \mathrm{~b}$ and $7.12 \mathrm{c}$ respectively. These figures should be compared to figure 7.2 derived on the basis of complete collisions. The most significant differences occur for large $\mathrm{k}$-values. For complete 


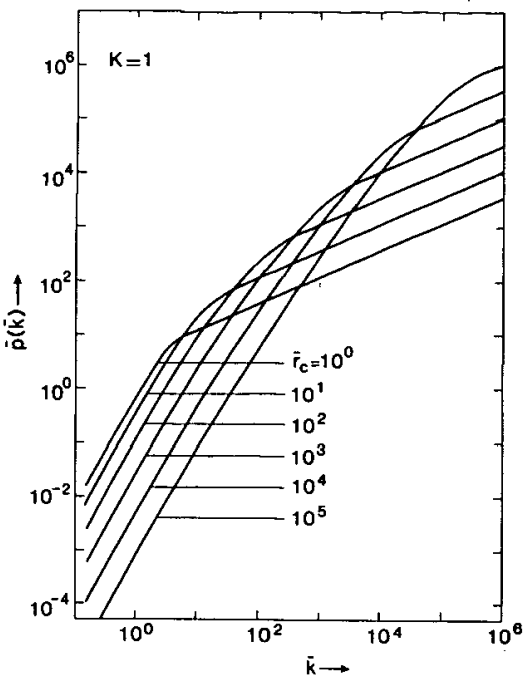

fig.7.12a

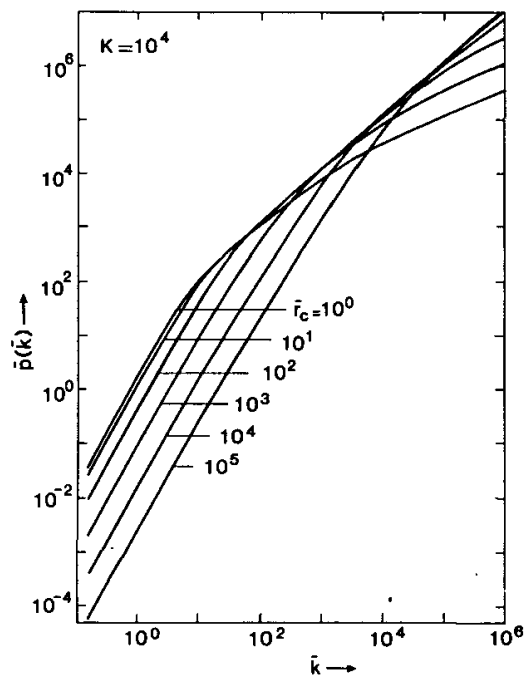

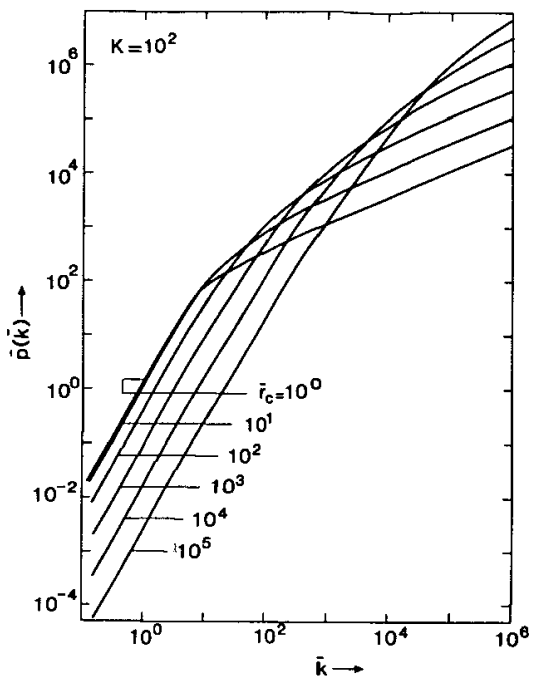

fig.7.12b

fig.7.12c

Pigure 7.12 The scaled function $\vec{p}(k)$ corresponding to the distribution of axial velocity displacements generated in a beam segment with a crossover in the middle, plotted for different values of the scaled crossover radius $\bar{r}_{\mathrm{c}}$. The figures $7.12 a, 7.12 b$ and $7.12 \mathrm{c}$ pertain to different values of the beam geometry parameter $\mathrm{K}=\alpha_{0} \mathrm{~L} / 2 \mathrm{r}_{\mathrm{c}}$, as is indicated. The plots should be compared to those of figure 7.2 , derived on the basis of complete collisions. 
collisions the large $\bar{k}$-behaviour is given by equation (7.3.19). All curves corresponding to different $\bar{x}_{c}$-values ultimately become linear in $\bar{k}$ for $\bar{k} \rightarrow \infty$. This dependency corresponds to the inverse-square behaviour of $\bar{\rho}\left(\Delta \bar{v}_{z}\right)$ found for small $\Delta \bar{v}_{z}$, see equation $(7.3 .12)$. For the general case studied in this section we found that $\bar{\rho}\left(\Delta \bar{v}_{z}\right)$ becomes ultimately proportional to $1 / \Delta \overline{\mathbf{v}}_{\mathrm{z}}{ }^{3 / 2}$ for $\Delta \overline{\mathbf{v}}_{\mathrm{z}} \rightarrow 0$, see equation (7.5.8). Substitution of this expression into equation $(7.3 .14)$ yields

$$
\bar{p}(\bar{k})=2\left(\bar{r}_{\mathrm{c}} \mathrm{KK}\right) 1 / 2 \int_{0}^{\infty} \mathrm{dx} \frac{\sin ^{2} \mathrm{x}}{\mathrm{x}^{3 / 2}}=\left(4 \pi \overline{\mathrm{r}}_{\mathrm{c}} \mathrm{K}\right) 1 / 2 \mathrm{~K}^{1 / 2} \quad(\bar{k}+\infty) \quad(7.5 .14)
$$

which is in agreement with the data of figures $7.12 \mathrm{a}, 7.12 \mathrm{~b}$ and $7.12 \mathrm{c}$.

By similar reasoning one finds that the behaviour described by equation (7.5.7) leads to a 3/2-power dependency on $k$

$$
\bar{p}(\bar{k})=\frac{8(K \bar{k})^{3 / 2}}{5 \bar{r}_{c} 1 / 2} \int_{0}^{\infty} d x \frac{\sin ^{2} x}{x^{5 / 2}}=\frac{32 \sqrt{\pi} K^{3 / 2}}{15 \bar{r}_{c} 1 / 2} k^{3 / 2}
$$

This dependency becomes manifest for intermediate $k$-values in the curves for which $K / \bar{r}_{c} 1 / 2<<1$, see figure $7.12 a$. For increasing $K$-values the intermediate $\bar{k}$-range ultimately becomes dominated by a linear K-dependency, see figure $7.12 \mathrm{c}$. This dependency corresponds to the $\Delta \bar{v}_{z}$-behaviour described by equation (7.5.13). Substitution of this expression into equation $(7.3 .14)$ yields

$$
\bar{p}(\bar{k})=8 k \int_{0}^{\infty} d x \frac{\sin ^{2} x}{x^{2}}=4 \pi k
$$

which is identical to equation (7.3.19).

For small $\mathrm{k}$-values, all curves shown in the figures $7.12 \mathrm{a}, 7.12 \mathrm{~b}$ and 7.12 become quadratic in $k$. In order to describe this behaviour we define the function $\bar{p}_{2}\left(\bar{r}_{c}, K\right)$ as

$$
\bar{p}(k)=\frac{1}{2} \bar{p}_{2}\left(\bar{r}_{c}, K\right) k^{2}
$$

The function $\bar{p}_{2}\left(\bar{r}_{c}, K\right)$ is the generalization of the function $\bar{p}_{2}\left(\bar{r}_{c}\right)$ defined by equation $(7,3,16)$. The function $\bar{p}_{2}\left(\bar{r}_{c}, K\right)$ is directly related to the second moment of the displacement distribution $\bar{\rho}\left(\Delta \bar{v}_{z}\right)$, as can be seen from equation $(7,3,30)$. We evaluated $\bar{p}_{2}\left(\bar{r}_{c}, K\right)$ numerically. The results are plotted in figure 7.13 .

In order to obtain an analytical expression for $\bar{p}_{2}\left(\overline{\mathbf{r}}_{\mathbf{c}}, K\right)$ we will consider its behaviour for $K+\infty$ and $K \rightarrow 0$. In the limit $K+\infty$ it should yield the result which was derived on the basis of complete collisions

$$
\lim _{\mathrm{K} \rightarrow \infty} \overline{\mathrm{p}}_{2}\left(\overline{\mathrm{r}}_{\mathrm{c}}, \mathrm{K}\right)=\overline{\mathrm{p}}_{2}\left(\overline{\mathrm{r}}_{\mathrm{c}}\right)
$$

in which $\bar{p}_{2}\left(\bar{r}_{c}\right)$ is defined by equation $(7.3 .16)$. For $K \rightarrow 0$ one should retrieve the result for a homocentric cylindrical beam segment, expressed by equation $(7.4 .14)$. By means of the scale-relations of equations $(7.5 .5)$ and $(7.5 .6)$ one obtains

$$
\lim _{\mathrm{K} \rightarrow 0} \overline{\mathrm{p}}_{2}\left(\overline{\mathrm{r}}_{\mathrm{c}}, \mathrm{K}\right)=16\left(2 \mathrm{~K}^{2} / \overline{\mathrm{r}}_{\mathrm{c}}\right)^{2 / 3} \mathrm{p}_{2} *\left[\left(\overline{\mathrm{r}}_{\mathrm{c}} / 16 \mathrm{~K}^{2}\right)^{1 / 3}\right]
$$

For $\bar{r}_{c} \gg 16 \mathrm{~K}$ one may approximate $p_{2} *\left[\left(\bar{r}_{c} / 16 K\right)^{1 / 3}\right] \approx p_{2} *(\infty) \approx 0.1513$, see equation $(7.4 .14)$. We recall that the functions $\bar{p}_{2}$ and $p_{2} *$, may in 


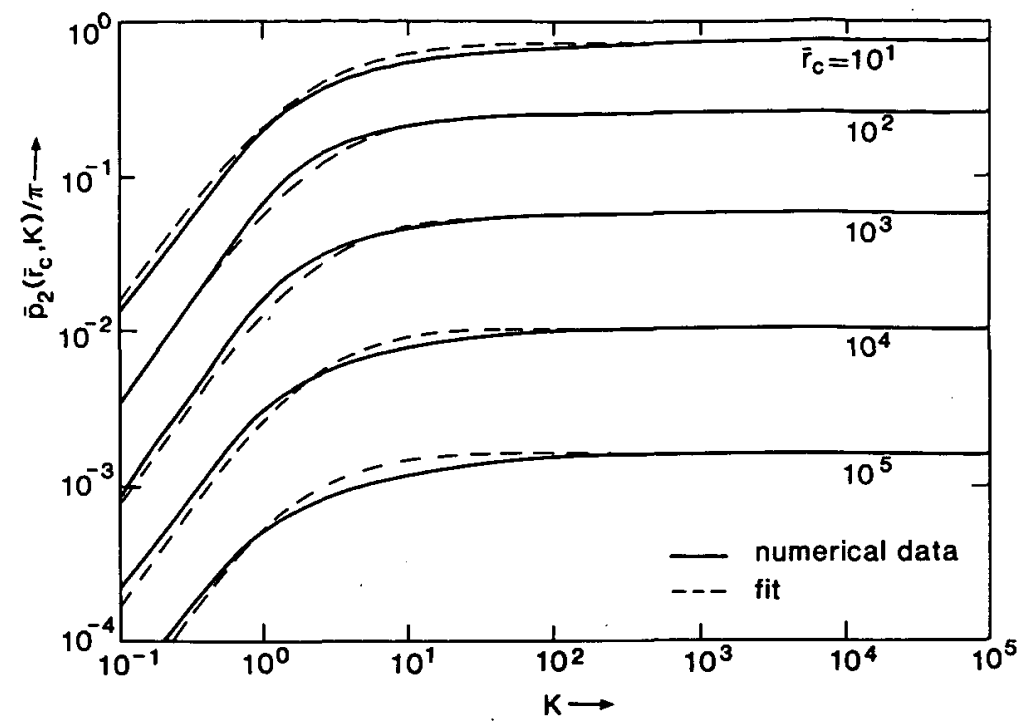

Figure 7.13 The function $\overline{\mathrm{p}}_{2}\left(\overrightarrow{\mathrm{r}}_{\mathrm{c}}, \mathrm{K}\right)$ defined by equation $(7.5 .17)$ and its approximation given by equation (7.5.20). This function gives the dependency on $\vec{r}_{c}$ and $\mathrm{K}$ of the function $\overline{\mathrm{p}}(\overline{\mathrm{K}})$, plotted in the figures $7.12 a, 7.12 b$ and $7.12 c$, for $k \rightarrow 0$.

general be approximated by the functions $\bar{p}_{2 a}$ and $p_{2 a} *$ defined by equations (7.3.17) and (7.4.16) respectively. As an approximation for the general function $\overline{\mathrm{p}}_{2}\left(\overline{\mathrm{r}}_{\mathrm{c}}, \mathrm{K}\right)$ we use

$\overline{\mathrm{p}}_{2 \mathrm{a}}\left(\overline{\mathrm{r}}_{\mathrm{c}}, \mathrm{K}\right)=\frac{\pi}{.788+.6\left(\frac{\overline{\mathrm{r}}_{\mathrm{c}}}{\mathrm{K}^{2}}\right)^{2 / 3}\left[1+.5\left(\frac{\mathrm{K}^{2}}{\overline{\mathrm{r}}_{\mathrm{c}}}\right)^{4 / 9}\right]^{3 / 2}+\frac{\pi \overline{\mathrm{r}}_{\mathrm{c}}}{\left\{2 \ln \left[.8673\left(114.6+\overline{\mathrm{r}}_{\mathrm{c}}\right)\right]\right\}^{2}}}$

$(7.5 .20)$

which shows the asymptotic behaviour described by equations (7.5.18) and (7.5.19), provided that $\overline{\mathrm{Y}}_{\mathrm{c}} \gg>16 \mathrm{~K}$. For comparison we included a plot of the function given by equation $(7.5 .20)$ in figure 7.13 .

Finally, we determined the FWHM of the displacement distribution $\bar{\rho}\left(\Delta \vec{v}_{z}\right)$ from the numerical data of the function $\bar{p}(\bar{k})$ by evaluating the integral of equation (7.3.21). The results for $K=1,10,100,1000$ and 10,000 are plotted in the figures $7.14 \mathrm{a}$ through $7.14 \mathrm{e}$. The following regimes should be distinguished :

I. Gaussian regime. For large values of $\pi$ the distribution $\bar{\rho}\left(\Delta \bar{v}_{z}\right)$ is determined by the small $k$-behaviour of $\bar{p}(\bar{k})$, expressed by equation (7.5.17). The quadratic $\mathrm{K}$-dependency leads to a Gaussian distribution with a FWHM which is given by equation (7.3.31). $P_{C E}$ now denotes a function which depends both on $\bar{r}_{c}$ and $K$

$$
\operatorname{PCE}\left(\bar{Y}_{c}, K\right)=\left[\bar{p}_{2}\left(\bar{r}_{c}, K\right) / \pi\right]^{1 / 2}
$$

where $\overline{\mathrm{p}}_{2}\left(\overline{\mathrm{r}}_{\mathrm{c}}, \mathrm{K}\right)$ can be approximated by equation $(7.5 .20)$. 

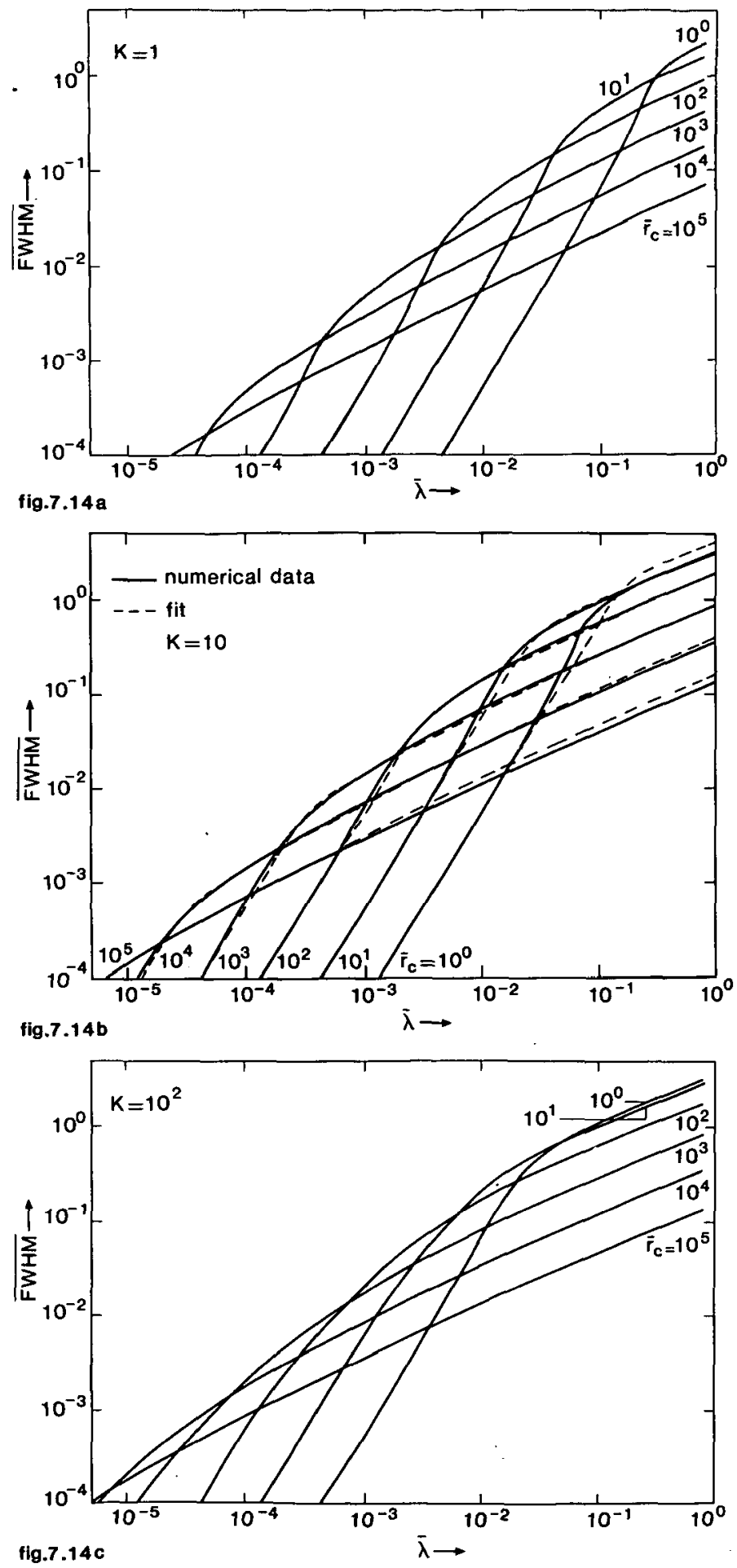


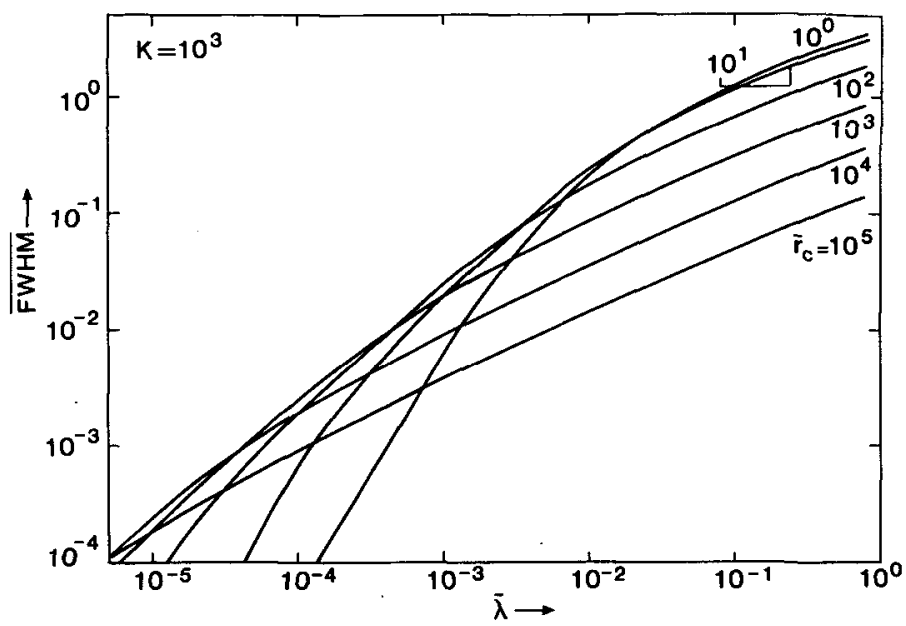

fig.7.14d

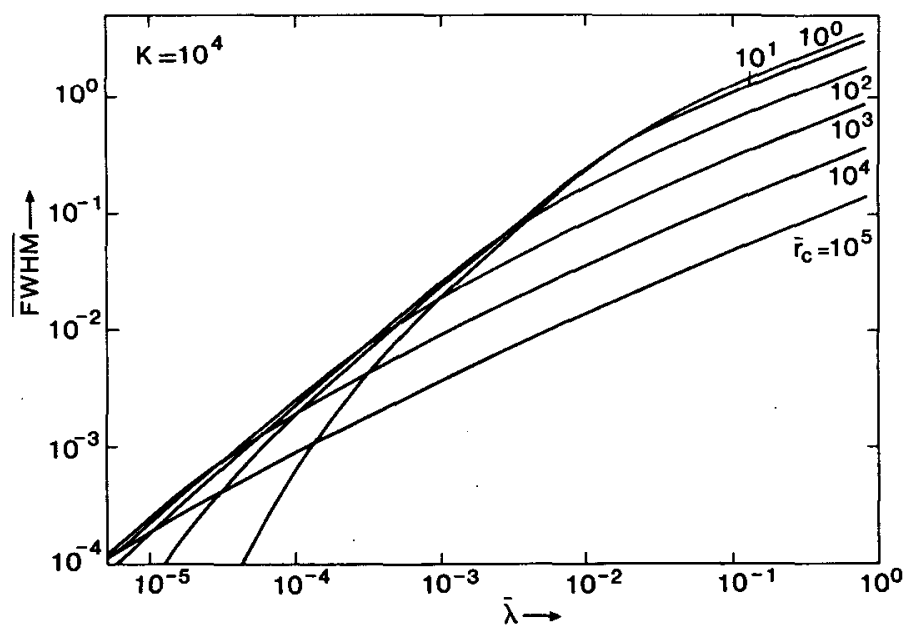

fig.7.140

Pigure 7.14 The FWHM of the scaled distribution of axial velocity displacements $\bar{\rho}\left(\Delta \bar{v}_{z}\right)$ generated in a beam segment with a crossover in the middle, plotted for different values of the scaled crossover radius $\bar{r}_{\mathrm{c}}$. The figures $7.14 \mathrm{a}, 7.14 \mathrm{~b}$, $7.14 \mathrm{c}, 7.14 \mathrm{~d}$ and $7.14 \mathrm{e}$ pertain to different values of the beam geometry parameter $\mathrm{K}=a_{0} \mathrm{~L} / 2 \mathrm{r}_{\mathrm{c}}$, as is indicated. The fit depicted in figure $7.14 \mathrm{~b}$ is defined by equations (7.5.30), $(7.5 .31)$ and (7.5.32). As the quality of the fit is about the same for all k-values, it is omitted in the other figures. The figures $7.14 a$ to 7.14 e should be compared to figure 7.5 , derived on the basis of complete collisions. 
II. Holtsmark regime. For intermediate values of $\lambda$ and small K-values the distribution $\bar{\rho}\left(\Delta \bar{v}_{z}\right)$ is dominated by the $3 / 2$ power-dependency of $\bar{p}(\bar{k})$, expressed by equation $(7.5 .15)$. The corresponding distribution is of the Holtsmark-type with a FWHM which is equal to

$$
\overline{\mathrm{FWHM}}_{\mathrm{H}}=2.87758\left(\frac{6 \pi}{25}\right)^{1 / 3} \mathrm{H}(\mathrm{K}) \pi^{2 / 3} / \overline{\mathrm{r}}_{\mathrm{c}} 1 / 3
$$

The function $H(K)$ can be estimated from the numerical data such as presented in the figures $7.11 \mathrm{a}$ through $7.11 \mathrm{e}$. As an approximation of the numerical data we use the function

$$
H(K)=\frac{1}{\left[1+9 K^{-2}+2 K^{-1 / 3}\right]^{1 / 2}}
$$

which has the following asymptotes

$$
\lim _{K \rightarrow 0} H(K)=K / 3 \quad, \quad \lim _{K \rightarrow \infty} H(K)=1-K-1 / 3
$$

We will demonstrate that these asymptotes can be derived from the analytical results. The limit $K \rightarrow 0$ corresponds to the conditions of $a$ homocentric cylindrical beam, which was studied in the previous section. In the Holtsmark regime one may use equation (7.4.24). By utilizing the scale-relations of equations $(7.5 .5)$ and $(7.5 .6)$ one can rewrite equation (7.4.24) in terms of the quantities $\pi, \bar{r}_{c}$ and $K$. By comparison with equation $(7.5 .22)$ one finds $H(K)=K / 3$, which is in agreement with the first relation of equations $(7.5 .24)$.

The behaviour for $K \rightarrow \infty$ can be verified from equation $(7.4 .24)$ by employing the slice-method, which was described in section 5.11. This method requires that the deviations of the particles from their unperturbed trajectories are small. For the Holtsmark regime this condition is always fulfilled (when the deviations would be large the resulting distribution is not Holtsmarkian). In order to perform the slice-method one should express the equations in terms of the experimental parameters. Using equation (7.3.36) one can rewrite equation (7.5.22) as

$$
\frac{F_{W H M}}{E}=C_{C H E} H(K) \frac{I^{2 / 3}}{V^{4 / 3} \mathrm{r}_{\mathrm{c}}{ }^{1 / 3} \alpha_{0}}, \quad C_{C H E}=2.8775 \frac{23^{1 / 3} \mathrm{~m}^{1 / 3}}{(5 \pi)^{2 / 3} \mathrm{E}_{0}}\left(=6 \mathrm{C}_{\mathrm{PHE}}\right)
$$

In the case of electrons one finds $\mathrm{C}_{\mathrm{CHE}}=14.488$ in sI-units. This equation refers to the energy spread generated in the entire beam segment. According to equation (7.4.31) the contribution of a single slice at axial position $z$ of length $\Delta z$ to the tota? generated energy distribution can be expressed as

$$
\Delta\left(\mathrm{FWHM}_{\mathrm{E}} / \mathrm{E}\right)=\mathrm{C}_{\mathrm{PHE}} \frac{\mathrm{I}^{2 / 3}}{\mathrm{~V}^{4 / 3} \mathrm{r}_{\mathrm{c}}^{4 / 3}} \frac{\Delta z}{\left[1+\alpha_{0}|z-2 \mathrm{c}| / \mathrm{r}_{\mathrm{C}}\right]^{4 / 3}}
$$

where $z_{c}$ denotes the $z$-coordinate of the crossover. Substitution into equation (5.11.1) and integration yields equation (7.5.25), where the function $H(K)$ is now given by

$$
H(K)=\left(1-\frac{1}{2\left(1+K_{1}\right)^{1 / 3}}-\frac{1}{2\left(1+K_{2}\right)^{1 / 3}}\right)
$$

in which $K_{1}$ and $K_{2}$ are given by equations (7.3.1). Here we study the case $K=K_{1}=K_{2}$. One directly sees that equation (7.5.27) satisfies the 
relations of equation $(7.5 .24)$. Thus equation $(7.5 .27)$ shows the same asymptotic behaviour for $K \rightarrow 0$ and $K \rightarrow \infty$ as equation (7.5.23), based on the numerical results. However, for intermediate $\mathrm{K}$-values equation $(7.5 .27)$ leads to somewhat smaller values than predicted by equation (7.5.23). This difference is due to the fact that the calculation based on the slice-method relies on the assumption that the particle density is uniform in every cross section of the beam. In the numerical evaluation, on the other hand, we assumed an uniform spatial and angular distribution in the crossover, see equation (7.2.6). Consequently, the density distribution is not entirely uniform in the planes which do not coincide with the crossover, the lowest density being reached at the edge of the beam.

III. Lorentzian regime. For intermediate $\pi$ and large $K$-values the distribution $\bar{\rho}\left(\Delta \bar{v}_{z}\right)$ is dominated by the linear part of $\bar{p}(\bar{k})$, described by equation (7.5.16). Accordingly, it takes on the Lorentzian form, given by equation $(7,3.26)$. The corresponding FWHM is given by equation (7.3.33). In terms of experimental parameters it leads to equation $(7.3 .40)$.

IV. Pencil beam regime. For small $\lambda$ values the distribution $\bar{\rho}\left(\Delta \bar{v}_{z}\right)$ is determined by the large $\bar{k}$-behaviour of the function $\bar{p}(\bar{k})$, which shows a square-root dependency, as expressed by equation (7.5.14). The corresponding distribution is characteristic for pencil beams, see equations (5.8.28) and (7.4.25). A beam with a crossover is referred to as pencil beam if it fulfils the condition $\chi_{c} \ll<1$. The quantity $\chi_{c}$ is called the pencil beam factor for a beam segment with a crossover

$$
\chi_{c}=2 K \bar{r}_{c} \bar{\lambda}=\alpha_{0} L \lambda
$$

which is the ratio between the distance $\alpha_{0} \mathrm{~L}$ and the average axial separation of the particles $1 / \lambda$. The FWHM of the distribution $\bar{\rho}\left(\Delta \bar{v}_{z}\right)$ generated in a pencil beam can be expressed as

$$
\overline{\mathrm{FWHM}}_{\mathrm{p}}=.447114 \pi \mathrm{K} \overline{\mathrm{r}}_{\mathrm{c}} \bar{\lambda}^{2}
$$

This relation can be obtained from equation $(7.4 .26)$ using the scaling relations of equations $(7.5 .5)$ and $(7.5 .6)$. Equation $(7.4 .33)$ gives the FWHM in terms of the experimental parameters. Notice that this equation is independent of the beam diameter $r_{0}$ and thus independent of the beam geometry.

In order to interpolate the results obtained for the different regimes we use

$$
\overline{F W H M}=\overline{F_{W H M}} G_{C E}\left(\bar{x}, \bar{r}_{c}, K\right), \quad \overline{F W H M}_{0}=2(2 \pi \ln 2) 1 / 2 \lambda^{1 / 2}
$$

similar to equation (7.3.34). The function $G_{C E}$ now also depends on $K$ and is defined as

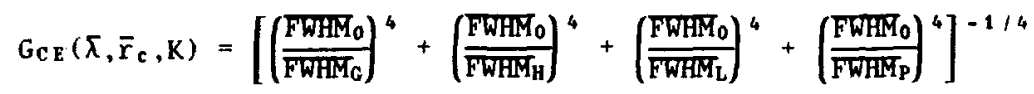

By substitution of equations (7.3.31), (7.5.22), (7.3.33) and (7.5.29) one obtains

$$
\mathrm{G}_{\mathrm{CE}}\left(\pi, \overline{\mathrm{r}}_{\mathrm{c}}, \mathrm{K}\right)=\left[\frac{1}{\operatorname{PCE}\left(\overline{\mathrm{r}}_{\mathrm{c}}, \mathrm{K}\right)^{4}}+\frac{\mathrm{A} \overline{\mathrm{r}}_{\mathrm{c}}^{4 / 3}}{\bar{\lambda}^{2 / 3} \mathrm{H}(\mathrm{K})^{4}}+\frac{\mathrm{B}}{\bar{\lambda}^{2}}+\frac{\mathrm{C}}{\bar{\lambda}^{6} \overline{\mathrm{r}}_{\mathrm{c}}^{4} \mathrm{~K}^{4}}\right]^{-1 / 4}
$$


in which the constants $A, B$ and $C$ are equal to

$$
A=1.57510^{-3}, \quad B=7.60610^{-4}, \quad C=0.3045
$$

The function $P_{C E}\left(\bar{r}_{C}, K\right)$ is defined by equations $(7.5 .21)$ and $(7.5 .20)$. and the function $H(K)$ by equation $(7.5 .23)$. For $K \rightarrow \infty$ one retrieves equation (7.3.35), using that $H(\infty)=1$ and $P_{C E}\left(\bar{r}_{C}, \infty\right)=P_{C E}\left(\bar{r}_{C}\right)$. This explains a posteriori the form of equation (7.3.35). For comparison the fit of equations $(7.3 .34)$ and $(7.5 .31)$ is included in figure $7.14 \mathrm{~b}$ $(K=10)$.

Equation (7.3.37) expresses the final result in terms of the experimental parameters. Equations $(7.3 .37)$ and $(7.5 .31)$ constitute the main results of this chapter. These expressions should yield accurate results for the FWHM energy spread generated in a beam segment of arbitrary geometry, provided that the theoretical parameters $\lambda_{,} \overline{\mathbf{r}}_{\mathrm{c}}$ and $K$, defined by equations $(7.3 .22),(7.3 .5)$ and $(7.3 .1)$ respectively, fulfil the conditions

$$
\bar{K}^{2}<1, \overline{\mathrm{r}}_{\mathrm{C}}<10^{6}
$$

which is usually the case for practical operating conditions.

Similar results can be derived for the Full width median (FW50) energy spread. Employing equation $(5.9 .15)$ and table 5.1 one finds from equation $(7.5 .30)$

$$
\overline{\mathrm{FW}}_{50}=.57288 \overline{\mathrm{FWHM}}_{\mathrm{O}} \mathrm{G}_{\mathrm{CE}}\left(\bar{\lambda}, \overline{\mathrm{r}}_{\mathrm{C}}, \mathrm{K}\right)
$$

The function $G_{C E}\left(\pi, \bar{r}_{c}, K\right)$ is again given by equation $(7.5 .31)$, now taking the constants $A, B$ and $C$ equal to

$$
A=8.24610^{-4}, \quad B=8.19210^{-5}, \quad C=3.01510^{-5}
$$

instead of the values given by equation (7.5.32). The results for the individual regimes can be obtained from the expressions for the FWHMvalues using

$$
\begin{aligned}
& \mathrm{FW}_{5} \text { OC }=.57288 \mathrm{FWHM}_{\mathrm{G}} \\
& \mathrm{FW}_{5 \text { OH }}=.67347 \mathrm{FWHM}_{\mathrm{H}} \\
& \mathrm{FW}_{5} \text { OL }=1.0000 \mathrm{FWHM}_{\mathrm{L}} \\
& \mathrm{FW}_{\text {S O }}=5.7429 \mathrm{FWHM}_{\mathrm{P}}
\end{aligned}
$$

as follows from the data of table 5.1 .

Finally, let us consider the case that the crossover is not located in the middle of the beam segment, thus $S_{c} \neq 0.5$. Accordingly, $\mathrm{K}_{1} \neq \mathrm{K}_{2}$, see equations $(7.3 .1)$. In good approximation one may determine the effective value of the function $G_{C E}\left(\bar{X}, \bar{r}_{c}, K\right)$ as

$$
G_{C E}\left(\pi, \bar{r}_{C}, K\right)_{\text {eff }}=\frac{1}{2}\left[G_{C E}\left(\pi, \bar{r}_{c}, K_{1}\right)+G_{C E}\left(\pi, \bar{r}_{c}, K_{2}\right)\right]
$$

The function $G_{C E}\left(\bar{r}, \bar{r}_{C}, K\right)$ becomes independent of $K$ for large $K$-values, especially in combination with large $\pi$-values. In that case equation $(7.5 .37)$ is trivial. For small and intermediate $K$ and $\lambda$-values one may assume that the energy spread generated in the second part of the beam segment (from the crossover to the exit plane) is not affected by the interaction in the first part of the beam segment (from the entrance plane to the crossover), which justifies equation $(7.5 .37)$. 
The validity of equation (7.5.37) can also be understood from the equations applying to the individual regimes. For the pencil-beam regime we found equation (7.5.29), which complies with equation $(7.5 .37)$. since $K=\left(K_{1}+K_{2}\right) / 2$. The same conclusion follows for the Holtsmark regime, see equations $(7.5 .22)$ and $(7.5 .27)$. In the Lorentzian regime the result becomes independent of $K$, see equation (7.3.33), and equation (7.5.37) is evident. In the Gaussian regime the approximation of equation (7.5.37) may become inaccurate, due to the contribution of strong interactions. However, these errors will be small since the dependency on $K$ is weak, as can be seen from equation (7.5.20).

\subsection{Results for Gaussian angular and spatial distributions}

The results of the previous sections apply to the case of an uniform spatial and an uniform angular distribution in the crossover, see equation $(7.2 .6)$. We will now investigate the required modifications in case either the angular or the spatial distribution or both distributions are Gaussian, utilizing equations (5.3.4). For a Gaussian angular distribution one has to replace the v-integration in equation $(7.2 .6)$ by

$$
\int_{0}^{\infty} \frac{2 v d v}{v_{0}^{2}} e^{-\left(v / v_{0}\right)^{2}}, \quad v_{0}=\sqrt{2} v_{z} \sigma_{\alpha}
$$

where $\sigma_{\alpha}$ is the $\sigma$-value of the angular distribution in the crossover. For this two-dimensional Gaussian distribution $\sigma_{\alpha}{ }^{2}=\left\langle\alpha^{2}\right\rangle / 2$. For a Gaussian spatial distribution one has to replace the $r_{\perp}$-integration in equation $(7,2.6)$ by

$$
\int_{0}^{\infty} \frac{2 r_{\perp} d r_{\perp}}{r_{c}^{2}} e^{-\left(r_{\perp} / r_{c}\right)^{2}}, \quad r_{c}=\sqrt{2} \sigma_{r}
$$

where $\sigma_{r}$ is the $\sigma$-value of the spatial distribution in the crossover (with $\sigma_{\mathrm{r}}^{2}=<r_{1}^{2}>/ 2$ ).

Starting from the modified distribution of field particles prescribed by equations $(7.6 .1)$ and $(7.6 .2)$ one can repeat the program outlined in the sections $7.3,7.4$ and 7.5 . As the principle aspects involved remain the same we will restrict the discussion to the presentation of the main results. We evaluated equations (7.2.6) and (7.2.7) on the basis of complete collisions, using equation (7.2.1). Analogous to equation $(7.3 .15)$ we obtained the following expressions for the scaled function $\bar{p}(\bar{k})$ :

- For a Gaussian angular distribution and a Gaussian spatial distribution

$$
\bar{p}_{B B}(k)=k \frac{16}{\sqrt{\pi}} \int_{0}^{\infty} d \bar{v} e^{-\bar{v}^{2}} \int_{0}^{\infty} d y e^{-y^{2}} \int_{0}^{1} d u \frac{\left(1-u^{2}\right)^{1 / 2}}{u} \sin \left(\frac{k \bar{v} u}{\left(4+\bar{v}^{4} y^{2} \bar{r}_{c}^{2}\right)^{1 / 2}}\right)
$$

- For a Gaussian angular distribution and an uniform spatial distribution 
$\bar{p}_{\mathrm{B}} u(\bar{k})=\bar{k} \frac{32}{\pi} \int_{0}^{\infty} d \bar{v} e^{-\bar{v}^{2}} \int_{0}^{1} d y \sqrt{1-y^{2}} \int_{0}^{1} d u \frac{\left(1-u^{2}\right)^{1 / 2}}{u} \sin \left(\frac{\bar{k} \bar{v} u}{\left(4+\bar{v}^{4} y^{2} \bar{r}_{c^{2}}\right)^{1 / 2}}\right)$

- For an uniform angular distribution and a Gaussian spatial distribution

$$
\bar{p}_{u g}(\bar{k})=\bar{k} \frac{16}{\sqrt{\pi}} \int_{0}^{1} d \bar{v} \int_{0}^{\infty} d y e^{-y^{2}} \int_{0}^{1} d u \frac{\left(1-u^{2}\right)^{1 / 2}}{u} \sin \left(\frac{k \bar{v} u}{\left(4+\bar{v}^{4} y^{2} \bar{r}_{\left.c^{2}\right)^{1 / 2}}\right)}\right.
$$

As before we will investigate the behaviour for $k \rightarrow 0$ and for $k \rightarrow \infty$.

For $k \rightarrow 0$ equations $(7.6 .3),(7.6 .4)$ and $(7.6 .5)$ show a quadratic dependency on $\bar{k}$, as described by the first equation of $(7.3 .16)$. For the function $\bar{p}_{2}\left(\bar{r}_{c}\right)$ one finds from equations $(7.6 .3),(7.6 .4)$ and $(7.6 .5)$ respectively:

$$
\begin{aligned}
& \overline{\mathrm{p}}_{2 \mathrm{zg}}\left(\overline{\mathrm{r}}_{\mathrm{c}}\right)=\frac{4 \sqrt{\pi}}{\overline{\bar{r}}_{\mathrm{c}}} \int_{0}^{\infty} \mathrm{dy} \frac{\exp \left(-\mathrm{y}^{2}\right)}{\mathrm{y}} N\left(\frac{\bar{r}_{c} y}{2}\right) \\
& \bar{p}_{28}\left(\bar{r}_{c}\right)=\frac{8}{\bar{r}_{c}} \int_{0}^{1} d y \frac{\sqrt{1-y^{2}}}{y} N\left(\frac{\bar{r}_{c} y}{2}\right) \\
& \bar{p}_{2 u_{B}}\left(\bar{r}_{c}\right)=\frac{4 \sqrt{\pi}}{\bar{r}_{c}} \int_{0}^{\infty} d y \frac{\exp \left(-y^{2}\right)}{y} \sinh ^{-1}\left(\frac{\bar{r}_{c} y}{2}\right)
\end{aligned}
$$

where the function $N(x)$ is defined as

$$
N(x)=\int_{0}^{\infty} d z \frac{\exp (-z / x)}{\left(1+z^{2}\right)^{1 / 2}}=\frac{1}{2} \int_{0}^{\infty} d z \exp [-\sinh (z) / x]
$$

For all practical purposes one may approximate this function by

$$
\mathrm{N}_{\mathrm{a}}(\mathrm{x})=\frac{1}{1 / \mathrm{x}+1 / \ln (17.58+1.180 \mathrm{x})}
$$

which has the same asymptotic behaviour for $x \rightarrow 0$ and $x+\infty$ as the original

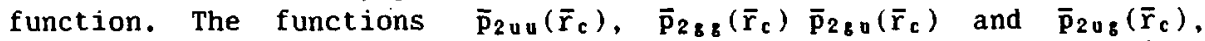
defined by equations $(7.3 .16),(7.6 .6),(7.6 .7)$ and $(7.6 .8)$ respectively, are plotted in figure 7.15 . Similar to equation (7.3.17) we approximate these functions by

$$
\overline{\mathbf{p}}_{2 \mathrm{a}}\left(\overline{\mathrm{r}}_{\mathrm{c}}\right)=\frac{\pi}{1+\pi F \overline{\mathrm{r}}_{\mathrm{c}} /\left\{2 \ln \left[.8673\left(\mathrm{E}+F \overline{\mathrm{r}}_{\mathrm{c}}\right)\right]\right\}^{2}}
$$

in which $E$ and $F$ are numerical constants, which are different for each plot

$$
\begin{aligned}
& E_{B B}=40.74 \quad, \quad F_{B B}=1.315 \\
& E_{B U}=49.19, \quad F_{8 U}=1.138 \\
& \mathbf{E}_{\mathbf{u}_{\mathrm{g}}}=100.0, \quad \mathbf{F}_{\mathbf{u}_{\mathrm{g}}}=1.160 \\
& \left(E_{u} u=114.6, \quad F_{u u}=1\right)
\end{aligned}
$$




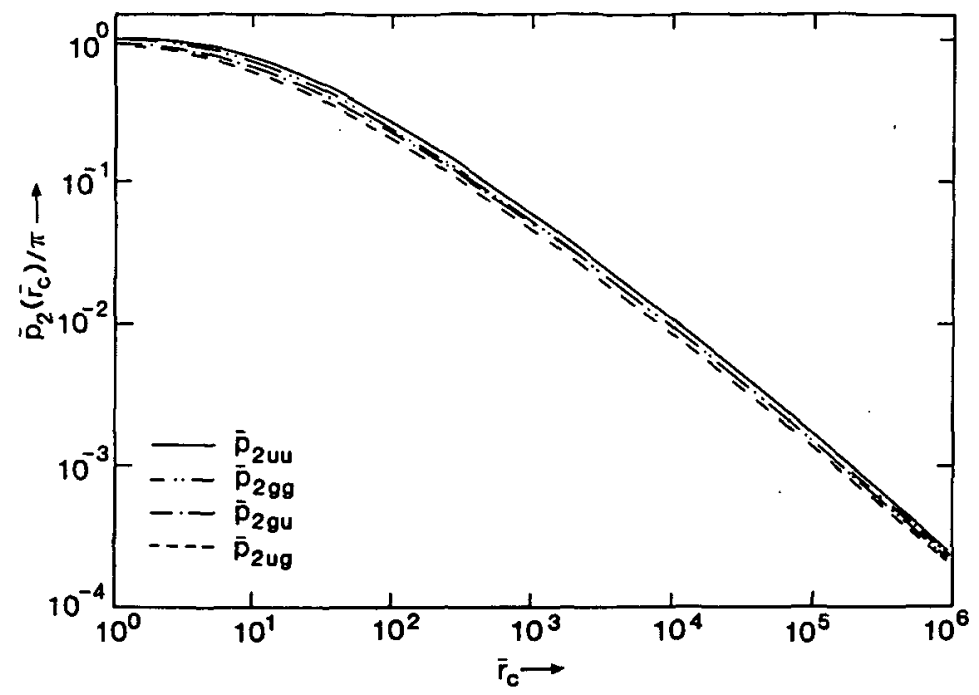

Pigure 7.15 The function $\overline{\mathrm{p}}_{2}\left(\overline{\mathrm{r}}_{\mathrm{c}}\right)$ for different types of angular and spatial distributions in the crossover. The first subscript refers to the type of angular distribution and the second subseript to the type of spatial distribution (u=uniform, g=Gaussian). The curve $\overline{\mathbf{p}}_{20 \mathrm{u}}$ is defined by equation $(7.3 .16)$ and is the same as plotted in figure 7.3. The other curves are defined by equations $(7.6 .6),(7.6 .7)$ and $(7.6 .8)$.

The constants $E_{u} u$ and $F_{u u}$ correspond to the values used in equation (7.3.17). The small $\bar{k}$-behaviour of the function $\bar{p}(\bar{k})$, which is specified by the function $\bar{p}_{2}\left(\bar{r}_{\mathrm{c}}\right)$, determines the mean square $\left\langle\Delta \mathrm{v}_{\mathrm{z}}{ }^{2}\right\rangle$ of the velocity distribution $\rho\left(\Delta \mathbf{v}_{2}\right)$, see equation $(7.3 .30)$. The quadratic $\bar{k}-$ dependency corresponds to a Gaussian distribution $\rho\left(\Delta v_{z}\right)$. The FWHM-value of this distribution is proportional to $\left[\bar{p}_{2}\left(\bar{r}_{\mathrm{c}}\right)\right]^{1 / 2}$, see equation (7.3.31).

For $k+\infty$ equations $(7.6 .3),(7.6 .4)$ and $(7.6 .5)$ show a linear dependency on $\bar{k}$, as described by equation (7.3.19). The constant $\bar{p}_{\infty}$ varies for different angular and spatial distributions :

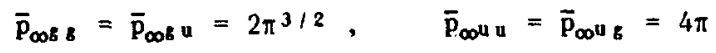

The linear $\mathrm{k}$-behaviour corresponds to a Lorentzian distribution $\rho\left(\Delta \mathrm{v}_{z}\right)$, see equations $(7.3 .26)$ and $(7.3 .24)$. Equation $(7.6 .13)$ indicates that the Lorentzian distribution is not affected by the type of spatial distribution, but depends only on the angular distribution. This is in agreement with the fact that the FWHM of the corresponding energy distribution does not depend on $r_{c}$, see equation (7.3.40). The physical argument is that the Lorentzian behaviour stems from weak complete collisions, which occur for a large axial separation $b_{z}$ and a large flight-distance Tv. The displacement $\Delta \mathrm{v}_{\mathrm{z}}$ caused by such a collision is insensitive for $r_{\perp}$, as can be seen from equation $(7,2.2)$.

It is of ten convenient to express the results obtained for nonuniform distributions in terms of the effective width measures $\alpha_{e f} f$ and ref $f$, which are defined as the widths for which the expressions obtained for uniform distributions yield the same results as those obtained within the full calculation taking the proper distribution(s) into 
account. From equation (7.6.13) and equation (7.3.40) it follows that the effective width of a Gaussian angular distribution is for the Lorentzian regime given by

$$
\alpha_{\text {eff }}=\frac{2}{\sqrt{\pi}} \alpha_{c}=\frac{2 \sqrt{2}}{\sqrt{\pi}} \sigma_{\alpha}=1.596 \sigma_{\alpha} \quad \text { (Lorentzian regime) }(7.6 .14 \text { ) }
$$

For the Gaussian regime the effective width measures depend on $\bar{r}_{c}$, as can be seen from figure 7.15. We ignore this dependency and approximate

$$
\alpha_{\text {eff }} \cong 1.6 \sigma_{\alpha}, \quad r_{\text {eff }} \cong 1.6 \sigma_{r} \quad \text { (Gaussian regime) }
$$

as can be understood from equations (7.6.11) and (7.6.12) with the definition of $\bar{r}_{c}$, see equation (7.3.5). The effective width measures of equation $(7.6 .15)$ yield very accurate results for $\bar{r}_{c} \gtrsim 100$, but are less suited to describe the results for smaller values of $\overline{\mathbf{r}}_{c}$.

The remaining cases to be investigated pertain to the Holtsmark regime and the pencil beam regime. For those cases the distribution $\rho\left(\Delta v_{z}\right)$ can be determined from the distribution of the axial interaction force $\rho\left(F_{\| /}\right)$occurring in the unperturbed beam. Accordingly, it is sufficient to determine in what way $\rho\left(F_{\| \prime}\right)$ is affected by the type of density distribution. The calculation of $\rho\left(F_{\|}\right)$for a eylindrical beam with an uniform density distribution was carried out in section 5.8 . The results are expressed by equations (5.8.25) and (5.8.28). We will reconsider this calculation, now starting from a Gaussian radial density distribution. Using equation $(7.6 .2)$ one may modify equation (5.8.21) as

$$
\rho_{2}\left(F_{\|}\right)=\int_{-\infty}^{\infty} d z \int_{0}^{\infty} \frac{2 r d r}{r_{0}^{2}} e^{-\left(r / r_{0}\right)^{2}} \delta\left(F_{/ /}-\frac{C_{0}}{r^{2}} \frac{(z / r)}{\left(1+(z / r)^{2}\right)^{3 / 2}}\right)
$$

Analogous to equation $(5.8 .22)$ this transforms to

$$
\rho_{2}\left(F_{/ /}\right)=\frac{C_{0} 3 / 2}{r_{0}^{2} F_{/ /}^{5 / 2}} \int_{0}^{\infty} d s \frac{s^{3 / 2}}{\left(1+s^{2}\right)^{9 / 4}} \exp \left(-\frac{C_{0}}{F_{/ /} r_{0}^{2}} \frac{s}{\left(1+s^{2}\right)^{3 / 2}}\right)
$$

As before we will investigate the extreme cases $r_{0}+\infty$ and $r_{0} \rightarrow 0$, which correspond to an extreme extended beam and a pencil beam respectively.

For $r_{0}+\infty$ one may replace the exponential function in equation (7.6.17) by unity and one retrieves equation (5.8.23). This expression leads to the one-dimensional variant of the Holtsmark distribution, given by equation $(5.8 .25)$. Thus one obtains the same equation as for an uniform density distribution. However $r_{0}$ now stands for $\sqrt{2} \times \sigma_{\mathrm{r}}$, instead of the outer beam radius, see equation (7.6.2). The FWHM of the corresponding energy distribution is for a homocentric cylindrical beam given by equation $(7.4 .31)$ and for a beam segment with a crossover by equation ( 7.5 .25$)$, In general, the equations for an uniform distribution are retrieved by taking

$$
\alpha_{\text {eff }}=\alpha_{0}=\sqrt{2} \sigma_{\alpha}, \quad r_{\text {eff }}=r_{c}=\sqrt{2} \sigma_{r} \quad \text { (Holtsmark regime) }
$$

For $r_{0 \rightarrow 0}$ one can approximate the integral of equation (7.6.16) by taking $1+\mathrm{s}^{2} \cong \mathrm{s}^{2}$, which is justified since the contribution to the integral comes from large $s$-values. This gives 


$$
\rho_{2}\left(F_{/ 1}\right)=\frac{C_{0}{ }^{3 / 2}}{r_{0}^{2} F_{/ 1}^{5 / 2}} \int_{0}^{\infty} d s \frac{1}{s^{3}} \exp \left(-\frac{C_{0}}{F_{\| /} r_{0}^{2}} \frac{1}{s^{2}}\right)=\frac{C_{0}{ }^{1 / 2}}{2 F_{/ /}^{3 / 2}}
$$

which is identical to equation $(5.8 .26)$. This result does not depend on $r_{0}$ and is therefore not affected by the type of current density distribution. This can also be seen from the expression for the FWHM of the corresponding energy distribution, which is given by equation $(7.4 .33)$. The physical explanation is that all particles are effectively on a row. Accordingly, the result depends only on the axial particle distribution and not on their radial distribution.

Equations $(7.6 .14),(7.6 .15)$ and $(7.6 .18)$ specify for the different regimes the values of the effective beam semi-angle $\alpha_{e} f$ of a Gaussian angular distribution and the effective crossover radius $r_{e} f$ of a Gaussian spatial distribution. For beams with a narrow crossover the generated energy distribution is either Gaussian or Lorentzian. In these regimes one should use $1.60 \times \sigma$ as an effective width for a Gaussian (angular or spatial) density distribution. For a (nearly) cylindrical extended beam the generated energy distribution is Holtsmarkian. As an effective width for a Gaussian density distribution one should in this case use $\sqrt{ } 2 \times \sigma$. The type of density distribution does not affect the results obtained for a pencil beam.

The FWHM of the energy distribution generated in a beam segment with a crossover is in the case of an uniform angular and spatial distribution given by equations (7.3.37) and (7.5.31). The different regimes are implicitly covered by these equations and the question arises how to implement the results of this section. One way is to assume that equation (7.6.15) provides a suitable approximation for all regimes. This leads to reasonable accurate results for the Gaussian, the Lorentzian and the pencil beam regime, but introduces a significant error in the Holtsmark regime. A more accurate approach is to use the full equations obtained in this section for Gaussian density distributions, instead of the various effective width measures $\alpha_{e f f}$ and $r_{\text {eff }}$. Starting from equations $(7.6 .11),(7.6 .12),(7.6 .13)$ and $(7.6 .18)$ one finds for each of the four combinations of an uniform or a Gaussian angular distribution and an uniform or a Gaussian spatial distribution (uu, $g g, g u$ and $u g$ ) a different version of the function $G_{C E}\left(\bar{\lambda}, \bar{r}_{c}, K\right)$, which is for the case of an uniform angular distribution and an uniform spatial distribution (uu) given by equation (7.5.31). For the resulting set of equations the reader is referred to section 16.4, which summarizes the analytical prescriptions for the calculation of the Boersch effect.

\subsection{Thermodynamic limits}

We wish to investigate whether the thermodynamic limits to the Boersch effect, considered in chapter 3, are reached for practical operating conditions. We distinguished two kinds of upper limits. One is associated with the "relaxation of kinetic energy" and given by equation (3.8.3). The other is associated with the "relaxation of potential energy" and given by equation (3.10.11). The former is relevant for a beam segment with a narrow crossover, in which one may neglect the contribution of the relaxation of potential energy (as long as complete collisions are dominant). The latter can best be examined in a homocentric beam segment, in which relaxation of kinetic energy does 
not occur.

It is convenient to express the thermodynamic limits in terms of the scaled parameters used in this chapter. Utilizing equation (7.3.36) one may rewrite equation $(3.8 .3)$ as (notation used in this chapter: $\mathrm{E}=\mathrm{eV}, \Delta \mathrm{E}=\Delta \mathrm{E}_{/}$)

$$
\left\langle\Delta \bar{v}_{z} 2>1 / 2=1 / \sqrt{6}\right.
$$

which specifies the thermodynamic limit of the relaxation of kinetic energy in a beam with a narrow crossover in terms of the rms-value of the distribution of scaled axial velocities $\bar{\rho}\left(\Delta \bar{v}_{z}\right)$. Assuming that this distribution is Gaussian, the corresponding FWHM-value is given by

$$
\overline{\mathrm{FWHM}}=\left(\frac{4 \ln 2}{3}\right)^{1 / 2}=.96135
$$

This upper limit is indicated in figure 7.5. One sees that in order to reach this limit one requires $\lambda_{\approx} 0.05$ and small values of $\bar{r}_{c}$. From figure 7.6 one should conclude that these conditions are beyond the range of normal operation. Thus for a single crossover the thermodynamic limit of kinetic energy relaxation will in general not be reached. However, the figures 7.5 and 7.6 also imply that the thermodynamic limit may possibly be reached for a succession of crossovers, in particular when the system employs a field emission gun of high brightness.

Let us now consider the thermodynamic limit of potential energy relaxation. Utilizing equations $(7.4 .29)$ and $(7.4 .18)$ one can rewrite equation $(3.10 .11)$ as

$$
<\Delta v_{z} * 2>1 / 2=(a / 2) 1 / 3 \lambda^{* 1 / 6}=.5224 \lambda^{* 1 / 6}
$$

which gives the thermodynamic limit of the relaxation of potential energy in a homocentric cylindrical beam segment in terms of the rmsvalue of the distribution of scaled axial velocities $\rho^{*}\left(\Delta v_{z}^{*}\right)$. Assuming that this distribution is Gaussian, the corresponding FWHM-value is given by

$$
\text { FWHM }^{*}=a^{1 / 3} 2^{7 / 6}(\ln 2) 1 / 2 \lambda * 1 / 6=1.230 \lambda * 1 / 6
$$

This upper limit is indicated in figure 7.10. One sees that in order to reach this limit one requires $\lambda * \approx 0.4$ and small values of $r_{c} *$. One may conclude this limit is not reached for normal operating conditions.

As the thermodynamic limit is in general not reached in either of the two extreme beam geometries considered, one may safely assume that the thermodynamic limit (of both kinetic and potential energy relaxation) is in general not reached in a single beam segment of any geometry. However, as was mentioned, the thermodynamic limit may possibly be reached in a beam consisting of a succession of beam segments, all operating at extreme high particle density. 


\subsection{Introduction}

In this chapter the extended two-particle approach will be used to calculate the distribution of transverse velocity displacements $\rho\left(\Delta v_{\perp}\right)$ generated by statistical coulomb interactions in a rotational symetric beam segment in drift space. The distribution $\rho\left(\Delta v_{\perp}\right)$ is equivalent to the distribution of angular deflections $\rho(\Delta \alpha)$. since $\Delta a z \Delta v_{\perp} / v_{z}$. The primary interest of this calculation is to obtain a better physical insight in the trajectory displacement effect, which is the subject of chapter 9.

The calculation of the distribution of transverse velocity displacements $\rho\left(\Delta v_{\perp}\right)$ is in many respects similar to the calculation of the distribution of axial velocity displacements $\rho\left(\Delta v_{z}\right)$, which was presented in chapter 7 . Consequently, the organization of this chapter is quite similar too. The chapter starts with a summary of the relevant results of the chapters 5 and 6 , which cover the basics of the statistical part of the model and the calculation of the two-particle velocity displacement $\Delta v_{\perp}$ respectively. This material is then used to perform the calculation of the statistical angular deflections in two specific beam geometries, which are most suited for an analytical treatment of the problem. One is a beam segment with a narrow crossover and the other is a homocentric cylindrical beam segment. The general case of a beam segment of arbitrary geometry is considered next. The angular and spatial distribution in the crossover are taken uniform in most calculations, but Gaussian distribution(s) are considered too. For all geometries the full angular deflection distribution is computed. Explicit expressions are presented for the Full width at Half Maximum (FWHM) of this distribution, as well as the Full width median value $\left(\mathrm{FW}_{50}\right)$ and the root mean square value ( $\mathrm{rms}$ ). Scaling is applied to reduce the number of independent parameters and to simplify the notation of the intermediate results. In the final results the scaling is removed in order to make the dependency on the experimental parameters explicit.

A computer program has been developed to perform the various steps of the calculation numerically, similar to the program used for the Boersch effect. The results show that one should in general distinguish four regimes, each corresponding to a different type of angular deflection distribution: The Gaussian regime, the weak complete collision regime, the Holtsmark regime and the pencil beam regime. The widths of the various distributions show different dependencies on the experimental parameters. It was found that the location of the crossover strongly influences the results, especially for low particle densities. This is due to the fact that the angular deflection experienced by a particle in the first part of the beam segment (from the entrance plane to the crossover) can partly or entirely be cancelled out in the second part of the beam segment (from the crossover to the exit plane), depending on the type of collision involved and the location of the crossover.

Where possible the numerical calculations are verified by analytical means. Analytical results for the width of the angular deflection distribution can be obtained for all limiting cases were a single regime is dominant. A direct analytical calculation of the behaviour in the transition areas between the different regimes seems not possible. However, the numerical data for the FWHM and FWso values is approximated by analytical expressions, covering the entire range of operating conditions. 
Consider a test particle running in axial direction along the central reference trajectory in a rotational symmetric beam segment. Due to the Coulomb interaction with its neighbour field particles it experiences a change in transverse velocity $\Delta v_{\perp}$ and ends up with a spatial displacement $\Delta r_{f}$. The displacements $\Delta v_{\perp}$ and $\Delta r_{f}$ experienced by a large number of test particles will be randomly distributed due to the stochastic nature of the distribution of field particles within the beam volume. In this chapter we will calculate the distribution $\rho\left(\Delta v_{\perp}\right)$. The distribution $\rho\left(\Delta \mathbf{v}_{\perp}\right)$ is equivalent to the distribution of angular deflections $\rho(\Delta \alpha)$ since, in paraxial approximation, $\Delta a \approx \Delta \mathbf{v}_{1} / \mathbf{v}_{\mathbf{Z}}$.

The trajectory displacement effect, which will be studied in the next chapter, corresponds to the occurrence of random lateral displacements $\Delta r$. The displacement $\Delta r$ of a test-particle is determined by extrapolating its final perturbed position along its final perturbed velocity towards some reference plane. Clearly, this procedure combines the lateral shift at the end of the beam segment $\Delta r_{f}$ and the change in lateral velocity $\Delta v_{\perp}$ into the single virtual shift $\Delta r$. The reference plane is assumed to be optically conjugated to the target plane of the system. The distribution $\rho(\Delta r)$ can therefore directly be related to the blurring observed at the target. It provides all information which is needed to evaluate the performance of a practical system. The calculation of the distribution $\rho\left(\Delta v_{\perp}\right)$ is mainly of theoretical importance. Our primary objective is to provide the theoretical means to handle the more complex calculation of the trajectory displacement distribution $\rho(\Delta r)$.

We will determine the distribution $\rho\left(\Delta v_{\perp}\right)$ by employing the extended two-particle approach, which was outlined in chapter 5 . The dynamical part of the problem consists of the calculation of the transverse velocity shift $\Delta v_{\perp}$ experienced by the test particle due to the interaction with a single field particle. This problem was studied in chapter 6 . The shift $\Delta v_{1}$ can be expressed as a function of the geometrical variables $\xi=\left(r_{1}, b_{2}, v, \Phi\right)$, the time of flight $T$ and the initial time $t_{i}=-S_{c} T$, see equations $(6.2 .1),(6.2 .2)$ and $(6.2 .3)$ respectively and figure 5.1 (note $\Phi=\psi-\varphi$ ). Explicit analytical equations can be determined for (nearly) complete collisions and for weak collisions. For a complete collision $(T \rightarrow \infty)$ we found

$$
\Delta v_{\perp} \cong \frac{v\left[1+\left(m v^{2} r_{\perp} \sin (\Phi) / 2 C_{0}\right)^{2}\right]^{1 / 2}}{1+\left(b_{z}^{2}+r_{\perp}{ }^{2} \sin ^{2} \Phi\right)\left(m v^{2} / 2 C_{0}\right)^{2}}
$$

see equations $(6.11 .3),(6.4 .16)$ and $(6.3 .4)$. The deviation $\Delta v_{\perp}$ caused by a weak collision is given by (reproducing equation $(6.11 .4)$ )

$$
\begin{aligned}
\Delta v_{\perp} & \equiv \frac{c_{0}}{m v b}\left[\left(\frac{b}{\left[\left(S_{c} T v+a_{0}\right)^{2}+b^{2}\right]^{1 / 2}}-\frac{b}{\left\{\left[\left(1-S_{c}\right) T v-a_{0}\right]^{2}+b^{2}\right\}^{1 / 2}}\right)^{2}+\right. \\
& \left.\left(\frac{r_{\perp} \sin (\Phi)}{b}\right)^{2}\left(\frac{S_{c} T v^{2} a_{0}}{\left[\left(S_{c} T v+a_{0}\right)^{2}+b^{2}\right]^{1 / 2}}+\frac{\left(1-S_{c}\right) T v-a_{0}}{\left\{\left[\left(1-S_{c}\right) T v-a_{0}\right]^{2}+b^{2}\right\}^{1 / 2}}\right)^{2}\right]^{1 / 2}
\end{aligned}
$$

in which

$$
a_{0}=-r_{\perp} \cos \Phi \quad, \quad b=\left(b_{z}^{2}+r_{\perp}^{2} \sin ^{2} \Phi\right) 1 / 2
$$

Notice that $\Delta v_{\perp}=0$ when both $r_{\perp}=0$ and $S_{c}=1 / 2$. This implies that weak collisions, taking place in a beam segment with a point crossover in the middle, do not generate angular deflections. On the other hand, complete 
collisions do cause angular deflections in such a geometry, as can be seen from equation $(8.2 .1)$.

Equations $(8.2 .1)$ and $(8.2 .2)$ apply to the case that the initial relative velocity of the particles is non-zero $(v \neq 0)$. In case $v=0$ we found for a half-complete collision (reproducing equation (6.11.6))

$$
\Delta v_{\perp} \cong\left(\frac{c_{0}}{m}\right)^{1 / 2} \frac{r_{\perp}}{\left(b_{z}^{2}+r_{\perp}^{2}\right)^{3 / 4}}-\frac{r_{\perp}}{4 T} \quad(v=0)
$$

and for a weak collision (reproducing equation (6.11.7))

$$
\Delta v_{\perp} \cong \frac{C_{0}}{m} \frac{r_{\perp} T}{\left(b_{z}^{2}+r_{\perp}^{2}\right)^{3 / 2}} \quad(v=0)
$$

Equations (8.2.1) and (8.2.2) will be used in the calculation of statistical angular deflections generated in a beam segment with a crossover, while equations $(8.2 .4)$ and $(8.2 .5)$ are relevant for a homocentric cylindrical beam segment.

The statistical part of the problem consists of the evaluation of equations $(5.7 .7),(5.7 .8)$ and $(5.7 .10)$, in which $\Delta \eta$ now represents $\Delta v_{\perp}$

$$
\begin{aligned}
& \rho_{2}\left(\Delta v_{\perp}\right)=\int_{0}^{v_{0}} \frac{2 v d v}{v_{0}^{2}} \int_{0}^{2 \pi} \frac{d \Phi}{2 \pi} \int_{0}^{r_{c}} \frac{2 r_{\perp} d r_{\perp}}{r_{c}{ }^{2}} \int_{-S_{c} L}^{\left(1-S_{c}\right) L} d b_{z} \delta\left[\Delta v_{\perp}-\Delta v_{\perp}\left(v, \Phi, r_{\perp}, b_{z}\right)\right] \\
& p(k)=\int_{0}^{\infty} d \Delta v_{\perp} \rho_{2}\left(\Delta v_{\perp}\right)\left[1-J_{0}\left(k \Delta v_{\perp}\right)\right] \\
& \rho\left(\Delta v_{\perp}\right)=\frac{1}{2 \pi} \int_{0}^{\infty} k d k J_{0}\left(k \Delta v_{\perp}\right) e^{-\lambda p(k)}
\end{aligned}
$$

in which we expressed $p(\xi) d \xi$ directly in terms $v, \Phi, r_{\perp}$ and $b_{z}$, using equations (5.4.6) and (5.3.2). The distribution in $v$ and $r_{\perp}$ are taken uniform with a cut-of $f$ at $v_{0}=\alpha_{0} v_{z}$ and $r_{c}$ respectively, as prescribed by equation (5.3.3). Due to the rotational symmetry of the beam and the choice of the central reference trajectory the displacement distribution $\rho\left(\Delta \mathbf{v}_{\perp}\right)$ will be rotational symmetric. This implies that $\rho\left(\Delta \mathbf{v}_{\perp}\right)$ depends only on the magnitude of the displacement $\Delta v_{\perp}$ and not on its direction. The probability of a displacement of size $\Delta v_{\perp}$ is equal to $\rho\left(\Delta v_{\perp}\right)=2 \pi \Delta v_{\perp}^{2} \rho\left(\Delta v_{\perp}\right)$.

The model represented by equations $(8.2 .6),(8.2 .7)$ and $(8.2 .8)$ can be considered as the two-dimensional equivalent of the model utilized in the analysis of the Boersch effect, see equations (7.2.6), $(7.2 .7)$ and $(7.2 .8)$. The fundamental aspects involved are the same and the reader is referred to section 7.2 for a summary of the underlying assumptions.

\subsection{Beam segment with a narrow crossover}

In this section we will calculate the distribution of transverse velocity displacements $\rho\left(\Delta v_{\perp}\right)$ generated in a beam segment with the characteristics $K_{1} \gg 1$ and $K_{2} \gg \gg 1$. The quantities $K_{1}$ and $K_{2}$ represent the ratio between the beam radius at the start and end of the segment respectively and the crossover radius, see equation (7.3.1). The 
expectation is that, in such a beam geometry, the main contribution to $\rho\left(\Delta v_{\perp}\right)$ stems from collisions which are complete or nearly complete. Accordingly, we will start from equation (8.2.1). We emphasize that this approach is only justified for a narrow crossover. The general case of a beam geometry with arbitrary values for $K_{1}$ and $K_{2}$ will be treated in section 8.5 .

For convenience of notation we will utilize the scalingquantities $d_{0}$ and $v_{0}$, defined by equation (7.3.2). Employing this scaling equation (8.2.1) transforms to

$$
\Delta \bar{v}_{\perp}=\frac{\Delta v_{1}}{v_{0}}=\frac{\bar{v}\left[1+\bar{r}_{1}^{2} \sin ^{2}(\Phi) \bar{v}^{4} / 4\right]^{1 / 2}}{1+\left(\bar{b}_{2}^{2}+\bar{r}_{\perp}{ }^{2} \sin ^{2} \Phi\right) \bar{v}^{4 / 4}}
$$

The scaled two-particle distribution $\bar{\rho}_{2}\left(\Delta \bar{v}_{1}\right)$ follows from equation $(8.2 .6)$

$$
\bar{\rho}_{2}\left(\Delta \bar{v}_{\perp}\right)=\frac{v_{0}}{d_{0}} \rho_{2}\left(\Delta v_{\perp}\right)=\int_{0}^{1} 2 \bar{v} \mathrm{~d} \bar{v} \int_{0}^{2 \pi} \frac{d \Phi}{2 \pi} \int_{0}^{\frac{\bar{r}_{f}}{2 \bar{r}_{\perp}} d \bar{r}_{\perp}} \frac{\bar{r}_{c}^{2}}{d} \int_{-\infty}^{\infty} \delta\left[\Delta \bar{v}_{\perp}-\Delta \bar{v}_{\perp}\left(\bar{v}, \Phi, \bar{r}_{\perp}, \bar{\sigma}_{z}\right)\right]
$$

where $\Delta \bar{v}_{\perp}\left(\bar{v}, \Phi, \bar{r}, \bar{b}_{z}\right)$ is given by equation $(8.3 .1)$. The quantity $\bar{r}_{c}=r_{c} / d_{0}$ is the scaled crossover radius specified by equation (7.3.5). We evaluated equation (8.3.2) numerically for different values of $\bar{r}_{c}$. The results are plotted in figure 8.1 .

For a number of extreme cases one can evaluate the two-particle distribution $\bar{\rho}_{2}\left(\Delta \bar{v}_{1}\right)$ by analytical means. Analogous to the derivation of equation $(7.3 .9)$ one finds from equations (8.3.1) and (8.3.2)

$$
\bar{\rho}_{2}\left(\Delta \bar{v}_{\perp}\right)=\frac{16}{\pi} \int_{0}^{1} \frac{d \bar{v}}{\bar{v}} \int_{0}^{1} d y \sqrt{1-y^{2}} \int_{0}^{1} \frac{R(\bar{v}, y) d u}{u^{3 / 2}(1-u)^{1 / 2}} \delta\left(\Delta \bar{v}_{\perp}-\frac{u \bar{v}}{2 R(\bar{v}, y)}\right)
$$

where the function $R(\bar{v}, y)$ is defined as

$$
R(\bar{v}, y)=\left(1+\bar{v}^{4} y^{2} \bar{r}_{c}^{2} / 4\right)^{1 / 2}
$$

Equation (8.3.3) can be rewritten as (for $0<\Delta \overline{\mathbf{v}}_{\perp}<1$ )

$$
\bar{\rho}_{2}\left(\Delta \bar{v}_{\perp}\right)=\frac{16}{\pi \Delta \bar{v}_{\perp}{ }^{3 / 2}} \int_{2 \Delta \bar{v}_{\perp}}^{1} \int_{0}^{\left.\min \left[1,\left(1-4 \Delta \bar{v}_{1}^{2} / \bar{v}^{2}\right) 1 / 2 / \Delta \bar{v}_{\perp} \bar{v} \bar{r}_{c}\right)\right]} d y \frac{\left(1-y^{2}\right)^{1 / 2} R(\bar{v}, y) 1 / 2}{\left[\bar{v}-\Delta \bar{v}_{\perp} R(\bar{v}, y)\right]^{1 / 2}}
$$

Equation (8.3.3) will be used to evaluate the function $p(k)$, while equation $(8.3 .5)$ is most suited to investigate the asymptotic behaviour of $\bar{\rho}_{2}\left(\Delta \bar{v}_{1}\right)$.

For a point-crossover $\left(\bar{r}_{c}=0\right)$ equation (8.3.5) becomes

$$
\bar{\rho}_{2}\left(\Delta \bar{v}_{\perp}\right)=\frac{8\left(1-\Delta \bar{v}_{\perp}\right) 1 / 2}{\Delta \bar{v}_{\perp}{ }^{3 / 2}} \quad\left(\bar{r}_{c}=0,0<\Delta \bar{v}_{\perp}<1\right)
$$

This seems the only case which leads to an exact analytical solution. However, a good approximation of $\bar{\rho}_{2}\left(\Delta \bar{v}_{1}\right)$ can be obtained for $\bar{r}_{c} \neq 0$ when $\overline{\mathbf{r}}_{\mathrm{c}} \Delta \overline{\mathrm{v}}_{\perp} \ll<1$ or oppositely when $\overline{\mathrm{r}}_{\mathrm{c}} \Delta \overline{\mathrm{v}}_{\perp} \gg>1$.

For small values of $\Delta \bar{v}_{f}$ and small values of $\bar{r}_{c} \quad\left(\bar{r}_{c} \Delta \bar{v}_{\perp} \ll 1\right)$ one may approximate equation $(8.3 .5)$ as 


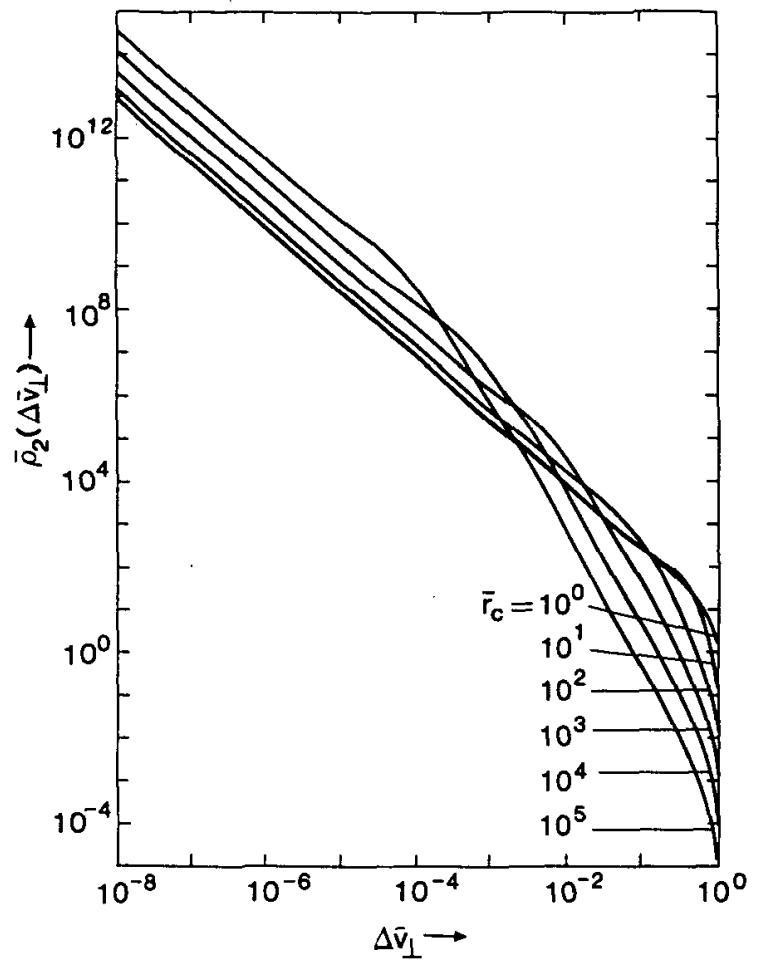

Figure 8.1 The scaled two-particle distribution $\bar{\rho}_{2}\left(\Delta \bar{v}_{1}\right)$ based on complete collisions, for different values of the scaled crossover radius $\bar{r}_{c}$.

$$
\bar{\rho}_{2}\left(\Delta \bar{v}_{\perp}\right)=\frac{8}{\Delta \bar{v}_{\perp} 3 / 2} f_{\infty}\left(\bar{r}_{c}\right) \quad\left(\bar{r}_{c} \Delta \bar{v}_{\perp} \ll 1,0<\Delta \bar{v}_{\perp}<1\right) \quad(8.3 .7)
$$

where the function $f_{\infty}\left(\bar{r}_{c}\right)$ is defined as

$$
f_{\infty}\left(\bar{r}_{c}\right)=\frac{2}{\pi} \int_{0}^{1} \frac{d \bar{v}}{\bar{v}^{1 / 2}} \int_{0}^{1} d y\left(1-y^{2}\right)^{1 / 2} R(\bar{v}, y)^{1 / 2}
$$

This function has the following properties

$$
\begin{aligned}
& \lim _{\mathbf{r}_{c} \rightarrow \infty} f_{\infty}\left(\bar{r}_{c}\right)=\frac{8}{15} \sqrt{\frac{2}{\pi}} \frac{\Gamma(3 / 4)}{\Gamma(1 / 4)} \bar{r}_{c} 1 / 2=.14383 \bar{r}_{c} 1 / 2 \\
& \lim _{c \rightarrow 0} f_{\infty}\left(\bar{r}_{c}\right)=1
\end{aligned}
$$

For later use we approximate the function $f_{\infty}\left(\bar{x}_{c}\right)$ by

$$
f_{\infty}\left(\bar{r}_{c}\right)=\left(1+.02069 \bar{r}_{c}\right)^{1} / 2
$$


which has the same asymptotic behaviour for $\bar{r}_{c} \rightarrow 0$ and $\bar{r}_{c \rightarrow \infty}$ as the original function $\mathrm{f}_{\infty}\left(\overrightarrow{\mathrm{r}}_{\mathrm{c}}\right)$.

For large values of $\Delta \bar{v}_{\perp}$ and large values of $\bar{r}_{c} \quad\left(\bar{r}_{c} \Delta \bar{v}_{\perp} \gg 1\right)$ equation $(8.3 .5)$ yields in good approximation

$$
\bar{\rho}_{2}\left(\Delta \bar{v}_{\perp}\right)=\frac{128}{\pi} \frac{1}{\bar{r}_{\mathrm{c}} \Delta \overline{\mathrm{v}}_{\perp}{ }^{5 / 2}}\left(\frac{1}{\Delta \overline{\mathrm{v}}_{\perp}{ }^{1 / 2}}-1\right) \quad\left(\overline{\mathrm{r}}_{\mathrm{c}} \Delta \overline{\mathrm{v}}_{\perp} \ll<, 0<\Delta \overline{\mathrm{v}}_{\perp}<1\right)
$$

The behaviour of the two-particle function $\bar{\rho}_{2}\left(\Delta \bar{v}_{1}\right)$ described by equations $(8.3 .6),(8.3 .7)$ and $(8.3 .11)$ is in agreement with the numerical data plotted in figure 8.1 .

We now proceed with the calculation of the function $p(k)$ and the displacement distribution $\rho\left(\Delta \mathrm{v}_{\perp}\right)$. By scaling equation (8.2.7) one obtains

$$
\bar{p}(k)=\frac{p(k)}{d_{0}}=\int_{0}^{\infty} d \Delta \bar{v}_{\perp} \bar{\rho}_{2}\left(\Delta \bar{v}_{\perp}\right)\left[1-J_{0}\left(k \Delta \bar{v}_{\perp}\right)\right]
$$

with $k=k v_{0}$. Substitution of equation (8.3.3) and partial integration with respect to $u$, using that $d J_{0}(z) / d z=-J_{1}(z)$, yields

$$
\bar{p}(\bar{k})=k \frac{32}{\pi} \int_{0}^{1} d \bar{v} \int_{0}^{1} d y \sqrt{1-y^{2}} \int_{0}^{1} d u\left(\frac{1}{u}-1\right)^{1 / 2} J_{1}\left(\frac{u k \bar{v}}{R(\bar{v}, y)}\right)
$$

in which $J_{1}$ the first order Bessel-function. We evaluated equation (8.3.13) numerically for different values of the scaled crossover radius $\overline{\mathbf{r}}_{c}$. The results are plotted in figure 8.2 . One sees that the function $\overline{\mathbf{p}}(\bar{k})$ shows a quadratic $\bar{k}$-dependency for small $\bar{k}$-values and a square-root dependency for large $\mathrm{k}$-values.

We will investigate the extreme cases $\bar{k} \rightarrow 0$ and $k \rightarrow \infty$ in more detail. For $\bar{k} \rightarrow 0$ one may approximate the Bessel function as $J_{1}(z) \approx z / 2$ and carry out the u-integration, which yields a factor $\pi / 8$. The $\bar{v}$-integration can be performed next and one obtains

$$
\bar{p}(\bar{k})=\frac{1}{4} \bar{p}_{2}\left(\bar{r}_{c}\right) k^{2}, \quad \bar{p}_{2}\left(\bar{r}_{c}\right)=\frac{8}{\bar{r}_{c}} \int_{0}^{1} d y \frac{\sqrt{1-y^{2}}}{y} \sinh ^{-1}\left(\frac{\bar{r}_{c y}}{2}\right) \quad(k \rightarrow 0)
$$

The function $\bar{p}_{2}\left(\bar{r}_{c}\right)$ is the same function as found for the axial velocity distribution $\rho\left(\Delta v_{z}\right)$, see equation 7.3.16. Notice, however, that its definition differs by a factor 2 , which is related to the fact that the distribution $\rho\left(\Delta v_{1}\right)$ is two-dimensional, while the distribution $\rho\left(\Delta v_{z}\right)$ is one-dimensional. The function $\overline{\mathbf{p}}_{2}\left(\overline{\mathrm{r}}_{\mathrm{c}}\right)$ can be approximated by the function $\overline{\mathbf{p}}_{2 \mathrm{a}}\left(\overline{\mathrm{r}}_{\mathrm{c}}\right)$, which is given by equation (7.3.17). See also figure 7.3 . using

$$
\text { For } \bar{k}+\infty \text { one may approximate the } u \text {-integral in equation }(8.3 .13)
$$

$$
\lim _{x \rightarrow \infty} \int_{0}^{1} d u\left(\frac{1}{u}-1\right)^{1 / 2} J_{1}(x u) \approx \int_{0}^{1} d u \frac{J_{1}(x u)}{u^{1 / 2}}=\frac{\Gamma(3 / 4)}{\Gamma(1 / 4)} \frac{2 \sqrt{2}}{\sqrt{x}}
$$

based on the argument that the contribution to the integral comes from small $u$-values. Accordingly, one finds for $\bar{k}+\infty$ 


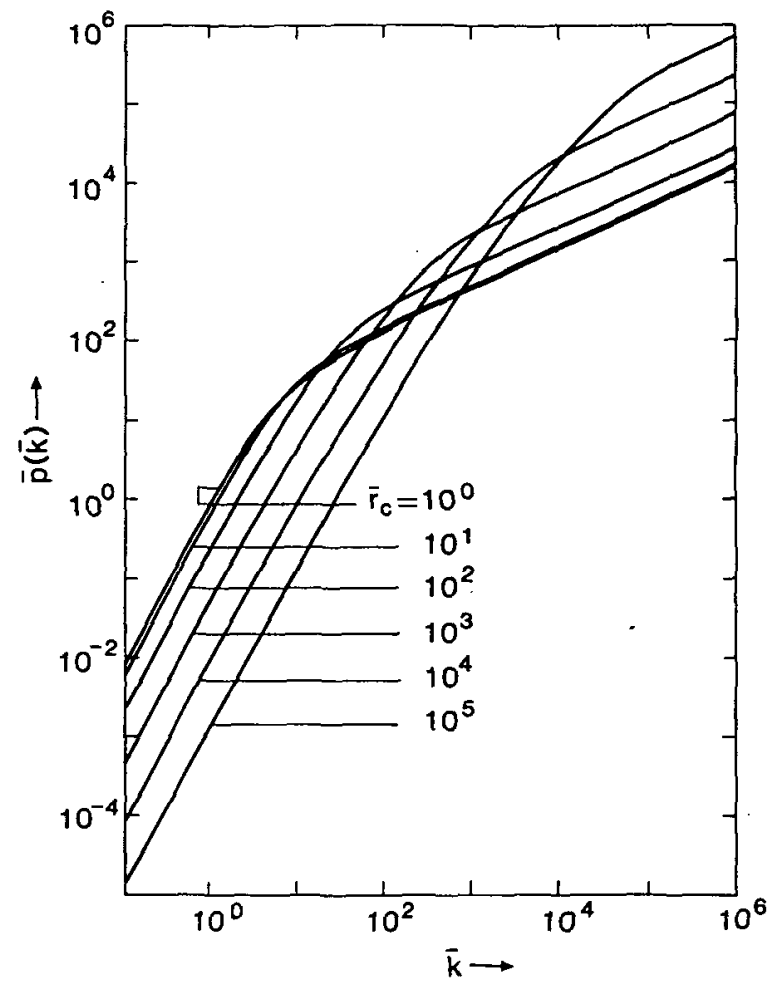

Figure 8.2 The scaled function $\overline{\mathrm{p}}(\overline{\mathrm{K}})$ corresponding to the distribution of transverse velocity displacements generated by complete collisions, for different values of the scaled crossover radius $\overline{\mathbf{r}}_{\mathbf{c}}$.

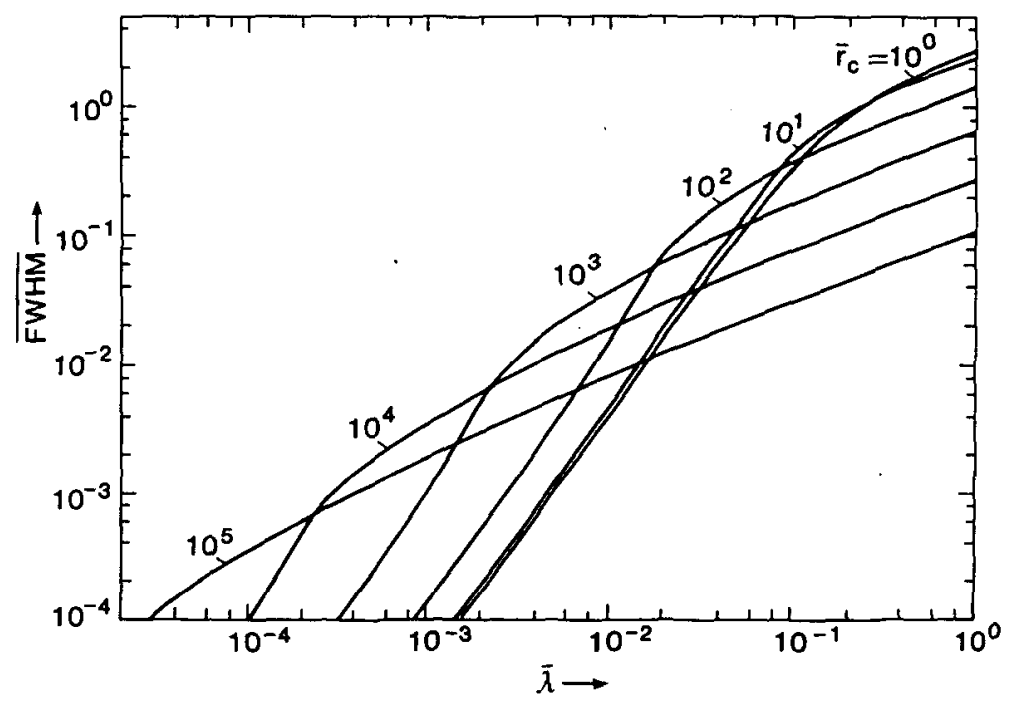

Figure 8.3 The FWHM of the scaled distribution of transverse velocity displacements $\bar{\rho}\left(\Delta \bar{v}_{\perp}\right)$ based on complete collisions, for different values of the scaled crossover radius $\bar{F}_{\mathrm{c}}$. 


$$
\overline{\mathrm{p}}(\mathrm{k})=\overline{\mathrm{p}}_{\infty}\left(\overline{\mathrm{r}}_{c}\right) \mathrm{K}^{1 / 2}, \quad \overline{\mathrm{p}}_{\infty}\left(\overline{\mathrm{r}}_{\mathrm{c}}\right)=\frac{32 \sqrt{2} \Gamma(3 / 4)}{\Gamma(1 / 4)} \mathrm{f}_{\infty}\left(\overline{\mathrm{r}}_{\mathrm{c}}\right) \quad(\bar{K} \rightarrow \infty)
$$

where the function $f_{\infty o}\left(\vec{r}_{c}\right)$ is given by equation (8.3.8). A good approximation of $f_{\infty o}\left(\bar{r}_{c}\right)$ is provided by equation $(8.3 .10)$.

The final step consists of the calculation of the distribution $\bar{\rho}\left(\Delta \mathbf{v}_{1}\right)$. By scaling equation $(8.2 .8)$ one obtains

$$
\bar{\rho}\left(\Delta \bar{v}_{\perp}\right)=v_{0}{ }^{2} \rho\left(\Delta \mathbf{v}_{\perp}\right)=\frac{1}{2 \pi} \int_{0}^{\infty} \overline{k d K} J_{0}\left(\bar{k} \Delta \bar{v}_{\perp}\right) e^{-\lambda \bar{p}(k)}
$$

with $\delta$ the scaled linear particle density for a beam segment with a crossover, specified by equation (7.3.22). The second moment of this distribution follows directly from the function $\vec{p}(\bar{k})$ using equations $(5.5 .4)$ (with $\mathrm{m}=2$ ) and $(8.3 .14$ )

$$
\left\langle\Delta \overline{\mathrm{v}}_{\perp}{ }^{2}\right\rangle=\overline{\mathrm{p}}_{2}\left(\overline{\mathrm{r}}_{\mathbf{c}}\right) \bar{\lambda}
$$

By comparison with equation $(7,3,30)$ one sees that $\left\langle\Delta \bar{v}_{1}^{2}\right\rangle=\left\langle\Delta \bar{v}_{z}{ }^{2}\right\rangle$. It should be emphasized that this result relies on the assumption that complete collisions are dominant.

The FWHM of the distribution $\bar{\rho}\left(\Delta \overline{\mathbf{v}}_{\perp}\right)$, given by equation (8.3.17), is plotted in figure 8.3 as function of $\pi^{\perp}$, for different values of $\bar{r}_{c}$. Two regimes should be distinguished :

I) Gaussian regime. For large $\lambda$-values $\bar{\rho}\left(\Delta \bar{v}_{1}\right)$ becomes equal to a twodimensional Gaussian distribution. This can be understood from the fact that the integral of equation $(8.3 .17)$ is for large $\lambda$-values dominated by the small $\bar{k}$-behaviour of the function $\bar{p}(\bar{k})$, which shows a quadratic dependency, see equation (8.3.14). The FWHM of $\bar{\rho}\left(\Delta \overline{\mathbf{v}}_{\perp}\right)$ is in the Gaussian regime given by

$$
\overline{\text { FWHM }_{G}}=2(\pi \ln 2) 1 / 2 \mathrm{P}_{\mathrm{CA}}\left(\overline{\mathrm{r}}_{\mathrm{c}}\right) \lambda^{1 / 2}
$$

$\left(\bar{\lambda}_{>}>0.17 \mathrm{P}_{\mathrm{CA}}\left(\overline{\mathrm{r}}_{\mathrm{c}}\right)^{2 / 3} / \mathrm{f}_{\infty}\left(\overline{\mathrm{r}}_{\mathrm{c}}\right)^{4 / 3}\right)$ where the function $\mathrm{P}_{C A}\left(\bar{r}_{c}\right)$ is defined as

$$
\operatorname{P}_{C_{A}}\left(\bar{r}_{c}\right)=P_{C E}\left(\bar{r}_{c}\right)=\left[\bar{p}_{2}\left(\bar{r}_{c}\right) / \pi\right]^{1 / 2}
$$

identical to equation (7.3.32). By removing the scaling by means of equation (7.3.2) one finds for the FWHM of the corresponding angular deflection distribution (using FWHM $\alpha_{\alpha}=\alpha_{0}$ FWHM)

$$
\text { FWHM }_{\alpha}=\mathrm{C}_{\mathrm{CGA}} \mathrm{PCA}_{\mathrm{CA}}\left(\overline{\mathrm{Y}}_{\mathrm{C}}\right) \sqrt{\frac{\mathrm{I}}{\mathrm{V}^{3 / 2}}}, \quad \mathrm{C}_{\mathrm{CGA}}=\left(\frac{(\ln 2)^{2} \mathrm{~m}}{8 \mathrm{E}_{0}^{2} \mathrm{e}}\right)^{1 / 4}
$$

In the case of electrons one finds $\mathrm{C}_{\mathrm{CGA}}=256.90$ in sI-units. The subscript CGA indicates that the constant refers to the Gaussian Angular deflection distribution generated in a beam segment with a crossover.

II) Weak complete collision regime. The distribution $\bar{\rho}\left(\Delta \bar{v}_{\perp}\right)$ is for small $\bar{\lambda}$-values determined by the large $\bar{k}$-behaviour of the function $\bar{p}(\bar{k})$, which shows a square-root dependency, as can be seen from equation (8.3.16). Substitution of equation (8.3.16) into equation (8.3.17) yields 


$$
\bar{\rho}\left(\Delta \overline{\mathbf{v}}_{\perp}\right)=\frac{1}{2 \pi} \int_{0}^{\infty} \mathrm{kdK} \mathrm{J}_{0}\left(\mathrm{k} \Delta \overline{\mathrm{v}}_{\perp}\right) \mathrm{e}^{-\bar{\lambda} \overline{\mathrm{P}}_{\infty}\left(\overline{\mathrm{r}}_{\mathrm{c}}\right) \mathrm{K}^{1 / 2}}
$$

This type of distribution is depicted in figure 5.4 (curve corresponding to $\gamma=1 / 2$ ). Its FWHM is given by

$$
\overline{\text { FWHM }}_{W}=.17997\left[\overline{\mathrm{p}}_{\infty}\left(\overline{\mathrm{r}}_{\mathrm{c}}\right)\right]^{2} \bar{\lambda}^{2}=42.105\left[\mathrm{f}_{\infty}\left(\overline{\mathrm{r}}_{\mathrm{c}}\right)\right]^{2} \bar{\lambda}^{2}
$$

$\left(\overline{\lambda<}<.17 \mathrm{P}_{\mathrm{CA}}\left(\overline{\mathrm{r}}_{\mathrm{c}}\right)^{2 / 3} / \mathrm{f}_{\infty}\left(\overline{\mathrm{r}}_{\mathrm{c}}\right)^{4 / 3}\right)$ as follows with equation $(5.9 .15)$ and table 5.1. It can be verified that the $k$-dependency given by equation (8.3.16) stems from weak complete collisions. Hence, this regime is called the "weak complete collision regime". Note that the analysis of the axial velocity distribution $\bar{\rho}\left(\Delta \bar{v}_{z}\right)$ resulted in a function $\bar{p}(\bar{k})$, which is linear for large $\bar{k}$, also due to the presence of weak complete collisions. The corresponding distribution $\bar{\rho}\left(\Delta \bar{v}_{z}\right)$ was found to be Lorentzian, see equation (7.3.26). Thus the similarity between the distributions $\bar{\rho}\left(\Delta \overline{\mathbf{v}}_{1}\right)$ and $\bar{\rho}\left(\Delta \overline{\mathbf{v}}_{\mathrm{z}}\right)$ disappears for small $\bar{\lambda}$-values.

The FWHM of the corresponding angular deflection distribution follows from equation (8.3.23) (using again $\mathrm{FWHM}_{\alpha}=\alpha_{0}$ FWHM)

$$
\mathrm{FWHM}_{\alpha}=\mathrm{C}_{\mathrm{CWA}} \mathrm{f}_{\infty}\left(\overline{\mathrm{r}}_{\mathrm{c}}\right)^{2} \frac{\mathrm{I}^{2}}{\alpha_{0} \mathrm{~V}^{3}}
$$

where the constant $C_{C w A}$ is equal to

$$
C_{c W A}=0.17997\left(\frac{4 \Gamma(3 / 4)}{\pi \Gamma(1 / 4)}\right)^{2} \frac{\mathrm{m}}{\epsilon_{0}^{2} \mathrm{e}}=0.033329 \frac{\mathrm{m}}{\epsilon_{0}^{2} \mathrm{e}}
$$

In the case of electrons one finds $C_{C W A}=2.4172 \times 10^{9}$ in sI-units.

\subsection{Homocentric cylindrical beam segment}

In this section we will determine the distribution of transverse velocity displacements $\rho\left(\Delta v_{1}\right)$ generated in a homocentric cylindrical beam segment. The calculation is quite similar to the calculation of the distribution of axial velocity displacements $\rho\left(\Delta \mathrm{v}_{z}\right)$ in this type of beam, see section 7.4 . The reader is referred to this section for the qualitative aspects involved.

We will utilize the $\delta, \nu$-scaling given by equations $(6,13,1)$. The scaled two-particle distribution $\rho_{2}{ }^{*}\left(\Delta v_{\perp}{ }^{*}\right)$ follows from equation $(8.2 .6)$

$$
\rho_{2} *\left(\Delta v_{1}{ }^{*}\right)=\frac{\nu r_{0} * 2}{\delta} \rho_{2}\left(\Delta v_{\perp}\right)=\int_{0}^{r_{0} *} 2 r^{*} d r^{*} \int_{0}^{\infty} d_{b_{2}}^{\infty} \delta\left[\Delta v_{\perp}^{*}-\Delta v_{\perp}^{*}\left(r^{*}, b_{2} *\right)\right](8.4 .1)
$$

We evaluated equation (8.4.1) numerically on the basis of the exact analysis of the function $\Delta v_{1}^{*}\left(r^{*}, b_{z}^{*}\right)$, following the procedure outlined in section 6.6. The results are presented in figure 8.4 . The function $\Delta v_{1}{ }^{*}\left(r^{*}, b_{2}{ }^{*}\right)$ can be determined analytically for half-complete collisions and for weak collisions, see equations $(8.2 .4)$ and $(8.2 .5)$ respectively. One might conjecture that the function $p_{2} *\left(\Delta v{ }^{*}\right)$ is determined by weak interactions for most of the $\Delta v^{*}$-range plotted in figure 8.4 , while its behaviour for very large $\frac{1}{\Delta v_{1}}{ }^{*}$-values stems from half-complete collisions. We will verify this statement by evaluating equation $(8.4 .1)$ analyticaliy, employing the approximations given by equations $(8.2 .4)$ and $(8.2 .5)$. 
Let us first consider the limit of weak interactions. Instead of using equation (8.2.5) we prefer to exploit the analysis of the twoparticle distribution of lateral forces $\rho_{2}\left(F_{1}\right)$ presented in section 5.8 . This is justified since equation $(8.2 .5)$ is based on the first order perturbation approximation

$$
\Delta \mathbf{v}_{\perp} \cong \mathrm{F}_{\perp} \mathrm{T} / \mathrm{m}
$$

Therefore, one may determine $\rho_{2}^{*}\left(\Delta v_{1}^{*}\right)$ from $\rho_{2}\left(F_{1}\right)$, using

$$
\rho_{2} *\left(\Delta v_{\perp}^{*}\right)=\frac{v r_{0} * 2}{\delta} \rho_{2}\left(\Delta v_{\perp}\right)=\frac{v r_{0} * 2}{\delta} \frac{\mathrm{m}}{\mathrm{T}} \rho_{2}\left(\mathrm{~F}_{\perp}=\Delta \mathrm{v}_{\perp} \mathrm{m} / \mathrm{T}\right)
$$

Equation (5.8.13) specifies $\rho_{2}\left(F_{\perp}\right)$ for an extended beam $\left(r_{0}+\infty\right)$. Substitution into equation $(8.4 .3)$ yields

$$
\rho_{2} *\left(\Delta v_{\perp}^{*}\right)=\frac{3 \sqrt{\pi} \Gamma(3 / 4)}{10 \Gamma(1 / 4)} \frac{1}{\Delta v_{\perp}^{* 5 !^{2}}} \quad\left(\Delta v_{\perp}^{*}<<1, r_{0}^{* 2} \Delta v_{\perp}^{*}>>1\right)
$$

in which we utilized the definitions of $\delta$ and $\nu$, given by equations (6.13.1). The numerical constant in the right hand sight of equation $(8.4 .4)$ is equal to 0.17973 .

For a pencil-beam $\left(r_{0} \rightarrow 0\right)$ one should use equation $(5.8 .18)$. Substitution into equation $(8.4 .3)$ yields

$$
\rho_{2}{ }^{*}\left(\Delta v_{\perp}^{*}\right)=\frac{42 / 3 r_{0}^{* 7 / 3}}{7 \Delta v_{\perp}^{* 4 / 3}} \quad\left(\Delta v_{\perp}^{*}<<1, r_{0}^{* 2} \Delta v_{\perp}^{*}<<1\right)
$$

One may verify from figure 8.4 that the small and intermediate $\Delta v_{1}{ }^{*}$ behaviour of $\rho_{2}{ }^{*}\left(\Delta v_{1}{ }^{*}\right)$ indeed follows equations $(8.4 .4)$ and $(8.4 .5)$. In order to investigate the large $\Delta v_{1}{ }^{*}$-behaviour of $\rho_{2}{ }^{*}\left(\Delta v_{1}{ }^{*}\right)$ we start from equation (8.2.4). It specifies the displacement $\Delta v_{1}$ for $a$ half-complete collision. By taking the limit $T \rightarrow \infty$ one may ignore the last term in this equation. After scaling one obtains with equation $(8.4 .1)$

$$
\rho_{2} *\left(\Delta v_{\perp}^{*}\right)=\int_{0}^{r_{0} *} 2 r^{*} d r^{*} \int_{0}^{\infty} 2 d b_{z}^{*} \delta\left(\Delta v_{\perp}^{*}-\frac{r_{\perp}^{*}}{2\left(r^{* 2}+b_{2}^{* 2}\right)^{3 / 4}}\right)
$$

By substituting

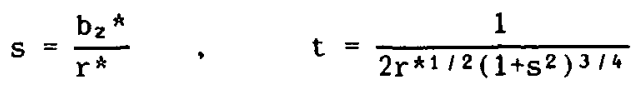

and carrying out the t-integration, equation (8.4.6) transforms to

$$
\rho_{2} *\left(\Delta v_{\perp}^{*}\right)=\frac{1}{8 \Delta v_{\perp}^{* 7}} \int_{0}^{\infty} \frac{d s}{\left(1+s^{2}\right)^{9 / 2}} \theta\left(\Delta v_{\perp}^{*}-\frac{1}{2 r_{0}^{* 1 / 2}\left(1+s^{2}\right)^{3 / 4}}\right)
$$

where $\boldsymbol{\theta}(\mathrm{x})$ is the step-function defined by equation (3.7.8). For an extended beam $\left(r_{0}^{*}+\infty\right)$ the argument of the step-function is positive for all positive values of $\Delta v_{\perp}{ }^{*}$ and one obtains

$$
\rho_{2} *\left(\Delta v_{\perp}^{*}\right)=\frac{1}{8 \Delta v_{1}^{* 7}} \int_{0}^{\infty} d s \frac{1}{\left(1+s^{2}\right)^{9 / 2}}=\frac{2}{35} \frac{1}{\Delta v_{\perp}^{* 7}}
$$
This asymptote gives a good approximation of $\rho_{2}^{*}\left(\Delta v_{\perp}{ }^{*}\right)$ for $\Delta v_{\perp} \gtrsim 1$, see
figure 8.4 . 


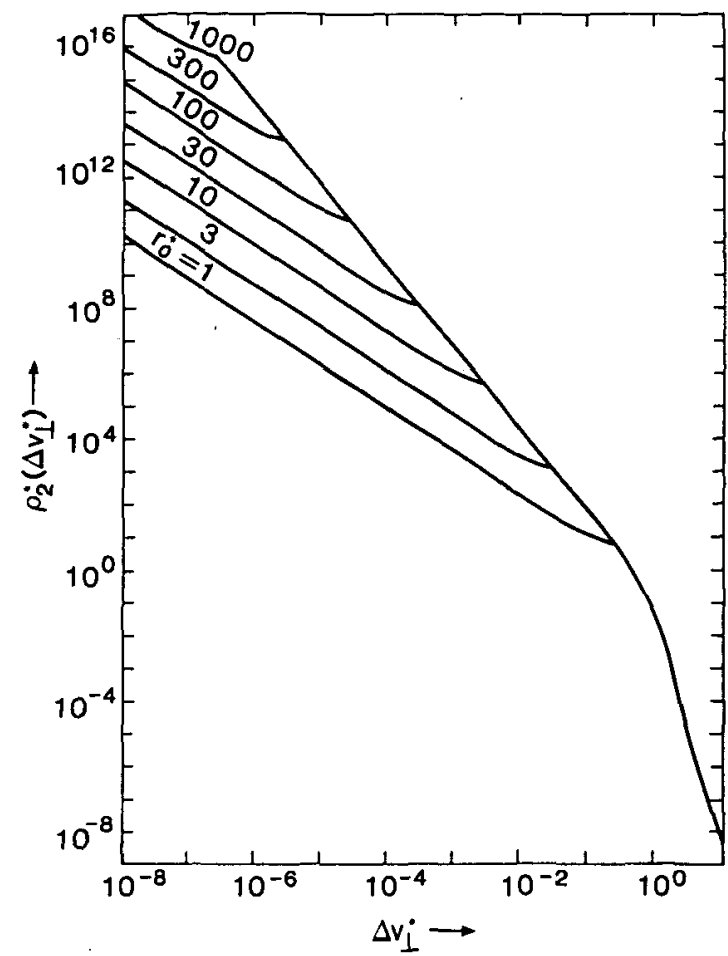

Figure 8.4 The scaled two-particle distribution $\rho_{2} *\left(\Delta v_{1}^{*}\right)$ for a homocentric cylindrical beam segment, plotted for different values of the scaled beam radius $\mathrm{r}_{0}{ }^{*}$.

For a pencil beam $\left(r_{0}^{*} \rightarrow 0\right)$ the argument of the step-function is positive for $s>s_{0 \approx}\left(2 \Delta v_{1}{ }^{*} r_{0} * 1 / 2\right)-2 / 3$. Thus the lower integration boundary of the integral in equation $(8.4 .8)$ should be replaced by $s_{0}$. As $s_{0} \gg 1$ one finds in good approximation

$$
\rho_{2}{ }^{*}\left(\Delta v_{\perp}{ }^{*}\right)=\frac{1}{8 \Delta v_{\perp}{ }^{* 7}} \int_{S_{0}=\left(2 \Delta v_{\perp}{ }^{*} r_{0} * 1 / 2\right)-2 / 3}^{\infty}=\frac{r_{0} * 8 / 3}{22 / 3 \Delta v_{\perp} * 5 / 3}
$$

However, this asymptote is not reached for normal operating conditions $\left(r_{0} * 1\right)$ and will be disregarded in the further analysis. Equations $(8.4 .4),(8.4 .5)$ and $(8.4 .9)$ provide a complete analysis of the behaviour of the two-particle distribution $\rho_{2}{ }^{*}\left(\Delta v_{\perp}{ }^{*}\right)$.

The next step is to determine the scaled function $p^{*}\left(k^{*}\right)$ which follows from equation $(8,2,7)$ 


$$
p^{*}\left(k^{*}\right)=\frac{r_{0}^{* 2}}{\delta} p(k)=\int_{0}^{\infty} d \Delta v_{\perp} * \rho_{2} *\left(\Delta v_{\perp}^{*}\right)\left[1-J_{0}\left(k^{*} \Delta v_{\perp}^{*}\right)\right]
$$

with $k^{*}=k v$. We evaluated the integral of equation $(8.4 .11)$ numerically from the numerical data of $\rho_{2}{ }^{*}\left(\Delta v_{1}{ }^{*}\right)$. The results are shown in figure 8.5 .

The behaviour of the function $p^{*}\left(k^{*}\right)$ can be understood from the analysis of the two-particle distribution $\rho_{2}{ }^{*}\left(\Delta v_{\perp}{ }^{*}\right)$. Substitution of equation $(8.4 .4)$ into equation $(8.4 .11)$ yields

$$
p^{*}\left(k^{*}\right)=\frac{3 \sqrt{\Gamma} \Gamma(3 / 4)}{10 \Gamma(1 / 4)} k^{* 3 / 2} \int_{0}^{\infty} d x \frac{1-J_{0}(x)}{x^{5 / 2}}=\frac{(2 \pi)^{1 / 2}}{15} k^{* 3 / 2}
$$

which provides an accurate approximation for $k^{*} \approx 10$ and $k^{*} \approx 10 \mathrm{r}_{0} * 2$. This result can also be obtained from equation $(5.8 .14)$, which refers to the distribution of lateral forces in an extended beam.

Substitution of equation $(8.4 .5)$ into equation $(8.4 .11)$ yields

$$
p^{*}\left(k^{*}\right)=\frac{24 / 3}{7} r_{0}^{* 7 / 3} k^{* 1 / 3} \int_{0}^{\infty} d x \frac{1-J_{0}(x)}{x^{3 / 2}}=\frac{36 \Gamma(5 / 6)}{7 \Gamma(1 / 6)} r_{0} * 7 / 3 k^{* 1 / 3}
$$

which provides an accurate approximation for $\mathrm{k} \gtrsim_{\approx} 10 \mathrm{r}_{0} * 2$. The numerical constant in the right hand side of equation $(8.4 .13)$ is equal to 1.0429 . Equation $(8.4 .13)$ is equivalent to equation $(5.8 .19)$, referring to the distribution of lateral forces in a pencil beam.

For small $k^{*}$-values the function $\mathrm{p}^{*}\left(\mathrm{k}^{*}\right)$ shows a quadratic $\mathrm{k}^{*}$-dependency, which can be described as

$$
\lim _{k^{*} \rightarrow 0} p^{*}\left(k^{*}\right)=\frac{1}{4} p_{2}^{*}\left(r_{0}^{*}\right) k^{* 2}, \quad, \quad p_{2}^{*}(\infty)=0.3026
$$

The small $k^{*}$-behaviour of the function $p^{*}\left(k^{*}\right)$ is directly related to the second moment $\left\langle\Delta v_{\perp} 2^{* 2}\right\rangle$ of the two-particle distribution $\rho_{2}{ }^{*}\left(\Delta v_{\perp}{ }^{*}\right)$

$$
\left\langle\Delta v_{1} 2^{* 2}\right\rangle=p_{2} *\left(r_{0}^{*}\right)
$$

The quantity $\left\langle\Delta v_{\perp} 2^{* 2}\right\rangle$ does not stem from either weak or half-complete collisions alone, but is built up over the entire $\Delta v^{*}$-range. This prohibits an analytical estimation of its value. We evaluated the function $\mathrm{p}_{2} *\left(\mathrm{r}_{0}{ }^{*}\right) / \mathrm{p}_{2} *(\infty)$ numerically. The result is depicted in figure 8.6. The data is fitted by the function

$$
p_{2 a}{ }^{*}\left(r_{0}{ }^{*}\right)=\frac{p_{2}^{*}(\infty)}{\left(1+.185 / r_{0}^{* 8 / 7}\right)^{7 / 2}} \quad\left(r_{0} * 1\right)
$$

which is also plotted in figure 8.6. The set of equations (8.4.12), (8.4.13) and (8.4.14) provides a complete analysis of the behaviour of the function $p^{*}\left(k^{*}\right)$.

The final step consist of the calculation of the displacement distribution $\rho^{*}\left(\Delta v_{1}^{*}\right)$. By scaling equation $(8.2 .8)$ one obtains 


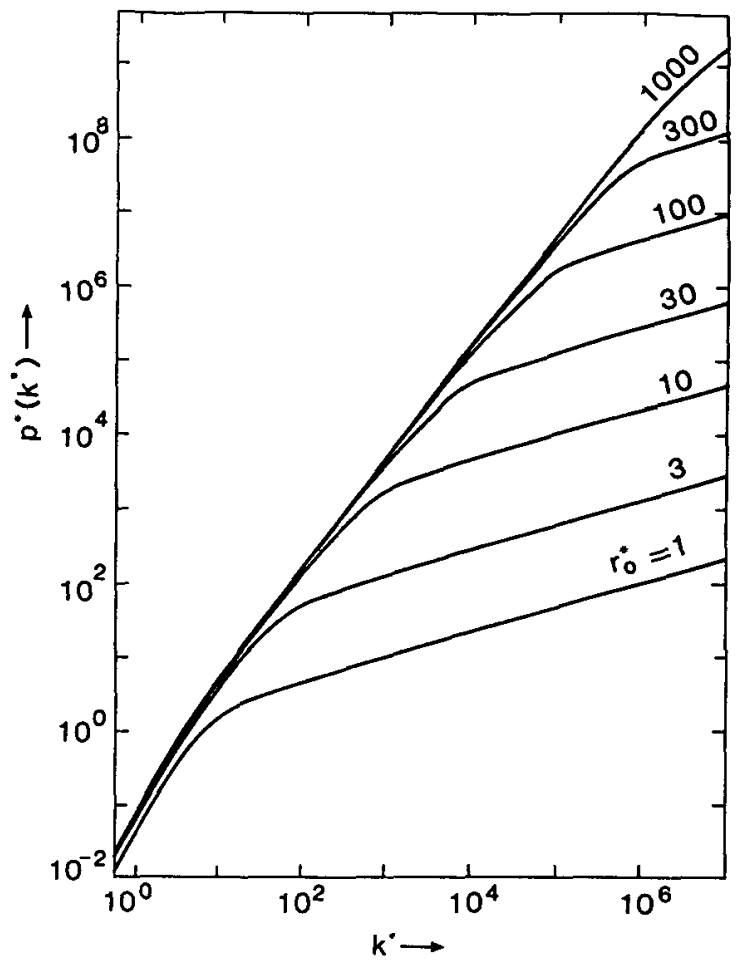

Figure 8.5 The scaled function $\mathrm{p}^{\star}\left(\mathrm{k}^{*}\right)$ corresponding to the distribution of transverse velocity displacements generated in a homocentric cylindrical beam segment, plotted for different values of the scaled beam radius $r_{0}{ }^{*}$.

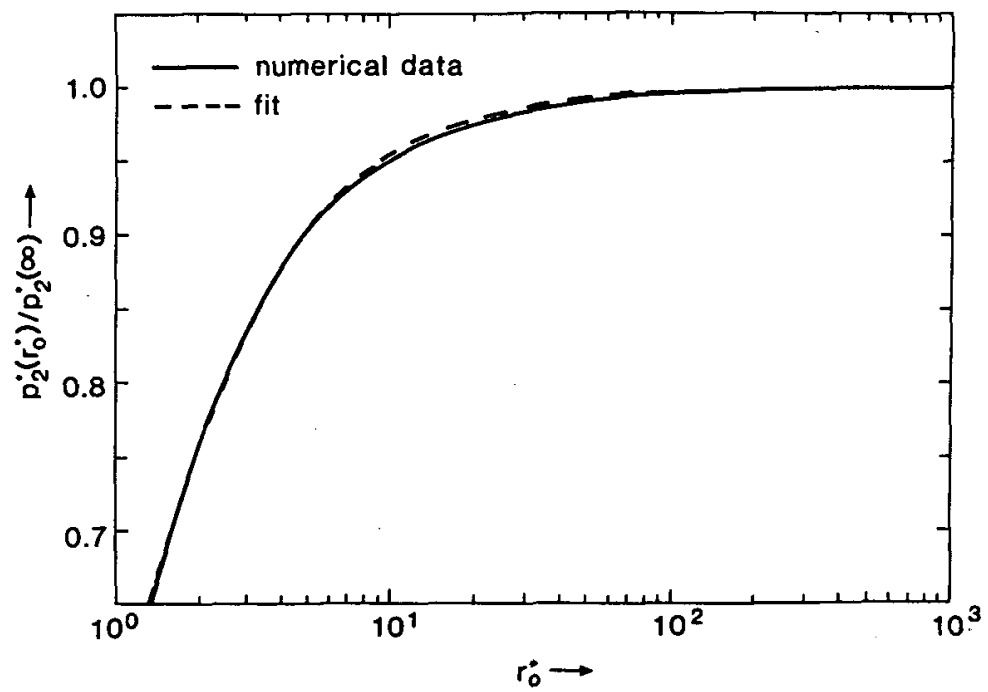

Pigure 8.6 The function $\mathrm{p}_{2}\left(\mathrm{r}_{\mathrm{o}^{*}}\right)$ defined by equation (8.4.14) and its approximation given by equation (8.4.16). This function gives the $r_{0}^{*}$-dependency of the function $p^{\star}\left(k^{*}\right)$, plotted in figure 8.5 , for $k^{\star} \rightarrow 0$. 


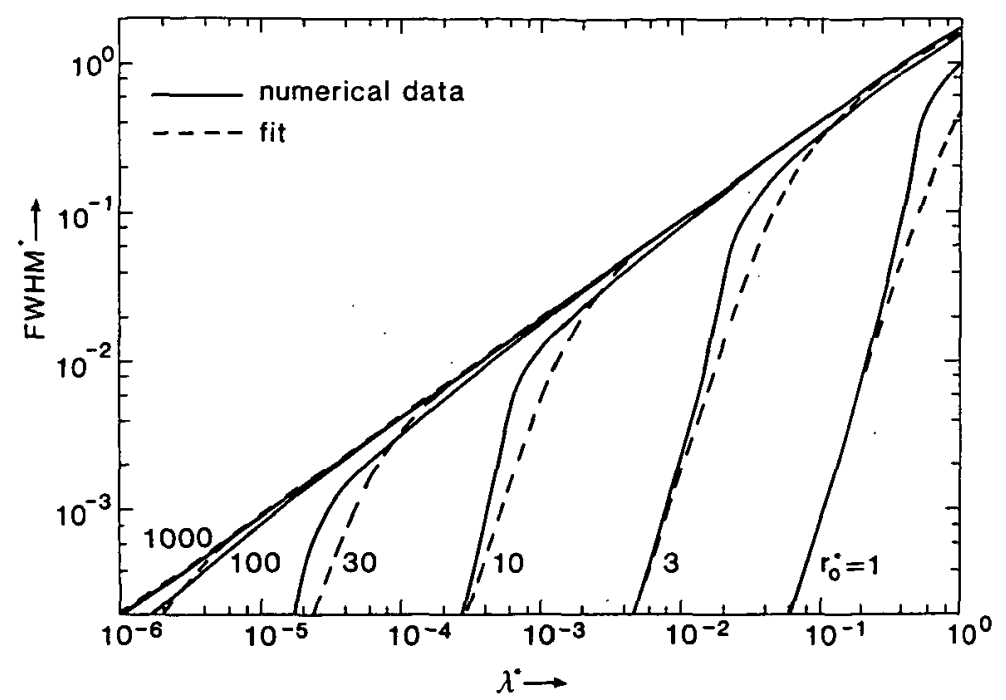

Figure 8.7 The FWHM of the scaled distribution of transverse velocity displacements $p^{*}\left(\Delta v_{\perp}{ }^{*}\right)$ generated in a homocentric cylindrical beam segment, for different values of the scaled beam radius $\mathrm{r}_{0}{ }^{*}$. The depicted fit is defined by equations $(8.4 .25)$ and $(8.4 .26)$.

$$
\rho^{*}\left(\Delta v_{\perp}^{*}\right)=v \rho\left(\Delta v_{\perp}\right)=\frac{1}{2 \pi} \int_{0}^{\infty} k^{*} d k^{*} J_{0}\left(k^{*} \Delta v_{\perp}^{*}\right) e^{-4 \lambda^{*} p^{*}\left(k^{*}\right)}
$$

where $\lambda^{*}$ is the scaled linear particle density for a cylindrical beam given by equation (7.4.18). The second moment of this distribution follows from the function $p^{*}\left(k^{*}\right)$ using equation $(5.5 .4)$ (with $m=2$ )

$$
\left\langle\Delta v_{\perp} * 2\right\rangle=4 p_{2} *\left(r_{0}^{*}\right) \lambda *
$$

This relation can also be obtained from equation (8.4.15), using equation (5.5.10) (with $\mathrm{m}=2$ ).

The FWHM of $\rho^{*}\left(\Delta v^{*}\right)$ was evaluated numerically from the numerical data of $p^{*}\left(k^{*}\right)$. The results are plotted in figure 8.7 . A keyparameter for the interpretation of this data is the pencil beam factor for a cylindrical beam $\chi_{p}$, given by equation $(7.4 .20)$. The following regimes should be distinguished :

I) Gaussian regime. For $\lambda^{*} \approx 1$ and $x_{p} \gg>1$ the distribution $\rho^{*}\left(\Delta v_{\perp}^{*}\right)$ is dominated by the quadratic behaviour of $p^{*}\left(k^{*}\right)$ for small $k^{*}$ values. This leads to a Gaussian distribution with a FWHM given by

$$
\begin{aligned}
\text { FWHMG }^{*} & =4\left[\mathrm{p}_{2}^{*}(\infty) \ln 2\right]^{1 / 2} \mathrm{P}_{\mathrm{PA}}\left(\mathrm{r}_{0}^{*}\right) \lambda^{* 1 / 2} \\
& =1.832 \mathrm{P}_{\mathrm{PA}}\left(\mathrm{r}_{0^{*}}\right) \lambda^{* 1 / 2}
\end{aligned}
$$

where the function $\mathrm{P}_{\mathrm{PA}_{\mathrm{A}}}\left(\mathrm{r}_{0}{ }^{*}\right)$ is defined as

$$
\operatorname{PPA}\left(r_{0}^{*}\right)=\left[p_{2} *\left(r_{0}^{*}\right) / p_{2} *(\infty)\right]^{1 / 2}
$$

in which $p_{2}{ }^{*}\left(r_{0} *\right)$ and $p_{2}{ }^{*}(\infty)$ are defined by equation $(8.4 .14)$. The 
subscript PA indicates that the result refers to the Angular deflection distribution generated in a cylindrical beam segment in which the particles run along Parallel trajectories.

II) Holtsmark regime. For $\lambda^{*}<<1$ and $\chi_{p} \gg>1$ the distribution $\rho^{*}\left(\Delta v_{1}{ }^{*}\right)$ becomes equal to the one-dimensional variant of the Holtsmark distribution, which is obtained by substituting equation (8.4.12) into equation $(8.4 .17)$

$$
\rho^{*}\left(\Delta v_{\perp}{ }^{*}\right)=\frac{1}{2 \pi} \int_{0}^{\infty} k^{*} d k^{*} J_{0}\left(k^{*} \Delta v_{\perp} *\right) e^{-(4 \sqrt{2 \pi} / 15) \lambda * k^{* 3} / 2}
$$

analogous to equation $(5.8 .16)$, specifying the distribution of the lateral force component $F_{\perp}$ in an extended cylindrical beam. The shape of this type of distribution is depicted in figure 5.4 (curve corresponding to $\gamma=3 / 2)$. The FWHM of the distribution of equation $(8.4 .21)$ is equal to

$$
\text { FWHM }_{H}^{*}=2.6554(4 \sqrt{2 \pi} / 15)^{2 / 3} \lambda * 2 / 3=2.0300 \lambda * 2 / 3
$$

as follows with equation $(5.9 .15)$ and table 5.1 .

III) Pencil beam regime. For $\chi_{p} \ll<$ the distribution $\rho^{*}\left(\Delta v_{\perp}{ }^{*}\right)$ is determined by the large $k^{*}$-behaviour specified by equation (8.4.13). Substitution into equation $(8.4 .17)$ yields

$$
\rho^{*}\left(\Delta v_{\perp}^{*}\right)=\frac{1}{2 \pi} \int_{0}^{\infty} k^{\star} d k^{*} J_{0}\left(k^{*} \Delta v_{\perp}^{*}\right) e^{-2 C_{1 / 3} r_{0}^{*} / 3 \lambda^{*} k^{* 1 / 3}}
$$

with $C_{1 / 3}=72 \Gamma(5 / 6) / 7 \Gamma(1 / 6)=2.0858$. Equation $(8.4 .23)$ is equivalent to equation $(5.8 .20)$, specifying the distribution of the lateral force component $F_{1}$ in a pencil beam. A plot of this type distribution is given by figure ${ }^{1} 5.4$ (curve for $\gamma=1 / 3$ ). The FWHM of the distribution of equation (8.4.23) follows again with equation (5.9.15) and table 5.1

$$
\text { FWHMP }_{P}^{*}=.013013\left(\frac{144 \Gamma(5 / 6)}{7 \Gamma(1 / 6)}\right)^{3} r_{0}^{* 7} \lambda^{* 3}=.94471 r_{0}^{* 7} \lambda^{* 3}
$$

It can directly be verified that equations (8.4.19), (8.4.22) and (8.4.24) provide a complete description of the different regimes shown in figure 8.7 .

In order to interpolate equations (8.4.19), (8.4.22) and $(8.4 .24)$ we use

$$
\text { FWHM }^{*}=\mathrm{FWHM}_{\mathrm{H}}^{*} \mathrm{H}_{\mathrm{PA}}\left(\lambda^{*}, \mathrm{r}_{0}{ }^{*}\right)
$$

where the function $H_{P A}\left(\lambda^{*}, r_{0}^{*}\right)$ is defined as

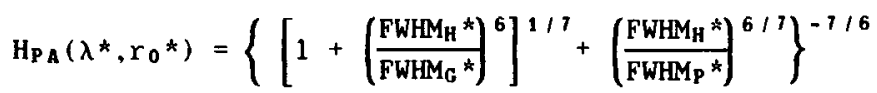

which is equal to

$$
H_{P A}\left(\lambda^{*}, r_{0}^{*}\right)=\left[\left(1+1.851 \frac{\lambda^{*}}{P_{P A}\left(r_{0}^{*}\right)^{6}}\right)^{1 / 7}+30.82 \frac{1}{x_{p}^{2}}\right]^{-7 / 6}(8.4 .26)
$$


with $\chi_{0}=4 \lambda^{*} r_{0} * 3$, as defined by equation 7.4.20. The function $P_{P A}\left(r_{0}^{*}\right)$ is specified by equations $(8.4 .20)$ and $(8.4 .16)$ and $F_{W H M_{H}}^{*}$ is given by equation (8.4.22). For comparison the fit of equation $(8.4 .26)$ is included in figure 8.7 .

$$
\text { Equations (8.4.19) through (8.4.26) give a complete }
$$
specification of the FWHM of the distribution of transverse velocity displacements $\rho^{*}\left(\Delta v^{*}\right)$ in terms of scaled coordinates defined by equations $(6.13 .1)$. The remaining task is to remove the scaling in order to make the dependency on the experimental parameters explicit. The properties of the scaled velocity distribution $\rho^{*}\left(\Delta v_{\perp}^{*}\right)$ can be related to the corresponding angular deflection distribution using

$$
\Delta \alpha=\frac{\Delta v_{\perp}^{*}}{v}=\left(\frac{\mathrm{e}}{2 \pi \varepsilon_{0}} \frac{1}{\mathrm{LV}}\right)^{1 / 3} \Delta \mathrm{v}_{\perp}^{*}
$$

The general expressions of equation (8.4.25) transforms to

$$
\mathrm{FWHM}_{\alpha}=\mathrm{C}_{\mathrm{PHA}} \mathrm{HPA}_{\mathrm{PA}}\left(\lambda^{*}, \mathrm{r}_{0^{*}}\right) \frac{\mathrm{I}^{2 / 3} \mathrm{~L}}{\mathrm{~V}^{4 / 3} \mathrm{r}_{0^{4 / 3}}}, \quad \mathrm{C}_{\mathrm{PHA}}=2.6554 \frac{\mathrm{m}^{1 / 3}}{2(15 \pi)^{2 / 3} \varepsilon_{0}} \quad(8.4 .28)
$$

In the case of electrons one finds $C_{P H A}=1.1142$ in SI-units (note: the subscript PHA indicates that the constant applies to a cylindrical beam in which the particles run along parallel trajectories and refers to the Holtsmark type of Angular deflection distribution).

For the Holtsmark-regime $\left(\lambda^{*}<<1\right.$ and $\left.x_{p} \gg>1\right)$ one may approximate $H_{P A}\left(\lambda^{*}, r_{0}^{*}\right)=1$. Accordingly, equation (8.4.28) reduces to

$$
\text { FWHM }_{\alpha}=\mathrm{C}_{P H A} \frac{\mathrm{I}^{2 / 3} \mathrm{~L}}{\mathrm{~V}^{4 / 3} \mathrm{r}_{0^{4 / 3}}} \quad\left(\lambda^{*}<<1, \chi_{\mathrm{p}}>>1\right)
$$

transforms to

For the Gaussian regime $\left(\lambda * \gtrsim 1\right.$ and $\left.\chi_{p} \gg>1\right)$ equation $(8.4 .28)$

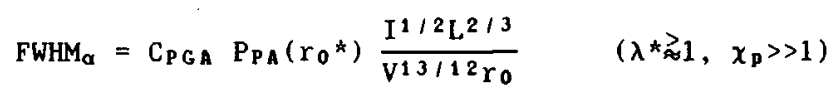

where the constant $\mathrm{C}_{P G A}$ is given by

$$
C_{P G A}=\frac{\left[p_{2}^{*}(\infty) \ln 2\right]^{1 / 2}}{2^{1 / 12 \pi^{5 / 6}}} \frac{e^{1 / 12 m^{1 / 4}}}{E_{0}^{5 / 6}}
$$

in which $\mathrm{p}_{2}{ }^{*}(\infty)$ given by equation $(8.4 .14)$. Equation $(8.4 .30)$ can also be derived directly from equations $(8.4 .19)$ and $(8.4 .27)$. In the case of electrons one finds $C_{P G A}=0.2269$ in SI-units.

For the pencil beam regime $\left(\chi_{p} \ll 1\right)$ equation $(8.4 .28)$ transforms to

$$
\mathrm{FWHM}_{\alpha}=\mathrm{C}_{\mathrm{PPA}} \frac{\mathrm{I}^{3} \mathrm{r}_{0} \mathrm{~L}}{\mathrm{~V}^{5 / 2}} \quad\left(x_{\mathrm{p}} \ll<1\right)
$$

where the constant $C_{P P A}$ is given by

$$
\mathrm{C}_{P P A}=.013013 \frac{\sqrt{2}}{\pi}\left(\frac{18 \Gamma(5 / 6)}{7 \Gamma(1 / 6)}\right)^{3} \frac{\mathrm{m}^{3 / 2}}{\varepsilon_{0} \mathrm{e}^{7 / 2}}=8.306110^{-4} \frac{\mathrm{m}^{3 / 2}}{\varepsilon_{0} \mathrm{e}^{7 / 2}}
$$

which can also be derived directly from equations (8.4.24) and (8.4.27). In the case of electrons one finds $C_{P P A}=4.9544 \times 10^{28}$ in SI-units.

It is interesting to compare the results for the distribution of transverse velocity displacements $\rho\left(\Delta v_{f}\right)$, presented in this section, with the results obtained for the distribution of axial velocity 
displacements $\rho\left(\Delta \mathbf{v}_{\mathbf{z}}\right)$, presented in section 7.4 . These results all apply to a monochromatic homocentric cylindrical beam segment, in which the particles are initially at rest in the frame of reference moving with the beam. Random velocities are generated during the flight due the conversion of potential energy into kinetic energy. On symmetry grounds one should expect that the generated velocity distribution (in the frame of reference moving with the beam) is rotational symmetric, provided that the dimensions of the beam are large compared to the average separation of the particles. Thus for an extended beam the distributions $\rho\left(\Delta v_{\perp}\right)$ and $\rho\left(\Delta v_{z}\right)$ should be equivalent. However, differences will occur for pencil beams in which the distribution of field particles around a certain test particle is non-rotational symmetric.

This reasoning is confirmed by our model as can best be observed from the various results for the $p^{*}\left(k^{*}\right)$-transform. The small $k^{*}-$ behaviour is for the separate cases given by equations (7.4.14) and (8.4.14). These equations are identical for $r_{0}^{*}+\infty$, which corresponds to an extended beam. As expected, different results are found for small $r_{0}{ }^{*}$-values, as can be seen from the approximations for the individual $\mathrm{p}_{2}{ }^{*}\left(\mathrm{r}_{0}{ }^{*}\right)$ functions given by equations $(7.4 .16)$ and $(8.4 .16)$ respectively. The intermediate $k^{*}$-behaviour of $p^{*}\left(k^{*}\right)$ is again identical for both cases, as can be seen from equations (7.4.12) and (8.4.12). This part of the $p^{*}\left(k^{*}\right)$ transform stems from weak interactions in an extended beam. The large $k^{*}$-behaviour stems from weak interactions in a pencil beam. The corresponding equations (7.4.13) and $(8.4 .13)$ are different, as was to be expected. Thus in general one finds that the $p^{*}\left(k^{*}\right)$ transforms of the distributions $\rho^{*}\left(\Delta v_{z}^{*}\right)$ and $\rho^{*}\left(\Delta v_{1}^{*}\right)$ coincide for that part of the function which represents the interactions in an extended beam segment. This can also directly be observed from the figures 7.8 and 8.5 .

It should be realized that identical $p^{*}\left(k^{*}\right)$-transforms do not necessarily lead to identical FWHM ${ }^{*}$-values of the corresponding distributions $\rho^{*}\left(\Delta v_{z}{ }^{*}\right)$ and $\rho^{*}\left(\Delta v_{\perp}{ }^{*}\right)$ since they are not of the same dimension. The former is one-dimensional, while the latter is twodimensional. A Gaussian distribution has the property that its FWHM value is independent of its dimension. Accordingly, equations (7.4.21) and $(8.4 .19)$ are identical, provided that $r_{0}^{*} \rightarrow \infty$. However, the FWHM*values obtained in the Holtsmark regime differ by a factor 0.9228 , as can be seen from equations $(7.4 .24)$ and $(8.4 .22)$. This factor follows also directly from table 5.1 .

\subsection{Beam segment with a crossover of arbitrary dimensions}

In the previous two sections the distribution of transverse velocity displacements $\rho\left(\Delta v_{\perp}\right)$ was calculated for the extreme cases of a beam segment with a narrow crossover and a homocentric cylindrical beam segment respectively. In this section we will consider the general case of a beam segment with arbitrary values of the geometry parameters $K_{1}$ and $K_{2}$, defined by equation (7.3.1). The analysis of this section is similar to that of section 7.5 , in which we considered the distribution of axial velocities $\rho\left(\Delta \mathrm{v}_{z}\right)$ generated in a beam segment of arbitrary dimensions. However, in the present calculation one can not simply restrict the problem to the case $S_{c}=0.5$ (crossover in the middle) and generalize the results afterwards. This is related to the fact that the displacement in transverse velocity $\Delta v_{1}$ experienced by the test particle in the first part of the beam segment (from the entrance plane to the crossover) can partly or entirely be cancelled out in the second part of 
the beam segment (from the crossover to the exit plane), depending on the location of the crossover as well as the type of collision involved. Complete cancellation occurs for a weak collision with a field particle which crosses the axis exactly in the crossover plane $\left(r_{\perp}=0\right)$, when this plane is located in the middle of the beam segment $\left(S_{c}=0.5\right)$, as can be seen from equation $(8.2 .2)$. In order to investigate the influence of the location of the crossover we will perform all the numerical calculations for two different locations, corresponding to $S_{c}=0.5$ and $S_{c}=0.75$ respectively. The results for other $S_{c}$-values follow from the analytical analysis.

As in section 8.3 we will employ the $d_{0}, v_{0}-s c a l i n g$ defined by equation $(7.3 .2)$. The scaled two-particle distribution $\bar{\rho}_{2}\left(\Delta \overline{\mathbf{v}}_{1}\right)$ is defined by equation $(8.3 .2)$. As the parameters $K_{1}$ and $K_{2}$ are not necessarily large only a fraction of the collisions will be complete. Accordingly, one may not apply equation (8.3.1) for the entire integration domain of equation $(8,3.2)$. The part of the integration domain which corresponds to complete collisions is indicated by the constraints of equation (7.5.1).

The other type of collision which can be treated by analytical means are weak collisions. The constraints of equation (7.5.3) indicate which part of the integration domain of equation (8.3.2) corresponds to weak collisions. The displacement $\Delta v_{1}$ caused by a weak collision follows from equation (8.2.2). Utilizing the $\mathrm{d}_{0}, \mathrm{v}_{0}$-scaling, this expression can be rewritten as

$$
\begin{aligned}
& \Delta \bar{v}_{\perp} \cong \frac{1}{\bar{v} \bar{b}}\left[\left(\frac{\bar{b}}{\left[\left(\bar{r}_{c} K_{1} \vec{v}+\vec{a}_{0}\right)^{2}+\bar{b}^{2}\right]^{1 / 2}}-\frac{\bar{b}}{\left[\left(\bar{r}_{c} K_{2} \bar{v}-\vec{a}_{0}\right)^{2}+\bar{b}^{2}\right]^{1 / 2}}\right)^{2}+\right. \\
& \\
& \left.\left[\frac{\bar{r}_{\perp} \sin (\Phi)}{\bar{b}}\right)^{2}\left(\frac{\bar{r}_{c} K_{1} \bar{v}+\bar{a}_{0}}{\left[\left(\bar{r}_{c} K_{1} \bar{v}+\bar{a}_{0}\right)^{2}+\bar{b}^{2}\right]^{1 / 2}}+\frac{\bar{r}_{c} K_{2} \bar{v}-\bar{a}_{0}}{\left[\left(\bar{r}_{c} K_{2} \bar{v}-\bar{a}_{0}\right)^{2}+\bar{b}^{2}\right]^{1 / 2}}\right)^{2}\right]^{1 / 2}
\end{aligned}
$$

The computer program which was used to evaluate $\bar{\rho}\left(\Delta \overline{\mathbf{v}}_{1}\right)$ from equation (8.3.2) checks for every two-particle collision whether all constraints of equation (7.5.1) are satisfied or not. If so the collision is (nearly) complete and it determines the corresponding displacement $\Delta \overline{\mathbf{v}}_{\perp}$ from equation $(8.3,1)$. If not it tests next whether one or more of the constraints of equation (7.5.3) are satisfied or not. If so the collision is weak and equation (8.5.1) is used to evaluate the corresponding displacement $\Delta \overline{\mathbf{v}}_{\perp}$. For collisions which are neither weak nor complete it determines the displacement $\Delta \overline{\mathbf{v}}_{\perp}$ by utilizing the numerical approach outlined in section 6.6 .

We have evaluated the distribution $\bar{\rho}\left(\Delta \overline{\mathbf{v}}_{1}\right)$ for $S_{c}=0.5$ and $S_{c}=0.75$, taking $k=0.1,0.2,0.5,1,10,100,1000$, and 10,000 . For every $S_{c}$ and $K$ we considered the cases $\bar{r}_{c}=10^{\circ}, 10^{1}, 10^{2}, 10^{3}, 10^{4}$ and $10^{5}$. Figures $8.8 \mathrm{a}, 8.8 \mathrm{~b}$ and $8.8 \mathrm{c}$ show the results obtained for $S_{c}=0.5$, with $\mathrm{K}=1,100$ and 10,000 respectively. Figures $8.9 \mathrm{~b}$ and $8.9 \mathrm{c}$ show the results for $S_{c}=0.75$, with $k=100$ and $k=10,000$ respectively. The results obtained for $K=1$ were found to be practically independent of $S_{c}$. For that reason we did not include a separate plot for $S_{c}=0.75$ and $K=1$. All figures should be compared to figure 8.1, which was calculated on the basis of complete collisions. One sees that the curves are identical for large values of $\Delta \bar{v}_{1}$, However, significant differences occur for small and intermediate $\Delta \bar{v}_{\perp}$-values, especially in combination with small $\bar{r}_{c}$-values. The differences become more pronounced for small K-values. Furthermore, it can be seen that results obtained for $S_{\mathbf{c}}=0.5$ and $S_{\mathbf{c}}=0.75$ show distinct differences, which become more extreme for large values of $\mathrm{K}$.

Let us investigate whether these results can be understood by analytical means. The comparison with figure 8.1 indicates that the 
large $\Delta \overline{\mathbf{v}}_{1}$-behaviour stems from strong complete collisions. Their total contribution to the distribution $\bar{\rho}\left(\Delta \overline{\mathbf{v}}_{1}\right)$ depends only weakly on the parameters $K$ and $S_{c}$. This is related to the fact that this type of collision corresponds to a small impact parameter $\bar{b}$, as is indicated by equation (7.5.1). The distribution of small b-values is not very sensitive to the geometry parameters $K$ and $S_{c}$. As the large $\Delta \bar{v}_{1}-$ behaviour stems from complete collisions, one can approximate this part of the curves by equation $(8.3 .11)$. The extreme case $\bar{r}_{c \approx 0}$ is covered by equation (8.3.6).

Small displacements $\Delta \overline{\mathbf{v}}_{1}$ correspond to weak, predominantly incomplete collisions with more distant field particles. Their contribution $\bar{\rho}\left(\Delta \bar{v}_{\perp}\right)$ is strongly affected by the value of the beam geometry parameters $K$ and $S_{c}$. In order to analyse the small $\Delta \overline{\mathbf{v}}_{\perp}-$ behaviour in a quantitative way, we will evaluate equation (8.3.2) on the basis of weak collisions, for which one may employ equation $(8.5 .1)$. An exact solution of this problem does appear to be feasible and the discussion will be restricted to the extreme cases $K \rightarrow 0$ and $K>>1$. For $\overline{\mathbf{r}}_{\mathrm{c}} \mathrm{K} \ll<1$ one may approximate equation $(8.5 .1)$ as

$$
\begin{gathered}
\Delta \bar{v}_{\perp} \cong \frac{1}{2 \bar{v}_{\left(\bar{r}_{l}^{2}+\bar{b}_{2}^{2}\right)^{3 / 2}}}\left[\left(-4 \bar{a}_{0} \bar{r}_{c} K \bar{v}+4\left(1-2 S_{c}\right)\left(\bar{r}_{c} K \bar{v}\right)^{2}+\cdots\right)^{2}+\right. \\
\left.\left(\frac{\bar{r}_{\perp} \sin (\Phi)}{\bar{b}^{2}}\right)^{2}\left(4 \bar{b}^{2} \bar{r}_{c} K \bar{v}-12 \bar{a}_{0}\left(1-2 S_{c}\right)\left(\bar{r}_{c} K \bar{v}\right)^{2}+\cdots\right)^{2}\right]^{1 / 2}
\end{gathered}
$$

utilizing equations (8.2.3) and (7.3.1). By ignoring the second and higher order terms in $\bar{x}_{c} K \bar{v}$ one obtains, after some reorganization of the remaining terms

$$
\Delta \bar{v}_{\perp} \cong \frac{2 \bar{r}_{c} K \bar{r}_{\perp}}{\left(\bar{r}_{\perp}^{2}+\bar{b}_{z}^{2}\right)^{3 / 2}}
$$

This result is identical to the scaled version of equation (8.2.5), which was derived for the case $v=0$. In the previous section this expression was used to evaluate the contribution of weak interactions in a homocentric cylindrical beam segment. The corresponding results for the two-particle distribution $\rho_{2}\left(\Delta v_{\perp}\right)$ are expressed by equations $(8.4 .4)$ and $(8.4 .5)$. Apparently, one may use these results to describe the limit $K \rightarrow 0$. The remaining task is therefore to transform the results from the $\delta, v$-scaling used in section 8.4 to the do, $v_{0}$-scaling used here. By means of equations $(7.5 .5)$ and $(7.5 .6)$ one obtains

$$
\begin{aligned}
& \bar{\rho}_{2}\left(\Delta \overline{\mathrm{v}}_{\perp}\right)=\frac{24 \sqrt{2 \pi} \Gamma(3 / 4) \mathrm{K}^{3 / 2}}{5 \Gamma(1 / 4) \overline{\mathrm{r}}_{\mathrm{c}}^{1 / 2} \Delta \overline{\mathrm{v}}_{\perp} 5 / 2} \\
& \begin{array}{r}
{\left[\Delta \bar{v}_{\perp}\left(\bar{r}_{c} \mathrm{~K} / 2\right) 1 / 3<<1, \Delta \bar{v}_{\perp} \bar{r}_{c} 2 / 3 /(32 \mathrm{~K}) 1 / 3>>1\right]} \\
(8.5 .3)
\end{array} \\
& \bar{\rho}_{2}\left(\Delta \bar{v}_{\perp}\right)=\frac{21 / 3 \bar{r}_{c}{ }^{2 / 3} K^{1 / 3}}{7 \Delta \bar{v}_{\perp}^{4 / 3}} \\
& {\left[\Delta \bar{v}_{\perp}\left(\bar{r}_{c} K / 2\right) 1 / 3 \ll<1, \Delta \bar{v}_{\perp} \bar{r}_{c} 2 / 3 /(32 \mathrm{~K}) 1 / 3 \ll 1\right]}
\end{aligned}
$$

We note that these equations can also be derived directly from equations (8.3.2) and (8.5.2). The asymptotic behaviour described by equation (8.5.4) provides an accurate approximation of the small $\Delta \bar{v}_{1}$-behaviour for small $\mathrm{K}$, as can be verified from figure $8.8 \mathrm{a}$. The behaviour described by equation $(8.5 .3)$ becomes manifest for intermediate $\Delta \bar{v}_{\perp}-$ values, combined with small $K$ and $\overline{\mathbf{r}}_{c}$ values.

For a narrow crossover $\left(K_{1} \gg 1\right.$ and $\left.K_{2} \gg>1\right)$ one may assume that $\bar{b}_{z} \gg \bar{r}_{\perp}$ for the majority of the weak collisions. Accordingly, the second 


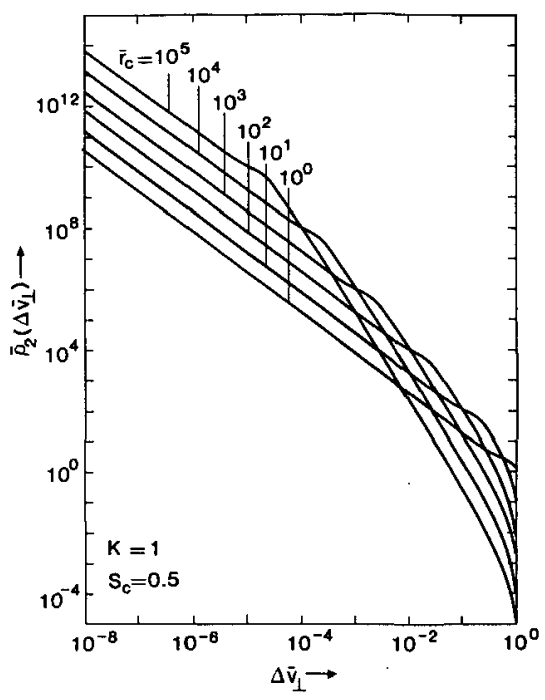

fig.8.8a

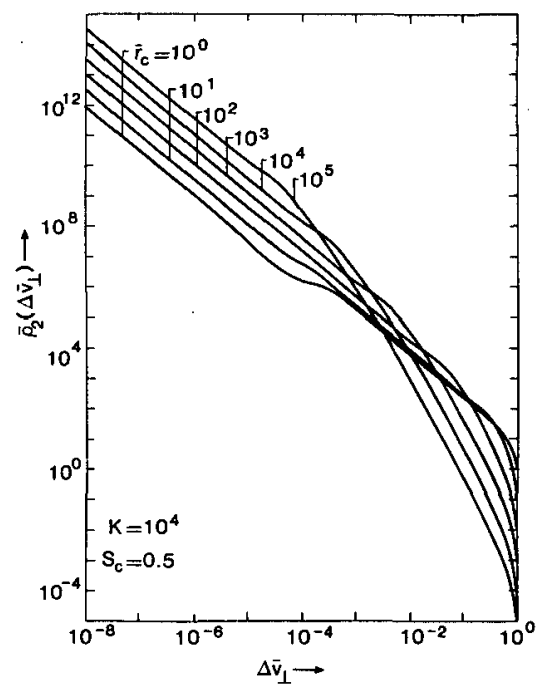

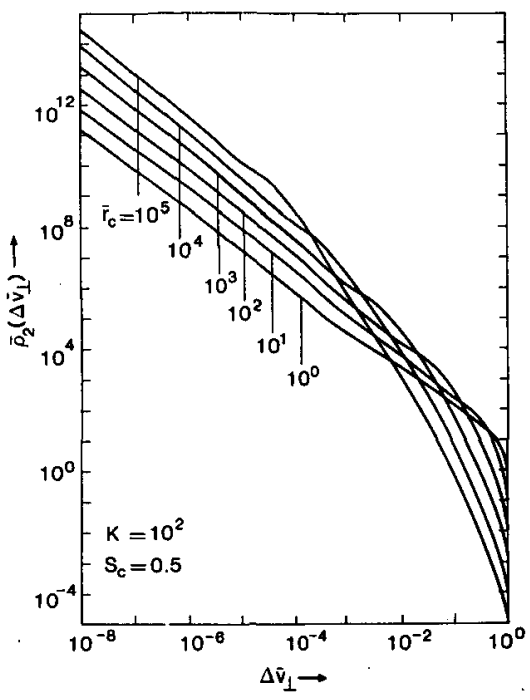

fig.8.8b

fig.8.8c

Figure 8.8 The scaled two-particle distribution $\bar{\rho}_{2}\left(\Delta \bar{v}_{\perp}\right)$ for a beam segment with a crossover in the middle $\left(S_{\mathrm{c}}=1 / 2\right)$, plotted for different values of the scaled crossover radius $\bar{r}_{\mathrm{c}}$. The figures $8.8 \mathrm{a}, 8.8 \mathrm{~b}$ and $8.8 \mathrm{c}$ pertain to different values of the beam geometry parameter $\mathrm{K}=\alpha_{0} \mathrm{~L} / 2 \mathrm{r}_{\mathrm{c}}, \mathrm{as}$ is indicated. The plots should be compared to those of figure 8.1 , derived on the basis of complete collisions. 


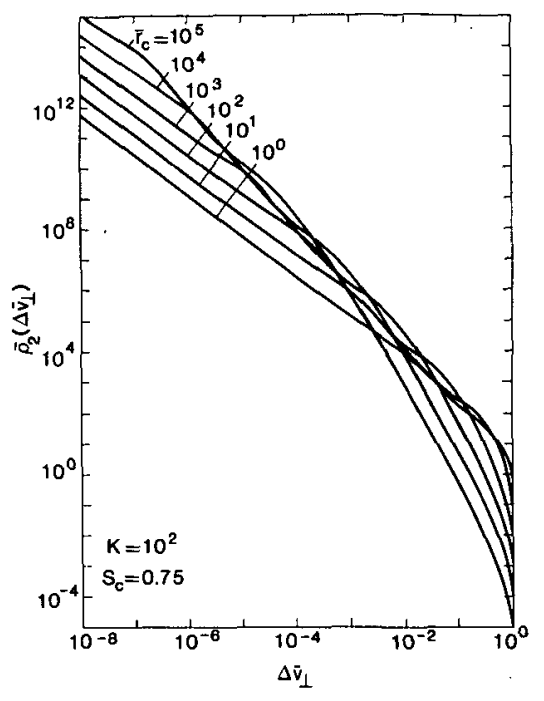

fig. $8.9 b$

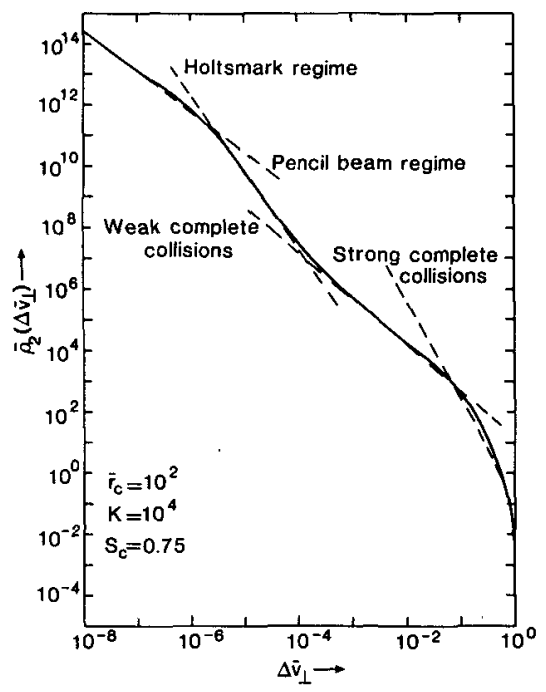

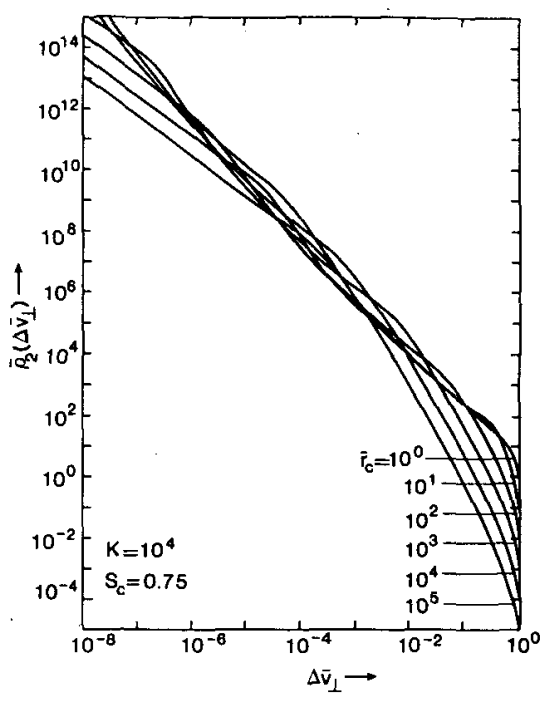

fig.8.9c

$\operatorname{flg} .8 .9 d$

Figure 8.9 The scaled two-particle distribution $\bar{\rho}_{2}\left(\Delta \bar{v}_{1}\right)$ for a beam segment with a crossover located at $3 / 4$ of the segment length $\left(S_{c}=3 / 4\right)$, plotted for different values of the scaled crossover radius $\overline{\mathrm{r}}_{\mathrm{c}}$. The curves should be compared to those of the figures $8.8 \mathrm{~b}$ and $8.8 \mathrm{c}$, which pertain to $S_{\mathrm{c}}=1 / 2$. The differences vanish for small $\mathrm{k}$-values. Therefore, there is no figure $8.9 a$ corresponding to $\mathrm{K}=1$ (which would be identical to figure 8.8a). Figure $8.9 d$ explains the shape of the curves from the asymptotic behaviour corresponding to the different regimes. The different asymptotes are (from left to right) specified by equations (8.5.16), $(8.5 .10),(8.3 .7)$ and $(8.3 .11)$. 
term in equation (8.5.1) can be ignored and one finds in good approximation for the remaining term

$$
\Delta \bar{v}_{\perp} \cong \frac{1}{\bar{v}}\left|\frac{1}{\left[\left(\bar{r}_{c} K_{1} \bar{v}\right)^{2}+\bar{b}_{2}^{2}\right]^{1 / 2}}-\frac{1}{\left[\left(\bar{r}_{c} K_{1} \bar{v}\right)^{2}+\bar{b}_{z^{2}}\right]^{1 / 2}}\right| \quad(K>>1)
$$

assuming that $\overline{\mathrm{v}} \neq 0$. The scaled two-particle distribution follows again with equation (8.3.2). As equation (8.5.5) does not depend on $\bar{r}_{\perp}$ or $\Phi$, equation (8.3.2) reduces to a two-dimensional integral, which can be expressed as

$$
\bar{\rho}_{2}\left(\Delta \bar{v}_{\perp}\right)=\int_{0}^{1} 2 \bar{v} d \bar{v} \int_{0}^{\infty} 2 d_{z} \delta\left(\Delta \bar{v}_{\perp}-\frac{F\left(\bar{b}_{z} / 2 \bar{r}_{c} K \bar{v}\right)}{2 \bar{r}_{c} K \bar{v}^{2}}\right)
$$

in which the function $F(z)$ is given by

$$
F(z)=\left|\frac{1}{\left[S_{c}{ }^{2}+z^{2}\right]^{1 / 2}}-\frac{1}{\left[\left(1-S_{c}\right)^{2}+z^{2}\right]^{1 / 2}}\right|
$$

where the definition of $K_{1}$ and $K_{2}$, given by equations $(7.3 .1)$, was used. By substituting

$$
z=\frac{\bar{b}_{z}}{2 \bar{r}_{c} K \bar{v}}, \quad \cdot t=\frac{F(z)}{2 \bar{r}_{c} K \bar{v}^{2}}
$$

and carrying out the t-integration, equation (8.5.6) transforms to

$$
\bar{\rho}_{2}\left(\Delta \bar{v}_{\perp}\right)=\frac{\sqrt{2}}{\left(\bar{r}_{c} K\right)^{1 / 2} \Delta \bar{v}_{\perp}^{5 / 2}} \int_{0}^{\infty} d z F(z)^{3 / 2} \theta\left(\Delta \bar{v}_{\perp}-\frac{F(z)}{2 \bar{r}_{c} K}\right)
$$

where $\theta(x)$ is the step-function. Equation (8.5.9) is similar to equation (7.5.12), specifying the two-particle distribution $\bar{\rho}\left(\Delta \bar{v}_{z}\right)$ for weak collisions in a narrow crossover. We will consider the cases $\overline{\mathbf{r}}_{\mathrm{c}} \mathrm{K} \Delta \overline{\mathrm{v}}_{\perp} \gg 1$ and $\overline{\mathbf{r}}_{\mathbf{c}} \mathrm{K} \Delta \overline{\mathrm{v}}_{1} \ll<1$.

For $\overline{\mathrm{r}}_{c} \mathrm{~K} \Delta \overline{\mathrm{v}}_{\perp} \gg>1$ the argument of the step-function in equation (8.5.9) is positive for all z-values and one obtains

$$
\bar{\rho}_{2}\left(\Delta \bar{v}_{\perp}\right)=\frac{I_{4} S_{H A}\left(S_{c}\right)^{3 / 2}}{\left(\bar{r}_{c} K\right)^{1 / 2} \Delta \bar{v}_{\perp}^{5 / 2}} \quad\left(K \gg>1, \bar{r}_{c} K \Delta \bar{v}_{\perp} \gg 1\right)
$$

in which

$$
S_{H A}\left(S_{C}\right)^{3 / 2}=\frac{\sqrt{2}}{I_{4}} \int_{0}^{\infty} d z F(z)^{3 / 2}
$$

and $I_{4}$ some arbitrary constant which will be used to scale the function $S_{\mathrm{HA}}\left(S_{\mathrm{C}}\right)$. The function $S_{\mathrm{HA}}\left(S_{\mathrm{c}}\right)$ has the following properties

$$
\begin{aligned}
& \lim _{S_{c} \rightarrow 0} S_{H A}\left(S_{c}\right)\left(2 S_{c}\right)^{1 / 3}=\lim _{S_{c} \rightarrow 1} S_{H A}\left(S_{c}\right)\left[2\left(1-S_{c}\right)\right]^{1 / 3}=\left(\frac{2 \sqrt{\pi} \Gamma(1 / 4)}{I_{4} \Gamma(3 / 4)}\right)^{2 / 3} \\
& \lim _{S_{c} \rightarrow 1 / 2} S_{H A}\left(S_{c}\right)=\left(\frac{24 \sqrt{2 \pi \Gamma(3 / 4)}}{5 I_{4} \Gamma(1 / 4)}\right)^{2 / 3}\left|1-2 S_{c}\right|
\end{aligned}
$$

As an approximation of the function $S_{H A}\left(S_{c}\right)$ we use 


$$
\mathrm{S}_{\mathrm{HAa}}\left(\mathrm{S}_{\mathrm{C}}\right) \cong \mathrm{C}\left|\frac{1}{\left(2 \mathrm{~S}_{\mathrm{c}}\right)^{1 / 3}}-\frac{1}{\left[2\left(1-\mathrm{S}_{\mathrm{C}}\right)\right]^{1 / 3}}\right|
$$

which has similar properties

$$
\begin{aligned}
& \lim _{S_{C} \rightarrow 0} S_{H A a}\left(S_{c}\right)\left(2 S_{c}\right)^{1 / 3}=\lim _{S_{c} \rightarrow 1} S_{H A a}\left(S_{C}\right)\left[2\left(1-S_{C}\right)\right]^{1 / 3}=C \\
& \lim _{S_{c} \rightarrow 1 / 2} S_{H A a}\left(S_{c}\right)=2 C / 3\left|1-2 S_{c}\right|
\end{aligned}
$$

Unfortunately, one can not choose the constants $C$ and $I_{4}$ such that equations (8.5.12) and (8.5.14) agree fully both for $S_{c} \rightarrow 0 \quad$ or $S_{c} \rightarrow 1$ ) and for $S_{c} \rightarrow 1 / 2$. Considering that the case $S_{c} \approx 1 / 2$ is the most relevant, we take

$$
\mathrm{C}=2 \quad, \mathrm{I}_{4}=\frac{3^{5 / 2}(2 \pi)^{1 / 2} \Gamma(3 / 4)}{5 \Gamma(1 / 4)}=2.6413
$$

This choice guarantees a good approximation of the real function $S_{H A}\left(S_{c}\right)$ for $S_{c} \approx 1 / 2$, but leads to some overestimation of $S_{H A}\left(S_{c}\right)$ for $S_{c} \rightarrow 0$ and $S_{c} \rightarrow 1$. We evaluated the function $S_{H A}\left(S_{c}\right)$ numerically. The results are plotted in figure 8.10. For comparison the approximation of equation (8.5.13) is included in the same figure.

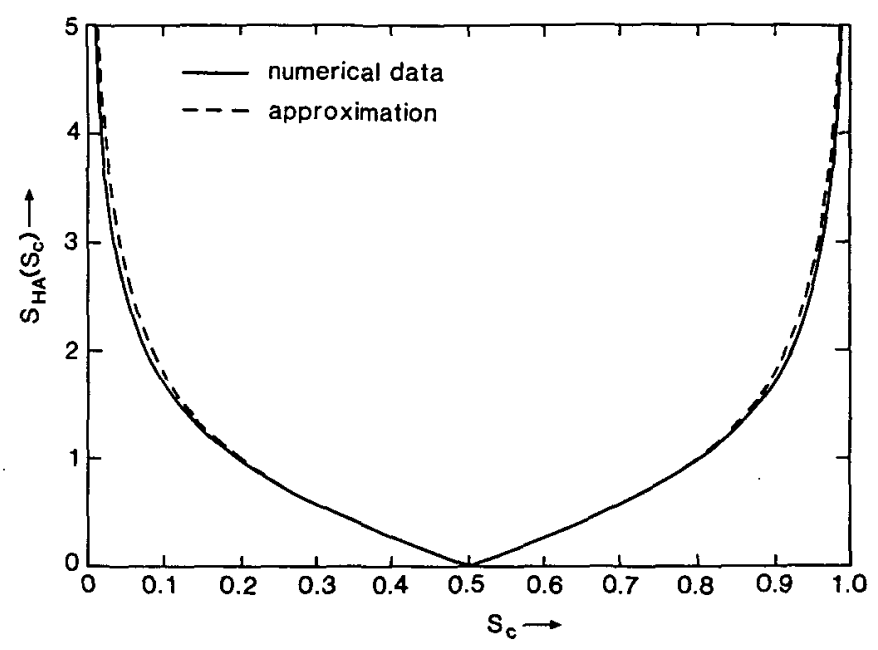

Figure 8.10 The function $\mathrm{S}_{\mathrm{HA}}\left(\mathrm{S}_{\mathrm{c}}\right)$ defined by equations $(8.5 .11)$, (8.5.7) and $(8.5 .15)$ and its approximation given by equations (8.5.13) and (8.5.15). This function specifies the dependency of the width of the angular displacement distribution in the Holtsmark regime. 
For $\overline{\mathbf{r}}_{\mathbf{c}} \mathrm{K} \Delta \overline{\mathrm{v}}_{1} \ll 1$ the argument of the step-function in equation (8.5.9) is only positive for large z-values. Accordingly, one may use the following asymptotic expansion of the function $F(z)$

$$
\lim _{z \rightarrow \infty} F(z)=\frac{\left|1-2 S_{c}\right|}{2 z^{3}}+0\left(z^{-5}\right)
$$

in which $0\left(z^{-5}\right)$ stands for terms of the order $z^{-5}$. Substitution into equation (8.5.9) yields

$$
\bar{\rho}_{2}\left(\Delta \bar{v}_{\perp}\right)=\frac{21 / 3}{7} \frac{S_{P A}\left(S_{c}\right)^{1 / 3}\left(\bar{r}_{c} K\right)^{2 / 3}}{\Delta \bar{v}_{\perp}^{4 / 3}} .\left(K \gg 1, \bar{r}_{c} K \Delta \bar{v}_{\perp} \ll<1\right)
$$

in which the function $S_{P A}\left(S_{c}\right)$ represents

$$
S_{P_{A}}\left(S_{c}\right)=\left|1-2 S_{c}\right|
$$

Equations (8.5.10) and (8.5.16) provide an accurate description of the small $\Delta \bar{v}_{1}$-behaviour of the distribution $\bar{\rho}_{2}\left(\Delta \bar{v}_{1}\right)$ for large K-values and $S_{c} \neq 0.5$, as can be verified from figures $8.9 \mathrm{~b}$ and $8.9 \mathrm{c}$. In order to demonstrate the significance of the various asymptotes derived for weak and complete collisions the curve in figure $8.9 \mathrm{c}$ corresponding to $\overline{\mathrm{r}}_{\mathrm{c}}=10^{2}$ is replotted separately in figure $8.9 \mathrm{~d}$. For very small $\Delta \overline{\mathbf{v}}_{\perp}$-values the curve follows the asymptote described by equation (8.5.16). This result can be associated with the pencil-beam regime as will be shown later on in this section. For larger $\Delta \vec{v}_{\perp}$-values the curve follows the asymptote described by equation $(8.5 .10)$, which is associated with the Holtsmark regime. It should be recalled that both equation (8.5.16) and equation (8.5.10) are based on weak collisions. With further increasing $\Delta \bar{v}_{\perp}$ the complete collision regime is reached. The curve now follows the asymptote described by equation (8.3.7), which corresponds to weak complete collisions. Finally, for very large $\Delta \bar{v}_{\text {-values the curve is }}$ dominated by strong, predominantly complete collisions and can be approximated by equation $(8.3 .11)$.

The previous analysis shows that the distribution $\bar{\rho}_{2}\left(\Delta \bar{v}_{1}\right)$ follows different sets of asymptotes for $K \rightarrow 0$ and $k \gg>1$. We wish to simplify the results somewhat by introducing some general equations which yield correct results for both $K \rightarrow 0$ and $K \gg>1$. By comparing equations $(8.5 .3)$ and $(8.5 .10)$ it is found that one may in general describe the small $\Delta \bar{v}_{\perp}$-behaviour of $\bar{\rho}_{2}\left(\Delta \bar{v}_{\perp}\right)$ by equation $(8.5 .10)$, provided that the function $S_{H A}\left(S_{C}\right)$ is replaced by some generalized function $S_{H A}\left(S_{c}, K\right)$ which has the following properties

$$
\begin{aligned}
& \lim _{K \rightarrow \infty} S_{H A}\left(S_{c}, K\right) \cong 2\left|\frac{1}{\left(2 S_{c}\right)^{1 / 3}}-\frac{1}{\left[2\left(1-S_{c}\right)\right]^{1 / 3}}\right| \\
& \lim _{K \rightarrow O} S_{H A}\left(S_{c}, K\right)=\frac{4}{3} K^{4 / 3}
\end{aligned}
$$

in which equations $(8.5 .13)$ and $(8.5 .15)$ were used. Similarly, one may combine equations $(8.5 .4)$ and $(8.5 .16)$ into a single expression which describes the intermediate $\Delta \bar{v}_{1}$-behaviour of $\bar{\rho}_{2}\left(\Delta \bar{v}_{1}\right)$ for arbitrary $K-$ values. This expression is the same as given by equation (8.5.16), provided that one replaces the function $S_{P A}\left(S_{C}\right)$ by some generalized function $S_{P A}\left(S_{C}, K\right)$ which has the following properties 


$$
\begin{aligned}
& \lim _{K \rightarrow \infty} S_{P A}\left(S_{c}, K\right)=\left|1-2 S_{c}\right| \\
& \lim _{K \rightarrow 0} S_{P A}\left(S_{c}, K\right)=\frac{1}{K}
\end{aligned}
$$

in which equation $(8.5 .17)$ was used. We mention that the slice-method (introduced in section 5.11) can be exploited to derive some suitable expressions to approximate the required generalized functions $S_{H_{A}}\left(S_{c}, K\right)$ and $S_{P_{A}}\left(S_{c}, K\right)$. This analysis is postponed to section 8.6. Here we suffice to present the results

$$
\begin{aligned}
& S_{H A a}\left(S_{c}, K\right) \cong 2\left|\frac{1}{\left(1 / K+2 S_{c}\right)^{1 / 3}}-\frac{1}{\left[1 / K+2\left(1-S_{c}\right)\right]^{1 / 3}}\right| \\
& S_{P A a}\left(S_{c}, K\right) \cong\left|1-2 S_{c}\right|\left(\frac{1}{K}+1\right)
\end{aligned}
$$

The reader might verify that the functions $S_{H A a}\left(S_{C}, K\right)$ and $S_{P a a}\left(S_{c}, K\right)$ indeed fulfil the conditions of equations $(8.5 .18)$ and $(8.5 .19)$ respectively, provided that one takes $S_{c}=0$ or $S_{c}=1$ in case $K \rightarrow 0$. This condition implies that a nearly cylindrical beam is represented as a beam segment with a crossover which is located either at the start or at the end of the beam segment. The results will be further discussed in section 8.6. It is postulated that equations $(8.5 .10)$ and $(8.5 .16)$ provide an accurate description of the intermediate and small $\Delta \bar{v}_{1}-$ behaviour of $\bar{\rho}_{2}\left(\Delta \bar{v}_{\perp}\right)$ respectively, which is valid for arbitrary values of $K$.

We now proceed with the calculation of the function $\vec{p}(\bar{k})$, which is defined by equation (8.3.12). We evaluated $\overline{\mathbf{p}}(\bar{K})$ numerically from the data obtained for $\bar{\rho}_{2}\left(\Delta \bar{v}_{1}\right)$. The results for $S_{c}=0.5$, with $K=1,100$ and 10,000 are plotted in the figures $8.11 \mathrm{a}, 8.11 \mathrm{~b}$ and $8.11 \mathrm{c}$ respectively. The results for $S_{c}=0.75$, with $K=100$ and 10,000 are plotted in the figures $8.12 \mathrm{~b}$ and $8.12 \mathrm{c}$. We recall that the results become independent of $S_{e}$ for small $K$. Thus figure 8.11a applies also to other $S_{c}$-values than $S_{c}=0.5$. The plots should be compared with figure 8.2 , which was derived on the basis of complete collisions. The most significant differences with the curves of figure 8.2 occur for intermediate and large $\bar{k}$-values. Figure 8.2 shows a square-root dependency on $\bar{k}$ for large $\bar{k}$, as described by equation $(8.3 .16)$. On the other hand, the curves in the figures $8.11 \mathrm{a}, 8.11 \mathrm{~b}, 8.11 \mathrm{c}, 8.12 \mathrm{~b}$ and $8.12 \mathrm{c}$ become for large $\mathbb{k}$ ultimately proportional to $\mathbf{k}^{1 / 3}$. This can be understood from the analysis of the two-particle distribution $\bar{\rho}_{2}\left(\Delta \bar{v}_{1}\right)$. Substitution of equation (8.5.16), with the generalized function $S_{P A}\left(S_{c}, K\right)$, into equation ( 8.3 .12 ) yields, after integration

$$
\overline{\mathrm{p}}(\mathrm{K})=\frac{72 \Gamma(5 / 6)}{7 \Gamma(1 / 6)} S_{P_{A}}\left(S_{\mathrm{c}}, \mathrm{K}\right)^{1 / 3}\left(\overline{\mathrm{r}}_{\mathrm{c}} \mathrm{K}\right)^{2 / 3} \mathrm{~K}^{1 / 3} \quad(\bar{K}+\infty)
$$

similar to derivation of equation $(5.8 .19)$. The behaviour of $S_{P A}\left(S_{C}, K\right)$ for $K \rightarrow 0$ and $K \gg>1$ is given by equation (8.5.19). For $K \rightarrow 0$ equation $(8.5 .22)$ transforms to

$$
\overline{\mathrm{p}}(\overline{\mathrm{K}})=\frac{72 \Gamma(5 / 6)}{7 \Gamma(1 / 6)} \overline{\mathrm{r}}_{\mathrm{c}} 2 / \mathrm{K}^{1 / 3} \mathrm{~K}^{1 / 3} \quad(\mathrm{~K} \rightarrow 0, \bar{K} \rightarrow \infty)
$$

which follows also directly by substitution of equation (8.5.4) into equation (8.3.12) and integration. Equation $(8.5 .23)$ provides an 


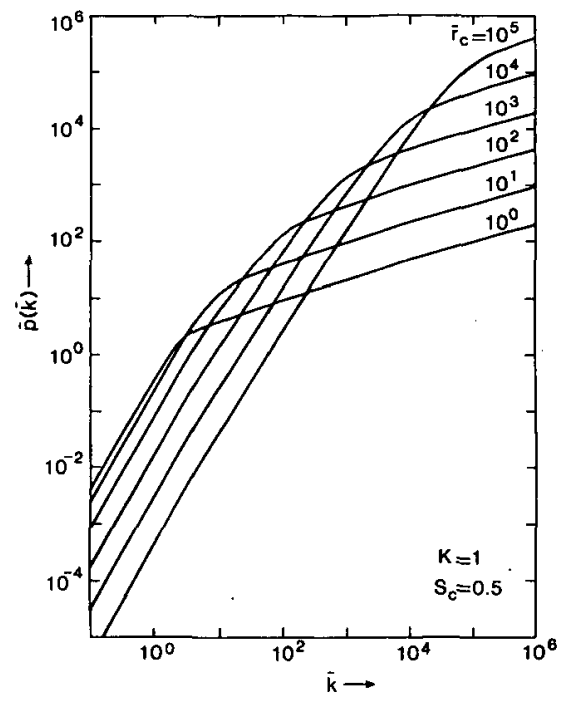

fig.8.11a

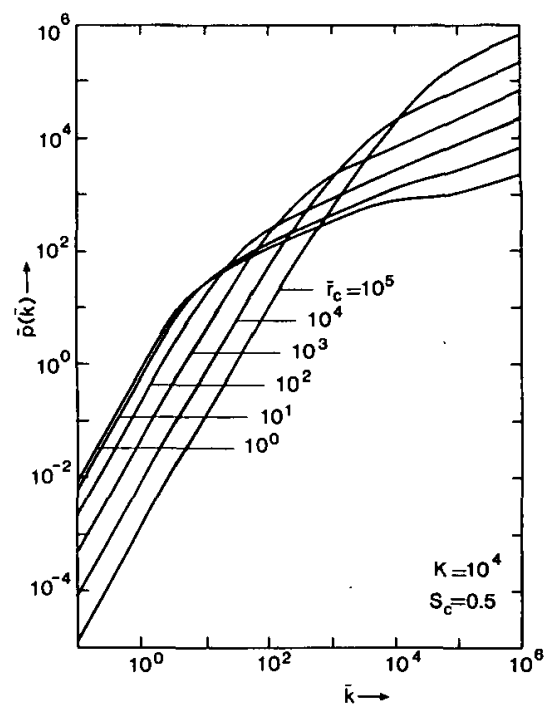

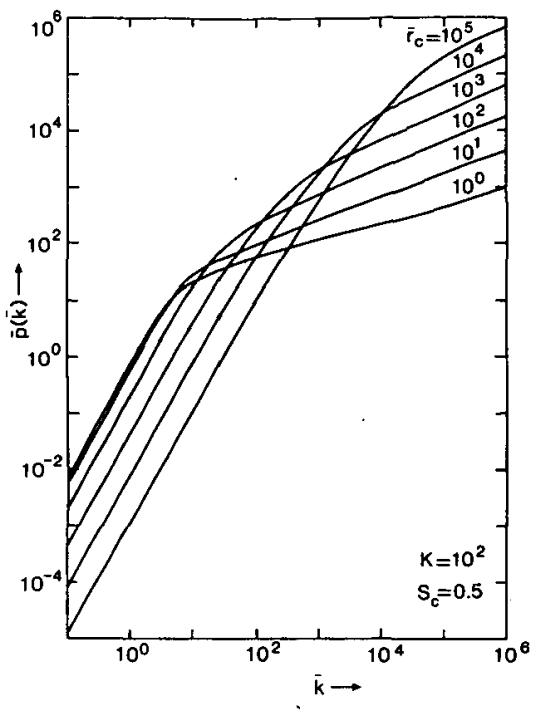

fig.8.11b

fig.8.11c

Figure 8.11 The scaled function $\overline{\mathrm{p}}(\overline{\mathrm{K}})$ corresponding to the distribution of transverse velocity displacements generated in a beam segment with a crossover in the middle $\left(S_{\mathrm{c}}=1 / 2\right)$, plot ted for different values of the scaled crossover radius $\bar{\gamma}_{\mathrm{c}}$. The figures $8.11 \mathrm{a}, 8.11 \mathrm{~b}$ and $8.11 \mathrm{c}$ pertain to different values of the beam geometry parameter $\mathrm{K}=\alpha_{0} \mathrm{~L} / 2 \mathrm{r}_{\mathrm{C}}$, as is indicated. The plots should be compared to those of figure 8.2 , derived on the basis of complete collisions. 


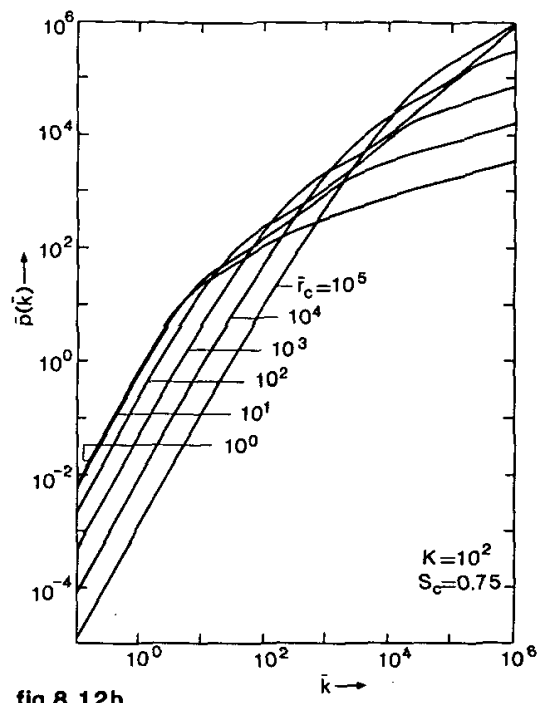

fig.8.12b

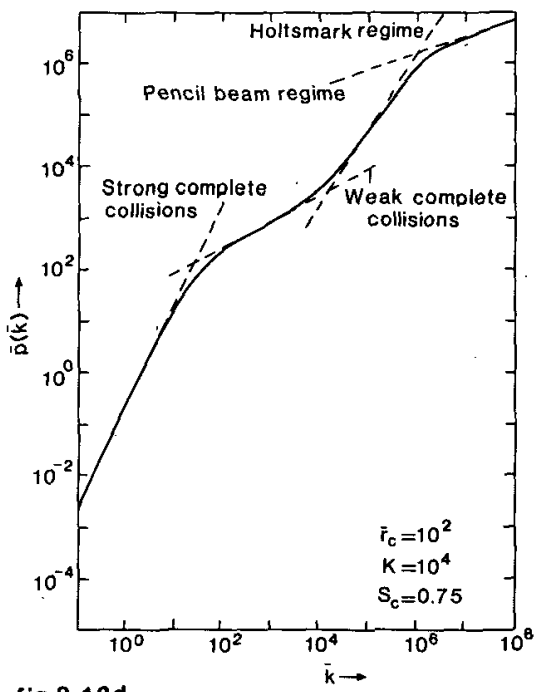

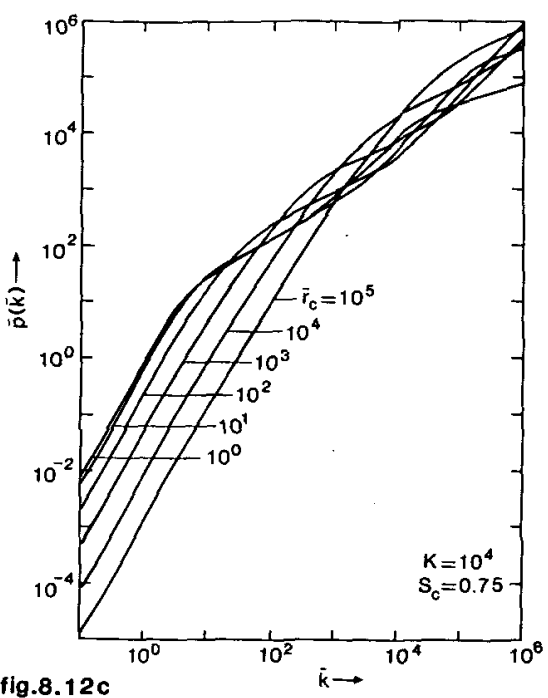

fig.8.12c

fig.8.12d

Figure 8.12 The scaled function $\bar{p}(\bar{k})$ corresponding to the distribution of transverse velocity displacements generated in a beam segment with a crossover located at $3 / 4$ of the segment length $\left(S_{\mathrm{c}}=3 / 4\right)$, plotted for different values of the scaled crossover radius $\overrightarrow{\mathrm{r}}_{\mathrm{c}}$. The curves should be compared to those of the figures $8.11 \mathrm{~b}$ and $8.11 \mathrm{c}$, which pertain to $\mathrm{s}_{\mathrm{c}}=1 / 2$. The differences vanish for small $\mathrm{K}$-values. Therefore, there is no figure $8.12 \mathrm{a}$ corresponding to $\mathrm{K}=1$ (which would be identical to figure 8.11 a). Figure $8.12 d$ explains the shape of the curves from the asymptotic behaviour corresponding to the different regimes. The different asymptotes are (from left to right) specified by equations (8.5.26), $(8.3 .16),(8.5 .24)$ and $(8.5 .22)$. 
accurate approximation of the large $k$ behaviour depicted in figure $8.11 \mathrm{a}$.

The behaviour described by equation (8.5.10) leads to a 3/2power dependency on $\bar{k}$ which becomes manifest for intermediate $\bar{k}$ values. Substitution of equation $(8.5 .10)$, with the generalized function $S_{\mathrm{HA}}\left(S_{\mathrm{C}}, \mathrm{K}\right)$, into equation (8.3.12) yields after integration

$$
\overline{\mathrm{p}}(\mathrm{K})=\frac{4(3 \pi)^{1 / 2}}{5} \frac{S_{\mathrm{HA}}\left(S_{\mathrm{c}}, K\right)^{3 / 2}}{\left(\overline{\mathrm{r}}_{\mathrm{c}} K\right)^{1 / 2}} \mathrm{k}^{3 / 2}
$$

similar to the derivation of equation (5.8.14). In equation (8.5.24) we used the expression for the numerical constant $I_{4}$, given by equation (8.5.15). The behaviour of $S_{H A}\left(S_{c}, K\right)$ for $K \rightarrow 0$ and $K \gg>1$ is given by equation (8.5.18). For $K \rightarrow 0$ equation (8.5.24) transforms to

$$
\overline{\mathrm{p}}(\mathrm{K})=\frac{32 \sqrt{\mathrm{I}}}{15} \frac{\mathrm{K}^{3 / 2}}{\overline{\mathrm{r}}_{\mathrm{c}}{ }^{1 / 2}} \mathrm{~K}^{3 / 2}
$$

which follows also directly by substitution of equation (8.5.3) into equation (8.3.12) and integration.

For small $\mathrm{k}$-values all curves in figures 8.11 and 8.12 become quadratic in $k$. In order to describe this behaviour, we define the function $\overline{\mathbf{p}}_{2}\left(\overline{\mathrm{r}}_{\mathrm{c}}, \mathrm{K}\right)$ as

$$
\overline{\mathrm{p}}(\mathrm{K})=\frac{1}{4} \overline{\mathrm{p}}_{2}\left(\overline{\mathrm{r}}_{\mathrm{C}}, \mathrm{K}\right) \mathrm{K}^{2}
$$

The function $\bar{p}_{2}\left(\bar{r}_{c}, K\right)$ is the generalization of the function $\bar{p}_{2}\left(\bar{r}_{c}\right)$, defined by equation $(8,3.14)$. The dependency of the small $k$-behaviour of $\bar{p}(k)$ on $S_{c}$ is very weak and will be discussed later on. We evaluated the function $\bar{p}_{2}\left(\bar{r}_{c}, K\right)$ numerically for $S_{c}=0.5$. The results are plotted in figure 8.13 .

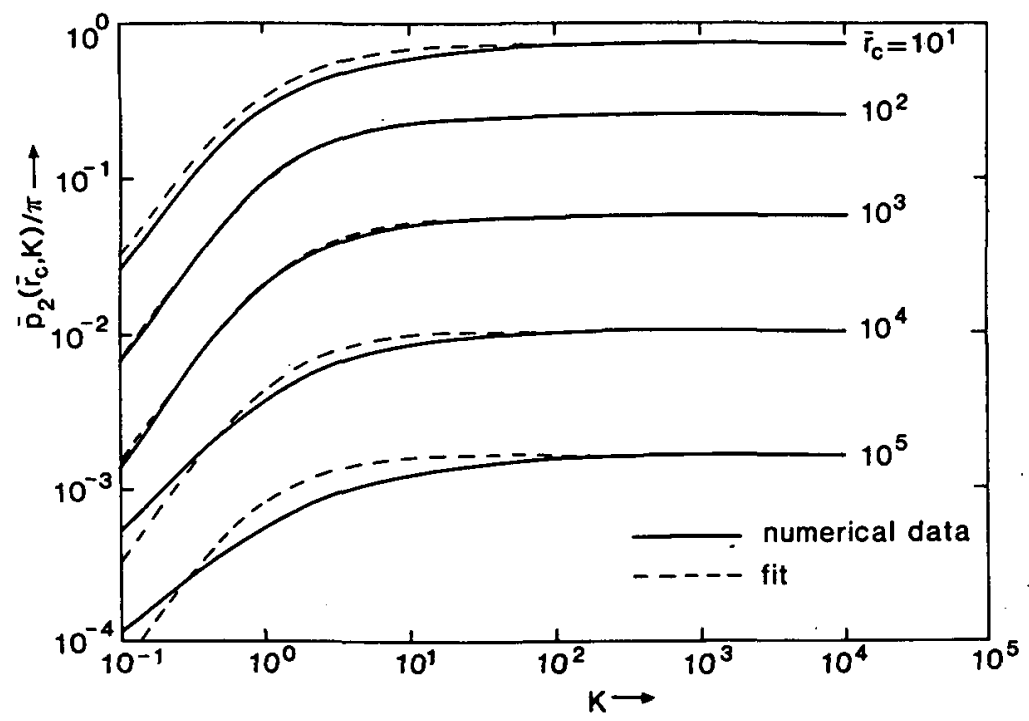

Figure 8.13 The function $\overline{\mathrm{p}}_{2}\left(\overline{\mathrm{r}}_{\mathrm{c}}, \mathrm{K}\right)$ defined by equation (8.5.26) and its approximation given by equation $(8.5,27)$. This function gives the dependency on $\bar{r}_{c}-$ and $k$ of the function $\bar{p}(\bar{k})$, plotted in the figures $8.11 \mathrm{a}, 8.11 \mathrm{~b}$ and $8.11 \mathrm{c}$, for $\mathrm{k} \rightarrow 0$. 
In the limit $K+\infty$ the function $\bar{p}_{2}\left(\bar{r}_{c}, K\right)$ should become identical to the function $\bar{p}_{2}\left(\bar{r}_{c}\right)$, as is formally expressed by equation (7.5.18). In the limit $K \rightarrow 0$ one should retrieve the result obtained for a homocentric cylindrical beam, which is given by equation $(8.4 .14)$. This condition leads to equation (7.5.19). Notice, however, that the saturation value $p_{2} *(\infty)=0.3026$ differs by a factor 2 from the result corresponding to the distribution of axial velocity displacements $\rho^{*}\left(\Delta \mathrm{v}_{\mathrm{z}}{ }^{*}\right)$. The corresponding approximations $\mathrm{p}_{\mathbf{z}}\left(\mathrm{r}_{0^{*}}\right)$ are different too, as can be seen from equations $(7.4 .16)$ and $(8.4 .16)$. As an approximation of the general function $\bar{p}_{2}\left(\bar{r}_{c}, K\right)$ we use in the present case

$$
\overline{\mathrm{p}}_{2 \mathrm{a}}\left(\overline{\mathrm{r}}_{\mathrm{c}}, \mathrm{K}\right)=\frac{\pi}{.894+.3\left(\frac{\overline{\mathrm{r}}_{\mathrm{c}}}{\mathrm{K}^{2}}\right)^{2 / 3}\left[1+.5\left(\frac{\mathrm{K}^{2}}{\overline{\mathrm{r}}_{\mathrm{c}}}\right)^{4 / 9}\right]^{3 / 2}+\frac{\pi \overline{\mathrm{r}}_{\mathrm{c}}}{\left\{2 \ln \left[.8673\left(114.6+\overline{\mathrm{r}}_{\mathrm{c}}\right)\right]\right\}^{2}}}
$$

which shows the asymptotic behaviour described by equations (7.5.18) and $(7.5 .19)$, provided that $\bar{r}_{c} \gg 16 \mathrm{~K}$. For comparison the approximation of equation (8.5.27) is included in figure 8.13 .

In order to demonstrate the significance of the various asymptotes the curve in figure $8.12 \mathrm{c}$ corresponding to $\overline{\mathrm{r}}_{c}=10^{2}$ is replotted separately in figure $8.12 \mathrm{~d}$. The features of the plot are comparable to those of figure $8.9 \mathrm{~d}$, showing the asymptotic behaviour of the two-particle distribution $\bar{\rho}\left(\Delta \bar{v}_{f}\right)$. For small $k$-values the curve follows the asymptotic behaviour given by equation $(8.5 .26)$, which is associated with the Gaussian regime. For intermediate kvalues the curve levels of $f$ to the asymptote predicted by equation (8.3.16), which corresponds to weak complete collisions. The large $k$-behaviour is determined by weak uncomplete collisions. The curve follows successively the asymptotes described by equations (8.5.24) and (8.5.22), which are associated with the Holtsmark regime and the pencil beam regime respectively.

The scaled displacement distribution $\bar{\rho}\left(\Delta \bar{v}_{1}\right)$ is defined by equation (8.3.17). We evaluated $\bar{\rho}\left(\Delta \bar{v}_{1}\right)$ numerically from the data obtained for $\bar{p}(K)$. The results for $S_{c}=0.5$, with $K=1,100$ and 10,000 are plotted in the figures $8.14 \mathrm{a}, 8.14 \mathrm{~b}$ and $8.14 \mathrm{c}$ respectively. The results for $S_{c}=0.75$, with $K=100$ and 10,000 are plotted in the figures $8.15 \mathrm{~b}$ and 8.15c. Figure 8.14a applies also to other $S_{c}$-values than $S_{c}=0.5$, since the results become for small $k$-values independent of $S_{c}$, as was mentioned previously. The plots should be compared with figure 8.3 , which was derived on the basis of complete collisions. The following regimes can be distinguished, as is explicitly shown for the case $K=10^{4}$ and $S_{c}=0.75$ in figure $8.15 d$ :

I. Gaussian regime. For large values of $\lambda$ the distribution of $\bar{\rho}\left(\Delta \bar{v}_{f}\right)$ is determined by the small $\bar{k}$-behaviour of $\bar{p}(\bar{k})$, which is specified by equation $(8.5 .26)$. The quadratic K-dependency implies that the distribution $\bar{\rho}\left(\Delta \bar{v}_{\perp}\right)$ is Gaussian. The FWHM of this distribution is given by equation $\left(8.3^{\perp} .19\right)$, where the function $P_{c_{A}}$ is now generalized to a function which depends on $\bar{r}_{c}, K$ and $S_{c}$

$$
P_{C a}\left(\bar{r}_{c}, K, S_{c}\right)=\frac{\bar{p}_{2}\left(\bar{r}_{c}, S_{c} K\right)^{1 / 2}}{2 \pi^{1 / 2}}+\frac{\bar{p}_{2}\left[\bar{r}_{c},\left(1-S_{c}\right) K\right]^{1 / 2}}{2 \pi^{1 / 2}}
$$

where $\overline{\mathbf{p}}_{2}\left(\overline{\mathrm{r}}_{\mathrm{c}}, \mathrm{K}\right)$ can be approximated by equation (8.5.27). Equation (8.5.28) is based on the assumption that, in the Gaussian regime, the contributions of the first and the second part of the beam segment 


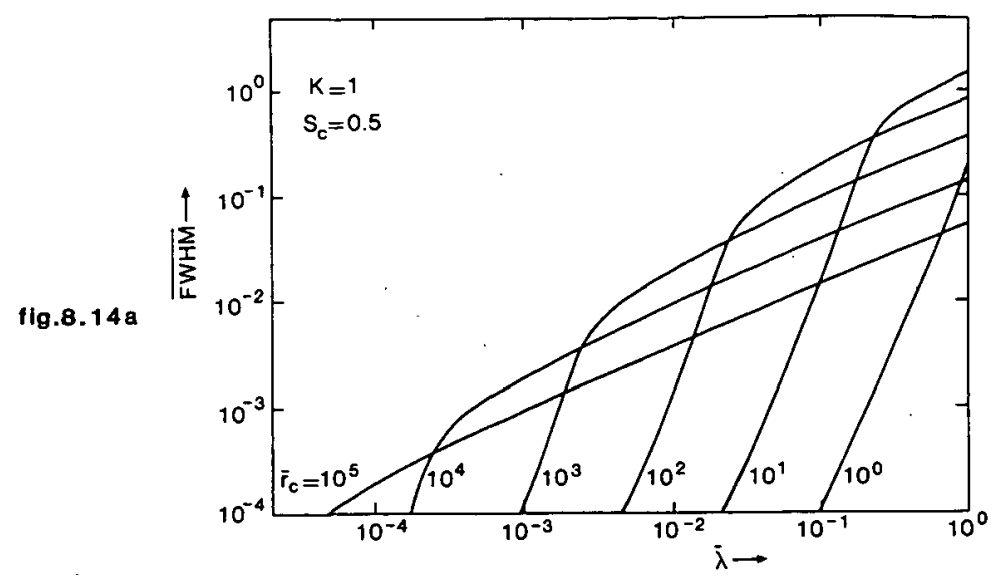

fig.8.14b
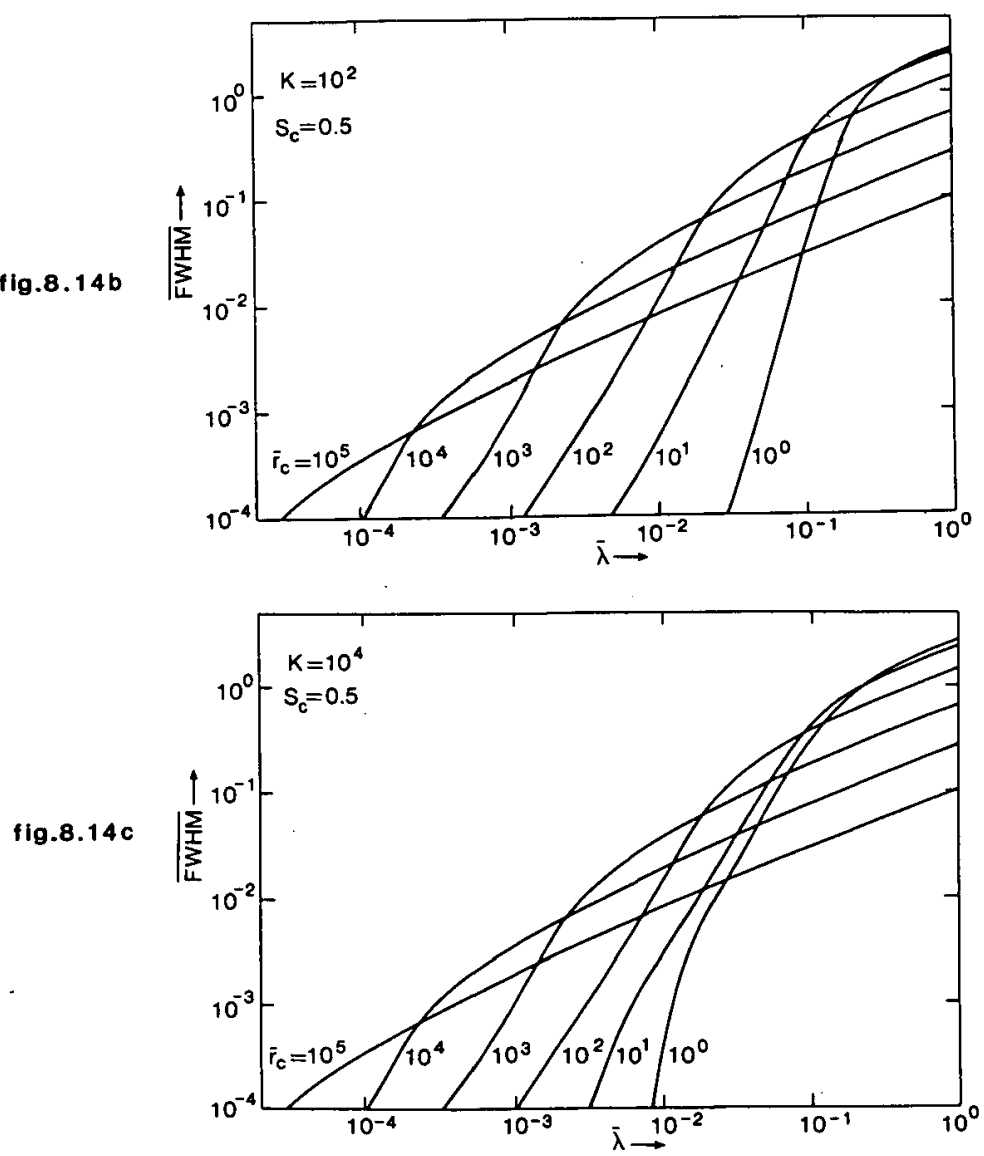

Pigure 8.14 The FWHM of the scaled distribution of transverse velocity displacements $\bar{\rho}\left(\Delta \bar{v}_{1}\right)$ generated in a beam segment with a crossover in the middle $\left(\mathrm{S}_{\mathrm{c}}=1 / 2\right)$, plotted for different values of the scaled crossover radius $\overline{\mathbf{r}}_{c}$. The figures $8.14 a, 8.14 b$ and $8.14 \mathrm{c}$ pertain to different values of the beam geometry parameter $\mathrm{K}=\alpha_{0} \mathrm{~L} / 2 \mathrm{r}_{\mathrm{c}}$, as is indicated. The figures should be compared to figure 8.3 , derived on the basis of complete collisions. 
should be added linearly. The form of equation (8.5.28) is similar to that of equation $(7.5 .37)$. It should be noted that the dependency of the function $P_{C A}\left(\bar{r}_{c}, K, S_{c}\right)$ on $S_{c}$ is in general very weak. In terms of the experimental parameters the result for the Gaussian regime is expressed by equation $(8.3 .21)$.

II. Weak complete collision regime. With decreasing $\bar{\lambda}$ and large $\mathrm{K}$-values the distribution $\bar{p}\left(\Delta \bar{v}_{1}\right)$ becomes dominated by the part of $\bar{p}(\bar{k})$ which is proportional to $\mathrm{K}^{1}{ }^{\frac{1}{2}}$, as described by equation $(8.3 .16)$. The corresponding FWHM is given by equation (3.3.23). In terms of experimental parameters it leads to equation (8.3.24).

III) Holtsmark regime. For an extended beam segment with a small or intermediate $\lambda$-value the distribution $\bar{\rho}\left(\Delta \bar{v}_{1}\right)$ is dominated by the $3 / 2$ power dependency of $\bar{p}(\bar{k})$, as expressed by equation $(8.5 .24)$. A beam segment is called extended when $\chi_{c} \gg>1$. The quantity $\chi_{c}$ is the pencil beam factor for a beam segment with a crossover and is defined by equation (7.5.28). Substitution of equation $(8.5 .24)$ into equation $(8.3 .17)$ yields

$$
\vec{\rho}\left(\Delta \bar{v}_{\perp}\right)=\frac{1}{2 \pi} \int_{0}^{\infty} k d K J_{0}\left(K \Delta \bar{v}_{\perp}\right) e^{-(4 / 5)\left(3 \pi / \bar{r}_{c} K\right) 1 / 2 \pi\left[S_{H A}\left(S_{c}, K\right) K\right]^{3 / 2}}
$$

which is the two-dimensional variant of the Holtsmark distribution, plotted in figure 5.4 (curve corresponding to $\gamma=3 / 2$ ). The FWHM of this distribution is given by

$$
\overline{F W H M}_{H}=2.6554 \frac{(48 \pi)^{1 / 3}}{52 / 3} \frac{S_{H A}\left(S_{c}, K\right) \pi^{2 / 3}}{\left(\bar{r}_{c} K\right)^{1 / 3}}
$$

as follows with equation (5.9.15) and table 5.1. The FWHM of the corresponding angular deflection distribution is given by (using FWHM $_{a}=\alpha_{0}$ FWHM)

$$
\mathrm{FWHM}_{\alpha}=\mathrm{C}_{\mathrm{CHA}} S_{H_{A}}\left(S_{\mathrm{C}}, \mathrm{K}\right) \frac{\mathrm{I}^{2 / 3}}{\mathrm{~V}^{4 / 3} \alpha_{0} / \mathrm{L}^{1 / 3}}
$$

where the constant $\mathrm{C}_{\mathrm{CHA}}$ is given by

$$
\mathrm{C}_{\mathrm{CHA}}=2.6554 \frac{31 / 3}{2^{5 / 3}(5 \pi)^{2 / 3}} \frac{\mathrm{m}^{1 / 3}}{\epsilon_{0}}=0.19233 \frac{\mathrm{m}^{1 / 3}}{\epsilon_{0}}
$$

In the case of electrons one finds $\mathrm{C}_{\mathrm{CHA}}=2.1057$ in SI-units.

$$
\text { For } K \rightarrow 0 \text { one may approximate } S_{H A}\left(S_{c}, K\right) \approx(4 / 3) K^{4 / 3}
$$
$=\left(2^{2} / 3 / 3\right)\left(\alpha_{0} L / r_{c}\right)^{4 / 3}$, as is stated by equation (8.5.18). Substitution into equation $(8.5 .31)$ leads back to equation $(8.4 .29)$, which was derived for a homocentric cylindrical beam segment. Notice that the constants $\mathrm{C}_{\mathrm{PHA}}$ and $\mathrm{C}_{\mathrm{CHA}}$ are related as

$$
C_{P H A}=\frac{22 / 3}{3} C_{C H A}
$$

as can be verified from their definitions.

IV) Pencil beam regime. In pencil beams $\left(\chi_{c} \ll<1\right)$ the distribution $\bar{\rho}\left(\Delta \bar{v}_{f}\right)$ is dominated by the $\bar{k}$-behaviour given by equation $(8.5 .22)$. Substitution into equation $(8.3 .17)$ yields 

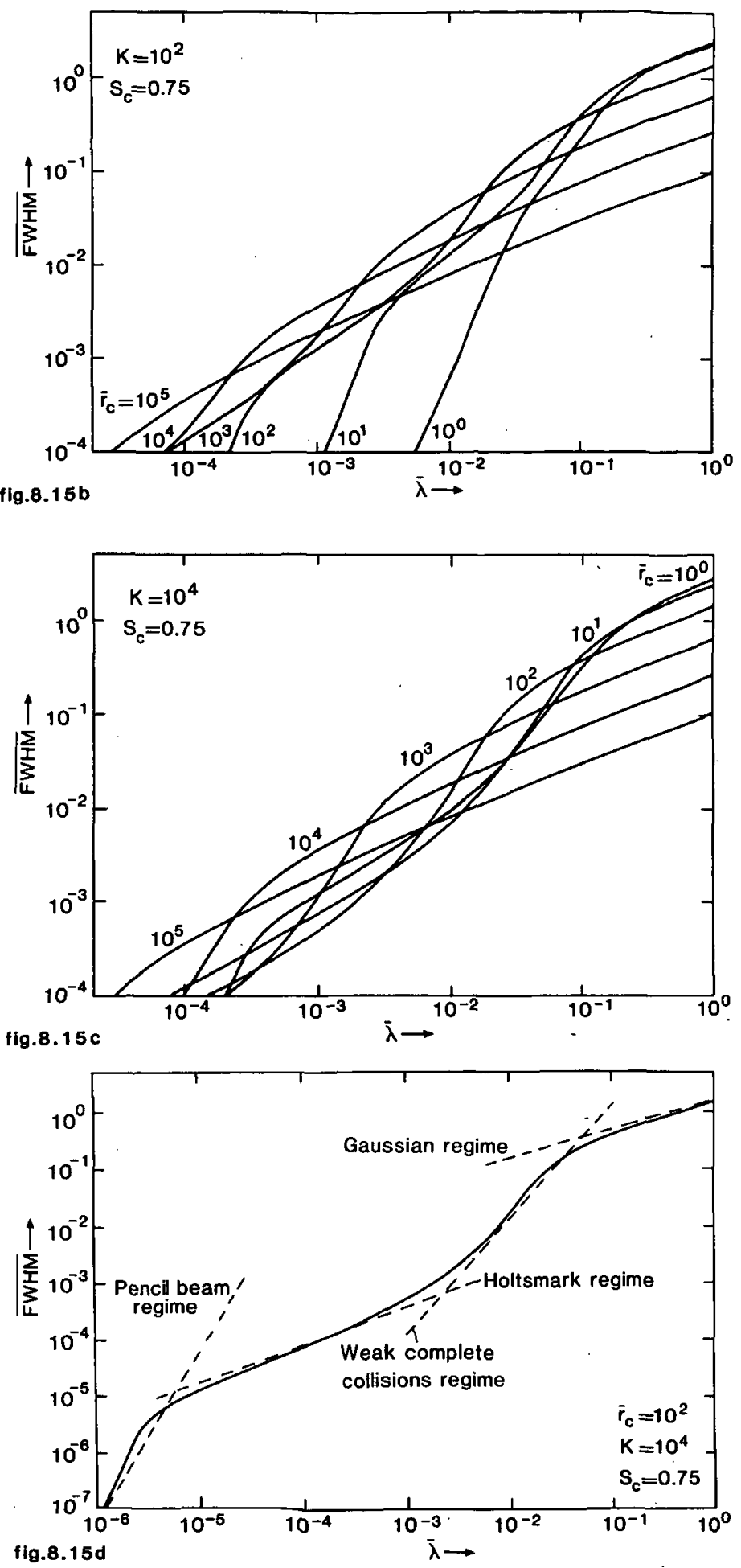


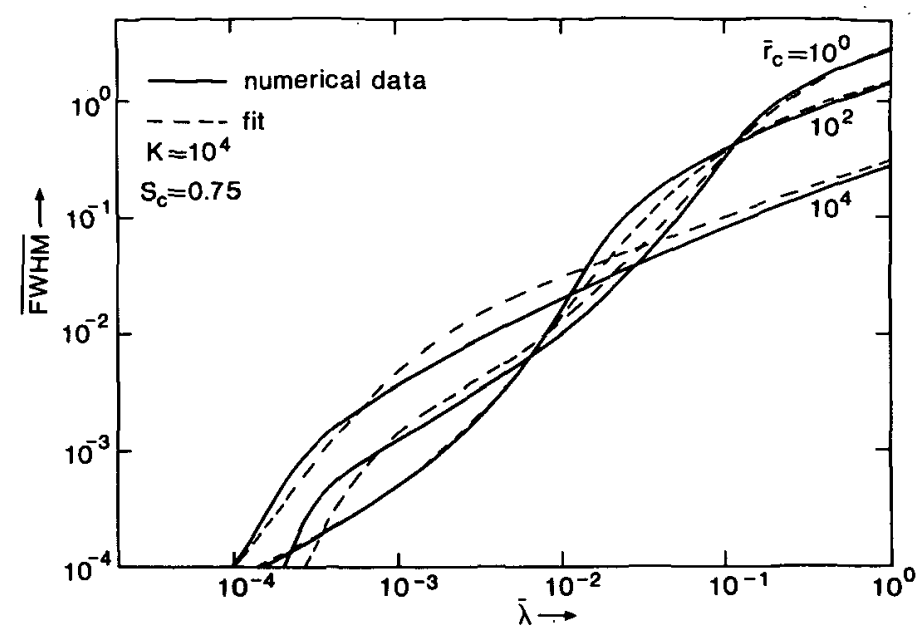

fíg.8.15e

Figure 8.15 The FWHM of the scaled distribution of transverse velocity displacements $\bar{\rho}\left(\Delta \bar{v}_{1}\right)$ generated in a beam segment with a crassover located at $3 / 4$ of the segment length $\left(S_{\mathrm{c}}=3 / 4\right)$, plotted for different values of the scaled crossover radius $\bar{r}_{c}$. The curves should be compared to those of the figures $8.14 b$ and $8.14 c$, which pertain to $S_{c}=1 / 2$. The differences vanish for small $\mathrm{K}$-values. Therefore, there is no figure 8.15 a corresponding to $\mathrm{K}=1$ (which would be identical to figure 8.14a). Figure $8.15 d$ explains the shape of the curves from the asymptotic behaviour corresponding to the different regimes. The different asymptotes are (from left to right) specified by equations $(8.5 .34),(8.5 .30)$, (8.3.23) and (8.3.19). Figure 8.15e is indicative for the quality of the fit given by equations (8.5.37) and $(8,5,38)$.

$$
\bar{\rho}\left(\Delta \overline{\mathbf{v}}_{\perp}\right)=\frac{1}{2 \pi} \int_{0}^{\infty} \mathrm{kdK} J_{0}\left(\mathrm{~K} \Delta \bar{v}_{\perp}\right) e^{-C_{1 / 3}\left(\bar{r}_{c} K\right)^{2 / 3} \lambda\left[S_{P A}\left(S_{c}, K\right) K\right]^{1 / 3}}
$$

where $C_{1 / 3}=72 \Gamma(5 / 6) / 7 \Gamma(1 / 6)=2.0858$. This type of distribution is referred to as the two-dimensional pencil beam distribution, see figure 5.4 (curve corresponding to $\gamma=1 / 3$ ). The FWHM of the distribution of equation $(8.5 .33)$ is given by

$$
\overline{\text { FWHM }}_{P}=.013013\left(\frac{72 \Gamma(5 / 6)}{7 \Gamma(1 / 6)}\right)^{3} S_{P A}\left(S_{c}, K\right)\left(\bar{r}_{c} K\right)^{2} \pi^{3}
$$

as follows with equation (5.9.15) and table 5.1. The numerical constant in the right hand sight of equation (8.5.34) is equal to 0.11809 . By removing the scaling one obtains

$$
\mathrm{FWHM}_{\alpha}=\mathrm{C}_{\mathrm{CPA}} S_{\mathrm{PA}}\left(\mathrm{S}_{\mathrm{C}}, \mathrm{K}\right) \frac{\mathrm{I}^{3} \alpha_{0} \mathrm{~L}^{2}}{\mathrm{~V}^{5 / 2}}
$$


where the constant $\mathrm{C}_{\mathrm{CPA}}$ is given by

$$
C_{C P A}=.013013\left(\frac{18 \Gamma(5 / 6)}{7 \Gamma(1 / 6)}\right)^{3} \frac{1}{\pi \sqrt{2}} \frac{\mathrm{m}^{3 / 2}}{E_{0} \mathrm{e}^{7 / 2}}=4.153110^{-4} \frac{\mathrm{m}^{3 / 2}}{\epsilon_{0} \mathrm{e}^{7 / 2}}
$$

In the case of electrons one finds $C_{C P A}=2.4772 \times 10^{28}$ in SI-units.

For $K \rightarrow 0$ one may approximate $S_{P_{A}}\left(S_{c}, K\right) \approx 1 / K=2 r_{c} / \alpha_{0} L$, as is stated by equation (8.5.19). Substitution into equation ( 8.5 .35 ) leads back to equation (8.4.31), which was derived for a homocentric cylindrical beam segment. Notice that the constants $C_{P P A}$ and $C_{C P A}$ are related as

$$
\mathrm{C}_{\mathrm{PPA}}=2 \mathrm{C}_{\mathrm{CPA}}
$$

as can be verified from their definitions.

In order to interpolate the results obtained for the different regimes we approximate

$$
\overline{F W H M}=\overline{F_{W H M}} G_{C A}\left(\pi, \bar{r}_{c}, K, S_{c}\right), \quad \overline{F_{H H M}}=2(2 \pi \ln 2) 1 / 2 \pi^{1 / 2}
$$

equivalent to equation $(7.5 .30)$. The function $\mathrm{G}_{\mathrm{CA}}$ is taken equal to

$$
G_{C A}\left(\bar{\lambda}, \bar{r}_{C}, K, S_{C}\right)=\left[\left(\frac{\text { FWHM }_{0}}{\text { FWHM }_{G}}+\frac{\text { FWHM }_{0}}{\text { FWHM }_{W}}\right)^{-1}+\left(\frac{\text { FWHM }_{0}}{\text { FWHM }_{H}}+\frac{F^{2} W_{H M}}{F_{W H M}}\right)^{-1}\right]^{-1}
$$

By substitution of equations (8.3.19), (8.3.23), (8.5.30) and (8.5.34) one obtains

$$
\begin{aligned}
\mathrm{G}_{\mathrm{CA}}\left(\bar{\lambda}, \bar{r}_{\mathrm{c}}, \mathrm{K}, \mathrm{S}_{\mathrm{c}}\right)= & {\left[\left(\frac{1}{\mathrm{P}_{\mathrm{CA}}\left(\overline{\mathrm{r}}_{\mathrm{c}}, \mathrm{K}, \mathrm{S}_{\mathrm{c}}\right)}+\frac{\mathrm{A}}{\bar{\lambda}^{3 / 2} \mathrm{f}_{\infty}\left(\bar{r}_{\mathrm{c}}\right)^{2}}\right)^{-1}\right.} \\
& \left.+\left(\frac{\mathrm{B}\left(\bar{r}_{\mathrm{c}} \mathrm{K}\right)^{1 / 3}}{\bar{\lambda}^{1 / 6} \mathrm{~S}_{\mathrm{HA}}\left(\mathrm{S}_{\mathrm{c}}, \mathrm{K}\right)}+\frac{\mathrm{C}}{\bar{\lambda}^{5 / 2}\left(\overline{\mathrm{r}}_{\mathrm{c}} \mathrm{K}\right)^{2} \mathrm{~S}_{\mathrm{PA}}\left(\mathrm{S}_{\mathrm{c}}, \mathrm{K}\right)}\right)^{-1}\right]^{-1}
\end{aligned}
$$

in which the constants $A, B$ and $C$ are given by

$$
A=7.00910^{-2}, \quad B=.6106, C=24.99
$$

The function $P_{C A}\left(\bar{r}_{c}, K, S_{C}\right)$ is defined by equations (8.5.28) and (8.5.27). The function $f_{\infty}\left(\bar{r}_{c}\right)$ is given by equation (8.3.8) and can be approximated by equation $(8,3.10)$. The functions $S_{H A}\left(S_{c}, K\right)$ and $S_{P A}\left(S_{c}, K\right)$ can be approximated by the functions $S_{H_{A a}}\left(S_{c}, K\right)$ and $S_{P A a_{a}}\left(S_{c}, K\right)$, given by equations $(8.5 .20)$ and $(8.5 .21)$ respectively. Figure $8.15 \mathrm{e}$ compares the approximation of equations $(8.5 .37)$ and $(8.5 .38)$ with the numerical data obtained for $S_{c}=0.75$ and $K=10^{4}$, with $\bar{r}_{c}=1,10^{2}$ and $10^{4}$. One sees that the fit is rather poor in the transition areas between the different regimes. rewritten as

In terms of the experimental parameters equation $(8.5 .37)$ can be

$$
\mathrm{FWHM}_{\alpha}=\mathrm{C}_{\mathrm{CGA}} \mathrm{G}_{\mathrm{CA}}\left(\bar{\lambda}, \overline{\mathrm{r}}_{\mathrm{c}}, \mathrm{K}, \mathrm{S}_{\mathrm{c}}\right) \sqrt{\frac{\mathrm{I}}{\mathrm{V}^{3 / 2}}}
$$

similar to equation (8.3.21), which also specifies the constant $C_{C G A}$. Equations $(8.5 .40)$ and $(8.5 .38)$ provide the most general description of the FWHM of the angular deflection distribution. It applies to a beam segment of arbitrary geometry and provides accurate results as long as the theoretical parameters fulfil the conditions expressed by equation $(7.5,33)$. 
Similar results can be derived for the Full width median ( FW $_{50}$ ) of the angular deflection distribution. For a two-dimensional Gaussian distribution the FWHM and FW50 are identical. Accordingly, one may write

$$
\overline{F W}_{50}=\overline{F W H M}_{0} G_{C A}\left(\pi, \bar{r}_{c}, K, S_{c}\right)
$$

similar to equation (8.5.37). The function $\mathrm{G}_{\mathrm{CA}^{A}}\left(\pi, \vec{r}_{\mathrm{c}}, \mathrm{K}, \mathrm{S}_{\mathrm{c}}\right)$ is again given by equation (8.5.38), now taking the constants $\mathrm{A}, \mathrm{B}$ and $\mathrm{C}$ equal to

$$
\mathrm{A}=2.65710^{-3}, \mathrm{~B}=.4883, \mathrm{C}=4.77610^{-2}
$$

instead of the values given by equation (8.5.39). The results for the individual regimes can be obtained from the expressions for the FWHMvalues using

$$
\begin{aligned}
& \mathrm{FW}_{5 \text { OG }}=1.0000 \mathrm{FWHM}_{\mathrm{G}} \\
& \mathrm{FW}_{5 \mathrm{OW}}=26.381 \mathrm{FWHM}_{\mathrm{W}} \\
& \mathrm{FW}_{5 \text { O }}=1.2505 \mathrm{FWHM}_{\mathrm{H}} \\
& \mathrm{FW}_{5 \text { O }}=523.29 \mathrm{FWHM}_{\mathrm{P}}
\end{aligned}
$$

as follows from the data of table 5.1.

\subsection{Application of the slice-method}

In this section we will verify the approximations of the functions $S_{H A}\left(S_{C}, K\right)$ and $S_{P A}\left(S_{C}, K\right)$, given by equations $(8.5 .20)$ and (8.5.21). We will show that these results can be established by means of the slice-method, which is described in section 5.11. The slice-method is used to relate the results obtained for a homocentric cylindrical beam segment to those obtained for a beam segment with a crossover. The essence of the procedure is expressed by equation (5.11.1). This equation states that one may calculate the total displacement generated in a beam segment by adding linearly the displacements generated in a large number of thin cylindrical slices. The basic assumption is that the contributions of the individual slices to the total displacement experienced by the test particle are entirely correlated. Such is to be expected when the deviations from the unperturbed trajectories are small. Accordingly, the slice-method is only applicable when the distribution $\rho\left(\Delta v_{1}\right)$ is dominated by weak collisions. This type of analysis is therefore restricted to the Holtsmark regime and the pencil beam regime.

Let us first consider the Holtsmark regime. According to equation (8.4.29) the contribution of a single slice at axial position 2 of length $\Delta z$ to the total distribution of angular deflections $\rho(\Delta \alpha)$ can be expressed as

$$
\Delta F W H M_{\alpha}=C_{P H A} \frac{I^{2 / 3}}{V^{4 / 3} r_{c}{ }^{4 / 3}} \frac{\Delta z}{\left[1+\alpha_{0}\left|z-z_{c}\right| / r_{c}\right]^{4 / 3}}
$$

in which $z_{c}$ the $z$-coordinate of the crossover. For the calculation of the distribution $\rho(\Delta \alpha)$ in a beam segment with a narrow crossover one should use a modified version of equation (5.11.1) 


$$
F W H M_{\alpha}=\left|\int_{z_{0}}^{z_{1}} d z \operatorname{sign}\left(z-z_{c}\right) \frac{\Delta F W H M_{\alpha}}{\Delta z}\right|
$$

The sign-function is included to account for the fact that the angular deflection experienced by the test particle during a weak collision with a single field particle changes of sign when the field particle crosses the beam axis (or in fact the reference trajectory, which is here identical to the beam axis). Equation (8.6.2) presupposes that the field particle crosses the axis at the location of the crossover. This approximation is only justified when the crossover is narrow $(K>>1)$. Equation (8.6.2) is therefore not suited to describe a nearly cylindrical beam segment. However, the extreme case of a cylindrical segment $(K=0)$ is covered by equation (8.6.2) if one takes $z_{c}=z_{0}$ or $z_{c}=z_{1}$. By this choice one accounts for the fact that in a homocentric cylindrical beam segment the particle trajectories do not cross each other.

Substitution of equation (8.6.1) into equation (8.6.2) and integration yields equation $(8.5 .31)$, where the function $S_{H A}\left(S_{c}, K\right)$ is now given by

$$
S_{H A}\left(S_{c}, K\right)=\frac{21 / 33 C_{P H A}}{C_{C H A}}\left|\frac{1}{\left(1 / K+2 S_{c}\right)^{1 / 3}}-\frac{1}{\left[1 / K+2\left(1-S_{c}\right)\right]^{1 / 3}}\right|
$$

Substitution of equation (8.5.32) yields the approximating function $\mathrm{S}_{\mathrm{HAa}}\left(\mathrm{S}_{\mathrm{C}}, \mathrm{K}\right)$ introduced by equation (8.5.20). From this derivation it becomes clear that equation $(8.5 .20)$ is only valid for $K \gg>1$, while correct results for $K \rightarrow 0$ are obtained if one takes $S_{c}=0$ or $S_{c}=1$.

Let us now consider the pencil beam regime. According to equation (8.4.31) the contribution of a single slice at axial position $z$ of length $\Delta z$ to the total distribution of angular deflections $\rho(\Delta \alpha)$ can be expressed as

$$
\Delta \mathrm{FWHM}_{\alpha}=\mathrm{C}_{\mathrm{PPA}} \frac{\mathrm{I}^{3} \mathrm{r}_{\mathrm{C}}}{\mathrm{V}^{5 / 2}}\left[1+\alpha_{0} \mid z-z_{c} / / \mathrm{r}_{c}\right] \Delta z
$$

in which $z_{c}$ the $z$-coordinate of the crossover. Substitution of equation (8.6.3) into equation (8.6.2) and integration yields equation (8.5.35), where the function $S_{P A}\left(S_{c}, K\right)$ is now given by

$$
\mathrm{S}_{P A}\left(S_{c}, K\right)=\frac{2 C_{P P A}}{C_{C P A}}\left|1-2 S_{c}\right|\left(\frac{1}{K}+1\right)
$$

Substitution of equation (8.5.36) yields the approximating function $\mathrm{S}_{\mathrm{PA} a}\left(\mathrm{~S}_{\mathrm{c}}, \mathrm{K}\right)$ introduced by equation (8.5.21). As for the Holtsmark regime, it be should noticed that this equation is only valid for $K \gg>1$, while correct results for $K \rightarrow 0$ are obtained by taking $S_{c}=0$ or $S_{c}=1$.

\subsection{Results for Gaussian angular and spatial distributions}

The results of the previous sections apply to the case of an uniform spatial and an uniform angular distribution in the crossover, see equation $(8.2 .6)$. We will now investigate the required modifications in case either the angular or the spatial distribution or both distributions are Gaussian. The analysis is similar to that of section 7.6 , pertaining to the Boersch effect. 
We repeated the program outlined in section 8.3 , now using the modified versions of equation $(8.2 .6)$ in which either the spatial or the angular distribution or both distributions are taken Gaussian, following the specifications of equation (7.6.1) and/or equation $(7,6.2)$. The principle aspects of the calculation remain the same and the discussion here is restricted to a presentation of the main results. We evaluated equations (8.2.6) and $(8.2 .7)$ on the basis of complete collisions, using equation (8.2.1). Analogous to equation (8.3.13) we found the following expressions for the scaled function $\bar{p}(\bar{k})$ :

- For a Gaussian angular distribution and a Gaussian spatial distribution

$$
\bar{p}_{b B}(k)=k \frac{16}{\sqrt{\pi}} \int_{0}^{\infty} d \bar{v} e^{-\bar{v}^{2}} \int_{0}^{\infty} d y e^{-y^{2}} \int_{0}^{1} d u\left(\frac{1}{u}-1\right)^{1 / 2} J_{1}\left(\frac{u \bar{k} \bar{v}}{R(\bar{v}, y)}\right)
$$

- For a Gaussian angular distribution and an uniform spatial distribution

$$
\bar{p}_{b u}(\bar{k})=k \frac{32}{\pi} \int_{0}^{\infty} d \bar{v} e^{-\bar{v}^{2}} \int_{0}^{1} d y \sqrt{1-y^{2}} \int_{0}^{1} d u\left(\frac{1}{u}-1\right)^{1 / 2} J_{1}\left(\frac{u \bar{k} \bar{v}}{R(\bar{v}, y)}\right)
$$

- For an uniform angular distribution and a Gaussian spatial distribution

$$
\bar{p}_{u s}(\bar{k})=k \frac{16}{\sqrt{\pi}} \int_{0}^{1} d \bar{v} \int_{0}^{\infty} d y e^{-y^{2}} \int_{0}^{1} d u\left(\frac{1}{u}-1\right)^{1 / 2} J_{1}\left(\frac{u k \bar{v}}{R(\bar{v}, y)}\right)
$$

Equations (8.7.1) through (8.7.3) are similar to equations (7.6.3) through $(7.6 .5)$. As in section 8.3 we will investigate the behaviour for $\mathrm{k} \rightarrow 0$ and for $\mathrm{k} \rightarrow \infty$.

For $k \rightarrow 0$ equations $(8.7 .1),(8.7 .2)$ and $(8.7 .3)$ show a quadratic dependency on $k$, as described by the first equation of (8.3.14). The functions $\overline{\mathbf{p}}_{2 \mathrm{~g}}\left(\overline{\mathrm{r}}_{\mathrm{c}}\right), \overline{\mathrm{p}}_{\mathrm{z} \mathrm{u}}\left(\overline{\mathrm{r}}_{\mathrm{c}}\right)$ and $\overline{\mathrm{p}}_{2 \mathrm{ug}_{\mathrm{g}}}\left(\overline{\mathrm{r}}_{\mathrm{c}}\right)$, which should be used in equation (8.3.14) for the different cases, are the same as obtained for the axial velocity distribution $\rho\left(\Delta \mathrm{v}_{z}\right)$, see equations $(7.6 .6),(7.6 .7)$ and $(7.6 .8)$ respectively. A similar result was found in section 8.3 for the case of an uniform angular and spatial distribution, see the second equation of (8.3.14). All functions can be approximated by equation (7.6.11), using different constants $E$ and $F$ for the individual cases, as prescribed by equation (7.6.12).

For $k \rightarrow \infty$ equations $(8.7 .1),(8.7 .2)$ and $(8.7 .3)$ show a square root dependency on $\bar{k}$, as described by equations (8.3.16). The function $f_{\infty}\left(\bar{r}_{c}\right)$ varies for the different angular and spatial distributions. Analogous to equation $(8.3 .8)$ one finds

$$
\begin{aligned}
& f_{\operatorname{cob} 8}\left(\bar{r}_{c}\right)=\frac{1}{\sqrt{\pi}} \int_{0}^{1} d \bar{v} \frac{\exp \left(-\bar{v}^{2}\right)}{\bar{v}^{1 / 2}} \int_{0}^{1} d y \exp \left(-y^{2}\right) R(\bar{v}, y)^{1 / 2} \\
& f_{\cos u}\left(\bar{r}_{c}\right)=\frac{2}{\pi} \int_{0}^{1} d \bar{v} \frac{\exp \left(-\bar{v}^{2}\right)}{\bar{v}^{1 / 2}} \int_{0}^{1} d y\left(1-y^{2}\right)^{1 / 2} R(\bar{v}, y)^{1 / 2}
\end{aligned}
$$




$$
\mathrm{f}_{\text {oou } \mathrm{b}}\left(\overline{\mathrm{r}}_{\mathrm{c}}\right)=\frac{1}{\sqrt{\pi}} \int_{0}^{1} \frac{\mathrm{d} \overline{\mathrm{v}}}{\overline{\mathrm{v}}^{1 / 2}} \int_{0}^{1} \mathrm{dy} \exp \left(-\mathrm{y}^{2}\right) R(\overline{\mathrm{v}}, \mathrm{y}) 1 / 2
$$

where the function $R(\bar{v}, y)$ is given by equation (8.3.4). These functions have the following properties

$$
\begin{aligned}
& \lim _{\bar{r}_{c} \rightarrow \infty} f_{\infty B s}\left(\bar{r}_{c}\right)=\frac{[\Gamma(3 / 4)]^{2}}{4(2 \pi)^{1 / 2}} \bar{r}_{c} 1 / 2=.14976 \bar{r}_{c}{ }^{1 / 2} \\
& \lim _{r_{c} \rightarrow \infty} f_{\infty o 8} u\left(\bar{r}_{c}\right)=\frac{2}{5} \sqrt{\frac{2}{\pi}} \frac{[\Gamma(3 / 4)]^{2}}{\Gamma(1 / 4)} \bar{r}_{c} 1 / 2=.13219 \bar{r}_{c} 1 / 2 \\
& \lim _{\bar{r}_{c \rightarrow \infty}} f_{\infty O B}\left(\bar{r}_{c}\right)=\frac{\Gamma(3 / 4)}{3(2 \pi)^{1 / 2}} \bar{r}_{c} 1 / 2=.16296 \bar{r}_{c} 1 / 2
\end{aligned}
$$

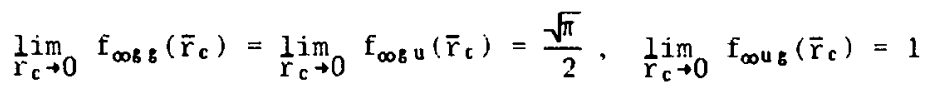

Similar to equation (8.3.10) one can approximate the functions $f_{\infty b s}\left(\bar{r}_{c}\right)$, $\mathrm{f}_{\text {cob } u}\left(\bar{r}_{\mathrm{c}}\right)$ and $\mathrm{f}_{\text {cou } \mathrm{g}}\left(\overline{\mathrm{r}}_{\mathrm{c}}\right)$ using

$$
\hat{f}_{\infty \mathrm{a}}\left(\bar{r}_{\mathrm{c}}\right)=\left(P+Q \bar{r}_{c}\right) 1 / 2
$$

in which $P$ and $Q$ are numerical constants which differ for the individual cases

$$
\begin{aligned}
& P_{B g}=P_{B}=\pi / 4 \quad, \quad P_{u g}\left(=P_{u u}\right)=1 \\
& Q_{B}=.02243, \quad Q_{B}=.01747 \\
& Q_{u_{\text {g }}}=.02655, \quad\left(Q_{u v}=.02069\right)
\end{aligned}
$$

The approximations for the functions $\bar{p}_{2}\left(\bar{r}_{c}\right)$ and $f_{\infty}\left(\bar{r}_{c}\right)$, given by equations $(7.6 .11)$ and (8.7.9), respectively contain the essential results of the analysis based on complete collisions for different types of spatial and angular distributions. The functions $\bar{p}_{2}\left(\bar{r}_{c}\right)$ and $f_{\infty}\left(\bar{r}_{c}\right)$ specify the $\bar{r}_{c}$-dependency of the FWHM of the angular deflection distribution in the Gaussian regime and the weak complete collision regime respectively as can be seen from equation (8.3.21) with equation $(8.3 .20)$ and equation (8.3.24).

It is often convenient to express the results obtained for nonuniform distributions in terms of the effective width measures $\alpha_{e f f}$ and $r_{\text {er }}$, which are defined as the widths for which the expressions obtained for uniform distributions yield the same results as those obtained within the full calculation taking the proper distribution(s) into account. The resulting expressions for the angular deflections in the Gaussian regime show the same functional dependency as was found for the Boersch effect in this regime. Accordingly, the effective widths $\alpha_{e f}$ and $r_{e f f}$ are the same as specified by equation (7.6.15). For the weak complete collision regime one should define $\alpha_{e f f}$ and $r_{e f r}$ such that the FWHM $\alpha$-values follow equations (8.3.24) and (8.7.9). For $\bar{r}_{c}=0$ the result depends only on the type of angular distribution and one finds

$$
\alpha_{\text {eff }}=\left(\frac{4}{\pi}\right)^{1 / 3} \alpha_{c}=\frac{2^{1 / 6}}{\pi^{1 / 3}} \sigma_{\alpha}=1.533 \sigma_{\alpha}\left(\bar{r}_{c}=0\right)
$$

(Weak complete coll. regime)

The effective width $r_{\text {eff }}$ follows by considering the case $\bar{r}_{c}+\infty$. The 
reader might verify that resulting values for $r_{e f}$ varies slightly for the different cases covered by equation (8.7.10). In general a reasonable approximation is obtained for all $\bar{r}_{c}$-values by taking

$$
\alpha_{\text {eff }} \cong 1.6 \sigma_{\alpha}, \quad r_{\text {eff }} \equiv 1.8 \sigma_{\mathrm{r}}
$$

(Weak complete coll. regime)

The exact values for $\alpha_{e f f}$ and $r_{e f f}$ obtained for the individual cases differ less than $5 \%$ from the values given by equation (8.7.12).

The remaining results to be investigated pertain to the Holtsmark regime and the pencil beam regime. For those regimes the distribution $\rho\left(\Delta \mathrm{v}_{\perp}\right)$ can be determined from the distribution of the lateral interaction force $\rho\left(\mathbf{F}_{\perp}\right)$ in a cylindrical beam. Accordingly, it is sufficient to determine in what way $\rho\left(F_{\perp}\right)$ is affected by the type of density distribution. The calculation of $\rho\left(F_{1}\right)$ for a cylindrical beam with an uniform density distribution was carried out in section 5.8. The results are expressed by equations $(5.8 .16)$ and $(5.8 .20)$. Let us reconsider this calculation, now starting from a Gaussian radial density distribution. Using equation (7.6.2) one may modify equation (5.8.9) as

$$
\rho_{2}\left(F_{1}\right)=\int_{0}^{\infty} 2 d z \int_{0}^{\infty} \frac{2 r d r}{r_{0}^{2}} e^{-\left(r / r_{0}\right)^{2}} \delta\left(F_{L}-\frac{C_{0}}{r^{2}} \frac{1}{\left(1+(z / r)^{2}\right)^{3 / 2}}\right)
$$

Analogous to equation $(5.8 .12)$ this transforms to

$$
\rho_{2}\left(F_{\perp}\right)=\frac{2 C_{0}^{3 / 2}}{r_{0}^{2} F_{\perp}^{5 / 2}} \int_{0}^{\infty} \frac{d s}{\left(1+s^{2}\right)^{9 / 4}} \exp \left(-\frac{C_{0}}{F_{\perp} r_{0}^{2}} \frac{1}{\left(1+s^{2}\right)^{3 / 2}}\right)
$$

As before we will investigate the extreme cases $r_{0} \rightarrow \infty$ and $r_{0} \rightarrow 0$, which correspond to an extreme extended beam and a pencil beam respectively. For $r_{0} \rightarrow \infty$ one may replace the exponential function in equation (8.7.14) by unity and one retrieves equation (5.8.13). This expression leads to the two-dimensional variant of the Holtsmark distribution, given by equation $(5.8 .16)$. Thus one obtains the same equation as for an uniform density distribution. However, $r_{0}$ now stands for $\sqrt{2} \times \sigma_{r}$, instead of the outer beam radius. The FWHM of the corresponding angular deflection distribution is for a homocentric cylindrical beam given by equation (8.4.29) and for a beam segment with a crossover by equation (8.5.31). In general, the same equations as for an uniform distribution are obtained if one takes

$$
\alpha_{\text {eff }}=\alpha_{0}=\sqrt{2} \sigma_{\alpha}, \quad r_{\text {eff }}=r_{c}=\sqrt{2} \sigma_{r} \quad \text { (Holtsmark regime) (8.7.15) }
$$

Notice that the same effective width measures were found for the Boersch effect, as expressed by equation $(7.6 .18)$.

For $r_{0} \rightarrow 0$ one may approximate the integral of $(8.7 .14)$ by taking $1+s^{2} \cong s^{2}$. This is justified since the contribution to the integral comes from large s-values. This gives

$$
\rho_{2}\left(F_{\perp}\right)=\frac{2 C_{0}^{3 / 2}}{r_{0}^{2} F_{/ /}^{5 / 2}} \int_{0}^{\infty} \frac{d s}{s^{9 / 2}} \exp \left(-\frac{C_{0}}{F_{\perp} r_{0}^{2}} \frac{1}{s^{3}}\right)=\frac{\Gamma(1 / 6)}{9} \frac{C_{0}^{1 / 3} r_{0} 1 / 3}{\vec{F}_{\perp}^{4 / 3}}(8.7 .16)
$$

which should be compared to equation (5.8.18) obtained for an uniform distribution. The FWHM of the corresponding angular deflection distribution is for a homocentric cylindrical beam given by equation 
(8.4.31) and for a beam segment with a crossover by equation (8.5.35). By comparison of equations $(8.7 .16)$ and $(5.8 .18)$ it is found that one may use the equations for an uniform distribution if one takes

$$
\alpha_{\text {erf }}=\left(\frac{7 \Gamma(1 / 6)}{36}\right)^{3} \sqrt{2} \sigma_{\alpha}=1.793 \sigma_{\alpha}, \quad r_{\text {eff }}=1.793 \sigma_{r} \quad \begin{array}{r}
(8.7 .17) \\
\text { (pencil beam regime) }
\end{array}
$$

which completes the analysis.

Equations $(7.6 .15),(8.7 .12),(8.7 .15)$ and $(8.7 .17)$ specify for the different regimes the values of the effective beam semi-angle $\alpha_{e r} f$ of a Gaussian angular distribution and effective crossover radius $r_{e} f$ of a Gaussian spatial distribution. The values of $\alpha_{e f f}$ and $r_{e f f}$ differ for the different regimes, varying between $\sqrt{2} \times \sigma$ and $1.8 \times \sigma$. 


\section{TRA.JECTORY DISPLACEMRNT EFFECT}

\subsection{Introduction}

In this chapter the extended two-particle approach will be used to calculate the trajectory displacement effect generated in a rotational symmetric beam segment in drift space. The objective is to determine the distribution of virtual displacements $\rho(\Delta r)$ observed in a certain reference plane. The virtual displacement $\Delta r$ of a test particle is determined by its spatial displacement at the end of the beam segment $\Delta r_{f}$, its velocity displacement $\Delta v_{\perp}$ and the location of the reference plane. The distribution of the transverse velocity displacements $\rho\left(\Delta v_{\perp}\right)$ was computed in chapter 8 . The results of that chapter are exploited to explain the mechanism of the trajectory displacement effect and to check the results in some limiting cases.

The chapter starts with a summary of the relevant results of the chapters 5 and 6 , covering the basics of the statistical part of the model and the calculation of the displacement $\Delta r$ caused by a twoparticle collision respectively. This material is then used to perform the calculation of $\rho(\Delta r)$ for different beam geometries. The specific cases of a homocentric beam segment with a crossover $\left(r_{\mathbf{c}}=0\right)$ and $a$ homocentric cylindrical beam segment $\left(\alpha_{0}=0\right)$ are studied successively. The slice-method, described in section 5.11, is exploited to the treat the general case of a beam segment with a crossover of arbitrary dimensions. For all geometries the full trajectory displacement distribution $p(\Delta r)$ is computed. Explicit expressions are presented for the Full Width at Half Maximum (FWHM) of this distribution, as well as the Full Width median (FW50) and the root mean square value (rms). The angular and spatial distribution in the crossover are assumed to be uniform in most calculations. Gaussian distributions are covered by introducing effective width measures for which the results obtained for uniform distributions can be used. Scaling is applied to reduce the number of independent parameters and to simplify the notation of the intermediate results. In the final results the scaling is removed in order to make the dependency on the experimental parameters explicit.

A computer program has been developed to perform the various steps of the calculation numerically. As for the angular deflection distribution one should in general distinguish four regimes, each corresponding to a different type of distribution: The Gaussian regime, the weak complete collision regime, the Holtsmark regime and the pencil beam regime. The weak complete collision regime becomes only manifest for a beam segment with a narrow crossover in which the reference plane does not coincide with the crossover plane. The widths of the various distributions show different dependencies on the experimental parameters.

The calculation of the trajectory displacement distribution $\rho(\Delta r)$ is in general more complex than the calculation of the distribution of the axial and transverse velocity displacements, $\rho\left(\Delta v_{z}\right)$ and $\rho\left(\Delta v_{\perp}\right)$, considered in the chapters 7 and 8 respectively. One reason is that an additional independent parameter is involved, namely the location of the reference plane. For a beam segment of relatively low particle density, with a narrow crossover in the middle, the dependency on this parameter is rather weak, since the angular deflections generated in such a beam are negligible. However, in all other cases the dependency on the location of the reference plane can not be disregarded. Another complication is that it is not permitted to use complete collision dynamics to evaluate the displacement $\Delta r$ caused by a two-particle encounter. One may use nearly complete collision dynamics, 
but the complexity of the resulting equation for the displacement $\Delta r$ prohibits an exact analytical treatment of the statistical part of the problem. For the Gaussian regime this implies that one has to rely entirely on numerical calculations, which makes a complete analysis rather involved. For all other regimes it is possible to verify the numerical results by analytical means. A direct calculation of the behaviour of the distribution in the transition areas between the regimes seems not possible. The numerical data for the FWHM and the $\mathrm{FW}_{5} 0$ of the trajectory displacement distribution is approximated by analytical expressions, which cover the entire range of operating conditions.

\subsection{General aspects}

The trajectory displacement effect corresponds to the generation of lateral displacements $\Delta r$ (observed in a certain reference plane) due to the effect of statistical coulomb interactions. These displacements are related to the stochastic fluctuations in the charge density, which occur due to the particle nature of the beam. We recall that the trajectory displacement effect should be distinguished from the space charge effect, which is related to the average (smoothed-out) charge density in the beam. Both phenomena are caused by the transverse component of the Coulomb interaction force, but their impact on the properties of the beam differs essentially. To isolate the trajectory displacement effect we consider the central reference trajectory in a rotational symmetric beam. The test particles running along this trajectory experience no systematic space charge force and their deviations are purely stochastic. A discussion of the impact of the space charge effect on the properties of the beam is presented in chapter 11 .

Our objective is to determine the distribution of virtual displacements $\rho(\Delta r)$ observed in a certain reference plane. The virtual displacement $\Delta r$ of the test-particle is found by extrapolating its final perturbed position along its final perturbed velocity towards the reference plane. This procedure combines the lateral shift at the end of the beam segment $\Delta r_{f}$ and the change in lateral velocity $\Delta v_{\perp}$ into the virtual shift $\Delta r$.

The location of the reference plane is specified by the parameter $S_{i}$, which is defined by equation (3.2.1). The reference plane is imaged down to the target. The virtual broadening in the reference plane is therefore representative for the broadening of the final probe. The reference plane will often coincide with the location of the crossover, which is specified by the parameter $S_{c}$. This parameter is also defined by equation (3.2.1). It should be emphasized that the reference plane and the crossover plane do not coincide in all cases of interest. For example, consider a shaped-beam lithography system employing Koehler-illumination, as described by Pfeiffer (1979) or Moore et al. (1981). The shaping-apertures, located in the upper part of the column, are imaged down to the target. The appropriate reference plane is therefore optically conjugated to these planes. However, the crossover between the shaping apertures is optically conjugated to the source crossover and is imaged by the second condenser and the demagnification lenses on the principal plane of the projector lens (and not to the target). Thus in the shaping-section of the column the crossover plane and the reference plane do not coincide. This example illustrates that the quantities $S_{c}$ and $S_{i}$ should in general be treated as independent parameters. 
The distribution $\rho(\Delta r)$ is in some extreme cases fully determined by the distribution of transverse velocity displacements $\rho\left(\Delta v_{1}\right)$, which was calculated in chapter 8 . One case is a beam segment with a crossover in which the reference plane is far removed from the crossover. In this situation the main contribution to the (virtual) displacement of a test particle $\Delta r$ stems from its angular deflection $\Delta \alpha=\Delta v_{\perp} / v_{z}$. The displacements $\Delta r$ and $\Delta v_{\perp}$ are therefore related by

$$
\lim _{\mathbf{S} \rightarrow+\infty} \Delta r=\left|S_{i} T \Delta v_{\perp}\right|
$$

in which the time of flight $\mathrm{T}=\mathrm{L} / \mathrm{v}_{\mathbf{z}}$. Another case is a cylindrical beam segment succeeded by a lens which focusses the beam in its focal plane. The transverse spatial shift $\Delta r_{f o c}$ in the focal plane of the lens is entirely determined by the transverse velocity shift $\Delta v_{\perp}$ of the test particle experienced in the cylindrical beam segment

$$
\Delta \mathrm{r}_{\mathrm{foc}}=\mathrm{f} \Delta \mathrm{v}_{\perp} / \mathrm{v}_{\mathbf{z}}
$$

with $f$ the focal length of the lens. Equations (9.2.1) and (9.2.2) will be exploited to relate the distributions $\rho(\Delta r)$ and $\rho\left(\Delta v_{1}\right)$.

As in the chapters 7 and 8 we will utilize the extended twoparticle approach, which was outlined in chapter 5 . The dynamical part of the problem consists of the calculation of the virtual radial displacement $\Delta r$ experienced by the test particle due to the interaction with a single field particle. This problem was studied in chapter 6 . The shift $\Delta r$ can be expressed as a function of the geometrical variables $\xi=\left(r_{\perp}, b_{z}, v, \Phi\right)$, the time of flight $T$, the initial time $t_{i}=-S_{c} T$, and the location of the reference plane specified by the parameter $S_{i}$, see equations $(6.2 .1),(6.2 .2)$ and $(6.2 .3)$ respectively and figure 5.1 (note $\Phi=\psi-\varphi)$. Explicit analytical equations can be determined for nearly complete collisions and for weak collisions. The virtual displacement $\Delta r$ caused by a nearly complete collision $\left(T \gg>/ b_{z}\right)$ is given by

$$
\begin{gathered}
\Delta r \cong \frac{1}{1+q_{c}} \mid\left(S_{i}-S_{c}\right) T v-\frac{b_{z}\left(1-q_{c}\right)}{2 \sqrt{q_{c}}}\left[2 \ln \left(\sqrt{\frac{q_{c}}{q_{c}+1}} \frac{2 v T}{b_{z}} \sqrt{S_{c}\left(1-S_{c}\right)}-2-\frac{S_{i}}{S_{c}}-\frac{1-S_{i}}{1-S_{c}}\right]\right. \\
+b_{z} \sqrt{q_{c}}\left[1-\frac{2}{1+q_{c}}\left(\frac{S_{i}}{S_{c}}-1\right)\right] \mid \quad\left(r_{\perp}=0\right)
\end{gathered}
$$

with

$$
q_{c}=\left(\frac{m v^{2} b_{z}}{2 c_{0}}\right)^{2}
$$$$
\left(r_{\perp}=0\right)
$$

as follows from equations $(6.12 .1),(6.12 .2),(6.7 .14)$ and $(6.4 .16)$. Equation $(9.2 .3)$ refers to the case $r_{\perp}=0$, which is relevant for a point crossover $\left(r_{c}=0\right)$. It is of importance to note that equation $(9.2 .3)$ diverges for $T \rightarrow \infty$, even when $S_{c}=S_{i}$. Accordingly, one can not perform the calculation of $\rho(\Delta r)$ on the basis of complete collisions, but is forced to account for the fact that $T$ is finite. Unfortunately, this prohibits an exact analytical calculation of the the statistical part of the problem. Therefore, one has to rely on numerical calculations when the contribution of nearly complete collisions to the distribution $p(\Delta r)$ becomes significant.

This observation also implies that an analysis of $\rho\left(\Delta v_{\perp}\right)$ based on complete collisions is insufficient to predict the behaviour of $\rho(\Delta r)$ for $S_{i} \rightarrow \pm \infty$. To illustrate this point consider equation $(9.2 .3)$ for $S_{i+\infty}$ 


$$
\lim _{S_{i} \rightarrow \infty} \frac{\Delta r}{S_{i} T} \cong \frac{v}{1+q_{c}}\left|1-\frac{2 C_{0}}{m v^{3} T}\left[\frac{1-q_{c}}{2}\left(\frac{1}{S_{c}}-\frac{1}{1-S_{c}}\right)-\frac{2 \sqrt{q_{c}}}{1+q_{c}} \frac{1}{S_{c}}\right]\right|
$$

in which we utilized equation $(9.2 .4)$. Equation $(9.2 .5)$ is identical to the expression for $\Delta v_{\perp}$ given by equation (6.11.2), substituting $r_{\perp}=0$. Thus equations $(9.2 .3)$ and $(6.11 .2)$ fulfil the relation given by equation (9.2.1). It is here essential that equation (6.11.2) includes the terms of the order $1 / T$. However, these terms were ignored in the analysis of the distribution $\rho\left(\Delta v_{\perp}\right)$, assuming that the collisions can be regarded as complete $(T+\infty)$. This assumption was introduced in order to permit an analytical calculation of the problem, see section 8.3. Unfortunately, one should at this point conclude that the results for $\rho\left(\Delta v_{\perp}\right)$ based on complete collisions are not suited to predict the behaviour of $\rho(\Delta r)$ for $S_{i} \rightarrow \pm \infty$ by means of equation (9.2.1).

The virtual displacement $\Delta r$ caused by a weak collision is for $r_{\perp}=0$ equal to (reproducing equation $(6.12 .6)$ )

$$
\Delta r \cong \frac{C_{0}}{m v^{2}}\left|\ln \left(\frac{\left[S_{c} v T+r_{i}\right]\left[\left(1-S_{c}\right) v T+r_{f}\right]}{b_{z}^{2}}\right)-v T\left(\frac{S_{i}}{r_{i}}+\frac{1-S_{i}}{r_{f}}\right)\right| \quad\left(r_{\perp}=0\right)
$$

where $r_{i}$ and $r_{f}$ are given by

$$
r_{i}=\left[\left(S_{c} v T\right)^{2}+b_{z}^{2}\right]^{1 / 2}, \quad r_{f}=\left\{\left[\left(1-S_{c}\right) v T\right]^{2}+b_{z}\right\}^{1 / 2}
$$

Notice that equation (9.2.6) and equation and (8.2.2) (for $r_{\perp}=0$ ) do satisfy equation $(9.2 .1)$. Thus the analysis of $\rho\left(\Delta v_{1}\right)$ based on weak collisions can be used to predict the behaviour of $\rho(\Delta r)$ for $S_{i} \rightarrow \pm \infty$.

Equations $(9.2 .3)$ and $(9.2 .6)$ apply to the case that the initial relative velocity of the particles is non-zero $(v \neq 0)$. In case $v=0$ we found for a half-complete collision (reproducing equation $(6.12 .8)$ )

$$
\Delta r \cong\left|\frac{\left(C_{0} / m\right)^{1 / 2} r_{\perp} T S_{i}}{\left(b_{z}^{2}+r_{\perp}^{2}\right)^{3 / 4}}-\frac{r_{\perp}}{4}\left[S_{i}+\ln \left(\frac{8\left(C_{0} / m\right)^{1 / 2} T}{\left(b_{z}^{2}+r_{\perp}^{2}\right)^{3 / 4}}\right)\right]\right| \quad(v=0)
$$

and for a weak collision (reproducing equation (6.12.9))

$$
\Delta r \cong\left|\frac{C_{0}}{m} \frac{r_{\perp} T^{2}\left(S_{i}-1 / 2\right)}{\left(b_{z}^{2}+r_{\perp}^{2}\right)^{3 / 2}}\right| \quad(v=0)
$$

Notice that equation (9.2.8) and equation (8.2.4) satisfy equation $(9.2 .1)$. The same holds true for equation (9.2.9) and equation (8.2.5). We recall that the case $v=0$ is relevant for a monochromatic homocentric cylindrical beam segment. Equation (9.2.2) gives the displacement $\Delta r_{\text {foc }}$ in the back focal plane of a lens located at the end of a cylindrical beam segment. This displacement is entirely determined by the velocity deviation $\Delta v_{\perp}$ only and does not depend on the shift $\Delta r_{f}$ of the test particle at the end of the segment. Instead of calculating $\Delta r_{f o c}$ from $\Delta v_{\perp}$ by means of equation $(9.2 .2)$ one can also determine this quantity directly from equations (9.2.8) and (9.2.9) using

$$
\Delta \mathrm{r}_{\mathrm{foc}}=\lim _{S_{\mathbf{i}}+\infty} \Delta r \frac{\mathbf{f}}{S_{\mathbf{i}} \mathrm{L}}
$$

This relation is in agreement with equations (9.2.1) and (9.2.2).

The statistical part of the problem consists of the evaluation of equations $(5.7 .7),(5.7 .8)$ and $(5.7 .10)$, in which $\Delta \eta$ now represents $\Delta r$ 


$$
\begin{aligned}
& \rho_{2}(\Delta r)=\int_{0}^{v_{0}} \frac{2 v d v}{v_{0}^{2}} \int_{0}^{2 \pi} \frac{d \Phi}{2 \pi} \int_{0}^{r_{c}} \frac{2 r_{\perp} d r_{\perp}}{r_{c}{ }^{2}} \int_{-S_{c} L}^{\left(1-S_{c}\right) L} d_{z} \delta\left[\Delta r-\Delta r\left(v, \Phi, r_{\perp}, b_{z}\right)\right] \\
& p(k)=\int_{0}^{\infty} d \Delta r \rho_{2}(\Delta r)\left[1-J_{0}(k \Delta r)\right] \\
& \rho(\Delta r)=\frac{1}{2 \pi} \int_{0}^{\infty} k d k J_{0}(k \Delta r) e^{-\lambda p(k)}
\end{aligned}
$$

similar to equations $(8.2 .6),(8.2 .7)$ and $(8.2 .8)$. We expressed $p(\xi) \mathrm{d} \xi$ directly in terms $v, \Phi, r_{\perp}$ and $b_{z}$, using equations $(5.4 .6)$ and $(5.3 .2)$. The distribution in $\mathrm{v}$ and $r_{1}$ are taken uniform with a cut-off at $v_{0}=\alpha_{0} v_{z}$ and $r_{c}$ respectively, as prescribed by equation (5.3.3). The displacement distribution $p(\Delta r)$ depends only on the magnitude of the displacement $\Delta r$ and not on its direction, which is a consequence of the rotational symmetry of the beam and the choice of the central reference trajectory. The probability of a displacement of size $\Delta r$ is equal to $\rho(\Delta r)=2 \pi \Delta r^{2} \rho(\Delta r)$. The reader is referred to section 7.2 for a summary of the assumptions underlying our model for statistical interactions.

\subsection{Homocentric beam segment with a crossover}

In this section we will calculate the distribution of transverse displacements $\rho(\Delta r)$ for a homocentric beam segment with a crossover $\left(r_{c}=0\right)$. We will use the $\delta, v$-scaling defined by equation $(6.13 .1)$. The scaled two-particle distribution $\rho^{\star} 2\left(\Delta r^{*}\right)$ follows from equation $(9.2 .11)$

$$
\rho^{*} 2\left(\Delta r^{*}\right)=v_{0}^{* 2} \rho_{2}(\Delta r)=\int_{0}^{v_{0} *} 2 v^{*} d v^{*} \int_{0}^{\infty} 2 \mathrm{db}^{*} \delta\left[\Delta r^{*}-\Delta r^{*}\left(r^{*}, b_{z^{*}}\right)\right]
$$

We evaluated this integral for different values of $v_{0}{ }^{*}$, on the basis of the exact solution for the function $\Delta r^{*}\left(r^{*}, b_{z}^{*}\right)$, following the analysis of section 6.6. The results for the case $S_{c}=S_{i}=1 / 2$ are plotted in figure 9.1.

Analytical expressions for the function $\Delta r^{*}\left(r^{*}, b_{z}^{*}\right)$ can be obtained for nearly complete collisions and for weak collisions, see equations $(9.2 .3)$ and $(9.2 .6)$ respectively. Unfortunately, the complexity of equation $(9.2 .3)$ prohibits an exact analytical treatment based on nearly complete collisions and in the analysis here we will only consider the contribution of weak collisions. This approach is appropriate to explain the data of figure 9.1 for small and medium $\Delta r^{*}$. By scaling equation $(9.2 .11)$ one obtains

$\Delta r^{*}\left(r^{*}, b_{z^{*}}\right) \cong \frac{1}{4 v^{* 2}}\left|\ln \left(\frac{\left[S_{c} v^{*}+r_{i}^{*}\right]\left[\left(1-S_{c}\right) v^{*}+r_{f}^{*}\right]}{b_{z}^{* 2}}\right)-v^{*}\left(\frac{S_{i}}{r_{i}^{*}}+\frac{1-S_{i}}{r_{f}^{*}}\right)\right|$

with $r_{i}^{*}=\left[\left(S_{c} V^{*}\right)^{2}+b_{z}^{* 2}\right]^{1 / 2}$ and $r_{f}^{*}=\left\{\left[\left(1-S_{c} v\right)^{*}\right]^{2}+b_{z}^{* 2}\right\}^{1 / 2}$. Similar to the derivation of equation (8.5.9) one finds from equations (9.3.1) and (9.3.2) 


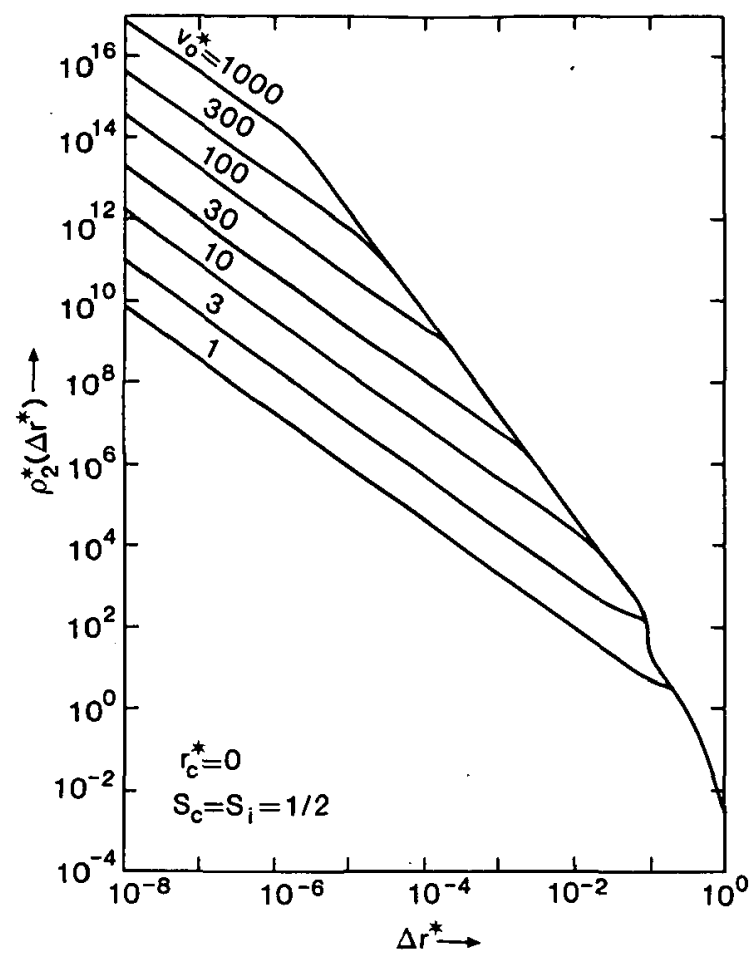

Figure 9.1 The scaled two-particle distribution $\rho_{2}{ }^{\star}\left(\Delta r^{\star}\right)$ for a homocentric beam segment with a crossover in the middle $\left(S_{c}=1 / 2\right)$, plotted for different values of the scaled maximum transverse velocity $\mathrm{v}_{0}{ }^{*}$. The results represent the virtual displacements observed in the crossover plane $\left(S_{1}=S_{c}\right)$.

$$
\rho^{\star}{ }_{2}\left(\Delta r^{*}\right)=\frac{2}{\Delta r^{* 5 / 2}} \int_{0}^{\infty} d z F(z)^{3 / 2} \theta\left(\Delta r^{*}-F(z) / v_{0} \star 2\right)
$$

in which the function $F(2)$ now stands for

$$
\begin{aligned}
F(z)=\frac{1}{4} & \ln \left(\frac{\left\{S_{c}+\left[S_{c}{ }^{2}+z^{2}\right]^{1 / 2}\right\}\left\{1-S_{c}+\left[\left(1-S_{c}\right)^{2}+z^{2}\right]^{1 / 2}\right.}{z^{2}}\right) \\
& -\frac{S_{i}}{\left[S_{c}{ }^{2}+z^{2}\right]^{1 / 2}}-\frac{1-S_{i}}{\left[\left(1-S_{c}\right)^{2}+z^{2}\right]^{1 / 2}} \mid
\end{aligned}
$$

We will consider the cases $v_{0}^{*+\infty}$ and $v_{0}^{*} \rightarrow 0$ separately.

For $v_{0}{ }^{*} \rightarrow \infty$ the argument of the step-function in equation (9.3.3) is positive for all $z$-values and one obtains 


$$
\rho^{*} 2\left(\Delta r^{*}\right)=I_{5} \frac{S_{H T}\left(S_{C}, S_{i}\right)^{3 / 2}}{\Delta r^{* 5 / 2}}, S_{H T}\left(S_{c}, S_{i}\right)^{3 / 2}=\frac{2}{I_{5}} \int_{0}^{\infty} d z \quad F(2)^{3 / 2}\left(V_{0}^{*+\infty}\right)(9.3 .5)
$$

We choose the constant $I_{5}$ such that $S_{H T}\left(S_{C}=1 / 2, S_{i}=1 / 2\right)=1$. This implies

$$
I_{5}=\frac{1}{2^{3 / 2}} \int_{0}^{\infty} d x\left[\ln \left(\frac{1+\left(1+x^{2}\right)^{1 / 2}}{x}\right)-\frac{1}{\left(1+x^{2}\right)^{1 / 2}}\right]^{3 / 2}=0.39507
$$

as follows by numerical integration. The integral for $S_{H T}\left(S_{C}, S_{i}\right)$ was evaluated numerically for different $S_{c}$ and $S_{i}$ and is plotted in figure 9.2. A reasonable approximation of the numerical data is given by

$$
S_{H T a}\left(S_{c}, S_{i}\right) \cong \frac{3 S_{c}-2 S_{i}}{\left[2 S_{c}\right]^{1 / 3}}+\frac{3\left(1-S_{c}\right)-2\left(1-S_{i}\right)}{\left[2\left(1-S_{c}\right)\right]^{1 / 3}}
$$

This equation can be derived by means of the slice-method as will be demonstrated in section 9.5. By fitting the numerical data it was found that a somewhat better approximation is given by

$$
S_{H T b}\left(S_{c}, S_{i}\right)=H\left(S_{C}\right)+\left|S_{H T a}\left(S_{c}, S_{i}\right)-H\left(S_{c}\right)\right|
$$

where $S_{\mathrm{HTa}_{\mathrm{a}}}\left(\mathrm{S}_{\mathrm{C}}, \mathrm{S}_{\mathrm{i}}\right)$ is given by equation (9.3.7) and the function $\mathrm{H}\left(\mathrm{S}_{\mathrm{c}}\right)$ is equal to

$$
H\left(S_{c}\right)=\ln \left\{1.1+\left[3 S_{c}\left(1-S_{c}\right)\right]^{4}\right\}
$$

However, equation (9.3.7) is, in general, sufficiently accurate and is preferred for its simplicity.

For the particular case that the reference plane and the crossover plane coincide $\left(S_{c}=S_{i}\right)$ equation $(9.3 .7)$ reduces to

$$
S_{\mathrm{HTa}}\left(S_{\mathrm{C}}\right)=\frac{1}{2}\left(\left[2 S_{\mathrm{c}}\right]^{2 / 3}+\left[2\left(1-S_{\mathrm{c}}\right)\right]^{2 / 3}\right) \quad\left(S_{\mathrm{c}}=\mathrm{S}_{\mathrm{i}}\right)
$$

This function has a maximum for $S_{\mathrm{c}}=1 / 2 \quad\left(S_{\mathrm{HT}}(1 / 2)=1\right)$. The total variation within the full $S_{c}$-range $\left(0<S_{c}<1\right)$ is approximately $20 \%$. $\left(S_{\mathrm{HTa}}(0)=S_{\mathrm{HTa}}(1)=2-1 / 3=0.7937\right)$. In general the function $S_{\mathrm{HTa}}\left(S_{c}, S_{i}\right)$ increases when the crossover and the reference plane do not coincide $\left(S_{c} \neq S_{i}\right)$, as can be seen from figure 9.2. The contribution of angular deflections becomes dominant when the crossover plane and the reference plane are separated by a large distance $\left(S_{i}<<S_{c}\right.$ or $\left.S_{i} \gg>S_{c}\right)$.

For $v_{0}{ }^{k} \rightarrow 0$ the argument of the step-function in equation (9.3.3) is only positive for large $z$-values and one may use the following asymptotic expansion of the function $F(z)$

$$
\lim _{z \rightarrow \infty} F(z)=\frac{S_{P I}\left(S_{C}, S_{i}\right)}{48 z^{3}}+0\left(z^{-4}\right)
$$

in which the function $S_{P_{T}}\left(S_{c}, S_{i}\right)$ is given by

$$
S_{P T}\left(S_{c}, S_{i}\right)=\left|4-6\left(S_{c}+S_{i}\right)+12 S_{c} S_{i}\right|
$$

The function $S_{P T}\left(S_{c}, S_{i}\right)$ was scaled such that $S_{P T}(1 / 2,1 / 2)=1$. It is plotted in figure 9.3. For the particular case that the reference plane and the crossover plane coincide $\left(S_{c}=S_{i}\right)$ one obtains 


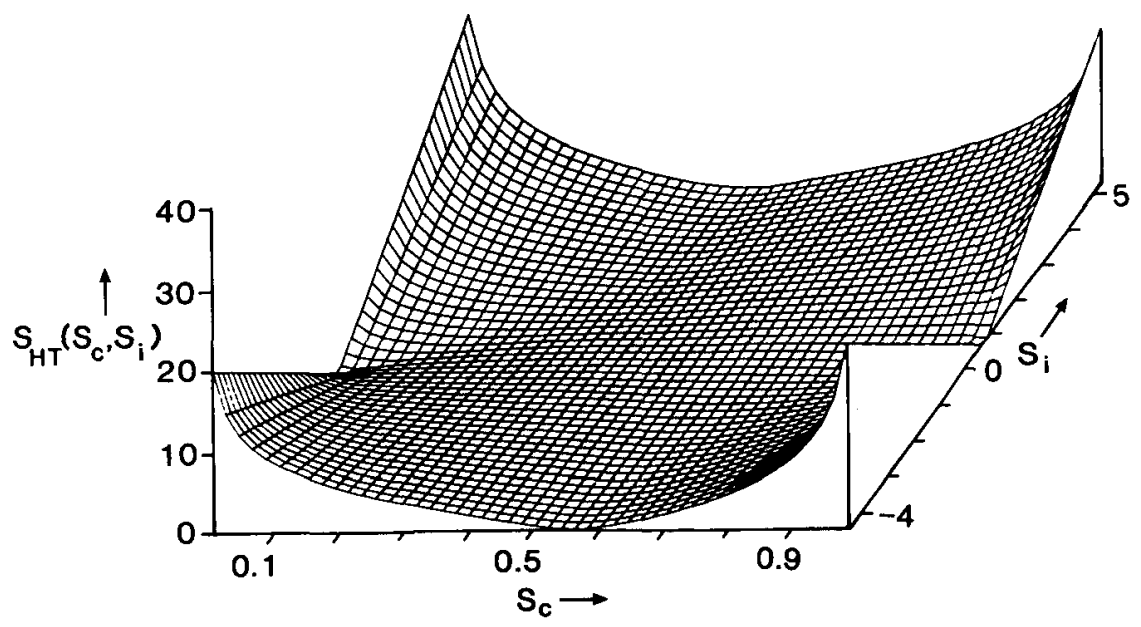

Pigure 9.2 The function $S_{\mathrm{HT}}\left(S_{c}, S_{i}\right)$ defined by equations (9.3.5), (9.3.4) and (9.3.6). This function specifies the dependency of the width of trajectory displacement distribution on the crossover position parameter $\mathrm{S}_{\mathrm{c}}$ and image plane position parameter $S_{i}$ in the Holtsmark regime and pertains to a stigmatic crossover $\left(r_{c}{ }^{\star}=0\right)$.

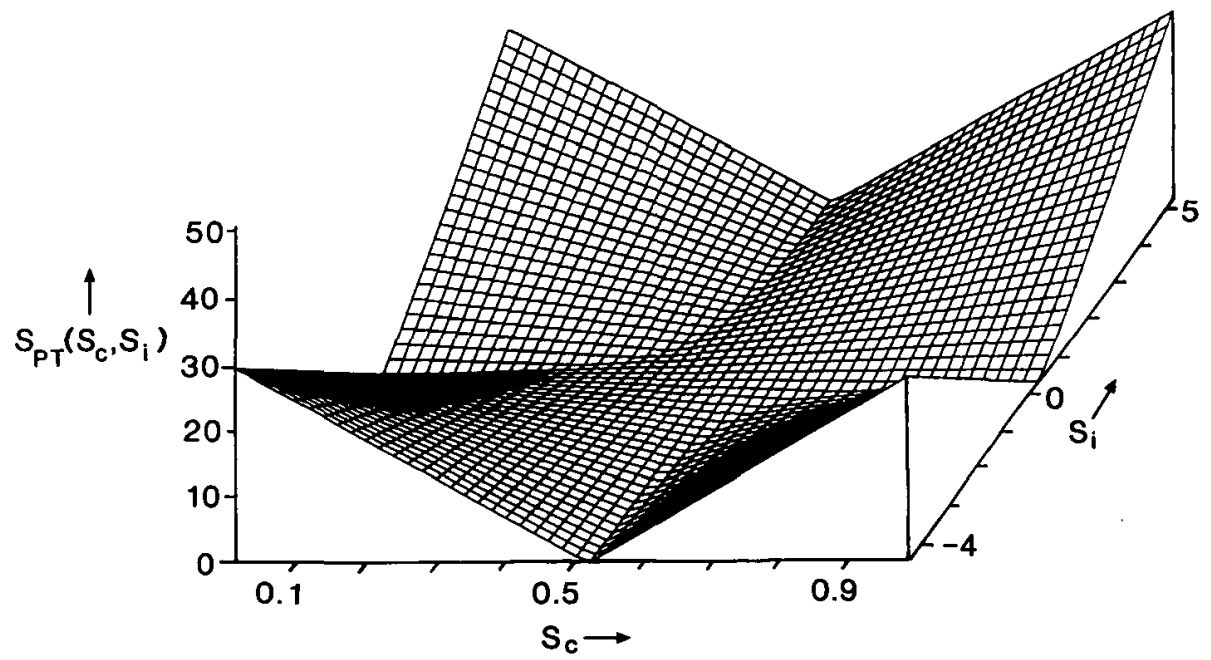

Figure 9.3 The function $S_{\mathrm{PT}}\left(S_{c}, S_{1}\right)$ given by equation (9.3.12). This function specifies the dependency of the width of trajectory displacement distribution on the crossover position parameter $S_{c}$ and image plane position parameter $S_{i}$ in the pencil beam regime and pertains to a stigmatic crossover $\left(r_{c}{ }^{\star}=0\right)$. 


$$
S_{P T}\left(S_{c}\right)=4\left|1+3 S_{c}\left(S_{c}-1\right)\right| \quad\left(S_{c}=S_{i}\right)
$$

This function has a minimum for $S_{c}=1 / 2 \quad\left(S_{P T}(1 / 2)=1\right)$. It is interesting to note that the behaviour of the function $S_{P_{T}}\left(S_{c}\right)$ is in this respect opposite to that of $S_{\mathrm{HTa}}\left(S_{c}\right)$, given by equation $(9.3 .10)$. The keyobservation here is that the magnitude of the trajectory displacement effect decreases with the beam radius in case of an extended beam, while it increases with the beam radius for a pencil beam. The behaviour of $S_{H T a}\left(S_{c}\right)$ and $S_{P T}\left(S_{C}\right)$ can now be understood from the fact that the maximum beam radius in the section becomes minimum for $S_{c}=1 / 2$. The total variation of the function $S_{\mathrm{PT}}\left(\mathrm{S}_{\mathrm{c}}\right)$ within the full $\mathrm{S}_{\mathrm{c}}$-range $\left(0<S_{c}<1\right)$ is a factor $4\left(S_{P T}(0)=S_{P T}(1)=4\right)$, which is considerably larger than the corresponding variation of the function $S_{H T a}\left(S_{c}\right)$.

Substitution of equation (9.3.11) into equation (9.3.3) yields

$$
\rho^{*}\left(\Delta r^{*}\right)=\frac{2}{6^{1 / 37}} \frac{S_{P T}\left(S_{c}, S_{i}\right)^{1 / 3} v_{0}^{* 7 / 3}}{\Delta r^{* 4 / 3}} \cdot\left(v_{0}^{*} \rightarrow 0\right)
$$

The reader might verify that the numerical data of $\rho^{*}{ }_{2}\left(\Delta r^{*}\right)$ for the case $S_{c}=S_{i}=1 / 2$, which is plotted in figure 9.1 , agrees with equations $(9.3 .5)$ and $(9.3 .14)$.

The scaled function $p^{*}\left(k^{*}\right)$ follows from equation $(9.2 .12)$

$$
p^{*}\left(k^{*}\right)=\frac{v_{0}^{* 2}}{\delta} p(k)=\int_{0}^{\infty} d \Delta r^{*} \rho_{2} *\left(\Delta r^{*}\right)\left[1-J_{0}\left(k^{*} \Delta r^{*}\right)\right]
$$

with $k^{*}=k \delta$. This integral was evaluated numerically' for the case $S_{c}=S_{i}=1 / 2$ starting from the numerical data for $\rho_{2}{ }^{*}\left(\Delta r^{*}\right)$. The results are plotted in figure 9.4 .

The behaviour of the function $p^{*}\left(k^{*}\right)$ can be understood from the analysis of the two particle distribution $\rho_{2}^{*}\left(\Delta r^{*}\right)$. For $v_{0}^{*} \rightarrow \infty$ one may use equation (9.3.5). By substitution into equation $(9.3 .15)$ one finds after integration

$$
p^{*}\left(k^{*}\right)=\frac{2 \sqrt{2} \Gamma(1 / 4) I_{5}}{9 \Gamma(3 / 4)} S_{H T}\left(S_{c}, S_{i}\right)^{3 / 2} k^{* 3 / 2}
$$

similar to the derivation of equation (5.8.14). The numerical factor in the right hand side of equation $(9.3 .16)$ is equal to 0.36734 , as follows with equation (9.3.6). Equation (9.3.16) constitutes a good approximation of $\mathrm{p}^{*}\left(\mathrm{k}^{*}\right)$ for intermediate $\mathrm{k}^{*}$-values, especially in combination with large $v_{0} *$.

Substitution of equation (9.3.14) into equation (9.3.15) yields after integration

$$
p^{*}\left(k^{*}\right)=\frac{21 / 318 \Gamma(5 / 6)}{3^{1 / 3} 7 \Gamma(1 / 6)} S_{P T}\left(S_{c}, S_{i}\right)^{1 / 3} v_{0}^{* 7 / 3} k^{* 1 / 3}
$$

similar to the derivation of equation (5.8.19). The numerical factor in the right hand side of equation (9.3.17) is equal to 0.45553 . Equation (9.3.17) constitutes a good approximation of $\mathrm{p}^{*}\left(\mathrm{k}^{*}\right)$ for large $k^{*}$-values, especially in combination with small $v_{0} \neq$. We define

For small $k^{*}$-values the function $p^{*}\left(k^{*}\right)$ becomes quadratic in $k^{\star}$.

$$
\lim _{k \neq 0} p^{*}\left(k^{*}\right)=\frac{1}{4} p_{2} *\left(v_{0}^{*}, s_{c}, s_{i}\right) 1 / 2 k^{* 2}
$$




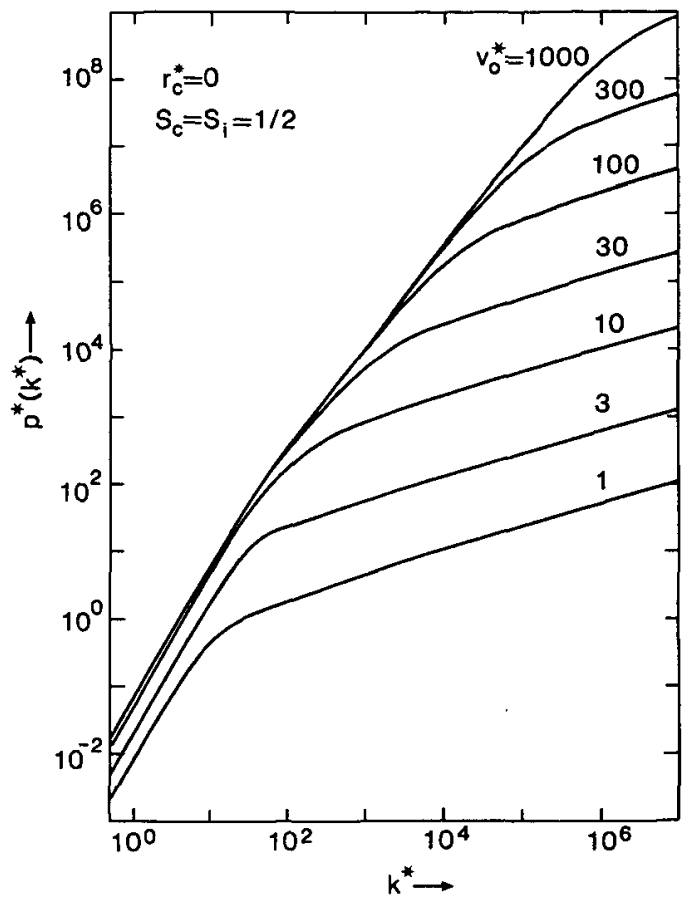

Figure 9.4 The scaled function $p^{*}\left(\mathrm{k}^{\star}\right)$ corresponding to the trajectory displacement distribution generated in a homocentric beam segment with a crossover in the middle $\left(S_{\mathrm{c}}=1 / 2\right)$, plotted for different values of the maximum transverse velocity $\mathrm{vo}^{*}$. The results pertain to the virtual displacements observed in the crossover plane $\left(S_{1}=S_{c}\right)$.

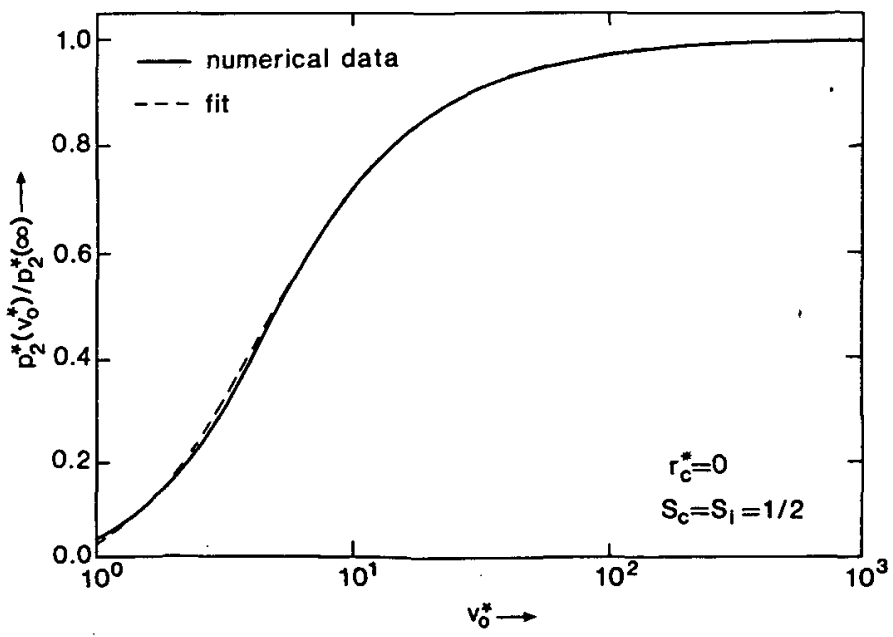

Figure 9.5 The function $\mathrm{p}_{2}\left(\mathrm{v}_{0}{ }^{*}\right)$ defined by equation (9.3.18) for $S_{c}=S_{1}=1 / 2$ and its approximation given by equation (9.3.21). This function gives the $v_{0}^{*}$-dependency of the function $\mathrm{p}^{\star}\left(\mathrm{k}^{\star}\right)$, plotted in figure 9.4 , for $\mathrm{k}^{\star} \rightarrow 0$. 
The small $\mathrm{k}^{*}$-behaviour can directly be related to the second moment of the two-particle distribution $\rho^{*}{ }_{2}\left(\Delta r^{*}\right)$

$$
\left\langle\Delta r_{2} * 2\right\rangle=p_{2}^{*}\left(v_{0}^{*}, S_{c}, S_{i}\right)
$$

as can be seen from equation (9.3.15) by expanding the Bessel function for small values of its argument $\left(\mathrm{J}_{0}(z) \approx 1-z^{2} / 4+\cdots\right)$. We evaluated the quantity $\left\langle\Delta \mathrm{r}_{2}{ }^{* 2}\right\rangle$ numerically as function of $v_{0} *, S_{c}$ and $S_{i}$. For $v_{0}{ }^{*+\infty}$ and $S_{c}=S_{i}=1 / 2$ it was found that

$$
\mathrm{p}_{2}{ }^{\star}(\infty, 1 / 2,1 / 2)=0.273
$$

The results obtained for $S_{c}=S_{i}=1 / 2$ and different $v_{0}{ }^{*}$-values are plotted in figure 9.5. The data can be fitted using

$$
\mathrm{p}_{2 \mathrm{a}} *\left(\mathrm{v}_{0} *, 1 / 2,1 / 2\right)=\frac{\mathrm{p}_{2} *(\infty, 1 / 2,1 / 2)}{\left(1+1,40 / \mathrm{v}_{0} * 8 / 7\right)^{7 / 2}}
$$

For comparison this approximation is included in figure 9.5. In order to evaluate the general behaviour of the function $p_{2} *\left(v_{0}^{*}, S_{c}, S_{i}\right)$ we computed the quantity $\left\langle\Delta r_{2} * 2\right\rangle$ for all combinations of $v_{0}^{*}, S_{c}$ and $S_{i}$ with $S_{c}=0.1,0.2, \cdots, 0.9 ; S_{i}=-0.5,-0.4, \cdots, 1.6$ and $v_{0}{ }^{*}=10,100$ and 1000 . The results obtained for $v_{0} * \geqslant 100$ and $S_{c}=S_{i}$ can be approximated by

$$
\mathrm{p}_{2 \mathrm{a}} *\left(\mathrm{v}_{0} *, S_{c}, S_{c}\right)=\mathrm{p}_{2} *(\infty, 1 / 2,1 / 2)\left[1+0.682\left(S_{c}-0.5\right)-0.739\left(S_{c}-0.5\right)^{2}\right]
$$

$$
\left(v_{0} * \geqslant 100\right) \quad(9.3 .22)
$$

which is a monotonically increasing function of $S_{c} \quad\left(0<S_{c}<1\right)$, which levels off for $S c \rightarrow 1$. The quantity $\left\langle\Delta r_{2} * 2\right\rangle$ increases strongly when $S_{c} \neq S_{i}$. For all considered $v_{0^{*}}$-values it was found that the results for $S_{c} \ll<S_{i}$ or $S_{c} \gg S_{i}$ can be approximated as

$$
\begin{array}{r}
\mathrm{p}_{2 \mathrm{a}} *\left(\mathrm{v}_{0}^{*}, \mathrm{~S}_{\mathrm{c}}, \mathrm{S}_{\mathrm{i}}\right)=\mathrm{p}_{2} *(\infty, 1 / 2,1 / 2) 113 \mathrm{v}_{0} * 1 / 2\left(S_{\mathrm{c}}-S_{i}\right)^{2} \\
\left(S_{c} \ll<S_{i} \text { or } S_{c} \gg S_{i}\right)
\end{array}
$$

On first sight the square root dependency on $v_{0}^{*}$ is somewhat surprising. The reader might verify, with the scaling relations of equation $(7.5 .6)$, that the result for $\left\langle\Delta v_{\perp}{ }^{2}\right\rangle$ given by equation (8.3.18) leads, according to the relation between $\Delta r$ and $\Delta v_{\perp}$ for $S_{i} \rightarrow \pm \infty$ given by equation (9.2.1), to a quadratic dependency on $v_{0}{ }^{*}$. However, it should be recalled that equation (8.3.18) is based on complete collisions. This renders equation (9.2.1) inapplicable as was discussed in section 9.2.

For further use it is convenient to define the function $\operatorname{PCT}_{\mathrm{T}}\left(\mathrm{v}_{0}^{*}, S_{\mathrm{C}}, S_{\mathrm{i}}\right)$ as

$$
\operatorname{Pct}\left(v_{0} *, S_{c}, S_{i}\right)=\left[p_{2} *\left(v_{0}^{*}, S_{c}, S_{i}\right) / p_{2} *(\infty, 1 / 2,1 / 2)\right]^{1 / 2}
$$

Combining the analysis of equations $(9.3 .21),(9.3 .22)$ and (9.3.23) we postulate that the function $\mathrm{P}_{\mathrm{CT}}\left(\mathrm{v}_{0}{ }^{*}, S_{c}, S_{i}\right)$ can in general be approximated as

$$
P_{c T a}\left(v_{0}^{*}, S_{c}, S_{i}\right)=\left[P_{C T 1}\left(v_{0}^{*}, S_{c}\right)^{2}+113 v_{0} * 1 / 2\left(S_{c}-S_{i}\right)^{2}\right]^{1 / 2}
$$

with 


$$
P_{C T 1}\left(v_{0}^{*}, S_{c}\right)=\frac{\left[1+0.682\left(S_{c}-0.5\right)-0.739\left(S_{c}-0.5\right)^{2}\right]^{1 / 2}}{\left(1+1.40 / v_{0}^{* 8 / 7}\right)^{7 / 4}}
$$

Equations $(9.3 .19),(9.3 .20),(9.3 .24),(9.3 .25)$ and $(9.3 .26)$ constitute a good approximation for the numerical data of $\left\langle\Delta r_{2} * 2\right\rangle$ for all considered values of the parameters $v_{0}{ }^{*}, S_{c}$ and $S_{i}$. To illustrate the dependency on the parameters $S_{c}$ and $S_{i}$ the exact numerical data obtained for the function $\mathrm{P}_{\mathrm{c} T}\left(\mathrm{v}_{0} *=1000, S_{c}, S_{1}\right)$ is plotted in figure 9.6.

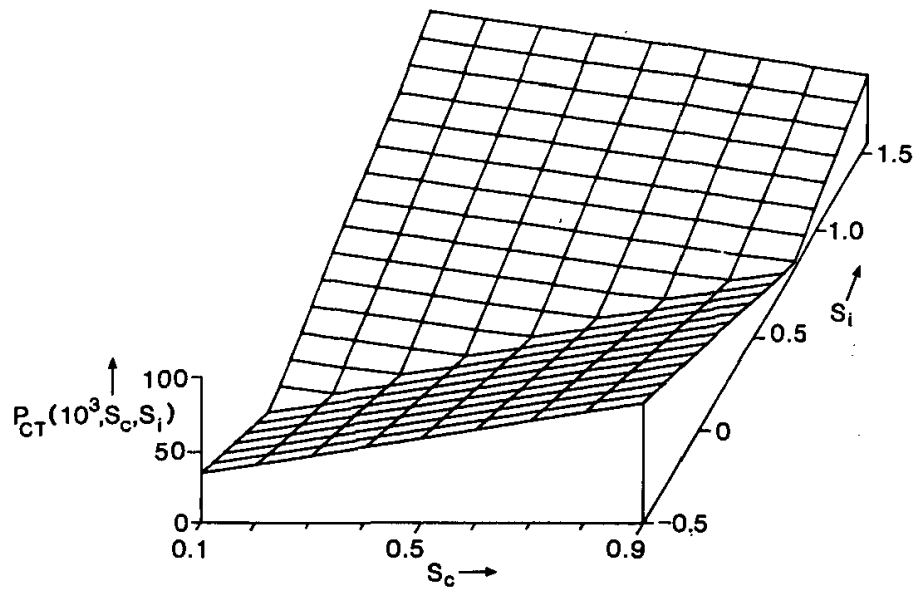

Figure 9.6 The function $\mathrm{P}_{\mathrm{c}}\left(\mathrm{v}_{0}{ }^{*}, \mathrm{~S}_{\mathrm{c}}, \mathrm{S}_{\mathrm{i}}\right)$ defined by equations (9.3.24), (9.3.19) and $(9.3 .20)$ for $v_{0}^{*}=1000$. This function specifies the dependency of the width of trajectory displacement distribution on the crossover position parameter $S_{c}$ and image plane position parameter $S_{1}$ in the Gaussian regime and pertains to a stigmatic crossover $\left(r_{c}{ }^{*}=0\right)$.

The scaled displacement distribution $\rho^{*}\left(\Delta r^{*}\right)$ follows from equation $(9.2 .13)$

$$
\rho^{*}\left(\Delta r^{*}\right)=\delta^{2} p(\Delta r)=\frac{1}{2 \pi} \int_{0}^{\infty} k^{*} d k^{*} J_{0}\left(k^{*} \Delta r^{*}\right) e^{-4 \lambda p^{*}\left(k^{*}\right)}
$$

The second moment of this distribution is determined by the small $k^{*}$ behaviour of the function $p^{*}\left(k^{*}\right)$. Using equations (5.5.4) (with $m=2$ ) and (9.3.18) one may write

$$
\left\langle\Delta r^{* 2}\right\rangle=4 p_{2} *\left(v_{0}^{*}, S_{c}, S_{i}\right) \pi
$$

This relation can also be obtained from equation (9.3.19), using equation (5.5.10) (with $\mathrm{m}=2$ ). We evaluated the FWHM of the distribution $\rho^{*}\left(\Delta r^{*}\right)$ numerically for $S_{c}=S_{i}=1 / 2$ and various $v_{0}^{*}$-values, starting from the numerical data obtained for $\mathrm{p}^{*}\left(\mathrm{k}^{*}\right)$. The results are plotted in figure 9.7. Figure 9.8 shows the results for $v_{0}{ }^{*}=100, S_{c}=1 / 2$ and various $S_{i}$ values. Different regimes should be distinguished by the type of the generated trajectory displacement distribution. The occurrence of the different regimes depends on the value of the scaled linear particle density $\bar{\lambda}$ and the pencil beam factor $\chi_{c}$, which are defined by equations 


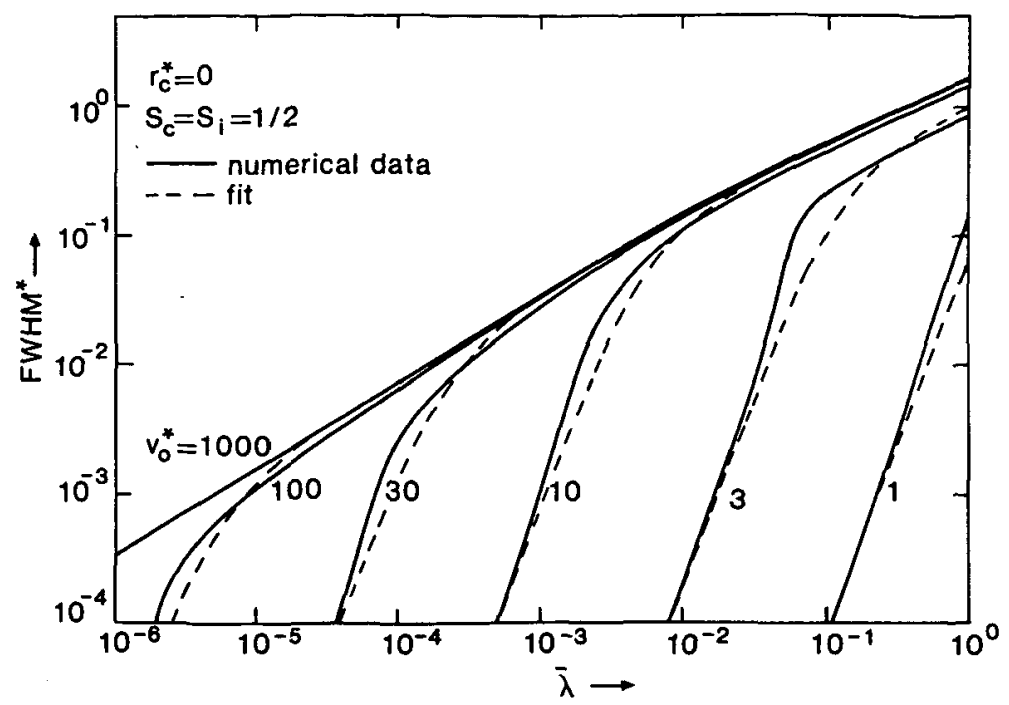

Figure 9.7 The FWHM of the trajectory distribution $\rho^{\star}\left(\Delta r^{\star}\right)$ generated in a homocentric beam segment with a crossover in the middle $\left(S_{\mathrm{c}}=1 / 2\right)$, plotted for different values of the maximum transverse velocity $\mathrm{v}_{0}{ }^{*}$. The results represent the virtual displacements observed in the crossover plane $\left(S_{i}=S_{c}\right)$. The depicted fit is defined equations (9.3.37), $(9.3 .40)$ and $(9.3 .39)$.

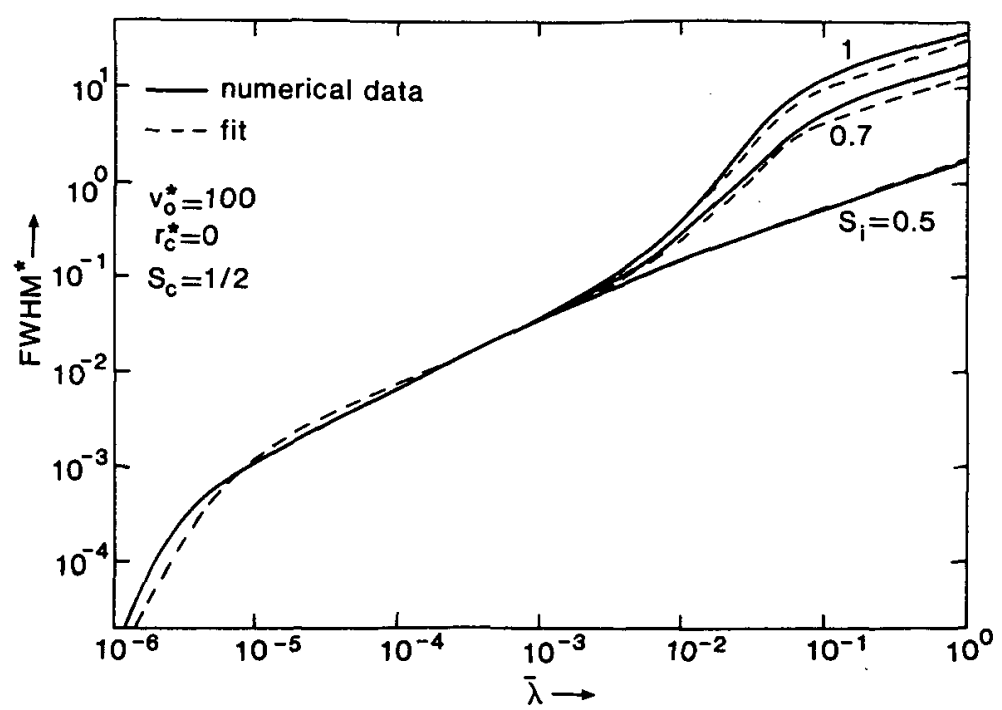

Figure 9.8 The FWHM of the trajectory distribution $\rho^{\star}\left(\Delta r^{\star}\right)$ generated in a homocentric beam segment with a crossover in the middle $\left(\mathrm{S}_{\mathrm{c}}=1 / 2\right)$, plotted for different locations of the image plane, which is specified by the parameter $S_{1}$. The results pertain to a beam for which the maximum transverse velocity $v_{0}^{*}=100$. The depicted fit is defined equations $(9.3 .37),(9.3 .38)$ and $(9.3 .39)$. 
$(7.3 .22)$ and $(7.5 .28)$ respectively :

I. Gaussian regime. For $\lambda \gg>10^{-3}$ and $\chi_{c} \gg 1$ the distribution $\rho^{*}\left(\Delta r^{*}\right)$ is dominated by the small $\mathrm{k}^{*}$. behaviour described by equations (9.3.18). This leads to a two-dimensional Gaussian distribution with a FWHM which is equal to

$$
\begin{aligned}
& \mathrm{FWHM}_{\mathrm{G}} *=4\left[\mathrm{p}_{2}{ }^{*}(\infty, 1 / 2,1 / 2) \ln 2\right]^{1 / 2} \mathrm{P}_{\mathrm{CT}}\left(\mathrm{v}_{0}^{*}, \mathrm{~S}_{\mathrm{C}}, \mathrm{S}_{\mathrm{i}}\right) \pi^{1 / 2} \\
& =1.740 \operatorname{PCT}\left(\mathrm{V}_{0}^{*}, S_{\mathrm{C}}, \mathrm{S}_{\mathrm{i}}\right) \lambda^{1 / 2}
\end{aligned}
$$

where the function $P_{C T}\left(v_{0} *, S_{c}, S_{i}\right)$ can be approximated by the function $\operatorname{Pcta}_{\mathrm{C}}\left(\mathrm{V}_{0^{*}}, S_{\mathrm{c}}, S_{i}\right)$, which is defined by equation $(9.3 .25)$. For $S_{c}=S_{i}$ one may approximate $\mathrm{FWHM}_{\mathrm{G}}^{*}=\mathrm{FWHM}_{\mathrm{G}}{ }^{*}$, with

$$
\text { FWHM }_{G_{1}} *=1.740 \operatorname{Pcr} 1\left(V_{0}^{*}, S_{C}\right) \pi^{1 / 2} \quad\left(S_{c}=S_{i}\right)
$$

where the function $P_{c_{1}}\left(v_{0} *, S_{c}, S_{i}\right)$ is given by $(9.3 .26)$. For $S_{c} \neq S_{i}$ one may approximate $\mathrm{FWHM}_{\mathrm{G}}{ }^{*}=\mathrm{FWHM}_{\mathrm{G}}{ }^{*}$, with

$$
\text { FWHM }_{G} *=18.5\left|S_{c}-S_{i}\right| V_{0} * 1 / 4 \lambda^{1 / 2} \quad\left(S_{c} \neq S_{i}\right)
$$

as follows from equations (9.3.29) and (9.3.25).

II Weak complete collision regime. This regime becomes only manifest for $S_{c} \neq S_{i}$. It can directly be related to the corresponding regime for the distribution of angular deflections, see equations (8.3.22) and $(8.3 .23)$. For the present case of a homocentric beam segment with a crossover $\left(r_{c}=0\right)$ it was found from the numerical data for FWHM* that

$$
\mathrm{FWHM}^{*}=42.105\left|S_{c}-S_{i}\right| v_{0} * \lambda^{2}
$$

which is in agreement with equation (8.3.23), utilizing equation $(9.2 .1)$ and the scale relations of equation (7.5.5).

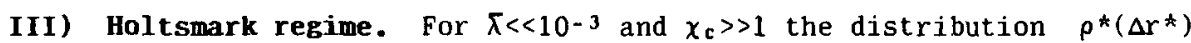
is dominated by the large $k^{*}$-behaviour described by equation $(9.3 .16)$. Substitution into equation $(9.3 .27)$ yields

$$
\rho^{\star}\left(\Delta r^{*}\right)=\frac{1}{2 \pi} \int_{0}^{\infty} k^{*} d k^{*} J_{0}\left(k^{*} \Delta r^{*}\right) e^{-C_{3} / 2 \pi\left[S_{H T}\left(S_{c}, S_{i}\right) k^{\star}\right]^{3 / 2}}
$$

with $C_{3 / 2}=8 \sqrt{2} \Gamma(1 / 4) I_{5} / 9 \Gamma(3 / 4)=1.4694$. This distribution is the twodimensional variant of the Holtsmark distribution, plotted in figure 5.4 (curve corresponding to $\gamma=3 / 2$ ). The FWHM of this distribution is given by

$$
\mathrm{FWHM}_{\mathrm{H}} * 2.6554 \frac{21 / 3 \Gamma(1 / 4)^{2 / 3} I_{5} 2 / 3}{3^{4 / 3} \Gamma(3 / 4)^{2 / 3}} S_{H T}\left(S_{C}, S_{1}\right) \pi^{2 / 3}
$$

as follows with equation $(5,9.15)$ and table 5.1. The numerical constant in the right hand sight of equation $(9.3 .34)$ is equal to 3.4320 , using the value of $I_{5}$ given by equation $(9.3 .6)$.

IV) Pencil beam regime For $\chi_{c} \ll<$ the distribution $\rho^{\star}\left(\Delta r^{*}\right)$ is dominated by the $k^{*}$-behaviour given by equation (9.3.17) 


$$
\left.\rho^{*}\left(\Delta r^{*}\right)=\frac{1}{2 \pi} \int_{0}^{\infty} k^{*} d k^{*} J_{0}\left(k^{*} \Delta r^{*}\right) e^{-C_{1} / 3 \pi\left[S_{P T}\left(S_{c}, S_{i}\right) V_{0} * 7\right.} k^{*}\right]^{1 / 3}
$$

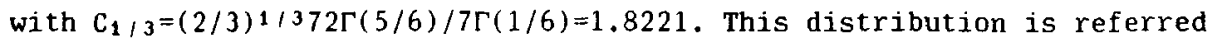
to as the two-dimensional pencil beam distribution, see figure 5.4 (curve corresponding to $\gamma=1 / 3$ ). The FWHM of this distribution is given by

$$
\text { FWHM }_{P} *=.013013 \frac{2}{3}\left(\frac{72 \Gamma(5 / 6)}{7 \Gamma(1 / 6)}\right)^{3} S_{P T}\left(S_{c}, S_{i}\right) v_{0} * 7 \pi^{3}
$$

as follows with equation (5.9.15) and table 5.1. The constant in the right hand sight of equation $(9.3 .36)$ is equal to 0.078729 .

In order to interpolate equations $(9.3 .29),(9.3 .32),(9.3 .34)$ and $(9.3 .36)$ we use

$$
\mathrm{FWHM}^{*}=\mathrm{FWHM}_{0} * \mathrm{H}_{\mathrm{CT}}\left(\pi, \mathrm{v}_{0}^{*}, \mathrm{~S}_{\mathrm{c}}, \mathrm{S}_{\mathrm{i}}\right), \mathrm{FWHM}_{0} *=3.4320 \pi^{2 / 3}
$$

where the function $H_{C T}\left(\lambda, v_{0}{ }^{*}, S_{c}, S_{i}\right)$ is defined as

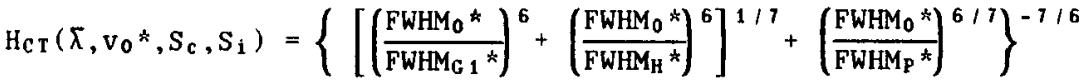

$$
\begin{aligned}
& +\left[\left(\frac{\text { FWHM }_{0}^{*}}{\text { FWHM }_{2^{*}}}\right)^{3}+\left(\frac{\text { FWHM }_{0}^{*}}{\text { FWHM }^{*}}\right)^{3}\right]^{1 / 3}
\end{aligned}
$$

which is identical to

$$
\begin{aligned}
& H_{C T}\left(\pi, v_{0}{ }^{*}, S_{c}, S_{i}\right)= \\
& \quad\left[\left(\frac{1}{S_{H T}\left(S_{c}, S_{i}\right)^{6}}+\frac{A \pi}{P_{C T 1}\left(v_{0}^{*}, S_{c}\right)^{6}}\right)^{1 / 7}+\frac{B}{V_{0}^{* 6} \lambda^{2} S_{P T}\left(S_{c}, S_{i}\right)^{6 / 7}}\right]^{-7 / 6} \\
& \quad+\left|S_{c}-S_{i}\right|\left(\frac{C \lambda^{1 / 2}}{V_{0}^{* 3 / 4}}+\frac{D}{V_{0}^{* 3} \lambda^{4}}\right)^{-1 / 3}
\end{aligned}
$$

in which the constants $A, B, C$ and $D$ are given by

$$
A=58.88, \quad B=25.42, \quad C=6.3810^{-3}, \quad D=5.41510^{-4}
$$

For the case $S_{c}=S_{i}$ equation $(9.3 .38)$ reduces to

$$
\begin{aligned}
& H_{\mathrm{CT}}\left(\pi, v_{0}{ }^{*}, S_{c}\right)=\left[\left(\frac{1}{S_{H T}\left(S_{c}\right)^{6}}+\frac{A}{P_{C T 1}\left(V_{0}{ }^{*}, S_{C}\right)^{6}}\right)^{1 / 7}+\frac{16 B}{\chi_{c}{ }^{2} S_{P T}\left(S_{c}\right)^{6 / 7}}\right]^{-7 / 6} \\
& \left(S_{c}=S_{i}\right)
\end{aligned}
$$

where the pencilbeam factor $\chi_{c}$ is defined by equation (7.5.28). The function $S_{\mathrm{HT}}\left(S_{\mathrm{c}}\right)$ can be approximated by the function $S_{\mathrm{HT}}\left(S_{c}\right)$ of equation $(9.3 .10)$. The function $S_{P T}\left(S_{c}\right)$ is given by equation (9.3.13). For comparison the fit of equations $(9.3 .37)$ and $(9.3 .38)$ is included in the figures 9.7 and 9.8 .

Similar results can be derived for the Full width median (FW5a) of the trajectory displacement distribution $\rho(\Delta r)$. The results for the individual regimes can be determined from the FWHM-values by means of equations (8.5.43). The general expression for the entire range of 
operating conditions becomes

$$
\mathrm{FW}_{50} *=1.2505 \mathrm{FWHM}_{0}^{*} \mathrm{H}_{\mathrm{C}} \mathrm{T}\left(\bar{\lambda}, \mathrm{V}_{0}^{*}, \mathrm{~S}_{\mathrm{c}}, \mathrm{S}_{\mathbf{1}}\right)
$$

similar to equation (9.3.37). The function $H_{C T}\left(\pi, V_{0} *, S_{C}, S_{i}\right)$ is again given by equation (9.3.38), now taking the constants $\mathrm{A}, \mathrm{B}, \mathrm{C}$ and $\mathrm{D}$ equal to

$$
A=225.2, \quad B=.1439, \quad C=1.2510^{-2}, \quad D=5.76810^{-8}
$$

instead of the values given by equation $(9,3.39)$.

By removing the scaling in equation (9.3.37) one obtains for the FWHM of the trajectory displacement distribution (using $\mathrm{FWHM}_{\mathrm{r}}=\delta \mathrm{FWHM}^{*}$ )

$$
\mathrm{FWHM}_{r}=\mathrm{C}_{\mathrm{CHT}} \mathrm{H}_{\mathrm{CT}}\left(\pi, \mathrm{v}_{0}^{*}, \mathrm{~S}_{\mathrm{C}}, \mathrm{S}_{i}\right) \frac{\mathrm{I}^{2 / 3} \mathrm{~L}^{2 / 3}}{\mathrm{~V}^{4 / 3} \alpha_{0^{4} / 3}}
$$

where the constant $\mathrm{C}_{\mathrm{CHT}}$ is given by

$$
\mathrm{C}_{\mathrm{CHT}}=2.6554 \frac{\Gamma(1 / 4)^{2 / 3} \mathrm{I}_{5}{ }^{2 / 3}}{2^{1 / 3} 3^{4 / 3} \pi \Gamma(3 / 4)^{2 / 3}} \frac{\mathrm{m}^{1 / 3}}{\epsilon_{0}}=0.17205 \frac{\mathrm{m}^{1 / 3}}{\mathrm{E}_{0}}
$$

using the numerical value of $I_{5}$ given by equation (9.3.6). In the case of electrons one finds $\mathrm{C}_{\mathrm{CHT}}=1.8837$ in SI-units. Equation (9.3.43), in combination with equation $(9.3 .38)$, constitutes the main result of this section. It gives the FWHM of the trajectory displacement distribution $\rho(\Delta r)$ generated in a homocentric beam segment with a crossover $\left(r_{c}=0\right)$ for the entire range of operating conditions.

We conclude this section by investigating the dependencies on the experimental parameters in the main regimes covered by equations $(9.3 .43)$ and $(9.3 .38)$. For $\lambda_{\approx 0.01}$ and $\chi_{c} \gg 1$ one may approximate

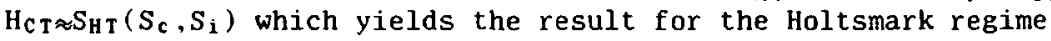

$$
F_{W H M}=C_{C H T} S_{H T}\left(S_{C}, S_{i}\right) \frac{I^{2 / 3} L^{2 / 3}}{V^{4 / 3} \alpha_{0}^{4 / 3}}
$$

which can also be found directly from equation (9.3.34).

For $\lambda_{\gtrsim 0.05}$ and $\chi_{c} \gg>1$ one obtains the result for the Gaussian regime

$$
\mathrm{FWHM}_{\mathrm{r}}=\mathrm{C}_{\mathrm{CGT}} \mathrm{P}_{\mathrm{CT}}\left(\mathrm{V}^{*} 0, S_{\mathrm{C}}, \mathrm{S}_{\mathrm{i}}\right) \frac{\mathrm{I}^{1 / 2} \mathrm{~L}^{2 / 3}}{\mathrm{~V}^{13 / 12 \alpha_{0}}}
$$

where the constant $\mathrm{C}_{\mathrm{CG}} \mathrm{T}$ is given by

$$
C_{C G T}=\frac{\left[P_{2}^{*}(\infty, 1 / 2,1 / 2) \ln 2\right]^{1 / 2}}{2^{1 / 12 \pi^{5 / 6}}} \frac{e^{1 / 12 m^{1 / 4}}}{\epsilon_{0}^{5 / 6}}
$$

in which the constant $p_{2} *(\infty, 1 / 2,1 / 2)$ is specified by equation $(9 \cdot 3.20)$. In the case of electrons one finds $\mathrm{C}_{\mathrm{cGT}}=0.215$ in SI-units.

For $S_{c} \neq S_{i}, \quad \chi_{c} \gg 1$ and intermediate $\lambda$-values one obtains the result for the weak complete collision regime. By removing the scaling in equation (9.3.32) it follows that

$$
\mathrm{FWHM}_{r}=\mathrm{C}_{C W A}\left|S_{c}-S_{i}\right| \frac{I^{2} L}{\alpha_{0}^{3} \mathrm{~V}^{3}}
$$

where the constant $\mathrm{C}_{\mathrm{CWA}}$ is defined in equation (8.3.24). Equation 
(9.3.46) and (8.3.24) fulfil equation (9.2.1), using that $\Delta \alpha=\Delta v_{1} / v_{z}$. This implies that the spatial broadening described by equation $\left(9 . \frac{1}{3} .46\right)$ is the result of the angular deflections produced by weak complete collisions.

For $\chi_{c} \ll<1$ equation $(9.3 .38)$ gives $H_{C T} \approx 9.033 \times 10^{-4} S_{P T}\left(S_{C}, S_{i}\right) \chi_{c} 7 / 3$. Substitution in equation (9.3.43) yields

$$
\mathrm{FWHM}_{\mathrm{r}}=\mathrm{C}_{C \mathrm{PT}} \mathrm{S}_{\mathrm{PT}}\left(\mathrm{S}_{\mathrm{C}}, S_{\mathrm{i}}\right) \frac{\mathrm{I}^{3} \alpha_{0} \mathrm{~L}^{3}}{\mathrm{~V}^{5} / 2}
$$

where the constant $\mathrm{C}_{\mathrm{CPT}}$ is given by

$$
\mathrm{C}_{\mathrm{CPT}}=.013013\left(\frac{18 \Gamma(5 / 6)}{7 \Gamma(1 / 6)}\right)^{3} \frac{1}{6 \pi \sqrt{2}} \frac{\mathrm{m}^{3 / 2}}{\varepsilon_{0} \mathrm{e}^{7 / 2}}=6.921810^{-5} \frac{\mathrm{m}^{3 / 2}}{\epsilon_{0} \mathrm{e}^{7 / 2}}
$$

Equation (9.3.47) can also be derived dirertly from equation (9.3.36). In the case of electrons one finds $\mathrm{C}_{\mathrm{CPT}}=4.1287 \times 10^{27}$ in SI-units.

\subsection{Homocentric cylindrical beam segment}

In this section we will consider the case of a monochromatic homocentric cylindrical beam, which is succeeded by a lens. The angular displacement $\Delta \alpha=\Delta v_{1} / v_{z}$ experienced by a particle in the cylindrical beam segment results in a spatial shift $\Delta \mathrm{r}_{\mathrm{foc}}$ in the backfocal plane of the lens which is given by equation (9.2.2). It should be emphasized that $\Delta r_{f_{0}}$ is entirely determined by $\Delta a$ only and does not depend on the spatial displacement experienced by the particle in the cylindrical beam segment. Accordingly, the trajectory displacement distribution $\rho\left(\Delta r_{f_{0}}\right)$ in the backfocal plane of the lens is fully determined by the distribution $\rho\left(\Delta v_{\perp}\right)$ generated in the cylindrical beam segment. This distribution was calculated in section 8.4 .

Combining equations $(9.2 .2)$ and $(8.4 .28)$ the FWHM of the distribution $\rho\left(\Delta r_{f_{0}}\right)$ can in general be expressed as

$$
\mathrm{FWHM}_{\mathrm{r}}=\mathrm{FWHM}_{\alpha} \mathrm{f}=\mathrm{C}_{\mathrm{PHA}} \mathrm{H}_{\mathrm{PA}}\left(\lambda^{*}, \mathrm{r}_{0^{*}}\right) \frac{\mathrm{I}^{2 / 3} \mathrm{Lf}}{\mathrm{V}^{4} / 3 \mathrm{r}_{0} / 3}
$$

in which $f$ denotes the focal distance of the lens. The constant $C_{P H A}$ and the function $\mathrm{H}_{\mathrm{PA}}\left(\lambda^{*}, \mathrm{v}_{0}{ }^{*}\right)$ are given by equations $(8.4 .28)$ and $(8.4 .26)$ respectively. Equation (9.4.1) covers the entire range of operating conditions. As described in section 8.4, three regimes should be distinguished by the type of distribution which is generated : The Gaussian regime, the Holtsmark regime and the pencil beam regime. The corresponding results for the individual regimes can directly be obtained from equations $(8.4 .30),(8.4 .29)$ and $(8.4 .31)$ respectively, again using $\mathrm{FWHM}_{\mathrm{r}}=\mathrm{FWHM}_{\alpha} \mathrm{f}$.

It is interesting to compare the effects generated in a homocentric cylindrical beam with radius $r_{0}$ to those generated in a homocentric beam segment with a crossover in the middle and beam semiangle $\alpha_{0}=2 r_{0} / L$. The length $L$ is the same for both geometries. This choice of parameters implies that the beam radius at the entrance and the exit plane of the beam segment is the same for both geometries, see figure 9.9. Assume that a lens focusses the beam in a spot at a distance $L / 2$ from the end of the beam segment. For the crossover this implies that the transverse magnification from the crossover to this image plane equals unity. For the cylindrical beam it implies that $f=L / 2$. We now define the ratio $R$ as 


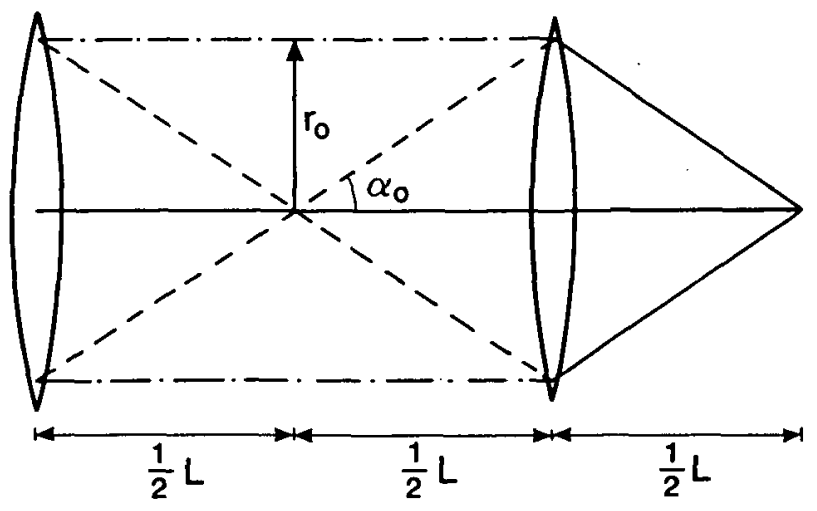

Figure 9.9 Schematic view of two beam geometries in a segment of length $\mathrm{L}$ between two lenses. One is a homocentric cylindrical beam $\left(\alpha_{0}=0\right)$ and the other is a homocentric beam segment with a crossover in the middle $\left(r_{\mathrm{c}}=0, S_{\mathrm{c}}=1 / 2\right)$. The corresponding contributions to the trajectory displacement effect observed in the succeeding crossover (located at a distance $\mathrm{L} / 2$ after the second lens) are compared, see equations (9.4.4) and (9.4.6).

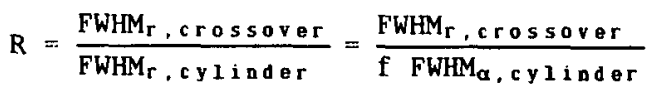

Substitution of equations (9.3.43) and (9.4.1) yields

$$
\mathrm{R}=\frac{\mathrm{C}_{C H T} H_{C T}\left(\pi, v_{0}^{*}, 1 / 2,1 / 2\right)}{21 / 3 C_{P H A} H_{P A}\left(4 \pi, v_{0}^{*} / 2\right)}=1.3418 \frac{H_{C T}\left(\pi, v_{0}^{*}, 1 / 2,1 / 2\right)}{H_{P A}\left(4 \pi, v_{0}^{*} / 2\right)}
$$

where it was used that $r_{0}=\alpha_{0} L / 2$ and $f=L / 2$, which implies $r_{0}^{*}=v_{0}^{*} / 2$ and $\lambda^{*}=4 \pi$. The last relation follows from the definitions of $\lambda$ and $\lambda^{*}$, given by equations $(7,3.22)$ and $(7.4 .18)$ respectively.

The results for the individual regimes can be determined from equation $(9.4 .3)$ or directly from equation $(9.4 .2)$ by substituting the corresponding results for the FWHM-values. For the Gaussian regime one finds

$$
\mathrm{R}_{\mathrm{G}}=.948 \frac{\mathrm{P}_{\mathrm{CT}}\left(\mathrm{V}_{0} * 1 / 2,1 / 2\right)}{\mathrm{P}_{\mathrm{PA}_{\mathrm{A}}}\left(\mathrm{V}_{0} * / 2\right)} \quad \text { (Gaussian regime) }
$$

Utilizing equations $(9.3 .25),(9.3 .26),(8.4 .20)$ and $(8.4 .16)$ this gives

$$
\lim _{V_{0^{*} \rightarrow \infty}} R_{G}=0.948, \quad \lim _{V_{0}^{*}+0} R_{G}=0.116
$$

Practical conditions correspond to $v_{0}{ }^{\star}>1$, for which $R_{G} \approx 0.948$. Similarly, one finds for the Holtsmark regime and the pencil beam regime 


$$
\begin{aligned}
& R_{H}=1.34 \\
& R_{P}=0.333
\end{aligned}
$$

(Holtsmark regime)

(Pencil beam regime)

$(9.4 .6)$

Equations (9.4.5) and (9.4.6) show that the ratio $R$ of the magnitude of the trajectory displacement effect,generated in the two different geometries, is in general a simple numerical constant, which differs slightly for the various regimes. However, for all regimes the ratio $R$ is close to unity, which implies that the size of the trajectory displacement effect generated in the beam segment with a crossover is of the same order of magnitude as the effect generated in the cylindrical beam.

On first sight this result is perhaps somewhat surprising. clearly, the particle density becomes much higher in the case of a crossover than for a cylindrical beam. For that reason one might conjecture that the trajectory displacement effect generated in the crossover is considerably larger too. Indeed, the deviations from the unperturbed trajectories will be larger in the case of a crossover than for a cylindrical beam. However, what counts is the virtual broadening in the reference plane. Here, one should realize that the final lateral spatial shift and velocity shift generated in the segment with a crossover are partly cancelled out by the extrapolation to a virtual position in the crossover plane. Our calculation shows that the resulting virtual broadening is of the same order of magnitude as the broadening generated in a cylindrical beam. It should be noted that the situation is quite different for the Boersch effect, as the reader might verify from the results presented in chapter 7 . The Boersch effect, which is related to the generated axial velocity spread, will be much larger for the crossover case than for the cylindrical beam.

\subsection{Beam segment with a crossover of arbitrary dimensions}

So far we considered the trajectory displacement effect generated in the extreme cases of a homocentric beam segment with a crossover and a homocentric cylindrical beam segment respectively. We now wish to consider the general case of a beam segment with a crossover which has some finite non-zero radius $r_{c}$. For a homocentric crossover $\left(r_{c}=0\right)$ it was found that the trajectory displacement effect is, in general, a function of four theoretical parameters, namely $\bar{\lambda}, v_{0} *, S_{c}$ and $S_{i}$, as is formally expressed by equation (9.3.37). For a crossover of arbitrary dimensions the results will, in addition, depend on the scaled crossover radius $r_{c}{ }^{*}=r_{c} / \delta$ and one ends up with five independent parameters. We note that it is sometimes more convenient to use the $d_{0}, v_{0}$-scaling, defined by equation (7.3.2), instead of the $\delta, v$-scaling, given by equation (6.13.1). In this representation the parameters $v_{0}$ * and $r_{c}{ }^{*}$ are replaced by the parameters $K$ and $\bar{r}_{c}$. The relation between both sets of parameters is given by equation (7.5.6). Clearly, in both representations one requires five independent theoretical parameters to describe the general case.

A straightforward analysis of the dependency on all five parameters is rather involved. In principle, one could extend the approach outlined in section 9.3 to include the dependency on $r_{c}{ }^{*}$. However, such an approach would require an extreme large number of numerical evaluations of the two-particle distribution $\rho^{*_{2}}\left(\Delta r^{*}\right)$, while each evaluation now requires the solution of a four-dimensional integral instead of a two-dimensional integral, as can be seen from equation (9.2.11). We therefore did not attempt a systematic approach to this 
problem. Instead we will use an approximate method starting from the results obtained for the extreme cases of a homocentric beam segment with a crossover and a homocentric cylindrical beam segment.

In order to get some indication of the dependency on the scaled crossover radius $r_{c}{ }^{*}$ we evaluated the case $S_{c}=S_{1}=1 / 2$ and $v_{0}^{*}=200$ for various values of $r_{c}$ * employing our numerical model. The results for the scaled FWHM of the trajectory displacement distribution $\rho^{*}\left(\Delta r^{*}\right)$ are plotted in figure 9.10. We recall that for practical operating conditions the scaling length $\delta$ is of the order $10^{-5} \mathrm{~m}$ (for $\mathrm{V}=10 \mathrm{kV}$ and $L=0.1 \mathrm{~m}$ one finds $\delta=14.2 \mu \mathrm{m})$. Accordingly, the data of figure 9.10 indicates that the dependency on $r_{c}$ * is rather weak for the investigated circumstances. Thus, contrary to the Boersch effect, the trajectory displacement effect generated in a narrow crossover is not very sensitive to the crossover radius.

We will now exploit the slice-method, which is outlined in section 5.11, to investigate the dependency on the parameters $v_{0} *$ and $r_{c}$ * (or $K$ and $\bar{r}_{c}$ ) in a more systematic way. The analysis presented here is similar to that of section 8.6 , concerning the distribution $\rho\left(\Delta v_{1}\right)$. The essence of the slice-method is expressed by equation (5.11.1). This equation states that one may calculate the total displacement generated in a beam segment by adding linearly the displacements generated in a large number of thin cylindrical slices. The basic assumption is that the contributions of the individual slices to the total displacement experienced by the test particle are entirely correlated. Such is to be expected when the deviations from the unperturbed trajectories are small. Accordingly, one should only apply the slice-method when the distribution $\rho(\Delta r)$ is dominated by weak collisions. This type of analysis is therefore restricted to the Holtsmark regime and the pencil beam regime.

Let us first consider the Holtsmark regime. According to equation (8.4.29) the contribution of a single slice at axial position $z$ of length $\Delta z$ to the FWHM of the total trajectory displacement distribution $\rho(\Delta r)$ can be expressed as

$$
\Delta \mathrm{FWHM}_{r}=C_{P H A} \frac{I^{2 / 3}}{V^{4 / 3} r_{c^{4 / 3}}} \frac{\left(z-z_{i}\right) \Delta z}{\left[1+\alpha_{0}\left|z-z_{c}\right| / r_{C}\right]^{4 / 3}}
$$

in which $z_{c}$ and $z_{i}$ denote the $z$-coordinates of the crossover and the image plane respectively. For the calculation of the distribution $\rho(\Delta r)$ in a beam segment with a narrow crossover we will use

$$
F W H M_{r}=\left|\int_{z_{0}}^{z_{1}} d z \operatorname{sign}\left(z-z_{c}\right) \frac{\Delta F W H M_{r}}{\Delta z}\right|
$$

The sign-function is included to account for the fact that the angular deflection experienced by the test particle during a weak collision with a single field particle changes of sign when the field particle crosses the beam axis (or in fact the reference trajectory which is here identical to the beam axis). The quantity $\triangle F W_{H M}$, given by equation (9.5.1), can become negative depending on the sign of the quantity $z-z_{i}$. Notice that the sign-changes of the quantities $z-z_{c}$ and $z-z_{i}$ cancel out when $z_{c}=z_{i}$. Equation (9.5.2) presupposes that the field particle crosses the axis exactly in the crossover. This approximation is only justified when the crossover is narrow $(K>>1)$. Equation (9.5.2) is therefore not suited to describe a nearly cylindrical beam segment. However, the extreme case of a cylindrical segment $(K=0)$ is covered too by taking $z_{c}=z_{0}$ or $z_{c}=z_{1}$.

Substitution of equation (9.5.1) into equation (9.5.2) and 
integration yields equation $(9.3 .44)$, where the function $S_{H} r\left(S_{c}, S_{i}\right)$ is now replaced by

$$
S_{H T a}\left(S_{c}, S_{i}, K\right)=\left|\frac{3 S_{c}-2 S_{i}+3 / 2 K}{\left(1 / K+2 S_{c}\right)^{1 / 3}}+\frac{3\left(1-S_{c}\right)-2\left(1-S_{i}\right)+3 / 2 K}{\left[1 / K+2\left(1-S_{c}\right)\right]^{1 / 3}}-\frac{3}{K^{2 / 3}}\right|
$$

in which we approximated

$$
\frac{C_{P H A}}{C_{C H T}} \frac{3}{2^{2 / 3}}=1.1179 \approx 1
$$

The constants $\mathcal{C}_{\mathrm{PHA}}$ and $\mathrm{C}_{\mathrm{CHT}}$ are defined in equations (8.4.28) and (9.3.43) respectively. The function $S_{H T a}\left(S_{c}, S_{i}, K\right)$ is the generalization of the function $S_{H T a}\left(S_{c}, S_{i}\right)$ given by equation (9.3.7). Both equations become identical for $K \rightarrow \infty$. As was mentioned previously, one should realize that the derivation of equation (9.5.3) presupposes $K>1$. However, correct results for $K \rightarrow 0$ are obtained if one takes $S_{c}=0$ or $S_{c}=1$. Let us now consider the pencil beam regime. According to equation ( 8.4 .31 ) the contribution of a single slice at axial position $z$ of length $\Delta z$ to the total distribution of trajectory displacements $\rho(\Delta r)$ can be expressed as

$$
\Delta \text { FWHM }_{r}=C_{P P A} \frac{I^{3} r_{c}}{V^{5 / 2}}\left[1+\alpha_{0}\left|z-z_{c}\right| / r_{c}\right]\left(z-z_{i}\right) \Delta z
$$

Substitution into equation (9.5.2) and integration yields equation (9.3.47), where the function $S_{P T}\left(S_{c}, S_{i}\right)$ is now replaced by

$$
S_{P_{\mathrm{Pa}}}\left(S_{\mathrm{c}}, S_{i}, K\right)=\left|4-6\left(S_{c}+S_{i}\right)+12 S_{c} S_{i}+\frac{3-6 S_{c}{ }^{2}-6 S_{i}\left(1-2 S_{c}\right)}{K}\right|
$$

in which we used that

$$
\mathrm{C}_{\mathrm{PPA}}=12 \mathrm{C}_{\mathrm{CPT}}
$$

as follows from the definitions of the constants $C_{C P A}$ and $C_{C P T}$. The function $S_{P T a}\left(S_{c}, S_{i}, K\right)$ is the generalization of the function $S_{P T a}\left(S_{c}, S_{i}\right)$ given by equation (9.3.12). These equations become identical for $K \rightarrow \infty$. As in the case of the Holtsmark regime it should be noted that equation (9.5.6) is only valid for $K \gg \gg 1$, while correct results for $K \rightarrow 0$ are obtained if one takes $S_{c}=0$ or $S_{c}=1$.

The remaining task is to extend the results for the Gaussian regime and the weak complete collision regime. For the latter it was found that the scaled FWHW follows equation (9.3.32). This result is directly related to the corresponding FWHM of the angular deflection distribution, which is specified by equation (8.3.24). Accordingly, it is reasonable to extend equation $(9.3 .32)$ for the case $r_{c} \neq 0$ as

$$
\text { FWHM }^{*}=42.105\left|S_{c}-S_{i}\right|\left(1+.08275 v_{0} * 2 r_{c} *\right) v_{0} * \lambda^{2}
$$

using the approximation of $f_{\infty}\left(\bar{r}_{c}\right)$ given by equation $(8.3 .10)$ and the relation $\overline{\mathrm{r}}_{\mathrm{c}}=4 \mathrm{v}_{0}{ }^{* 2} \mathrm{r}_{\mathrm{c}} *$.

In order to obtain a systematic analysis of the dependency of the FWHM in the Gaussian regime on the parameters $v_{0}, r_{c} * S_{c}$ and $S_{i}$ one should extend the numerical calculations of the mean square value $\langle\Delta r * 2\rangle$ to include non-zero $r_{c}{ }^{*}$-values. As we did not perform such a systematic analysis and we will just present some provisional results to cover this case. The data of figure 9.10 shows that the FWHM of the 


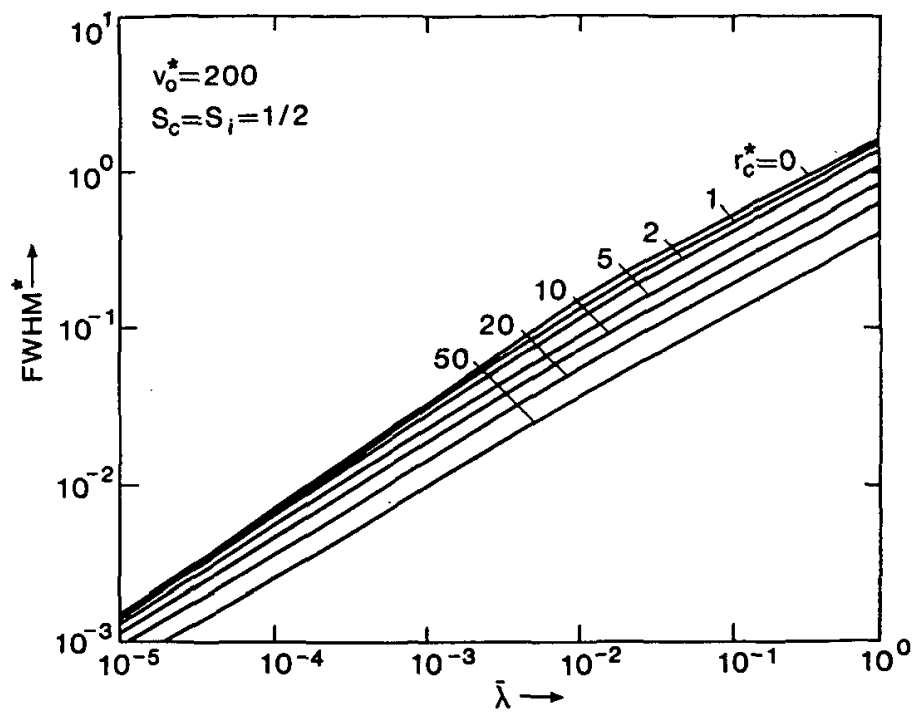

Pigure 9.10 The FWHM of the trajectory distribution $\rho^{*}\left(\Delta \mathrm{r}^{*}\right)$ generated in a beam segment with a crossover in the middle $\left(S_{\mathrm{c}}=1 / 2\right)$, plotted for different values of the scaled crossover radius $r_{c}{ }^{*}$. The results represent the virtual displacements observed in the crossover plane $\left(S_{i}=S_{c}\right)$ and pertain to a beam for which the maximum transverse velocity $v_{0} * 200$.

scaled trajectory displacement distribution falls of with $r_{c}$ as

$$
\text { FWHM }_{G 1} *=\frac{\text { FWHM }_{G 1}^{*}\left(r_{c}^{*}=0\right)}{\left(1+0.30 r_{c}^{*}\right)^{1 / 2}} \quad\left(S_{c}=S_{i}, v_{0}^{*} * 200\right)
$$

Therefore, we generalize the function $\mathrm{P}_{\mathrm{CT}}$ specified by equation $(9.3 .26)$ as

$$
\operatorname{PcT~} 1_{1}\left(v_{0}^{*}, r_{c}^{*}, S_{c}\right)=\frac{\left[1+0.682\left(S_{c}-0.5\right)-0.739\left(S_{c}-0.5\right)^{2}\right]^{1 / 2}}{\left(1+1.40 / v_{0}^{* 8 / 7}\right)^{7 / 4}\left(1+0.30 r_{c}^{*}\right)^{1 / 2}}
$$

which should yield accurate results for most conditions, but may overestimate the exact result for small $v_{0}^{*}$-values in combination with large $r_{c}{ }^{*}$-values. These conditions pertain to a nearly cylindrical extended beam. Equation $(9.3 .26)$ is relevant for the case $S_{c}=S_{i}$. We did not further investigate the case $S_{c} \neq S_{i}$ and we will use equation (9.3.31) also for non-zero $r_{c}{ }^{*}$-values.

The modifications outlined above have to be introduced in the general expression for the FWHM of the trajectory displacement distribution given by equation (9.3.43). This equation remains valid for a beam segment of arbitrary dimensions, provided that one replaces the original function $H_{C T}\left(\lambda, v_{0}^{*}, S_{c}, S_{i}\right)$ by a function $H_{C T}\left(\pi, v_{0}^{*}, r_{c}^{*}, S_{c}, S_{i}\right)$ which depends also on $r_{c}{ }^{*}$. Similar to the derivation of equation (9.3.38) it was found that 
$H_{C T}\left(\pi, v_{0}^{*}, r_{c}{ }^{*}, S_{c}, S_{i}\right)=$

$$
\begin{aligned}
& {\left[\left(\frac{1}{S_{H T}\left(S_{c} ; S_{i}, K\right)^{6}}+\frac{A \lambda}{P_{\mathrm{CT} 1}\left(V_{0}^{*}, r_{c}^{*}, S_{c}\right)^{6}}\right)^{1 / 7}+\frac{B}{V_{0}^{* 6} \lambda^{2} S_{P T}\left(S_{c}, S_{i}, K\right)^{6 / 7}}\right]^{-7 / 6}} \\
& \quad+\left|S_{c}-S_{i}\right|\left(\frac{C \lambda^{1 / 2}}{V_{0}^{* 3 / 4}}+\frac{D}{\left(1+.08272 v_{0}^{* 2} r_{c}\right)^{3} v_{0}{ }^{* 3} \lambda^{4}}\right)^{-1 / 3}
\end{aligned}
$$

The constants $\mathrm{A}, \mathrm{B}, \mathrm{C}$ and $\mathrm{D}$ remain the same and are specified by equation (9.3.39). The functions $S_{H T}\left(S_{c}, S_{i}, K\right), S_{P T}\left(S_{c}, S_{i}, K\right)$ can be approximated by the expressions given by equations $(9.5 .3)$ and $(9.5 .6)$ respectively, while the function $\mathrm{P}_{\mathrm{Cr}_{1}}\left(\mathrm{v}_{0}{ }^{*}, \mathrm{r}_{\mathrm{c}}{ }^{*}, S_{\mathrm{c}}\right)$ is defined by equation (9.5.10). Notice that $K=v_{0}^{*} / 2 r_{c}{ }^{*}$, as expressed by equation (7.5.6). Correct results for $K \rightarrow 0$ are obtained by substituting $S_{c}=0$ or $S_{c}=1$, as was mentioned in the derivation of the functions $S_{\mathrm{HT}}\left(\mathrm{S}_{\mathrm{c}}, \mathrm{S}_{\mathrm{i}}, \mathrm{K}\right)$ and $\mathrm{SPTa}_{\mathrm{P}}\left(\mathrm{S}_{\mathrm{c}}, \mathrm{S}_{\mathrm{i}}, \mathrm{K}\right)$. Equations (9.3.43) and (9.5.11) constitute the main results of this chapter. The modifications required to obtain the Full width median value $F_{5} 0$ are indicated by equations (9.3.41) and $(9.3 .42)$.

\subsection{Trajectory displacement and angular deflection distribution}

In this section we will investigate the results of the previous section for the limiting cases $S_{i}+\infty$ and $K \rightarrow 0$. Our objective is to clarify the relation with the equations for the FWHM of the distribution of angular deflections presented in chapter 8 . The results for the different regimes involved will be investigated separately.

According to equation (9.2.1) the relation between FWHM $_{r}$ and $\mathrm{FWHM}_{\alpha}$ can in general be expressed as

$$
\text { FWHM }_{\alpha}=\lim _{S_{i} \rightarrow \pm \infty} \frac{\text { FWHM }_{r}}{\left|S_{i} L\right|}
$$

utilizing that $T=L / V_{z}$ and $\Delta a n \Delta v_{1} / V_{2}$. Equation (9.6.1) expresses that the trajectory displacement effect observed in a far removed reference plane is entirely the result of angular deflections.

For the Holtsmark regime the FWHM of the trajectory displacement distribution is given by equation (9.3.44). For the general case the function $S_{H T}$ can be approximated by the function $S_{H T a}$, which is given by equation $(9.5 .3)$. Substitution into equation $(9.6 .1)$ yields

$$
F_{W H M}=C_{C H ~}\left|\frac{2}{\left(1 / K+2 S_{C}\right)^{1 / 3}}-\frac{2}{\left[1 / K+2\left(1-S_{C}\right)\right]^{1 / 3}}\right| \frac{I^{2 / 3}}{V^{4 / 3} \alpha_{0}^{4 / 3} L^{1 / 3}}
$$

which is in agreement with equations (8.5.31) and (8.5.20), approximating that

$$
\frac{C_{C H A}}{C_{C H T}}=\left(\frac{3^{5} \pi \Gamma(3 / 4)}{2^{4} 5^{2} I_{5}^{2} \Gamma(1 / 4)^{2}}\right)^{1 / 3}=1.1179 \approx 1
$$

equivalent to equation (9.5.4). The constant $I_{5}$ is given by equation $(9.3 .6)$.

For the pencil beam regime the FWHM of the trajectory displacement distribution is given by equation (9.3.47). The function $S_{P T}$ can be approximated by the function $S_{P T a}$, which is given by equation (9.5.6). Substitution into equation $(9.6 .1)$ yields 


$$
\mathrm{FWHM}_{\alpha}=6 \mathrm{C}_{\mathrm{CPT}}\left|1-2 \mathrm{~S}_{\mathrm{c}}\right|\left(\frac{1}{\mathrm{~K}}+1\right) \frac{\mathrm{I}^{3} \alpha_{0} \mathrm{~L}^{2}}{\mathrm{~V}^{5} / 2}
$$

which is in agreement with equations (8.5.35) and (8.5.21), using that

$$
6 \mathrm{C}_{\mathrm{CPT}}=\mathrm{C}_{\mathrm{CPA}}
$$

as follows from the definition of the constants $\mathrm{C}_{\mathrm{CPT}}$ and $\mathrm{C}_{\mathrm{CPA}}$.

For the weak complete collision regime one may verify that substitution of equation (9.3.46) into equation (9.6.1) directly leads to the FWHM $\alpha$ given by equation $(8.3 .24)$. Notice that equation $(9.3 .46)$ pertains to the case $r_{c}=0$. For the general case the function $f_{\infty}\left(\vec{r}_{c}\right)$ was included, using that $\bar{r}_{c}=4 v_{0} * 2 r_{c} *$, as expressed by equation (9.5.8). For the Gaussian regime one can not simply relate the results obtained for FWHM $_{r}$ to those obtained for FWHM $\alpha$, as was discussed in section 9.2.

Another extreme case which is of interest is that of a nearly cylindrical beam. In terms of the parameter $K$, which is defined by equation (7.3.1), this case corresponds to $K \rightarrow 0$. We will demonstrate that the following relation between FWHM $\alpha$ and $\mathrm{FWHM}_{r}$ should exist when weak collisions are dominant

$$
\mathrm{FWHM}_{\mathrm{r}}=\left|\mathrm{S}_{\mathbf{i}}-1 / 2\right| \mathrm{L} \mathrm{FWHM}_{\alpha} \quad(K \rightarrow 0 \text {, weak collisions })
$$

This equation can be understood from the observation that a weak collision in a homocentric cylindrical beam segment leads to a virtual spatial displacement $\Delta r$ which is proportional to the distance from the reference plane to the middle of the beam segment $\left(=\left|S_{i}-1 / 2\right| L\right)$ times the total angular displacement $\Delta \alpha$ caused by the collision. This observation can be understood as follows. Assume that a field particle exerts a lateral force $F_{\perp}$ on the test particle during the flight through the beam segment from $z_{0}$ to $z_{1}$. As the particles are initially running along parallel trajectories and only experience a small deviation from these trajectories the force $F_{\perp}$ will approximately be constant. The angular displacement $\Delta \alpha$ of the test particle can therefore be expressed as

$$
\Delta \alpha=\int_{z_{0}}^{z_{1}} \mathrm{dz} \frac{F_{\perp}}{m_{z_{2}}{ }^{2}}=\frac{F_{\perp} L}{m v_{z}{ }^{2}}
$$

with $L=z_{1}-z_{0}$. Similarly, the virtual displacement $\Delta r$ in a reference plane at $z=z_{r}$ can be expressed as

$$
\Delta r=\int_{z_{0}}^{z_{1}} d z\left|z_{r}-z\right| \frac{F_{\perp}}{m v_{z}^{2}}=\left|\left(z_{0}-z_{r}\right)^{2}-\left(z_{1}-z_{r}\right)^{2}\right| \frac{F_{\perp}}{2 m v_{z}^{2}}=\left|S_{i}-1 / 2\right| \frac{F_{\perp} L^{2}}{m_{z_{2}}^{2}}
$$

using that $z_{r}-z_{0}=S_{1} L$. The derivation of equation (9.6.8) is equivalent to that of equation (5.3.7). From equations $(9.6 .7)$ and $(9.6 .8)$ one finds

$$
\Delta r=\left|S_{i}-1 / 2\right| L \Delta \alpha
$$

By assuming that this result applies to all interactions of the test particle with the individual field particles one obtains equation 
$(9.6 .6)$.

Let us now verify whether our results based on weak interactions fulfil equation (9.6.6). The analysis of the previous sections showed that weak incomplete interactions in an extended beam generate a Holtsmark type of distribution with a FWHM which is given by equation (9.3.44). The FWHM of the distribution generated by weak incomplete interactions in a pencil beam is given by equation (9.3.47). Weak complete collisions are absent for $\mathrm{K} \rightarrow 0$ and the corresponding regime becomes only manifest for $K \gg>1$. The Gaussian regime is dominated by strong collisions and the corresponding results will not fulfil equation (9.6.6). Accordingly, the analysis here should be restricted to the Holtsmark regime and the pencil beam regime.

The dependency of FWHM $_{r}$ on $S_{c}, S_{i}$ and $K$ is for the Holtsmark regime described by the function $S_{H T a}\left(S_{c}, S_{i}, K\right)$, specified by equation $(9.5 .3)$. The derivation of this function presupposes that $S_{c}=0$ or $S_{c}=1$ for $K \rightarrow 0$. By power expansion one finds

$$
\lim _{\mathrm{K} \rightarrow 0} S_{\mathrm{HT}}\left(S_{\mathrm{c}}, S_{\mathrm{i}}, \mathrm{K}\right)=\frac{4}{3} \mathrm{~K}^{4 / 3}\left|\frac{1}{2}-S_{\mathrm{c}}{ }^{2}-S_{\mathrm{i}}+2 \mathrm{~S}_{\mathrm{c}} \mathrm{S}_{\mathrm{i}}\right|
$$

Taking $S_{c}=0$ or $S_{c}=1$ one obtains with equation $(9.3 .44)$

$$
\lim _{\mathrm{k} \rightarrow 0} \mathrm{FWHM}_{\mathrm{r}}=\mathrm{C}_{\mathrm{CHT}} \frac{2^{2 / 3}}{3}\left|\frac{1}{2}-\mathrm{S}_{\mathrm{i}}\right| \frac{\mathrm{I}^{2 / 3} \mathrm{~L}^{2}}{\mathrm{~V}^{4 / 3} \mathrm{r}_{\mathrm{c}}{ }^{4 / 3}}
$$

Similarly, one obtains from equation (8.5.20)

$$
\lim _{\mathrm{K} \rightarrow 0} S_{\mathrm{HAa}}\left(S_{c}, K\right)=\frac{4}{3} \mathrm{~K}^{4 / 3}\left|1-2 S_{\mathrm{c}}\right|
$$

Taking $S_{\mathbf{c}}=0$ or $S_{\mathbf{c}}=1$ this gives with equation $(8,5.31)$

$$
\lim _{\mathrm{R} \rightarrow 0} \mathrm{FWHM}_{\alpha}=\mathrm{C}_{\mathrm{CHA}} \frac{2^{2 / 3}}{3} \frac{\mathrm{I}^{2 / 3} \mathrm{~L}^{2}}{\mathrm{~V}^{4 / 3} \mathrm{Y}_{\mathrm{c}}{ }^{4 / 3}}
$$

Indeed equations $(9.6 .10)$ and $(9.6 .11)$ satisfy equation (9.6.6) within the approximation of equation $(9.6 .3)$.

The dependency of $\mathrm{FWHM}_{r}$ on $S_{c}, S_{i}$ and $K$ is for the pencil beam regime described by the function $S_{P T}\left(S_{c}, S_{i}, K\right)$, specified by equation $(9.5 .6)$. By power expansion one finds

$$
\lim _{\mathrm{K} \rightarrow 0} S_{P T a}\left(S_{c}, S_{i}, K\right)=\frac{6}{K}\left|\frac{1}{2}-S_{c} 2-S_{i}\left(1-2 S_{c}\right)\right|
$$

Taking $S_{c}=0$ or $S_{c}=1$ one obtains with equation $(9.3 .47)$

$$
\lim _{\mathrm{k} \rightarrow 0} \mathrm{FWHM}_{\mathrm{r}}=12 \mathrm{C}_{\mathrm{CPT}}\left|\frac{1}{2}-\mathrm{S}_{\mathrm{i}}\right| \frac{\mathrm{I}^{3} \mathrm{r}_{\mathrm{C}} \mathrm{L}^{2}}{\mathrm{~V}^{5 / 2}}
$$

From equation $(8.5 .21)$ one obtains

$$
\lim _{K \rightarrow 0} S_{P A a}\left(S_{c}, K\right)=\frac{1}{K}\left|1-2 S_{c}\right|
$$

Taking $S_{c}=0$ or $S_{c}=1$ this gives with equation (8.5.35)

$$
\lim _{\mathrm{R} \rightarrow 0} \mathrm{FWHM}_{\alpha}=2 \mathrm{C}_{C P A} \frac{\mathrm{I}^{3} \mathrm{r}_{\mathrm{C}} \mathrm{L}}{\mathrm{V}^{5 / 2}}
$$

Utilizing the relation between the constants $\mathrm{C}_{C P T}$ and $\mathrm{C}_{C \mathrm{PA}}$ given by equation $(9.6 .5)$ one sees that equations $(9.6 .12)$ and $(9.6 .13)$ satisfy 
equation $(9.6 .6)$.

\subsection{Results for Gaussian angular and spatial distributions}

The results of the previous sections apply to the case of an uniform spatial and an uniform angular distribution. Similar to the analysis of the sections 7.6 and 8.7 we will now determine the effective width measures, $r_{e f f}$ and $\alpha_{e f f}$ of a Gaussian spatial and angular distribution respectively for which the equations obtained for uniform distributions yield accurate results. We repeated the calculations outlined in this chapter, now starting from the modified versions of equation (9.2.11) which are obtained by replacing either the uniform distribution in $v$ or the uniform distribution in $r_{\perp}$ or both distributions by their Gaussian counterparts given by equations (7.6.1) and (7.6.2) respectively. As the results are quite similar to those of section 8.6 we will not go into detail.

For the Gaussian regime the numerical calculations of the mean square broadening $\left\langle\Delta r^{*}\right\rangle$ were reconsidered. It was found that the equations obtained for the case of an uniform angular and an uniform spatial distribution can be retrieved by using

$\alpha_{\text {eff }}=\sqrt{2} \sigma_{\alpha}, \quad r_{\text {eff }}=\sqrt{2} \sigma_{r} \quad$ (Gaussian regime)

The weak complete collision regime is entirely determined by angular deflections, as was discussed previously. Accordingly, one should use equation $(8,7.12)$ for the calculation of the effective width measures of the Gaussian spatial and angular distribution.

For the Holtsmark regime and the pencil beam regime the trajectory displacement effect is directly related to the distribution of the lateral interaction force $\rho\left(F_{t}\right)$ occurring in the beam. As the same holds true to for the corresponding regimes of the angular deflection distribution we may take over the analysis of section 8.7. For the Holtsmark regime the effective width measures should therefore be calculated from equation $(8.7 .15)$. For the pencil beam regime one should use equation $(8.7 .17)$.

The FWHM of the trajectory displacement distribution generated in a beam segment with a crossover is in the case of an uniform angular and an uniform spatial distribution given by equations $(9.3 .43)$ and (9.5.11). The different regimes are implicitly covered by these equations and the question arises how to implement the results of this section. The best approach is to use the full equations found for each of the four combinations of an uniform or a Gaussian angular distribution and an uniform or a Gaussian spatial distribution (uu, $\mathbf{g g}$, gu and ug) instead of employing the effective width measures $\alpha_{\text {eff }}$ and $r_{e f f}$. This approach leads, for each of these cases, to a different version of the function $H_{C T}\left(\pi, v_{0}{ }^{*}, r_{c}{ }^{*}, S_{c}, S_{i}\right)$, which is for an uniform angular and an uniform spatial distribution (uu) given by equation (9.5.11). For the resulting set of equations the reader is referred to section 16.5, which summarizes the analytical prescriptions for the calculation of the trajectory displacement effect. 


\subsection{Introduction}

This chapter reports on some further investigations on statistical Coulomb interactions in particle beams. Part of these investigations concern the verification of the validity of the assumptions underlying the calculations presented in the previous chapters. The other investigations study the feasibility to extend the model to beams located in an uniform acceleration field and to relativistic beams.

The extended two-particle model was outlined in chapter 5 and applied to the Boersch effect, the effect of statistical angular deflections and the trajectory displacement effect in the chapters 7,8 and 9 respectively. These calculations pertain to the displacements experienced by the test particles running along the central (on-axis) reference trajectory in a rotational symmetric beam segment in drift space. Furthermore, the calculations were restricted to initially monochromatic beams with respect to the normal energy of the particles. These simplification were introduced to facilitate the calculations, assuming that they do not affect the results in any significant way. This will be verified here, within the framework of the extended twoparticle model, by taking a more general approach.

First, we will treat the problem of including of $f$-axis reference trajectories. Rather than evaluating the effect for a specific on- or off-axis reference trajectory we will evaluate the average result for all possible reference trajectories in the beam. In general, the exact solution of this problem requires the evaluation of an 8-dimensional integral. However, for a homocentric beam the problem reduces to the evaluation of a 4-dimensional integral, which can be handled by numerical means. The cases of a homocentric cylindrical beam and a homocentric beam segment with a crossover are studied. The expectation is that if the central reference trajectory approximation is justified for those extreme beam geometries it will be justified for any other beam geometry as well. For Boersch effect produced in a beam segment with a crossover one can not rely on this approach, since the case of a homocentric crossover is not representative. As an alternative, an approximating method will be used, which is in fact extremely accurate for paraxial beams. The analysis shows that the average results for all reference trajectories are in general 10\% to $20 \%$ smaller than those obtained for the central reference trajectory.

Next, the interaction effects generated in non-monochromatic beams will be studied. The calculations are restricted to the extreme cases of a homocentric cylindrical beam and a homocentric beam segment with a crossover. The analysis shows that the impact of the initial energy spread on the statistical interaction effects is negligible for practical operating conditions.

Finally, some preliminary results will be presented of an analysis to extend the model for statistical interactions to beams which are accelerated or retarded and to high energy beams. It will be shown that the extended two-particle model can straightforwardly be modified to handle beams which are located in an uniform axial electrostatic field. The modifications concern the dynamical part of the problem only. The essential point is to treat the two-particle dynamics at the entrance and the exit of the field in a proper way. The chapter concludes with a brief discussion of the relativistic corrections required to treat high energy beams, in which the particle velocities, observed in the laboratory system, are comparable to the speed of light. 
For a paraxial beam in drift space, one may assume that the relative velocities of the particles are small compared to the speed of light and the required modifications only concern the coordinate transformation between the laboratory system and the frame of reference moving with the beam. The analysis shows that it is sufficient to replace the beam potential $V$, appearing in the final equations for the various interaction effects, by the well-known relativistic potential $\mathrm{V}^{*}$.

\subsection{Exact approach for off-axis reference trajectories}

In section 5.6 we studied the required modifications of the basic equations of the extended two-particle model to cover of $f$-axis reference trajectories. It was shown that the function $p(k)$, defined by equation $(5.4 .8)$, will have a non-vanishing imaginary part $p_{i}(k)$ when the reference trajectory is of $f$-axis. It was found that the impact of the imaginary part $p_{i}(k)$ on the displacement distribution $\rho(\Delta \eta)$ is twofold. The first order term in $k$ of the imaginary part $p_{i}(k)$ leads to an uniform shift of size $\langle\Delta \eta\rangle$, which represents the space charge effect. The third and higher order terms in $\mathbf{k}$ of the imaginary part $\mathbf{p}_{\mathbf{i}}(\mathbf{k})$ cause the shifted distribution $\rho(\Delta \eta-\langle\Delta \eta\rangle)$ to become asymmetric, which means that $\rho(\Delta \boldsymbol{\eta}-\langle\Delta \boldsymbol{\eta}\rangle) \neq \rho(-\Delta \boldsymbol{\eta}+\langle\Delta \boldsymbol{\eta}\rangle)$.

The symmetry in the problem can be restored by taking the average over all possible reference trajectories. The odd-moments of the resulting displacement distribution $\rho(\Delta \eta)$ will be zero, as for the onaxis reference trajectory, and the function $p(k)$ will only have a real part. In this section we will perform this type of calculation for some extreme beam geometries and compare the results with those obtained previously for the on-axis reference trajectory. The objective of this analysis is to show that the differences with the results obtained for the on-axis reference trajectory are minor. This would, a posteriori, justify the approach taken in the previous chapters. The space charge shift $\langle\Delta \eta\rangle$ occurring for the individual of $f$-axis reference trajectories will be calculated separately in chapter 11 .

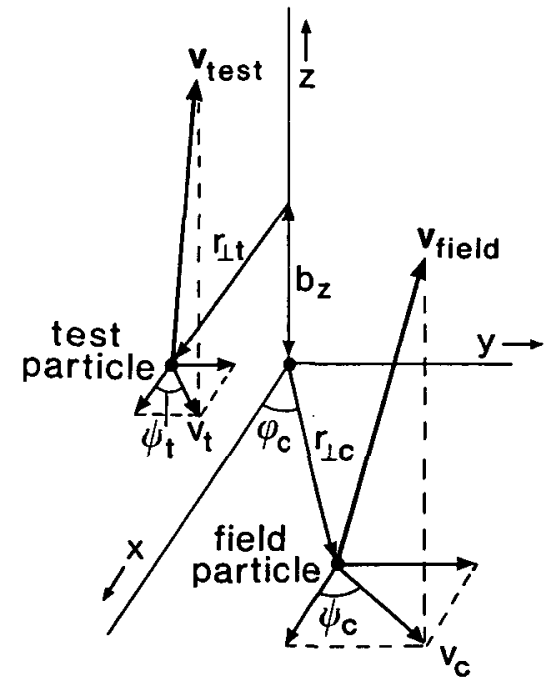

Pigure 10.1 Unperturbed coordinates of an off-axis test particle and a field particle at the moment that the field particle passes the $x, y-p l a n e$ of the laboratory system. 
Figure 10.1 shows the unperturbed trajectories of a test and a field particle at the moment that the field particle passes the $x, y$-plane of the laboratory system. The z-axis of this system coincides with the beam axis, while the $x, y$-plane coincides with the crossover plane (for a cylindrical beam the choice of the $x, y$-plane is arbitrary). The $x$-axis is chosen parallel to $r_{\perp t}$ (thus $\varphi_{t}=0$ ). The set of coordinates defining the unperturbed positions and velocities of the test and field (or colliding) particle is equal to

$$
\xi=\left(\xi_{t}, \xi_{c}\right) \quad \text { with } \quad \begin{aligned}
\xi_{c} & =\left(r_{\perp c}, \varphi, v_{c}, \psi_{c}, b_{z}\right) \\
\xi_{t} & =\left(r_{\perp t}, v_{t}, \psi_{t}\right)
\end{aligned}
$$

Equation (10.2.1) should be compared with equation (5.2.1), which applies to the on-axis reference trajectory $\left(r_{\perp t}=0, v_{t}=0\right)$.

For a specific (on- or of $f$-axis) reference trajectory the integral over the distribution of field particles, appearing in the definition of the two-particle distribution $\rho(\Delta \xi)$ given by equation $(5.4 .10)$, can be expressed as

$$
\begin{aligned}
& \int d \xi p(\xi)=\int d \xi_{c} p\left(\xi_{c}\right) \\
& =\int_{0}^{\infty} d v_{c} f_{v}\left(v_{c}\right) \int_{0}^{2 \pi} \frac{d \psi_{c}}{2 \pi} \int_{0}^{\infty} d r_{\perp c} f_{r}\left(r_{\perp c}\right) \int_{0}^{2 \pi} \frac{d \varphi_{c}}{2 \pi} \int_{-S_{c} L}^{\left(1-S_{c}\right) L} d b_{z}
\end{aligned}
$$

in which the distributions $f_{v}$ and $f_{r}$ correspond to distribution of transverse velocities and the density distribution in the crossover respectively. Equation (5.3.3) specifies these functions in case of uniform distributions and equation (5.3.4) in case of Gaussian distributions. Notice in equation (10.2.2) that the integration over the angles $\psi_{c}$ and $\varphi_{c}$ are kept separate. For the on-axis reference trajectory they can be combined into a single integration over the relative angle $\Phi=\psi-\varphi$, due to the rotational symmetry of the problem. In that case equation (10.2.2) leads back to equations (5.3.2) and (5.4.6).

In order to determine the average over all reference trajectories one should use

$$
\begin{aligned}
& \int d \xi p(\xi)=\int d \xi_{t} p\left(\xi_{t}\right) \int d \xi_{c} p\left(\xi_{c}\right) \\
& =\int_{0}^{\infty} d v_{t} f_{v}\left(v_{t}\right) \int_{0}^{2 \pi} \frac{d \psi_{t}}{2 \pi} \int_{0}^{\infty} d r_{\perp t} f_{r}\left(r_{\perp c}\right) \int d \xi_{c} p\left(\xi_{c}\right)
\end{aligned}
$$

in which the integral over $\xi_{c}$ represents the 5-dimensional integral given by equation $(10.2 .2)$. Equation $(5.4 .10)$ can now be expressed as

$$
\rho_{2}(\Delta \boldsymbol{\eta})=\int \mathrm{d} \xi_{\mathrm{t}} \mathrm{p}\left(\boldsymbol{\xi}_{\mathrm{t}}\right) \int \mathrm{d} \xi_{\mathrm{c}} \mathrm{p}\left(\boldsymbol{\xi}_{\mathrm{c}}\right) \delta\left[\Delta \boldsymbol{J}-\Delta \boldsymbol{\eta}_{2}\left(\boldsymbol{\xi}_{\mathrm{t}}, \xi_{\mathrm{c}}\right)\right]
$$

which represents an 8-dimensional integral. For short we will refer to the results based on equation $(10.2 .4)$ as the results for the "average reference trajectory", as opposed to the results for the "on-axis reference trajectory" presented previously.

In order to facilitate the numerical calculations we will concentrate on two extreme cases for which equation $(10.2 .4)$ reduces to a 4-dimensional integral. One case is that of a homocentric beam segment 
with a crossover with an uniform angular distribution, for which one finds

$\int d \xi_{t} p\left(\xi_{t}\right) \int d \xi_{c} p\left(\xi_{c}\right)=\int_{0}^{v_{0}} \frac{2 v_{r} d v_{r}}{v_{0}^{2}} \int_{0}^{2 \pi} \frac{d \Phi_{v}}{2 \pi} \int_{0}^{v_{0}} \frac{2 v_{c} d v_{c}}{v_{0}^{2}} \int_{-S_{c} L}^{\left(1-S_{c}\right) L} d b_{z}$

with $\Phi_{v}=\psi_{c}-\psi_{t}$ and $v_{0}=\alpha_{0} V_{z}$. The other case is that of a cylindrical homocentric beam segment with an uniform current density distribution, which leads to

$\int d \xi_{t} p\left(\xi_{t}\right) \int d \xi_{c} p\left(\xi_{c}\right)=\int_{0}^{r_{0}} \frac{2 r_{\perp r} d r_{\perp} r}{r_{0}{ }^{2}} \int_{0}^{2 \pi} \frac{d \Phi_{r}}{2 \pi} \int_{0}^{r_{0}} \frac{2 r_{\perp c} d r_{1, c}}{r_{0}{ }^{2}} \int_{-S_{c} L}^{\left(1-S_{c}\right) L} d b_{z}$

with $\Phi_{r}=\varphi_{c}-\varphi_{t}$ and $r_{0}$ the beam radius.

Let us first consider the case of a homocentric cylindrical beam segment. The distribution of axial velocity displacements $\rho\left(\Delta v_{z}\right)$ for the on-axis reference trajectory was calculated in section 7.4. We repeated the calculation of the scaled two-particle distributions $\rho_{2} *\left(\Delta v_{z}^{*}\right)$, now using the distribution of field and test particles given by equation $(10.2 \cdot 6)$. Figure $10.2 \mathrm{a}$ shows the results for different values of the scaled beam radius $r_{0}{ }^{*}$. For comparison we included, in the same figure, the results obtained previously for the on-axis reference trajectory. The second step is the calculation of the $p^{*}\left(k^{*}\right)$-transform, defined by equation (7.4.11). The results of this. step are shown in figure $10.2 \mathrm{~b}$. The third and final step is the evaluation of the FWHM of the scaled displacement distribution $\rho^{*}\left(\Delta \mathrm{v}_{z} *\right)$, defined by equation (7.4.17), as function of the scaled linear particle density $\lambda^{*}$. The results of this step are given in figure $10.2 \mathrm{c}$.

Similar calculations were carried out for the distribution of transverse velocities $\rho\left(\Delta v_{1}\right)$ generated in a homocentric cylindrical beam segment. The results for the scaled two-particle distribution $\rho_{2} *\left(\Delta v_{\perp} *\right)$, the $\mathrm{p}^{*}\left(\mathrm{k}^{*}\right)$-transform and the FWHM of the scaled displacement distribution $\rho^{*}\left(\Delta v_{\perp} *\right)$ are shown in the figures $10.3 \mathrm{a}, 10.3 \mathrm{~b}$ and $10.3 \mathrm{c}$ respectively. The functions $p^{*}\left(k^{*}\right)$ and $p^{*}\left(\Delta v_{\perp}^{*}\right)$ are defined by equations $(8.4 .11)$ and $(8.4 .17)$ respectively. For comparison we included in the same figures the results obtained for the on-axis reference trajectory, which were presented previously in section 8.4 .

The figures $10.2 \mathrm{c}$ and $10.3 \mathrm{c}$, pertaining to the distributions $\rho^{*}\left(\Delta v_{z}{ }^{*}\right)$ and $\rho^{*}\left(\Delta v_{\perp}^{*}\right)$ respectively, show similar features. For very large values of the scaled beam radius $r_{0}$ * the two types of calculations lead to identical results. This reflects that in a very wide beam most of the reference trajectories are sufficiently far removed from the edge of the beam to be treated as a central trajectory in an infinite wide beam. For less wide beams, which are nevertheless extended $\left(\chi_{p}=4 \lambda^{*} r_{0} * 3=\lambda r_{0} \gg>1\right)$, the calculation for an average reference trajectory leads to smaller results than that for the on-axis reference trajectory. This is due to the fact that the impact on a test particle moving along the edge of the beam is smaller than on a central test particle. For pencil beams $\left(\chi_{p}<1\right)$ one finds different results for $\rho^{*}\left(\Delta v_{z}{ }^{*}\right)$ and $\rho^{*}\left(\Delta v_{\perp^{*}}\right)$. The displacements in axial velocity $\Delta v_{z}$ are now fully determined by axial separation of the particles, which is the same for on- and of $\mathrm{f}$-axis test particles. Accordingly, figure $10.2 \mathrm{c}$ shows in the pencil beam regime equal results for the average and the on-axis reference trajectory. However, the transverse velocity displacements $\Delta \mathrm{v}_{\perp}$ become zero when all particles are on a row, and increase proportional to $r_{0}$ for small, but non-zero $r_{0}$. Therefore, one should expect that the impact on a particle moving along the edge of a pencil 


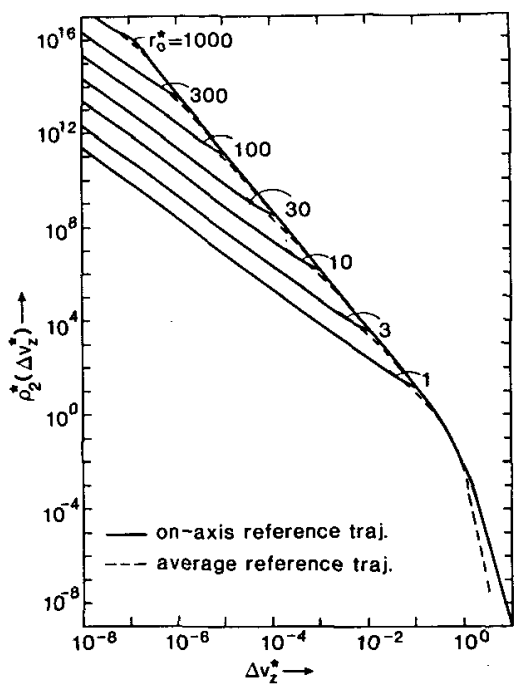

fig. 10.2a

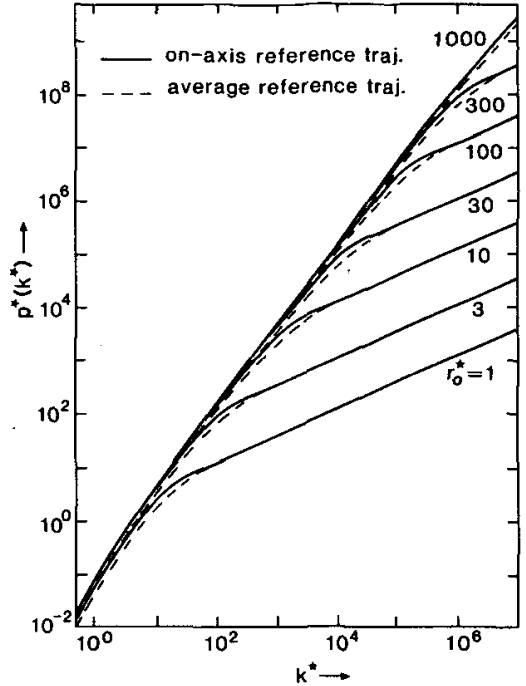

fig.10.2b

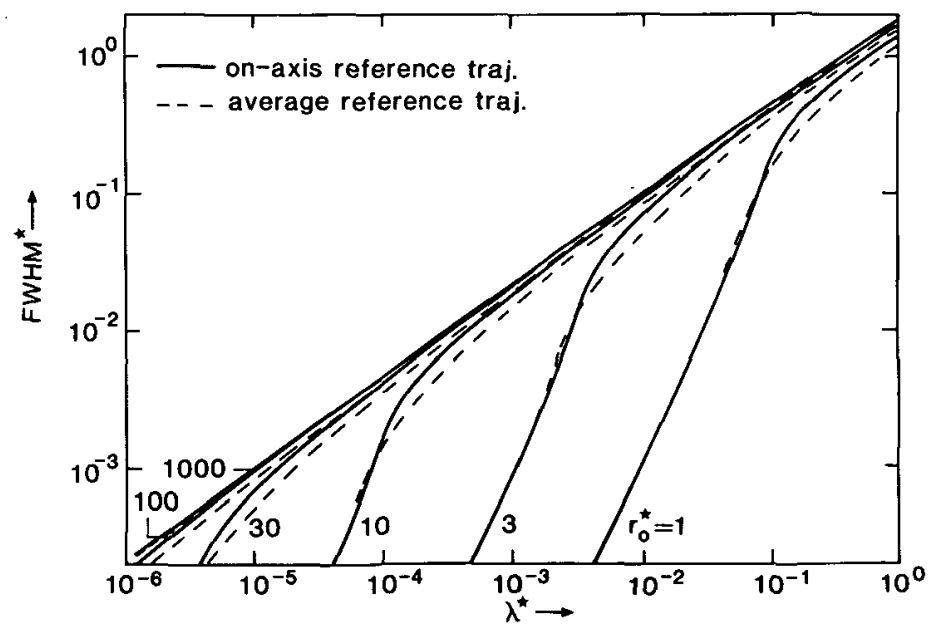

fig.10.2c

Figure 10.2 Comparison of the results for the on-axis and the average reference trajectory for the axial velocity displacements generated in a homocentric cylindrical beam segment. The figures $10.2 \mathrm{a}, 10.2 \mathrm{~b}$ and $10.2 \mathrm{c}$ show the scaled two-particle distribution $p_{2}{ }^{*}\left(\Delta \mathrm{v}_{2}{ }^{\star}\right)$, its transform $\mathrm{p}^{\star}\left(\mathrm{k}^{\star}\right)$ and the FWHM of the scaled displacement distribution $\rho^{*}\left(\Delta \mathrm{v}_{2}{ }^{*}\right)$ respectively. The results for the on-axis reference trajectory were plotted previous1y in the figures $7.7,7.8$ and 7.10 . 


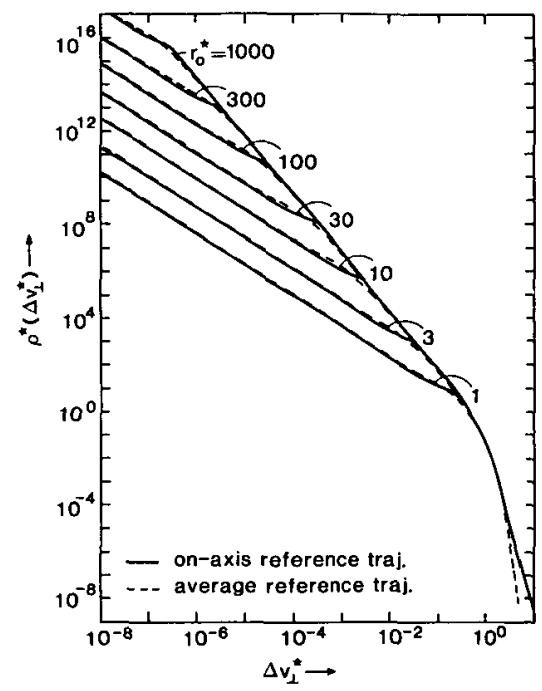

tig. 10.3a

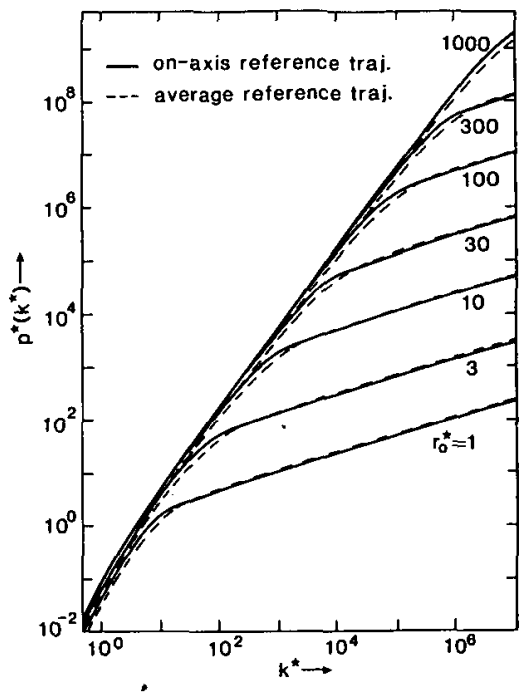

fig. 10.3b

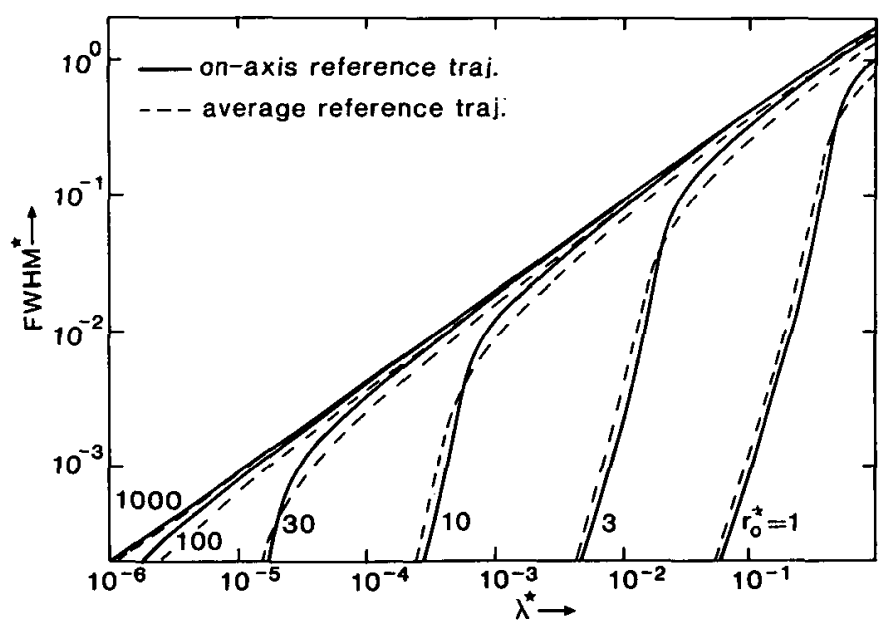

fig.10.3c

Pigure 10.3 Comparison of the results for the on-axis and the average reference trajectory for the transverse velocity displacements generated in a homocentric cylindrical beam segment. The figures $10.3 \mathrm{a}, 10.3 \mathrm{~b}$ and $10.3 \mathrm{c}$ show the scaled two-particle distribution $\rho_{2} *\left(\Delta v_{1}{ }^{*}\right)$, its transform $p^{\star}\left(k^{*}\right)$ and the FWHM of the scaled displacement distribution $\rho^{*}\left(\Delta v_{1}^{*}\right)$ respectively. The results for the on-axis reference trajectory were plotted previously in the figures $8.4,8.5$ and 8.7 . 
beam is larger than for a central particle. Accordingly, figure $10.3 \mathrm{c}$ shows in the pencil beam regime larger results for the average reference trajectory than for the on-axis reference trajectory.

Let us now consider the case of a homocentric beam segment with a crossover. The scaled trajectory displacement distribution $\rho^{*}\left(\Delta r^{*}\right)$ was for the on-axis reference trajectory calculated in section 9.3. We repeated this calculation, now using the distribution of field and test particles given by equation (10.2.5). The final results for the FWHM of $\rho^{*}\left(\Delta r^{*}\right)$ are presented in figure 10.4 . The reader might verify that the characteristics of this plot are similar to that of figure $10.3 \mathrm{c}$, pertaining to the distribution of transverse velocity displacements generated in a homocentric cylindrical beam. The plots of the twoparticle distributions $\rho^{*}\left(\Delta r^{*}\right)$ and the $p^{*}\left(k^{*}\right)$ are omitted since they are also quite similar to the corresponding plots obtained for a homocentric cylindrical beam, which are shown in the figures $10.3 \mathrm{a}$ and $10.3 \mathrm{~b}$ respectively.

The Boersch effect occurring in a beam segment with a crossover could in principle be handled in the same way by evaluating the distribution of axial velocities $\rho\left(\Delta v_{z}\right)$ for a homocentric beam. However, since the Boersch effect depends strongly on the size of the crossover the case of a homocentric beam $\left(r_{c}=0\right)$ is not representative. In order to study the more practical case of an extended crossover we will follow an alternative method, which is the subject of the next section.

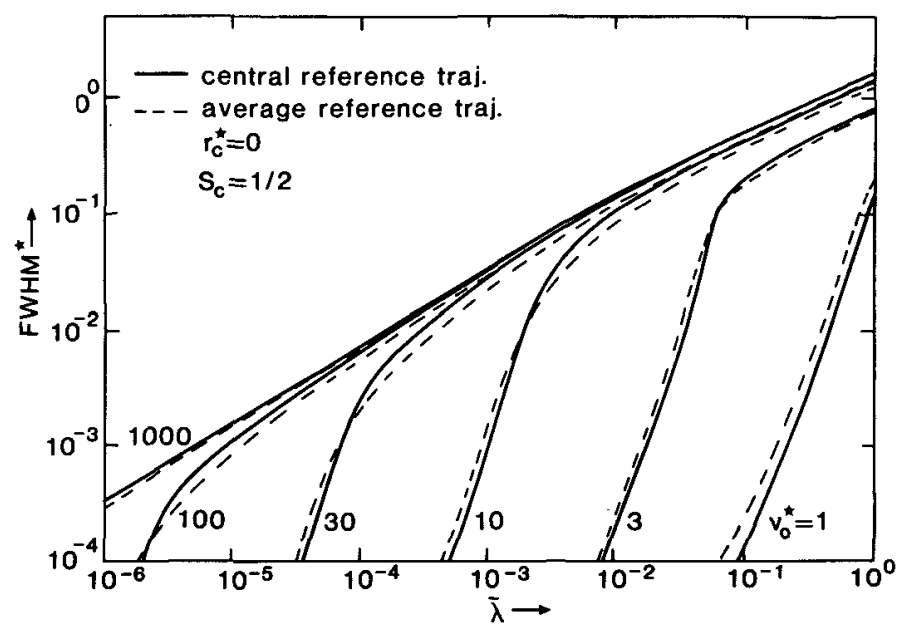

Figure 10.4 Comparison of the results for the on-axis and the average reference trajectory for the FWHM of the scaled trajectory displacement distribution $\rho^{*}(\Delta \mathbf{r} *)$ generated in a homocentric beam segment with a crossover in the middle $\left(S_{c}=1 / 2\right)$. The results for the on-axis reference trajectory were plotted previously in figure 9.7 .

\subsection{Approximating approach for off-axis reference trajectories}

In this section a method will be outlined to reduce the dimension of the integral for the two-particle distribution $\rho(\Delta \eta)$, given by equation (10.2.4). For paraxial beams one may in good approximation replace the exact dynamical solution for an off-axis test particle $\Delta \boldsymbol{\eta}=\Delta \eta_{2}\left(\xi_{\mathrm{c}}, \xi_{\mathrm{t}}\right)$ by the solution for an on-axis test particle $\Delta \boldsymbol{\eta}=\Delta \boldsymbol{\eta}_{2}\left(\boldsymbol{\xi}^{\prime}\right)$, 
with $\xi^{\prime}=\left\{r_{\perp}^{\prime}, \varphi^{\prime}, v^{\prime}, \psi^{\prime}, b_{z}{ }^{\prime}\right\}$ and

$$
\begin{aligned}
& r_{\perp} \cong\left[r_{\perp c}{ }^{2}+r_{\perp t^{2}}{ }^{2}-2 r_{L c} r_{L t} \cos \left(\varphi_{c}\right)\right]^{1 / 2} \\
& \varphi^{\prime} \cong \arctan *\left[\frac{r_{\perp c} \sin \varphi_{c}}{r_{\perp c} \cos \varphi_{c}-r_{\perp t}}\right]-\psi_{t} \\
& v^{\prime}=\left[v_{c}^{2}+v_{t}^{2}-2 v_{c} v_{t} \cos \left(\psi_{c}-\psi_{t}\right)\right]^{1 / 2} \\
& \psi^{\prime}=\arctan *\left[\frac{v_{c} \sin \left(\psi_{c}-\psi_{t}\right)}{v_{c} \cos \left(\psi_{c}-\psi_{t}\right)-v_{t}}\right] \\
& b_{z} \equiv b_{z}
\end{aligned}
$$

in which

$$
\arctan *\left(\frac{y}{x}\right)=\left(1-\frac{x}{|x|}\right) \frac{\pi}{2}+\arctan \left(\frac{y}{x}\right)
$$

Equations (10.3.1) represent the coordinate transformation from the original coordinate system $R=(x, y, z)$, which is drawn in figure 10.1 , to the coordinate system $R^{\prime}=\left(x^{\prime}, y^{\prime}, z^{\prime}\right)$ in which the $z^{\prime}$-axis is directed along the velocity of the test particle $v_{t e s t}$ and the $x^{\prime}, y^{\prime}-p l a n e$ coincides with the plane through the original z-axis and the vector

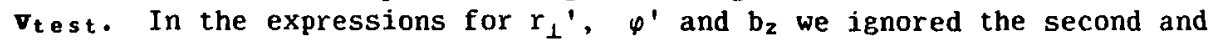
higher order terms in the angle $\alpha_{t} \approx v_{t} / v_{z}$, which is allowed for paraxial beams.

As the integrant in equation (10.2.4) now depends on $\xi_{t}$ and $\xi_{c}$ only through $\xi^{\prime}$ one may replace the probability $p\left(\xi_{t}\right) p\left(\xi_{c}\right)$ by the probability $p\left(\xi^{\prime}\right)$, defined as

$$
p\left(\xi^{\prime}\right)=\int \mathrm{d} \xi_{\mathrm{t}} \mathrm{p}\left(\boldsymbol{\xi}_{\mathrm{t}}\right) \int \mathrm{d} \boldsymbol{\xi}_{\mathrm{c}} \mathrm{p}\left(\boldsymbol{\xi}_{\mathrm{c}}\right) \delta\left[\boldsymbol{\xi}^{\prime}-\boldsymbol{\xi}^{\prime}\left(\boldsymbol{\xi}_{\mathrm{t}}, \boldsymbol{\xi}_{\mathrm{c}}\right)\right]
$$

with $\xi^{\prime}\left(\xi_{t}, \xi_{c}\right)$ representing the coordinate transformation of equation (10.3.1). The two-particle distribution can now be expressed as

$$
\rho_{2}(\Delta \boldsymbol{\eta})=\int \mathrm{d} \xi^{\prime} p\left(\xi^{\prime}\right) \delta\left[\Delta \boldsymbol{\eta}-\Delta \eta_{2}\left(\xi^{\prime}\right)\right]
$$

as follows with equation $(10.2 .4)$.

The problem of determining $\rho_{2}(\Delta \eta)$ is now reduced to the evaluation of the 5 -dimensional integral of equation (10.3.4), provided that the function $p\left(\xi^{\prime}\right)$ can be evaluated from equation $(10.3 .3)$. Let us consider this problem first. Due to the rotational symmetry of the beam one may conjecture that, for any given value of $r_{1}^{\prime}$ and $v^{\prime}$, the distribution over the angles $\varphi^{\prime}$ and $\psi^{\prime}$ is uniform over $2 \pi$. The main problem is therefore to determine the distributions over $r_{\perp}{ }^{\prime}$ and $v^{\prime}$. Equations (10.3.1) and (10.3.3) show that the calculation of both distributions requires the solution of the same mathematical problem. Given the distribution $f(r)$ one should evaluate the function $p(r)$, using

$$
p(r)=\int_{0}^{\infty} d r_{1} f\left(r_{1}\right) \int_{0}^{\infty} d r_{2} f\left(r_{2}\right) \int_{0}^{2 \pi} \frac{d \varphi}{2 \pi} \delta\left[r-\left\{r_{1}^{2}-r_{2}^{2}-2 r_{1} r_{2} \cos \varphi\right\}^{1 / 2}\right]
$$

The function $p(r)$ can be visualized as the distribution of the distance $r=\left|r_{1}-r_{2}\right|$ between two points, $r_{1}$ and $r_{2}$, in a plane, when both points are distributed as $f(|r|)$ with respect to the origin and uniform over an 
angle $2 \pi$. In appendix 10.A it is demonstrated that when $f(r)$ is a twodimensional uniform distribution, thus $f(r)=f_{u}(r)$, with

$$
f_{u}(r)= \begin{cases}2 r / R^{2} & \text { for } 0<r<R \\ 0 & \text { for } r>R\end{cases}
$$

equation $(10.3 .5)$ yields $p(r)=p_{u}(r)$, with

$$
p_{u}(r)= \begin{cases}\frac{4 r}{\pi R^{2}}\left[\arccos \left(\frac{r}{2 R}\right)-\frac{r}{2 R} \sqrt{\left.1-\left(\frac{r}{2 R}\right)^{2}\right]}\right. & \text { for } 0<r<2 R \\ 0 & \text { for } r>2 R\end{cases}
$$

When $f(r)$ is a two-dimensional Gaussian distribution, thus $f(r)=f_{b}(r)$, with

$$
f_{t}(r)=\frac{2 r}{R^{2}} e^{-(r / R)^{2}}
$$

one finds from equation $(10.3 .5)$ that $p(r)=p_{5}(r)$, with

$$
p_{\varepsilon}(r)=\frac{r}{R^{2}} e^{-(r / R)^{2} / 2}
$$

which is also demonstrated in appendix 10.A. Notice that the resulting distribution $\mathrm{p}_{\mathrm{g}}(x)$ is again Gaussian, but its width is a factor $\sqrt{2}$ larger than the original distribution $f_{B}(r)$.

The probability distribution $p\left(\xi^{\prime}\right)$, occurring in equation (10.3.4) can now be expressed as

$$
\int d \xi^{\prime} p\left(\xi^{\prime}\right)=\int_{0}^{\infty} d v^{\prime} p_{v}\left(v^{\prime}\right) \int_{0}^{2 \pi} \frac{d \Phi^{\prime}}{2 \pi} \int_{0}^{\infty} d r_{\perp} p_{r}\left(r_{\perp}^{\prime}\right) \int_{-S_{c} L}^{\left(1-S_{c}\right) L} d b_{z}^{\prime}
$$

with $\Phi^{\prime}=\psi^{\prime}-\varphi^{\prime}$. For the functions $p_{v}$ and $p_{r}$ one should use equation (10.3.7) when the corresponding distribution $f_{v}$ and $f_{r}$ are uniform and equation (10.3.9) when they are Gaussian, taking the proper measure for the width $R$. With equations $(10.3 .4)$ and $(10.3 .10)$ the calculation of the average two-particle distribution $\rho_{2}(\Delta \eta)$ for all possible reference trajectories is reduced to the same form as the calculation of $\rho_{2}(\Delta \boldsymbol{\eta})$ for the on-axis reference trajectory. For the dynamical part one may use the same function $\Delta \eta_{2}(\xi)$ as used for the on-axis reference trajectory. The differences between both calculations concern the distributions of $v^{\prime}$ and $r_{\frac{1}{1}}$, only.

We will concentrate on the case that the distributions in $v$ and $r$ are uniform, leading to distributions in $r^{\prime}$ and $\mathrm{v}^{\prime}$ of the type given by equation (10.3.7). The case of a Gaussian distribution in $v$ or $r$ does not require any separate calculation. Equation (10.3.9) shows that when the distribution in $v$ or $r$ is Gaussian the distribution in $v^{\prime}$ or $r^{\prime}$ is Gaussian too, with a width which is larger by a factor $\sqrt{2}$. Accordingly, for this case one should expect that a calculation based on equations $(10.3 .4)$ and $(10.3 .10)$ leads to the same final equations as obtained for the on-axis reference trajectory, but with the crossover radius $r_{c}$ and the beam semi-angle $\alpha_{0}$ replaced by some effective quantities which are $\sqrt{2}$ times larger than the original ones.

The correctness of the approximating method outlined above can be verified by applying it to the extreme cases of a homocentric cylindrical beam segment and a homocentric beam segment with a 
crossover, which were studied in the previous section using the exact approach. For a homocentric beam equation (10.3.4), defining the twoparticle distribution $\rho_{2}(\Delta \boldsymbol{\eta})$, reduces to a 2-dimensional integral. We evaluated this integral for each of the cases studied in the previous section. The results were found to be in very close agreement with those presented in the figures $10.2,10.3$ and 10.4, justifying the use of the approximating method.

The approximating method to treat of $f$-axis reference trajectories was utilized to evaluate the distribution of axial velocity displacements $\rho\left(\Delta v_{z}\right)$ generated in a beam segment with an extended, but narrow crossover, assuming an uniform angular and spatial distribution. The corresponding calculation for the on-axis reference trajectory was presented in section 7.3. The calculation for the average reference trajectory is quite similar, but starts from the modified distribution of field particles defined by equations (10.3.10) and $(10.3 .7)$. As the principle aspects involved are the same we will restrict the discussion to the presentation of the main results.

Figure 10.5a shows the results for the scaled two-particle distributions $\bar{\rho}_{2}\left(\Delta \bar{v}_{z}\right)$ for different values of the scaled beam radius $\bar{r}_{c}$. For comparison the data obtained previously for the on-axis reference trajectory is included in the same figure. The next step is the calculation of the $\bar{p}(\bar{k})$ transform, defined by equation $(7.3 .14)$. The results are shown in figure 10.5b. Finally, the FWHM of the scaled displacement distribution $\bar{\rho}\left(\Delta \bar{v}_{z}\right)$ was evaluated, which is defined by equation (7.3.21). The results of this step are presented in figure $10.5 \mathrm{c}$.

Following the calculation scheme of section 7.3 the reader might verify that the scaled $\bar{p}(\bar{k})$ transform for the average reference trajectory, shown in figure $10.5 \mathrm{~b}$, is given by

$$
\bar{p}(\bar{k})=16 \bar{k} \int_{0}^{2} d \bar{v} F(\bar{v}) \int_{0}^{2 \pi} \frac{d \Phi}{2 \pi} \int_{0}^{2} d t F(t) \int_{0}^{1} d u \frac{\left(1-u^{2}\right)^{1 / 2}}{u} \sin \left(\frac{\bar{k} \bar{v} u}{\left(4+\bar{v}^{4} t^{2} \bar{r} c^{2} \sin ^{2} \Phi\right)^{1 / 2}}\right)
$$

with

$$
F(x)=\frac{2}{\pi}\left[\arccos (x / 2)-(x / 2) \sqrt{1-(x / 2)^{2}}\right]
$$

We will investigate the behaviour of $\bar{p}(\bar{k})$ for $\bar{k} \rightarrow 0$ and for $\bar{k} \rightarrow \infty$.

For $\bar{k} \rightarrow 0$ equation (10.3.11) shows a quadratic dependency on $\bar{k}$, which can be described by the first equation of $(7.3 .16)$, taking the function $\bar{p}_{2}\left(\bar{r}_{c}\right)$ equal to

$$
\begin{aligned}
\bar{p}_{2}\left(\bar{r}_{c}\right) & =2 \int_{0}^{2} d \vec{v} F(\bar{v}) \int_{0}^{2 \pi} d \Phi \int_{0}^{2} d t F(t)\left[1+\bar{v}^{4} t^{2} \bar{r}_{c} \sin ^{2} \Phi / 4\right]^{-1 / 2} \\
& =8 \int_{0}^{2} d \bar{v} F(\bar{v}) \int_{0}^{2} d t F(t) \frac{1}{\left[1+\bar{v}^{4} t^{2} \bar{r}_{c}^{2} / 4\right]^{1 / 2}} \times\left(\frac{\bar{v}^{2} t \bar{r}_{c} / 2}{\left[1+\bar{v}^{4} t^{2} \bar{r}_{c}{ }^{2} / 4\right]^{1 / 2}}\right)
\end{aligned}
$$

with $K$ the complete elliptic integral of the first kind. This function 


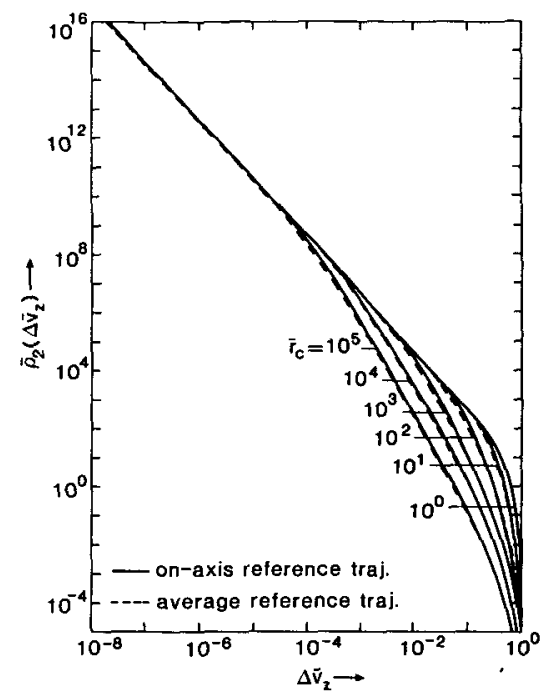

fig. 10.5a

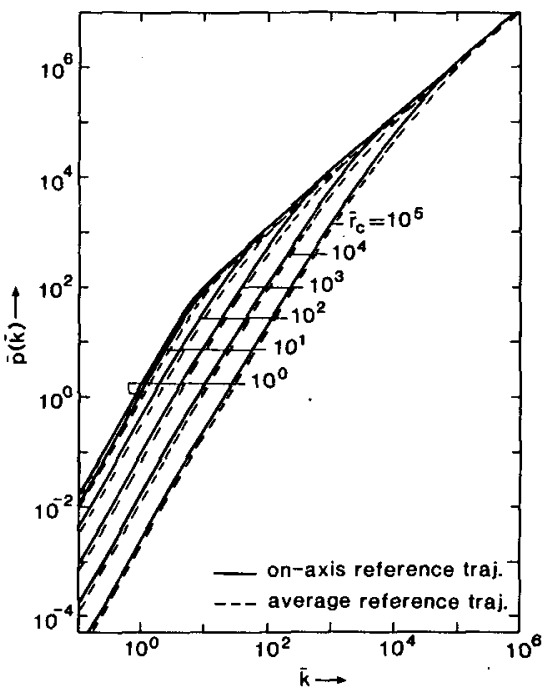

fig. 10.5b

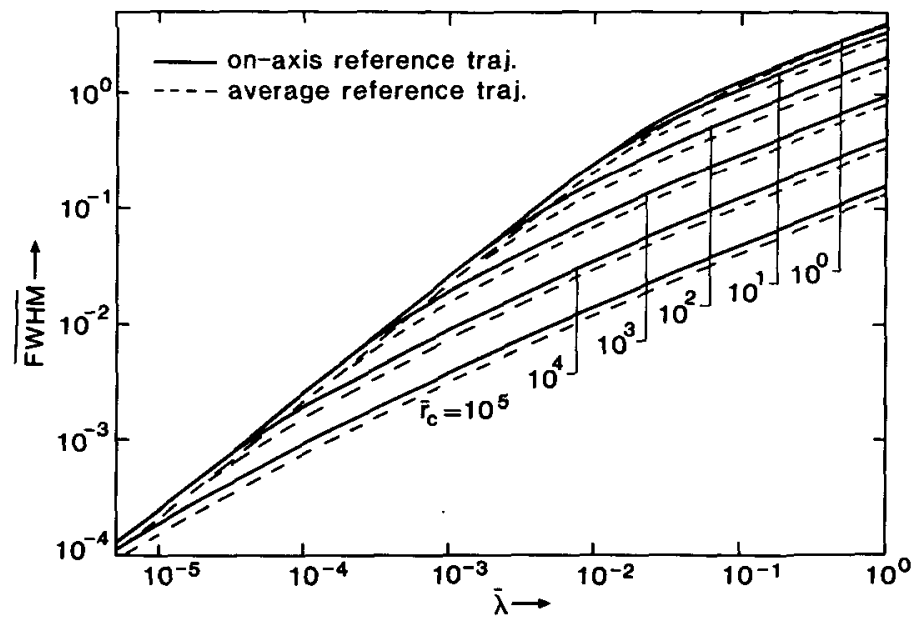

fig. 10.5c

Figure 10.5 Comparison of the results for the on-axis and the average reference trajectory for the axial velocity displacements generated by complete collisions in a beam segment with a crossover. The figures 10.5a, $10.5 b$ and $10.5 \mathrm{c}$ show the scaled two-particle distribution $\bar{\rho}_{2}\left(\Delta \bar{v}_{z}\right)$, its transform $\overline{\mathrm{p}}(\bar{k})$ and the FWHM of the scaled displacement distribution $\bar{\rho}(\Delta \bar{x})$ respectively. The results for the on-axis reference trajectory were plotted previously in the figures $7.1,7.2$ and 7.5 . 
is plotted in figure 10.6. For comparison we also plotted the result for the on-axis reference trajectory, given by the second equation of (7.3.16). The function $\overline{\mathrm{p}}_{2}\left(\overline{\mathrm{r}}_{\mathrm{c}}\right)$ given by equation $(10.3 .13)$ can be approximated by equation (7.6.11), taking

$$
E=37.38, \quad F=1.471
$$

which should be compared to the values $E_{u v}$ and $F_{u} u$ given by equation $(7.6 .12)$.

For $k \rightarrow \infty$ equation (10.3.11) shows a linear dependency on $k$, which is given by

$$
\overline{\mathbf{p}}(\bar{k})=\overline{\mathbf{p}}_{\infty} \mathrm{K}, \quad \overline{\mathbf{p}}_{\infty}=32 / 3 \quad(\bar{k}+\infty)
$$

This result should be compared to equation (7.3.19), pertaining to the on-axis reference trajectory.

The analysis of section 10.2 and the present section shows that the results obtained for the average reference trajectory are for most conditions somewhat smaller than those obtained for the on-axis reference trajectory. However, in general the differences are within $20 \%$ and in many cases even within $10 \%$. The analysis justifies the assumption that the results obtained for the on-axis reference trajectory are representative for other trajectories as well.

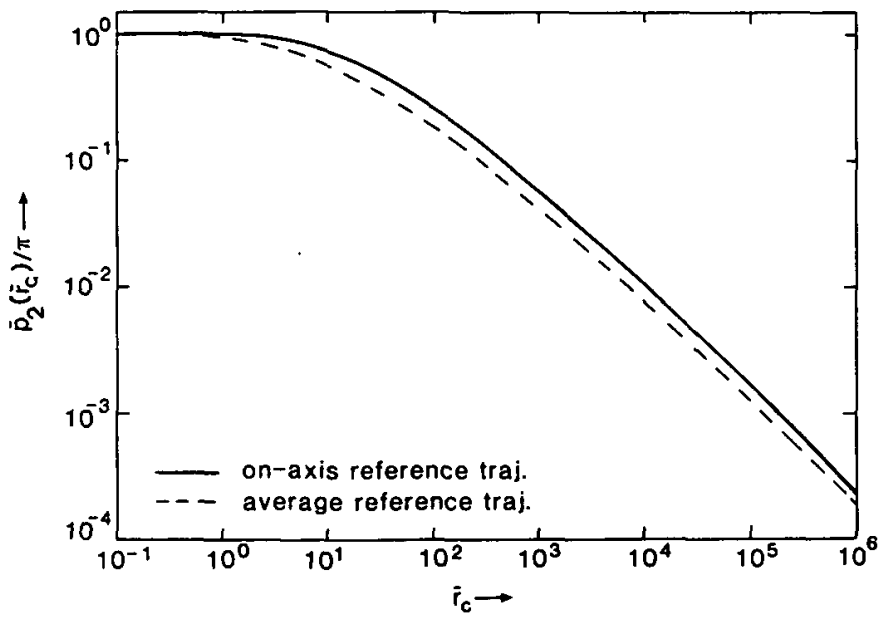

Figure 10.6 Comparison of the functions $\overline{\mathrm{p}}_{2}\left(\bar{r}_{\mathrm{c}}\right)$ for the on-axis and the average reference trajectory, defined by equations (7.3.16) and (10.3.13) respectively. The function for the on-axis reference trajectory was plotted previously in figure 7.3 .

\subsection{Non-monochromatic beans}

The calculations carried out in the chapters 7,8 and 9 rely on the assumption that the beam is initially monochromatic with respect to the normal energy. This means that it is assumed that all particles initially have the same axial velocity. In this section we will study the more realistic case of a beam in which the particles start with a 
certain axial velocity spread. The objective of this analysis is to show that the differences with the results obtained for an initially monochromatic beam are minor for practical conditions.

Let us assume that the initial axial velocity spread of the field particles with respect to the test particle is given by the Gaussian distribution

$$
f\left(\Delta v_{z}\right)=\frac{1}{\sqrt{\pi} \Delta v_{\mathbf{z} 0}} e^{-\left(\Delta v_{z} / \Delta v_{z_{0}}\right)^{2}}
$$

which has a FWHM equal to

$$
\text { FWHM }_{\mathbf{v}}=2(\ln 2)^{1 / 2} \Delta \mathrm{v}_{\mathbf{z}} \mathrm{O}
$$

For the on-axis reference trajectory the set of coordinates defining the unperturbed position and velocity of the field particle with respect to the test particle is now given by

$$
\xi=\left(r_{\perp}, \Phi, v_{\perp}, \Delta v_{z}, b_{z}\right)
$$

similar to equation $(5.2 .1)$. The angles $\varphi$ and $\psi$ were combined as $\Phi=\psi-\varphi$, using that the size of the displacement $|\Delta \eta|$ depends only on $\varphi$ and $\psi$ through the relative angle $\Phi$.

For the integral over the distribution of field particles in equation (5.4.10), which defines the two-particle distribution $\rho_{2}(\Delta \eta)$, one should now use

$$
\int d \xi p(\xi)=\int_{0}^{v_{0}} \frac{2 v_{\perp} d v_{\perp}}{v_{0}{ }^{2}} \int_{-\infty}^{\infty} \frac{d \Delta v_{z}}{\sqrt{\pi} \Delta v_{z 0}} e^{-\left(\Delta v_{z} / \Delta v_{z}\right)^{2}} \int_{0}^{2 \pi} \frac{d \Phi}{2 \pi} \int_{0}^{r_{c}} \frac{2 r_{\perp} d r_{\perp}}{r_{c}{ }^{2}} \int_{-S_{c} L}^{\left(1-S_{c}\right) L} d b_{z}
$$

$(10.4 .4)$

We will restrict the analysis to the extreme cases of a homocentric beam segment with a crossover $\left(r_{c}=0\right)$ and a homocentric cylindrical beam segment $\left(v_{0}=0\right)$. For the former, one may omit the integration over $r_{\perp}$ and $\Phi$. For the latter, the integration over $v$ and $\Phi$ can be left out. In both cases the remaining integral is 3-dimensional.

We will again employ the $\delta, v$-scaling defined by equation (6.13.1). The scaled axial velocity displacement $\Delta v_{z}{ }^{*}$ is related to the relative energy displacement $\Delta E / E$ as

$$
\Delta v_{z}^{*}=\left(\frac{\pi \varepsilon_{0}}{4 e}\right)^{1 / 3} V^{1 / 3} L^{1 / 3} \frac{\Delta E}{E}
$$

using that $\Delta E \cong m_{\mathrm{z}} \Delta \mathrm{v}_{\mathrm{z}}$. For electrons the physical constant in front of the right hand side of equation $(10.4 .5)$ is equal to 351.43 in SI units. Taking as a numerical example an electron beam with $\mathrm{V}=10 \mathrm{kV}, \mathrm{L}=0.1 \mathrm{~m}$ and $\Delta E / E=10^{-4}$ (which implies $\Delta E=1 \mathrm{eV}$ ) one finds $\Delta \mathrm{V}_{\mathrm{z}}{ }^{*}=0.35$. Thus for practical beams the scaled FWHM of the initial distribution of axial velocities $\mathrm{FWHM}_{v} *\left(=\mathrm{FWHM}_{\mathrm{v}} / \nu\right)$ is of the order 1 .

Let us first consider the case of a homocentric cylindrical beam segment. We repeated the calculations described in the sections 7.4 and 8.4, now including the integration over $\Delta v_{z}$ prescriped by equation $(10.4 .4)$. For the scaled beam radius $r_{0} *$ we considered the values 1000 , 100,10 and 1 and for each $r_{0}{ }^{*}$ we took FWHM ${ }^{*}=0,0.2,0.4,1,2,4,10$ and 20 .

The figures $10.7 \mathrm{a}, 10.7 \mathrm{~b}$ and $10.7 \mathrm{c}$ show part of the results 

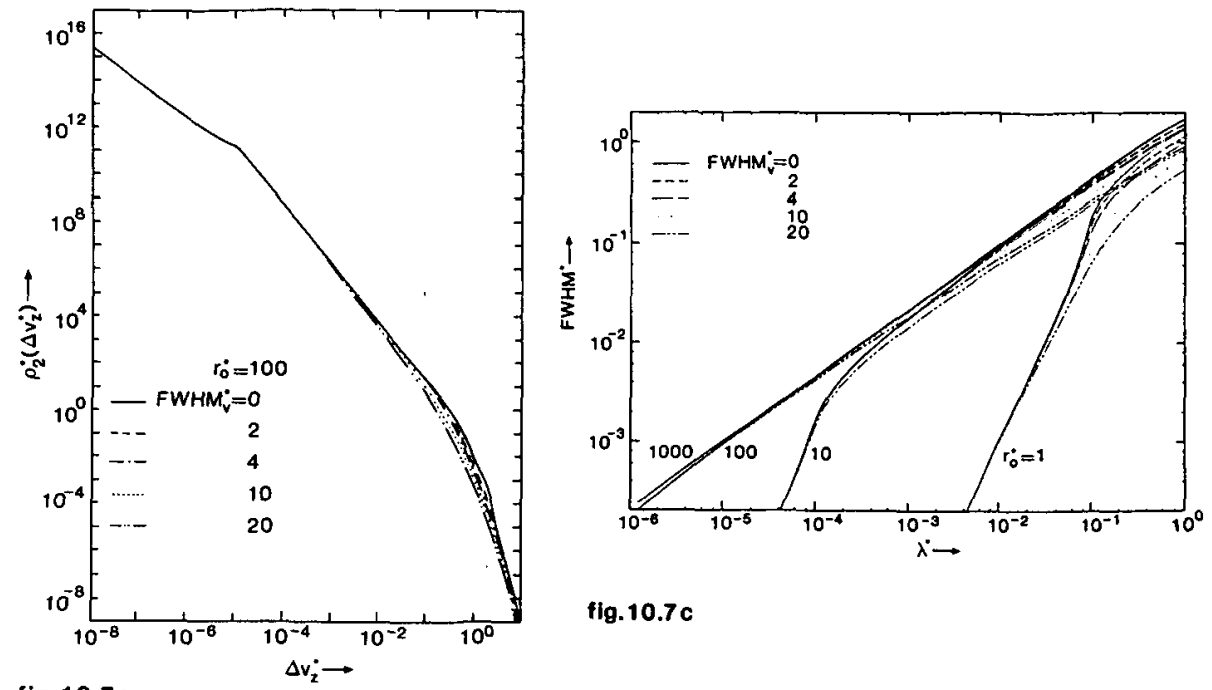

fig.10.7 c

fig. 10.7 a
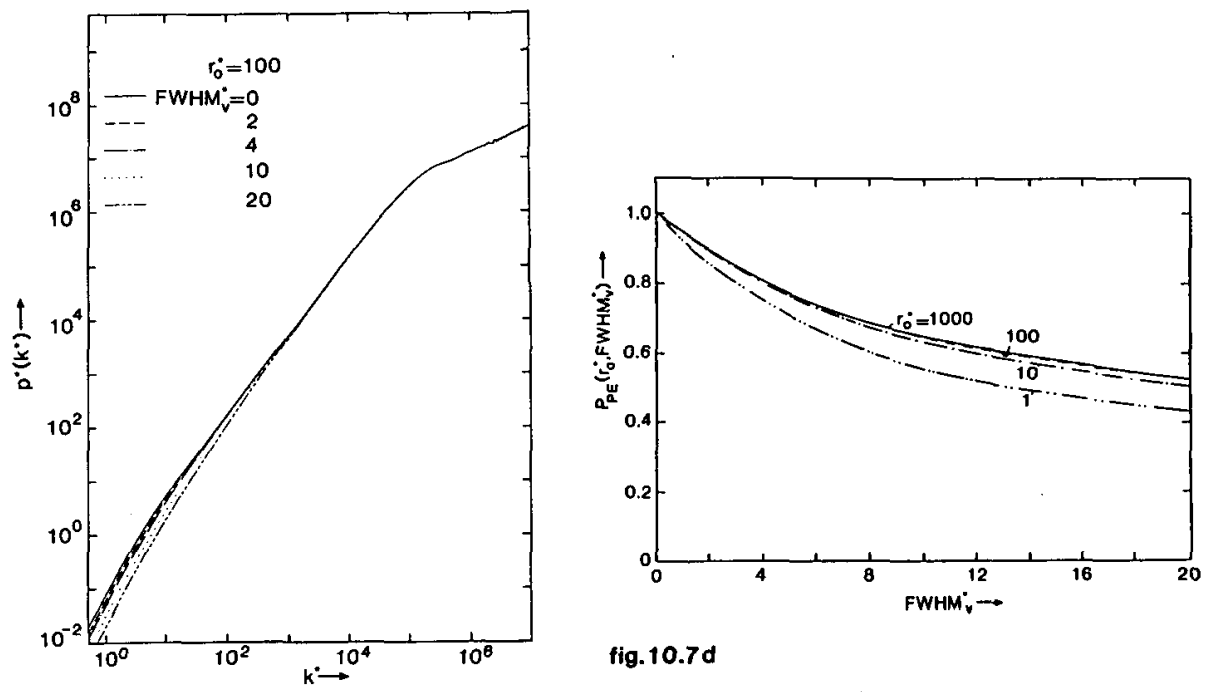

fig.10.7d

flg. $10.7 \mathrm{~b}$

Figure 10.7 Results for the axial velocity displacements generated in a homocentric cylindrical beam segment for different values of the initial axial velocity spread FWH. *. The figures $10.7 \mathrm{a}, 10.7 \mathrm{~b}$ and $10.7 \mathrm{c}$ show the scaled two-particle distribution $\rho_{2}{ }^{*}\left(\Delta v_{z}{ }^{*}\right)$, its transform $p^{*}\left(k^{*}\right)$ and the FWHY of the scaled displacement distribution $\rho^{\star}\left(\Delta \mathrm{v}_{2}^{*}\right)$ respectively. The curves for the monochromatic case $\left(\mathrm{FWHM}_{\mathrm{\gamma}}{ }^{*}=0\right.$ ) were plotted previously in the figures $7.7,7.8$ and 7.10 . Figure $10.7 d$ gives the results for the function $\mathrm{PPE}_{\mathrm{P}}\left(\mathrm{r}_{\mathrm{O}}{ }^{*}, \mathrm{FWHM}_{\mathrm{V}}{ }^{*}\right)$, which is defined by equation $(10.4 .6)$. This function specifies the dependency on $r_{0}{ }^{*}$ and FWHM * of the function $p^{\star}\left(k^{\star}\right)$, plotted in figure $10.7 b$, for $k^{\star} \rightarrow 0$. 

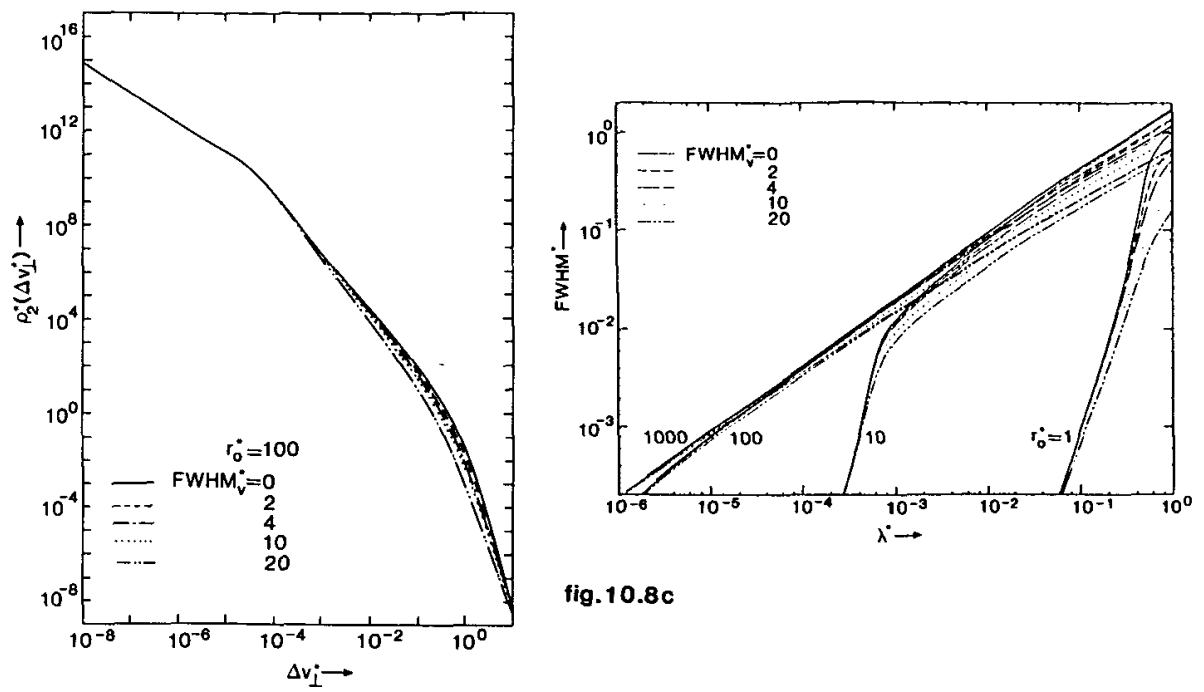

fig. 10.8c

fig. 10.8a
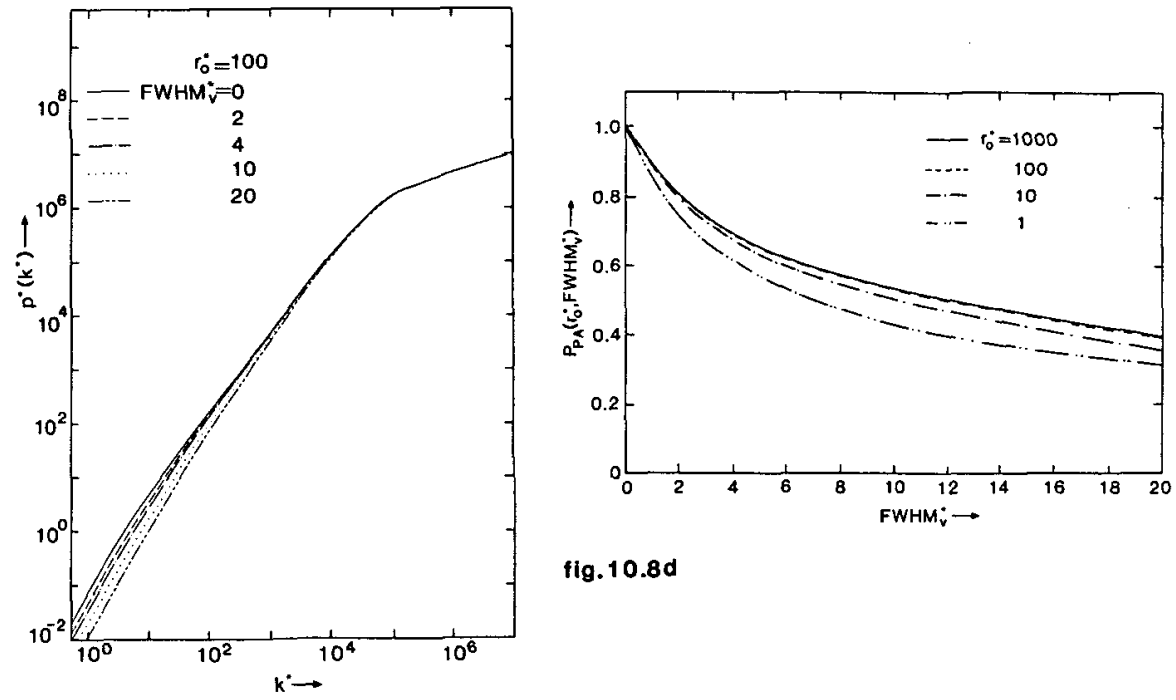

fig. 10.8d

fig. $10.8 b$

Figure 10.8 Results for the transverse velocity displacements generated in a homocentric cylindrical beam segment for different values of the initial axial velocity spread FWHM *. The figures $10.8 \mathrm{a}, 10.8 \mathrm{~b}$ and $10.8 \mathrm{c}$ show the scaled two-particle distribution $\rho_{2} *\left(\Delta v^{*}\right)$, its transform $\mathrm{p}^{*}\left(\mathrm{k}^{*}\right)$ and the $F W H M$ of the scaled displacement distribution $\rho^{\star}\left(\Delta v_{1}{ }^{*}\right)$ respectively. The curves for the monochromatic case $\left(\mathrm{FWHM}_{v}{ }^{\star}=0\right.$ ) were plotted previously in the figures $8.4,8.5$ and 8.7. Figure 10.8d gives the results for the function $\mathrm{P}_{\mathrm{PA}}\left(\mathrm{r}_{0^{*}}, \mathrm{FWHM}_{\mathrm{v}}{ }^{*}\right)$, which is the generalization of the function Ppa $\left(\mathrm{r}_{0}^{*}\right)$ defined by equation (8.4.20). It specifies the dependency on $\mathrm{r}_{0}{ }^{*}$ and $\mathrm{FWHM}_{\mathrm{v}}$ * of the function $\mathrm{p}^{\star}\left(\mathrm{k}^{*}\right)$, plotted in figure $10.8 \mathrm{~b}$, for $\mathrm{k}^{*} \rightarrow 0$. 
obtained for the scaled two-particle distribution $\rho_{2} *\left(\Delta v_{2}^{*}\right)$, the corresponding $p^{*}\left(k^{*}\right)$ transform and the FWHM of the scaled displacement distribution $\rho^{*}\left(\Delta \mathbf{v}_{z} *\right)$ respectively. One sees that the magnitude of the Boersch effect in a cylindrical beam decreases with increasing FWHM ${ }^{*}$. The effect is the strongest in the Gaussian regime $\left(\lambda * 2\right.$ and $\left.\chi_{p} \gg>1\right)$ and becomes larger for small $r_{0}^{*}$-values. In the Gaussian regime the FWHM of $\rho^{*}\left(\Delta v_{z}^{*}\right)$ can be expressed as

$$
\text { FWHM }^{*}=1.832 \mathrm{P}_{\mathrm{PE}}\left(\mathrm{r}_{0} *, \mathrm{FWHM}_{\mathrm{V}}^{*}\right) \lambda^{* 1 / 2}
$$

similar to equation (7.4.21). In figure $10.7 \mathrm{~d}$ the function $P_{P E}\left(r_{0}{ }^{*}, F_{W H M}{ }^{*}\right)$ is plotted as function of $F_{W H M} *$, for different values of the scaled beam radius $r_{0}^{*}$.

The figures $10.8 \mathrm{a}, 10.8 \mathrm{~b}$ and $10.8 \mathrm{c}$ show part of the results obtained for the scaled two-particle distribution $\rho_{2}^{*}\left(\Delta v_{1}^{*}\right)$, the corresponding $\mathbf{p}^{*}\left(\mathbf{k}^{*}\right)$ transform and the FWHM of the scaled displacement distribution $\rho^{*}\left(\Delta v^{*}\right)$ respectively. The function $P_{P_{A}}\left(r_{0^{*}}, F W H M_{y}^{*}\right)$, which is plotted in figure $10.8 \mathrm{~d}$, is the generalization of the function $P_{P a}\left(r_{0}{ }^{*}\right)$, defined by equation $(8.4 .20)$. It specifies the dependency of the FWHM of $\rho^{*}\left(\Delta v_{1}^{*}\right)$ on $r_{0^{*}}$ and FWHM $_{4}^{*}$ in the Gaussian regime, as can be seen from equation (8.4.19). All figures show the same characteristics as the equivalent figures obtained for the axial velocity displacements, but the decrease of the FWHM of $\rho^{*}\left(\Delta v_{\perp}{ }^{*}\right)$ with increasing FWHM ${ }_{v}^{*}$ is stronger.

We also studied the impact of the initial energy spread on the trajectory displacement distribution $\rho^{*}\left(\Delta r^{*}\right)$ generated in a homocentric bean segment with a crossover in the middle $\left(S_{c}=0.5\right)$. The displacements refer to the crossover plane $\left(S_{i}=0.5\right)$. For the scaled maximum transverse velocity $v_{0}$ * we considered the values $1000,100,10$ and 1 and for each $v_{0}{ }^{*}$ we took FWHM $^{*}=0,0.2,0.4,1,2,4,10$ and 20. Part of the results obtained for the two-particle distribution $\rho_{2}^{*}\left(\Delta v^{*}\right)$, the $p^{*}\left(k^{*}\right)$ transform and the FWHM of the displacement distribution $\rho^{*}\left(\Delta r^{*}\right)$ are plotted in the figures $10.9 \mathrm{a}, 10.9 \mathrm{~b}$ and $10.9 \mathrm{c}$ respectively. In general, the results are not very sensitive to FWHM $^{*}$. Figure $10.9 \mathrm{c}$ shows that the trajectory displacement effect generated in a homocentric beam segment with a crossover increases slightly with FWHM ${ }^{*}$. The strongest increase is found for small $v_{0}$ *values. It also shows that the deviations from the monochromatic case $\left(\mathrm{FWHM}_{\mathrm{v}}{ }^{\mathrm{x}}=0\right.$ ) are the largest for those operating conditions which correspond to the transition area between the pencil beam and the extended beam regimes (that is the Holtsmark and the Gaussian regime). For the Gaussian regime the dependencies on $V_{0}{ }^{*}$ and $F_{W H M} *$ are represented by the function $\operatorname{Pct}\left(v_{0}{ }^{*}, F_{H M}{ }^{*}\right)$, which is shown in figure 10.9d. This function is the equivalent of $P_{C T}\left(v_{0}{ }^{*}, S_{c}, S_{i}\right)$ for $S_{c}=S_{i}=0.5$ and non-zero $F_{W H M_{v}}$. The function $\operatorname{PCr}\left(v_{0}{ }^{*}, S_{c}, S_{i}\right)$ is defined by equation $(9.3 .24)$ and is used in equation $(9.3 .29)$, specifying the FWHM of $\rho^{*}\left(\Delta r^{*}\right)$ in the Gaussian regime.

Some physical appreciation for the results of this section can be gained by studying the contributions of the different types of collisions involved. Consider the figures $10.7 \mathrm{a}$ and $10.8 \mathrm{a}$ showing the scaled two-particle distributions $\rho_{2}{ }^{*}\left(\Delta v_{2}{ }^{*}\right)$ and $\rho_{2}{ }^{*}\left(\Delta v_{1}{ }^{*}\right)$ respectively for a cylindrical beam with $r_{0}{ }^{*}=100$. One sees that an increase of $F W_{H M}$ * causes a decrease in the distributions for large displacements. For $\mathrm{FWHM}_{\mathrm{v}}{ }^{*}=0$ the large displacements stem from half-complete collisions as is shown by equations $(7.4 .9)$ and $(8.4 .9)$. For such a collision the initial kinetic energy in the centre of mass system is zero and the initial potential energy is fully converted into kinetic energy during the flight. With increasing $\mathrm{FWHM}_{\mathrm{v}}$ * the initial kinetic energy in the 

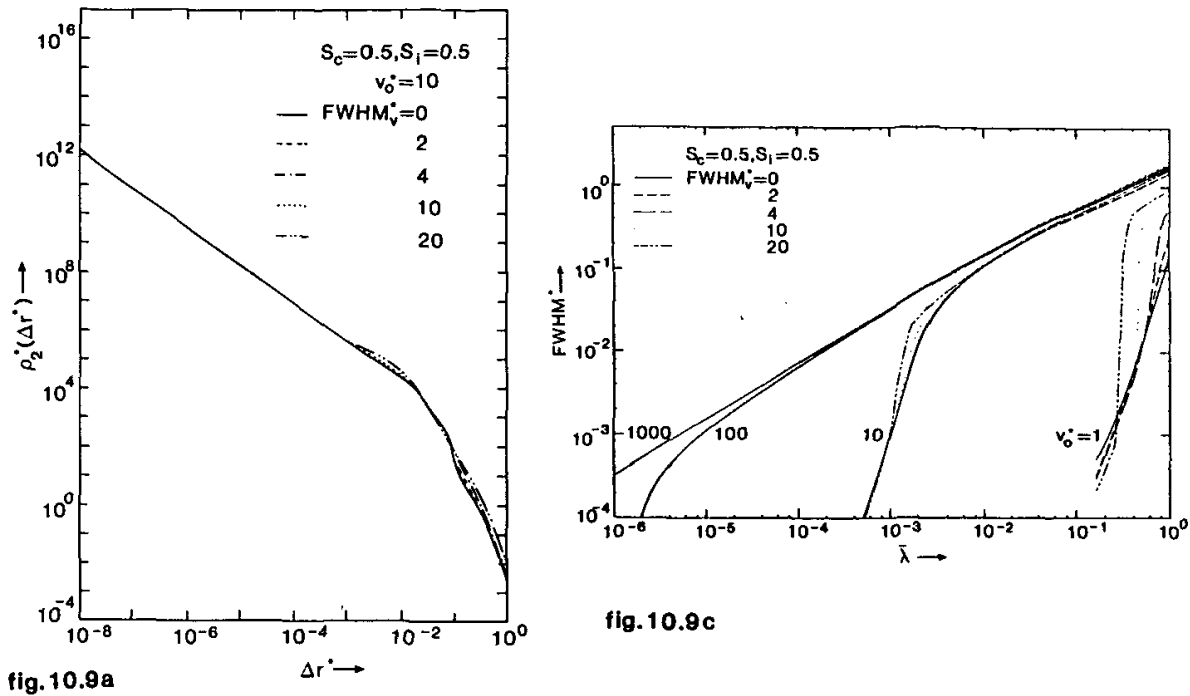

fig. 10.9c
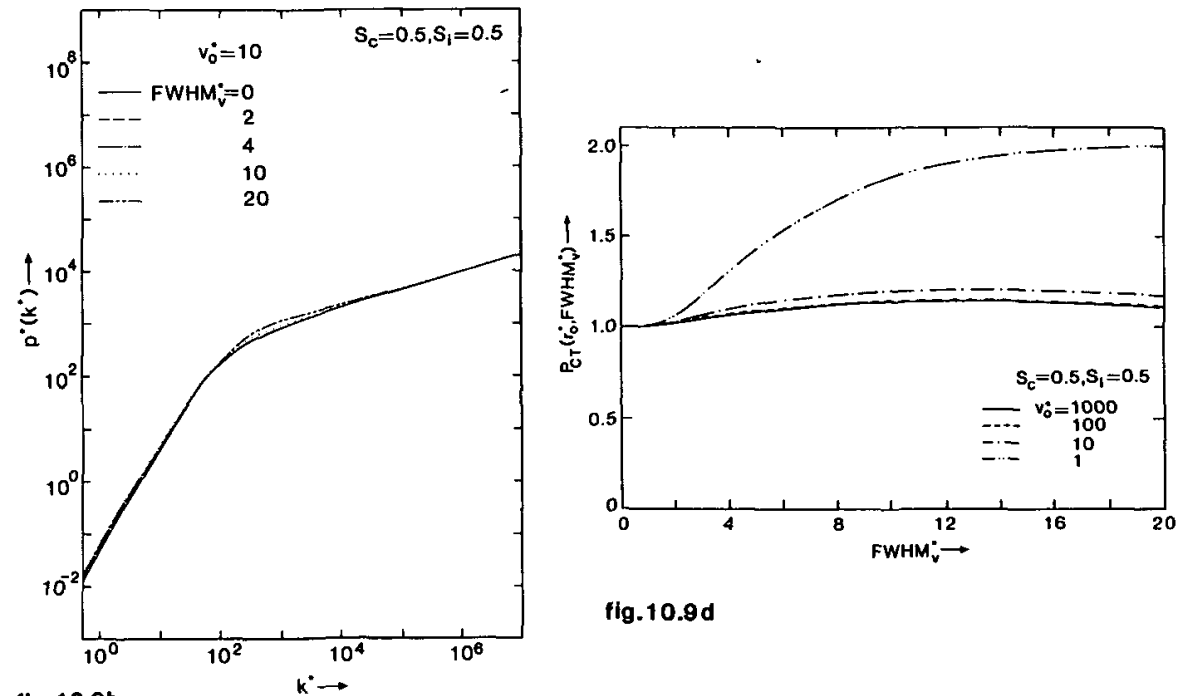

fig.10.9d

flg. 10.9b

Figure 10.9 Results for the trajectory displacement effect generated in a homocentric beam segment with a crossover in the middle $\left(S_{c}=1 / 2\right)$, for different values of the initial axial velocity spread FWHM *. The results pertain to the virtual displacements observed in the crossover plane $\left(S_{t}=S_{c}\right)$ and pertain to a beam for which the maximum transverse velocity $v_{0}{ }^{*}=10$. The figures $10.9 a, 10.9 b$ and $10.9 c$ show the scaled two-particle distribution $\rho_{2}{ }^{*}\left(\Delta r^{*}\right)$, its transform $p^{*}\left(k^{*}\right)$ and the FWHM of the scaled displacement distribution $p^{\star}\left(\Delta r^{*}\right)$ respectively. The curves for the monochromatic case (FWHM ${ }^{\star}=0$ ) were plotted previously in the figures $9.1,9.4$ and 9.7. Figure 10.9d gives the results for the function $\mathrm{PcT}\left(\mathrm{r}_{0^{*}}, \mathrm{FWH}_{\mathrm{V}^{*}}\right)$, which is the equivalent of the function $\mathrm{P}_{\mathrm{C}}\left(\mathrm{r}_{\mathrm{O}^{\star}}, \mathrm{S}_{\mathrm{C}}, \mathrm{S}_{1}\right)$, defined by equation $(9,3,2.4)$, and pertains to $S_{\mathrm{c}}=\mathrm{S}_{\mathrm{i}}=1 / 2$ and non-zero $\mathrm{FWHM}_{\mathrm{v}}{ }^{*}$. It specifies the dependency on $\mathrm{r}_{0^{*}}$ and $\mathrm{FWHM}_{\mathrm{V}^{*}}$ of the function $\mathrm{p}^{\star}\left(\mathrm{k}^{\star}\right)$, plotted in figure $10.9 b$, for $k^{\star} \rightarrow 0$. 
centre of mass system will in general be non-zero. For a collision in which the initial potential energy is fully converted into kinetic energy the change in relative velocity $\Delta v$ is determined by

$$
4 \mathrm{C}_{0} / \mathrm{mr}_{\mathrm{i}}=\left(\mathrm{v}_{\mathrm{i}}+\Delta \mathrm{V}\right)^{2}-\Delta \mathrm{v}^{2}=2 \mathrm{v}_{\mathrm{i}} \Delta \mathrm{v}+\Delta \mathrm{v}^{2}
$$

where $r_{i}$ is the initial distance between the particles and $v_{i}$ is their initial relative velocity. One sees that the velocity change $\Delta v$ decreases with increasing $v_{i}$, which is a direct consequence of the quadratic dependency of kinetic energy on velocity.

Small displacements stem from weak uncomplete collisions. Using first order perturbation dynamics one finds for the relative velocity displacement $\Delta v$ caused by such a collision

$$
\Delta \mathrm{v} \cong 2 \mathrm{C}_{0} \mathrm{~T} / \mathrm{mr}_{\mathrm{i}}{ }^{2}
$$

in which $T$ is the time of flight. This result does not depend on the initial relative velocity $v_{i}$, which explains why the distribution of small displacements in the figures $10.7 \mathrm{a}$ and $10.8 \mathrm{a}$ is hardly affected by the magnitude of initial axial velocity spread represented by FWHM $_{v}$.

Figure $10.9 \mathrm{a}$ gives the scaled two-particle distributions $\rho_{2}{ }^{*}\left(\Delta r^{*}\right)$ in a homocentric beam segment with a crossover with $v_{0}^{*}=10$. The curves show substantially less variation with FWHM $_{\mathrm{y}}{ }^{*}$ than the corresponding curves obtained for a cylindrical beam segment. The physical reason is that even for $\mathrm{FWHM}_{\mathrm{v}} *=0$ almost all collisions start with a large initial relative velocity $v_{i}$ due to the lateral motion of the particles. Therefore, the additional axial velocity components $\Delta v_{z_{\text {, }}}$ occurring for non-zero FWHM $_{v}$, hardly change the initial conditions of the various collisions, unless the relative transverse velocity $v_{\text {}}$ and/or the impact parameter $b$ are very small. Increasing the value of FWHM $_{v}$ * has some effect for large displacements, which stem predominantly from collisions with small $b$ and/or $v_{\perp}$.

From the figures $10.7 \mathrm{c}, 10.8 \mathrm{c}$ and $10.9 \mathrm{c}$ one sees that for values of FWHM $_{v}$ * of the order 1 or less and practical particle densities ( $\lambda$ or $\lambda^{*}$ smaller than 10-2) the differences with the results obtained for the monochromatic case $\left(\mathrm{FWHM}_{\mathrm{v}}{ }^{*}=0\right)$ are negligible. This data supports the assumption that, as far as the calculation of statistical interactions is concerned, practical beams can be treated as monochromatic.

\subsection{Beams in an external uniform axial electrostatic field}

In this section we will investigate how the model for statistical interactions can be extended to cover beams which are accelerated or retarded by an uniform electrostatic field. Consider the system schematically drawn in figure 10.10. In the area between the two grids, located at $z=z_{0}$ and $z=z_{1}$, the beam is accelerated from a potential $v_{0}$ to a potential $v_{1}$. The electrostatic field produced by the grids is assumed to be perfectly uniform. Furthermore, it is assumed that the beam is initially monochromatic with respect to the normal energy, which means that all particles have the same initial axial velocity $\mathrm{v}_{\mathbf{z}}$.

One way to evaluate the effect of statistical interactions in presence of an acceleration field is by means of the slice method described in section 5.11. The function $\mathrm{FWHM}_{\mathrm{P}}\left(\mathrm{I}, \mathrm{V}, \mathrm{r}_{0}, \Delta z\right)$ appearing in the integrant of equation (5.11.1) depends on the beam voltage. Instead of using a constant $V$ one now takes $V=V(z)$, with 


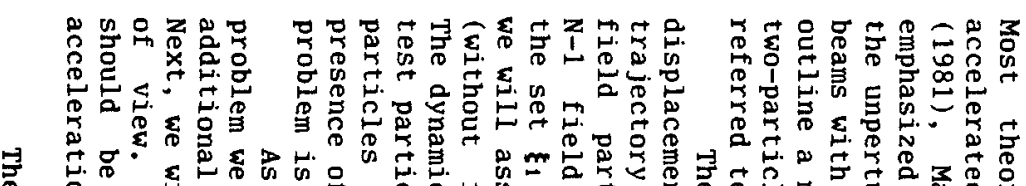
을

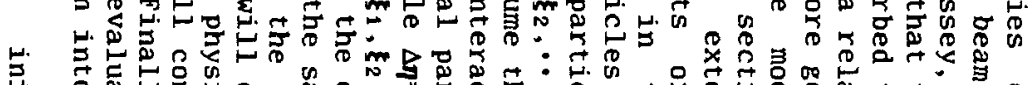

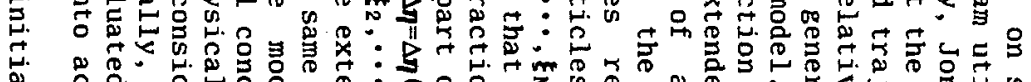
2 ऽ

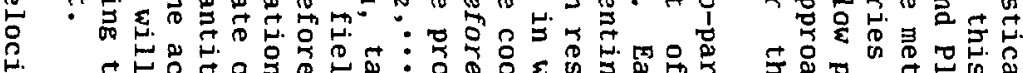

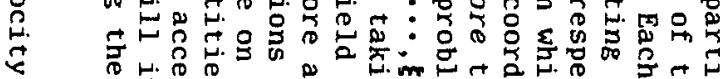

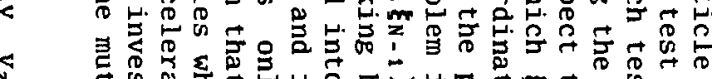

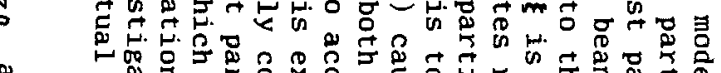
落

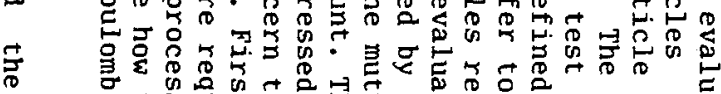

离.

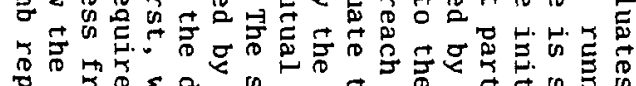

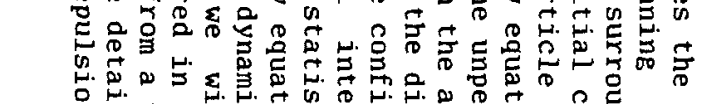

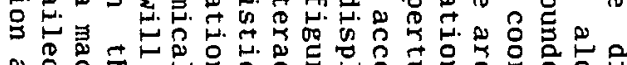

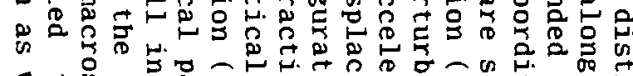

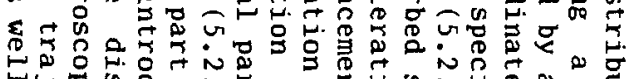

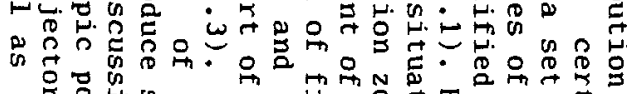

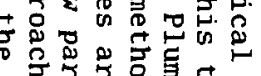

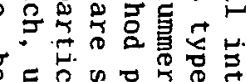

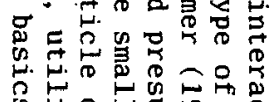

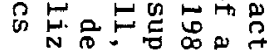

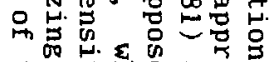

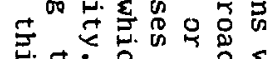

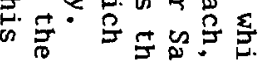

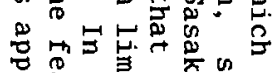

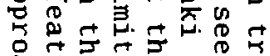

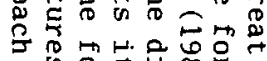
(n) $+x^{\infty}$

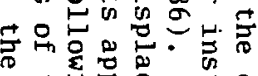

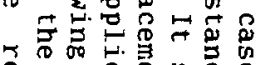

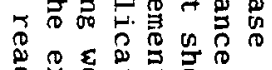

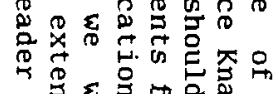

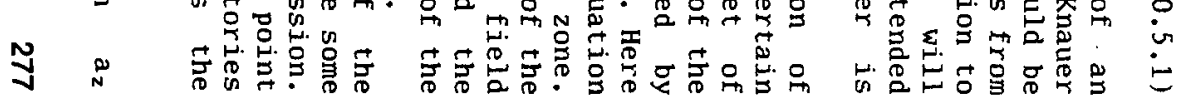

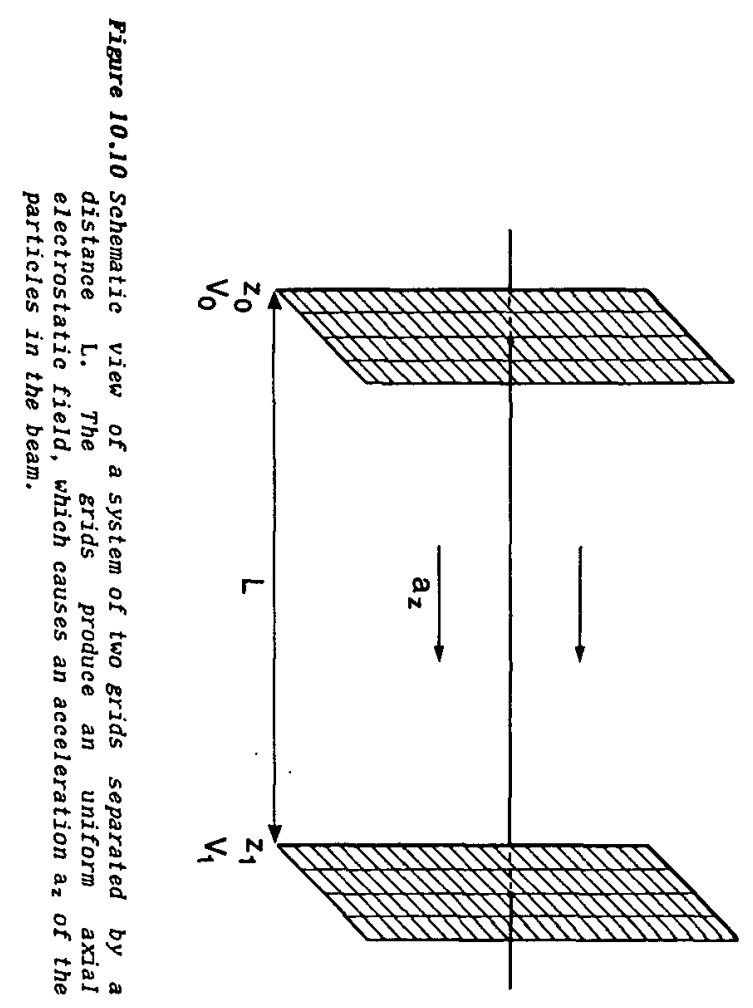


experienced by a particle with mass $m$ and charge $e$ are equal to

$$
v_{z 0}=\sqrt{\frac{2 e V_{0}}{m}} \quad, \quad a_{z}=\frac{e\left(v_{1}-v_{0}\right)}{m L}
$$

with $L=z_{1}-z_{0}$, the distance between the grids. In case of deceleration $V_{1}<V_{0}$ and $a_{z}$ is negative. The time of flight $T$ in presence of acceleration $\left(V_{1} \neq V_{0}\right)$ and the time of flight $T_{0}$ in absence of acceleration $\left(V_{1}=V_{0}\right)$ are given by

$$
T=\frac{v_{z 0}}{a_{z}}\left(\sqrt{1+\frac{2 a_{z} L}{v_{z 0}{ }^{2}}}-1\right) \quad, \quad T_{0}=\frac{L}{V_{z 0}}
$$

It is convenient to introduce the acceleration coefficient $\kappa$, defined as

$$
\kappa=\left(V_{1} / V_{0}\right) 1 / 2
$$

The relation between the flight times $T$ and $T_{0}$ can now be expressed as

$$
\frac{T}{T_{0}}=\frac{2}{\kappa \cdot+1}
$$

which can directly be verified from equations (10.5.3).

The impact of the acceleration on the macroscopic properties of the beam can be summarized as follows :

- The time of flight $T$ is reduced by a factor $(k+1) / 2$, as shown by equation (10.5.5).

- The linear particle density $\lambda$ is decreased by a factor $k$, as can be seen from equation $(3.2 .4)$.

- The angular distribution of the particles shrinks with a factor $k$ since the axial velocities increase with a factor $x$, while the transverse velocities are not affected by the acceleration field. Notice also that a transverse velocity displacement $\Delta v_{\perp}$ occurring after acceleration corresponds to a $k$ times smaller angular displacement as the same velocity displacement before acceleration.

- Any initial axial velocity spread is reduced by a factor $\kappa$, as follows from equation (3.7.14). Notice also that an axial velocity displacement $\Delta \mathrm{v}_{\mathrm{z}}$ occurring after acceleration corresponds to a $\kappa$ times larger energy displacement as the same velocity displacement before acceleration.

The description of the detailed particle trajectories should be consistent with these macroscopic observations.

The model for statistical interactions is based on a reduction of the full $\mathrm{N}$-particle dynamical problem into $\mathrm{N}-1$ two-particle problems, as described in section 5.4. The main problem of this section is therefore to describe a two-particle collision in the presence of an uniform external field. In chapter 6 we considered the dynamical problem of two free particles interacting through the coulomb force. We will show that this analysis requires only some minor modifications to treat the present case. The essential observation is that, once both particles have entered the acceleration zone, the motion in their centre of mass system is the same as for two free particles, due to the uniformity of the external field. Accordingly, one can employ the same set of equations, but now using the adjusted time of flight $T$ specified by equation (10.5.5). The remaining problem is to treat the dynamics of the particles at the entrance and the exit plane in a proper way. 
Consider two particles axially separated by a distance $b_{z 0}$, which enter the acceleration zone with an axial velocity $v_{z o}$. The particle in front will enter the acceleration field earlier than the other particle. This time difference $\Delta t_{0}$ is equal to $b_{z o} / v_{z o}$. At the moment that the second particle enters the acceleration zone the first particle has already increased its axial velocity by an amount $\delta v_{z} 0$, while the axial separation of the two particles $b_{z o}$ has increased by an amount $\delta b_{z} 0$, where

$$
\delta \mathrm{b}_{\mathrm{z} 0}=\frac{1}{2} \mathrm{a}_{\mathrm{z}}\left(\frac{\mathrm{b}_{\mathrm{zO}}}{\mathrm{v}_{\mathrm{z} 0}}\right)^{2}, \quad \delta \mathrm{v}_{\mathrm{zO}}=\mathrm{a}_{\mathrm{z}} \frac{\mathrm{b}_{\mathrm{zO}}}{\mathrm{v}_{\mathrm{z} 0}}
$$

Using equations $(10.5 .2)$ and $(10.5 .4)$ these quantities can be expressed as

$$
\frac{\delta \mathrm{b}_{\mathrm{z} 0}}{\mathrm{~b}_{\mathrm{z} 0}}=\left(\kappa^{2}-1\right) \frac{\mathrm{b}_{\mathrm{z} 0}}{4 \mathrm{~L}}, \quad \frac{\delta \mathrm{v}_{\mathrm{z} 0}}{\mathrm{v}_{\mathrm{z} 0}}=\left(\kappa^{2}-1\right) \frac{\mathrm{b}_{\mathrm{z} 0}}{2 \mathrm{~L}}
$$

Due to the velocity difference $\delta v_{z o}$ the axial separation of the particles will increase linearly with time during the period that both particles are in the acceleration zone. The particle in front will leave the acceleration earlier than the other particle. This time difference $\Delta t_{1}$ is equal to $\Delta t_{0}$ and the corresponding reduction in the axial separation and relative axial velocity compensates the differences $\delta b_{z} 0$ and $\delta \mathrm{v}_{z 0}$ produced at the entrance plane. The net increase in axial separation $\delta \mathrm{b}_{\mathrm{z}}$ is therefore equal to $T \times \delta \mathrm{v}_{\mathbf{z}} \mathrm{O}$ and the final axial separation $b_{z 1}$ is given by

$$
b_{21}=b_{z 0}+T \delta v_{z_{0}}=\kappa b_{z_{0}}
$$

as follows with equations (10.5.5) and (10.5.7). This result is consistent with the observation that the linear particle density $\lambda$ decreases with a factor $k$ due to the acceleration.

We will assume that the average axial separation $\langle s\rangle=1 / \lambda$ is very small compared to the length $L$ of the acceleration zone. This implies that the time intervals $\Delta t_{0}$ and $\Delta t_{1}$, defined above, are in general small compared to the total flight time $T$. Accordingly, during the major part of the flight both particles are in the acceleration region. The calculation of the two-particle displacement $\Delta \boldsymbol{\eta}(\boldsymbol{\xi})$ now proceeds as follows :

- The relative initial coordinates $\xi=\left(\mathrm{r}_{\perp}, \varphi, \mathrm{b}_{\mathrm{z} 0}, \mathrm{v}_{\perp}, \psi\right)$ are adjusted to account for the acceleration of the foremost particle during the time interval $\Delta t_{0}$ that the other particle has not reached the acceleration zone yet. In this step the coordinates $\xi$ are replaced by $\xi$ ', with

$$
\xi^{\prime}=\left(r_{\perp}, \varphi, b_{z 0}+\delta b_{20}, v_{\perp}, \delta v_{z 0}, \psi\right)
$$

where $\delta \mathrm{b}_{\mathbf{z}} \mathrm{o}$ and $\delta \mathrm{v}_{\mathrm{z} 0}$ are given by equation (10.5.7). Contrary to $\xi$ the coordinates $\xi$ include a relative axial velocity.

- The displacement $\Delta \boldsymbol{\eta}\left(\boldsymbol{\xi}^{\prime}\right)$ is evaluated using the analysis for two interacting particles in drift space presented in chapter 6 . For the flight time $T$ one should now use equation (10.5.5) instead of equation (6.2.2). Special care should be taken with the calculation of the virtual spatial displacement $\Delta r$ in the reference plane. The final coordinates should be extrapolated (backwards) to the reference plane along a trajectory which is curved, due to the presence of the acceleration field.

- The axial velocity displacement $\Delta v_{z}$ is corrected for the fact that interaction causes the actual flight time through the acceleration. 
zone to be shorter than $\mathrm{T}$ for the particle ahead and longer than $\mathrm{T}$ for the particle behind. If $\Delta z$ is the relative axial displacement caused by the interaction, the correction term involved is equal to

$$
\delta \Delta v_{z}=-v_{z 1}\left(\sqrt{1+\frac{2 a_{z} \Delta z}{v_{z 1}{ }^{2}}}-1\right)=-v_{z \circ K}\left(\sqrt{1+\frac{2 \Delta z(\kappa-1)}{T v_{z} 0 K^{2}}}-1\right)
$$

using equation $(10.5 .4)$.

The function $\Delta \boldsymbol{\eta}(\xi)$ now specifies the two-particle displacement in presence of an uniform acceleration field, which is characterized by the coefficient $\kappa$. As for the case without an external field the two particle distribution $\rho_{2}(\Delta \eta)$ follows from equation (5.4.10). The next step is to evaluate the $p(\mathbf{k})$ transform from equation (5.4.11). Finally, the required $N$-particle displacement distribution $\rho(\Delta \eta)$ follows with equation $(5.4 .9)$.

\subsection{Relativistic beams}

In this section we will investigate, in general terms, what modifications are required to extend the applicability of the models for statistical and space charge interactions to high energy beams, in which the average velocity of the particles is comparable to the speed of 1ight. It is assumed that the reader is familiar with the basic notions of the theory of special relativity.

The analysis will be restricted to paraxial beams in drift space. Conform the paraxial condition it is justifiable to assume that the relative velocities of the particles are small compared to their average velocity in the laboratory system. This implies that it is permitted to use non-relativistic equations to describe the relative motion of the particles and the required modifications only concern the coordinate transformation between the laboratory system $S$ and the frame of reference $S^{\prime}$ moving with beam. For beams in drift space, the system $S^{\prime}$ moves with a constant axial velocity $v_{z}$ relative to $S$. Accordingly, the problem is covered by the theory of special relativity. For high energy beams the Galileo transformation, which was used so far to relate the coordinates in the systems $S$ and $S^{\prime}$, has to be replaced by the Lorentz transformation. We will investigate the corresponding changes of the basic equations of our model.

For a relativistic beam, equation (3.2.2) is not suited to evaluate the average axial velocity of the particles observed in the laboratory system $S$. Instead, one should use the relativistic relation between momentum $p$ and energy $E$, which follows from the covariance of the energy-momentum four vector

$$
p^{2}=E^{2} / c^{2}-m_{0}^{2} c^{2}
$$

where $c$ is the speed of light, mo is the rest mass of the particles and $\mathrm{E}$ their average total energy, which is equal to

$$
\mathrm{E}=\mathrm{m}_{0} \mathrm{c}^{2}+\mathrm{eV}
$$

Substitution into equation $(10.6 .1)$ yields 


$$
p^{2}=2 m_{0} e V\left(1+\frac{e V}{2 m_{0} c^{2}}\right)
$$

which is the relativistic equivalent of equation (3.2.2). For paraxial beams, the lateral momentum $p_{t}$ is extremely small compared to the axial momentum $p_{z}$ and in the remaining analysis we will identify $p_{z}$ with $p$.

In particle optics one usually accounts for relativistic effects by introducing the so-called relativistic beam potential $\mathrm{V}^{*}$, which is defined such that the non-relativistic relation $\mathrm{p}^{2}=2 \mathrm{~m}_{0} \mathrm{eV}^{*}$ yields the same result as the relativistic equation (10.6.3). Accordingly, it is given by

$$
V^{*}=v\left(1+\frac{e V}{2 m a c^{2}}\right)
$$

We will come back to the use of the relativistic potential $V^{*}$ at the end of this section.

The time of flight $T$ (or $T_{f}$ ) is in the non-relativistic model given by $T=L / v_{z}$, as expressed by equation (3.2.3) (where $v_{z}$ is denoted as $\left.\left\langle v_{\mu}\right\rangle\right)$. In the relativistic model, the time of flight differs for the observers in the systems $S$ and $S^{\prime}$

$$
T=\frac{\gamma_{r} m_{0} L}{p}, \quad T^{\prime}=\frac{m_{0} L}{p}
$$

where $\gamma_{r}$ can be expressed as

$$
\gamma_{r}=\frac{E}{m_{0} c^{2}}=1+\frac{e V}{m_{0} c^{2}}
$$

conform the celebrated relation $E=m c^{2}$. Equation (10.6.2) was exploited in the second step. The proper measure for the duration of the interaction, to be used in the dynamical part of the calculation, is $T^{\prime}$, measured by the observer in $S^{\prime}$. It is specified by the second equation of $(10.6 .5)$. This equation provides the relativistic equivalent of equation (3.2.3). Notice that $\mathrm{L}$ is the length of the beam segment measured in the laboratory system $S$.

The linear particle densities $\lambda$ and $\lambda^{\prime}$, observed in $S$ and $S^{\prime}$ respectively, are equal to

$$
\lambda=\frac{\gamma_{r} m_{0} I}{\text { ep }}, \quad \lambda^{\prime}=\frac{m_{0} I}{\text { ep }}
$$

where the beam current $I$ is observed in the laboratory system $S$. The measure for the linear particle density to be used in the statistical part of the calculation is $\lambda^{\prime}$, which is observed in $S^{\prime}$. The second equation of $(10.6 .7)$ is the relativistic equivalent of equation (3.2.4).

Finally, it should be realized that the lateral velocities of the particles differ for the observers in $S$ and $S^{\prime}$. However, the transverse momentum $p_{\perp}$ of a particle is the same for both observers. The maximum transverse momentum $p_{0}$ is, in paraxial approximation, given by

$$
p_{0} \approx \alpha_{0} p
$$

where $\alpha_{0}$ is the beam semi-angle observed in the laboratory system $S$. Equation (10.6.8) replaces the non-relativistic equation $v_{0} \approx \alpha_{0} v_{z}$, which is used throughout the model. Notice that the lateral dimensions of the beam are the same for the observers in $S$ and $S^{\prime}$, thus the beam radius $r_{0}$ and the crossover radius $r_{c}$ can be used in the same way as in the non- 
relativistic model.

The macroscopic condition of the beam, observed in the reference system $S^{\prime}$, is now specified in terms of the experimental parameters, measured in the laboratory system $S$. The calculations can be carried out as before, using the appropriate values for the time of flight ' $T^{\prime}$, the linear particle density $\lambda^{\prime}$ and the maximum transverse momentum $p_{0}$, specified by equations $(16.6 .5),(16.6 .7)$ and (16.6.8) respectively. The results of the model should be expressed in terms of the generated distributions of axial and transverse momentum, $\rho\left(\Delta \mathrm{p}_{z}{ }^{\prime}\right)$ and $\rho\left(\Delta \mathrm{p}_{\perp}^{\prime}\right)$, and the trajectory displacement distribution $\rho\left(\Delta r^{\prime}\right)$, all observed in ' $S^{\prime}$. The transformation to the desired energy distribution $\rho(\Delta E)$, the angular deflection distribution $\rho(\Delta \alpha)$ and the trajectory displacement. distribution $\rho(\Delta r)$, observed in the laboratory system $S$, can be performed with

$$
\begin{aligned}
& \Delta E \approx p \Delta p_{z}{ }^{\prime} / m_{0} \\
& \Delta \alpha \approx \Delta p_{\perp}^{\prime} / p \\
& \Delta r=\Delta r^{\prime}
\end{aligned}
$$

which completes the analysis.

The discussion given above shows that the essential equations of the model can simply be adjusted to the relativistic case by expressing them in terms of the components of the momentum vectors $p$ and $p^{\prime}$, instead of the velocity components of the particles. The value of $p$ should be evaluated from the relativistic equation (10.6.3). As an alternative, one may use the original equations and account for the relativistic effects by means of the relativistic potential $V^{*}$, specified by equation (10.6.4). Accordingly, we conclude that for a paraxial beam in drift space, it is sufficient to replace the beam potential $\mathrm{v}$, appearing in the final equations for the various interaction effects, by its relativistic counterpart $V^{*}$.

\section{Appendix 10.A Distributions for the average reference trajectory}

This appendix discusses the derivation of the distributions
$\mathrm{pu}_{\mathrm{u}}(\mathrm{r})$ and $\mathrm{p}_{8}(\mathrm{r})$, given by equations $(10.3 .7)$ and $(10.3 .9)$
respectively. The evaluation of equation $(10.3 .5)$ can be split into two
steps by introducing the auxiliary function $h\left(r, r_{1}\right)$ $h\left(r, r_{1}\right)=\int_{0}^{\infty} d r_{2} f\left(r_{2}\right) \int_{0}^{2 \pi} \frac{d \varphi}{2 \pi} \delta\left[r-\left\{r_{1}^{2}-r_{2} 2-2 r_{1} r_{2} \cos \varphi\right\}^{1 / 2}\right] \quad$ (10.A.1)

This function will be evaluated first. The function $p(r)$ then follows from $h\left(r, r_{1}\right)$ as

$$
p(r)=\int_{0}^{\infty} d r_{1} f\left(r_{1}\right) h\left(r, r_{1}\right)
$$

As mentioned in section 10.3, the function $p(r)$ represents the distribution of the distance $r=\left|r_{1}-r_{2}\right|$ between two points, $r_{1}$ and $r_{2}$, in a plane when the distribution of the distance between the individual points and the origin is for both points given by the same distribution $f(r)$. The function $h\left(r, r_{1}\right)$ represents the distribution of the distance 
$r=\left|r_{1}-r_{2}\right|$ for a given value of $r_{1}$. We will successively treat the cases of an uniform $f(r)$ and a Gaussian $f(r)$.

Let $f(r)=f_{u}(r)$, where $f_{u}(r)$ is the uniform distribution given by equation (10.3.6). Substitution into equation (10.A.1) and integration yields

$$
h_{u}\left(r, r_{1}\right)= \begin{cases}2 r / R^{2} & \text { for } 0<r<R-r_{1} \\ \frac{2 r}{\pi R^{2}} \arccos \left(\frac{r_{1}{ }^{2}+r^{2}-R^{2}}{2 r_{1} r}\right) & \text { for } R-r_{1}<r<R+r_{1} \\ 0 & \text { for } r>R+r_{1}\end{cases}
$$

The reader might verify that this result can straightforwardly be understood from geometrical arguments. By substitution of equation (10.A.3) into equation (10.A.2) and integration one finds equation $(10.3 .7)$. We notice that an alternative way to derive equation (10.3.7) is to prove that the quantity $p_{u}(r) R^{2} / 2 r$ is equal to the ratio $A_{1} / A_{2}$, where $A_{1}$ is the mutual surface of two circles, both with radius $R$, whose centres are separated by a distance $r$ and $A_{2}=\pi R^{2}$, the surface of one circle.

Let $f(r)=f_{B}(r)$, where $f_{g}(r)$ is the Gaussian distribution given by equation $(10.3 .8)$. Substitution into equation (10.A.1) yields

$$
h_{6}\left(r, r_{1}\right)=\frac{2 r}{R^{2}} e^{-(r / R)^{2}-\left(r_{1} / R\right)^{2}} I_{0}\left(\frac{2 r r_{1}}{R^{2}}\right)
$$

using the integral

$$
\int_{a}^{b} d x \frac{e^{-q x}}{[(x-a)(b-x)]^{1 / 2}}=\pi e^{-(a+b) q / 2} I_{0}[(b-a) q / 2]
$$

where $I_{0}$ is the modified Bessel function of the first kind and zero order. From equations (10.A.4) and (10.A.2) one obtains equation (10.3.9), using the Laplace transform

$$
\int_{0}^{\infty} d x I_{0}\left[2(a x)^{1 / 2}\right] e^{-q x}=\frac{1}{q} e^{a / q}
$$

which is given by Erdélyi et al. (1954). 


\section{SPACE CHARGE RFFECT IN LOW DENSITY PARTICLE BEAMS}

\subsection{Introduction}

This chapter discusses an analytical model for the space charge effect in a beam segment in drift space. The model is based on first order perturbation theory. This approximation should be accurate for beams of relatively low particle density in which the particles experience only small deviations from their unperturbed trajectories. The theory applies to the same types of beams as the model for statistical interactions discussed in the previous chapters.

It will be demonstrated that the impact of the space charge effect on the lateral properties of the beam corresponds to the action of a negative lens. This space-charge lens is ideal if the current density distribution is uniform in every cross section of the beam. Accordingly, it causes a defocussing of the image as well as some (de)magnification. These effects can be compensated by proper lens adjustment and do not influence the resolution of the system. For nonuniform current density distributions the space-charge lens is nonideal. In addition to a defocussing and a (de)magnification, it produces aberrations causing a non-refocusable blurring of the image. We will describe the space-charge lens in terms of its first and third order properties, analogous to the description of conventional lenses used in geometrical optics.

\subsection{General aspects}

The space charge effect in charged particles beams is studied by many authors during the past 60 years. The reader is referred to Kirstein et al. (1967), Nagy and Szilagyi (1974) or Pierce (1954) for a general discussion of the subjects involved. The majority of the contributions deal with the beam spreading observed in high intensity beams or study the space charge effect occurring in the vicinity of the cathode surface of a thermionic gun. In this chapter we will restrict ourselves to the impact of space charge on the lateral properties of low and medium density beams in drift space.

Both the (deterministic) space charge effect and the effect of statistical interactions are generated by the mutual coulomb repulsion of the charged particles constituting the beam. In order to emphasize this common basis we will start from a general description with covers both phenomena, using the terminology and notation introduced in chapter 5. The specific aspects of an analytical model for the space charge effect will be considered next.

The displacement distribution $\rho(\Delta \eta)$ contains all information regarding the different manifestations of Coulomb particle-particle interactions. It specifies the probability that a test particle experiences a displacement $\Delta \eta$ due to the coulomb interaction with the other particles in the beam, referred to as field particles. Equivalently, one may describe $\rho(\Delta \eta)$ as the distribution of displacements $\Delta \eta$ experienced by a large set of test-particles, which would, in absence of Coulomb interactions, all run along the same trajectory in the beam. The unperturbed trajectory of the test particles is called the reference trajectory. In this chapter we are primarily interested in the lateral properties of the beam, thus we consider the displacements 


$$
\Delta \boldsymbol{\eta}=\Delta \mathbf{v}_{\perp} \quad \text { or } \quad \Delta \boldsymbol{\eta}=\Delta \boldsymbol{r}_{\perp}
$$

The quantities $\Delta v_{1}$ and $\Delta r_{1}$ specify the lateral displacement in velocity and position of a test particle at the end of the beam segment.

In chapter 5 the following representation of the distribution $\rho(\Delta \eta)$ was introduced (reproducing equation $(5.2 .3)$ )

$$
\rho(\Delta \eta)=\int d \xi_{1} d \xi_{2} \cdots d \xi_{N-1} P_{N}\left(\xi_{1}, \xi_{2}, \cdots, \xi_{N-1}\right) \delta\left[\Delta \eta-\Delta \eta\left(\xi_{1}, \xi_{2}, \cdots, \xi_{N-1}\right)\right]
$$

in which the set of generalized coordinates $\xi_{1}, \xi_{2}, \cdots, \xi_{N-1}$ specifies the initial configuration of field particles relative to the test particle, see equation (5.2.1) and figure 5.1. Given this set of coordinates one can in principle determine the deviation of the test particle $\Delta \boldsymbol{\eta}=\Delta \boldsymbol{\eta}\left(\boldsymbol{\xi}_{1}, \boldsymbol{\xi}_{2}, \cdots, \boldsymbol{k}_{\mathrm{N}-1}\right)$ using Coulomb's law and classical mechanics. This evaluation is referred to as the dynamical part of the problem. The statistical part of the problem is the determination of the probability $P_{N}\left(\xi_{1}, \xi_{2}, \cdots, \xi_{N-1}\right)$ of the configuration $\xi_{1}, \xi_{2}, \cdots, \xi_{N-1}$ and the evaluation of the distribution of displacements $\rho(\Delta \boldsymbol{\eta})$ from equation $(11.2 .2)$. We note that equation (11.2.2) expresses the distribution $\rho(\Delta \eta)$ as an ensemble average. The ensemble average is assumed to be equivalent to a time average, which corresponds to the distribution of displacements of a large set of test-particles successively arriving at the end of the beam segment. The reader should be alert that we will often describe a quantity in terms of a time-average, while the corresponding equation represents an ensemble-average.

Assuming that one is somehow able to determine the distribution $\rho(\Delta \eta)$, the average shift of the test particles $\langle\Delta \eta\rangle$ can be calculated as

$$
\begin{aligned}
\langle\Delta \eta\rangle & =\int \mathrm{d} \Delta \boldsymbol{\eta} \rho(\Delta \boldsymbol{\eta}) \Delta \eta \\
& =\int \mathrm{d} \xi_{1} \mathrm{~d} \xi_{2} \cdots \mathrm{d} \xi_{N-1} \mathrm{P}_{N}\left(\xi_{1}, \xi_{2}, \cdots, \xi_{N-1}\right) \Delta \eta\left(\xi_{1}, \xi_{2}, \cdots, \xi_{N-1}\right)
\end{aligned}
$$

This shift depends strongly on the choice of the reference trajectory. For the central reference trajectory in a rotational symmetric beam one expects that $\langle\Delta \eta\rangle=0$, since there is no average space charge force acting on a particle which is located on the axis. In the previous chapters this phenomenon was exploited to isolate the effect of statistical interactions. However, this chapter focusses on the calculation of the average shift $\langle\Delta \eta\rangle$ and we should consider of $f$-axis reference trajectories in order to obtain non-vanishing results.

The average shift $\langle\Delta\rangle\rangle$ is directly related to the average electrostatic force acting on the test particles during their flight. In order to make this relation explicit we express the displacement $\Delta \boldsymbol{\eta}\left(\xi_{1}, \xi_{2}, \cdots, \xi_{N-1}\right)$ of the test particle caused by a given set of field particles $\xi_{1}, \xi_{2}, \cdots, \xi_{N-1}$ as an integral over time (reproducing equation $(5.3 .5))$

$$
\Delta \eta\left(\xi_{1}, \xi_{2}, \cdots, \xi_{N-1}\right)=\int_{t_{i}}^{t_{f}} d t G_{N}\left(t_{1}, t_{2}, \cdots, \xi_{N-1}, t\right)
$$

in which $t_{i}$ and $t_{f}$ the initial and final time of interaction, defined by the moments that the test particle enters and leaves the beam segment respectively. For the displacement in transverse velocity $\left(\Delta \boldsymbol{\eta}=\Delta \boldsymbol{v}_{\perp}\right)$ the function $G_{N}\left(t_{1}, t_{2}, \cdots, t_{N}-1, t\right)$ is given by

$$
G_{N}\left(\xi_{1}, \xi_{2}, \cdots, \xi_{N-1}, t\right)=F_{\perp}\left(\xi_{1}, \xi_{2}, \cdots, \xi_{N-1}, t\right) / m
$$


in which $F_{1}\left(\xi_{1}, \xi_{2}, \cdots, \xi_{N-1}, t\right)$ represents the transverse component of the total interaction force acting on the test particle at time $t$. For the displacement in transverse position $\left(\Delta \boldsymbol{\eta}=\Delta \mathbf{r}_{1}\right)$ one finds (reproducing equation $(5.3 .7))$

$$
\mathbf{G}_{N}\left(\xi_{1}, \xi_{2}, \cdots, \xi_{N}-1, t\right)=\left(t_{f}-t\right) F_{\perp}\left(\xi_{1}, \xi_{2}, \cdots, \xi_{N-1}, t\right) / m
$$

Equations (11.2.5) and (11.2.6) follow directly from the definition of the function $G_{N}\left(\xi_{1}, \xi_{2}, \cdots, \xi_{N-1}, t\right)$ given by equation $(11.2 .4)$.

We will now consider the cases $\Delta \boldsymbol{\eta}=\Delta \mathbf{v}_{\perp}$ and $\Delta \boldsymbol{\eta}=\Delta \mathbf{r}_{\perp}$ separately. By substituting equation (11.2.4) and (11.2.5) into equation (11.2.3) and interchanging the integration over $t$ and the integration over $\xi_{1}, \xi_{2}, \cdots, \xi_{\mathrm{N}-1}$ one obtains

$$
\left\langle\Delta v_{\perp}\right\rangle=\int_{t_{i}}^{t_{f}} d t \frac{\left\langle\mathbf{F}_{\perp}(t)\right\rangle}{m}
$$

Similarly, one finds with equation $(11.2 .6)$

$$
\left\langle\Delta r_{\perp}\right\rangle=\int_{t_{i}}^{t_{f}} d t\left(t_{f}-t\right) \frac{\left\langle P_{\perp}(t)\right\rangle}{m}
$$

The quantity $\left\langle\boldsymbol{F}_{\perp}(t)\right\rangle$ denotes the average transverse component of the interaction force experienced by the test particles at time $t$

$$
\left\langle\boldsymbol{P}_{\perp}(t)\right\rangle=\int \mathrm{d} \xi_{1} \mathrm{~d} \xi_{2} \cdots \mathrm{d} \xi_{N-1} P_{N}\left(\xi_{1}, \xi_{2}, \cdots, \xi_{N-1}\right) F_{\perp}\left(\xi_{1}, \xi_{2}, \cdots, \xi_{N-1}, t\right)
$$

Equations $(11.2 .7),(11.2 .8)$ and (11.2.9) constitute the basic equations for the space charge effect within the formalism of chapter 5. An important difference with the theory for the statistical effects is that one may determine the average over all configurations $\xi_{1}, \xi_{2}, \cdots, \boldsymbol{k}_{\mathrm{N}-1}$ first and carry out the time-integration next, without loss of generality. This is simply due to the fact that the characteristic quantities for the space charge effect $\left\langle\Delta \mathbf{r}_{\perp}\right\rangle$ and $\left\langle\Delta \nabla_{\perp}\right\rangle$ are linear in the interaction force, while the characteristic quantities for the statistical effects $\left\langle\Delta v_{z}{ }^{2}\right\rangle,\left\langle\Delta v_{\perp}{ }^{2}\right\rangle$ and $\left\langle\Delta r_{\perp}{ }^{2}\right\rangle$ are quadratic in the interaction force.

A straightforward analytical evaluation of equations (11.2.7), $(11.2 .8)$ and $(11.2 .9)$ does not appear to be feasible. In order to determine the function $F_{1}\left(\xi_{1}, \xi_{2}, \cdots, \xi_{N-1}, t\right)$ one needs to know the actual positions of all particles at time $t$. However, the computation of these positions requires the knowledge of the particle trajectories under influence of the interaction force $F_{\perp}$ up to that time. In order to disentangle this many-body problem by analytical means one is forced to introduce some fundamental assumptions.

The essential observation is that one is not really interested in the value of $\boldsymbol{F}_{1}\left(\xi_{1}, \xi_{2}, \cdots, \xi_{N-1}, t\right)$ for any specific configuration, but rather in the ensemble average $\left\langle\mathbf{F}_{\perp}(t)\right\rangle$. One may conjecture that the calculation of $\left\langle\mathbf{F}_{\perp}(t)\right\rangle$ does not require any detailed knowledge of the coordinates of the individual field particles in the separate configurations constituting the ensemble, but only involves some smoothed-out distribution of charge which can directly be expressed in terms of the macroscopic properties of the beam. Let us investigate this idea more closely. 
Consider a single configuration of field particles. Let $\rho(r, t)$ be the microscopic density distribution at time $t$

$$
\rho(r, t)=\sum_{i=1}^{N-1} \text { e } \delta\left[r-r_{i}(t)\right]
$$

where $\delta(\mathbf{r})$ is the 3-dimensional delta-Dirac function. We recall that the quantity $e$ denotes the particle charge (not the unit of elementary charge). The charge density distribution $\rho(r, t)$ contains all microscopic information of the individual particle positions as function of time. If one knows this distribution one can directly calculate the force $\boldsymbol{F}(\boldsymbol{r}, t)$ acting at time $t$ on the test particle located at position $r$, using

$$
F(r, t)=\frac{e}{4 \pi \varepsilon_{0}} \int d r_{1} \rho\left(r_{1}, t\right) \frac{r-r_{1}}{\left|r-r_{1}\right|^{3}}
$$

It is sometimes convenient to express this equation in an alternative form. By exploiting the vector relation

$$
\frac{\partial}{\partial r} \frac{r-r_{1}}{\left|r-r_{1}\right|^{3}}=4 \pi \delta\left(r-r_{1}\right)
$$

equation (11.2.11) can be transformed to

$$
\int_{S} r(r, t) \cdot d S=\frac{e}{\epsilon_{0}} \int_{V} \rho(r, t) d r
$$

in which $S$ is a closed surface surrounding the volume $V$. Equation $(11.2 .13)$ is known as Gauss's theorem for the electrostatic force produced by a configuration of charges. Gauss's theorem states that the total flux of $F$ through the surface $S$ depends only on the total charge within volume $\mathrm{v}$, whatever the exact location of the individual charges within this volume.

Equations $(11.2 .11)$ and $(11.2 .13)$ are true for every configuration of particles in the ensemble. Accordingly, one should expect that the same relations do apply to the corresponding ensemble averaged quantities. By taking the ensemble average of both sides of equation $(11.2 .11)$ one directly obtains

$$
\langle\mathbf{P}(\mathbf{r}, \mathrm{t})\rangle=\frac{e}{4 \pi \varepsilon_{0}} \int d \mathbf{r}_{1}\left\langle\rho\left(r_{1}, t\right)\right\rangle \frac{r-r_{1}}{\left|r-r_{1}\right|^{3}}
$$

in which $\langle\rho(r, t)\rangle$ is called the smoothed-out distribution of charge. Employing equation (11.2.12) this result can be transformed to

$$
\int_{S}\langle\mathbf{F}(\mathbf{r}, \mathrm{t})\rangle \cdot \mathrm{d} \mathbf{S}=\frac{\mathbf{e}}{\varepsilon_{0}} \int_{V}\langle\rho(\mathbf{r}, \mathrm{t})\rangle \mathrm{dr}
$$

which can also be obtained from equation $(11.2 .13)$ by taking the ensemble average of both sides. Equation (11.2.15) relates the average force $\langle\mathbf{F}(\mathbf{r}, \mathrm{t})\rangle$ directly to the smoothed-out distribution of charge $\langle\rho(r, t)\rangle$. Equation $(11.2 .15)$ is of ten identified with Gauss's theorem. However, strictly speaking Gauss's theorem pertains to a specific configuration of particles and not to the ensemble average.

From now on we will restrict ourselves to the case of a rotational symmetric beam. Let $r_{0}(z)$ be a measure for the beam radius at 
For an uniform distribution it is sufficient to specify the outer beam radius $r_{m}(z)$, taking $r_{0}(z)=r_{m}(z)$. For a Gaussian distribution one should take $r_{m}(z)=\infty$ and relate $r_{0}(z)$ to some characteristic width measure, for instance the rms-value of the spatial distribution at position $z$. In general we will express the smoothed-out distribution of charge in a rotational symmetric beam as

$\langle p(r, z)\rangle= \begin{cases}\frac{e \lambda}{\pi r_{0}(z)^{2}}\left[a(z)+b(z)\left(\frac{r}{r_{0}(z)}\right)^{2}+c(z)\left(\frac{r}{r_{0}(z)}\right)^{4}+\cdots\right] & \text { for } r<r_{m}(z) \\ 0 & \text { for } r>r_{m}(z)\end{cases}$

$(11.2 .16)$

in which $\lambda$ is the linear particle density defined by equation (3.2.4). The type of distribution is specified by the quantities $a(z), b(z)$, $c(z), \cdots$, which may still depend on the axial coordinate $z$. For all $z$ these quantities are related through the normalization of $\langle p(r, z)\rangle$

$$
1=\frac{1}{e \lambda} \int_{0}^{r_{m}(z)}\langle\rho(r, z)\rangle 2 \pi r d r
$$

In the representation of equation $(11.2 .16)$ an uniform current density distribution corresponds to

$$
a(z)=1, b(z)=c(z)=\cdots=0, r_{m}(z)=r_{0}(z)
$$

while a Gaussian current density distribution is represented as

$$
a(z)=1, \quad b(z)=\frac{-1}{2 !}, \quad c(z)=\frac{1}{3 !}, \cdots, \quad r_{m}(z)=\infty, \quad r_{0}(z)=\sqrt{2} \sigma(z)
$$

with $\sigma(z)=\left[\left\langle\mathrm{r}(z)^{2}\right\rangle / 2\right]^{1 / 2}$, the well-known sigma-value of the Gaussian distribution at axial position z. Finally, a parabolic current density distribution is specified by

$$
a(z)=2, b(z)=-2,=c(z)=\cdots=0, r_{m}(z)=r_{0}(z)
$$

The advantage of the form of equation (11.2.16) is that one can study the space charge effect on a general basis without making any a priori assumptions on the exact distribution of charge in the beam. We will study the impact of each term in $\langle\rho(r, z)\rangle$ separately. For an uniform distribution one only has to consider the first term. For non-uniform distributions one has to consider higher order terms as well.

We will now evaluate equation (11.2.15) using the representation of $\langle\rho(r, z)\rangle$ given by equation $(11,2,16)$. Consider a cylinder of length $\Delta z$ and radius $r$ centred around the beam axis of a rotational symmetric beam. The average lateral force component $\left\langle\mathbf{F}_{1}\right\rangle$ is directed perpendicular to the cylinder surface. We will ignore any variation in the axial component of the average force (thus assuming $\left\langle F_{/ /}\right\rangle=0$ or $\left\langle F_{\| /}\right\rangle=$constant). Accordingly, the left hand side of equation (11.2.15) is equal to $\left\langle\mathbf{P}_{\perp}\right\rangle \times 2 \pi r \Delta z$, which gives

$$
\left\langle\mathbf{P}_{\perp}(r, z)\right\rangle=\frac{e}{\epsilon_{0} r} \int_{0}^{r}\left\langle\rho\left(r_{1}, z\right)\right\rangle r_{1} d r_{1}
$$

By substitution of equation (11.2.16) into equation $(11.2 .21)$ one 
obtains for the average value of the transverse component of the force acting on a test particle in the beam $(r<\mathrm{rm}(z))$

$$
\left\langle\boldsymbol{F}_{\perp}(r, z)\right\rangle=\frac{\mathrm{e}^{2} \lambda}{2 \pi \varepsilon_{0}}\left[a(z) \frac{\mathrm{r}}{\mathrm{r}_{0}(z)^{2}}+b(z) \frac{\mathrm{r}^{3}}{2 \mathrm{r}_{0}(z)^{4}}+c(z) \frac{\mathrm{r}^{5}}{3 \mathrm{r}_{0}(z)^{6}}+\cdots\right]
$$

The first term between brackets in equation (11.2.22) is proportional to the distance to the axis $r$. For an uniform current density distribution this is the only non-vanishing term, as follows with equation (11.2.18). Notice that an ideal lens has the property that it deflects the particles by an angle which is proportional to the radial distance $r$. Hence, for an uniform current density distribution the space charge force $\left\langle F_{\perp}(r, z)\right\rangle$ acts as an ideal lens. For non-uniform distributions the remaining terms in equation $(11.2 .22)$ do not all vanish and the space charge force $\langle F(r, z)\rangle$ acts as a non-ideal lens. Besides its imaging action it introduces aberrations as well. The second term between brackets in equation (11.2.22) corresponds to third-order aberrations, similar to those generated by a conventional electrostatic or magnetic lens.

The radial equation of motion of a test particle moving under influence of a lateral force $\left\langle F_{\perp}(r, z)\right\rangle$ is given by

$$
\frac{d^{2} r(t)}{d t^{2}}=\frac{\left\langle F_{\perp}[r(t), z(t)]\right\rangle}{m}
$$

In general one may write

$$
\frac{d^{2} r}{d t^{2}}=\frac{d^{2} r}{d z^{2}}\left(\frac{d z}{d t}\right)^{2}+\frac{d r}{d z} \frac{d^{2} z}{d t^{2}}
$$

As we restrict ourselves to beam segments in drift space, the second term can be disregarded (since $z$ is then linear in $t: z=z_{0}+v\left(t-t_{0}\right)$ ). With equations $(11.2 .22)$ and $(11.2 .23)$ one now obtains

$$
\frac{d^{2} r}{d z^{2}}=k\left[a(z) \frac{r}{r_{0}(z)^{2}}+b(z) \frac{r^{3}}{2 r_{0}(z)^{4}}+c(z) \cdot \frac{r^{5}}{3 r_{0}(z)^{6}}+\cdots\right]
$$

where the quantity $k$ is given by

$$
\mathrm{k}=\frac{\mathrm{e} \lambda}{4 \pi \varepsilon_{0} V}=\frac{1}{4 \pi \varepsilon_{0}}\left(\frac{\mathrm{m}}{2 \mathrm{e}}\right)^{1 / 2} \frac{\mathrm{I}}{\mathrm{V}^{3 / 2}}
$$

Equation (11.2.24) is the ray-equation for a rotational symetric beam in drift space, which takes the interaction force curresponding to the smoothed-out distribution of charge into account. It should be emphasized that this ray-equation pertains to the average trajectory of a large set of test particles starting with the same initial condition.

The ray-equation $(11.2 .24)$ relies on an essential assumption which might not be obvious from its derivation. The average trajectory of the test particle is calculated as if it is moving in some external electrostatic field. This "external" field is produced by the smoothedout distribution of charge of the field particles. In this approach one considers only the perturbation of the test particle and ignores the influence of the test particle on the field particles surrounding it. Due to this lack of feedback (from the coordinates of the test particle to the coordinates of the field particles) the model becomes unrealistic in case the space charge effect is produced by strong interactions between a limited number of particles. 
It is instructive to consider the space charge effect occurring in a beam with a narrow waist of radius $r_{c}$ (we use the term "waist" in stead of crossover, since the particles do not necessarily cross the axis). Let $\alpha_{0}$ be the beam semi-angle and assume that the current density distribution is approximately uniform in every cross section of the beam. Consider a particle moving along the edge of the beam towards the waist of the beam. Thus $r=r_{0}(2)$ and $\alpha=\alpha_{0}$. From equations (11.2.22) and $(11.2,18)$ one finds for the space charge force acting on this particle

$$
\left\langle F_{\perp}\right\rangle=\frac{e^{2} \lambda}{2 \pi \varepsilon_{0} r_{0}(z)}
$$

According to this equation the space charge force $\left\langle\mathrm{F}_{\perp}\right\rangle$ acting on the test particle is expected to diverge for a waist radius which approaches zero (thus $r_{0}(z)=r_{c} \rightarrow 0$ ). At this point one should notice that $r_{0}(z)$ represents the beam radius in presence of the space charge repulsion, which will in general be larger than the unperturbed beam radius. When studying the particle motion in the vicinity of the waist under influence of the space charge force $\left\langle F_{t}\right\rangle$ it is useful to consider the extreme cases of a beam of very high particle density and a beam of low particle density. Let us study the implications of equation (11.2.26) for these cases separately.

In a beam of very high particle density the particles running along the edge of the beam are expected to remain at the edge of the beam (without crossing the axis) due to the repulsion of the total space charge present in the inner part of the beam. The corresponding waist radius $r_{c}$ will be non zero even for a homocentric beam. Accordingly, the space charge force $\left\langle F_{\perp}\right\rangle$ computed from equation $(11.2 .26)$ will be finite provided that one accounts for the impact of the space charge repulsion on the distribution of field particles. If the stream of particles is such that non of the particle trajectories do cross each other, one speaks of "laminar flow". This condition implies that all particles located at a certain position have the same velocity. This type of flow will be further investigated in the next section.

In a beam of low particle density, a particle running along the edge of the beam is expected to cross the axis unless it happens to collide with some other particle travelling with approximately the same axial coordinate, but diametrically opposite. In this type of beam the space charge effect may cause an axial shift and a slight (de)magnification of the crossover, but it will not change the characteristics of the beam drastically. Accordingly, a homocentric beam is expected to have a crossover of zero radius and a straightforward application of equation (11.2.26) to compute the space charge force $\left\langle F_{1}\right\rangle$ will lead to divergent results at the location of this crossover. clearly, in reality the space charge force acting on the test particle is always finite due to the fact that the minimum distance between the test and a field particle is limited by their mutual interaction. The divergence of equation (11.2.26) for this case is a consequence of the decoupling of the coordinates of the test particle from the coordinates of the field particles. However, anticipating on the results of section 11.7, we mention that this decoupling does not represent any problem in the actual calculation of the space charge effect in beams of low particle density with a narrow crossover.

In order to specify the distinction between beams of low and beams of high particle density let us consider the number of particles simultaneously present in a cylindrical volume in the beam with some characteristic length $\Delta z$ and some characteristic radius $r$. A beam of high particle density is now defined as a beam for which this number is large compared to unity. Taking $r=r_{m}(z)$ and $\Delta z$ of the order $r_{m}(z)$ this 
condition can be expressed as

$$
\frac{r_{m}(z)}{e} \int_{0}^{r_{m}(z)}\langle\rho(r, z)\rangle 2 \pi r d r \gg 1
$$

We will refer to equation $(11.2 .27)$ as the continuum condition. It is postulated that the continuum condition should be fulfilled in order to obtain laminar flow. However, we add that this condition does not guarantee laminar flow.

For a beam with an uniform current density distribution equation $(11.2 .27)$ transforms to

$$
\lambda r_{0}(z) \gg 1
$$

as follows with equations (11.2.16) and (11.2.18). This equation expresses that the beam radius $r_{0}(z)$ should be large compared to the average axial separation of the particles $\langle s\rangle=1 / \lambda$. It should be fulfilled for all $z$, including the location of the waist $\left(r_{0}(z)=r_{c}\right)$. In order to verify equation (11.2.28) one needs to know the actual dimensions of the beam which occur under influence of the space charge force.

The classic approach to the space charge effect is to assume laminar flow. We will briefly review this approach in section 11.3. However, as it turns out the continuum condition of equation (11.2.27) is in general not fulfilled for the particle beams used in lithography and microscopy instruments. Therefore, the laminar flow model is not suited to describe the space charge effect occurring in the drift sections of such systems. The remaining part of the chapter concerns an alternative approach which is appropriate for this case. In this model the ray-equation $(11.2 .24)$ is solved in first order perturbation approximation. The basic philosophy of the model is described in section 11.4. The actual calculations are presented in section 11.5 and 11.6 , treating the first order optical properties of the space-charge lens and its third order aberrations respectively. In sections 11.7 and 11.8 the results of the model are examined for the specific cases of a beam segment with a narrow crossover and a homocentric cylindrical beam segment respectively. Finally, section 11.9 considers the problem of adding the contributions of the individual beam segments to determine the total effect generated in the entire beam.

\subsection{Beans with laminar flow}

The classic approach to the problem of calculating the spreading of a beam under influence of its own space charge is to assume laminar flow. The first contributions to the subject were given by McGreggorMorris and Mines (1925) and Watson (1927). Detailed discussions and extensive references can be found in the standard textbooks on electron optics, for instance see Glaser (1952), Klemperer (1953), El-Kareh and El-Kareh (1970) or Hutter (1967). We will only briefly investigate the subject here. Our main interest is to determine the range of validity of the laminar-flow approach.

Consider a rotational symmetric homocentric converging beam with initial radius $r_{i}$ and slope $r_{i}$. Assume that the current density distribution in the initial plane $\left(z=z_{i}\right)$ is uniform. The reader might verify that the assumption of laminar flow in a homocentric beam implies that the distribution will be uniform in other planes as well. The space 
charge force, which is for an uniform distribution directly proportional to the distance to the axis, may change the size of the beam but it does not alter its shape. This type of laminar flow is referred as congruent flow. In case of congruent flow it is sufficient to determine the trajectory of a particle moving along the edge of the beam, thus taking $r=r_{0}(z)$.

The appropriate ray equation for the case of an uniform beam follows from equations (11.2.24) and (11.2.18). Multiplication of both sides of equation $(11.2 .24)$ with $(\mathrm{d} r / \mathrm{dz})$ and integration results in

$$
\frac{1}{2}\left(\frac{d r_{0}}{d z}\right)^{2}=k \ln \left(\frac{r_{0}}{r_{i}}\right)+\frac{1}{2} r_{1}^{2}
$$

The minimum beam radius $r_{c}$ follows by taking $\left(d r_{0} / d z\right)=0$. Equation (11.3.1) now yields

$$
r_{c}=r_{i} \exp \left(-r_{i}^{\prime} / 2 k\right)=r_{i} \exp (-1 / 4 \pi)
$$

where $\lambda$ is the scaled linear particle density, given by

$$
\bar{\lambda}=\frac{k}{2 \alpha_{0}{ }^{2}}=\frac{m^{1 / 2}}{2^{7 / 2} \pi \varepsilon_{0} \mathrm{e}^{1 / 2}} \frac{\mathrm{I}}{\alpha_{0} \mathrm{~V}^{3 / 2}}
$$

as was previously defined by equation (7.3.22). In equation (11.3.2) we approximated $r_{i} \alpha_{0}$, with $\alpha_{0}$ the beam semi-angle at the initial plane. This is justified since we consider only paraxial beams. The $z-$ coordinate for which the beam radius reaches its minimum value follows by integrating equation (11.3.1)

$$
z_{c}-z_{i}=\int_{r_{i}}^{r_{c}}\left[2 k \ln \left(\frac{r_{0}}{r_{i}}\right)+r_{i}^{2}\right]^{-1 / 2} d r_{0}
$$

Substituting $r=r_{i} \exp \left(5^{2}-r_{i}^{\prime 2} / 2 k\right)$ this transforms to

$$
z_{c}-z_{i}=\sqrt{\frac{2}{k}} r_{c} \int_{0}^{\left|r_{i}^{\prime}\right| / \sqrt{2 k}} \exp \left(\zeta^{2}\right) d \zeta=\frac{r_{c}}{\alpha_{0} \pi^{1 / 2}} \int_{0}^{1 / 2 \pi} \exp \left(\zeta^{2}\right) d \zeta
$$

where $r_{c}$ is given by equation (11.3.2) and the linear particle density $\pi$ by equation (11.3.3). Equations (11.3.2) and (11.3.4) specify the size and axial position of the minimum cross section of an uniform homocentric beam, assuming laminar flow.

In the design of oscilloscope tubes one likes to determine the radius $r_{s}$ of the smallest spot which can be obtained at the screen, given a certain beam current $I$, beam voltage $V$ and distance to the screen $L=z_{s}-z_{i}$. Given these parameters the spot size at the screen can be minimized by varying $r_{i}^{\prime}$. Schwartz (1957) presented an universal curve from which the ratio $r_{s} / r_{i}$ can be determine as function of $\pi^{1 / 2}$ (our notation). He pointed out that the minimum spot at the screen is obtained by focussing the beam in such a manner that its minimum cross section occurs somewhat ahead of the screen. For high $\lambda$-values the spot radius $r_{s}$ can be appreciable smaller than the spot radius $r_{c}$ obtained when the minimum cross section is made to occur at the screen. The reader is referred to the original publication of Schwartz (1957) or to El-Kareh and E1-Kareh (1970) for a more detailed discussion of this phenomenon. One should keep in mind that the so-called universal beani spreading curve is, in fact, not universal at all. It relies on the 
continuum approximation, which breaks down for low particle densities, as was pointed out earlier. Van den Broek (1984) investigated the influence of the current density distribution on the spot growth. The results are presented in graphs similar to those of Schwartz, showing that the spot growth for non-uniform beams is larger than for uniform beams.

We conclude this section by examining the conditions for which the laminar flow model is justified. Substitution of equation (11.3.2) into the continuum condition of equation $(11.2 .28)$ yields

$$
\bar{r}_{i} \gg \frac{\exp (1 / 4 \pi)}{\pi}
$$

in which $\overline{\mathbf{r}}_{\mathbf{i}}$ is given by

$$
\bar{r}_{i}=\frac{r_{i}}{d_{0}}=\frac{8 \pi \varepsilon_{0}}{\mathrm{e}} \alpha_{0}^{2} \mathrm{Vr}_{i}
$$

where the scaling quantity $d_{0}$ is defined by equation (7.3.2). The right hand side of equation (11.3.5) increases exponentially with decreasing $\lambda$. Given a certain value of $\bar{r}_{i}$ equation $(11.3 .5)$ specifies a lower limit for $\bar{\lambda}$. For an electron beam with $V=10 \mathrm{kV}, \quad \alpha_{0}=10 \mathrm{mR}$ and $r_{1}=100 \mu \mathrm{m}$ one finds from equation $(11.3 .6) \bar{r}_{1}=1.39 \times 10^{5}$. According to equation (11.3.5) one should demand $\lambda_{* 5} \neq 0 \times 10^{-2}$, which implies $\mathrm{I}>0.66 \mathrm{~mA}$, as follows with equation (11.3.3). This is a typical value of the minimum beam current necessary to fulfil the continuum condition at the smallest cross section. Clearly, this type of current is so large that it is beyond the scope of this monograph, see section 1.1. It is interesting to note that a transition region may occur in which the continuum approximation is justified for the particles at the edge of the beam, but not for the inner-beam particles. In that case the outer beam particles still form a waist, while the inner-beam particles already cross over.

It should be emphasized that our model for statistical interactions, described in chapter 5 , breaks down in case equation (11.3.5) would be satisfied. The assumption that the field particles can be considered as statistically independent, as expressed by equation (5.3.1), would clearly not be justified. Similarly, it would be incorrect to represent the total deviation of the test particle as the sum of independent two particle effects, as expressed by equation $(5,3.12)$. We recall that our model relies on the assumption that the test particle is not involved in more than one strong interaction during its flight. The majority of all interactions are assumed to generate only small deviations from the unperturbed trajectory. In the next section we will present a method to calculate the space charge effect in beams to which our model of statistical interactions applies, that is beams of low or medium current density.

\subsection{First order perturbation theory}

Consider a rotational symmetric paraxial beam segment with a crossover. Let the beam radius at axial position $z$ be characterized by $r_{0}(z)$, which is, in absence of particle interactions, given by

$$
\tilde{r}_{0}(z)=r_{c}+\alpha_{0}\left|z-z_{c}\right|
$$

The tilde ( $\sim$ ) refers to the unperturbed condition of the beam. The axial 
coordinate $z_{c}$ specifies the location of the crossover plane. The unperturbed trajectory of a test particle can be represented as

$$
\tilde{r}(z)=\left[r_{\perp}^{2}+\alpha^{2}\left(z-z_{c}\right)^{2}+2 r_{\perp} \alpha\left(z-z_{c}\right) \cos (\psi-\varphi)\right]^{1 / 2}
$$

as can be understood from figure 5.1. A meridian trajectory (which is a trajectory which intersects the beam axis) corresponds to $\psi=\varphi$ or $\psi=\varphi+\pi$. For these trajectories equation $(11.4 .2)$ transforms to

$$
\widetilde{r}(z)=\left|r_{\perp} \pm \alpha\left(z-z_{c}\right)\right|
$$

the + sign referring to the case $\psi=\varphi$ and the $-\operatorname{sign}$ to $\psi=\varphi+\pi$. By allowing negative values for $\tilde{r}(z), r_{\perp}$, and $\alpha$, one can in general represent a meridian trajectory as

$$
\tilde{\mathbf{r}}(z)=r_{\perp}-\alpha\left(z-z_{c}\right)
$$

where $\alpha$ is defined such that a positive value corresponds to a trajectory which runs from large $r_{\perp}$ to small $r_{\perp}$ values with increasing $z$, see figure 11.1. Notice that a meridian trajectory in a rotational symmetric beam remains meridian also in presence of the space charge repulsion. From now on we will restrict ourselves to trajectories described by equation $(11.4 .3)$ and assume that the results apply to other trajectories as well.

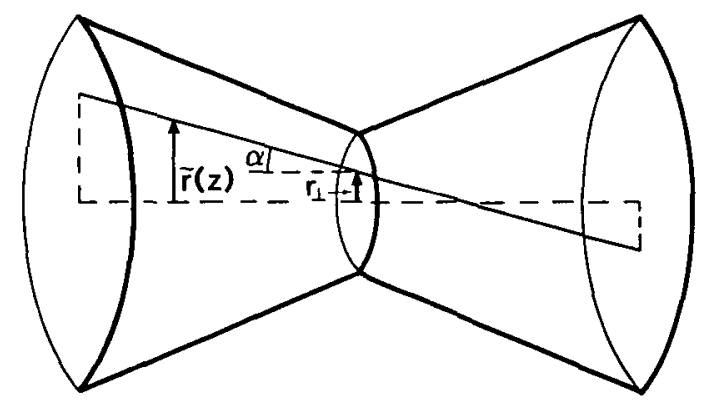

Figure 11.1 Rotational symmetric beam segment with a crossover. The radial coordinate $\hat{r}(z)$ of an (unperturbed) meridian trajectory is specified by the angle $\alpha$ and the radial coordinate $r_{1}$ in the crossover plane.

The model presented in this section starts from the ray-equation $(11.2 .24)$. In the first order perturbation approximation the total space charge force acting on the test particle is evaluated from the unperturbed dimensions of the beam and the unperturbed trajectory of the test particle, specified by $\tilde{r}_{0}(z)$ and $\tilde{r}(z)$ respectively. By replacing $r$ and $r_{0}(z)$ in the right hand side of the ray-equation $(11.2 .24)$ by $r_{0}(z)$ and $\tilde{r}(z)$ respectively one finds for the angular and spatial displacements of the test particle 


$$
\begin{aligned}
& \Delta \alpha_{f} \approx \frac{\Delta v_{\perp} r}{v_{z}}=k \int_{z_{i}}^{z_{S}} d z\left[a \frac{\tilde{r}(z)}{\tilde{r}_{0}(z)^{2}}+b \frac{\tilde{r}(z)^{3}}{2 \tilde{r}_{0}(z)^{4}}+c \frac{\tilde{r}(z)^{5}}{3 \tilde{r}_{0}(z)^{6}}+\cdots\right] \\
& \Delta r_{\perp} r=k \int_{z_{i}}^{2 r} d z\left(z_{f}-z\right)\left[a \frac{\tilde{r}(z)}{\tilde{r}_{0}(z)^{2}}+b \frac{\tilde{r}(z)^{3}}{2 \tilde{r}_{0}(z)^{4}}+c \frac{\tilde{r}(z)^{5}}{3 \tilde{r}_{0}(z)^{6}}+\cdots\right]
\end{aligned}
$$

similar to equations $(11.2 .7)$ and $(11.2 .8)$. The axial coordinates $z_{i}$ and $z_{f}$ refer to the initial (or entrance) and final (or exit) plane of the beam segment. The coefficients $a, b, c, \cdots$ are from now on assumed to be independent of $z$, which means that the shape of the current density distribution is assumed to be the same in every cross section of the beam. Notice that the quantities $\Delta \alpha_{f}$ and $\Delta r_{f}$ may become negative.

The virtual displacement $\Delta r_{1}$ in a reference plane with axial coordinate $z_{r}$ follows from

$$
\Delta r_{\perp}=\Delta r_{\perp} f-\left(z_{f}-z_{r}\right) \Delta \alpha_{f}
$$

By substituting equations (11.4.4) into equation (11.4.5) one obtains

$$
\Delta r_{\perp}=k \int_{z_{i}}^{z_{r}} d z\left(z_{r}-z\right)\left[a \frac{\tilde{r}(z)}{\tilde{r}_{0}(z)^{2}}+b \frac{\tilde{r}(z)^{3}}{2 \tilde{r}_{0}(z)^{4}}+c \frac{\tilde{r}(z)^{5}}{3 \tilde{r}_{0}(z)^{6}}+\cdots\right]
$$

similar to the second equation of (11.4.4). The difference with this equation is that $z_{f}$ is replaced by $z_{r}$. The integral in equation $(11.4 .6)$ can be evaluated term by term. Accordingly, one may write

$$
\Delta r_{\perp}=a \Delta r_{\perp}+b \Delta r_{\perp} 2+\cdots
$$

in which

$$
\begin{aligned}
& \Delta r_{\perp^{1}}=k \int_{z_{i}}^{z_{f}} d z\left(z_{r}-z\right) \frac{\tilde{r}(z)}{\tilde{r}_{0}(z)^{2}} \\
& \Delta r_{\perp}=k \int_{z_{i}}^{z_{f}} d z\left(z_{r}-z\right) \frac{\tilde{r}(z)^{3}}{2 \tilde{r}_{0}(z)^{4}}
\end{aligned}
$$

In the next section we will evaluate the quantity $\Delta r_{\perp^{1}}$, given by equation (11.4.8). It specifies the first order optical properties of the space charge lens. These properties can be expressed in terms of a defocussing distance and a magnification. The quantity $\Delta \mathrm{r}_{\perp} 2$, given by equation (11.4.9), specifies the third order aberration properties of the space charge lens. It will be evaluated in section 11.6 .

\subsection{First order optical properties of the space charge lens}

In this section we will evaluate the quantity $\Delta r_{1}{ }_{1}$, given by equation $(11.4 .8)$. Let us define $z_{c}=0$, which implies that the $x, y$-plane of the laboratory system coincides with the crossover plane. 
Substitution of equations $(11.4 .1)$ and (11.4.3) into equation (11.4.8) yields

$$
\frac{\Delta r_{\perp 1}}{r_{c}}=2 \pi\left(R \frac{r_{\perp}}{r_{c}}+A \frac{\alpha}{\alpha_{0}}\right)
$$

in which $\mathrm{R}$ and $\mathrm{A}$ represent

$$
R=\int_{x_{i}}^{x_{f}} \frac{\left(x_{r}-x\right)}{[1+|x|]^{2}} d x, \quad A=\int_{x_{i}}^{x_{f}} \frac{x\left(x-x_{r}\right)}{[1+|x|]^{2}} d x
$$

where $x$ is defined as

$$
x=\frac{\alpha_{0} 2}{r_{c}}
$$

The scaled linear particle density $\lambda$ is given by equation By carrying out the integrals in equations (11.5.2) one finds

$(11.3 .3)$

$$
\begin{aligned}
& R=\ln \left(\frac{1+K_{1}}{1+K_{2}}\right)+\frac{1}{1+K_{1}}-\frac{1}{1+K_{2}}+x_{r}\left[2-\frac{1}{1+K_{1}}-\frac{1}{1+K_{2}}\right] \\
& A=2 K+2-\frac{1}{1+K_{1}}-\frac{1}{1+K_{2}}-2 \ln \left[\left(1+K_{1}\right)\left(1+K_{2}\right)\right]+x_{r}\left[\ln \left(\frac{1+K_{1}}{1+K_{2}}\right)+\frac{1}{1+K_{1}}-\frac{1}{1+K_{2}}\right]
\end{aligned}
$$

in which the quantities $K, K_{1}$ and $K_{2}$ are defined by (as in equation $(7.3 .1))$

$$
K_{1}=\frac{\alpha_{0} L_{1}}{r_{c}}, \quad K_{2}=\frac{\alpha_{0} L_{2}}{r_{c}}, \quad K=\frac{K_{1}+K_{2}}{2}=\frac{\alpha_{0} L}{2 r_{c}}
$$

where $L_{1}=-2_{i}$ is the distance between the initial plane and the crossover, $\mathrm{L}_{2}=\mathrm{z}_{\mathrm{f}}$ is the distance between the crossover and the final plane and $L=L_{1}+L_{2}$ is the total beam length.

We will now assume that the reference plane is located in the vicinity of the crossover. This implies that $x_{r} \ll<1$ and one may neglect the terms in equations (11.5.4) and (11.5.5) which are proportional to $x_{r}$. The resulting expressions can be written as

$$
\begin{array}{ll}
R \approx R_{1}\left(K_{1}\right)-R_{1}\left(K_{2}\right), & R_{1}(K)=\ln (1+K)-\frac{K}{1+K} \\
A \approx A_{1}\left(K_{1}\right)+A_{1}\left(K_{2}\right), & A_{1}(K)=K+\frac{K}{1+K}-2 \ln (1+K)
\end{array}
$$

Notice that one may add an arbitrary constant to $R_{1}(K)$ without affecting the outcome of $R$. The functions $R_{1}(K)$ and $A_{1}(K)$ are plotted in figure 11.2. For the case of a crossover located in the middle of the beam segment (thus $K_{1}=K_{2}=K$ ) one finds $R=0$ and $A=2 A_{1}(K)$.

We like to express the results given by equations (11.5.1), (11.5.7) and $(11.5 .8)$ in terms of a defocussing distance $\Delta z_{f}$ and a magnification $M$, both referring to the crossover. For that, we have to determine the axial position $z_{r}$ of the plane in which the crossover is imaged by the space charge lens. The radial coordinate of the perturbed trajectory of the test particle can be expressed as

$$
r(z)=r(z)+\Delta r_{\perp}=r_{\perp}+\alpha z+a \Delta r_{\perp}+b \Delta r_{\perp}+\cdots
$$




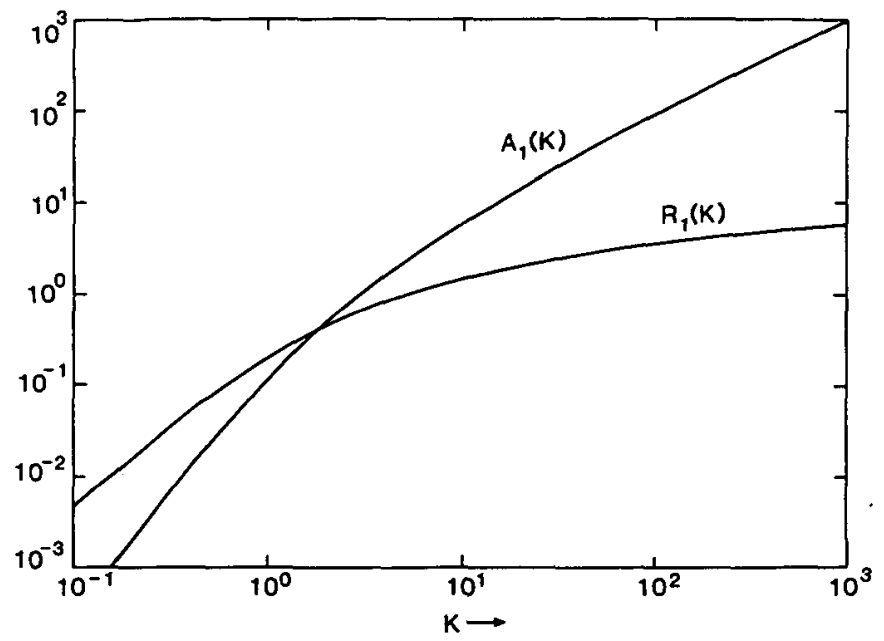

Figure 11.2 The first order optical functions $\mathrm{R}_{1}(\mathrm{~K})$ and $\mathrm{A}_{1}(\mathrm{~K})$ of the spacè charge lens, which are specified by equations $(11.5 .7)$ and $(11.5 .8)$ respectively.

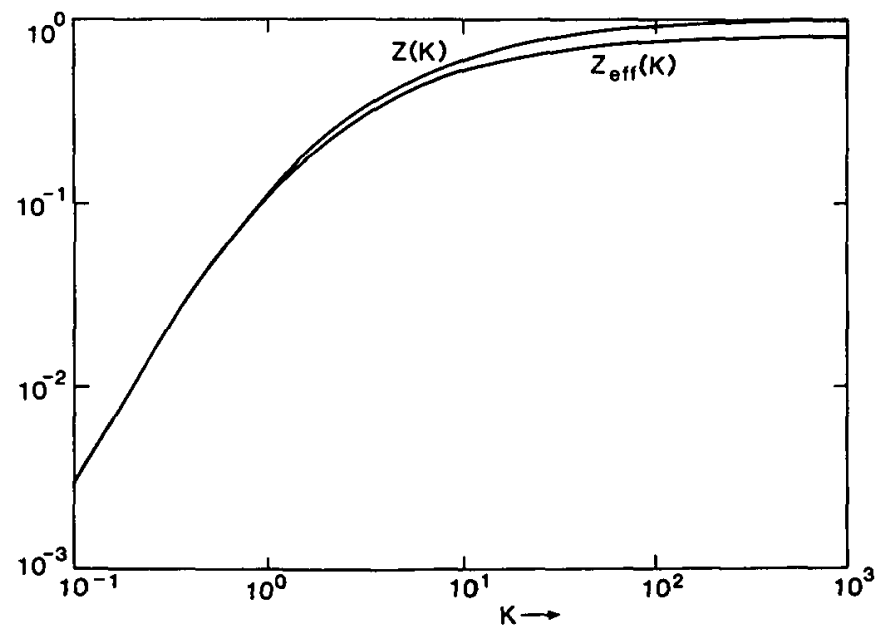

Pigure 11.3 The defocussing functions $Z(K)$ and $Z_{e f}(K)$ of the space charge lens, which are specified by equations (11.5.13) and (11.6.13) respectively. 
as follows with equations $(11.4 .3)$ and $(11.4 .7)$, using that $z_{c}=0$. The axial coordinate $z_{r}$ of the image plane follows by solving

$$
\left[\frac{\partial r(z)}{\partial \alpha}\right]_{z=z_{r}}=0
$$

With equations $(11.5 .9),(11.5 .1)$ and (11.5.8) one obtains (note that $\left.x_{r}=\alpha_{0} z_{r} / r_{c}\right)$

$$
x_{r} \approx a 2 \pi A \approx a 2 \pi\left[A_{1}\left(K_{1}\right)+\dot{A}_{1}\left(K_{2}\right)\right]
$$

ignoring the terms $b \Delta \mathrm{H}_{\perp} \mathrm{z}^{+\cdots}$ in equation (11.5.9). With equation (11.5.3) one finds for the defocussing distance $\Delta z_{r} \quad\left(=z_{r}-z_{c}=z_{r}\right)$

$$
\Delta z_{\mathrm{f}} \approx a 2 \delta \mathrm{L} \frac{1}{2 \mathrm{~K}}\left(\mathrm{~K}_{1} \mathrm{Z}\left(\mathrm{K}_{1}\right)+\mathrm{K}_{2} Z\left(\mathrm{~K}_{2}\right)\right)
$$

in which the function $Z(K)$ is defined as

$$
Z(K)=\frac{A_{1}(K)}{K}=1+\frac{1}{1+K}-\frac{2}{K} \ln (1+K)
$$

This function is plotted in figure 11.3. For $K \geqq 100$ one may approximate $Z(K)$ w within $10 \%$ accuracy. From equation (11.5.12) it now follows that for $K_{1} \gtrsim 100$ and $K_{2} \gtrsim 100$ the defocussing distance $\Delta z_{f}$ is practically independent of the position of the crossover. Notice that the $\Delta z_{f}$ is always positive. Thus the virtual crossover, which is imaged by the lens at the end of the beam segment, is always located after the crossover which would occur in the unperturbed beam.

Utilizing equation (11.3.3) one can express equation (11.5.12) in terms of the experimental parameters

$$
\Delta z_{f} \approx C_{S c} \frac{1}{2 K}\left(K_{1} Z\left(K_{1}\right)+K_{2} Z\left(K_{2}\right)\right) \frac{a I L}{\alpha_{0}^{2} V^{3 / 2}}
$$

where the constant $\mathrm{C}_{S \mathrm{C}}$ is given by

$$
\mathrm{C}_{S \mathrm{c}}=\frac{\mathrm{m}^{1 / 2}}{2^{5 / 2} \pi \epsilon_{0} \mathrm{e}^{1 / 2}}
$$

In the case of electrons one finds $\mathrm{C}_{S \mathrm{C}}=1.5154 \times 10^{4}$ in SI units.

Equations (11.5.14) shows that the defocussing distance increases linearly with the beam current. If one does not refocus the spot while changing the beam current, the space charge effect causes a blurring of the image. The point-spread function representing the blurring at the location of the original (unperturbed) crossover is given by

$$
\rho\left(\Delta r_{\perp}\right)=\frac{1}{\Delta z_{f}} f_{\alpha}\left(\Delta r_{\perp} / \Delta z_{f}\right)
$$

where $f_{\alpha}(\alpha)$ is the angular distribution at the crossover. The FWHM of this distribution is equal to

$$
\text { FWHM }_{r}=\Delta z_{\mathbf{f}} \text { FWHM }
$$

where FWHM $_{\alpha}$ is the FWHM of the angular distribution $f_{\alpha}(\alpha)$. The corresponding blurring observed at the target follows by multiplying FWHMr with the magnification of the system from the crossover to the 
target .

The magnification $M$ of the crossover due to action of the space charge lens follows with equation (11.5.9)

$$
M=\frac{r\left(z_{r}\right)}{r_{\perp}} \approx 1+a \frac{z_{r}}{r_{\perp}}+a \frac{\Delta r_{\perp}\left(z_{r}\right)}{r_{\perp}}
$$

By substituting equations (11.5.1), (11.5.7) and (11.5.11) one obtains

$$
M \approx 1+a 2 \lambda\left[R_{1}\left(K_{1}\right)-R_{1}\left(K_{2}\right)\right]
$$

in which the function $R_{1}(K)$ is given by equation (11.5.7). Equation $(11.5 .19)$ shows that, in a beam segment with a crossover halfway $\left(K_{1}=K_{2}\right)$, the magnification generated by the space charge effect in the first half of the beam is exactly compensated in the second half. Thus for this particular geometry one $f$ inds $M=1$. Furthermore, one sees that $M>1$ when $K_{1}>K_{2}$ and $M<1$ when $K_{1}<K_{2}$.

\subsection{Third order geometrical aberrations of the space charge lens}

In this section we will evaluate the quantity $\Delta \mathrm{r}_{\perp} 2$, given by equation $(11.4 .9)$. We define again $z_{c}=0$. Substitution of equations $(11.4 .1)$ and $(11.4 .3)$ into equation $(11.4 .9)$ yields

$$
\frac{\Delta r_{\perp} 2}{r_{c}}=2 \pi\left[D\left(\frac{r_{\perp}}{r_{c}}\right)^{3}+F \frac{\alpha}{\alpha_{0}}\left(\frac{r_{\perp}}{r_{c}}\right)^{2}+C\left(\frac{\alpha}{\alpha_{0}}\right)^{2} \frac{r_{\perp}}{r_{c}}+S\left(\frac{\alpha}{\alpha_{0}}\right)^{3}\right]
$$

in which $D, F, C$ and $S$ represent

$$
\begin{array}{ll}
D=\int_{x_{i}}^{x_{f}} \frac{\left(x_{r}-x\right)}{2[1+|x|]^{4}} d x, & F=\int_{x_{i}}^{x_{f}} \frac{3 x\left(x-x_{r}\right)}{2[1+|x|]^{4}} d x \\
C=\int_{x_{i}}^{x_{f}} \frac{3 x^{2}\left(x_{r}-x\right)}{2[1+|x|]^{4}} d x, \quad S=\int_{x_{i}}^{x_{f}} \frac{x^{3}\left(x-x_{r}\right)}{2[1+|x|]^{4}} d x
\end{array}
$$

where $x$ is defined by equation (11.5.3) and the scaled linear particle density $\lambda$ is given by equation (11.3.3). By carrying out the integrals in equations $(11.6 .2)$ one finds

$$
\begin{aligned}
& D=\frac{1}{6\left(1+K_{1}\right)^{3}}-\frac{1}{6\left(1+K_{2}\right)^{3}}-\frac{1}{4\left(1+K_{1}\right)^{2}}+\frac{1}{4\left(1+K_{2}\right)^{2}} \\
& +\frac{x_{r}}{6}\left[2-\frac{1}{\left(1+K_{1}\right)^{3}}-\frac{1}{\left(1+K_{2}\right)^{3}}\right] \\
& F=\frac{-1}{2\left(1+K_{1}\right)^{3}}-\frac{1}{2\left(1+K_{2}\right)^{3}}+\frac{3}{2\left(1+K_{1}\right)^{2}}+\frac{3}{2\left(1+K_{2}\right)^{2}}-\frac{3}{2\left(1+K_{1}\right)}-\frac{3}{2\left(1+K_{2}\right)}+1 \\
& +x_{r}\left[\frac{1}{2\left(1+K_{1}\right)^{3}}-\frac{1}{2\left(1+K_{2}\right)^{3}}-\frac{3}{4\left(1+K_{1}\right)^{2}}+\frac{3}{4\left(1+K_{2}\right)^{2}}\right] \\
& C=\frac{1}{2\left(1+K_{1}\right)^{3}}-\frac{1}{2\left(1+K_{2}\right)^{3}}-\frac{9}{4\left(1+K_{1}\right)^{2}}+\frac{9}{4\left(1+K_{2}\right)^{2}}+\frac{9}{2\left(1+K_{1}\right)}-\frac{9}{2\left(1+K_{2}\right)}-\frac{3}{2} \ln \left(\frac{1+K_{1}}{1+K_{2}}\right) \\
& -x_{r}\left[\frac{1}{2\left(1+K_{1}\right)^{3}}+\frac{1}{2\left(1+K_{2}\right)^{3}}-\frac{3}{2\left(1+K_{1}\right)^{2}}-\frac{3}{2\left(1+K_{2}\right)^{2}}+\frac{3}{2\left(1+K_{1}\right)}+\frac{3}{2(1+K 2)}-1\right]
\end{aligned}
$$




$$
\begin{aligned}
S= & -\frac{1}{6\left(1+K_{1}\right)^{3}}-\frac{1}{6\left(1+K_{2}\right)^{3}}+\frac{1}{\left(1+K_{1}\right)^{2}}+\frac{1}{\left(1+K_{2}\right)^{2}}-\frac{3}{1+K_{1}}-\frac{3}{1+K_{2}} \\
& +\frac{13}{3}-2 \ln \left[\left(1+K_{1}\right)\left(1+K_{2}\right)\right]+K \\
& +\frac{x_{r}}{2}\left[\ln \left(\frac{1+K_{1}}{1+K_{2}}\right)+\frac{1}{3\left(1+K_{1}\right)^{3}}-\frac{1}{3\left(1+K_{2}\right)^{3}}-\frac{3}{2\left(1+K_{1}\right)^{2}}+\frac{3}{2\left(1+K_{2}\right)^{2}}+\frac{3}{1+K_{1}}-\frac{3}{1+K_{2}}\right]
\end{aligned}
$$

in which the quantities $K, K_{1}$ and $K_{2}$ are defined by equation (11.5.6). We will now assume $x_{r} \ll 1$ and ignore the terms proportional to $x_{r}$. This way one obtains

$$
\begin{array}{ll}
D \approx D_{1}\left(K_{1}\right)-D_{1}\left(K_{2}\right), & F \approx F_{1}\left(K_{1}\right)+F_{1}\left(K_{2}\right) \\
C \approx C_{1}\left(K_{1}\right)-C_{1}\left(K_{2}\right), &
\end{array}
$$

in which the functions $D_{1}(K), F_{1}(K), C_{1}(K)$ and $S_{1}(K)$ can be expressed as

$$
\begin{aligned}
& D_{1}(K)=\frac{3 K^{2}+K^{3}}{12(1+K)^{3}} \\
& F_{1}(K)=\frac{K^{3}}{2(1+K)^{3}} \\
& C_{1}(K)=\frac{3}{2} \ln (1+K)-\frac{6 K+15 K^{2}+11 K^{3}}{4(1+K)^{3}} \\
& S_{1}(K)=\frac{1}{2} K-2 \ln (1+K)+\frac{9 K+21 K^{2}+13 K^{3}}{6(1+K)^{3}}
\end{aligned}
$$

These functions are plotted in figure 11.4. For the case of a crossover located in the middle of the beam segment (thus $K_{1}=K_{2}=K$ ) one finds from equations $(11.6 .3)$ that $D=C=0, F=2 F_{1}(K)$ and $S=2 S_{1}(K)$.

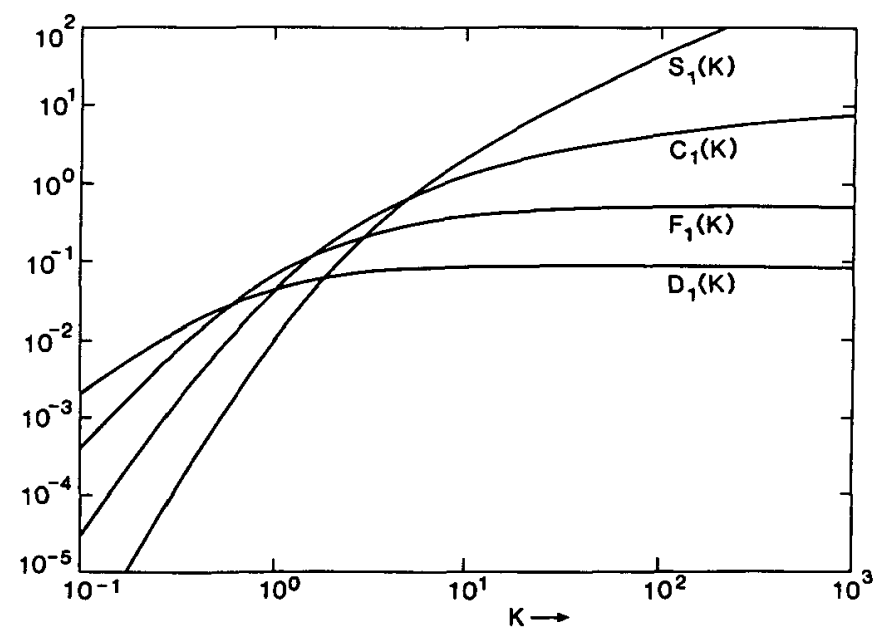

Figure 11.4 The third order aberration functions $\mathrm{D}_{1}(\mathrm{~K}), \mathrm{F}_{1}(\mathrm{~K}), \mathrm{C}_{1}(\mathrm{~K})$ and $\mathrm{S}_{1}(\mathrm{~K})$ of the space charge lens, which are specified by equations (11.6.4), (11.6.5), (11.6.6) and (11.6.7) respectively. 
Figure 11.4 shows that the term proportional to $S$ dominates equation $(11.6 .1)$ for $K \gg 1$. This term corresponds to the spherical aberration of the space charge lens. Let us investigate the properties of this term in more detail. The coefficient of spherical aberration $C_{s}$ of a lens is usually defined as

$$
\Delta r_{\perp}=-c_{s} \alpha^{3}
$$

The minus sign implies that a lens with a positive $C_{s}$ deflects the outer rays by a larger angle then required for ideal imaging. The corresponding deviation $\Delta r$ is negative.

With equations $(11.4 .7),(11.6 .1)$ and $(11.6 .3)$ one obtains from equation (11.6.8) for the coefficient of spherical aberration of the space charge lens

$$
\mathrm{C}_{s}=-\mathrm{b} \frac{2 \pi \mathrm{L}}{\alpha_{0}^{2}} \frac{1}{2 \mathrm{~K}}\left(\mathrm{~S}_{1}\left(\mathrm{~K}_{1}\right)+\mathrm{S}_{1}\left(\mathrm{~K}_{2}\right)\right)
$$

where $2 K=K_{1}+K_{2}$ and the function $S_{1}(K)$ is given by equation (11.6.7). Substitution of the expression for $\lambda$, given by equation (11.3.3), yields

$$
\mathrm{C}_{s}=\mathrm{C}_{S \mathrm{c}} \frac{1}{2 \mathrm{~K}}\left(\mathrm{~S}_{1}\left(\mathrm{~K}_{1}\right)+\mathrm{S}_{1}\left(\mathrm{~K}_{2}\right)\right) \frac{-\mathrm{bIL}}{\alpha_{0^{4} \mathrm{~V}^{3 / 2}}}
$$

where the constant $C_{5}$ is given by equation (11.5.15). We recall that the parameter $b$ is a measure for the non-uniformity of the current density distribution, see equation (11.2.16). A Gaussian distribution has the properties $a=1, b=-1 / 2$, as is specified by equation (11.2.19). Equation (11.6.10) shows that the coefficient of spherical aberration $C_{s}$ is positive when $b$ is negative. A negative value of $b$ implies that the current density at the edge of the beam is smaller than in the centre of the beam, as can be seen from equation (11.2.16). This is usually the case in practical beams. In order to exploit the space charge lens to compensate the spherical aberration of the system components (which is negative) . one should produce a beam which has the property b>0. This means that the current density at the edge of the beam should be larger than in the centre of the beam.

As is well known from geometrical optics the disk of least confusion of a beam which is dominated by spherical aberrations is located at a distance $(3 / 4) \mathrm{C}_{5} \alpha_{0}{ }^{2}$ ahead of the Gaussian image plane and has a diameter $d_{s} \approx(1 / 2) c_{s} \alpha_{0}{ }^{3}$. The effective defocussing distance $\Delta z_{e} f$ of the space charge lens is now defined as

$$
\Delta z_{\text {eff }}=\Delta z_{f}-\frac{3}{4} C_{s} \alpha_{0}{ }^{2}
$$

By substitution of equations $(11.6 .10)$ and $(11.5 .14)$ one finds an expression for $\Delta z_{\text {eff }}$ which has the same form as equation (11.5.14), but with the function $Z(K)$ replaced by a function $Z_{e f}(K)$, which is given by

$$
\begin{aligned}
Z_{\text {eff }}(K) & =Z(K)+\frac{3 b}{4 a} \frac{s_{1}(K)}{K} \\
& =1+\frac{3 b}{8 a}+\frac{1}{1+K}+\frac{b}{a} \frac{9+21 K+13 K^{2}}{8(1+K)^{3}}-\left(2+\frac{3 b}{2 a}\right) \frac{\ln (1+K)}{K}
\end{aligned}
$$

For a Gaussian current density distribution one obtains with equation $(11.2 .19)$ 


$$
Z_{\text {eff }}(K)=\frac{13}{16}+\frac{7+11 K+3 K^{2}}{16(1+K)^{3}}-\frac{5 \ln (1+K)}{4 K}
$$

which is plotted in figure 11.3. The diameter of the disk of least confusion $d_{s}$ is equal to

$$
\mathrm{d}_{s}=\frac{1}{2} \mathrm{C}_{s} \alpha_{0}{ }^{3}=\mathrm{C}_{\mathrm{Sc}} \frac{1}{2 \mathrm{~K}}\left(\mathrm{~S}_{1}\left(\mathrm{~K}_{1}\right)+S_{1}\left(\mathrm{~K}_{2}\right)\right) \frac{-\mathrm{bIL}}{2 \alpha_{0} \mathrm{~V}^{3 / 2}}
$$

as follows with equation (11.6.10).

\subsection{Beam segment with a narrow crossover}

In this section we will study the results of the previous two sections for the case of a beam segment with a narrow crossover. This geometry corresponds to $r_{c} \rightarrow 0$, thus $K \rightarrow \infty$. We will start by investigating the first order optical properties of the space charge lens and will discuss its third order properties next. For $K \rightarrow \infty$ one may expand equations $(11.5 .4),(11.5 .13)$ and $(11.6 .12)$ in the following power series

$$
\begin{aligned}
& R_{1}(K)= \ln (K)-1+\frac{2}{K}-\frac{3}{2 K^{2}}+\frac{4}{3 K^{3}}+0(K-4) \quad(K+\infty) \quad(11.7 .1) \\
& Z(K)=1-\frac{2 \ln (K)}{K}+\frac{1}{K}-\frac{3}{K^{2}}+\frac{2}{K^{3}}+0(K-4) \quad(K+\infty) \quad(11 \cdot 7.2) \\
& Z_{\text {ef }(K)=} 1+\frac{3 b}{8 a}-\left(2+\frac{3 b}{2 a}\right) \frac{\ln (K)}{K}+\left(1+\frac{13 b}{8 a}\right) \frac{1}{K}-\left(3+\frac{15 b}{4 a}\right) \frac{1}{K^{2}} \\
&+\left(2+\frac{15 b}{4 a}\right) \frac{1}{K^{3}}+0(K-4)
\end{aligned}
$$

in which $0\left(\mathrm{~K}^{-4}\right)$ represents terms of the order $\mathrm{K}^{-4}$. From equations $(11.7 .2),(11.5 .14)$ and $(11.5 .17)$ one obtains for the extreme case of a homocentric beam with a crossover $\left(r_{c}=0, \quad k=\infty\right)$ with an uniform current density distribution in every cross section of the beam $(a=1)$

$$
\Delta z_{\mathrm{f}}=\mathrm{C}_{\mathrm{SC}} \frac{\mathrm{IL}}{\alpha_{0} \mathrm{~V}^{3 / 2}}, \quad \mathrm{FWHM}_{\mathrm{r}}=\mathrm{C}_{S \mathrm{C}} \frac{2 \mathrm{IL}}{\alpha_{0} \mathrm{~V}^{3 / 2}} \quad(\mathrm{a}=1, \mathrm{~K} \rightarrow \infty)
$$

where the constant $C_{5} c$ is defined by equation (11.5.15). For a Gaussian current density distribution $(a=1, b=-1 / 2)$ one finds an effective defocussing distance which is smaller by a factor $13 / 16$

$$
\Delta z_{\text {eff }}=\mathrm{C}_{\mathrm{Sc}} \frac{13 \mathrm{IL}}{16 \alpha_{0} \mathrm{~V}^{3 / 2}} \quad(\mathrm{a}=1, \mathrm{~b}=-1 / 2, \mathrm{~K}+\infty)
$$

as follows from equations (11.5.14) and (11.7.3). For an electron beam with $I=1 \mu \mathrm{A}, L=0.1 \mathrm{~m}, V=10 \mathrm{kV}$ and $\alpha_{0}=10 \mathrm{mR}$ equations $(11.7 .4)$ and $(11.7 .5)$ yield $\Delta z_{\mathrm{f}}=15 \mu \mathrm{m}, \Delta z_{\mathrm{ef} g}=12 \mu \mathrm{m}$ and $F W H M_{\mathrm{r}}=0.30 \mu \mathrm{m}$.

The magnification of a narrow crossover $M$ can be determined from equations (11.5.19) and (11.7.1). For a converging beam with an uniform current density distribution $\left(a=1, K_{1}+\infty\right.$ and $\left.K_{2}=0\right)$ one obtains 


$$
(M-1) r_{c} \approx C_{S C} \frac{I L}{\alpha_{0} V^{3 / 2}} \frac{\ln \left(K_{1}\right)}{K_{1}} \approx \Delta z_{f} \alpha_{0} \frac{\ln \left(K_{t}\right)}{K_{1}}
$$

utilizing equations $(11.3 .3)$ and $(11.7 .4)$. Equation (11.7.6) shows that the lateral displacement $(M-1) r_{c}$, associated with the magnification effect, is for the considered geometry $(K \rightarrow \infty)$ typically much smaller than the displacement $\Delta z_{\mathrm{f}} \alpha_{0}$, associated with the defocussing effect. This can also directly be observed from figure 11.2, employing equations (11.5.1), (11.5.7) and (11.5.8). The ratio $\Delta z_{f} \alpha_{0} / M_{c}$ becomes even larger for a beam segment with a narrow crossover which is located somewhere in the middle $\left(\mathrm{K}_{1} \mathrm{~K}_{2}\right)$ due to the cancellation of the contributions to $M$ of the first and the second part of the beam segment, see equation (11.5.7).

The third order aberrations of the space charge lens are described by equation (11.6.1). The functions D, F, C, S are defined by equations $(11.6 .3)$ to $(11,6.7)$. For $k+\infty$ these functions can be expanded as

$$
\begin{aligned}
D_{1}(K)=\frac{1}{12}-\frac{4}{K^{2}}-\frac{2}{3 K^{3}}+0\left(K^{-4}\right) & (K+\infty) \\
F_{1}(K)=\frac{1}{2}-\frac{3}{2 K}+\frac{3}{K^{2}}-\frac{5}{K^{3}}+0\left(K^{-4}\right) & (K+\infty) \\
C_{1}(K)=\frac{3}{2} \ln (K)-\frac{11}{4}+\frac{6}{K}-\frac{15}{2 K^{2}}+\frac{10}{K^{3}}+0(K-4) & (K+\infty) \\
S_{1}(K)=\frac{1}{2} K-2 \ln (K)+\frac{13}{16}-\frac{5}{K}+\frac{5}{K^{2}}-\frac{35}{6 K^{3}}+0(K-4) & (K+\infty)
\end{aligned}
$$

These equations show that for large $K$-values the dominating third order term is spherical aberration, as was mentioned previously. This can also be seen from figure 11.4. For the extreme case of a homocentric beam segment with a crossover $\left(K_{1}+\infty\right.$ and $\left.K_{2}+\infty\right)$ with a Gaussian current density distribution $(a=1, b=-1 / 2)$ one obtains from equations $(11.6 .10)$, $(11.6 .14)$ and $(11.7 .10)$

$$
\mathrm{C}_{s}=\mathrm{Csc}_{\mathrm{Sc}} \frac{\mathrm{IL}}{4 \alpha_{0} \mathrm{~V}^{3 / 2}}, \quad \mathrm{~d}_{\mathrm{s}}=\mathrm{CSc}_{\mathrm{Sc}} \frac{\mathrm{IL}}{8 \alpha_{0} \mathrm{~V}^{3 / 2}}(\mathrm{a}=1, \mathrm{~b}=-1 / 2, \mathrm{~K}+\infty)
$$

We recall that $d_{s}$ represents the diameter of the disk of least confusion. The corresponding axial location is specified by equation $(11.7 .5)$. For an electron beam with $\mathrm{I}=1 \mu \mathrm{A}, \mathrm{L}=0.1 \mathrm{~m}, \mathrm{~V}=10 \mathrm{kV}$ and $\alpha_{0}=10 \mathrm{mR}$ these equations yield $C_{s}=38 \mathrm{~mm}$ and $d_{s}=19 \mathrm{~nm}$.

\subsection{Homocentric cylindrical beam segment}

We will now consider the case of a homocentric cylindrical beam segment with a narrow crossover. This geometry corresponds to $a_{0}=0$, thus $\mathrm{K}=0$. As in the previous section we will start by investigating the first order optical properties of the space charge lens and will discuss its third order properties next. For $K \rightarrow 0$ one may expand equations (11.5.4) and $(11.5 .13)$ and $(11.6 .12)$ in the following power series

$$
\mathrm{R}_{1}(\mathrm{~K})=\frac{1}{2} \mathrm{~K}^{2}-\frac{2}{3} \mathrm{~K}^{3}+\frac{3}{4} \mathrm{~K}^{4}-\frac{4}{5} \mathrm{~K}^{5}+\mathrm{O}\left(\mathrm{K}^{6}\right)
$$




$$
\begin{aligned}
& Z(K)=\frac{1}{3} K^{2}-\frac{1}{2} K^{3}+\frac{3}{5} K^{4}-\frac{2}{3} K^{5}+0\left(K^{6}\right) \quad(K \rightarrow 0) \\
& Z_{\text {eff }}(K)=\frac{1}{3} K^{2}-\frac{1}{2} K^{3}+\left(\frac{3}{5}+\frac{3 b}{40 a}\right) K^{4}-\left(\frac{2}{3}+\frac{b}{8 a}\right) K^{5}+0\left(K^{6}\right) \\
& (K \rightarrow 0)
\end{aligned}
$$

Equations (11.8.2) and (11.8.3) show that for $K \rightarrow 0$ one may approximate $Z_{\text {eff }}(K) \approx Z(K)$, which is correct up to fourth order terms in $K$. This behaviour is confirmed by figure 11.2. For the extreme case that $\alpha_{0}=0$ (thus $K=0$ ) one obtains from equations (11.5.1), (11.5.7), (11.5.8), $(11.5 .13),(11.8 .1),(11.8 .2)$ and $(11.5 .6)$

$$
\Delta r_{\perp}{ }^{1}=2 \lambda^{*}\left(S_{c}-1 / 2\right) r_{\perp} \quad\left(\alpha_{0}=0\right)
$$

in which the scaled linear particle density for a cylindrical beam $\lambda^{*}$ is defined as (as stated previously by equation $(7.4 .18)$ )

$$
\lambda^{*}=4 \pi \mathrm{K}^{2}=\frac{\mathrm{kL}^{2}}{2 \mathrm{Y}_{0}^{2}}=\frac{\mathrm{m}^{1 / 2}}{2^{7 / 2} \pi \varepsilon_{0} \mathrm{e}^{1 / 2}} \frac{\mathrm{IL}^{2}}{\mathrm{r}_{0}^{2} \mathrm{~V}^{3 / 2}}
$$

in which $r_{0}$ denotes the radius of the beam. As the crossover disappears for $\alpha_{0} \rightarrow 0$ the parameter $S_{c}$ in equation (11.8.4) merely defines the location of the image plane. In fact $S_{c}$ replaces here the parameter $S_{1}$, defined by equation (3.2.1). Equation (11.8.4) can also be obtained directly by performing the integral in equation (11.4.8), taking $\widetilde{\mathbf{r}}(z)=r_{\perp}$ and $\vec{r}_{0}(z)=r_{0}$, independent of $z$,

The third order aberration functions $D_{1}, F_{1}, C_{1}$ and $S_{1}$, given by equations $(11.6 .4)$ to $(11.6 .7)$, can for $K \rightarrow 0$ be expanded as

$$
\begin{aligned}
& D_{1}(K)=\frac{1}{4} K^{2}-\frac{2}{3} K^{3}+\frac{5}{4} K^{4}-2 K^{5}+O\left(K^{6}\right) \\
& F_{1}(K)=\frac{1}{2} K^{3}-\frac{3}{2} K^{4}+3 K^{5}+O\left(K^{6}\right) \\
& C_{1}(K)=\frac{3}{8} K^{4}-\frac{6}{5} K^{5}+0\left(K^{6}\right) \\
& S_{1}(K)=\frac{1}{10} K^{5}+O\left(K^{6}\right)
\end{aligned}
$$

Substitution into equation (11.6.1), using equation (11.6.3), yields for for the extreme case $\alpha_{0}=0$

$$
\Delta r_{\perp^{2}}=\lambda^{*}\left(S_{c}-1 / 2\right) \frac{r_{\perp}^{3}}{r_{0}^{2}} \quad\left(\alpha_{0}=0\right)
$$

utilizing equation (11.8.5). Equation (11.8.10) can also be obtained directly by performing the integral in equation (11.4.9), taking $\widetilde{r}(z)=r_{\perp}$ and $\tilde{r}_{0}(z)=r_{0}$.

Equations (11.8.4) and (11.8.10) specify the first and third order optical properties of the space charge lens associated with a cylindrical beam segment in terms of the displacements $\Delta r_{1}$ and $\Delta r_{2}$ in a certain plane determined by the parameter $S_{c}$. We will now translate these quantities in terms of the corresponding properties of the beam in the back-focal plane of the lens succeeding the beam segment. Figure 11.5 shows a cylindrical beam segment of length $L$ between two-lenses. 


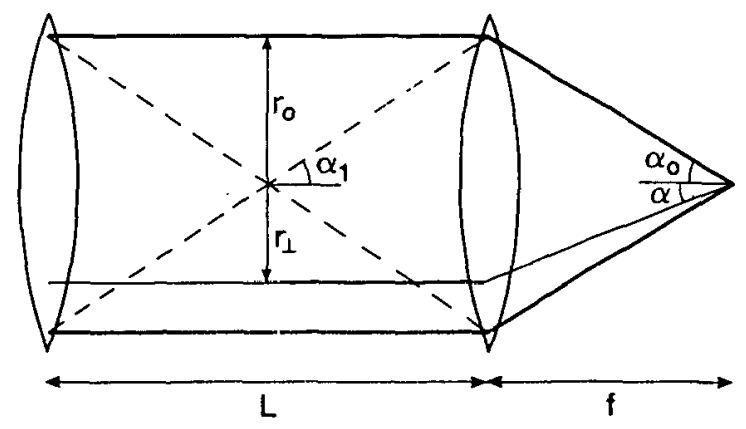

Figure 11.5 Schematic view of a homocentric cylindrical beam segment (solid lines) and a homocentric beam segment with a crossover in the middle (dashed lines). The corresponding space charge effect, observed in the crossover after the second lens, is the same for both geometries.

The beam radius is $r_{0}$. The focal distance of the second lens is $f$. The displacement $\Delta r_{f}$ in the back-focal plane of the lens can be expressed as

$$
\Delta r_{\mathrm{f}}=\lim _{\mathrm{S}_{\mathrm{c}} \rightarrow-\infty}\left|\frac{\mathrm{f}}{\mathrm{S}_{\mathrm{c}} \mathrm{L}}\right| \Delta r
$$

in which $\Delta r$ is the displacement in the plane defined by $S_{c}$. Equation $(11.8 .11)$ is equivalent to equation (9.2.10) (We recall that the parameter $S_{c}$ is for the present case identical to $S_{i}$ ). It expresses that the displacement $\Delta r_{f}$ is entirely the result of the angular displacement generated in the cylindrical beam segment. $(11.8 .10)$

With equation (11.8.11) one obtains from equations (11.8.4) and

$$
\begin{aligned}
& \Delta r_{1, f}=2 \lambda * \frac{f r_{1}}{L} \\
& \Delta r_{2, f}=\lambda * \frac{f r_{\perp}^{3}}{L r_{0}^{2}}
\end{aligned}
$$

The displacement $\Delta r_{1}, f$ is directly proportional to $r_{\perp}$ and can therefore be expressed in terms of a defocussing distance $\Delta z_{f}$. We define

$$
\Delta z_{\mathrm{f}}=a \Delta r_{1}, \frac{f}{r_{\perp}}
$$

utilizing equation (11.4.7). The constant a depends on the type of current density distribution in the cross section of the beam, as can be seen from equation (11.2.16). Substituting equations (11.8.12) and $(11.8 .5)$ into equation $(11.8 .14)$ yields

$$
\Delta z_{f}=a 2 \lambda * \frac{f^{2}}{L}=C_{s c} \frac{a I L f 2}{r_{0}^{2} V^{3} / 2}
$$

where the constant $C_{s c}$ is defined by equation (11.5.15). Similarly, one can express the third order aberration specified by equation $(11.8 .13)$, as

$$
\Delta r=c_{s c} \frac{-b I L f r_{1}^{3}}{2 r_{0} V^{3 / 2}}=c_{s c} \frac{-b I L f^{4} a^{3}}{2 r_{0} V^{3 / 2}}
$$


using that for a paraxial beam $r_{1} \approx \alpha$, as can be seen from figure 11.5 . The minus sign was included to express that a positive value of $b$ leads to an aberration which corresponds to a too strong focussing of the outer rays. Utilizing equation $(11.6 .8)$ one can express this result in terms of a coefficient of spherical aberration $C_{s}$

$$
\mathrm{C}_{s}=\mathrm{C}_{\mathrm{sc}} \frac{-\mathrm{bILf}^{4}}{2 \mathrm{ro}^{4} \mathrm{~V}^{3 / 2}}
$$

We recall that a Gaussian current density distribution corresponds to $a=1$ and $b=-1 / 2$, as specified by equation (11.2.19).

Equations (11.8.15) and (11.8.17) constitute the main results of this section. It is interesting to compare these results to the corresponding results of the previous section. Assume that the cylindrical beam segment in figure 11.5 is replaced by a homocentric beam segment with a crossover, as indicated in the figure by the dashed lines. One may now use equations (11.7.4) and (11.7.11) to calculate the defocussing distance $\Delta z_{f}, c$ and the coefficient of spherical aberration $\mathrm{C}_{s, c}$, both referring to the crossover. In order to compare the outcome with the results of this section one has to translate these quantities to the image plane of the second lens, using

$$
\begin{aligned}
& \Delta z_{\mathrm{f}}=\left(\frac{\mathrm{f}}{2 \mathrm{~L}}\right)^{2} \Delta z_{\mathrm{f}, \mathrm{c}} \\
& \mathrm{C}_{\mathrm{s}}=\frac{\mathrm{f}}{2 \mathrm{~L}} \frac{\alpha_{\mathrm{I}}{ }^{3}}{\alpha_{0}{ }^{3}} \mathrm{C}_{\mathrm{s}, \mathrm{c}}=\left(\frac{\mathrm{f}}{2 \mathrm{~L}}\right)^{4} \mathrm{C}_{\mathrm{s}, \mathrm{c}}
\end{aligned}
$$

The quantity $\mathrm{f} / 2 \mathrm{~L}$ is equal to the transverse magnification from the crossover to image plane of the second lens. Substitution of equations $(11.7 .4)$ and $(11.7 .11)$ into equations $(11.8 .18)$ yields

$$
\begin{aligned}
& \Delta z_{f}=C_{s c} \frac{I L}{\alpha_{1}^{2} V^{3 / 2}}\left(\frac{f}{2 L}\right)^{2}=C_{S c} \frac{I L}{\alpha_{0}^{2} V^{3 / 2}} \\
& C_{S}=C_{S C} \frac{I L}{4 \alpha_{1}{ }^{4} V^{3 / 2}}\left(\frac{f}{2 L}\right)^{4}=C_{S c} \frac{I L}{4 \alpha_{0} V^{3 / 2}}
\end{aligned}
$$

using that $\alpha_{1}=f \alpha_{0} / 2 \mathrm{~L}$. Since $r_{0}=\alpha_{0} f$ one finds that equations (11.8.19) are identical to equations (11.8.15) and (11.8.17), taking $a=1$ and $b=-1 / 2$. Thus the space charge effect generated in the homocentric beam segment with a crossover is identical to that generated in the homocentric cylindrical beam segment as far as the impact on the spot in the image plane of the second lens is concerned.

\subsection{Addition of the effects generated in individual beam segments}

We conclude this chapter by investigating how to add the space charge effects generated in the different segments of a beam. We have demonstrated that space charge effect generated in a single beam segment corresponds to action of a negative lens. Accordingly, the total effect generated in the entire beam can be determined by combining the different space charge lenses associated with the individual beam segments, employing the laws of geometrical optics. As the solution of this problem is straightforward we will restrict the discussion to some general remarks.

The defocussing distance $\Delta z_{\mathrm{f}}$ of a crossover is directly 
proportional to the factor $2 \pi \mathrm{L}$ and a function which depends on the geometry parameters $K_{1}$ and $K_{2}$, see equation (11.5.12). In order to obtain the corresponding defocussing of the final probe one has to multiply $\Delta z_{f}$ with $M^{2}$, in which $M$ denotes the transverse magnification of the crossover to the final probe (Notice that the axial magnification is the square of the transverse magnification). The quantity $\bar{\lambda}$ depends on the beam semi-angle in the crossover $\alpha_{0}$, as well as the beam current I and beam potential $\mathrm{V}$, see equation (11.3.3). In absence of acceleration areas and apertures one may assume that $I$ and $V$ are constant. The semiangle $\alpha_{0}$ is related to the semi-angle at the final probe $\alpha_{p}$ as $a_{0}=M \alpha_{p}$. Accordingly, one finds that the total defocussing of the final probe for a beam consisting of $N_{b}$ beam segments is proportional to

$$
\sum_{i=1}^{N_{b}} 2 \lambda_{i} M_{i}^{2}=C_{S c} \frac{I}{V^{3 / 2} a_{p}^{2}} \sum_{i=1}^{N_{b}} L_{i}
$$

where $L_{i}$ is the length of segment number $i$. For a narrow crossover $(K>>1)$ the geometry-dependent factor in equation (11.5.12) is equal to unity. Equation (11.9.1) indicates that if the condition $K>>1$ is fulfilled for all crossovers the total defocussing is given by the same equation as obtained for a single beam segment, provided that the segment length.L is replaced by the length of the total beam. The same holds true if one or more beam segments are nearly cylindrical $(K \rightarrow 0)$, as follows from the analysis of the previous section. However, for intermediate $K$ values one has to account for the geometry dependent factors of each individual beam segment. In general one should evaluate the quantity

$$
\sum_{i=1}^{N_{b}} L_{i} \frac{1}{2 K_{i}}\left(K_{1 i} Z\left(K_{1 i}\right)+K_{2 i} Z\left(K_{2 i}\right)\right)
$$

in which $K_{i}, K_{1 i}$ and $K_{2 i}$ represent the parameters $K, K_{1}$ and $K_{2}$ for beam segment number $i$. The function $Z(K)$ is specified by equation (11.5.13). The third order aberration properties associated with the space charge effect in a single beam segment are described by equation (11.6.1). When the displacement $\Delta r_{1} 2$ is magnified with a factor $M$ to the target one can express the corresponding displacement $\Delta r_{\perp}{ }^{2}, p$ in the final probe as

$$
\frac{\Delta r_{\perp}, p}{r_{p}}=2 \lambda M^{2}\left[\frac{D}{M^{2}}\left(\frac{r_{\perp}}{r_{c}}\right)^{3}+\frac{F}{M^{2}} \frac{\alpha}{\alpha_{0}}\left(\frac{r_{\perp}}{r_{c}}\right)^{2}+\frac{C}{M^{2}}\left(\frac{\alpha}{\alpha_{0}}\right)^{2} \frac{r_{\perp}}{r_{c}}+\frac{S}{M^{2}}\left(\frac{\alpha}{\alpha_{0}}\right)^{3}\right]
$$

using that $r_{p}=M r_{c}$. The ratios $r_{\perp} / r_{c}$ and $\alpha / \alpha_{0}$ are not affected by the imaging system. The quantity $2 \pi \mathrm{M}^{2}$ is directly proportional to the segment length $L$, as is shown by equation (11.9.1). Accordingly, one should calculate the total aberration $X(=D, F, C$ or $S)$ as

$$
X_{p}=\sum_{i=1}^{N_{b}} \frac{X_{i} L_{i}}{M_{i}^{2}} / \sum_{i=1}^{N_{b}} L_{i}
$$

in which $L_{i}$ and $X_{i}$ denote the length and the aberration constant respectively of segment number $i$, while $M_{i}$ is the magnification of crossover number $i$ to the target. 


\subsection{Introduction}

This chapter is concerned with the calculation of a number of different measures for the resolution of probe forming instruments, which are often used in experiment. In Gaussian beam systems, in which the source is imaged on the target, the spot-width is usually evaluated by scanning the beam across a knife-edge. The transmitted current is measured as function of the position of the beam. The spot-width follows as the distance between two characteristic current levels, for instance those corresponding to $10 \%$ and $90 \%$ of the total beam current.

In shaped beam systems, in which an aperture is imaged on the target, one is primarily interested in the edge-width of the spot. As a measure for the edge-width, one usually considers the distance between the points corresponding to two characteristic intensity levels, for instance those corresponding to $10 \%$ and $90 \%$ of the intensity level at the centre of the spot. It will be demonstrated that this type of edgewidth is equivalent to the spot-width of a Gaussian spot, determined by knife-edge scans. The corresponding profiles can both be interpreted as the convolution of a step-function with the point-spread function, representing the total aberrations of the system. In general, we will denote this type of width as $d_{p, 1-p}$, where $p$ refers to the choice of the characteristic intensity level $(0<p<1 / 2)$.

In some applications, one likes to know the Full width of the spot which contains a certain fraction $f$ of the total beam current $(0<f<1)$. This width is here denoted as $\mathrm{FW}_{\mathrm{f}}$. The Full width median $\mathrm{FW}_{50}$ value corresponds to $\mathrm{f}=0.5$.

The material of this chapter serves to express the results of the previous chapters in terms of the corresponding $d_{p, 1-p}$ and $F W_{f}$ values. The main part of the chapter is concerned with the trajectory displacement effect and the effect of axial chromatic aberration, related to the energy spread produced by the Boersch effect. The contribution of spherical aberration and (uncompensated) space charge defocussing is also studied. The resulting chromatic aberration, spherical aberration and blurring due to uncompensated space charge defocussing depends on the type of angular distribution at the target. The cases of an uniform, a Gaussian and a truncated Gaussian distribution are considered. The latter corresponds to a Gaussian angular distribution, which is limited by an aperture.

\subsection{Spot $\rightarrow$ width obtained by knife-edge scans}

Consider a spot, characterized by a current-density distribution $\mathrm{j}(\mathrm{x}, \mathrm{y})$, like the one depicted in figure 12.1. Assume that $j(x, y)$ is rotational symmetric with respect to the origin $(x=0, y=0)$ and has a maximum at the origin. Let $j(x, y)$ be normalized to unity

$$
\int_{-\infty}^{\infty} d x \int_{-\infty}^{\infty} d y j(x, y)=1
$$

The associated one-dimensional current density distribution $j(x)$ is defined as 


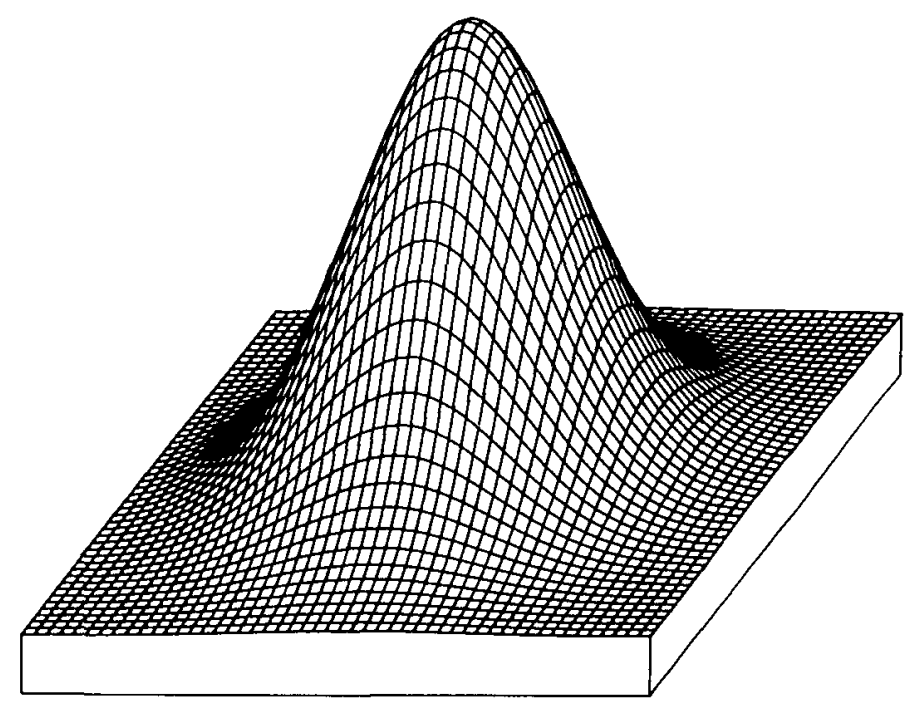

Figure 12.1 Current density distribution of a Gaussian spot.

$$
j(x)=\int_{-\infty}^{\infty} d y j(x, y)
$$

which can be considered as a projection of the two-dimensional distribution $j(x, y)$ on the $y$-direction. The edge-integral $I_{E}(x)$ is defined as

$$
I_{E}(x)=\int_{-\infty}^{x} d x^{\prime} j\left(x^{\prime}\right)
$$

It represents the fraction of the total current which is passed along a knife-edge, when the centre of the spot is located at a distance $x$ from the edge. This situation is shown in figure 12.2. The general behaviour of $I_{E}(x)$ is depicted in figure 12.3a. It is convenient to express $I_{E}(x)$ in terms of the auxiliary function $I(x)$, using

$$
I_{E}(x)=\frac{1}{2}[1+I(x)]
$$

where

$$
I(x)=2 \int_{0}^{x} d x^{\prime} j\left(x^{\prime}\right)
$$

The function $I(x)$ is anti-symmetric: $I(-x)=-I(x)$. Its general behaviour for positive $x$ is shown in figure $12.3 \mathrm{~b}$.

The width measure $d_{p, 1-p}$ is defined as the distance between the points for which $I_{E}(x)$ equals $p$ and $1-p$ respectively, as is indicated in figure 12.3a. Accordingly, one finds 


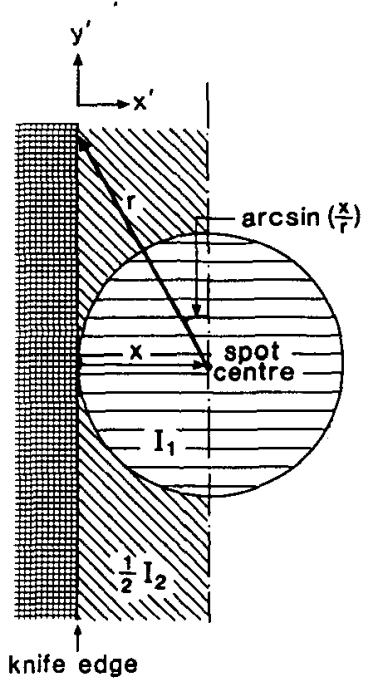

Figure 12.2 Schematic view of a spot which is scanned over a knifeedge. The centre of the spot is located at a distance $x$ from the knife-edge. The fraction $\mathrm{I}_{\mathrm{E}}(\mathrm{x})$ of the total current which passes through is equal to $\left[1+I_{1}(x)+I_{2}(x)\right] / 2$, as expressed by equations (12.2.4) and (12.2.9). The quantities $\mathrm{I}_{1}(\mathrm{x})$ and $\mathrm{I}_{2}(\mathrm{x})$ are specified by equations $(12.2 .10)$ and $(12.2 .11)$ respectively.

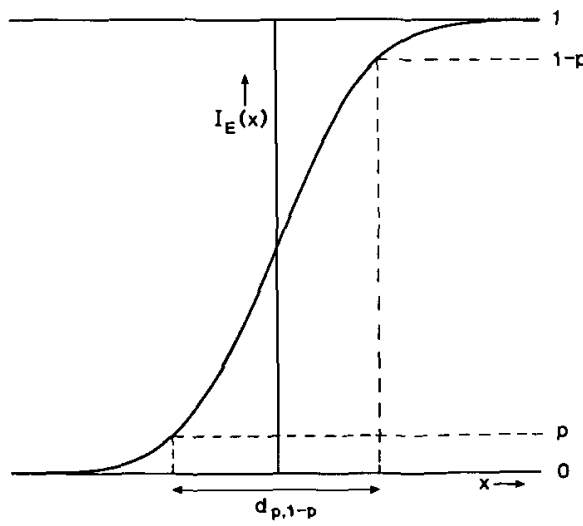

Fig. 12.3a

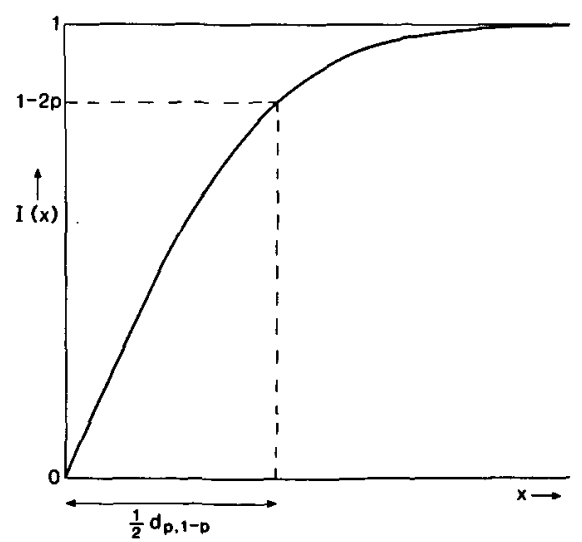

Fig. 12.3b

Pigure 12.3 Behaviour of the functions $I_{E}(x)$ and $I(x)$, defined by equations $(12.2 .4)$ and $(12.2 .5)$. 


$$
d_{p, 1-p}=I_{E}-1(1-p)-I_{E}^{-1}(p)=2 I-1(1-2 p)
$$

where $I_{E^{-1}}$ and $I^{-1}$ denote the inverse functions of $I_{E}$ and $I$ respectively. Equations $(12.2 .6),(12.2 .5)$ and $(12.2 .2)$ specify the width $d_{p, 1-p}$, obtained by scanning the beam across a knife-edge, in terms of the current density distribution $j(x, y)$.

In the actual calculations, we will employ cylindrical coordinates, which is convenient since $j(x, y)$ is assumed to be rotational symmetric. The cylindrical current-density distribution $j(r)$ is related to $\mathrm{j}(\mathrm{x}, \mathrm{y})$ as

$$
j(r)=2 \pi r j(x, y)
$$

where $r^{2}=x^{2}+y^{2}$. When $j(x, y)$ is normalized, conform equation (12.2.1), $j(r)$ is normalized too

$$
\int_{0}^{\infty} d r j(r)=1
$$

Employing cylindrical coordinates, equation (12.2.5) transforms to

$$
I(x)=I_{1}(x)+. I_{2}(x)
$$

where $I_{1}(x)$ and $I_{2}(x)$ are defined as

$$
\begin{aligned}
& I_{1}(x)=\int_{0}^{x} d r j(r) \\
& I_{2}(x)=\frac{2}{\pi} \int_{x}^{\infty} d r \arcsin (x / r) j(r)
\end{aligned}
$$

as can be understood from figure 12.2. $I_{1}(x)$ represents the fraction of the total current contained within the circle of radius $r . I_{2}(x)$ is twice the fraction of the current contained in the area, outside this circle, between the lines in y-direction through $x^{\prime}=0$ and $x^{\prime}=x$. Equations $(12.2 .6)$ and $(12.2 .9)$, in combination with equations $(12.2 .10)$ and $(12.2 .11)$, specify the width $d_{p, 1, p}$ in terms of the cylindrical current density distribution $j(r)$.

The function $I_{1}(x)$ will be used to calculate the Full width of the current density distribution FW, which contains a certain fraction $f$ of the total current

$$
\mathrm{FW}_{\mathrm{f}}=2 \mathrm{I}_{1}-1(\mathrm{f})
$$

in which $I_{1}-1$ denotes the inverse function of $I_{1}$. The case $f=1 / 2$ corresponds to the Full width median value $\mathrm{FW}_{50}$, introduced in section 5.9. We will use equations $(12.2 .10)$ and (12.2.12) to calculate the width $\mathrm{FW}_{\mathrm{f}}$ from the current density distribution $j(r)$. 
Figure 12.4 shows the current density distribution $j(x, y)$ of a shaped spot. The profile can be represented as the convolution of an ideal rectangular distribution $A(x, y)$, with a point spread function $S(x, y)$, which is determined by the aberrations of the system

$$
j(x, y)=\int_{-\infty}^{\infty} d x^{\prime} \int_{-\infty}^{\infty} d y^{\prime} A\left(x^{\prime}, y^{\prime}\right) s\left(x-x^{\prime}, y-y^{\prime}\right)
$$

where $A(x, y)$ is defined as

$$
A(x, y)=\frac{1}{d_{x} d_{y}} \theta\left(d_{x} / 2-|x|\right) \theta\left(d_{y} / 2-|y|\right)
$$

in which $d_{x}$ and $d_{y}$ are the full width-values of the ideal spot, in $x-$ and $y$-direction respectively. $\theta(x)$ is the step-function, defined by equation (3.7.8). Equation (12.3.1) presupposes that the aberrations, represented by $S(x, y)$, are independent of the (lateral) location in the image plane. The distribution $A(x, y)$ is normalized to unity. The corresponding distribution $j(x, y)$, specified by equation (12.3.1), will be normalized too, provided that $s(x, y)$ is normalized.

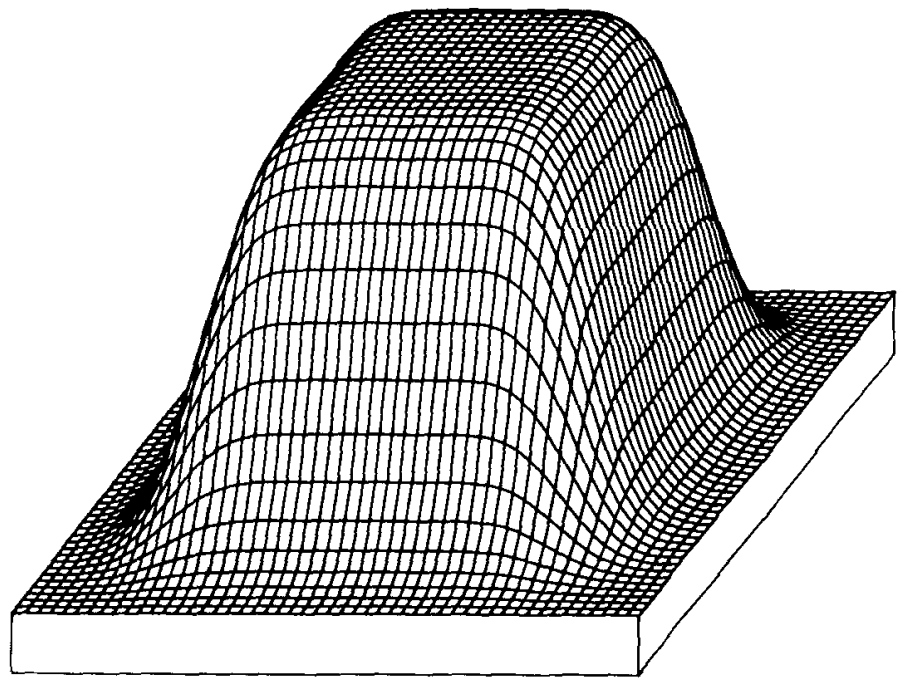

Pigure 12.4 Current density distribution of a shaped spot.

We will assume that the width of the point spread function $S(x, y)$ is small compared to both $d_{x}$ and $d_{y}$. This implies that the rectangular shape of the ideal spot is practically maintained. The main effect of the aberrations, is an increase of the edge-width, which is small compared to the dimensions of the spot. Furthermore, we will assume that $S(x, y)$ is rotational symmetric. Under these circumstances, it is sufficient to study the one-dimensional equivalent of equation $(12.3 .1)$ 


$$
j(x)=\int_{-\infty}^{\infty} d y j(x, y)=\int_{-\infty}^{\infty} d x^{\prime} A\left(x^{\prime}\right) s\left(x-x^{\prime}\right)
$$

where $A(x)$ and $S(x)$ are defined as

$$
\begin{aligned}
& A(x)=\int_{-\infty}^{\infty} d y A(x, y)=\frac{1}{d x} \theta\left(d_{x} / 2-|x|\right) \\
& S(x)=\int_{-\infty}^{\infty} d y S(x, y)
\end{aligned}
$$

The functions $j(x), A(x)$ and $S(x)$ can be considered as the projection of their two-dimensional counterparts on the y-direction. Figure 12.5 shows the general behaviour of $j(x)$ and $A(x)$.

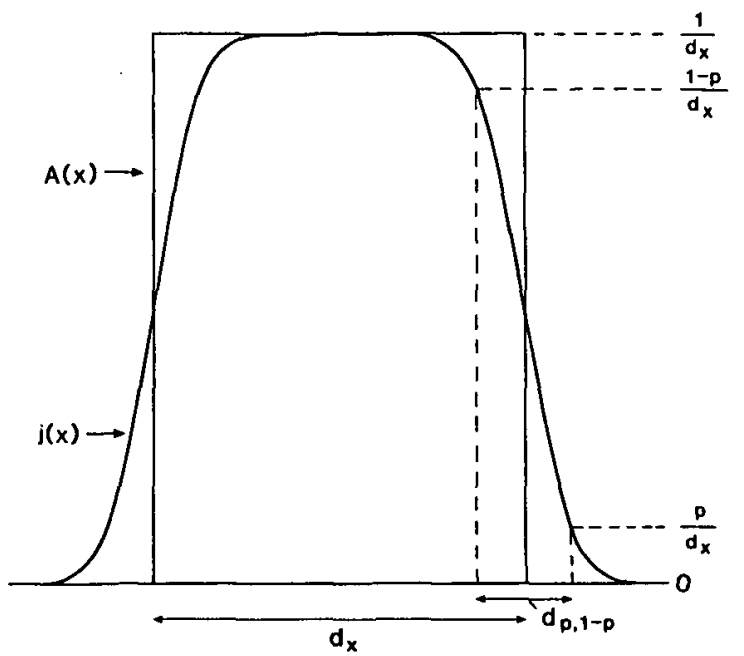

Figure 12.5 Behaviour of the functions $\mathrm{f}(\mathrm{x})$ and $\mathrm{A}(\mathrm{x})$, defined by equations (12.3.3) and (12.3.4). The edge-width $d_{\mathfrak{p}, 1-p}$ is defined as the distance between the intensity levels (within the edge) which are equal to $\mathrm{p}$ and $1-\mathrm{p}$ times the intensity level at the centre of the spot.

By substitution of equations $(12.3 .4)$ and (12.3.5) into equation $(12.3 .3)$, one obtains

$$
j(x)=\frac{1}{2 d_{x}}\left[I\left(d_{x} / 2+x\right)+I\left(d_{x} / 2-x\right)\right]
$$

in which $I(x)$ is defined as

$$
I(x)=2 \int_{0}^{x} d x^{\prime} S\left(x^{\prime}\right)
$$

Notice that equation $(12.3 .6)$ relies on the fact that $S(x)$ is a symmetric function, which means $S(-x)=S(x)$. This property follows from our assumption that $S(x, y)$ is rotational symetric. 
Let us consider the right edge of the profile $j(x)$ plotted in figure 12.5. This location corresponds to $x \approx d_{x} / 2$. As the width of $S(x)$ is assumed to be small compared to $d_{x}$, one may now approximate $I\left(d_{x} / 2+x\right) \approx I\left(d_{x}\right) \approx I(\infty)=1$ and equation $(12.3 .6)$ transforms to

$$
j(x) \approx \frac{1}{2 d_{x}}\left[1+I\left(d_{x} / 2-x\right)\right] \quad\left(x \approx d_{x} / 2\right)
$$

The $d_{p, 1-p}$ edge-width is defined as the distance between the points for which the current density $j(x)$ equals $p \times j(0)$ and $(1-p) \times j(0)$ respectively, where $j(0)$ is the current density at the centre of the spot, see again figure 12.5. Utilizing equation (12.3.8), one can express $d_{p}, i-p$ as

$$
d_{p, 1-p}=j^{-1}\left[(1-p) / d_{x}\right]-j^{-1}\left[p / d_{x}\right]=2 I^{-1}(1-2 p)
$$

similar to equation $(12.2 .6), j^{-1}$ and $I^{-1}$ denote the inverse functions of $j(x)$ and $I(x)$ respectively. Given the point spread function $S(x, y)$, the edge-width $d_{p, 1-p}$ is fully specified by equations $(12.3 .5),(12.3 .7)$ and $(12.3 .9)$.

Equations (12.3.9) and (12.3.7) represent the same mathematical problem as equations $(12.2 .6)$ and (12.2.5). Therefore, we conclude that the calculation of the spot-width, obtained by scanning the beam across a knife-edge, is mathematically equivalent to calculation of the edgewidth of a shaped spot. The point spread function $S(x, y)$, used to describe the edge-blurring of the shaped spot, plays the same role as the current density distribution $j(x, y)$, used for the Gaussian spot. Both quantities are determined by the brightness of the source, the aberrations of the optical components in the column and the effect of Coulomb interactions between the beam particles.

From now on, we will employ the notation used in equations $(12.2 .6)$ and $(12.2 .5)$. We emphasize that the resulting $d_{p, 1-p}$ value represents the edge-width of a shaped spot, as well as the spot-width of a Gaussian spot, determined by knife-edge scans. Equations (12.2.9), $(12.2 .10)$ and $(12.2 .11)$ are exploited to perform the calculation in cylindrical coordinates. In the next sections, we will successively study the extreme cases that the distribution $f(r)$ is entirely determined by the trajectory displacement effect, chromatic aberration, spherical aberration and space-charge defocussing respectively. Thus, in each case, it is assumed that the ideal image is a point, while the blurring of this point is the result of the considered aberration only. Accordingly, one may regard $j(r)$ as the cylindrical point-spread function, associated with that aberration.

In all cases, the calculation proceeds as follows. First, we will determine the distribution $j(r)$, corresponding to the considered aberration. Next, we will use $j(r)$ to evaluate the integrals $I_{1}(x)$ and $I(x)$ defined by equations (12.2.9), (12.2.10) and $(12.2 .11)$. Finally, the $F W_{f}$ value will be determined from $I_{1}(x)$, by solving equation $(12.2 .12)$, while the $d_{p, 1-p}$ value follows from $I(x)$, by solving equation (12.2.6).

It should be noticed that, in practical systems, the pointspread function $j(r)$ will not be determined by a single aberration, but rather by the simultaneous action of a number of aberrations. The calculation of the corresponding $j(r)$ is more involved and will not be considered here. Some related problems in the image formation of Scanning Transmission Electron Microscopy (STEM) instruments are discussed by Mory (1985) and Mory, Colliex and Cowley (1987). We note that, given the function $j(r)$ for certain combination of aberrations, one can again use the procedure outlined above, to evaluate the 
corresponding $F_{f}$ and $d_{p}, 1-p$ values.

\subsection{Trajectory displacement effect}

Let us assume that the current density distribution $j(r)$ of the spot is entirely determined by the trajectory displacement effect. Utilizing the representation of equation $(5.7 .10)$, one can now express $j(r)$ as

$$
j(r)=\int_{0}^{\infty} d k k r J_{0}(k r) e^{-\lambda p(k)}
$$

where $\lambda$ is the linear particle density, defined by equation $(3.2 .4)$, and $p(k)$ the function representing $j(r)$ in the k-domain. Following the analysis of section 5.9 , we approximate $\lambda p(k)$ as (reproducing equation $(5.9 .1))$

$$
\lambda p(k)=A_{\gamma} k^{\gamma}
$$

where $\gamma$ is a numerical constant and $A_{\gamma}$ is a physical quantity, which is determined by the experimental parameters. This representation permits a straightforward discussion of the different type of displacement distributions involved, as was shown in section 5.9. We recall that, for the trajectory displacement effect, the parameter $\gamma$ can take on the values $\gamma=2$ (Gaussian distribution), $\gamma=3 / 2$ (Holtsmark type of distribution), $\gamma=1$ (Lorentzian distribution) and $\gamma=1 / 3$ (twodimensional pencil beam distribution).

Substitution of equation $(12.4 .1)$ into equation $(12.2 .10)$ and integration over $r$ yields

$$
I_{1}(x)=\int_{0}^{\infty} d k x J_{1}(k x) e^{-\lambda p(k)}
$$

using the identity

$$
z J_{0}(z)=\frac{d}{d z}\left(z J_{1}(z)\right)
$$

In fact, equation $(12.4 .3)$ was utilized in section 5.9 , to calculate the median Full width FWso for the case of a two-dimensional distribution $(n=2)$.

Substitution of equation (12.4.1) into equation $(12.2 .11)$ yields

$$
I_{2}(x)=\int_{0}^{\infty} d k x A_{2}(k x) e^{-\lambda p(k)}
$$

in which the function $A_{2}(z)$ is defined as

$$
A_{2}(z)=\frac{2}{\pi} \int_{1}^{\infty} d t z t \quad J_{0}(z t) \arcsin (1 / t)
$$

Partial integration, employing equation (12.4.4), gives 


$$
A_{2}(z)=-J_{1}(z)+\frac{2}{\pi} \frac{\sin (z)}{z}
$$

in which we utilized the integral

$$
\int_{1}^{\infty} d t \frac{J_{1}(t z)}{\left(t^{2}-1\right)^{1 / 2}}=\frac{\sin (z)}{z}
$$

By combining equations $(12.4 .3),(12.4 .5)$ and $(12.4 .6)$, one finds for the function $I(x)$, defined by equation (12.2.9)

$$
I(x)=\frac{2}{\pi} \int_{0}^{\infty} d k \frac{\sin (k x)}{k} e^{-\lambda p(k)}
$$

The function $I(x)$ is used in the calculation of the $d_{p, 1-p}$ width, from equation $(12 \cdot 2 \cdot 6)$.

We note that the derivation of equation (12.4.8) can be performed in a more direct way, by using rectangular coordinates. According to equation (5.7.6), one may express the one dimensional distribution $\mathrm{j}(\mathrm{x})$ as

$$
j(x)=\frac{1}{\pi} \int_{0}^{\infty} d k \cos (k x) e^{-\lambda p(k)}
$$

where $p(k)$ is the same function as appearing in equation (12.4.1). Substitution into equation $(12.2 .5)$ and integration over $x$, directly yields equation $(12.4 .8)$.

We will now replace the function $\lambda p(k)$ by the expression given by equation $(12.4 .2)$. In order to bring the resulting expression(s) in a form which is independent of the experimental parameters (represented by the quantity $A_{\gamma}$ ), we will employ the scaled quantities

$$
\bar{k}=A_{\gamma}{ }^{1 / \gamma} k, \bar{x}=A_{\gamma}{ }^{-1 / \gamma} x
$$

first introduced by equation (5.9.13). Equations (12.4.3) and (12.4.8) now transform to

$$
\begin{aligned}
& I_{1}(\bar{x})=\int_{0}^{\infty} d \bar{k} \bar{x} J_{1}(\bar{k} \bar{x}) e^{-\bar{k}^{\gamma}} \\
& I(\bar{x})=\frac{2}{\pi} \int_{0}^{\infty} d \bar{k} \frac{\sin (\bar{k} \bar{x})}{\bar{k}} e^{-\bar{k}^{\gamma}}
\end{aligned}
$$

We evaluated equations (12.4.11) and (12.4.12) numerically, as function of $\bar{x}$, for $\gamma$ is $2,3 / 2,1,1 / 2$ and $1 / 3$. The results are plotted in the figures 12.6 and 12.7 .

Explicit analytical expressions for $I_{1}(\bar{x})$ and $I(\bar{x})$ can be obtained in the cases $\gamma=2$ (Gaussian trajectory displacement distribution) and $\gamma=1$ (Lorentzian trajectory displacement distribution). For $\gamma=2$, one obtains from equations $(12.4 .11)$ and $(12.4 .12)$ 


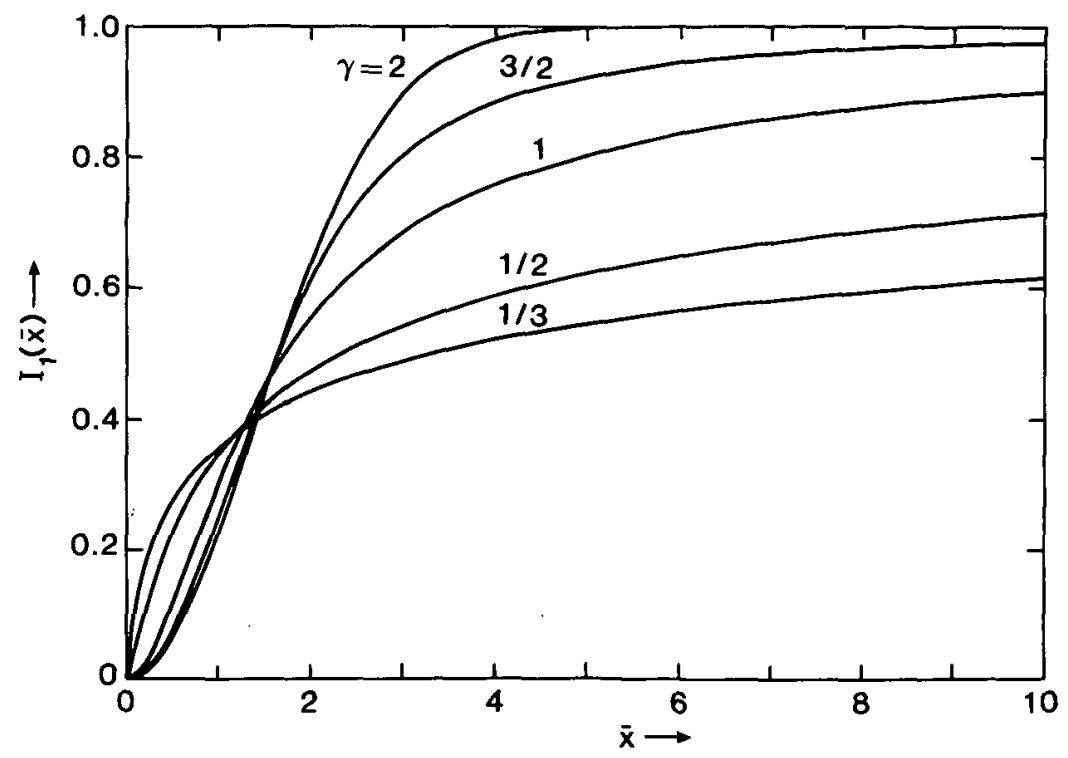

Pigure 12.6 The function $I_{1}(\bar{x})$ for a spot which is limited by the trajectory displacement effect, see equation (12.4.11).

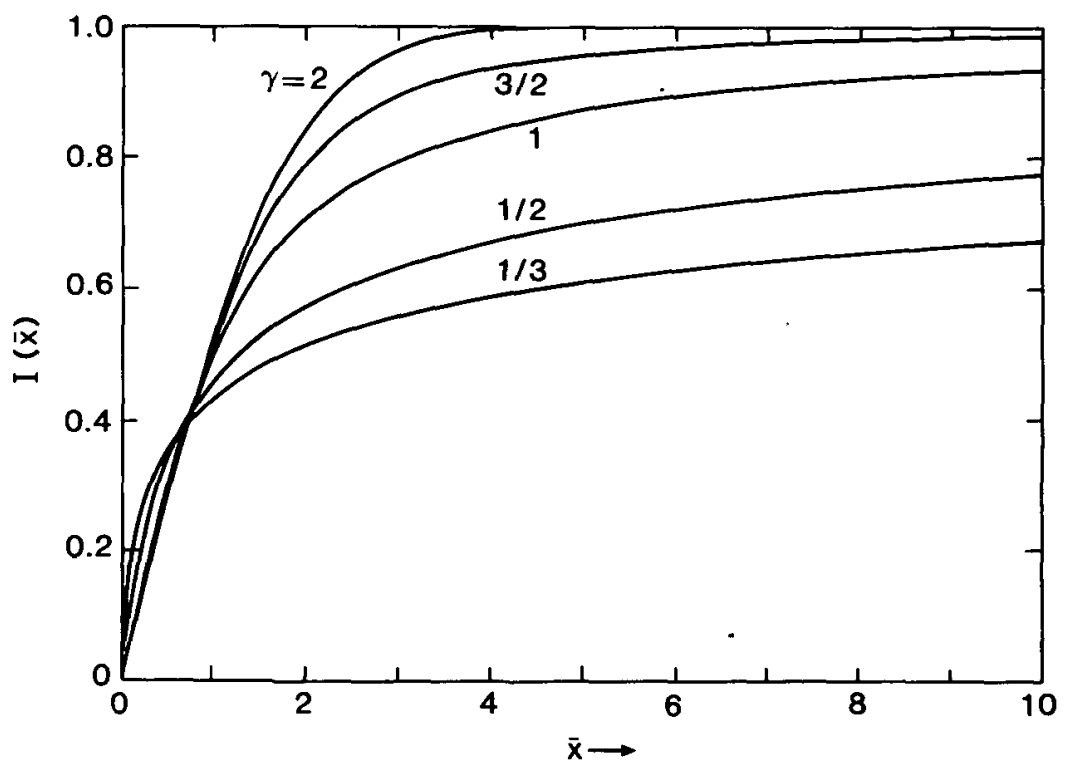

Figure 12.7 The function $I(\bar{x})$ for a spot which is limited by the trajectory displacement effect, see equation (12.4.12). 


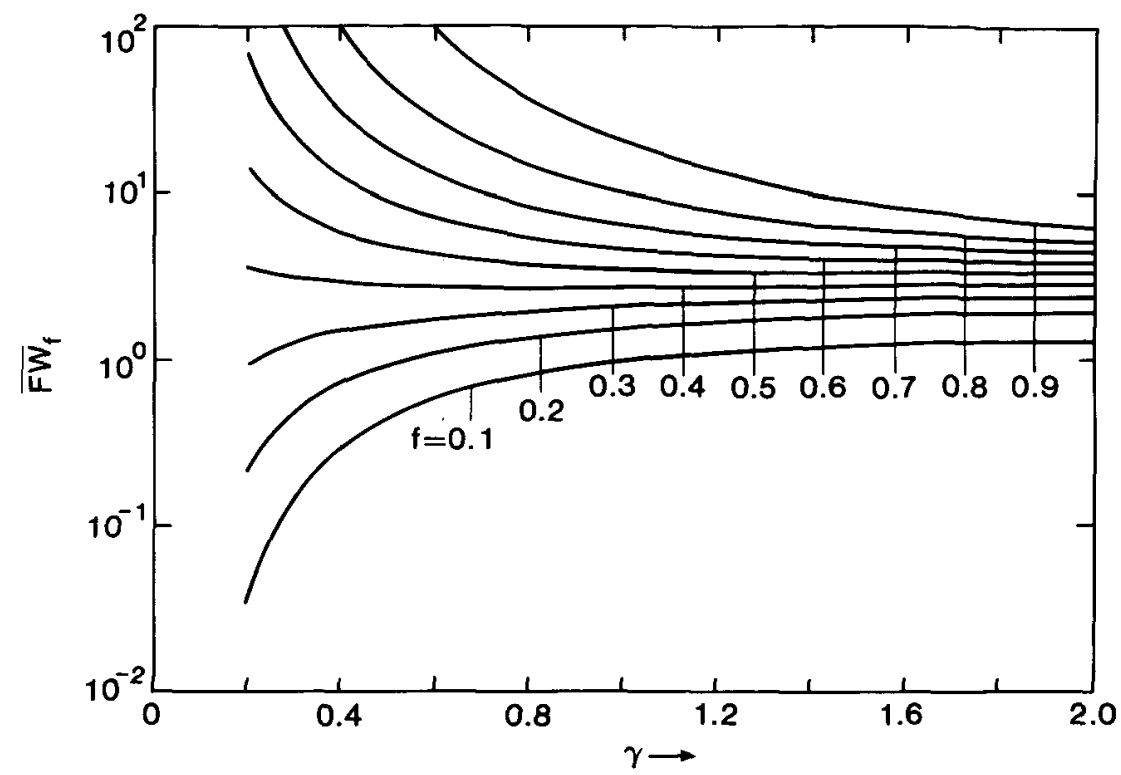

Figure 12.8 The scaled width $\overline{\mathrm{FW}}_{\mathrm{f}}$ for a spot which is limited by the trajectory displacement effect as function, of the type of trajectory displacement distribution, represented by the parameter $\gamma$. See equations (12.2.12), (12.4.10) and (12.4.11).

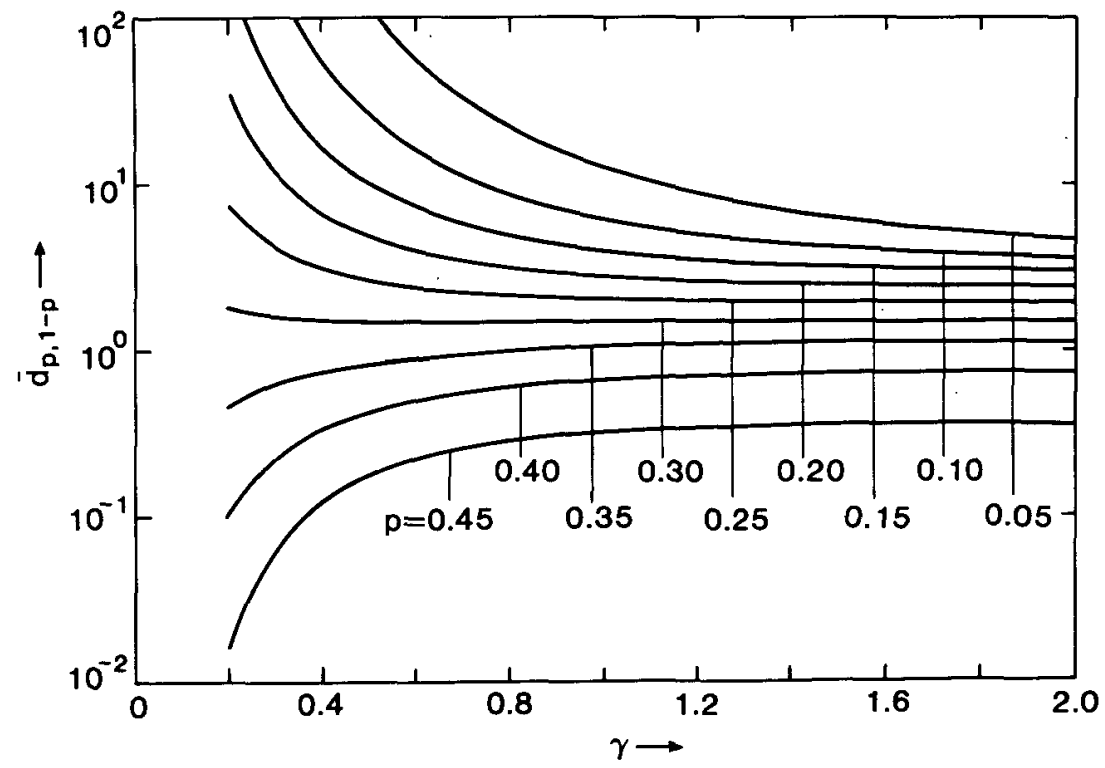

Pigure 12.9 The scaled width $\overline{\mathrm{d}}_{\mathrm{p}, 1-\mathrm{p}}$ for a spot which is limited by the trajectory displacement effect, as function of the type of trajectory displacement distribution, represented by the parameter $\gamma$. See equations (12.2.6), (12.4.10) and $(12.4 .12)$. 


$$
\begin{array}{ll}
I_{1}(\bar{x})=1-e^{-(\bar{x} / 2)^{2}} & (\gamma=2) \\
I(\bar{x})=\operatorname{erf}(\bar{x} / 2) & (\gamma=2)
\end{array}
$$

where $\operatorname{erf}(x)$ is the Fresnel error-function, defined by equation (4.5.9). Notice that equations $(12.4 .13)$ and $(12.4 .14)$ can also be derived from equations (5.9.3) (taking $n=2$ and $\Delta \eta=r),(12.2 .9),(12.2 .10)$ and (12.2.11), using the scaling of equation (12.4.10). For $\gamma=1$, one obtains from equations $(12.4 .11)$ and $(12.4 .12)$

$$
\begin{array}{ll}
I_{1}(\bar{x})=1-\frac{1}{\left(1+\bar{x}^{2}\right)^{1 / 2}} & (\gamma=1) \\
I(\bar{x})=\frac{2}{\pi} \arctan (\bar{x}) & (\gamma=1)
\end{array}
$$

which can also be derived from equations (5.9.6) (taking $\Delta \eta=r$ ), $(12.2 .9),(12.2 .10)$ and $(12.2 .11)$, using the scaling of equation $(12.4 .10)$.

The next step, is the calculation of the $d_{p, 1-p}$ width from equation (12.2.6) and the $\mathrm{FW}_{f}$ value from equation $(12.2 .12)$. For $\gamma=2$, one finds with equations $(12.4 .13)$ and $(12.4 .14)$

$$
\begin{array}{ll}
\overline{F W}_{f}=4 \sqrt{\ln \left(\frac{1}{1-f}\right)} & (\gamma=2) \\
\bar{d}_{p, 1-p}=4 \operatorname{erf}-1(1-2 p) & (\gamma=2)
\end{array}
$$

in which erf-1 denotes the inverse of the error function. For $\gamma=1$, one finds with equations $(12.4 .15)$ and $(12.4 .16)$

$$
\begin{array}{ll}
\overline{F W}_{f}=2 \sqrt{\left(\frac{1}{1-f}\right)^{2}-1} & (\gamma=1) \\
\bar{d}_{p, 1-p}=2 \tan [\pi(1-2 p) / 2] & (\gamma=1)
\end{array}
$$

In fact, equations $(12.4 .17)$ and $(12.4 .19)$ were exploited in equations (5.9.4) and (5.9.8), to calculate the FW50 value (which corresponds to $f=1 / 2)$ of the two-dimensional $(n=2)$ Gaussian and Lorentzian distribution respectively.

In order to determine the $\mathrm{FW}_{\mathrm{f}}$ and $\mathrm{d}_{\mathrm{p}, 1-\mathrm{p}}$ values for arbitrary $\gamma$-values, we followed a numerical approach. Equation (12.2.12) was solved for $\gamma$ values in the range $0.2<\gamma<2$, using the expression for $I_{1}(x)$ given by equation (12.4.11). For the parameter $f$, we considered the values $0.1,0.2, \cdots, 0.9$. The results are plotted in figure 12.8 . Equation (12.2.6) was solved for the same range of $\gamma$-values, using the expression for $I(x)$ given by equation (12.4.12). For the parameters $p$, we considered the values $0.05,0.10, \cdots, 0.45$. The results are presented in figure 12.9 .

We note that the Full Width at Half Maximum (FWHM) of the distribution $j(r)$, determined by the trajectory displacement effect, can directly be obtained from the analysis presented in section 5.9. The quantity FWHM, which is the FWHM of the scaled distribution given by equation (5.9.14), is plotted in figure 5.5a. Table 5.1 summarizes the numbers for some specific cases. The scaling, which is defined by equation (5.9.13), is the same as used in this section, see equation $(12.4 .10)$. This allows a direct comparison of the figures $12.8,12.9$ and 5.5 a (curve corresponding to a two-dimensional distribution). Figure 
$5.5 \mathrm{~b}$ gives the corresponding values of the scaled Full width median value $F W_{50}$. The curve in this figure given for a two-dimensional distribution $(n=2)$ corresponds to the curve presented in figure 12.9 for $\mathrm{f}=0.5$.

\subsection{Chromatic aberration}

In this section we will study the properties of the probe in a chromatically limited system. Let us assume that the energy distribution is entirely the result of statistical particle-particle interactions (Boersch effect). According to equation (5.7.6), the energy distribution can then be expressed as

$$
\rho(\Delta \mathrm{E})=\frac{1}{\pi} \int_{0}^{\infty} \mathrm{dk} \cos (k \Delta E) \mathrm{e}^{-\lambda p(k)}
$$

which is a symmetric function in $\Delta \mathrm{E}$. The function $p(k)$ represents the energy distribution in the k-domain.

Before embarking on the actual calculations, we will briefly consider some related subjects. In order to determine the fraction of the particles, contained within a certain energy range, one has to evaluate the following integral

$$
E_{1}(x)=2 \int_{0}^{x} d \Delta E \rho(\Delta E)
$$

similar to equation (12.2.10). The Full Width energy spread $F_{k}, f$, which contains a fraction $f$ of the particles, follows by solving the equation

$$
F W_{E, f}=2 E_{1}^{-1}(f)
$$

similar to equation (12.2.12). $E_{1}-1$ denotes the inverse function of $\mathbb{E}_{1}$. By substitution of equation (12.5.1) into equation (12.5.2) and integration over $\Delta E$, one obtains

$$
E_{1}(x)=\frac{2}{\pi} \int_{0}^{\infty} d k \frac{\sin (k x)}{k} e^{-\lambda p(k)}
$$

In fact, this equation was used in section 5.9 , to calculate the median Full Width FW5o for the general case of an one-dimensional displacement distribution $(n=1)$. By comparison with equation $(12.4 .8)$, one sees that the function $E_{1}(x)$ is identical to the function $I(x)$ obtained for the trajectory displacement effect. Accordingly, the solution of equation (12.5.3) can be obtained from the results plotted in figure 12.9, using that $F W_{E}, f$ is here equivalent to $d_{p}, 1-p$, where $f=1-2 p$.

The relation between the energy deviation $\Delta \mathrm{E}$ of a particle and its corresponding deviation $\Delta v_{z}$, from the average axial velocity $v_{z}$, is given by

$$
\Delta E \cong \mathrm{mv}_{\mathbf{2}} \Delta \mathrm{v}_{\mathbf{2}}
$$

provided that $v_{z} \gg>\Delta v_{z}$. The energy distribution $\rho(\Delta E)$ and the velocity distribution $\rho_{v}\left(\Delta v_{z}\right)$ are, therefore, related as 


$$
\rho(\Delta \mathrm{E})=\frac{1}{\mathrm{mV}_{\mathbf{z}}} \rho_{\mathrm{v}}\left(\Delta \mathrm{E} / \mathrm{mV}_{\mathrm{z}}\right)
$$

The corresponding functions in the $k$-domain are related by

$$
p(k)=p_{v}\left(k m v_{z}\right)
$$

as can be seen from the structure of equation (12.5.1). Utilizing the representation of equation $(12.4 .2)$, one finds

$$
A_{\gamma}=A_{\gamma} \cdot v\left(m_{z}\right)^{\gamma}
$$

where $A_{\gamma}$ represents the energy distribution and $A_{\gamma} \cdot v$ the distribution of axial velocities. In general, the reader should be alert that $A_{\gamma}$ may represent different physical quantities.

Let us now return to the main subject of this section and investigate the distribution $j(r)$ of a probe which is dominated by chromatic aberration. Consider the single thin-lens system of figure 12.10. The distance between the object plane and the lens is $q$; the distance between the lens and the image pl`ne is p. A particle leaving the object plane from the axis, with a certain angle $\alpha_{1}$ and an energy equal to the average energy $E$, will cross the axis in the image-plane, with an angle $\alpha=\alpha_{1} p / q$. Another particle, which starts out along the same trajectory, but with an energy smaller than the average energy $E$, will be deflected stronger and crosses the axis before reaching the image plane, as is shown by the dashed line in figure 12.10. In general, one finds that the radial position $r$ of a particle in the vicinity of the image plane, which left the object from the axis, depends on its energy deviation $\Delta \mathrm{E}$, its angle with the axis a and the distance to the image plane $\Delta z$, as

$$
r=\left|\Delta z \alpha+C_{c} \frac{\Delta E}{E}\left(1+\frac{\Delta z}{q}\right) \alpha\right|
$$

where $C_{c}$ is the constant of chromatic aberration of the lens. The radial position in the (Gaussian) image plane follows by taking $\Delta z=0$

$$
r=C_{c} \frac{|\Delta E|}{E} \alpha
$$

From this equation, one sees that the distribution of $r$ in the Gaussian image plane, denoted as $j(r)$, depends on the energy distribution $\rho(\Delta E)$ and the angular distribution $f_{\alpha}(\alpha)$ near the image plane. Equation (12.5.9) shows that the distribution $j(r)$ also depends on the axial position, represented by $\Delta z$. However, we will restrict the analysis to the case $\Delta z=0$, that is to the calculation of $j(r)$ in the Gaussian image plane.

Assuming that the the energy distribution $\rho(\Delta E)$ and the angular distribution $f_{\alpha}(\alpha)$ are independent, one can express $j(r)$ as

$$
j(r)=\int d \Delta E \rho(\Delta E) \int d \alpha f_{\alpha}(\alpha) \delta\left(r-C_{c} \frac{|\Delta E|}{E} \alpha\right)
$$

where $\delta(x)$ is the delta-Dirac function. For the angular distribution $f_{\alpha}(\alpha)$ we write

$$
f_{\alpha}(\alpha)=\frac{2 \alpha}{\alpha_{0}^{2}} F_{\alpha}(\alpha) \quad(a>0)
$$




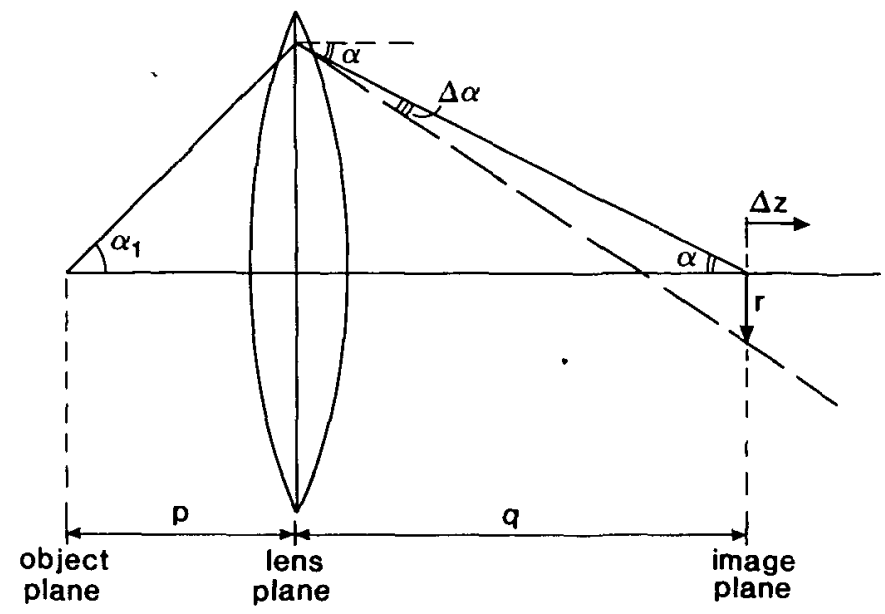

\begin{abstract}
Figure 12.10 Schematic view of a single lens system. The image is affected by the aberrations of the lens. An angular shift $\Delta \alpha$ in the lens plane translates into a spatial shift $r$ in the image plane.
\end{abstract}

in which the function $F_{\alpha}$ is for an uniform angular distribution given by

$$
F_{\alpha}(\alpha)=\theta\left(1-\alpha / \alpha_{0}\right)
$$

where $\theta(x)$ denotes the step function. In case of a Gaussian angular distribution, we use

$$
\mathbf{F}_{\alpha}(\alpha)=\exp \left[-\left(\alpha / \alpha_{0}\right)^{2}\right]
$$

Finally, a truncated Gaussian distribution is represented as

$$
F_{\alpha}(\alpha)=\frac{\exp \left[-\left(\alpha / \alpha_{0}\right)^{2}\right] \theta\left(1-\alpha / \alpha_{m}\right)}{1-\exp \left[-\left(\alpha_{m} / \alpha_{0}\right)^{2}\right]}
$$

This type of distribution corresponds to a Gaussian illumination of the image, which is limited by an aperture. The angle $\alpha_{m}$ denotes the maximum angle of the rays passing through the aperture.

By substituting equations (12.5.1) and (12.5.12) into equation (12.5.11) and eliminating the $\delta$-function by means of its definition, one obtains

$$
j(r)=\frac{4 E^{2} r}{\pi C_{c}^{2} \alpha_{0}^{2}} \int_{0}^{\infty} d k e^{-\lambda p(k)} \int_{0}^{\infty} d \Delta E \frac{\cos (k \Delta E)}{\Delta E^{2}} F_{\alpha}\left(\frac{E r}{C_{c} \alpha_{0} \Delta E}\right)
$$

We will exploit the representation of $p(k)$, given by equation (12.4.2), to rewrite equation $(12.5 .16)$. In addition, we introduce the following scaling 


$$
\begin{array}{ll}
\bar{r}=\frac{E}{C_{c} \alpha_{0} A_{\gamma}{ }^{1 / \gamma}} r & , \bar{j}(\bar{r})=\frac{C_{c} \alpha_{0} A_{\gamma}{ }^{1 / \gamma}}{E} j(r) \\
\bar{k}=A_{\gamma}{ }^{1 / \gamma_{k}} & , \Delta \bar{E}=A_{\gamma}{ }^{-1 / \gamma} \Delta E
\end{array}
$$

Equation (12.5.16) now transforms to

$$
\bar{j}(\bar{r})=\frac{4 \bar{r}}{\pi} \int_{0}^{\infty} d \bar{k} e^{-\bar{k}^{\gamma}} \cdot \int_{0}^{\infty} d \Delta \bar{E} \frac{\cos (\bar{k} \Delta \bar{E})}{\Delta \bar{E}^{2}} F_{\alpha}(\bar{r} / \Delta \bar{E})
$$

This equation can be rewritten as

$$
\bar{j}(\bar{r})=\frac{4}{\pi} \int_{0}^{\infty} d \bar{k} \frac{C(\bar{k} \bar{r})}{\bar{k}} e^{-\bar{k}^{\gamma}}
$$

where the function $C$ depends on the type of angular distribution. For an uniform angular distribution $\left(\mathrm{C}=\mathrm{C}_{\mathrm{u}}\right)$, one finds with equation $(12.5 .13)$

$$
C_{u}(z)=\int_{1}^{\infty} d x \frac{\cos (x z)}{x^{2}} \quad \text { (uniform } f_{\alpha}(\alpha) \text { ) }
$$

For a Gaussian angular distribution $\left(C=C_{8}\right)$, one finds with equation $(12.5 .14)$

$$
\left.C_{g}(z)=\int_{0}^{\infty} d x \frac{\cos (x z)}{x^{2}} e^{-1 / x^{2}} \quad \text { (Gaussian } f_{\alpha}(\alpha)\right)
$$

The case of a truncated Gaussian distribution will be considered separately in section 12.8 .

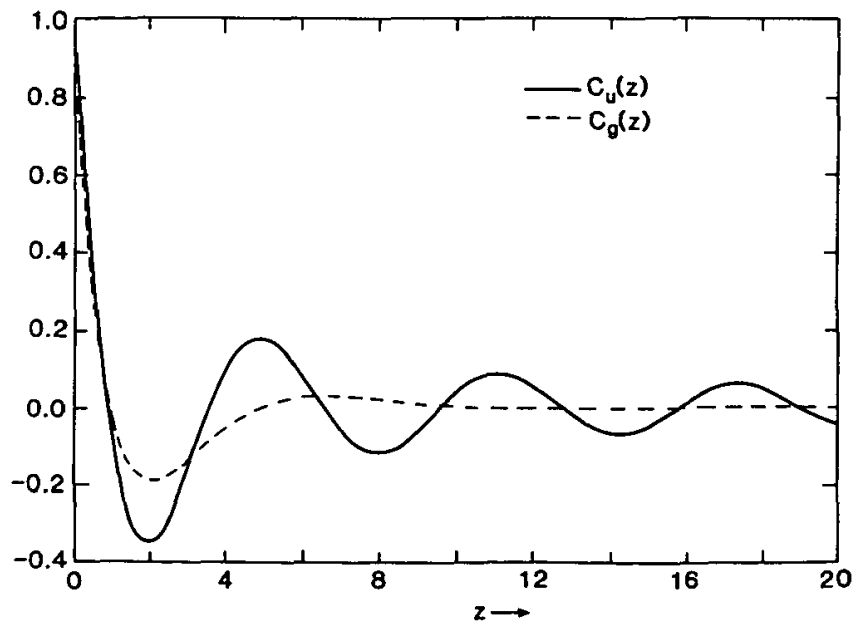

Figure 12.11 The functions $\mathrm{C}_{0}(\mathrm{z})$ and $\mathrm{C}_{\mathrm{z}}(\mathrm{z})$, defined by equations

$(12.5 .20)$ and $(12.5 .21)$ respectively. 
The functions $C_{u}(z)$ and $C_{B}(z)$ were evaluated numerically and are plotted in figure 12.11. For further numerical calculations, we use the following approximations

$$
C_{u, a}(z)=\frac{1-0.1751 z+2.121 z \cos (z)}{1+3.584 z+1.116 z^{2}+z^{3}}-\frac{z \sin (z)}{0.2976+0.9307 z+z^{2}}
$$

$$
C_{B, a}(z)=\frac{\sqrt{\pi} / 2+1.842 z-0.5902 z^{2}+6.362 z^{3} \sin (3.932 \sqrt{z+5.326}) e^{-z / 8}}{1+3.877 z+4.831 z^{2}++9.105 z^{3}+3.155 z^{4}+z^{5}}
$$

The form of equations (12.5.22) was chosen such that the behaviour for $z \rightarrow 0$ and $z \rightarrow \infty$ agrees with the behaviour of the exact functions, given by equations $(12.5 .20)$ and $(12.5 .21)$. This also explains some of the numerical constants in equations (12.5.22). The remaining constants were determined by fitting the functions to the numerical data. The accuracy. of these approximations is, in general, better than $0.5 \%$.

Equation (12.5.19) was evaluated numerically, using the approximations of equation (12.5.22). The relevant values of $\gamma$ are 2 (Gaussian energy distribution), $3 / 2$ (Holtsmark type of energy distribution), 1 (Lorentzian energy distribution) and $1 / 2$ (energy distribution generated in a pencil beam). The corresponding distributions found for an uniform angular distribution are plotted in figure $12.12 \mathrm{a}$, while the results for a Gaussian angular distribution are plotted in figure $12.12 \mathrm{~b}$.

For the cases $\gamma=2$ and $\gamma=1$, one can evaluate $\bar{j}(\bar{r})$ analytically without introducing any approximations. From equations (12.5.19) and (12.5.20), one finds for the case of an uniform angular distribution

$$
\bar{j}(\bar{r})=\frac{2}{\sqrt{\pi}} e^{-(\bar{r} / 2)^{2}}+\bar{r}[\operatorname{erf}(\bar{r} / 2)-1] \quad\left(\gamma=2 \text {, uniform } f_{\alpha}(\alpha)\right)
$$

$$
\bar{j}(\bar{r})=\frac{4}{\pi}\left[1+\bar{r}\left(\arctan (\bar{r})-\frac{\pi}{2}\right)\right] \quad\left(\gamma=1 \text {, uniform } f_{\alpha}(\alpha)\right)
$$

From equations (12.5.19) and $(12.5 .21)$, one finds for the case of a Gaussian angular distribution

$$
\begin{array}{lr}
\bar{j}(\bar{r})=e^{-\bar{r}} & \left(\gamma=2, \text { Gaussian } f_{\alpha}(\alpha)\right) \\
\bar{j}(\bar{r})=\frac{2}{\sqrt{\pi}}+2 \bar{r}[\operatorname{erf}(\bar{r})-1] e^{\bar{r}^{2}} & \left(\gamma=1, \text { Gaussian } f_{\alpha}(\alpha)\right)
\end{array}
$$

The analytical results of equations (12.5.23) and (12.5.24) were exploited to check the accuracy of the numerical calculations based on the approximations of equations (12.5.22). The numerical accuracy of the results plotted in figures $12.12 \mathrm{a}$ and $12.12 \mathrm{~b}$ was found to be better than $1 \%$.

The next step is the evaluation of the functions $I_{1}(x)$ and $I(x)$ from equations $(12.2 .9),(12.2 .10)$ and $(12.2 .11)$. We will scale the quantity $x$ in the same way as $r$ and the quantities $I$ and $I_{1}$ in the same way as $j$, see equation (12.5.17). From equations (12.2.10) and $(12.5 .19)$, one obtains 

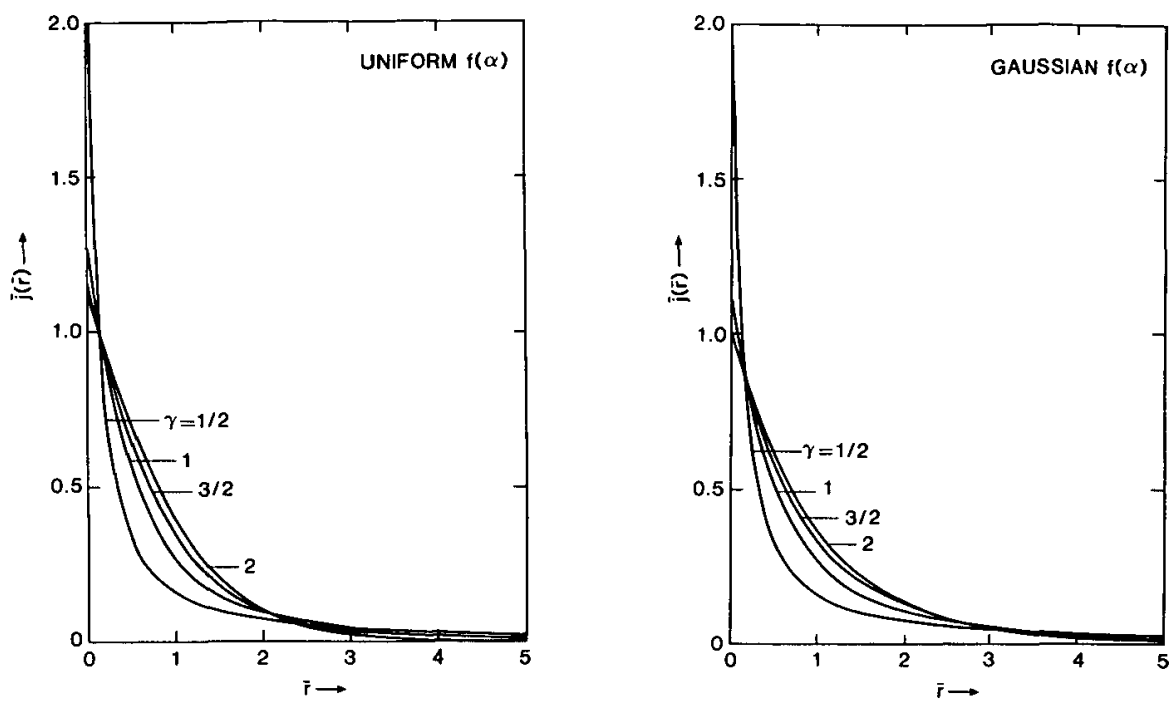

Figure 12.12 The scaled current density distribution $\bar{j}(\bar{r})$ of a chromatically limited spot, for different types of energy distributions, represented by the parameter $\gamma$. The figures $12.12 a$ and $12.12 b$ pertain to an uniform and a Gaussian angular distribution respectively and are evaluated from equations (12.5.19) and (12.5.22). Notice that the distributions are normalized conform equation (12.2.8).

$$
\bar{I}_{1}(\bar{x})=\int_{0}^{\bar{x}} d \bar{r} \bar{j}(\bar{r})=\int_{0}^{\infty} d \bar{k} \frac{B_{1}(\bar{k} \bar{x})}{\bar{k}} e^{-\bar{k}^{\gamma}}
$$

in which the function $B_{1}(z)$ is defined as

$$
B_{1}(z)=\frac{4}{\pi} \int_{0}^{z} d t C(t)
$$

Similarly, one finds from equations $(12.2 .9),(12.2 .10),(12.2 .11)$ and $(12.5 .19)$

$$
\overline{\mathrm{I}}(\overline{\mathrm{x}})=\overline{\mathbf{I}}_{1}(\overline{\mathrm{x}})+\frac{2}{\pi} \int_{\overline{\mathrm{x}}}^{\infty} \mathrm{d} \overline{\mathrm{r}} \arcsin (\overline{\mathrm{x}} / \overline{\mathrm{r}}) \overline{\mathrm{j}}(\overline{\mathrm{r}})=\int_{0}^{\infty} \mathrm{d} \overline{\mathrm{k}} \frac{\mathrm{B}(\overline{\mathrm{k}} \overline{\mathrm{x}})}{\overline{\mathrm{k}}} \mathrm{e}^{-\overline{\mathrm{k}}^{\gamma}}
$$

in which the function $B(z)$ is defined as

$$
B(z)=B_{1}(z)+\frac{8}{\pi^{2}} \int_{z}^{\infty} d t \arcsin (z / t) C(t)
$$

By substitution of equation (12.5.20) into equations (12.5.26) and $(12.5 .28)$, one finds, after some straightforward transformations, for an uniform angular distribution 


$$
\begin{aligned}
& B_{1 u}(z)=\frac{2}{\pi}\left(\sin z+z C_{u}(z)\right) \\
& B_{u}(z)=\frac{8}{\pi^{2}} \int_{1}^{\infty} d t \frac{\sin (t z)}{t^{3}} \sqrt{t^{2}-1}
\end{aligned}
$$

where the function $C_{u}(z)$ is given by equation (12.5.20). Similarly, one obtains with equation (12.5.21), for a Gaussian angular distribution

$$
\begin{aligned}
& B_{1_{B}}(z)=\frac{2 z}{\pi} \int_{0}^{\infty} d t \cos (t z)\left(1-e^{-1 / t^{2}}\right) \\
& B_{B}(z)=\frac{4}{\pi^{3 / 2}} \int_{0}^{\infty} d t \frac{\sin (t z)}{t^{2}} e^{-1 / t^{2}} .
\end{aligned}
$$

(Gaussian $\left.f_{\alpha}(\alpha)\right)(12,5.30)$

The functions $B_{u}(z), B_{1 g}(z)$ and $B_{g}(z)$ were evaluated numerically and are plotted in figure 12.13. For further numerical calculations, we use the following approximations

$B_{18, a}(z)=\frac{2 z / \sqrt{1+1}+120 z+21.09 z^{3} \sin (3.51 \sqrt{z+2.91}+0.751) e^{-z / 8}}{1+2.298 z+6.979 z^{2}+28.47 z^{3}+8.066 z^{4}+z^{5}}$

$B_{u} . a(z)=\frac{1.80 z^{5 / 6}-1.395 z+1.617 z \cos (z-0.582)}{1+3.709 z+1.098 z^{2}+z^{3}}-\frac{z^{3 / 2} \cos (z+0.857)}{3.067+9.538 z+2.312 z^{2}+z^{3}}$

$B_{B}, a(z)=\frac{1.62 z^{5 / 6}-0.1801 z-.04237 z^{2}+4.637 z^{3} \sin (3.70 \sqrt{z+3.763}+0.0869) e^{-z / 8}}{1+2.345 z+2.722 z^{2}+7.616 z^{3}+4.250 z^{4}+z^{5}}$

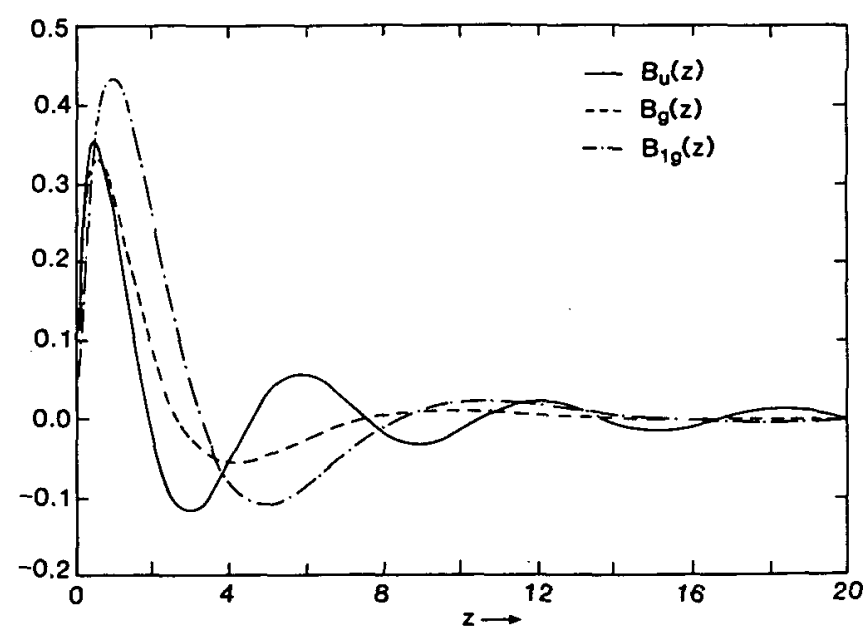

Figure 12.13 The functions $\mathrm{B}_{\mathrm{v}}(\mathrm{z}), \mathrm{B}_{\mathrm{z}}(\mathrm{z})$ and $\mathrm{B}_{1 \mathrm{z}}(\mathrm{z})$, defined by equations (12.5.29) and $(12.5 .30)$. 
The form of equations (12.5.31) was chosen such that the behaviour for $z \rightarrow 0$ and $z+\infty$ agrees with the behaviour of the exact functions, given by equations (12.5.29) and (12.5.30). This also explains some of the numerical constants in equations $(12.5 .31)$. The remaining constants were determined by fitting the functions to the numerical data. The accuracy of these approximations is, in general, better than $0.5 \%$. The first equation of $(12.5 .29)$ shows that the function $B_{1 v}(z)$ is directly related to the function $\mathrm{C}_{\mathrm{u}}(2)$, for which one may use the approximation given by the first equation of $(12.5 .22)$.

Equations $(12.5 .25)$ and $(12.5 .29)$, which apply to calculation of $\overline{\mathrm{I}}_{1}(\overline{\mathrm{x}})$ for an uniform angular distribution, were evaluated numerically using the approximation of $\mathrm{C}_{\mathrm{u}, \mathrm{a}}(z)$, given by of equation (12.5.22). The results are plotted in figure 12.14a. Similar results were obtained for a Gaussian angular distribution, using equation (12.5.25) and the first equation of $(12.5 .31)$. These results are plotted in figure $12.14 \mathrm{~b}$. The function $\overline{\mathrm{I}}(\overline{\mathrm{x}})$ was evaluated from equation $(12.5 .27)$, using the approximations given by the second and third equation of (12.5.31). The results for an uniform and a Gaussian angular distribution are plotted in the figures $12.15 \mathrm{a}$ and $12.15 \mathrm{~b}$ respectively.

The cases $\gamma=2$ (Gaussian energy distribution) and $\gamma=1$ (Lorentzian energy distribution) permit a more detailed analytical treatment. For $\gamma=2$ and an uniform angular distribution, one finds

$$
\begin{aligned}
\overline{\mathrm{I}}_{1}(\overline{\mathrm{x}}) & =\frac{\overline{\mathrm{x}}}{\sqrt{\pi}} \mathrm{e}^{-(\overline{\mathrm{x}} / 2)^{2}}+\operatorname{erf}(\overline{\mathrm{x}} / 2)+\frac{1}{2} \overline{\mathrm{x}}^{2}[\operatorname{erf}(\overline{\mathrm{x}} / 2)-1] \\
\overline{\mathrm{I}}(\overline{\mathrm{x}}) & =\frac{4}{\pi} \int_{1}^{\infty} \mathrm{dt} \frac{\sqrt{\mathrm{t}^{2}-1}}{\mathrm{t}^{3}} \operatorname{erf}\left(\frac{\mathrm{t} \overline{\mathrm{x}}}{2}\right)= \\
& =1-\frac{2 \overline{\mathrm{x}}}{\pi^{3 / 2}} \int_{1}^{\infty} \mathrm{dt}\left[\arccos (1 / \mathrm{t})-\frac{\sqrt{\mathrm{t}^{2}-1}}{\mathrm{t}^{2}}\right] \mathrm{e}^{-(\mathrm{t} \overline{\mathrm{x}} / 2)^{2}}
\end{aligned}
$$

For $\gamma=1$ and an uniform angular distribution, one finds

$$
\begin{aligned}
& \overline{\mathrm{I}}_{1}(\overline{\mathrm{x}})=\frac{2}{\pi}\left[\overline{\mathrm{x}}+\left(\overline{\mathrm{x}}^{2}+1\right) \arctan (\overline{\mathrm{x}})-\overline{\mathrm{x}}^{2}\right] \\
& \overline{\mathrm{I}}(\overline{\mathrm{x}})=\frac{8}{\pi^{2}} \int_{1}^{\infty} \mathrm{dt} \frac{\sqrt{\mathrm{t}^{2}-1}}{\mathrm{t}^{3}} \arctan (\mathrm{t} \overline{\mathrm{x}})
\end{aligned}
$$

For $\gamma=2$ and a Gaussian angular distribution, one finds

$$
\begin{aligned}
& \overline{\mathrm{I}}_{1}(\overline{\mathrm{x}})=1-\mathrm{e}^{-\overline{\mathrm{x}}} \\
& \overline{\mathrm{I}}(\overline{\mathrm{x}})=\frac{2}{\sqrt{\pi}} \int_{0}^{\infty} \mathrm{dt} \operatorname{erf}(\overline{\mathrm{x}} / 2 \mathrm{t}) \mathrm{e}^{-\mathrm{t}^{2}}=1-\frac{2 \overline{\mathrm{x}}}{\pi} \int_{1}^{\infty} \mathrm{dt} \arccos (1 / \mathrm{t}) \mathrm{e}^{-\mathrm{t} \overline{\mathrm{x}}}
\end{aligned}
$$

Finally, for $\gamma=1$ and a Gaussian angular distribution, one finds 


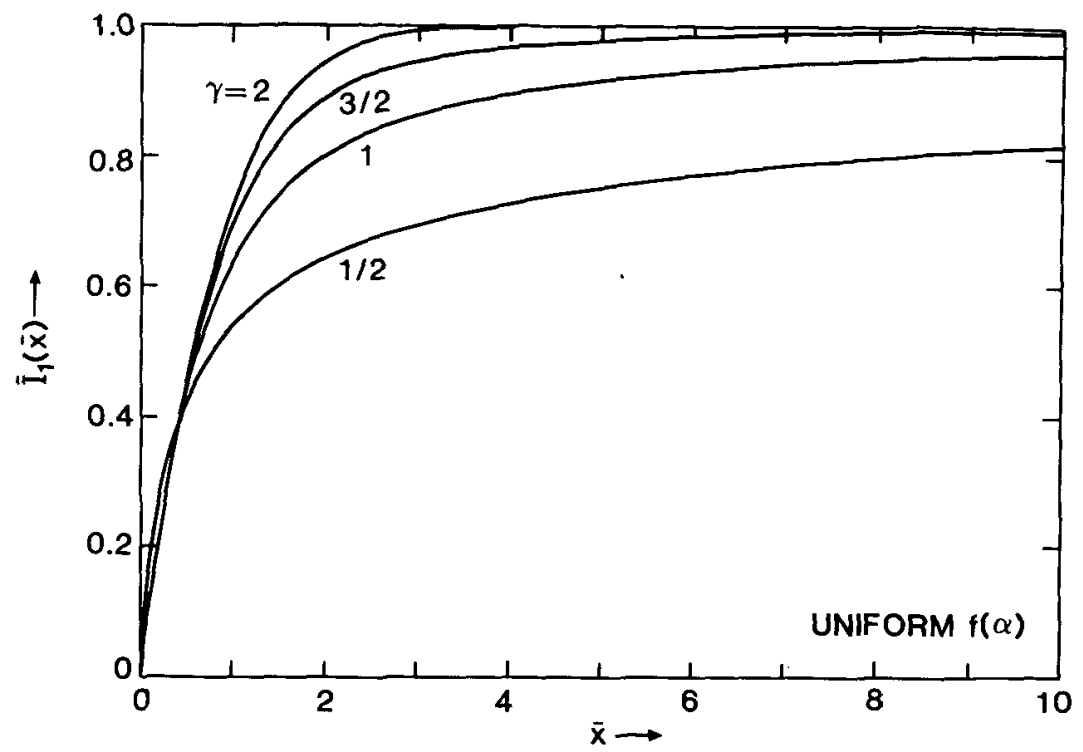

fig.12.14a

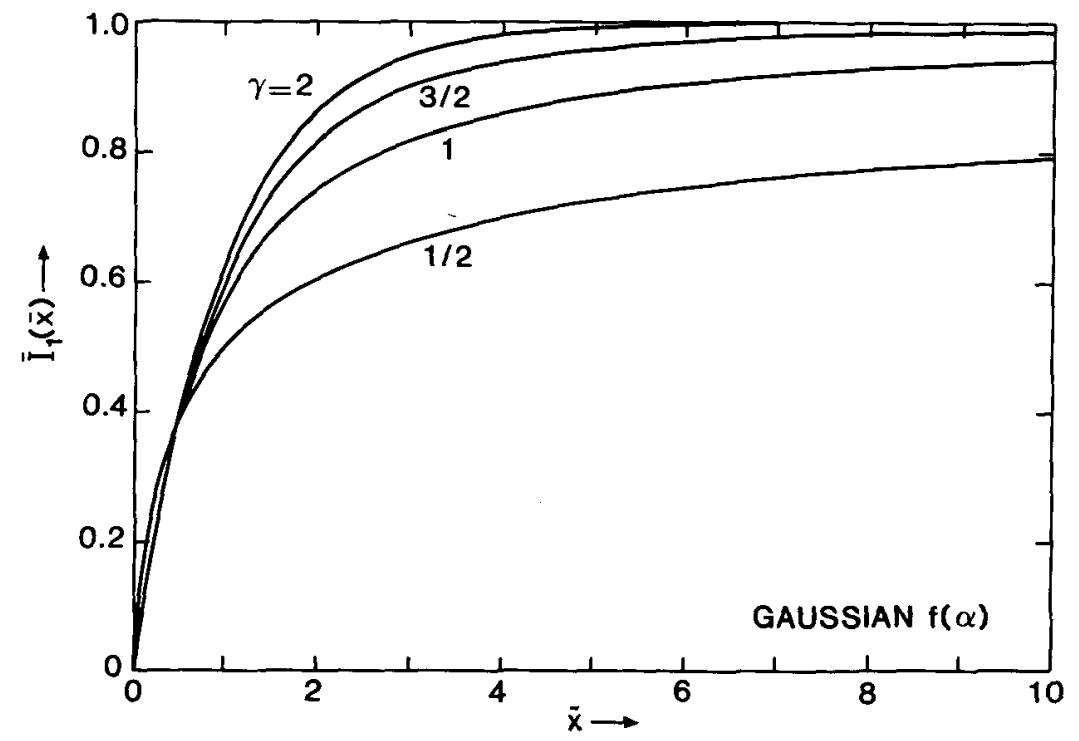

fig.12.14b

Figure 12.14 The function $\overline{\mathrm{I}}_{1}(\overline{\mathrm{x}})$ for a chromatically limited spot, for different types of energy distributions, represented by the parameter $\gamma$. The figures $12.14 \mathrm{a}$ and $12.14 \mathrm{~b}$ pertain to an uniform and a Gaussian angular distribution respectively. They are evaluated from equation (12.5.25), using the approximations for the function $\mathrm{B}_{1}(2)$ given by equations (12.5.29) and (12.5.22) for figure $12.14 \mathrm{a}$ and the first of equations (12.5.31) for figure 12.14b. 


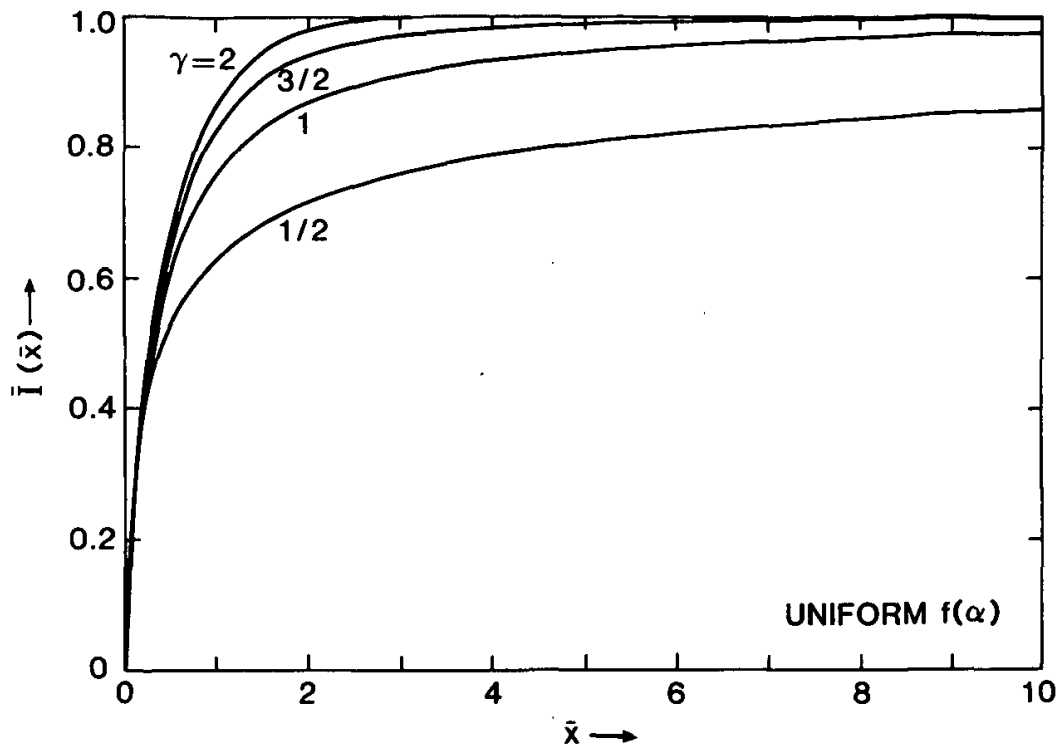

fig. $12.15 a$

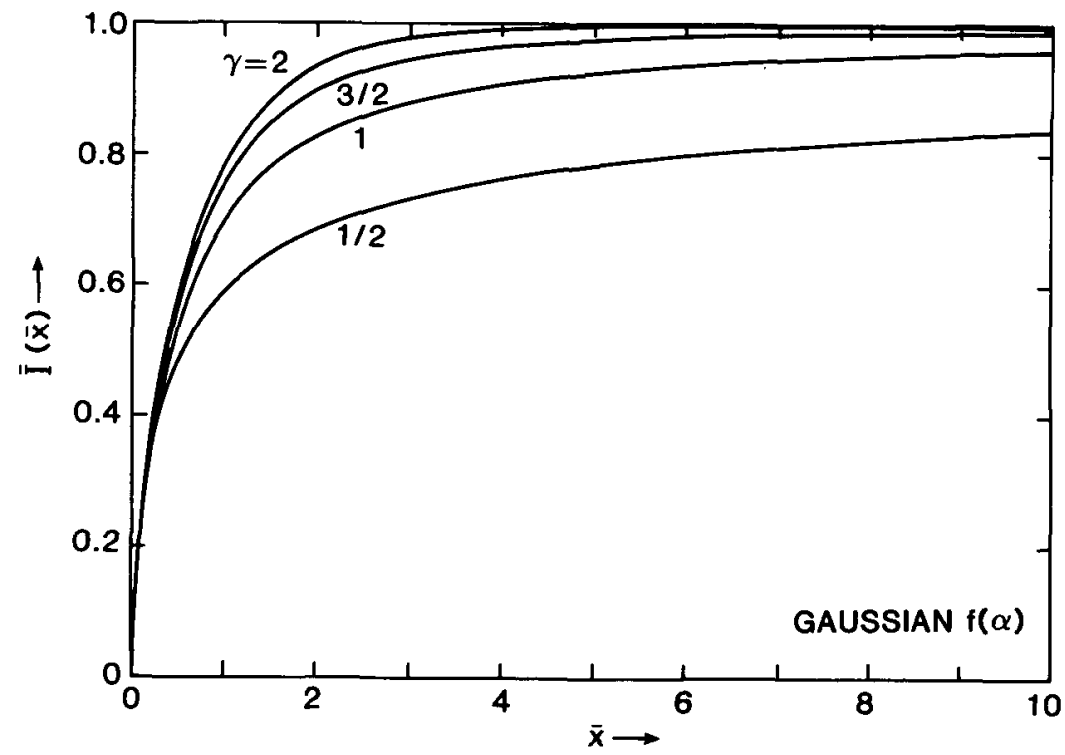

fig. 12.15b

Figure 12.15 The function $\overline{\mathrm{I}}(\overline{\mathrm{x}})$ for a chromatically 1 imited spot, for different types of energy distributions, represented by the parameter $\gamma$. The figures $12.15 \mathrm{a}$ and $12.15 \mathrm{~b}$ pertain to an uniform and a Gaussian angular distribution respectively. They are evaluated from equation (12.5.27), using the approximations for the function $\mathrm{B}(\mathrm{z})$ given by the second and the third of equations (12.5.31) respectively. 

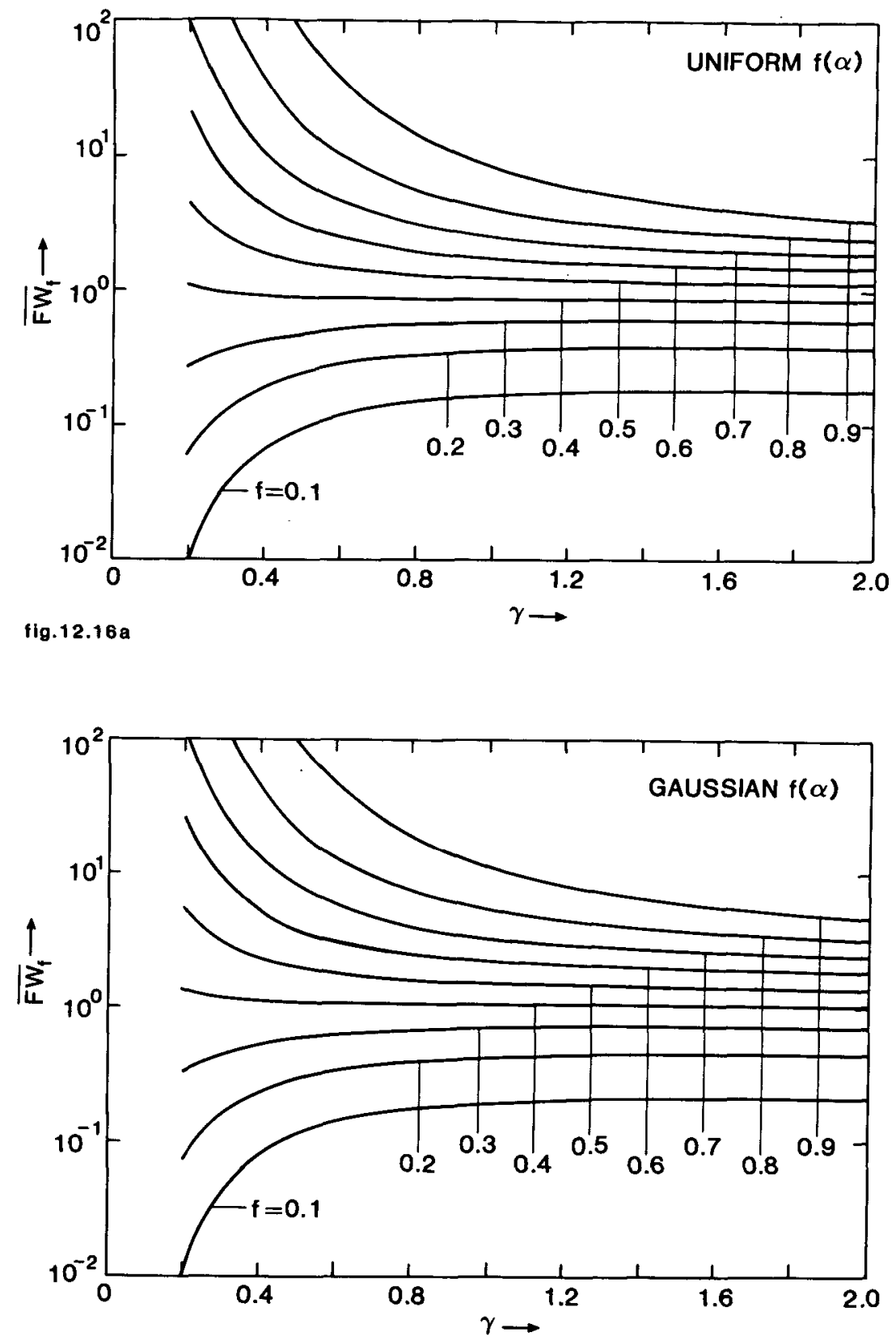

fig. 12.16b

Figure 12.16 The scaled width $\overline{F_{F}}$ for a chromatically limited spot as function of the type of energy distribution, represented by the parameter $\gamma$. The figures $12.16 \mathrm{a}$ and $12.16 \mathrm{~b}$ pertain to an uniform and a Gaussian angular distribution respectively. See equations (12.2.12) and (12.5.17) and the caption of figure 12.14 . 


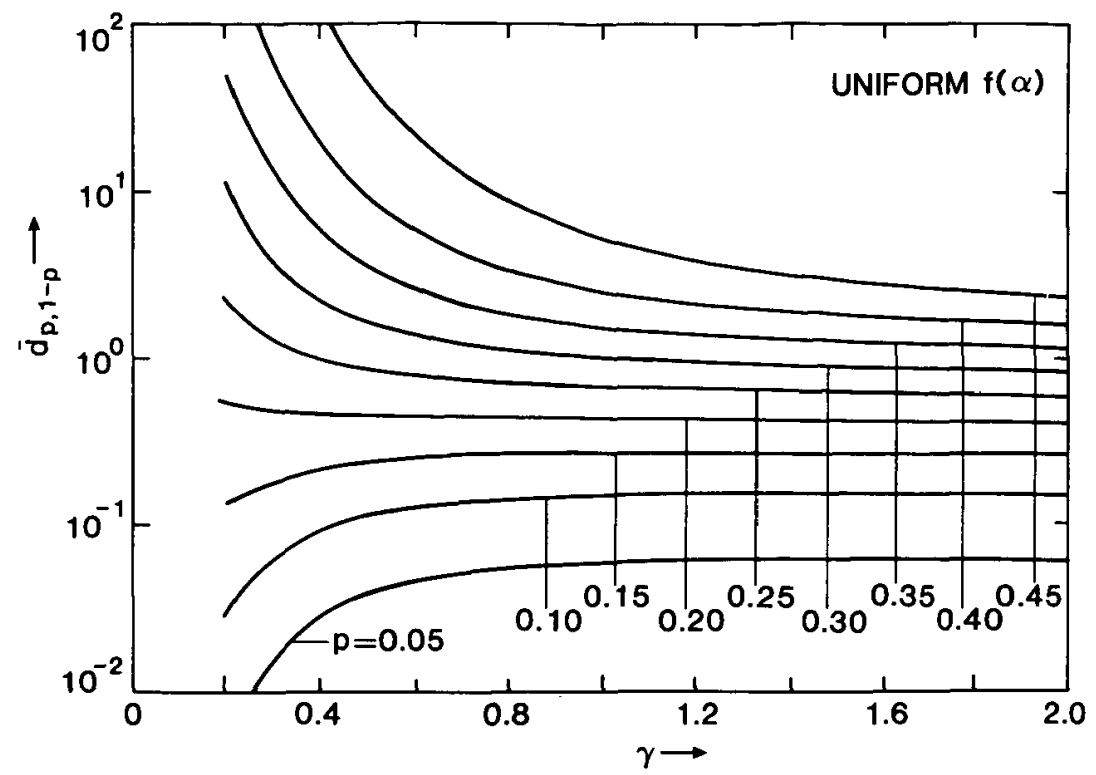

fig.12.17a

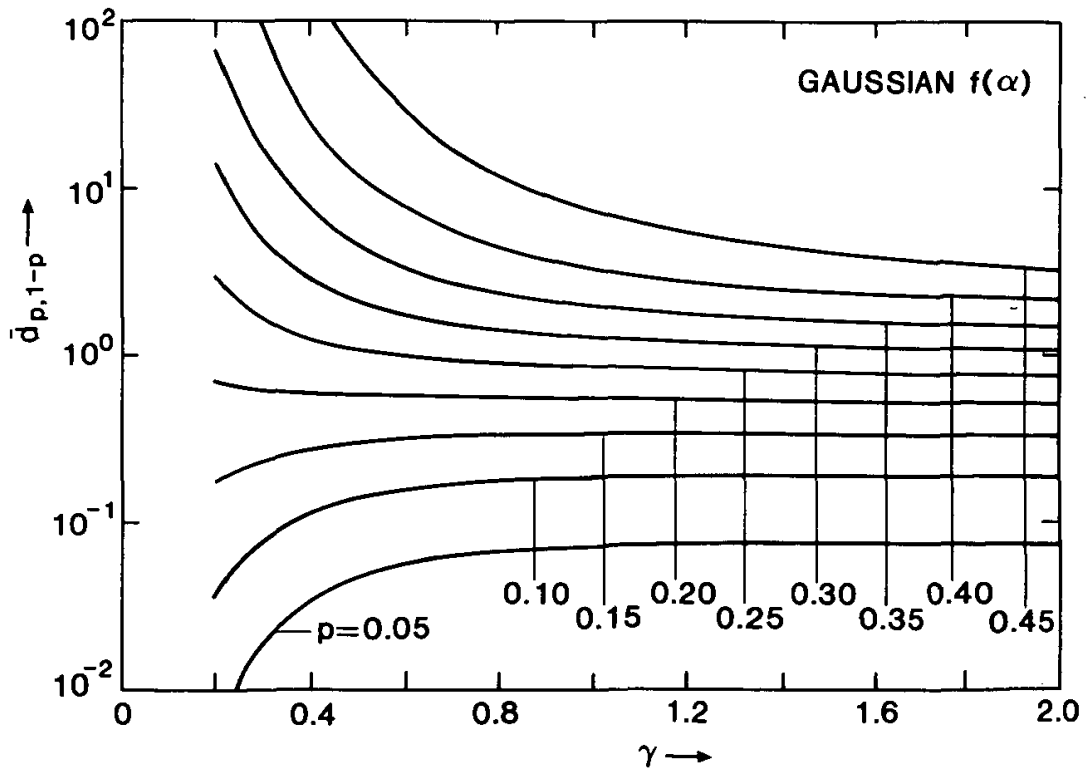

fig. $12.17 b$

Figure 12.17 The scaled width $\bar{d}_{\mathrm{p} .1-\mathrm{p}}$ for a spot which is 1imited by chromatic aberration as function of the type of energy distribution, represented by the parameter $\gamma$. The figures $12.17 \mathrm{a}$ and $12.17 \mathrm{~b}$ pertain to an uniform and a Gaussian angular distribution respectively. See equations (12.2.6) and (12.5.17) and the caption of figure 12.15 . 


$$
\begin{aligned}
& \bar{I}_{1}(\bar{x})=1+[\operatorname{erf}(\bar{x})-1] e^{\bar{x}^{2}} \\
& \bar{I}(\bar{x})=\frac{4}{\pi^{3 / 2}} \int_{0}^{\infty} d t \arctan (\bar{x} / t) e^{-t^{2}}
\end{aligned}
$$$$
\left(\gamma=1, \text { Gaussian } \mathbf{f}_{\alpha}(\alpha)\right)(12.5 .35)
$$

The results of equations (12.5.32) to (12.5.35) were exploited to check the accuracy of the numerical calculations based on the approximations given by equations $(12.5 .31)$. The accuracy of the results plotted in figures $12.14 \mathrm{~b}, 12.15 \mathrm{a}$ and $12.15 \mathrm{~b}$ was found to be better than $1 \%$.

The last step, is the calculation of the $d_{p}, 1-p$ width from equation $(12.2 .6)$ and the $\mathrm{FW}_{f}$ value from equation (12.2.12). The only case for which we found an explicit analytical expression, is the calculation of the $F_{W_{f}}$ value of the current density distribution corresponding to a Gaussian energy distribution $(\gamma=2)$ and a Gaussian angular distribution. From equation (12.2.12) and the first equation of $(12.5 .34)$, it follows for this particular case

$$
\overline{F W}_{\mathrm{f}}=2 \ln \left(\frac{1}{1-\mathrm{f}}\right)
$$

$\left(\gamma=2\right.$, Gaussian $\left.f_{\alpha}(\alpha)\right)(12.5 .36)$

We determined the $F W_{f}$ and $d_{p, 1-p}$ values for arbitrary $\gamma$-values, using a numerical approach. Equation (12.2.12) was solved for $\gamma$ values in the range $0.2<\gamma<2$, using the expression for $I_{1}(x)$ given by equations $(12.5 .25)$. For the function $B_{1}(z)$, we used the approximation given by equations (12.5.29) and (12.5.22). For the function $B_{1 \mathrm{~g}}(z)$, we exploited the approximation given by the first equation of $(12.5 .31)$. For the parameter $f$, we considered the values $0.1,0.2, \cdots, 0.9$. The results are plotted in the figures $12.16 \mathrm{a}$ and $12.16 \mathrm{~b}$, for an uniform and a Gaussian angular distribution respectively. Equation (12.2.6) was solved for the same range of $\gamma$-values, using the expression for $I(x)$ given by equation (12.5.27) and the approximations of equation (12.5.31). For the parameters $\mathrm{p}$, we considered the values $0.05,0.10, \cdots, 0.45$. The results are presented in the figures $12.17 \mathrm{a}$ and $12.17 \mathrm{~b}$, for an uniform and a Gaussian distribution respectively.

In order to relate the $d_{p, 1-p}$ and $F_{F}$ values, presented in this section, to the Full Width at Half Maximum of the energy distribution (FWHM $M_{\mathrm{B}}$ one has to remove the scaling, defined by equation (12.5.17). In addition, one should express the quantity $A_{\gamma}$ in terms of FWHM $_{E}$. According to equation (5.9.15), one may write

$$
\text { FWHM }=A_{\gamma}{ }^{1 / \gamma} F W H M_{\gamma}
$$

The quantity FWHM is plotted in figure 5.5a (curve corresponding to the one-dimensional distribution). Table 5.1 summarizes the numbers for some specific cases. From equations (12.5.17) and (12.5.37), one finds

$$
\begin{aligned}
\mathrm{FW}_{\mathrm{f}} & =\frac{\overline{F W}_{\mathrm{f}}}{F W H M_{\gamma}} \mathrm{C}_{\mathrm{c}} \alpha_{0} \frac{F W H M_{\mathrm{E}}}{\mathrm{E}} \\
\mathrm{d}_{\mathrm{p}, 1-\mathrm{p}} & =\frac{\overline{\mathrm{d}}_{\mathrm{p}, 1-\mathrm{p}}}{F W H M_{\gamma}} \mathrm{C}_{\mathrm{c}} \alpha_{0} \frac{F W H M_{\mathrm{E}}}{\mathrm{E}}
\end{aligned}
$$

The numerical factors $\overline{F W}_{\mathrm{f}} / F W H M_{\gamma}$ and $\overline{\mathrm{d}}_{\mathrm{p}} 1-\mathrm{p} / F W H M_{\gamma}$ can be determined from the figures 12.16, 12.17 and 5.5a (or table 5.1). For example, in case of a Gaussian energy distribution $(\gamma=2)$ and $p=0.1$ and $f=0.5$, one 
finds for an uniform and a Gaussian angular distribution respectively

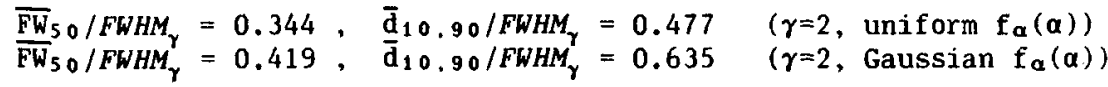

Finally, we note that one can not define a FWHM of the current density distribution corresponding to a chromatically limited spot, since the quantity $j(r) / 2 \pi r$ diverges for $r \rightarrow 0$. This can, for instance, be seen from equations $(12.5 .23)$ and $(12.5 .24)$. We recall, that $j(r)$ represents $a$ point spread function. For a practical spot, the ideal image is not a point and the resulting distribution, which is a convolution of the ideal image and the point spread function, will be finite for $r \rightarrow 0$.

\subsection{Spherical aberration}

In this section we will study the properties of a probe which is limited by spherical aberration. Consider again the single thin-lens system of figure 12.10. Assume that all particles have the same energy, which implies that chromatic aberration is absent. For an ideal lens, a particle leaving the object plane from the axis, with a certain angle $a_{1}$, will cross the axis in the image-plane, irrespective of the value of $\alpha_{1}$. However, in presence of spherical aberration, the outer rays (with a large angle $\alpha_{1}$ ) are focussed more strongly than the rays near the axis. Therefore, the outer rays will cross the axis at a location closer to the lens. In general, one finds that the radial position $r$ of a particle in the vicinity of the image plane, which left the object from the axis, depends on its angle with the axis $\alpha$ and the distance to the image plane $\Delta z$, as

$$
r=\left|-\Delta z \alpha-C_{s}\left(1+\frac{\Delta z}{q}\right) \alpha^{3}\right|
$$

where $C_{s}$ is the constant of spherical aberration of the lens. The radial position in the (Gaussian) image plane follows by taking $\Delta z=0$

$$
r=C_{s} \alpha^{3}
$$

From this equation, one sees that the distribution of $r$ in the Gaussian image plane, denoted as $j(r)$, depends on the angular distribution $f_{\alpha}(\alpha)$ near the image plane. Equation (12.6.1) shows that the distribution $j(r)$ also depends on the axial position, represented by $\Delta z$. However, we will restrict the analysis to the case $\Delta z=0$, that is to the calculation of $j(r)$ in the Gaussian image plane. It is well known that, when the probe is dominated by spherical aberration, the plane of best focus is located at a distance $\Delta z=-0.75 \mathrm{C}_{s} \alpha_{0}{ }^{3}$ ahead of the Gaussian image plane, where $\alpha_{0}$ represents the beam semi-angle subtended at the image. The radius of the spot in this plane is equal to $0.25 \mathrm{C}_{s} \alpha_{0}{ }^{3}$, which is four times smaller than the radius in the Gaussian image plane. It should be noted that the shape of the current density distribution $j(r)$ is entirely different for both planes. When spherical aberration is not dominant, it is more appropriate to evaluate the contribution to the probe size in the Gaussian image plane. We will concentrate on this case and start from equation (12.6.2).

Similar to equation (12.5.11), one can express the distribution $j(r)$ associated with spherical aberration as 


$$
j(r)=\int d \alpha f_{\alpha}(\alpha) \delta\left(r-\mathcal{C}_{s} \alpha^{3}\right)
$$

The angular distribution $f_{\alpha}(\alpha)$ is again given by equation (12.5.12), in combination with either equation (12.5.13), for an uniform distribution or equation (12.5.14), for a Gaussian distribution. We will investigate these two cases.

For convenience of notation, we introduce the following scaling

$$
\bar{r}=\frac{r}{C_{s} \alpha_{0}{ }^{3}} \quad, \bar{j}(\bar{r})=C_{s} \alpha_{0}{ }^{3} j(r)
$$

For an uniform angular distribution, one obtains from equations $(12.6 .3),(12.5 .12)$ and $(12.5 .13)$

$$
\left.\bar{j}(\bar{r})=\int_{0}^{1} d t 2 t \delta\left(\bar{r}-t^{3}\right)=\frac{2}{3 r^{1 / 3}} \quad \text { (uniform } f_{\alpha}(\alpha)\right) \quad \text { (12.6.5) }
$$

For a Gaussian angular distribution, one finds from equations (12.6.3), $(12.5 .12)$ and $(12.5 .14)$

$$
\bar{j}(\bar{r})=\int_{0}^{\infty} \mathrm{dt} 2 t \mathrm{e}^{-\mathrm{t}^{2}} \delta\left(\bar{r}-\mathrm{t}^{3}\right)=\frac{2}{3 \overline{\mathrm{r}}^{1 / 3}} \mathrm{e}^{-\overrightarrow{\mathrm{r}}^{2 / 3}}\left(\text { Gaussian } \mathrm{f}_{\alpha}(\alpha)\right)
$$

similar to equation $(12.6 .5)$.

The next step is to evaluate the functions $I_{1}(x)$ and $I(x)$ from equations $(12.2 .9),(12.2 .10)$ and $(12.2 .11)$. We will scale the quantity $x$ in the same way as $r$ and the quantities $I$ and $I_{1}$ in the same way as $j$, see equation (12.6.4). With equation (12.6.5), one obtains for an uniform angular distribution (with $0<\bar{x}<1$ )

$$
\begin{aligned}
\bar{I}_{1}(\bar{x}) & =\bar{x}^{2 / 3} \\
\bar{I}(\bar{x}) & =\frac{2}{\pi} \arcsin (\bar{x})+\frac{6}{\pi} \bar{x}^{2 / 3} \int_{\bar{x}^{1 / 3}}^{1} d t \frac{1}{\left(1-t^{6}\right)^{1 / 2}} \\
& \left.=1-\frac{6}{\pi} \int_{\bar{x}^{1 / 3}}^{1} d t \frac{t^{2}-\bar{x}^{2 / 3}}{\left(1-t^{6}\right)^{1 / 2}} \quad \text { (uniform } f_{\alpha}(\alpha)\right)
\end{aligned}
$$

Similarly, one obtains with equation (12.6.6), for a Gaussian angular distribution

$$
\begin{array}{rlrl}
\overline{\mathrm{I}}_{1}(\overline{\mathrm{x}}) & =1-\mathrm{e}^{-\overline{\mathrm{x}}^{2 / 3}} & \text { (Gaussian } \left.\mathrm{f}_{\alpha}(\alpha)\right) & \text { (12.6.9) } \\
\overline{\mathrm{I}}(\overline{\mathrm{x}}) & =1-\frac{3}{\pi} \int_{1}^{\infty} \mathrm{du} \frac{\mathrm{e}^{-\mathrm{u} \overline{\mathrm{x}}^{2 / 3}}}{\mathrm{u}\left(\mathrm{u}^{3}-1\right)^{1 / 2}} & \left.\quad \text { (Gaussian } \mathrm{f}_{\alpha}(\alpha)\right) & (12.6 .10) \\
& =1-\frac{6}{\pi} \int_{0}^{1} \mathrm{dt} \frac{\mathrm{t}^{2} \mathrm{e}^{-\overline{\mathrm{x}}^{2} / 3 / \mathrm{t}^{2}}}{\left(1-\mathrm{t}^{6}\right)^{1 / 2}} \quad &
\end{array}
$$

The integrals of equations (12.6.8) and (12.6.10) were evaluated numerically. In order to improve the numerical accuracy, we 
replaced the t-integration by an integration over $z$, using

$$
z=\left(1 / t^{2}-1\right)^{1 / 2} \Rightarrow t^{2}=1 /\left(1+z^{2}\right), d t /\left(1-t^{6}\right)^{1 / 2}=d z /\left(3+3 z^{2}+z^{4}\right)^{1 / 2}
$$

The numerical results obtained for the functions $\bar{I}(\bar{x})$, given by equations $(12.6 .8)$ and $(12.6 .10)$, are plotted in figure 12.18.

The last step is the calculation of the $d_{p, 1-p}$ width from equation (12.2.6) and the $F_{f}$ value from equation (12.2.12). For the $\mathrm{FW}_{\mathrm{f}}$ value, one directly finds from equations (12.6.7) and (12.6.9)

$$
\begin{array}{ll}
\overline{F W}_{f}=2 f^{3 / 2} & \text { (uniform } f_{\alpha}(\alpha) \text { ) } \\
\overline{F W}_{f}=2\left[\ln \left(\frac{1}{1-f}\right)\right]^{3 / 2} & \text { (Gaussian } f_{\alpha}(\alpha) \text { ) }
\end{array}
$$

We determined the $d_{p, 1-p}$ values from equations $(12.2 .6),(12.6 .8)$ and $(12.6 .10)$ by numerical means. The results are plotted in figure 12.19 .

\subsection{Space charge effect}

In chapter 11 it was demonstrated that the space charge effect, occurring in the drift space of a column, can be described in terms of the properties of a negative lens. The first order properties of the space charge lens cause a defocussing of the image, as well as some (de)magnification. These effects can, in principle, be compensated by proper adjustment of the lenses in the column. It was also found that the space charge lens will be non-ideal when the lateral current density distribution in the beam is non-uniform. For those conditions the space charge lens shows non-vanishing third-order properties, which cause a non-refocusable blurring of the image. In general, the dominant contribution to the blurring stems from spherical aberration.

The impact of spherical aberration on the $\mathrm{FW}_{\mathrm{f}}$ and $\mathrm{d}_{p, 1-p}$ width measures is covered by the analysis of the previous section. In this section, we will study the impact of the first order properties of the space charge lens, assuming that the corresponding defocussing of the probe is not compensated. This situation is usually encountered in variable shaped spot lithography columns, in which it is difficult to adjust the projector lens on a spot-by-spot basis.

The current density distribution $j(r)$, associated to a defocussing of the spot by a distance $\Delta z_{r}$, is equal to

$$
j(r)=\frac{1}{\Delta z_{f}} f_{\alpha}\left(r / \Delta z_{f}\right)
$$

which is also expressed by equation (11.5.16), however using a somewhat different notation. The function $f_{\alpha}$ specifies, as before, the angular distribution near the target. For convenience of notation, we introduce the following scaling

$$
\bar{r}=\frac{r}{\Delta z_{f} \alpha_{0}} \quad, \quad \bar{j}(\bar{r})=\Delta z_{f} \alpha_{0} j(r)
$$

For an uniform angular distribution, one obtains from equations $(12.7 .1),(12.5 .12)$ and $(12.5 .13)$

$$
\vec{j}(\bar{r})=2 \bar{r} \theta(1-\bar{r})
$$




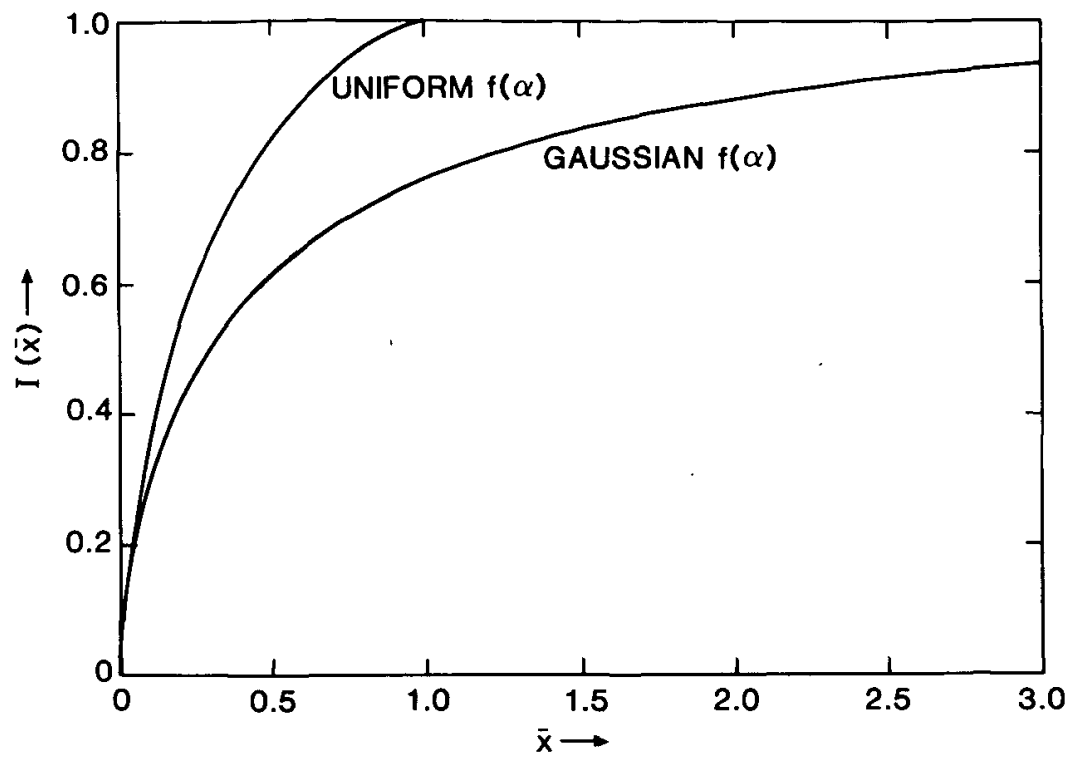

Figure 12.18 The function $\mathrm{I}(\overline{\mathrm{x}})$ for a spot which is 1imited by spherical aberration. See equations (12.6.8) and (12.6.10) for the case of an uniform and a Gaussian angular distribution respectively.

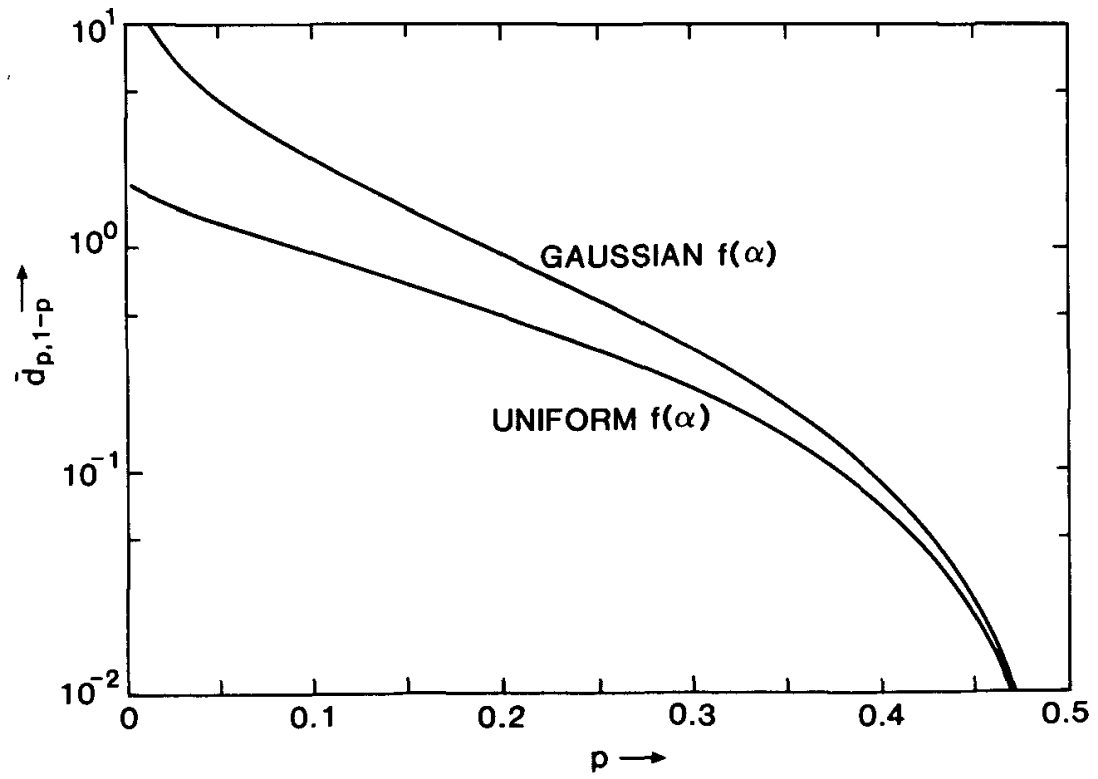

Pigure 12.19 The scaled width $\bar{d}_{p, 1-p}$ of a spot which is 1imited by spherical aberration. It is evaluated from equation (12.2.6), using equations (12.6.8) and (12.6.10) for the case of an uniform and a Gaussian angular distribution respectively and the scaling of equation (12.6.4). 
Similarly, one obtains with equation (12.5.14), for a Gaussian angular distribution

$$
\bar{j}(\bar{r})=2 \bar{r} e^{-\bar{r}^{2}}
$$

As before, the functions $I_{1}(x)$ and $I(x)$ follow with equations (12.2.9), $(12.2 .10)$ and $(12.2 .11)$. We will scale the quantity $x$ in the same way as $r$ and the quantities $I$ and $I_{1}$ in the same way as $j$, see equation $(12.7 .2)$. From equation (12.7.3), one obtains for an uniform angular distribution

$$
\begin{array}{ll}
\overline{\mathrm{I}}_{1}(\overline{\mathrm{x}})=\overline{\mathrm{x}}^{2} & \text { (uniform } \left.\mathrm{f}_{\alpha}(\alpha)\right) \\
\overline{\mathrm{I}}(\overline{\mathrm{x}})=\frac{2}{\pi}\left(\arcsin (\overline{\mathrm{x}})+\overline{\mathrm{x}} \sqrt{1-\mathrm{x}^{2}}\right) & \text { (uniform } \left.\mathrm{f}_{\alpha}(\alpha)\right)
\end{array}
$$

where $0<\bar{x}<1$. From equation (12.7.4), one obtains for a Gaussian angular distribution

$$
\begin{array}{ll}
\tilde{\mathrm{I}}_{1}(\overline{\mathrm{x}})=1-\mathrm{e}^{-\overline{\mathrm{x}}^{2}} & \text { (Gaussian } \left.\mathrm{f}_{\alpha}(\alpha)\right) \\
\overline{\mathrm{I}}(\overline{\mathrm{x}})=\operatorname{erf}(\overline{\mathrm{x}}) & \text { (Gaussian } \left.\mathrm{f}_{\alpha}(\alpha)\right)
\end{array}
$$

The final step is the calculation of the corresponding $d_{p, 1-p}$ and $F_{f}$ values. From equations $(12.2 .12),(12.7 .5)$ and $(12.7 .7)$, one obtains for the $\mathrm{FW}_{\mathrm{f}}$ value, in case of an uniform and a Gaussian angular distribution respectively

$$
\begin{array}{ll}
\overline{F W}_{\mathrm{f}}=2 \mathrm{f}^{1 / 2} & \text { (uniform } \mathrm{f}_{\alpha}(\alpha) \text { ) } \\
\overline{F W}_{\mathrm{f}}=2\left[\ln \left(\frac{1}{1-\mathrm{f}}\right)\right]^{1 / 2} & \text { (Gaussian } f_{\alpha}(\alpha) \text { ) }
\end{array}
$$

From equations $(12.2 .6)$ and $(12.7 .8)$, one obtains for a Gaussian a angular distribution

$$
\bar{d}_{p, 1-p}=2 \operatorname{erf}-1(1-2 p) \quad \text { (Gaussian } f_{\alpha}(\alpha) \text { ) }
$$

The inverse of the function $\overline{\mathrm{I}}(\overline{\mathrm{x}})$, specified by equation $(12.7 .6)$, can not be made explicit. In order to determine the $d_{p, 1-p}$ value for an uniform angular distribution, we solved equation (12.2.6), combined with equation (12.7.6), numerically. The results are plotted in figure 12.20, which also shows the relation given by equation (12.7.11), applying to the case of a Gaussian angular distribution.

\subsection{Results for a truncated Gaussian angular distribution}

So far we restricted our calculations to an uniform and a Gaussian angular distribution. In this section, we will outline an method to approximate the intermediate case of a truncated Gaussian angular distribution. This type of distribution is defined by equations $(12.5 .12)$ and $(12.5 .15)$.

Suppose that some arbitrary width measure w of the spot is, for an uniform angular distribution, given by

$$
w=C \alpha_{m}^{q}
$$

where $\alpha_{m}$ denotes the maximum (cut-off) beam semi-angle and $c$ some 


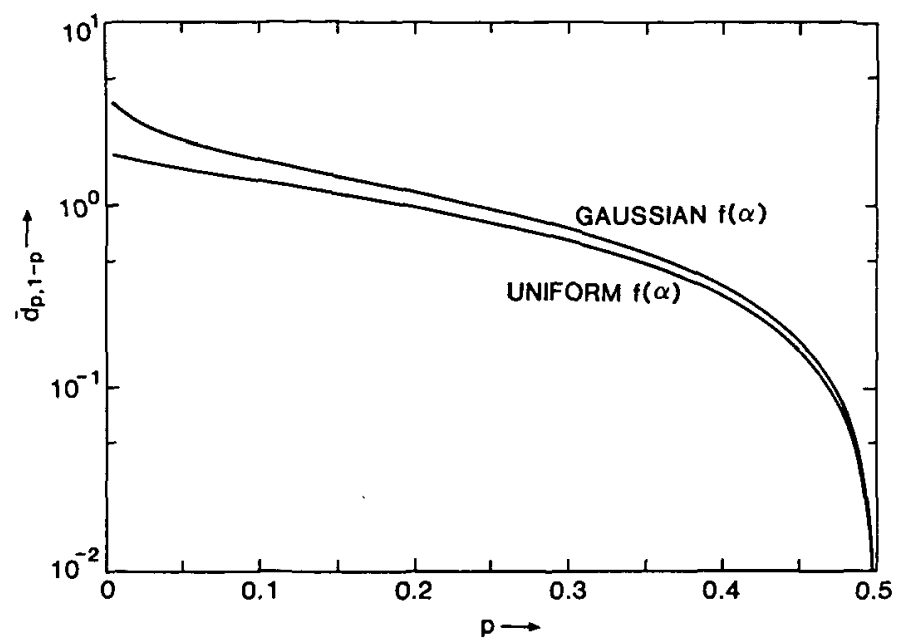

Figure 12.20 The scaled width $\bar{d}_{0.1-p}$ for a spot which is limited by (uncompensated) space charge defocussing. The curve for an uniform angular distribution is evaluated from equations (12.2.6) and (12.7.6), using the scaling of equation (12.7.2). The curve for a Gaussian angular distribution is given by equation $(12.7 .11)$.

constant. The parameter $q$ is characteristic for the aberration considered. For example, chromatic aberration corresponds to $q=1$ and spherical aberration to $q=3$, as can be seen from equations (12.5.10) and $(12,6.2)$ respectively.

We now define the effective angle $\alpha_{e f f}$ of an arbitrary angular distribution $f_{\alpha}(\alpha)$, to be used in equation (12.8.1), as

$$
\alpha_{\text {eff }}=\left[\frac{q+2}{2} \int_{0}^{\alpha_{m}} d \alpha f_{\alpha}(\alpha) \alpha q\right]^{1 / q}
$$

The factor in front of the integral was chosen such that $\alpha_{\text {eff }}=\alpha_{m}$ when the angular distribution $f_{\alpha}(\alpha)$ is uniform. For a truncated Gaussian angular distribution, one obtains by substitution of equations (12.5.12) and $(12.5 .15)$ into equation $(12.8 .2)$

$$
\frac{\alpha_{e f f}}{\alpha_{0}}=\left[\frac{q+2}{1-\exp \left(-\alpha_{m}^{2} / \alpha_{0}^{2}\right)} \int_{0}^{\alpha_{m} / \alpha_{0}} d t t q+1 e^{-t^{2}}\right]^{1 / q}
$$

which specifies $\alpha_{e f f} / \alpha_{0}$ as function of $\alpha_{m} / \alpha_{0}$. We evaluated equation (12.8.3) numerically, for different values of $q$. The results are plotted in figure 12.21. For $\alpha_{m} / \alpha_{0} \ll 1$ the angular distribution is nearly uniform and one finds $\alpha_{\text {ef } f} / \alpha_{0} \approx \alpha_{m} / \alpha_{0}$, thus $\alpha_{e f f} \approx \alpha_{m}$. For $\alpha_{m} / \alpha_{0} \gg 1$ the angular distribution is nearly Gaussian and the ratio $\alpha_{e f} / \alpha_{0}$ saturates to a value which is characteristic for this type of distribution.

We note that the integral in equation $(12.8 .3)$ can be expressed in elementary functions (and the error function) when $q$ is a positive integer $(q=1,2,3, \cdots)$. For instance, for $q=1,2$ and 3 one finds respectively 


$$
\begin{aligned}
& \frac{\alpha_{\text {eff }}}{\alpha_{0}}=\frac{3 \sqrt{\pi}}{4} \frac{\operatorname{erf}\left(\alpha_{m} / \alpha_{0}\right)-(2 / \sqrt{\pi})\left(\alpha_{m} / \alpha_{0}\right) \exp \left(-\alpha_{m}^{2} / \alpha_{0}^{2}\right)}{1-\exp \left(-\alpha_{m}^{2} / \alpha_{0}^{2}\right)} \\
& \frac{\alpha_{\text {eff }}}{\alpha_{0}}=\sqrt{2}\left[\frac{1-\left[1+\left(\alpha_{m} / \alpha_{0}\right)^{2}\right] \exp \left(-\alpha_{m}^{2} / \alpha_{0}^{2}\right)}{1-\exp \left(-\alpha_{m}^{2} / \alpha_{0}^{2}\right)}\right]^{1 / 2} \quad(q=1) \\
& \frac{\alpha_{\text {eff }}}{\alpha_{0}}=\frac{(15 \sqrt{\pi})^{1 / 3}}{2}\left[\frac{\operatorname{erf}\left(\alpha_{m} / \alpha_{0}\right)-(4 / 3 \sqrt{\pi})\left[3 \alpha_{m} / 2 \alpha_{0}+\left(\alpha_{m} / \alpha_{0}\right)^{3}\right] \exp \left(-\alpha_{m}^{2} / \alpha_{0}^{2}\right)}{1-\exp \left(-\alpha_{m}^{2} / \alpha_{0}^{2}\right)}\right]^{1 / 3}
\end{aligned}
$$

as follows straightforwardly by integration.

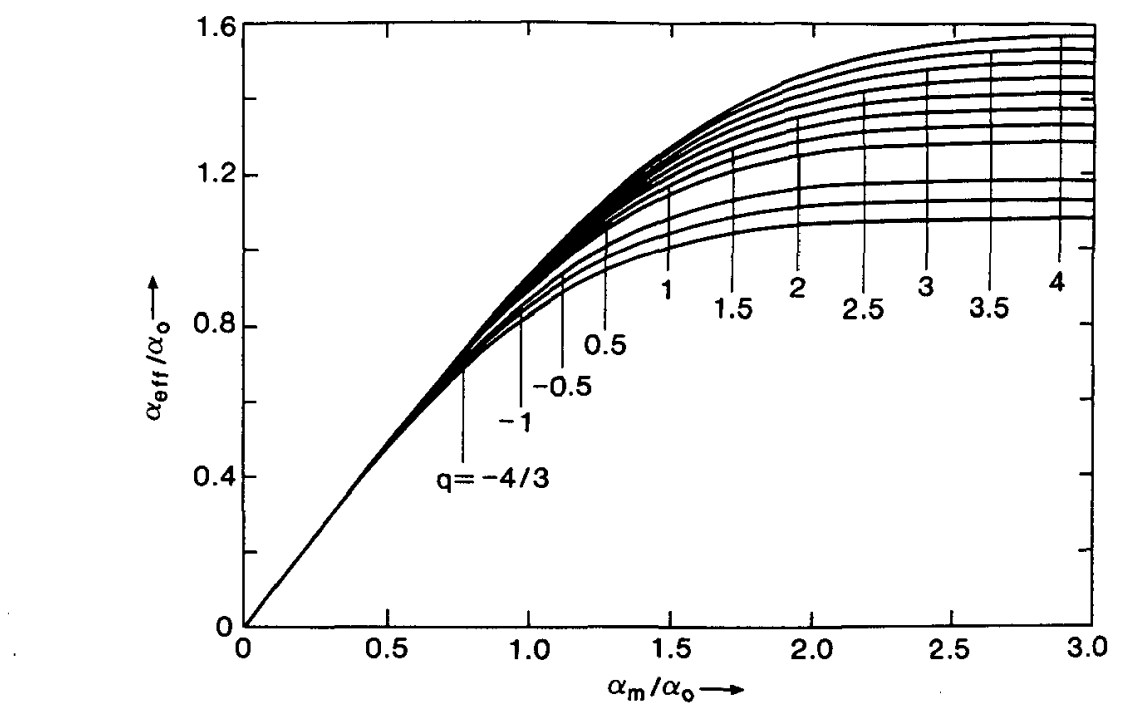

Figure 12.21 The effective angle $\alpha_{\mathrm{e}}$ (1) of a truncated Gaussian distribution as defined by equation (12.8.3).

It should be emphasized that the calculation of $\alpha_{e f f}$ by means of equations (12.8.3) provides only a rough method to calculate the $\mathrm{FW}_{\mathrm{f}}$ and $d_{p, 1-p}$ values for a truncated Gaussian angular distribution. The extreme cases $\boldsymbol{\alpha}_{m} / \boldsymbol{\alpha}_{0} \rightarrow 0$ (uniform distribution) and $\boldsymbol{\alpha}_{m} / \boldsymbol{\alpha}_{0}+\infty$ (Gaussian distribution) were explicitly considered in the previous sections and can therefore be used to check the accuracy of equation (12.8.3). As an example, let us consider the calculation of the $F W_{50}\left(F_{f}\right.$ for $\left.f=0.5\right)$ and $d_{10,90}\left(d_{p, 1-p}\right.$ for $\left.p=0.1\right)$ values for a chromatically limited spot. From the first equation of $(12.8 .4)$, one finds for $\alpha_{m} / \alpha_{0}+\infty$ that $\alpha_{\text {ef } f} / \alpha_{0}=3 \sqrt{1} / 4=1.33$, which can also be observed from figure 12.21 (curve for $q=1)$. As the width measures $F_{f}$ and $d_{p, 1-p}$ increase linearly with $\alpha$ (since $q=1$ ), this analysis predicts that the results obtained for a Gaussian angular distribution are approximately a factor 1.33 larger than the results obtained for an uniform angular distribution (for the same value $\left.\alpha_{0}\right)$. The results of the exact analysis, carried out in section 12.5, are plotted in the figures $12.16 \mathrm{a}, 12.16 \mathrm{~b}, 12.17 \mathrm{a}$ and $12.17 \mathrm{~b}$. Let us consider the cases $\gamma=2$ (Gaussian energy distribution) and $\gamma=1$ (Lorentzian energy distribution). For $\gamma=2$, one finds the following 
values for the ratio of the results obtained for a Gaussian and an uniform angular distribution

$$
\frac{F_{50: 8}}{F W_{50 ; u}}=\frac{1.39}{1.15}=1.21 \quad, \quad \frac{d_{10.90 ; 8}}{d_{10.90 ; u}}=\frac{2.10}{1.61}=1.30 \quad(\gamma=2) \quad(12.8 .5)
$$

Similarly, one obtains for $\gamma=1$

$$
\frac{\text { FW50:6 }}{\text { FW }_{50 ; u}}=\frac{1.55}{1.24}=1.25 \quad, \quad \frac{d_{10.90: 8}}{d_{10.90 ; u}}=\frac{3.35}{2.52}=1.33 \quad(\gamma=1) \quad(12.8 .6)
$$

Equations (12.8.5) and (12.8.6) shows that the exact ratio $\alpha_{e f f} / \alpha_{0}$ depends on the width measure considered, as well as the type of energy distribution. This particular example shows that the approximating value $\alpha_{\text {eff }} / \alpha_{0}=1.33$ is within $10 \%$ of the exact values. One may expect that the results for a truncated Gaussian angular distribution $\left(\alpha_{m} / \alpha_{0}\right.$ finite) are at least as accurate as the results presented above, for a Gaussian angular distribution $\left(\alpha_{m} / \alpha_{0}+\infty\right)$. 


\section{MONTE CARLO SIMULATION OF PARTICle beamS}

\subsection{Introduction}

This chapter discusses the basics of numerical Monte Carlo (MC) simulation of charged particle beams. This technique provides a direct method to determine the impact of Coulomb interactions on the properties of the beam. The essential part of a MC simulation program is a raytracing routine, which computes the particle trajectories by numerical integration of the equations of motion, taking the mutual coulomb repulsion into account. The solution of the $\mathrm{N}$-body problem, obtained this way, does not rely on any fundamental physical approximations. Therefore, one application of the MC simulation method, is to verify the validity of the assumptions underlying the analytical models. A comparison of the results of $M C$ simulations with the analytical theory presented in the previous chapters, is reported in chapter 14.

In general, a MC simulation program contains the following elements: A source routine to simulate a random initial condition of an ensemble (sample/bunch) of particles; routines to simulate lenses and other optical components; ray-tracing routines; routines to translate the final coordinates of the particles in terms of some characteristic quantities, such as the width of the energy distribution, the defocussing distance and the width of the spatial distribution in the plane of best focus. We will successively describe each of these elements. Various concepts presented here are based on the ideas of Groves et al. (1979) and Groves $(1981 a, b)$. The actual realization of our program was considerably facilitated by the fact that Dr. T.R. Groves has made a basic version of his program available.

The different methods to perform the ray-tracing will be considered in some detail. We will discuss a numerical third order integration procedure, which uses a variable time step. A similar procedure is proposed by Meisburger (1983) and Munro (1987). As a spinof $f$ of the analysis of the two-particle dynamical problem, which was presented in chapter 6 , we were able to produce a semi-analytical raytracing method. This method constitutes an alternative to the conventional numerical ray-tracing routine. The analytical ray-tracing method was found to be extremely accurate for the operating conditions encountered in practical columns, while it increases the speed of the program with as much as two orders' of magnitude. This technique is, therefore, referred to as Fast Monte Carlo simulation (FMC). Part of the material presented here, was reported previously by Jansen (1987) and van der Mast and Jansen (1987).

The accuracy of the MC simulation method is limited by raytracing errors, statistical errors and errors related to the finite-size of the sample. Several provisions have been introduced in the program to estimate the impact of these errors on the accuracy the final results. A correction of the final velocities is described, which reduces the error in the generated energy spread due to finite-size effects. Under normal conditions, statistical errors and finite-size errors are dominant, leading to an error in the final results which is typically $10 \%$ for the median width of the energy and trajectory displacement distribution and somewhat larger for the Full width at Half Maximum (FWHM) of these distributions and the edge-width of shaped spots.

The main part of the chapter is dedicated to the fundamental subjects involved. The actual organization of our program is briefly outlined at the end of this chapter, where we also give some simulation examples. The column, which is to be simulated, can be specified by 
editting the so-called system-file. A command structure has been developed for a flexible definition of the various elements, constituting the column. The first command should define a single- or multi-source. It can be succeeded by any combination of commands, specifying drift spaces, thin lenses, deflectors, quadrupoles and round or rectangular apertures. Other commands are available to instruct the program how to analyse the final coordinates. The so-called MC data-file contains the variable beam-properties (such as the beam current, the beam voltage and the initial energy spread) as well as a number of general program parameters. Separate output files are used for the storage of the final coordinates, the positions in the target plane and the final energy and spatial distribution. A separate graphics program is available to plot the data on screen or paper.

\subsection{Source routine}

The source routine generates the initial coordinates of a sample of particles, representing the beam in the vicinity of the emission surface. The number of particles in the sample $\mathbf{N}_{s}$ am is typically chosen between 100 and 1000. A pseudo-random number generator is used to assign a position and a velocity to every particle, in such a way that their distribution in phase space resembles the macroscopic properties of the beam.

The set of quantities, used to specify the macroscopic condition of the beam, depends on the type of source which is to be simulated. In our program, we have implemented two different types of single sources. The essential difference between the two algorithms concerns the formation of the velocity distribution. The formation of the spatial distribution is the same. Type one is a somewhat artificial source in which the particles start at full speed. The velocity distribution of the particles is specified in terms of their angular distribution and their energy distribution. Type two is a thermionic source in which the particles are emitted according to the half Maxwell-Boltzmann distribution. In this algorithm the particles may start at full speed, as in the type-one source, but can also start at zero beam potential and be accelerated afterwards in the ray-tracing routine. The ray-tracing is the subject of sections 13.4 and 13.5 . We note that it allows for an uniform external acceleration field only. For that reason, we did not attempt to model field emitters, which require the incorporation of a spherical symmetric field. An array of sources, all of the same type, can be simulated by the program. This possibility was included to model columns employing multiple emitters, as proposed by van der Mast, Jansen and Barth (1985). In this section we will discuss the algorithms used to model a single source. The extension to multiple sources is straightforward and will not be considered here.

For the type-one source one has to specify the initial velocity distribution in terms of the angular distribution and the energy distribution of the particles at emission. The angular distribution can be chosen circular uniform or circular Gaussian. The energy distribution can be chosen uniform, Gaussian or Lorentzian. The user has to specify the Half Width at Half Maximum (HWHM) of both distributions. Finally, the beam potential $\mathrm{V}$ defines the average energy of the particles, which is equal to $\mathrm{eV}$. From this information, the program selects for each particle randomly an angle $a$ with the axial direction, an polar angle $\varphi_{v}$ (between 0 and $2 \pi$ ) and an energy deviation $\Delta E$ from the average energy eV. A description of the random number generator, which serves this purpose, is presented in appendix 13.A. Once the values $\alpha, \varphi_{\mathrm{v}}$ and $\Delta \mathrm{E}$ 
have been selected for a certain particle, the program calculates the corresponding total velocity $\mathrm{v}$ as

$$
v=\sqrt{\frac{2 e(e V+\Delta E)}{m}}
$$

while the individual velocity components follow, using

$$
\begin{aligned}
& \mathbf{v}_{\mathbf{x}}=\mathrm{v} \cos \left(\varphi_{\mathbf{v}}\right) \sin (\alpha) \\
& \mathbf{v}_{\mathbf{y}}=\mathrm{v} \sin \left(\varphi_{\mathbf{v}}\right) \sin (\alpha) \\
& \mathbf{v}_{\mathbf{z}}=\mathrm{v} \cos (\alpha)
\end{aligned}
$$

The $z$-axis here coincides with the beam axis. Alternatively, one can instruct the program (by setting ICONS=2) to associate the energy distribution entirely with the z-component of velocity, using

$$
\begin{aligned}
& \mathbf{v}_{\mathbf{x}}=\mathbf{v} \cos \left(\varphi_{\mathbf{v}}\right) \tan (\alpha) \\
& \mathbf{v}_{\mathbf{y}}=\mathbf{v} \sin \left(\varphi_{\mathbf{v}}\right) \tan (\alpha) \\
& \mathbf{v}_{\mathbf{z}}=\mathbf{v}
\end{aligned}
$$

This feature is primarily intended to generate a beam which is monochromatic with respect to the axial velocity of the particles. This case is considered in our analytical model.

In the thermionic source (the type-two source) the velocity distribution is fully determined by the temperature of the cathode surface $T_{c a}$ only, as is expressed by equation (3.7.7). Defining the quantities $\varepsilon_{/ /}$and $E_{\perp}$ as

$$
\epsilon_{/ /}=\frac{m v_{/ /}^{2}}{2 \mathrm{k}_{\mathrm{b}} \mathrm{T}_{\mathrm{ca}}} \quad, \quad \epsilon_{\perp}=\frac{\mathrm{mv}_{\perp}^{2}}{2 \mathrm{k}_{\mathrm{B}} \mathrm{T}_{\mathrm{ca}}}
$$

one can rewrite equation $(3.7 .7)$ as

$$
f_{i}\left(\varepsilon_{/ /}, \varepsilon_{\perp}, \varphi_{v}\right) d \epsilon_{/ /} d \varepsilon_{\perp} d \varphi_{v}=\frac{1}{2 \pi} e^{-\left(\varepsilon_{\| /}+\varepsilon_{\perp}\right)} d \varepsilon_{/ /} d \varepsilon_{\perp} d \varphi_{v}
$$

in which $\varphi_{v}$ represents, again, the polar angle, which is uniformly distributed over $2 \pi$. Notice that both $\varepsilon_{/ /}$and $\varepsilon_{\perp}$ are distributed according to the one-dimensional exponential distribution $f_{1 e}(x)$

$$
f_{10}(x)=e^{-x}
$$

This distribution can easily be generated, as is discussed in appendix 13.A. Given a certain value for $\varepsilon_{\| /}$and $\varepsilon_{\perp}$, the program determines the total velocity of the particle $v$ and $i t s$ angle $\alpha$ with the axial direction, using

$$
v=\sqrt{\frac{2 \mathrm{e}\left(\varepsilon_{\| /}+\varepsilon_{\perp}\right) \mathrm{k}_{\mathrm{B}} \mathrm{T}_{\mathrm{Ca}}}{\mathrm{m}}}, \quad \alpha=\sqrt{\frac{\varepsilon_{\perp}}{\varepsilon_{\| /}}}
$$

The individual velocity components follow again with equations (13.2.2) or equations $(13,2,3)$.

The specification of the current density distribution at the emission surface is the same for both types of sources. It can be chosen circular uniform, circular Gaussian or rectangular uniform. The last possibility is included to facilitate the simulation of shaped beam lithography systems. The source dimensions are expressed in terms of the HWHM of the current density distribution. In case of a circular current 
density distribution, the program selects for each particle randomly a radius $r$ and a polar angle $\varphi_{p}$. The $x$ and $y$ component of the particle position follow from

$$
\begin{aligned}
& x=r \cos \left(\varphi_{p}\right) \\
& y=r \sin \left(\varphi_{p}\right)
\end{aligned}
$$

In case of a rectangular uniform distribution, the $\mathrm{x}$ and $\mathrm{y}$ coordinates are chosen uniform in the intervals $\left(-\mathrm{HWHM}_{\mathbf{x}},+\mathrm{HWHM}_{\mathbf{x}}\right)$ and $\left(-\mathrm{HWHM}_{\mathbf{y}},+\mathrm{HWHM}_{\mathbf{y}}\right)$ respectively. The program allows for a shift in the $x$ and $y$ direction, which can be exploited to simulate some misalignment of the source.

The axial distribution of the particles is determined by the total beam current $I$. For particles of charge $e$, the average time $\mathrm{T}_{\mathrm{s} a \mathrm{~m}}$, required to emit $\mathrm{N}_{\mathrm{s} a \mathrm{~m}}$ particles, is given by

$$
\mathrm{T}_{\text {sam }}=\frac{e \mathrm{~N}_{\text {sam }}}{I}
$$

Each particle is assigned a time $T$, which is randomly chosen in the interval $\left[-T_{s a m} / 2, T_{s a m} / 2\right]$, using an uniform distribution. The source routine and ray-tracing routines are organized such that the particles are effectively emitted one by one from the cathode surface.

The nominal length of the sample $L_{s a m}$ is a function of the beam current $I$, the beam potential $V$ and the number of particles in the sample $\mathbf{N}_{\text {sam }}$

$$
L_{s a m}=N_{s a m}\langle S\rangle=\sqrt{\frac{2 e^{3}}{m}} \frac{N_{s a m} \sqrt{V}}{I}
$$

where <s> denotes the average axial separation of the particles, which is specified by equation (3.2.6). In the case of an electron beam with $V=10 \mathrm{kV}, I=1 \mu \mathrm{A}$ and a sample size $\mathrm{N}_{\mathrm{sam}}=100$ one obtains $\mathrm{L}_{\mathrm{s}} \mathrm{m}=95 \mu \mathrm{m}$.

The actual sample length will always differ somewhat from the nominal length $L_{s a m}$, since the positions of the particles are chosen randomly. Moreover, the sample length may increase during the flight. Firstly, because the energy spread of the particles causes some spatial dispersion. Secondly, because the collective space charge force is unbalanced for particles near the edge of the bunch. The particles at the front of the bunch experience an artificial acceleration, while the particles at the back of the sample are artificially retarded. This so-called finite-size effect will be further discussed in section 13.7. In general, one should choose the sample length $\mathrm{L}_{s}$ am large enough to render finite-size effects negligible. In this respect, problems may arise with the simulation of high current beams operating at a low beam potential. Equation (13.2.10) shows that such beams require an extreme large $\mathrm{N}_{\mathrm{s} a \mathrm{~m}}$ in order to obtain a sufficient sample length $\mathrm{L}_{\mathrm{s} a \mathrm{~m}}$. However, the value of $\mathrm{N}_{\mathrm{sam}}$ is, in general, limited by cpu-time constraints and in some cases by the available memory space. An alternative way to model a high-current beam in the vicinity of the source will be discussed in section 13.6 .

\subsection{Optical elements}

Lenses, deflectors and quadrupoles can be simulated by the program in thin-lens approximation. Round and rectangular apertures are incorporated too. We will refer to these elements as optical components. 
The first order imaging properties of a thin-lens are fully specified by the axial position of the lens and its focal distance $f$. Consider a particle which reaches the lens-plane with an axial velocity $v_{z}$ and transverse velocity $v_{\perp}$. Let $r_{\perp}$ be its radial distance to the axis when it crosses the lens-plane. The first order imaging properties of the lens are simulated by adding a lateral velocity $\Delta \mathbf{v}_{\perp 1}$ to the velocity of the particle, which is chosen equal to

$$
\Delta \mathbf{v}_{\perp 1}=-\frac{\mathbf{v}_{\mathbf{z}}}{\mathbf{f}} \mathbf{r}_{\perp}
$$

A lens is called ideal when it shows only first order imaging properties. Equation (13.3.1) expresses that such a lens can be simulated by shifting the transverse velocity of each particle opposite to the direction of $r_{\perp}$, by an amount which is directly proportional to the length of this vector.

The performance of actual lenses is limited by third and higher order geometrical aberrations as well as chromatic aberrations. In addition, magnetic lenses may introduce rotational errors. These effects are incorporated within the thin-lens approximation. The chromatic aberration of a lens causes an additional shift in the transverse velocity $\Delta v_{\perp c}$, which is equal to

$$
\Delta v_{\perp c}=C_{c} \frac{v_{z}}{f^{2}}\left(\frac{v^{2}}{\left\langle v^{2}\right\rangle}-1\right) r_{\perp}
$$

where $\left\langle v^{2}\right\rangle$ represents the mean square velocity of all particles and $C_{c}$ is the constant of chromatic aberration of the lens, for an object which is located at infinity. Notice that the term between brackets is equal to $\Delta \mathrm{E} / \mathrm{E}$.

Third order geometrical aberrations are taken into account by introducing the additional shift in transverse velocity $\Delta v_{13}$, with

$$
\Delta \mathbf{v}_{\perp 3}=-\mathrm{C}_{\mathrm{s}} \frac{\mathbf{v}_{\mathrm{z}} \mathbf{r}_{\perp}^{2}}{\mathbf{f}^{4}} \mathbf{r}_{\perp}
$$

where $C_{s}$ is the constant of spherical aberration of the lens, for an object which is located at infinity.

The program does not account for the rotation of the beam by a magnetic lens. However, the aberrations caused by the non-uniformity of the rotation are included within the thin lens approximation. The lateral velocity and position of a particle are rotated around the centre of the lens over an angle $\Delta \varphi$, which depends on the radial distance to the axis $r_{\perp}$ and the total velocity $v$, as

$$
\Delta \varphi=\frac{R_{d}}{f^{2}} r_{\perp}^{2}-R_{c}\left(\frac{v^{2}}{\left\langle v^{2}\right\rangle}-1\right)
$$

where $R_{d}$ represents the rotational distorsion of the lens and $R_{c}$ represents the chromatic error in the rotation. The rotation over the angle $\Delta \varphi$ is incorporated by shifting the $x$ and $y$ coordinates of the particle with

$$
\begin{aligned}
& \Delta \mathrm{x}=-\mathrm{y} \Delta \varphi \quad, \quad \Delta \mathrm{y}=\mathrm{x} \Delta \varphi \\
& \Delta v_{x}=-v_{y} \Delta \varphi, \quad \Delta v_{y}=v_{x} \Delta \varphi
\end{aligned}
$$

This scheme, which was proposed by Barth (1988), guarantees conservation of the (canonical) angular momentum of the particle around the $z$-axis.

The lens routine accounts for the rotational shifts given by equation (13.3.5) first and computes the total velocity shift $\Delta v_{\perp}$. 
specified by equations $(13.3 .1),(13.3 .2)$ and $(13.3 .3)$ next, using

$$
\begin{aligned}
\Delta v_{\perp} & =\Delta v_{\perp 1}+\Delta v_{\perp c}+\Delta v_{\perp} 3 \\
& =-\frac{v_{z}}{f}\left[1-c_{c}\left(\frac{v^{2}}{\left\langle v^{2}\right\rangle}-1\right)+C_{s} \frac{r_{\perp}{ }^{2}}{f^{3}}\right] r_{\perp}
\end{aligned}
$$

The user has to specify the axial position of the lens, its focal distance $f$ and the aberration constants $C_{c}, C_{s}, R_{d}$ and $R_{c}$, In addition, one can specify a shift of the centre of the lens relative to the beam axis in order to simulate some misalignment.

The lens procedure described by equations (13.3.5) and (13.3.6) affects the transverse velocity of a particle. Its axial velocity remains the same. This implies that the total kinetic energy of the particle is changed in this scheme. However, the user may instruct the program (by setting ICONS=1) to compensate this effect, by multiplying each of the final $x, y$ and $z$ velocity components with a factor

$$
F=\frac{\left(v_{x, i}{ }^{2}+v_{y, i}{ }^{2}+v_{z, i}\right)^{1 / 2}}{\left(v_{x, f^{2}}+v_{y, f^{2}}+v_{z, f^{2}}\right)^{1 / 2}}
$$

where $v_{x, i}, v_{y}, i$ and $v_{z}, i$ are the initial velocity components of the particle (before entering the lens) and $v_{x}, f, v_{y}, f$ and $v_{z}, f$ are the final velocity components (when leaving the lens).

So far, we considered the action of a rotational symmetric thin lens. Similarly, one can describe the action of thin-deflector by specifying its axial position, the deflection angles $\Delta \alpha_{x}$ and $\Delta \alpha_{y}$ in $x$ and $y$ direction respectively and a constant $D_{c}$, which covers the dispersion of the deflector. The change in the transverse velocity of a particle, caused by the deflector, is computed by

$$
\begin{aligned}
& \Delta v_{x}=\tan \left(\alpha_{x}\right) v_{z}\left[1-D_{c}\left(\frac{v^{2}}{\left\langle v^{2}\right\rangle}-1\right)\right] \\
& \Delta v_{y}=\tan \left(\alpha_{y}\right) v_{z}\left[1-D_{c}\left(\frac{v^{2}}{\left\langle v^{2}\right\rangle}-1\right)\right]
\end{aligned}
$$

As for the lens, the user may instruct the program to multiply the final velocity by the factor $F$, defined by equation (13.3.7).

Quadrupoles are also included in the program, in thin-lens approximation. A quadrupole is specified by its axial position, the focal distances $f_{x}$ and $f_{y}$, the constants of chromatic aberration $c_{c x}$ and $\mathrm{C}_{\mathrm{c} y}$ and the spherical aberration constants $\mathrm{C}_{30}, \mathrm{C}_{03}, \mathrm{C}_{21}$ and $\mathrm{C}_{12}$. The change in transverse velocity of a particle, caused by the quadrupole, is computed by

$$
\begin{aligned}
& \Delta v_{x}=-\frac{v_{z}}{f_{x}}\left[1-C_{c x}\left(\frac{v^{2}}{\left\langle v^{2}\right\rangle}-1\right)+\frac{1}{f_{x}{ }^{3}}\left(C_{30} x^{2}+C_{12} y^{2}\right)\right] x \\
& \Delta v_{y}=-\frac{v_{z}}{f_{y}}\left[1-C_{c y}\left(\frac{v^{2}}{\left\langle v^{2}\right\rangle}-1\right)+\frac{1}{f_{y}{ }^{3}}\left(C_{03} y^{2}+C_{21} x^{2}\right)\right] y
\end{aligned}
$$

similar to equation (13.3.6), describing the action of a thin rotational symmetric lens. For a weak lens, one may assume that $\mathrm{C}_{30}=\mathrm{C}_{03}$ and $\mathrm{C}_{21}=\mathrm{C}_{12}$. If specified, the final velocity of the particle is multiplied with the factor $F$, defined by equation (13.3.7). The centre of the quadrupole can be shifted in $x$ and $y$ direction.

Apertures are modelled by means of an index table, which contains the indices of the particles present in the sample. The 
aperture-routine checks for every particle, whether its perturbed trajectory passes through the aperture or not. The parameter $\mathbf{N}_{s}$ am is then set to the actual number of particles which have passed the aperture and the indices of these particles are stored. in the first $\mathrm{N}_{\mathrm{sam}}$ positions of the index table. The routine which is called next by the program, retrieves the coordinates of the particles by reading the indices from the first $\mathrm{N}_{\mathrm{s}} \mathrm{m}$ positions in the index-table. The remaining coordinates are ignored.

The user has to specify the axial location of the aperture, its shape and its size. The shape can be round or rectangular. The centre of the aperture can be shifted in $x$ and $y$ direction. An option is included to "invert" the aperture, which means that the program keeps track of the particles which normally do not pass through the aperture and omits the others. This feature can, for example, be used to simulate a ring source, by combining a round source and a somewhat smaller round inverted aperture, both located at the same axial position.

\subsection{Numerical ray-tracing}

The ray-tracing routine calculates the trajectories of the particles during the passage from one optical component to the next, taking the mutual coulomb repulsion of the particles into account. An uniform axial accelerating or retarding external field can be included. Two different ray-tracing methods are implemented in the program. The numerical method (in the program referred to as DRIFT1) is described here, while the so called analytical ray-tracing method (in the program referred to as DRIFT2) is the subject of the sections 13.5 and 13.6 .

The total force exerted on particle $i$ in a sample, consisting of $\mathrm{N}_{\text {sam }}$ particles, is equal to

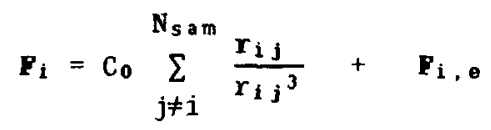

where $\mathbf{r}_{\mathbf{i}}=\mathbf{r}_{\mathbf{i}}-\mathbf{r}_{j}$, the relative position vector of particles $i$ and $j$ and

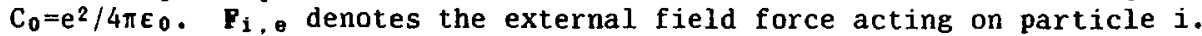
For the moment, we will disregard the external field and concentrate on the first term in equation $(13.4 .1)$.

The equation of motion of particle number $i$ is, in absence of external fields, given by

$$
r_{i}(I I)=\frac{C_{0}}{m} \sum_{j \neq i}^{N_{s a m}} \frac{r_{i j}}{r_{i j}{ }^{3}}
$$

in which $\mathbf{r}_{\mathbf{i}}$ (II) denotes the second derivative of $\mathbf{r}_{\mathbf{i}}$ with respect to time. By differentiating equation (13.4.2) once, one obtains

$$
r_{i}(\text { III })=\frac{c_{0}}{m} \sum_{j \neq i}^{N_{s a m}}\left(\frac{v_{i j}}{r_{i j}{ }^{3}}-\frac{\left(r_{i j} \cdot v_{i j}\right) r_{i j}}{r_{i j}}\right)
$$

where $\nabla_{i j}=\dot{r}_{i j}=\nabla_{i}-\nabla_{j}$, the relative velocity vector of particles $i$ an $j$. Given the positions $r_{i}$ and velocities $v_{i}$ of each particle at time $t$, the second and third time derivatives $r_{i}(I)$ and $r_{i}$ (III' can be calculated, for each particle, from equations (13.4.2) and (13.4.3) respectively. 
The new positions and velocities, of each particle, at time $t+\Delta t$ are determined next, using the following approximations

$$
\begin{aligned}
& r_{i}(t+\Delta t) \cong r_{i}(t)+v_{i}(t) \Delta t+\frac{1}{2} r_{i}(I I)(t) \Delta t^{2}+\frac{1}{6} r_{i}(I I I)(t) \Delta t^{3} \\
& v_{i}(t+\Delta t) \cong v_{i}(t)+r_{i}(I I)(t) \Delta t+\frac{1}{2} r_{i}(I I I)(t) \Delta t^{2}
\end{aligned}
$$

Thus, a third order expansion is used for updating the positions and a second order expansion for updating the velocities.

The time step size $\Delta t$ is adjusted after every step, in order to maintain a certain local integration error. Two different types of step size algorithms are implemented. In the first type, the program evaluates the average absolute value of the second and third order derivatives $r_{i}(I I)$ and $r_{i}$ (III), from

$$
\langle r|I|\rangle=\frac{1}{N_{s a m}} \sum_{i=1}^{N_{s a m}}\left|r_{i}\langle I I\rangle, \quad\langle r(I I i)\rangle=\frac{1}{N_{s a m}} \sum_{i=1}^{N_{s a m}}\right| r_{i}(I I I) \mid
$$

The nominal time steps $\Delta t_{2}$ and $\Delta t_{3}$ are evaluated next, defined as the the time steps which lead to a contribution of the second and third order term in the first expansion of equation $(13.4 .4)$, which are equal to the predefined values $\varepsilon_{2}$ and $\varepsilon_{3}$ respectively

$$
\Delta t_{2}=\left(\frac{2 \varepsilon_{2}}{\left\langle r^{(I I)}\right\rangle}\right)^{1 / 2}, \quad \Delta t_{3}=\left(\frac{6 \varepsilon_{3}}{\left\langle r^{(I I I)\rangle}\right.}\right)^{1 / 3}
$$

The next time step $\Delta t$ is taken equal to the minimum of $\Delta t_{2}$ and $\Delta t_{3}$.

The second type of time step algorithm evaluates the maximum absolute value of the second and third order derivatives, denoted as $r^{(I I)} \max$ and $r^{(I I I)^{\prime}}$ ax respectively. The nominal step sizes $\Delta t_{2}$ and $\Delta t_{3}$ follow again by equation $(13.4 .6)$, in which $\left\langle Y^{(I I)}\right\rangle$ and $\left\langle Y^{(I I I)}\right\rangle$ are now

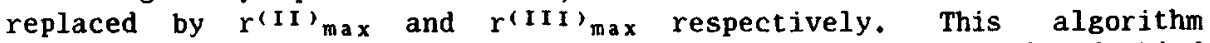
guarantees that the perturbations, corresponding to the second and third order terms in the expansion for $\mathbf{r}_{\mathbf{i}}(t+\Delta t)$, do not exceed the predefined values $\varepsilon_{1}$ and $\varepsilon_{2}$ for any of the particles. This algorithm was proposed by Meisburger (1983) and reported by Munro (1987). We note that in most applications, one is not really interested in the exact coordinates of a few strongly deflected particles and the first algorithm is therefore preferred.

The values for the parameters $E_{2}$ and $\varepsilon_{3}$ are estimated by the program from the input parameter $\mathrm{N}_{s t e p}$, which specifies the nominal number of steps per meter, which should on the average be maintained during the ray-tracing. This quantity can directly be related to the run-time of the program, as will be discussed later. For normal conditions it is sufficient to set $\mathrm{N}_{s t e p}=1000$. The program keeps track of the total integration error in the positions and the velocities, denoted as $\epsilon_{\mathrm{p}}$ and $\epsilon_{\mathrm{v}}$, using

$$
\begin{aligned}
& \varepsilon_{v}(t+\Delta t) \approx \varepsilon_{v}(t)+\frac{\left|r^{(I I I)}(t+\Delta t)-r^{(I I I)}(t)\right|}{6 \Delta t} \Delta t^{3} \\
& \varepsilon_{p}(t+\Delta t) \approx E_{p}(t)+\frac{\mid r^{(I I I)}(t+\Delta t)-r^{(I I I)(t) \mid}}{24 \Delta t} \Delta t^{4}+\varepsilon_{v}(t) \Delta t
\end{aligned}
$$

where $\mathrm{YIII}$ represents the same measure for the third order derivative as used in the step-size algorithm. Thus, $r(I I)=\langle Y(I I I)\rangle$ when using the 
first type of algorithm and $\mathrm{rIII}^{\prime}=\mathrm{rIII}$ max when using the second type. As the global integration error in the velocities $\varepsilon_{v}(t)$ is of the second order in $\Delta t$, one sees from the second equation of $(13.4 .7)$ that the local integration error in the positions is of the third order in $\Delta t$ (and not of the fourth order as equation (13.4.4) suggests). The global integration errors $E_{v}(t)$ and $\varepsilon_{p}(t)$ are therefore both of the second order in $\Delta t$.

In principle, one can use even higher order schemes than the one described by equation (13.4.4). Tang (1983) proposed a procedure which includes the evaluation of terms proportional to the fourth order derivative $\mathbf{r}_{\mathbf{i}}(\mathrm{IV})$. Clearly, this permits to take larger time steps $\Delta t$, while maintaining a certain local integration error. However, at the accuracy level which is normally pursued (typically $1 \%$ total integration error in the positions and velocities) it appears to be questionable whether this gain counterbalances the additional cpu-time required per step. Furthermore, we note that Tang uses a time-step algorithm in which $\Delta t$ is taken proportional to the smallest value found for the quantity $\left[\mid r_{i}\left(\text { II) }|/| r_{i} \text { (IV) } \mid\right]^{1 / 2}\right.$ (with $i=1,, 2, \cdots, N_{s a m}$ ). He claims that the terms associated with $\mathbf{r}_{\mathbf{i}}$ (II) always cause the largest perturbations, while every term in the Taylor expansion is, in general, smaller than its precessor. In our experience, this is not necessarily true for all conditions and his algorithm is expected to fail occasionally.

Equation (13.4.1) shows that the presence of an uniform external electrostatic field can simply be taken into account in equations $(13.4 .4)$, by replacing

$$
r_{1}(I I) \rightarrow r_{i}(I)+P_{e} / m
$$

where $F_{e}$ is the external field force acting on all particles. In our program, $F_{e}$ is always directed along the axial direction and is specified as

$$
F_{e}=\frac{e\left(V_{1}-V_{0}\right)}{z_{1}-z_{0}}
$$

where $V_{0}$ is the beam potential at the initial axial position $z_{0}$ (entrance plane) and $v_{1}$ is the beam potential at the final axial position $z_{1}$ (exit plane). As the field is uniform, the scheme of equation (13.4.4) provides an exact calculation of the trajectories, as far as the influence of the external force is concerned. For that reason, the program does not account for the external force in the time step algorithm. Thus, the measure for the acceleration $r^{(I I)}$, considered in equation $(13.4 .6)$, stems from the interaction between the particles only, even in presence of an external field.

Special care has been taken to handle the calculation of the trajectories at the entrance plane and the exit plane of the beam section. At the start of the ray tracing routine, all particles are projected backwards along their unperturbed trajectories, over a time interval $T_{1}, T_{1}$ is here the minimum value of all. $T_{1 i}$, with

$$
\mathrm{T}_{1 \mathrm{i}}=\frac{z_{\mathrm{i}}-z_{0}}{\mathrm{v}_{\mathrm{zi}}} \quad, \quad \mathrm{i}=1,2, \cdots, \mathrm{N}_{\mathrm{sam}}
$$

After this operation, the foremost particle is located in the entrance plane. The ray tracing is now started. Step by step all particles will pass the entrance plane. In the normal mode of operation (specified by setting INT=1) a particle $i$, which has passed this plane $\left(z_{0}<z_{1}<z_{1}\right)$, experiences the interaction of all other particles, irrespective of 
their location. The interaction of particle $i$ (with all other particles) continues until it has passed the exit plane $\left(z_{i}>z_{1}\right)$. The ray-tracing is stopped when all particles have passed this plane.

In order to clarify this procedure, assume that the ray-tracing routine is called again to compute the trajectories from $z=z_{1}$ to $z=z_{2}$. The procedure is now repeated: The particles are projected backwards such that all particles are behind the new entrance plane $z=z_{1}$. Next, they are traced step-wise through the section until all particles have passed the new exit plane $z=z_{2}$. Instead of calling the ray-tracing routine twice, one could have called it only once to perform the raytracing directly from $z=z_{0}$ to $z=z_{2}$. The procedure followed by the program guarantees that both calculations yield the same final coordinates.

A somewhat different procedure is implemented to calculate the trajectories near the source (specified by setting INT=2). In this procedure, two particles only interact when they have both passed the entrance plane. As the entrance plane is here assumed to coincide with the emission surface, this seems the most realistic approach. Effectively, the particles are now emitted one by one from the source and start interacting directly after emission. We note that the timestep algorithm always evaluates the perturbation of all particles, whether they are actually taken into account in the ray-tracing or not. This way, sudden changes in the time step size $\Delta t$ are avoided, which might occur otherwise when a particle passes the entrance plane.

In presence of an external field, special care has to be taken to treat the passage of a particle through the entrance plane or exit plane. The program calculates the exact time at which the particle has passed the plane and adjusts the acceleration of the particle experienced during the last time step accordingly. The significance of this procedure becomes clear when one realizes that, for a typical acceleration field of $100 \mathrm{kV} / \mathrm{m}$ and a number of steps $\mathrm{N}_{\mathrm{step}}=1000 / \mathrm{m}$, a particles gains on the average an energy of $10 \mathrm{eV}$ per step. Consequently, if one does not account for the exact time of entrance or exit, but just starts or stops accelerating in the next time-step after this event has taken place, the final energy of a particle can easily be off by $10 \mathrm{eV}$. This is clearly unacceptable.

In order to avoid the occurrence of large numbers, the coordinates of all particles are scaled at the start of the ray-tracing routine, employing the $\delta, v$-scaling, defined by equation (6.13.1). This procedure also leads to some minor reduction of the number of computations in the evaluation of the quantities $\mathbf{r}_{\mathbf{i}}$ (II) and $\mathbf{r}_{\mathbf{i}}$ (III) from equations $(13.4 .2)$ and $(13.4 .3)$, since the physical parameter $C_{0} / m$ does not appear in the scaled equations. Before leaving the ray-tracing routine, the coordinates are converted to unscaled quantities, which are used in the other routines of the program.

The total cpu-time $t_{p}$, used by the numerical ray-tracing routine, for the calculation of the trajectories of a single sample, consisting of $\mathrm{N}_{\mathrm{sam}}$ particles, through a single section of the system, is equal to

$$
t_{n}=n_{s t e p} \frac{1}{2} N_{s a m}\left(N_{s a m}-1\right) \Delta t_{n}
$$

where $n_{s t e p}$ is the actual number of time-steps used in the ray-tracing through the section and $\Delta t_{n}$ is the cpu-time required to evaluated the interaction per pair, per time-step. The quantity $\mathrm{N}_{s a m}\left(\mathrm{~N}_{\mathrm{sam}}-1\right) / 2$ is equal to the number of pairs in the sample. For the routine implemented in the program, running on an IBM 3083 mainframe computer, it was found that $\Delta t_{n} \neq 10^{-5} s$. Thus, for $N_{s a m}=100$ and $n_{s t e p}=100$, one obtains $t_{n} \approx 5 s$. 
In order to estimate the total run-time of the program, one has to consider that an actual column consists of a number of beam sections. In addition, one usually runs a number of samples in order to obtain sufficient statistics, each sample starting with a different "seed" of initial conditions. Denoting the number of beam sections (or drift sections) as $\mathrm{N}_{\mathrm{d}} \mathrm{ift}$ and the number of seeds as $\mathrm{N}_{\mathrm{sed}}$, one finds for the total run time $T_{n}$ required for the numerical ray-tracing

$$
T_{n}=N_{\text {seed }} N_{d r i f t}\left\langle n_{s t e p}\right\rangle \frac{1}{2} N_{s a m}\left(N_{s a m}-1\right) \Delta t_{n}
$$

in which $\left\langle n_{s t e p}\right\rangle$ is the average number of steps per drift section. The quantity $N_{d r i f t} x\left\langle n_{s t e p}>\right.$ is equal to the total number of time-steps used to calculate the trajectories through the entire column. Taking this number equal to $1000, \mathrm{~N}_{\text {seed }}=10$ and $\mathrm{N}_{s \text { am }}=100$, one obtains $T_{n} \approx 5 \times 10^{2} \mathrm{~s} \approx 8 \mathrm{~min}$ on an IBM 3083 computer. The cpu-time used by the other routines of the program is, in general, negligible compared to the cpu-time required for the ray-tracing.

The ray-tracing can be speeded up by limiting the interaction range of the particles to some value $r_{i a t}$. This reduces the number of pairs which has to be considered in the calculation of the interaction force acting on each particle in the sample. The interaction radius $r_{i n t}$ is, in our program, specified by the input parameter $N_{i n t}$, as $r_{i n t}=N_{i n t}\langle s\rangle$, where $\langle s\rangle$ is the average axial separation of the particles. In case $r_{i n t}$ is large compared to the beam radius, the number of interactions per particle is roughly equal to $2 \mathrm{~N}_{\mathrm{int}}$. The total number of interacting pairs in the sample is then equal to $\mathbf{N}_{\mathrm{int}_{\mathrm{t}}} \times \mathrm{N}_{\mathrm{sam}}$, versus $\mathrm{N}_{\mathrm{s} \text { am }}\left(\mathrm{N}_{\mathrm{sam}}-1\right) / 2$, when the interaction range is not limited. Accordingly, the total computation time reduces with a factor $2 \mathrm{~N}_{\text {int }} / \mathrm{N}_{\mathrm{s} a m}$. In case $\mathrm{r}_{\mathrm{int}}$ is not large compared to beam radius, the number of interactions considered per particle per step will be smaller than $2 \mathrm{~N}_{\mathrm{int}}$ and the computation time reduces even more.

In practice, it is not very convenient to use the feature of interaction range limitation, since one can not predict beforehand the appropriate value for the parameter $\mathrm{N}_{\mathrm{int}}$. Not only does it depend on the beam geometry, but it also differs for the various phenomena of particle interactions. For simplicity one better avoids any restriction of the interaction range and tries to keep the total cpu-time within reasonable limits by choosing suitable values for $\mathrm{N}_{s a m}$ and $\mathrm{N}_{s e e d}$. However, for the simulation of high-current and/or low voltage beams, one may prefer a restriction of the interaction range above the choice of an "economical" sample size, in order to reduce finite-size effects. We will come back to this subject in section 13.7 .

\subsection{Analytical ray-tracing}

The analytical ray-tracing routine (in the program referred to as DRIFT2) is based on a decomposition of the full $\mathrm{N}$-body problem into a sum of two-particle interactions, which are evaluated from analytically derived expressions. In many respects this method can be considered as the Monte Carlo variant of the extended two-particle approximation, which was described in section 5.3 and utilized in the chapters 6 to 10 . The dynamical aspects of the problem are handled alike in both approaches. However, the systematic integration over the coordinates of a field particle relative to a test particle, carried out within the analytical model, is in the $\mathrm{MC}$-approach replaced by a random selection of initial conditions of a large set of particles. We emphasize that the 
fundamental assumptions, underlying the concept of analytical raytracing, are the same as for the extended two-particle approximation. Accordingly, the reader is referred to section 5.3 for the basic issues involved and the justification of the approach. Here we will concentrate on the actual realization of the concept within the MC-program. The analytical ray-tracing routine is written such, that it is fully compatible with the numerical ray-tracing routine, described in the previous section. The user can easily switch from one method to the other and compare the results.

The reason for implementing the analytical ray-tracing routine into the MC-program was to verify the premisses of the extended twoparticle approximation. The practical significance of the routine was first recognized, when it was found that, for practical operating conditions, it is as accurate as its numerical counterpart, while it increases the speed of the program with one to two orders of magnitude. It therefore constitutes a very useful enhancement of the MC-program. Fast Monte Carlo (FMC) simulations, based on analytical ray-tracing, can be carried out on a fast micro-computer, keeping the cpu-time within reasonable limits (typically in the order of an hour). A brief description of the method and some examples to verify its validity were reported by Jansen (1987). Here we will outline the method in more detail.

In the analytical ray-tracing routine, the final coordinates of the particles (near the exit-plane) are directly determined from their initial coordinates (near the entrance-plane), using the equations

$$
\begin{aligned}
& \mathbf{r}_{i}\left(t_{0}+T\right)=\tilde{r}_{i}\left(t_{0}+T\right)+\sum_{j \neq i}^{N_{s a m}} \Delta \mathbf{r}_{i j}\left(t_{0}, t_{0}+T\right) \\
& \mathbf{v}_{i}\left(t_{0}+T\right)=\tilde{v}_{i}\left(t_{0}+T\right)+\sum_{j \neq i}^{N_{s} a m} \Delta \mathbf{v}_{i j}\left(t_{0}, t_{0}+T\right)
\end{aligned}
$$

in which $\tilde{\mathbf{r}}_{i}\left(t_{0}+T\right)$ and $\tilde{v}_{i}\left(t_{0}+T\right)$ are the final unperturbed position and velocity of particle $i$ respectively. T denotes the total time of flight and $\Delta \mathbf{r}_{\mathbf{i} j}$ and $\Delta \mathbf{v}_{\mathbf{i} j}$ are the displacements, in position and velocity respectively, of particle $i$, due to the interaction with particle $j$ during the flight. In absence of external fields, the unperturbed coordinates are simply given by

$$
\begin{aligned}
& \tilde{\mathbf{r}}_{i}\left(t_{0}+T\right)=r_{i}\left(t_{0}\right)+\mathbf{v}_{i}\left(t_{0}\right) T \\
& \tilde{\mathbf{v}}_{1}\left(t_{0}+T\right)=\mathbf{v}_{i}\left(t_{0}\right)
\end{aligned}
$$$$
\left(V_{1}=V_{0}\right)
$$

Equation (13.5.1) is equivalent to equation (5.3.12), which is exploited in the extended two-particle approximation. The underlying assumption is that the displacements $\Delta \mathbf{r}_{i j}$ and $\Delta \mathbf{v}_{i j}$ are in general small. As was pointed out in section 5.3 , the method breaks down when the particles are involved in two or more strong collisions during their flight. However, such events are exceptional for normal operating conditions. In addition, one should realize that such events mainly affect the tails of the generated energy and trajectory displacement distributions and not the central region. We emphasize that equation (13.5.2) refers to a field free drift space. Modifications are required to accomodate external fields, as will be discussed later on in this section.

Equations (13.5.1) reduce the many particle problem to the 
calculation of the displacements $\Delta \boldsymbol{r}_{i j}$ and $\Delta \mathbf{v}_{i j}$. This is a problem of two-particle dynamics, which was considered in chapter 6 , Given the initial relative position $\mathbf{r}_{\mathbf{i} j}=\mathbf{r}_{\mathbf{i}}-\mathbf{r}_{\mathbf{j}}$, the initial relative velocity $v_{i j}=v_{i}-v_{j}$ and the time of flight $T$, one has to compute the perturbation of the final coordinates of the particles $i$ and $j$. As was pointed out in chapter 6, it is not possible to obtain the exact solution of this problem in an explicit form. However, it is possible to obtain accurate approximations, in an explicit form, for two limiting cases, namely for weak collisions and for nearly complete collisions. If none of these approximations is allowed, the exact solution should be obtained by a numerical procedure.

For convenience of notation, we will from now on denote $\mathbf{r}_{i j}$ as $r$ and $v_{i j}$ as $v$ and take the initial time $t_{i}=0$. The coordinates referring to the initial condition are indicated with the subscript 0 . The program uses the following scheme to evaluate a two-particle collision:

- The $(\hat{a}, \hat{b})$ coordinate system in the orbital plane is determined from the initial relative position and velocity $r_{0}$ and $v_{0}$, using equations (6.3.1).

- The parameters $a_{0}$ and $b$ are evaluated from the vectors $r_{0}, \hat{a}$ and $\hat{b}$. The eccentricity related parameter $q_{c}$ is determined from equation $(6.4 .16)$.

- The type of collision is determined and the final displacements in the orbital plane are evaluated, using the appropriate collision dynamics. The program proceeds as follows. The collision is considered as weak if

$$
q_{c}>10^{6} \text { or } a_{0}<-50 b \text { or } \quad v_{0} T-a_{0}<-50 b
$$

conform equation (6.8.1). When one or more of the inequalities in equation (13.5.3) are satisfied, it is allowed to employ first order perturbation dynamics. The displacements $\Delta \mathrm{a}_{\mathrm{f}}, \Delta \mathrm{b}_{\mathrm{f}}, \Delta \dot{\mathrm{a}}_{\mathrm{f}}$ and $\Delta \dot{b}_{\mathrm{f}}$ are evaluated from equations $(6.8 .6)$ and $(6.8 .5)$. If equation $(13.5 .3)$ is not satisfied, the program verifies whether the collision can be considered as nearly complete. This is the case if

$$
\mathrm{a}_{0}>50 \mathrm{~b} \text { and } \quad \mathrm{v}_{0} \mathrm{~T}-\mathrm{a}_{0}>50 \mathrm{~b} \text { and } \quad \frac{1}{2} \mathrm{mv}_{0}^{2}>50 \frac{\mathrm{C}_{0}}{\mathrm{r}_{0}}
$$

conform equation (6.7.7). When all inequalities in equation (13.5.4) are satisfied, it is allowed to use nearly complete collision dynamics and the displacements $\Delta a_{f}, \Delta b_{f}, \Delta \dot{a}_{f}$ and $\Delta b_{f}$ are evaluated from equations $(6.7 .5)$ and $(6.7 .6)$. Otherwise, the program utilizes a numerical procedure to evaluate these quantities, which is referred to as full collision dynamics. The first step, of this procedure, is the evaluation of the initial value of the polar coordinates $r$ and $\theta$ and the sign parameter $\sigma_{i}$, from equations (6.3.5). Micro-scaling is applied, using the prescriptions of equations $(6.4 .3)$ and $(6.4 .4)$. In the next steps the program follows the analysis of section 6.5 , which leads, eventually, to the final polar coordinates $r_{f}$ and $\theta_{f}$ and their derivatives with respect to time $\dot{r}_{f}$ and $\dot{\theta}_{f}$, see equations $(6.5 .11)$ and (6.5.12). A key-step is the evaluation of the final polar coordinate $\rho_{f}=r_{f} / d_{s}$ from equation (6.5.7). This is done by numerical inversion, following the scheme of section $6 ; 6$. After removing the micro-scaling, the displacements $\Delta a_{f}, \Delta b_{f}, \Delta \dot{a}_{f}$ and $\Delta b_{f}$ follow, finally, from equations $(6.3 .9)$ and $(6.3 .7)$. Figure 6.4 contains a flow diagram of the essential steps of the calculation in the orbital plane.

- Having evaluated the displacements $\Delta a_{f}, \Delta b_{f}, \Delta \dot{a}_{f}$ and $\Delta b_{f}$, the remaining part is to express the results in terms of the displacements 
of the individual particles in the laboratory system. This is done by means of equations $(6.3 .8)$ and $(6.3 .11)$. Identifying particle $i$ with the colliding particle (subscript $c$ ) and particle $j$ with the test particle (subscript $t$ ), these equations yield in the present notation

$$
\begin{aligned}
& \Delta r_{i j}=\frac{1}{2}\left(\Delta a_{f} \hat{a}+\Delta b_{f} \hat{b}\right), \quad \Delta v_{i j}=\frac{1}{2}\left(\Delta \dot{a}_{f} \hat{a}+\Delta \dot{b}_{f} \hat{b}\right) \\
& \Delta \mathbf{r}_{j i}=-\Delta \mathbf{r}_{i j} \quad, \quad \Delta \mathbf{v}_{j i}=-\Delta \mathbf{v}_{i j}
\end{aligned}
$$

which completes the calculation for a single two-particle interaction. When the interaction has been evaluated for every pair in the sample, the final positions and velocities of the particles follow by means of equations $(13.5 .1)$.

In the analytical ray-tracing routine, the start and the end of the interaction are handled somewhat different than in the numerical ray-tracing routine, since one can not switch the interaction on and of $f$ for each particle separately. The routine is organized such that all pairs interact during the same period $T$, while the absolute time of the start of the interaction may vary per pair. As for the numerical raytracing, two modes of operation have been incorporated. In the normal mode of operation (specified by setting INT=1), a pair of particles is shifted along the unperturbed trajectories, such that the the foremost particle is located in the entrance plane $z=z_{0}$. The displacements, generated by their mutual repulsion, is then calculated over the next period $T$. In the other mode of operation (specified by setting INT=2), the particles are shifted along the unperturbed trajectories, such that the last particle is located in the entrance plane $z=z_{0}$. This seems more realistic when the entrance plane coincides with the emission surface of the source. In this connection, we should mention that the final results are, in general, insensitive to the choice of the exact initial and final conditions. Finally, we emphasize that the time shifts only concern the calculation of the displacements $\Delta \mathbf{r}_{i j}$ and $\Delta \mathbf{v}_{i j}$ and do not involve the calculation of the unperturbed trajectories. This guarantees that the final coordinates all refer to the same time.

Uniform acceleration fields can be taking into account within the analytical ray-tracing routine. The essential observation is that, in the accelerated frame of reference moving with the beam, the particles can be considered as free, that is not influenced by external fields. The situation is similar to the case of an elevator which is in free-fall in a homogeneous gravitation field. In the elevator-system the laws of mechanics can be applied, as if the system is an inertial system in field free space. For the analytical ray-tracing routine, this observation implies that the scheme for the calculation of the displacements $\Delta \mathbf{r}_{\mathbf{i}}$ and $\Delta \mathbf{v}_{\mathbf{i j}}$, from the initial relative coordinates $\mathbf{r}_{\mathbf{i}}$ and $v_{i j}$, remains the same in presence of an uniform acceleration field. However, the field impacts the displacements $\Delta \mathbf{r}_{i j}$ and $\Delta \mathbf{v}_{i j}$ through the flight time $T$ and the initial coordinates $r_{i j}$ and $v_{i j}$. In addition, one has to modify equations (13.5.2), for a correct calculation of the unperturbed trajectories under influence of the external field. We will now consider these aspects in more detail.

The flight time $T_{i}$ of particle $i$, with initial axial velocity $v_{2 i}\left(t_{0}\right)$, which is accelerated by an external force $F_{e}$ along the axial direction, is equal to

$$
T_{1}=\frac{m v_{z^{\prime} i}\left(t_{0}\right)}{F_{e}}\left(\operatorname{sign}\left[v_{z_{1}}\left(t_{0}\right)\right] \sqrt{1+\frac{2 F_{0}\left(z_{1}-z_{0}\right)}{m v_{z_{i}}\left(t_{0}\right)^{2}}}-1\right)
$$


assuming that $2 F_{e}\left(z_{1}-z_{0}\right) / m_{z_{i}}\left(t_{0}\right)^{2}>-1$, which means that the final axial velocity of the particle is expected to be positive. If this is not true, the particle is removed from the sample. The initial velocity $v_{z_{i}}\left(t_{0}\right)$ may here be negative. The force $F_{\theta}$, which is specified by equation (13.4.9), can be positive or negative, corresponding to an acceleration or a deceleration of the particle respectively.

The flight time $T$ of the sample as a whole, is evaluated from equation (13.5.6), taking $v_{z i}\left(t_{0}\right)$ equal to the average axial velocity $\left\langle v_{z}\right\rangle$ of all particles. The final unperturbed coordinates of particle $i$ now follow as

$$
\begin{aligned}
& \tilde{\mathbf{r}}_{i}\left(t_{0}+T\right)=r_{i}\left(t_{0}\right)+v_{i}\left(t_{0}\right) T+\frac{P_{e} T_{i}^{2}}{2 m}+\frac{P_{e} T_{i}}{m}\left(T-T_{i}+\frac{z_{i}\left(t_{0}\right)-z_{0}}{v_{z_{i}}\left(t_{0}\right)}\right) \\
& \tilde{\mathbf{v}}_{i}\left(t_{0}+T\right)=\mathbf{v}_{i}\left(t_{0}\right)+\frac{\mathbf{P}_{e} T_{i}}{m}
\end{aligned}
$$

which replaces equations (13.5.2). The last term in the expression for $\tilde{\mathbf{r}}_{i}\left(t_{0}+T\right)$ accounts for the fact that the initial drift-velocity of the particle (before reaching the entrance plane) differs by a term $P_{e} T_{i} / m$ from its final drift velocity (after passing through the exit-plane).

The displacements $\Delta \mathbf{r}_{i j}$ and $\Delta \mathbf{v}_{i j}$ are calculated in the same way as in absence of acceleration, using the appropriate flight time $T$, which can be obtained from equation (13.5.6), by replacing $v_{z i}\left(t_{0}\right)$ by $\left\langle v_{z}\right\rangle$. With respect to the initial coordinates $r_{i j}$ and $\mathbf{v}_{i j}$, the program accounts for the fact that the first particle is accelerated during some time $\Delta t$, while the last particle has not yet reached the acceleration zone. The time $\Delta t$ is calculated as

$$
\Delta t=\left\{\begin{array}{lll}
\frac{\left|z_{i}\left(t_{0}\right)-z_{j}\left(\tau_{0}\right)\right|}{v_{z_{i}}\left(t_{0}\right)} & \text { if } & z_{j}\left(t_{0}\right) \geqslant z_{i}\left(t_{0}\right) \\
\frac{\left|z_{i}\left(t_{0}\right)-z_{j}\left(\tau_{0}\right)\right|}{v_{z_{j}}\left(t_{0}\right)} & \text { if } & z_{j}\left(t_{0}\right)<z_{i}\left(t_{0}\right)
\end{array}\right.
$$

The relative position and velocity, $\mathbf{r}_{\mathbf{i j}}$ and $\mathbf{v}_{\mathbf{i j}}$, are adjusted to account for the acceleration of the first particle during the time $\Delta \mathrm{t}$.

Finally, it should be recognized that the flight time $T_{i}$, of particle $i$ through the acceleration zone between $z_{0}$ and $z_{1}$, is affected by the interaction with the other particles in the sample. The total axial shift $\Delta z_{i}$ of particle $i$, due to the interaction with the other particles is computed by

$$
\Delta z_{i}=\sum_{j \neq i}^{N_{s a m}} \Delta z_{i j}\left(t_{0}, t_{0}+T\right)
$$

conform equation (13.5.1). When $\Delta z_{i}$ is positive, the actual flight time $T_{i}$ in the acceleration zone will be shorter than predicted by equation (13.5.6) and when $\Delta z_{i}$ is negative, the actual flight time $T_{i}$ will be longer. This effect is compensated by adjusting the flight time in the calculation of the unperturbed trajectories, by means of equations $(13.5 .7)$.

As in the numerical-ray tracing routine, the coordinates of all particles are scaled at the start of the ray-tracing routine, employing the $\delta, v$-scaling defined by equation $(6.13 .1)$. This way, the occurrence of large numbers is avoided. Another advantage is that the parameters $\mathrm{C}_{0} / \mathrm{m}$ and the time of flight $\mathrm{T}$ do not appear explicitly in the scaled 
equations, which leads to some simplification of the code. Before terminating the ray-tracing routine, the scaling is removed.

The gain in computation speed using analytical ray-tracing stems from the ability to determine the final coordinates from the initial coordinates in a single step. The total computation time $T_{a}$, required for the analytical ray-tracing of $\mathrm{N}_{\text {sed }}$ samples, through $\mathrm{N}_{\mathrm{d}} \mathrm{ift}$ sections, is equal to

$$
T_{a}=N_{\text {seed }} N_{d r i f t} \frac{1}{2} N_{s a m}\left(N_{s a m}-1\right)\left\langle\Delta t_{a}\right\rangle
$$

where $\left\langle\Delta t_{a}\right\rangle$ is the average cpu-time, required to evaluate the displacements corresponding to a single two-particle interaction. The actual computation time per two-particle interaction depends on the type of dynamics which is employed. Full collision dynamics requires typically five times more computation time than weak or nearly complete collision dynamics. On an IBM 3083 mainframe computer, it was found that, typically, $\left\langle\Delta t_{a}>=5 \times 10^{-5} \mathrm{~s}\right.$. As for the numerical ray-tracing, one can increase the computation speed by restricting the interaction range of the particles to some distance $r_{i n t}$. For the analytical-ray tracing routine, this implies that the interactions of the pairs with an impact parameter $b>r_{i n t}$ are disregarded.

By comparing equations (13.4.12) and (13.5.10), one finds that the ratio of the cpu-times required for analytical and numerical raytracing $T_{a} / T_{n}$, is equal to

$$
\frac{T_{a}}{T_{n}}=\frac{\left\langle\Delta t_{a}\right\rangle}{\left\langle N_{\text {step }}\right\rangle \Delta t_{n}} \approx \frac{5}{\left\langle N_{\text {step }}\right\rangle}
$$

Thus, the computation speed improvement over the numerical method depends on the average number of steps per section $\left\langle N_{s t e p}\right\rangle$, required for the numerical ray-tracing. Typically this number varies between 10 and $10^{3}$, depending on the length of the section, the particle density and the specified integration accuracy. Accordingly, the required cputime reduces with one to two orders of magnitude when switching to analytical ray-tracing.

\subsection{Simulation of large currents near the source}

In section 13.2 it was pointed out that an accurate simulation of high-current beams, operating at a low beam potential, requires an extreme large number $\mathrm{N}_{\mathrm{sam}}$ of particles in the sample. Equations $(13.4 .12)$ and $(13.5 .10)$ show that the total computation time required for the ray-tracing increases with the square of $\mathrm{N}_{\mathrm{s}} \mathrm{m}$. In order to keep the total cpu-time within practical limits, $\mathbf{N}_{\mathbf{s}}$ am should be smaller than typically $10^{3}$ for numerical ray-tracing and smaller than $10^{4}$ for analytical ray-tracing. When the interaction range is limited to $r_{\text {int }}=N_{i n t}\langle S\rangle$, the maximum $N_{s a m}$ can be chosen larger by a factor $\mathrm{N}_{\mathrm{sam}} / 2 \mathrm{~N}_{\mathrm{int}}$, as far as cpu-constraints are concerned. However, the memory space required for the storage of the coordinates is (at least) $6 \times 8=48$ bytes per particle, leading to a practical upper limit for $\mathrm{N}_{\mathrm{s}} \mathrm{m}$ in the order of $10^{4}$.

It is important to observe that one does not need that many particles to obtain acceptable statistics. Therefore, one might as well delete a part of the sample after it has passed through the critical (high current/ low voltage) section of the beam. This consideration lead us to the introduction of so-called field particles, which are only 
used to represent the beam and are not stored to memory. The particles in the sample, now referred to as test particles, are traced throughout the system, while the field particles are generated at the start and deleted at the end of a beam section. This concept can most easily be implemented within the analytical ray-tracing routine. As the trajectories are calculated in a single time-step, analytical raytracing does not require the storage of intermediate coordinates. This implies that one can generate a field particle, determine its impact on all the test particles and delete its coordinates. The next field particle is then generated and these steps are repeated, etc. As the field particles are generated one-by-one, their number can be arbitrarily large, without requiring any additional memory space. In this set-up, the interaction between the test-particles is ignored. Thus, every test-particle is independent from the other test-particles and experiences only the interaction with the field particles. The reader should notice that this model comes very close to the analytical theory, which also considers a number of independent test-particles, experiencing the interaction with the field-particles, constituting the beam. The main difference with the analytical model, used in the chapters 7,8 and 9 , is that the motion of the test particles is not confined to the beam axis, but resembles the actual properties of the beam.

The gain in computation speed, relative to the normal analytical ray-tracing routine, arises from the fact that every field particle interacts only with a relatively small number of test particles. Let $\mathrm{N}_{\mathrm{field}}$ be the number of field particles and $\mathrm{N}_{s}$ am the number of test particles. The computation time is now proportional to $\mathrm{N}_{\mathrm{f} \text { ield }} \times \mathrm{N}_{\mathrm{sam}}$, instead of $\mathrm{N}_{\mathrm{s} a \mathrm{~m}}\left(\mathrm{~N}_{\mathrm{s} a m}-1\right) / 2$. Taking $\mathrm{N}_{\mathrm{s} a \mathrm{~m}}$ of the order $10^{2}$, one may choose Nfield as large as $10^{6}$, while keeping the cpu-time within reasonable limits.

The MC-program contains a separate analytical ray-tracing routine (referred to as DRIFT3) which was developed along these lines of thought. It is especially intended to simulate the beam in the vicinity of the source. The field particles are generated one by one, following the specifications of the source. Each field particle interacts with every test particle in the sample and is deleted afterwards. The set of field particles are centered around the same axial coordinate as the sample of test particles. The final coordinates of the test particles are stored in the usual way and can be traced throughout the remaining part of the column, using one of the other ray-tracing routines. The analytical ray-tracing with field particles has been used to simulate temperature-limited thermionic sources with currents up to $100 \mu \mathrm{A}$. In general, the results have to be viewed with some caution, since the deviations from the unperturbed trajectories can become quite large for a significant fraction of the particles, contradicting the premisses of the analytical ray-tracing method. Clearly, the method is not suited to simulate space charge limited emission, in which the trajectories of the individual test particles are strongly affected by the simultaneous action of many field particles.

\subsection{Correction of finite-size effects}

The qualification "finite-size effect" is used to denote all apparent interaction effects, present in the final results of a MCsimulation, which are related to the inadequate representation of the beam by a sample of particles of finite size. The following finite-size effects are distinguished : 
- Underestimation of the interaction effects due to an insufficient sample length $\mathrm{L}_{\mathrm{s} a \mathrm{~m}}$. In this case, a particle does not have enough "neighbours" to represents the total beam. To put it differently, the sample length does not exceed the effective interaction range of the particles by a large factor. The effective interaction range is here defined as the maximum distance for which two particles still affect each other strong enough to yield a significant contribution to the macroscopic measure for the considered interaction phenomenon, such as the defocussing distance, the energy spread and the spatial broadening. The effective interaction range depends on the linear particle density $\lambda$ and the beam geometry. In addition, it differs for the various interaction phenomena. For instance, the total Boersch effect, generated in a beam segment with a narrow crossover, stems predominantly from the crossover area, as was discussed in chapter 7 . For the simulation of this effect in this geometry, it is therefore sufficient to take $\mathrm{L}_{\mathrm{s} a \mathrm{~m}}$ an order of magnitude larger than the crossover radius. However, the trajectory displacement effect, generated in the same beam geometry, is not necessarily dominated by the contribution of the crossover area. A significant contribution may stem from the dilute parts of the beam and a proper simulation requires a larger $L_{s} a m$ than for the Boersch effect. The space charge effect has even a longer effective interaction range and a proper simulation requires a larger sample than for the statistical effects. The situation is again different for a nearly cylindrical beam segment, in which all parts of the beam contribute equally to the various interaction phenomena, leading to longer effective interaction distances than for a beam segment with a narrow crossover. In all cases the effective interaction range decreases when the average axial separation of the particles $\langle s\rangle(=1 / \lambda)$ decreases. However, both quantities do not change at the same rate and the number of "neighbours", having a significant impact, increases with the linear density $\lambda$. This implies that the minimum required $N_{s}$ am increases with $\lambda$, but, - in general, less than directly proportional. It should be noted that the particles near the edge of the beam, always experience a deficiency of interacting neighbours. $\mathrm{N}_{\mathrm{s} \text { am }}$ should be large enough to render their contribution insignificant, relative to the contribution of the particles which have sufficient neighbours.

- Improper estimation of the interaction effects due to the unbalanced space charge force acting on the particles near the edge of the bunch. It may cause an underestimation as well as an overestimation of the interaction effects. The Boersch effect is, in general, the most strongly affected by this kind of finite-size effect. The particles at the front of the bunch experience an artificial acceleration, while the particles in the back are artificially retarded. Accordingly, the generated axial velocity spread (Boersch effect) is overestimated. However, the unbalanced space charge force also causes an increase of the sample length during the flight, which leads to an artificial reduction of the linear particle density and a corresponding reduction of all interaction effects.

It is difficult to formulate general rules for the minimum $\mathrm{N}_{s a m}$ required for a proper simulation of a certain interaction phenomenon, as function of the beam geometry and the particle density in the beam. To verify the validity of an MC-simulation, one has to check whether an increase of $\mathrm{N}_{s} \mathrm{~m}$ affects the outcome of the simulation significantly or not. If so, the sample was too small and one has to perform the simulation again with a larger sample. This aspect of running MCsimulations requires a certain skill of the operator. A clear 
understanding of the mechanisms involved is indispensable to obtain reliable results, without using enormeous amounts of cpu-time.

Various approaches have been suggested to minimize finite-size effects. Jones et. al (1983) presented a method in which two so-called "ghost" charges travel with the bunch, each at one side, located on the axis. The value of the ghost charge and its distance to the bunch should be chosen such that the space charge force, acting on the particles at the edge of the bunch, is just balanced. This is the case when one takes the ghost charge equal to the total charge in the bunch and the distance to the edge of the bunch equal to half the sample length $L_{s a m}$. The drawback of the ghost charge approach is that those particles in the bunch which are off-axis, experience a lateral interaction force from the ghost charges, which causes an artificial change in the lateral properties of the bunch. Errors are therefore introduced in the simulation of the trajectory displacement effect and space charge defocussing. The remedy would be to consider an extended ghost charge, with a lateral dimension which reflects the local width of the beam. However, this seems rather difficult to implement within the ray-tracing algorithm.

The approach taken in our program is to introduce a routine (referred to as PROCCO) which processes the final velocities to compensate the artificial acceleration of the particles, caused by the unbalanced space charge effect. The routine evaluates the correlation in the final coordinates, between the z-velocities and the z-position within the bunch. When specified by the user, the final velocities are adjusted such that the $z$-dependency of the $z$-velocities is removed. The major part of the correlation is assumed to be the result of the unbalanced space charge force acting on the particles. However, some correlation is induced by the dispersion of the particles during the time of flight, as a consequence of the variation in the initial axial velocities. To put it simply, slow particles have a tendency to run behind, while fast particles have a tendency to run ahead. This source of correlation can be isolated by examining the final coordinates in absence of interaction. When specified by the user, the routine performs this calculation and compensates only for the correlation caused by the interaction between the particles.

The z-dependent velocity correction of the final coordinates removes the direct impact of the second kind of finite size effect on the axial velocity distribution. The main use of this procedure is to prevent an overestimation of the generated energy spread. One can instruct the program to calculate the energy spread both with and without processing of the final coordinates. This provides some insight in the significance of finite-size effects and is helpful in the choice of a proper sample length. It is interesting to study the dependency of the resulting energy spread on the number of particles in the sample $\mathrm{N}_{\mathrm{sam}}$. For very small $\mathrm{N}_{\mathrm{sam}}$, the energy spread will be underestimated. This can be understood from the fact that the interaction force, between the particles in a thin slice, is directed perpendicular to the beam axis. With increasing $\mathrm{N}_{\mathrm{s}} \mathrm{m}$ and in absence of correction of the final coordinates, the spread increases to a value which is too large, due to the artificial deceleration and acceleration of the particles near the edges of the bunch. With further increasing $\mathrm{N}_{\mathrm{sam}} \mathrm{m}$, their contribution gradually becomes insignificant and the observed energy spread levels off to the correct value. In presence of correction of the final coordinates, the energy spread is expected to increase monotonically with the number of particles in the sample $\mathrm{N}_{\mathrm{sam}}$ and reaches the correct value for a smaller sample-size than without correction. By studying the results both with and without correction, one obtains a lower and an upper limit for the correct energy spread at a relative small $\mathrm{N}_{\mathrm{s} a \mathrm{~m}}$. 
In extreme cases, the sample can significantly be deformed during the flight, due to the unbalanced space charge force. Clearly, the resulting errors in the simulation can not be removed by processing the final coordinates. Some improvement can be obtained by correcting the velocities during the ray-tracing, after every time step. A facility is included in the program to perform this task (which can be activated by setting the parameter IPROC=1). As for the correction of the final coordinates, this procedure gives an indication of the error caused by finite size effects and provides a partial correction.

\subsection{Data analysis}

The source routine, ray-tracing routines and optical element routines provide the means to calculate the trajectories of a sample of interacting particles through an user defined column. The output of these routines is a set of $6 \times N_{s}$ am coordinates, specifying the final positions and velocities of the $\mathrm{N}_{\mathrm{s} a \mathrm{~m}}$ particles. In order to obtain sufficient statistics, the calculation is repeated for a number of seeds $N_{\text {seed, }}$ leading to a set of $6 \times N_{\text {seed }} \times N_{\text {sam }}$ final coordinates. Typically, the total number of particles $\mathrm{N}_{\text {tot }}\left(=\mathrm{N}_{\text {seed }} \times \mathrm{N}_{5 \mathrm{am}}\right)$ is chosen between 1000 and 2000, which corresponds to 6000 to 12000 final coordinates. Clearly, some data-analysis is required to reduce this information to a limited set of numbers, specifying the characteristic properties of the beam at the target.

Several routines are implemented in the program which serve this purpose. Two routines (referred to as SYMEBR and ASYEBR) analyse the energy broadening of the particles. The first one presupposes that the energy distribution is symmetric and computes the distribution of absolute energy deviations relative to the mean energy of the particles. The second one computes the full energy distribution, which may possibly be asymmetric. The energy distribution generated by the Boersch effect is always symmetric and the first routine (SYMEBR) is appropriate for its analysis. However, the simulation of a thermionic source leads to an asymmetric initial energy distribution and one needs the second routine (ASYEBR) for an adequate analysis. For both routines, the user can specify, whether to inspect the total energy distribution (by setting ICONS $=1$ ) or the energy distribution associated with the $z$-component of the velocities (by setting ICONS $=2$ ).

Three routines (referred to as TBR, RNDTBR and RECTBR) concern the transverse broadening of the beam. The first one is a general routine, which considers the displacements from the unperturbed trajectories in a spot of arbitrary shape, while the other two are taylored for the analysis of a round (Gaussian) spot and a rectangular (shaped) spot respectively. The program is organized such that it calculates the trajectories of the particles twice, for every sample, one time with the interaction switched of $f$ and one time with the interaction switched on (unless specified otherwise by the user). Both the perturbed and the unperturbed final coordinates of the particles are stored and can be used for further analysis. The general routine (TBR) utilizes both sets of coordinates to evaluate the lateral displacements from the unperturbed trajectories in the target-plane. The other routines (RNDTBR and RECTBR) consider the perturbed positions in the target plane only. All routines have a facility to seek the plane of best focus, which is usually located somewhat ahead of the Gaussian image plane, as a result of the defocussing action associated with the space charge effect.

The basic strategy of the analysis is the same for the various 
routines. A width-measure of a distribution which can easily be evaluated is the rms-width. The rms spread of a set of $N$ scalar quantities $\eta_{i}$, is given by

$$
\left\langle\Delta \eta^{2}\right\rangle=\frac{1}{N} \sum_{i=1}^{N}\left(\eta_{i}-\frac{1}{N} \sum_{i=1}^{N} \eta_{i}\right)^{2}=\frac{1}{N} \sum_{i=1}^{N} \eta_{i}{ }^{2}-\left(\frac{1}{N} \sum_{i=1}^{N} \eta_{i}\right)^{2}(13.8 .1)
$$

The second form permits a straightforward computation of the rms-value in a single loop over the index $i$, which performs the summation of the quantities $\eta_{1}, \eta_{2}, \cdots, \eta_{N}$, as well as the quantities $\eta_{1}{ }^{2}, \eta_{2}{ }^{2}, \cdots \eta_{N}{ }^{2}$.

Unfortunately, the rms-width is, in general, not adequate to characterize the various distributions, obtained within the MC-program. It is usually dominated by a few large displacements. Therefore, it is rather sensitive to statistical fluctuations. In addition, one should realize that, particularly, the large displacements are susceptible to various kind of errors. One error is the integration error (when using DRIFT1) or the model error (when using DRIFT2) associated with the raytracing. Another error is related to the modelling of the source. Large displacements are partly generated by pairs of particles which are initially very close to each other. The question arises, whether the fully random selection of initial conditions performed by the source routine, reflects the actual emission process of a real source. The statistics of near neighbour particles is not known and the $\mathrm{MC}-$ simulation may, in this respect, be inappropriate, as will be further discussed in section 13.9. Summarizing, the rms-value is sensitive to statistical errors, ray-tracing errors and source-modelling errors and the numbers obtained from equation (13.8.1) should be viewed with caution. From the experimental point of view, one should add, that predicting a rms-value is not very practical either, since it can not directly be measured.

A reliable width-measure, which can straightforwardly be obtained within the MC-program, is the smallest Full width $\mathrm{FW}_{\mathrm{f}}$ which contains a certain fraction $f$ of the particles. The program considers the cases $f=0.1,0.3,0.5,0.7$ and 0.9 . The width corresponding to $\mathrm{f}=0.5$ is referred to as the Full width median value ( $\mathrm{FW}_{50}$ ). For the computation of this type of width measures, the program has to sort the displacements (in energy or position relative to some mean value) on size in ascending order. After sorting, the $\mathrm{FW}_{\mathrm{f}}$ value of a symmetric distribution can simply be obtained as

$$
F W_{f}=2\left|\Delta \eta\left(\mathrm{fN}_{\mathrm{tot}}\right)\right|
$$

in which $\left|\Delta \eta\left(f N_{t o t}\right)\right|$ denotes the absolute displacement (from the mean value) of the particle with the nearest integer number to $f \times N_{t o t}$ in the array of sorted displacements. For a distribution, which is possibly asymmetric, the program evaluates the set of quantities

$$
F W_{f}, i=\Delta \eta\left(i+f N_{t o t}\right)-\Delta \eta(i), \quad i=1,2, \cdots,(1-f) N_{t o t}
$$

and takes $\mathrm{FW}_{\mathrm{f}}$ equal to the minimum value.

Sorting algorithms are described in detail by knuth (1973). A comprehensive discussion is given by Press et al. (1986). The evaluation of equation (13.8.2) does not require a completely ordered array of displacements. It only requires that element $f \times N_{t} t$ is on the right position in the array. The MC-program exploits a modified "quicksort" routine to perform this task. The routine differs from the normal quicksort algorithm in the respect that it does not order every subfile defined during the sorting process, but proceeds with that subfile which 
contains element number $\mathbf{f} \times \mathrm{N}_{\mathrm{tot}} \mathrm{t}$ and ignores the others. This procedure provides an extreme fast evaluation of equation (13.8.2), even for large $\mathrm{N}_{\mathrm{tot}}$. This speed is required to perform the focussing of the spot within acceptable computation time. The focussing is carried out by an iteration-loop, which evaluates equation (13.8.2) for various $z$-values in order to determine the plane for which the width of the spatial distribution becomes minimum. Contrary to equation (13.8.2), the evaluation of equation (13.8.3) requires a completely ordered array of displacements. Shell's method is used to perform this task. This algorithm is fast enough, since it is only called once by the routine used for the evaluation of an asymmetric energy distribution (ASYEBR).

other width-measures than the rms-value and the $\mathrm{FW}_{\mathrm{f}}$ value are, in general, more difficult to evaluate. The program has a provision to calculate the FWHM, utilizing a least square polynomial fit of the corresponding histogram. A similar method is employed to estimate the edge-width of a shaped spot. Histograms are formed by the program, following the specifications of the user. The input required by the program is the number of divisions (or strips) in the histogram and the largest displacement to be included in the histogram. The last value is specified in terms of the fraction of particles to be represented in the histogram or in terms of the ratio between the maximum displacement in the histogram and the Half Width at Half Maximum (HWHM) value of the distribution. The program automaticly selects a division width, conform these specifications. In the case of a shaped spot, one may alternatively select the number of divisions in the edge of the spot, instead of the total number of divisions.

A dedicated least square fit procedure has been developed to provide an optimum fit of the histograms. Chosen is for a polynomial fit function, for reasons of flexibility and speed. The general form of the polynomial used by the program, is given by

$$
\left.y(x)-y_{s}=\sum_{j=1}^{M} a_{j}\left(x-x_{s}\right)\left[n_{1}(M-j)+n_{2}\right)\right] / n_{3}
$$

where $n_{1}, n_{2}$ and $n_{3}$ are constants. The user has to specify the number of terms $M$ and the type of polynomial function. The following types are included :

$\begin{array}{llll}\text { normal } & \text { polynomial : } & n_{1}=1, n_{2}=0, n_{3}=1 \\ \text { even } & \text { polynomial : } & n_{1}=2, n_{2}=0, n_{3}=1 \\ \text { odd } & \text { polynomial : } & n_{1}=2, n_{2}=1, n_{3}=1 \\ \text { reduced } & \text { polynomial : } & n_{1}=1, n_{2}=0, n_{3}=(M-1) \\ \text { reduced odd polynomial : } & n_{1}=2, n_{2}=1, n_{3}=(2 M-1)\end{array}$

Even polynomials are recommended to fit a symmetric distribution. Odd polynomials, reduced polynomials and reduced odd polynomials are included to fit the edge of a shaped spot. The shifts $x_{s}$ and $y_{s}$ are chosen by the program. In most cases the program takes $x_{s}=y_{s}=0$, but for the $f i t$ of the edge of a shaped spot, the values for $x_{s}$ and $y_{s}$ are chosen such that the origin is centred in the edge. The expectation is that the edge follows an odd function with respect to this origin. An additional feature of the fit algorithm is that one may specify a value $y\left(x_{0}\right)$, of the polynomial at $x_{0}$ and a value $y^{\prime}\left(x_{1}\right)$, of its derivative at $x_{1}$, which are imposed on the fit. This feature is included for a shaped spot, to obtain a continuous and smooth joint of the fit through the edge and the intensity level at the centre of the spot. For the fit of a central distribution, this feature can be used to impose that the fitfunction becomes zero and/or has zero derivative in the last division of 
the histogram. A description of the fit-algorithm is presented in appendix 13.B.

The program has a facility to estimate the statistical error in the calculated rms-width and $\mathrm{FW}_{50}$-width of the energy distribution and the distribution of transverse displacements. It estimates the distribution width for a number of sub-ensembles, selected from the total ensemble of $\mathrm{N}_{\mathrm{t}} \mathrm{t}$ particles. The variance in the results, obtained for the individual sub-ensembles, is used to estimate the statistical error of the result for the total ensemble. The number of sub-ensembles $N_{s u b}$ is taken equal to the nearest integer of $N_{t} t^{1 / 2}$. This way, the number of sub-ensembles $\mathbf{N}_{\mathbf{s u b}}$ equals, approximately, the number of particles per sub-ensemble $\mathrm{N}_{\mathrm{p}}=\mathrm{N}_{\mathrm{tot}} / \mathrm{N}_{\mathrm{sub}}$. The minimum total number of particles $N_{\text {tot }}$, required to activate this procedure, is 100 . For each sub-ensemble, the program calculates the corresponding rms-width and FWso-width value, using equations similar to (13.8.1) and (13.8.2) respectively. The error in the rms-value, obtained for the total ensemble, is now estimated as

$$
\sigma_{r m s}=\left(\frac{\left\langle r m s^{2}\right\rangle_{s e}-\left\langle r m s s_{s e}\right.}{N_{s u b}}\right)^{1 / 2}
$$

where $\langle. .\rangle_{s e}$ denotes an average over the results obtained for the individual sub-ensembles. Equation (13.8.6) exploits that the statistical error in the rms-value is expected to decrease with the square root of the number of particles in the ensemble. The program prints out the relative 3 -sigma error $3 \sigma_{\mathrm{rms}} / \mathrm{rms}$. The same procedure is used tot estimate the relative 3-sigma error in the FW50-width, obtained for the total ensemble.

Finally, we should mention that there is some subtlety in the algorithm used to determine the plane of best focus from the distribution of transverse displacements (which is incorporated in TBR). Denoting the perturbed and the unperturbed lateral position at $z$ as $\mathbf{r}_{\perp}(z)$ and $\tilde{\mathbf{r}}_{\perp}(z)$ respectively, the program seeks the $z$-coordinate for which the width of the distribution of displacements $\rho\left(\left|\mathbf{r}_{\perp}(z)-\tilde{r}_{\perp}\left(z_{\mathbf{r} e f}\right)\right|\right)$ becomes minimum. The axial coordinate $z_{r}$ f defines the location of the Gaussian image plane and should be specified by the user. Notice that the algorithm computes the transverse displacement of a particle, as the lateral distance between its perturbed position at $z$ and its unperturbed position in the original Gaussian image plane, which is located at $z_{r}$ f .

\subsection{Accuracy limitations of the MC-program}

The accuracy of an MC-simulation is limited by the occurrence of several types of errors. Assuming that trivial errors, such as errors in the program code, errors in the input data and errors due to program abuse by ignorant operators are absent, the following error-categories remain :

- Mode1 errors. The representation of the beam and the simulation of optical components within the MC-program relies on a number of simplifying assumptions. All optical components are assumed to be thin and fully described by their first and third order optical properties and chromatic aberration constants. External fields, other than an uniform acceleration field, are assumed to be absent. Stray-fields, mechanical vibrations and electrical charging effects are ignored. Clearly, this list can easily be extended with a number of other obvious simplifications. Less trivial is the model-error in the formation of the sample in the vicinity of the cathode surface. The 
particles are assumed to be randomly distributed over phase-space, conform the macroscopic properties of the beam. Any correlation in the initial coordinates of the particles is ignored. Various authors have questioned the validity of MC-simulations on the ground that this leads to an unrealistic distribution of the potential energy of the particles. For instance, Rose and Spehr (1983) stated that the MC program allows more than one particle in the same initial position simultaneously, which obviously does not correspond to reality. However, since the source-routine exploits a pseudo-random number generator with a cycle of the order $10^{9}$, there is no risk of selecting two identical initial positions as long as the total number of particles is smaller than say $10^{8}$. Nevertheless, the initial positions can be very close to each other, leading to the generation of pairs of particles with an extreme large potential energy. For that reason one should disregard large displacements in the data-analysis. This is done by evaluating those width-measures, which do note depend on the exact tails of the distribution, such as the Full Width median value $\left(\mathrm{FW}_{50}\right.$ ) and the Full Width at Half Maximum (FWHM).

- Ray-tracing errors. The numerical ray-tracing is affected by an integration error, which depends on the size of the time-step $\Delta t$. The local integration error, in the positions and velocities, is of the third order in the time step $\Delta t$, as can be seen from equations (13.4.7). Therefore, the total integration error is of the second order in $\Delta t$. This implies that an increase of the integration accuracy by a factor $A$, requires a factor $A^{1 / 2}$ more time steps, leading to a corresponding increase in cpu-time. The total integration error is estimated by the program from equation (13.4.7) and the user can directly verify, whether the accuracy of the ray-tracing has been sufficient or not. The errors generated by the analytical ray-tracing are related to the reduction to pair interactions. The calculation of the displacements, within the two-particle model, can be considered as exact. The reduction to pair interactions is justified as long as the average number of strong interactions per particle, experienced during the flight, is small. This number is counted by the analytical ray-tracing routine and is printed out, which provides an indication of the validity of the Fast Monte Carlo (FMC) approach. In this connection, it should be noted that the errors in the final coordinates, due to the occurrence of strong collisions, are of a stochastic nature, which has favorable effect on the overall accuracy of the FMC approach. Furthermore, it was found that the error in the prediction of the statistical properties of the beam (like the width of the energy and trajectory displacement distribution) is significantly smaller than the error in the exact final coordinates of the individual particles. See Jansen (1987), for a more detailed discussion on this topic.

- Statistical errors. The accuracy of the estimation of the statistical properties of the beam is related to the total number of particles $\mathrm{N}_{\text {tot }}$, accumulated in all seeds $\left(\mathrm{N}_{\mathrm{tot}}=\mathrm{N}_{\text {sam }} \times \mathrm{N}_{\text {seed }}\right)$. The statistical error in the rms-value and the $\mathrm{FW}_{50} \mathrm{v}$ value of the energy and trajectory displacement distribution are estimated within the program, which allows a direct verification by the user. As an additional test, one can run the same simulation a number of times, using different start values for the random number generator. By observing the variation of the final results, one obtains some idea of the statistical error involved. In order to improve the statistical accuracy by a factor $A$ one has to increase $N_{t o t}$ by a factor $A^{2}$. This should be done by running more seeds. Accordingly, the cpu-time increases with a factor $A^{2}$, as can be seen from equations $(13.4 .12)$ and $(13.5 .10)$.

- Finite size errors. The errors related to the finite size of the 
sample were discussed in section 13.7. The application of z-dependent velocity correction, on the intermediate coordinates or the final coordinates, leads to some reduction of these effects and provides insight in their magnitude. In general, one should choose the number of particles in the sample $\mathbf{N}_{s}$ am large enough to assure that these effects are insignificant. An increase of $\mathrm{N}_{s} \mathrm{~m}$ by a factor $\mathrm{A}$, leads to an increase of the required cpu-time by factor $A^{2}$, assuming that the interaction range of the particles is not limited.

As far as cpu-time is concerned, one sees that the reduction of finite-size errors and statistical errors both lead to an quadratic increase with a certain accuracy improvement factor $A$, whereas the integration error of the numerical ray-tracing shows a square-root dependency on $A$. Therefore, when using numerical ray-tracing, it is a good strategy to choose the nominal number of steps per meter $\mathrm{N}_{\mathrm{step}}$ large enough, to rule out the contribution of integration errors. In order to run the program economically, one should take $\mathrm{N}_{\mathrm{sam}}$ and $\mathrm{N}_{\mathrm{sead}}$ such, that the finite size error and the statistical error are of the same magnitude.

Clearly, model errors are more difficult to estimate. The major problems occur with the simulation of the beam in the source area, as can be understood from the preceding analysis. Disregarding model errors, the accuracy, obtained for the defocussing distance and the FW50 values of the energy and spatial distribution, is estimated to be better than $10 \%$, for normal operating conditions. The accuracy of the calculated FWHM values, is in general somewhat worse, which is related to the use of the polynomial fit procedure and the reliability of the estimation of the central height of the distribution. The accuracy of the predictions for the edge-width of a shaped spot, depends strongly on the number of particles constituting the edge. In general, an accurate estimation of the edge-width requires a substantially larger number of particles $\mathbf{N}_{t_{0}}$, than the estimation of the FW $_{50}$ or FWHM width of a central distribution.

\subsection{MC-simulation versus analytical modelling}

Our main objective to develop a MC simulation program, was to obtain a method to verify the fundamental assumptions underlying the analytical theory. However, the MC program is, in itself, a very useful tool in the design of electron and ion beam columns. Comparing the pro's and con's of the analytical theory and the numerical MC-modelling, brings out the following conclusions:

- An accurate analytical theory provides insight into the mechanism of the interaction effects and explicitly shows the dependency on the experimental parameters. The MC-approach is less suited to gain any physical appreciation for the different phenomena of particle interactions.

- When implemented in a program, the analytical theory can be used for a practically instantaneous evaluation of a column design. It is therefore very suited for the process of design and optimization. This is a clear advantage over the MC-simulation, which is in fact a rather cumbersome tool for the optimization of a column. Every change in the system parameters requires a new simulation. Performing somekind of optimization, therefore takes a lot of cpu-time (and patience of the operator).

- The analytical theory is less suited to provide accurate predictions for the impact of particle interactions in a complex beam geometry, consisting of many, possibly non-rotational symmetric beam segments. 
The MC method is for that purpose preferred.

- The combination of an accurate analytical theory and a MC simulation program is very convenient in the design of high-current and/or lowvoltage particle beam systems. The analytical theory is indispensable in the process of optimizing a column. The MC simulation program is most suited to produce an accurate evaluation of the final design and may serve as a backup of the analytical predictions.

\subsection{Program organization and examples}

In order to demonstrate the organization and performance of the MC program we will, as an example, consider the simulation of the Perkin-Elmer AEBLE 150 shaped beam lithography column. The column is schematically drawn in figure 13.1. Most of the input data, required for the MC program, was obtained from Veneklasen (1985). However, some numbers had to be guessed and the column dimensions and lens settings used in the simulation may differ slightly from the actual values.

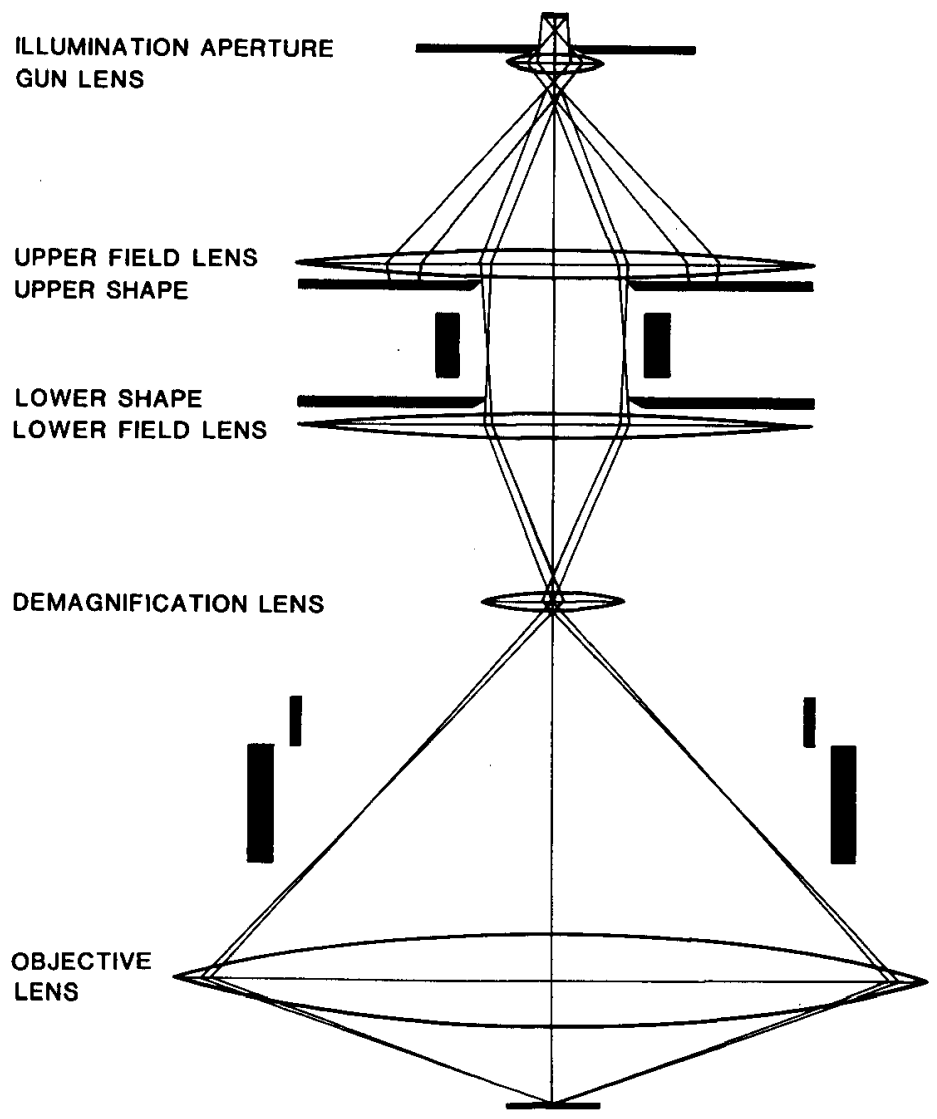

Pigure 13.1 Schematic view of the Perkin-E1mer AEBLE 150 Column. 


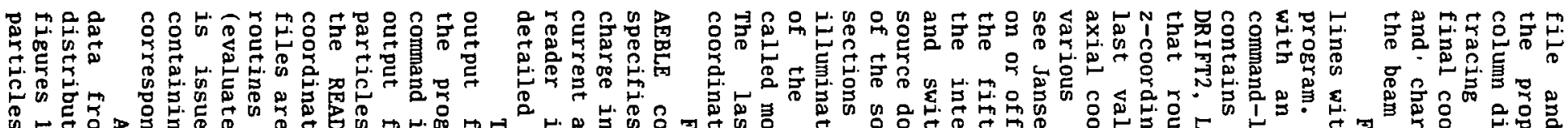

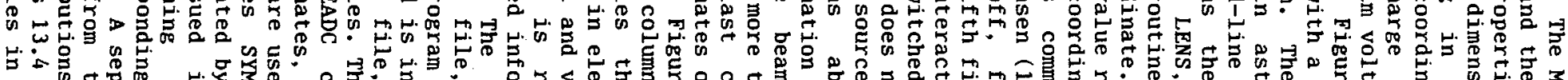

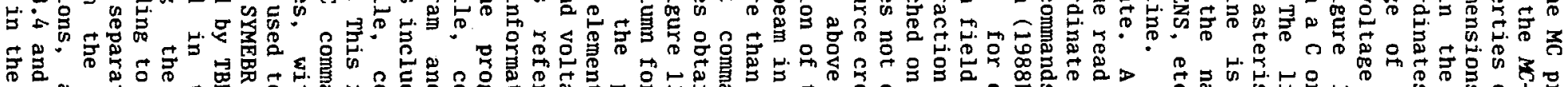

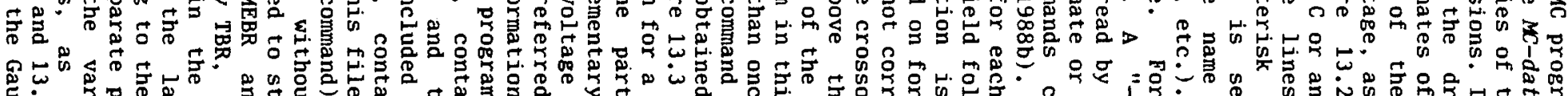

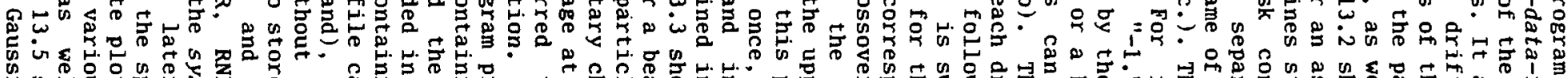

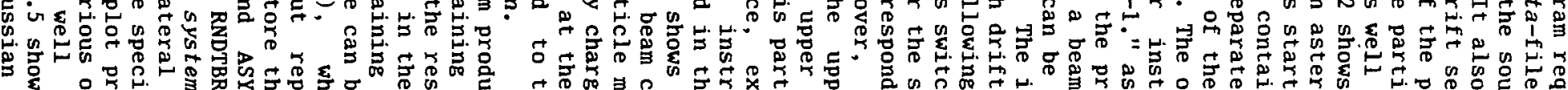

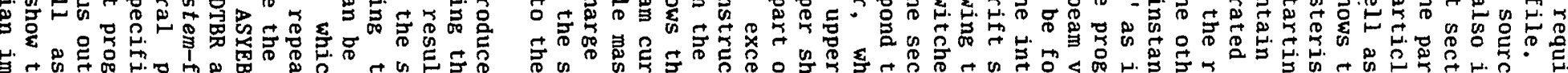

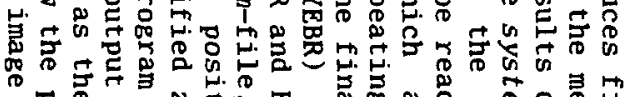

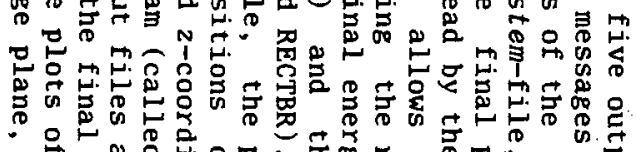

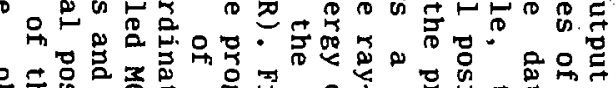

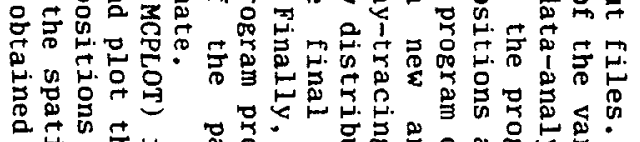

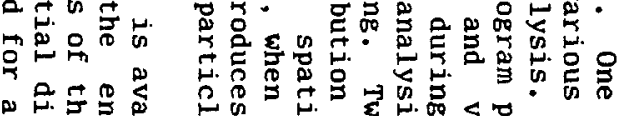

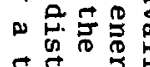

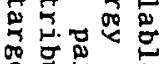

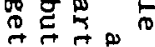

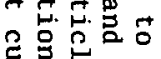

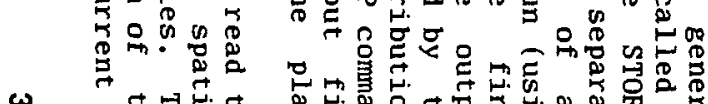

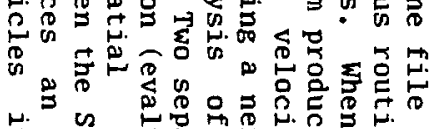

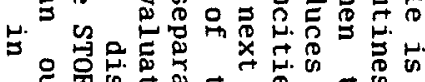

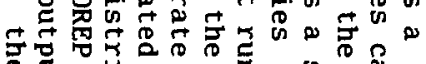

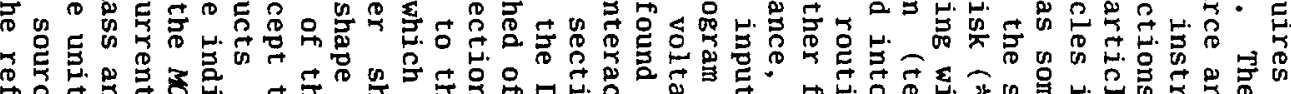

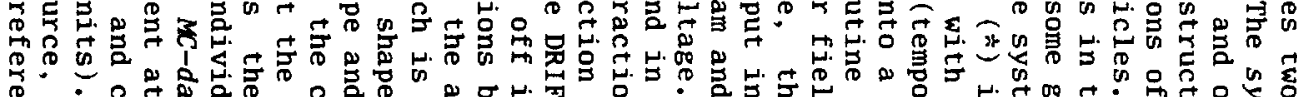

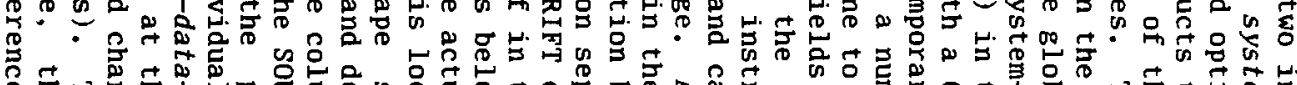

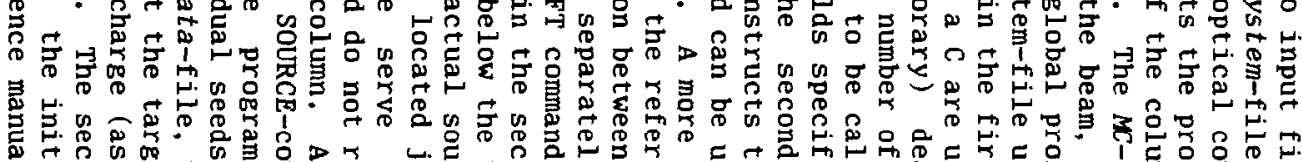

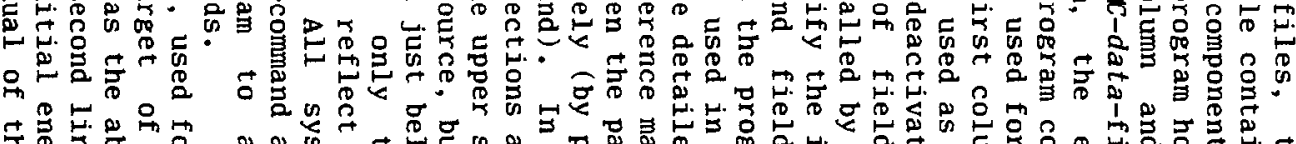

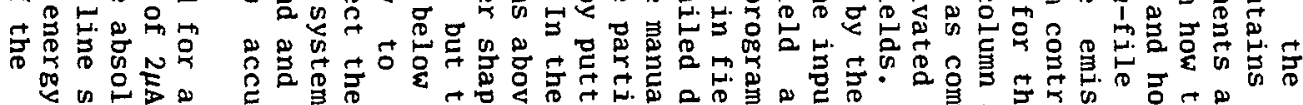

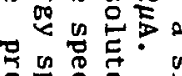

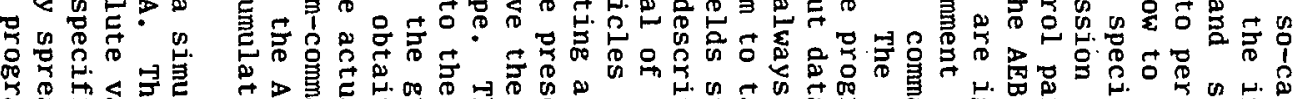

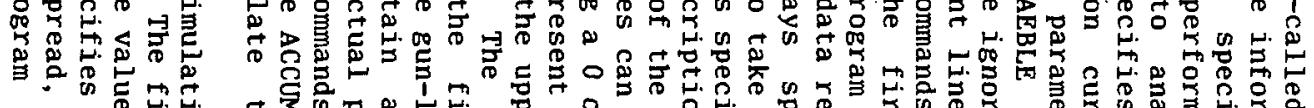

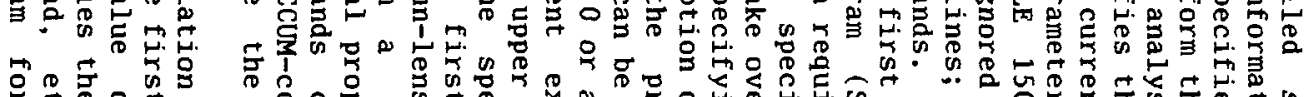

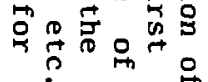

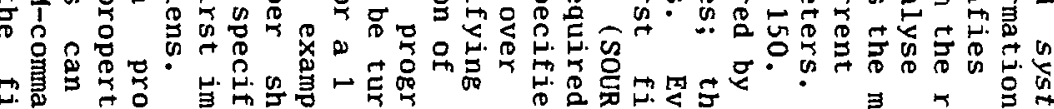

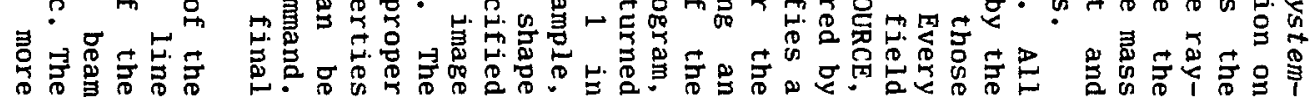




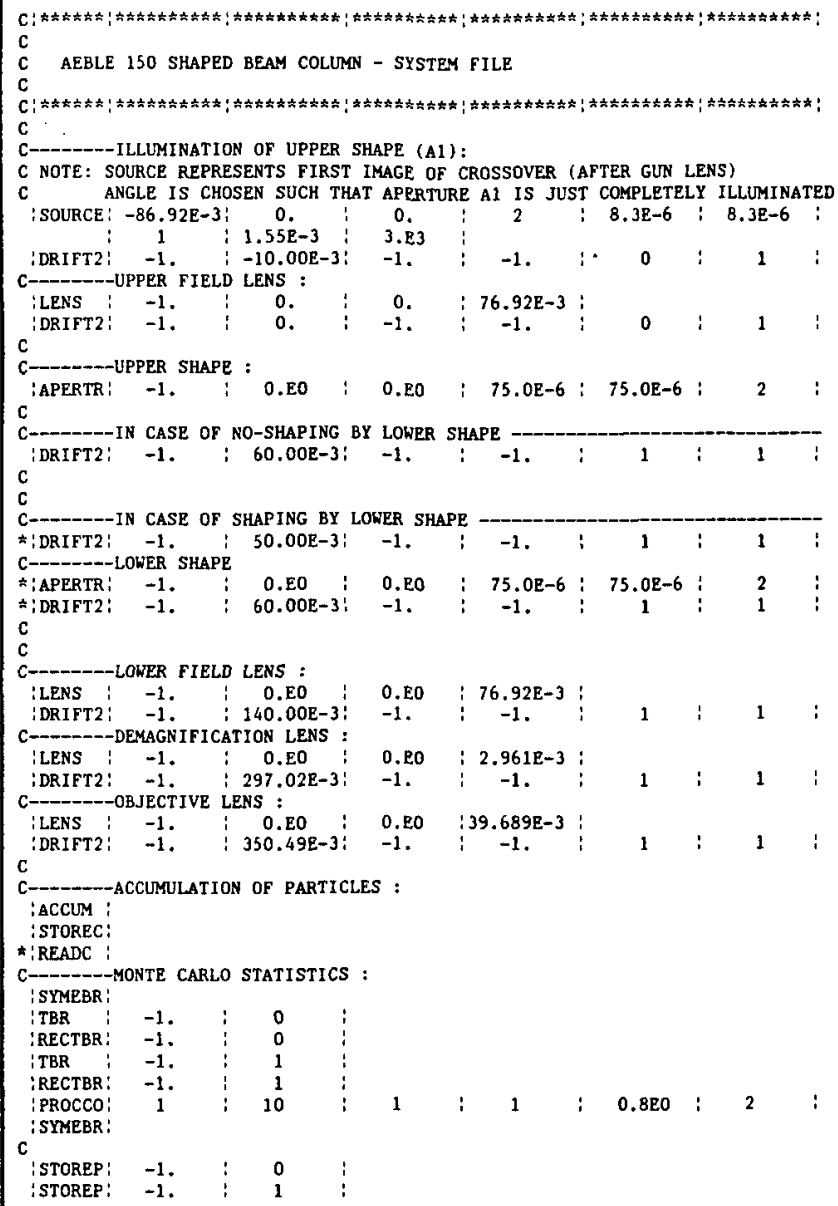

Figure 13.2 System-file for the MC simulation of the AEBLE 150 Column.

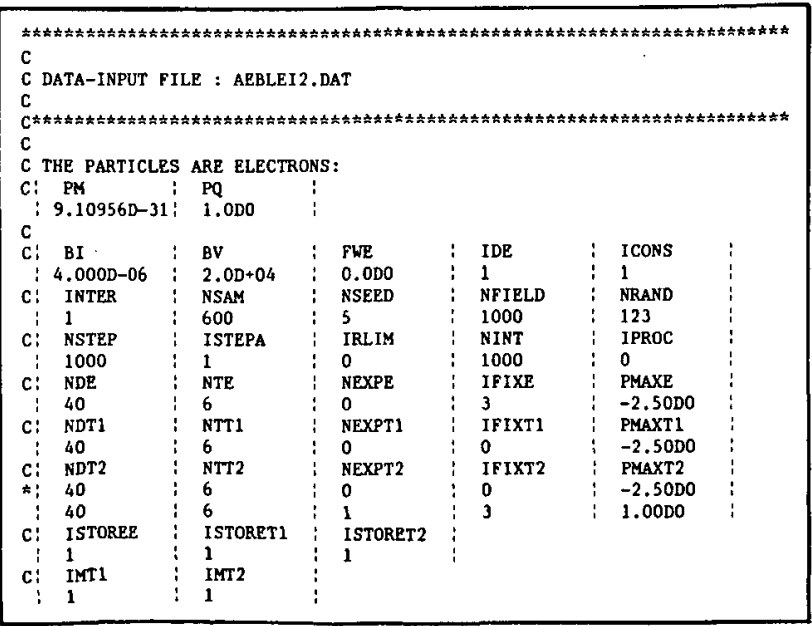

Figure 13.3 M-data-file for the MC simulation of the AEBLE 150 Column. 
2 and $5 \mu \mathrm{A}$ respectively. The edge-width of the spot is determined by the combined action of the space charge and the trajectory displacement effect. There are no other contributions to the edge-width, since all aberration coefficients of all lenses are taken equal to zero. Figure 13.6 compares the intensity distribution in the Gaussian image plane and the plane of best focus, for the considered cases of a beam current of 2 and $5 \mu \mathrm{A}$ at the target. The remaining edge-width in the plane of best focus is mainly due to the trajectory displacement effect. Figure 13.7 shows the energy distribution at the target for the 2 and $5 \mu \mathrm{A}$ case. As the energy spread at the source was taken equal to zero, the broadening is entirely the result of the interaction between the particles in the part of the beam below the upper shape. More results on the performance of the AEBLE 150, as well as the IBM EL3 shaped beam column, are presented by Jansen (1988a).
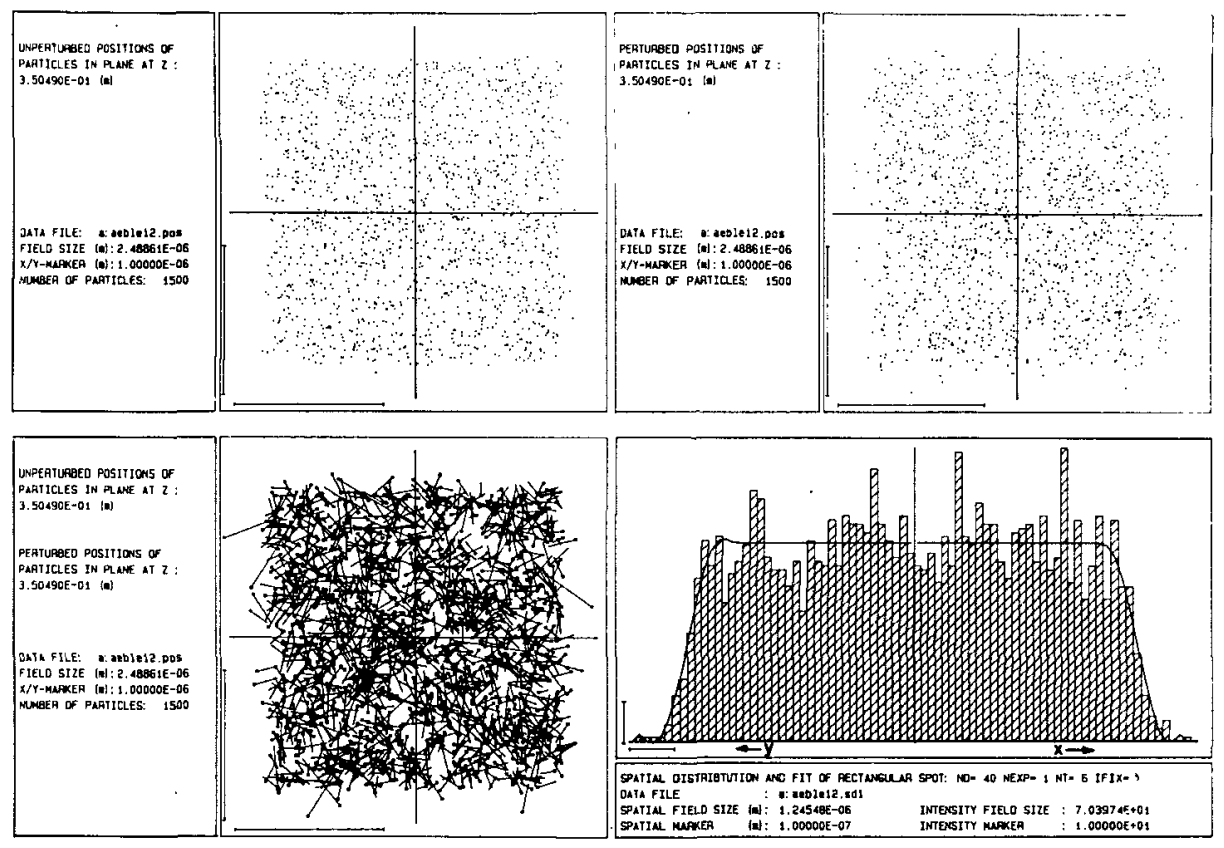

Figure 13.4 Particle positions in the Gaussian image plane of the AEBLE 150 Column for a $2 \mu \mathrm{A}$ beam current. The plots at the upper left and the upper right show the unperturbed and the perturbed positions respectively. The plot at the lower left shows the displacements from the unperturbed positions (the squares indicate the perturbed positions). The bars in the lower left corner of these plots is equal to $1 \mu \mathrm{m}$. The histogram at the lower right is formed by projecting the perturbed positions to the $x$-axis (left side of the histogram) and the y-axis (right side of the histogram) and folding the projected positions with respect to the origin. The horizontal bar in the left corner is equal to $0.1 \mu \mathrm{m}$. The vertical bar represents 10 particles. The edges are fitted by the $x$ program using a normal 6 th order polynomial function, imposing that the function itself as well as its derivative are connected continuously to the centre intensity level of the spot. 


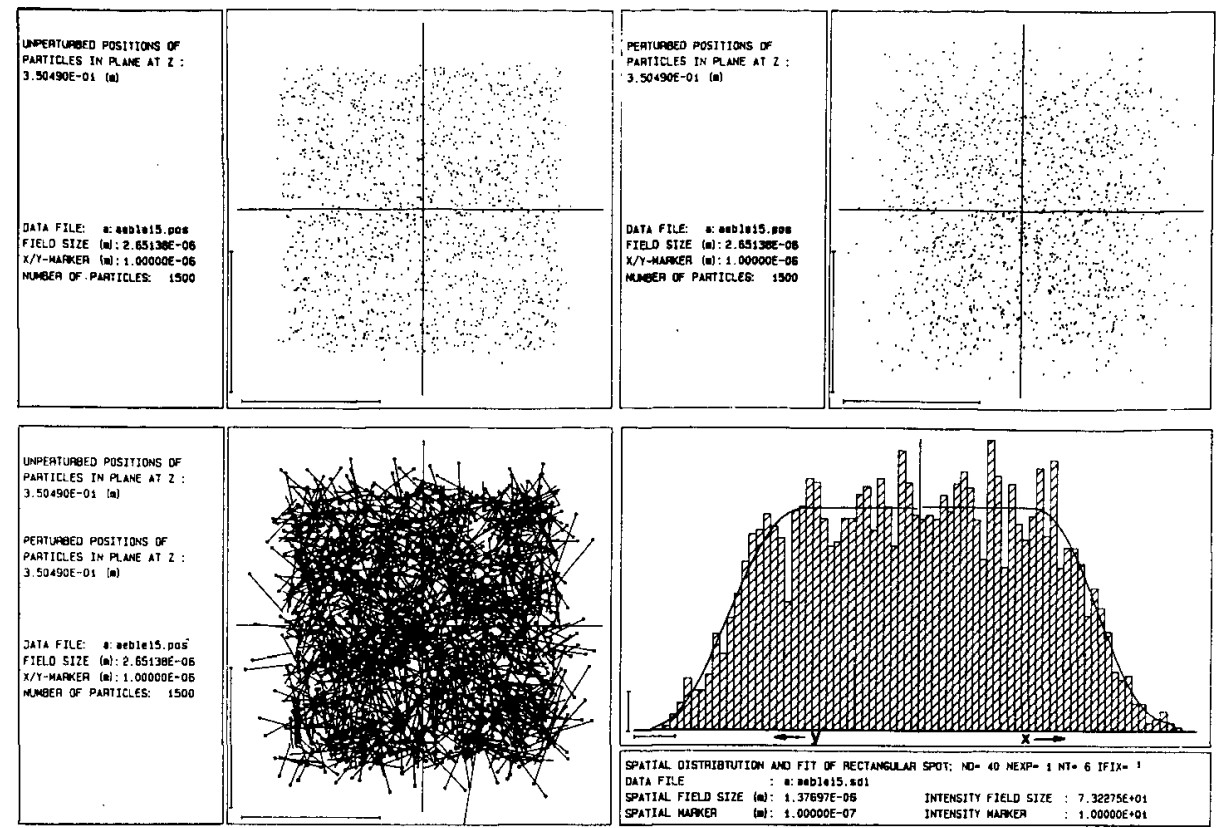

Figure 13.5 Particle positions in the Gaussian image plane of the AEBLE 150 Column for a $5 \mu \mathrm{A}$ beam current. See the caption of figure 13.4 for further details.
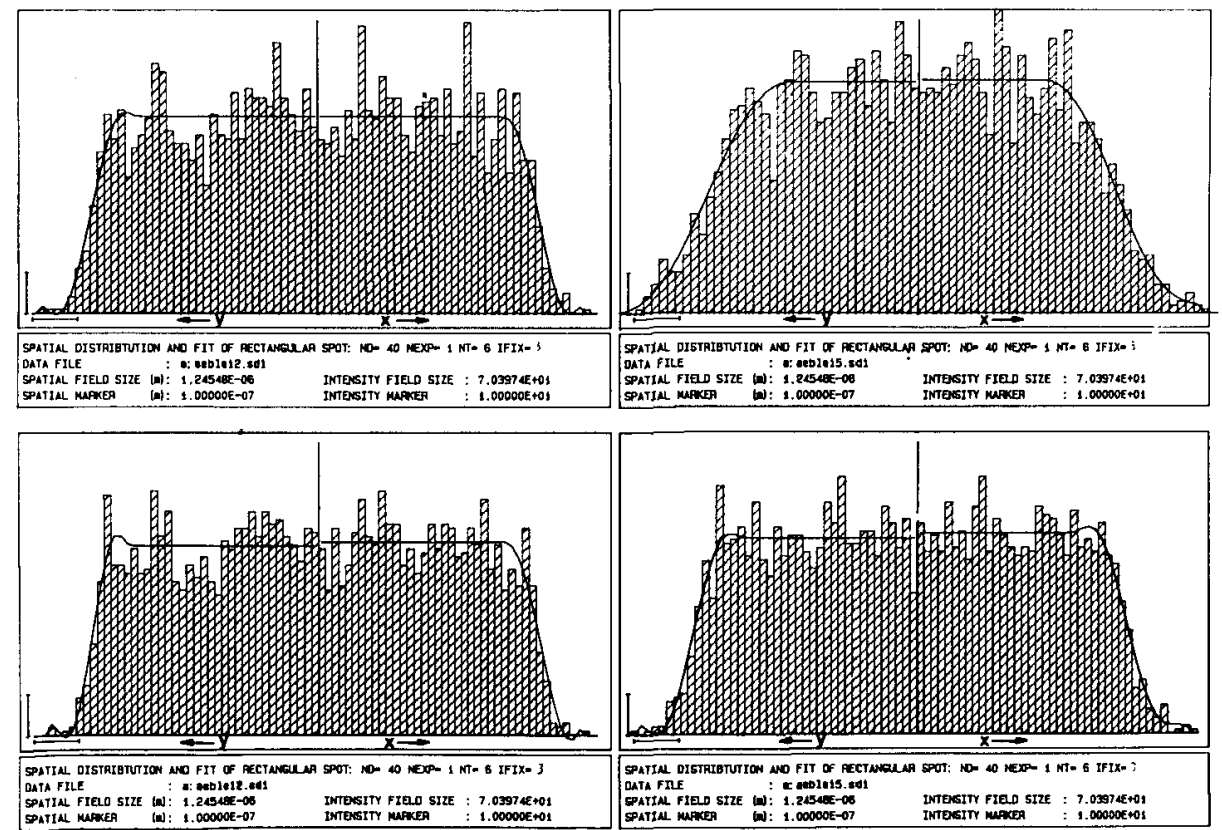

Figure 13.6 Histograms for the final particle positions in the AEBLE 150 Column. The histograms at the left pertain to a $2 \mu \mathrm{A}$ beam current. Those at the right to a $5 \mu \mathrm{A}$ beam current. The upper histograms are formed in the Gaussian image plane and the lower ones in the plane of best focus, which is evaluated by the MC program by seeking the plane for which the edge-width becomes minimum. 

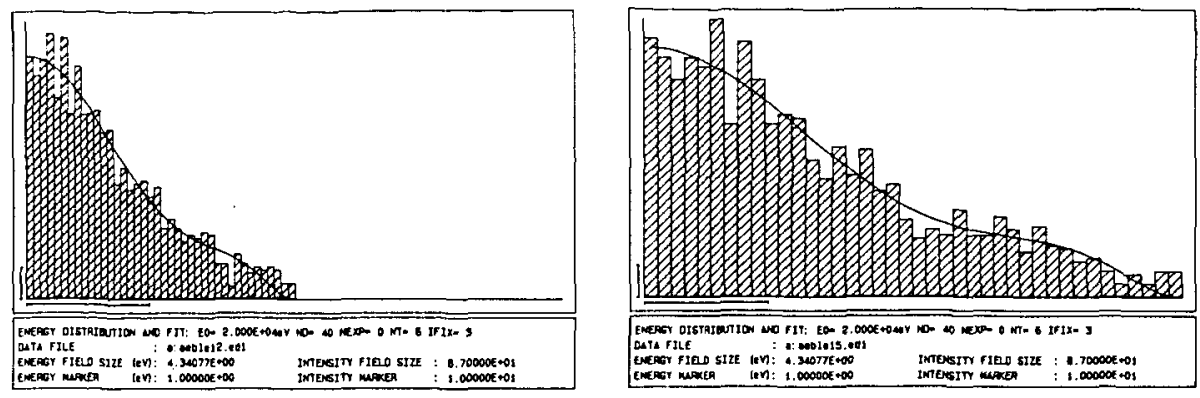

Fieure 13.7 Energy distributions generated in the section of the AEBLE 150 Column below the upper shape for a $2 \mu \mathrm{A}$ beam current (left plot) and a $5 \mu \mathrm{A}$ beam current (right plot). The distributions are plotted on the same scale. The horizontal bar indicates a $1 \mathrm{eV}$ energy spread. The vertical bar represents 10 particles. The energy range included in the histograms is chosen by the $M$ program such that the maximum energy is equal to 2.5 times the Half Width at half Maximum (HWHM) energy width (Thus, the histograms do not contain all particles). The distributions are fitted by the $M$ program using an even polynomial function of 6 terms, imposing that the function itself as well as its derivative are zero in the last division of the histogram.

\section{Appendix 13.A Random number routine}

For the generation of pseudo-random numbers the program employs the so-called linear congruential method, which is described in various textbooks on computer programming. See, for instance, Knuth (1969). Given a number $X_{1}$, the next number $X_{i+1}$ in the sequence is determined, using

$$
X_{i+1}=\left(a X_{i}+c\right) \bmod m
$$

where $a, c$ and $m$ represents some carefully chosen "magic numbers". The constant $a$ is called the multiplier, $c$ the increment and $\mathrm{m}$ the modulus. The program uses

$$
a=65539, c=0, m=2^{31}-1=2147483647
$$

which has a period (or repeating cycle) equal to $\mathrm{m}$. For the start value $\mathrm{X}_{0}$, one has to choose some number between 1 and $\mathrm{m}$. In order to obtain numbers $R_{1}$, which are uniformly distributed between 0 and 1 , the program computes

$$
R_{i+1}=X_{i+1} / m
$$

Double precision reals are used to represent the quantities $a, m$ and $R_{i}$, which guarantees that the routine is machine independent. Clearly, this causes some loss in computation speed, but this is irrelevant in our application.

Equations (13.A.1), (13.A.2) and (13.A.3) constitute the scheme for the generation of an one-dimensional uniform distribution. In 
addition to this distribution, the source routine requires the simulation of a Gaussian, a Lorentzian and an exponential distribution. Furthermore, these distributions can either be one-dimensional or rotational-symmetric two-dimensional. For the latter, one wishes to simulate the distribution of the radial coordinate. In the remaining part of this appendix, we will outline a method to transform a sequence of random numbers $R$, which are produced by the scheme of equations (13.A.1) and (13.A.3), into either a sequence of random numbers $R_{1}$, which follows some one-dimensional distribution $f_{1}\left(R_{1}\right)$, or into a sequence of random numbers $R_{2}$, which follows some two-dimensional distribution $f_{2}\left(R_{2}\right)$. Let us denote the one-dimensional uniform distribution generated by equations (13.A.1) and (13.A.3) as $f_{1 u}(R)$

$$
f_{1 u}(R)= \begin{cases}1 & \text { for } 0<R<1 \\ 0 & \text { for } R<0 \text { or } R \geqslant 1\end{cases}
$$

The problem is to generate the following distributions

$$
\begin{aligned}
& f_{2 u}\left(R_{2}\right)=\left\{\begin{array}{ll}
1 & \text { for } 0<R_{2}<1 \\
0 & \text { for } R_{2}<0 \text { or } R_{2}>1
\end{array} \quad\right. \text { (2-dim. uniform) } \\
& f_{1 G}\left(R_{1}\right)=\frac{1}{(2 \pi)^{1 / 2} \sigma} e^{-R_{1} 2 / 2 \sigma^{2}} \quad(1-\text { dim. Gaussian }) \\
& f_{2 G}\left(R_{2}\right)=\frac{1}{2 \pi \sigma^{2}} e^{-R_{2} 2 / 2 \sigma^{2}} \\
& \text { (2-dim. Gaussian) } \\
& f_{1 L}\left(R_{1}\right)=\frac{1}{\pi A_{1}\left[1+\left(R_{1} / A_{1}\right)^{2}\right]} \quad(1-\text { dim. Lorentzian) } \\
& f_{2 L}\left(R_{1}\right)=\frac{1}{2 \pi A_{2}^{2}\left[1+\left(R_{1} / A_{2}\right)^{2}\right]^{3 / 2}} \text { (2-dim. Lorentzian) } \\
& f_{1 e}\left(R_{1}\right)=\frac{1}{B} e^{-R_{1} / B} \quad \text { for } R_{1}>0 \quad \text { (1-dim. exponential) (13.A.10) }
\end{aligned}
$$

For each of the one-dimensional distributions, an auxiliary function $I_{1}\left(R_{1}\right)$ is defined as

$$
I_{1}\left(R_{1}\right)=\int_{0}^{R_{1}} f_{1}(x) 2 d x
$$

Similarly, for each of the two-dimensional distributions, an auxiliary function $I_{2}\left(R_{2}\right)$ is defined as

$$
I_{2}\left(R_{2}\right)=\int_{0}^{R_{2}} f_{2}(x) 2 \pi x d x
$$

For the one-dimensional uniform distribution, specified by equation (13.A.4), one finds from equation (13.A.11) that $I_{1}(R)=R$. In order to transform a random number $R$ into $R_{1}$ or into $R_{2}$, we will exploit the fact that each distribution is normalized to unity. This implies

$$
\begin{array}{lll}
I_{1}\left(R_{1}\right)=I_{1}(R)=R & \Rightarrow & R_{1}=I_{1}-1(R) \\
I_{2}\left(R_{2}\right)=I_{1 u}(R)=R & \Rightarrow & R_{2}=I_{2}-1(R)
\end{array}
$$


where the functions $I_{1}^{-1}$ and $I_{2}-1$ denote the inverse functions of $I_{1}$ and $\mathrm{I}_{2}$ respectively. With the equations (13.A.11) and (13.A.12), one obtains from equations (13.A.5) to (13.A.10)

$$
\begin{aligned}
& I_{2 u^{-1}}(R)=\sqrt{R} \\
& \text { (2-dim. uniform) } \\
& I_{1 G^{-1}}(R)=\sqrt{2} \sigma \operatorname{erf}^{-1}(R) \quad(1-\operatorname{dim}, \text { Gaussian }) \\
& I_{2 G^{-1}}(R)=\sigma \sqrt{-2 \ln (1-R)} \quad \text { (2-dim. Gaussian) } \\
& I_{1 L}-1(R)=A_{1} \tan (\pi R / 2) \\
& \text { (1-dim. Lorentzian) } \\
& I_{2 L}-1(R)=A_{2} \sqrt{(1-R)^{-2}-1} \\
& \text { (2-dim. Lorentzian) } \\
& \mathrm{I}_{1 \mathrm{e}^{-1}(\mathrm{R})}=-\mathrm{B} \ln (1-R) \\
& \text { (1-dim. exponential) (13.A.19) }
\end{aligned}
$$

which solves the problem. Random numbers $R_{1}$ or $R_{2}$ can be generated from a sequence of uniformly distributed numbers $R$, using equation (13.A.13) in combination with the appropriate function chosen from equations (13.A.14) to (13.A.19).

The transformations outlined above, involve only elementary functions, except for the one-dimensional Gaussian distribution. The corresponding equation (13.A.15) contains the inverse of the Fresnel error function erf-1, which is defined by equation (4.5.9). In order to avoid the use of an analytical approximation for erf-1, an alternative two-step approach is employed to generate numbers which follow an onedimensional Gaussian distribution. In the first step, the program selects a number $R$ and evaluates $R_{2}$ from equation (13.A.16), which refers to a two-dimensional Gaussian distribution. In the second step, the program selects another random number $R$ and computes $R_{1}$ from $R$ and $\mathbf{R}_{2}$ as

$$
R_{1}=R_{2} \cos (2 \pi R)
$$

which represents the projection of the radius $R_{2}$ on an axis which is rotated over an angle $2 \pi R$ with respect to the radius $R_{2}$. The numbers $R_{1}$ produced this way follow an one-dimensional Gaussian distribution.

In order to standardize the input of the random number routine, the different width measures $\sigma, A$ and $B$ are expressed in terms of the corresponding Half Width at Half Maximum (HWHM) values, using

$$
\begin{aligned}
& \sigma=H W H M / \sqrt{2 \operatorname{In} 2} \\
& A_{1}=H W H M, \quad A_{2}=\frac{H W H M}{\left(2^{2 / 3}-1\right)^{1 / 2}} \\
& B=H W H M / \ln 2
\end{aligned}
$$

as follows from equations (13.A.15) to (13.A.19). The input of the random number routine now consist out of four parameters: $X_{i}, I_{d}$ is,$I_{d i m}$ and HWHM. $X_{1}$ is the last random number which was generated by equation (13.2.3) or the start value $X_{0}$ (in the first call). $I_{d i s}$ selects the type of distribution (1=uniform, 2=Gaussian, 3 =Lorentzian, $4=$ exponential). I $I_{\mathrm{d} m}$ specifies the dimension of the distribution ( 1 or 2 ). The routine returns a random number $R$, which is selected according to the specified distribution. 
In this appendix we will outline a method to fit a set of data points $\left\{\left(x_{i}, y_{i}\right) \mid i=1,2, \cdots, N\right\}$ with the polynomial function of $M$ terms

$$
y(x)=\sum_{j=1}^{M} a_{j} x^{e_{j}}, \quad e_{j}=\frac{n_{1}(M-j)+n_{2}}{n_{3}}
$$

where $n_{1}, n_{2}$ and $n_{3}$ are constants and $a_{j}$ (for $j=1, \cdots, M$ ) are the parameters to be determined by the fit procedure. The different sets of values used for $n_{1}, n_{2}$ and $n_{3}$ are specified by equation (13.8.5). We wish to determine the set of parameters $a_{j}$, for which the merit function

$$
S=\sum_{i=1}^{N}\left[y_{i}-y\left(x_{i}\right)\right]^{2}
$$

has a minimum. This implies

$$
\frac{\partial S}{\partial a_{k}}=0 \quad \text { for } \quad k=1,2, \cdots, M
$$

Solving equations (13.B.3) constitutes a common least square fit problem. The particular problem we like to investigate here, is to solve the least square problem in the subspace of parameters $a_{j}$, for which the function $y(x)$ fulfils one of the following conditions

$$
\begin{array}{lll}
\text { (a) } y\left(x_{0}\right)=y_{0} & & \left(x_{0} \neq 0\right) \\
\text { (b) } y^{\prime}\left(x_{1}\right)=y_{1}^{\prime} & & \left(x_{1} \neq 0\right) \\
\text { (c) } y\left(x_{0}\right)=y_{0} \text { and } y^{\prime}\left(x_{1}\right)=y_{1}, & \left(x_{0} \neq 0 \text { and } x_{1} \neq 0\right)
\end{array}
$$

where $y^{\prime}(x)$ is the derivative of $y(x)$. The constants $x_{0}$ and $x_{1}$ are assumed to be non-zero. We will investigate each of the three cases given by equation (13.B.4) separately. The reader may notice that equation (13.B.1) differs from equation (13.8.4), by the fact that it assumes $x_{s}=y_{s}=0$. This choice simplifies the notation, without loss of generality. The shifts $x_{s}$ and $y_{s}$ can be incorporated afterwards.

Let us define the auxiliary fit function $y^{\star}(x)$ as

$$
y^{*}(x)=y_{0} f_{0}(x)+y_{1} f_{1}(x)+\sum_{j=1}^{M-g} a_{j} *\left(x^{e_{j}}-x_{0}{ }^{e_{j}} f_{0}(x)-e_{j} x_{1}{ }^{e_{j}-1} f_{1}(x)\right)
$$

Our aim is to find functions $f_{0}(x)$ and $f_{1}(x)$ for which $y^{*}(x)$ fulfils the additional constraint (a), (b) or (c), for any set of parameters $a_{j}{ }^{*}$. The number of terms in the summation is diminished by $g$, which reflects the reduction of the number of degrees of freedom caused by the additional constraints imposed on the fit. For the cases (a) and (b) $g=1$, while $g=2$ for case (c). Equation (13.B.5) is organized such that the conditions (a), (b) and (c) are fulfilled for those functions $f_{o}(x)$ and $f_{1}(x)$, which show the following properties

$$
\begin{aligned}
& \text { (a) } f_{0}\left(x_{0}\right)=1, f_{1}(x)=0 \text { for all } x \\
& \text { (b) } f_{1}\left(x_{1}\right)=1, f_{0}(x)=0 \text { for all } x \\
& \text { (c) } f_{0}\left(x_{0}\right)=1, f_{0}^{\prime}\left(x_{1}\right)=0 \\
& f_{1}\left(x_{0}\right)=0, \quad f_{1}{ }^{\prime}\left(x_{1}\right)=1
\end{aligned}
$$

Thus case (a) only concerns $f_{0}(x)$, taking $f_{1}(x)=0$ for all $x$; case (b) 
only concerns $f_{1}(x)$, taking $f_{0}(x)=0$ for all $x$, while case (c) involves both functions. An additional constraint, which should be introduced at this point, is that the functions $y^{*}(x)$ and $y(x)$ are of the same type. This means that for any given set of parameters $a_{j}{ }^{*}$ one should be able to find a corresponding set of parameters $a_{j}$, such that

$$
y(x)=y^{*}(x)
$$

This conditions determines the order of the functions $f_{0}(x)$ and $f_{1}(x)$, as will explicitly be shown later. Assuming, that one can indeed find functions $f_{0}(x)$ and $f_{1}(x)$, which fulfil the conditions of equations (13.B.6) and (13.B.7), the next step would be to solve the set of equations

$$
\frac{\partial S}{\partial a_{k}{ }^{*}}=0 \quad \text { for } \quad k=1,2, \cdots, M-g
$$

in which the merit function $S$ is now given by

$$
S=\sum_{i=1}^{N}\left[y_{i}-y^{*}\left(x_{i}\right)\right]^{2}
$$

similar to equation (13.B.2). By solving equations (13.B.8), one obtains a set of parameters $a_{k}{ }^{*}$. Using equation (13.B.7) one can express this solution in terms of a set of parameters $a_{k}$ and the problem is solved.

Our strategy is now explained and the remaining problem is the choice of the functions $f_{0}(x)$ and $f_{1}(x)$. For the separate cases (a), (b) and $(c)$ we found that the following functions $f_{0}(x)$ and $f_{1}(x)$ meet the requirements, expressed by equations (13.B.6) and (13.B.7)

$$
\begin{array}{lll}
\text { (a) } f_{0}(x)=\left(\frac{x}{x_{0}}\right)^{p} & , f_{1}(x)=0 & p=\frac{n_{2}}{n_{3}} \\
\text { (b) } f_{0}(x)=0 & f_{1}(x)=\frac{x^{q}}{q x_{1}^{q-1}} & q=\frac{n_{1}-n_{2}}{n_{3}} \\
\text { (c) } f_{0}(x)=\frac{q x_{1}^{q} x^{p}-p x_{1} p x^{q}}{q x_{1}^{q} x_{0}^{p}-p x_{1}^{p} x_{0}^{q}}, & f_{1}(x)=\frac{x_{0}^{p} x^{q}-x_{0}^{q} x^{p}}{q x_{0}^{p} x_{1}^{q-1}-p x_{0}^{q} x_{1}^{p-1},}, p=\frac{n_{2}}{n_{3}}, \\
q=\frac{n_{1}+n_{2}}{n_{3}} & \text { (13.B.10) }
\end{array}
$$

Notice that $p=e_{M}$, where $e_{M}$ is defined by equation (13.B.1). Thus $p$ is equal to the order of the lowest order term in $y(x)$. In case (c), we took $q=e_{M-1}$, equal to the order of the next term in $y(x)$. To understand the choice of $q$ in case (b), one has to consider the situations $n_{2}=0$ and $n_{2}=1$ separately. For $n_{2}=0$, we have $q=n_{1} / n_{3}=e_{M-1}$. For $n_{2}=1$, we have $q=\left(n_{1}-1\right) / n_{3}=1 / n_{3}=e_{M}$, using that $n_{1}=2$ whenever $n_{2}=1$, as can be seen from equation (13.8.5). Thus in both cases, $q$ is chosen equal to the order of the lowest order term in $y(x)$, which is not a constant.

The reader can directly verify that the functions $f_{0}(x)$ and $f_{1}(x)$, defined by equations (13.B.10), fulfil the conditions of equation (13.B.6). One also sees that the functions $y(x)$ and $y^{*}(x)$ are of the same type, as required by equation (13.B.7). One finds that a given set of parameters $a_{j}{ }^{*}$ is equivalent to a set of parameters $a_{j}$, which is for the separate cases (a), (b) and (c) specified by 

(a), (c), (b) for $n_{2}=1$
$a_{j}=a_{j} *$
for $j=1,2, \cdots, M-g$
(b) for $n_{2}=0$
$a_{j}=a_{j}{ }^{*}$
for $j=1,2, \cdots, M-2, M$

(a) $a_{M}=\frac{1}{x_{0} p}\left(y_{0}-\sum_{j=1}^{M-1} a_{j}^{*} x_{0} e_{j}\right)$

(b) $a_{k}=\frac{1}{q x_{1} q-1}\left(y_{1},-\sum_{j=1}^{M-1} a_{j}^{*} e_{j} x_{1} e_{j}-1\right) \quad$ with $k= \begin{cases}M & \text { for } n_{2}=0 \\ M-1 & \text { for } n_{2}=1\end{cases}$

(c) $a_{M}=\frac{1}{q x_{1} q x_{0} p-p x_{1}{ }^{p} x_{0}^{q}}\left[y_{0} q x_{1} q-y_{1}{ }^{\prime} x_{0}^{q} x_{1}-\sum_{j=1}^{M-2} a_{j}{ }^{*}\left(x_{0}{ }^{e_{j}} q x_{1}{ }^{q}-e_{j} x_{1} e_{j} x_{0}^{q}\right)\right]$

$$
a_{M-1}=\frac{1}{q x_{1} q x_{0} p-p x_{1}^{p} x_{0} q}\left[-y_{0} p x_{1} p^{p}+y_{1}{ }^{p} x_{0} p^{p} x_{1}-\sum_{j=1}^{M-2} a_{j} *\left(-x_{0}{ }^{e_{j}}{ }_{p x_{1}}{ }^{p}+e_{j} x_{1}{ }^{e_{j}} x_{0} p\right)\right]
$$

which gives the resulting fit-function in the form of equation (13.B.1).

The set of parameters $a_{k}$ is obtained from equations (13.B.8), in the usual way, by solving the normal equations

$$
0=\sum_{i=1}^{N}\left(y_{1}-y^{*}\left(x_{i}\right)\right)\left(x_{i}{ }^{e_{j}}-x_{0} e_{j} f_{0}\left(x_{i}\right)-e_{j} x_{i}{ }^{e_{j}-1} f_{i}\left(x_{i}\right)\right)
$$

which follow by substitution of equation (13.B.9) into equations (13.B.8), utilizing equation (13.B.5). Alternatively, one may express the normal equations as

$$
\sum_{j=1}^{M-g} T_{k j} a_{j} *=b_{k}
$$

where $\mathrm{T}$ is an $(\mathrm{M}-\mathrm{g}) \times(\mathrm{M}-\mathrm{g})$ matrix and $\mathrm{b}$ is a vector of length $(\mathrm{M}-\mathrm{g})$, with elements

$$
\begin{aligned}
& T_{k j}=\sum_{i=1}^{N}\left(x_{i}{ }^{e_{k}}-x_{0} e^{e} f_{0}\left(x_{i}\right)-e_{k} x_{i} e_{k}-1 f_{i}\left(x_{i}\right)\right)\left(x_{i} e_{j}-x_{0}{ }^{e} f_{0}\left(x_{i}\right)-e_{j} x_{i} e_{j}^{-1} f_{i}\left(x_{i}\right)\right) \\
& b_{k}=\sum_{i=1}^{N}\left(y_{i}-y_{0} f_{0}\left(x_{i}\right)-y_{1} f_{1}\left(x_{i}\right)\right)\left(x_{i}{ }^{e_{k}}-x_{0}{ }^{e} f_{0}\left(x_{i}\right)-e_{k} x_{i}{ }^{e-1} f_{1}\left(x_{i}\right)\right)
\end{aligned}
$$

Notice that the elements $T_{k}$ depend only on $x_{i}$ and not on $y_{i}$, while the elements $b_{k}$ depend on both $x_{j}$ and yi. Equations (13.B.13) are solved by means of LU-decomposition and backsubstitution. For this, the reader is referred to the standard textbooks on numerical programing, see for instance Press et al. (1986). As the matrix $T$ depends only on the set $x_{i}$, the decomposition only has to be performed once for a given set $x_{i}$ and can then be used to solve $a_{k}$ f for a number of sets $y_{i}$, having the same arguments $x_{i}$. In our problem the quantities $x_{i}$ are equally spaced with a distance $h=x_{i+1}-x_{i}$, which is equal to the division width of the histogram. The fit-routine uses the scaled arguments $x_{i} / h$ as input, instead of the arguments $x_{i}$ themselves. The advantage of this approach is, that one knows beforehand, that the element $T_{11}$ is, in general, the largest element in the matrix $T$ and the element $T(M-g)(M-g)$ the smallest, since $x_{i} / h>1$. This knowledge is exploited to simplify the pivot-strategy in the LU-decomposition, which should be incorporated to guarantee an optimum numerical accuracy of the fit. 


\subsection{Introduction}

In this chapter we will compare the analytical theory for the Boersch effect, the trajectory displacement effect and the space charge effect with the results of numerical Monte Carlo simulations. The Monte Carlo technique provides the capability to verify some of the basic assumptions underlying the analytical theory, particularly the reduction of the $\mathrm{N}$-particle dynamical problem to a two-particle problem. It also provides an independent check on the overall correctness of the final equations obtained within the analytical theory.

The analysis of this chapter is restricted to a single segment of an electron beam with a crossover in the middle. The current density distribution and angular distribution in the crossover are assumed to be uniform. The study is based on the results of 276 runs of the Monte Carlo program, covering a wide range of operating conditions. The simulated beam currents varied from $0.01 \mu \mathrm{A}$ to $50 \mu \mathrm{A}$ and the beam potentials from $1 \mathrm{kV}$ to $50 \mathrm{kV}$. A number of different beam geometries were considered, taking as extreme cases a nearly cylindrical beam with a beam semi-angle $\alpha_{0}=0.5 \mathrm{mR}$ and a crossover radius $r_{c}=20 \mu \mathrm{m}$ and a beam segment with a narrow crossover with $\alpha_{0}=10 \mathrm{mR}$ and $r_{\mathrm{c}}=1 \mu \mathrm{m}$. The beam length $L$ was in all simulations equal to $0.1 \mathrm{~m}$.

In general, the Monte Carlo results are in excellent agreement with the predictions of the analytical theory. A good qualitative and quantitative agreement is found for low-current beams as well as for high-current beams, while some quantitative differences occur for intermediate beam currents. These differences are probably caused partly by the inaccuracy of the fit-functions employed in the analytical model and partly by the fact that the analytical results refer to the displacements experienced by particles running along the beam axis, while the Monte Carlo program takes the displacements of all particle into account. There also exists some disagreement in the predictions of the space charge defocussing distance for low beam currents due to the incompatibility of the different criteria used in the models to determine the plane of best focus. However, as the defocussing distances are extremely small for these currents, the differences are irrelevant from an experimental point of view.

The chapter starts with a discussion of the fundamental issues involved. The results of the various Monte Carlo simulations are presented next and are compared with the predictions of the analytical theory. The chapter concludes with a discussion of the results.

\subsection{General aspects}

In the previous chapter the technique of numerical Monte Carlo (MC) simulation of charged particle beams was discussed. The Monte Carlo program provides the means to verify some of the basic assumptions underlying our analytical model for Coulomb interactions in particle beams. The principle aspects of the extended two-particle approach used to calculate the Boersch effect and the trajectory displacement effect were outlined in chapter 5, while the main results of the actual calculations were presented in the chapters 7 and 9 respectively. The analytical model for the space charge effect was outlined in chapter 11. A summary of the final equations for the Boersch effect, the trajectory displacement effect and the space charge effect will be given in chapter 
16. The final equations of the analytical theory were implemented in an computer program called INTERAC, which provides a convenient tool to evaluate the analytical predictions for a certain set of experimental parameters. For short we will indicate the predictions of the analytical theory sometimes as the results from INTERAC. In this chapter the outcome of the INTERAC program is compared to the results of the MC program for the case of a single segment of an electron beam.

The main purpose of the comparison is to verify the accuracy of the results of the analytical theory. It should be recalled that the analytical theory for statistical interactions relies on the following assumptions :

(1) The problem can be modelled by considering the displacements experienced by a set of test particles running along a certain reference trajectory due to the interaction with a set field particles representing the beam. The results obtained for the central reference trajectory (which is the beam axis) are representative for other (off-axis) trajectories as well.

(2) The initial condition of the beam can be considered as monochromatic with respect to the normal energy. In other words, one may assume the initial axial velocity is the same for all particles.

(3) The total displacement of the test particle is equal to the sum of all displacements experienced in the two-particle interactions with the individual field particles, as expressed by equation (5.3.12).

(4) The field particles can be considered as statistically independent, as expressed by equation (5.3.1).

(5) Magnetic interactions and relativistic effects can be ignored.

Some of these assumptions can be verified within the framework of the analytical model itself. In chapter 10 we investigated the changes in the final results occurring for off-axis reference trajectories and non-monochromatic beams respectively. According to assumptions (1) and (2) these differences should be minor. With respect to assumption (2) it was found that the results obtained by averaging over all possible reference trajectories are in general somewhat smaller than those pertaining to the central reference trajectory. The differences are in general less than $20 \%$ and for many conditions even less than $10 \%$. The validity of assumption (2) was confirmed by the fact that the width of the initial energy distribution had no significant impact on final results for practical operating conditions.

Assumption ( 3 ), which concerns the dynamical part of the problem, can not directly be verified within the analytical model. For that purpose we will use the MC program. As was mentioned in chapter 13, two types of ray-tracing algorithms are implemented in the $M C$ program, referred to as numerical ray-tracing (by the routine DRIFT1) and analytical ray-tracing (by the routine DRIFT2) respectively. The analytical ray-tracing algorithm utilizes the same dynamics as the analytical theory. Accordingly, it relies also on assumption (3). Thus, numerical ray-tracing is required to verify the validity of assumption (3). The approach taken in this chapter is to perform all MC simulations twice, one time using numerical ray-tracing and one time using analytical ray-tracing. By comparing the results of both $M C$ simulations one gets a direct insight in the errors caused by the reduction of the full dynamical $\mathrm{N}$-body problem to pair interactions.

The source-routine of the MC program, which generates the initial condition of a bunch of particles, ignores any correlation in the initial coordinates of the particles. This approach is equivalent to assumption (4) underlying the analytical theory. Accordingly, assumption (4) can not directly be verified with the Monte Carlo program. In this connection it should be noticed that the MC program 
could be used to study the correlation in the particle coordinates originating from the mutual Coulomb interaction in the beam. For instance, one could investigate to what extent the interaction in a certain beam segment is affected by the correlation in the initial coordinates arising from the Coulomb interaction in the preceding beam segment. This "source" of correlation is ignored in the analytical theory, which treats every beam segment as independent. However, we did not pursue this problem and restricted the simulations to a single beam segment.

Assumption (5) can not be verified either with our MC program since the ray-tracing algorithms are non-relativistic and account only for the Coulomb interaction between the particles. However, the implications of this assumption can be studied from first principles, as was shown in section 10.6 .

Summarizing, as far as the fundamental aspects are concerned, the main use of a comparison between analytical theory and MCsimulations is to verify assumption (3). One could use MC simulations to verify the validity of assumptions (1) and (2). However, these assumptions were already verified within the analytical model itself, which provides a more elegant method.

Apart from testing some of the premisses of the analytical model the comparison with MC simulations is also useful as an independent check on the overall correctness of the final equations. The MC method is relatively straightforward and its correct implementation can more easily be verified than the extensive calculations carried out within the analytical model. It is therefore suited as back-up for the analytical predictions.

For the comparison between analytical theory and MC simulations we selected a large number of different cases, varying over a wide range of experimental conditions. For each case we obtained three predictions for each of the interaction effects: One from the MC program using numerical ray-tracing (referred to as DRIFT1), another one from the MC program using analytical ray-tracing (referred to as DRIFT2) and the third one from the analytical theory (referred to as INTERAC). Any differences between the results of DRIFT1 and DRIFT2 would imply that assumption (3) is inappropriate for that specific case. Differences between the results of DRIFT2 and INTERAC can have a variety of causes. Firstly, the MC simulation may be of $f$ due to the inherent statistical errors or due to the model-errors related to the finite-size of the particle bunch. These errors were discussed in section 13.9. Secondly, it should be noticed that the final equations of the theory include functions which are obtained by parameter-fitting of numerical data. This introduces some deviations from the exact theoretical predictions. Finally, there are some fundamental differences between both approaches in the way the lateral displacements are separated into a space charge and a trajectory displacement component. We will return to these subjects in section 14.5, where the results of the comparison are discussed in some detail.

\subsection{Voltage and current dependencies for a fixed geometry}

Table 14.1 gives an overview of the cases selected to verify the dependencies of the Coulomb interaction effects on the beam voltage $v$ and the beam current $I$ for a fixed geometry with a beam semi-angle $a_{0}=5 \mathrm{mR}$ and a crossover radius $r_{c}=5 \mu \mathrm{m}$. The table also gives the selected values for the MC parameters $N_{s}$ am and $N_{s e a d}$, which represent the number particles per sample and the number of seeds respectively. The total 
Table 14.1. Overview of the cases selected to compare the analytical theory with $\mathrm{MC}$ simulations, for various beam voltages $\mathrm{V}$ and beam currents I (and a fixed geometry). For all cases the beam segment length $\mathrm{L}=0.1 \mathrm{~m}$ and crossover position parameter $\mathrm{S}_{\mathrm{c}}=0.5$. The values chosen for the beam current I are .01, .02,.05, $0.1,0.2,0.5,1,2,5,10,20 \mu \mathrm{A}$.

\begin{tabular}{|c|c|c|c|c|c|c|c|c|c|c|}
\hline \multicolumn{4}{|c|}{ Experimental parameters } & \multicolumn{2}{|c|}{ MC param. } & \multicolumn{5}{|c|}{ Theoretical parameters } \\
\hline $\mathrm{I}(\mu \mathrm{A})$ & $\mathrm{v}(\mathrm{kv})$ & $\alpha_{0}(\mathrm{mR})$ & $r_{c}(\mu \mathrm{m})$ & $N_{s}$ a m & $N_{\text {seed }}$ & $\pi$ & $\overline{\mathbf{r}}_{\mathbf{c}}$ & K & $r_{c}{ }^{*}$ & $\mathbf{v}_{0}{ }^{*}$ \\
\hline $\begin{array}{l}.01 \\
0 . \\
20 .\end{array}$ & 1 & 5 & 5 & $\begin{array}{l}100 \\
600\end{array}$ & $\begin{array}{l}15 \\
2\end{array}$ & $\begin{array}{l}9.58 \times 10^{-5} \\
\because .9 \\
.192\end{array}$ & 274 & 50 & .163 & 16.3 \\
\hline $\begin{array}{l}.01 \\
\cdots \\
20 .\end{array}$ & 2 & 5 & 5 & $\begin{array}{l}100 \\
600\end{array}$ & $\begin{array}{l}15 \\
2\end{array}$ & $\begin{array}{l}3.39 \times 10^{-5} \\
\cdots \\
6.78 \times 10^{-2}\end{array}$ & 347 & 50 & .206 & 20.6 \\
\hline $\begin{array}{l}.01 \\
\cdots \\
20\end{array}$ & 5 & 5 & 5 & $\begin{array}{l}50 \\
600\end{array}$ & $\begin{array}{l}30 \\
2\end{array}$ & $\begin{array}{l}8.57 \times 10^{-6} \\
\cdots \\
1.71 \times 10^{-2}\end{array}$ & 868 & 50 & .279 & 27.9 \\
\hline $\begin{array}{l}.01 \\
\cdots \\
20 .\end{array}$ & 10 & 5 & 5 & $\begin{array}{l}50 \\
600\end{array}$ & $\begin{array}{l}30 \\
2\end{array}$ & $\begin{array}{l}3.03 \times 10^{-6} \\
6.06 \times 10^{-3}\end{array}$ & 1736 & 50 & .351 & 35.1 \\
\hline $\begin{array}{l}.01 \\
\ddot{2} \\
20\end{array}$ & 20 & 5 & 5 & $\begin{array}{l}50 \\
600\end{array}$ & $\begin{array}{l}30 \\
2\end{array}$ & $\begin{array}{l}1.07 \times 10^{-6} \\
\cdots \\
2.14 \times 10^{-3}\end{array}$ & 3472 & 50 & .443 & 44.3 \\
\hline $\begin{array}{l}.01 \\
\cdots \\
20 .\end{array}$ & 50 & 5 & 5 & $\begin{array}{l}50 \\
600\end{array}$ & $\begin{array}{l}30 \\
2\end{array}$ & $\begin{array}{l}2.71 \times 10^{-7} \\
\cdots \\
5.42 \times 10^{-4}\end{array}$ & 8681 & 50 & .601 & 60.1 \\
\hline
\end{tabular}

number of particles $\mathrm{N}_{\mathrm{sam}} \times \mathrm{N}_{\text {seed }}$ is 1500 in the simulations for small beam currents $(<2 \mu \mathrm{A})$ and 1200 in the simulations for large beam currents $(\geqslant 5 \mu \mathrm{A})$. The theoretical parameters $\bar{\lambda}, \bar{r}_{\mathrm{c}}$ and $\mathrm{K}$ are relevant for the Boersch effect, while the parameters $\bar{\lambda}, r_{c}^{*}$ and $v_{0}{ }^{*}$ apply to the trajectory displacement effect. An overview of the main theoretical parameters can be found in table 16.1 .

The results for the FW5 5 energy spread, the $F_{5} 0$ trajectory displacement effect and the space charge defocussing distance $\Delta z$ are given in the figures 14.1a, $14.1 \mathrm{~b}$ and 14.1c. In these figures the data is plotted as function of the beam current $I$. The different sets of curves correspond to the beam voltages 1,10 and $50 \mathrm{kV}$. In the figures $14.2 \mathrm{a}, 14.2 \mathrm{~b}$ and $14.2 \mathrm{c}$ the data is plotted as function of the beam potential $\mathrm{V}$ and the different sets of curves correspond to beam currents of $0.01,0.1,1$ and $10 \mu \mathrm{A}$.

Consider the curves for the $\mathrm{FW}_{50}$ energy spread presented in the figures $14.1 \mathrm{a}$ and $14.2 \mathrm{a}$. The results obtained with DRIFT1 and DRIFT2 are in general indistinguishable, except for very high particle densities. This confirms the validity of the reduction to two-particle dynamics (assumption 3). The deviations between MC-simulations (DRIFT1 and DRIFT2) and the analytical theory (INTERAC) are larger. All curves in figure 14.1a show the transition from the pencil beam regime for small beam currents ( $I^{2}$-dependency), via the Lorentzian regime (linear Idependency) and the Holtsmark regime ( $\mathrm{I}^{2 / 3}$-dependency) to the Gaussian regime for large beam currents ( $I^{1 / 2}$-dependency). These dependencies are explicitly predicted by equations $(7.4 .33),(7.5 .25),(7.3 .40)$ and (7.3.37) respectively. The corresponding voltage dependencies are predicted to be $V^{-1}$ for the pencil beam regime, $V-1 / 3$ for the Holtsmark regime, $V^{-1 / 2}$ for the Lorentzian regime and $V-1 / 4$ for the Gaussian regime (provided that $\bar{r}_{c}>100$ ). These dependencies are confirmed by the 
plots of figure $14,2 \mathrm{a}$.

While the agreement by trend is excellent over the full range of operating conditions, one finds for intermediate beam currents somewhat larger values from the analytical theory than from the MC program. A good quantitative agreement is found for small as well as large beam currents. The differences found for intermediate beam currents will be further investigated in section 14.5.

Similar characteristics are found for the FWso trajectory displacement broadening, given in figures $14.1 \mathrm{~b}$ and $14.2 \mathrm{~b}$. The curves show the transition from the pencil beam regime for small beam currents ( $\mathrm{I}^{3}$-dependency), via the Holtsmark regime ( $\mathrm{I}^{2 / 3}$-dependency) to the Gaussian regime for large beam currents ( $1^{1 / 2}-$ dependency). These dependencies are explicitly predicted by equations (9.3.47), (9.3.44) and $(9.3 .45)$ respectively. The corresponding voltage dependencies are predicted to be $V-5 / 2$ for the pencil beam regime, $V-4 / 3$ for the Holtsmark regime and $v^{-13 / 12}$ for the Gaussian regime, which is confirmed by the curves of figure $14.2 \mathrm{~b}$. As for the energy spread one finds that the analytical theory predicts somewhat larger values than the MC program for intermediate beam currents, while the quantitative agreement is excellent for the full range of operating conditions.

The analytical theory for the space charge effect predicts that the defocussing distance $\Delta z$ is proportional to the perveance $I / V^{3 / 2}$, as can be seen from equation (11.5.14). This behaviour is confirmed by the data plotted in the figures $14.1 \mathrm{c}$ and $14.2 \mathrm{c}$. The figures show a good qualitative and quantitative agreement between theory and MC simulations for most operating conditions. However, for very small and for very large beam currents the MC simulations predict smaller $\Delta z$ than the analytical theory. The interpretation of these results is postponed to section 14.5 .

Table 14.2. Overview of the cases selected to compare the analytical theory with MC simulations, for various beam geometries (defined by $\alpha_{0}$ and $r_{c}$ ) and beam eurrents I (for a fixed beam potential v). For all cases the beam segment length $\mathrm{L}=0.1 \mathrm{~m}$ and crossover position parameter $S_{\mathrm{c}}=0.5$. The values chosen for the beam current $I$ are $.01, .02, .05,0.1$, $0.2,0.5,1,2,5,10,20 \mu \mathrm{A}$.

\begin{tabular}{|c|c|c|c|c|c|c|c|c|c|c|}
\hline \multicolumn{4}{|c|}{ Experimental parameters } & \multicolumn{2}{|c|}{ MC param. } & \multicolumn{5}{|c|}{ Theoretical parameters } \\
\hline $\mathrm{I}(\mu \mathrm{A})$ & $\mathrm{V}(\mathrm{kV})$ & $\alpha_{0}(\mathrm{mR})$ & $r_{c}(\mu \mathrm{m})$ & $N_{s} \mathbf{m}$ & $N_{\text {seod }}$ & $\bar{\lambda}$ & $\overline{\mathbf{r}}_{\mathbf{c}}$ & $\mathbf{K}$ & $r_{c}{ }^{*}$ & $v_{0}{ }^{*}$ \\
\hline $\begin{array}{l}.01 \\
\cdots \\
20 .\end{array}$ & 10 & 10 & 1 & $\begin{array}{l}50 \\
600\end{array}$ & $\begin{array}{l}30 \\
2\end{array}$ & $\begin{array}{l}7.58 \times 10^{-1} \\
\because .5 \\
1.52 \times 10^{-3}\end{array}$ & 1389 & 500 & .0703 & 70.3 \\
\hline $\begin{array}{c}.01 \\
\cdots \\
20\end{array}$ & 10 & 7.5 & 1.333 & $\begin{array}{l}50 \\
600\end{array}$ & $\begin{array}{l}30 \\
2\end{array}$ & $\begin{array}{l}1.35 \times 10^{-6} \\
2.6 \\
2.69 \times 10^{-3}\end{array}$ & 1042 & 281 & .0937 & 52.7 \\
\hline \begin{tabular}{l}
.01 \\
\hdashline. \\
20.
\end{tabular} & 10 & 5 & 2 & $\begin{array}{l}50 \\
600\end{array}$ & $\begin{array}{l}30 \\
2\end{array}$ & $\begin{array}{l}3.03 \times 10^{-6} \\
6.06 \times 10^{-3}\end{array}$ & 695 & 125 & .141 & 35.1 \\
\hline $\begin{array}{l}.01 \\
\cdots \\
20\end{array}$ & 10 & 2.5 & 4 & $\begin{array}{l}50 \\
600\end{array}$ & $\begin{array}{l}30 \\
2\end{array}$ & $\begin{array}{l}1.21 \times 10^{-5} \\
2.43 \times 10^{-2}\end{array}$ & 347 & 31.3 & .281 & 17.6 \\
\hline $\begin{array}{l}.01 \\
\ddot{\cdots} \\
20\end{array}$ & 10 & 1.25 & 8 & $\begin{array}{l}50 \\
600\end{array}$ & $\begin{array}{l}30 \\
2\end{array}$ & $\begin{array}{l}4.85 \times 10^{-5} \\
\cdots \\
9.70 \times 10^{-2}\end{array}$ & 174 & 7.81 & .562 & 8.79 \\
\hline $\begin{array}{l}.01 \\
\cdots \\
20\end{array}$ & 10 & 0.5 & 20 & $\begin{array}{l}50 \\
600\end{array}$ & $\begin{array}{l}30 \\
2\end{array}$ & $\begin{array}{l}3.03 \times 10^{-4} \\
\cdots \\
.606\end{array}$ & 69.5 & 1.25 & 1.41 & 3.51 \\
\hline
\end{tabular}




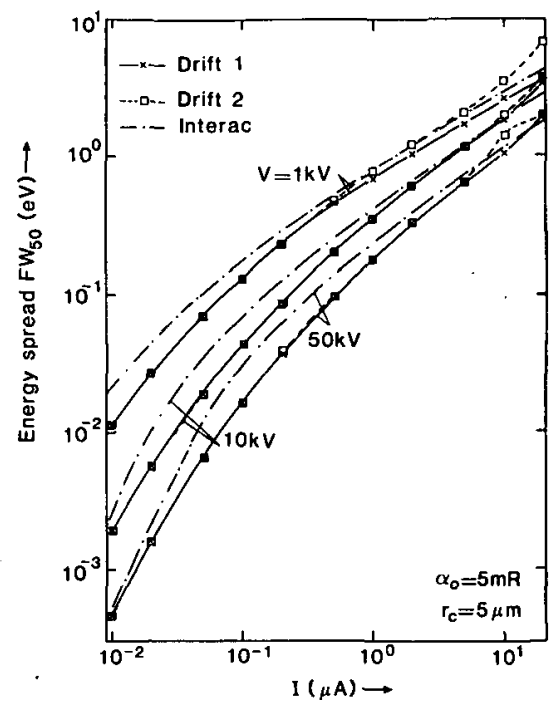

fig. 14.1 a

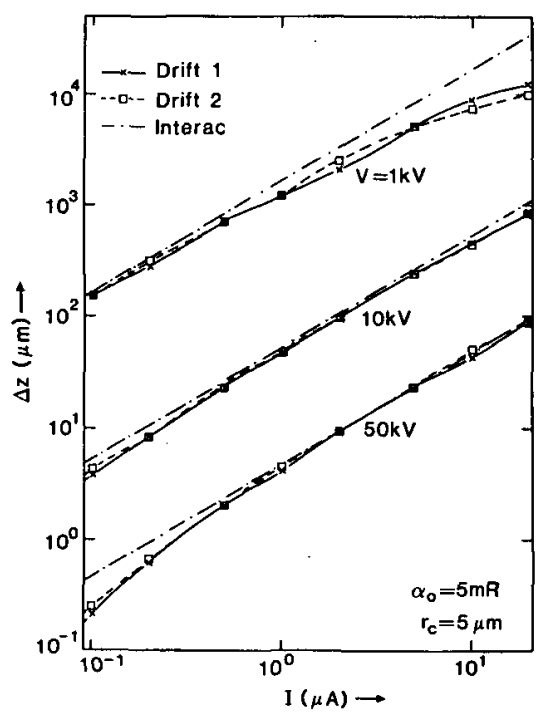

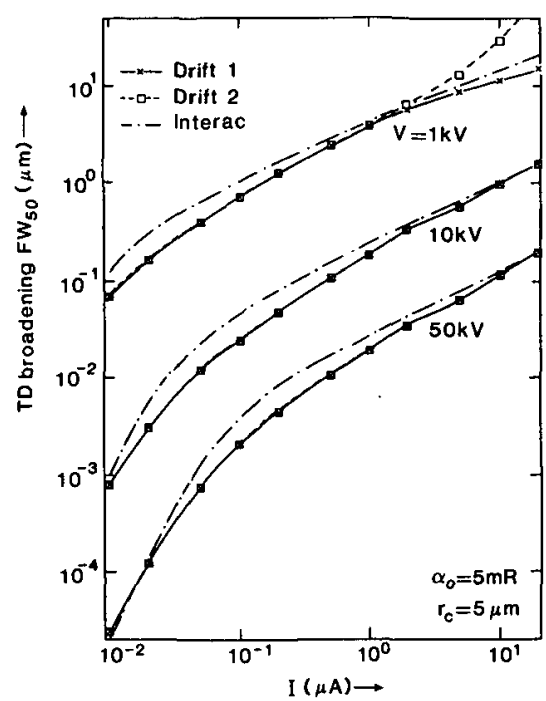

fig.14.1b

fig.14.1c

Figure 14.1 Comparison of the different predictions for the FWso energy spread (figure 14.1a), the FWso trajectory displacement broadening (figure 14.1b) and the space charge defocussing distance $\Delta z$ (figure 14.1 $\mathrm{c}$ ), produced in a beam segment with $\mathrm{L}=0.1 \mathrm{~m}, \alpha_{0}=5 \mathrm{mR}$ and $\mathrm{r}_{c}=5 \mu \mathrm{m}$, as function of the beam current I, for different beam voltages V. See table 14.1 for the detailed input. 


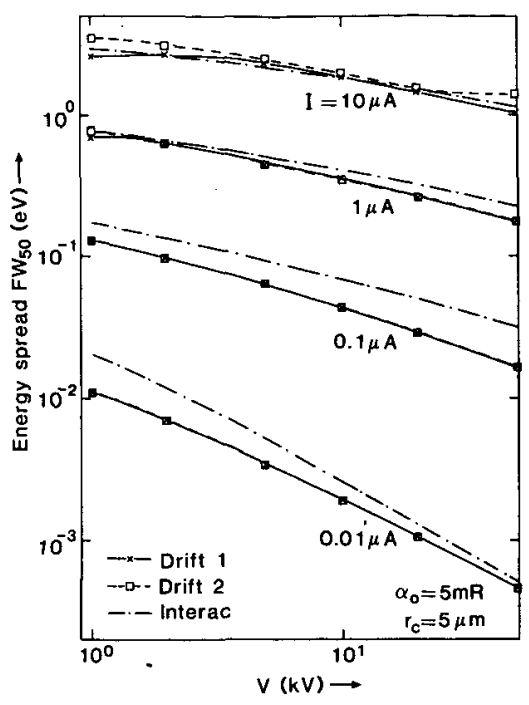

fig. 14.2a

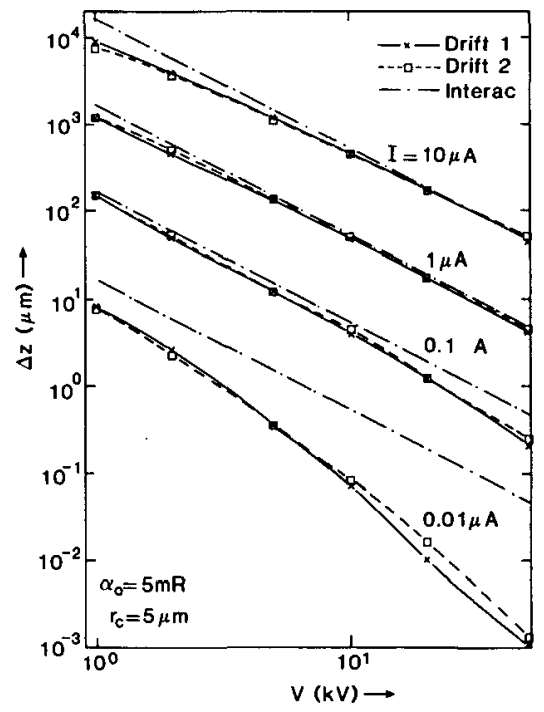

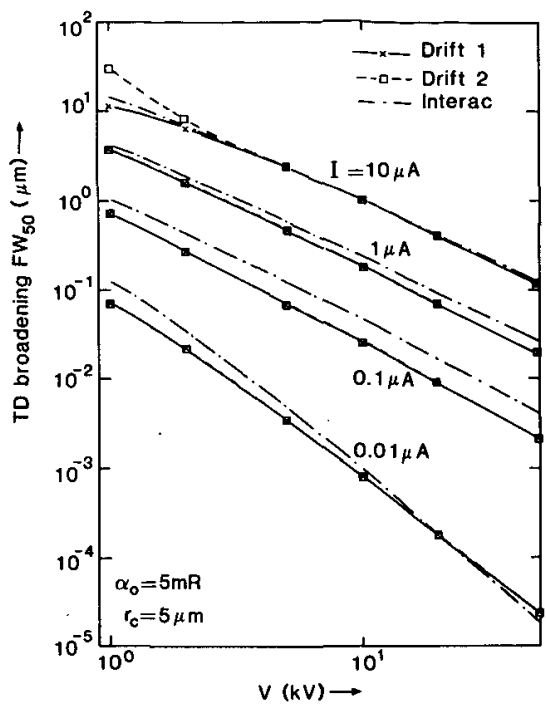

fig. 14.2b

$1 \mathrm{ig} .14 .2 \mathrm{c}$

Figure 14.2 Comparison of the different predictions for the $\mathrm{FW}_{5}$ o energy spread (figure 14.2a), the FWso trajectory displacement broadening (figure $14.2 \mathrm{~b}$ ) and the space charge defocussing distance $\Delta z$ (figure 14.2c), produced in a beam segment with $\mathrm{L}=0.1 \mathrm{~m}, \quad \alpha_{0}=5 \mathrm{mR}$ and $\mathrm{r}_{\mathrm{c}}=5 \mu \mathrm{m}$, as function of the beam voltage $\mathrm{v}$, for different beam currents $\mathrm{I}$. This data is equivalent to that of figure 14.1 . 


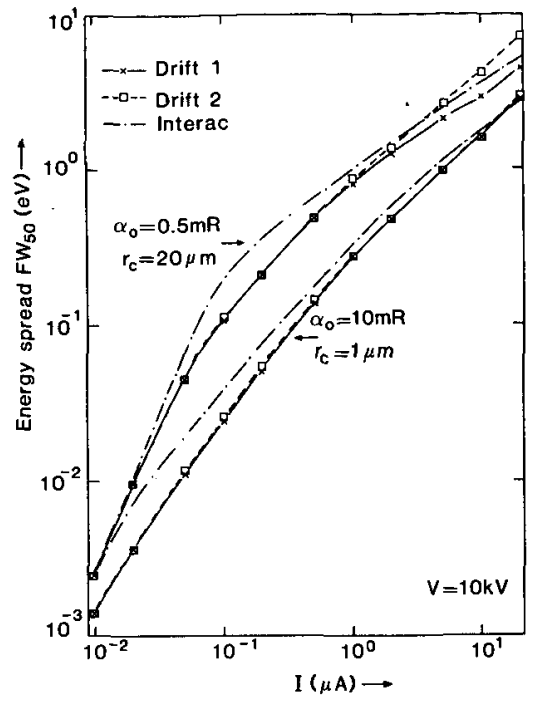

fig. 14.3a

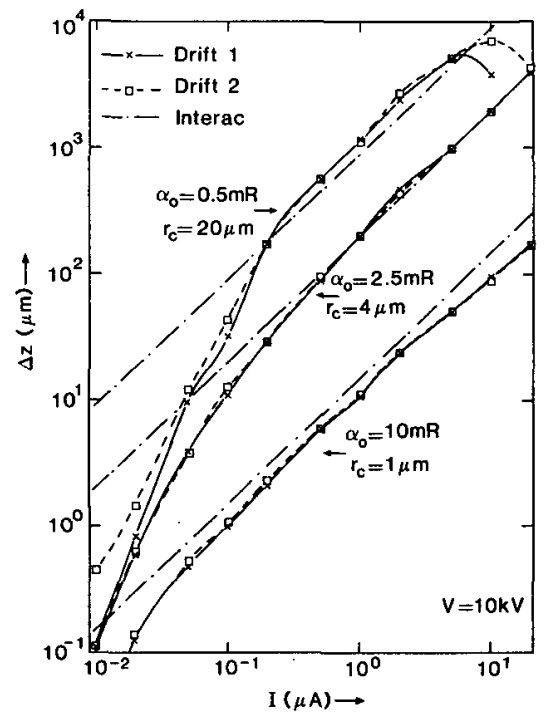

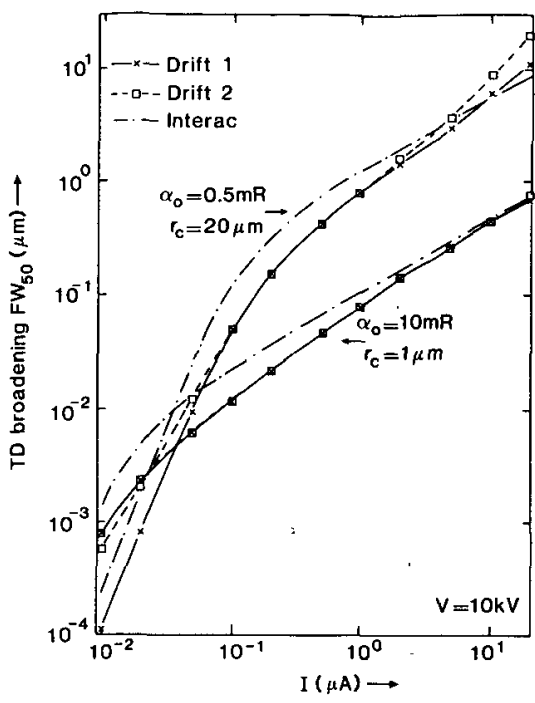

fig. 14.3b

fig.14.3c

Pigure 14.3 Comparison of the different predictions for the FWso energy spread (figure 14.3a), the $\mathrm{FW}_{50}$ trajectory displacement broadening (figure 14.3b) and the space charge defocussing distance $\Delta 2$ (figure $14.3 \mathrm{c}$ ), produced in a beam segment with $\mathrm{L}=0.1 \mathrm{n}$ and $\mathrm{V}=10 \mathrm{kV}$, as function of the beam current $\mathrm{I}$, for different combinations of $\alpha_{0}$ and $r_{c}$. See table 14.2 for the detailed input. 

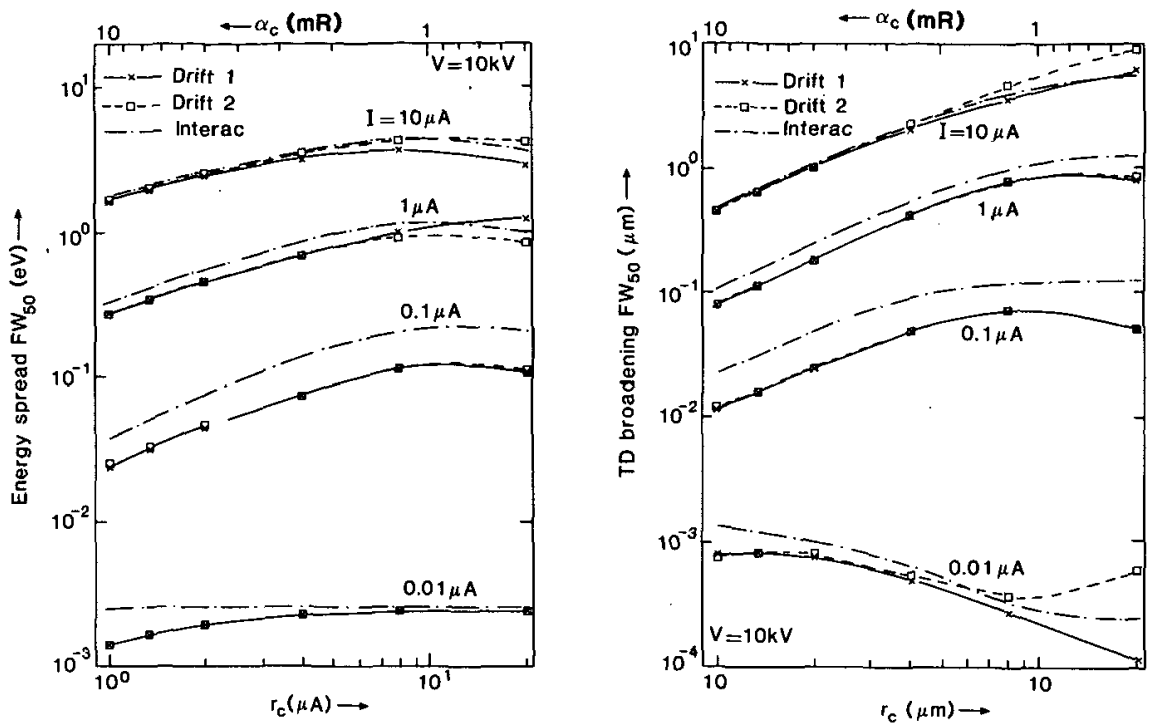

fig. 14.4a

fig. $14.4 b$

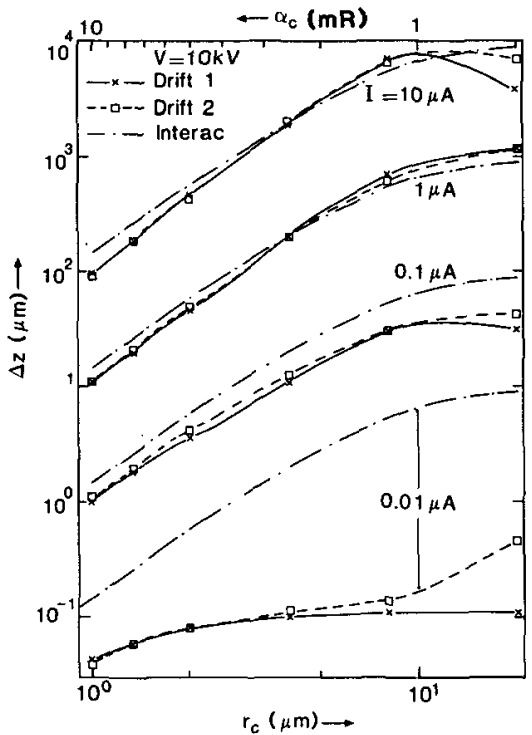

fig. 14.4c

Figure 14.4 Comparison of the different predictions for the FWs energy spread (figure 14.4a), the FW 50 trajectory displacement broadening (figure 14.4b) and the space charge defocussing distance $\Delta z$ (figure $14.4 \mathrm{c}$ ), produced in a beam segment with $\mathrm{L}=0.1 \mathrm{~m}$ and $\mathrm{V}=10 \mathrm{kV}$, as function of the beam geometry represented by $\alpha_{0}$ and $r_{c}$ (which are chosen such that $\alpha_{0} \times r_{c}$ is constant), for different beam currents $I$. This data is equivalent to that of figure 14.3 . 
Table 14.2 gives an overview of the cases selected to verify the dependencies of the Coulomb interaction effects on the beam current I for different geometries and a fixed beam potential $\mathrm{V}$ of $10 \mathrm{kV}$. The beam semi-angle $\alpha_{0}$ and crossover radius $r_{c}$ were chosen such that their product is the same for every case. As in table 14.1. we included the corresponding values of some theoretical parameters as well as the chosen values for the MC parameters $N_{s a m}$ and $N_{s e e d}$. Notice that the characteristic beam diameter ratio $K=2 \alpha_{0} L / 2 r_{c}$ varies between 1.25 and 500 for the different cases.

The results for the $\mathrm{FW}_{50}$ energy spread, the $\mathrm{FW}_{50}$ trajectory displacement effect and the space charge defocussing distance $\Delta z$ are given in the figures $14.3 \mathrm{a}, 14.3 \mathrm{~b}$ and $14.3 \mathrm{c}$ respectively. In these figures the data is plotted as function of the beam current $I$. The different sets of curves correspond to the cases $\left(\alpha_{0}=10 \mathrm{mR}, r_{c}=1 \mu \mathrm{m}\right)$ and $\left(\alpha_{0}=0.5 \mathrm{mR}, \quad r_{c}=20 \mu \mathrm{m}\right)$, while fig $14.3 \mathrm{c}$ also includes a set of curves for $\left(\alpha_{0}=2.5 \mathrm{mR}, r_{\mathrm{c}}=4 \mu \mathrm{m}\right)$. In the figures $14.4 \mathrm{a}, 14.4 \mathrm{~b}$ and $14.4 \mathrm{c}$ the data is plotted as function of the beam geometry, defined by the values for $\alpha_{0}$ and $r_{c}$. The different sets of curves correspond to beam currents of $0.01,0.1,1$ and $10 \mu \mathrm{A}$.

As for the cases discussed in section 14.3 the agreement by trend is excellent, while some quantitative differences between the analytical theory and the MC simulations occur for some operating conditions.

The figures $14.5 \mathrm{a}$ and $14.5 \mathrm{~b}$ give the energy distribution obtained by MC simulation for a current of $0.01 \mu \mathrm{A}$ and $10 \mu \mathrm{A}$ respectively in a beam segment with a semi-angle $\alpha_{0}=5 \mathrm{mR}$ and a crossover radius $r_{c}=2 \mu \mathrm{m}$. Both plots give the distribution of the absolute value of the energy displacement with respect to the average energy of $10 \mathrm{keV}$ and contain $90 \%$ of the particles. The $\mathrm{FW}_{50}$ energy spread is $1.9 \times 10^{-3} \mathrm{eV}$ and $2.5 \mathrm{eV}$ for the figures $14.5 \mathrm{a}$ and $14.5 \mathrm{~b}$ respectively. The distribution of figure 14.5a shows distinct non-Gaussian features, as predicted by the analytical model. It has a narrow core and extremely long tails.

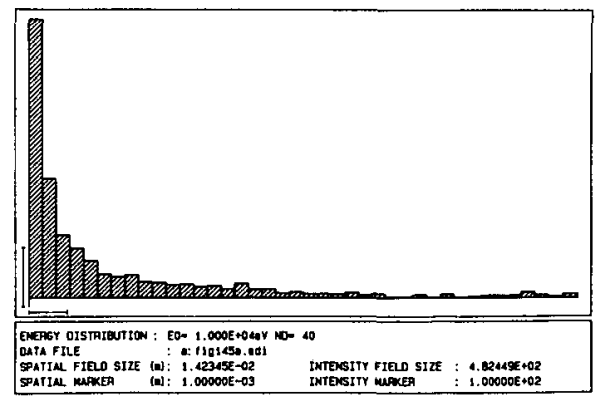

fig. 14.6a

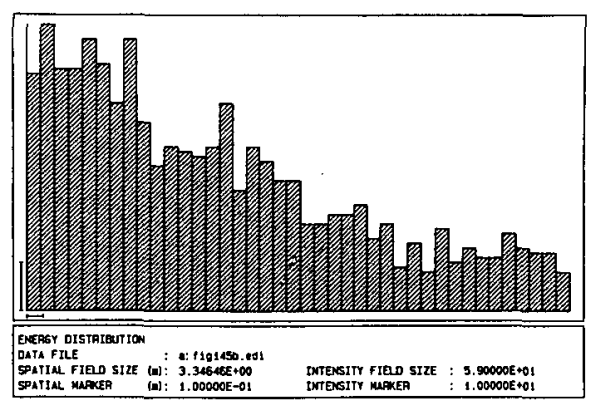

fig. 14.5b

Figure 14.5 Energy distribution obtained by $M$ simulation (using DRIFTI) of a beam segment with $\mathrm{L}=0.1 \mathrm{~m}, \alpha_{0}=5 \mathrm{mR}, \mathrm{r}_{\mathrm{c}}=2 \mu \mathrm{m}$ and $\mathrm{V}=10 \mathrm{kV}$, for a beam current of $0.01 \mu \mathrm{A}$ (figure 14.5a) and a beam current of $10 \mu \mathrm{A}$ (figure $14.5 \mathrm{~b}$ ). The horizontal bars in the figures $14.5 \mathrm{a}$ and $14.5 \mathrm{~b}$ indicate an energy width of $1 \mathrm{mev}$ and $0.1 \mathrm{eV}$ respectively, while the vertical bars represent 100 and 10 particles respectively. 
gone

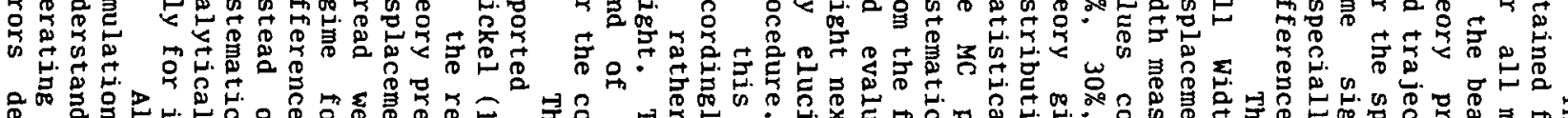

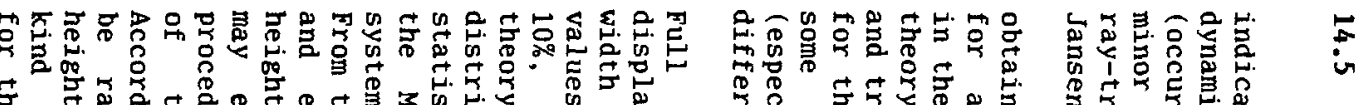

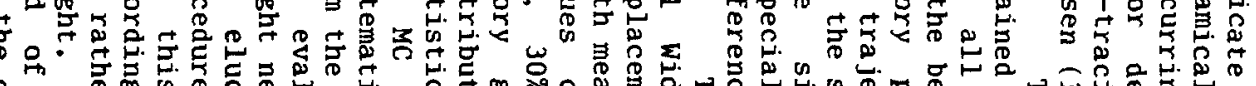

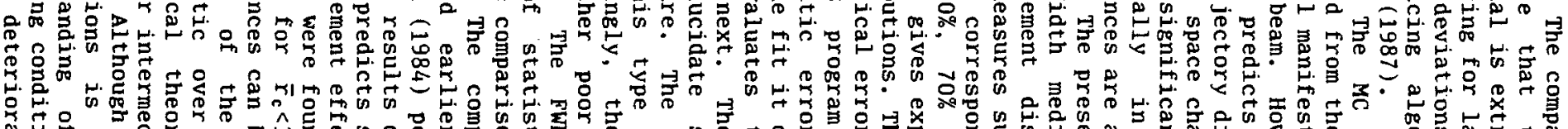

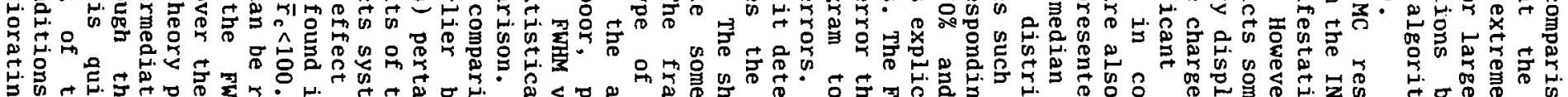

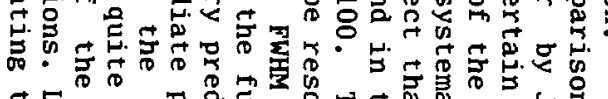

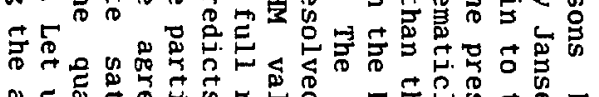

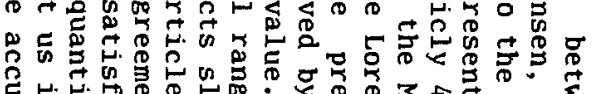

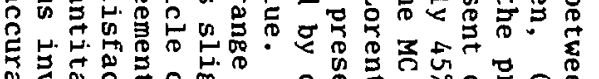

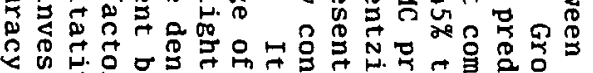

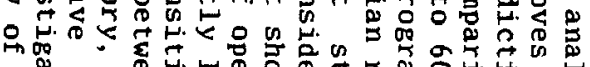

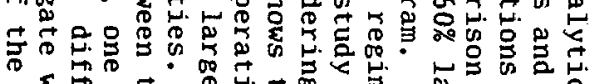

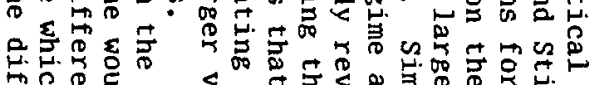

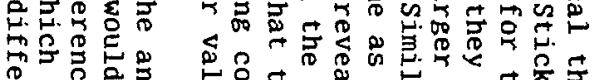

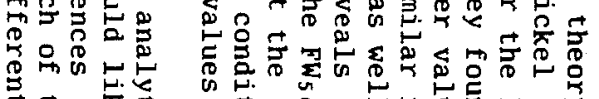

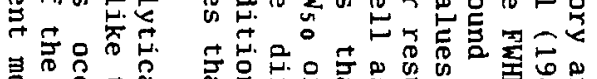

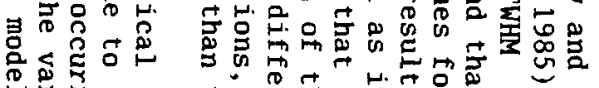

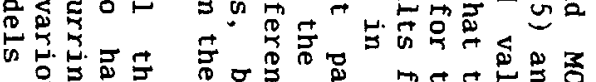

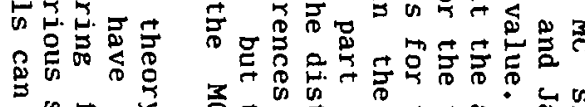

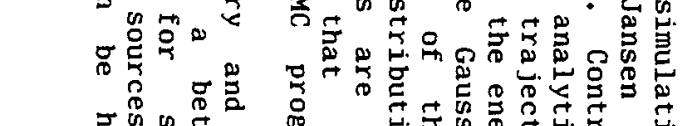

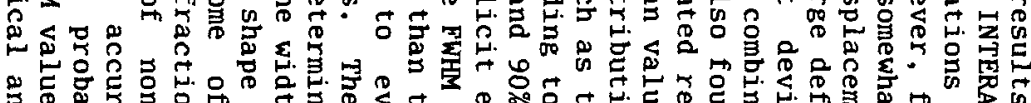

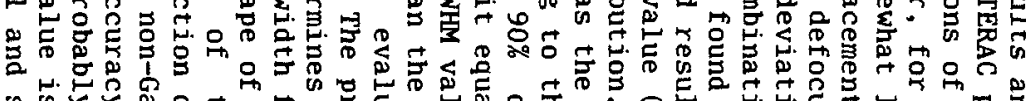

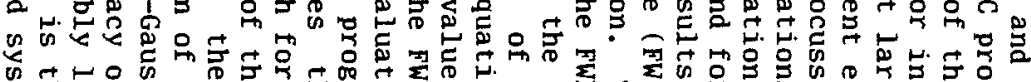

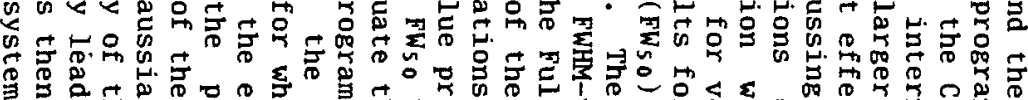

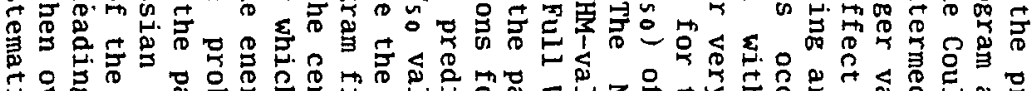

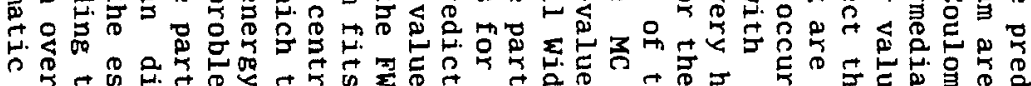

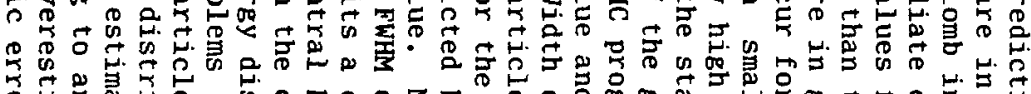

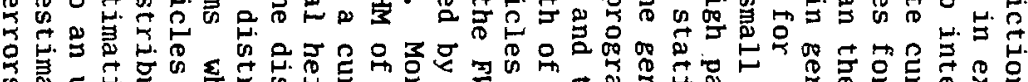

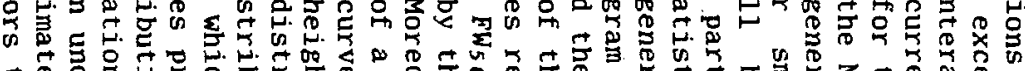

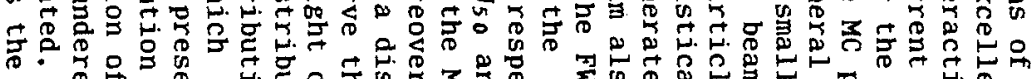

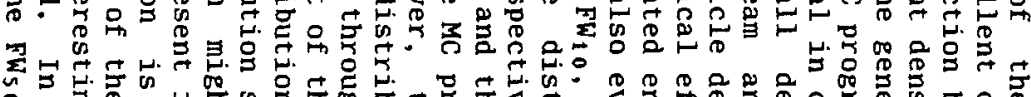

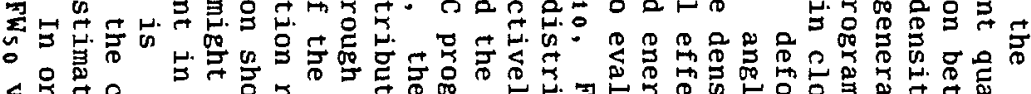

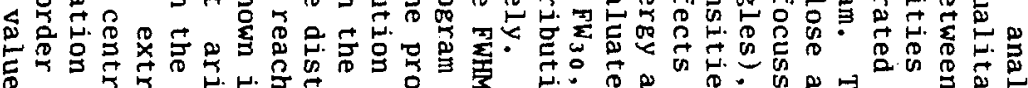

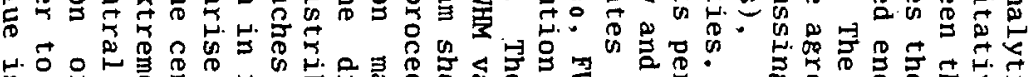

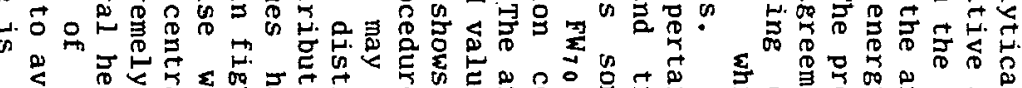

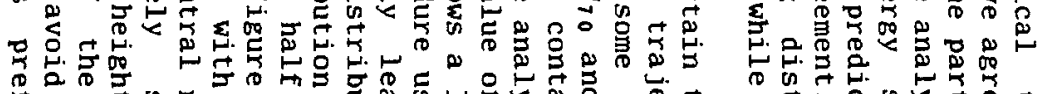

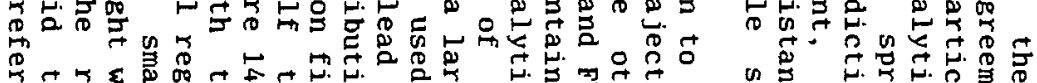

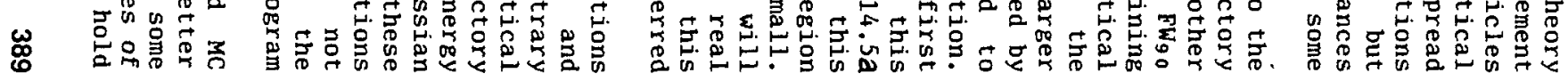

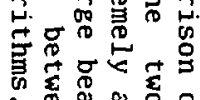

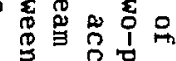

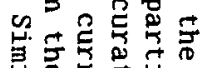

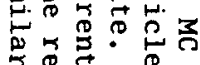

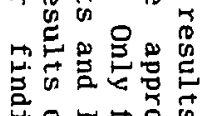

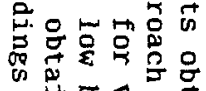

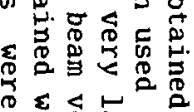

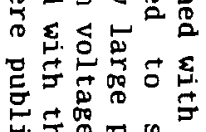

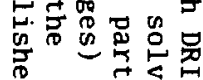

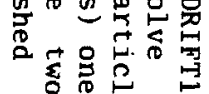

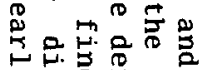

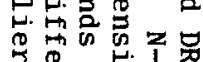

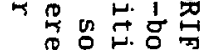

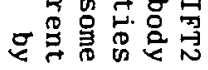




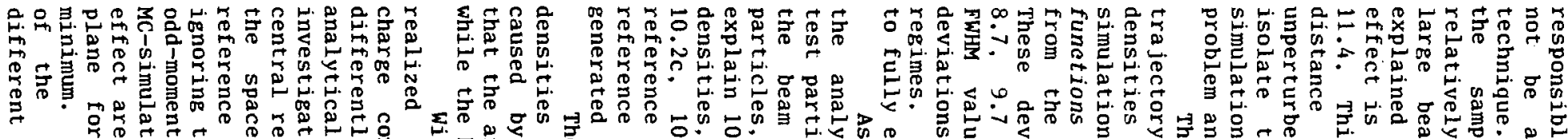

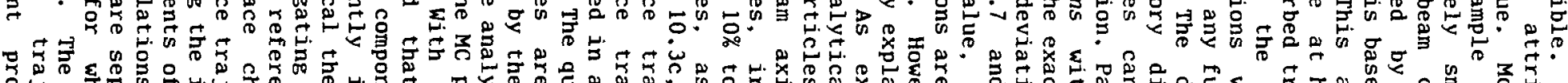

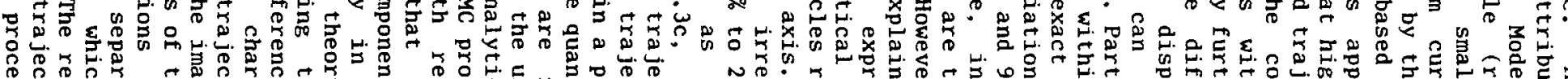

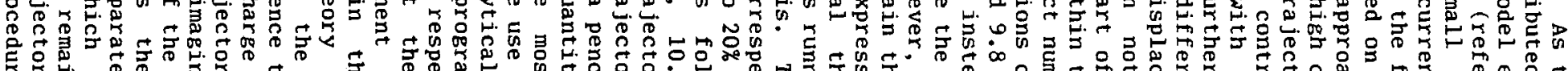

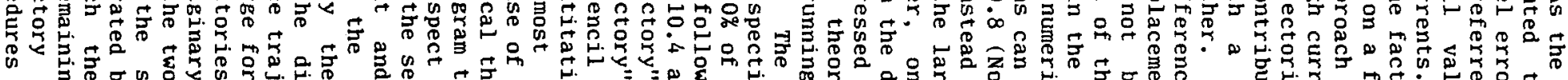

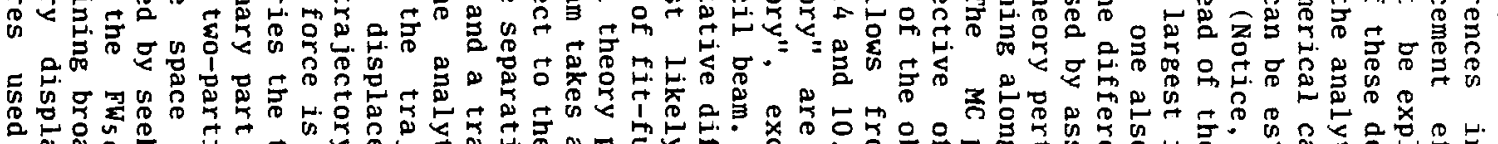

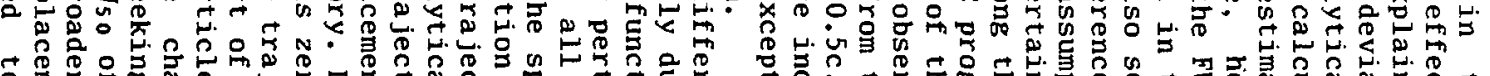

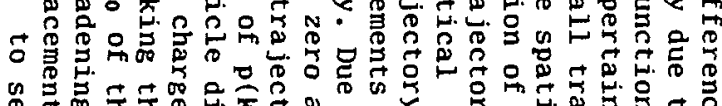

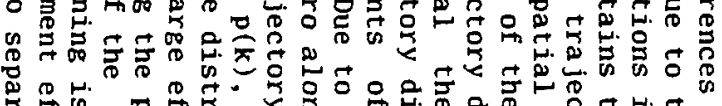

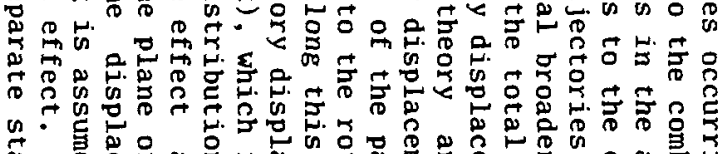

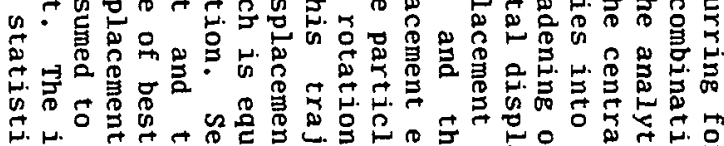

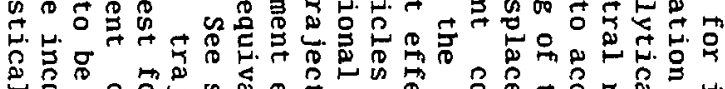

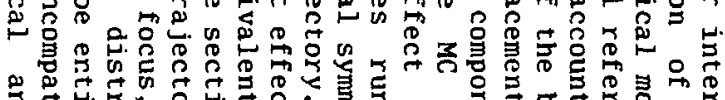

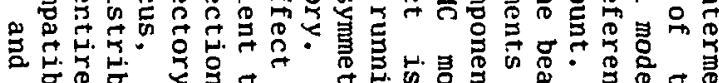

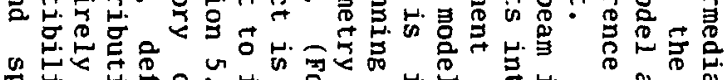

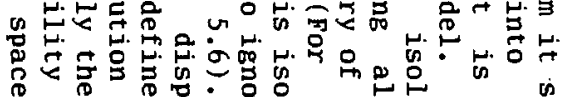

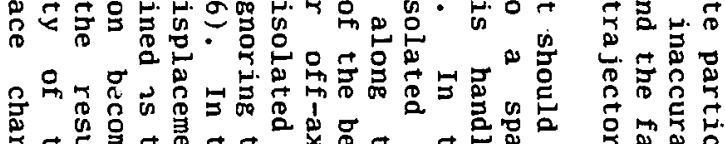

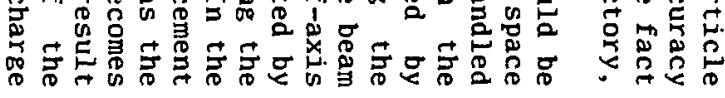

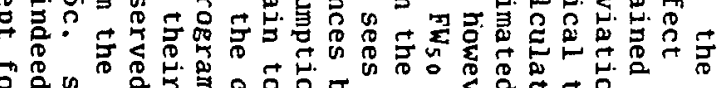

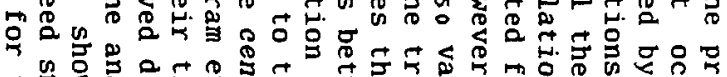

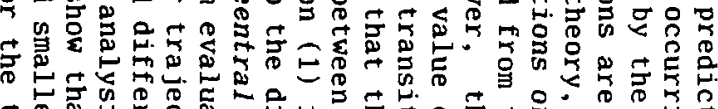

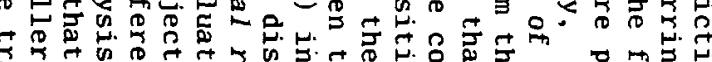

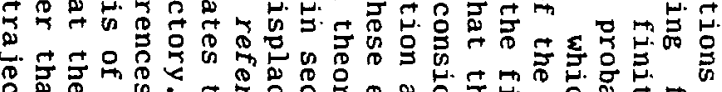

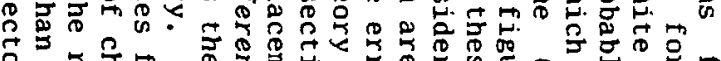

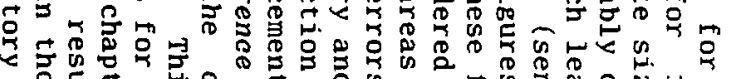

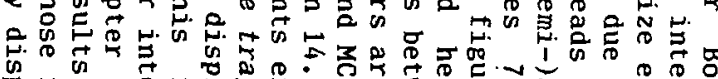

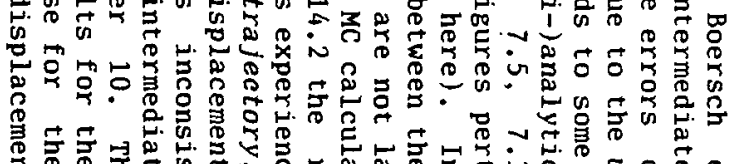

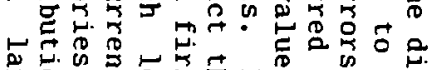

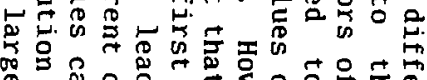

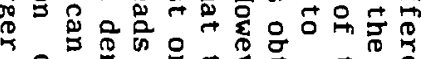

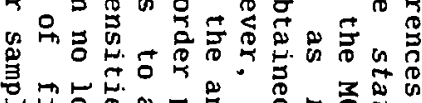

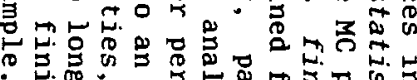
范哭

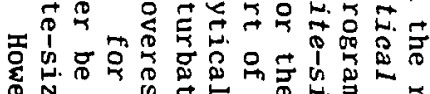

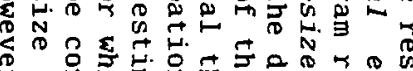

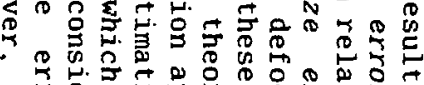

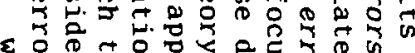

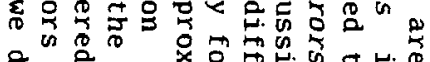

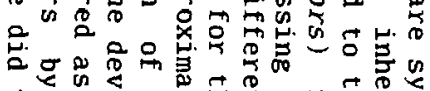

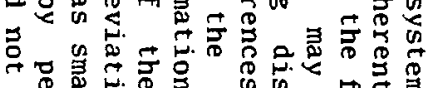

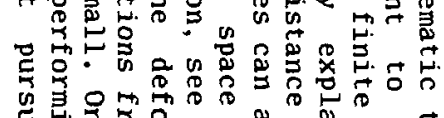

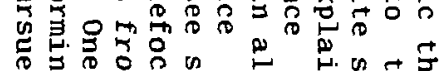

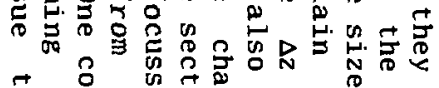

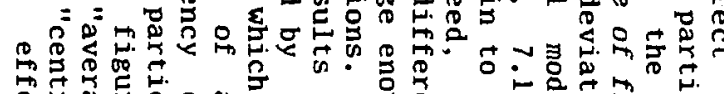

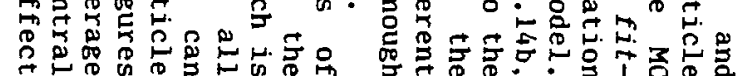

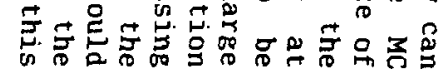


effects may lead to different predictions for both the space charge defocussing distance and the trajectory displacement effect, particularly for small beam currents when the displacements are small compared to lateral dimensions of the beam. This explains the disagreement in the predictions for the space charge defocussing distance at small beam currents.

In order to get some appreciation for the size of the displacements relative to the beam dimensions the figures $14.6 \mathrm{a}$ and $14.6 \mathrm{~b}$ show the displacements in the plane of best focus found by $M C$ simulation of a beam segment with $\alpha_{0}=5 \mathrm{mR}, r_{\mathrm{c}}=2 \mu \mathrm{m}$ and $\mathrm{V}=10 \mathrm{kV}$ for a beam current of $0.01 \mu \mathrm{A}$ and $10 \mu \mathrm{A}$ respectively. Figure $14.6 \mathrm{a}$ illustrates that the exact value of the space charge defocussing distance and the trajectory displacement effect is for such operating conditions irrelevant from an experimental point of view, since the contribution of the particle interactions to the spot-width can be ignored.

Finally, it should be noted that the analytical model indicates that the space charge effect also causes some non-refocusable blurring if the current density distribution in the cross sections of the beam is non-uniform. In the MC program this blurring will show up as a contribution to the trajectory displacement effect, which is identified as the remaining blurring after refocussing. This may lead to a larger apparent trajectory displacement effect in the MC-simulation than predicted by the analytical theory. However, for the comparison we considered a beam segment with an uniform angular and spatial distribution in the crossover. The non-refocusable space charge blurring is for this case negligible.

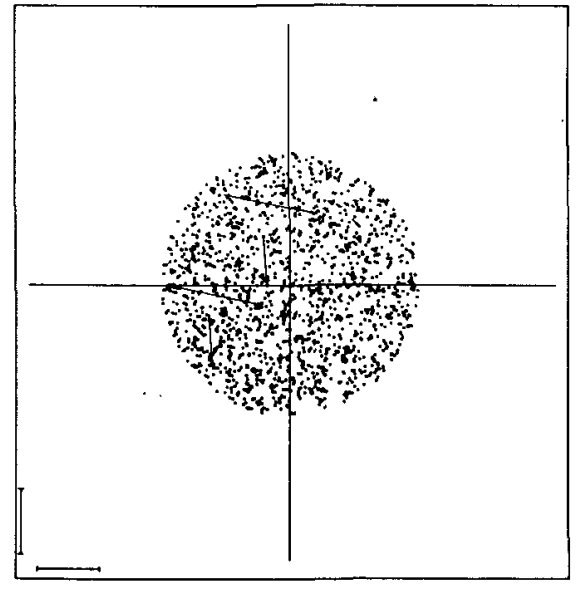

fig. 14.6a

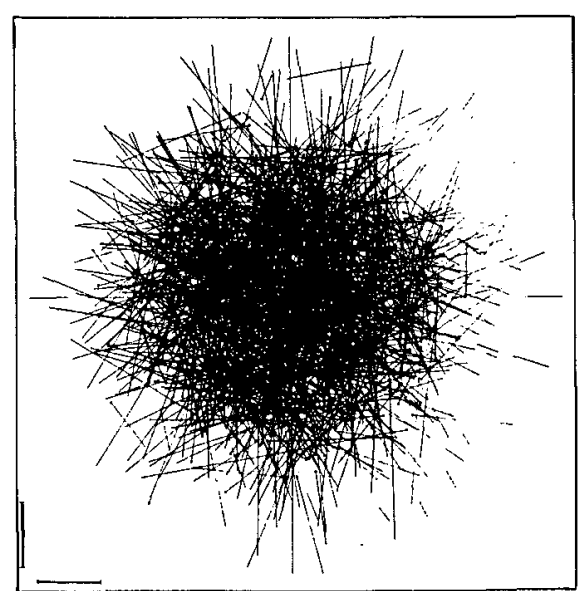

fig.14.6b

Figure 14.6 Displacements in the plane of best focus obtained by $M$ simulation (using DRIFTI) of a beam segment with $\mathrm{L}=0.1 \mathrm{~m}$, $\alpha_{0}=5 \mathrm{mR}, \quad r_{\mathrm{c}}=2 \mu \mathrm{m}$ and $\mathrm{v}=10 \mathrm{kV}$, for a beam current of $0.01 \mu \mathrm{A}$ (figure 14.6a) and a beam current of $10 \mu \mathrm{A}$ (figure $14.6 \mathrm{~b}$ ). The bars in the figures $14.6 \mathrm{a}$ and $14.6 \mathrm{~b}$ represent $a$ distance of $1 \mathrm{\mu m}$. The perturbed positions are indicated by a square. 


\subsection{Introduction}

This chapter gives a quantitative comparison of some recent theories on statistical coulomb interactions. A qualitative description of the various models was given in chapter 2, approaching the subject from a historical perspective. In chapter 5, a systematic overview was presented of the alternative approaches to the $\mathrm{N}$-body dynamical problem and the statistical part of the problem. Some of the models are based on the theoretical concepts developed in the field of plasma physics and stellar dynamics. These concepts were reviewed in chapter 4.

The comparison of this chapter is restricted to those models which yield their final results in terms of analytical expressions. Numerical models, such as Monte Carlo models and the model proposed by Tang (1987), are therefore disregarded. Furthermore, only those models are considered which treat beams in drift space. This excludes the approaches of Knauer (1981) and Sasaki (1986), dealing with the interaction phenomena occurring in guns with spherical cathodes. In order to cover the results presented by Massey, Jones and Plummer (1981) for a cylindrical beam in an uniform acceleration field their equations were recalculated for the case that acceleration is absent. The analysis for cylindrical beams is restricted to (nearly) homocentric beams. This excludes cylindrical beams in which the lateral thermal motion is magnetically confined, such as discussed by Zimmermann (1970) and Knauer (1979a). Most theories treat the case of an uniform current density distribution in the cross sections of the beam. For the comparison we will therefore study this case.

The models developed for the Boersch effect, statistical angular deflections and the trajectory displacement effect will be discussed separately in the successive sections of this chapter. Our approach to these phenomena, which is based on the so-called extended two-particle model, was described in the chapters 7,8 and 9 respectively. The strategy followed here is to express the final results of the different theories in terms of the scaled quantities employed in our model. This leads to a reduction of the number of parameters involved, which facilitates the comparison.

The extended two-particle model is unique in its ability to predict the full energy, angular and spatial displacement distributions. The model shows that different types of distributions are generated, depending on the operating conditions. Due to the variation in the shape of the generated distributions the choice of the measure to characterize their width is critical. Our model gives explicit expressions for the Full Width at Half Maximum (FWHM), the Full width median (FW50) and the root mean square (rms) value. Most other models evaluate the root mean square (rms) broadening only. More practical width-measures are usually obtained from the rms-value by assuming that the displacement distribution is Gaussian. However, our model shows that Gaussian behaviour occurs only for high particle densities. In the non-Gaussian regimes the rms-value is entirely dominated by the tails of the distribution or in other words by a few large displacements. For those conditions the rms-value provides no information of the central part of the displacement distribution and a straightforward conversion to more practical width measures as the FWHM and $\mathrm{FW}_{50}$ is not possible. Theories predicting a rms-value only are therefore essentially incomplete.

Since most theories evaluate a rms-value the comparison focusses on the different predictions for this width-measure. The model presented by Loeffler (1969) and Loeffler and Hudgin (1970) requires a separate 
treatment since it expresses the final results in terms of the half width median of the displacement distributions. For the comparison these results will be converted to a rms-value employing Loeffler's claim that the distribution is Gaussian. In addition, the results of Loeffler and Hudgin will be compared directly to the median width predicted by our model, which is more appropriate when the displacement distribution is non-Gaussian.

\subsection{Boersch effect}

We will first review the predictions of our model for the rms energy spread caused by the Boersch effect and next discuss the results of some other theories. As some of the theories make use of the thermodynamic upper limit to the Boersch effect we will include this limit in the analysis.

With equations $(7.3 .30),(7.3 .32),(7.5 .20)$ and $(7.5 .37)$ the rms-value of the distribution of axial velocities generated in a beam segment with a crossover of arbitrary dimensions can be expressed as (using the $\mathrm{d}_{0}, \mathrm{v}_{0}$-scaling of equation $(7,3.2)$ )

$$
\left\langle\Delta \bar{v}_{z} 2>1 / 2=(\pi \bar{X}) 1 / 2 P_{C E}\left(\bar{r}_{c}, K_{1}, K_{2}\right)\right.
$$

where $P_{C E}\left(\bar{r}_{C}, K_{1}, K_{2}\right)=\left[P_{C E}\left(\bar{r}_{C}, K_{1}\right)+P_{C E}\left(\bar{r}_{C}, K_{2}\right)\right] / 2$ and

$$
\begin{aligned}
& P_{C E}\left(\vec{r}_{c}, K\right)= \\
& \quad\left[.788+.6\left(\frac{\bar{r}_{c}}{\mathrm{~K}^{2}}\right)^{2 / 3}\left[1+.5\left(\frac{\mathrm{K}^{2}}{\bar{r}_{c}}\right)^{4 / 9}\right]^{3 / 2}+\frac{\pi \bar{r}_{c}}{\left\{2 \ln \left(.8673\left(114.6+\bar{r}_{c}\right)\right]\right\}^{2}}\right]^{-1 / 2}
\end{aligned}
$$

The quantities $\bar{\lambda}, \bar{r}_{c}, K_{1}$ and $K_{2}$ are defined by equations (7.3.22), $(7.3 .5)$ and (7.3.1) respectively. For large K-values the function $P_{C E}\left(\bar{r}_{c}, K\right)$ becomes independent of $K$. For $\bar{r}_{c \rightarrow 0}$ it becomes equal to unity. By removing the scaling in equation (15.2.1), using equation (7.3.36), one finds for the relative rms-energy spread

$$
\frac{<\Delta E^{2}>1 / 2}{E}=\left(\frac{m}{8 E_{0}^{2} e}\right)^{1 / 4} \operatorname{PCE}\left(\bar{r}_{c}, K_{1}, K_{2}\right) \frac{I^{1 / 2}}{V^{3 / 4}}
$$

If the energy distribution is Gaussian, the FWHM and the rms are related as $F_{W H M_{E}}=(8 \ln 2) 1 / 2\left\langle\Delta E^{2}>1 / 2\right.$ and equation $(15.2 .3)$ becomes equivalent to equation (7.3.37) (notice that in the Gaussian regime the functions $\mathrm{G}_{\mathrm{CE}}$ and $P_{C E}$ are identical).

In section 3.8 the thermodynamic upper limit of kinetic energy relaxation was introduced, which is relevant for a beam segment with a narrow crossover. For an uniform angular distribution the corresponding rms-energy spread is given by equation (3.8.3). By scaling this expression one obtains equation (7.7.1). Using the form of equation $(15.2 .1)$ the thermodynamic limit of kinetic energy relaxation can now be expressed in terms of an upper limit $\mathrm{P}_{\mathrm{CET}}(\bar{\lambda})$ for the function $\mathrm{P}\left(\overline{\mathrm{r}}_{\mathrm{c}}, \bar{\lambda}, \mathrm{K}_{1}, \mathrm{~K}_{2}\right)$, which is equal to

$$
\operatorname{PCET}(\pi)=\frac{1}{(6 \pi \pi)^{1 / 2}}
$$

This upper limit increases with decreasing $\lambda$. For $\pi=1 / 6 \pi=0.053$ one finds $P_{C E T}=1$, which is equal to the maximum of the function $P_{C E}\left(\bar{r}_{C}, K_{1}, K_{2}\right)$ given by equation (15.2.2). Thus, according to our model thermodynamic 
equilibrium is not reached in a beam with a single crossover for practical particle densities $\left(\pi<10^{-2}\right)$.

With equations $(7.4 .19),(7.4 .22)$ and $(7.4 .16)$ the rms-value of the distribution of axial velocities generated in a homocentric cylindrical beam segment can be expressed as (using the $\delta, \nu$-scaling of equation $(6.13 .1))$

$$
\left\langle\Delta V^{*} z^{2}>1 / 2=2\left[p_{2}^{*}(\infty) \lambda^{*}\right]^{1 / 2} \operatorname{PPE}\left(r_{0}^{*}\right)\right.
$$

where $p_{2} *(\infty)=0.1513$ and

$$
\operatorname{PPE}\left(r_{0}^{*}\right)=\left(1+0.240 / r_{0} * 8 / 7\right)-7 / 8
$$

and $\lambda^{*}$ is defined by equation $(7,4,18)$. For an extended cylindrical beam $\left(r_{0}^{*}>>1\right)$ one finds $P_{P E}\left(r_{0}^{*}\right)=1$. Removing the scaling in equation $(15.2 .5)$, using equation $(7.4 .29)$, yields

$$
\frac{<\Delta E^{2}>1 / 2}{E}=0.1414 \frac{e^{1 / 12 m^{1 / 4}}}{E_{0}^{5 / 6}} \operatorname{PPE}\left(r_{0}{ }^{*}\right) \frac{I^{1 / 2} L^{2 / 3}}{V^{13 / 12} r_{0}}
$$

If the energy distribution is Gaussian, the FWHM and rms are related as $\mathrm{FWHM}_{E}=(8 \ln 2)^{1 / 2} / 2 \Delta E^{2}>1 / 2$ and equation $(15.2 .6)$ leads back to equation $(7.4 .32)$.

In section 3.10 the thermodynamic upper limit of potential energy relaxation was discussed, which is relevant for a homocentric cylindrical beam segment. The corresponding rms-energy spread is given by equation (3.10.11). By scaling one obtains equation (7.7.3). Using the form of equation (15.2.5) one can now express this result in terms of an upper limit $P_{P E T}\left(\lambda^{*}\right)$ for the function $P_{P E}\left(r_{0}{ }^{*}\right)$, which is equal to

$$
\operatorname{PPET}\left(\lambda^{*}\right)=\frac{1.34}{\lambda^{* 1 / 3}}
$$

One finds $\mathrm{PPET}_{\mathrm{P}}=1$ for $\lambda^{*}=2.41$, while PPET increases for smaller $\lambda^{*}$ values. Thus, the upper limit of potential energy relaxation is not reached for practical conditions $\left(\lambda^{*}<10^{-2}\right)$.

To the best of our knowledge the only other theory treating the case of a homocentric beam segment is that of Massey, Jones and Plumer (1981). We will review their result at the end of this section. For all theories treating a beam segment with a crossover it was found that the resulting rms energy spread can be expressed in the form of equation $(15,2.3)$, provided that the function $\operatorname{PCE}_{C E}\left(\bar{r}_{c}, K_{1}, K_{2}\right)$ is generalized to a function $P\left(\bar{r}_{c}, \bar{\lambda}, K_{1}, K_{2}\right)$ which may also depend on $\bar{\lambda}$. In order to compare the theories it is therefore sufficient to compare the corresponding functions $P\left(\bar{r}_{c}, \bar{\lambda}, K_{1}, K_{2}\right)$. We will first determine this function for each of the theories and carry out the comparison next. The discussion presented in this section forms an extension to the work presented previously by Jansen, Groves and Stickel (1985).

\section{Zimmermann and Knauer}

Zimmermann (1970) and Knauer (1979) attribute the Boersch effect to the relaxation of internal kinetic energy from the longitudinal to the lateral degree of freedom by multiple weak complete two-particle collisions. This approach is closely related to the FokkerPlanck model used in plasma physics and stellar dynamics. In chapter 4 it was shown that the results presented by Zimmermann and Knauer for a 
cylindrical beam can directly be obtained from the velocity-diffusion coefficient appearing in the Fokker-Planck equation, see equation $(4.8 .4)$. The models of Zimmermann and Knauer are less suited to treat non-cylindrical beams in which the current density $\mathrm{J}$ and and lateral beam temperature $T_{1}$ varies with the axial coordinate. For the case of a beam segment with a crossover Zimmermann (1970) takes over the analysis given by Loeffler (1969), which will be discussed later on in this section. Knauer's approach for a beam segment with a crossover is to start from the result obtained for a thin cylindrical beam slice and integrate this expression over the axial coordinate $z$, taking the local current density $J(z)$ and local lateral temperature $T_{1}(z)$ equal to

$$
J(z)=J_{c}\left(\frac{r_{c}}{r_{0}(z)}\right)^{2}, \quad T_{\perp}(z)=T_{\perp c}\left(\frac{r_{0}(z)}{r_{c}}\right)^{2}
$$

in which $J_{c}$ and $T_{1}$ refer to the crossover plane. The first relation is trivial, while the second one was clarified in chapter 3 , see equation (3.7.17). In SI-units Knauer's result can be expressed as (Knauer (1979a), equation (33))

$$
\frac{<\Delta E^{2}>1 / 2}{E}=\frac{(\mathrm{em})^{1 / 4}}{(4 \pi)^{3 / 4} E_{0}} \frac{\mathrm{I}^{1 / 2}}{\alpha_{0} \mathrm{Y}_{\mathrm{c}}{ }^{1 / 2 V^{5 / 4}}}\left[\ln \Lambda_{\mathrm{c}} \ln \left(\mathrm{K}_{1} \mathrm{~K}_{2}\right)-\frac{2}{3} \ln ^{2} \mathrm{~K}_{1}-\frac{2}{3} \ln ^{2} \mathrm{~K}_{2}\right]^{1 / 2}
$$

where

$$
\Lambda_{c}=\frac{2^{13 / 6} \pi^{4 / 3} \varepsilon_{0}}{e^{1 / 2} m^{1 / 6}} \frac{\alpha_{0}^{2} r_{c}^{2 / 3} V^{7 / 6}}{I^{1 / 3}}
$$

Knauer separates the lateral velocities in thermal velocities and crossover velocities. He argues that the impact of both should be combined in a proper way and denotes the total energy broadening as the "composite" Boersch effect. However, one can not consider thermal and crossover motion as independent quantities. They both originate from the initial thermal motion near the cathode surface and are both affected by the focussing action of the optical components thereafter. One should either express the lateral velocities in terms of geometrical properties (like the beam semi-angle $\alpha_{0}$ ) or in terms of thermodynamical properties (like the beam temperature $T_{1}$ ). In equations $(15.2 .10)$ and $(15.2 .11)$ we therefore ignored Knauer's thermal component by taking $T=0$.

Knauer truncates the integration when the thermodynamic limit of kinetic energy relaxation is reached. In equation $(15.2 .10)$ we took the integration limits equal to the physical boundaries of the beam, assuming that the thermodynamic limit is not reached. This assumption should be verified afterwards. Furthermore, one should note that Knauer presupposes a Gaussian angular distribution and defines $\alpha_{0}=\left\langle\alpha^{2}\right\rangle 1 / 2$. For the uniform angular distribution with a cut-of $f$ angle $\alpha_{0}$, considered in the comparison, one finds $\left\langle\alpha^{2}\right\rangle 1 / 2=\alpha_{0} / \sqrt{2}$. Thus one might argue that equations $(15.2 .10)$ and $(15.2 .11)$ should be modified by replacing $\alpha_{0}$ by $\alpha_{0} / \sqrt{2}$. However, we prefer to use Knauer's original result and take the angle $\alpha_{0}$, appearing in equations (15.2.10) and (15.2.11), equal to the cut-of $f$ angle of the uniform angular distribution.

Equations $(15.2 .10)$ and $(15.2 .11)$ can be expressed in the form of equation (15.2.3), replacing the function $P_{C R}\left(\bar{r}_{C}, K_{1}, K_{2}\right)$ by

$$
\mathrm{P}_{\mathrm{K}}\left(\overline{\mathrm{r}}_{\mathrm{c}}, \bar{\lambda}, \mathrm{K}_{1}, \mathrm{~K}_{2}\right)=\left(\frac{8}{9 \pi \bar{r}_{\mathrm{c}}{ }^{2}}\right)^{1 / 4}\left[\frac{1}{2} \ln \left(\frac{\pi \bar{r}_{\mathrm{c}}{ }^{2}}{8 \pi}\right) \ln \left(\mathrm{K}_{1} \mathrm{~K}_{2}\right)-\ln ^{2} \mathrm{~K}_{1}-\ln ^{2} \mathrm{~K}_{2}\right]^{1 / 2}
$$

In order to comply with Knauer's thermodynamic limit the function $P_{k}$ 
should not exceed the upper value $P_{C E T}(\bar{l})$ given by equation $(15.2 .4)$.

\section{Loeffler}

Loeffler (1969) evaluated the energy spread generated in a beam segment with a narrow crossover by considering the effect of weak interactions on a reference particle moving along the beam axis. Loeffler's statistical treatment of the problem was outlined in section 5.3, see equations $(5.3 .14)$ to $(5.3 .16)$. He claims that this procedure leads to a median energy spread rather than a rms energy spread, which is not obvious at all. Moreover, some of the mathematical steps in his derivation are not entirely straightforward. In the approximation for the probability density function of the coordinates of the colliding particles, given by Loeffler's equation (19), one would expect that the boundaries are chosen equal to $r_{0}$ and $-r_{0}$, instead of $2 r_{0}$ and $-2 r_{0}$. Furthermore, for the case $8 \lambda r_{0}>1$ Loeffler replaces the sum of $N^{\text {th }}$ largest contributions to the total displacement of the reference particle by a "suitable" integral, which can be evaluated analytically. The resulting expression (Loeffler (1969) equation (22)) appears to be wrong, as was pointed out by Rose and Spehr (1983). Despite these obscurities we will use Loeffler's final results in their unmodified form.

Loeffler's result applies to a beam segment of infinite length, in which all collisions can be considered as complete. Loeffler and Hudgin (1970) presented a correction factor, which should be included for beams in which $\mathrm{K}=\alpha_{0} \mathrm{~L} / 2 \mathrm{r}_{\mathrm{c}}$ is not infinitely large. The resulting equation for the median energy spread generated in a beam segment with a crossover can be expressed as (Loeffler (1969) equations (22), (23) and (24); Loeffler and Hudgin (1970) equation (5))

$$
\frac{F W_{50, E}}{E}=\frac{e}{2 \pi \varepsilon_{0}} \frac{1}{\alpha_{0} r_{c} V} \Omega\left(\lambda r_{c}\right) \frac{1}{2}\left(F_{L}\left(K_{1}\right)+F_{L}\left(K_{2}\right)\right)
$$

where

$$
\begin{aligned}
& \Omega\left(\lambda r_{c}\right)=\left\{\begin{array}{l}
\left(8 \lambda r_{c}\right)^{1 / 2}\left(3+\ln 2+2 \ln \left(8 \lambda r_{c}\right)+\frac{1}{4} \ln 2\left(8 \lambda r_{c}\right)\right)^{1 / 2} \text { for } 8 \lambda r_{c}>1 \\
\frac{\pi}{\sqrt{2}} 8 \lambda r_{c} \text { for } 8 \lambda r_{c} \ll<1
\end{array}\right. \\
& F_{L}(K)=\left(1+\frac{11+0.4 /\left(\lambda r_{c}\right)^{2}}{K^{2}}\right)^{-1 / 2}
\end{aligned}
$$

Loeffler assumed that the resulting energy distribution is Gaussian. In that case the rms energy spread follows as

$$
\left\langle\Delta \mathrm{E}^{2}>1 / 2=\mathrm{FW}_{50, \mathrm{E}} / 2^{3 / 2} \operatorname{erf}^{-1}(1 / 2)=0.74130 \mathrm{FW}_{50, \mathrm{E}}\right.
$$

Equation (15.2.13) can now be expressed in the form of equation $(15.2 .3)$, replacing the function $P_{C E}\left(\bar{r}_{c}, K_{1}, K_{2}\right)$ by 


$$
\begin{aligned}
& \operatorname{Pl}_{L}\left(\overline{\mathrm{r}}_{\mathrm{c}}, \pi, \mathrm{K}_{1}, \mathrm{~K}_{2}\right)= \\
& = \begin{cases}\frac{4.55}{\overline{\mathrm{r}}_{\mathrm{c}} 1 / 2}\left(1+.54 \ln \left(8 \pi \overline{\mathrm{r}}_{\mathrm{c}}\right)+.0677 \ln ^{2}\left(8 \pi \overline{\mathrm{r}}_{\mathrm{c}}\right)\right)^{1 / 2} \frac{1}{2}\left(\mathrm{~F}_{\mathrm{L}}\left(\mathrm{K}_{1}\right)+\mathrm{F}_{\mathrm{L}}\left(\mathrm{K}_{2}\right)\right) & \text { for } 8 \overline{\mathrm{r}_{\mathrm{c}}>1} \\
14.9 \pi^{1 / 2} \frac{1}{2}\left(\mathrm{~F}_{\mathrm{L}}\left(\mathrm{K}_{1}\right)+\mathrm{F}_{\mathrm{L}}\left(\mathrm{K}_{2}\right)\right) & \text { for } 8 \pi \overline{\mathrm{r}}_{\mathrm{c}} \ll<1\end{cases}
\end{aligned}
$$

where the function $F_{L}(K)$ is defined by the second equation of (15.2.14). Our model shows that the energy distribution generated in an extended beam with a narrow crossover $(K>>1)$ is Gaussian when $\bar{\lambda} \gg>P_{C E}\left(\bar{r}_{C}\right)^{2} / 16 \pi$ and Lorentzian when $\pi<<P_{C E}\left(\bar{r}_{C}\right)^{2} / 16 \pi$, see section 7.3 . It is interesting to note that the Gaussian and Lorentzian regime roughly coincide with the regimes distinguished by Loeffler, corresponding to $8 \pi \bar{r}_{\mathrm{c}}>1$ and $8 \pi \bar{r}_{\mathrm{c}}<<1$ respectively. In the Lorentzian regime we found for the Full width median energy spread

$$
\frac{F W_{50, E}}{E}=\left(\frac{2 m}{\varepsilon_{0}^{2} e}\right)^{1 / 2} \frac{I}{\alpha_{0} V^{3 / 2}}
$$

as follows from equation (7.3.40), using that for an 1-dimensional Lorentzian distribution $\mathrm{FW}_{50}=\mathrm{FWHM}$. Loeffler's result for $8 \lambda \mathrm{r}_{\mathbf{c}} \ll<1$ given by equations $(15.2 .13)$ and $(15.2 .14)$ gives for $K \rightarrow \infty$ the same functional dependency, as can be seen by substituting the expression for $\lambda$ given by equation (3.2.4), but is larger by a factor $\sqrt{2}$.

For a pencil beam our model predicts

$$
\frac{F W_{50 . E}}{E}=0.64193 \frac{\mathrm{m}}{\varepsilon_{0} \mathrm{e}^{2}} \frac{I^{2} \mathrm{~L}}{\mathrm{~V}^{2}}
$$

as follows from equations $(7.4 .33)$ and $(7.5 .36)$. The same functional dependency follows from Loeffler's result for $8 \lambda r_{c}<<1$ and $k \rightarrow 0$ (using that $\left.F_{\mathrm{L}}(\mathrm{K}) \approx 1.58 \lambda \mathrm{r}_{\mathrm{c}} \mathrm{K}\right)$, but this equation predicts values which are $a$ factor 1.74 larger. The single regime which is not covered by Loeffler's equations for the Boersch effect is the Holtsmark regime, corresponding to a $2 / 3$ power-dependency on the beam current. This regime applies to extended beams with small K-values in which weak collisions are dominant.

\section{Crewe}

Crewe (1978a) estimated the energy deviation of an on-axis reference particle due to a single complete collision with a particle running with an angle $\alpha=\alpha_{0}$ and axial separation $b_{2}=1 / \lambda$ with respect to the reference particle. His result can be expressed as

$$
\frac{\Delta E}{E}=\left(\frac{m}{8 \varepsilon_{0}^{2} e}\right)^{1 / 2} \frac{I}{\pi^{2} \alpha_{0} V^{3 / 2}}\left[1+\frac{m}{2^{7} \varepsilon_{0}^{2} e^{2}} \frac{I^{2}}{\alpha_{0}^{4} V^{3}}\right]^{-1}
$$

Since Crewe ignores the statistical nature of the problem it is not obvious what measure the width $\Delta \mathrm{E}$ represents. For the comparison we will take $\left\langle\Delta E^{2}\right\rangle 1 / 2=\Delta E$. Equation $(15.2 .19)$ can now be expressed in the form of equation $(15.2 .3)$, replacing the function $P_{C E}\left(\vec{r}_{c}, K_{1}, K_{2}\right)$ by 


$$
\operatorname{Pc}(\pi)=\frac{2}{\pi^{3 / 2}} \frac{\pi^{1 / 2}}{1+\bar{\lambda}^{2}}
$$

which is independent of $\overline{\mathbf{r}}_{\mathrm{c}}, \mathrm{K}_{1}$ and $\mathrm{K}_{2}$.

\section{Rose and Spehr}

Rose and Spehr (1980) introduced the so-called "closest encounter approach" to evaluate the energy spread generated in a beam segment with a narrow circular or astigmatic crossover. This approach relies on the assumption that the displacement of a reference particle is dominated by the complete collision with its nearest neighbour, which is defined as the particle with the smallest impact parameter with respect to this particle. The statistical procedure followed by Rose and Spehr was outlined in section 5.3, see equations (5.3.19) and (5.3.20). The final equations of Rose and Spehr do not pertain to a specific reference trajectory, but represent an average over all possible reference trajectories in the beam. For that they followed the method described in section 10.3.

The scaling used by Rose and Spehr is similar to our do, voscaling defined by equation $(7,3.2)$. Their results can be converted to our notation, using

$$
\begin{aligned}
\left\langle\mathrm{u}^{2}\right\rangle & =\left\langle\Delta \overline{\mathrm{v}}_{\mathrm{z}}{ }^{2}\right\rangle=\left\langle\Delta \mathrm{E}^{2}\right\rangle /\left(2 \alpha_{0} \mathrm{E}\right)^{2} \\
\chi & =\lambda / 2 \\
\lambda & =4 \overline{\mathrm{r}}_{\mathrm{c}} \\
\omega & =\overline{\mathrm{v}} / 2 \\
\eta & =\overline{\mathrm{r}}_{\perp} / 2 \overline{\mathrm{r}}_{\mathrm{c}}
\end{aligned}
$$

The equation of Rose and Spehr for the rms energy spread generated in an extended circular crossover with an uniform angular and spatial distribution can directly be expressed in the form of equation (15.2.1), provided that the function $\mathrm{P}_{\mathrm{CE}}\left(\overline{\mathrm{r}}_{\mathrm{c}}, \mathrm{K}_{1}, \mathrm{~K}_{2}\right)$ is replaced by the function $\mathrm{P}_{\mathrm{RS}}\left(\overline{\mathrm{r}}_{\mathrm{c}}\right)$, given by (Rose and Spehr $(1980)$, equations (38), (40), (22) and (24))

$$
\operatorname{PRS}\left(\bar{r}_{c}\right)^{2}=\frac{8}{\pi} \int_{0}^{2} d \bar{v} F(\bar{v}) \int_{0}^{2} d t F(t) \frac{1}{\left[1+\bar{v}^{4} t^{2} \bar{r}_{c}^{2} / 4\right]^{1 / 2}} K\left(\frac{\bar{v}^{2} t \bar{r}_{c} / 2}{\left[1+\bar{v}^{4} t^{2} \bar{r}_{c}^{2} / 4\right]^{1 / 2}}\right)
$$

where the function $K$ is the complete elliptic integral of the first kind and

$$
F(x)=\frac{2}{\pi}\left[\arccos (x / 2)-(x / 2) \sqrt{1-(x / 2)^{2}}\right]
$$

This result is identical to our result obtained for an average reference trajectory, as expressed by equations (10.3.13) and (10.3.12), using that $P_{C E}\left(\bar{r}_{c}\right)=\left[p_{2}\left(\bar{r}_{c}\right) / \pi\right]^{1 / 2}$.

One may wonder why the closest encounter approach yields identical results as our model, in which the total displacement of the test particle is calculated as the sum of the displacements caused by all surrounding field particles. The answer can be found in the derivation given by Rose and Spehr (1980) by considering the approximation involved in the transition of equation (34) to equation (38). They argue that for practical beams, in which $x(=\pi / 2)<<1$, it is 
allowed to replace the exponential function in equation (34) by unity. Doing so, they remove the factor in the probability distribution which suppresses the contribution of the particles which are not the nearest neighbour of the reference particle. By this approximation their model becomes mathematically identical to ours and therefore yields the same results. The fact that this approximation is allowed implies that the rms energy spread generated in a beam segment with a narrow crossover is indeed dominated by collisions between nearest neighbours. Since the impact of more distant neighbours is minor one may as well include their contribution in the calculation. This is exactly what Rose and Spehr do by ignoring the exponential function in their equation (34).

The approximation for the integral of equation $(15.2 .23)$ given by Rose and Spehr (their equation $(42 . \mathrm{b})$ ) overestimates the numerical results and we prefer our approximation given by equations (7.6.11) and (10.3.14). Accordingly, we take

$$
\operatorname{PRS}\left(\bar{r}_{c}\right) \approx\left(1+\frac{1.471 \pi \bar{r}_{c}}{4 \ln ^{2}\left(32.43+1.276 \bar{r}_{c}\right)}\right)^{-1 / 2}
$$

which is sufficiently accurate for all $\bar{r}_{c}$ values. The calculation of Rose and Spehr relies on the assumption that the dominant collisions are (nearly) complete, which restricts its applicability to the case $K_{1} \gg 1$ and $K_{2} \gg 1$. Our calculation also covers the case of small K-values. The function $P_{C E}$ defined by equation (15.2.2) therefore depends also on $K_{1}$ and $K_{2}$. Notice that even for $K_{1} \gg 1$ and $K_{2} \gg 1$ equations (15.2.2) and (15.2.24) differ slightly, since the former pertains to the on-axis reference trajectory while the latter represents an average over all possible reference trajectories.

\section{De Chambost and Hennion}

De Chambost and Hennion (1979) estimated the Boersch effect from the mean square fluctuations in the electrostatic force acting on a reference particle. The basics of this type of approach were discussed in section 5.3 , see equations $(5.3 .21)$ to $(5.3 .26)$. De Chambost and Hennion studied the case of a cylindrical beam in an uniform acceleration zone and the case of a half-crossover in drift space. We will consider the latter. The rms energy spread generated in a full crossover follows in their model by summing the contributions of the two half-crossovers in quadrature. As the two contributions are identical the rms energy broadening of a full crossover is $\sqrt{2}$ times that of $a$ half-crossover. We checked the derivation given by De Chambost and Hennion and found an error in the arithmatics. Their result is a factor $2^{1 / 8}$ too large. After correction of this error one finds (De Chambost and Hennion (1979), equation (21) $\times \sqrt{2 \times 2-1 / 8)}$

$$
\frac{\left\langle\Delta E^{2}>1 / 2\right.}{E}=\frac{m^{3 / 16} e^{1 / 16}}{18^{3 / 16}\left(\pi E_{0}\right)^{5 / 8}} \frac{I^{3 / 8}}{\left(\alpha_{0} r_{c}\right)^{1 / 4} V^{13 / 16}}
$$

This result can be expressed in the form of equation (15.2.3), replacing the function $P_{C E}\left(\bar{r}_{c}, K_{1}, K_{2}\right)$ by

$$
\operatorname{PCH}_{\mathrm{CH}}\left(\overline{\mathrm{r}}_{\mathrm{C}}, \bar{\lambda}\right)=\frac{2^{7 / 8}}{3^{3 / 8} \sqrt{\pi}} \frac{1}{\bar{\lambda}^{1 / 8} \overline{\mathrm{r}}_{\mathrm{c}}^{1 / 4}}
$$

This equation is independent of $K_{1}$ and $K_{2}$, which are assumed to be large compared to unity. 
Sasaki (1984) presented a model for the Boersch effect produced in a beam segment with a crossover which is similar to that of De Chambost and Hennion. However, Sasaki's approach is somewhat more refined in the choice of the cut-off of the interaction force at small interparticle distances and differs in the calculation of the time intervals between successive independent states. His final result can be expressed as (Sasaki (1984), equation (4.29), (4.30) and (4.27))

$$
\frac{<\Delta E^{2}>1 / 2}{E}=0.143 \frac{m^{1 / 4}}{\varepsilon_{0}^{1 / 2} e^{1 / 4}} \frac{I^{1 / 2}}{V^{3 / 4}} G\left[0.881\left(\lambda r_{c}\right)^{1 / 3}\right]^{1 / 2}
$$

where

$$
G(\gamma)=\sum_{k=0}^{N(\gamma)-1}\left[\left(1-\frac{2 k}{3 \gamma}\right)^{3 / 2}-\left(1-\frac{2(k+1)}{3 \gamma}\right)^{3 / 2}\right]
$$

and $N(\gamma)$ the integer part of $3 \gamma / 2$. The plot of the function $G(\gamma)$ given by Sasaki is not consistent with the definition of $G(\gamma)$, reproduced above. It appears that the plot represents the first term only $(k=0)$, which is indeed monotonically decreasing with $\gamma$. The function of $G(\gamma)$ shown in the plot can be approximated as

$$
G(\gamma) \approx \frac{1}{1 / 3+\gamma}
$$

We will use this approximation in the further analysis. Equations (15.2.27) and $(15.2 .29)$ can be expressed in the form of equation (15.2.3), replacing the function $\operatorname{PcE}_{C}\left(\bar{r}_{\mathrm{c}}, \mathrm{K}_{1}, \mathrm{~K}_{2}\right)$ by

$$
P_{S}\left(\bar{r}_{c}, \bar{\lambda}\right)=\frac{0.240}{\left[1 / 3+.881\left(\bar{\lambda} \bar{r}_{c}\right)^{1 / 3}\right]^{1 / 2}}
$$

This results is independent of $K_{1}$ and $K_{2}$, which are assumed to be large compared to unity.

\section{Massey, Jones and Plummer}

Massey, Jones and Plummer (1981) studied the space charge effect and the statistical interaction effects occurring in the acceleration area of a laser illuminated photo-electron microscope. For the statistical effects they distinguish two mechanisms, namely potential energy relaxation and velocity (internal kinetic energy) relaxation. For the latter they followed the approach of Knauer (1979a), discussed previously in this section. Here we will investigate their model for potential energy relaxation in a cylindrical beam. As the model of De Chambost and Hennion and the model of Sasaki the model of Massey et al. starts from the mean square of the fluctuations in the electrostatic force acting on a reference particle. However, their statistical approach differs by the fact that the energy displacements produced in the successive states are added linearly instead of quadraticly, as was discussed in section 5.3, see equation (5.3.27). Furthermore, they account for the fact that the smallest interparticle distance will increase during the flight.

We recalculated their final equation for the energy broadening 


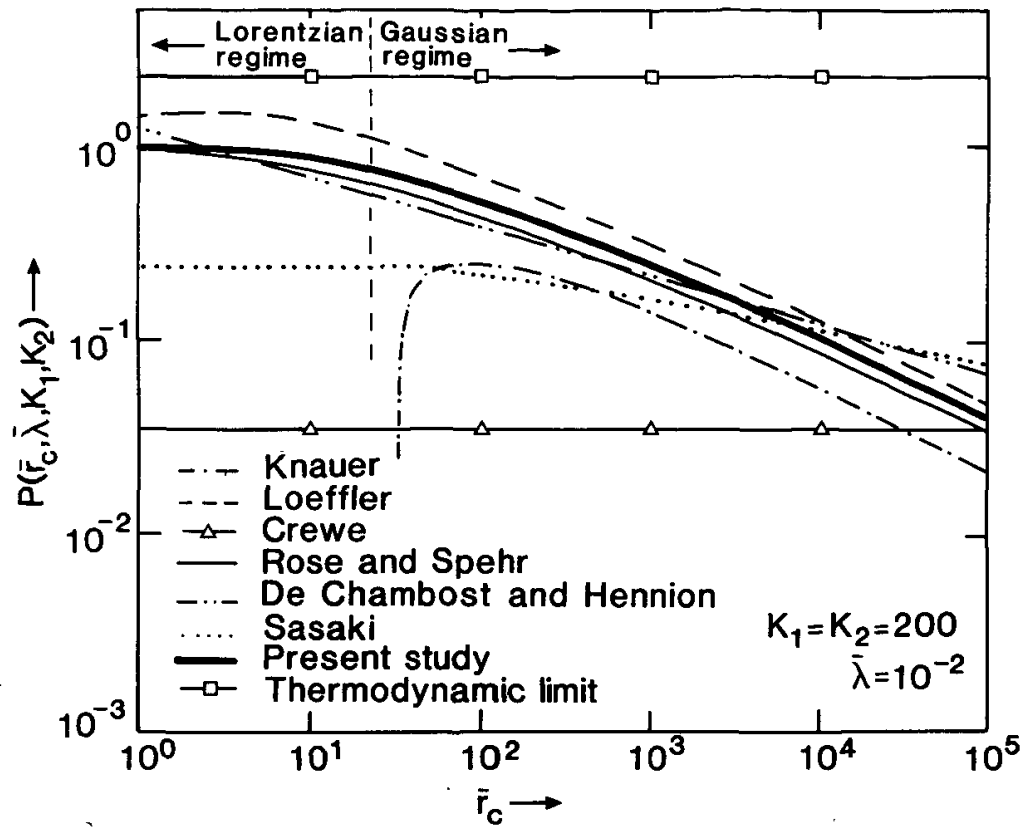

fig. 15.1a

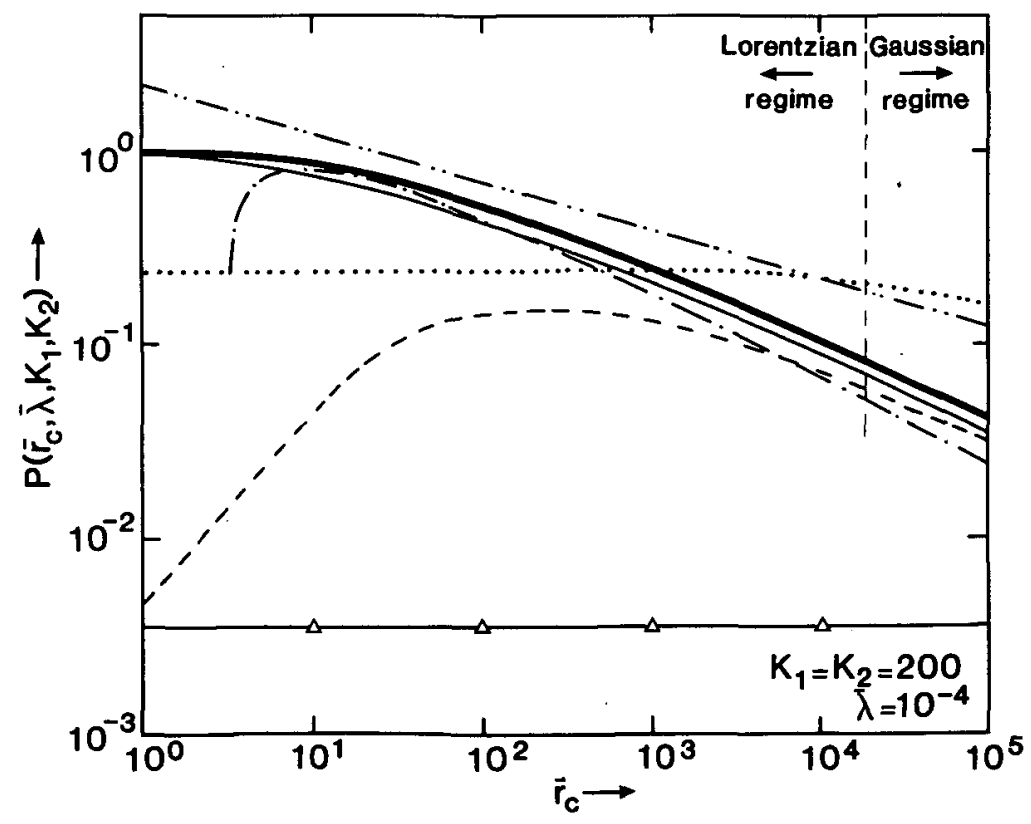

fig. 15.1b 


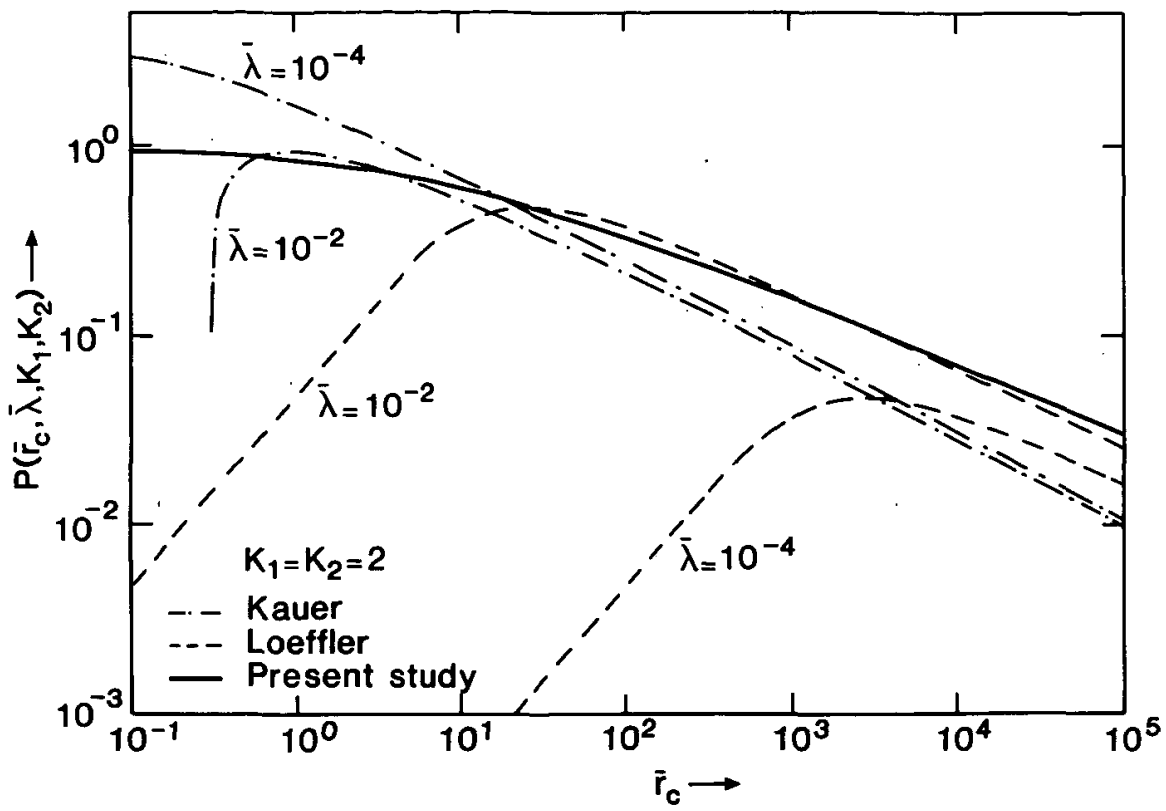

fig.15.1c

Figure 15.1 Comparison of the predictions of different theories for the rms energy spread generated in a beam segment with a crossover in the middle. Each theory is represented by a different function $\mathrm{P}\left(\overrightarrow{\mathrm{r}}_{\mathrm{c}}, \bar{\lambda}, \mathrm{K}_{1}, \mathrm{~K}_{2}\right)$, which is proportional to the rms energy spread, see equation (15.2.3). Figures 15.1 a and $15.1 \mathrm{~b}$ give the results for a narrow crossover $\left(\mathrm{K}_{1}=\mathrm{K}_{2}=200\right)$ and a scaled linear particle density $\bar{\lambda}$ equal to 10-2 and 10-4 respectively. Figure $15.1 \mathrm{c}$ gives the results for a more cylindrical shaped beam segment $\left(K_{1}=\mathrm{K}_{2}=2\right)$ and includes only those theories which apply to this case. The thermodynamic limit indicated in figure $15.1 \mathrm{a}$, pertains to the relaxation of kinetic energy, see equation (15.2.4). For smaller $\lambda$-values it exceeds the plotted P-range and it is therefore not shown in figure $15.1 \mathrm{~b}$. The present study reveals that the energy distribution generated in a narrow crossover (figures 15.1a and 15.1b) is either Gaussian or Lorentzian. These regimes are indicated. 
in a (nearly) homocentric cylindrical beam for the case that acceleration is absent. The final result shows the same functional dependency as the results obtained with acceleration, but is smaller by a factor 7/10. It can be expressed as (Massey, Jones and Plummer (1981), equation (27) $\times 7 / 10$, using equations (22) and (25))

$$
\frac{<\Delta \mathrm{E}^{2}>1 / 2}{E}=0.09325 \frac{\mathrm{e}^{1 / 12} \mathrm{~m}^{1 / 4}}{\varepsilon_{0}^{5 / 6}} \frac{\mathrm{I}^{1 / 2} \mathrm{~L}^{2 / 3}}{\mathrm{r}_{0} \mathrm{~V}^{13 / 12}}
$$

Our result for the rms energy spread generated in a homocentric cylindrical beam is given by equation $(15.2 .7)$. For an extended beam $\left(r_{0} * \gg 1\right)$ one may take $\operatorname{PPE}\left(r_{0}{ }^{*}\right) \approx 1$. One sees that the resulting equation shows the same functional dependency as equation $(15.2 .31)$, but is larger by a factor 1.52 .

\section{Comparison of theories for a beam segment with a crossover}

We have expressed the energy spread predicted by the various theories for a beam segment with a crossover in terms of the equivalent of the function $P_{C E}\left(\bar{Y}_{\mathbf{C}}, K_{1}, K_{2}\right)$ appearing in equation $(15.2 .3)$. In order to compare the theories it is sufficient to compare these functions, which are for the models of Knauer (1979a), Loeffler (1969), Crewe (1978a), Rose and Spehr (1980), De Chambost and Hennion (1979) and Sasaki (1984) given by equations (15.2.12), (15.2.16), (15.2.20), $(15.2 .24),(15.2 .26)$ and $(15.2 .30)$ respectively. our model is represented by the function $P_{C E}\left(\bar{r}_{C}, K_{1}, K_{2}\right)$ given by equation (15.2.2). In the figures $15.1 \mathrm{a}$ and $15.1 \mathrm{~b}$ all functions are plotted as function of $\overline{\mathbf{r}}_{\mathrm{c}}$ for the case $K_{1}=K_{2}=200$ and $\lambda=10^{-2}$ and $10^{-4}$ respectively. Figure $15.1 \mathrm{c}$ shows the corresponding results for $K_{1}=K_{2}=2$, leaving out those functions which do not depend on $K_{1}$ and $K_{2}$. In figure $15.1 \mathrm{a}$ the thermodynamic upper limit given by equation $(15.2 .4)$ is also plotted. One sees that it is not reached by any of the curves. The thermodynamic limit goes up for smaller $\lambda$-values and exceeds all predictions even further.

The occurrence of Gaussian and Lorentzian type of energy distributions, as predicted by our model, is indicated in the figures $15.1 \mathrm{a}$ and $15.1 \mathrm{~b}$. For a given $\lambda$-value the transition between both regimes is determined by $\bar{r}_{\mathfrak{c}}$ only, as can, for instance, be seen from figure 7.6. It ' should be recalled that the situation is more complicated for small K-values, for which the energy distribution can also be Holtsmarkian or of the pencil beam type. For this the reader is referred to section 7.5. From the figures $15.1 \mathrm{a}$ and $15.1 \mathrm{~b}$ one sees that there is some agreement between the theories in the Gaussian regime, while significant differences exist in the Lorentzian regime. The discrepancy in this regime between our result and that of Loeffler is mainly due to the conversion of Loeffler's median width to a rms value by means of equation (15.2.15), which requires the energy distribution to be Gaussian. Direct comparison of the predictions for the median energy spreads leads to a better agreement, see equation $(15.2 .17)$.

\subsection{Statistical angular deflections}

In chapter 8 the effect of statistical angular deflections was discussed, which is related to the distribution of transverse velocity displacements $\rho\left(\Delta v_{\perp}\right)$. The calculation is comparable to that of Boersch effect, which is related to the distribution of axial velocity displacements $\rho\left(\Delta v_{z}\right)$. It should be emphasized that the concept of 
statistical angular deflections is mainly of theoretical significance. In practice, one likes to know the trajectory displacements occurring in the final probe, which are only in a few special cases fully determined by angular deflections. The relation between the effect of statistical angular deflections and the trajectory displacement effect was investigated in section 9.6.

The organization of this section is quite similar to that of the previous section. The predictions of our model for the generated rms angular spread will be reviewed first and the results of some other theories will be considered next. The section ends up with a comparison of the various predictions.

With equations $(8.3 .18),(8.3 .20)$ and (8.5.27) the rms-value of the distribution of transverse velocity displacements generated in a beam segment with a crossover of arbitrary dimensions can be expressed as (using the $d_{0}, v_{0}$-scaling of equation $(7.3 .2)$ )

$$
<\Delta \bar{v}_{\perp} 2>1 / 2=\frac{<\Delta \alpha^{2}>1 / 2}{\alpha_{0}}=(\pi \overline{1}) 1 / 2 \mathrm{P}_{\mathrm{CA}_{\mathrm{A}}}\left(\overline{\mathrm{r}}_{\mathrm{c}}, \mathrm{K}_{1}, \mathrm{~K}_{2}\right)
$$

where $\mathrm{P}_{\mathrm{CA}}\left(\overline{\mathrm{r}}_{\mathrm{c}}, \mathrm{K}_{1}, \mathrm{~K}_{2}\right)=\left[\mathrm{P}_{\mathrm{CA}}\left(\overline{\mathrm{r}}_{\mathrm{c}}, \mathrm{K}_{1}\right)+\mathrm{P}_{\mathrm{CA}}\left(\overline{\mathrm{r}}_{\mathrm{c}}, \mathrm{K}_{2}\right)\right] / 2$ and

$$
\begin{aligned}
& P_{C A}\left(\bar{r}_{c}, K\right)= \\
& \quad\left[.894+.3\left(\frac{\bar{r}_{c}}{K^{2}}\right)^{2 / 3}\left[1+.5\left(\frac{K^{2}}{\bar{r}_{c}}\right)^{4 / 9}\right]^{3 / 2}+\frac{\pi \bar{r}_{c}}{\left\{2 \ln \left[.8673\left(114.6+\bar{r}_{c}\right)\right]\right\}^{2}}\right]^{-1 / 2}
\end{aligned}
$$

For large $K$-values the function $\mathrm{P}_{\mathrm{CA}}\left(\overline{\mathrm{r}}_{\mathrm{C}}, \mathrm{K}\right)$ becomes independent of $\mathrm{K}$ and identical to the function $P_{c k}\left(\vec{r}_{c}, K\right)$, defined by equation (15.2.2). For $\overline{\mathbf{r}}_{\mathrm{c}} \rightarrow 0$ it becomes equal to unity. By removing the scaling in equation (15.3.1), using that $\Delta \alpha=\alpha_{0} \Delta \bar{v}_{1}$, one finds for the rms-angular spread

$$
\left\langle\Delta \alpha^{2}>1 / 2=\frac{1}{2}\left(\frac{\mathrm{m}}{8 \varepsilon_{0}^{2} \mathrm{e}}\right)^{1 / 4} \mathrm{P}_{\mathrm{CA}}\left(\overline{\mathrm{r}}_{\mathrm{c}}, \mathrm{K}_{1}, \mathrm{~K}_{2}\right) \frac{\mathrm{I}^{1 / 2}}{\mathrm{~V}^{3 / 4}}\right.
$$

similar to equation (15.2.3). If the angular distribution is Gaussian, the FWHM and rms are related as FWHM $_{\alpha}=2(\ln 2)^{1 / 2} / 2\left\langle\Delta a^{2}>1 / 2\right.$ and equation (15.3.3) becomes equivalent to equation (8.3.21).

With equations $(8.4 .18),(8.4 .20)$ and $(8.4 .16)$ the rms-value of the distribution of transverse velocity displacements generated in a homocentric cylindrical beam segment can be expressed as (using the $\delta, \nu-$ scaling of equation (6.13.1))

$$
\left\langle\Delta V^{*}{ }_{\perp}{ }^{2} 1 / 2=2\left[P_{2}{ }^{*}(\infty) \lambda^{*}\right]^{1 / 2} P_{P A}\left(r_{0}^{*}\right)\right.
$$

where $\mathrm{p}_{2} *(\infty)=0.3026$ and

$$
\mathrm{P}_{P A}\left(\mathrm{r}_{0}{ }^{*}\right)=\left(1+.185 / \mathrm{r}_{0} * 8 / 7\right)-7 / 4
$$

and $\lambda^{*}$ is defined by equation $(7.4 .18)$. For an extended cylindrical beam $\left(r_{0}{ }^{*}>1\right)$ one finds $P_{P_{A}}\left(r_{0}{ }^{*}\right)=1$. By removing the scaling in equation (15.3.4), using equation $(8.4 .27)$, one finds for the rms angular spread

$$
\left\langle\Delta Q^{2}>1 / 2=0.1000 \frac{e^{1 / 12} \mathrm{~m}^{1 / 4}}{\varepsilon_{0}^{5 / 6}} \mathrm{P}_{\mathrm{PA}^{5}}\left(\mathrm{r}_{0}{ }^{*}\right) \frac{I^{1 / 2} \mathrm{~L}^{2 / 3}}{\mathrm{~V}^{13 / 12} \mathrm{r}_{0}}\right.
$$

If the angular distribution is Gaussian, the FWHM follows as $F W H M=2(\ln 2)^{1 / 2<\Delta \alpha^{2}>1 / 2}$, in agreement with equation $(8.4 .30)$.

The rms angular spread and the rms energy spread generated in an extended cylindrical beam, in which the interaction effects are not 
limited by the beam boundaries, are related by

$$
<\Delta \alpha^{2}>1 / 2=\frac{\left\langle\Delta E^{2}>1 / 2\right.}{\sqrt{2} E}
$$

as can directly be verified from equations (15.3.6) and (15.2.7), taking $r_{0} * \gg 1$. From equations (15.3.3) and (15.2.3) one sees that a similar relation holds true for the interaction effects occurring in a beam segment with a narrow crossover (thus $K_{1}$ and $K_{2} \gg 1$ ), which differs, however, by a factor $\sqrt{2}$.

For the theories treating a beam segment with a crossover it was found that the resulting rms angular spread can be expressed in the form of equation $(15.3 .3)$, provided that the function $P_{C_{A}}\left(\bar{r}_{c}, K_{1}, K_{2}\right)$ is generalized to a function $P\left(\bar{r}_{c}, \bar{\lambda}, K_{1}, K_{2}\right)$ which may also depend on $\bar{\lambda}$. We will first determine this function for each of the theories. The comparison between the theories will be carried out next by comparing these functions.

\section{Loeffler and Hudgin}

Loeffler and Hudgin (1970) extended the model of Loeffler (1969) to include the impact of the lateral components of the coulomb force acting on a reference particle running along the axis in a beam segment with a narrow crossover. First order perturbation dynamics is used to evaluate the impact of a single weak two-particle collision analytically. They separate the lateral force exerted by a colliding particle on the reference particle in a component in the direction of the relative transverse velocity $\left(\mathbf{F}_{\perp 1}\right)$ and a component perpendicular to this direction $\left(P_{12}\right)$, see figure 2.1. They state that the latter is responsible for an angular deflection of the reference particle $\Delta \alpha$, while the former causes a lateral shift $\Delta r$ only. Their reasoning is that this component changes of sign when the particles pass the point of closest approach, causing a cancellation of the angular deflections picked up in the first and the second half of the flight.

One may wonder to what image plane the shift $\Delta r$ evaluated by Loeffler and Hudgin refers. As it is not accompanied by a net angular shift the size of the corresponding virtual shift will be the same for any image plane. However, the force component causing the angular deflection $\Delta a$ will also produce a spatial shift $\Delta r$. The corresponding virtual shift will only be negligible for an image plane which coincides with the crossover plane, as can be understood from the symmetry of the trajectory with respect to the crossover. Accordingly, we conclude that the trajectory displacement effect predicted by Loeffler and Hudgin pertains to the virtual broadening observed in the crossover plane, while the virtual spatial broadening will be larger for other planes.

It should also be noticed that the force component producing the angular deflection will be zero when the colliding electron follows a meridian trajectory. The effect of angular deflections is therefore expected to vanish in a homocentric beam segment $\left(r_{c}=0\right)$. Finally, it should be emphasized that the reasoning of Loeffler and Hudgin is only valid for weak collisions and requires that the crossover is narrow and located somewhere near the middle of the beam segment.

Loeffler and Hudgin solved the statistical part of the problem by numerical means, but presented their final results in an analytical form. For the Full width median of the distributions of angular changes $\Delta a$ and trajectory displacements $\Delta r$ they give respectively (Loeffler and Hudgin (1970), equations (2) and (3) after correction of a misprint) 


$$
\begin{aligned}
& F W_{50 \alpha}=\frac{e}{4 \pi \varepsilon_{0}} \frac{1}{\alpha_{0} r_{c} V} \wedge\left(\lambda r_{c}\right) \\
& F W_{50 r}=\frac{e}{4 \pi \varepsilon_{0}} \frac{1}{\alpha_{0}^{2} V} \Gamma\left(\lambda \alpha_{0} L\right)
\end{aligned}
$$

From the plot of the functions $\Lambda$ and $\Gamma$ one finds

$$
\begin{aligned}
& \Lambda(x) \approx\left\{\begin{array}{lll}
5.0 x^{2 / 3} & \text { for } & x>>1 \\
13 x^{2} & \text { for } & x<1
\end{array}\right. \\
& \Gamma(x) \approx\left\{\begin{array}{lll}
2.8 x^{5 / 6} & \text { for } & x<1 \\
10 x^{3} & \text { for } & x<1
\end{array}\right.
\end{aligned}
$$

which should be compared to the function $\Omega(x)$ given by the first of equations $(15.2 .14)$.

In this section we will consider the result of Loeffler and Hudgin for the angular displacements; while the result for the trajectory displacements will be discussed in the next section. By naively assuming that the distribution of angular displacements is a (2dimensional) Gaussian distribution one obtains the following relation

$$
\mathrm{FW}_{50, \alpha}=\mathrm{FWHM}_{\alpha}=2(\ln 2) 1 / 2<\Delta \alpha^{2}>1 / 2
$$

Equations $(15.3 .8)$ and $(15.3 .10)$ can now be expressed in the form of equation (15.3.3), replacing the function $\mathrm{P}_{\mathrm{CA}}\left(\overline{\mathrm{r}}_{\mathrm{c}}, \mathrm{K}_{1}, \mathrm{~K}_{2}\right)$ by

$$
P_{L H}\left(\bar{r}_{c}, \bar{\lambda}\right)^{\prime}= \begin{cases}\frac{5.0}{(\pi \ln 2)^{1 / 2}} \frac{\pi^{1 / 6}}{\bar{r}_{c}^{1 / 3}} & \text { for } \lambda \bar{r}_{c} \gg 1 \\ \frac{13}{(\pi \ln 2)^{1 / 2}} \pi^{3 / 2} \bar{r}_{c} & \text { for } \lambda \bar{r}_{c} \ll 1\end{cases}
$$

This equation is independent of $K_{1}$ and $K_{2}$, which are assumed to be large.

As for the Boersch effect our model indicates that distribution becomes non-Gaussian for low and moderate particle densities. For moderate $\pi$-values and $K_{1}$ and $k_{1} \gg 1$ one finds from equations $(8.3 .24)$ and (8.5.43) for the Full Width median angular displacement generated in a beam segment with a crossover

$F_{50, \alpha}=.01819 \frac{\mathrm{m}}{\varepsilon_{0}{ }^{2} \mathrm{e}}\left(1+.02069 \bar{\gamma}_{\mathrm{c}}\right) \frac{\mathrm{I}^{2}}{\alpha_{0}{ }^{3} \mathrm{~V}^{3}}=\left\{\begin{array}{l}.4571 \frac{\mathrm{m}}{\varepsilon_{0} \mathrm{e}^{2}} \frac{\mathrm{I}^{2} \mathrm{r}_{\mathrm{c}}}{\alpha_{0} \mathrm{~V}^{2}} \text { for } \bar{r}_{\mathrm{c}} \gg>100 \\ .01819 \frac{\mathrm{m}}{\varepsilon_{0} \mathrm{e}_{\mathrm{e}}} \frac{\mathrm{I}^{2}}{\alpha_{0}{ }^{3} \mathrm{~V}^{3}} \text { for } \bar{r}_{c} \ll<100\end{array}\right.$

using the approximation for $f_{o p}\left(\bar{r}_{c}\right)$ given by equation (8.3.10) and the definition of $\bar{r}_{c}$ given by equation (7.3.5). The functional dependency of equation (15.3.14) occurs when weak complete collisions are dominant and the corresponding regime is called the "weak complete collision regime". The result of Loeffler and Hudgin expressed by equations (15.3.8) and (15.3.10) leads for $\lambda r_{c} \ll<$ to the same functional dependency as equation (15.3.14) for $\overline{\mathbf{r}}_{\mathrm{c}} \gg>100$, but is larger by a factor 1.13 . We 
conclude that their result for $\lambda r_{c} \ll<$ is not generally valid, but predicts accurate results for the weak complete collision regime if $\bar{r}_{c} \gg 100$. It should be noticed that for very small particle densities the angular displacement distribution will change to the pencil beam type, leading to the functional dependency given by equation (8.5.35). This regime is not covered by the results of Loeffler and Hudgin.

Let us now investigate the result of Loeffler and Hudgin given for $\lambda r_{c} \gg 1$. From equations $(15.3 .8)$ and $(15.3 .10)$ one obtains by using the definition of $\lambda$, given by equation $(3.2 .4)$

$$
\mathrm{FW}_{50, \alpha}=0.32 \frac{\mathrm{m}^{1 / 3}}{\epsilon_{0}} \frac{\mathrm{I}^{2 / 3}}{\mathrm{~V}^{4 / 3} \alpha_{0} \mathrm{r}_{\mathrm{c}}{ }^{1 / 3}}=0.40 \frac{\mathrm{m}^{1 / 3}}{\epsilon_{0}} \frac{\mathrm{I}^{2 / 3} \mathrm{~K}^{1 / 3}}{V^{4 / 3} \alpha_{0} /{ }^{3} \mathrm{~L}^{1 / 3}}
$$

with $\mathrm{K}=\alpha_{0} \mathrm{~L} / 2 \mathrm{r}_{\mathrm{c}}$. The $2 / 3$ power dependency on the beam current suggest that this regime coincides with the Holtsmark regime distinguished in our model. This regime occurs for an extended beam in which the effect is dominated by weak incomplete interactions. From equations (8.5.31), $(8.5 .20)$ and $(8.5 .43)$ one finds for the Holtsmark regime

$$
\text { FW }_{5, \alpha}=0.4810 \frac{\mathrm{m}^{1 / 3}}{\varepsilon_{0}}\left|\frac{1}{\left(1 / \mathrm{K}+2 \mathrm{~S}_{\mathrm{c}}\right)^{1 / 3}}-\frac{1}{\left[1 / \mathrm{K}+2\left(1-\mathrm{S}_{\mathrm{c}}\right)\right]^{1 / 3}}\right| \frac{\mathrm{I}^{2 / 3}}{\mathrm{~V}^{4 / 3} \alpha_{0}^{4 / 3} \mathrm{~L}^{1 / 3}}
$$

in which one should take $S_{c}=0$ or $S_{c}=1$ when $K \rightarrow 0$. For $K \rightarrow \infty$ the $F W_{50}, \alpha$ value predicted by equation $(15.3 .16)$ vanishes for a crossover in the middle $\left(S_{c}=1 / 2\right)$, contrary to the result given by Loeffler and Hudgin. For a half-crossover $\left(S_{c}=0\right.$ or $\left.S_{c}=1\right)$ and $K \gg>1$ one $f$ inds the same functional dependency as predicted by Loeffler and Hudgin. Finally, one should realize that for very large particle densities the angular displacement distribution will become Gaussian, leading to the functional dependency given by equation (8.3.21). This regime is not covered by the results of Loeffler and Hudgin.

\section{Weidenhausen, Spehr and Rose}

Weidenhausen, Spehr and Rose (1985) employed the "closest encounter approach" of Rose and Spehr (1980) to evaluate the mean square angular spread generated in a beam segment with a narrow circular or astigmatic crossover. The angular and current density distribution in the crossover are assumed to be Gaussian. Using the relations of equation (15.2.21) their final result can directly be expressed in the form of equation (15.3.1), provided that the the function $P_{c A}\left(\bar{r}_{c}, K_{1}, K_{2}\right)$ is replaced by (Weidenhausen, Spehr and Rose (1985), equations (20), (22) and (23))

$$
\operatorname{PWSR}_{\mathrm{W}}\left(\overline{\mathrm{r}}_{\mathrm{c}}\right)=\left\{\begin{array}{l}
(4 / \pi-1)^{1 / 2} \text { for } \overline{\mathrm{r}}_{\mathrm{c}} \rightarrow 0 \\
\frac{(4 / \pi-1)^{1 / 2}}{2 \overline{\mathrm{r}}_{\mathrm{c}}{ }^{1 / 2}}\left(32.4-33.6 \ln \overline{\mathrm{r}}_{\mathrm{c}}+16.5 \ln ^{2} \overline{\mathrm{r}}_{\mathrm{c}}\right)^{1 / 2} \text { for } \overline{\mathrm{r}}_{\mathrm{c}}>25
\end{array}\right.
$$

This result applies to the case $\lambda \ll<1$ and $\bar{x}_{\mathrm{c}}<1 / 2$. The numerical data plotted by Weidenhausen et al. shows that the function PwSR $\left(\bar{r}_{c}\right)$ has a maximum for $\bar{r}_{c} \approx 5$, which exceeds the value for $\bar{r}_{c}=0$ with about $30 \%$. 
Massey, Jones and Plummer (1981) evaluated the impact of the fluctuations in the transverse component of the electrostatic force acting on a reference particle which runs through an extended (nearly) homocentric beam segment in an uniform acceleration zone. Using this model we evaluated the rms angular spread for the case that acceleration is absent. We found that the resulting expression for the angular spread $\left\langle\Delta \alpha^{2}>1 / 2\right.$ can directly be obtained from the energy spread $\left\langle\Delta E^{2}>1 / 2\right.$ predicted by equation (15.2.31) by employing equation (15.3.7). As the same relation holds true for the corresponding equations derived within our model we end up with the same conclusions as reached for the Boersch effect, namely that the predictions of both models show the same functional dependency for the case of an extended homocentric cylindrical beam, but differ by a factor 1.52 .

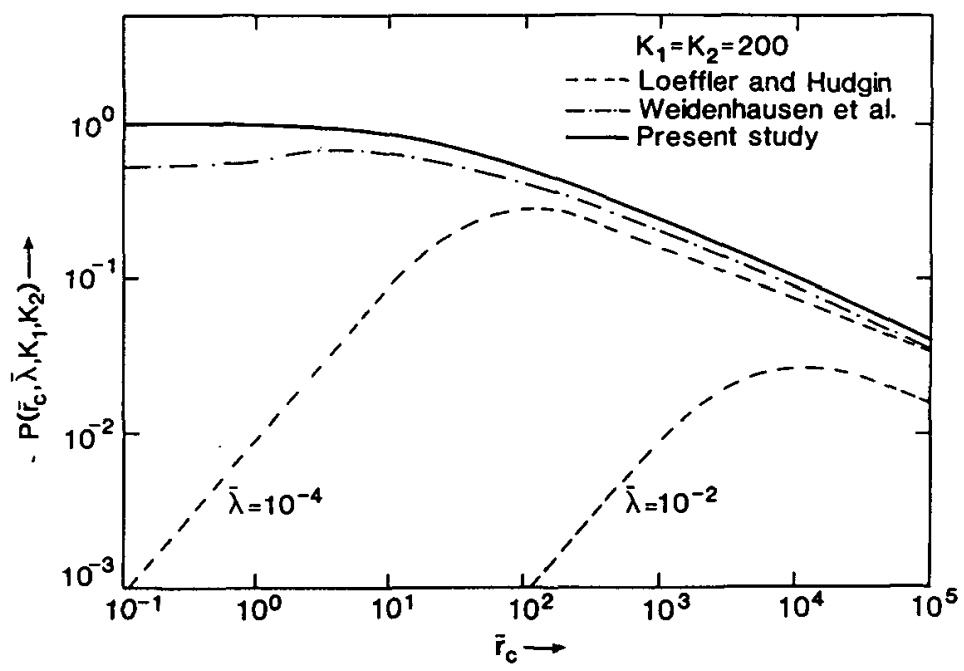

Pigure 15.2 Comparison of the predictions of different theories for the rms angular spread generated in a beam segment with a crossover in the middle. Each theory is represented by a different function $\mathrm{P}\left(\overline{\mathrm{r}}_{\mathrm{c}}, \bar{\lambda}, \mathrm{K}_{1}, \mathrm{~K}_{2}\right)$, which is proportional to the rms angular spread, see equation (15.3.3). All theories, apart from the present one, assume a narrow crossover and the comparison is therefore restricted to the case $\mathrm{K}_{\mathrm{L}}=\mathrm{K}_{2}=200$. The result of Loeffler and Hudgin is the only one which depends on the scaled linear particle density $\bar{\lambda}$. The values $\bar{\lambda}=10^{-2}$ and $\bar{\lambda}=10^{-4}$ are considered.

\section{Comparison of theories for a beam segment with a crossover}

The theories of Loeffler and Hudgin (1970) and Weidenhausen, Spehr and Rose (1985) give a prediction for the angular spread generated in a beam segment with a crossover. We have expressed their results in terms of the equivalent of the function $P_{C A}\left(\bar{r}_{C}, K_{1}, K_{2}\right)$ appearing in equation (15.3.3), see equations (15.3.13) and (15.3.17) respectively. Our model is represented by the function defined by equation (15.3.2). Contrary to the other models it is also valid for beams with small $\mathrm{K}-$ values. The comparison will be restricted to the case $K_{1}$ and $K_{2} \gg 1$.

In figure 15.2 the functions $P\left(\bar{r}_{c}, \bar{\lambda}, K_{1}, K_{2}\right)$ representing the different theories are plotted as function of $\bar{r}_{c}$ for the case 
$K_{1}=K_{2}=200$. The function representing the theory of Loeffler and Hudgin is the only one which depends on $\lambda$. It is plotted for the cases $\bar{\lambda}=10^{-2}$ and $10^{-4}$. As for the different predictions for the rms energy spread, which were compared in the previous section, one finds some agreement between the different theories for large values of $\bar{r}_{c}$. For these $\bar{r}_{c}$ values the angular displacement distribution is of the Gaussian type.

The substantial disagreement for smaller $\overline{\mathbf{r}}_{\mathrm{c}}$-values between our result and that of Loeffler and Hudgin is partly due to the conversion of their result from a median width to a rms value by means of equation (15.3.12), which requires the angular displacement distribution to be Gaussian. As the distribution is non-Gaussian for small $\bar{r}_{c}$-values the direct comparison of the predictions for the median angular spreads is more appropriate and shows a better agreement, see equation-(15.3.14).

The result of Weidenhausen et al, based on the closest encounter approach is in reasonable agreement with our prediction for most $\overline{\mathbf{r}}_{c}$ values, but leads to a 0.52 times smaller value for $\bar{r}_{c} \rightarrow 0$. Due to the approximations made within this model for practical particle densities $(\bar{\lambda}<<1)$ it is mathematically equivalent to our model, as was pointed out in the previous section. The differences between both results arise from the fact that our result pertains to an on axis reference trajectory and assumes an uniform angular and spatial distribution in the crossover, while that of Weidenhausen et al. represents an average over all possible reference trajectories and assumes a Gaussian angular and spatial distribution in the crossover. For $\overline{\mathbf{r}}_{\mathrm{c}} \rightarrow 0$ one would expect that the differences in the results become negligible (as is the case for the Boersch effect) and the disagreement in this limit is somewhat surprising.

In experimental systems one is mainly concerned with the spatial broadening of the final probe and the angular spread has to be translated into a spatial broadening in some reference plane, which is imaged on the target. The angular spread should therefore be multiplied with some distance, which will be proportional to the beam segment length L. In section 9.2 it was pointed out that this procedure implies that one can not use complete collision dynamics to evaluate the angular spread, since this dynamics neglects the terms proportional to $1 / \mathrm{L}$ (or equivalently $1 / \mathrm{T}$ ). See the discussion of equation (9.2.5). This is apparently not recognized by Weidenhausen, Spehr and Rose (1985) who claim that their result for the rms angular spread (which is based on complete collisions) can be used to evaluate the spatial blurring in some image plane which is located at some distance from the crossover.

\subsection{Trajectory displacement effect}

In chapter 9 the extended two-particle model was employed to describe the trajectory displacement effect, which is related to the distribution of virtual displacements $\rho(\Delta r)$ observed in a certain reference (or image) plane. With equations $(9.3 .28),(9.3 .24),(9.3 .25)$ and $(9.5 .10)$ the rms-value of the distribution of virtual displacements generated in a beam segment with a crossover of arbitrary dimensions can be expressed as (using the $\delta, v$-scaling of equation (6.13.1))

$$
\left\langle\Delta r^{* 2}\right\rangle 1 / 2=2\left[p_{2}{ }^{*}(\infty, 1 / 2,1 / 2) \pi\right]^{1 / 2} \mathrm{Pcr}_{\mathrm{cr}}\left(\mathrm{v}_{0}{ }^{*}, \mathrm{r}_{\mathrm{c}}{ }^{*}, \mathrm{~S}_{\mathrm{c}}, \mathrm{S}_{\mathrm{i}}\right) \quad(15.4 .1)
$$

where $p_{2} *(\infty, 1 / 2,1 / 2)=.273$ and 
$\operatorname{PCT}\left(v_{0}^{*}, r_{c}^{*}, S_{c}, S_{i}\right) \approx$

$$
\left(\frac{1+0.682\left(S_{c}-0.5\right)-0.739\left(S_{c}-0.5\right)^{2}}{\left(1+1.40 / v_{0} * 8 / 7\right)^{7 / 2}\left(1+0.30 r_{c}^{*}\right)}+113 v_{0} * 1 / 2\left(S_{c}-S_{i}\right)^{2}\right)^{1 / 2}
$$

By removing the scaling in equation (15.4.1), using equation (6.13.1), one obtains

$$
<\Delta r^{2}>1 / 2=9.5010^{-2} \frac{e^{1 / 12 m^{1 / 4}}}{\epsilon_{0}^{5 / 6}} P_{C T}\left(v_{0}^{*}, r_{c}^{*}, S_{c}, S_{i}\right) \frac{I^{1 / 2} L^{2 / 3}}{\alpha_{0} V^{1 / 1 / 2}}
$$

If the trajectory displacement distribution is Gaussian, the FWHM and rms-value are related as $F_{W H M}=2(\ln 2)^{1 / 2} / \Delta r^{2}>1 / 2$ and equation (15.4.3) becomes equivalent to equation $(9.3 .45)$. It should be noted that the Gaussian regime occurs only for relatively large particle densities $(\bar{\lambda}>.05)$. The practical use of the rms-value to characterize the trajectory displacement effect is therefore even further restricted than the use of the rms energy spread and rms angular broadening.

Similar to the approach taken in the previous sections our strategy is to express the results of the different theories for the trajectory displacement effect in the form of equation (15.4.3), replacing the function $P_{c r}\left(v_{0}{ }^{*}, r_{c}{ }^{*}, S_{c}, S_{i}\right)$ by a generalized version, which will be different for each theory. The comparison of the theories will be carried out at the end of the section, by comparing these functions.

\section{Loeffler and thudgin}

In the previous section we reproduced the results of Loeffler and Hudgin (1970) for the trajectory displacement generated in a beam segment with a narrow crossover, see equations (15.3.9) and (15.3.11). By naively assuming that the trajectory displacement distribution is a (2-dimensional) Gaussian distribution the rms value can be calculated from the $F W_{50}$ value as $\left\langle\Delta r^{2}>1 / 2=F_{50, r} / 2(\ln 2) 1 / 2\right.$, similar to equation $(15.3 .12)$. The results of Loeffler and Hudgin can now be expressed in the form of equation $(15.4 .3)$, replacing the function $P_{C T}\left(v_{0}{ }^{*}, r_{c}^{*}, S_{c}, S_{i}\right)$ by

$$
\operatorname{PLH}_{L}\left(v_{0}^{*}, \bar{\lambda}\right)= \begin{cases}2.6 v_{0} * 1 / 2 \lambda^{1 / 3} & \text { for } 4 \pi v_{0} * 3>>1 \\ 1810-2 \lambda^{5 / 2 v_{0} * 7} & \text { for } 4 \pi v_{0} * 3<<1\end{cases}
$$

This equation pertains to the case $S_{c}=S_{i} \approx 1 / 2$ and requires a narrow crossover.

Our model shows that the trajectory displacement distribution will be non-Gaussian for most operating conditions. Accordingly, it is more appropriate to compare the $\mathrm{FW}_{50, r}$-value predicted by Loeffier and Hudgin directly to our results obtained for this width measure. Loeffler and Hudgin give for $\lambda \alpha_{0} \mathrm{~L} \ll<1$

$$
\mathrm{FW}_{50 . r}=0.28 \frac{\mathrm{m}^{3 / 2}}{\varepsilon_{0} \mathrm{e}^{7 / 2}} \frac{\mathrm{I}^{3} \alpha_{0} \mathrm{~L}^{3}}{\mathrm{~V}^{5 / 2}}
$$

as follows from equations (15.3.9) and (15.3.11). Our model predicts a similar functional dependency for the pencil beam regime. From equation $(9.3 .47),(9.5 .6)$ and $(8.5 .43)$ one finds for this regime in case $S_{c}=S_{i}$ 


$$
F W_{50, r}=0.03622 \frac{\mathrm{m}^{3 / 2}}{\varepsilon_{0} \mathrm{e}^{7 / 2}}\left|4-12 \mathrm{~S}_{\mathrm{c}}\left(\mathrm{S}_{\mathrm{c}}-1\right)+\frac{3-6 \mathrm{~S}_{\mathrm{c}}\left(1-\mathrm{S}_{\mathrm{c}}\right)}{\mathrm{K}}\right| \frac{\mathrm{I}^{3} \alpha_{0} \mathrm{~L}^{3}}{\mathrm{~V}^{5 / 2}}(15.4 .6)
$$

For $K \rightarrow 0$ one should take $\dot{S}_{c}=0$ or $S_{c}=1$. For $K \rightarrow \infty$ and $S_{c}=1 / 2$ we obtain the same functional dependency as Loeffler and Hudgin, but their result is larger by a factor 7.8 .

For $\lambda \alpha_{0} L \gg>1$ one obtains from equations $(15.3 .9)$ and $(15.3 .11)$

$$
F_{50, r}=0.17 \frac{m^{5 / 12}}{E_{0} e^{1 / 4}} \frac{I^{5 / 6} L^{5 / 6}}{V^{17 / 12} \alpha_{0}^{7 / 6}}
$$

This functional dependency is not confirmed by any of the regimes distinguished within our model. According to our model the trajectory displacement distribution is Holtsmarkian for moderate particle densities and Gaussian for large particle densities, leading to the functional dependencies of equations (9.3.44) and (9.3.45) respectively. These regimes are not covered by the results of Loeffler and Hudgin.

\section{De Chambost}

De Chambost (1982) relates the trajectory displacement effect generated in a beam segment with a crossover to the energy spread generated in such a beam segment. For the energy spread he utilizes the result given by De Chambost and Hennion (1979). Following this approach, but using the corrected equation $(15.2 .25)$, one finds

$$
\begin{aligned}
\left\langle\Delta r^{2}>1 / 2\right. & =\frac{\left\langle\Delta E^{2}>1 / 2 L\right.}{2 E} F_{C}\left(K_{1}, K_{2}\right) \\
& =\frac{m^{3 / 16} e^{1 / 16}}{18^{3 / 16} 2\left(\pi \varepsilon_{0}\right)^{5 / 8}} \frac{I^{3 / 8} L}{\left(\alpha_{0} r_{c}\right)^{1 / 4} V^{13 / 16}} F_{C}\left(K, S_{c}\right)
\end{aligned}
$$

where

$$
\begin{aligned}
& F_{c}\left(K, S_{c}\right)=\left(S_{c}{ }^{2} h\left(2 S_{c} K\right)^{2}+\left(1-S_{c}\right)^{2} h\left[2\left(1-S_{c}\right) K\right]^{2}\right)^{1 / 2} \\
& h(x)=\frac{x^{1 / 2}}{(x-1)^{3 / 2}}\left(x-\frac{1}{x}-2 \ln x\right)^{1 / 2}
\end{aligned}
$$

Equation (15.4.8) can be expressed in the form of equation (15.4.3), replacing the function $\mathrm{P}_{\mathrm{ct}}\left(\mathrm{v}_{\mathrm{o}}{ }^{*}, \mathrm{r}_{\mathrm{c}}{ }^{*}, \mathrm{~S}_{\mathrm{C}}, \mathrm{S}_{\mathrm{i}}\right)$ by

$$
P_{c}\left(v_{0}^{*}, r_{c}{ }^{*}, S_{c}, \lambda\right)=0.822 \frac{v_{0}{ }^{* 1 / 2}}{\pi^{1 / 8} r_{c}{ }^{* 1 / 4}} F_{c}\left(v_{0}^{*} / 2 r_{c}{ }^{*}, S_{c}\right)
$$

using that $K=\mathrm{v}_{0} * / 2 \mathrm{r}_{\mathrm{c}}{ }^{*}$. This equation pertains to the case $S_{c}=S_{i}$ and requires that $\mathrm{K}>>1$.

\section{Spehr}

Spehr (1985a) relates the trajectory displacement effect to a transfer of internal kinetic energy from the longitudinal to the lateral degree of freedom by weak complete collisions. According to Spehr this 
transfer occurs in the dilute parts of the beam where the longitudinal beam temperature exceeds the transverse beam temperature. In this model the trajectory displacement effect stems mainly from the initial energy spread. The magnitude of the initial energy spread, which plays no role in the other models, is therefore an essential parameter in Spehr's model.

For the broadening of an initially homocentric crossover $\left(r_{c}=0\right)$ Spehr gives (Spehr (1985a), equations (19), (20), (21) and (17))

$$
\Delta r=\left(\frac{\mathrm{em}}{2^{9} \pi^{3} \varepsilon_{0}^{4}}\right)^{1 / 4} \frac{\mathrm{I}^{1 / 2} \mathrm{~L}^{1 / 2}}{\mathrm{~V}^{5 / 4} \alpha_{0}} \frac{\mathrm{E}^{1 / 2}}{\left\langle\Delta \mathrm{E}^{2}\right\rangle^{1 / 4}}\left[3.22+\ln 2\left(\frac{4 \pi \varepsilon_{0}}{7.98 \mathrm{e}} \mathrm{Va}_{0} \mathrm{~L} \frac{\left\langle\Delta \mathrm{E}^{2}\right\rangle}{\mathrm{E}^{2}}\right)\right]^{1 / 2}
$$

provided that

$$
\frac{\left\langle\Delta \mathrm{E}^{2}\right\rangle}{\mathrm{E}^{2}}>100 \frac{\mathrm{C}_{0}}{\alpha_{0} \mathrm{LE}} \quad \text { and } \quad \pi<\frac{\left\langle\Delta \mathrm{E}^{2}\right\rangle}{24 \alpha_{0} \mathrm{E}^{2}}
$$

By assuming that we may identify $\Delta r$ with a rms-value, this result can be expressed in, the form of equation (15.4.3), replacing the function $P_{C T}\left(v_{0}{ }^{*}, r_{c}{ }^{*}, S_{c}, S_{i}\right)$ by

$$
\operatorname{Ps}\left(v_{0} *,\left\langle\Delta v_{z} * 2\right\rangle\right)=\frac{0.901}{\left\langle\Delta v_{z}^{* 2}\right\rangle 1 / 4}\left[3.22+\ln ^{2}\left(1.00 v_{0}^{*}\left\langle\Delta v_{z} * 2\right\rangle\right)\right]^{1 / 2}(15.4 .13)
$$

The conditions of equation (15.4.12) can be expressed as

$$
<\Delta v_{z} * 2>>12.5 / v_{0}^{*} \text { and } \pi<\alpha_{0}<\Delta v_{z} * 2>/ 6 v_{0} * 2
$$

Spehr's result pertains to the virtual broadening of an originally homocentric crossover $\left(r_{c} *=0, S_{i}=S_{c}\right)$. It is independent of the location of the crossover, which is here specified by $S_{c}$. The initial energy spread is in equation $(15.4 .13)$ represented by the parameter $\left\langle\Delta v_{z}^{* 2>1 / 2}\right.$. The relation with the experimental parameters can be obtained from equation $(10.4 .5)$. For practical beams the value of $\left\langle\Delta v_{z} * 2\right\rangle t / 2$ is typically in the range .1 to 1 . When the first constraint of equation (15.4.14) is not fulfilled, it is not permitted to use the approximating result of equation (15.4.13). Instead one has to evaluate the integral appearing in Spehrs model by numerical means (Spehr (1985a), equation (20)). The outcome will be smaller than predicted by equation (15.4.13) and leads to a zero broadening for $\left\langle\Delta v_{2} * 2>1 / 2+0\right.$.

\section{Comparison of theories}

The theories of Loeffler and Hudgin (1970), De Chambost (1982) and Spehr (1985a) give a prediction for the trajectory displacement effect generated in a beam segment with a crossover. We have expressed their results in terms of the equivalent of the function $P_{c T}\left(v_{0}^{*}, r_{c}^{*}, S_{c}, S_{1}\right)$ appearing in equation $(15.4 .3)$, see equations $(15.4 .4), \quad(15.4 .10)$ and $(15.4 .13)$ respectively. our model is represented by the function defined by equation (15.4.2). This function is valid for the full range of operating conditions represented by the parameters $v_{0}^{*}, r_{c}{ }^{*}, S_{c}$ and $S_{i}$. The other models apply to large values of $K=v_{0} * / 2 r_{c} *$ (narrow crossover) and $S_{i}=S_{c}$ (image plane in the crossover). The result of Loeffler and Hudgin requires in addition that the crossover is located somewhere near the middle of the beam segment $\left(S_{c} \approx 1 / 2\right)$.

In figure 15.3a the functions $\mathrm{P}\left(\mathrm{v}_{0} *, \mathrm{r}_{\mathrm{c}}^{*}, \mathrm{~S}_{\mathrm{c}}, \mathrm{S}_{i}, \pi,\left\langle\Delta \mathrm{v}_{z}^{* 2}\right\rangle \mathbf{1} / 2\right)$ representing the different theories are plotted as function of $v_{0}^{*}$ for 


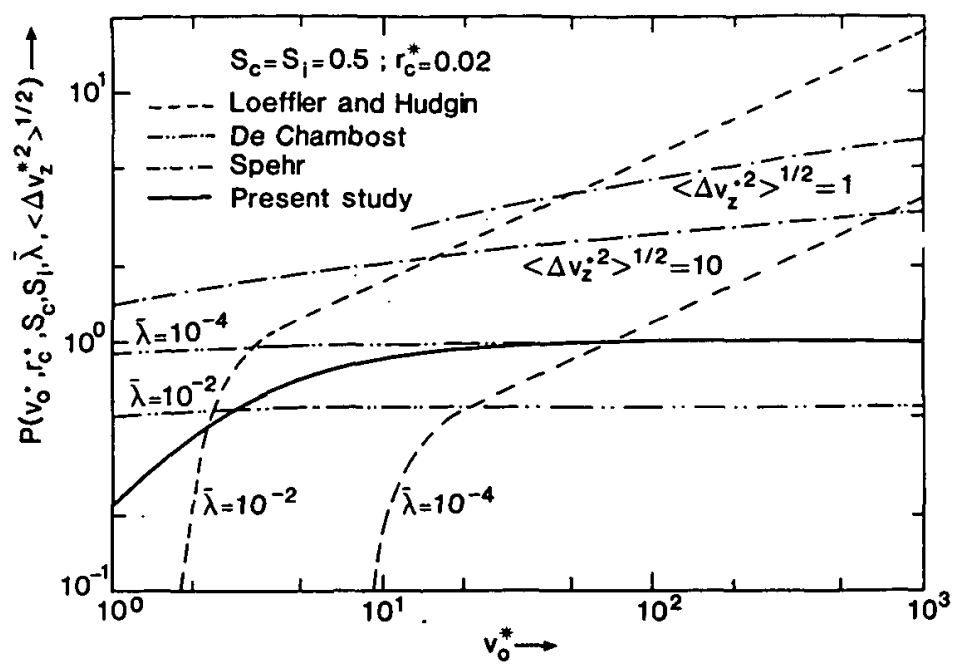

fig. 15.3a

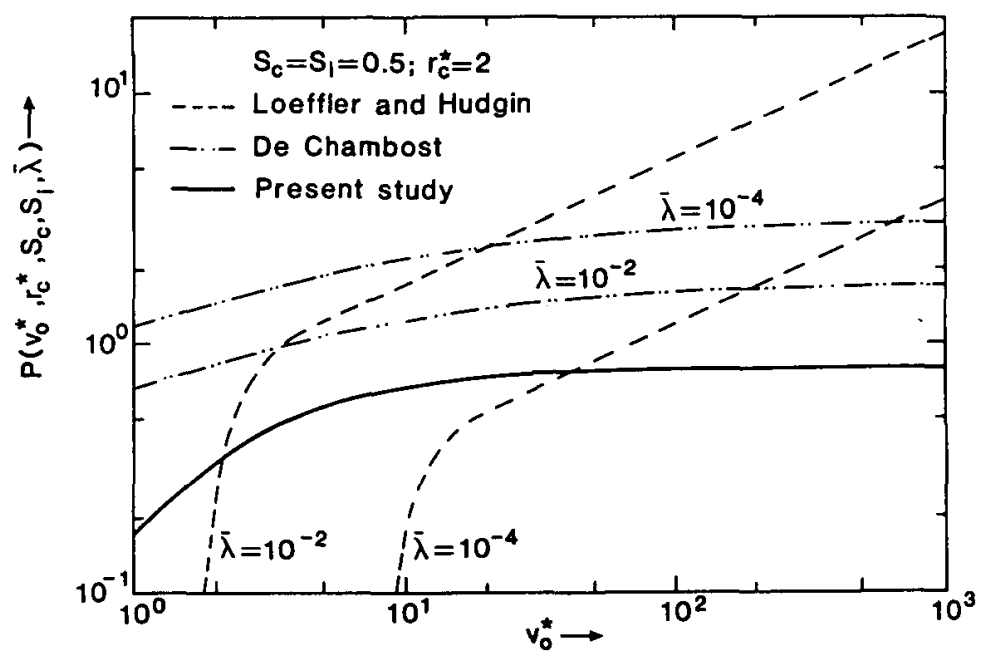

fig. 15.3b

Figure 15.3 Comparison of the predictions of different theories for the rms trajectory displacement broadening generated in a beam segment with a crossover in the middle $\left(S_{c}=1 / 2\right)$. Each theory is represented by a different function $\mathrm{P}\left(\mathrm{v}_{\mathrm{o}}{ }^{\star}, \mathrm{r}_{\mathrm{c}}{ }^{*}, \mathrm{~S}_{\mathbf{c}}, \mathrm{S}_{\mathbf{i}}, \bar{\lambda}\right)$, which is proportional to the rms angular spread, see equation (15.4.3). The results pertain to the virtual broadening observed in the crossover plane $\left(\mathrm{S}_{\mathrm{i}}=\mathrm{S}_{\mathrm{c}}\right)$. The figures $15.3 \mathrm{a}$ and $15.3 \mathrm{~b}$ apply to a sma11 crossover $\left(r_{\mathrm{c}}{ }^{*}=0.02\right)$ and a large crossover $\left(\mathrm{r}_{\mathrm{c}}^{*}=2\right)$ respectively. Spehr's result pertains to a (nearly) homocentric crossover and is therefore not included in figure 15.3b. The model of Loeffler and Kudgin and the model of De Chambost depend on the scaled linear particle density $\bar{\lambda}$. The values $\bar{\lambda}=10^{-2}$ and $\bar{\lambda}=10^{-4}$ are considered. Spehr's result depends on the initial energy spread, which is represented by the parameter $\left\langle\Delta \mathrm{v}_{\mathrm{z}} \star 2\right\rangle 1 / 2$. The cases of $a$ normal energy spread $\left(\left\langle\Delta v_{z} * 2>1 / 2=1\right)\right.$ and an extreme large energy spread $\left(\left\langle\Delta v_{z} * 2>1 / 2=10\right)\right.$ are considered. 
the case $S_{c}=S_{i}=1 / 2$ and $r_{c}^{*}=.02$. Figure $15.3 \mathrm{~b}$ gives the corresponding results for $r_{c}{ }^{*}=2$. The function representing Spehr's model is not included in this figure since its use is restricted to a nearly homocentric crossover $\left(r_{c} *<<1\right)$. For the parameter $\left\langle\Delta v_{z}^{* 2>1 / 2}\right.$ appearing in Spehr's model we considered the values 1 and 10, which correspond to a normal and an extreme large initial energy spread respectively. The functions representing the theory of Loeffler and Hudgin and the theory of De Chambost depend on $\lambda$. They are plotted for the cases $\lambda=10^{-2}$ and $10^{-4}$.

The figures $15.3 \mathrm{a}$ and $15.3 \mathrm{~b}$ show that there exists a significant disagreement between the predictions of the various theories. It should be noticed that for the theory of Loeffler and Hudgin it is more appropriate to compare the predictions for the median width of the trajectory displacement distribution. This leads to a better agreement with our theory for some conditions, as was pointed out previously in this section.

\subsection{Conclusions}

A number of recent theories for the different manifestations of the statistical Coulomb interactions in a beam in drift space have been compared with each other and with the predictions of the extended twoparticle model. Most theories handle the case of beam segment with a crossover, which is usually assumed to be narrow $(\mathrm{K}>>1)$. Massey, Jones and Plumer (1981) considered a (nearly) homocentric beam segment. The Boersch effect is the most studied manifestation of statistical interactions, for which seven other theories were considered. For the effect of statistical angular deflections we studied three other theories. The same number of theories were investigated for the trajectory displacement effect. Apart from the present study only two models cover all manifestations of statistical interactions. One is that of Loeffler (1969) and Loeffler and Hudgin (1970) and the other that of the Darmstadt group, presented by Rose and Spehr (1980,1983), Weidenhausen, Spehr and Rose (1984) and Spehr (1985a,1985b). Most theories evaluate the rms width of the displacement distribution. Loeffler and Hudgin expresses their final result in terms of a median width.

The models of 2immermann (1970) and Knauer (1979) for the Boersch effect are based on the concepts developed in the field of plasma physics and stellar dynamics. In this type of approach, the effect is attributed to a large number of independent weak collisions. Accordingly, the generated energy distribution is assumed to be Gaussian. Although not explicitly stated by the authors the typical duration of a collision is assumed to be short compared to the time scale at which the macroscopic properties of a bunch of particles in the beam change. Furthermore, the lateral dimensions of such a bunch are assumed to be large enough to treat it as an extended three dimensional system, ignoring the influence of the beam boundaries.

In actual beams the particle motion can not be represented as a random thermal motion superimposed on a smooth systematic motion since the typical collision duration is of the same order as the total flight time through the beam segment. Furthermore, the number of collisions is not necessarily large and the contribution of strong interactions can not always be ignored. The validity of the models of Zimmermann and Knauer is typically restricted to beams with a relatively narrow crossover ( $\mathrm{K}$ of the order $10^{2}$ ) and high particle densities. The models do not apply to beams of low particle density and/or nearly cylindrical beams (for which the displacement of a particle is determined by only a 
few collisions which are predominantly incomplete), neither to beams with an extreme small crossover (for which the influence of the beam boundary can not be ignored). These conclusions are confirmed by the plots of the figures $15.1 \mathrm{a}, 15.1 \mathrm{~b}$ and $15.1 \mathrm{c}$.

The approach of Loeffler (1969) and Loeffler and Hudgin (1970) is based on a more realistic representation of the particle motion in the beam. Their collision dynamics is based on first order perturbation theory. Both weak complete collisions and weak incomplete collisions are therefore evaluated in a proper way. However, this type of dynamics is not suited for strong collisions, which restricts the approach to beams of moderate and low particle density. By evaluating a median instead of a rms value their results are suited to characterize the width of the non-Gaussian distributions generated in beams of low and moderate particle densities, which distinguishes this model from all others. The comparison with the median values predicted by our model shows that the results of Loeffler and Hudgin are not generally valid, as they suggest, but do provide reasonable accurate predictions for some operating conditions. See equations (15.2.17), (15.2.18), (15.3.14) and $(15.4 .5)$. Unfortunately, the discussion presented by Loef $f$ ler and Hudgin is extremely brief and does not give any physical explanation for the different predicted parameter dependencies. It appears that the relation between the parameter dependency of the median width and the type of displacement distribution involved was not fully understood by these authors. With respect to the comparison of the rms values in the figures $15.1,15.2$ and 15.3 , it should be noted that the conversion from a median to a rms value is not correct for the non-Gaussian regimes. The direct comparison of the median values is for these regimes preferred.

The model presented by De Chambost and Hennion (1979) and De Chambost (1982) and the model of Sasaki (1984) are not based on a reduction to two-particle collisions, but start from the mean square of the fluctuations in the electrostatic force in the beam. The time development of the distribution of field particles surrounding a test particle is represented as a succession of independent states. The electrostatic interaction force experienced by the test particle varies randomly from state to state. This approach ignores any correlation in the particle coordinates in successive independent states, which leads to a rather artificial truncation of the interaction for small interparticle distances. Furthermore, the size of the time intervals between successive independent states represents a critical parameter in these models, but its choice seems rather arbitrary. Accordingly, one can not expect that these approaches predict accurate absolute values, which is indeed confirmed by the comparison. Nevertheless, there is some agreement by trend with the results of our model for extended beams with a narrow crossover.

The model of Massey, Jones and Plummer (1981) for the rms energy spread and rms angular spread generated by potential energy relaxation in a nearly homocentric cylindrical beam also starts from the mean square fluctuations in the electrostatic force in the beam, but accounts for the correlation between the successive states. Their results are in exact agreement by trend with our results, but smaller by a factor of approximately $2 / 3$. It should be noticed that the practical use of the rms width is restricted to the Gaussian regime, which occurs in (nearly) homocentric cylindrical beams only for rather high particle densities $(\lambda * \mathbb{* 1})$

Crewe (1978a) evaluated the energy change caused by a single complete collision, taking some representative initial condition. This approach entirely ignores the statistical nature of the problem. The figures $14.5 \mathrm{a}$ and $14.5 \mathrm{~b}$ show that Crewe's equation strongly underestimates the Boersch effect generated in a beam segment with a 


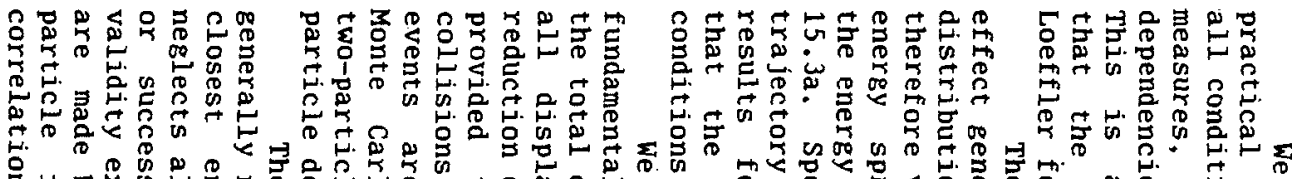

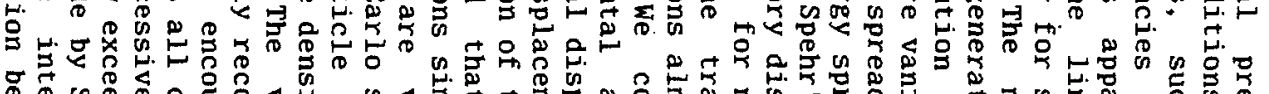

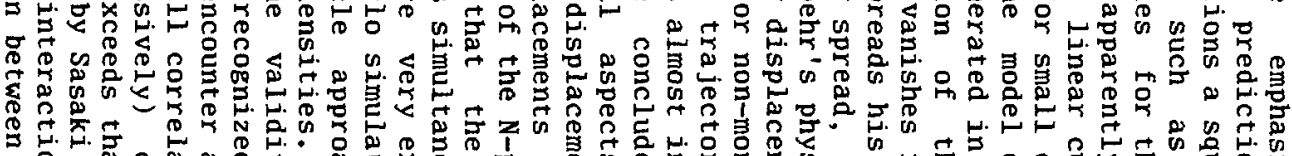
3.

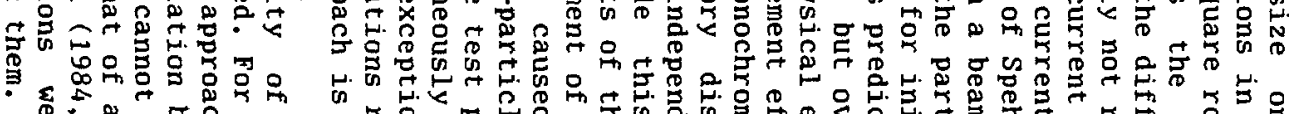

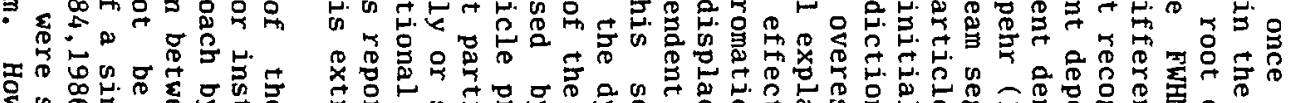

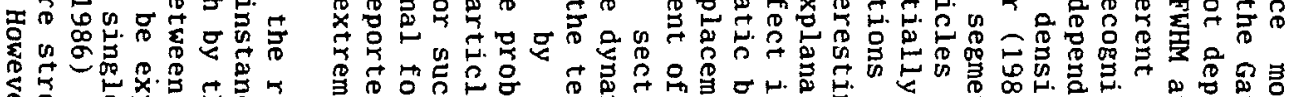

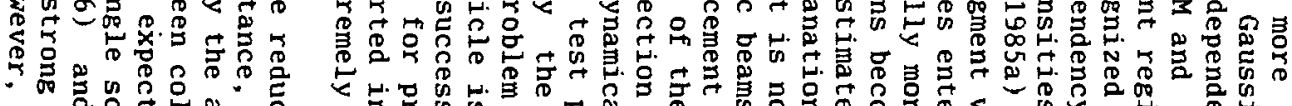

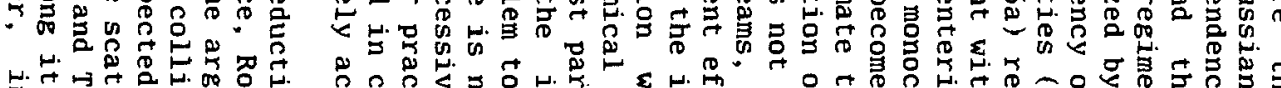

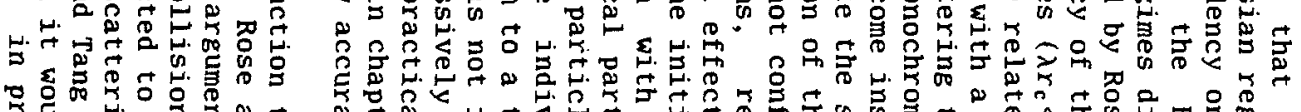

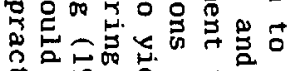

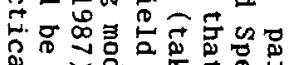

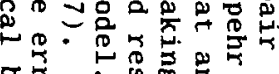

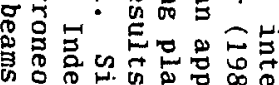

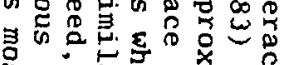

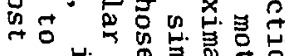

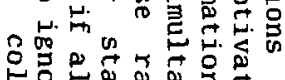

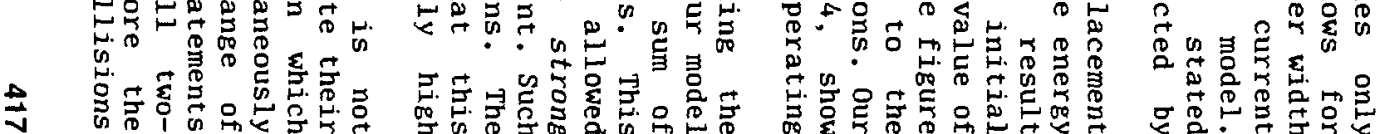

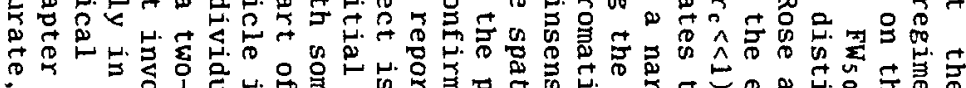

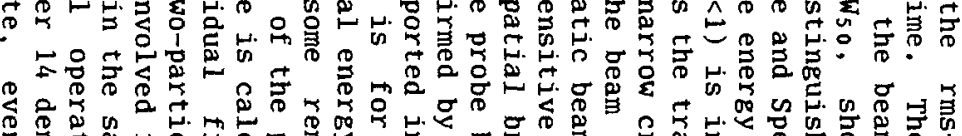

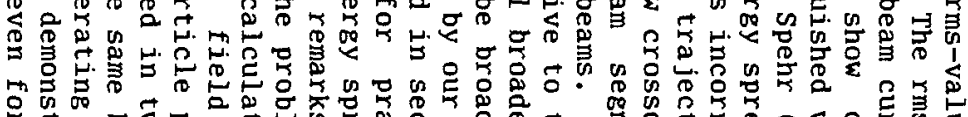
天.

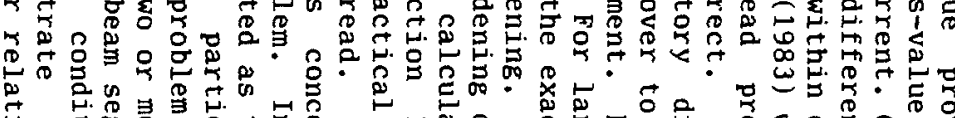

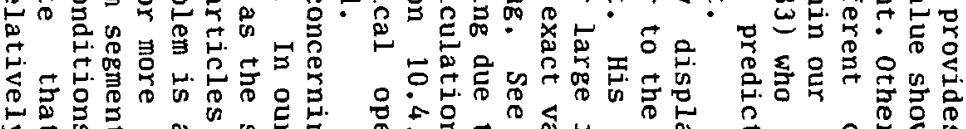

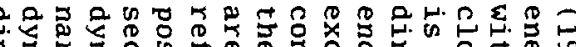
管

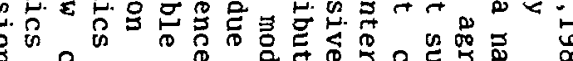

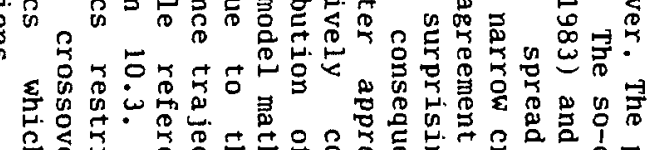

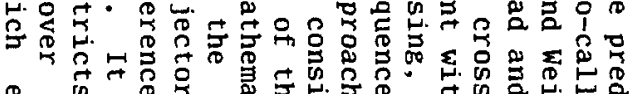

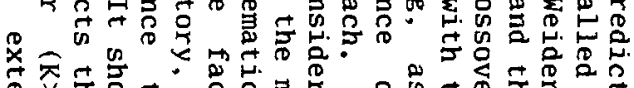

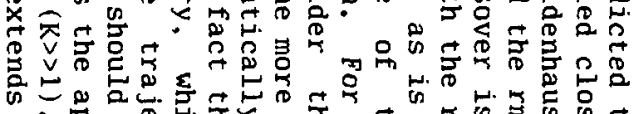

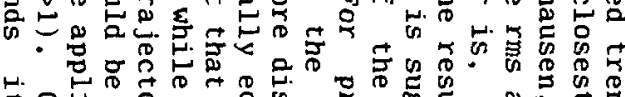

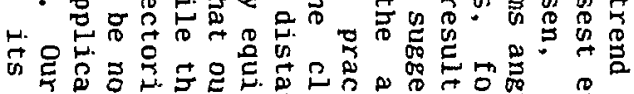

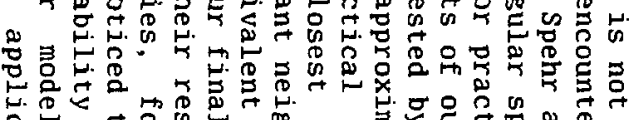

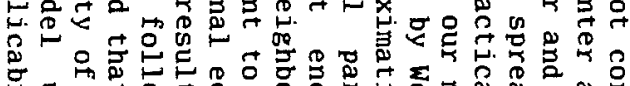

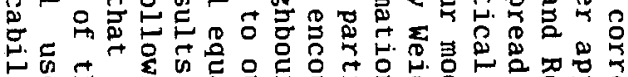

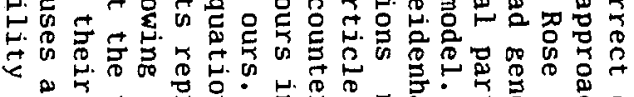

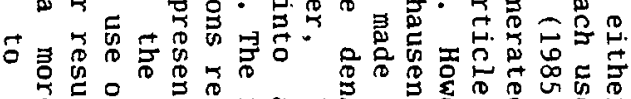

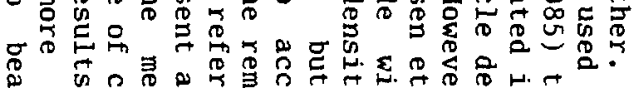

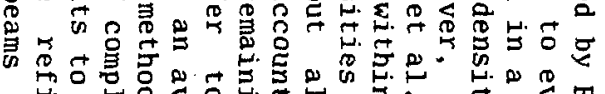

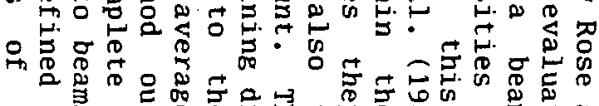

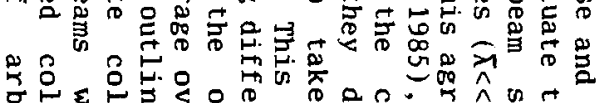

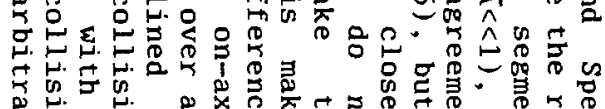

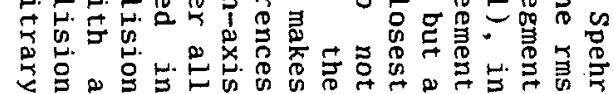


are weak and the total displacement of a particle can in good approximation be calculated as the sum of all contributions of the individual two-particle encounters, based on the argument that forces are additive. The inaccuracy of some theories based on two-particle collisions is due to the fact that the two-particle dynamical problem is oversimplified. In order to obtain results which are valid for arbitrary beam geometries, it is essential to solve the two-particle problem without making any assumptions on the strength or the completeness of the collision. 


\subsection{Introduction}

This chapter summarizes the results of this monograph which are relevant for the designer of charged particle beam systems. The physical aspects of our model for Coulomb interactions in particles beams are reviewed with the objective to provide insight in the essential steps of the calculation and the basic assumptions involved. The extended twoparticle approach, used for the modelling of the Boersch effect and the trajectory displacement effect (statistical interactions), can predict the detailed shape of the generated energy and trajectory displacement distribution. Different regimes of operating conditions are found leading to different types of distributions. Gaussian behaviour occurs at relatively high particle densities. Each of the various types of nonGaussian distributions arising at low and moderate particle densities can be related to a different type of collision, which is dominant for those conditions.

A number of measures are used to characterize the width of the distributions. The root mean square ( $r m s$ ) is only suited for a Gaussian distribution. We therefore prefer to use the Full width at Half Maximum (FWHM) and the Full Width median $\left(F_{5} W_{5}\right.$ ) value. In order to evaluate the performance of a shaped beam lithography system the trajectory displacement broadening is also expressed in terms of the corresponding $d_{1288}$ and $d_{2575}$ edge-width. The relation between the different widthmeasures depends on the type of distribution involved. The theory provides explicit equations for the FWH, the $\mathrm{FW}_{50}$, the $\mathrm{d}_{1288}$ and the $\mathrm{d}_{2575}$ values.

The dependency of the width of the distributions on the experimental parameters differs for the various regimes. The different dependencies can be understood on the basis of some elementary physical arguments. A more detailed analysis is required to obtain expressions which are also quantitatively correct. The first two sections of this chapter focus on the qualitative analysis. The next three sections review the detailed analytical prescriptions obtained for the Boersch effect, the trajectory displacement effect and the space charge effect. These results pertain to a single beam segment in drift space. The method of adding the contributions of the different segments constituting the beam is outlined in the last section.

The equations resulting from the analytical model are particularly suited for the implementation in a computer program. Such a program has been developed and is called INTERAC. It allows for an interactive evaluation of the impact of Coulomb interactions on the performance of a column. Due to the speed of this approach it provides a powerful tool for the optimization of a certain column design.

\subsection{Physical aspects}

The model schematizes the beam as a succession of beam segments, separated by thin optical components. The impact of particle interactions on the properties of the beam is studied for a single beam segment. The basic expectation of this approach is that the correlation between the contributions of the various segments can be ignored. Figure 3.1. Shows an individual rotational symmetric beam segment in drift space. Its macroscopic condition is determined by the experimental parameters: The beam current $I$, beam voltage $V$, length $L$, crossover 
radius $r_{c}$, beam semi-angle $\alpha_{0}$, crossover position parameter $S_{c}=L_{1} / L$, image plane position parameter $S_{i}=L_{i} / L$ and initial energy spread $\Delta E_{0} . L_{1}$ is the distance between entrance plane and crossover plane and $\mathrm{L}_{i}$ the distance between entrance plane and image plane. The image plane is optically conjugated to the target plane of the column. The (virtual) spatial broadening of the beam observed in this plane can therefore directly be related to the broadening of the probe at the target. In most applications, the image plane coincides with the crossover plane $\left(S_{i}=S_{c}\right)$. However, this not true for all beam segments in a shaped beam lithography column, which employs Koehler illumination. The current density distribution and angular distribution in the crossover are in general assumed to be uniform, but Gaussian distributions have been considered too.

Given the spatial and velocity distribution of the particles at the entrance of the beam segment, the final distribution has to be computed, taking the mutual coulomb repulsion into account. Consider a system of $\mathrm{N}$ identical particles of charge $\mathrm{e}$ and mass $\mathrm{m}$. The particle density is assumed to be low enough to treat the "gas" as a classic system. neglecting quantum mechanical effects. Given the positions $\mathbf{r}_{\mathbf{j}}(t)$ of all particles $(j=1,2, \cdots, N)$ at time $t$ the total coulomb force acting on a particle $i$ can be evaluated as

$$
\mathbf{F}_{\mathbf{i}}(t)=\mathrm{C}_{0} \sum_{j \neq i}^{N} \frac{\mathbf{r}_{\mathbf{i}}(t)-\mathbf{r}_{\mathbf{j}}(t)}{\left|\mathbf{r}_{\mathbf{i}}(t)-\mathbf{r}_{\mathbf{j}}(t)\right|^{3}}
$$

in which $C_{0}=e^{2} / 4 \pi \varepsilon_{0}$. Magnetic interactions and relativistic effects are ignored.

In order to compute the trajectories of the individual particles one has to solve Newton's equations of motion

$$
\frac{d^{2} r_{i}(t)}{d t^{2}}=\frac{r_{i}(t)}{m} \quad(i=1,2, \cdots, N)
$$

in which the interaction force $\mathbf{F}_{\mathbf{i}}(t)$ is given by equation $(16.2 .1)$. Notice that the evaluation of $F_{i}(t)$ requires the knowledge of the trajectories of all particles up to time $t$. The coupling of equations (16.2.1) and (16.2.2) constitutes a many particle problem which seems impossible to solve by analytical means for $\mathrm{N}>2$. Even for $\mathrm{N}=2$ one can not obtain the particle trajectories $r_{1}(t)$ and $r_{2}(t)$ in an explicit form, as will be discussed later on.

A detailed calculation of all particle trajectories is not required for all manifestations of particle interactions. Consider a set of test particles running successively along a specific trajectory in the beam, called the reference trajectory. Each of the test particles experiences the Coulomb repulsion of the particles by which it is surrounded, referred to as field particles. Due to the statistical nature of the beam the configuration of field particles will vary randomly for the different test particles. As a result the displacements of the test particles arriving at the exit plane of the segment will be statistically distributed. It can be demonstrated that the average displacement of the test particles is related to the average electrostatic force experienced along the reference trajectory. This force is strictly deterministic. Using a continum model it can be represented as the force generated by the smoothed-out distribution of charge, referred to as the space charge force. On the other hand, the widths of the distributions of displacements in position and velocity 
experienced by the test particles are directly related to the discrete nature of the beam. They are determined by the stochastic fluctuations in the charge density, rather than the average (smoothed-out) charge density. The distribution of displacements relative to the average shift of the test-particles represent the so-called statistical coulomb interactions. For the calculation of statistical interactions one has to consider the individual particle trajectories, whereas for the calculation of the space charge effect it is sufficient to consider the smoothed-out distribution of charge.

The lateral component of the space charge force acting on a test particle in a rotational symmetric beam with an uniform current density distribution is proportional to its radial distance to the beam axis. This implies that the impact of the space charge effect on the lateral properties of the beam corresponds to the action of a negative lens, causing a defocussing of the final probe as well as some (de)magnification. These effects can be compensated by proper lens adjustment and do not affect the resolution of the system. However, for a non-uniform current density distribution the space charge lens will also introduce aberrations, leading to a non-refocusable blurring of the probe. In general, the impact of the space charge effect can be described by specifying the first- and third-order optical properties of the corresponding space charge lens. These properties have been calculated in first order perturbation approximation, assuming that particle density is low enough to assure that the perturbations are small.

It should be emphasized that the model for the space charge effect (as well as the model for statistical interactions) applies to beams of low or moderate density. It is not suited to describe the beam spreading observed in high intensity beams, for instance, used in oscilloscope tubes. It does not cover the space charge effects which may occur in the vicinity of the cathode of a thermionic gun either.

Our model for statistical interactions, called the extended twoparticle model, is based on a decomposition of the full $\mathrm{N}$-particle problem into a sum of two-particle effects. For convenience of notation we will denote the total displacement of particle $i$ in position and velocity, $\Delta \mathbf{r}_{\mathbf{i}}$ and $\Delta \mathbf{v}_{i}$, in general as $\Delta \boldsymbol{\eta}_{i}$. The Boersch effect corresponds to a broadening of the axial velocities. Thus, for this effect one should consider $\Delta \eta=\Delta v_{z}$. The trajectory displacement effect corresponds to random (virtual) shifts observed in the image plane, thus $\Delta \eta=\Delta r_{\perp}$. In some cases the shift $\Delta r_{\perp}$ is entirely determined by the change in transverse velocity of the test particle. In that case it is sufficient to consider $\Delta \eta=\Delta v_{\perp}$. The reduction of the $N$-particle dynamical problem, used within the model, can be expressed as

$$
\Delta \boldsymbol{\eta}_{i}=\sum_{j \neq i}^{N} \Delta \boldsymbol{\eta}_{i j}
$$

in which $\Delta \boldsymbol{\eta}_{i j}$ is the displacement of particle $i$ due to the two-particle interaction with particle $j$. The validity of equation (16.2.3) will be discussed later on in this section.

The calculation of $\Delta \boldsymbol{\eta}_{\mathbf{i}}$ constitutes a two-particle problem, which is defined by equations (16.2.1) and (16.2.2), taking $\mathrm{N}=2$. One would like to have an explicit analytical expression for the displacement $\Delta \boldsymbol{\eta}_{\mathbf{i}} \mathbf{j}$ in terms of the initial coordinates $\mathbf{r}_{\mathbf{i}}, \mathbf{r}_{\mathbf{j}}, \mathbf{v}_{\mathbf{i}}, \mathbf{v}_{\mathbf{j}}$ and the time of flight $T$. However, in general, such a solution can not be obtained. The classic solution of the Kepler problem specifies the relative trajectory of the particles in the orbital plane, which is in 
polar coordinates represented by a function $r(\theta)$. Our problem requires a more detailed analysis which takes the time of flight $T$ through the beam segment into account. For that one needs the coordinates of the particles explicitly as function of time $t$. In terms of the polar coordinates in the orbital plane one requires the functions $r(t), \theta(t)$ and their derivatives with respect to time, $\dot{r}(t)$ and $\dot{\theta}(t)$. As it turns out one can obtain the time $t$ explicitly as function of the relative distance $r$, thus $t=t(r)$, but the function involved can not be inverted. This prohibits an exact explicit analytical calculation of the particle trajectories as function of time $t$.

A good approximation of the exact final coordinates of the particles involved in a two-particle collision can be obtained for two types of collisions. One is a complete collision (or nearly complete collision), in which the particles effectively come from infinity and recede to infinity. For this type of collision it is sufficient to consider the asymptotic values of the polar coordinates. The other type is a weak collision in which the deviations from the unperturbed trajectories are small. First order perturbation dynamics can be used to describe such a collision. In this approach one approximates the actual interaction force by the force which would occur when the particles would follow their unperturbed trajectories. The remaining collisions, those which are both strong and incomplete, can not be handled analytically. For these collisions one has to perform the inversion of the function $t(r)$ by numerical means. This procedure is referred to as full collision dynamics.

We note that the calculation of the spatial displacements of the particles, $\Delta \mathbf{r}_{1}$ and $\Delta \mathbf{r}_{2}$, caused by a nearly complete collision is more involved than calculation of the corresponding velocity displacements $\Delta \mathbf{v}_{1}$ and $\Delta \mathbf{v}_{2}$. The displacements $\Delta \mathbf{v}_{1}$ and $\Delta \mathbf{v}_{2}$ caused by a complete collision are finite, despite the fact that the corresponding time of flight $T$ is infinitely large. However, the results for $\Delta r_{1}$ and $\Delta \mathbf{r}_{2}$ diverge when $T$ is taken infinitely large. One has to account for the fact that the collision is nearly, but not entirely complete. In other words, the initial and final state of the collision may not be identified with the asymptotic conditions. The type of dynamics which takes this into account is referred to as nearly complete collision dynamics.

Let us return to the general $\mathrm{N}$-particle problem. The validity of the decomposition of equation (16.2.3) depends on the strength of the two-particle interactions. The decomposition is entirely justified when all interactions are weak. First order perturbation dynamics is then appropriate to calculate all displacements $\Delta \eta_{i j}$, using the interaction force which would occur when the particles would follow their unperturbed trajectories. Since forces are additive the decomposition of equation $(16.2 .3)$. is then justified. Equation $(16.2 .3)$ also provides accurate results when the total total displacement of a particle is dominated by a single strong interaction. The particle will experience a significant deviation from its unperturbed trajectory, which should be calculated using nearly complete collision dynamics or full collision dynamics. As the deviation from the unperturbed trajectory is large, the calculation of the weak interactions with the other particles will be inaccurate. However, as the total displacement of the particle is dominated by that single strong interaction, the contributions of the weak interactions can be ignored and equation (16.2.3) is still valid. Clearly, the decomposition of equation (16.2.3) breaks down when the particles are involved in two or more strong collisions simultaneously or successively. However, such events are rare for the particle densities encountered in systems used for lithography and microscopy 
applications.

Equation (16.2.3) reduces the dynamical part of the manyparticle problem to a proper analysis of the two-particle problem. The statistical part of the problem is to determine the probability that a test particle is surrounded by a certain configuration of field particles and given this probability distribution, to compute the corresponding distribution of displacements of the test particle $\rho(\Delta \eta)$. Let us denote the set of unperturbed coordinates of field particle $i$ relative to the test particle as $\boldsymbol{\xi}_{i}$. A certain configuration of field particles relative to the test particles is then specified by the set $\xi_{1}, \xi_{2}, \cdots, \xi_{N-1}$. In order to determine the probability $P_{N}\left(\xi_{1}, \xi_{2}, \cdots, \xi_{N-1}\right)$ of the configuration of field particles $\xi_{1}, \xi_{2}, \cdots, \xi_{N-1}$ we assume that the field particles are statistically independent

$$
P_{N}\left(\xi_{1}, \xi_{2}, \cdots, \xi_{N-1}\right)=\prod_{i=1}^{N-1} P_{2}\left(\xi_{i}\right)
$$

in which $\mathrm{P}_{2}(\xi)$ is the probability that a field particle has coordinates $\xi$ relative to the test particle. Equation (16.2.4) ignores any correlation in the coordinates of the field particles. Such correlations might arise from the emission process at the cathode or the coulomb interaction in the beam segments preceding the one of consideration. Ignoring the last "source" of correlation is equivalent to the assumption that Debeye screening is absent. This is indeed the case for practical operating conditions, as can be verified by comparing the relaxation time involved to the time of flight through the column.

The model concentrates on the displacements experienced along the central (on-axis) reference trajectory in a rotational symmetric beam. The space charge force is zero on this trajectory and the resulting displacements are purely statistical, which facilitates the analysis. In addition, it is assumed that the beam is initially monochromatic with respect to the normal energy, which means that all particles initially have the same axial velocity. This assumption is reasonable since the velocity spread of particles at emission is strongly reduced during the succeeding acceleration. As a result of these two simplifications the number of independent components of the vector $\xi$ is only four, whereas the general case would require six components to describe the position and velocity of a field particle relative to the test particle. The validity of these assumptions has been verified within the framework of the model by performing the full calculation for some specific beam geometries. It was found that average broadening for all possible reference trajectories is in general somewhat smaller than the broadening obtained for the central reference trajectory. In general, the differences are within $20 \%$ and for many cases even within 10\%. Furthermore, it was found that the impact of the initial energy distribution on the magnitude of the statistical effects can be ignored for practical operating conditions.

Equation (16.2.4) enables us to express the distribution of field particles relative to the test particle in terms of the experimental parameters which were specified previously. The problem is now to compute the distributions of displacements of the test particle $\rho(\Delta \eta)$ from the distribution of field particles, employing the solution of the dynamical problem expressed by equation (16.2.3). This problem can be solved using a procedure which is well known from statistical mechanics (sometimes referred to as Markoff's method, for instance see Chandrasekhar (1943)). This method permits a calculation of the detailed shape of the full distributions. It was found that the type of distribution depends on the beam geometry as well as the particle 
density. For large particle densities the total deviation of the test particle is built up by a large number of independent collisions. The corresponding distribution is Gaussian, as can be understood from the central limit theorem. For lower particle densities the distributions become non-Gaussian, showing more pronounced tails. It was also found that different types of displacement distributions are generated in extended beams and pencil beams. A beam is called extended (or threedimensional) if its transverse dimensions are large compared to the average axial separation of the particles. A pencil beam represents the opposite case. In such a beam all particles are nearly on a row.

Without going into a detailed discussion we wish to clarify the the occurrence of the different types of non-Gaussian displacement distributions and indicate their relation with the beam geometry and the particle density. As an example, let us consider an extended monochromatic cylindrical beam. Assume that the beam is homocentric, which means that the particles run along parallel trajectories. In the frame of reference moving with the beam, the particles are initially in rest. Assume that the particle density is low enough to assure that the deviations from the unperturbed trajectories are small. Thus, first order perturbation dynamics can be used to compute the displacements. The displacements in transverse and axial velocity now follow as

$$
\Delta \mathbf{v}_{\perp}=\frac{\mathbf{F}_{\perp} \mathrm{T}}{\mathrm{m}}, \quad \Delta \mathbf{v}_{\mathbf{z}}=\frac{\mathbf{F}_{\mathbf{z}} \mathrm{T}}{\mathrm{m}}
$$

where $T$ is the time of flight. Clearly, if first order perturbation dynamics holds true, the distribution of displacements is directly related to the distribution of the fluctuating component of the interaction force which would occur in the unperturbed beam. For the specific case of particles which are at rest relative to each other, as in our example, this distribution was first calculated by Holtsmark (1919). He considered the problem of determining the probability $\rho(\mathbf{F})$ that a given electric field strength $P$ acts at a point in a gas of density $n_{d}$ composed of randomly distributed ions. His result can be expressed as

$$
\rho(F)=\frac{1}{2 \pi^{2} F} \int_{0}^{\infty} k d k \sin (F k) e^{-A_{3} / 2 k^{3 / 2}}
$$

in which $A_{3} / 2$ depends on the particle mass $m$, particle charge $e$ and the density $n_{d}$

$$
A_{3 / 2}=\frac{4}{15}\left(2 \pi C_{0}\right)^{3 / 2} n_{d}
$$

where $C_{0}=e^{2} / 4 \pi \varepsilon_{0}$. It can be demonstrated that the distribution of the longitudinal component of the interaction force $F_{z}$ is given by the onedimensional variant of the Holtsmark distribution

$$
\rho\left(F_{z}\right)=\frac{1}{\pi} \int_{0}^{\infty} \mathrm{dk} \cos \left(k F_{z}\right) e^{-A_{3} / 2 k^{3 / 2}}
$$

while the distribution of the lateral component $P_{\perp}$ is given by 


$$
\rho\left(\mathbf{F}_{\perp}\right)=\frac{1}{2 \pi} \int_{0}^{\infty} k d k J_{0}\left(k F_{\perp}\right) e^{-A_{3} / 2 k^{3} / 2}
$$

in which $J_{0}$ is the Bessel function of the first kind and zero order. Notice that the argument of the exponential function is the same in equations $(16.2 .6),(16.2 .8)$ and $(16.2 .9)$. The $3 / 2$ power dependency on the integration variable $k$ is characteristic for the Holtsmark distribution. For the beam of our example it follows from equation (16.2.5) that the generated distributions of transverse and axial velocities, $\rho\left(\Delta v_{\perp}\right)$ and $\rho\left(\Delta v_{z}\right)$, are of the same type as the distributions $\rho\left(\mathbf{F}_{\perp}\right)$ and $\rho\left(\mathrm{F}_{z}\right)$ respectively.

A more general analysis shows that the distributions $\rho\left(\Delta \mathbf{v}_{\perp}\right)$ and $\rho\left(\Delta \mathrm{v}_{z}\right)$ are Holtsmarkian for any beam geometry, provided that the beam is extended and provided that the perturbations of the particles are small. Other types of distributions will be generated when these conditions are not fulfilled. However, the form of equations (16.2.8) and $(16.2 .9)$ can be generalized to include those distributions as well. Denoting the displacements in position or velocity again as $\Delta \eta$, one can express the one-dimensional distribution of axial displacements, in general, as

$$
\rho(\Delta \eta)=\frac{1}{\pi} \int_{0}^{\infty} d k \cos (k \Delta \eta) e^{-A \gamma k^{\gamma}}
$$

and the two-dimensional distribution of transverse displacements as

$$
\rho(\Delta \eta)=\frac{1}{2 \pi} \int_{0}^{\infty} k d k J_{0}(k \Delta \eta) e^{-A_{\gamma} k^{\gamma}}
$$

The numerical constant $\gamma$ is characteristic for the type of distribution involved, while the parameter Ar specifies the dependency on the experimental parameters. The different types of distributions encountered in the calculation of statistical effects can be summarized as

$$
\begin{array}{lll}
\gamma=2 & - & \text { Gaussian distribution } \\
\gamma=3 / 2 & - & \text { Holtsmark distribution } \\
\gamma=1 & - & \text { Lorentzian distribution } \\
\gamma=1 / 2 & - & \text { distribution of } F_{z} \text { in a pencil beam } \\
\gamma=1 / 3 & - & \text { distribution of } F_{\perp} \text { in a pencil beam }
\end{array}
$$

The shape of the corresponding one- and two-dimensional distributions are depicted in the figures 5.3 and 5.4 respectively. These figures clearly show that a small $\gamma$-value leads to a distribution with a narrow core and long tails.

The representation of equations (16.2.10) and (16.2.11) still contains some degree of approximation. The extended two-particle approach, which is based on a strict derivation of the distribution $\rho(\Delta \eta)$ starting from the assumptions expressed by equations (16.2.3) and $(16.2 .4)$, shows that the argument of the exponential function appearing in equations $(16.2 .10)$ and $(16.2 .11)$ should in general be expressed as a function $\lambda p(k)$, with $\lambda$ the linear particle density. Thus equations $(16.2 .10)$ and $(16.2 .11)$ rely on the approximation

$$
\lambda p(k) \approx A \gamma k^{\gamma}
$$




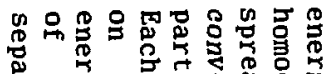

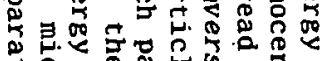

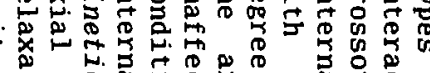

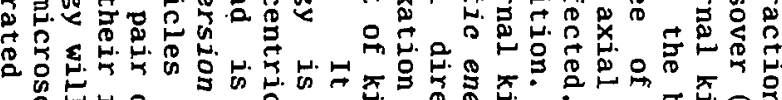

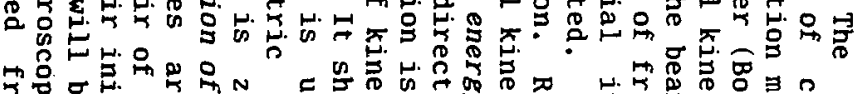

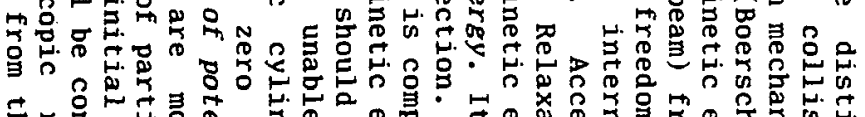

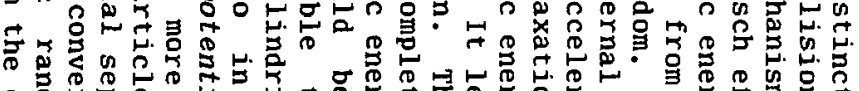

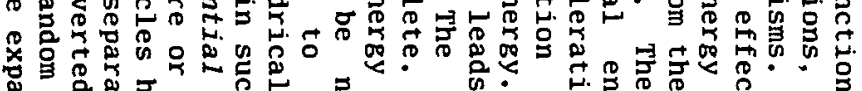

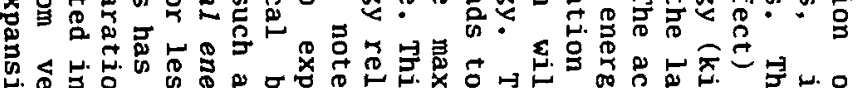

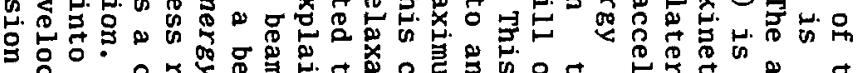

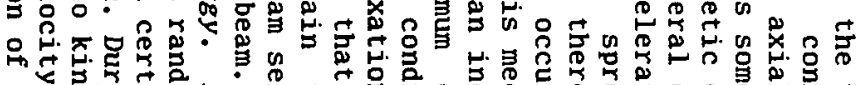

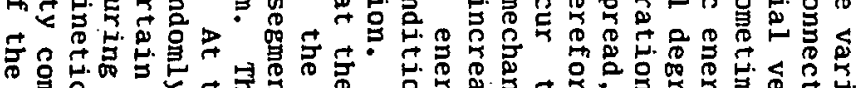

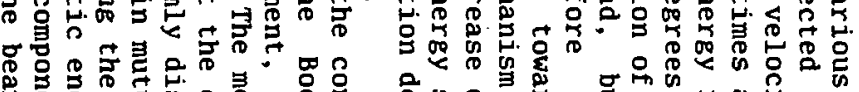

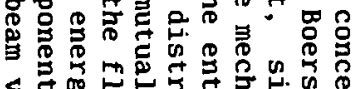

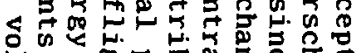

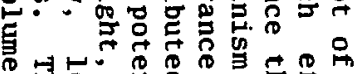

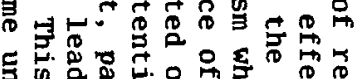

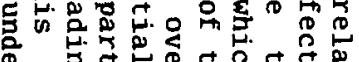

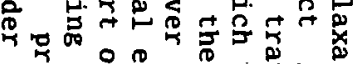

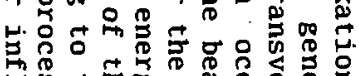

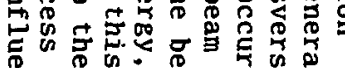

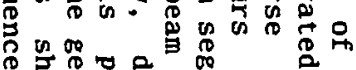

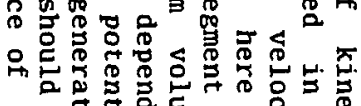

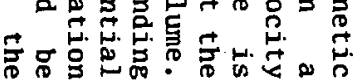

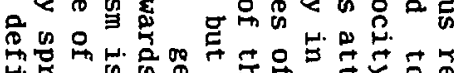

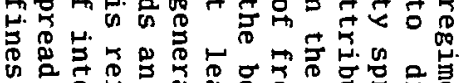

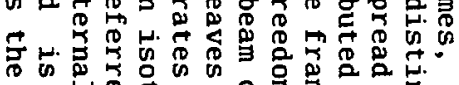

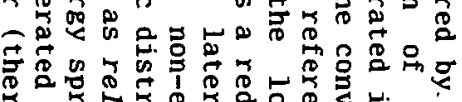

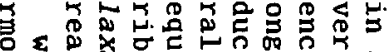

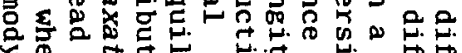

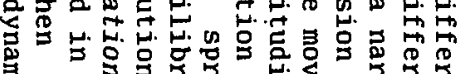

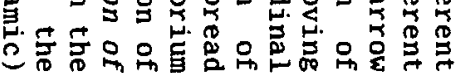

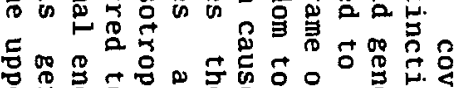

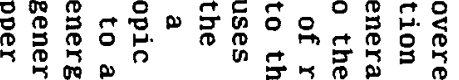

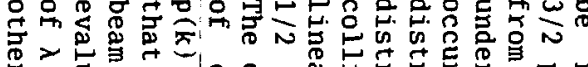

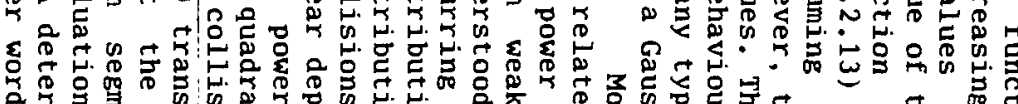

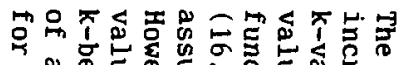

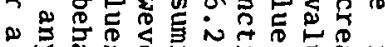

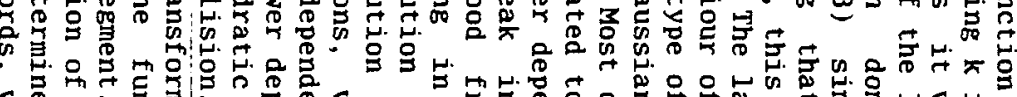

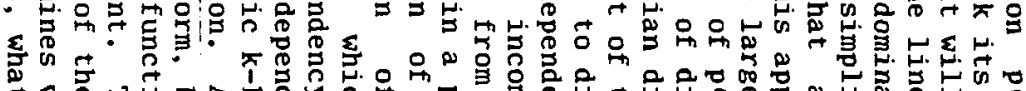

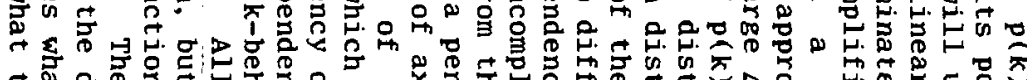

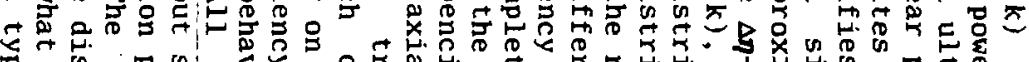

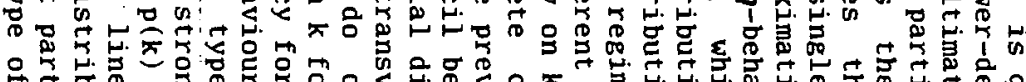

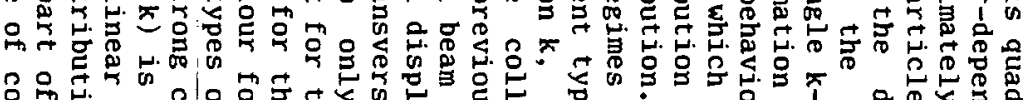

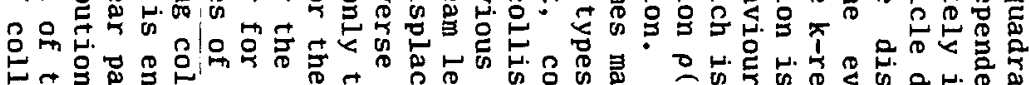

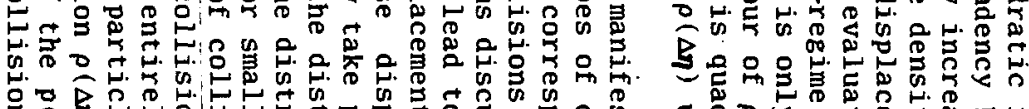

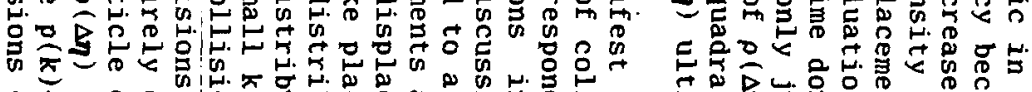

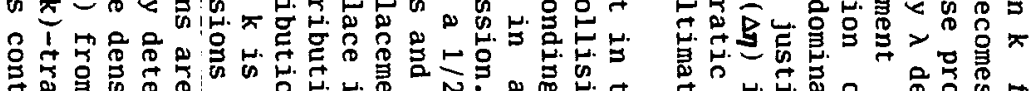

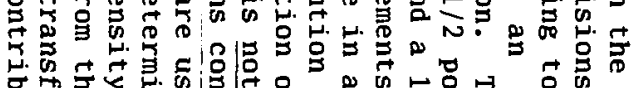

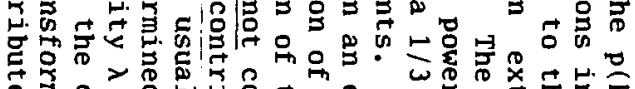

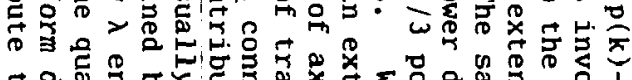

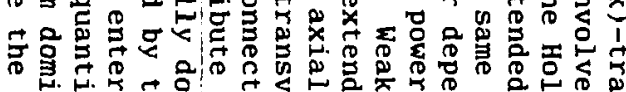

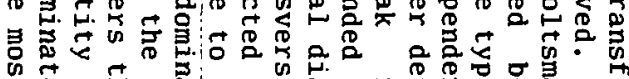

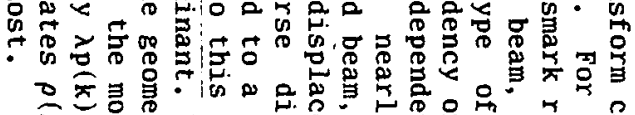

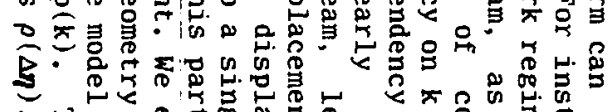
ᄂ

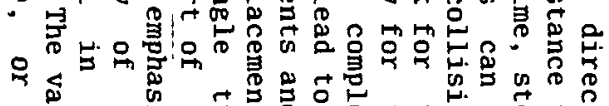

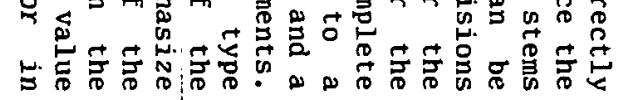

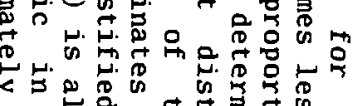

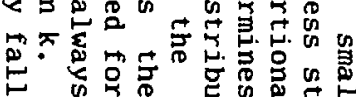

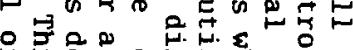

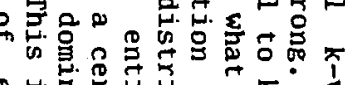

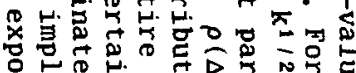

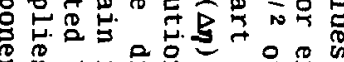

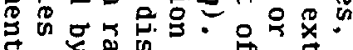

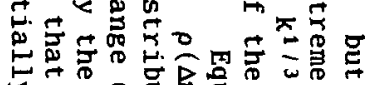

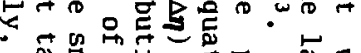

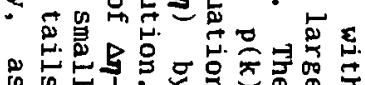


space charge force, which corresponds to the conversion of potential energy into a macroscopic systematic motion. The upper (thermodynamic) limit of potential energy relaxation is reached when the microscopic density distribution obeys Maxwell-Boltzmann statistics. In this description the kinetic energy of the particles is represented by a beam temperature.

Our model implicitly accounts for the different mechanisms through the detailed treatment of the two-particle problem. The potential energy of the particles involved in a nearly complete collision is effectively zero in both the initial and the final state. The main effect of such a collision is a directional change of the particles and a transition of kinetic energy from one particle to the other. Thus, when nearly complete collisions are dominant, the mechanism involved is relaxation of kinetic energy. Conversion of potential energy into kinetic energy (and visa versa) occurs in incomplete collisions. When weak incomplete collisions are dominant, the mechanism involved is relaxation of potential energy.

The width of the distributions specified by equations $(16.2 .10)$ and (16.2.11) depends on the parameter $A r$. The Full Width at Half Maximum (FWHM) and the Full Width median ( $\mathrm{FW}_{50}$ ) can be expressed as

$$
\mathrm{FWHM}=\mathrm{Ar}^{1 / \gamma} \mathrm{FWHM \gamma}, \quad \mathrm{FW}_{50}=\mathrm{Ar}^{1 / \gamma} \mathrm{FW}_{50 \gamma}
$$

in which FWHMr and $F W_{5}$ or are numerical constants, which depend on the dimension $n$ of the distribution as well as the value of $\gamma$. Table 5.1 gives the values of $F W H M$ and $F W_{5}$ or for $n=1,2$ and 3 and the $\gamma$-values listed in equation (16.2.12). We note that the distributions of equations (16.2.10) and (16.2.11) have no finite second or higher moment for $\gamma<2$, due to their long tails. However, if one calculates the distribution $\rho(\Delta \eta)$ from the full $p(k)$ transform the tails will ultimately fall of exponentially, as was mentioned previously. This leads to a finite value of second and higher order moments. In this context it should be recognized that the root mean square (rms) is not a practical width-measure for the non-Gaussian distributions generated by statistical interactions. It is entirely determined by the tails, which show a.different dependency on the experimental parameters as the bulkpart of the distribution.

The parameter $\gamma$ is a key-parameter in the theory. It specifies the type of distribution which is generated. Equation (16.2.14) shows that it also determines the dependency of the FWHM and FW5 width measures on the experimental parameters, which are represented by the quantity $A_{\gamma}$. For instance, $A_{\gamma}$ is directly proportional to the beam current I. Thus if one knows $\gamma$ one can directly say that FWHM and $\mathrm{FW}_{5} 0$ value of the displacement distribution $\rho(\Delta \eta)$ will be proportional to $I^{1 / \gamma}$. The other way around, if one knows that the FWHM or FW 5 a increases as IP, one may conclude that $\gamma=1 / p$. We emphasize that the rms-width is dominated by the tails of $\rho(\Delta \eta)$, which ultimately follow a Gaussian type of behaviour $(\gamma=2)$. Accordingly, the rms-width is for all conditions proportional to $\mathrm{I}^{1 / 2}$.

The parameter $\gamma$ also determines in what way the effects generated in the individual segments, constituting the beam, have to be added. Assuming that the displacements experienced by the test particle in the successive beam segments are not correlated and that all distributions are of the same type (same $\gamma$ ), one finds for the FWHM of total displacement distribution $\rho(\Delta \eta)$, generated in the entire beam 


$$
\text { FWHMT }_{T}=\left(\sum_{j=1}^{N_{b}} \text { FWHM }_{j}^{\gamma}\right)^{i / \gamma}
$$

where $\mathrm{FWHM}_{j}$ is the FWHM of $\rho(\Delta \eta)$ generated in segment number $j$ and $\mathrm{N}_{b}$ is the total number of beam segments. The same equation holds true for the median width FW5 $_{5}$, while rms-values should always be added quadraticly $(\gamma=2)$. If the distributions are not all of the same type (same $\gamma$ ) one should in principle add the corresponding $\lambda p(k)$ functions and determine the FWHM from the resulting total $\lambda p(k)$ function. However, in practice it is more convenient to define somekind of effective $\gamma$-value for which equation (16.2.15) holds approximately. This approach will be discussed in some detail in section 16.7 .

Equation (16.2.15) presupposes that the displacements generated in the individual beam segments are uncorrelated. In the opposite case that the contributions are entirely correlated their widths should be added linearly. This situation is relevant for the so-called slice-method. In this approach one subdivides a beam segment into thin cylindrical slices and calculates the total FWHM as a linear sum of the contribution of the individual slices

$$
F W H M=\sum_{S=1}^{N_{S}} \operatorname{FWHM}_{P}\left(I, V, r_{0}, \Delta z_{s}\right) \approx \int_{z_{0}}^{z_{1}} \mathrm{dz}\left(\frac{\operatorname{FWHM}_{P}\left(I, V, r_{0}, \Delta z\right)}{\Delta z}\right)
$$

where FWHMP $_{P}\left(I, V, r_{0}, \Delta z\right)$ is the FWHM of the distribution generated in a cylindrical slice (with Parallel rays) of length $\Delta z$, radius $r_{0}$, carrying a current $I$ at a potential $V$. This expression is expected to be linear in $\Delta z$. Accordingly, the integrant $\operatorname{FWHM}_{P}\left(I, V, r_{0}, \Delta z\right) / \Delta z$ will only depend on $z$ through the beam radius $r_{0}$. The quantities $z_{0}$ and $z_{1}$ are the axial coordinates of the entrance and exit plane of the beam segment respectively, thus $L=z_{1}-z_{0}$. The validity of equation (16.2.16) requires the particle density in the beam to be low enough to guarantee that the displacements from the unperturbed trajectories are small. In that case the displacements of the test particle experienced in the individual slices will indeed be entirely correlated, since they are generated by the same complexion of neighbour field particles. The slice method will be employed in the next section to derive the parameter dependencies in a beam segment with a crossover at low particle density from the results obtained for a cylindrical beam segment.

\subsection{Parameter dependencies}

The extended two-particle model has the capability to predict the detailed shape of the full displacement distribution $\rho(\Delta \eta)$, generated by the statistical interactions and includes a calculation of different width measures of $\rho(\Delta \eta)$. In the previous section it was pointed out that different types of distributions may occur, depending on the beam geometry and the particle density in the beam. The dependency on the experimental parameters of the characteristic width measures, such as the FWHM and the FW50 value, will be different in the various regimes. Prior to the presentation of the detailed equations we like to show that the dependency on the experimental parameters can be predicted on the basis of some elementary physical arguments. We will first consider a beam of low particle density in which the deviations from the unperturbed trajectories are small. In terms of "collisions" 
this implies that weak incomplete collisions are dominant. Beams of moderate particle density, in which the contribution of weak complete collisions as well as strong collisions may become significant, will be considered next.

In order to specify the qualifications "low", "moderate" and "high" particle density let us first introduce the dimensionless quantities $\lambda$ and $\lambda^{*}$. For a beam segment with a crossover the particle density can best be expressed in terms of the scaled linear particle density $\pi$, defined as

$$
\pi=\frac{\mathrm{m}^{1 / 2}}{2^{7 / 2} \pi \varepsilon_{0} \mathrm{e}^{1 / 2}} \frac{\mathrm{I}}{\alpha_{0}^{2} \mathrm{~V}^{3 / 2}}
$$

For a cylindrical beam segment $\left(\alpha_{0}=0\right)$ one can not use $\pi$. Instead, we use the scaled linear particle density $\lambda^{*}$, defined as

$$
\lambda^{*}=\frac{\mathrm{m}^{1 / 2}}{27 / 2 \pi \epsilon_{0} \mathrm{e}^{1 / 2}} \frac{\mathrm{IL}^{2}}{r_{0}^{2} \mathrm{~V}^{3 / 2}}
$$

As a rough indication, low particle densities correspond to values for $\lambda$ and $\lambda^{*}$ below $10^{-4}$; moderate particle densities to values between $10^{-4}$ and $10^{-2}$ and high particle densities to values above $10^{-2}$.

The Boersch effect corresponds to a broadening of the axial velocity distribution. It is usually expressed in terms of the generated energy spread. An energy displacement $\Delta E$ is related to an axial velocity displacement $\Delta v_{z}$ as

$$
\frac{\Delta \mathrm{E}}{\mathrm{E}} \approx \frac{\mathrm{mv_{z }} \Delta \mathrm{v}_{\mathrm{z}}}{1 / 2 \mathrm{mv}_{\mathrm{z}}{ }^{2}}=2 \frac{\Delta \mathrm{v}_{\mathrm{z}}}{\mathrm{v}_{\mathrm{z}}}
$$

provided that $\Delta v_{z}<<v_{z}$. The trajectory displacement effect corresponds to the generation of random displacements in position and velocity in the lateral direction of the beam. An angular displacement $\Delta \alpha$ is related to a transverse velocity displacement $\Delta v_{\perp}$ as

$$
\Delta \alpha \approx \frac{\Delta v_{\perp}}{v_{z}}
$$

provided that $\Delta v_{1}<<v_{2}$. The corresponding virtual spatial displacement $\Delta r$ in the image plane (which is optically conjugated to the target plane) is given by

$$
\Delta r \approx \Delta \alpha\left|z-z_{i}\right|
$$

in which $\left|z-z_{i}\right|$ is the distance between the location where the deflection $\Delta a$ occurs and the image plane.

Consider a homocentric cylindrical beam segment with radius $r_{0}$. Given a force $F=\left(F_{\perp}, F_{z}\right)$ acting on the test particle, the corresponding velocity displacements $\Delta v_{\perp}$ and $\Delta v_{z}$ follow in first order perturbation approximation from equation $(16.2 .5)$. The time of flight $T=L / v_{z}$. We emphasize that equation (16.2.5) presupposes that particles are initially at rest relative to each other, while the deviations from the unperturbed trajectories are small. The force component $F_{z}$ will scale with $C_{0} d_{z} / d^{3}$, where $d$ and $d_{z}$ denote the average distance and the average axial distance between neighbour particles respectively and $C_{0}=e^{2} / 4 \pi \varepsilon_{0}$. Employing equation $(16.3 .3)$ we therefore expect that 


$$
\frac{\Delta E}{E} \sim \frac{C_{0}}{m} \frac{L d_{z}}{v_{z}^{2} d^{3}}
$$

For an extended beam, both $d$ and $d_{z}$ will scale with $n_{d}-1 / 3$, where $n_{d}$ denotes the particle density, which is given by

$$
n_{d}=\frac{I}{\pi r_{0}^{2}} \sqrt{\frac{m}{2 e^{3} V}}
$$

Taking $d \sim n_{d}-1 / 3$ and $d_{z} \sim n_{d}-1 / 3$ equation $(16.3 .6)$ yields

$$
\frac{\Delta E}{E} \sim \frac{\mathrm{m}^{1 / 3}}{\varepsilon_{0}} \frac{\mathrm{I}^{2 / 3} \mathrm{~L}}{\mathrm{~V}^{4 / 3} \mathrm{ro}^{4 / 3}}
$$

This equation specifies the dependency on the experimental parameters of the energy spread generated in an extended homocentric cylindrical beam segment in which the deviations from the unperturbed trajectories are small. From the dependency on the beam current I one may conclude that $\gamma=3 / 2$. Thus the distribution $\rho(\Delta E)$ is of the Holtsmark type, as specified by equation $(16.2 .12)$. The reader may verify that equation (16.3.8) can also be obtained from the Holtsmark distribution $\rho\left(\mathbf{F}_{\mathbf{z}}\right)$ given by equation $(16.2 .8)$, employing equations $(16.2 .14),(16.2 .7)$, $(16.2 .5)$ and $(16.3 .3)$.

In a pencil beam, both $d$ and $d_{z}$ will scale with $1 / \lambda$, where the linear particle density $\lambda$ is given by

$$
\lambda=I \sqrt{\frac{m}{2 e^{3} V}}
$$

Taking $d \sim 1 / \lambda$ and $d_{z} \sim 1 / \lambda$, equation (16.3.6) yields

$$
\frac{\Delta E}{E} \sim \frac{m}{\varepsilon_{0} e^{2}} \frac{I^{2} L}{V^{2}}
$$

This equation specifies the dependency on the experimental parameters of the energy spread generated in a pencil beam in which the deviations from the unperturbed trajectories are small. Notice that equation (16.3.10) does not depend on the radial dimensions of the beam, which are assumed to be small compared to the average axial separation of the particles.

For the angular displacement $\Delta \alpha$ one finds from equations $(16.3 .4)$ and $(16.2 .5)$

$$
\Delta a \sim \frac{C_{0}}{m} \frac{L d_{r}}{v_{z}^{2} d^{3}}
$$

similar to equation (16.3.6). The quantity $d_{r}$ is the average radial distance between neighbour particles. For an extended beam both $d$ and $d_{r}$ will scale with $n_{d}-1 / 3$, where $n_{d}$ is the particle density given by equation $(16 \cdot 3.7)$. Accordingly, one finds

$$
\Delta a \sim \frac{\mathrm{m}^{1 / 3}}{\mathrm{E}_{0}} \frac{\mathrm{I}^{2 / 3} \mathrm{~L}}{\mathrm{~V}^{4 / 3} \mathrm{r}_{0}^{4 / 3}}
$$

This equation is similar to equation (16.3.8) and applies to the same conditions. The similarities between the results obtained for $\Delta E / E$ and $\Delta \alpha$ can be understood from the fact that the particles are initially at 
rest in the frame of reference moving with the beam. Random velocity components are generated during the flight due to the relaxation of potential energy. The generated velocity distribution will be rotational symmetric (in the frame of reference moving with the beam), provided that the beam is extended.

Differences between the results for $\Delta \mathrm{E} / \mathrm{E}$ and $\Delta \alpha$ are expected to occur in pencil beams, in which the distribution of field particles around a test particle is non-rotational symmetric. In a pencil beam the distance $d$ scales with $1 / \lambda$, where $\lambda$ is the linear particle density given by equation (16.3.9). However, the radial distance $d_{r}$ is expected to scale with the beam radius $r_{0}\left(r_{0}<<1 / \lambda\right)$. Substitution into equation (16.3.11) yields

$$
\Delta \alpha \sim \frac{\mathrm{m}^{3 / 2}}{\epsilon_{0} \mathrm{e}^{7 / 2}} \frac{\mathrm{I}^{3} \mathrm{Lr}_{0}}{\mathrm{~V}^{5 / 2}}
$$

which specifies the dependency on the experimental parameters of the angular deflections generated in a cylindrical pencil beam in which the deviations from the unperturbed trajectories are small. In the extreme case that all particles are on a line $\left(r_{0}=0\right)$, there is no transverse component of the interaction force and $\Delta \alpha$ becomes zero. From the dependency on the beam current I one may conclude that $\gamma=1 / 3$, which defines the type of two-dimensional distribution involved.

In order to express the angular displacement $\Delta \alpha$ into a spatial displacement $\Delta r$ one should realize that a cylindrical beam segment is usually succeeded by a lens, which focusses the beam in its back-focal plane. Let $f$ be the focal distance of the lens. The spatial displacement in the back focal plane of the lens $\Delta r$ caused by an angular displacement $\Delta \alpha$ is equal to

$$
\Delta r \approx f \Delta \alpha
$$

The parameter dependency of the trajectory displacement effect generated in an extended cylindrical beam follows by substitution of equation (16.3.12), while the result for a pencil beam follows with equation $(16.3 .13)$.

Now consider a beam segment with a narrow crossover of radius $r_{c}$, with a beam semi-angle $\alpha_{0}$. We will employ the slice-method of equation (16.2.16) to determine the parameter dependencies for $\Delta E / E$ and $\Delta \alpha$ from the results obtained for a cylindrical beam segment. The characteristic beam geometry quantities $K, K_{1}$ and $K_{2}$ are defined as

$$
\mathrm{K}=\frac{\alpha_{0} \mathrm{~L}}{2 \mathrm{r}_{\mathrm{c}}}, \quad \mathrm{K}_{1}=2 \mathrm{KS}_{\mathrm{c}}, \quad \mathrm{K}_{2}=2 \mathrm{~K}\left(1-\mathrm{S}_{\mathrm{c}}\right)
$$

where $S_{c}=L_{1} / L$ is the crossover position parameter. The restriction that the crossover is narrow implies that $K>>1$. We will assume that both $K_{1} \gg 1$ and $K_{2} \gg 1$, which is justified for $K \gg \gg 1$ when the crossover is located somewhere near the middle of the beam segment $\left(S_{c} \approx 1 / 2\right)$. Let us define the axial coordinate of the crossover as $z=0$. The beam radius at position 2 is then approximately given by

$$
r_{0}(z) \approx r_{c}+\alpha_{0}|z|
$$

For the energy spread generated in an extended beam one should start from equation (16.3.8), where $r_{0}$ is now given by equation $(16.3 .16)$. Substitution into equation $(16.2 .16)$ yields 


$$
\frac{\Delta E}{E} \sim \frac{m^{1 / 3}}{E_{0}} \frac{I^{2 / 3}}{V^{4 / 3}} \int_{z_{0}}^{z_{1}} \frac{d z}{\left[r_{c}+\alpha_{0}|z|\right]^{4 / 3}} \sim \frac{m^{1 / 3}}{E_{0}} \frac{I^{2 / 3}}{V^{4 / 3} r_{c} 1 / 3 \alpha_{0}}
$$

using that $K_{1} \gg 1$ and $K_{2} \gg 1$. This equation specifies the dependency on the experimental parameters of the energy spread generated by weak incomplete collisions in an extended beam segment with a narrow crossover. The energy spread generated in a pencil beam does not depend on $r_{0}$, as is expressed by equation $(16.3 .10)$. Accordingly, this equation applies also to a pencil beam with a narrow crossover.

The virtual spatial displacement $\Delta r$ in the crossover $\left(S_{i}=S_{c}\right)$ of an extended beam follows from equation (16.2.16), substituting equations $(16.3 .5),(16.3 .12)$ and $(16.3 .16)$

$$
\begin{aligned}
\Delta r & \sim \frac{m^{1 / 3}}{\varepsilon_{0}} \frac{I^{2 / 3}}{V^{4 / 3}} \int_{z_{0}}^{z_{1}} \frac{|z| d z}{\left[r_{c}+a_{0}|z|\right]^{4 / 3}} \\
& \sim \frac{m^{1 / 3}}{\varepsilon_{0}} \frac{I^{2 / 3} L^{2 / 3}}{V^{4 / 3} \alpha_{0} / 3}\left|S_{c} 2 / 3+\left(1-S_{c}\right)^{2 / 3}\right| \quad\left(S_{c}=S_{i}\right)
\end{aligned}
$$

using that $K_{1} \gg 1$ and $K_{2} \gg 1$. This equation applies to the same conditions as equation (16.3.17). Similarly, one finds with equation (16.3.13) for a pencil beam

$$
\Delta r \sim \frac{\mathrm{m}^{3 / 2}}{E_{0} \mathrm{e}^{7 / 2}} \frac{\mathrm{I}^{3} \mathrm{~L}^{3} \alpha_{0}}{\mathrm{~V}^{5 / 2}}\left|3 \mathrm{~S}_{\mathrm{c}}\left(1-\mathrm{S}_{\mathrm{c}}\right)-1\right| \quad\left(\mathrm{S}_{\mathrm{c}}=\mathrm{S}_{\mathrm{i}}\right)
$$

using that $K_{1} \gg>1$ and $K_{2} \gg>1$. This equation specifies the dependency of the trajectory displacement effect generated by weak incomplete collisions in a pencil beam with a narrow crossover.

So far we restricted the analysis to beams of low particle density, in which the interaction effects are dominated by weak incomplete collisions. With increasing particle density other type of collisions will become significant. Weak complete collision can occur in beams with a narrow crossover in which the lateral dimensions of the beam in the entrance plane $\left(\sim \alpha_{0} L S_{c}\right)$ and exit plane $\left(\sim \alpha_{0} L\left[1-S_{c}\right]\right)$ are large compared to the average axial separation of the particles $1 / \lambda$. These conditions are typical for moderate particle densities. With further increasing particle density the contribution of strong complete and strong incomplete collisions becomes significant.

The velocity shifts of an on-axis test particle involved in a complete collision are given by

$$
\begin{aligned}
& \Delta v_{z} \cong \frac{m \alpha^{3} v_{z}{ }^{3} b_{z}}{2 C_{0}\left[1+\left(b_{z}^{2}+r_{\perp}^{2} \sin ^{2} \Phi\right)\left(m \alpha^{2} v_{z}^{2} / 2 C_{0}\right)^{2}\right]} \\
& \Delta v_{\perp} \cong \frac{\alpha v_{z}\left[1+\left(m \alpha^{2} v_{z}{ }^{2} r_{\perp} \sin (\Phi) / 2 C_{0}\right)^{2}\right]^{1 / 2}}{\left[1+\left(b_{z}{ }^{2}+r_{\perp}{ }^{2} \sin ^{2} \Phi\right)\left(m \alpha^{2} v_{z}{ }^{\left.\left.2 / 2 C_{0}\right)^{2}\right]}\right.\right.}
\end{aligned}
$$

in which the coordinates $\xi=\left(\alpha, b_{z}, r_{1}, \Phi\right)$ specify the unperturbed coordinates of the field particle relative to those of the test particle, see figure 5.1. Equations (16.3.20) and (16.3.21) follow from the angle of deflection caused by a complete collision (which is the angle between the asymptotes of the relative trajectory in the orbital plane) and the transformation to coordinates in the laboratory system.

For a weak complete collision one may neglect the term 1 between 
the brackets in the denominator of equations (16.3.20) and (16.3.21). In addition we assume that $r_{1} \ll<b_{z}$, which is justified for a narrow crossover. This gives

$$
\Delta v_{z} \cong \frac{2 C_{0}}{m \alpha v_{z} b_{z}}, \quad \Delta v_{\perp} \cong \frac{4 C_{0}^{2}}{m^{2} \alpha^{3} v_{z}^{3} b_{z}^{2}}
$$

The distance $b_{z}$ will scale with $1 / \lambda$ and the angle $\alpha$ will scale with the beam semi-angle $\alpha_{0}$. Accordingly, one finds, with equation (16.3.3), for the parameter dependency of the energy spread generated by weak complete collisions in a beam segment with a narrow crossover

$$
\frac{\Delta E}{E} \sim \frac{m^{1 / 2}}{\epsilon_{0} \mathrm{e}^{1 / 2}} \frac{I}{\alpha_{0} V^{3 / 2}}
$$

using that $v_{z}=(2 \mathrm{eV} / \mathrm{m})^{1 / 2}$. Similarly, it follows from equations $(16.3 .4)$ and $(16.3 .22)$ that

$$
\Delta \alpha \sim \frac{\mathrm{m}}{\varepsilon_{0}^{2} \mathrm{e}} \frac{\mathrm{I}^{2}}{\alpha_{0} \mathrm{~V}^{3}}
$$

which refers to the same conditions as equation (16.3.23). The corresponding virtual shift in the image plane follows by multiplication with the distance between crossover and image plane

$$
\Delta r \sim \frac{m}{\varepsilon_{0}^{2} e} \frac{I^{2} L}{\alpha_{0}{ }^{3} V^{3}}\left|S_{c}-S_{i}\right|
$$

This equation expresses that the virtual shift generated by a weak complete collision is zero when crossover and image plane coincide $\left(S_{c}=S_{i}\right)$. For that condition the spatial and angular shift cancel out.

With further increasing particle densities the contribution of strong collisions will become significant and the deviations from the unperturbed trajectories can no longer be ignored. The total displacement of each test particle will be built up by a number of uncorrelated interactions, leading to a Gaussian type of displacement distribution $\rho(\Delta \eta)$. In general, this distribution is not dominated by a single type of collision. In order to determine the dependency on the experimental parameters one has to carry out the statistical calculation of the rms value, which now provides a proper width-measure. For a narrow crossover $(K>>1)$ one may assume that the main contribution to the distribution of axial and transverse velocity displacements comes from (nearly) complete collisions and employ equations (16.3.20) and (16.3.21). For an on-axis test particle and an uniform spatial and angular distribution the calculation of the mean square velocity displacement $\left\langle\Delta v^{2}\right\rangle$ consists of the evaluation of the following integral

$$
\left\langle\Delta v^{2}\right\rangle=\lambda \int_{0}^{v_{0}} \frac{2 v d v}{v_{0}^{2}} \int_{0}^{2 \pi} \frac{d \Phi}{2 \pi} \int_{0}^{r_{c}} \frac{2 r_{\perp} d r_{\perp}}{r_{c}{ }^{2}} \int_{-S_{c} L}^{\left(1-S_{c}\right) L} d b_{z}\left[\Delta v\left(v, \Phi, r_{\perp}, b_{z}\right)\right]^{2}
$$

where $\Delta v\left(v, \Phi, r_{1}, b_{2}\right)$ denotes the expression given by either equation (16.3.20) or equation (16.3.21), for the calculation of the quantities $\left\langle\Delta v_{z}{ }^{2}\right\rangle$ and $\left\langle\Delta v_{\perp}{ }^{2}\right\rangle$ respectively. In both cases all integrals can be solved analytically but one. Using equations (16.3.3) and (16.3.4) the results can be expressed as 


$$
\frac{\left\langle\Delta E^{2}>1 / 2\right.}{E}=2\left\langle\Delta \alpha^{2}>1 / 2=\left(\frac{m}{8 \varepsilon_{0}^{2} e}\right)^{1 / 4} P_{C}\left(\bar{r}_{c}\right) \frac{I^{1 / 2}}{V^{3 / 4}}\right.
$$

where the scaled crossover radius $\vec{r}_{c}$ and the function $P_{c}$ are given by

$$
\begin{aligned}
& \bar{r}_{c}=\frac{8 \pi \varepsilon_{0}}{e} \alpha_{0}{ }^{2} V_{c} \\
& P_{c}\left(\bar{r}_{c}\right)=\left[\frac{8}{\pi \bar{r}_{c}} \int_{0}^{1} d y \frac{\sqrt{1-y^{2}}}{y} \operatorname{sinh-1}\left(\frac{1}{2} \bar{r}_{c} y\right)\right]^{1 / 2}
\end{aligned}
$$

A good approximation of $\mathrm{P}_{\mathrm{c}}\left(\overline{\mathrm{r}}_{\mathrm{c}}\right)$ is given by

$$
\mathrm{Pca}_{\mathrm{a}}\left(\overline{\mathrm{r}}_{\mathrm{c}}\right)=\left(\frac{1}{1+\pi \bar{r}_{\mathrm{c}} /\left\{2 \ln \left[.8673\left(114.6+\overline{\mathrm{r}}_{\mathrm{c}}\right)\right]\right\}^{2}}\right)^{1 / 2}
$$

Equation (16.3.27) shows a square root dependency on the beam current $I$. Thus $\gamma=1 / 2$, confirming that the corresponding distribution is Gaussian.

The dependency on the other experimental parameters depends on the value of the scaled crossover radius $\bar{r}_{c}$. For $\bar{r}_{c} \rightarrow 0$ one finds from equation $(16.3 .29)$ or equation (16.3.30) that $\operatorname{Pc}\left(\bar{r}_{c}\right)=1$. The corresponding relative energy spread $\left\langle\Delta E^{2}>1 / 2 / E\right.$ and angular spread $<\Delta \alpha^{2}>1 / 2$ are proportional to the square root of the perveance $I / V^{3} / 2$, as can be seen from equation (16.3.27). For $\bar{r}_{c}+\infty$ one finds from equations $(16.3 .27),(16.3 .28)$ and $(16.3 .30)$

$$
\frac{<\Delta E^{2}>1 / 2}{\mathrm{E}} \approx .616 \frac{(\mathrm{me})^{1 / 4}}{\varepsilon_{0}}\left[1+.217 \ln \left(1+\frac{\bar{r}_{\mathrm{c}}}{114.6}\right)\right] \frac{\mathrm{I}^{1 / 2}}{\mathrm{~V}^{5 / 4} \mathrm{r}_{\mathrm{c}}{ }^{1 / 2} \alpha_{0}}
$$

The same result applies to $2<\Delta \alpha^{2}>1 / 2$, conform equation (16.3.27). Equation (16.3.31) provides an accurate approximation for $\bar{r}_{c}>100$.

It should be emphasized that equation (16.3.27) relies on the assumption that complete collision are dominant. For high particle densities this will be true if $K_{1} \gg 1$ and $K_{2} \gg>1$, which requires $K \gg \gg 1$ and $S_{c \approx 1} / 2$. The analysis for smaller $K$ values is more complex since one has to account for strong incomplete collisions, which can not be handled analytically, as was discussed in the previous section. Accordingly, the integral of equation $(16.3 .26)$ has to be performed numerically, employing the numerical solution of the two-particle problem. This was done for various $K$ and $\bar{r}_{c}$ values. The result can again be expressed by equation $(16.3 .27)$, provided that the function $P_{c}\left(\bar{r}_{c}\right)$ is replaced by a function $P_{c}\left(\bar{r}_{c}, K_{1}, K_{2}\right)$, which depends also on $K_{1}$ and $K_{2}$. An analytical approximation for this function was obtained by fitting the numerical data. Different functions $P_{c}\left(\bar{r}_{c}, K_{1}, K_{2}\right)$ are required to describe the small K-behaviour of $\left\langle\Delta E^{2}\right\rangle 1 / 2$ and $\left\langle\Delta \alpha^{2}\right\rangle 1 / 2$.

The trajectory displacement distribution $\rho(\Delta r)$ also becomes Gaussian for high particle densities. In this regime the contribution of strong collisions is significant, leading to substantial deviations from the unperturbed trajectories. Contrary to the analysis of the velocity shifts $\Delta v_{\perp}$ and $\Delta v_{z}$, one can not use complete collision dynamics to calculate the shift $\Delta r$ caused by a two-particle collision, as was discussed in the previous section. One has to account for the fact that the interaction time $T$ is finite. Unfortunately, the complexity of the resulting equation for $\Delta r$ prohibits an exact analytical evaluation of the mean square displacement $\left\langle\Delta r^{2}\right\rangle$. The corresponding integral, which is similar to equation $(16.3 .26)$, has to be performed numerically. At this 
point it is advantageous to scale the equations in order to reduce the number of independent parameters. In addition to the quantities $S_{c}$ and $S_{i}$ and the scaled linear particle density $\bar{\lambda}$, the resulting equation depends on the scaled crossover radius $r_{c}{ }^{*}$ and scaled transverse velocity $v_{0}{ }^{*}$, which are defined as

$$
\begin{aligned}
& r_{c} *=\left(\frac{2 \pi \varepsilon_{0}}{e}\right)^{1 / 3} \frac{V^{1 / 3} r_{c}}{L^{2} / 3} \\
& v_{0} *=\left(\frac{2 \pi \varepsilon_{0}}{e}\right)^{1 / 3} V^{1 / 3} L^{1 / 3} \alpha_{0}
\end{aligned}
$$

Notice that the scaling quantities differ from the ones used for the Boersch effect $\left(\bar{r}_{c}\right.$ and $\left.K\right)$. However, in both cases the quantity $\lambda$ is used as the measure for the particle density. For the case $S_{c}=S_{i}=1 / 2$, $r_{c}{ }^{*}=0$ and $v_{0}^{*}+\infty$, the $r m s$ value of $\rho(\Delta r)$ is given by

$$
<\Delta r^{2}>1 / 2=.0950 \frac{\mathrm{e}^{1 / 12} \mathrm{~m}^{1 / 4}}{\varepsilon_{0}^{5 / 6}} \frac{I^{1 / 2} \mathrm{~L}^{2 / 3}}{V^{13 / 12} \alpha_{0}}
$$

In general, the width of the distribution increases strongly when $S_{i} \neq S_{c}$. When the crossover plane and the image plane do coincide $\left(S_{c}=S_{i}\right)$, the rms value shows a slight increase with $S_{c} \quad\left(0<S_{c}<1\right)$. The dependency on the scaled crossover radius $r_{c}{ }^{*}$ is very weak and can practically be ignored for $r_{c}^{*}<1$. The $r m s$ is independent of $v_{0}^{*}$ for large $v_{0}^{*}$-values (typically $v_{0}^{*}>100$ ) and decreases with $v_{0}^{*}$ for small $v_{0}^{*}$.

This completes our qualitative analysis. In the next sections, the detailed equations are reviewed which yield accurate quantitative predictions for the various manifestations of coulomb particle-particle interactions in a rotational symmetric beam segment in drift space. The form of each of these equations is derived from the analytical analysis performed for the extreme cases that a single regime is dominant. Some of the constants are determined by parameter-fitting of the numerical data obtained for the general case, using a suitable fit-function. The final equations explicitly show the functional dependency corresponding to the regime which is dominant for most practical operating conditions. The dependencies of the other regimes are not explicitly represented, but can be retrieved from the asymptotic behaviour of the fit-functions. These functions are expressed in terms of dimensionless parameters. Table 16.1 gives an overview of the scale measures and the dimensionless parameters used in the model. The various functional dependencies and physical constants appearing in the final equations for the Boersch effect, the effect of statistical angular deflections and the trajectory displacement effect are summarized by the tables $16.2 \mathrm{a}, 16.2 \mathrm{~b}$ and $16.2 \mathrm{c}$ respectively.

\subsection{Bquations for the Boersch effect}

The theory for the Boersch effect is presented in chapter 7. Here we will restrict ourselves to a review of the essential results. For the case of a homocentric cylindrical beam segment we will add an equation for the calculation of the FW50 value, which follows straightforwardly from the analysis of section 7.4 .

The Full Width (FWHM or FWso) of the normal energy distribution, generated in a beam segment with a crossover, is given by 
Table 16.1. Overview of the scale measures and scaled parameters used in the extended two-particle model for Coulomb interactions in particle beams.

\begin{tabular}{|c|c|c|c|c|c|}
\hline & & unit & definition & $\begin{array}{l}\text { functional } \\
\text { dependency }\end{array}$ & $\begin{array}{l}\text { value physical const. } \\
\text { for electrons (SI) }\end{array}$ \\
\hline \multirow{4}{*}{$\begin{array}{l}S \\
C \\
A \\
L \\
\text { E } \\
M \\
\text { E } \\
A \\
S \\
\text { U } \\
R \\
\text { E } \\
\text { S } \\
\text { S }\end{array}$} & $d_{0}$ & m & $\frac{\mathrm{e}^{2}}{4 \pi \varepsilon_{0} \pi v_{0}^{2}}$ & $\frac{e}{8 \pi \varepsilon_{0}} \frac{1}{a_{0}^{2} V}$ & $7.1999 \times 10^{-10}$ \\
\hline & $v_{0}$ & $\frac{\mathrm{m}}{\mathrm{s}}$ & $\alpha_{0} v_{z}$ & $\left(\frac{2 \mathrm{e}}{\mathrm{m}}\right)^{1 / 2} \alpha_{0} \mathrm{~V}^{1} \mathrm{i}^{2}$ & $5.9309 \times 10^{5}$ \\
\hline & $\delta$ & $\mathrm{m}$ & $\left(\frac{e^{2} L^{2}}{\pi E_{0} m v_{z}^{2}}\right)^{1 / 3}$. & $\left(\frac{\mathrm{e}}{2 \pi \varepsilon_{0}}\right)^{1 / 3} \frac{\mathrm{L}^{2 / 3}}{\mathrm{~V}^{1 / 3}}$ & $1.4228 \times 10^{-3}$ \\
\hline & $\nu$ & $\frac{\mathrm{m}}{\mathrm{s}}$ & $\left(\frac{e^{2} v_{2}}{\pi \varepsilon_{0} m L}\right)^{1 / 3}$ & $\frac{21 / 6 \mathrm{e}^{5 / 6}}{\left(\pi \varepsilon_{0}\right)^{1 / 3} \mathrm{~m}^{1 / 2}} \frac{\mathrm{V}^{1 / 6}}{\mathrm{~L}^{1 / 3}}$ & $8.4382 \times 10^{2}$ \\
\hline \multirow{5}{*}{$\begin{array}{l}\mathrm{D} \\
\mathbf{Z} \\
\mathbf{N} \\
\mathbf{S} \\
\mathrm{I} \\
\mathbf{T} \\
\mathbf{Y}\end{array}$} & $\lambda$ & $\frac{1}{m}$ & $\frac{\mathrm{I}}{\mathrm{ev} v_{z}}$ & $\left(\frac{\mathrm{m}}{2 \mathrm{e}^{3}}\right)^{1 / 2} \frac{\mathrm{I}}{\mathrm{V}^{1 / 2}}$ & $1.0524 \times 10^{13}$ \\
\hline & $\bar{\lambda}$ & - & $\lambda d_{0}$ & $\frac{\mathrm{m}^{1 / 2}}{21 / 2 \pi \varepsilon_{0} e^{1 / 2}} \frac{\mathrm{I}}{\alpha_{0}^{2} \mathrm{~V}^{3 / 2}}$ & $7.5768 \times 10^{3}$ \\
\hline & $\lambda$ * & - & $\lambda \frac{\delta^{3}}{4 r_{0}{ }^{2}}$ & $\frac{\mathrm{m}^{1 / 2}}{2^{I / 2} \pi \varepsilon_{0} \mathrm{e}^{1 / 2}} \frac{\mathrm{IL}^{2}}{r_{0}{ }^{2} \mathrm{~V}^{3 / 2}}$ & $7.5768 \times 10^{3}$ \\
\hline & $x_{c}$ & - & $\lambda \alpha_{0} L$ & $\left(\frac{m}{2 e^{3}}\right)^{1 / 2} \frac{I a_{0} L}{V^{1 / 2}}$ & $1.0524 \times 10^{13}$ \\
\hline & $x_{p}$ & - & $\lambda r_{0}$ & $\left(\frac{\mathrm{m}}{2 \mathrm{e}^{3}}\right)^{1 / 2} \frac{\mathrm{Ir}_{0}}{\mathrm{~V}^{1 / 2}}$ & $1.0524 \times 10^{13}$ \\
\hline \multirow{4}{*}{$\begin{array}{l}\mathrm{M} \\
\mathrm{A} \\
\mathrm{I} \\
\mathrm{N} \\
\mathrm{P} \\
\mathrm{A} \\
\mathrm{R} \\
\mathrm{A} \\
\mathrm{M} \\
\mathrm{E} \\
\mathrm{T} \\
\mathrm{E} \\
\mathrm{R} \\
\mathrm{S}\end{array}$} & $\overline{\mathbf{r}}_{\mathrm{c}}$ & - & $\frac{r_{c}}{d_{0}}$ & $\frac{8 \pi \varepsilon_{0}}{e} \dot{a}_{0}^{2} r_{c} v$ & $1.3889 \times 10^{9}$ \\
\hline & $\mathrm{K}$ & - & $\frac{\alpha_{0} L}{2 r_{c}}$ & $\frac{a_{0} L}{2 r_{c}}$ & - \\
\hline & $r_{c}{ }^{*}$ & - & $\frac{r_{c}}{\delta}$ & $\left(\frac{2 \pi \epsilon_{0}}{e}\right)^{1 / 3} \frac{r_{c} V^{1 / 3}}{L^{2 / 3}}$ & $7.0286 \times 10^{2}$ \\
\hline & $v_{0}{ }^{\star}$ & - & $\frac{v_{0}}{v}$ & $\left(\frac{2 \pi \varepsilon_{0}}{\mathrm{e}}\right)^{1 / 3} \alpha_{0} V^{1 / 3} L^{1 / 3}$ & $7.0286 \times 10^{2}$ \\
\hline
\end{tabular}

\section{related parameters}

\begin{tabular}{|l|l|}
\hline$S_{c}=\frac{L_{1}}{L}$ & $S_{1}=\frac{L_{1}}{L}$ \\
\hline$K_{1}=2 S_{c} K$ & $K_{2}=2\left(1-S_{c}\right) K$ \\
\hline
\end{tabular}


Table 16.2a Overview of the results for the relative energy spread $\mathrm{FWH}_{\mathrm{E}} / \mathrm{E}$ in the different regimes, for a beam segment with a crossover and a homocentric cylindrical beam segment. The equations for the cylindrical beam apply to an uniform current density distribution. Those for a beam with a crossover apply to an uniform spatial and an uniform angular distribution in the crossover and $\mathrm{K}_{1}=\mathrm{K}_{2}=\mathrm{K}$ (crossover in the middle). Effective measures for Gaussian density distributions are presented in section 7.6 . The results for the beam segment with a crossover can be generalized for $\mathrm{K}_{1} \neq \mathrm{K}_{2}$, using the approach of equation $(7.5 .37)$.

\begin{tabular}{|c|c|c|c|c|c|c|}
\hline \multirow[b]{2}{*}{$\frac{F W H M_{E}}{E}$} & \multicolumn{3}{|c|}{ beam segment with a crossover } & \multicolumn{3}{|c|}{ homocentric cylindrical beam } \\
\hline & $\begin{array}{l}\text { code }+ \\
\text { equation }\end{array}$ & $\begin{array}{l}\text { factor + value } \\
\text { for electrons }\end{array}$ & $\begin{array}{l}\text { funet. dependency } \\
+ \text { add. equations }\end{array}$ & $\begin{array}{l}\text { code }+ \\
\text { equation }\end{array}$ & $\begin{array}{l}\text { factor + value } \\
\text { for electrons }\end{array}$ & $\begin{array}{l}\text { funet. dependeney } \\
+ \text { add. equations }\end{array}$ \\
\hline $\mathbf{G}$ & $\begin{array}{l}\text { CGE } \\
7.3 .37\end{array}$ & $\begin{array}{l}\left(\frac{8(\ln 2)^{2} \mathrm{~m}}{\mathrm{E}^{2} \mathrm{e}}\right)^{1 / 4} \\
726.62\end{array}$ & $\begin{array}{l}\operatorname{PCE}\left(\bar{r}_{C}, K\right) \frac{I^{1 / 2}}{V^{3 / 4}} \\
7.5 .21,7.5 .20\end{array}$ & $\begin{array}{l}\text { PGE } \\
7.4 .32\end{array}$ & $\begin{array}{l}.3330 \frac{\mathrm{e}^{1 / 12 \mathrm{~m}^{1 / 4}}}{\varepsilon_{0} / 6} \\
.4537\end{array}$ & $\begin{array}{l}P_{P E}\left(r_{0}{ }^{*}\right) \frac{I^{1 / 2} L^{2 / 3}}{r_{0} V^{13 / 12}} \\
7.4 .22,7.4 .16\end{array}$ \\
\hline H & $\begin{array}{l}\text { CHE } \\
7.5 .25\end{array}$ & $\begin{array}{l}1.3233 \frac{\mathrm{m}^{1 / 3}}{\epsilon_{0}} \\
14.488\end{array}$ & $\begin{array}{l}H(K) \frac{I^{2 / 3}}{r_{c}^{1 / 3} \alpha_{0} V^{6 / 3}} \\
7.5 .23\end{array}$ & $\begin{array}{l}\text { PHE } \\
7.4 .31\end{array}$ & $\begin{array}{l}.22056 \frac{\mathrm{m}^{1 / 3}}{\varepsilon_{0}} \\
2.4147\end{array}$ & $\frac{I^{2 / 3} L}{r_{0} / 3 V^{4} / 3}$ \\
\hline$L$ & $\begin{array}{l}\text { CLE } \\
7.3 .40\end{array}$ & $\begin{array}{l}\left(\frac{2 \mathrm{~m}}{\varepsilon_{0}^{2} \mathrm{e}}\right)^{1 / 2} \\
3.8085 \times 10^{5}\end{array}$ & $\frac{I}{\alpha_{0} V^{3 / 2}}$ & not a & applicable & \\
\hline $\mathbf{P}$ & equal to & a result for cy & lindrical beam & $\begin{array}{l}\text { PPE } \\
7.4 .33\end{array}$ & $\begin{array}{l}.11178 \frac{\mathrm{m}}{\varepsilon_{0} \mathrm{e}^{2}} \\
4.4800 \times 10^{11}\end{array}$ & $\frac{I^{2} L}{V^{2}}$ \\
\hline
\end{tabular}

Table 16.2b overview of the results for the angular spread $\mathrm{FWHM}_{\alpha}$ in the different regimes, for a beam segment with a crossover and a homocentric cylindrical beam segment. The equations apply to the same conditions as described in the caption of table 16.2a. Effective measures for Gaussian density distributions are presented in section 8.7 .

\begin{tabular}{|c|c|c|c|c|c|c|}
\hline \multirow[b]{2}{*}{ FWHM $_{\mathbf{a}}$} & \multicolumn{3}{|c|}{ beam segment with a crossover } & \multicolumn{3}{|c|}{ homocentric cylindrical beam } \\
\hline & $\begin{array}{l}\text { code }+ \\
\text { equation }\end{array}$ & $\begin{array}{l}\text { factor + value } \\
\text { for electrons }\end{array}$ & $\begin{array}{l}\text { funct. dependency } \\
+ \text { add. equations }\end{array}$ & $\begin{array}{l}\text { code }+ \\
\text { equation }\end{array}$ & $\begin{array}{l}\text { factor + value } \\
\text { for electrons }\end{array}$ & $\begin{array}{l}\text { funct. dependency } \\
+ \text { add. equations }\end{array}$ \\
\hline G & $\begin{array}{l}\text { CGA } \\
8.3 .21\end{array}$ & $\begin{array}{l}\left(\frac{(\ln 2)^{2} m}{8 \varepsilon_{0}^{2} e}\right)^{1 / 4} \\
256.90\end{array}$ & $\begin{array}{l}\operatorname{Pca}_{\mathrm{Ca}}\left(\bar{r}_{\mathrm{c}}, \mathrm{K}\right) \frac{\mathrm{I}^{1 / 2}}{\mathrm{~V}^{3 / 4}} \\
8.5 .28,8.5 .27\end{array}$ & $\begin{array}{l}\text { PGA } \\
8.4 .30\end{array}$ & $\begin{array}{l}.1665 \frac{\mathrm{e}^{1 / 12 \mathrm{~m}^{1 / 4}}}{\epsilon_{0}^{5 / 6}} \\
.2269\end{array}$ & $\begin{array}{l}P_{P A}\left(r_{0} *\right) \frac{I^{1 / 2} L^{2 / 3}}{r_{0} V^{13 / 12}} \\
8.4 .20,8.4 .16\end{array}$ \\
\hline H & $\begin{array}{l}\text { CHA } \\
8.5 .31\end{array}$ & $\begin{array}{l}.19233 \frac{\mathrm{m}^{1 / 3}}{\varepsilon_{0}} \\
2.1057\end{array}$ & $\begin{array}{l}S_{\mathrm{HA}} \frac{I^{2 / 3}}{a_{0} / 3 \mathrm{~V}^{4} / 3 \mathrm{~L}^{1 / 3}} \\
8.5 .20\end{array}$ & $\begin{array}{l}\text { PHA } \\
8.4 .29\end{array}$ & $\begin{array}{l}.10177^{\mathrm{m}^{1 / 3}} \frac{\mathrm{E}_{0}}{1.1142}\end{array}$ & $\frac{I^{2 / 3} L}{r_{0} / 3^{3} V^{6 / 3}}$ \\
\hline w & $\begin{array}{l}\text { CWA } \\
8.3 .24\end{array}$ & $\begin{array}{l}.033329 \frac{\mathrm{m}}{\varepsilon_{0}^{2} \mathrm{e}} \\
2.4172 \times 10^{9}\end{array}$ & $\begin{array}{l}f_{\infty}\left(\bar{r}_{c}\right)^{2} \frac{I^{2}}{a_{0}^{3} V^{3}} \\
8.3 .10\end{array}$ & not & applicable & \\
\hline $\mathbf{P}$ & $\begin{array}{l}\text { CPA } \\
8.5 .35\end{array}$ & $\begin{array}{l}4.1531 \frac{10-4 \mathrm{~m}^{3 / 2}}{\varepsilon_{0} \mathrm{e}^{7 / 2}} \\
2.4772 \times 10^{20}\end{array}$ & $\begin{array}{l}S_{P A} \frac{I^{3} \alpha_{0} L^{2}}{V^{5 / 2}} \\
8.5 .21\end{array}$ & $\begin{array}{l}\text { PPA } \\
8.4 .32\end{array}$ & $\begin{array}{l}8.3061 \frac{10^{-6} \mathrm{~m}^{3 / 2}}{\varepsilon_{0} \mathrm{e}^{7 / 2}} \\
4.9544 \times 10^{28}\end{array}$ & $\frac{I^{3} r_{0 L}}{v^{5 / 2}}$ \\
\hline
\end{tabular}


rable 16.2e overview of the results for the FWHM trajectory displacement effect in the different regimes, for a beam segment with a crossover. The equations apply to the case of an uniform spatial and an uniform angular distribution in the crossover. Effective measures for Gaussian density distributions are presented in section 9.7. For a cylindrical beam the spatial broadening in the backfocal plane of a lens, which is located at the end of the segment, is equal to FWHM $_{\alpha} \times f$, where $f$ is the focal length of the lens. The reader is referred to table $16.2 \mathrm{~b}$. for the functional dependency of the angular spread $\mathrm{FWH}_{\alpha}$ generated in a homocentric cylindrical beam.

\begin{tabular}{|c|c|c|c|}
\hline \multirow[b]{2}{*}{ FWHMr } & \multicolumn{3}{|c|}{ beam segment with a crossover } \\
\hline & $\begin{array}{l}\text { code }+ \\
\text { equation }\end{array}$ & $\begin{array}{l}\text { factor + value } \\
\text { for electrons }\end{array}$ & $\begin{array}{l}\text { funct. dependency } \\
+ \text { add. equations }\end{array}$ \\
\hline \multirow{3}{*}{ G } & \multirow{2}{*}{ CGT } & \multirow{2}{*}{$.158 \frac{\mathrm{e}^{1 / 12} \mathrm{~m}^{1 / 4}}{\varepsilon 0^{5 / 6}}$} & $P_{C T} I^{1 / 2} L^{2 / 3}$ \\
\hline & & & $\operatorname{ctt} \overline{a_{0} V^{13 / 12}}$ \\
\hline & 9.3 .45 & .215 & $9.3 .25,9.5 .10$ \\
\hline \multirow{3}{*}{ H } & \multirow{3}{*}{$\begin{array}{l}\text { CHT } \\
9.3 .44\end{array}$} & \multirow{3}{*}{$\begin{array}{l}.17205 \frac{\mathrm{m}^{1 / 3}}{\varepsilon_{0}} \\
1.8837\end{array}$} & \multirow{3}{*}{$\begin{array}{l}S_{H T} \frac{I^{2 / 3} L^{2 / 3}}{a_{0^{4} / 3} V^{4 / 3}} \\
9.5 .3\end{array}$} \\
\hline & & & \\
\hline & & & \\
\hline \multirow{3}{*}{ W } & & m & \multirow{3}{*}{$\begin{array}{l}\left|S_{c}-S_{i}\right| f_{\infty}{ }^{2} \frac{I^{2} L}{a_{0}^{3} V^{3}} \\
8.3 .10\end{array}$} \\
\hline & & $.033329 \overline{E_{0}^{2} \mathrm{e}}$ & \\
\hline & 9.3 .46 & $2.4172 \times 10^{9}$ & \\
\hline \multirow{3}{*}{$\mathbf{p}$} & & $10^{-5} \mathrm{~m}^{3 / 2}$ & \multirow{3}{*}{$\begin{array}{l}S_{\mathrm{PT}} \frac{I^{3} \alpha_{0} L^{3}}{V^{5 / 2}} \\
9.5 .6\end{array}$} \\
\hline & $\mathrm{CPT}$ & $6 . 9 2 1 8 \longdiv { \epsilon o _ { 0 } / 1 2 }$ & \\
\hline & 9.3 .47 & $4.1287 \times 10^{21}$ & \\
\hline
\end{tabular}

$$
\frac{F W_{E}}{E}=G_{F W} C_{C G E} \frac{1}{2}\left[G_{C E}\left(\pi, \bar{r}_{c}, K_{1}\right)+G_{C E}\left(\lambda, \bar{r}_{c}, K_{2}\right)\right] \sqrt{\frac{I}{V^{3 / 2}}}
$$

where the constant $C_{C G E}$ is equal to

$$
C_{C G E}=1.4002 \frac{m^{1 / 4}}{\varepsilon_{0}^{1 / 2} \mathrm{e}^{1 / 4}}
$$

In the case of electrons one finds $C_{C G E}=726.62$ in SI-units. The function $G_{C E}\left(\pi, \bar{r}_{c}, K\right)$ represents

$$
G_{C E}\left(\pi, \bar{r}_{c}, K\right)=\left[\frac{1}{P_{C E}\left(\bar{r}_{c}, K\right)^{4}}+\frac{A \bar{r}_{c}^{4 / 3}}{\bar{\lambda}^{2 / 3} \mathrm{H}(K)^{4}}+\frac{B}{D \lambda^{2}}+\frac{C}{\bar{\lambda}^{6} \bar{r}_{c}{ }^{4} K^{4}}\right]^{-1 / 4}
$$

where the functions $P_{C E}\left(\bar{r}_{c}\right)$ and $H(K)$ are given by

$$
\begin{array}{r}
\operatorname{PCE}\left(\overline{\mathrm{r}}_{\mathrm{c}}\right)=\left[.788+.6\left(\frac{\overline{\mathrm{r}}_{\mathrm{c}}}{\mathrm{K}^{2}}\right)^{2 / 3}\left[1+\frac{1}{2}\left(\frac{\mathrm{K}^{2}}{\overline{\mathrm{r}}_{\mathrm{c}}}\right)^{4 / 9}\right]^{3 / 2}+\frac{\pi \overline{\mathrm{r}}_{\mathrm{c}}}{\left\{2 \ln \left[.8673\left(\mathrm{E}+\mathrm{F} \overline{\mathrm{r}}_{\mathrm{c}}\right)\right]\right\}^{2}}\right]^{-1 / 2} \\
\mathrm{H}(\mathrm{K})=\frac{1}{\left[1+9 \mathrm{~K}^{-2}+2 \mathrm{~K}^{-1 / 3}\right]^{1 / 2}}
\end{array}
$$

In order to obtain a FWHM value, the constant Grw in equation (16.4.1) and the constants $A, B$ and $C$ in equation (16.4.3) should be taken equal to 


$$
\mathrm{G}_{\mathrm{PW}}=1, \quad \mathrm{~A}=1.57510^{-3}, \mathrm{~B}=7.60610^{-4}, \mathrm{C}=.3045
$$

while for a FWso, one should use

$$
\mathrm{G}_{\mathrm{FW}}=.57288, A=8.24610^{-4}, B=8.19210^{-5}, \mathrm{C}=3.01510^{-5} \quad\left(F_{50}\right) \quad(16.4 .7)
$$

The constant $D$ in equation (16.4.3) and the constants $E$ and $F$ in equation (16.4.4) are for an uniform angular and spatial distribution given by

$$
\mathrm{D}=1, \quad \mathrm{E}=114.6, \quad \mathrm{~F}=1
$$

Gaussian angular and spatial distributions can in reasonable approximation be represented by the same set of equations, provided that one replaces $\alpha_{0}$ and $r_{c}$ by the effective values $\alpha_{e f f}$ and $r_{e f f}$, defined as

$$
\alpha_{\text {eff }} \cong 1.6 \sigma_{\alpha}, \quad r_{\text {eff }} \cong 1.6 \sigma_{r}
$$

where $\sigma_{\alpha}{ }^{2}=<\alpha^{2}>/ 2$ and $\sigma_{r}{ }^{2}=\left\langle r^{2}>/ 2\right.$. A more accurate approach is to take $\alpha_{0}=\sqrt{2} \sigma_{\alpha}$ and $r_{c}=\sqrt{2} \sigma_{r}$ and use the following modified values for the constants $D, E$ and $F$

$$
\begin{aligned}
& \mathrm{D}_{\mathrm{Bg}}=.6169, \quad \mathrm{E}_{\mathrm{gB}}=40.74, \quad \mathrm{~F}_{\mathrm{Bg}}=1.315 \\
& \mathrm{D}_{\mathrm{Bu}}=.6169, \quad \mathrm{E}_{\mathrm{Bu}}=49.19, \quad \mathrm{~F}_{\mathrm{BU}}=1.138 \\
& \mathrm{D}_{\mathrm{ug}}=1 \quad, \quad \mathrm{E}_{\mathrm{ug}_{\mathrm{g}}}=100.0, \quad \mathrm{~F}_{\mathrm{ug}}=1.160 \\
& \left(D_{u u}=1 \quad, \quad E_{u u}=114.6, \quad F_{u u}=1\right)
\end{aligned}
$$

in which the first subscript indicates the type of angular distribution and the second subscript the type of spatial distribution (u=uniform, $\mathbf{g}=$ Gaussian).

In order to obtain an effective $\gamma$-value which characterizes the type of distribution; one should evaluate

$$
\gamma_{\text {eff }}=\frac{\gamma\left(\pi, \bar{r}_{c}, K_{1}\right)+\gamma\left(\pi, \bar{r}_{c}, K_{2}\right)}{G_{C E}\left(\pi, \bar{r}_{c}, K_{1}\right)+G_{C E}\left(\bar{r}, \bar{r}_{c}, K_{2}\right)}
$$

where

$\gamma\left(\bar{r}, \bar{r}_{c}, K\right)=G_{C E}\left(\bar{r}, \bar{r}_{c}, K\right)\left[\frac{2^{4}}{P_{C E}\left(\bar{r}_{c}, K\right)^{4}}+\frac{3^{4}}{2^{4}} \frac{A \bar{r}_{c}{ }^{4 / 3}}{\bar{\lambda}^{2 / 3} \mathrm{H}(K)^{4}}+\frac{B}{D \bar{\lambda}^{2}}+\frac{1}{2^{4}} \frac{C}{\bar{\lambda}^{6} \bar{r}_{c}{ }^{4} K^{4}}\right]^{1 / 4}$

and the function $G_{C E}\left(\pi, \bar{r}_{\mathfrak{c}}, K\right)$ is given by equation $(16.4 .3)$. For the constant $A, B$ and $C$ we use the values for the $F_{50}$ value, specified by equation (16.4.7). The constant $D$ depends on the type of distribution, as specified by equation $(16.4 .10)$. The outcome of equation (16.4.11) is a value for $\gamma_{\text {eff }}$ between $1 / 2$ and 2. The parameter $\gamma_{\text {eff }}$ serves as input for the algorithm used for the addition of the results obtained for the individual beam segments, which is the subject of section 16.7 .

The Full Width (FWHM or $F_{50}$ ) of the normal energy distribution, generated in a homocentric cylindrical beam segment, is given by

$$
\frac{F W_{E}}{E}=H_{F W} C_{P H E} H_{P E}\left(\lambda^{*}, r_{0}^{*}\right) \frac{I^{2 / 3} L}{V^{4} / 3 r_{0}^{4 / 3}}
$$

where the constant $C_{P H E}$ is equal to 


$$
C_{P H E}=0.22056 \frac{\mathrm{m}^{1 / 3}}{E_{0}}
$$

For electrons one finds $\mathrm{C}_{\mathrm{PBE}}=2.4147$ in SI-units. The function $H_{P E}\left(\lambda^{*}, r_{0}{ }^{*}\right)$ represents

$$
\mathrm{HPE}_{\mathrm{PE}}\left(\lambda^{*}, \mathrm{r}_{0}^{*}\right)=\left[\left(1+\frac{A \lambda^{*}}{\operatorname{PPE}\left(\mathrm{r}_{0}^{*}\right)^{6}}\right)^{1 / 4}+\frac{\mathrm{B}}{\chi_{\mathrm{p}}{ }^{2}}\right]^{-2 / 3}
$$

where $\chi_{p}$ is the pencil beam factor for a cylindrical beam $\left(\chi_{p}=4 \lambda^{*} r_{0} * 3\right)$ and the function $\operatorname{PPE}\left(r_{0}{ }^{*}\right)$ is given by

$$
\mathrm{PPE}_{\mathrm{PE}}\left(\mathrm{Y}_{0}{ }^{*}\right)=\left(1+0.240 / \mathrm{r}_{0}^{* 8 / 7}\right)-7 / 8
$$

In order to obtain a FWHM value, the constant $H_{P} w$ in equation (16.4.13) and the constants $A$ and $B$ in equation (16.4.15) should be taken equal to

$$
\mathrm{H}_{\mathrm{FW}}=1, \quad \mathrm{~A}=2.997, \quad \mathrm{~B}=1.386
$$

(FWHM)

$(16.4 .17)$

while for a FW5o, one should use

$$
\mathrm{H}_{\mathrm{FW}}=.67347, \mathrm{~A}=7.911, \mathrm{~B}=5.56610^{-2} \quad\left(\mathrm{FW}_{50}\right)
$$

A Gaussian current density distribution leads to the same set of equations, provided that one takes $r_{0}=\sqrt{2} \sigma_{r}$, with $\sigma_{r}{ }^{2}=\left\langle r^{2}\right\rangle / 2$.

In order to obtain an effective $\gamma$-value which characterizes the type of distribution, one should evaluate

$$
\gamma_{Q C f}=H_{P E}\left(\lambda^{*}, r_{0^{*}}\right)\left[\left(\frac{3^{6}}{2^{6}}+\frac{2^{6} A \lambda^{*}}{\operatorname{PPE}_{\mathrm{PE}}\left(r_{0^{*}}\right)^{6}}\right)^{1 / 4}+\frac{1}{2^{3 / 2}} \frac{B}{\chi_{p^{2}}}\right]^{2 / 3}
$$

where the function $H_{P E}\left(\lambda^{*}, r_{0}{ }^{*}\right)$ is given by equation $(16.4 .15)$. For the constant $A$ and $B$ we use the values for the $F_{5} 0$ value, specified by equation (16.4.18). The outcome of equation (16.4.19) is a value for $\gamma_{e f f}$ between $1 / 2$ and 2 .

\subsection{Equations for the trajectory displacement ef fect}

The theory for the trajectory displacement effect is presented in chapter 9. Chapter 8 covers the related effect of statistical angular deflections. Here we will restrict ourselves to a review of the essential results and will add some equations for the calculation of the $d_{1288}$ and $d_{2575}$ edge-width of shaped spots, utilizing the analysis of chapter 12. For the case of a homocentric cylindrical beam we will add an equation for the $\mathrm{FW}_{5}$ a value, which follows straightforwardly from the analysis of sections 8.4 and 9.4 .

The Full Width (FWHM, FW50, $d_{1288}$ or $d_{2575}$ ) of the trajectory displacement distribution, generated in a beam segment with a crossover, is given by

$$
\mathrm{FW}_{\mathrm{r}}=\mathrm{H}_{\mathrm{FW}} \mathrm{C}_{\mathrm{CHT}} \mathrm{H}_{\mathrm{CT}}\left(\pi, \mathrm{V}_{0}{ }^{*}, \mathrm{r}_{\mathrm{c}}{ }^{*}, \mathrm{~S}_{\mathrm{c}}, \mathrm{S}_{\mathrm{i}}\right) \frac{\mathrm{I}^{2 / 3} \mathrm{~L}^{2 / 3}}{\mathrm{~V}^{4 / 3} \alpha_{0} \alpha^{4 / 3}}
$$

where the constant $\mathrm{C}_{\mathrm{CH}}$ is equal to

$$
\mathrm{C}_{\mathrm{CHT}}=0.17205 \frac{\mathrm{m}^{1 / 3}}{\varepsilon_{0}}
$$


For electrons $\mathrm{C}_{\mathrm{CHT}}=1.8837$ in SI-units. The function $\mathrm{H}_{\mathrm{CT}}\left(\pi, \mathrm{v}_{0}{ }^{*}, \mathrm{r}_{\mathrm{c}}{ }^{*}, S_{\mathrm{c}}, \mathrm{S}_{\mathrm{i}}\right)$ is given by

$$
H_{C T}\left(\pi, v_{0}^{*}, r_{c}^{*}, S_{c}, S_{i}\right)=H_{C T 1}+\left|S_{c}-S_{i}\right| H_{C T 2}
$$

where the functions $H_{C_{1}}$ and $H_{C_{T}}$ are equal to

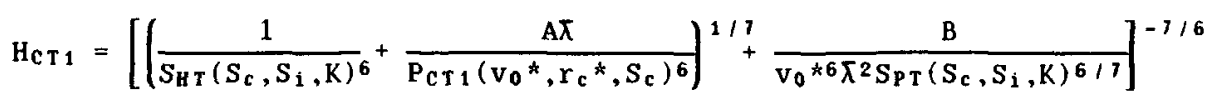

$H_{C T 2}=\left(\frac{C \lambda^{1 / 2}}{V_{0}^{* 3 / 4}}+\frac{D}{\left(E+F V_{0}^{* 2} Y_{C}^{*}\right)^{3} V_{0}^{* 3} \lambda^{4}}\right)^{-1 / 3}$

The functions $\operatorname{PCT}_{1}\left(v_{0}{ }^{*}, r_{c} *, S_{c}\right), S_{H T}\left(S_{c}, S_{i}, K\right)$ and $S_{P T}\left(S_{c}, S_{i}, K\right)$ represent

$$
\begin{aligned}
& \operatorname{P}_{\mathrm{CT} 1}\left(\mathrm{~V}_{0}{ }^{*}, \mathrm{r}_{\mathrm{c}}{ }^{*}, S_{\mathrm{c}}\right)=\frac{\left[1+0.682\left(\mathrm{~S}_{\mathrm{c}}-0.5\right)-0.739\left(\mathrm{~S}_{\mathrm{c}}-0.5\right)^{2}\right]^{1 / 2}}{\left(1+1.40 / \mathrm{v}_{0}^{* 8 / 7}\right)^{1 / 4}\left(1+0.30 \mathrm{r}_{\mathrm{c}}{ }^{*}\right)^{1 / 2}} \quad(16.5 .5) \\
& S_{H T}\left(S_{c}, S_{i}, K\right)=\left|\frac{3 S_{c}-2 S_{i}+3 / 2 K}{\left(1 / K+2 S_{c}\right) 1 / 3}+\frac{3\left(1-S_{c}\right)-2\left(1-S_{i}\right)+3 / 2 K}{\left[1 / K+2\left(1-S_{c}\right)\right]^{1 / 3}}-\frac{3}{K^{2 / 3}}\right| \\
& S_{P T}\left(S_{c}, S_{i}, K\right)=G\left|4-6\left(S_{c}+S_{i}\right)+12 S_{c} S_{i}+H \frac{3-6 S_{c}^{2}-6 S_{i}\left(1-2 S_{c}\right)}{K}\right|
\end{aligned}
$$

In order to obtain a FWHM value, the constant $H_{F W}$ in equation (16.5.1) and the constants $A, B, C$ and $D$ in equations $(16.5 .4)$ should be taken equal to

$$
\mathrm{H}_{\mathrm{PW}}=1 \quad, \mathrm{~A}=58.88, \mathrm{~B}=25.42, \mathrm{C}=.00638, \mathrm{D}=5.41510^{-4} \quad(\mathrm{FWHM}) \quad(16.5 .8)
$$

while for a FWo, $d_{128 B}$ or $d_{1275}$ value, one should use respectively

$H_{F W}=1.2505, A=225.2, B=.1439, C=.0125, D=5.76810-8 \quad\left(F W_{50}\right)$

$\mathrm{H}_{\mathrm{F} W}=1.3836, A=418.2, B=.02458, C=.0170, D=1.75810^{-9} \quad\left(d_{1288}\right)$

$H_{p W}=.72980, A=251.7, B=.1576, C=.0132, D=7.24810^{-8} \quad\left(d_{2575}\right) \quad(16.5 .9)$

The constants $\mathrm{E}$ and $\mathrm{F}$ in the expression for $\mathrm{H}_{\mathrm{Cr}}$, given by equation (16.5.4) and the constants $G$ and $H$ in equation (16.5.7) should for an uniform angular and spatial distribution be taken equal to

$$
E=1, \quad F=.08275, \quad G=1, \quad H=1
$$

Gaussian angular and spatial distributions can best be represented by taking $\alpha_{0}=\sqrt{2} \sigma_{\alpha}$ and $r_{c}=\sqrt{2} \sigma_{r}$ (with $\sigma_{\alpha}{ }^{2}=\left\langle\alpha^{2}>/ 2\right.$ and $\sigma_{r}{ }^{2}=\left\langle r^{2}\right\rangle / 2$ ) and using the following modified values for the constants $E, F, G$ and $H$

$$
\begin{aligned}
& \mathrm{E}_{\mathrm{Bg}}=.7854, \quad \mathrm{~F}_{\mathrm{gB}}=.08971, \quad \mathrm{G}_{\mathrm{Bg}}=1.268, \quad \mathrm{H}_{\mathrm{Bg}}=1 \\
& \mathrm{E}_{\mathrm{Bu}}=.7854, \quad \mathrm{~F}_{\mathrm{Bu}}=.06990, \quad \mathrm{G}_{\mathrm{gu}}=1.268, \quad \mathrm{H}_{\mathrm{Bu}}=.7887 \\
& \mathrm{E}_{\mathrm{u}_{8}}=1, \quad \mathrm{~F}_{\mathrm{u}_{8}}=.1062, \mathrm{G}_{\mathrm{u} 8}=1, \mathrm{H}_{\mathrm{u}}=1.268 \\
& \left(E_{u}=1 \quad, F_{u} u=.08275, G_{u v}=1 \quad, \quad H_{u} u=1\right)
\end{aligned}
$$

in which the first subscript indicates the type of angular distribution and the second subscript the type of spatial distribution (u=uniform, $g=$ Gaussian). 
In order to obtain an effective $\gamma$-value which characterizes the type of distribution, one should evaluate

$\gamma_{\text {eff }}=H_{C T 1}\left[\left(\frac{(3 / 2)^{6}}{S_{H T}\left(S_{C}, S_{i}, K\right)^{6}}+\frac{2^{6} A X}{P_{C T 1}\left(V_{0}^{*}, r_{c}{ }^{*}, S_{c}\right)^{6}}\right)^{1 / 7}+\frac{B / 36 / 7}{V_{0}{ }^{6} \lambda^{2} S_{P T}\left(S_{C}, S_{i}, K\right)^{6 / 7}}\right]^{7 / 6}$

$(16.5 .12)$

where the function $\mathrm{H}_{\mathrm{C} t \mathrm{t}}$ is given by equation (16.5.4). For the constant $A$ and $B$ we use the values for the $F_{50}$ value, specified by the first line of equation (16.5.9). The outcome of equation $(16.5 .12)$ is a value for $\gamma_{\mathrm{ef}} \mathrm{between} 1 / 3$ and 2 .

The angular deflections generated in a homocentric cylindrical beam segment produce a trajectory displacement distribution in the back focal plane of the lens succeeding the beam segment, which has a Full Width (FWHM, FW50, $d_{1288}$ or $d_{2575}$ ) given by

$$
F W_{r}=F W_{\alpha} f=H_{F W} C_{P H A} H_{P A}\left(\lambda^{*}, r_{0}{ }^{*}\right) \frac{I^{2 / 3} L f}{V^{4 / 3} r_{0}^{4 / 3}}
$$

where $f$ is the focal distance of the lens and the constant $C_{P H A}$ is equal to

$$
\mathrm{C}_{\mathrm{PHA}}=0.10177 \frac{\mathrm{m}^{1 / 3}}{\varepsilon_{0}}
$$

For electrons one finds $\mathrm{C}_{\mathrm{PHA}}=1.1142$ in SI-units. The function $\mathrm{HPA}_{\mathrm{PA}}\left(\lambda^{*}, r_{0}{ }^{*}\right)$ represents

$$
\mathrm{H}_{\mathrm{PA}}\left(\lambda^{*}, \mathrm{r}_{0}^{*}\right)=\left[\left(1+\frac{\mathrm{A} \lambda^{*}}{\mathrm{PPA}\left(\mathrm{r}_{0}^{*}\right)^{6}}\right)^{1 / 7}+\frac{\mathrm{B}}{\mathrm{C} \chi_{\mathrm{p}}{ }^{2}}\right]^{-7 / 6}
$$

where $\chi_{p}$ is the pencil beam factor for a cylindrical beam $\left(x_{p}=4 \lambda^{*} r_{0} * 3\right)$ and the function $\mathrm{PPA}_{\mathrm{PA}}\left(\mathrm{r}_{0}{ }^{*}\right)$ is given by

$$
P_{P A}\left(r_{0}{ }^{*}\right)=\left(1+0.185 / r_{0} * 8 / 1\right)-7 / 4
$$

In order to obtain a FWHM value, the constant $\mathrm{H}_{\mathrm{FW}}$ in equation (16.5.13) and the constants $A$ and $B$ in equation (16.5.15) should be taken equal to

$$
\mathrm{H}_{\mathrm{FW}}=1, \quad \mathrm{~A}=1.851, \quad \mathrm{~B}=30.82 \quad \text { (FWHM) }
$$

while for a FW50, $d_{1288}$ or $d_{1275}$ value, one should use respectively

$$
\begin{aligned}
& \mathrm{H}_{\mathrm{FW}}=1.2505, \mathrm{~A}=7.078, \mathrm{~B}=.1745 \quad \text { (FW50) } \\
& H_{F W}=1.3836, \quad A=13.15, \quad B=2.98010^{-2} \quad\left(d_{1288}\right) \\
& H_{P W}=.72980, A=7.914, B=.1910 \quad\left(d_{25}, 5\right)
\end{aligned}
$$

For an uniform current density distribution $\mathrm{C}=1$. For a Gaussian current density distribution one should take $C=1.226$ and $r_{0}=\sqrt{2} \sigma_{r}$, with $\sigma_{\mathrm{r}}{ }^{2}=\left\langle\mathrm{r}^{2}\right\rangle / 2$.

In order to obtain an effective $\gamma$-value which characterizes the type of distribution, one should evaluate

$$
\gamma_{\text {eff }}=H_{P A}\left(\lambda^{*}, r_{0}^{*}\right)\left[\left(\frac{3^{6}}{2^{6}}+\frac{64 A \lambda^{*}}{P_{P A}\left(r_{0}^{*}\right)^{6}}\right)^{1 / 1}+\frac{1}{3^{6 / 7}} \frac{B}{x_{p}^{2}}\right]^{1 / 6}
$$

where the function $\mathrm{HPA}_{\mathrm{PA}}\left(\lambda^{*}, \mathrm{r}_{0}{ }^{*}\right)$ is given by equation $(16.5 .15)$. For the constant $A$ and $B$ we use the values for the $F_{50}$ value, specified by the 
first line of equation (16.5.18). The outcome of equation (16.5.19) is a value for $\gamma_{e f f}$ between $1 / 3$ and 2 .

\subsection{Equations for the space charge effect}

The theory of the space charge effect occurring in beams of low particle density is presented in chapter 11 . We will review the essential results here. For a beam segment with a crossover, the defocussing distance $\Delta z_{f}$ and magnification $M$ of the crossover due to the space charge effect are given by

$$
\begin{aligned}
& \Delta z_{f}=C_{S c} \frac{1}{2 K}\left(K_{1} Z\left(K_{1}\right)+K_{2} Z\left(K_{2}\right)\right) \frac{I L}{\alpha_{0}^{2} V^{3 / 2}} \\
& M=1+C_{S c}\left[R_{1}\left(K_{1}\right)-R_{1}\left(K_{2}\right)\right] \frac{I}{\alpha_{0}^{2} V^{3 / 2}}
\end{aligned}
$$

where the constant $\mathrm{C}_{\mathrm{SC}}$ is equal to

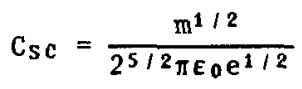

For electrons one finds $\mathrm{C}_{S \mathrm{c}}=1.5154 \times 10^{4}$ in SI-units. The functions $\mathrm{Z}(\mathrm{K})$ and $R_{1}(K)$ are given by

$$
\begin{aligned}
& Z(K)=1+\frac{1}{1+K}-\frac{2}{K} \ln (1+K) \\
& R_{1}(K)=\ln (1+K)-\frac{K}{1+K}
\end{aligned}
$$

The functions $R 1(K)$ and $Z(K)$ are plotted in the figures 11.2 and 11.3 respectively. Equations $(16.6 .1)$ to $(16.6 .5)$ are valid for uniform as well as Gaussian angular and spatial distributions. For the latter one should use $\alpha_{0}=\sqrt{2} \sigma_{\alpha}$ and $r_{c}=\sqrt{2} \sigma_{r}$, where $\sigma_{\alpha}{ }^{2}=\left\langle\alpha^{2}>/ 2\right.$ and $\sigma_{r}{ }^{2}=\left\langle r^{2}>/ 2\right.$.

For non-uniform distributions, the space charge effect will also introduce aberrations. When the current density distribution is Gaussian in every cross section of the beam, the virtual transverse displacement in the crossover $\Delta r_{1}$, caused by the third order aberration of the space charge lens is, for a trajectory with unperturbed coordinates $\alpha$ and $r_{\perp}$, equal to

$$
\frac{\Delta r_{\perp}}{r_{c}}=\frac{1}{2} C_{S c}\left[D\left(\frac{r_{1}}{r_{c}}\right)^{3}+F\left(\frac{r_{\perp}}{r_{c}}\right)^{2} \frac{\alpha}{\alpha_{0}}+C\left(\frac{\alpha}{\alpha_{0}}\right)^{2} \frac{r_{\perp}}{r_{c}}+S\left(\frac{\alpha}{\alpha_{0}}\right)^{3}\right] \frac{I L}{\alpha_{0}^{4} V^{3 / 2}} \quad(16.6 .6)
$$

where the functions $D, F, C$ and $S$ are defined as

$$
\begin{array}{ll}
D=D_{1}\left(K_{1}\right)-D_{1}\left(K_{2}\right), & D_{1}(K)=\frac{3 K^{2}+K^{3}}{12(1+K)^{3}} \\
F=F_{1}\left(K_{1}\right)+F_{1}\left(K_{2}\right), & F_{1}(K)=\frac{K^{3}}{2(1+K)^{3}} \\
C=C_{1}\left(K_{1}\right)-C_{1}\left(K_{2}\right), & C_{1}(K)=\frac{3}{2} \ln (1+K)-\frac{6 K+15 K^{2}+11 K^{3}}{4(1+K)^{3}}
\end{array}
$$




$$
S=S_{1}\left(K_{1}\right)+S_{1}\left(K_{2}\right), \quad S_{1}(K)=\frac{1}{2} K-2 \ln (1+K)+\frac{9 K+21 K^{2}+13 K^{3}}{6(1+K)^{3}}
$$

The functions $D_{1}(K), F_{1}(K), C_{1}(K)$ and $S_{1}(K)$ are plotted in figure 11.4 . For $K \gg>1$ the major contribution stems spherical aberration, described by the term proportional to $\mathrm{S}^{3}$. The corresponding coefficient of spherical aberration $\mathrm{C}_{s}$ can be expressed as

$$
C_{s}=C_{S c} \frac{1}{4 K}\left(S_{1}\left(K_{1}\right)+S_{1}\left(K_{2}\right)\right) \frac{I L}{\alpha_{0}^{4} V^{3 / 2}}
$$

where the constant $\mathrm{C}_{S \mathrm{C}}$ is given by equation $(16.6 .3)$.

The space charge effect generated in a homocentric cylindrical beam segment leads to a defocussing $\Delta z_{f}$ of the crossover formed by the succeeding lens, which is equal to

$$
\Delta z_{f}=C_{s c} \frac{I L f^{2}}{r_{0}^{2} V^{3 / 2}}
$$

where $f$ is the focal distance of the lens and the constant $C_{s c}$ is given by equation (16.6.3). This equation applies to an uniform as well as a Gaussian spatial distribution. For the latter one should use $r_{c}=\sqrt{2} \sigma_{r}$, where $\sigma_{r}^{2}=\left\langle r^{2}\right\rangle / 2$.

Since the beam is homocentric, there is only one third order aberration term. The corresponding aberration in the crossover formed by the succeeding lens is proportional to $C_{s} \alpha^{3}$, where $\alpha$ is the angle of the unperturbed trajectory at the crossover. For a Gaussian current density distribution in the cylindrical beam, the coefficient of spherical aberration $C_{s}$ is equal to

$$
C_{s}=C_{s c} \frac{I L f^{4}}{4 r_{0}^{4} V^{3 / 2}}
$$

where the constant $C_{s}$ is given by equation $(16.6 .3)$.

\subsection{Addition of the effects generated in individual beam segments}

Equation (16.2.15) specifies how to add the FWHM of the distributions of displacements $\rho(\Delta E)$ and $\rho(\Delta r)$ generated in the individual beam segments constituting the beam. Denoting, in general, the FWHM, FW $50, d_{1288}$ or $d_{2515}$ value of the distribution generated in beam segment $j\left(j=1,2, \cdots, N_{b}\right)$ as $F W_{j}$, one can express the summation rule as

$$
F W_{T}=\left(\sum_{j=1}^{N_{b}} F_{j}^{\gamma}\right)^{1 / \gamma}
$$

It presupposes that the individual displacement distributions are uncorrelated and all of the same type, that is described by the same parameter $\gamma$. The corresponding one- and two-dimensional distribution are specified by equation $(16.2 .10)$ and $(16.2 .11)$ respectively.

In section 16.2 it was pointed out that the representation of equations $(16.2 .10)$ and $(16.2 .11)$ is only approximate. The actual distributions can not fully be characterized by a single $\gamma$-value. For all conditions, the distribution will ultimately fall of $f$ exponentially 
for large displacements. Such a fall off corresponds to $\gamma=2$. Accordingly, the core and the tails of the displacement distribution are of ten determined by different $\gamma$-values. The effective parameter $\gamma_{\text {ef }}$, which is for the different distributions defined by equations $(16.4 .11)$, (16.4.19), (16.5.12) and (16.5.19), roughly corresponds to the $\gamma$-value of the distribution at the boundary of the $50 \%$ volume. For the summation rule of equation (16.7.1), we now take $\gamma=\gamma_{e f} f$ for all width measures.

There is still another problem in the application of equation (16.7.1). It presupposes that all beam segments give the same $\gamma_{\text {ef }}$ value. However, it should be anticipated that this is usually not the case. A way to handle this problem is to define a total effective value $\gamma_{e f} f, T$ as a weighted-average of the individual $\gamma_{e f f-v a l u e s}$

$$
\gamma_{\text {eff }, T}=\frac{\sum_{j=1}^{N_{b}} F W_{50, j} \gamma_{e f f, j}}{\sum_{j=1}^{N_{b}} F W_{50, j}}
$$

where FW5o,j and $\gamma_{e f f, j}$ are the Full width median and the effective $\gamma$-value for beam segment $j$ respectively. The summation can now be performed with equation (16.7.1), taking $\gamma=\gamma_{\text {eff.T. A somewhat more }}$ sophiscated approach is to subdivided the full $\gamma$-range into a number of intervals and perform the summation in two steps. In the first step, one adds those $\mathrm{FW}_{j}$ with a $\gamma_{\mathrm{ef}} \mathrm{f}, j$ which are in the same $\gamma$-interval, using equation (16.7.1), with $\gamma$ equal to the midpoint of the $\gamma$-interval. In the second step, the resulting FW values for each $\gamma$-interval are used to compute $\gamma_{\text {eff,T }}$ from equation (16.7.2) and summed next by means of equation $(16.7 .1)$, taking $\gamma=\gamma$ ef, $\mathrm{t}$.

For the trajectory displacement effect as well as the space charge effect one has to take the transverse magnification $M_{j}$ of the image plane in segment $\mathrm{j}$ to the target into account. The $\mathrm{FW}_{\mathrm{j}}$ trajectory displacement values used in equations (16.7.1) and $(16.7 .2)$ should all refer to the target plane. Thus the quantities $\mathrm{FW}_{j}$ are assumed to include a factor $M_{j}$. The space charge defocussing distances $\Delta z_{j}$ should be added as

$$
\Delta z_{T}=\sum_{j=1}^{N_{b}} M_{j}{ }^{2} \Delta z_{j}
$$

using that the axial magnification is the square of the transverse magnification. The third-order space charge aberrations $\Delta r_{1}, j$, given by equation $(16.6 .6)$, should be multiplied with $M_{j}$, as for the trajectory displacement effect. When adding the coefficients of spherical aberration $C_{s, j}$ one should account for the (de)magnification of the beam semi-angle $\left(\alpha_{0}, j=M_{j} \alpha_{0}, r\right)$. This gives

$$
C_{s T}=\sum_{j=1}^{N_{b}} M_{j}{ }^{4} C_{s, j}
$$

similar to summation of the spherical aberration of the electrostatic and magnetic lenses in the column. 
Aarseth,S.J. (1972). In "Gravitational N-Body Problem", p.373 Proc. IAU Coll. 10. (M.Lecar, ed.), D. Reidel Publ. Comp. Dordrecht-Hol land Abramowitz,M. and Stegun,S. (1965). "Handbook of Mathematical Functions" Dover Publ., Inc., New York

Allee,D.R., Pehoushek,J.D. and Pease,R.F.W. (1988). J.Vac. Sci. Technol. to be published

Alles,D.S. and Thomson,M.G.R. (1987). In "VLSI Electronics

Microstructure Science", Vol. 16,p.57, Academic Press, New York

Andersen,W.H.J. (1967). Brit. J.Appl. Phys. 18,1573

Andersen,W.H.J. and Mol,A. (1968). Proc. European Reg. Conf. Electron Microsc., 4th,p.339.

Andretta,M., Marini,M. and Zanarini,G. (1986). IEEE Transactions on Electron Devices ED-33,1084

Ash,E.A. and Gabor,D. (1954). Proc. Roy. Soc. (London) A228,477

Beck,A.H. (1973). Int. J. Electronincs 36,121

Bell,A.E. and Swanson L.W. (1979). Physical Review B 19,3353

Boersch,H. (1954). Zeitschr. Phys. 139,115

Bohm,D. and Pines,D. (1951). Physical Review 82,625

Borries, D. von, Dosse,J. (1938). Arch. Electrotech. 32,221

Brody, I. et al. (1981). IEEE Trans. Electron Dev., Vol. ED-28,11,p.1422

Brody,I. (1987). Int. J. Electronics, Vol.62,1,p.1

Broek, M.H.L.M. van den (1984). Optik 47,69

Broek,M.H.L.M. van den (1986a). J. Appl. Phys. 59,3923

Broek,M.H.L.M. van den (1986b). Ph.D. Thesis Delft University of Technol.

Broers,A.N. (1981). J. Electrochem. Soc. Solid. State. Sci. Technol. $128, \mathrm{p} .166$

Broers,A.N. (1981). IEEE Trans. ED-28,p.1268

Busch, H.(1926). Zeitschr. Phys. 81,974

Chambost,E. De and Hennion,C. (1979). Optik 55,357

Chambost,E. De (1982). Optik 62,189

Chambost,E. De, Frichet,A., Chartier,M. Ta The,H. and Trotel J. (1986). J. Vac. Sci. Technol. B 4,78

Chandrasekhar,S. (1941). Astrophys. J. 94,511

Chandrasekhar,S. (1942a). "Principles of Stellar Dynamics", University of Chicago Press.

Chandrasekhar,S. and Neumann, J. von, (1942b). Astrophys. J. 95,489

Chandrasekhar,S. and Neumann, J. von, (1943). Astrophys. J. 97,1

Chandrasekhar,S. (1943). Rev. Modern Phys. 15,1

Chang, T.H.P., Wilson,A.D., Speth,A.J. and Ting,C.H. (1976). Proc.

Electron Ion Sci. Technol. 7th Int. Conf.,p.392

Chapman,S. and Cowling,T.G. (1970). "The Mathematical Theory of NonUniform Gases",p.359 Cambridge University Press.

Chu,H.C. and Munro,E. (1982). Optik 61,121

Contopoulos,G. (1972). In "Gravitational N-Body Problem", p.169 Proc.IAU

Col1.10. (M.Lecar, ed.), D. Reidel Publ. Comp. Dordrecht-Holland

Cottrel1, G.A. (1981). Rev. Sci. Instrum. 52,1174

Crewe,A.v. (1978a). Optik 50,205

Crewe,A.V. (1978b). Optik 52,337

Cummings, K.D., Harriot,L.R., Chi,G.C. and Ostermayer,F.W. (1986) SPIE $632, \mathrm{p} .93$

Davis, D.E., Gillespie,S.J., Silverman, S.L. and Stickel, W. (1983). J. Vac. Sci. Technol. B. B 1 (4), p.1003

Dayan,P.S. and Jones,G.A.C. (1981). J. Vac. Sci. Technol. 19,1094

Debeye,P. and Hückel,E. (1923). Zeitschr. Physik. 24,185

Degenhardt,V. and Koops,H. (1982). Optik 61,395

Dietrich,w. (1958). Zeitschr. Phys, 152,306

Ditchfield,R.W. and Whelan,M.J. (1977). Optik 48,163 
Doran, S., Perkins,M. and Stickel W. (1975). J. Vac. Sci. Technol., Vol.12, 6, p.1174

El-Kareh,A.B. and El-Kareh,J.C.J. (1970). "Electron beams, Lenses and Optics",p.250, Academic Press, New York.

E1-Kareh,A.B, and Smither,M.A. (1979), J. Appl. Phys, 50,5596

Eng, G. (1985). J. Appl. Phys. 58 (11), p.4365

Epstein, B. (1958). Compt. Rend. 246,586

Erdélyi,A., Magnus,W., oberhettinger,F. and Tricomi,F.G. (1954), Tables of integral transforms (Cal. Inst. Technol. Bateman manuscript project), McGraw-Hill Book Company Inc., New York

Fack,H. (1955). Physikalischer. Verh, 6,6

Feller,J. (1966). "An Introduction to Probability Theory and its Applications" Vol 2,p165 J. Wiley \& Sons Inc., New York

Fischer, M. (1970). J. App 1. Phys. 41,3615

Fowler, R. D. and Gibson , G.E. (1934). Phys. Rev. 46, 1075.

Franzen, W. and Porter, J.H. (1975). In "Adv. Electronics Electron Phys." (L. Marton, ed.), Vol. 39, p.73, Academic Press, New York.

Fujinami, M., Shimazu,N. Hosokawa,T. and Shibayama,A. (1987). J. Vac. Sci. Technol. B 5(1), p.61

Furukawa,Y. (1986). J. Vac. Sci. Technol. A 4,1908

Gadzuk,W. and Plummer,E.w. (1973). Rev. Modern Phys. 45,487

Gaukler,K,H., Speidel,R. and Vorster,F. (1975). Optik 42,391

Gesley M.A. and Swanson L.W. (1984). J. de Physique C9 45,167

Gilbert, H. (1972). In "Gravitational N-Body Problem", p.5 Proc. IAU Coll. 10. (M.Lecar, ed.), D. Reidel Publ. Comp. Dordrecht-Holl land

Glaser,W. (1952). "Grundlagen der Elektronenoptik", p.66, SpringerVerlag, Vienna.

Goldstein (1980). "Classical mechanics", 2nd ed., Addison-Wesley, Reading, Massachusetts, USA.

Gorkum, van G.G.P. and Hoeberechts, A.M.E. (1986), J. Vac. Sci. Technol. B, 4, p.108

Goto,E. and Soma,T. (1977). Optik 3,p.255

Gradshteyn, I.S. and Ryzhik,I.M. (1980). "Table of Integrals Series and Products", Academic Press, New York

Grivet,P. (1965). "Electron Optics",p.277 Pergamon Press, Oxford

Grivet,P. (1971). "Electron Optics",2nd ed., Pergamon Press, Oxford

Groves,T., Hammond, D.L. and Kuo,H. (1979). J. Vac. Sci. Technol. 16,1680

Groves, T. (1981a). J. Vac. Sci. Technol. 19,110.

Groves,T. (1981b). J. Vac. Sci. Technol. 19,1106.

Groves, T. (1984). Private Communications

Gryzinski,M. (1964a). Physical Review 138,A305

Gryzinski,M. (1964b). Physical Review 138,A322

Gryzinski,M. (1964c). Physical Review 138,A336

Haberstroh,G. (1956). Zeitschr. Phys, 145,20

Haef, A.V. (1939). Proc. Inst. Radio Engrs, 27,586

Hamaguchi,S. Kai,J. and Yasuda,H. (1988). J. Vac. Sci. Techno1. B 6(1), p. 204

Hamish,H., Loeffler,K.H. and Kaiser,H.J. (1964). Proc. European Reg. Conf. Electron Microsc., 3th,p.11

Hanson,G.R. and Siegel,B.M. (1979). J. vac. sci. Technol. 16,1875

Hanszen,K.J. and Lauer,R. (1967). Zeitschr. Naturforsch. 22a,238

Hanszen, K.J. and Lauer,R. (1969). Zeitschr. Naturforsch. 24a, 214

Haeringen, H. van (1985). "Charged Particle Interactions", Coulomb Press Leyden, Leiden, The nether lands

Hart1,W.A.M. (1966). Zeitschr. Phys, 191,33

Hartwig, D. and Ulmer,K. (1963a). Zeitschr. Angew. Phys. 15,309

Hartwig,D. and Ulmer,K. (1963b). Zeitschr. Phys. 173,294

Hasse 1 Shearer,M., Takemura,H., Isobe,M., Goto,N.. Tanaka,K., Miyauchi,S. (1986). J.Vac.Sci.Technol. B 4(1),p.64 
Hauke, R. (1977). Ph.D. thesis, University of Tubingen

Heinrich,H., Essig,M. and Geiger,J. (1977). J. Appl. Phys. 12,197

Herriot,D.R. (1982). J. Vac. Sci. Technol, 20(3),p.781

Hertz,P. (1909). Math. Annalen 67,387

Hines, M.E. (1951). J. App1. Phys. 22,1385

Hoeberechts, A.M.E. and Gorkum, van G.G.P. (1986). J. Vac. Sci. Technol. B, 4,p.105

Holtsmark, J. (1919). Annalen Phys. 58,38

Hosokawa,T. and Morita,H. (1983). J. Vac. Sci. Technol, B 1(4), p.1293

Hutter, R. (1967). In "Focussing of Charged Particles" (A. Septier, ed.), Vol. 2, p.3, Academic Press, New York

Ichimaru,S. (1973). "Basic Principles of Plasma Physics", W.A. Benjamin Inc., London

Ichinokawa,T. (1968), Jap. J. App l. Phys. 7,799

Ichinokawa, T. (1969). Jap. J. Appl. Phys. 8,137

Ishitani,T., Umemura,K., Hosoki,S. Takayama,S and Tamura,H. (1984).J. Vac. Sci. Technol. A 2(3), p.1365

Ishitani,T., Kawanami,Y. and Shukuri, S. (1987a). Japanese J. Appl. Phys., Vol.26,10,0.1777

Ishitani,T., Umemura,K. and Kawanami,Y. (1987b). J. Appl. Phys. $61(2), p .748$

Ishitani,T., Kawanami,Y., Oshinishi,T. and Umemura,K. (1987c). App1. Phys. A. 44,p233

Ishitani,T., Umemura,K. and Aida,T. (1987d). J. Vac. Sci. Technol. A 5(5),P.2907

Jansen,G.H., Leeuwen,J.M.J van, and Mast,K.D. van der (1983). Proc. Microcirc. Eng. p.99

Jansen,G.H. and Stickel,W. (1984). Proc. Microcirc. Eng. p.167

Jansen,G.H., Groves,T.R. and Stickel,W. (1985). J. Vac Sci. Technol. B 3,190

Jansen,G.H. (1987). J. Vac Sci. Technol. B 5,146

Jansen,G.H. (1988a). J. Vac Sci. Technol. B, to be published.

Jansen,G.H. (1988b). Reference Manual MC-simulation program, Delft University of Technology

Jansen,G.H. (1988c). Reference Manual INTERAC program, Delft University of Technology

Jones, G.A.C., Rao, V.R.M., Sun, H.T. and Ahmed, H. (1983). J. Vac. SCi. Technol. B 1, 1298.

Jones,G.A.C., Sargent,P.M., Norris,T.S. and Ahmed,H. (1985). J. Vac.Sci. Technol. B 3(1),p.124

Kampen, N.G. van, (1981). "Stochastic Processes in Physics and Chemistry", North-Holland Publ. Comp., Amsterdam.

Kasper E. (1982). In "Adv. Optical Electron Microsc." (R.Barer and V.E. Cosslett, ed.), Vol. 8, p.207, Academic Press, New York.

Katsuhiro,K., Ozasa,S. and Komoda T. (1983). J. Vac. SCi. Technol. B $1(4), p .1303$

Kelly,J., Groves,T. and Kuo,H.P. (1981). J. Vac, Sci. Technol. 19,936

Kirstein, P.T., Kino,G.S., and Walters, W.E. (1967). "Space Charge "Flow" McGraw-Hil 1, New York

Kittel,C.(1958). "Elementary Statistical Mechanics", J.Wiley\&Sons,Inc., New York

Klemperer,0. (1953). "Electron 0ptics", Cambridge University Press.

Klemperer,0. and Barnett,M.E. (1971). "Electron Optics", 3rd edn., Cambridge University Press.

Knauer,W. (1979a). Optik 54,211

Knauer,W.(1979b). J. Vac. Sci. Technol. 16,1676

Knauer,W. (1981). Optik 59,335

Knuth, D.E. (1969). "The Art of Computer Programming", Vol. 2 Addison-Wesley company, Reading, Massachusetts 
Knuth, D.E. (1973). "The Art of Computer Programming", Vol. 3 Addison-Wesley company, Reading, Massachusetts

Komuro,M., Kanayama,T., Hiroshima,H. and Tanoue,H. (1983). Appl. Phys. Lett. 42,908

Krohn,V.E. and Ringo,G.R. (1975). Appl. Phys. Lett. 27,249

Kuroda,K. (1984), J.Vac.Sci.Technol. a 2(1),p.68

Langmuir, D.B. (1937). Proc. Inst. Radio Engrs. 25,977

Lauer, R. (1982). In "Adv. Optical Electron Microsc." (R.Barer and V.E. Cosslett, ed.), Vol. 8, p.137, Academic Press, New York.

Lawson, J.D. (1977) "The Physics of Charged-Particle Beams", Clarendon Press, Oxford.

Leeuwen, J.M.J. van, and Jansen,G.H. (1983). Optik 65,179

Le jeune,C. and Aubert,J. (1980). In "Applied Charged Particle Optics" (A. Septier, ed.), Part A,p.159 Academic Press, New York

Lenz,F. (1958). Proc. Int. Conf. Electron Microsc, 4th,p.39

Lindsay,P.A. (1960). In "Adv. Electronics Electron Phys." Vol. 13, p.181

Livesay,W.R., Greeneich,J.S., Wolfe,J.E. and Felker,R.J. (1983). Solid State Technol., 9-83,p.137

Loeff ler K.H. (1963). Physikalischer Verh. 3,104

Loeffler K.H. (1964). Ph.D. Thesis, University of Berlin

Loeffler K.H, (1969). Zeitschr. Angew. Phys, 27,145

Loeffler K.H. and Hudgin, R.H. (1970). Proc. Int. Conf. Electron Microsc. 7 th, p. 67

Mair, G.L.R. and Mulvey,T. (1985). Proc. Microcirc.Eng. p. 133

Mayer,H.P. (1985). Appl. Phys. Lett. 47,1247

Markoff, A.A. (1912). "Wahrscheinlichkeitsrechung", Leipzig

Mast,K.D. van der, Jansen G.H. and Barth J.E.. (1985). Proc. Microcirc. Eng. p.43.

Mast,K.D. van der and Jansen G.H. (1987). Proc. Microcirc. Eng. p.93

Massey,G.A., Jones, M.D. and Plummer,B.P. (1981). J. Appl. Phys, 52,3780

McClelland,J.J., Ratliff,J.M. and Fink,M. (1981). J. Appl. Phys. 52,7039

McGregor-Morris, J.T. and Mines, R. (1925). J. Instn. Elect. Engrs., 63, 1065 .

Meisburger, W.D. (1983). Private Communications.

Melngailis, J. (1987). J. Vac. Sci. Technol. b 5 (2), p.469

Meyer,W.e. (1958). Optik 15,398

Mihran, T.G. (1966). J. Appl. Phys. 38,159

Miller,M.H. and Dow W.G. (1961). J. Appl. Phys 32,274

Moore,R.D., Caccoma,G.A., Pfeiffer,H.C., Weber,E.V. and woodard,0.C. (1981). J. Vac. Sci. Technol. 19 (4), p.950

Moriya,S., Komatsu,K., Harada,K. and Kitayama,T. (1983). J. Vac. Sci. Techno1. B 1(4), p.990

Morita, H., Hosokawa,T. and Fujinama,M. (1985). Proc. Microcirc. Eng. 3, p. 53

Mory, C. (1985). These d'Etat, Orsay (1985).

Mory, C., Colliex C. and Cowley, J.M. (1987). Ultramicroscopy 21,171

Mott-Smith,H.M. (1952). J. Appl. Phys. 24,249

Munro, E. (1987). Nucl. Instm. Meth. Phys. Res. A258, p. 443.

Murata,K. and Kyser,D.F. (1987). In "Adv. Electronics Electron Phys.", Vol. 69,p.175, Academic Press., New York.

Newton, I (1687). "Philosophiae Naturalis Principia Mathematica". Reprint of the third edition (1726) (editor A. Koyre and I.B.

Cohen), Harvard Univ. Press 1972

Nagy, G.A. and Szilagyi,M. (1974). "Introduction to the Theory of Space Charge Optics", Macmillan Press LTD, London

Namkung,W. and Chon jnacki,E.P. (1986). Rev. Sci. Instrum. 57,341

Narum,D.H, and Pease,R.F.W. (1986). J. Vac. Sci. Technol. B 4(1),p.154

Norris, T.S., Jones, G.A.C., Ahmed, H. (1987). Proc. Microcirc. Eng. p. 99 
Nottingham,W.B. (1956). In "Handbuck der Physik" (s.Flugge, ed.), Vol XXI,p.1, Springer-Verlag, Berlin

Orl off,J. (1985). J. Microsc. 140,303

Parzen,P. and Goldstein L. (1950). J. Appl. Phys. 22,398

Petric,P. and Woodard,0. (1983). Solid State Technol., 9-83,p.154

Pfeiffer,H.C. and Loeffler,K.H. (1970). Proc. Int. Conf, Electron Microsc. 17 th, p.63

Pfeiffer,H.C. (1971). Rec. Symp. Electron, Ion, Laser Beam Technol. 11 th, p. 239

Pfeiffer,H.C. (1972). Proc. Scanning Electron Microsc. 5th,p.113

Pfeiffer,H.C. (1979). Recent advances in electron beam lithography for the high volume production of VLSI devices, IEEE Trans. Electron Dev., Vol. ED-26, no 4, p.663

Pfeiffer,H.C. (1984). Solid State Technol., 9-84, p223.

Pierce,J.R. (1954). "Theory and Design of Electron Beams", D. van Nostrand Comp. Inc, New York.

Pines,D. and Bohm,D. (1952). Physical Review 85,338

Pipes, L.A. and Harvill, L.R. (1983). "Applied mathematics for engineers and physisists", $9_{\text {th }}$ printing. McGraw-Hill, New York

Piwczyk,B.P. and Williams,A.E. (1983). Solid State Technol., 9-83,p.145

Press, W.H., Flannery, B.P., Teukolsky, S.A., Vetterling, W.T. (1986). "Numerical Recipes", Cambridge University Press, Cambridge.

Prewett,P.D. and Kellogg,E.M. (1985). Nuclear Instrum. Meth. Phys. Research B 6,135

Rehmet,M. (1965). Ph.D. thesis University of Berlin.

Reimer,L. (1984). "Transmission Electron Microscopy", Springer-Verlag, Berlin.

Rose,H. and Spehr,R. (1980). Optik 57,339

Rose,H. and Spehr,R. (1983). In "Adv. Electronics Electron Phys.", Supp. 13c,p. 475, Academic Press., New York.

Saitou,N., Ozasa,S., Komoda,T. Tatsuno,G. and Uno,Y. (1981). J. Vac. Sci. Technol. 19(4), p.1087

Saitou,N., Okumura,M., Matsuoka,G., Matsuzaka,T., Komoda,T. and Sakitani,Y. (1985). J. Vac. Sci. Technol. B 3(1),p.98

Saitou,N., Hosoki,S., Okumura,M., Matsuzaka,T.,Matsuoka,G. and Ohyama,M. (1986). Proc. Microcirc. Eng, 5,p.123.

Saitou,N., Okazaki,S., Murai,F. Ozasa,S. and Konishi,T. (1986). J.Vac. Sci. Technol. B 4(1), p.265

Saitou,N., Okazaki,S. and Nakamura,K. (1987). Solid State Technol, 87$11, p, 65$

Sasaki,T. (1979). Conf. VLS1: Architect., Design, Fabr. Cal.Tech.

Sasaki,T. (1982). J. Vac. Sci. Technol. 21,695

Sasaki,T. (1984). J. Vac. Sci. Technol. A 2,1352

Sasaki,T. (1986). J. Vac. Sci. Technol, B 4,135

Schiske,P. (1961). Physikalischer Verh, 12,143

Schiske,P. (1962). Proc. Int. Conf. Electron Microsc. 5th,p.KK-9

Schwartz,J.W. (1957). RCA Review 18,3

Seliger,R.L., Ward,J.W., Wang,V, and Kubena,R.L. (1978). Appl. Phys. Lett. 34,310

Septier, A. (1967). "Focussing of Charged Particles", Vol. I and II, Academic Press, Inc., New York

Simpson,J.A. and Kuyatt,C.E. (1966) J. Appl. Phys, 37,3805

Sivukhin,D.v. (1966). In "Reviews of Plasma Physics", Vol.4,p.93, (M.A. Leontovich, ed.) Consultants Bureau Enterprises, Inc., New York

Smith, L.P. and Hartman, P.L. (1940). J. Apply. Ohys, 11,220

Spehr,R. and Rose,H. (1979) Annual EMSA Meeting 37th,p.570

Spehr,R. (1985a). Optik 70,100

Spehr,R. (1985b). Proc. Microcirc, Eng. p.61 
Speidel,R. and Gaukler,K.H. (1968). Zeitschr. Phys. 208,419

Speidel,R., Kurz,D. and Gaukler,K.H. (1979). Optik 54,257

Speidel,R., Brauchle,P., Kramer,B. and Schwab,U. (1985). Optik 71,167

Spizer,L. (1962). "Physics of Fully Ionized Gases", J. Wiley \& Sons, New York

Steffen,K.G. (1965). "High Energy Beam Optics", J.Wiley \& Sons, New York Stickel, W. and Pfeiffer, H.C. (1978). Proc. Symp. Electron, Ion Beam Sci. Techno1. p.32

Stickel,W. and Langner,G.0. (1983), J. Vac. SCi. Technol. B 1(4),p.1007

Stumpff, K. (1959), "Himmelsmechanik", Band I, VEB Deutscher Verlag der Wissenschaften, Berlin.

Swanson,L.W. (1975). J. Vac. Sci. Technol. 12,1228

Swanson,L.W., Schwind,G,A., Bell,A.E. and Brady,J.E. (1979).'J. Vac. Sci. Technol. 16,1864

Swanson,L.W., Schwind,G.A. and Bell,A.E. (1980). J. Appl. Phys. 51,3453

Szilagyi (1988),M. (1988). "Electron and Ion Optics", Plenum Press, New York

Takaoka,A. and Ura,K. (1986). Optik 74,71

Tang, T.T. (1983). Optik 64,237

Thompson, B.J. and Headrick, L.B. (1940). Proc. Inst. Radio Engrs., 28, 319.

Troyon,M. (1976). Optik 46,439

Troyon,M. and Zinzindohoue,P. (1986). Proc. Int. Conf. Electron Microsc. 11 th, 27

Troyon,M. (1987). J. Microsc. Spectrosc. Electr., Vol.12, p.431

Troyon,M. (1988). J. Microsc. Spectrosc. Electr., Vol.13, p.49

Trubnikov,B.A. (1965). In "Reviews of Plasma Physics", Vol.1,p.105, (M.A. Leontovich, ed.) Consultants Bureau Enterprises, Inc., New York

Tscharnuter, W. (1972). In "Gravitational N-Body Problem", Proc. IAU Coll. 10. (M.Lecar, ed.), D. Reidel Publ. Comp. Dordrecht-Holland, p. 10

Tuggle,D.W. and Swanson L.W. (1985). J. Microsc., 140,293

Tuggle,D.W., Swanson L.W. and Gesley,M.A. (1986). J. Vac. Sci. Technol. B 4,131

Ulmer,K. and Zimmermann B. (1964). Zeitschr. Phys. 182,194

Umemura,K., Ishitani,T and Tamura,H. (1986). Japanese J. Appl. Phys. Vol.25, 11, p.L885

Varnell,G.L., Spicer,D.F., Hebley,J., Robbins,R. Carpenter,C. and Malone,M. (1979). J. Vac. Sci. Technol. 16(6),p.1787

Veith, w. (1955). Zeitschr. Phys. 152,306

Venables,J.A. and Janssen,A.P. (1980). Ultramicrosc. 5,297

Venables,J.A, and Cox,G. (1987). Ultramicrosc. 21, 33

Veneklasen, L.H, (1985). J. Vac. Sci. Technol. B 3,185

Ward, J.W. (1984). J.Vac. Sci. Technol.B 3,207

Ward, J.W., Utlaut M.W. and Kubena,R.L. (1987). J.Vac. Sci, Technol.B 5,169

Ward, J.W., Utlaut M.W. and Kubena,R.L. (1988). J.Vac. Sci. Technol.B to be published

Watson,E.E. (1927). Phil. Mag. 3,849

Watson,K.W. (1955). Physical Review 102,12

Weidenhausen,A., Spehr,R. and Rose,H. (1984). Optik 69,126

Wendt, G. (1942). Zeitschr. Phys. 119,423

Wendt, G. (1943). Zeitschr. Phys. 120,720

Wendt, G. (1948). Annalen. Phys. 2,256

Wilson,A.D. (1983). Proc. IEEE, Vol.71, 5, p.575

Wolf,D.A. (1985a). J. Appl. Phys, 58,3692

Wolf,D.A. (1985b). J. Appl. Phys. 58,3697

Wolf.E.D. (1983). Proc. IEEE, Vol.71 5, p.589 
Yau,Y.W., Pease,R.F.W. and Groves,T.R. (1983). J. Vac. Sci. Technol. B $1, \mathrm{p} .1141$

Zimmermann,B. (1968). Ph.D. Thesis, University Karlsruhe

Zimmermann,B. (1969). Rec. Symp. Electron, Ion, Laser Beam Technol. 10th, p. 297

Zimmermann,B. (1970). In "Adv. Electronics Electron Phys." Vol 29, p.257, Academic Press, New York.

Zinzindohoue, $P$. and Troyon,M. (1986a). Proc. Int. Conf. Electron Microsc. 11th, p.271

Zinzindohoue,P. (1986b). Optik 74,131

Zvorykin,V.K., Morton,G.A., Ramberg,E.G., Hillier,J. and Vance,A.W.

(1961). "Electron Optics and the Electron Microscope", Sec. 16.9, J. Wiley \& Sons, Inc., New York 
Dit proefschrift geeft een theoretische beschrijuing van de Coulomb wisselwerking tussen identieke geladen deelt jes (electronen of ionen) in een gefocuseerde bundel. Zowel het ruimteladings effect als de verschillende statistische wisselwerkings effecten, bekend als het Boersch effect en het "trajectory displacement effect" " worden behandeld. Ter introductie wordt er een literatuur overzicht gegeven. Daaruit blijkt dat er grote verschillen van inzicht bestaan in de vereiste theoretische aanpak. Vervolgens worden de methoden onderzocht die men in de plasma fysica en de stellaire dynamica gebruikt om vergelijkbare problemen te bestuderen. Deze blijken slechts voor bepaalde extreme condities op deeltjes bundels toepasbaar. De uiteindelijk in dit onderzoek gekozen aanpak is tweevoudig. Enerzijds wordt er gebruik gemaakt van een semi-analytisch model, waarin de statistische en dynamische aspecten van het $\mathrm{N}$-deeltjes probleem worden gereduceerd tot een twee-deeltjes probleem. Dit model resulteert in een aantal expliciete vergelijkingen in de experimentele parameters, waarmee de invloed van de wisselwerkingen op macroscopische eigenschappen van de bundel direct bepaald kan worden. Anderzijds is er gebruik gemaakt van een puur numeriek Monte Carlo model, waarin de bewegingsvergelijkingen van een ensemble wisselwerkende deeltjes met "random" gekozen startconditie exact worden opgelost. Dit model leidt niet tot algemene uitdrukkingen, maar geeft een specifieke numerieke voorspelling voor elke gesimuleerde experimentele situatie. De resultaten van beide modellen blijken goed met elkaar overeen te stemmen. Dit levert een consistente theorie op, die de bestaande kennis van de deeltjes optica aanvult en het mogelijk maakt om systemen te beschrijven waarin de wisselwerking tussen de deeltjes niet verwaarloosd mag worden. De voorspellingen van deze theorie zijn kwalitatief en kwantitatief vergeleken met die van enkele andere, recent in de literatuur gerapporteerde, modellen.

Een probe-vormend deeltjes optisch apparaat bestaat uit een bron, warin geladen deeltjes worden vrijgemaakt en versneld en een afbeeldend systeem, opgebouwd uit electrostatische en/of magnetische lenzen. In dit systeem zijn gewoonlijk één of meer diafragma's aangebracht om de bundel te begrenzen. Nabij de laatste lens bevinden zich electrostatische of magnetische afbuigers om de probe (of spot) te kunnen rasteren (of "scannen") over het beeldveld. Voorbeelden van zulke apparaten zijn de Scanning Electronen Microscoop (SEM) en de electronen lithografische machines die in de halfgeleiderindustrie gebruikt worden om patronen te genereren voor de productie van "integrated circuits".

De standaard theorie van de deeltjes optica, zoals die zich van af het midden van de twintiger jaren ontwikkeld heeft, houdt zich voornamelijk bezig met de berekening van de afbeeldende eigenschappen van electrostatische en magnetische velden. De theorie concentreert zich daarbij op de berekening van de banen van geladen deeltjes in een extern veld en verwaarloost de wisselwerking tussen de deeltjes onderling. Dit resulteert in een beschrijuing die nauw verwant is aan de geometrische optica, gebruikt voor licht-optische systemen. De analogie met de lichtoptica houdt evenwel geen stand wanneer de wisselwerking tussen de geladen deeltjes een significante invloed krijgt op de macroscopische eigenschappen van de bundel.

De verschijnselen, die veroorzaakt worden door de coulomb wisselwerking tussen de deeltjes onderling, worden in twee categorieën onderscheiden, namelijk ruimteladings en statistische effecten. Deze 
begrippen kunnen het best worden verklaard door een verzameling ruimtelijk gescheiden test-deeltjes te beschouwen, die langs een bepaalde baan in de bundel bewegen. Deze baan wordt aangeduid als de referentie baan. Elk test-deeltje ondervindt de wisselwerking met de deeltjes die hem omringen, aangeduid als veld-deeltjes. Door deze wisselwerking ondergaat het test-deeltje een bepaalde afwijking van de referentie baan ( $z i j n$ ongestoorde baan), welke volledig bepaald is door de initiele configuratie van de veld-deeltjes ten opzichte van het testdeeltje. Door de stochastische eigenschappen van de bundel verschilt deze configuratie per test-deeltje en zijn de afwijkingen van een verzameling test-deeltjes zodoende statistisch verdeeld. Het kan worden bewezen dat de gemiddelde verandering, in de positie en de snelheid, van een groot aantal test-deeltjes, bepaald is door de gemiddelde (of uitgesmeerde) lading in de bundel. Deze grootheden worden daarom geclassificeerd als manifestaties van het ruimteladingseffect. De gegenereerde spreiding in de posities en de snelheden houdt verband met de statistische fluctuaties in de ruimteladingsdichtheid, die optreden als gevolg van het deeltjes-karakter van de bundel. Deze grootheden worden daarom geclassificeerd als statistische effecten.

De statistische effecten worden voorts onderscheiden in het Boersch effect en het "trajectory displacement effect". Het Boersch effect correspondeert met de gegenereerde spreiding in de axiale component van de snelheden, welke uitgedrukt kan worden als een energiespreiding. Het beinvloedt de afmetingen van de spot via de chromatische aberraties van de lenzen en de afbuigers in het systeem. Het trajectory displacement effect correspondeert met de gegenereerde spreiding in de transversale component van de posities en de snelheden, welke een directe verbreding van de spot veroorzaken.

Dat de ruimtelading een significante invloed kan hebben op de eigenschappen van de bundel, werd al onderkend in de eerste dagen van de deelt jes optica. Men begreep dat dit effect, bij hoge bundel stromen, de minimaal te bereiken spotgrootte in oscilloscoop buizen beperkt en dat het ook een dominerende rol kan spelen bij de emissie in . thermische electronenbronnen. De toename van de energie spreiding in bundels met een hoge stroomdichtheid, die heden ten dagen aan de statistische wisselwerkingen wordt toegeschreven, werd het eerst door Boersch (1954) systematisch onderzocht. Dit effect draagt daarom zijn naam. De invloed van de statistische effecten op de transversale eigenschappen van de bundel (trajectory displacement effect) werd het eerst vastgesteld door Loeffler (1964). Het onderzoek aan deze verschijnselen werd sterk gestimuleerd door de opkomst van de electronen lithografie in de zeventiger jaren, welke in de halfgeleider technologie gebruikt wordt om patronen te genereren voor de vervaardiging van "Very Large Scale Integrated (VLSI) circuits". Ook in de laagspannings SEM's, die in opkomst zijn voor de inspectie en het testen van electronische circuits, spelen statistische effecten een belangrijke rol, daar deze effecten sterk toenemen met een afname van de versnelspanning. Een goede theorie voor de beschrijuing van alle manifestaties van de deeltjes wisselwerkingen is essentieel om tot een optimaal ontwerp van dit soort apparatuur te komen. Dit verklaart de aanzienlijke stroom van publicaties, gewijd aan de berekening van deze effecten. Tot op heden is er evenwel geen concensus bereikt over de vereiste theoretische aanpak en de verschillende modellen leveren voorspellingen op die zowel kwantitatief als kwalitatief sterk verschillen.

Dit proefschrift beoogt enige helderheid te scheppen in dit gebied en tevens tot een consistente beschrijving te komen, die een nauwkeurige voorspelling van de verschillende wisselwerkings effecten mogelijk makk, voor praktische systemen. In hoofdstuk 2 . wordt een 
historisch overzicht gegeven van het theoretisch en experimenteel werk dat verricht is aan het Boersch effect, het trajectory displacement effect en het ruimteladings effect. Hoofdstuk 3 introduceert enkele begrippen die veel gebruikt worden om de macroscopische eigenschappen van de bundel te beschrijven. Liouville's theorema, uit de statistische mechanica, wordt gebruikt om het principe van emittantie en helderheids invariantie af te leiden. Het begrip bundel-temperatuur wordt ingevoerd, dat een centrale rol speelt in de thermodynamische beschrijving van de bundel. De thermodynamische beschrijving wordt gebruikt om bovengrenzen te bepalen voor de grootte van de statistische effecten, waarbij de relaxatie van kinetische en de relaxatie van potentiele energie wordt onderscheiden. Hoofdstuk 4 behandelt de Fokker-Planck benadering, die in de plasma fysica en de stellaire dynamica wordt gebruikt om het gedrag van systemen te beschrijuen waarvan de deeltjes wisselwerken door een Coulomb-type kracht, dat wil zeggen een kracht die evenredig is met het omgekeerde kwadraat van de onderlinge afstand tussen de deeltjes. De Fokker-Planck benadering is in het algemeen niet geschikt om de statistische wisselwerkingen in deeltjes-bundels te beschrijven, omdat deze, in vergelijking tot een plasma, een relatief lage dichtheid en temperatuur hebben, terwijl de vluchttijd in het algemeen kort is ten opzichte van de karakteristieke relaxatietijden. Vervolgens wordt de Holtsmark verdeling behandeld. Dit is de waarschijnlijkheids verdeling van de fluctuerende component van de electrostatische veldkracht die op een punt werkt in een gas dat samengesteld is uit random verdeelde identieke puntladingen. Het wordt aangetoond dat de gegenereerde axiale en transversale snelheids verdelingen in een cylindervormige bundel, met een gematigde deelt jesdichtheid, van dit type $2 i j n$.

De fundamentele aspecten van een analytisch model voor de beschrijving van de statistische Coulomb wisselwerkingen tussen de deeltjes van een bundel, worden belicht in hoofdstuk 5 . Het probleem wordt daarbij, in eerste instantie, beperkt tot het bestuderen van één bundelsegment in een veldvrije ruimte. De verschillende, in de literatuur voorgestelde, manieren om dit type $\mathrm{N}$-deeltjes probleem te reduceren worden bestudeerd. Gekozen wordt voor een model dat werd geintroduceerd door van Leeuwen en Jansen (1983) en hier wordt aangeduid als het "extended two-particle model". In dit model wordt de totale verplaatsing, in positie en snelheid, van een test-deelt je berekend als de som van alle verplaatsingen veroorzaakt door de twee-deeltjes wisselwerkingen met de afzonderlijke veld-deeltjes (merk op dat het begrip "verplaatsing" hier in algemene $z$ in wordt gebruikt en niet specifiek betrekking heeft op een verandering van de ruimtelijke coördinaten). Voorts worden de veld-deeltjes als statistisch onafhankelijk beschouwd. Met deze twee aannamen is het mogelijk om de volledige gegenereerde energie verdeling (Boersch effect) en trajectory displacement verdeling te bepalen. De berekening, waarvan in hoofdstuk 5 slechts de principes worden aangegeven, verloopt in drie stappen. In de eerste stap wordt de zogenaamde twee-deelt jes verdeling geëvalueerd. Dit is de verdeling van verplaatsingen voor het geval dat er slechts één veld-deeltje in de bundel aanwezig is. De tweede stap bestaat uit een Fourier-type integraaltransformatie naar het zogenaamde $k$-domein (of spatiële frequentie domein). De derde en laatste stap, is een Fouriertransformatie terug naar het verplaatsings-domein welke de gevraagde $\mathrm{N}$ deeltjes veplaatsings verdeling oplevert. Als een demonstratie van de werking van het model wordt de Holtsmark verdeling binnen het model afgeleid, evenals de verdeling van de electrostatische veldkracht die optreedt in een "pencil beam". Dit is een bundel waarvan de dwarsafmetingen klein $z i j n$ ten opzichte van de gemiddelde axiale afstand tussen de deeltjes. Tenslotte wordt aangegeven hoe de effecten die berekend $z i j n$ voor de afzonderlijke bundelsegmenten moeten worden 
opgeteld om het totale effect, voor de bundel in zijn geheel, te bepalen.

Hoofdstuk 6 behandelt het dynamische probleem van twee deelt jes die wisselwerken door middel van de Coulomb kracht. Het doel is om een expliciete uitdrukking te krijgen voor de verplaatsingen, in positie en snelheid, die de deeltjes als gevolg van de wisselwerking ondergaan, uitgedrukt in hun start posities en snelheden en de vluchttijd $T$. Een dergelijke uitdrukking kan alleen in goede benadering verkregen worden voor twee typen botsingen, te weten bijna volledige botsingen en zwakke botsingen. In een bijna volledige botsing benaderen de begin- en eindtoestand de asymptoten van de baan die de deeltjes zouden volgen indien de vluchttijd T oneindig lang zou zijn. Voor zulke botsingen kunnen de vergelijkingen voor de begin- en eindtoestand ontwikkeld worden in een machtreeks in $1 / \mathrm{T}$, hetgeen uiteindelijk tot de gevraagde expliciete uitdrukkingen leidt. In een zwakke botsing zijn de verplaatsingen klein en mag men de baan berekenen door middel van een eerste orde storings benadering. Voor de overige botsingen, dat zijn de botsingen die ên sterk én onvolledig zijn, is men aangewezen op een numerieke methode.

Met het materiaal uit de hoofdstukken 5 en 6 kunnen de verschillende statistische effecten berekend worden. Het Boersch effect wordt behandeld in hoofdstuk 7. De berekening van het trajectory displacement effect is verdeeld over de hoofdstukken 8 en 9 , waarin respectievelijk de transversale snelheids verplaatsingen (die zich vertalen in hoek-verplaatsingen) en de transversale positionele verplaatsingen worden besproken. In praktische systemen is men uitsluitend in de transversale positionele verplaatsingen in de spot geinteresseerd. De berekening van de transversale snelheids verplaatsingen is slechts bedoeld om het theoretisch inzicht te vergroten. Voor elk van de effecten is een computer programma ontwikkeld, dat de drie stappen van het "extended two-particle model" numeriek uitvoerd. De resultaten worden analytisch geverifieerd door middel van een asymptotische analyse van het probleem, uitgaande van de uitdrukkingen voor de twee-deeltjes effecten die bepaald $2 i j n$ in hoofdstuk 6. Teneinde expliciete vergelijkingen te verkrijgen, die nauwkeurig zijn over het volledige bereik van experimentele condities, worden de numerieke resultaten voor de verschillende effecten benaderd met een geschikte fit-functie. De vorm van de gebruikte fit-functies volgt uit de asymptotische analyse van het probleem.

Het meest opzienbarende resultaat van deze berekeningen is dat de vorm van de verplaatsings verdelingen sterk afhankelijk blijkt te zijn van de bundel geometrie en de deelt jesdichtheid. Gauss-verdelingen worden slechts aangetroffen bij hoge stroomdichtheden, waarbij de totale verplaatsing van een test-deelt je wordt veroorzaake door een groot aantal onafhankelijke botsingen. Holtsmark-verdelingen treden op in bundels waarin de verplaatsingen van de ongestoorde banen klein zijn en de begrenzing van de bundel geen rol speelt ("extended beams"). Voor bundels waarin de begrenzing wel een rol speelt ("pencil beams") treedt weer een ander type verdeling op, welke aangeduid wordt als de "pencil beam" verdeling. Een vierde regime treedt op wanneer zwakke volledige botsingen dominant zijn. Voor het Boersch effect leidt dit tot een Lorentz-verdeling. In de hoofdstukken 7,8 en 9 worden expliciete uitdrukkingen gegeven voor twee breedtematen van de gegenereerde verdelingen, namelijk de "Full width at Half Maximum" (FWHM) en de "Full Width median" (FW50). De afhankelijkheid die deze grootheden vertonen van de experimentele parameters (zoals de bundelstroom, de versnelspanning, de bundellengte, de openingshoek en de straal van het "crossover") is verschillend voor elk van de bestudeerde effecten, maar verschilt bovendien per regime. De "root mean square" (rms) breedte 
wordt gedomineerd door de staart van de verdeling en vertoont onder alle omstandigheden de zelfde parameterafhankelijkheid.

In hoofdstuk $10 \mathrm{zijn}$ een aantal aanvullende berekeningen aan de statistische effecten verzameld. In de hoofdstukken 7,8 en 9 is ter vereenvoudiging uitgegaan van een referentie baan die samenvalt met de as van de bundel en is bovendien aangenomen dat de axiale beginsnelheid van alle deeltjes gelijk is. De berekeningen van hoofdstuk 10 rechtvaardigen deze vereenvoudigingen. Voorts wordt er aangeven hoe het model uitgebreid kan worden tot bundel segmenten waarin de deeltjes versneld worden door een uniform axiaal electrostatisch veld. Tenslotte worden de relativistische correcties onderzocht, die nodig zijn voor bundels waarin de deelt jes met een snelheid bewegen die vergelijkbaar is met die van het licht.

Hoofdstuk 11 behandelt het ruimteladings effect in een bundel segment in een veldvrije ruimte. De klassieke aanpak, die gebruikt wordt om de spotverbreding in oscilloscoop buizen te berekenen, is niet geschikt voor de relatieve lage stroomdichtheden die men aantreft in lithografische systemen en microscopen. Eerste orde storings theorie biedt een geschikt alternatief, zolang de verplaatsingen van de ongestoorde banen klein zijn. Aangetoond wordt dat het ruimteladings effect correspondeert met de werking van een negatieve lens. Deze lens is ideaal indien de stroomdichtheid uniform is in elke doorsnede van de bundel en veroorzaakt dan uitsluitend een defocusering en een verandering van de vergroting van de spot. Indien de stroomdichtheidsverdeling niet uniform is introduceert het ruimteladings effect daarnaast aberraties, die een niet-refocuseerbare onscherpte veroorzaken. De eerste en derde orde optische eigenschappen van de ruimteladings lens worden, met behulp van de eerste orde storings benadering, uitgedrukt in de experimentele parameters.

In hoofdstuk 12 worden de resultaten van de voorgaande hoofdstukken vertaald in enkele experimentele maten voor de spotgrootte. In systemen waarin de spot een afbeelding is van de bron ("Gaussian beam systems") bepaalt men de grootte van de spot gewoonlijk door deze over een meskant te "scannen" en de doorgelaten stroom te meten als functie van de positie van de spot. De spotgrootte volgt dan als de afstand tussen twee karakteristieke intensiteits waarden, bijvoorbeeld de waarden die corresponderen met $10 \%$ en $90 \%$ van de totale bundelstroom. In systemen waarin de spot een afbeelding is van één of meerdere vierkante diafragma's ("shaped beam systems") is men geinteresseerd in de randbreedte van de spot, die gedefinieerd wordt als de afstand tussen twee karakteristieke intensiteits waarden, bijvoorbeeld de waarden die corresponderen met $10 \%$ en $90 \%$ van de stroomdichtheid in het centrum van de spot. Het wordt aangetoond dat de berekening van de randbreedte van een "shaped spot" equivalent is aan de berekening van de grootte van een "Gaussian spot", bepaald met de meskant-methode. Deze maat wordt aangeduid als de $d_{p, 1-p}$-breedte, waarbij $p$ refereert naar de keuze van het karakteristieke intensiteitsniveau $(0<p<1 / 2)$. In sommige toepassingen is men daarnaast geinteresseerd in de breedte van de spot FW die een zekere fractie $f(0<f<1)$ van de totale bundelstroom bevat. De grootheden $d_{p, 1-p}$ en $F_{f}$ worden berekend voor het geval dat de spot volledig gedomineerd is door slechts één aberratie. Achtereenvolgens worden daarvoor het trajectory displacement effect, de chromatische aberratie ten gevolge van het Boersch effect, sferische aberratie en de onscherpte veroorzaakt door (niet gecompenseerde) ruimteladings defocusering beschouwd.

In hoofdstuk 13 wordt de techniek van numerieke Monte carlo simulatie van een bundel geladen deeltjes besproken. In deze techniek worden de banen berekend van een ensemble geladen deeltjes, met random gekozen startcoordinaten. De verschillende routines van het daartoe 
ontwikkelde computer programma (MONTEC) worden in enig detail beschreven. De "source"-routine genereert de beginconditie van het ensemble. Voorts zijn er routines om dunne lenzen, dunne vierpolen, dunne afbuigers en diafragma's te simuleren en routines om de eindcoördinaten te vertalen in de relevante experimentele grootheden, zoals de energiespreiding, de spotgrootte en de randscherpte van een "shaped spot". De baan-berekenings (of "ray-tracing") routines vormen de kern van het programma. Daarin worden de banen van de deeltjes onder invloed van de onderlinge coulomb wisselwerking berekend, mogelijk in aanwezigheid van een uniform axiaal electrostatisch veld. Naast een puur numerieke baan-berekenings routine, die gebruik makt van een derde orde integratie schema met variabele stapgrootte, bevat het programma ook een (semi-) analytische baan-berekenings routine. Deze routine is gebaseerd op de zelfde reductie van het dynamische $\mathrm{N}$-deeltjes probleem als het analytische model voor de statistische wisselwerkingen en makt gebruik van de resultaten voor een twee-deeltjes botsing, welke $z i j n$ afgeleid in hoofdstuk 6. Deze aanpak blijkt zeer nauwkeurig voor alle praktische omstandigheden en versnelt het programma met één of twee ordes van grootte, dit afhankelijk van de bundelgeometrie en de deeltjesdichtheid. Voorts worden enkele methoden besproken om bepaalde simulatiefouten te onderdrukken en de techniek geschikt te maken voor de simulatie van bundels met een relatief hoge deeltjesdichtheid, zoals die in bronnen aangetroffen worden.

In hoofdstuk 14 worden de resultaten van de analytische modellen voor de statistische en de ruimteladings effecten vergeleken met de uitkomsten van Monte Carlo simulaties. Daarmee kunnen sommige benaderingen van de analytische theorie op hun nauwkeurigheid onderzocht worden, met name de reductie tot twee-deeltjes botsingen. Ook levert dit een onafhankelijke, globale test op van de eindresultaten van de analytische theorie. De overeenkomst tussen de analytische theorie en de Monte Carlo simulaties is in het algemeen uitstekend, maar bij middelmatige stromen voorspelt de theorie iets grotere waarden voor de mediane breedte (FW50) van de gegenereerde energie verdeling en trajectory displacement verdeling dan het Monte Carlo programma. Deze verschillen worden waarschijnlijk veroorzaakt door het gebruik van fitfuncties in het analytische model, alsmede doordat de resultaten van dit model betrekking hebben op de verplaatsingen van de deeltjes die langs de as van de bundel lopen, terwijl het Monte Carlo programma de verplaatsingen van alle deeltjes beschouwt.

Hoofdstuk 15 confronteert de resultaten van het "extended twoparticle model" met de resultaten van enkele andere, uit de literatuur bekende modellen voor de berekening van de statistische wisselwerkings effecten. De meeste van deze modellen beperken zich tot de berekening van één karakteristieke breedte van de verdelingen, gewoonlijk de rmsbreedte. De rms-breedte geeft uitsluitend praktische voorspellingen voor het geval dat de verdeling Gaussisch is. Voor de andere, door het "extended two-particle model" voorspelde verdelingen is de rms waarde volledige bepaald door de staart van de verdeling en geeft geen informatie over het centrale deel. Dit soort modellen zijn zodoende inherent onvolledig. Een uitzondering vormt het werk van Loeffler (1969) en Loeffler en Hudgin. $(1969,1970)$ warin een mediane waarde van de verdelingen wordt afgeleid. De vergelijking met de uitkomsten van het "extended two-particle model" geeft aan dat hun resultaten niet algemeen geldig zijn, zoals gesuggereerd wordt, maar niettemin onder bepaalde omstandigheden tot redelijk nauwkeurige voorspellingen leiden. De rms energie spreiding gegeven door Rose en Spehr (1980) en de rms van de hoek-verplaatsingen gegeven door Weidenhausen, Rose en Spehr (1985), voor een bundel met een nauw crossover, komen goed overeen met de voorspellingen van het "extended two-particle model". In het algemeen 
verschillen de uitkomsten van de onderzochte theorieen significant, terwijl enige kwalitatieve overeenstemming wordt gevonden in de Gaussische regimes.

De resultaten van dit onderzoek welke van direct belang zijn voor de ontwerpers van probe-vormende systemen, worden samengevat in hoofdstuk 16. De fysische aspecten van het probleem worden kort weergegeven en de afhankelijkheid van de experimentele parameters wordt voor elk van de regimes verklaard vanuit elementaire fysische overwegingen. Tenslotte wordt een overzicht gegeven van de vergelijkingen waarmee het Boersch effect, het trajectory displacement effect en het ruimteladings effect direct berekend kunnen worden voor een gegeven experimentele situatie. Deze vergelijkingen zijn geimplementeerd in het computer programma INTERAC, waarmee de invloed van de coulomb interacties in een bepaald systeemontwerp interactief geèvalueerd kan worden. Deze evaluaties vergen een praktisch verwaarloosbare rekentijd, hetgeen dit programma zeer geschikt maakt voor optimalisatie doeleinden. Ter verificatie, kan het ontwerpproces besloten worden met enkele Monte Carlo simulaties, uitgevoerd door het programma MONTEC. De uitkomsten van dit programma kunnen grafisch worden weergegeven met het daartoe ontwikkelde plot-programma MCPLOT. Alle programma's $2 i j n$ geschreven in FORTRAN 77 en draaien, onder meer, op een IBM PC/AT. 
De schrijver van dit proefschrift werd op 21 april 1958 geboren te Hilversum. In de periode 1970 - 1976 volgde hij de opleiding Atheneum $\beta$ aan de Scholengemeenschap "De Amersfoortse Berg", te Amersfoort, waar hij op veertienjarige leeftijd voor het eerst met het concept electron geconfronteerd werd. Kort daarop volgde de wet van Coulomb en Newton's mechanica wetten, welke de basis van dit proefschrift vormen. Het was ook in die periode dat hij zich, voor het eerst, de vraag stelde wat voor een kleur het electron heeft, waarop hij evenwel, tot op heden, nog geen bevredigend antwoord heeft gevonden. Niettemin sloot hij zijn eindexamen in 1976 met positief gevolg af en schreef zich in als student bij de afdeling der Technische Natuurkunde van de TH Delft. De studie verliep, ondanks het feit dat hij hoofd-en nevenactiviteiten nogal eens verwarde, tamelijk voorspoedig, mede dankzij een scherpe selectie van de te volgen colleges. Dit ontlokte nu en dan wat hoofdschuddend gemompel bij sommige hoogleraren en het werd hem voorgehouden dat de voorbereiding van een tentamen onder het motto "even een vakje doen" ten ene male niet tot een diepgeworteld inzicht in de fysica kan leiden. In 1983 studeerde hij (ondanks alles Cum Laude) af bij de vakgroep Deeltjes Optica onder begeleiding van zijn huidige promotoren, alsmede professor Le Poole. Aansluitend werkte hij als "post-graduate" research medewerker bij de General Technology Division van IBM East Fishkill te New York. Bij de afdeling electronen lithografie werd hij geconfronteerd met de harde industriële praktijk van de ontwikkeling van "high throughput variable shaped e-beam machines". Zijn manager vond een paar theoretische vergelijkingen wel aardig, maar zag een geschikt fysisch model toch meer als een schroevendraaier ("screw driver"), hetgeen, naar de schrijver van dit proefschrift pas later begreep, overdrachtelijk bedoeld was. In deze omgeving werd een belangrijk deel van het hier neergelegde onderzoek uitgevoerd. In 1985 meende de schrijver de rust van het landelijke East Fishkill en de aangrenzende gehuchten Beacon en Hopewell Junction voldoende op zijn waarde te kunnen beoordelen en keerde terug naar Nederland, waar hij zijn werk voortzette als Toegevoegd onderzoeker aan de TH Delft, gefinancierd door de Stichting Technische Wetenschappen (S.T.W.). Dit proefschrift werd samengesteld in de periode van mei 1987 tot en met september 1988, in de hoop dat de lezer van deze tekst het niet laat zitten bij het bestuderen van de omslag, de samenvatting en het curriculum vitae. 


\section{STRLIRGES}

behorende bij het proefschrift

"Coulomb Interactions in Particle Beass" 
16. De ontwikkeling die de reclameslogan voor de anti-iaagpi jntabletten van het merk "Rennie" vertoont geeft een goede indicatie van het tempo waarin de Nederlandse samenleving de Noord-Amerikaanse samenleving volgt in de ujtbanning van het "gezond verstand" in algemeen anvaarde opvattingen. Op dit moment luidt de slogan. in Nederland "als Uw maag Uw levenstempo niet kan bi jhouden", terwijl men aan de overzijde van de Atlantische Oceaan nog een stap verder is pet "for the stress that comes with success".

17. Indien de toename:- gedurende de afgelopen tien jaar, van het aantal verkeerslichten op het traject Westvest, Phoenixstraat en Wateringsevest in Delft zich ongewijzigd doorzet dan $2 a 1$ de straatverlichting daar binnenkort overbodig worden en het begrip "red light district" een nieuwe betekenis krijgen. 


\section{THESISSES}

connected with the diss"...: ,

"Coulamb Interactions in :

G.H. Jansen

De:- : : $3: \cdots 1959$. 
1. Optimization of the design of the state of the art : high-throughput electron beam lithography pattern generators by means of the theory presented in this dissertation may lead to a throughput improvement with (at least) a factor of 2 , which corresponds to a cost reduction of the order of $10^{\prime}$ uss per two machines, assuming a full use of their capacity.

2. For the particle beams used in electron-microscopes and lithography pattern generators, the perturbation experienced by a particle in a certain beam segment due to the coulomb interaction with the surrounding particles can, in good approximation, be computed as a direct sum of the perturbations caused by pair-interactions.

3. In order to obtain a model for statistical Coulomb interactions in particle beams which yjelds accurate results over a wide range of operating conditions it is essential to treat the two-particle problem in an exact manner, without making any. assumptions on the completeness or the strength of the two-particle collision.

4. The Fokker-Planck approximation, of ten exploited in plasma physics and stellar dynamics to describe the impact of particle collisions on the macroscopic properties of the studied system, is, in general, not suited to describe the impact of statistical interactions in the particle beams mentioned in thesis no. 2.

5. The energy, angular displacement and trajectory displacement distributions, generated by statistical coulomb interactions, are only for high particle densities of the Gaussian type, while, depending on the beas geometry, various other types of distributions occur for lower particle densities, which are all characterized by a relatively narrow core and long tajls.

6. The root mean square (rms) of the displacement distributions mentioned in thesis no. 5 is in the non-Gaussian regimes dominated by the tails of the distribution and provides no information of the centre part. Therefore, models which consider rms-values only are essentially incomplete.

7. The integral representation of the displacement distribution $p(\Delta \eta)$ which is introduced in section 5.9 of this dissertation should, in the terminology employed by Mandelbrot, be denoted as the Levi stable distribution with exponent $\gamma$. However, the phenomenon of statistical coulomb interactions of the particles in a beam segment, which leads to this type of distribution, can (for $r<2$ ) not be represented by a generalized (fractional) Brown function.

Mandelbrot. B.B. (1983). "The Fractal Geometry of Nature", p.351-372, H.H.Freeman New York 
8. The belief of some designers of particle optical pattern generators for lithography applications that the trajectory displacement effect can be minimized by reducing the column length as much as possible is, in its generality, not correct.

9. Since the energy spread generation caused by Coulomb interactions is, after its discoverer, called the Boersch effect, it should be recommended to denote the "trajectory displacement effect" as the Loeffler effect.

Loeffler, K.H. (1964). Ph.D. Thesis, University of Berlin

10. The poor controllability of the bit trajectory in the deviated drilling for oil and gas is primarily a consequence of the lack of feedback from the "trajectory displacement" to the driller and not so much his lack of inside in the mechanics of the drilling process.

11. The quality of the physics education (on the technical universities) would improve by paying more attention to the problems in physics which have not been solved (yet).

12. The difference in attractiveness of universities en industry respectively for talented scientist is stronger determined by social-cultural factors than by the height of the offered salary.

13. It is a misconception of the Minister of Education and Science that the abolition of the prolongation of the study enrolment duration for voluntary administrators of student corporations will lead to an increased effectivity of the budget spent for higher education.

Document no. 20469 of the "Tweede Kaner der Staten Generaal"; Meetings of the year 1987-1988 ("Harmonisatje wet").

14. Environmental protection is not merely a duty of the-government, but should also by the industry be considered as a primary objective.

15. The fact that Sir Isaac Newton published his "Philosphiae Naturalis Principia Mathematica" not until he reached the age of 44 should for many scientist be regarded as encouraging. 
16. The development of the advertisement slogan for the anti-stomachache tablets of the brand "Rennie" gives a good indication of the rapidity at which the Dutch society follows the Northern-American society in the loss of "common sense" in generally accepted viewpolnts. At present, the slogan used in the Netherlands is (translated to English) "when your stomach cannot keep up with the pace of your daily life". while across the Atlantic Ocean one is still one step ahead with "for the stress that comes with success".

17. In case the increase, during the past ten years, of the number of traffic lights on the traject Westvest, Phoenixstrat and Wateringsevest in Delft carries on at the same rate, the streetlighting will soon become superfluous, while the notion of "red light district" will acquire a new meaning. 